\title{
Transportation of Hazardous Materials Emergency Preparedness Hazards Assessment
}

by

A. Blanchard

Westinghouse Savannah 'River Company

Savannah River Site

Aiken, South Carolina 29808

T. I. Brown Jr.
RECEIVED

MAR 132000

OSTI

This paper was prepared in connection with work done under the above contract number with the U.S. Department of Energy. By acceptance of this paper, the publisher and/or recipient acknowledges the U. S. Government's right to retain a nonexclusive, royalty-free license in and to any copyright covering this paper, along with the right to reproduce and to authorize others to reproduce all or part of the copyrighted paper. 


\section{DISCLAIMER}

This report was prepared as an account of work sponsored by an agency of the United States Government. Neither the United States Government nor any agency thereof, nor any of their employees, makes any warranty, express or implied, or assumes any legal liability or responsibility for the accuracy, completeness, or usefulness of any information, apparatus, product or process disclosed, or represents that its use would not infringe privately owned rights. Reference herein to any specific commercial product, process or service by trade name, trademark, manufacturer, or otherwise does not necessarily constitute or imply its endorsement, recommendation, or favoring by the United States Government or any agency thereof. The views and opinions of authors expressed herein do not necessarily state or reflect those of the United States Government or any agency thereof.

This report has been reproduced directly from the best available copy.

Available for sale to the public, in paper, from: U.S. Department of Commerce, National Technical Information Service, 5285 Port Royal Road, Springfield, VA 22161, phone: (800) 553-6847, fax: (703) 605-6900

email: orders@ntis.fedworld.gov

online ordering: http://www.ntis.gov/ordering.htm

Available electronically at http://www.doe.gov/bridge

Available for a processing fee to U.S. Department of Energy and its contractors, in paper, from:

U.S. Department of Energy, Office of Scientific and Technical Information, P.O. Box 62,

Oak Ridge, TN 37831-0062,

phone: (865)576-8401,

fax: (865)576-5728

email : reportseadonis.osti.gov 


\section{DISCLAIMER}

Portions of this document may be illegible in electronic image products. Images are produced from the best available original document. 
S-EHA-G-00003

Revision 1

\section{TRANSPORTATION OF HAZARDOUS MATERIALS (U) EMERGENCY PREPAREDNESS HAZARDS ASSESSMENT SAVANNAH RIVER SITE}

N. E. Baucom

B. K. Tharakan

December 31, 1998
Westinghouse Safety Management Solutions, Inc.

Westinghouse Safety Management Solutions, Inc.

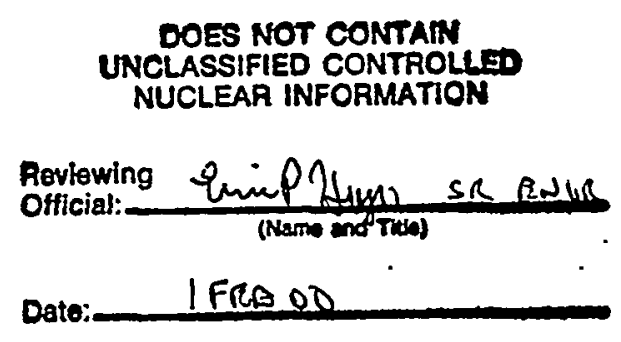

Westinghouse Savannah River Company Safety Engineering Department Aiken, SC 29808

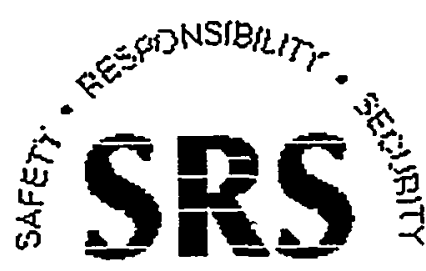


S-EHA-G-00003, Rev.1

Key Words: Transportation Emergency Management Emergency Preparedness Hazardous Materials

APPROVALS:

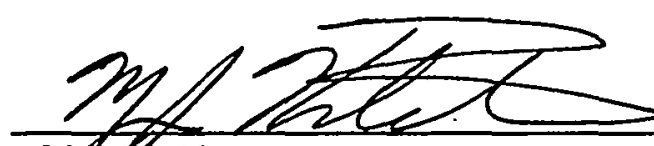

M.J. Jitchler

Safety Engineering Department-SAES Manager

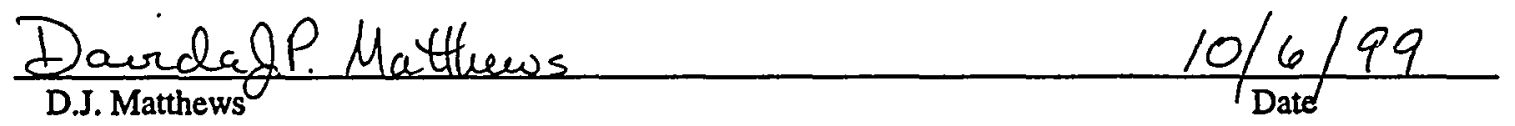

Site Emergency Programs Manager 
1.0 INTRODUCTION

1.1 PURPOSE

1.2 SCOPE

1.3 BACKGROUND

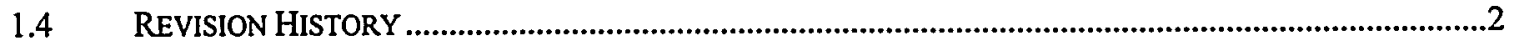

\subsection{SUMMARY 3}

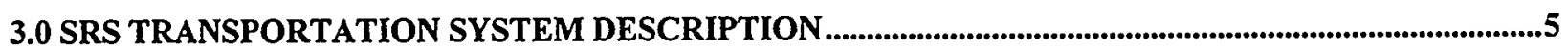

3.1 SEGMENT 1, SMALL VEHICLES AND CONTAINERS .............................................................................5

3.2 SEGMENT 2, LARGE VEHICLES AND CONTAINERS ...............................................................................5

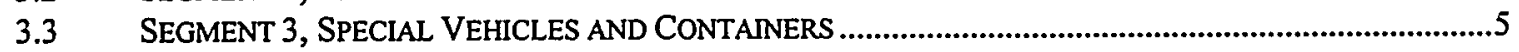

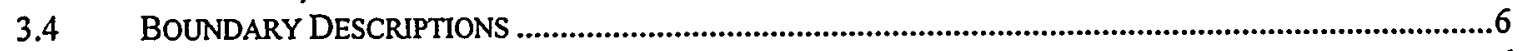

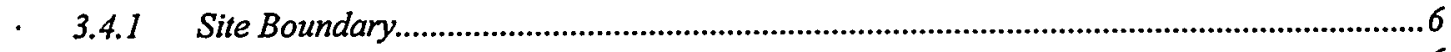

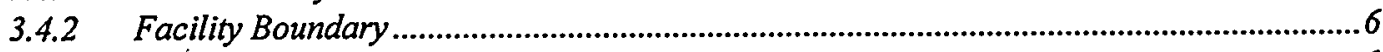

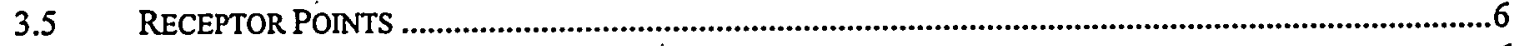

3.5.1 Notification of Unusual Event ..........................................................................................6

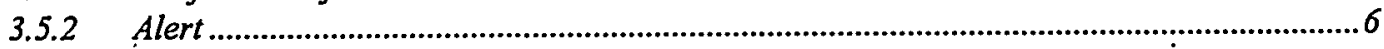

3.5.3 Site Area Emergency......................................................................................................

3.5.4 General Emergency .................................................................................................

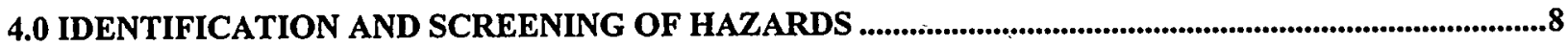

4.1 HAZARDOUS CHEMICAL IDENTIFICATION \& SCREENING..................................................................

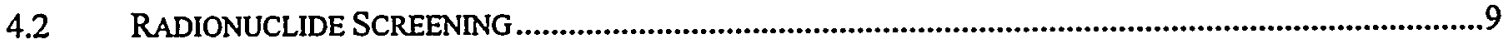

4.2.1 Sum of Ratios ...................................................................................................................... 10

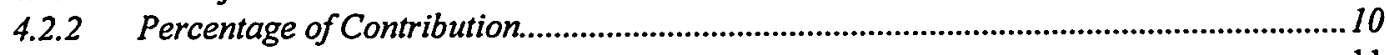

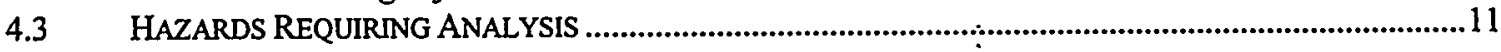

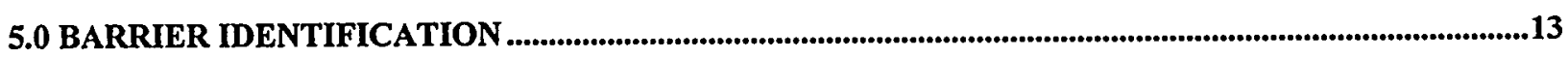

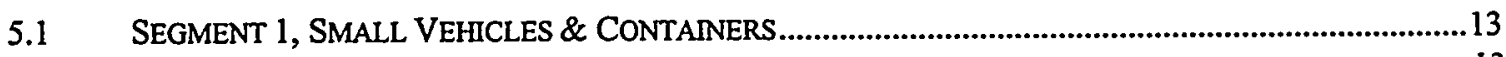

5.1.1 Chemical Sources .......................................................................................................13

5.1.2 Radiological Sources.................................................................................................13

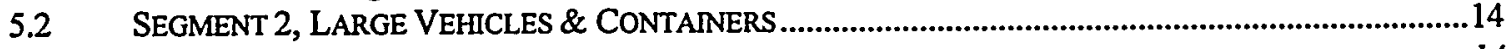

5.2.1 Chemical Sources ...............................................................................................................14

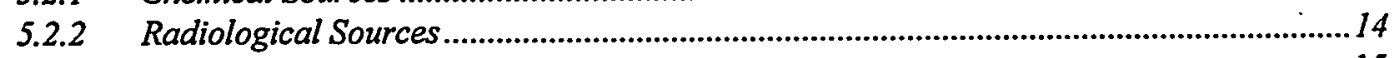

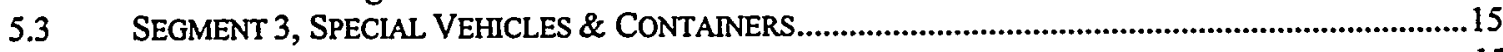

5.3.1 Chemical Sources ....................................................................................................... 15

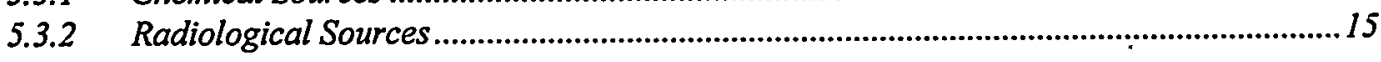

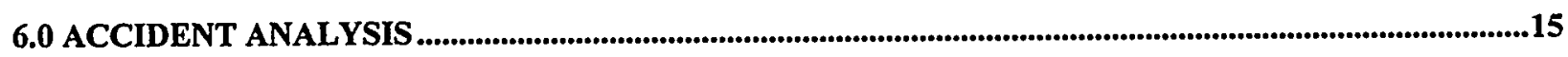

6.1 SEGMENT 1, SMALL VEHICLES AND CONTAINERS ...........................................................................15

6.1.1 Chemical Releases.....................................................................................................15

6.1.2 Radiological Releases...............................................................................................16

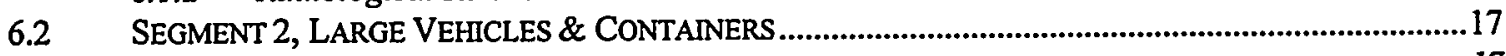

6.2.1 Chemical Releases .......................................................................................................17

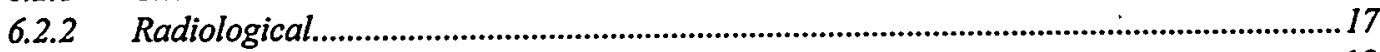

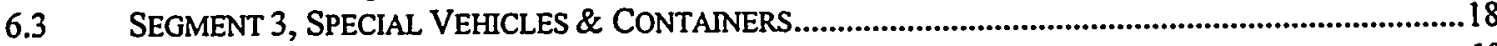

6.3.1 Chemical Releases....................................................................................................... 18 


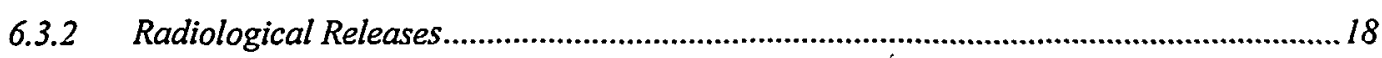

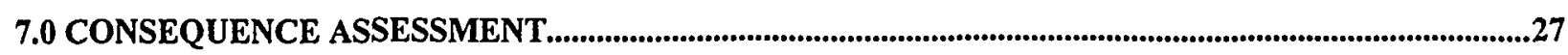

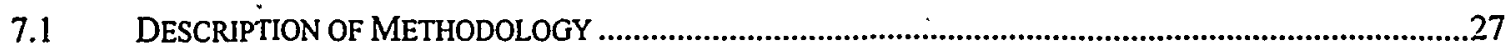

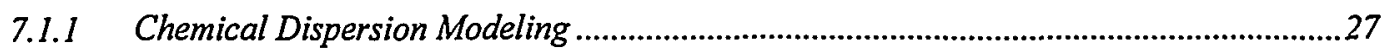

7.1.2 Radionuclide Dispersion Modeling .................................................................................2.

7.1.3 Dispersion Modeling Parameters .................................................................................27

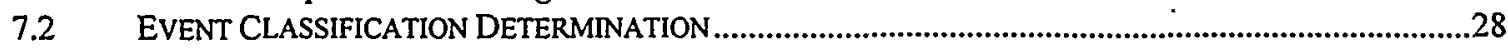

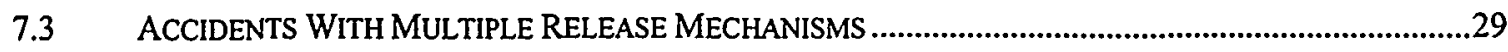

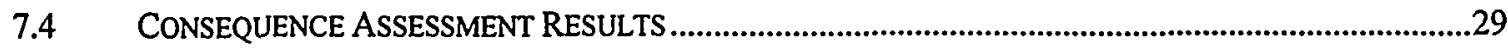

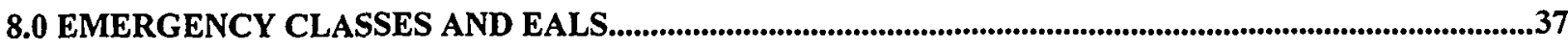

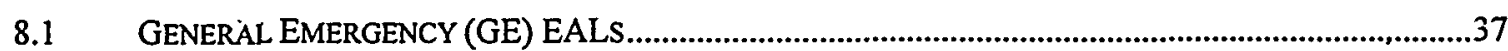

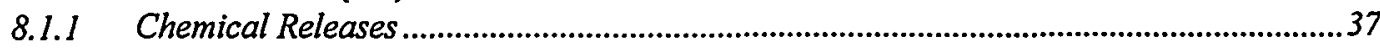

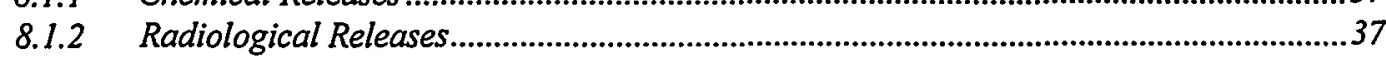

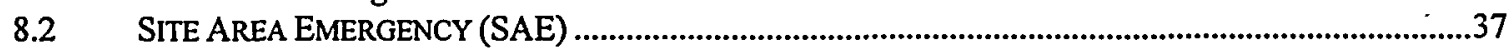

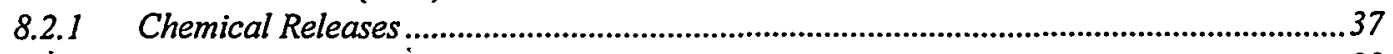

8.2.2 Radiological Releases..................................................................................................... 39

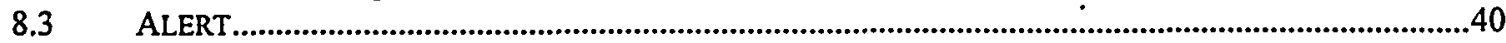

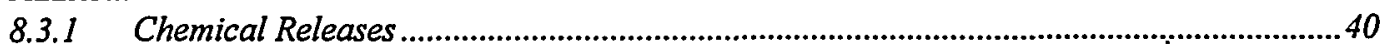

8.3.2 Radiological Releases..................................................................................................4

9.0 EMERGENCY PLANNING ZONE (EPZ) DETERMINATION..................................................................44

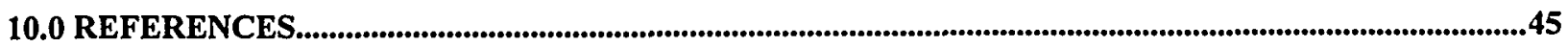


Appendix A: $\quad$ Figures

Appendix B: Calculations

Appendix C: Correspondence

Appendix D: Emergency Preparedness Hazards Assessment Definitions

\section{LIST OF TABLES}

TABLE 4.1 TRANSPORTERS

TABLE 4.2 HAZARDS REQUIRING FURTHER ANALYSIS 11

TABLE 5.1 BARRIER IDENTIFICATION - SEGMENT 1

TABLE 5.2 BARRIER IDENTIFICATION - SEGMENT 2 14

TABLE 5.3 BARRIER IDENTIFICATION - SEGMENT 3

TABLE 6.1 SEGMENT 1 CHEMICALS, ASSOCIATED ERPG, AND STATE 19

TABLE 6.2 EQUIVALENT SOURCE TERMS FOR SEGMENT I RADIONUCLIDES, AND ASSOCIATED RELEASE DESIGNATIONS

TABLE 7.1 EPHA 95\% DISPERSION METEOROLOGY

TABLE 7.2 EPHA 50\% DISPERSION METEOROLOGY

TABLE 7.3 95\% ADVERSE MET CONSEQUENCE ASSESSMENT RESULTS - CHEMICAL 30

TABLE 7.4 95\% ADVERSE MET CONSEQUENCE ASSESSMENT RESULTS - RADIOLOGICAL 32

TABLE 7.5 50\% AVERAGE MET CONSEQUENCE ASSESSMENT RESULTS - CHEMICAL

TABLE 7.6 50\% AVERAGE MET CONSEQUENCE ASSESSMENT RESULTS - RADIOLOGICAL

TABLE 8.1 CHEMICAL SPILL TARGET SURFACE AREAS FOR SITE AREA EMERGENCIES 37

TABLE 8.2 CHEMICAL SPILL TARGET SURFACE AREAS FOR ALERT EMERGENCIES 


\section{LIST OF ACRONYMS}

ALOHA Areal Locations of Hazardous Atmospheres

ALS Analytical Laboratories Section

ARCHIE Automated Resource for Chemical Hazard Incident Evaluation

BIO Basis for Interim Operation

CEDE Committed Effective Dose Equivalent

CFR Code of Federal Regulations

CIIS Chemical Inventory Information System

CLAB Central Laboratory

DOE Department of Energy

DOT Department of Transportation

EAL Emergency Action Level

EMG Emergency Management Guide

EMPP Emergency Management Program Procedure

EPHA Emergency Preparedness Hazards Assessment

EPIP Emergency Plan Implementing Procedure

EPZ Emergency Planning Zone

ERPG Emergency Response Planning Guide

GE General Emergency

HAD Hazards Assessment Document

HEPA High Efficiency Particulate Air

IDLH Immediately Dangerous to Life and Health

ILNTV Intermediate Level Non Tritium Vault

ILTV Intermediate Level Tritium Vault

LLW Low Level Waste

LLWSB Long-Lived Waste Storage Building

LNTV Low Non Tritium Vault 


\section{LIST OF ACRONYMS}

NoUE Notification of Unusual Event

NMSP Nuclear Materials Stabilization Program

OTR Over The Road

PAC Protective Action Criteria

RCRA Resource Conservation and Recovery Act

$\mathrm{RD} \quad$ Release Designation

SAE Site Area Emergency

SAR Safety Analysis Report

SRS Savannah River Site

TEDE Total Effective Dose Equivalent

THM Transportation of Hazardous Materials

TPCIC Technical Programs Common Industrial Chemical

TPQ Threshold Planning Quantity

TPSQ Technical Programs Screening Quantity

TPQ Threshold Planning Quantity

TRU Transuranic

UNH Uranyl Nitrate Hexahydrate

WAC Waste Acceptance Criteria

WINDs Weather Information and Display System

WSI Wackenhut Services Incorporated 


\section{ABBREVIATIONS}

\begin{tabular}{ll}
$\mathrm{C}$ & Celsius (temperature) \\
$\mathrm{Ci}$ & Curies \\
$\mathrm{cpm}$ & counts per minute \\
$\mathrm{d} / \mathrm{m} / \mathrm{ml}$ & disintegrations per minute per milliliter \\
$\mathrm{F}$ & Fahrenheit (temperature) \\
$\mathrm{ft}$ & feet \\
$\mathrm{ft} / \mathrm{sec}$ & feet per second \\
$\mathrm{gal}$ & gallons \\
$\mathrm{hr}$ & hour \\
$\mathrm{in} \mathrm{H}_{2} \mathrm{O}$ & inches of water column (pressure) \\
$\mathrm{lbm}$ & pounds-mass \\
$\mathrm{min}$ & minutes \\
$\mathrm{mm} \mathrm{Hg}$ & millimeters of mercury (pressure) \\
$\mathrm{m}$ & meter, or milli (10-3) \\
$\mathrm{n}$ & nano (10-9) \\
$\mathrm{ppm}$ & parts per million \\
$\mathrm{Vol} \%$ & volume percent (concentration) \\
wt\% & weight percent (concentration) \\
$\mu$ & micro (10 \\
\hline-6
\end{tabular}




\subsection{INTRODUCTION}

\subsection{Purpose}

This report documents the Emergency Preparedness Hazards Assessment (EPHA) for the Transportation of Hazardous Materials (THM) at the Department of Energy (DOE) Savannah River Site (SRS). This hazards assessment is intended to identify and analyze those transportation hazards significant enough to warrant consideration in the SRS Emergency Management Program. This guidance for the conduct of an EPHA was based primarily on the Emergency Management Program Procedure (EMPP) 6Q-001 (Ref. 1).

\subsection{Scope}

The EPHA for the THM scope includes the transportation of hazardous materials (radiological and chemical) to include the following (Ref. 2):

- Offsite shipments of DOE owned hazardous materials.

- Receipt of DOE owned hazardous materials.

- Receipt of non-DOE owned (e.g., DOD) hazardous materials.

- Non-DOE owned hazardous materials routinely shipped via truck on South Carolina State Route 125/278 and SRS Route $1 \& 1 \mathrm{~A}$.

- Non-DOE owned hazardous materials routinely shipped via rail through SRS.

Because Safe Secure Transport (SST) transportation incidents are under the jurisdiction of DOE Albuquerque, NM and spent fuel transportation is addressed in the Reactors EPHA, the scope of the THM EPHA specifically excludes any Safe Secure Transport (SST) and the transportation of spent fuel, both offsite and Inter/Intra-Area (Ref. 2). The only exception to this is a scenario involving malevolent acts to an offsite spent fuel shipment, in a Type B Container, to the Receiving Basin for Offsite Fuel (RBOF) or L-Reactor disassembly basin.

\subsection{Background}

This EPHA is intended to provide an emergency planning basis that is in compliance with the requirements of DOE Emergency Management Guide (EMG) (Ref. 3). This document does not address the EMG beyond what is necessary to develop Emergency Action Levels (EALs). Those issues, such as evacuation route planning and protective actions, will be addressed by an SRS site level consolidation document.

The EPHA methodology specified in EMPP 6Q-001 (Ref. 1) differs from existing safety documentation [e. g., Hazard Assessment Document (HAD), Safety Analysis Report (SAR) - Addendum]. The specified consequence analysis methodology utilizes the concept of a barrier approach to hazardous material releases as opposed to a risk/probability assessment of potential accidents or analyses as totally unmitigated releases. The EPHA involves the compilation and screening of radiological and chemical inventories with respect to their airborne hazard potentials. For materials failing this screening process, a detailed hazard characterization and barrier identification process is applied. Then, event scenarios are defined for potential releases of the materials (ranging from minor to beyond design basis), including information on release mechanism, duration of release, initiator identification, and source term calculations. A consequence assessment is performed from the scenarios to determine downwind dose/concentrations at the defined receptor locations. 
Because this EPHA deals with transportation incidents, the consequence assessment will be utilized to also determine the distance to defined Protective Action Criteria (PAC). This distance will be utilized for determining if events are defined as Site Area Emergencies (SAE) opposed to General Emergencies (GE) based on the location of a particular accident. This differs slightly from the methodology utilized for stationary facilities as their distances are fixed. Finally, the EALs and Emergency Planning Zones (EPZs) are determined from these results.

Events capable of exceeding defined PAC at the receptor locations are assigned an emergency classification. Based on the events, corresponding classification, and indications of each event, a Emergency Plan Implementing Procedure (EPIP) will be written for use by personnel to accurately (and quickly) classify events and to recommend protective actions.

Because the distance to PAC is not unique no true site boundary receptor point exists for potential General Emergency (GE) classification. For the THM EPHA a distance of 6,000 meters was selected as an analysis point to make preliminary GE determinations. This corresponds to the downwind distance for one hour during adverse meteorological $(1.7 \mathrm{~m} / \mathrm{s}$ wind speed) conditions.

The EPHA is required to address malevolent acts within the accident analysis. The DOE Emergency Management Guide (EMG) assumes two types of malevolent acts, moderate and extreme (Ref. 3). Moderate events are those that could be initiated by a single individual using materials or tools readily available in the facility, or small quantities of flammables. Extreme events, such as those used in Vulnerability Assessments and Radiological/Toxicological Sabotage assessments, should provide an upper bound on the severity of potential consequences. In most cases, malevolent acts would have consequences similar to those caused by other initiators. For example, catastrophic failure of a chemical storage tank may be postulated due to a seismic event. If the same level of damage may be caused by an act of sabotage, such as running a truck into the tank, the malevolent act would simply by considered another initiator for the failure of the tank.

\subsection{Revision Summary}

This revision to the EPHA concentrated primarily on updating the consequence assessment of chemical releases and incorporating the changes resulting from revisions made to the ERPG values since the completion of Revision 0 of this document. Also the layout and format of the document has been updated to conform to current EPHA formatting. 


\subsection{SUMMARY}

An EPHA was conducted for the transportation activities involving hazardous materials in accordance with EMPP 6Q-001 (Ref. 1). The analysis included the following:

- A compilation, screening, and hazard characterization of both chemical and radiological materials

- Barrier identification

- Accident scenario development for potential releases of identified hazardous materials

- Consequence assessment of the identified scenarios

- Emergency classification development

Based on this EPHA, THM requires an emergency plan and implementation procedure.

The materials present within the scope of this EPHA requiring full consequence assessment are:

- Chemicals:

$\begin{array}{llll}\text { Acetaldehyde } & \text { Diphenylmethane-4,4- } & \text { Methylene Chloride } & \text { Tetrahydrofuran } \\ \text { Acetonitrile } & \text { diisocyanate } & \text { Methyol Cellosolve } & \text { Titanium Dioxide } \\ \text { Acetic Acid } & \text { Ethyl Acetate } & \text { Monoethanolamine } & \text { Toluene } \\ \text { Alpha-Pinene } & \text { Ethyl Benzene } & \text { Morpholine } & \text { Toluidine-0 } \\ \text { Ammonia } & \text { Ethylene Oxide } & \text { N Butyl Alcohol } & \text { Trichloroethane-1,1,1 } \\ \text { Ammonium Hydroxide } & \text { Fluorosilicic Acid } & \text { N Methyl Pyrrolidone } & \text { Trichloroethylene } \\ \text { Benzene } & \text { Fluorosilicic Acid 25\% } & \text { Nitric Acid } & \text { Triethylamine } \\ \text { Benzoic Acid } & \text { Formaldehyde } & \text { Onyxide } & \text { Trimethylamine } \\ \text { Benzyl Alcohol } & \text { Formic Acid } & \text { Perchloroethylene } & \text { Vinyl Acetate } \\ \text { Benzyl Chloride } & \text { Glycol Ether DE } & \text { Petroleum Sulfonate } & \text { Xylenes } \\ \text { Butyraldehyde } & \text { Glycol Ether EB } & \text { Phenol, Molten } & \\ \text { Carbon disulfide } & \text { Hydrochloric Acid 31\% } & \text { Propagyl Alcohol } & \\ \text { Carbon Monoxide } & \text { Hydrofluoric Acid 70\% } & \text { Propionic Acid } & \\ \text { Chloracetone } & \text { Hydrogen Chloride } & \text { Rodine 50 (formaldehyde) } & \\ \text { Chlorine } & \text { Hydrogen Peroxide } & \text { Silica (as respirable dust) } & \\ \text { Chlorosulfonic Acid } & \text { Isopropyl Alcohol } & \text { Sodium Hypochlorite 13- } & \\ \text { Cyclohexane } & \text { Magnesium Oxide } & \text { Sodium Sulfhydrate } & \\ \text { Cyclohexanone } & \text { Methyl Alcohol } & \text { Sulfur Dioxide } & \\ \text { Diethylamine } & \text { Methyl Bromide } & \text { Sulfur, Molten } & \\ \text { Diethylene Glycol } & \text { Methyl Ethyl Ketone } & \text { Sulfuric Acid 66\% } & \\ \text { Dioxane } & \text { Methyl Isobutyl Ketone } & \text { Sulfuric Acid, Fuming } & \end{array}$


- Radionuclides:

$\begin{array}{llll}\text { H-3 } & \text { I-129 } & \text { Pu-239 } & \text { Cm-244 } \\ \text { C-14 } & \text { Cs-137 } & \text { Pu-240 } & \text { Cf-252 } \\ \text { Cr-51 } & \text { Ce-144 } & \text { Am-241 } & \\ \text { Co-60 } & \text { U-235 } & \text { Pu-241 } & \\ \text { Sr-90 } & \text { Np-237 } & \text { Pu-242 } & \\ \text { Ru-106 } & \text { Pu-238 } & \text { Am-243 }\end{array}$

For the materials identified above, accident scenarios were identified that may exceed the specified PAC at the downwind receptor points of interest. For each scenario identified, a consequence assessment and corresponding . EAL determination was made. The results show the potential for the following:

- General Emergencies (GE):

- $\quad$ Site Area Emergencies (SAE):

- Alert:

- Notification of Unusual Events (NoUE):

- The EPZ corresponds to the General Site Boundary.
Radiological
Chemical

0 


\subsection{SRS TRANSPORTATION SYSTEM DESCRIPTION}

The SRS is managed by Westinghouse Savannah River Company (WSRC) for the DOE. Facilities have been operated primarily to produce radioactive material products for United States defense activities, energy activities, and other federal government programs. The site is located in South Carolina, bordered by the Savannah River to the south-southwest. It is approximately twelve miles south of Aiken, SC; 15 miles east-southeast of Augusta, GA; and eight miles west of Barnwell, SC. South Carolina State Highway 278 and SRS Road 1 parallel the site along the site boundary and pass through small sections along the site perimeter. Access to the site is strictly controlled by Wackenhut Services Incorporated; however, SC State Route 125 passes through the site property, running north to south along the Savannah River starting in Jackson, SC and SRS Road 1A allows public traffic during regular business hours to portions of $3 / 700$ area. Additionally, CSX Transportation has a right of way for their tracks passing through the site adjacent to SC State Route 125, Figure 1 - Appendix A. Materials, Freight On Board (FOB) delivery point, are delivered to the site by common carrier and materials belonging to others may be transported on and through the site as portions of other loads. The roads and railways onsite were developed to provide safe and efficient transportation of personnel, supplies, products, and wastes in support of facility operations. Vehicles transporting hazardous materials include those operated by DOE, WSRC, DOE contractors on site, as well as vehicles operated by vendors and commercial carriers transporting materials to, from, and through the site.

For the THM EPHA special rules are applied for determining boundaries (receptor points) for postulated releases. Additionally, segmentation is generally utilized to isolate incidents that do not propagate into other areas. In this case, segmentation will be utilized as a work control tool for scenario development and to properly estimate inventories released when barriers fail. Segments and boundaries are described in the following sections.

\subsection{Segment 1, Small Vehicles and Containers}

For purposes of this EPHA, Small Vehicles and Containers are defined as any transporting device $\leq 6,000$ gallons and/or 800 cubic feet.

These limits are chosen because the majority of vehicles and containers utilized for inter-site transportation would fall within this segment. Additionally, materials shipped in less than tanker quantities and less than tractor/trailer rigs will also fall within this segment. This segment is intended to capture shipments of smaller quantities such as drums, carboys, B-25s, boxed material, etc.

\subsection{Segment 2, Large Vehicles and Containers}

Large Vehicles or Containers transporting liquids in quantities $>6,000$ gallons and $\leq 10,000$ gallons or transporting solids in volumes $>800$ cubic feet and $\leq 2,880$ cubic feet.

These limits are chosen because the majority of Over The Road (OTR) vehicles (tank trucks and tractor trailers) will fall within this segment. Due to Department of Transportation (DOT) regulations (Ref. 4), Transporters typically limit OTR tankers to hold between 6,000 \& 10,000 gallons with the typical size being 8,600 gallons. Box trailer units pulled by OTR tractors generally do not exceed 45 feet in length and 8 feet in width with an effective interior load height of 8 feet. This segment is intended to capture bulk shipments of materials.

\subsection{Segment 3, Special Vehicles and Containers}

For purposes of this EPHA, Special Vehicles and Containers are defined as any transporting device $>10,000$ gallons for liquids, $>2,880$ cubic feet for solids and rail cars of all sizes. 
These limits are chosen because shipments of these quantities are either by rail or are issued special permits by DOT. OTR shipments of this size are typically escorted and utilize special pre-planned routes. This segment is intended to capture abnormally large quantities of material and rail shipments.

\subsection{Boundary Descriptions}

Boundaries are utilized to determine event classification during emergencies. During movement of hazardous materials on roadways and rail, the distance to personnel that may be impacted will vary greatly. For this reason fixed boundary distances are established from the point of release per EMPP 6Q-001 as described below and illustrated in Figure 2 - Appendix A.

\subsubsection{Site Boundary}

The site boundary is defined as the perimeter of the DOE-owned and controlled land at SRS (Ref. 1). The site boundary is utilized for stationary facilities to determine if PAC is exceeded off site for and is a fixed distance. For transportation incidents, the distance to the site boundary varies based on the specific location of an incident. The THM EPHA analysis is based on a site boundary of 6,000 meters from the point of release, to reflect a one hour downwind distance during adverse meteorological conditions.

\subsubsection{Facility Boundary}

The facility boundary is defined as 100 meters from the point of release per EMPP-6Q-001 (Ref. i).

\subsection{Receptor Points}

The downwind receptor points of interest are utilized to determine if the specified PAC is exceeded, requiring emergency declarations as described in the following sections.

\subsubsection{Notification of Unusual Event}

This applies to Radiological releases only and is determined by comparing the distance to 0.1 mrem Total Effective Dose Equivalent (TEDE) with the distance to the site boundary. Information regarding the distance to $0.1 \mathrm{mrem}$ TEDE is not developed in this EPHA. The distance is accident specific and will have to be calculated at the time of accident utilizing information and tools available. That distance will have to be compared with the distance to site boundary for the specific incident to determine if a NoUE shall be declared.

\subsubsection{Alert}

This applies to radiological and chemical events regardless of location. ALERTS are declared anytime the PAC for the particular material(s) is exceeded 30 meters from the release point but less than 100 meters from the release point. 


\subsubsection{Site Area Emergency}

This applies to radiological and chemical events regardless of location. SAEs are declared anytime the PAC is exceeded at one hundred meters from the release point and is not exceeded at or beyond the site boundary.

\subsubsection{General Emergency}

Applies to radiological and chemical events regardless of location. GEs are declared anytime that the PAC is exceeded at or beyond the site boundary. The site boundary distance will vary depending upon the location of the incident. Information regarding the distance to PAC will be developed in this EPHA and that distance will have to be compared with the distance to site boundary for the specific incident to determine if a GE shall be declared. For calculational purposes in this EPHA a distance of 6,000 meters is utilized to make initial GE determinations. 


\subsection{IDENTIFICATION AND SCREENING OF HAZARDS}

Maximum inventories of radioactive and non radioactive hazardous materials are identified in Calculations 1 through 4 - Appendix B. Included as applicable are both physical limits and associated administrative limits. External hazards are identified in Calculation 2 - Appendix B and were developed by obtaining inventories from a representative sample of Transporters in the area or those known to move material across the site boundary. Each of the hazardous materials found was analyzed utilizing the criteria specified in EMPP-6Q-001 (Ref. 1) to eliminate insignificant hazards; those remaining were characterized. Based on characterization, additional materials presenting insignificant hazards were eliminated from further analysis. The results of the screening and characterization are presented in Calculations 3 and 4 - Appendix B.

\subsection{Hazardous Chemical Identification \& Screening}

Chemical inventories for SRS fixed facilities were obtained from the SRS Chemical Inventory Information System (CIIS) downloaded per S-ESR-G-00001 (Ref. 5). Shipping and Receiving records provided by the Transportation Department, a review of inventories of hazardous waste from the Mixed Hazardous Waste Storage facilities EPHA, and a review of inventories presented in revision 1 of "Evaluation of Accident Risks in the Transportation of Hazardous Materials by Truck and Rail at the Savannah River Site, WSRC-RP-715" (Ref. 6) were utilized to formulate inventories of materials routinely transported.

Additionally, to obtain a representative sample of non-DOE owned hazardous materials routinely shipped across the site boundary via truck on State Routes 125/278, SRS Routes $1 \& 1 \mathrm{~A}$, and Rail; inventories were requested from the following:

Table 4.1 Transporters

\begin{tabular}{|c|c|c|}
\hline $\begin{array}{l}\text { Air Products \& Chemicals } \\
\text { Hazardous Material Manager } \\
\text { 403 Carline Rd. } \\
\text { Langley, SC } 29834\end{array}$ & $\begin{array}{l}\text { AIRCO } \\
\text { Hazardous Materials Manager } \\
3702 \text { Clanton Rd. } \\
\text { Augusta, GA } 30906\end{array}$ & $\begin{array}{l}\text { Amoco Chemicals \& Polymers } \\
\text { Hazardous Materials Manager } \\
3702 \text { Clanton Rd. } \\
\text { Augusta, GA } 30906\end{array}$ \\
\hline $\begin{array}{l}\text { Chemical Leman Tank Lines } \\
\text { Hazardous Materials Manager } \\
\text { Columbia Nitrogen Rd. } \\
\text { Augusta, GA } 30906\end{array}$ & $\begin{array}{l}\text { Cryogenic Transportation Inc. } \\
\text { Hazardous Materials Manager } \\
113 \text { Laney Walker Blvd. } \\
\text { Augusta, GA } 30901\end{array}$ & $\begin{array}{l}\text { CSX Rail } \\
\text { Director-Hazardous Materials } \\
500 \text { Water Street } \\
\text { Jacksonville, FL } 32202\end{array}$ \\
\hline $\begin{array}{l}\text { Eka Nobel Inc., NYACOL } \\
\text { Hazardous Materials Manager } \\
\text { 2432 New Savannah Rd. } \\
\text { Augusta, GA } 30906\end{array}$ & $\begin{array}{l}\text { Federal Express } \\
\text { Hazardous Materials Manager } \\
1070 \text { Bertram Rd. } \\
\text { Augusta, GA } 30909\end{array}$ & $\begin{array}{l}\text { Fleet Transport } \\
\text { Hazardous Materials Manager } \\
1507 \text { Jordan Rd } \\
\text { Augusta, GA } 30904\end{array}$ \\
\hline $\begin{array}{l}\text { Florida Rock \& Tank Lines } \\
\text { Hazardous Materials Manager } \\
1814 \text { Carmichael Rd. } \\
\text { Augusta, GA } 30906\end{array}$ & $\begin{array}{l}\text { HOLOX } \\
\text { Hazardous Materials Manager } \\
1221 \text { New Savannah Rd. } \\
\text { Augusta, GA } 30906 \text {. }\end{array}$ & $\begin{array}{l}\text { Infinger } \\
\text { Hazardous Materials Manager } \\
\text { 1016 Atomic Rd } \\
\text { North Augusta, SC } 29841\end{array}$ \\
\hline $\begin{array}{l}\text { Kenan } \\
\text { Hazardous Materials Manager } \\
\text { Sweetwater Rd } \\
\text { Belvedere, SC } 29841\end{array}$ & $\begin{array}{l}\text { Liquid Carbonic } \\
\text { Hazardous Materials Manager } \\
\text { Columbia Nitrogen Rd. } \\
\text { Augusta, GA } 30906\end{array}$ & $\begin{array}{l}\text { Monsanto } \\
\text { Hazardous Materials Manager } \\
1610 \text { Marvin Griffin Rd } \\
\text { Augusta, GA } 30906\end{array}$ \\
\hline
\end{tabular}


Table 4.1 cont. Transporters

\begin{tabular}{|l|l|l|}
\hline $\begin{array}{l}\text { Montgomery Tank Lines } \\
\text { Hazardous Materials Manager } \\
\text { 2421 Old Savannah Rd. } \\
\text { Augusta, GA 30906 }\end{array}$ & $\begin{array}{l}\text { North American Van Lines } \\
\text { Hazardous Materials Manager } \\
700 \text { Twiggs St. } \\
\text { Augusta, GA 30901 }\end{array}$ & $\begin{array}{l}\text { OLIN } \\
\text { Hazardous Materials Manager } \\
\text { 2402 Old Savannah Rd. } \\
\text { Augusta, GA 30906 }\end{array}$ \\
\hline $\begin{array}{l}\text { Roadway Express } \\
\text { Hazardous Materials Manager } \\
725 \text { Laney Walker Blvd. } \\
\text { Augusta, GA 30901 }\end{array}$ & $\begin{array}{l}\text { Schwerman } \\
\text { Hazardous Materials Manager } \\
\text { 1601 Marvin Griffin Rd. } \\
\text { Augusta, GA 30906 }\end{array}$ & $\begin{array}{l}\text { Transport South } \\
\text { Hazardous Materials Manager } \\
121 \text { Murrah Rd. } \\
\text { North Augusta, SC 29841 }\end{array}$ \\
\hline $\begin{array}{l}\text { UPS } \\
\text { S315 Summit Parkway } \\
\text { San Antonio, TX 78228 }\end{array}$ & $\begin{array}{l}\text { Van Waters \& Rogers } \\
\text { Hazardous Materials Manager } \\
\text { 1455 Columbia Nitrogen Rd. } \\
\text { Augusta, GA 30906 }\end{array}$ & $\begin{array}{l}\text { Yellow Freight System } \\
\text { Hazardous Materials Manager } \\
\text { Augusta, GA } 30907\end{array}$ \\
\hline
\end{tabular}

Those Transporters listed above that responded to the inventory request are bolded and their responses are incorporated in this document as Appendix $\mathrm{C}$.

The chemical inventory for transportation was analyzed by dividing it into the three segments developed in Section 3.

- Segment 1, Small Vehicles and Containers $\leq 6,000$ gallons and/or 800 cubic feet.

- Segment 2, Large Vehicles or Containers transporting liquids in quantities $>6,000$ gallons and $\leq 10,000$ gallons or transporting solids in volumes $>800$ cubic feet and $\leq 2,880$ cubic feet.

- Segment 3, Special Vehicles \& Containers $>10,000$ gallons for liquids, $>2,880$ cubic feet for solids, and Rail Cars of all sizes.

\subsection{Radionuclide Screening}

The radiological inventory and screening for the THM EPHA was conducted in accordance with EMPP 6Q-001 (Ref. 1). The radionuclides were screened using quantities listed in 10 CFR 30.72 (Ref. 7) and employing the methodology identified in DOE STD-1027. If the sum of all the ratios of the inventory of each nuclide to that nuclide's Screening Quantity (SQ) from 10 CFR 30.72 is less than one, the entire inventory was screened (eliminated from analysis). The radioactive materials analyzed in this hazards assessment were extracted from and quantities based on the maximum inventories of radionuclides transported to and/or across the site based on individual facility EPHAs, SARs, BIOs, JCOs, various transportation documents, Hazardous Materials Shipment Public Access Roads Forms, Waste Acceptance Criteria Manual, approximate inventories from the Inter-Site Transportation Radiological and Toxicological Sabotage Vulnerability Assessment (Ref. 9 through 18), and from the inventories produced by those responding to written request as described in section 4.1 . 
The radionuclide inventory for transportation was completed by compiling the entire inventory into the three segments developed in section 3 .

- Segment 1 , Small Vehicles and Containers $\leq 6,000$ gallons and/or 800 cubic feet.

- Segment 2, Large Vehicles or Containers transporting liquids in quantities $>6,000$ gallons and $\leq 10,000$ gallons or transporting solids in volumes $>800$ cubic feet and $\leq 2,880$ cubic feet.

- Segment 3, Special Vehicles \& Containers $>10,000$ gallons, $>2,880$ cubic feet, and Rail Cars of all sizes.

Two criteria are applied to the radionuclide inventories for screening purposes and are explained below.

\subsubsection{Sum of Ratios}

Radionuclides are screened using the screening quantities listed in 10 CFR 30.72 (Ref. 7) and employing the methodology identified in DOE STD-1027 (Ref. 8). If the sum of all the ratios of the inventory of each nuclide to that nuclide's Screening Quantity from 10 CFR 30.72 is less than one, the entire inventory can be screened:

$$
\sum_{i=1}^{n} \frac{r_{i}}{S Q_{i}}<1.0
$$

where:

$\mathrm{r}=\mathrm{Ci}$ of individual nuclide

$\mathrm{n}=$ number of nuclides

$\mathrm{SQ}=$ Screening Quantity for individual nuclide (Ci)

The nuclides in the THM inventory cannot be screened out if the ratio of at least one nuclide in the inventory is found to exceed the above criteria. An example of the calculation for an inventory of $200 \mathrm{Ci}$ of Plutonium-238 is as follows :

- Radionuclide: Pu-238

- $\mathrm{SQ}(\mathrm{Pu}-238): 2 \mathrm{Ci}$

- $r(\mathrm{Pu}-238) / \mathrm{SQ}(\mathrm{Pu}-238)=2.00 \mathrm{E} 02 \mathrm{Ci} / 2 \mathrm{Ci}=\underline{1.00 \mathrm{E}+02}$

- Since $1.00 \mathrm{E}+02$ is greater than 1 , this precludes screening radionuclides by calculating the sum of all the ratios.

\subsubsection{Percentage of Contribution}

To aid in the accident scenario/consequence assessment analysis, some of the radionuclides present are eliminated because they contribute only a small percentage to the resulting dose. The percentage of dose contribution is found using the total segment inventory. Refer to Calculation 4 - Appendix B for the detailed analysis. 


\subsection{Hazards Requiring Analysis}

After applying the above described methodologies to the chemical and radionuclide inventories the results were summarized in Calculations 3 and 4 - Appendix B. Table 4.2 indicates those hazards requiring further analysis. Carbon monoxide, diethylamine, ethylene oxide, hydrogen chloride, methyl bromide, and sulfur dioxide are shipped as compressed gases in containers of varying sizes and different pressures. This EPHA cannot evaluate the different conditions to accurately develop consequences from these materials. It is determined that emergency classification for the release of these chemicals be made based on the DOT guidance (Ref. 28). These chemicals, therefore, are eliminated from the analyses contained in this document.

Table 4.2 Hazards Requiring Further Analysis

\begin{tabular}{|c|c|}
\hline Chemicals & Radionuclides \\
\hline Acetaldehyde & $\mathrm{H}-3$ \\
\hline Acetonitrile & $\mathrm{C}-14$ \\
\hline Acetic Acid & Co-60 \\
\hline Alpha-Pinene & Sr-90 \\
\hline Ammonia & $\mathrm{Ru}-106$ \\
\hline Ammonium Hydroxide & $\mathrm{I}-129$ \\
\hline Benzene & Cs-137 \\
\hline Benzoic Acid & $\mathrm{Ce}-144$ \\
\hline Benzyl Alcohol & $\mathrm{U}-235$ \\
\hline Benzyl Chloride & Np-237 \\
\hline Butyraldehyde & $\mathrm{Pu}-238$ \\
\hline Carbon disulfide & Pu-239 \\
\hline Chloracetone & Pu-240 \\
\hline Chlorine & Am-241 \\
\hline Chlorosulfonic Acid & $\overline{\mathrm{Pu}-241}$ \\
\hline Cyclohexane & Pú-242 \\
\hline Cyclohexanone & Am-243 \\
\hline Diethylene Glycol & $\mathrm{Cm}-244$ \\
\hline Dioxane & $\mathrm{Cf}-252$ \\
\hline Diphenylmethane-4,4-Diisocyanate & - \\
\hline Ethyl Acetate & - \\
\hline Ethyl Benzene & - \\
\hline Fluorosilicic Acid & - \\
\hline Fluosilicic Acid 25\% & - \\
\hline Formaldehyde (Rodine) & - \\
\hline Formic Acid & - \\
\hline Glycol Ether DE & - \\
\hline Glycol Ether EB & - \\
\hline Hydrochloric Acid $31 \%$ & - \\
\hline Hydrofluoric Acid $70 \%$ & - \\
\hline Hydrogen Peroxide & - \\
\hline Isopropyl Alcohol & - \\
\hline Magnesium Oxide & - \\
\hline Methyl Alcohol & - \\
\hline Methyl Ethyl Ketone & - \\
\hline Methyl Isobutyl Ketone / & - \\
\hline Methylene Chloride & - \\
\hline Methyol Cellosolve & - \\
\hline Monoethanolamine & - \\
\hline Morpholine & - \\
\hline
\end{tabular}


Table 4.2 cont. Hazards Requiring Further Analysis

\begin{tabular}{|l|c|}
\hline \multicolumn{1}{|c|}{ Chemicals } & Radionuclides \\
\hline N Butyl Alcohol & - \\
\hline N Methyl Pyrrolidone & - \\
\hline Nitric Acid & - \\
\hline Onyxide & - \\
\hline Perchloroethylene & - \\
\hline Phenol, Molten & - \\
\hline Propagyl Alcohol & - \\
\hline Propionic Acid & - \\
\hline Rodine 50 (formaldehyde) & - \\
\hline Silica (as respirable dust) & - \\
\hline Sodium Sulfhydrate & - \\
\hline Sulfuric Acid & - \\
\hline Sulfuric Acid, Fuming & - \\
\hline Tetrahydrofuran & - \\
\hline Titanium Dioxide & - \\
\hline Toluene & - \\
\hline Toluidine-o & - \\
\hline Trichloroethane-1,1,1 & - \\
\hline Trichloroethylene & - \\
\hline Triethylamine & - \\
\hline Trimethylamine & - \\
\hline Vinyl Acetate & - \\
\hline Xylenes & - \\
\hline
\end{tabular}




\subsection{BARRIER IDENTIFICATION}

From the Identification and Screening of Hazards, Section 4.0, some of the materials present require barrier identification. Sections 5.1 through 5.3 contain a summary of the systems in each segment and a barrier identification analysis for those materials that failed the screening. These materials will be evaluated further for their possible release modes and downwind consequences.

\subsection{Segment 1 , Small Vehicles \& Containers}

\subsubsection{Chemical Sources}

The chemicals not eliminated in Segment 1 by screening are listed in Table 5.1 along with the associated barriers, mitigative features, and administrative controls.

\subsubsection{Radiological Sources}

The radionuclides not eliminated in segment 1 by screening are listed in Table 5.1 along with the associated barriers, mitigative features, and administrative controls.

Table 5.1 Barrier Identification - Segment 1

\begin{tabular}{|c|c|c|c|}
\hline Material & $\begin{array}{l}\text { Description of } \\
\text { Barriers }\end{array}$ & $\begin{array}{l}\text { Description of } \\
\text { Mitigative } \\
\text { Features }\end{array}$ & $\begin{array}{l}\text { Description of } \\
\text { Administrative } \\
\text { Features }\end{array}$ \\
\hline $\begin{array}{l}\text { Radionuclides: } \\
\text { H-3 } \\
\text { C-14 } \\
\text { Cr-51 } \\
\text { Co-60 } \\
\text { Sr-90 } \\
\text { Ru-106 } \\
\text { I-129 } \\
\text { Cs-137 } \\
\text { Ce-144 } \\
\text { U-235 } \\
\text { Np-237 } \\
\text { Pu-238 } \\
\text { Pu-239 } \\
\text { Pu-240 } \\
\text { Am-241 } \\
\text { Pu-241 } \\
\text { Pu-242 } \\
\text { Am-243 } \\
\text { Cm-244 } \\
\text { Cf-252 }\end{array}$ & $\begin{array}{l}\text { B-25, B-012, } 21 \text { inch } \\
\text { by } 21 \text { inch cardboard } \\
\text { box, } 55 \text { gallon drums, } \\
\text { Concrete Culvert, } \\
\text { Type B casks } \\
\text { (estimated as less than } \\
800 \text { cubic feet), } \\
\text { Sample Container } \\
\text { (max. about } 200 \mathrm{ml} \text { ), } \\
\text { SRTC Material } \\
\text { Container, Tank } \\
\text { trucks estimated as } \\
\text { less than } 5000 \\
\text { gallons, } 70 \text { ton cask } \\
\text { metal scrap, Basin } \\
\text { Deionizer, Reactor } \\
\text { Samples Container } \\
\text { (1000 ml per sample) }\end{array}$ & $\begin{array}{l}\text { Container } \\
\text { Specifications }\end{array}$ & $\begin{array}{l}\text { State \& Federal } \\
\text { regulatory limitations } \\
\text { on type material, routes } \\
\text { containers and vehicles } \\
\text { DOT Placard } \\
\text { Regulations } \\
\text { SRS Transportation } \\
\text { Procedures.19Q }\end{array}$ \\
\hline
\end{tabular}




\begin{tabular}{|l|l|l|l|}
\hline Chemicals & $\begin{array}{l}\text { Drums, boxes, } \\
\text { carboys, glass jars } \\
\text { All Chemicals } \\
\text { Listed in } \\
\text { Table 4.2 }\end{array}$ & $\begin{array}{l}\text { Container } \\
\text { Specifications } \\
\text { tanks, etc. }\end{array}$ & $\begin{array}{l}\text { State \& Federal } \\
\text { regulatory limitations } \\
\text { on type material, routes } \\
\text { containers and vehicles }\end{array}$ \\
& & & $\begin{array}{l}\text { DOT Placard } \\
\text { Regulations } \\
\end{array}$ \\
& & & $\begin{array}{l}\text { SRS Transportation } \\
\text { Procedures 19Q }\end{array}$ \\
\hline
\end{tabular}

\subsection{Segment 2, Large Vehicles \& Containers}

\subsubsection{Chemical Sources}

The chemicals not eliminated in Segment 2 by screening are listed in Table 5.2 along with the associated barriers, mitigative features, and administrative controls.

\subsubsection{Radiological Sources}

None

Table 5.2 Barrier Identification - Segment 2

\begin{tabular}{|l|l|l|l|}
\hline \multicolumn{1}{|c|}{ Material } & \multicolumn{1}{|c|}{$\begin{array}{c}\text { Description of } \\
\text { Barriers }\end{array}$} & $\begin{array}{l}\text { Description of } \\
\text { Mitigative } \\
\text { Features }\end{array}$ & \multicolumn{1}{|c|}{$\begin{array}{c}\text { Description of } \\
\text { Administrative } \\
\text { Features }\end{array}$} \\
\hline Chemicals & $\begin{array}{l}\text { Drums, boxes, } \\
\text { carboys, glass jars } \\
\text { All Chemicals } \\
\text { Listed in } \\
\text { Table 4.2 } \\
\text { buckets, cylinders; } \\
\text { tanks, etc. }\end{array}$ & $\begin{array}{l}\text { Container } \\
\text { Specifications }\end{array}$ & $\begin{array}{l}\text { State \& Federal } \\
\text { regulatory limitations } \\
\text { on type material, routes } \\
\text { containers and vehicles }\end{array}$ \\
& & & $\begin{array}{l}\text { DOT Placard } \\
\text { Regulations }\end{array}$ \\
& & & $\begin{array}{l}\text { SRS Transportation } \\
\text { Procedures 19Q }\end{array}$ \\
\hline
\end{tabular}




\subsection{Segment 3, Special Vehicles \& Containers}

\subsubsection{Chemical Sources}

The chemicals not eliminated in Segment 3 by screening are listed in Table 5.3 along with the associated barriers, mitigative features, and administrative controls.

\subsubsection{Radiological Sources}

None

Table 5.3 Barrier Identification - Segment 3

\begin{tabular}{|c|c|c|c|}
\hline Material & $\begin{array}{c}\text { Description of } \\
\text { Barriers }\end{array}$ & $\begin{array}{c}\text { Description of } \\
\text { Mitigative } \\
\text { Features }\end{array}$ & $\begin{array}{c}\text { Description of } \\
\text { Administrative } \\
\text { Features }\end{array}$ \\
\hline $\begin{array}{l}\text { Chemicals } \\
\text { All Chemicals } \\
\text { Listed in } \\
\text { Table } 4.2\end{array}$ & $\begin{array}{l}\text { Drums, boxes, } \\
\text { carboys, glass jars } \\
\text { \& bottles, bags, } \\
\text { buckets, cylinders, } \\
\text { tanks, etc. }\end{array}$ & $\begin{array}{l}\text { Container } \\
\text { Specifications }\end{array}$ & $\begin{array}{l}\text { State \& Federal } \\
\text { regulatory limitations } \\
\text { on type material, routes } \\
\text { containers and vehicles } \\
\text { DOT Placard } \\
\text { Regulations } \\
\text { SRS Transportation } \\
\text { Procedures } 19 Q\end{array}$ \\
\hline
\end{tabular}

\subsection{ACCIDENT ANALYSIS}

The barriers that maintain control over the radionuclides / chemicals identified in Section 5.0 have been analyzed and the possible failure modes considered. The barrier analyses, its results, and resulting release designations are described in this section

The use of the barrier challenge/failure analysis method of EPHA accident analysis implicitly analyzes the vast majority of moderate malevolent acts. Moderate malevolent acts are defined as events that could be initiated by a single individual using materials or tools readily available in the facility, or small quantities of flammables. Within Section 6.0 , the potential events that would challenge/fail a barrier are not normally detailed, as the list can become quite long. However, if events are identified where the only initiator to a release is a malevolent act, it will be explicitly stated in the EPHA. Otherwise, no notation is made.

\subsection{Segment 1, Small Vehicles and Containers}

\subsubsection{Chemical Releases}

Table 6.1 lists those chemicals requiring hazards analysis in Segment 1 of the THM EPHA.

\subsubsection{Failure of Primary Barrier}

The effects of the failure of the primary barrier differ greatly depending on the container, its size/type, its contents as well as the physical form. The primary barrier for release is the container in which the material is transported in. 
Failure of a container for liquids would allow any free liquids to flow into diked areas and/or the soil, allowing the material to evaporate directly to the atmosphere. The eventual surface area of a liquid spill is an important factor in the rate of evaporation.

Failure of the containers which house powders would allow materials to migrate directly to the atmosphere via resuspension, and other release mechanisms. For failure of the container which houses particles or granules, materials may fall to the ground forming a mound. Releases to the atmosphere via evaporation would be minimal.

\subsubsection{Effects of Other Barriers/Mitigative. Features}

Although various mitigative features are available, they depend largely upon the specific transport container, location, and contents. As a measure of conservatism, no mitigative effects were credited. Examples of such mitigative barriers and features could include shipping trays for samples, secondary barriers, and actions taken by response personnel.

\subsubsection{Range of Possible Releases}

Leaks or ruptures release the contents from a container and or multiple containers. The releases are limited to the container size involved in the accident. However, due to the segment limitations the container size for Segment 1 will not exceed 6000 gallons or $800 \mathrm{ft}^{3}$. Each chemical to be analyzed and its associated release designation (RD number) are shown in Table 6.1.

Released liquid materials will produce a spill surface area available for evaporation of $4.074 \mathrm{ft}^{2}$ per gallon or $30.5 \mathrm{ft}^{2}$ per $\mathrm{ft}^{3}$ of liquid. The maximum surface area would be $24,420 \mathrm{ft}^{2}$.

Solids that are in the form of particles or granules will produce surface areas available for aerosolization of unpredictable size and shape. Accidents involving solids will utilize an Airborne Release Fraction of $1 \mathrm{E}-03$ which will be applied to the entire inventory released. The maximum quantity for solids is $800 \mathrm{ft}^{3}$.

\subsubsection{Radiological Releases}

Table 6.2 lists the radionuclide transport containers, the calculated source term, and the release designation for each release in Segment 1.

\subsubsection{Failure of Primary Barrier}

The effects of the failure of the primary barrier differ greatly depending on the container, its size/type, its contents as well as the contents and physical form. The primary barrier for release is the container in which the material is transported.

Failures of containers resulting in fire will cause the materials involved to be carried into the atmosphere through convection currents.

Failure of a container for liquids would first cause a release due to impact and then allow any free liquids to flow into diked areas and or the soil allowing the material to enter the atmosphere via resuspension with the exception of Heavy Water which would evaporate directly to the atmosphere (Ref. 21). The eventual surface area of a liquid spill is an important factor in the rate of evaporation.

Failure of a container for powders would first cause a release due to impact and then allow materials to migrate directly to the atmosphere via resuspension.

Failure of a container of gases would release the gases directly to the environment and can be approximated as an instantaneous release. 


\subsubsection{Effects of Other Barriers/Mitigative Features}

Although various mitigative features are available, depending largely of the specific transport container, location, and contents. As a measure of conservatism no mitigative effects were credited. Examples of such mitigative barriers and features could include shipping trays, secondary barriers, and actions taken by response personnel.

\subsubsection{Range of Possible Releases}

For transportation incidents releases are categorized into two potential types, fire and impact/resuspension. Calculation 5 - Appendix develops the maximum source terms

\subsection{Segment 2, Large Vehicles \& Containers}

\subsubsection{Chemical Releases}

Table 6.1 lists those materials requiring hazards analysis in Segment 2 of the THM EPHA.

\subsubsection{Failure of Primary Barrier}

The effects of the failure of the primary barrier are the same as listed in section 6.1.1.1.

\subsubsection{Effects of Other Barriers/Mitigative Features}

The effects of other barriers/mitigative features are the same as section 6.1.1.2.

\subsubsection{Range of Possible Releases}

Range of possible releases is the same as section 6.1.1.3, except that the maximum quantities are increased as follows:

Leaks or ruptures release the contents from a container and or multiple containers. The releases are limited to the container size involved in the accident. However, due to the segment limitations the container size for Segment 1 will not exceed 10,000 gallons or $2,880 \mathrm{ft}^{3}$.

Released liquid materials will produce a spill surface area available for evaporation of $4.074 \mathrm{ft}^{2}$ per gallon or $30.5 \mathrm{ft}^{2}$ per $\mathrm{ft}^{3}$ of liquid. The maximum surface area would be $40,700 \mathrm{ft}^{2}$.

Solids, which are in the form of particles or granules, will produce surface areas available for aerosolization of unpredictable size and shape. Accidents involving solids will utilize an Airborne Release Fraction of $1 \mathrm{E}-03$ which will be applied to the entire inventory released. The maximum quantity for solids is $2,880 \mathrm{ft}^{3}$.

\subsubsection{Radiological}

There are no Segment 2 radiological releases. 


\subsection{Segment 3, Special Vehicles \& Containers}

\subsubsection{Chemical Releases}

Table 6.1 lists those materials requiring hazards analysis in Segment 3 of the THM EPHA.

\subsubsection{Failure of Primary Barrier}

The effects of the failure of the primary barrier are the same as listed in section 6.1.1.1.

\subsubsection{Effects of Other Barriers/Mitigative Features}

The effects of other barriers/mitigative features are the same as section 6.1.1.2.

\subsubsection{Range of Possible Releases}

Range of possible releases is the same as section 6.1.1.3, except that the maximum quantities are increased as follows:

Leaks or ruptures release the contents from a container and or multiple containers. The releases are limited to the container size involved in the accident. Due to the segment limitations the container sizes for Segment 3 exceed 10,000 gallons or $2,880 \mathrm{ft}^{3}$.

Released liquid materials will produce a spill surface area available for evaporation of $4.074 \mathrm{ft}^{2}$ per gallon or $30.5 \mathrm{ft}^{2}$ per $\mathrm{ft}^{3}$ of liquid. For calculational purposes, the ALOHA default maximum puddle size of $10,000 \mathrm{~m}^{2}$ will be utilized for segment 3. A $10,000 \mathrm{~m}^{2}$ puddle is equal to an $\sim 28,000$ gallon spill, instantaneously spreading to a $1 \mathrm{~cm}$ depth covering an area of $\sim 2.25$ acres. This assumes the surface is perfectly flat, there are zero obstructions, and none of the material is absorbed into the ground. The default $10,000 \mathrm{~m}^{2}$ is sufficiently conservative to address segment 3 scenarios.

Solids that are in the form of particles or granules will produce surface areas available for aerosolization of unpredictable size and shape. Accidents involving solids will utilize an Airborne Release Fraction of 1E-03 that will be applied to the entire inventory released. The maximum quantity for solids utilized for segment 3 is $5,760 \mathrm{ft}^{3}$ the standard rail car size.

\subsubsection{Radiological Releases}

There are no Segment 3 radiological releases.

Based on size none of the transport containers can be considered to be in Segment 3. However, some of the containers may be shipped by rail and therefore are considered as Segment 3 hazards. They are shown in Table 6.1. 
Emergency Preparedness Hazards Assessment for the

Transportation of Hazardous Materials

S-EHA-G-00003, Rev. 1

October 11, 1996

Page 19 of 46

Table 6.1 Segment 1 Chemicals, Associated ERPG, and State

\begin{tabular}{|c|c|c|c|}
\hline $\begin{array}{l}\text { Chemical Name / } \\
\text { Molecular Weight }\end{array}$ & $\begin{array}{c}\text { ERPG or } \\
\text { Equiv } \\
\text { ppm }\end{array}$ & $\begin{array}{l}\text { State } \\
\text { (S)olid } \\
\text { (L)iquid } \\
\text { (G)as }\end{array}$ & $\begin{array}{c}\text { RELEASE } \\
\text { DESIGNATION }\end{array}$ \\
\hline Acetaldehyde / 44.1 & 200 & $\mathrm{~L}$ & $1-R D-1$ \\
\hline Acetic Acid & 35 & $\mathrm{~L}$ & $1-R D-2$ \\
\hline Acetonitrile /41.1 & 60 & $\bar{L}$ & $1-R D-3$ \\
\hline Alpha-Pinene /136.3 & $300\left(\mathrm{mg} / \mathrm{m}^{3}\right)$ & $\mathrm{L}$ & 1-RD-4 \\
\hline Ammonium Hydroxide & 35 & $\mathbf{L}$ & 1-RD-5 \\
\hline Benzene /78.1 & 150 & $\mathrm{~L}$ & $1-R D-6$ \\
\hline Benzyl Alcohol & 100 & $\mathbf{L}$ & 1-RD-7 \\
\hline Benzy! Chloride & 10 & L & $1-\mathrm{RD}-8$ \\
\hline Butyraldehyde & $500\left(\mathrm{mg} / \mathrm{m}^{3}\right)$ & $\mathbf{L}$ & 1-RD-9 \\
\hline Carbon disulfide $/ 76.1$ & 50 & $\overline{\mathbf{L}}$ & 1-RD-10 \\
\hline Chloracetone /92.5 & 3.8 & L & $1-\mathrm{RD}-11$ \\
\hline Chlorosulfonic Acid / 116.52 & 10 & $\mathrm{~L}$ & $1-R D-12$ \\
\hline Cyclohexane / 84.2 & 1300 & $\mathbf{L}$ & $1-R D-13$ \\
\hline Cyclohexanone $/ 98.2$ & 125 & L & 1-RD-14 \\
\hline Diethylene Glycol / 106.1 & 100 & $\mathrm{~L}$ & 1-RD-15 \\
\hline Dioxane /88.1 & 75 & $\mathrm{~L}$ & I-RD-16 \\
\hline $\begin{array}{l}\text { Diphenylmethane-4,4-Difisocyanate ! } \\
250.3\end{array}$ & $2\left(\mathrm{mg} / \mathrm{m}^{3}\right)$ & $\mathrm{L} / \mathrm{S}$ & I-RD-17 \\
\hline Ethyl Acetate /88.1 & 2000 & $\bar{L}$ & 1-RD-18 \\
\hline Ethyl benzene / 106.2 & 125 & $\bar{L}$ & 1-RD-19 \\
\hline $\begin{array}{l}\text { Ethylene Glycol Monobutyl Ether (Glycol } \\
\text { Ether EB) } / 118.2\end{array}$ & 125 & $\bar{L}$ & I-RD-20 \\
\hline Fluorosilicic Acid / 144.1 as Hf & $12.5\left(\mathrm{mg} / \mathrm{m}^{3}\right)$ & $\bar{L}$ & $1-R D-21$ \\
\hline Formic Acid /46.0 & 10 & $\mathrm{~L}$ & $1-\mathrm{RD}-22$ \\
\hline Glycol Ether DE / 134.2 & $500\left(\mathrm{mg} / \mathrm{m}^{3}\right)$ & $\mathrm{L}$ & $1-R \bar{D}-23$ \\
\hline Hydrochloric Acid 31\%/36.5 & 20 & $\mathrm{~L}$ & l-RD-24 \\
\hline Hydrofluoric Acid $70 \% / 20.0$ & 20 & $\mathrm{~L}$ & 1-RD-25 \\
\hline Hydrogen Peroxide $/ 34.0$ & 50 & $\mathrm{~L}$ & $1-\mathrm{RD}-26$ \\
\hline Isopropyl Alcohol 60.1 & 400 & $\overline{\mathrm{L}}$ & 1-RD-27 \\
\hline Methyl Alcohol / 32.0 & 1000 & $\overline{\mathrm{L}}$ & $1-\mathrm{RD}-28$ \\
\hline Methyl Ethyl Ketone /72.1 & 300 & $\bar{L}$ & l-RD-29 \\
\hline Methyl Isobutyl Ketone /100.2 & 500 & $\bar{L}$ & $1-R D-30$ \\
\hline Methylene Chloride / 84.9 & 750 & $\mathrm{~L}$ & $1-R D-31$ \\
\hline Methyol Cellosolve /76.1 & 25 & L & $1-R D-32$ \\
\hline Monoethanolamine $/ 61.1$ & 30 & $\bar{L}$ & 1-RD-33 \\
\hline Morpholine / 87.1 & 100 & $\overline{\mathrm{L}}$ & 1-RD-34 \\
\hline N Butyl Alcohol /74.1 & 50 & $\mathrm{~L}$ & $1-\mathrm{RD}-35$ \\
\hline N Methyl Pyrrolidone / 99.15 & $500\left(\mathrm{mg} / \mathrm{m}^{3}\right)$ & $\bar{L}$ & $1-R D-36$ \\
\hline Nitric Acid / 63.0 & 15 & $\overline{\mathrm{L}}$ & 1-RD-37 \\
\hline Onyxide & $60\left(\mathrm{mg} / \mathrm{m}^{3}\right)$ & - & 1-RD-38 \\
\hline Perchloroethylene / 165.8 & 200 & $\mathrm{~L}$ & $1-R D-39$ \\
\hline Phenol, Molten / 94.1 & 50 & L/S & $1-R D 40$ \\
\hline Propagyl Alcohol / 56.1 & 5 & $\mathrm{~L}$ & $1-\mathrm{RD}-41$ \\
\hline Propionic Acid / 74.1 & 15 & $\overline{\mathrm{L}}$ & $1-\mathrm{RD}-42$ \\
\hline Rodine 50 (formaldehyde) $/ 30.0$ & 10 & $\bar{L}$ & $1-R D-43$ \\
\hline Sulfuric Acid /98.1 & 10 & $\mathrm{~L}$ & l-RD-44 \\
\hline Sulfuric Acid, Fuming / 98.1 & 10 & $\bar{L}$ & 1-RD-45 \\
\hline Tetrahydrofuran $/ 72.1$ & 2000 & $\bar{L}$ & $1-R D-46$ \\
\hline Toluene $/ 92.1$ & 300 & $\mathrm{~L}$ & $1-R D-47$ \\
\hline Toluidine-0 / 107.2 & 10 & $\bar{L}$ & $1-\mathrm{RD}-48$ \\
\hline Trichloroethane-1,1,1/133.4 & 700 & $\bar{L}$ & 1-RD-49 \\
\hline Trichloroethylene / 131.4 & 500 & $\mathbf{L}$ & l-RD-50 \\
\hline Triethylamine / 101.22 & 3 & $\mathbf{L}$ & 1-RD-51 \\
\hline Vinyl Acetate / 86.1 & 75 & $\mathbf{L}$ & $1-\mathrm{RD}-52$ \\
\hline Xylenes / 106.2 & 200 & $\mathbf{L}$ & $1-\mathrm{RD}-53$ \\
\hline
\end{tabular}


Emergency Preparedness Hazards Assessment for the

S-EHA-G-00003, Rev. 1

Transportation of Hazardous Materials

October 11, 1996

Page 20 of 46

Table 6-1 cont. Segment 1 Chemicals, Associated ERPG, and State

\begin{tabular}{|c|c|c|c|}
\hline $\begin{array}{l}\text { Chemical Name / } \\
\text { Molecular Weight }\end{array}$ & $\begin{array}{c}\text { ERPG or } \\
\text { Equiv } \\
\text { ppm }\end{array}$ & $\begin{array}{l}\text { State } \\
\text { (S)olid } \\
\text { (L)iquid } \\
\text { (G)as }\end{array}$ & $\begin{array}{c}\text { RELEASE } \\
\text { DESIGNATION }\end{array}$ \\
\hline Benzoic Acid & $75\left(\mathrm{mg} / \mathrm{m}^{3}\right)$ & $\mathbf{S}$ & 1-RD-54 \\
\hline Magnesium Oxide $/ 40.3$ & 50 & $\mathrm{~S}$ & 1-RD-55 \\
\hline Silica (as respirable dust) & $0.5\left(\mathrm{mg} / \mathrm{m}^{3}\right)$ & $\bar{S}$ & $1-\mathrm{RD}-56$ \\
\hline Sodium Sulfhydrate / 56.06 & $4\left(\mathrm{mg} / \mathrm{m}^{3}\right)$ & $\mathbf{S}$ & 1-RD- $\overline{2} 7$ \\
\hline Titanium Dioxide / 79.9 & .50 & $\bar{S}$ & 1-RD-58 \\
\hline & $\therefore$ & & 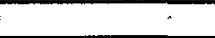 \\
\hline Ammonia /17.0 & 200 & $\mathrm{G}^{*}$ & $1-R D-59$ \\
\hline Chlorine / 70.9 & 3 & $\mathrm{G}^{*}$ & 1-RD-60 \\
\hline Trimethylamine / 59.1 & 100 & $\mathrm{G}^{*}$ & l-RD-6I \\
\hline
\end{tabular}

* Liquefied gas 
Table 6.2 Equivalent Source Terms for Segment 1 Radionuclides, and Associated Release Designations Container of LLW SWDF (Waste)

\begin{tabular}{|c|c|c|c|}
\hline \multirow{2}{*}{$\begin{array}{c}\text { Pu-238 equiv } \\
(\mathrm{Ci})\end{array}$} & \multirow{2}{*}{ Form } & \multicolumn{2}{|c|}{ Source Terms (Ci) } \\
\cline { 3 - 4 } & & Fire & $\begin{array}{c}\text { Impact + } \\
\text { Resuspension } \\
1-\mathrm{RD}-68\end{array}$ \\
\hline $5.65 \mathrm{E}+03$ & & $1-\mathrm{RD}-67$ & $6.10 \mathrm{E}+00$ \\
\hline
\end{tabular}

Container of LLW Compactor (253-H)

\begin{tabular}{|c|c|c|c|}
\hline \multirow{2}{*}{$\begin{array}{c}\text { Pu-238 } \text { equiv } \\
\text { (Ci) }\end{array}$} & Form & \multicolumn{2}{|c|}{ Source Terms (Ci) } \\
\cline { 3 - 4 } & & Fire & $\begin{array}{c}\text { Impact + } \\
\text { Resuspension } \\
1-\mathrm{RD}-70\end{array}$ \\
\hline $4.20 \mathrm{E}-02$ & & $1-\mathrm{RD}-69$ & $4.54 \mathrm{E}-05$ \\
\hline
\end{tabular}

MW Waste - Tritium Bearing Container (Waste)

\begin{tabular}{|c|c|c|c|}
\hline $\mathrm{H}-3$ & \multirow{2}{*}{ Form } & \multicolumn{2}{|c|}{ Source Terms (Ci) } \\
\cline { 3 - 4 }$(\mathrm{Ci})$, & & Fire & $\begin{array}{c}\text { Impact + } \\
\text { Resuspension } \\
1-\mathrm{RD}-72\end{array}$ \\
\hline $5.00 \mathrm{E}+03$ & & $1-\mathrm{RD}-71$ & $5.90 \mathrm{E}+00$ \\
\hline
\end{tabular}

Container of MW Waste - Beta/Gamma (Waste)

\begin{tabular}{|c|c|c|c|}
\hline \multirow{2}{*}{$\begin{array}{c}\text { Pu-238 } \\
\text { cquuv }\end{array}$} & Form & \multicolumn{2}{|c|}{ Source Terms (Ci) } \\
\cline { 3 - 4 } & & Fire & $\begin{array}{c}\text { Impact }+ \\
\text { Resuspension } \\
1-R D-74\end{array}$ \\
\hline $4.30 \mathrm{E}-03$ & & $1-\mathrm{RD}-73$ & $5.08 \mathrm{E}-06$ \\
\hline
\end{tabular}

Container of MW WASTE -

Alpha (Waste)

\begin{tabular}{|c|c|c|c|}
\hline \multirow{2}{*}{$\begin{array}{c}\text { Pu-238 } 8_{\text {equiv }} \\
\text { (Ci) }\end{array}$} & & Form & \multicolumn{2}{|c|}{ Source Terms (Ci) } \\
\cline { 3 - 4 } & & Fire & $\begin{array}{c}\text { Impact + } \\
\text { Resuspension } \\
1-\mathrm{RD}-76\end{array}$ \\
\hline $2.91 \mathrm{E}+00$ & Waste & $1.45 \mathrm{E}-03$ & $3.43 \mathrm{E}-03$ \\
\hline
\end{tabular}

Container of TRU (Waste)

\begin{tabular}{|c|l|c|c|}
\hline Pu-238 cquvv & Form & \multicolumn{2}{|c|}{ Source Terms (Ci) } \\
\cline { 3 - 4 } & & Fire. & $\begin{array}{c}\text { Impact }+ \\
\text { Resuspension } \\
\text { (Ci) }\end{array}$ \\
& & $1-R D-78$ \\
\hline $5.03 \mathrm{E}-01$ & Waste & $2.52 \mathrm{E}-04$ & $5.43 \mathrm{E}-04$ \\
\hline
\end{tabular}

Container for ILNTV Combustibles (E Area Vaults)

\begin{tabular}{|c|c|c|c|}
\hline \multirow{2}{*}{$\begin{array}{l}\text { Pu-238 cquiv } \\
\text { (Ci) }\end{array}$} & \multirow[t]{2}{*}{ Form } & \multicolumn{2}{|c|}{ Source Terms (Ci) } \\
\hline & & $\begin{array}{c}\text { Fire } \\
\text { 1-RD-79 }\end{array}$ & $\begin{array}{c}\text { Impact }+ \\
\text { Resuspension } \\
\text { 1-RD-80 }\end{array}$ \\
\hline $1.52 \mathrm{E}+00$ & Waste & $7.62 \mathrm{E}-04$ & $1.65 \mathrm{E}-03$ \\
\hline
\end{tabular}


Table 6.2 Continued.

Container for LNTV Noncombustibles (E Areá)

\begin{tabular}{|c|c|c|c|}
\hline \multirow{2}{*}{$\begin{array}{c}\text { Pu-238 equiv } \\
\text { (Ci) }\end{array}$} & \multirow[t]{2}{*}{ Form } & \multicolumn{2}{|c|}{ Source Terms (Ci) } \\
\hline & & $\begin{array}{c}\text { Fire } \\
\text { l-RD-81 }\end{array}$ & $\begin{array}{c}\text { Impact + } \\
\text { Resuspension } \\
\text { 1-RD-82 }\end{array}$ \\
\hline $1.62 \mathrm{E}+01$ & Waste & $8.11 E-03$ & $1.75 \mathrm{E}-02$ \\
\hline
\end{tabular}

ILTV Bulk Container (E Area Vaults)

\begin{tabular}{|l|c|c|c|}
\hline Pu-238 & \multirow{2}{*}{ Form } & & \multicolumn{2}{|c|}{ Source Terms (Ci) } \\
\cline { 3 - 4 } & & Fire & $\begin{array}{c}\text { Impact }+ \\
\text { Resuspension } \\
1-\mathrm{RD}-84\end{array}$ \\
\hline & & $1-\mathrm{RD}-83$ & $2.04 \mathrm{E}-03$ \\
\hline
\end{tabular}

ILTV Crucibles Container (E Area Vaults)

\begin{tabular}{|c|c|c|c|}
\hline H-3 & Form & \multicolumn{2}{|c|}{ Source Terms (Ci) } \\
\cline { 3 - 4 }$(\mathrm{Ci})$ & & Fire & $\begin{array}{c}\text { Impact }+ \\
\text { Resuspension } \\
1-\mathrm{RD}-86\end{array}$ \\
\hline $4.00 \mathrm{E}+04$ & & $1-\mathrm{RD}-85$ & $1.97 \mathrm{E}-03$ \\
\hline
\end{tabular}

LLWSB Container (E Area Vaults)

\begin{tabular}{|c|c|c|c|}
\hline \multirow{2}{*}{$\begin{array}{l}\mathrm{H}-3 \\
(\mathrm{Ci})\end{array}$} & Form & \multicolumn{2}{|c|}{ Source Terms (Ci) } \\
\cline { 3 - 4 } & & Fire & $\begin{array}{c}\text { Impact }+ \\
\text { Resuspension } \\
1-\mathrm{RD}-88\end{array}$ \\
\hline $1.00 \mathrm{E}+04$ & & $1-\mathrm{RD}-87$ & $1.66 \mathrm{E}-03$ \\
\hline
\end{tabular}

$\mathrm{Pu} \mathrm{O} 2$ Container (235F and 247F)

\begin{tabular}{|c|c|c|c|}
\hline $\begin{array}{c}\text { Pu-238 cquviv } \\
(\mathrm{Ci})\end{array}$ & Form & \multicolumn{2}{|c|}{ Source Terms (Ci) } \\
\cline { 3 - 4 } & & Fire & $\begin{array}{c}\text { Impact }+ \\
\text { Resuspension } \\
1-\mathrm{RD}-90\end{array}$ \\
\hline $9.16 \mathrm{E}+02$ & & $1-\mathrm{RD}-89$ & $1.91 \mathrm{E}+00$ \\
\hline
\end{tabular}

H Canyon Sample Container (221H to SRTC)

\begin{tabular}{|l|c|c|c|}
\hline Pu-238 cquk & Form & \multicolumn{2}{|c|}{ Source Terms (Ci) } \\
\cline { 3 - 4 } & & Fire & $\begin{array}{c}\text { Impact }+ \\
\text { Resuspension } \\
1-\mathrm{RD}-92\end{array}$ \\
\hline $1.54 \mathrm{E}+01$ & & $1-\mathrm{RD}-91$ & $3.36 \mathrm{E}-02$ \\
\hline
\end{tabular}

H Canyon Sample Container (221H to SRTC)

\begin{tabular}{|l|l|c|c|}
\hline $\begin{array}{l}\text { Pu-238 equiv } \\
\text { (Ci) }\end{array}$ & \multicolumn{2}{|c|}{ Form } & \multicolumn{2}{c|}{ Source Terms (Ci) } \\
\cline { 3 - 5 } & & Fire & $\begin{array}{c}\text { Impact } t \\
\text { Resuspension } \\
1-\mathrm{RD}-94\end{array}$ \\
\hline & & $1-\mathrm{RD}-93$ & $4.91 \mathrm{E}-08$ \\
\hline
\end{tabular}


Table 6.2 Continued.

H Canyon Sample Container (221H to SRTC)

\begin{tabular}{|l|c|c|c|}
\hline \multirow{2}{*}{\begin{tabular}{l|c|} 
Pu-238 \\
(Ci)
\end{tabular}} & Form & \multicolumn{2}{|c|}{ Source Terms (Ci) } \\
\cline { 3 - 4 } & & Fire & $\begin{array}{c}\text { Impact + } \\
\text { Resuspension } \\
1-R D-96\end{array}$ \\
\hline $7.51 \mathrm{E}-04$ & & $1-\mathrm{RD}-95$ & $1.64 \mathrm{E}-06$ \\
\hline
\end{tabular}

F Canyon Sample Container (221F to SRTC)

\begin{tabular}{|l|c|c|c|}
\hline $\begin{array}{l}\text { Pu-238 } \\
\text { (Ci) }\end{array}$ & \multirow{2}{*}{ Form } & \multicolumn{2}{|c|}{ Source Terms (Ci) } \\
\cline { 3 - 4 } & & $\begin{array}{c}\text { Fire } \\
\text { Impact }+ \\
\text { Resuspension } \\
1-R D-98\end{array}$ \\
\hline $1.20 \mathrm{E}-01$ & Liquid & $2.40 \mathrm{E}-04$ & $2.62 \mathrm{E}-04$ \\
\hline
\end{tabular}

F Canyon Sample Container (221F to SRTC)

\begin{tabular}{|l|c|c|c|}
\hline Pu-238 & \multirow{2}{*}{ Formis } & \multicolumn{2}{|c|}{ Source Terms (Ci) } \\
\cline { 3 - 4 } & & Fire & $\begin{array}{c}\text { Impact }+ \\
\text { Resuspension } \\
1-R D-100\end{array}$ \\
\hline & & $1-R D-99$ & $4.90 \mathrm{E}-05$ \\
\hline
\end{tabular}

F Canyon Sample Container (221F to SRTC)

\begin{tabular}{|l|c|c|c|}
\hline Pu-238 cquv & Form & \multicolumn{2}{|c|}{ Source Terms (Ci) } \\
\cline { 3 - 4 } & & Fire & $\begin{array}{c}\text { Impact }+ \\
\text { Resuspension } \\
1-R D-102\end{array}$ \\
\hline & & $1-R D-101$ & $1.04 \mathrm{E}-02$ \\
\hline
\end{tabular}

HB Line Sample Container (221H to 772-1F)

\begin{tabular}{|l|c|c|c|}
\hline Pu-238 & \multirow{2}{*}{ Form } & \multicolumn{2}{|c|}{ Source Terms (Ci) } \\
\cline { 3 - 4 } & & Fire & $\begin{array}{c}\text { Impact }+ \\
\text { Resuspension } \\
1-\mathrm{RD}-104\end{array}$ \\
\hline & & $1-\mathrm{RD}-103$ & $1.7 \mathrm{E})$ \\
\hline $8.55 \mathrm{E}-01$ & Liquid & $1.71 \mathrm{E}-03$ & $1.86 \mathrm{E}-03$ \\
\hline
\end{tabular}

HB Line Sample Container (221H to SRTC)

\begin{tabular}{|l|c|c|c|}
\hline Pu-238 & Form & \multicolumn{2}{|c|}{ Source Terms (Ci) } \\
\cline { 3 - 4 } & & Fire & $\begin{array}{c}\text { Impact }+ \\
\text { Resuspension } \\
\text { (Ci) }\end{array}$ \\
& & $1-\mathrm{RD}-106$ \\
\hline $8.55 \mathrm{E}-01$ & Liquid & $1.71 \mathrm{E}-03$ & $1.86 \mathrm{E}-03$ \\
\hline
\end{tabular}

FB Line Oxide Container (221F to Inter Area)

\begin{tabular}{|l|c|c|c|}
\hline Pu-238 equiv & Form & \multicolumn{2}{|c|}{ Source Terms (Ci) } \\
\cline { 3 - 4 } & & Fire & $\begin{array}{c}\text { Impact }+ \\
\text { Resuspension } \\
\text { (Ci) }\end{array}$ \\
& & $1-$ RD-108 \\
\hline $6.19 \mathrm{E}+03$ & Powder & $3.71 \mathrm{E}-01$ & $1.11 \mathrm{E}-02$ \\
\hline
\end{tabular}


Table 6.2 Continued.

FB Line Sample Container (221F to SRTC)

\begin{tabular}{|l|c|c|c|}
\hline Pu-238 oquiv & Form & \multicolumn{2}{|c|}{ Source Terms (Ci) } \\
\cline { 3 - 4 } & & Fire & $\begin{array}{c}\text { Impact } t \\
\text { Resuspension } \\
1-R D-110\end{array}$ \\
\hline & & $1-R D-109$ & $1.50 \mathrm{E}-03$ \\
\hline
\end{tabular}

235F Material Container (235F to 221H)

\begin{tabular}{|l|c|c|c|}
\hline $\begin{array}{l}\text { Pu-238 equiv } \\
\text { (Ci) }\end{array}$ & Form* & \multicolumn{2}{|c|}{ Source Terms (Ci) } \\
\cline { 3 - 4 } & & Fire & $\begin{array}{c}\text { Impact }+ \\
\text { Resuspension } \\
1-\mathrm{RD}-112\end{array}$ \\
\hline $3.79 \mathrm{E}+04$ & Powder & $2.27 \mathrm{E}+00$ & $6.10 \mathrm{E}-01$ \\
\hline
\end{tabular}

235F Material Container (235F to $221 \mathrm{~F}$ )

\begin{tabular}{|l|c|c|c|}
\hline Pu-238 equav $_{\text {(Ci) }}$ & Form & \multicolumn{2}{|c|}{ Source Terms (Ci) } \\
\cline { 3 - 4 } & & Fire & $\begin{array}{c}\text { Impact }+ \\
\text { Resuspension } \\
1-\mathrm{RD}-114\end{array}$ \\
\hline $1.84 \mathrm{E}+02$ & & $1-\mathrm{RD}-113$ & $3.31 \mathrm{E}-02$ \\
\hline
\end{tabular}

SRTC Material Container (SRTC to DWPF)

\begin{tabular}{|l|c|c|c|}
\hline Pu-238 cquiv & \multirow{2}{*}{ Form } & \multicolumn{2}{|c|}{ Source Terms (Ci) } \\
\cline { 3 - 4 } & & Fire & $\begin{array}{c}\text { Impact }+ \\
\text { Resuspension } \\
1-\mathrm{RD}-116\end{array}$ \\
\hline $7.60 \mathrm{E}+00$ & & $1-\mathrm{RD}-115$ & $8.21 \mathrm{E}-03$ \\
\hline
\end{tabular}

A Line Material (A Line)

\begin{tabular}{|l|c|c|c|}
\hline Pu-238 & Form & \multicolumn{2}{|c|}{ Source Terms (Ci) } \\
\cline { 3 - 4 }$(\mathrm{Ci})$ & & Fire & $\begin{array}{c}\text { Impact }+ \\
\text { Resuspension } \\
1-\mathrm{RD}-118\end{array}$ \\
\hline & & $1-\mathrm{RD}-117$ & $1.84 \mathrm{E}-06$ \\
\hline
\end{tabular}

UNH Trailer (H Area Outside)

\begin{tabular}{|l|c|c|c|}
\hline $\begin{array}{l}\text { Pu-238 cquiv } \\
\text { (Ci) }\end{array}$ & Form & \multicolumn{2}{|c|}{ Source Terms (Ci) } \\
\cline { 3 - 4 } & & Fire & $\begin{array}{c}\text { Impact }+ \\
\text { Resuspension } \\
1-R D-120\end{array}$ \\
\hline $3.99 \mathrm{E}+01$ & Liquid & $7.98 \mathrm{E}-02$ & $7.98 \mathrm{E}-02$ \\
\hline
\end{tabular}

Container, Pail, Casket, Charge Can, Type A for Storage Vault (235-F)

\begin{tabular}{|l|c|c|c|}
\hline $\begin{array}{l}\text { Pu-238 equiv } \\
\text { (Ci) }\end{array}$ & Form & \multicolumn{2}{|c|}{ Source Terms (Ci) } \\
\cline { 3 - 4 } & & Fire & $\begin{array}{c}\text { Impact + } \\
\text { Resuspension } \\
\text { l-RD-122 }\end{array}$ \\
\hline $2.74 \mathrm{E}+05$ & Surface & $8.20 \mathrm{E}+-01$ & $1.48 \mathrm{E}-02$ \\
\hline
\end{tabular}


Table 6.2 Continued.

RBOF Portable Deionizer Column Transport (RBOF)

\begin{tabular}{|l|c|c|c|}
\hline Pu-238 & \multirow{2}{*}{$\begin{array}{c}\text { cquiv } \\
(\mathrm{Ci})\end{array}$} & & \multicolumn{2}{|c|}{ Source Terms (Ci) } \\
\cline { 3 - 4 } & & Fire & $\begin{array}{c}\text { Impact }+ \\
\text { Resuspension } \\
1-\mathrm{RD}-124\end{array}$ \\
\hline $8.57 \mathrm{E}-02$ & L/Resin & $8.57 \mathrm{E}-04$ & $1.01 \mathrm{E}-04$ \\
\hline
\end{tabular}

RBOF Spent Deoinizer Resin Transport (RBOF)

\begin{tabular}{|l|c|c|c|}
\hline Pu-238 & \multirow{2}{*}{ Form } & \multicolumn{2}{|c|}{ Source Terms (Ci) } \\
\cline { 3 - 4 } & & Fire & $\begin{array}{c}\text { Impact }+ \\
\text { Resuspension } \\
1-R D-126\end{array}$ \\
\hline & & $1-$ RD-125 & $8.19 \mathrm{E}-04$ \\
\hline
\end{tabular}

Disassembly Basin Deionizer \& Purif Resin Cask (Reactors)

\begin{tabular}{|l|c|c|c|}
\hline $\begin{array}{l}\text { Pu-238 } \\
\text { (Ci) }\end{array}$ & Form & \multicolumn{2}{|c|}{ Source Terms (Ci) } \\
\cline { 3 - 4 } & & Fire & $\begin{array}{c}\text { Impact }+ \\
\text { Resuspension } \\
1-R D-128\end{array}$ \\
\hline $5.51 E-04$ & L/Resin & $5.51 \mathrm{E}-06$ & $6.50 \mathrm{E}-07$ \\
\hline
\end{tabular}

Reactor Samples Container (Reactors to 771-D/F)

\begin{tabular}{|l|c|c|c|}
\hline $\begin{array}{l}\text { Pu-238 equity } \\
(\mathrm{Ci})\end{array}$ & \multirow{2}{*}{ Form } & \multicolumn{2}{|c|}{ Source Terms (Ci) } \\
\cline { 3 - 4 } & & Fire & $\begin{array}{c}\text { Impact + } \\
\text { Resuspension } \\
1-\mathrm{RD}-130\end{array}$ \\
\hline 5.5lE-04 & Liquid & $1.10 \mathrm{E}-06$ & $1.20 \mathrm{E}-06$ \\
\hline
\end{tabular}

Sample Truck (Inter Area)

\begin{tabular}{|l|c|c|c|}
\hline \multirow{2}{*}{\begin{tabular}{l|l|} 
Pu-238 \\
$(\mathrm{C} i)$
\end{tabular}} & Form & \multicolumn{2}{|c|}{ Source Terms (Ci) } \\
\cline { 3 - 4 } & & Fire & $\begin{array}{c}\text { Impact }+ \\
\text { Resuspension } \\
1-\mathrm{RD}-132\end{array}$ \\
\hline $5.92 \mathrm{E}-02$ & & $1-\mathrm{RD}-13 \mathrm{I}$ & $1.29 \mathrm{E}-04$ \\
\hline
\end{tabular}

Moderator Transport in 55 Gallon Drums (100-400 Areas)

\begin{tabular}{|l|c|c|c|}
\hline \multirow{2}{*}{\begin{tabular}{l|l|} 
Pu-238 \\
$(\mathrm{Ci})$
\end{tabular}} & Form & \multicolumn{2}{|c|}{ Source Terms (Ci) } \\
\cline { 3 - 4 } & & Fire & $\begin{array}{c}\text { Impact }+ \\
\text { Resuspension } \\
\end{array}$ \\
\cline { 3 - 4 } & & $1-\mathrm{RD}-134$ \\
\hline $2.26 \mathrm{E}+00$ & Liquid & $4.52 \mathrm{E}-03$ & $4.93 \mathrm{E}-03$ \\
\hline
\end{tabular}


Table 6.2 Continued.

SRTC 776A to F Tank Farm 5000 Gallon Truck (776A

to Tank Farm)

\begin{tabular}{|l|c|c|c|}
\hline Pu-238 equiv & \multirow{2}{*}{ Form } & \multicolumn{2}{|c|}{ Source Terms (Ci) } \\
\cline { 3 - 4 } & & Fire & $\begin{array}{c}\text { Impact } t \\
\text { Resuspension } \\
1-\mathrm{RD}-136\end{array}$ \\
\hline $6.91 \mathrm{E}-02$ & Liquid & $1.38 \mathrm{E}-04$ & $1.51 \mathrm{E}-04$ \\
\hline
\end{tabular}

Contaminated Water Transport (100 Area to 211-F)

\begin{tabular}{|c|c|c|c|}
\hline \multirow{2}{*}{$\begin{array}{l}\text { Pu-238 } \\
\text { (Ciuli } \\
\text { (Ci) }\end{array}$} & \multirow[t]{2}{*}{ Form } & \multicolumn{2}{|c|}{ Source Terms (Ci) } \\
\hline & & $\begin{array}{r}\text { Fire } \\
\text { 1-RD-137 }\end{array}$ & $\begin{array}{c}\text { Impact + } \\
\text { Resuspension } \\
\text { 1-RD-138 }\end{array}$ \\
\hline $2.26 \mathrm{E}+00$ & Liquid & $4.52 \mathrm{E}-03$ & $4.93 \mathrm{E}-03$ \\
\hline
\end{tabular}

100 Area Sludge Transport (100 Area to 24I-F)(2000

gallons)

\begin{tabular}{|l|c|c|c|}
\hline $\begin{array}{l}\text { Pu-238 } \\
\text { (Ci) }\end{array}$ & Form & \multicolumn{2}{|c|}{ Source Terms (Ci) } \\
\cline { 3 - 4 } & & Fire & $\begin{array}{c}\text { Impact }+ \\
\text { Resuspension } \\
1-\mathrm{RD}-140\end{array}$ \\
\hline $6.52 \mathrm{E}-04$ & L/Sludge & $1.30 \mathrm{E}-06$ & $3.78 \mathrm{E}-07$ \\
\hline
\end{tabular}

Sabotage attack on offsite fuel being transported in a NON-SRS Cask (Type B).

\begin{tabular}{|l|c|c|}
\hline Pu-238 couli $_{\text {(Ci) }}$ & Form & Source Terms (Ci) \\
\cline { 3 - 3 } & & $1-\mathrm{RD}-141$ \\
\hline & & Explosion \\
\hline & & \\
\hline
\end{tabular}




\subsection{CONSEQUENCE ASSESSMENT}

This section provides an overview of the methodology and presents the results of the analysis used to transform the accident scenarios identified in Section 6.0 into projected ground-level concentration/doses at the previously identified receptor locations.

\subsection{Description of Methodology}

\subsubsection{Chemical Dispersion Modeling}

The release of hazardous chemicals was modeled using the Areal Locations of Hazardous Atmospheres (ALOHA) (Ref. 19) dispersion program. ALOHA utilizes both a straight line Gaussian model and a heavy gas computation model. Parameters (spill area required for ERPG-2 concentrations to reach receptor points, material at risk, maximum concentration for segment maximum inventories, maximum distance PAC for segment maximum source terms) for each postulated accident were individually calculated (Calculation 7 - Appendix B).

\subsubsection{Radionuclide Dispersion Modeling}

The HOTSPOT computer code (Ref. 20) was used for dispersion modeling of all radionuclide releases. Several HOTSPOT data sheets (Calculation 6 - Appendix 2) were developed and reviewed to calculate the dispersion of radionuclides. Each sheet is based on a series HOTSPOT runs for a single isotope. Calculation 5 - Appendix B converts the radionuclides of concern to a single source term. This allows several isotopes to be evaluated at the same time utilizing the ratio of dose conversion factors for the various isotopes to the base line isotope used in the HOTSPOT runs. Meteorological conditions utilized are consistent with procedure TP-95-002 (Ref. 21) for the various release heights along with comparisons utilizing average and adverse met conditions.

\subsubsection{Dispersion Modeling Parameters}

Distances to receptor locations:

- 30 meters from the release point

- 100 meters from the release point

- Distance to PAC from the release point 
Meteorology (Ref. 21)

For the THM EPHA all releases are ground releases and utilize the dispersion meteorology shown in tables 7.1 and 7.2

Table 7.1 EPHA 95\% Dispersion Meteorology

\begin{tabular}{|l|l|l|l|}
\hline Release Height & $\begin{array}{c}\text { 30 meter to Facility } \\
\text {.Boundary }\end{array}$ & $\begin{array}{c}\text { Facility Boundary to } \\
\text { Site Boundary }\end{array}$ & $\begin{array}{c}\text { Site Boundary and } \\
\text { Beyond }\end{array}$ \\
\hline \hline Ground & Class E, $1.7 \mathrm{~m} / \mathrm{s}$ & Class $\mathrm{E}, 1.7 \mathrm{~m} / \mathrm{s}$ & Class $\mathrm{E}, 1.7 \mathrm{~m} / \mathrm{s}$ \\
\hline
\end{tabular}

Table 7.2 EPHA 50\% Dispersion Meteorology

\begin{tabular}{|l|l|l|l|}
\hline Release Height & $\begin{array}{c}30 \text { meter to Facility } \\
\text { Boundary }\end{array}$ & $\begin{array}{c}\text { Facility Boundary to } \\
\text { Site Boundary }\end{array}$ & $\begin{array}{c}\text { Site Boundary and } \\
\text { Beyond }\end{array}$ \\
\hline \hline Ground & Class $\mathrm{C}, 2.5 \mathrm{~m} / \mathrm{s}$ & Class $\mathrm{C}, 2.5 \mathrm{~m} / \mathrm{s}$ & Class $\mathrm{C}, 2.5 \mathrm{~m} / \mathrm{s}$ \\
\hline
\end{tabular}

\section{Default Values (Ref. 21)}

- Deposition velocity of $1 \mathrm{~cm} / \mathrm{sec}$.

- $\quad$ Receptor height of 0 meters

- Sample time of 30 minutes for fires and 10 minutes for all other releases

\subsection{Event Classification Determination}

Consequence Assessment results are evaluated against the following criteria to determine the appropriate emergency class for the event scenario (Ref. 1).

(1) Thirty meters from the release point or edge of the spill. The consequence (e.g., radiation dose or peak concentration of a toxic chemical) at this receptor location provides the demarcation between an accident that would require emergency response organization involvement (e.g., ALERT) and one that would not.

(2) One hundred meters from the release point or edge of the spill. The consequence (e.g., radiation dose or peak concentration of a toxic chemical) at this receptor location provides the demarcation between an ALERT and SAE declaration. 
(3) Maximum distance to PAC from the release point or edge of the spill. This distance is compared to the closest site boundary. The consequence (e.g., radiation dose or peak concentration of a toxic chemical) at this receptor location is the demarcation for a GE declaration.

(4) Notification of Unusual Event (NoUE) - The NoUE emergency classification is below an Alert and is not recognized by DOE. Radiological NoUEs are required by the states of Georgia and South Carolina to be identified and reported at a level of 0.1 mrem (TEDE) at the site boundary. The NoUE aligns with the Unusual Occurrence category in DOE Order 232.1A.

In each of the zones the releases are evaluated to determine if the PAC has been exceeded. The last zone where the PAC has been exceeded determines the event classification. The PACs are a personnel radiation exposure level (1 rem TEDE) (Ref. 22) or toxic chemical concentration (ERPG-2) (Ref. 22, 25 \& 27) above which protective action is considered.

Although the radiological PAC is defined in terms of TEDE (external dose plus internal dose), the radiological dispersion model only calculates Committed Effective Dose Equivalent (CEDE). The CEDE is the 50 year committed dose from inhalation of radionuclides. For non-reactor type accidents, as in this EPHA, the CEDE is by far the major portion of the TEDE and can be considered equivalent for purposes of the EPHA.

\subsection{Accidents With Multiple Release Mechanisms}

Some accident events involve multiple release mechanisms; an instantaneous release(s) followed by a longer term release(s). In these cases, the following conservative assumption was used:

- The dominating release mechanism (e.g., impact) is used for the release duration with other contributing mechanisms (e.g., spill, resuspension) added together to form one release amount. .

\subsection{Consequence Assessment Results}

EPHAs are typically developed for facilities having fixed hazards. Due to the nature of transportation incidents, accident specific information cannot be predicted nor developed in advance. It is practical that this EPHA developed consequences based on segment maximums for distance to PAC as well as developing maximum chemical source terms required to exceed PAC at 30 and 100 meters. The results from the consequence assessments. performed for the EPHA are presented in the following tables.

- Table 7.3 Lists the 95\% Adverse results for all THM EPHA chemical releases.

- Table 7.4 Lists the 95\% Adverse results for all THM EPHA radiological releases.

- Table 7.5 Lists the 50\% Average results for all THM EPHA chemical releases.

- Table 7.6 Lists the 50\% Average results for all THM EPHA radiological releases. 
Table 7.3

95\% Adverse met Consequence Assessment Results - Chemical

\begin{tabular}{|c|c|c|c|c|c|}
\hline & $\begin{array}{c}\text { Puddle Size Required } \\
\text { to produce ERPG-2 } \\
\text { Concentration @ } \\
30 \text { meters (ALERT) } \\
\left(\mathrm{FT}^{2}\right)\end{array}$ & $\begin{array}{c}\text { Puddle Size Required } \\
\text { to produce ERPG-2 } \\
\text { Concentration @ } 100 \\
\text { meters (SAE) } \\
\left(\mathrm{FT}^{2}\right)\end{array}$ & $\begin{array}{l}\text { Maximum Distance to } \\
\text { PAC for Segment } 1 \\
\text { Maximum Source } \\
\text { Term }\left(24,420 \mathrm{ft}^{2} \text { ) }\right. \\
\text { (meters) }\end{array}$ & $\begin{array}{l}\text { Maximum Distance to } \\
\text { PAC for Segment } 2 \\
\text { Maximum Source } \\
\text { Term }\left(40,700 \mathrm{ft}^{2}\right) \\
\text { (meters) }\end{array}$ & $\begin{array}{l}\text { Maximum Distance to } \\
\text { PAC for Segment } 3 \\
\text { Maximum Source } \\
\text { Term (10,000 } \mathrm{m}^{2} \\
\text { Puddle) }{ }^{\star} \\
\text { (meters) }\end{array}$ \\
\hline I-RD-1 & 60 & 672 & 635 & 824 & 1400 \\
\hline 1-RD-2 & 115 & 1425 & 428 & 568 & 1200 \\
\hline I-RD-3 & 21 & 265 & 1100 & 1400 & 2200 \\
\hline I-RD-4 & 491 & 5941 & 202 & 261 & 432 \\
\hline $1-\mathrm{RD}-5$ & \multicolumn{5}{|c|}{ Previously analyzed chemical which screens as not a severe airbome hazard. } \\
\hline l-RD-6 & 68 & 695 & 549 & 708 & 1200 \\
\hline 1-RD-7 & $>30000$ & $>100000$ & 27 & 34 & 56 \\
\hline l-RD-8 & 730 & 9200 & 163 & 213 & 360 \\
\hline 1-RD-9 & 84 & 777 & 532 & 686 & 1100 \\
\hline 1-RD-10 & 4.4 & 31 & 2200 & 2800 & 4500 \\
\hline 1-RD-11 & 0.5 & 7 & 8900 & $>10000$ & $>10000$ \\
\hline 1-RD-12 & $>30000$ & $>100000$ & 27 & 34 & 56 \\
\hline 1-RD-13 & 1045 & 11000 & 135 & 196 & 332 \\
\hline I-RD-14 & 7750 & NA & 52 & 67 & 109 \\
\hline I-RD-15 & $>30000$ & $>100000$ & 27 & 34 & 56 \\
\hline 1-RD-16 & 98 & 1400 & 501 & 643 & 1000 \\
\hline I-RD-17 & 17237 & $>100000$ & 35 & 45 & 72 \\
\hline l-RD-18 & 1885 & 18400 & 103 & 150 & 255 \\
\hline 1-RD-19 & 3300 & 24086 & 147 & 189 & 306 \\
\hline l-RD-20 & $>30000$ & $>100000$ & 27 & 34 & 56 \\
\hline I-RD-2! & 24600 & $>100000$ & 21 & 38 & 61 \\
\hline I-RD-22 & 7 & 101 & 2400 & 3100 & 5000 \\
\hline 1-RD-23 & 8750 & $>100000$ & 50 & 64 & 103 \\
\hline 1-RD-24 & 3230 & 39400 & 79 & 102 & 165 \\
\hline 1-RD-25 & 24600 & $>100000$ & 2.18 & 38 & 61 \\
\hline 1-RD-26 & 5175 & 62200 & 63 & 81 & 131 \\
\hline 1-RD-27 & 848 & 4930 & 217 & 282 & 468 \\
\hline 1-RD-28 & 1115 & 14000 & 132 & 253 & 442 \\
\hline 1-RD-29 & 250 & 2125 & 334 & 436 & 679 \\
\hline 1-RD-30 & 6034 & 32500 & 87 & 112 & 187 \\
\hline l-RD-3! & 96 & 1110 & 450 & 592 & 1000 \\
\hline 1-RD-32 & 145 & 1810 & 377 & 498 & 1200 \\
\hline 1-RD-33 & 18500 & $>100000$ & 34 & 44 & 70 \\
\hline 1-RD-34 & 191 & 2380 & 328 & 586 & 936 \\
\hline 1-RD-35 & 288 & 3630 & 262 & 436 & 701 \\
\hline 1-RD-36 & $>30000$ & NA & 27 & 34 & 56 \\
\hline I-RD-37 & 12 & 141 & 1700 & 2200 & 3500 \\
\hline I-RD-38 & Insufficient info for $\mathrm{EAL}$ & Insufficient info for EAL & Insufficient info for EAL & Insufficient info for EAL & Insufficient info for EAL \\
\hline 1-RD-39 & 1175 & 8575 & 166 & 217 & $\cdot 363$ \\
\hline l-RD-40 & 4550 & 53500 & 68 & 88 & 142 \\
\hline I-RD-41 & 6 & 71 & 2700 & 3700 & 5900 \\
\hline 1-RD-42 & 283 & 3600 & 263 & 346 & 597 \\
\hline 1-RD-43 & 18 & 226 & 1200 & 1600 & 2900 \\
\hline 1-RD-44 & $>30000$ & $>100000$ & 27 & 34 & .56 \\
\hline 1-RD-45 & 37 & 437 & 936 & 1200 & 1900 \\
\hline 1-RD-46 & 1080 & 13300 & 138 & 183 & 310 \\
\hline I-RD-47 & 1810 & 7730 & 174 & 226 & 376 \\
\hline 1-RD-48 & 6260 & 73000 & 58 & 75 & 122 \\
\hline 1-RD-49 & 472 & 6060 & 208 & 275 & 466 \\
\hline
\end{tabular}


Table 7.3 cont.

95\% Adverse met Consequence Assessment Results - Chemical

\begin{tabular}{|c|c|c|c|c|c|}
\hline & $\begin{array}{c}\text { Puddle Size Required } \\
\text { to produce ERPG-2 } \\
\text { Concentration @ } \\
30 \text { meters (ALERT) } \\
\left(\text { FT }^{2} \text { ) }\right.\end{array}$ & $\begin{array}{c}\text { Puddle Size Required } \\
\text { to produce ERPG-2 } \\
\text { Concentration @ 100 } \\
\text { meters (SAE) } \\
\left(\mathrm{FT}^{2}\right)\end{array}$ & $\begin{array}{l}\text { Maximum Distance to } \\
\text { PAC for Segment } 1 \\
\text { Maximum Source } \\
\text { Term }\left(24,420 \mathrm{ft}^{2}\right) \\
\text { (meters) }\end{array}$ & $\begin{array}{l}\text { Maximum Distance to } \\
\text { PAC for Segment } 2 \\
\text { Maximum Source } \\
\left.\text { Term (40,700 } \mathrm{ft}^{2}\right) \\
\text { (meters) }\end{array}$ & $\begin{array}{l}\text { Maximum Distance to } \\
\text { PAC for Segment } 3 \\
\text { Maximum Source } \\
\text { Term (10,000 } \mathrm{m}^{2} \\
\text { Puddle) } \\
\text { (meters) }\end{array}$ \\
\hline $1-R D-50$ & 522 & 5760 & 206 & 271 & 456 \\
\hline 1-RD-51 & 1.7 & 20 & 5300 & 6900 & $>10000$ \\
\hline 1-RD-52 & 25.6 & 225 & 914 & 1200 & 1900 \\
\hline \multirow[t]{2}{*}{ 1-RD-53 } & 6720 & $>32140$ & 56 & 111 & 182 \\
\hline & $\begin{array}{l}\text { Pounds Required to } \\
\text { produce ERPG-2 } \\
\text { Concentration @ } \\
30 \text { meters (ALERT) } \\
\left(\mathrm{lb}_{\mathrm{m}}\right) \\
\end{array}$ & $\begin{array}{l}\text { Pounds Required to } \\
\text { produce ERPG-2 } \\
\text { Concentration @ } 100 \\
\text { meters (SAE) } \\
\left(\mathrm{lb}_{\mathrm{m}}\right)\end{array}$ & $\begin{array}{l}\text { Maximum Distance to } \\
\text { PAC for Segment } 1 \\
\text { Maximum Source } \\
\left.\text { Term (37,600 lb } b_{m}\right) \\
\text { (meters) }\end{array}$ & $\begin{array}{l}\text { Maximum Distance to } \\
\text { PAC for Segment } 2 \\
\text { Maximum Source } \\
\text { Term }\left(135,000 \mathrm{lb}_{\mathrm{m}}\right) \\
\text { (meters) }\end{array}$ & $\begin{array}{l}\text { Maximum Distance to } \\
\text { PAC for Segment } 3 \\
\text { Maximum Source } \\
\text { Term }\left(263,000 \mathrm{lb}_{\mathrm{m}}\right) \\
\text { (meters) }\end{array}$ \\
\hline I-RD-54 & 285 & 3000 & 344 & 580 & 755 \\
\hline $1-\overline{R D}-55$ & 490 & $5 \overline{100}$ & 260 & 437 & 567 \\
\hline $1-R D-56$ & 1.9 & 20 & 2500 & 4300 & 5800 \\
\hline 1-RD-57 & 15 & 160 & 1100 & 1800 & 2400 \\
\hline \multirow[t]{2}{*}{ I-RD-58 } & 490 & 5100 & 260 & 437 & 567 \\
\hline & $\begin{array}{c}\text { Pounds Required to } \\
\text { produce ERPG-2 } \\
\text { Concentration @ } \\
30 \text { meters (ALERT) } \\
\left(\mathrm{lb}_{\mathrm{m}}\right) \\
\end{array}$ & $\begin{array}{c}\text { Pounds Required to } \\
\text { produce ERPG-2 } \\
\text { Concentration @ } 100 \\
\text { meters (SAE) } \\
\left(1 \mathrm{~b}_{\mathrm{m}}\right)\end{array}$ & $\begin{array}{l}\text { Maximum Distance to } \\
\text { PAC (GE) for Segment } \\
1 \text { Maximum Source } \\
\text { Term (1 ton) }{ }^{\star \star} \\
\text { (meters) }\end{array}$ & $\begin{array}{l}\text { Maximum Distance to } \\
\text { PAC (GE) for } \\
\text { Segment } 2 \text { Maximum } \\
\text { Source Term } \\
\text { (10 tons) })^{\star \star} \text { (meters) }\end{array}$ & $\begin{array}{l}\text { Maximum Distance to } \\
\text { PAC (GE) for Segment } \\
3 \text { Maximum Source } \\
\text { Term (17.5 tons) } \\
\text { (meters) }\end{array}$ \\
\hline 1-RD-59 & 7 & 77 & 550 & 1800 & 2400 \\
\hline $1-\mathrm{RD} \cdot 60$ & 0.3 & 3 & 4000 & 6300 & 6400 \\
\hline 1-RD-61 & 1.3 & 6.7 & 1200 & 3200 & 4000 \\
\hline
\end{tabular}

- ALOHA default maximum (see section 6.3.1.1) 
Table 7.4 95\% Adverse met Consequence Assessment Results - Radiological

\begin{tabular}{|c|c|c|c|c|}
\hline $\begin{array}{c}\text { Release } \\
\text { Designation }\end{array}$ & $\begin{array}{c}\text { Total Dose @ } \\
30 \text { meters } \\
\text { (rem) }\end{array}$ & $\begin{array}{c}\text { Total Dose @ } \\
100 \text { meters } \\
\text { (rem) }\end{array}$ & $\begin{array}{c}\text { Maximum Distance } \\
\text { to PAC } \\
\text { (meters) }\end{array}$ & $\begin{array}{c}\text { Possible Event } \\
\text { Class* }\end{array}$ \\
\hline $1-\mathrm{RD}-67$ & $6.4 \mathrm{E}-01^{* *}$ & $3.2 \mathrm{E}-01^{* *}$ & N/A & NONE \\
\hline $1-\mathrm{RD}-68$ & $3.8 \mathrm{E}-01^{* *}$ & $1.9 \mathrm{E}-02^{* *}$ & N/A & NONE \\
\hline $1-\mathrm{RD}-69$ & $8.00 \mathrm{E}-02$ & $1.20 \mathrm{E}-02$ & N/A & NONE \\
\hline $1-\mathrm{RD}-70$ & $1.80 \mathrm{E}-01$ & $3.10 \mathrm{E}-02$ & N/A & NONE \\
\hline I-RD-71 & $1.90 \mathrm{E}-03$ & $4.00 \mathrm{E}-04$ & N/A & NONE \\
\hline $1-\mathrm{RD}-72$ & $5.70 \mathrm{E}-03$ & $1.20 \mathrm{E}-03$ & N/A & NONE \\
\hline $1-\mathrm{RD}-73$ & $7.00 \mathrm{E}-02$ & $1.20 \mathrm{E}-03$ & N/A & NONE \\
\hline $1-\mathrm{RD}-74$ & $2.10 \mathrm{E}-02$ & $3.50 \mathrm{E}-03$ & N/A & NONE \\
\hline $1-\mathrm{RD}-75$ & $4.70 \mathrm{E}+00$ & $1.00 \mathrm{E}+00$ & $1.00 \mathrm{E}+02$ & ALERT \\
\hline $1-\mathrm{RD}-76$ & $1.40 \mathrm{E}+01$ & $2.40 \mathrm{E}+00$ & $1.60 \mathrm{E}+02$ & SAE \\
\hline $1-\mathrm{RD}-77$ & $8.20 \mathrm{E}-01$ & $1.40 \mathrm{E}-01$ & N/A & NONE \\
\hline $1-\mathrm{RD}-78$ & $2.20 \mathrm{E}+00$ & $3.70 \mathrm{E}-01$ & $5.0 \mathrm{E}+01$ & ALERT \\
\hline $1-\mathrm{RD}-79$ & $2.50 \mathrm{E}+00$ & $4.20 \mathrm{E}-01$ & $6.00 \mathrm{E}+01$ & ALERT \\
\hline $1-\mathrm{RD}-80$ & $6.70 \mathrm{E}+00$ & $1.10 \mathrm{E}+00$ & $1.10 \mathrm{E}+02$ & SAE \\
\hline $1-\mathrm{RD}-81$ & $2.60 \mathrm{E}+01$ & $4.50 \mathrm{E}+00$ & $2.30 \mathrm{E}+02$ & SAE \\
\hline $1-\mathrm{RD}-82$ & $7.10 \mathrm{E}+01$ & $1.20 \mathrm{E}+01$ & $3.80 \mathrm{E}+02$ & SAE \\
\hline $1-\mathrm{RD}-83$ & $3.10 \mathrm{E}+00$ & $5.20 \mathrm{E}-01$ & $7.00 \mathrm{E}+01$ & ALERT \\
\hline $1-\mathrm{RD}-84$ & $8.20 \mathrm{E}+00$ & $1.40 \mathrm{E}+00$ & $1.20 \mathrm{E}+02$ & SAE \\
\hline $1-\mathrm{RD}-85$ & $7.10 \mathrm{E}-07$ & $1.50 \mathrm{E}-07$ & N/A & NONE \\
\hline $1-\mathrm{RD}-86$ & $1.96 \mathrm{E}-06$ & $3.90 \mathrm{E}-07$ & N/A & NONE \\
\hline $1-\mathrm{RD}-87$ & $6.00 \mathrm{E}-07$ & $1.20 \mathrm{E}-07$ & N/A & NONE \\
\hline $1-\mathrm{RD}-88$ & $1.60 \mathrm{E}-06$ & $3.30 \mathrm{E}-07$ & N/A & NONE \\
\hline $1-\mathrm{RD}-89$ & $1.80 \mathrm{E}+02$ & $3.00 \mathrm{E}+01$ & $6.30 \mathrm{E}+02$ & SAE \\
\hline $1-\mathrm{RD}-90$ & $7.70 \mathrm{E}+03$ & $1.30 \mathrm{E}+03$ & $5.16 \mathrm{E}+03$ & SAE \\
\hline $1-\mathrm{RD}-91$ & $1.00 \mathrm{E}+02$ & $1.70 \mathrm{E}+01$ & $4.60 \mathrm{E}+02$ & SAE \\
\hline $1-\mathrm{RD}-92$ & $1.40 \mathrm{E}+02$ & $2.30 \mathrm{E}+01$ & $5.40 \mathrm{E}+02$ & SAE \\
\hline
\end{tabular}

* For transportation event class estimations 6,000 meters was utilized for General Emergencies

** These values were taken directly from the EAV EPHA (Ref.29) because the basis for the high source term originally used could not be determined and is deemed to be in error. 
Emergency Preparedness Hazards Assessment for the

S-EHA-G-00003, Rev. I

Transportation of Hazardous Materials

October 11, 1996

Page 33 of 46

Table 7.4 cont. $95 \%$ Adverse met Conséquence Assessment Results - Radiological

\begin{tabular}{|c|c|c|c|c|}
\hline \multicolumn{5}{|c|}{ Protective Action Criteria = 1 rem TEDE } \\
\hline $\begin{array}{c}\text { Release } \\
\text { Designation }\end{array}$ & $\begin{array}{c}\text { Total Dose@ } \\
30 \text { meters } \\
\text { (rem) }\end{array}$ & $\begin{array}{c}\text { Total Dose@ } \\
100 \text { meters } \\
\text { (rem) }\end{array}$ & \begin{tabular}{|c}
$\begin{array}{c}\text { Maximum Distance } \\
\text { to PAC } \\
\text { (meters) }\end{array}$ \\
\end{tabular} & $\begin{array}{l}\text { Possible Event } \\
\text { Class * }\end{array}$ \\
\hline 1-RD-93 & $1.50 \mathrm{E}-04$ & $2.50 \mathrm{E}-05$ & N/A & NONE \\
\hline I-RD-94 & $2.00 \mathrm{E}-04$ & $3.40 \mathrm{E}-05$ & N/A & NONE \\
\hline 1-RD-95 & $4.90 \mathrm{E}-03$ & $8.30 \mathrm{E}-04$ & N/A & NONE \\
\hline 1-RD-96 & $6.60 \mathrm{E}-03$ & $1.10 \mathrm{E}-03$ & N/A & NONE \\
\hline 1-RD-97 & $7.80 \mathrm{E}-01$ & $1.30 \mathrm{E}-01$ & N/A & NONE \\
\hline 1-RD-98 & $1.10 \mathrm{E}+00$ & $1.80 \mathrm{E}-01$ & $3.00 \mathrm{E}+01$ & ALERT \\
\hline 1-RD-99 & $1.50 \mathrm{E}-01$ & $2.50 \mathrm{E}-02$ & N/A & NONE \\
\hline 1-RD-100 & $2.00 \mathrm{E}-0 \mathrm{I}$ & $3.40 \mathrm{E}-02$ & N/A & NONE \\
\hline 1-RD-101 & $3.10 \mathrm{E}+01$ & $5.30 \mathrm{E}+00$ & $2.50 \mathrm{E}+02$ & SAE \\
\hline 1-RD-102 & $4.20 \mathrm{E}+01$ & $7.20 \mathrm{E}+00$ & $2.90 \mathrm{E}+02$ & SAE \\
\hline 1-RD-103 & $5.50 \mathrm{E}+00$ & $9.50 \mathrm{E}-01$ & $9.00 \mathrm{E}+01$ & ALERT \\
\hline 1-RD-104 & $7.50 \mathrm{E}+00$ & $1.30 \mathrm{E}+00$ & $1.10 \mathrm{E}+02$ & SAE \\
\hline 1-RD-105 & $5.50 \mathrm{E}+00$ & $9.50 \mathrm{E}-01$ & $9.00 \mathrm{E}+01$ & ALERT \\
\hline 1-RD-106 & $7.50 \mathrm{E}+00$ & $1.30 \mathrm{E}+00$ & $1.10 \mathrm{E}+02$ & SAE \\
\hline I-RD-107 & $1.20 \mathrm{E}+03$ & $2.10 \mathrm{E}+02$ & $1.74 \mathrm{E}+03$ & SAE \\
\hline 1-RD-108 & $4.40 \mathrm{E}+01$ & $7.60 \mathrm{E}+00$ & $3.00 \mathrm{E}+02$ & SAE \\
\hline 1-RD.109 & $4.40 \mathrm{E}+00$ & $7.60 \mathrm{E}-01$ & $8.00 \mathrm{E}+01$ & ALERT \\
\hline 1-RD-110 & $6.10 \mathrm{E}+00$ & $1.00 \mathrm{E}+00$ & $1.00 \mathrm{E}+02$ & $\mathrm{SAE}$ \\
\hline 1-RD-111 & $7.40 \mathrm{E}+03$ & $1.30 \mathrm{E}+03$ & $5.00 \mathrm{E}+03$ & SAE \\
\hline $1-R D-112$ & $2.50 \mathrm{E}+03$ & $4.20 \mathrm{E}+02$ & $2.70 \mathrm{E}+03$ & SAE \\
\hline $1-R D-113$ & $3.60 \mathrm{E}+01$ & $6.10 \mathrm{E}+00$ & $2.70 \mathrm{E}+02$ & SAE \\
\hline 1-RD-114 & $1.30 \mathrm{E}+02$ & $2.30 \mathrm{E}+01$ & $5.40 \mathrm{E}+02$ & $\mathrm{SAE}$ \\
\hline 1-RD-115 & $1.50 \mathrm{E}+02$ & $2.50 \mathrm{E}+01$ & $5.70 \mathrm{E}+02$ & SAE \\
\hline 1-RD-116 & $3.30 \mathrm{E}+0 \mathrm{I}$ & $5.70 \mathrm{E}+00$ & $2.60 \mathrm{E}+02$ & SAE \\
\hline 1-RD-117 & $5.50 \mathrm{E}-03$ & $9.30 \mathrm{E}-04$ & N/A & NONE \\
\hline 1-RD-118 & $7.40 \mathrm{E}-03$ & $1.30 \mathrm{E}-03$ & N/A & NONE \\
\hline 1-RD-119 & $2.60 \mathrm{E}+02$ & $4.40 \mathrm{E}+01$ & $7.70 \mathrm{E}+02$ & $\mathrm{SAE}$ \\
\hline 1-RD-120 & $3.20 \mathrm{E}+02$ & $5.50 \mathrm{E}+0 \mathrm{I}$ & $8.60 \mathrm{E}+02$ & SAE \\
\hline $1-R D-121$ & $2.70 \mathrm{E}+03$ & $4.50 \mathrm{E}+02$ & $2.80 \mathrm{E}+03$ & $\mathrm{SAE}$ \\
\hline $1-R D-122$ & $6.00 \mathrm{E}+01$ & $1.00 \mathrm{E}+01$ & $3.60 \mathrm{E}+02$ & SAE \\
\hline $1-R D-123$ & $2.80 \mathrm{E}+00$ & $4.70 \mathrm{E}-01$ & $6.00 \mathrm{E}+01$ & ALERT \\
\hline 1-RD-124 & $4.10 \mathrm{E}-01$ & $7.00 \mathrm{E}-02$ & N/A & NONE \\
\hline $1-\mathrm{RD}-125$ & $2.30 \mathrm{E}+01$ & $3.80 \mathrm{E}+00$ & $2.10 \mathrm{E}+02$ & SAE \\
\hline 1-RD-126 & $3.30 \mathrm{E}+00$ & $5.60 \mathrm{E}-01$ & $7.00 \mathrm{E}+01$ & ALERT \\
\hline 1-RD-127 & $1.80 \mathrm{E}-02$ & $3.00 \mathrm{E}-03$ & N/A & NONE \\
\hline 1-RD-128 & $2.60 \mathrm{E}-03$ & $4.50 \mathrm{E}-04$ & N/A & NONE \\
\hline 1-RD-129 & $3.60 \mathrm{E}-03$ & $6.10 \mathrm{E}-04$ & N/A & NONE \\
\hline 1-RD-130 & $4.90 \mathrm{E}-03$ & $8.30 \mathrm{E}-04$ & N/A & NONE \\
\hline 1-RD-131 & $3.80 \mathrm{E}-01$ & $6.50 \mathrm{E}-02$ & N/A & NONE \\
\hline 1-RD-132 & $5.20 \mathrm{E}-01$ & $8.90 \mathrm{E}-02$ & N/A & NONE \\
\hline 1-RD-133 & $1.50 \mathrm{E}+01$ & $2.50 \mathrm{E}+00$ & $1.60 \mathrm{E}+02$ & SAE \\
\hline I-RD-134 & $2.00 \mathrm{E}+0 \mathrm{I}$ & $3.40 \mathrm{E}+00$ & $1.90 \mathrm{E}+02$ & $\mathrm{SAE}$ \\
\hline $1-R D-135$ & $4.50 \mathrm{E}-01$ & $7.60 \mathrm{E}-02$ & N/A & NONE \\
\hline l-RD-136 & $6.10 \mathrm{E}-01$ & $1.00 \mathrm{E}-01$ & N/A & NONE \\
\hline I-RD-137 & $1.50 \mathrm{E}+01$ & $2.50 \mathrm{E}+00$ & $1.60 \mathrm{E}+02$ & SAE \\
\hline 1-RD-138 & $2.10 \mathrm{E}+01$ & $3.40 \mathrm{E}+00$ & $1.90 \mathrm{E}+02$ & SAE \\
\hline 1-RD-139 & $4.20 \mathrm{E}-03$ & $7.20 \mathrm{E}-04$ & N/A & NONE \\
\hline $1-R D-140$ & $1.50 \mathrm{E}-03$ & $2.60 \mathrm{E}-04$ & N/A & NONE \\
\hline 1-RD-141 & $1.10 \mathrm{E}+04$ & $1.50 \mathrm{E}+03$ & $1.80 \mathrm{E}+04$ & $\mathrm{GE}$ \\
\hline
\end{tabular}

* For transportation event class estimations 6,000 meters was utilized for General Emergencies

** These values were taken directly from the EAV EPHA (Ref.29) because the basis for the high source term originally used could not be determined and is deemed to be in error. 
Table 7.5 50\% Average met Consequence Assessment Results - Chemical

\begin{tabular}{|c|c|c|c|c|c|}
\hline & $\begin{array}{c}\text { Puddle Size Required } \\
\text { to produce ERPG-2 } \\
\text { Concentration @ } \\
30 \text { meters (ALERT) } \\
\left.\text { (FT }{ }^{2}\right)\end{array}$ & $\begin{array}{c}\text { Puddle Size Required } \\
\text { to produce ERPG-2 } \\
\text { Concentration @ } 100 \\
\text { meters (SAE) } \\
\left(\mathrm{FT}^{2}\right)\end{array}$ & $\begin{array}{l}\text { Maximum Distance to } \\
\text { PAC (GE) for Segment } \\
1 \text { Maximum Source } \\
\text { Term }\left(24,420 \mathrm{ft}^{2}\right) \\
\text { (meters) }\end{array}$ & $\begin{array}{c}\text { Maximum Distance to } \\
\text { PAC (GE) for } \\
\text { Segment } 2 \text { Maximum } \\
\text { Source Term }(40,700 \\
\left.\mathrm{ft}^{2}\right) \\
\text { (meters) }\end{array}$ & $\begin{array}{l}\text { Maximum Distance to } \\
\text { PAC (GE) for Segment } \\
3 \text { Maximum Source } \\
\text { Term (10,000 } \mathrm{m}^{2} \\
\text { Puddle) } \\
\text { (meters) }\end{array}$ \\
\hline 1-RD-1 & 410 & 4600 & 601 & 780 & 1300 \\
\hline 1-RD-2 & 895 & 11850 & 140 & 178 & 280 \\
\hline I-RD-3 & 177 & 2325 & 303 & 386 & 1600 \\
\hline I-RD-4 & 3341 & 42582 & 77 & 98 & 155 \\
\hline $1-R D-5$ & \multicolumn{5}{|c|}{ Previously analyzed chemical which screens as not a severe airbome hazard. } \\
\hline $1-\mathrm{RD}-6$ & 535 & $>4000$ & 499 & 641 & 1000 \\
\hline 1-RD-7 & $>30000$ & $>100000$ & 27 & 34 & 56 \\
\hline I-RD-8 & 3700 & 50000 & 72 & 91 & 143 \\
\hline I-RD-9 & 737 & 6485 & 471 & 603 & 994 \\
\hline 1-RD-10 & 32.3 & $>260$ & 1800 & 2400 & 3900 \\
\hline 1-RD-11 & 4 & 50 & 5400 & 690 & $>10000$ \\
\hline 1-RD-12 & $>30000$ & $>100000$ & 27 & 34 & 56 \\
\hline $1-\mathrm{RD}-13$ & $>2950$ & 18700 & 115 & 149 & 247 \\
\hline I-RD-14 & $>30000$ & $>100000$ & 27 & 34 & 56 \\
\hline $1-R D-15$ & $>30000$ & $>100000$ & 27 & 34 & 56 \\
\hline $1-R D-16$ & 997 & 13000 & 135 & 556 & 907 \\
\hline 1-RD-17 & $>30000$ & $>100000$ & 16 & 20 & 32 \\
\hline 1-RD-18 & 3140 & 35500 & 82 & 107 & 180 \\
\hline I-RD-19 & 17700 & $>100000$ & 35 & 45 & 72 \\
\hline 1-RD-20 & $>30000$ & $>100000$ & 27 & 34 & 56 \\
\hline $1-R D-21$ & $>30000$ & $>100000$ & 27 & 34 & 56 \\
\hline 1-RD-22 & 59 & 760 & 528 & 677 & 1100 \\
\hline 1-RD-23 & $>30000$ & $>100000$ & 27 & 34 & 56 \\
\hline 1-RD-24 & 13080 & $>100000$ & 41 & 52 & 82 \\
\hline 1-RD-25 & $>30000$ & $>100000$ & $<10$ & $<10$ & $<100$ \\
\hline 1-RD-26 & 26800 & $>100000$ & 29 & 37 & 58 \\
\hline I-RD-27 & 9600 & $>36000$ & 47 & 248 & 408 \\
\hline 1-RD-28 & 12200 & $>100000$ & 42 & 53 & 85 \\
\hline l-RD-29 & 3165 & 9384 & 313 & 400 & 540 \\
\hline 1-RD-30 & $>30000$ & 61426 & 27 & 34 & 173 \\
\hline |-RD-31 & 132 & 1712 & 380 & 494 & 819 \\
\hline l-RD-32 & 1950 & 27750 & 94 & 119 & 186 \\
\hline 1-RD-33 & $>30000$ & $>100000$ & 27 & 34 & 56 \\
\hline 1-RD-34 & 1450 & 19600 & 111 & 140 & 220 \\
\hline 1-RD-35 & 1995 & 26900 & 96 & 121 & 191 \\
\hline l-RD-36 & $>30000$ & $>100000$ & 27 & 34 & 56 \\
\hline 1-RD-37 & 82 & 1015 & 469 & 603 & 2200 \\
\hline 1-RD-38 & Insufficient infor for EAL & Insufficient infor for EAL & Insufficient infor for EAL & Insufficient infor for $\mathrm{EAL}$ & Insufficient infor for EAL \\
\hline 1-RD-39 & 9850 & 12900 & 113 & 133 & 177 \\
\hline $1-R D-40$ & 14550 & $>100000$ & 39 & 49 & 78 \\
\hline I-RD-4I & 37 & 447 & 696 & 895 & 1500 \\
\hline l-RD 42 & 2050 & 28500 & 93 & 118 & 185 \\
\hline $1-R D-43$ & 116 & 1440 & 392 & 501 & 805 \\
\hline 1-RD-44 & $>30000$ & $>100000$ & $<10$ & $<10$ & $<100$ \\
\hline $1-R D-45$ & 189 & 2300 & 313 & 402 & 647 \\
\hline 1-RD-46 & 3010 & 21100 & 108 & 140 & 232 \\
\hline l-RD-47 & 11950 & $>22350$ & 118 & 144 & 191 \\
\hline 1-RD-48 & 23880 & $>100000$ & 30 & 39 & 56 \\
\hline 1-RD-49 & 875 & 7840 & 176 & 229 & 381 \\
\hline 1-RD-50 & $>1700$ & 8200 & 174 & 225 & 375 \\
\hline l-RD-51 & 20 & 245 & 2400 & 3200 & 5100 \\
\hline l-RD-52 & 11.3 & 143 & 3200 & 4100 & 6700 \\
\hline 1-RD-53 & 30300 & $>100000$ & 27 & 34 & 56 \\
\hline
\end{tabular}




\begin{tabular}{|c|c|c|c|c|c|}
\hline & $\begin{array}{c}\text { Pounds required to } \\
\text { produce ERPG-2 } \\
\text { Concentration at } 30 \\
\text { meters (ALERT) } \\
\left(1 \mathrm{~b}_{\mathrm{m}}\right) \\
\end{array}$ & $\begin{array}{c}\text { Pounds required to } \\
\text { produce ERPG-2 } \\
\text { Concentration at } 30 \\
\text { meters (SAE) } \\
\left(\mathrm{Ib}_{\mathrm{m}}\right) \\
\end{array}$ & $\begin{array}{l}\text { Maximum Distance to } \\
\text { PAC for Segment } 1 \\
\text { Maximum Source } \\
\text { Term }\left(37,600_{m}\right) \\
\text { (meters) }\end{array}$ & $\begin{array}{l}\text { Maximum Distance to } \\
\text { PAC for Segment } 2 \\
\text { Maximum Source } \\
\left.\text { Term (135,000 l } \mathrm{lb}_{\mathrm{m}}\right) \text { ) } \\
\text { (meters) }\end{array}$ & $\begin{array}{c}\text { Maximum Distance to } \\
\text { PAC for Segment } 3 \\
\text { Maximum Source } \\
\text { Term (263,000 lb } b_{m} \text { ) } \\
\text { (meters) }\end{array}$ \\
\hline 1-RD-54 & 1900 & 21300 & 133 & 252 & 353 \\
\hline 1-RD-55 & 3400 & 37200 & 100 & 191 & 267 \\
\hline 1-RD-56 & 12.8 & 142 & 1300 & 2000 & 2600 \\
\hline 1-RD-57 & 100 & 1135 & 569 & 948 & 1200 \\
\hline 1-RD-58 & 3400 & 37200 & 100 & 191 & 267 \\
\hline & $\begin{array}{c}\text { Pounds Required to } \\
\text { produce ERPG-2 } \\
\text { Concentration @ } \\
30 \text { meters (ALERT) } \\
\left(\mathrm{lb}_{\mathrm{m}}\right) \\
\end{array}$ & $\begin{array}{c}\text { Pounds Required to } \\
\text { produce ERPG-2 } \\
\text { Concentration @ } 100 \\
\text { meters (SAE) } \\
\left(\mathrm{lb}_{\mathrm{m}}\right)\end{array}$ & $\begin{array}{l}\text { Maximum Distance to } \\
\text { PAC (GE) for Segment } \\
1 \text { Maximum Source } \\
\text { Term (1 ton) ** } \\
\text { (meters) }\end{array}$ & $\begin{array}{l}\text { Maximum Distance to } \\
\text { PAC (GE) for } \\
\text { Segment } 2 \text { Maximum } \\
\text { Source Term } \\
\text { (10 tons) }{ }^{\star \star} \text { (meters) }\end{array}$ & $\begin{array}{l}\text { Maximum Distance to } \\
\text { PAC (GE) for Segment } \\
3 \text { Maximum Source } \\
\text { Term (17.5 tons) } \\
\text { (meters) }\end{array}$ \\
\hline 1-RD-59 & 47 & 558 & 190 & 570 & 750 \\
\hline 1-RD-60 & 2 & 10 & 2700 & 7500 & 9300 \\
\hline I-RD-61 & 9 & 110 & 1000 & 2800 & 3600 \\
\hline
\end{tabular}

* ALOHA default maximum (see section 6.3.1.1)

Table 7.6 50\% Average met Consequence Assessment Results - Radiological

\begin{tabular}{|c|c|c|c|}
\hline \multicolumn{4}{|c|}{ Protective Action Criteria = 1 rem TEDE } \\
\hline $\begin{array}{c}\text { Release } \\
\text { Designation }\end{array}$ & $\begin{array}{c}\text { Total Dose @ } \\
30 \text { meters } \\
\text { (rem) }\end{array}$ & $\begin{array}{c}\text { Total Dose@ } \\
100 \text { meters } \\
\text { (rem) }\end{array}$ & \begin{tabular}{|c|} 
Maximum Distance \\
to PAC \\
(meters) \\
\end{tabular} \\
\hline 1-RD-67 & $1.9 \mathrm{E}-01^{* *}$ & $7.2 \mathrm{E}-03^{* *}$ & N/A \\
\hline $1-\mathrm{RD}-68$ & $1.1 \mathrm{E}-01^{* *}$ & $4.4 \mathrm{E}-03^{* *}$ & N/A \\
\hline 1-RD-69 & $2.00 \mathrm{E}-02$ & $2.70 \mathrm{E}-03$ & N/A \\
\hline 1-RD-70 & $5.40 \mathrm{E}-02$ & $7.73 \mathrm{E}-03$ & N/A \\
\hline I-RD-7I & $5.00 \mathrm{E}-04$ & $7.20 \mathrm{E}-05$ & N/A \\
\hline 1-RD-72 & $1.50 \mathrm{E}-03$ & $2.10 \mathrm{E}-04$ & N/A \\
\hline 1-RD-73 & $2.00 \mathrm{E}-03$ & $2.80 \mathrm{E}-04$ & N/A \\
\hline 1-RD-74 & $6.00 \mathrm{E}-03$ & $8.20 \mathrm{E}-04$ & N/A \\
\hline 1-RD-75 & $1.40 \mathrm{E}+00$ & $1.00 \mathrm{E}+00$ & $1.00 \mathrm{E}+03$ \\
\hline 1-RD-76 & $4.10 \mathrm{E}+00$ & $1.00 \mathrm{E}+00$ & $1.00 \mathrm{E}+02$ \\
\hline I-RD-77 & $2.40 \mathrm{E}-01$ & $3.30 \mathrm{E}-02$ & N/A \\
\hline 1-RD-78 & $6.40 \mathrm{E}-01$ & $8.80 \mathrm{E}-02$ & N/A \\
\hline 1-RD-79 & $7.20 \mathrm{E}-01$ & $9.90 \mathrm{E}-02$ & N/A \\
\hline I-RD-80 & $2.00 \mathrm{E}+00$ & $1.00 \mathrm{E}+00$ & $1.00 \mathrm{E}+02$ \\
\hline l-RD-81 & $7.70 \mathrm{E}+00$ & $1.10 \mathrm{E}+00$ & $1.00 \mathrm{E}+02$ \\
\hline 1-RD-82 & $2.10 \mathrm{E}+01$ & $2.80 \mathrm{E}+01$ & $1.70 \mathrm{E}+02$ \\
\hline 1-RD-83 & $9.00 \mathrm{E}-01$ & $1.20 \mathrm{E}-01$ & N/A \\
\hline I-RD-84 & $2.40 \mathrm{E}+00$ & $3.30 \mathrm{E}-01$ & $5.00 \mathrm{E}+1$ \\
\hline 1-RD-85 & $1.80 \mathrm{E}-07$ & $2.60 \mathrm{E}-08$ & N/A \\
\hline 1-RD-86 & $5.00 \mathrm{E}-07$ & $7.10 \mathrm{E}-08$ & N/A \\
\hline 1-RD-87 & $1.60 \mathrm{E}-07$ & $2.20 \mathrm{E}-08$ & N/A \\
\hline 1-RD-88 & $4.20 \mathrm{E}-07$ & $6.00 \mathrm{E}-08$ & N/A \\
\hline 1-RD-89 & $5.20 \mathrm{E}+01$ & $7.10 \mathrm{E}+00$ & $2.80 \mathrm{E}+02$ \\
\hline 1-RD-90 & $2.30 \mathrm{E}+03$ & $3.10 \mathrm{E}+02$ & $2.05 E+03$ \\
\hline 1-RD-91 & $2.90 \mathrm{E}+01$ & $4.00 \mathrm{E}+00$ & $2.10 \mathrm{E}+02$ \\
\hline 1-RD-92 & $4.00 \mathrm{E}+01$ & $5.40 \mathrm{E}+00$ & $2.50 \mathrm{E}+02$ \\
\hline 1-RD-93 & $4.30 \mathrm{E}-05$ & $5.90 \mathrm{E}-06$ & N/A \\
\hline 1-RD-94 & $5.80 \mathrm{E}-05$ & $7.90 \mathrm{E}-06$ & N/A \\
\hline 1-RD-95 & $1.40 \mathrm{E}-03$ & $1.90 \mathrm{E}-04$ & N/A \\
\hline 1-RD.96 & $1.90 \mathrm{E}-03$ & $2.70 \mathrm{E}-04$ & N/A \\
\hline 1-RD-97 & $2.30 \mathrm{E}-01$ & $3.10 \mathrm{E}-02$ & N/A \\
\hline l-RD-98 & $3.10 \mathrm{E}-01$ & $4.20 \mathrm{E}-02$ & N/A \\
\hline 1-RD-99 & $4.20 \mathrm{E}-02$ & $5.80 \mathrm{E}-03$ & N/A \\
\hline
\end{tabular}

** These values were taken directly from the EAV EPHA (Ref. 29) because the basis for the high source term originally used could not be determined and is deemed to be in error 
Table 7.6 cont. $\quad 50 \%$ Average met Consequence Assessment Results - Radiological

\begin{tabular}{|c|c|c|c|}
\hline \multicolumn{4}{|c|}{ Protective Action Criteria $=1$ rem TEDE } \\
\hline $\begin{array}{c}\text { Release } \\
\text { Designation }\end{array}$ & $\begin{array}{c}\text { Total Dose@ } \\
30 \text { meters } \\
\text { (rem) }\end{array}$ & $\begin{array}{c}\text { Total Dose@ } \\
100 \text { meters } \\
\text { (rem) }\end{array}$ & $\begin{array}{c}\text { Maximum Distance } \\
\text { to PAC } \\
\text { (meters) }\end{array}$ \\
\hline I-RD-100 & $5.80 \mathrm{E}-02$ & $7.90 \mathrm{E}-03$ & $\mathrm{~N} / \mathrm{A}$ \\
\hline 1-RD-101 & $9.00 \mathrm{E}+00$ & $1.20 \mathrm{E}+00$ & $1.10 \mathrm{E}+02$ \\
\hline 1-RD-102 & $1.20 \mathrm{E}+01$ & $1.70 \mathrm{E}+00$ & $1.30 \mathrm{E}+02$ \\
\hline I-RD-103 & $1.60 \mathrm{E}+00$ & $1.00 \mathrm{E}+00$ & $1.00 \mathrm{E}+02$ \\
\hline I-RD-104 & $2.20 \mathrm{E}+00$ & $1.00 \mathrm{E}+00$ & $1.00 \mathrm{E}+02$ \\
\hline I-RD-105 & $1.60 \mathrm{E}+00$ & $1.00 \mathrm{E}+00$ & $1.00 \mathrm{E}+02$ \\
\hline l-RD-106 & $2.20 \mathrm{E}+00$ & $1.00 \mathrm{E}+00$ & $1.00 \mathrm{E}+02$ \\
\hline $1-\mathrm{RD}-107$ & $3.50 \mathrm{E}+02$ & $4.80 \mathrm{E}+01$ & $7.60 \mathrm{E}+02$ \\
\hline I-RD-108 & $1.30 \mathrm{E}+01$ & $1.80 \mathrm{E}+00$ & $1.40 \mathrm{E}+02$ \\
\hline 1-RD-109 & $1.30 \mathrm{E}+00$ & $1.00 \mathrm{E}+00$ & $1.00 \mathrm{E}+02$ \\
\hline l-RD-110 & $1.80 \mathrm{E}+00$ & $1.00 \mathrm{E}+00$ & $1.00 \mathrm{E}+02$ \\
\hline I-RD-111 & $2.20 \mathrm{E}+03$ & $2.90 \mathrm{E}+02$ & $1.99 \mathrm{E}+03$ \\
\hline $1 \cdot \mathrm{RD}-112$ & $7.20 \mathrm{E}+02$ & $9.80 \mathrm{E}+01$ & $1.10 \mathrm{E}+03$ \\
\hline $1-\mathrm{RD}-113$ & $1.00 \mathrm{E}+01$ & $1.40 \mathrm{E}+00$ & $1.20 \mathrm{E}+02$ \\
\hline $1-R D-114$ & $3.90 \mathrm{E}+01$ & $5.40 \mathrm{E}+00$ & $2.40 \mathrm{E}+02$ \\
\hline I-RD-115 & $4.30 \mathrm{E}+01$ & $5.90 \mathrm{E}+00$ & $2.50 \mathrm{E}+02$ \\
\hline 1-RD-116 & $9.70 \mathrm{E}+00$ & $1.30 \mathrm{E}+00$ & $1.10 \mathrm{E}+02$ \\
\hline $1-\mathrm{RD}-117$ & $1.60 \mathrm{E}-03$ & $2.20 \mathrm{E}-04$ & N/A \\
\hline $1-\mathrm{RD}-118$ & $2 . \overline{20 E}-03$ & $3.00 \mathrm{E}-04$ & N/A \\
\hline 1-RD-119 & $7.60 \mathrm{E}+01$ & $1.00 \mathrm{E}+01$ & $3.40 \mathrm{E}+02$ \\
\hline I-RD-120 & $9.50 \mathrm{E}+01$ & $1.30 \mathrm{E}+0 \mathrm{I}$ & $3.80 \mathrm{E}+02$ \\
\hline 1-RD-121 & $7.80 \mathrm{E}+02$ & $1.10 \mathrm{E}+02$ & $1.15 \mathrm{E}+03$ \\
\hline l-RD-122 & $1.80 \mathrm{E}+01$ & $2.40 \mathrm{E}+00$ & $1.69 \mathrm{E}+02$ \\
\hline I-RD-123 & $8.10 \mathrm{E}-01$ & $1.10 \mathrm{E}-01$ & N/A \\
\hline 1-RD-124 & $1.20 \mathrm{E}-0 \mathrm{I}$ & $1.60 \mathrm{E}-02$ & $\mathrm{~N} / \mathrm{A}$ \\
\hline $1-\mathrm{RD}-125$ & $6.60 \mathrm{E}+00$ & $1.00 \mathrm{E}+00$ & $1.00 \mathrm{E}+02$ \\
\hline $1-R D-126$ & $9.70 \mathrm{E}-01$ & $1.30 \mathrm{E}-01$ & N/A \\
\hline 1-RD-127 & $5.20 \mathrm{E}-03$ & $7.20 \mathrm{E}-04$ & N/A \\
\hline l-RD-128 & $7.70 \mathrm{E}-04$ & $1.10 \mathrm{E}-04$ & N/A \\
\hline 1-RD-129 & $1.00 \mathrm{E}-03$ & $1.40 \mathrm{E}-04$ & N/A \\
\hline $1-R D-130$ & $1.40 \mathrm{E}-03$ & $1.90 \mathrm{E}-04$ & N/A \\
\hline I-RD-131 & $1.10 \mathrm{E}-01$ & $1.50 \mathrm{E}-02$ & N/A \\
\hline $1-R D-132$ & $1.50 \mathrm{E}-01$ & $2.10 \mathrm{E}-02$ & N/A \\
\hline $1-R D-133$ & $4.30 \mathrm{E}+00$ & $1.00 \mathrm{E}+00$ & $1.00 \mathrm{E}+02$ \\
\hline 1-RD-134 & $5.80 \mathrm{E}+00$ & $1.00 \mathrm{E}+00$ & $1.00 \mathrm{E}+02$ \\
\hline $1-\mathrm{RD}-135$ & $1.30 \mathrm{E}-01$ & $1.80 \mathrm{E}-02$ & N/A \\
\hline 1-RD-136 & $1.80 \mathrm{E}-01$ & $2.40 \mathrm{E}-02$ & N/A \\
\hline 1-RD-137 & $4.30 \mathrm{E}+00$ & $1.00 \mathrm{E}+00$ & $1.00 \mathrm{E}+02$ \\
\hline 1-RD-138 & $5.80 \mathrm{E}+00$ & $1.00 \mathrm{E}+00$ & $1.00 \mathrm{E}+02$ \\
\hline 1-RD-139 & $1.20 \mathrm{E}-03$ & $1.70 \mathrm{E}-04$ & N/A \\
\hline I-RD-140 & $4.50 \mathrm{E}-04$ & $6.10 \mathrm{E}-05$ & $\mathbf{N} / \mathbf{A}$ \\
\hline
\end{tabular}




\subsection{EMERGENCY CLASSES AND EALS}

This section documents the correlation of the consequence assessment (section 7) with the requirements to classify events. Table 7.5 presents a summary of all classifications, however, additional rationale for specific EALs is required. If an identified initiator has the potential, through barrier analysis, to cause the release of hazardous materials resulting in consequences greater than or equal to PAC at the identified receptor locations, then EALs are required (Ref. 25)

Various materials are continually shipped to, from and through the SRS. Therefore, materials are transported in many different types of containers by a variety of methods. For this reason initiators such as tornadoes, earthquakes handling accidents, projectiles, corrosion, and sabotage were evaluated and found credible on any given day. For this reason, specific initiators are not listed for each consequence, rather they are grouped as being generic. With generic initiators and the special circumstances of the THM EPHA release mechanisms for chemical incidents are considered to be spills to the ground.

\subsection{General Emergency (GE) EALs}

\subsubsection{Chemical Releases}

Leakage or rupture of any container resulting in a spill producing a puddle with a surface area available for evaporation capable of producing a chemical concentration greater than ERPG-2 to a distance greater than the distance of the accident to the site boundary.

\subsubsection{Radiological Releases}

Extreme malevolent act against a Type B shipping container transporting spent fuel that results in a breach of the container 1-RD-141.

\subsection{Site Area Emergency (SAE)}

\subsubsection{Chemical Releases}

Leakage or rupture of any container resulting in a spill producing a puddle with a surface area available for evaporation in the range of the target surface area in table 8.2

Table 8.1 Chemical Spill Target Surface Areas for Site Area Emergencies

\begin{tabular}{|l|c|c|c|}
\hline Chemical & $\begin{array}{l}\text { ft } \\
\text { Surface Area }\end{array}$ & Gallons & Release Designation \\
\hline Acetaldehyde /44.1 & 672 & 165 & $1-\mathrm{RD}-1$ \\
\hline Acetic Acid & 1425 & 350 & $1-\mathrm{RD}-2$ \\
\hline Acetonitrile /41.1 & 265 & 65 & $1-\mathrm{RD}-3$ \\
\hline Alpha-Pinene /136.3 & 5941 & 1458 & $1-\mathrm{RD}-4$ \\
\hline Ammonium Hydroxide & Should have been screened out. & $1-\mathrm{RD}-5$ \\
\hline Benzene /78.1 & 695 & 171 & $1-\mathrm{RD}-6$ \\
\hline Benzyl Alcohol & $>100000$ & 24541 & $1-\mathrm{RD}-7$ \\
\hline Benzyl Chloride & 9200 & 2258 & $1-\mathrm{RD}-8$ \\
\hline Butyraldehyde & 777 & 191 & $1-\mathrm{RD}-9$ \\
\hline Carbon disulfide /76.1 & 31 & 8 & $1-\mathrm{RD}-10$ \\
\hline Chloracetone /92.5 & 7 & 2 & $1-\mathrm{RD}-11$ \\
\hline Chlorosulfonic Acid/116.52 & $>100000$ & 24546 & $1-\mathrm{RD}-12$ \\
\hline Cyclohexane /84.2 & 11000 & 2700 & $1-\mathrm{RD}-13$ \\
\hline Cyclohexanone /98.2 & $\mathrm{NA}$ & $\mathrm{NA}$ & $1-\mathrm{RD}-14$ \\
\hline Diethylene Glycol/106.1 & $>100000$ & 24541 & $1-\mathrm{RD}-15$ \\
\hline Dioxane /88.1 & 1400 & 344 & $1-\mathrm{RD}-16$ \\
\hline Diphenylmethane-4,4-Diisocyanate/250 & $>100000$ & 24541 & $1-\mathrm{RD}-17$ \\
\hline
\end{tabular}


Table 8.1 cont. Chemical Spill Target Surface Areas for Site Area Emergencies

\begin{tabular}{|c|c|c|c|}
\hline Chemical & $\begin{array}{l}\mathrm{ft}^{2} \text { Target } \\
\text { Surface Area }\end{array}$ & Gallons & Release Designation \\
\hline UNLISTED CHEMICAL & See DOT Guidebook & N/A & N/A \\
\hline Ethyl Acetate /88.1 & 18400 & 4516 & $1-\mathrm{RD}-18$ \\
\hline Ethyl benzene / 106.2 & 24086 & 5911 & 1-RD-19 \\
\hline $\begin{array}{l}\text { Ethelene Glycol Monobutyl Ether I } \\
118.2\end{array}$ & $>100000$ & 24541 & $1-R D-20$ \\
\hline Fluorosilicic Acid / 144.1. & $>100000$ & 24541 & $1-\mathrm{RD}-21$ \\
\hline Formic Acid / 46.0 & 101 & 25 & $1-\mathrm{RD}-22$ \\
\hline Glycol Ether DE / 134.2 & $>100000$ & 24541 & 1-RD-23 \\
\hline Hydrochloric Acid 31\% / 36.5 & 39400 & 9671 & 1-RD-24 \\
\hline Hydrofluoric Acid $70 \% / 20.0$ & $>100000$ & 24546 & $1-\mathrm{RD}-25$ \\
\hline Hydrogen Peroxide $/ 34.0$ & 62200 & 15264 & $1-\mathrm{RD}-26$ \\
\hline Isopropyl Alcohol 60.1 & 4930 & 1210 & 1-RD-27 \\
\hline Methyl Alcohol / 32.0 & 14000 & 3436 & l-RD-28 \\
\hline Methyl Ethyl Ketone /72.1 & 2125 & 521 & 1-RD-29 \\
\hline Methyl Isobutyl Ketone / 100.2 & 32500 & 7976 & 1-RD-30 \\
\hline Methylene Chloride / 84.9 & 1110 & 272 & $1-\mathrm{RD}-31$ \\
\hline Methyol Cellosolve / 76.1 & 1810 & 444 & $1-\mathrm{RD}-32$ \\
\hline Monoethanolamine $/ 6 \mathrm{l} .1$ & $>100000$ & 24541 & 1-RD-33 \\
\hline Morpholine /87.1 & 2380 & 584 & $1-\mathrm{RD}-34$ \\
\hline N Butyl Alcohol / 74.1 & 3630 & 891 & $1-\mathrm{RD}-35$ \\
\hline N Methyl Pyrrolidone /99.15 & $\mathrm{NA}$ & NA & 1-RD-36 \\
\hline Nitric Acid / 63.0 & 141 & 35 & 1-RD-37 \\
\hline Onyxide & See DOT Guidebook & N/A & 1-RD-38 \\
\hline Perchloroethylene / 165.8 & 8575 & 2105 & I-RD-39 \\
\hline Phenol, Molten / 94.1 & 53500 & 13132 & $1-\mathrm{RD}-40$ \\
\hline Propagyl Alcohol / 56.1 & 71 & 17 & 1-RD-4l \\
\hline Propionic Acid /74.1 & 3600 & 883 & $1-\mathrm{RD}-42$ \\
\hline Rodine 50 (formaldehyde) $/ 30.0$ & 226 & 55 & $1-\mathrm{RD}-43$ \\
\hline Sulfuric Acid /98.1 & $>100000$ & 24546 & 1-RD-44 \\
\hline Sulfuric Acid, Fuming / 98.1 & 437 & 107 & 1-RD-45 \\
\hline Tetrahydrofuran /72.1 & 13300 & 3264 & 1-RD46 \\
\hline Toluene $/ 92.1$ & 7730 & 1897 & 1-RD-47 \\
\hline Toluidine-o / 107.2 & 73000 & 17919 & $1-\mathrm{RD}-48$ \\
\hline Trichloroethane-1,1,1/133.4 & 6060 & 1487 & $1-\mathrm{RD}-49$ \\
\hline Trichloroethylene /131.4 & 5760 & 1414 & 1-RD-50 \\
\hline Triethylamine / 101.22 & 20 & 5 & 1-RD-51 \\
\hline Vinyl Acetate /86.1 & 225 & 55 & $1-\mathrm{RD}-52$ \\
\hline Xylenes & $>32140$ & 7889 & 1-RD-53 \\
\hline
\end{tabular}


Table 8.1 Cont. Chemical Spill Target Weight for Site Area Emergencies

\begin{tabular}{|l|c|c|c|}
\hline Chemical & Weight in Pounds & Gallons & Release Designation \\
\hline Benzoic Acid & 3000 & NA & $1-R D-54$ \\
\hline Magnesium Oxide /40.3 & 5100 & NA & I-RD-55 \\
\hline Silica (as respirable dust) & 20 & NA & $1-\mathrm{RD}-56$ \\
\hline Sodium Sulfhydrate /56.06 & 160 & NA & $1-R D-57$ \\
\hline Titanium Dioxide /79.9 & 5100 & NA & $1-$ RD-58 \\
\hline Ammonia / 17.0 & 77 & NA & $1-$ RD-59 \\
\hline Chlorine /70.9 & 3 & NA & $\cdot 1-$ RD-60 \\
\hline Trimethylamine & 6.7 & NA & $1-$ RD-61 \\
\hline
\end{tabular}

\subsubsection{Radiological Releases}

Transportation Accidents resulting in

- Containers MW Waste - Alpha (waste) ruptured as a result of impact

1-RD-76

- Containers of ILNTV Combustibles (E Area Vaults) ruptured as a result of impact

1-RD-80

- Containers of LNTV Noncombustibles (E Area) burning, or ruptured as a result of impact 1-RD-81 \& 1-RD-82

- ILTV Bulk containers (E Area Vaults) ruptured as a result of impact $1-R D-84$

- $\mathrm{PuO}_{2}$ Containers (235F and 247F) burning, or ruptured as a result of impact 1-RD-89 \& 1-RD-90

- H Canyon Sample Container (221H to SRTC) burning, or ruptured as a result of impact 1-RD-91 \& 1-RD-92

- F Canyon Sample Container (221H to SRTC) burning, ruptured as a result of impact l-RD-101 \& 1-RD-102

- HB Line Sample Container (221H to $772-1 \mathrm{~F})$ ruptured as a result of impact 1-RD-104

- HB Line Sample Container (221H to SRTC) ruptured as a result of impact 1-RD-106

- FB Line Oxide Container ( $221 \mathrm{~F}$ to Inter Area) burning, or ruptured as a result of impact 1-RD-107 \& 1-RD-108

- $235 \mathrm{~F}$ Material Container (235F to $221 \mathrm{H})$ ruptured as a result of impact 1-RD-112

- FB Line Sample Container ( $221 \mathrm{H}$ to SRTC) ruptured as a result of impact 1-RD-110

- $235 \mathrm{~F}$ Material Container $(235 \mathrm{~F}$ to $221 \mathrm{H})$ burning $1-R D-111$

- $235 \mathrm{~F}$ Material Container ( $235 \mathrm{~F}$ to $221 \mathrm{~F}$ ) burning 1-RD-113 
- 235F Material Container (235F to $221 \mathrm{~F}$ ruptured as a result of impact

1-RD-114

- SRTC Material Container (SRTC to DWPF) burning, or ruptured as a result of impact

1-RD-115 \& 1-RD-116

- UNH Trailer (H Area outside) burning, or ruptured as a result of impact

1-RD-119 \& 1-RD-120

- Container, Pail, Casket, Charge Can, Type A for Storage Vault (235-F) burning, or ruptured as a result of impact

1-RD-121 \& 1-RD-122

- RBOF Spent Deionizer Resin Transport (RBOF) burning

1-RD-125

- Moderator Transport in 55 gallon Drums (100-400 Areas) burning, or ruptured as a result of impact $1-R D-133 \& 1-R D-134$

- Contaminated Water Transport (100 Area to $211 \mathrm{~F}$ ) burning, or ruptured as a result of impact

$1-R D-137 \&$ 1-RD-138

\subsection{Alert}

\subsubsection{Chemical Releases}

Leakage or rupture of any container resulting in a spill producing a puddle with a surface area available for evaporation in the range of the target surface area in table 8.2 
Table 8.2 Chemical Spill Target Surface Areas for Alert Emergencies

\begin{tabular}{|c|c|c|c|}
\hline Chemical & $\begin{array}{l}\mathrm{ft}^{2} \text { Target Surface } \\
\text { Area }\end{array}$ & Gallons & Release Designation \\
\hline UNLISTED CHEMICAL & See DOT Guidebook & N/A & N/A \\
\hline Acetaldehyde / 44.1 & 60 & 15 & $1-\mathrm{RD}-1$ \\
\hline Acetic Acid & 115 & 2855 & $1-\mathrm{RD}-2$ \\
\hline Acetonitrile /41.I & 21 & 5 & $1-\mathrm{RD}-3$ \\
\hline Alpha-Pinene / 136.3 & 491 & 120 & $1-\mathrm{RD}-4$ \\
\hline Ammonium Hydroxide & \multicolumn{2}{|c|}{ Should have been screened out. } & $1-\mathrm{RD}-5$ \\
\hline Benzene /78.1 & 68 & 17 & $1-\mathrm{RD}-6$ \\
\hline Benzyl Alcohol & $>30000$ & 7362 & 1-RD-7 \\
\hline Benzyl Chloride & 730 & 179 & 1-RD-8 \\
\hline Butyraldehyde & 84 & 21 & $1-\mathrm{RD}-9$ \\
\hline Carbon disulfide $/ 76.1$ & 4.4 & 1 & $1-R D-10$ \\
\hline Chloracetone /92.5 & 0.5 & 1 & $1-R D-11$ \\
\hline Chlorosulfonic Acid / 116.52 & $>30000$ & 7364 & $1-\mathrm{RD}-12$ \\
\hline Cyclohexane /84.2 & 1045 & 257 & $1-R D-13$ \\
\hline Cyclohexanone /98.2 & 7750 & 1902 & $1-R D-14$ \\
\hline Diethylene Glycol / 106.1 & $>30000$ & 7362 & $1-R D-15$ \\
\hline Dioxane $/ 88.1$ & 98 & 24 & $1-\mathrm{RD}-16$ \\
\hline Diphenylmethane-4,4-Dilisocyanate/ 250 & 17237 & 4260 & $1-\mathrm{RD}-17$ \\
\hline Ethyl Acetate / 88.I & 1885 & 463 & $1-\mathrm{RD}-18$ \\
\hline Ethyl benzene / 106.2 & 3300 & 810 & 1-RD-19 \\
\hline Ethylene Glycol Monobutyl Ether & $>30000$ & 7362 & I-RD-20 \\
\hline Fluorosilicic Acid / 144.1 & 24600 & 6037 & $1-\mathrm{RD}-21$ \\
\hline Formic Acid / 46.0 & 7 & 2 & $1-\mathrm{RD}-22$ \\
\hline Glycol Ether DE / 134.2 & 8750 & 2147 & 1-RD-23 \\
\hline Hydrochloric Acid $31 \% / 36.5$ & 3230 & 793 & 1-RD-24 \\
\hline Hydrofluoric Acid $70 \% / 20.0$ & 24600 & 6038 & $1-R D-25$ \\
\hline Hydrogen Peroxide $/ 34.0$ & 5175 & 1270 & $1-\mathrm{RD}-26$ \\
\hline Isopropyl Alcohol 60.1 & 848 & 208 & I-RD-27 \\
\hline Methyl Alcohol /32.0 & 1115 & 274 & 1-RD-28 \\
\hline Methyl Ethyl Ketone /72.1 & 250 & 61 & 1-RD-29 \\
\hline Methyl Isobutyl Ketone / 00.2 & 6034 & 1481 & $\mathrm{I}-\mathrm{RD}-30$ \\
\hline Methylene Chloride / 84.9 & 96 & 24 & $1-R D-31$ \\
\hline Methyol Cellosolve /76.1 & 145 & 35.6 & $1-\mathrm{RD}-32$ \\
\hline Monoethanolamine /61.1 & 18500 & 4540 & 1-RD-33 \\
\hline Morpholine / 87.1 & 191 & 47 & $1-R D-34$ \\
\hline NButyl Alcohol / 74.1 & 288 & 71 & $1-R D-35$ \\
\hline
\end{tabular}


Table 8.2 cont. Chemical Spill Target Surface Areas for Alert Emergencies

\begin{tabular}{|l|c|c|c|}
\hline Chemical & $\begin{array}{l}\mathrm{ft}^{2} \text { Target Surface } \\
\text { Area }\end{array}$ & Gallons & Release Designation \\
\hline NMethyl Pyrrolidone /99.15 & $>30000$ & 7362 & $1-\mathrm{RD}-36$ \\
\hline Nitric Acid / 63.0 & 12 & 3 & $1-\mathrm{RD}-37$ \\
\hline Onyxide & See DOT Guidebook & $\mathrm{N} / \mathrm{A}$ & $1-\mathrm{RD}-38$ \\
\hline Perchloroethylene /165.8 & 1175 & 288 & $1-\mathrm{RD}-39$ \\
\hline Phenol, Molten /94.1 & 4550 & 1117 & $1-\mathrm{RD}-40$ \\
\hline Propagyl Alcohol /56.1 & 6 & 1 & $1-\mathrm{RD}-41$ \\
\hline Propionic Acid /74.1 & 283 & 69 & $1-\mathrm{RD}-42$ \\
\hline Rodine 50 (formaldehyde)/30.0 & 18 & 4 & $1-\mathrm{RD}-43$ \\
\hline Sulfuric Acid /98.1 & $>30000$ & 7364 & $1-\mathrm{RD}-44$ \\
\hline Sulfuric Acid, Fuming/98.1 & 37 & 9 & $1-\mathrm{RD}-45$ \\
\hline Tetrahydrofuran /72.1 & 425 & 104 & $1-\mathrm{RD}-46$ \\
\hline Toluene /92.1 & 1810 & 444 & $1-\mathrm{RD}-47$ \\
\hline Toluidine-0 /107.2 & 6260 & 1537 & $1-\mathrm{RD}-48$ \\
\hline Trichloroethane-1,1,1/133.4 & 690 & 169 & $1-\mathrm{RD}-49$ \\
\hline Trichloroethylene /131.4 & 522 & 128 & $1-\mathrm{RD}-50$ \\
\hline Triethylamine /101.22 & 1.7 & 0.4 & $1-\mathrm{RD}-51$ \\
\hline Vinyl Acetate /86.1 & 25.6 & 6 & $1-\mathrm{RD}-52$ \\
\hline Xylenes & 6720 & 1649 & $1-\mathrm{RD}-53$ \\
\hline
\end{tabular}

Table 8.2 Chemical Spill Target Weight for Alert Emergencies

\begin{tabular}{|l|c|c|c|}
\hline Chemical & Weight in Pounds & Gallons & Release Designation \\
\hline Benzoic Acid & 285 & NA & I-RD-54 \\
\hline Magnesium Oxide /40.3 & 490 & NA & I-RD-55 \\
\hline Silica (as respirable dust) & 1.9 & NA & $1-\mathrm{RD}-56$ \\
\hline Sodium Sulfhydrate /56.06 & 15 & NA & $1-R D-57$ \\
\hline Titanium Dioxide /79.9 & 490 & NA & $1-R D-58$ \\
\hline Ammonia /17.0 & 7 & NA & $1-R D-59$ \\
\hline Chlorine /70.9 & 0.3 & NA & $1-R D-60$ \\
\hline Trimethylamine & 1.3 & NA & $1-\mathrm{RD}-61$ \\
\hline
\end{tabular}




\subsubsection{Radiological Releases}

Transportation Accidents resulting in

- Containers of MW Waste - Alpha (waste) burning 1-RD-75

- Containers TRU waste leaking as a result of impact 1-RD-78

- Containers of ILNTV Combustibles (E Area Vaults) burning

1-RD-79

- ILTV Bulk Container (E Area Vaults) burning 1-RD-83

- Containers of ILNTV Combustibles (E Area Vaults) leaking as a result of impact 1-RD-98

- HB Line Sample Container (221H to $772 \mathrm{1F})$ burning 1-RD-103

- HB Line Sample Container (221H to SRTC) burning 1-RD-105

- FB Line Sample Container (221H to SRTC) burning 1-RD-109

- RBOF Portable Deionizer Column Transport (RBOF) burning 1-RD-123

- $\quad$ RBOF Spent Deionizer Resin Transport (RBOF) leaking as a result of impact 1-RD-126 


\subsection{EMERGENCY PLANNING ZONE (EPZ) DETERMINATION}

The facility EPZ is defined as an area within which special planning and preparedness efforts are warranted as a means of apportioning preparedness resources. The methodology used in determining the EPZ is outlined in EMPP 6Q-001 (Ref. 1).

The EMG (Ref. 3) directs the EPZ for a site should not be extended beyond the site boundary solely on the basis of potential consequences of a transportation accident if transportation activity is comparable (in terms of materials, quantities, and mode of shipment) to that normally conducted on public routes. Transportation activities analyzed in this EPHA meet this test, therefore, The EPZ is the site boundary and does not require calculation for public routes. 


\subsection{REFERENCES}

1. Hadlock, D. J., Standards For Development and Maintenance of a Facility Hazards Assessment, 6Q Vol. 2, EMPP 6Q-001, Rev. 1, November 1, 1995.

2. Proposed Phase 1Scope for Transportation EPHA (U), ECS-EST-95-0021, Inter Office Memorandum, Westinghouse Savannah River Company, Savannah River Site, 03/20/95.

3. Emergency Management Guide - Vol. II, Hazards Surveys and Hazards Assessment, DOE G 151.1-1, U. S. Department of Energy (DOE), August 1997.

4. Code of Federal Regulations, 40 CFR - Transportation, National Archives and Records Administration, Washington, DC, 1992.

5. Nadeau, M., L. and Henderson, S., A., Electronic Screening of Chemical Inventory Information (U), SESR-G-00001, Rev. 0, Westinghouse Savannah River Company, Aiken SC, 12/28/93.

6. Evaluation of Accident Risks in the Transportation of Hazardous Materials by Truck and Rail at the Savannah River Site (U), WSRC-RP-89-715, Rev. 1, Westinghouse Savannah River Company, Aiken, SC, September 1992.

7. Code of Federal Regulations, Quantities of Radioactive Materials Requiring Consideration of the Need for an Emergency Plan for Responding to a Release., 10 CFR 30.72 Schedule C, National Archives and Records Administration, Washington, DC, July 1, 1991.

8. DOE Standard, Hazard Categorization and Accident Analysis, DOE-STD-1027-92, U. S. Department of Energy 2 December 1992.

9. Moyer, R.A., SP91-039, SRTS Packagings for Onsite Transportation of Radioactive Materials, March 29, 1991, Savannah River Laboratory, SAIC to Westinghouse Savannah River Company, Aiken, South Carolina 29808.

10. Evaluation of Accident Risks in the Transportation of Hazardous Materials by Truck and rail at the Savannah River Site (U)," WSRC-RP-89-715, Revision 1, September 1992

11. Hazards Assessment for HWSF \& MWSF, S-EHA-G-00001, Revision 1, October, 1996.

12. Emergency Preparedness Hazards Assessment for the Central Laboratory Facility, S-EHA-F-00001, Buildings 772-F, 772-1F, and 772-4F (U), Rev. 1, August 26, 1997.

13. K and L Areas (old Reactor Areas), S-EHA-K-00001, Revision 0, April 1998.

14. Emergency Preparedness Hazards Assessment for the RBOF/RRF Facility (U), S-EHA-H-00003, Rev. 1, $07 / 98$.

15. Emergency Preparedness Hazards Assessment for the Consolidated Incineration Facility(CIF) / Solvent Storage Facility (SSF) / Effluent Treatment Facility (ETF) / Saltstone (U), S-EHA-G-00004, Revision 0, March 31, 1998. 
16. Emergency Preparedness Hazards Assessment For the ITP/ESP Facility (U), S-EHA-H-00002, Revision 0, July 20, 1995.

17. SRS Intra-Site Transportation Radiological and Toxicological Sabotage Vulnerability Assessment (Secret), WSRC-RP-95-624, May 1995, Westinghouse Savannah River Company, Savannah River Site, Aiken SC 29808.

18. Savannah River Site Waste Acceptance Criteria Manual, Procedure Manual 1S, 1/17/95, Westinghouse Savannah River Company, Savannah River Site, Aiken, SC 29808.

19. Areal Locations of Hazardous Atmospheres (ALOHA), Version 5.2.1S, NOAA and EPA, June 1995.

20. Homann, S. G., Hazards Control Department \& the Emergency Preparedness and Response Program, Nonproliferation, Arms Control and International Security Directorate, HOTSPOT Health Physics Codes for the PC; Lawrence Livermore National Laboratory UCRL-MA-106315, March 1994.

21. Hadlock, D. J., Consequence Assessment for Emergency Preparedness Hazards Assessments, TP-95-002 Rev. 3, January 20, 1997.

22. Guidance for Event Classification and Emergency Action Levels $s_{2}$ DOE Emergency Management Guide, U.S. Department of Energy, Washington, DC, June 26, 1992.

23. Craig, D. K., Integrated ERPG or ERPG Equivalent List of Chemicals, EPD-CAT-94-0060, Rev. 1, Westinghouse Savannah River Company, Savannah River Site, Aiken, SC, September 28, 1994.

24. Craig, D. K., ERPG-Equivalent Concentration-Limit Values for 83 Selected Chemicals, SRT-RAM940037, Westinghouse Savannah River Company, Savannah River Site, Aiken, SC, March 29, 1994.

25. Craig, D. K., Description of Methodology for Deriving ERPG-equivalents for Chemicals, S-CLC-G00074, Rev. 0 and SWMF Chemicals on "Master File" Requiring Independent Verification, S-CLC-G00079 Rev. 0, Westinghouse Savannah River Company, Aiken, SC, July 10, 1995.

26. Standards for Development and Maintenance of Emergency Action Level (EAL) Procedures ${ }_{2}$ Manual 6Q Vol. 2, EMPP 6Q-002, Rev. 1, December 2, 1996.

27. D. K. Craig, ERPGs and TEELs for Chemicals of Concern, WSMS-SEA-99-00101, Rev 14, August 4, 1998. Addendum to Rev. 14, ERPGs and TEELs for Chemicals of Concern, WSMS Inter-Office Memorandum, WSMS-SAE-M-98-00100, September 3, 1998.

28. A Guidebook for First Responders During the Initial Phase of a Hazardous Materials / Dangerous Goods Incident - 1996 North American Emergency Response Guidebook, U.S. Department of Transportation Research and Special Programs Administration.

29. Emergency Preparedness Hazards Assessment for the E-Area Vaults Facility (U), N-HAD-E-00001, Rev. 1, October 1996. 
Figure 1

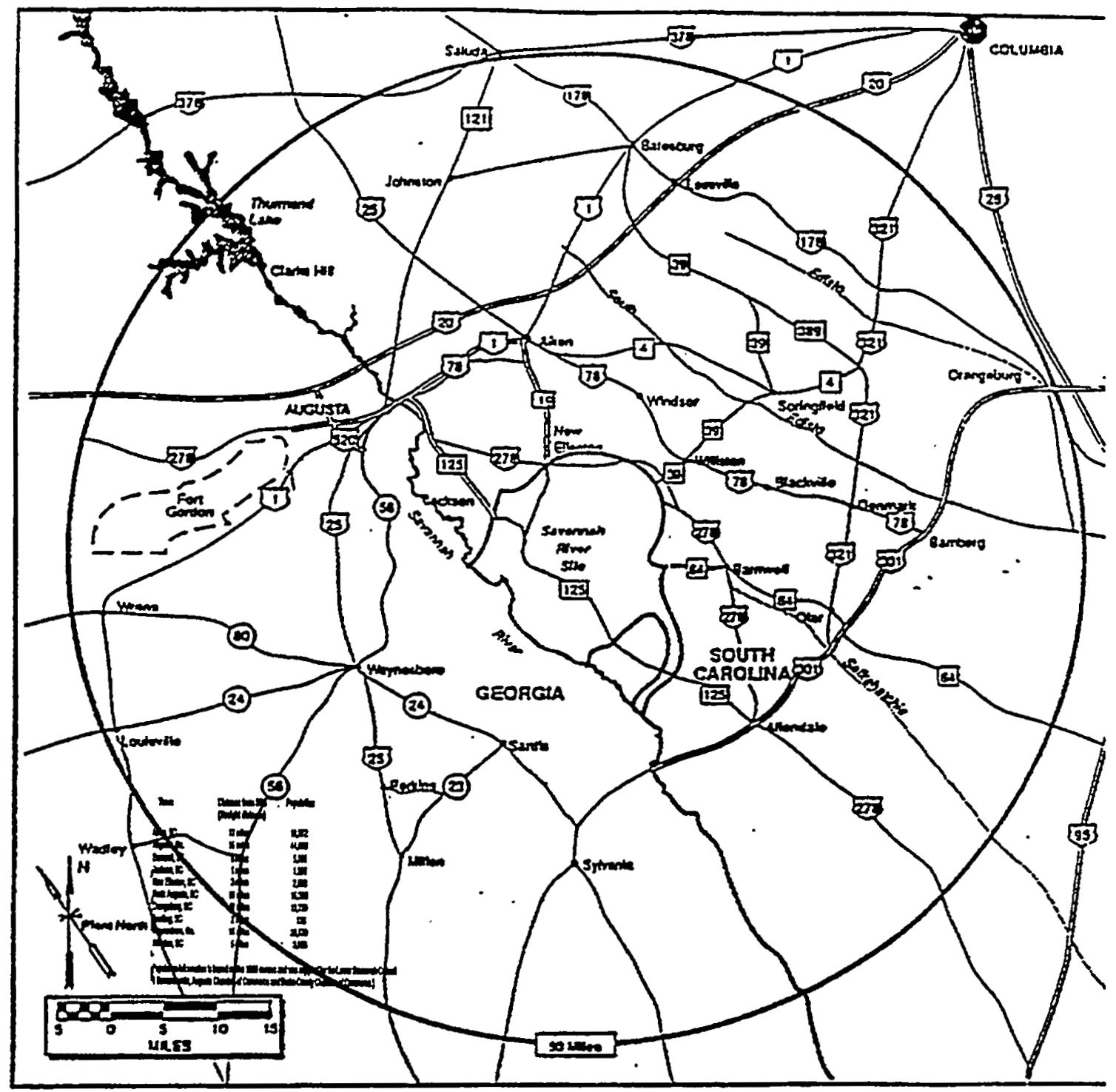


Figure 2 Isodose Concept

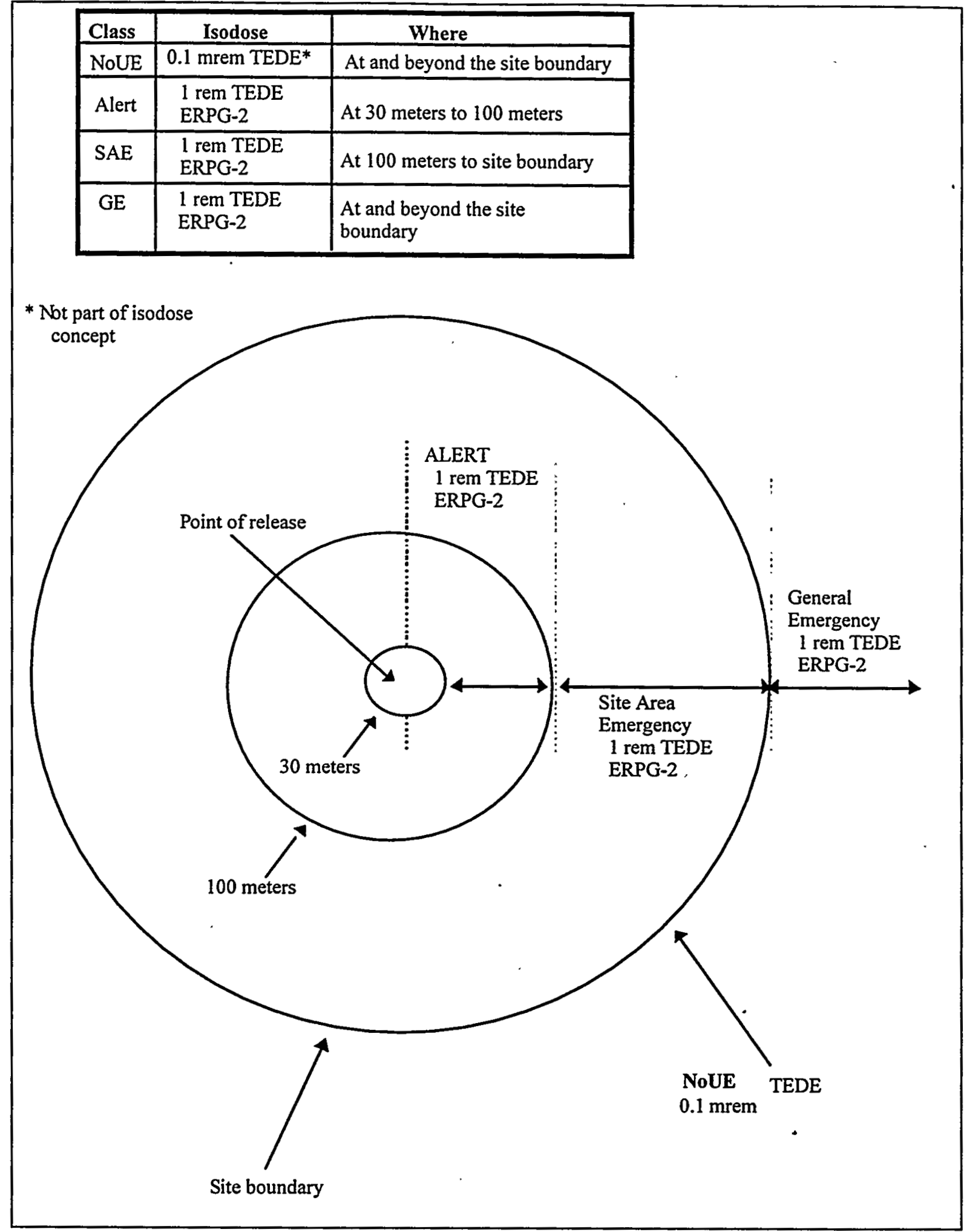


S-EHA-G-00003

EPHA for the Transportation of Hazardous Materials

Rev. 1

October 11,1996

Page A-4 of 3 


\section{APPENDIX B}

\section{CALCULATIONS}

Calculation 1

Calculation 2

Calculation 3

Calculation 4

Calculation 5

Calculation 6

Calculation 7
CIIS Chemical Screening

Inventory, Screening/Characterization of External Hazards

Characterization/Screening of THM EPHA Chemicals

Screening of Radionuclides

Source Terms and Release Designation for Radionuclides

Radiological Consequence Assessment

Concentration Analysis for THM Hazardous Chemicals 
This page intentionally left blank. 
Title of Project THM EPHA

Reviewed by S. A. Henderson

Subject Inventory, Screening/CIIS Chemical s

Works Calculation 1

Computer M. Nadeau

Date 09/14/95

Sheet No. 1 of 5 (163 pp attch)

\section{INTRODUCTION}

This calculation presents the results of a review of the Savannah River Site (SRS) chemical inventory as reported on the Chemical Information and Inventory System (CIIS) database. The purpose of the review is to eliminate chemicals with insignificant hazards as defined in the Emergency Management Program Procedure (EMPP) 6Q-001 (Ref. 1).

This review was concerned with evaluating the chemicals as an airborne chemical hazard to humans. The materials were not evaluated to determine if they represented a non-airborne hazard (e.g. liquid spill into a water supply) or if they represent a potential initiator or promoter of a release of some other material, e.g., a flammable chemical or chemicals that may form hazardous reaction products. In addition, this review evaluates materials in the chemical state they were purchased. It does not include chemicals created as part of the process nor chemicals considered to be waste.

The chemical inventory entries from the CIIS that will require further characterization are summarized in Calculation 3. The remaining chemical inventory entries were eliminated as insignificant hazards.

\section{DISCUSSION / ASSUMPTIONS}

Engineering Studies Report S-ESR-G-00001 presents the methodology used to review the chemical inventory information (Ref. 2). It presents guidance on how to obtain chemical inventory information from the CIIS, the loading of the information into a FILEMAKER-PRO database and the screening of the information to determine if any chemicals require further evaluation.

The "Reasons" referred to in the "Screening Comments" of the FILEMAKER-PRO databases correspond to portions of Section A.3.e(1) of EMPP 6Q-001 as follows:

(a) Chemical is not present in quantities greater than a Technical Programs Screening Quantity (TPSQ) established for that material. A current list of TPSQs is available from Emergency Services Technical Programs-Safety Analysis Engineering Section (ESTP-SAES).

(b) Chemical has already been identified as posing an insignificant hazard by ESTP- SAES, and there is nothing to indicate that a more detailed evaluation is required.

(c) One or more of the following is valid:

- A "zero" amount is presented as the maximum quantity in the CIIS report and it has been verified that the use of the material has been discontinued by the facility

- The material is identified as a sample

- The material is identified as part of the radionuclide inventory

- The material is used in a laboratory setting and in laboratory quantities 
Title of Project THM ĖPHA

Subject Inventory, Screening/CIIS Chemical s

Reviewed by S. A. Henderson

Computer M. Nadeau

Date 09/14/95

Works Calculation 1

Sheet No. 2 of 5 (163 pp attch)

(d) One or more of the following is valid:

- The material is commonly used in industry and/or by the general public. This includes any substance to the extent it is used for personal, family or household purposes or is present in the same form and concentration as a product packaged for distribution and use by the general public, e.g., motor oil, gasoline, diesel fuel.

- The material is a true solid,i.e., not a finely divided powder under normal circumstances and does not present an airborne concern.

- The material is not hazardous to humans as a result of inhalation.

- The material has a vapor pressure of $\leq 0.5 \mathrm{~mm} \mathrm{Hg} @ 25^{\circ} \mathrm{C}$ and an ERPG-2 or equivalent $\geq 1$ ppm.

\section{CALCULATION}

1. Approximately 44,000 entries were compiled from the CIIS for the SRS. To reduce the number of chemicals to be analyzed to a manageable quantity, only chemicals reported in $1000 \mathrm{lbs}$ or greater are analyzed in this calculation (approximately 1600 entries).

2. This screening does not include chemicals considered to be hazardous waste.

3. Other materials and hazard sources, such as flammable or explosive materials and energy sources are not considered if they do not exhibit acute or chronic toxicity via the inhalation pathway. Consideration of their potential for initiating releases of radioactive or chemically toxic materials, contributing to dispersal of those materials, or degrading the effectiveness of safety systems is considered during barrier identification and event scenario development.

4. This review was concerned with evaluating the chemicals as an airborne chemical hazard to humans. The materials were not evaluated to determine if the chemical represents a non-airborne hazard, e.g., liquid spill into a water supply.

5. This review evaluates materials in the chemical state they were purchased. It does not include chemicals created as part of a site process.

6. Previously Analyzed Chemical Identification

The FILEMAKER-PRO database ESPAC.FM presents a list of the chemicals which have been defined by ESTP as being an "Insignificant Hazard" (Ref. 3). This database was used to identify the materials already analyzed in the chemical inventory information from previously conducted EPHAs. 
Title of Project THM EPHA

Subject Inventory, Screening/CIS Chemical s

Computer M. Nadeau

Date 09/14/95

Reviewed by $\underline{\text { S. A. Henderson }}$

Works Calculation 1

Sheet No. 3 of 5 (163 pp attch)

\section{RESULTS}

A FILEMAKER-PRO database of the chemical inventory from the CIIS in accordance with the guidance in S-ESR-G-00001 and EMPP-001 (Refs. 1,2). The buildings associated with each areas were identified and all listings for those buildings were imported into a new database, named "(area)DUMP.FM" with more than 8,800 records that were matched with Material Safety Data Sheets (MSDS) initially.

The screening of the chemical inventory was conducted, using the criteria in EMPP-001, as follows:

(a) Eliminate a chemical if it is not present in quantities greater than a Technical Programs Screening Quantity (TPSQ) established for that material (Refs. 4, 5). An approved list of TPSQs is maintained by ESTG. All entries where the total amount in a building was below the TPSQ for that material were exported to a database named "TRANTPSQ.FM."

(b) Eliminate a chemical if it has been previously analyzed to be insignificant hazards and there is nothing to indicate that a more detailed evaluation is required (e.g., the material is present in greater than end-user quantities). The listings in (area)DUMP.FM were compared to the Emergency Services Previously Analyzed Chemical (ESPAC.FM) database to find those materials already identified from previously conducted EPHA's as posing insignificant threats (Ref. 3). All previously identified materials were transferred to a database named "TRANPAC.FM".

(c) Eliminate the chemical if one or more of the following is valid:

- A "zero" amount is shown as the maximum quantity in the CIIS report and it is verified that the use of the chemical has been discontinued by the facility or inventory is $\leq 0.5 \mathrm{lbs}$.

- The material is identified as a sample.

- The material is used in a laboratory setting and in laboratory scale quantities. Materials whose maximum amount at a given location or segment was under approximately twenty pounds were designated as being a laboratory quantity. A database named "TRANLABQ.FM" was created.

(d) Propose elimination of the chemical if it satisfies one or more of the following criteria. Upon completion of the previous screening steps MSDSs and references were consulted to determine the properties of the chemicals remaining in the database TRANDUMP.FM. The below listed criteria was utilized to determine if a material should be screened or retained for further evaluation.

- The material is commonly used in industry and/or by the general public: Materials such as vehicle fuel and common industrial solvents are normally screened. These materials can be event initiators for hazardous materials in close proximity. Consider and document their possible effects on other more hazardous materials, e.g., as initiators or promoters of a release. This includes any substance to the extent it is used for personal, family, or household purpose or is present in the same form and concentration as a product packaged for distribution and use by the general public, e.g., motor oil, gasoline, diesel fuel. Materials that identified as being a common material were transferred to a database named "TRANCOM.FM." Materials proposed as common are reviewed by the Hazard Assessment Team and ESTP-SAES. Materials accepted as common are added to the data base of common materials maintained by ESTP-SAES. 
Title of Project THM EPHA

Subject Inventory, Screening/CIIS Chemicals

Computer M. Nadeau

Date 09/14/95

Reviewed by S. A. Henderson

Works Calculation 1

Sheet No. 4 of 5 (163 pp attch)

- The material is a true solid, i.e., not a finely divided powder under normal circumstances and does not present an airborne concern.

- The material cannot cause harm via the inhalation pathway from an acute exposure. Materials such as sodium hydroxide solution that do not fit the conventional definition of "hazards" for airborne human heaith or safety impacts, could cause a significant environmental impact. Consider the effects on the environment, the necessary response actions, and the time within which actions need to be taken when determining whether to include such materials within the scope of the hazard assessment for a given facility.

- The material has a vapor pressure of $\leq 0.5 \mathrm{~mm} \mathrm{Hg} @ 25^{\circ} \mathrm{C}$ and an ERPG-2 or equivalent of $\geq 1 \mathrm{ppm}$

Materials exhibiting the above qualities were transferred to a database named "TRANDEL.FM".

The final set of records that could be eliminated using screening criteria were transferred to a database named "1kinventory.FM".

Filemaker Pro data sheets 1 through 163 (attachment) list the screening results for the CIIS Database. Materials not screened shall undergo further evaluation and characterization in Calculation 3 to determine if they require hazards analysis.

\section{REFERENCES}

1. Standards for Development and Maintenance of Hazard Assessments. EMPP 6Q-001, Revision 1, Manual 6Q, Vol. II, November 1, 1995.

2. Nadeau, M. L. and Henderson, S. A., Electronic Screening of Chemical Inventory Information (U). S-ESR-G-00001, Revision 0, February 28, 1993.

3. Nadeau, M. L., Implementation of the Emergency Services Previously Analyzed Chemical Database. EPD-EST-94-0069,January 24, 1995.

4. Henderson, S. A., Technical Program Screening Quantity (TPSQ) Values For Use In Facility Hazards Assessment (U). S-ESR-G-00002, Revision 0, May 23, 1995.

5. Henderson, S. A., Technical Programs Screening Quantity For Methyl Isobutyl Ketone, $\left(\mathrm{CH}_{3}\right)_{2} \mathrm{CHCH}_{2} \mathrm{COCH}_{3}(U)$. EPD-EST-94-0023, August 7, 1994. 


\section{ENGINEERING COMPUTATION SHEET}

Title of Project THM EPHA

Reviewed by S. A. Henderson

Subject Inventory, Screening/ CIIS Chemical s

Works Calculation 1

Computer M. Nadeau

Date 09/14/95

Sheet No. 5 of 5 (163 pp attch)

Attachment

Site Chemical Screening Results (163 pages) 
Title of Project EPHA for THM

Subject Inventory. Screening/Characterization of External Hazards

Computer S. A. Henderson
Reviewed by D. J. Hadlock

Works Salculation 2

Sheet No. 1 of 11

\section{INTRODUCTION}

This calculation tabulates the inventories of chemical materials transported to and/or across the site based on the information received from a representative sampling of transporters moving hazardous materials in the area. This calculation applies the screening methodology as per procedure 6Q-001 (Ref. 1) resulting in a tabulated listing of chemicals to be further evaluated in the Transportation of Hazardous Materials (THM) Emergency Preparedness Hazards Assessment (EPHA). Each of the chemicals requiring further evaluation will be evaluated by EPHA segment.

\section{DISCUSSION/ASSUMPTIONS}

Requests for inventories (Ref. 2) of all hazardous materials transported to and/or through the site were made of a representative sample of transporters in the area and/or known to transport across the site boundary. Of the twenty requests made, fourteen replies were received. Of these fourteen replies (Sheets 10 through 104), one was from CSX transportation and another from Van Waters \& Rogers. These two companies collectively reported in excess of six hundred $(600)$ separate hazardous chemicals transported across the site. The other eleven respondents, on average reported less than four hazardous chemicals. It is assumed the information provided by the fourteen respondents is correct and is a representative sampling of the non-DOE owned chemicals that may cross the site. The Chemical Abstract Service (CAS) registry numbers indicated are those supplied by the transporter and randomly verified utilizing SAX (Ref. 3). Materials listed from the transporters with multiple CAS registry numbers indicate the material is made up of several constituents with the CAS registry numbers representing the primary materials. These materials were separated and evaluated as individual chemicals, as it would be impossible to ascertain the quantity in each solution on any specific load. Certain items with multiple CAS registry numbers may be screened as common industrial materials (Ref. 4) i. e. gasoline, although their independent CAS registry numbers would indicate a mixture of chemicals that would not screen in pure form.

\section{CALCULATION}

The replies received (see correspondence Appendix - C) present the hazardous chemical inventories as shown in the table below, from each of the respondents. The information from these inventories was tabulated and sorted by CAS registry number, as this is the only unique identifier for each of the chemicals presented. Duplicate entries were eliminated and the remaining names and CAS registry numbers were utilized to present the following table of chemicals reported by the representative sampling of transporters as crossing the site boundary. The table lists the chemical name, CAS registry number, and an indication whether material is screened from further analysis per $6 Q$ 001 (Ref. 1) with the reason indicated. Because multiple containers could be involved in the same load it was assumed quantities could equal the maximum volume of the shipping devise, therefore TPSQ screening will be performed by segment only in the specific segments outlined in the THM EPHA. The SAX (Ref. 3) "Entry Number" is provided for reference purposes where applicable.

\begin{tabular}{|l|r|c|c|l|}
\hline Chemical Name & $\begin{array}{c}\text { CAS registry } \\
\text { number }\end{array}$ & $\begin{array}{c}\text { SAX Entry } \\
\text { number }\end{array}$ & $\begin{array}{c}\text { Screened from } \\
\text { further analysis } \\
(x)\end{array}$ & $\begin{array}{l}\text { Reason for screening } \\
(\text { Ref. 3, 5, 6, \& 7) }\end{array}$ \\
\hline Acetaldehyde & $75-07-0$ & AAG250 & & \\
\hline Acetic Acid & $64-19-7$ & AAT250 & & \\
\hline Acetone & $67-64-1$ & ABC750 & $X$ & Common Material \\
\hline
\end{tabular}


Title of Project EPHA for THM

Subject Inventory. Screening/Characterization of Extemal Hazards

Computer $\underline{\mathrm{S}}, \mathrm{A}, \mathrm{Henderson}$

Date $09 / 14 / 95$
Reviewed by D. J. Hadlock

Works Calculation 2

Sheet No. 2 of 11

\begin{tabular}{|c|c|c|c|c|}
\hline Chemical Name & $\begin{array}{c}\text { CAS registry } \\
\text { number }\end{array}$ & $\begin{array}{l}\text { SAX Entry } \\
\text { number }\end{array}$ & $\begin{array}{l}\text { Screened from } \\
\text { further analysis } \\
(\mathrm{x})\end{array}$ & $\begin{array}{l}\text { Reason for screening } \\
\text { (Ref. } 3,5,6, \text { \& } 7 \text { ) }\end{array}$ \\
\hline Acrylamide & $79-06-1$ & ADS250 & $\mathrm{X}$ & Solid in pure form \\
\hline Activated Carbon & $7440-44-0$ & CBT500 & $\mathrm{X}$ & Common Non-Hazardous Material \\
\hline Adipic Acid & $124-04-9$ & AEN250 & $\mathrm{X}$ & Solid in pure form \\
\hline Aerosol 501 & $37294-49-8$ & $\mathrm{DJCOOO}$ & & \\
\hline Aerosol A-102 & $39354-45-5$ & CAKOOO & & \\
\hline Aerosol QTS & $64741-65-7$ & - & & \\
\hline Alpha-Pinene & $80-56-8$ & PIH250 & & \\
\hline Aluminum Hydroxide & $21645-51-2$ & $\mathrm{AHCOOO}$ & $\mathrm{X}$ & Solid in pure form \\
\hline Aluminum Sulfate & $10043-01-3$ & AHG750 & $\mathrm{X}$ & Solid in pure form \\
\hline Ammonia & $7664-41-7$ & AMY500 & & \\
\hline Aqua ammonia liquid & $1336-21-6$ & ANK250 & & \\
\hline Ammonium Bicarbonate & $1066-33-7$ & ANB250 & $\mathbf{X}$ & Solid in pure form \\
\hline Ammonium Bifluoride & $1341-49-7$ & ANJ000 & $\mathrm{X}$ & Solid in pure form \\
\hline Ammonium Hydroxide & $1336-21-6$ & ANK250 & & \\
\hline Ammonium Nitrate & $6484-52-2$ & ANNOOO & $\bar{X}$ & Solid in pure form \\
\hline Ammonium Persulfate & $7727-54-0$ & ANR000 & $\mathbf{X}$ & Solid in pure form \\
\hline Ammonium Sulfate & $7783-20-2$ & ANU750 & $\mathbf{X}$ & Solid in pure form \\
\hline Ammonyx & $8001-54-5$ & AFP250 & & \\
\hline Amphosol & $139-10-6$ & AOB500 & $\mathrm{X}$ & Solid in pure form \\
\hline Argon & $7440-37-1$ & AQW250 & $\mathrm{X}$ & Not a toxicological inhalation hazard \\
\hline Arlacel 80 & $8007-43-0$ & SKV100 & $\mathrm{x}$ & Solid in pure form \\
\hline Ascorbic Acid & $50-81-7$ & ARN000 & $\mathrm{x}$ & Solid in pure form \\
\hline Benzoflex 9-88 & 94-51-9 & DWS800 & $\mathbf{X}$ & Not an EPHA inhalation hazard \\
\hline Benzoic Acid & $65-85-0$ & BCL750 & & Respirable powder \\
\hline Benzyl Alcohol & $100-51-6$ & BDX500 & & \\
\hline Benzyl Chloride & $100-44-7$ & BEE375 & & \\
\hline Borates, tetra Sodium Salts & $1330-43-4$ & DXG035 & $\mathbf{x}$ & Solid in pure form \\
\hline Boric Acid & $10043-35-3$ & $\mathrm{BMCOOO}$ & $\mathbf{X}$ & Solid in pure form \\
\hline Brij 30 & $9002-92-0$ & DXY000 & & \\
\hline Brij 97 & $9004-98-2$ & OIG000 & $\mathrm{X}$ & Solid in pure form \\
\hline Bubble Breaker 748 & $64741-88-4$ & MQV850 & $\mathrm{X}$ & Common Material \\
\hline Butene Oxide-1 & $106-88-7$ & BOX750 & & \\
\hline Butyraldehyde & $123-72-8$ & BSU250 & & \\
\hline Calcium Carbonate & $471-34-1$ & CAT775 & $\mathrm{X}$ & Solid in pure form \\
\hline Calcium Hydroxide & $1305-62-0$ & CAT225 & $\mathrm{X}$ & Solid in pure form \\
\hline Calcium Hypochlorite & $7778-54-3$ & HOV 500 & $\mathrm{X}$ & Solid in pure form \\
\hline Calcium Oxide & $1305-78-8$ & CAU500 & $\mathbf{X}$ & Solid in pure form \\
\hline Carbon Dioxide & $124-38-9$ & CBU250 & $\mathrm{X}$ & Not a toxocological inhalation hazard \\
\hline Carbon Monoxide & $630-08-0$ & CBW750 & & \\
\hline Caustic Potash & $1310-58-3$ & PLJ500 & $\mathbf{X}$ & Solid in pure form \\
\hline Chloracetone & $78-95-5$ & $\mathrm{CDN} 200$ & & \\
\hline Chlorine & $7782-50-5$ & CDV750 & & \\
\hline Chloronitrobenzene & $25167-93-5$ & CJA950 & & \\
\hline Chlorosulfonic Acid & $7790-94-5$ & CLG500 & & \\
\hline Citric Acid & $77-92-9$ & CMS750 & $\mathrm{X}$ & Solid in pure form \\
\hline
\end{tabular}


ENGINEERING COMPUTATION SHEET

Title of Project EPHA for THM Reviewed by D. J. Hadlock Works Calculation 2

Subject Inventory. Screening/Characterization of External Hazards

Computer S. A. Henderson

Date 09/14/95

Sheet No. 3 of 11

\begin{tabular}{|c|c|c|c|c|}
\hline Chemical Name & $\begin{array}{l}\text { CAS registry } \\
\text { number }\end{array}$ & $\begin{array}{l}\text { SAX Entry } \\
\text { number }\end{array}$ & $\begin{array}{l}\text { Screened from } \\
\text { further analysis } \\
\text { (x) }\end{array}$ & $\begin{array}{l}\text { Reason for screening } \\
\text { (Ref. } 3,5,6, \& 7)\end{array}$ \\
\hline Copper Sulfate & $7758-99-8$ & CNP500 & & \\
\hline Cumene & $98-82-8$ & COE750 & 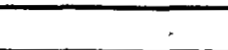 & \\
\hline Cyclohexane & $108-94-1$ & $\mathrm{CPCOO0}$ & & \\
\hline Di Sodium Sulfosuccinate & $577-11-7$ & DJLOOO & $\bar{x}$ & Solid in pure form \\
\hline Di-Tertobutyl-p-cresol-2,6 & $128-37-0$ & BFW750 & $\mathrm{X}$ & Solid in pure form \\
\hline Diammonium Phosphate & $7783-28-0$ & ANR500 & $\mathrm{X}$ & Solid in pure form \\
\hline Diatomaceous Earth & $68855-54-9$ & DCJ800 & $\mathrm{X}$ & Solid in pure form \\
\hline Dicalcium Phosphate & $7757-93-9$ & CAW100 & $\mathrm{X}$ & Solid in pure form \\
\hline Diesel Fuel & $68476-34-6$ & FOPOOD & $\mathbf{X}$ & Common material \\
\hline Diethanolamine & $111-42-2$ & DMF000 & $\mathrm{X}$ & Solid in pure form \\
\hline Diethyl Ethane Phosphonite & $2651-85-6$ & DJH500 & $\bar{x}$ & Not an EPHA inhalation hazard \\
\hline Diethylamine & $124-40-3$ & $\mathrm{DOQ} 800$ & & \\
\hline Diethylene Glycol & $111-46-6$ & DJD600 & & \\
\hline Dioxane & $123-91-1$ & DVQ000 & & \\
\hline $\begin{array}{l}\text { Diphenyimethane-4,4- } \\
\text { Diisocyanate }\end{array}$ & $101-68-8$ & MJP400 & & Melts @ 37.29 C \\
\hline Dipropylene Glycol & $25265-71-8$ & OQM000 & $\mathbf{X}$ & $\begin{array}{l}V_{p}<0.5 \& \text { not a severe inhalation } \\
\text { hazard }\end{array}$ \\
\hline Disodium Phosphate & $7558-79-4$ & SJH090 & $\mathbf{X}$ & Solid in pure form \\
\hline Dowfax 2A1 & $119345-04-9$ & & & \\
\hline Dowicide & $6152-33-6$ & BGJ750 & $\mathbf{X}$ & Solid in pure form \\
\hline Dowicil & $4080-31-3$ & CEG550 & $\mathrm{X}$ & Solid in pure form \\
\hline Dursban & $2921-88-2$ & CMA100 & & \\
\hline Ethyl Acetate & $141-78-6$ & EFR000 & & \\
\hline Ethyl Alcohol & $64-17-5$ & EFUOOO & $\mathrm{X}$ & Common material \\
\hline Ethyl Benzene & $100-41-4$ & EGP500 & & \\
\hline Ethylene & $75-85-1$ & EIO000 & $\mathrm{X}$ & Not a toxicological inhalation hazard \\
\hline Ethylene Glycol & $107-21-1$ & EJC500 & $\mathbf{x}$ & Common material \\
\hline Ethylene Oxide & $75-21-8$ & EJN500 & & \\
\hline $\begin{array}{l}\text { Ethylenediaminediacetic Acid } \\
\text { Tetra Sodium Salt -N,N }\end{array}$ & $64-02-8$ & EIV000 & $\bar{X}$ & Solid in pure form \\
\hline Ethylhexanol -2 & $104-76-7$ & EKQ000 & & \\
\hline Ferric Chloride & $7705-08-0$ & FAUO00 & $\mathbf{X}$ & Solid in pure form \\
\hline Ferrous Sulfate & $7720-78-7$ & FBN100 & $\mathrm{X}$ & Solid in pure form \\
\hline Ferrous Sulfate & $7782-63-0$ & FBOOO0 & $\mathrm{X}$ & Solid in pure form \\
\hline Fluorosilicic Acid & $16961-83-4$ & SCO500 & & \\
\hline Fluosilicic Acid 25\% & $16961-83-4$ & $5 \operatorname{COS} 00$ & & \\
\hline Forestall & $61791-34-2$ & - & & \\
\hline Formaldehyde & $50-00-0$ & FMV000 & & \\
\hline Formic Acid & $64-18-6$ & FNA000 & & \\
\hline Freon MCA & $76-13-14$ & FOO000 & $\mathbf{X}$ & Common material \\
\hline Fumaric Acid & $110-17-8$ & FOUO00 & $\mathrm{X}$ & Solid in pure form \\
\hline Gasoline & $8006-61-9$ & GBYOOO & $\mathrm{X}$ & Common material \\
\hline Glycerine & $56-81-5$ & GGA000 & & \\
\hline Glycol Ether DB & $112-34-5$ & DJF200 & $\mathrm{X}$ & Not an EPHA inhalation hazard \\
\hline
\end{tabular}


ENGINEERING COMPUTATION SHEET

Title of Project EPHA for THM

Subject Inventory. Screening/Characterization of External Hazards

Computer S.A. Henderson

Date 09/14/95
Reviewed by D. J. Hadlock

Works Calculation 2

Sheet No. 4 of 11

\begin{tabular}{|c|c|c|c|c|}
\hline Chemical Name & $\begin{array}{c}\text { CAS registry } \\
\text { number }\end{array}$ & $\begin{array}{l}\text { SAX Entry } \\
\text { number }\end{array}$ & $\begin{array}{l}\text { Screened from } \\
\text { further analysis } \\
(\mathrm{x})\end{array}$ & $\begin{array}{l}\text { Reason for screening } \\
\text { (Ref. } 3,5,6, \& 7 \text { ) }\end{array}$ \\
\hline Glycol Ether DE & $111-90-0$ & CBR000 & & \\
\hline Glycol Ether EB & $111-76-2$ & BPJ850 & & \\
\hline Glycolic Acid & $79-14-1$ & GHOOOO & $\mathbf{X}$ & Solid in pure form \\
\hline Hazardous Waste NOS & - & - & & \\
\hline Hexamethylenediamine & $124-09-4$ & HEOOOO & $\bar{X}$ & Solid in pure form \\
\hline Hydrated Amorphous Silica & $7631-86-9$ & SCIO00 & $\mathbf{X}$ & Not a toxicological hazard \\
\hline Hydrochloric Acid & $7647-01-0$ & $\mathrm{HHCOOO}$ & & \\
\hline Hydrochloric Acid $31 \%$ & $7647-01-0$ & $\mathrm{HHCOOO}$ & & \\
\hline Hydrofluoric Acid $70 \%$ & $7664-39-3$ & HHU500 & & \\
\hline Hydrogen Peroxide & $7722-84-1$ & HIB000 & & \\
\hline Hydroquinone & $123-31-9$ & HIHOOO & $\mathrm{X}$ & Solid in pure form \\
\hline Igepal CA 630 & $9002-93-1$ & PKF500 & $\mathrm{X}$ & Common material \\
\hline Igepal CO 630 & $68412-54-4$ & PKF000 & $\mathrm{X}$ & Common material \\
\hline Isopar & $64742-47-8$ & - & & \\
\hline Isopropyl Alcohol & $67-63-0$ & INJ000 & & \\
\hline Lactic Acid & $50-21-5$ & LAG000 & $\bar{x}$ & Not an EPHA inhalation hazard \\
\hline Lecithin & $8002-43-5$ & LEF200 & & \\
\hline Liquefied Petroleum Gases & $68476-85-7$ & LGM000 & $\mathbf{X}$ & Common material \\
\hline Magnesium Carbonate & $7760-50-1$ & MAC650 & $\mathrm{X}$ & $\begin{array}{l}\text { Solid in pure form, as a respirable } \\
\text { powder does not present inhalation } \\
\text { hazard }\end{array}$ \\
\hline Magnesium Oxide & $1309-48-4$ & MAH500 & & Respirable powder \\
\hline Magnesium Sulfate & $7487-88-9$ & MAJ250 & $\mathbf{X}$ & Solid in pure form \\
\hline Malic Acid & 6915-15-7 & MAN000 & $\mathrm{X}$ & Solid in pure form \\
\hline Methocel & $9004-67-5$ & - & & \\
\hline Methoxyacetic Acid & $625-45-6$ & MDW275 & $\mathrm{X}$ & Solid in pure form \\
\hline Methyl Alcohol & $67-56-1$ & MGB150 & & \\
\hline Methyl Bromide & $74-83-9$ & MHR200 & & \\
\hline Methyl Ethyl Ketone & $78-93-3$ & MKA400 & & \\
\hline Methyl Isobutyl Ketone & $108-10-1$ & HFG500 & & 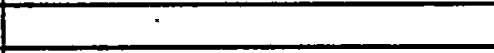 \\
\hline Methylene Chloride & $75-09-2$ & MJP450 & & \\
\hline Methyol Cellosolve & $109-86-4$ & EJH500 & & \\
\hline Mineral Oil & $8042-47-5$ & MQV875 & $\mathrm{X}$ & Common material \\
\hline Mineral Spirits & $8052-41-3$ & SLU500 & $\mathrm{X}$ & Common material \\
\hline Monoethanolamine & $141-43-5$ & EEC600 & & \\
\hline Morpholine & $110-91-8$ & MRP750 & & \\
\hline Myrj & 9004-99-3 & PJV250 & $\mathbf{X}$ & Not an EPHA inhalation hazard \\
\hline N Butyl Alcohol & $71-36-3$ & BPW500 & & ind \\
\hline N Methyl Pyrrolidone & $872-50-4$ & MPF200 & & \\
\hline Naphtha & $64742-89-8$ & NAI500 & & \\
\hline Napthalene & $91-20-3$ & NAJ500 & $\bar{X}$ & Common material \\
\hline Natrasol & $9004-62-0$ & - & & \\
\hline Natural Gas & - & - & $\bar{X}$ & Common material \\
\hline Neodol 25-12 & $68131-39-5$ & - & & \\
\hline Neodol $91-8$ & $68439-46-3$ & - & & \\
\hline Neutronyx & $9016-45-9$ & NND500 & $\bar{X}$ & \begin{tabular}{|l|} 
Solid in pure form \\
\end{tabular} \\
\hline Nitric Acid & $7697-37-2$ & NEDS00 & & \\
\hline
\end{tabular}


Title of Project EPHA for THM

Subject Inventory, Screening/Characterization of Extemal Hazards

Computer S.A. Henderson

Date 09/14/95
Reviewed by D. J. Hadlock

Works Calculation 2

Sheet No. 5 of 11

\begin{tabular}{|c|c|c|c|c|}
\hline Chemical Name & $\begin{array}{l}\text { CAS registry } \\
\text { number }\end{array}$ & $\begin{array}{l}\text { SAX Entry } \\
\text { number }\end{array}$ & $\begin{array}{l}\text { Screened from } \\
\text { further analysis } \\
(\mathrm{x})\end{array}$ & $\begin{array}{l}\text { Reason for screening } \\
\text { (Ref. } 3,5,6, \& 7 \text { ) }\end{array}$ \\
\hline Nitrogen & $7727-37-9$ & NGP500 & $\mathrm{X}$ & Not a toxicological inhalation hazard \\
\hline Nitromethane & $75-52-5$ & NHM500 & $\mathrm{X}$ & Common material \\
\hline Nitroosylsulfuric Acid & $7782-78-1$ & - & & \\
\hline Onyxide & $4719-04-4$ & BBA625 & & \\
\hline Oxalic Acid & $144-62-7$ & OLA000 & $\mathbf{X}$ & Solid in pure form \\
\hline Oxodiacetic Acid & $110-99-6$ & ONQ100 & $\mathrm{X}$ & Solid in pure form \\
\hline Oxygen & $7782-44-7$ & OQW000 & $\mathbf{X}$ & Not a toxicological inhalation hazard \\
\hline Perchloroethylene & $127-18-4$ & PCF275 & & \\
\hline Percofloc & $9002-86-2$ & PKQ059 & $\mathrm{X}$ & Solid in pure form \\
\hline Pertrolatum Snow White min oil & 8009-03-8 & MQV750 & $\bar{X}$ & Common material \\
\hline Petroleum Oil & $8002-05-9$ & PCS250 & $\bar{X}$ & Common material \\
\hline Petroleum Sulfonate & $68608-26-4$ & - & & \\
\hline Phenol, Molten & $108-95-2$ & PDN750 & & Shipped as liquid @ 45×C \\
\hline Phosphoric Acid & $7664-38-2$ & PHB250 & & \\
\hline Phosphoric Acid 75-85\% & $7664-38-2$ & PHB250 & & \\
\hline Phosphoric Anhydride & $1314-56-3$ & PHS250 & $\mathbf{X}$ & Solid in pure form \\
\hline Phosphorus, White (in water) & $7723-14-0$ & PHPOOO & & Solid, spontaneously burns in air \\
\hline Phosphorus, Yellow & $7723-14-0$ & PHO740 & & Solid, spontaneously burns in air \\
\hline Pluronic & $9003-11-6$ & PJH630 & $\mathbf{X}$ & Not an EPHA inhalation hazard \\
\hline Polyethylene Glycols & 9038-95-3 & GHYOOO & $\mathrm{X}$ & Solid in pure form \\
\hline Polyethylene Sorbitol & $9002-88-4$ & PJS750 & $\mathbf{X}$ & Solid in pure form \\
\hline Polyoxyethylene Sorbitol & $25322-68-3$ & PJT000 & $X$ & Common material \\
\hline Polyphosphoric Acid & $8017-16-1$ & - & & \\
\hline Potassium Benzoate & $582-25-2$ & PKW760 & $\mathbf{X}$ & Solid in pure form \\
\hline Potassium Carbonate & 584-08-7 & PLA000 & $\mathbf{X}$ & Solid in pure form \\
\hline Potassium Nitrate & $7757-79-1$ & PLL500 & $\bar{X}$ & Solid in pure form \\
\hline Potassium Permanganate & $7722-64-7$ & PLPOOO & $\mathbf{X}$ & Solid in pure form \\
\hline Potassium Persulfate & $7727-21-1$ & DWQ000 & $\mathrm{X}$ & Solid in pure form \\
\hline Potassium Tripolyphosphate & $13845-36-8$ & PLW400 & $\mathbf{X}$ & $\begin{array}{l}\text { Solid, respirable powder not an EPHA } \\
\text { inhalation hazard }\end{array}$ \\
\hline Propagyl Alcohol & 107-19-7 & PMN450 & & \\
\hline Propane & $74-98-6$ & PMJ750 & $\mathbf{X}$ & Common material \\
\hline Propionic Acid & $79-09-4$ & PMU750 & & \\
\hline Propyl Gallate-n & $121-79-9$ & PNM750 & $X$ & Solid in pure form \\
\hline Propylene Glycol & $57-55-6$ & PMLOOO & $\mathbf{X}$ & Not an EPHA inhalation hazard \\
\hline Radioactive Material NOS & - & - & & \\
\hline Rhodacal UB & 151-21-3 & SIB 600 & $\mathbf{X}$ & Solid in pure form \\
\hline Rocket Motors & Reactive Solid & - & $\mathrm{X}$ & Solid in pure form \\
\hline Rodine 50 (formaldehyde) & $50-00-0$ & FMV000 & & \\
\hline Rodine 213 & $143106-84-7$ & RCF000 & $\mathbf{X}$ & Solid in pure form \\
\hline Salicylamide & $65-45-2$ & SAHOOO & $\mathbf{X}$ & Solid in pure form \\
\hline Silica (as respirable dust) & 14808-60-7 & SCJ500 & & Respirable powder \\
\hline Silica, Crystalline-Cristobalite & $14464-46-1$ & SCJ000 & $\mathbf{X}$ & Solid in pure form \\
\hline Silicone 45 & $63148-62-9$ & DUB600 & & \\
\hline Silicone 323 & $70131-67-8$ & - & & \\
\hline Silicone 462 & $7732-18-5$ & WAT259 & $\mathrm{X}$ & Common material \\
\hline Silicone 471 & $25322-69-4$ & PKI500 & $\mathrm{X}$ & Not an EPHA inhalation hazard \\
\hline
\end{tabular}


Title of Project EPHA for THM

Reviewed by D. J. Hadlock

Subject Inventory, Screening/Characterization of Extemal Hazards

Works Calculation 2

Computer S.A. Henderson

Date 09/14/95

Sheet No. 6 of 11

\begin{tabular}{|c|c|c|c|c|}
\hline Chemical Name & $\begin{array}{l}\text { CAS registry } \\
\text { number }\end{array}$ & $\begin{array}{l}\text { SAX Entry } \\
\text { number }\end{array}$ & $\begin{array}{l}\text { Screened from } \\
\text { further analysis } \\
\text { (x) }\end{array}$ & $\begin{array}{l}\text { Reason for screening } \\
\text { (Ref. } 3,5,6, \text { \& } 7 \text { ) }\end{array}$ \\
\hline Silicone Oil 45 & $48-62-9$ & SCR400 & $\mathrm{X}$ & Not an EPHA inhalation hazard \\
\hline Soda Ash & $497-19-8$ & SFO000 & $\mathrm{X}$ & Solid in pure form \\
\hline Sodium Acetate & $6131-90-4$ & SEG500 & $\mathrm{X}$ & Solid in pure form \\
\hline Sodium Acid Pyrophosphate & $7758-16-9$ & DXF800 & $\mathrm{X}$ & Solid in pure form \\
\hline Sodium Aluminum Phosphate & $7785-88-8$ & AHG500 & $\mathrm{X}$ & Solid in pure form \\
\hline Sodium Benzoate & $532-32-1$ & SFBO00 & $\mathrm{X}$ & Solid in pure form \\
\hline Sodium Bicarbonate & $144-55-8$ & SFC500 & $\mathrm{X}$ & Solid in pure form \\
\hline Sodium Bisulfite & $7681-57-4$ & SH000 & $\mathbf{X}$ & Solid in pure form \\
\hline Sodium Chlorate & $7775-09-9$ & SFSOOO & $\bar{X}$ & Solid in pure form \\
\hline Sodium Chloride & $7647-14-5$ & SFT000 & $\bar{X}$ & Solid in pure form \\
\hline Sodium Erythorbate & $6381-77-7$ & $\cdot$ & & \\
\hline Sodium Fluoride & $7681-49-4$ & SHF500 & $\mathrm{X}$ & Solid in pure form \\
\hline Sodium Formate & $141-53-7$ & SHJOOO & $\mathrm{X}$ & Solid in pure form \\
\hline Sodium Hexametaphosphate & $68915-31-1$ & SHM500 & $\mathrm{X}$ & Solid in pure form \\
\hline Sodium Hydrosulfite & $7775-14-6$ & SHR500 & $\mathrm{X}$ & Solid in pure form \\
\hline Sodium Hydroxide & $1310-73-2$ & SHSOOO & $\mathbf{X}$ & Solid in pure form \\
\hline Sodium Hydroxyacetate & $2836-32-0$ & SHT000 & $\mathbf{X}$ & Solid in pure form \\
\hline Sodium Hypochlorite $13-15 \%$ & $7681-52-9$ & SHU500 & & Highly Reactive \\
\hline Sodium Nitrite & $7632-00-0$ & SIQ500 & $\mathrm{X}$ & Solid in pure form \\
\hline Sodium Perborate Tetrahydrate & $10486-00-7$ & SJB350 & $\mathrm{X}$ & Solid in pure form \\
\hline Sodium Persulfate & $7775-27-1$ & SJE000 & $\mathrm{X}$ & Solid in pure form \\
\hline Sodium Phosphate, Tribasic & $7601-54-9$ & SJH200 & $\mathbf{X}$ & Solid in pure form \\
\hline Sodium Propionate & $137-40-6$ & SJL500 & $\mathrm{X}$ & Solid in pure form \\
\hline Sodium Silicate & $1344-09-8$ & SJU000 & $\mathrm{X}$ & Solid in pure form \\
\hline Sodium Silicofluoride & $16893-85-9$ & DXE000 & $\mathrm{X}$ & Solid in pure form \\
\hline Sodium Sulfate & $7757-82-6$ & SJYOOO & $\mathrm{X}$ & Solid in pure form \\
\hline Sodium Sulfhydrate & $16721-80-5$ & SHROOO & & Solid, spontaneously combusts in air \\
\hline Sodium Sulfide & $1313-82-2$ & SJY500 & $\mathbf{X}$ & Solid in pure form \\
\hline Sodium Sulfite & $7757-83-7$ & SJZO00 & $\mathrm{X}$ & Solid in pure form \\
\hline Sodium Tetraborate Penta & $12179-04-3$ & $\therefore$ & & \\
\hline Sodium Thiosulfate & $7772-98-7$ & SKIOOO & $\bar{X}$ & Solid in pure form \\
\hline Sodium Trimetaphospháte & $7785-84-4$ & SKM500 & $\mathrm{X}$ & Solid in pure form \\
\hline Sodium Tripolyphosphate & $7758-29-4$ & SKNOOO & $\mathrm{X}$ & \begin{tabular}{|l|} 
Solid in pure form \\
\end{tabular} \\
\hline Sodium(I) Nitrate $1: 1$ & $7631-99-4$ & SIO900 & $\mathrm{X}$ & Solid in pure form \\
\hline Sorbic Acid & $110-44-1$ & SKUO00 & $\mathbf{X}$ & Solid in pure form \\
\hline Sorbitan Monooleate & $1338-43-8$ & SKV100 & $\mathrm{X}$ & Solid in pure form \\
\hline Stearic Acid & $57-11-4$ & SLKOOO & $\bar{X}$ & Solid in pure form \\
\hline Stearic Acid & $1323-39-3$ & SLLOOO & $\mathrm{X}$ & Common material \\
\hline Stepanol & $151-21-3$ & SIB600 & $\mathrm{X}$ & Solid in pure form \\
\hline Sulfamic Acid & $5329-14-6$ & SNK500 & $\mathrm{X}$ & Solid in pure form \\
\hline Sulfur Dioxide & $7446-09-5$ & SOH500 & & \\
\hline Sulfur, Molten & $7704-34-9$ & SOD500 & & Shipped as liquid @ 12009 C, Reactive \\
\hline Sulfuric Acid & $7664-93-9$ & SOI500 & & \\
\hline Sulfuric Acid $66 \%$ & $7664-93-9$ & SOI500 & & \\
\hline Sulfuric Acid, Fuming & $8014-95-7$ & SOI520 & & \\
\hline Tergitol & $139-88-8$ & EMT500 & $\mathrm{X}$ & Solid in pure form \\
\hline
\end{tabular}


Title of Project EPHA for THM

Reviewed by D. J. Hadlock. Subject Inventory, Screening/Characterization of Extemal Hazards Computer S.A.Henderson
Works Calculation?

Sheet No. 7 of 11

\begin{tabular}{|l|r|r|r|l|}
\hline Chemical Name & $\begin{array}{c}\text { CAS registry } \\
\text { number }\end{array}$ & $\begin{array}{c}\text { SAX Entry } \\
\text { number }\end{array}$ & $\begin{array}{c}\text { Screened from } \\
\text { further analysis } \\
(\mathrm{x})\end{array}$ & $\begin{array}{l}\text { Reason for screening } \\
\text { (Ref. 3, 5, 6, \& 7) }\end{array}$ \\
\hline Tergitol 15-S-9 (nonionic) & $68131-40-8$ & TAY250 & $\mathrm{X}$ & Solid in pure form \\
\hline Tetraborate Decahydrate & $1303-96-4$ & SFE500 & $\mathrm{X}$ & Solid in pure form \\
\hline Tetrahydrofuran & $109-99-9$ & TCR750 & & \\
\hline Tetrasodium Pyrophosphate & $7722-88-5$ & TEE500 & $\mathrm{X}$ & Solid in pure form \\
\hline Titanium Dioxide & $13463-67-7$ & TGG760 & & respirable powder \\
\hline Toluene & $108-88-3$ & TGK750 & & \\
\hline Toluidine-0 & $95-53-4$ & TGQ750 & & \\
\hline Tree/weed killing compounds & Herbicides & - & & CSX Descriptive Term \\
\hline Trichloroethane-1,1,1 & $71-55-6$ & MIH275 & & \\
\hline Trichloroethylene & $79-01-6$ & TIO750 & & \\
\hline Triethanolamine 85\% & $102-71-6$ & TKP500 & & Vp 0.5 \\
\hline Triethanolamine 99\% & $102-71-6$ & TKP500 & & Vp<0.5 \\
\hline Triethylamine & $121-44-8$ & TJO000 & & \\
\hline Trimethylamine & $75-50-3$ & TLD500 & & \\
\hline Trimethylbenzene-1,2,4 & $95-63-6$ & TLL750 & & \\
\hline Trisodium Phosphate & $10101-89-0$ & SJH200 & $\mathrm{X}$ & Solid in pure form \\
\hline Triton CF-10 & $60864-33-7$ & - & & \\
\hline Triton N-57 \& 101 & $127087-87-0$ & - & & \\
\hline Triton X-100 \& 114 & $9036-19-5$ & GHS000 & $\mathrm{X}$ & Common material \\
\hline Triton X-207 & $68891-21-4$ & - & & \\
\hline Tween & $9005-64-5$ & PKL000 & $\mathrm{X}$ & Not an EPHA inhalation hazard \\
\hline UREA 46\% Nitrogen & $57-13-6$ & USS000 & $\mathrm{X}$ & Not a toxicological inhalation hazard \\
\hline Versene Acid & $60-00-4$ & ELX000 & $\mathrm{X}$ & Solid in pure form \\
\hline Versenex & $140-01-2$ & - & & \\
\hline Vinyl Acetate & $108-05-4$ & VLU250 & & polymerizes when exposed to light \\
\hline Wax & $25155-30-0$ & DXW200 & $\mathrm{X}$ & Common material \\
\hline Whitconol & $37220-82-9$ & - & & \\
\hline Witconate & $9007-48-1$ & AFO250 & $\mathrm{X}$ & Solid in pure form \\
\hline Xylenes & $1330-20-7$ & XGS000 & & \\
\hline & & & & \\
\hline
\end{tabular}


Title of Project EPHA for THM

Subject Inventory. Screening/Characterization of Extermal Hazards

Computer S. A. Henderson

Date $\underline{09 / 14 / 95}$
Reviewed by D. J. Hadlock

Works Calculation 2

Sheet No. 8 of 11

\section{RESULTS}

The following table lists those materials that would not screen out based on initial properties and will be carried forward to calculation 3 for full characterization and screening based on charecteristics.

\begin{tabular}{|c|c|c|c|}
\hline Chemical Name & $\begin{array}{c}\text { CAS registry } \\
\text { number }\end{array}$ & $\begin{array}{l}\text { SAX Entry } \\
\text { number }\end{array}$ & $\begin{array}{l}\text { Comments } \\
\text { (Ref. } 3,5,6, \& 7) \\
\end{array}$ \\
\hline Acetaldehyde & $75-07-0$ & $\mathrm{AAG} 250$ & \\
\hline Acetic Acid & $64-19-7$ & AAT250 & \\
\hline Aerosol 501 & $37294-49-8$ & DJCOOO & \\
\hline Aerosol A-102 & $39354-45-5$ & CAKOOO & \\
\hline Aerosol QTS & $64741-65-7$ & - & \\
\hline Alpha-Pinene & $80-56-8$ & PIH250 & possibly most severe conseq chem. \\
\hline Ammonia & $7664-41-7$ & AMY500 & \\
\hline Ammonium Hydroxide & $1336-21-6$ & ANK250 & \\
\hline Ammonyx & $8001-54-5$ & AFP250 & \\
\hline Benzoic Acid & $65-85-0$ & BCL750 & Respirable powder \\
\hline Benzyl Alcohol & $100-51-6$ & BDX500 & \\
\hline Benzyl Chloride & $100-44-7$ & BEE375 & \\
\hline Brij 30 & $9002-92-0$ & DXY000 & \\
\hline Butene Oxide-1 & $106-88-7$ & BOX750 & \\
\hline Butyraldehyde & $123-72-8$ & BSU250 & \\
\hline Carbon Monoxide & $630-08-0$ & CBW750 & \\
\hline Chloracetone & $78-95-5$ & CDN200 & \\
\hline Chlorine & $7782-50-5$ & CDV750 & \\
\hline Chloronitrobenzene & $25167-93-5$ & CJA950 & \\
\hline Chlorosulfonic Acid & $7790-94-5$ & CLG500 & \\
\hline Copper Sulfate & $7758-99-8$ & CNP500 & \\
\hline Cumene & $98-82-8$ & COE750 & \\
\hline Cyclohexane & $108-94-1$ & $\mathrm{CPCOOO}$ & \\
\hline Diethylamine & $124-40-3$ & DOQ800 & \\
\hline Diethylene Glycol & $111-46-6$ & DJD600 & \\
\hline Dioxane & 123-91-1 & DVQ000 & \\
\hline $\begin{array}{l}\text { Diphenylmethane-4,4- } \\
\text { Diisocyanate }\end{array}$ & $101-68-8$ & MJP400 & Melts @ 37.2a C \\
\hline Dowfax 2A1 & $119345-04-9$ & - & \\
\hline Dursban & $2921-88-2$ & CMA100 & \\
\hline Ethyl Acetate & $141-78-6$ & EFRO00 & \\
\hline Ethyl Benzene & $100-41-4$ & EGP500 & \\
\hline Ethylene Oxide & $75-21-8$ & EJN500 & \\
\hline Ethylhexanol -2 & $104-76-7$ & EKQ000 & \\
\hline Fluorosilicic Acid & $16961-83-4$ & $S C 0500$ & \\
\hline Fluosilicic Acid 25\% & $16961-83-4$ & $S C 0500$ & \\
\hline Forestall & $61791-34-2$ & - & \\
\hline Formaldehyde & $50-00-0$ & FMV000 & \\
\hline Formic Acid & $64-18-6$ & FNA000 & \\
\hline Glycerine & $56-81-5$ & GGA000 & \\
\hline Glycol Ether DE & $111-90-0$ & CBROOO & \\
\hline Glycol Ether EB & $111-76-2$ & BPJ850 & \\
\hline Hydrochloric Acid & $7647-01-0$ & $\mathrm{HHCOOO}$ & \\
\hline Hydrochloric Acid 31\% & $7647-01-0$ & $\mathrm{HHCOOO}$ & \\
\hline
\end{tabular}


Title of Project EPHA for THM Subject Inventory, Screening/Characterization of Extemal Hazands

Computer S. A. Hienderson
Reviewed by D. J. Hadlock Works Calculation 2

Sheet No. 9 of 11

\begin{tabular}{|c|c|c|c|}
\hline Chemical Name & $\begin{array}{c}\text { CAS registry } \\
\text { number }\end{array}$ & $\begin{array}{c}\text { SAX Entry } \\
\text { number }\end{array}$ & $\begin{array}{l}\text { Comments } \\
\text { (Ref. } 3,5,6, \& 7)\end{array}$ \\
\hline Hydrofluóric Acid 70\% & 7664-39-3 & HHU500 & \\
\hline Hydrogen Peroxide & $7722-84-1$ & HIB 000 & \\
\hline Isopar & $64742-47-8$ & - & \\
\hline Isopropyl Alcohol & $67-63-0$ & INJ000 & \\
\hline Lecithin & $8002-43-5$ & LEF200 & \\
\hline Magnesium Oxide & $1309-48-4$ & MAH500 & Respirable powder \\
\hline Methocel & $9004-67-5$ & - & \\
\hline Methyl Alcohol & $67-56-1$ & MGB150 & \\
\hline Methyl Bromide & $74-83-9$ & MHR200 & \\
\hline Methyl Ethyl Ketone & $78-93-3$ & MKA400 & \\
\hline Methyl Isobutyl Ketone & $108-10-1$ & HFG500 & \\
\hline Methylene Chloride & $75-09-2$ & MJP450 & \\
\hline Methyol Cellosolve & $109-86-4$ & EJH500 & \\
\hline Monoethanolamine & $141-43-5$ & EEC600 & \\
\hline Morpholine & $110-91-8$ & MRP750 & \\
\hline N Butyl Alcohol & $71-36-3$ & BPW500 & \\
\hline N Methyl Pyrrolidone & $872-50-4$ & MPF200 & \\
\hline Naphtha & $64742-89-8$ & NAI500 & \\
\hline Natrasol & $9004-62-0$ & - & \\
\hline Neodol 25-12 & $68131-39-5$ & - & \\
\hline Neodol 91-8 & $68439-46-3$ & - & \\
\hline Nitric Acid & $7697-37-2$ & NED500 & \\
\hline Nitroosylsulfuric Acid & $7782-78-1$ & - & \\
\hline Onyxide & $4719-04-4$ & BBA625 & \\
\hline Perchloroethylene & $127-18-4$ & PCF275 & \\
\hline Petroleum Sulfonate & $68608-26-4$ & - & \\
\hline Phenol, Molten & $108-95-2$ & PDN750 & Shipped as liquid @ 45a C \\
\hline Phosphoric Acid & $7664-38-2$ & PHB250 & \\
\hline Phosphoric Acid 75 - 85\% & $7664-38-2$ & PHB250 & \\
\hline Phosphorus, White (in water) & $7723-14-0$ & PHPODO & Solid, spontaneously combusts in air \\
\hline Phosphorus, Yellow & $7723-14-0$ & PHO740 & Solid, spontaneously combusts in air \\
\hline Polyphosphoric Acid & $8017-16-1$ & - & \\
\hline Propagyl Alcohol & $107-19-7$ & PMN450 & \\
\hline Propionic Acid & $79-09-4$ & PMU750 & \\
\hline Radioactive Material NOS & - & - & \\
\hline Rodine 50 (formaldehyde) & $50-00-0$ & FMV000 & \\
\hline Silica (as respirable dust) & $14808-60-7$ & SCJ500 & Respirable powder \\
\hline Silicone 45 & $63148-62-9$ & DUB600 & \\
\hline Silicone 323 & $70131-67-8$ & & \\
\hline
\end{tabular}


Title of Project EPHA for THM

Subject Inventory, Screenino/Characterization of Extemal Hazards

Computer S. A. Henderson
Date $09 / 14 / 95$
Reviewed by D. J. Hadlock

Works Calculation 2

Sheet No. 10 of 11

\begin{tabular}{|l|r|r|l|}
\hline $\begin{array}{l}\text { Chemical Name } \\
\text { Sodium Erythorbate }\end{array}$ & $\begin{array}{c}\text { CAS registry } \\
\text { number }\end{array}$ & $\begin{array}{c}\text { SAX Entry } \\
\text { number }\end{array}$ & $\begin{array}{l}\text { Comments } \\
\text { (Ref. 3,5,6, \& 7) }\end{array}$ \\
\hline Sodium Hypochlorite 13-15\% & $7681-77-7$ & - & \\
\hline Sodium Sulfhydrate & $16721-82-9$ & SHU500 & Highly Reactive \\
\hline Sodium Tetraborate Penta & $12179-04-3$ & - & \\
\hline Sulfur Dioxide & $7446-09-5$ & SOH500 & \\
\hline Sulfur, Molten & $7704-34-9$ & SOD500 & Shipped as liquid @ 120: C, Reactive \\
\hline Sulfuric Acid & $7664-93-9$ & SOI500 & \\
\hline Sulfuric Acid 66\% & $7664-93-9$ & SOI500 & \\
\hline Sulfuric Acid, Fuming & $8014-95-7$ & SOI520 & \\
\hline Tetrahydrofuran & $109-99-9$ & TCR750 & \\
\hline Titanium Dioxide & $13463-67-7$ & TGG760 & respirable powder \\
\hline Toluene & $108-88-3$ & TGK750 & \\
\hline Toluidine-0 & $95-53-4$ & TGQ750 & \\
\hline Tree/weed killing compounds & Herbicides & - & CSX Descriptive Term \\
\hline Trichloroethane-1,1,1 & $71-55-6$ & MIH275 & \\
\hline Trichloroethylene & $79-01-6$ & TIO750 & \\
\hline Triethanolamine 85\% & $102-71-6$ & TKP500 & Vp<0.5 \\
\hline Triethanolamine 99\% & $102-71-6$ & TKP500 & Vp<0.5 \\
\hline Triethylamine & $121-44-8$ & TJO000 & \\
\hline Trimethylamine & $75-50-3$ & TLD500 & \\
\hline Trimethylbenzene-1,2,4 & $95-63-6$ & TLL750 & \\
\hline Triton CF-10 & $60864-33-7$ & - & \\
\hline Triton N-57 \& 101 & $127087-87-0$ & - & \\
\hline Triton X-207 & $68891-21-4$ & - & \\
\hline Versenex & $140-01-2$ & - & \\
\hline Vinyl Acetate & $108-05-4$ & VLU250 & polymerizes when exposed to light \\
\hline Whitconol & $37220-82-9$ & - & \\
\hline Xylenes & $1330-20-7$ & XGS000 & \\
\hline
\end{tabular}




\section{REFERENCES}

1. Hadlock, D. J.. Standards For Development and Maintenance of a Facility Hazards Assessment, 6Q-001, Rev. 1, Emergency Management Program Procedures (EMPPs) Manual, WSRC Procedure Manual 6Q Vol. II, Westinghouse Savannah River Company, Savannah River Site, Aiken, SC, May 2, 1994.

2. Henderson; S. A. Request For Complete List of all Hazardous Materials Transported To or Through the SRS. Emergency Services Technical Group, Westinghouse Savannah River Company, Savannah River Site, Aiken, SC, July 27, 1995.

3. Lewis, Richard J.. SAX's Dangerous Properties of Industrial Materials, Eighth Edition, Volumes 1-4. Van Nostrand Reinhold, New York, NY, 1992.

4. Nadeau, M., L. and Henderson, S., A.. Electronic Screening of Chemical Inventory Information (U). SESR-G-00001, Rev. 0, Westinghouse Savannah River Company, Savannah River Site, Aiken, SC, December.

5. Emergency Handling of Hazardous Materials in Surface Transportation. Association of American Railroads, Bureau of Explosives, Washington, DC, July 1989.

6. Craig, D. K. Description of Methodology for Deriving ERPG-equivalents for Chemicals. S-CLC-G-00074, Rev. 0 and SWMF Chemicals on "Master File" Requiring Independent Verification, S-CLC-G-00079 Rev. 0, Westinghouse Savannah River Company, Savannah River Site, Aiken, SC, July 10, 1995.

7. Pocket Guide to Chemical Hazards. NIOSH, US. Department of Health and Human Services, Washington, DC, June, 1994 
Title of Project EPHA for THM

Subject Characterization/Screening of THM EPHA chemicals

Computer S. A. Henderson

Date 09/29/95
Reviewed by D. J. Hadlock

Works Calculation 3

Sheet No. 1 of 5

\section{INTRODUCTION}

This calculation characterizes the un-screened materials from calculations 1 and 2, and screens out additional materials based on their characteristics and volume restrictions. Materials not screened out during initial screening require further characterization and limited analysis to ascertain if they will screen based on characteristics and segmentation. For the THM EPHA additional screening is done based on the different transporting container types and sizes within each segment.

\section{DISCUSSION / ASSUMPTIONS}

THM EPHA calculations 1 and 2 developed chemical materials that do not screen out from further analysis based on their initial limited information. These materials are separated and characterized as individual chemicals and are assumed to be in each segment. Certain criteria (vapor pressure, state, hazards etc...) is developed during characterization that allows for screening out additional materials per 6Q-001 (Ref. 1).

\section{CALCULATION}

The following table lists the chemical name, molecular weight, CAS registry number, SAX entry number (Ref. 2), the ERPG-2 or equivalent value where possible (Ref. 3), ambient state, boiling point in degrees centigrade, and the vapor pressure at 20 degrees centigrade or temperature indicated (Ref. $2,3,4, \& 5$ ). From this information each chemical is evaluated and a determination is made weather it can be screened out from further evaluation in the segment based on the chemical characteristics and hazards involved. Because multiple containers could be involved in the same load it was assumed quantities could equal the maximum volume of the shipping devise, therefore any TPSQ screening is not performed.

\begin{tabular}{|c|c|c|c|c|c|c|c|c|}
\hline $\begin{array}{l}\text { Chemical Name / } \\
\text { Molecular Weight. }\end{array}$ & CAS \# & SAX\# & $\begin{array}{l}\text { ERPG } \\
\text { or } \\
\text { Equiv } \\
\text { ppm }\end{array}$ & $\begin{array}{l}\text { State } \\
\text { (S)olid } \\
\text { (L)uqu } \\
\text { (G)as }\end{array}$ & $\begin{array}{c}\text { BP } \\
\text { Deg. C }\end{array}$ & $\begin{array}{c}\mathrm{Pv} \\
\mathrm{mmHg} \\
20 \mathrm{C}\end{array}$ & $\begin{array}{l}\text { Screen } \\
\text { from } \\
\text { further } \\
\text { Analysis }\end{array}$ & Comments \\
\hline Acetadehyde /44.1 & $75-07-0$ & AAG250 & TBD & L & 20.8 & 740 & & \\
\hline Acetic Acid & $64-19-7$ & AAT250 & TBD & $\mathbf{L}$ & 118.1 & 11.4 & & \\
\hline Acetonitrile /41.1 & $75-05-8$ & ABESOO & 35 & L & 81.6 & 73 & & \\
\hline Aerosol $501 / 307.3$ & $37294-49-8$ & $\mathrm{DJCOOO}$ & $\cdot$ & - & - & - & $\mathbf{X}$ & Trace amount \& not a severe hazard \\
\hline Aerosol A.102/112.4 & $39354-45-5$ & CAK000 & - & $\mathbf{S}$ & - & - & $\mathbf{X}$ & Trace quantity in a common material \\
\hline Acrosol QTS & $64741-65-7$ & - & - & - & - & - & $\mathbf{X}$ & Common material. Vendor conf \\
\hline AJpha-Pinene / 136.3 & $80-56-8$ & PII250 & TBD & $\mathbf{L}$ & 155 & $10 @ 37$ & & possibly most severe conseq chem. \\
\hline Ammonia / 17.0 & $7664-41-7$ & AMY500 & 200 & $\mathbf{G}$ & - & $>760$ & & \\
\hline Ammonium Hydroxide & $1336-21-6$ & ANK250 & TBD & $\mathbf{L}$ & - & Var & & $<44 \%$ ammonia \\
\hline Ammonyx & $8001-54-5$ & AFP250 & - & $\bar{L}$ & - & - & $\mathbf{X}$ & Trace quantity in a common material \\
\hline Benzene /78.1 & $71-43-2$ & BBL250 & 150 & $\overline{\mathbf{L}}$ & 80 & 75 & & \\
\hline Benzoic Acid & $65-85-0$ & BCL750 & TBD & $\mathbf{S}$ & 249 & $1996 \mathrm{C}$ & & Respirable powder \\
\hline Benzyl Alcohol & $100-51-6$ & BDX500 & $\overline{\text { TBD }}$ & $\overline{\mathbf{L}}$ & 205 & 1 & & \\
\hline Benzyl Chloride & $100-44-7$ & BEE375 & TBD & $\mathbf{L}$ & 179 & - & & TLV=1ppm \\
\hline Brij 30 & $9002-92-0$ & DXY000 & - & - & - & - & $\bar{x}$ & Trace quantity in a common material \\
\hline Butene Oxide-1 & $106-88-7$ & $80 \times 750$ & - & $\mathbf{L}$ & 63 & - & $\bar{x}$ & Not a severe inhalation hazard \\
\hline Butyraldehyde & $123-72-8$ & BSU250. & TBD & $\mathbf{L}$ & 74.7 & - & & 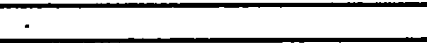 \\
\hline Carbon disulfide /76.1 & $75-15-0$ & CBV500 & 50 & L & 46.6 & 297 & & \\
\hline Carbon Monoxide / 28.0 & $630-08-0$ & CBW750 & 350 & $\mathbf{G}$ & $(-191.3)$ & $>760$ & & \\
\hline Chloracetone / 92.5 & $78-95-5$ & CDN200 & TBD & $\mathbf{L}$ & 119 & - & & $T L V=1 p p m$ \\
\hline Chlorine /70.9 & $7782-50-5$ & CDV750 & 3 & $\mathbf{G}$ & $(-34)$ & 4800 & & \\
\hline Chloronitrobenzene / 157.96 & $25167-93-5$ & CJA950 & - & $\mathbf{S}$ & - & - & $\bar{x}$ & $\begin{array}{l}\text { Solid trace amnt. in a common } \\
\text { material }\end{array}$ \\
\hline Chlorosulfonic Acid / 116.52 & $7790-94-5$ & CLG500 & TBD & $\mathbf{L}$ & 151 & $1932 \mathrm{C}$ & & \\
\hline
\end{tabular}


Title of Project EPHA for THM

Subject Characterization/Screening of THM EPHA chemicals

Computer $\underline{S}$. A. Henderson
Date 09/29/95
Reviewed by D. J. Hadlock

Works Calculation 3

Sheet No.

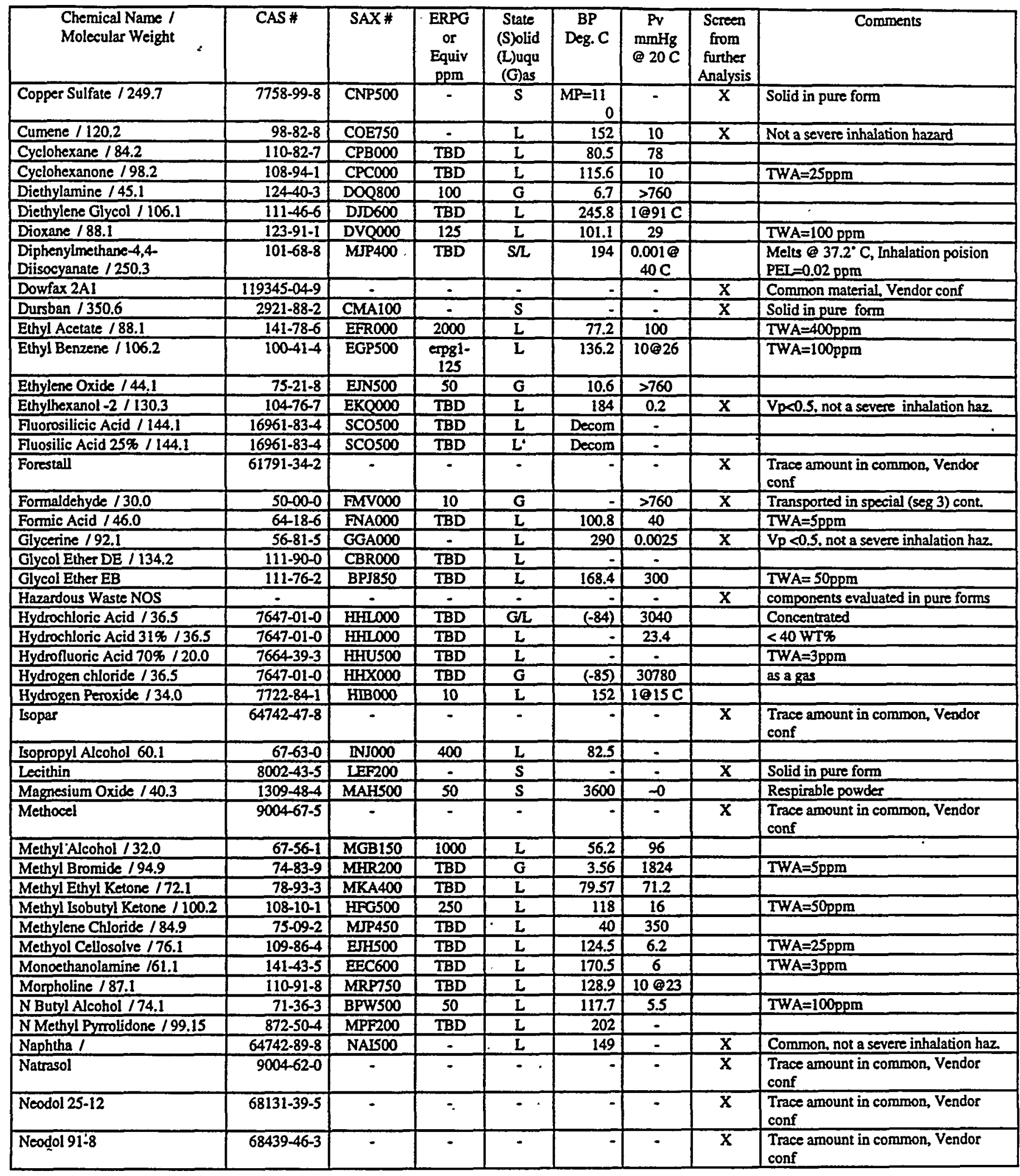


Title of Project EPHA for THM

Subject Characterization/Screening of THM EPHA chemicals

Computer S. A. Henderson

Date $\underline{09 / 29 / 95}$

Reviewed by D. J. Hadlock

Works Calculation 3

Sheet No.

3 of 5

\begin{tabular}{|c|c|c|c|c|c|c|c|c|}
\hline $\begin{array}{l}\text { Chemical Name / } \\
\text { Molecular Weight }\end{array}$ & CAS \# & SAX\# & $\begin{array}{l}\text { ERPG } \\
\text { or } \\
\text { Equiv } \\
\text { ppm }\end{array}$ & $\begin{array}{l}\text { State } \\
\text { (S)olid } \\
\text { (L)uqu } \\
\text { (G)as }\end{array}$ & $\begin{array}{c}\text { BP } \\
\text { Deg. C }\end{array}$ & $\begin{array}{c}\mathrm{Pv} \\
\mathrm{mmHg} \\
\Theta 20 \mathrm{C}\end{array}$ & $\begin{array}{l}\text { Screen } \\
\text { from } \\
\text { further } \\
\text { Analysis }\end{array}$ & Comments \\
\hline Nitric Acid / 63.0 & $7697-37-2$ & NED500 & 15 & $\mathrm{~L}$ & 86 & $w t \%$ & & \\
\hline Nitroosylsulfuric Acid & $7782-78-1$ & $\cdot$ & $\cdot$ & - & $\cdot$ & - & $\mathbf{X}$ & $\begin{array}{l}\text { Trace amount in common. Vendor } \\
\text { conf }\end{array}$ \\
\hline Onyxide & $4719-04-4$ & BBA625 & TBD & - & - & - & & NIF \\
\hline Perchloroethylene / 165.8 & $127-18-4$ & PCF275 & 200 & $\mathbf{L}$ & 121.1 & 14 & & \\
\hline Petroleum Sulfonate & $68608-26-4$ & - & TBD & - & - & - & & NIF \\
\hline Phenol, Molten /94.1 & $108-95-2$ & PDN750 & 50 & $S / L$ & 181.7 & 0.4 & & Shipped as liquid @ $45^{\circ} \mathrm{C}$ \\
\hline Phosphoric Acid /98.0 & $7664-38-2$ & PHB250 & 5 & $\mathrm{~L} / \mathrm{S}$ & 213 & 0.028 & $x$ & $V_{p}<0.5$. ERPG $>1$ \\
\hline Phosphoric Acid 75 - 85\% /98 & $7664-38-2$ & PHB250 & 5 & L & 213 & 0.028 & $\mathbf{X}$ & $V_{p}<0.5$ ERPG $>1$ \\
\hline Phosphorus, White (in water) & $7723-14-0$ & PHPOOO & TBD & $\mathbf{S}$ & 280 & $1876 \mathrm{C}$ & $\mathbf{x}$ & $\begin{array}{l}\text { Solid. spontaneously bums in air } \\
\text { Transported in special cont. (seg. 3) }\end{array}$ \\
\hline Phosphorus, Yellow /123.88 & $7723-14-0$ & PHO740 & TBD & $\mathbf{S}$ & 280 & $1 \otimes 76 \mathrm{C}$ & $\bar{x}$ & $\begin{array}{l}\text { Solid, spontaneously bums in air } \\
\text { Transported in special cont. (seg } 3 \text { ) }\end{array}$ \\
\hline Polyphosphoric Acid & $8017-16-1$ & - & - & - & - & - & $\mathbf{X}$ & $\begin{array}{l}\text { Trace amount in common, Vendor } \\
\text { conf }\end{array}$ \\
\hline Propagyl Alcohol / 56.1 & $107-19-7$ & PMN450 & 5 & $\mathrm{~L}$ & 114.5 & 11.6 & & TWA $=1 \mathrm{ppm}$ \\
\hline Propionic Acid /74.1 & $79-09-4$ & - PMU750 & TBD & $\mathbf{L}$ & 141.1 & 10039 & & TWA=10ppm \\
\hline Radioactive Material NOS & - & - & - & $\therefore$ & - & - & $\mathbf{x}$ & Analyzed with radionuclides \\
\hline Rodine 50 (formaldehyde) $/ 30.0$ & $50-00-0$ & FMV000 & 10 & L & Infl. & Infl & & In solution \\
\hline Silica (as respirable dust) & $14808-60-7$ & SCJ500 & TBD & $\mathbf{S}$ & - & - & & Respirable dust, $T W A=10 \mathrm{mg} / \mathrm{m}^{\wedge} 3$ \\
\hline Silicone 45 & $63148-62-9$ & DUB600 & - & SS & - & - & $\mathbf{x}$ & Semi solid \& common \\
\hline Silicone 323 & $70131-67-8$ & - & - & & $=$ & $=$ & $\mathbf{x}$ & \\
\hline Sodium Erythorbate & $6381-77-7$ & 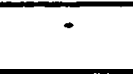 & - & & - & - & $\bar{x}$ & $\begin{array}{l}\text { Trace amount in common, Vendor } \\
\text { conf }\end{array}$ \\
\hline $\begin{array}{l}\text { Sodium Hypochlorite 13-15\% } \\
75.45\end{array}$ & $7681-52-9$ & SHU500 & TBD & L & Decom & - & $\mathbf{x}$ & $\begin{array}{l}\text { Rail car only in this Wts } \\
\text { Highly Reactive. Initiator only }\end{array}$ \\
\hline $\begin{array}{l}\text { Sodium Sulfhydrate / } 56.06 \\
\text {. }\end{array}$ & $16721-80-5$ & SHROOO & TBD & $\mathbf{s}$ & - & - & & $\begin{array}{l}\text { Solid, spontaneously combusts in air } \\
\text { Initiator only }\end{array}$ \\
\hline Sodium Tetraborate Penta & $12179-04-3$ & $\cdot$ & 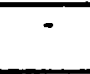 & - & $\cdot$ & - & $\bar{x}$ & $\begin{array}{l}\text { Trace amount in common, Vendor } \\
\text { conf }\end{array}$ \\
\hline Sulfur Dioxide / 64.1 & $7446-09-5$ & SOH500 & 3 & $\mathbf{G}$ & $(-10)$ & $>760$ & & \\
\hline Sulfur, Molten / 32.06 & $7704-34-9$ & SOD500 & TBD & $\mathbf{S} / \mathbf{L}$ & 444.6 & 19188 & $\bar{x}$ & $\begin{array}{l}\text { Special containet only, (seg 3) } \\
\text { Shipped as liquid } \odot 120^{\circ} \mathrm{C} \text {. Reactive }\end{array}$ \\
\hline Sulfuric Acid /98.1 & 7664-93-9 & SOI500 & 10 & $\mathbf{L}$ & 290 & 19145 & & \\
\hline Sulfuric Acid 66\% / 98.1 & $7664-93-9$ & SOL500 & 10 & L & 290 & 19145 & & \\
\hline Sulfuric Acid, Fuming / 98.1 & $8014-95-7$ & SOIS20 & 10 & $\mathbf{L}$ & 290 & 18145 & & \\
\hline Tetrahydrofuran / 72.1 & $109-99-9$ & TCR750 & 1000 & L & 65.4 & 132 & & TWA $=200 \mathrm{ppm}$ \\
\hline Titanium Dioxide / 79.9 & $13463-67-7$ & TGG760 & TBD & $\mathbf{S}$ & - & $\cdot$ & & respirable powder. TWA $=5 \mathrm{mg} / \mathrm{m}^{\wedge} 3$ \\
\hline Toluene / 92.1 & $108-88-3$ & TGK750 & 300 & $\mathbf{L}$ & 111.1 & 20 & & \\
\hline Toluidine-o / 107.2 & $95-53-4$ & TGO750 & 10 & L & 200 & 0.3 & & \\
\hline Tree/weed killing compounds & Herbicides & $\cdot$ & $\cdot$ & - & - & - & $\mathbf{x}$ & $\begin{array}{l}\text { CSX Descriptive Term, evaluated in } \\
\text { pure form }\end{array}$ \\
\hline Trichloroethane-1,1,1/133.4 & $71-55-6$ & MIH275 & 1000 & $\mathbf{L}$ & 74.1 & 100 & & TWA $=350 \mathrm{ppm}$ \\
\hline Trichloroethylene / 131.4 & $79-01-6$ & T10750 & 500 & $\mathbf{L}$ & 87.2 & 58 & & \\
\hline Triethanolamine $85 \%$ & $102-71-6$ & TKP500 & - & $\mathrm{L}$ & 360 & $10 @ 205$ & $\mathbf{X}$ & $V_{p}<0.5$ \\
\hline Triethanolamine $99 \%$ & $102-71-6$ & TKP500 & - & L & 360 & $10 @ 205$ & $\mathbf{X}$ & $V_{p}<0.5$ \\
\hline Triethylamine / 101.22 & $121-44-8$ & TJO000 & TBD & $\mathbf{L}$ & 89.5 & - & & \\
\hline Trimethylamine / 59.1 & $75-50-3$ & TLD500 & TBD & $\mathbf{G}$ & 2.87 & $>760$ & & TWA $=10 \mathrm{ppm}$ \\
\hline Trimethylbenzene-1.2.4/120.2 & $95-63-6$ & TLL750 & - & $\mathbf{L}$ & 168.89 & - & $\mathbf{x}$ & Not a severe inhalation hazard \\
\hline Triton CF-10 & $60864-33-7$ & - & - & - & - & - & $\mathrm{x}$ & \\
\hline Triton N-57 \& 101 & $127087-87-0$ & - & - & - & - & - & $\mathbf{x}$ & \\
\hline Triton X-207 & $68891-21-4$ & - & - & - & - & - & $\mathbf{X}$ & \\
\hline Versenex & $140-01-2$ & $\cdot \quad-$ & - & - & - & - & $\mathbf{x}$ & \\
\hline Vinyl Acetate / 86.1 & $108-05-4$ & VLU250 & 75 & $\mathbf{L}$ & 73 & $100 \Theta 21$ & & polymerizes when exposed to light \\
\hline Whitconol & $37220-82-9$ & $\cdot$ & $\cdot$ & 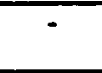 & - & - & $\bar{x}$ & $\begin{array}{l}\text { Trace amount in common, Vendor } \\
\text { conf }\end{array}$ \\
\hline Xylenes / 106.2 & $1330-20-7$ & $\mathrm{XGSO00}$ & 200 & L & 138.5 & 6.72 & & \\
\hline
\end{tabular}


Title of Project EPHA for THM

Subject Characterization/Screening of THM EPHA chemicals

Computer S. A. Henderson

Date 09/29/95

\section{RESULTS}

The following table lists those materials requiring full hazards analysis in the THM EPHA.

\begin{tabular}{|c|c|}
\hline $\begin{array}{l}\text { Chemical Name I } \\
\text { Molecular Weight }\end{array}$ & CAS \# \\
\hline Acetadehyde / 44.1 & $75-07-0$ \\
\hline Acetic Acid & $64-19-7$ \\
\hline Acetonitrile /41.1 & $75-05-8$ \\
\hline Alpha-Pinene / 136.3 & $80-56-8$ \\
\hline Ammonia / 17.0 & $7664-41-7$ \\
\hline Ammonium Hydroxide & $1336-21-6$ \\
\hline Benzene /78.1 & $71-43-2$ \\
\hline Benzoic Acid & $65-85-0$ \\
\hline Benzyl Alcohol & $100-51-6$ \\
\hline Benzyl Chloride & $100-44-7$ \\
\hline Butyraldehyde & $123-72-8$ \\
\hline Carbon disulfide /76.1 & $75-15-0$ \\
\hline Carbon Monoxide / 28.0 & $630-08-0$ \\
\hline Chloracetone /92.5 & $78-95-5$ \\
\hline Chlorine / 70.9 & $7782-50-5$ \\
\hline Chlorosulfonic Acid / 116.52 & $7790-94-5$ \\
\hline Cyclohexane / 84.2 & $110-82-7$ \\
\hline Cyclohexanone / 98.2 & $108-94-1$ \\
\hline Diethylamine / 45.1 & $124-40-3$ \\
\hline Diethylene Glycol / 106.1 & $111-46-6$ \\
\hline Dioxane / 88.1 & $123-91-1$ \\
\hline $\begin{array}{l}\text { Diphenylmethane-4,4- } \\
\text { Diisocyanate / } 250.3\end{array}$ & $101-68-8$ \\
\hline Ethyl Acetate / 88.1 & $141-78-6$ \\
\hline Ethyl Benzene / 106.2 & $100-41-4$ \\
\hline Ethylene Oxide / 44.1 & $75-21-8$ \\
\hline Fluorosilicic Acid / 144.1 & $16961-83-4$ \\
\hline Fluosilicic Acid 25\% / 144.1 & $16961-83-4$ \\
\hline Formic Acid / 46.0 & $64-18-6$ \\
\hline Glycol Ether DE / 134.2 & $111-90-0$ \\
\hline Glycol Ether EB & $111-76-2$ \\
\hline Hydrochloric Acid / 36.5 & $7647-01-0$ \\
\hline Hydrochloric Acid 31\% / 36.5 & $7647-01-0$ \\
\hline Hydrofluoric Acid 70\% / 20.0 & $7664-39-3$ \\
\hline Hydrogen chloride (gas) / 36.5 & $7647-01-0$ \\
\hline Hydrogen Peroxide / 34.0 & $7722-84-1$ \\
\hline Isopropyl Alcohol 60.1 & $67-63-0$ \\
\hline Magnesium Oxide / 40.3 & $1309-48-4$ \\
\hline Methyl Alcohol / 32.0 & $67-56-1$ \\
\hline Methyl Bromide / 94.9 & $74-83-9$ \\
\hline Methyl Ethyl Ketone / 72.1 & $78-93-3$ \\
\hline Methyl Isobutyl Ketone / 100.2 & $108-10-1$ \\
\hline Methylene Chloride / 84.9 & $75-09-2$ \\
\hline Methyol Cellosolve /76.1 & $109-86-4$ \\
\hline
\end{tabular}

Reviewed by D. J. Hadlock

Works Calculation 3

Sheet No. 4 of 5 
Title of Project EPHA for THM

Subject Characterization/Screening of THM EPHA chemicals

Computer S. A. Henderson
Date $\underline{09 / 29 / 95}$
Reviewed by D. J. Hadlock

Works Calculation 3

Sheet No.

5 of 5

\begin{tabular}{|l|r|}
\hline \multicolumn{1}{|c|}{$\begin{array}{c}\text { Chemical Name / } \\
\text { Molecular Weight }\end{array}$} & \multicolumn{1}{c|}{ CAS \# } \\
\hline Monoethanolamine /61.1 & $141-43-5$ \\
\hline Morpholine / 87.1 & $110-91-8$ \\
\hline N Butyl Alcohol /74.1 & $71-36-3$ \\
\hline N Methyl Pyrrolidone /99.15 & $872-50-4$ \\
\hline Nitric Acid / 63.0 & $7697-37-2$ \\
\hline Onyxide & $4719-04-4$ \\
\hline Perchloroethylene / 165.8 & $127-18-4$ \\
\hline Petroleum Sulfonate & $68608-26-4$ \\
\hline Phenol, Molten / 94.1 & $108-95-2$ \\
\hline Propagyl Alcohol / 56.1 & $107-19-7$ \\
\hline Propionic Acid /74.1 & $79-09-4$ \\
\hline Rodine 50 (formaldehyde) /30.0 & $50-00-0$ \\
\hline Silica (as respirable dust) & $14808-60-7$ \\
\hline Sodium Sulfhydrate / 56.06 & $16721-80-5$ \\
\hline Sulfur Dioxide / 64.1 & $7446-09-5$ \\
\hline Sulfuric Acid / 98.1 & $7664-93-9$ \\
\hline Sulfuric Acid 66\% /98.1 & $7664-93-9$ \\
\hline Sulfuric Acid, Fuming /98.1 & $8014-95-7$ \\
\hline Tetrahydrofuran /72.1 & $109-99-9$ \\
\hline Titanium Dioxide /79.9 & $13463-67-7$ \\
\hline Toluene / 92.1 & $108-88-3$ \\
\hline Toluidine-0 / 107.2 & $95-53-4$ \\
\hline Trichloroethane-1,1,1/133.4 & $71-55-6$ \\
\hline Trichloroethylene / 131.4 & $79-01-6$ \\
\hline Triethylamine / 101.22 & $121-44-8$ \\
\hline Trimethylamine / 59.1 & $75-50-3$ \\
\hline Vinyl Acetate / 86.1 & $108-05-4$ \\
\hline Xylenes / 106.2 & $1330-20-7$ \\
\hline
\end{tabular}

\section{REFERENCES}

1. Hadlock, D. J.Standards For Development and Maintenarice of a Facility Hazards Assessment, $6 \mathrm{Q}$ Vol. 2, EMPP 6Q-001, Rev. 1, Westinghouse Savannah River Company, Savannah River Site, Aiken, SC, May 2, 1994.

2. Lewis, Richard J., SAX's Dangerous Properties of Industrial Materials, Eighth Edition, Volumes 1-4, Van Nostrand Reinhold, New York, NY, 1992.

3. Craig, D. K., Description of Methodology for Deriving ERPG-equivalents for Chemicals, S-CLC-G00074, Rev. 0 and SWMF Chemicals on "Master File" Requiring Independent Verrification, S-CLC-G00079 Rev. 0, Westinghouse Savana River Company, Aiken, SC, July 10, 1995.

4. NIOSH, Pocket Guide to Chemical Hazards, U.S. Department of Health and Human Services, Washington, DC, June, 1994.

5. Association of American Railroads, Bureau of Explosives, Emergency Handling of Hazardous Materials in Surface Transportation, Washington, DC, July 1989. 
Title of Project EPHA for THM

\section{INTRODUCTION}

The purpose of this calculation is to screen and eliminate radioactive isotopes that do not contribute significantly to the total dose. Quantities are based on the maximum inventories of radionuclides transported to and/or across the site based on individual facility EPHAs, SARs, BIOs, JCOs, various transportation documents, and Hazardous Materials Shipment Public Access Roads Forms, the Waste Acceptance Criteria Manual and approximate inventories from the Inter-Site Transportation Radiological and Toxicological Sabotage Vulnerability Assessment (Ref. 1-11). The radionuclides were screened using quantities listed in 10 CFR 30.72 (Ref. 10) and employing the methodology identified in DOE STD-1027. If the sum of all the ratios of the inventory of each nuclide to that nuclide's Screening Quantity (SQ) from 10CFR30.72 is less than one, the entire inventory was screened (eliminated from analysis):

$$
\sum_{i=1}^{n} \frac{r_{i}}{S Q_{i}}<1.0
$$

where:

$\mathrm{r}=\mathrm{Ci}$ of individual nuclide

$\mathrm{n}=$ number of nuclides

$\mathrm{SQ}=$ Screening Quantity for individual nuclide (Ci)

Per this analysis, all the nuclides fail the screening methodology. The nuclides were further characterized by their contribution to dose upon release. Therefore radioactive isotopes that do not contribute significantly to the total dose were eliminated. The remaining radionuclides contributed greater than or equal to $95 \%$ of the total dose as required in the EMPP-6Q-001 (Ref. 12).

\section{INPUT/ASSUMPTIONS}

The projected maximum radionuclide inventory was developed using various sources as discussed above. Where information was incomplete or unavailable, approximations were used. Some of the inventory lists only one or two of the isotopes. In those cases, the distribution was considered to be made of only those isotopes. The following facilities/missions were considered:

235-F
F Canyon
A Line
HB Line
Tritium Facilities
RBOF
SRTC Operations
SRTC TNX Operations
Central Labs
Tank Farms
TRU Wastes
Mixed Wastes
SREL
D\&D Operations

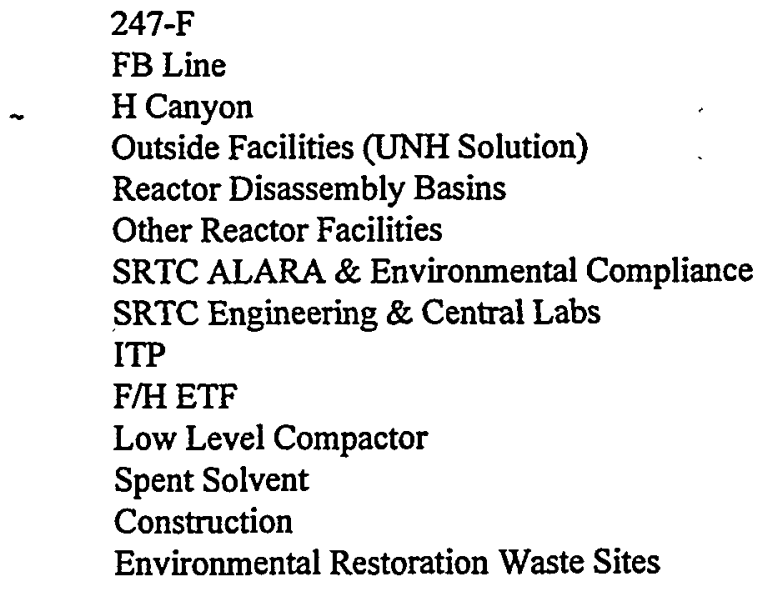

As part of this calc-note SST and spent fuel were not considered in the analysis as they were specifically excluded from the scope of the transportation EPHA. 
Title of Project EPHA for THM Reviewed bv S. A. Henderson

Subject Screening of Radionuclides Works Calculation 4

Computer E.T. Ketusky Date 09/95

\section{CALCULATION}

The release fractions (RF) (Ref. 13) and inhalation Dose Conversion Factors (DCF) (Ref. 14) were used to evaluate the radionuclides of interest. The radionuclide screening for the transportation associated with each of the above facilities are shown in Table 1.

\section{Table 1 Radionuclide Screening}

Note: Release Fractions (RF) extracted from DOE Standard 1027.92.

Inhalation Dose Conversion Factors (DCF) extracted from DOE/EH-0071.

DCF for $\mathrm{H}-3$ increased by $50 \%$ to account for absorption.

Undesignated alpha radiation considered as Am-241.

Undesignated Beta/Gamma radiation considered as Co-60.

Tank Farm Sludge (25 liters) worst case was from HM-Process.

Depleted Uranium Oxide was considered all U-235 for conservatism.

DTotal $=\mathrm{D}$ for that container.

Container for LLW SWDF (Waste)

\begin{tabular}{|l|l|l|l|l|l|}
\hline Radionuclides & RF & $\begin{array}{l}\text { DCF } \\
(\text { rem/uCi })\end{array}$ & $\begin{array}{l}\text { Inventory } \\
(\mathrm{Ci})\end{array}$ & $\begin{array}{l}\text { Total Release } \\
(\text { rem })\end{array}$ & Percent \\
\hline & A & B & C & D=ABC*1E6 & E=D/D TOTAL \\
\hline H-3 & $1.00 E+01$ & $9.50 \mathrm{E}-05$ & $1.00 \mathrm{E}+05$ & $9.50 \mathrm{E}+07$ & $0.36 \%$ \\
\hline Co-60 & $1.00 \mathrm{E}-02$ & $1.50 \mathrm{E}-01$ & $6.75 \mathrm{E}+04$ & $1.01 \mathrm{E}+08$ & $0.39 \%$ \\
\hline Sr-90 & $1.00 \mathrm{E}-03$ & $1.30 \mathrm{E}+00$ & $5.00 \mathrm{E}+01$ & $6.50 \mathrm{E}+04$ & $0.00 \%$ \\
\hline Ru-106 & $1.00 \mathrm{E}-02$ & $4.40 \mathrm{E}-01$ & $7.50 \mathrm{E}+03$ & $3.30 \mathrm{E}+07$ & $0.13 \%$ \\
\hline $\mathrm{Cs}-137$ & $1.00 \mathrm{E}-02$ & $3.20 \mathrm{E}-02$ & $5.00 \mathrm{E}+01$ & $1.60 \mathrm{E}+04$ & $0.00 \%$ \\
\hline $\mathrm{U}-234$ & $1.00 \mathrm{E}-03$ & $1.30 \mathrm{E}+02$ & $4.00 \mathrm{E}+04$ & $5.20 \mathrm{E}+09$ & $19.83 \%$ \\
\hline Am-241 & $1.00 \mathrm{E}-03$ & $5.20 \mathrm{E}+02$ & $4.00 \mathrm{E}+04$ & $2.08 \mathrm{E}+10$ & $79.30 \%$ \\
\hline
\end{tabular}


Title of Project EPHA for THM Reviewed by S. A. Henderson

Container for LLW Compactor $(253-\mathrm{H})$

\begin{tabular}{|c|c|c|c|c|c|}
\hline Radionuclides & RF & $\begin{array}{l}\text { DCF } \\
(\text { rem/uCi) }\end{array}$ & $\begin{array}{l}\text { Inventory } \\
\text { (Ci) }\end{array}$ & $\begin{array}{l}\text { Total Release } \\
(\mathrm{rem})\end{array}$ & Percent \\
\hline & $A$ & $B$ & C & $D=A B C^{*} 1 E 6$ & $E=D / D_{\text {TOTAL }}$ \\
\hline $\mathrm{H}-3$ & $1.00 \mathrm{E}+00$ & $9.50 \mathrm{E}-05$ & $8.30 \mathrm{E}-01$ & $7.89 \mathrm{E} \div 01$ & $0.34 \%$ \\
\hline $\mathrm{C}-14$ & $1.00 \mathrm{E}-02$ & $2.10 \mathrm{E}-03$ & $2.80 \mathrm{E}-04$ & $5.88 \mathrm{E}-03$ & $0.00 \%$ \\
\hline Ni-59 & $1.00 \mathrm{E}-02$ & 2.70E-03 & $5.30 \mathrm{E}-03$ & $1.43 \mathrm{E}-01$ & $0.00 \%$ \\
\hline Co-60 & 1.00E-02 & 1.50E-01 & 2.09E+00 & $3.14 E+03$ & $13.39 \%$ \\
\hline Se-79 & 1.00E-02 & $8.90 E-03$ & 4.30E-05 & $3.83 E-03$ & $0.00 \%$ \\
\hline Sr-90 & $1.00 \mathrm{E}-03$ & $1.30 \mathrm{E}+00$ & $9.20 \mathrm{E}-02$ & $1.20 \mathrm{E}+02$ & $0.51 \%$ \\
\hline Tc-99 & 1.00E-02 & 7.50 E-03 & 1.40E-05 & $1.05 \mathrm{E}-03$ & $0.00 \%$ \\
\hline $5 n-126$ & 1.00E-02 & $8.60 \mathrm{E}-02$ & $2.40 \mathrm{E}-05$ & $2.06 \mathrm{E}-02$ & $0.00 \%$ \\
\hline $1-129$ & $5.00 \mathrm{E}-01$ & $1.80 E-01$ & $6.90 E-09$ & 6.21E-04 & $0.00 \%$ \\
\hline Cs-137 & $1.00 E-02$ & $3.20 \mathrm{E}-02$ & $6.30 \mathrm{E}-02$ & $2.02 E+01$ & $0.09 \%$ \\
\hline U-233 & $1.00 \mathrm{E}-0.3$ & $1.30 \mathrm{E}+02$ & $1.80 \mathrm{E}-06$ & 2.34E-01 & $0.00 \%$ \\
\hline U-234 & 1.00E-03 & $1: 30 \mathrm{E}+02$ & 6.30 E-04 & $8.19 E+01$ & $0.35 \%$ \\
\hline U-235 & 1.00E-03 & $1.20 \mathrm{E}+02$ & $9.20 \mathrm{E}-06$ & $1.10 \mathrm{E}+00$ & $0.00 \%$ \\
\hline U-236 & $1.00 \mathrm{E}-03$ & $1.20 \mathrm{E}+02$ & $1.20 E-04$ & $1.44 \mathrm{E}+01$ & $0.06 \%$ \\
\hline U-238 & 1.00E-03 & $1.20 E+02$ & $1.90 \mathrm{E}-04$ & $2.28 E+01$ & $0.10 \%$ \\
\hline Np-237 & 1.00 E-03 & $4.90 \mathrm{E}+02$ & $1.80 \mathrm{E}-06$ & 8.82E-01 & $0.00 \%$ \\
\hline Pu-238 & $1.00 \mathrm{E}-03$ & $4.60 E+02$ & $7.60 \mathrm{E}-03$ & $3.50 E+03$ & $14.94 \%$ \\
\hline $\mathrm{Pu}-239$ & 1.00E-03 & $5.10 E+02$ & $7.60 \mathrm{E}-03$ & $3.88 \mathrm{E}+03$ & $16.56 \%$ \\
\hline Pu-240 & $1.00 E-03$ & $5.10 E+02$ & 7.60 E-03 & $3.88 E+03$ & $16.56 \%$ \\
\hline Pu-241 & $1.00 \mathrm{E}-03$ & $1.00 E+01$ & $9.20 E-02$ & $9.20 E+02$ & $3.93 \%$ \\
\hline Pu-242 & $1.00 E-03$ & $4.80 \mathrm{E}+02$ & 7.60E-03 & $3.65 E+03$ & $15.59 \%$ \\
\hline Am-241 & 1.00E-03 & $5.20 E+02$ & $7.91 E-03$ & 4.11E+03 & $17.57 \%$ \\
\hline
\end{tabular}

Container for MW Waste - Tritium Bearing (Waste)

\begin{tabular}{|c|c|c|c|c|c|}
\hline Radionuclides & RF & $\mid \begin{array}{l}\text { DCF } \\
(\text { rem/uCi) }\end{array}$ & $\begin{array}{l}\text { Inventory } \\
\text { (Ci) }\end{array}$ & \begin{tabular}{|l} 
Total Release \\
(rem)
\end{tabular} & Percent \\
\hline & A & $B$ & $\mathrm{C}$ & $D=A B C * 1 E 6$ & $E=D / D_{\text {TOTAL }}$ \\
\hline $\mathrm{H}-3$. & $1.00 E+00$ & 9.50E-05 & $5.00 E+03$ & $4.75 E+05$ & $100 \%$ \\
\hline
\end{tabular}

Container for MW Waste - Beta/Gamma (Waste)

\begin{tabular}{|l|l|l|l|l|l|}
\hline Radionuclides & RF & $\begin{array}{l}\text { DCF } \\
(\text { rem/uCi })\end{array}$ & $\begin{array}{l}\text { Inventory } \\
(\mathrm{Ci})\end{array}$ & $\begin{array}{l}\text { Total Release } \\
\text { (rem) }\end{array}$ & Percent \\
\hline & A & B & C & D=ABC*1E6 & E=D/D TOTAL \\
\hline $\mathrm{C} 0-60$ & $1.00 \mathrm{E}-02$ & $1.50 \mathrm{E}-01$ & $1.00 \mathrm{E}+00$ & $1.50 \mathrm{E}+03$ & $24 \%$ \\
\hline $\mathrm{Sr}-90$ & $1.00 \mathrm{E}-02$ & $1.30 \mathrm{E}+00$ & $1.00 \mathrm{E}-03$ & $1.30 \mathrm{E}+01$ & $0 \%$ \\
\hline $\mathrm{Y}-90$ & $1.00 \mathrm{E}-02$ & $8.20 \mathrm{E}-03$ & $1.00 \mathrm{E}-03$ & $8.20 \mathrm{E}-02$ & $0 \%$ \\
\hline $\mathrm{Cs}-137$ & $1.00 \mathrm{E}-02$ & $3.20 \mathrm{E}-02$ & $1.50 \mathrm{E}+01$ & $4.80 \mathrm{E}+03$ & $75 \%$ \\
\hline $\mathrm{Ba}-137 \mathrm{~m}$ & $1.00 \mathrm{E}-02$ & $4.40 \mathrm{E}-04$ & $1.50 \mathrm{E}+01$ & $6.60 \mathrm{E}+01$ & $1 \%$ \\
\hline
\end{tabular}


Title of Project EPHA for THM

Reviewed by S. A. Henderson

Subject Screening of Radionuclides Works Calculation 4

Computer E. T. Ketusky Date $09 / 95$

Container for MW Waste - Alpha (Waste)

\begin{tabular}{|c|c|c|c|c|c|}
\hline Radionuclides & RF & $\begin{array}{l}\text { DCF } \\
\text { (rem/uCi) }\end{array}$ & $\begin{array}{l}\text { Inventory } \\
\text { (Ci) }\end{array}$ & $\begin{array}{l}\text { Total Release } \\
\text { (rem) }\end{array}$ & Percent \\
\hline & A & B & C & $D=A B C^{*} 1 E 6$ & $\mathrm{E}=\mathrm{D} / \mathrm{D}_{\text {TOTAL }}$ \\
\hline Pu-239 & 1.00E-03 & $5.10 E+02$ & $1.20 \mathrm{E}+00$ & $6.12 \mathrm{E}+05$ & $44 \%$ \\
\hline Pu-240 & $1.00 \mathrm{E}-03$ & $5.10 \mathrm{E}+02$ & $3.00 \mathrm{E}-01$ & $1.53 \mathrm{E}+05$ & $11 \%$ \\
\hline Pu-241 & 1.00 E-03 & $1.00 \mathrm{E}+01$ & $5.50 \mathrm{E}+00$ & $5.50 \mathrm{E}+04$ & $4 \%$ \\
\hline Am-241 & $1.00 E-03$ & $5.20 \mathrm{E}+02$ & $1.10 \mathrm{E}+00$ & $5.72 E+05$ & $41 \%$ \\
\hline
\end{tabular}

Container for LAWW (Waste)

\begin{tabular}{|c|c|c|c|c|c|}
\hline Radionuclides & $\mathrm{RF}$ & $\begin{array}{l}\text { DCF } \\
\text { (rem/uCi) }\end{array}$ & $\begin{array}{l}\text { Inventory } \\
\text { (Ci) }\end{array}$ & $\begin{array}{l}\text { Total Release } \\
\text { (rem) }\end{array}$ & Percent \\
\hline & $A$ & $B$ & C & $D=A B C^{*} 1 E 6$ & $E=D / D_{\text {TOTAL }}$ \\
\hline $\mathrm{H}-3$ & $1.00 E+00$ & $9.50 \mathrm{E}-05$ & $1.00 E+01$ & $9.50 E+02$ & $0.34 \%$ \\
\hline$\overline{C-14}$ & $1.00 \mathrm{E}-02$ & 2.10E-03 & 3.30E-03 & 6.93E-02 & $0.00 \%$ \\
\hline Ni-59 & 1.00E-02 & $2.70 E-03$ & $6.30 \mathrm{E}-02$ & $1.70 E+00$ & $0.00 \%$ \\
\hline Co-60 & 1.00E-02 & $1.50 \mathrm{E}-01$ & $2.53 E+01$ & $3.80 E+04$ & $13.52 \%$ \\
\hline Se-79 & 1.00 E-02 & $8.90 \mathrm{E}-03$ & $5.20 \mathrm{E}-04$ & 4.63E-02 & $0.00 \%$ \\
\hline Sr-90 & $1.00 \mathrm{E}-03$ & $1.30 \mathrm{E}+00$ & $1.10 \mathrm{E}+00$ & $1.43 E+03$ & $0.51 \%$ \\
\hline Tc-99 & $1.00 E-02$ & $7.50 \mathrm{E}-03$ & 1.70E-04 & $1.28 \mathrm{E}-02$ & $0.00 \%$ \\
\hline Sn-126 & $1.00 \mathrm{E}-02$ & $8.60 \mathrm{E}-02$ & $2.90 \mathrm{E}-04$ & 2.49E-01 & $0.00 \%$ \\
\hline $1-129$ & 5.00E-01 & $1.80 E-01$ & $8.30 E-08$ & $7.47 \mathrm{E}-03$ & $0.00 \%$ \\
\hline Cs-137 & $1.00 \mathrm{E}-02$ & 3.20E-02 & $7.60 \mathrm{E}-01$ & $2.43 E+02$ & $0.09 \%$ \\
\hline U-233 & $1.00 \mathrm{E}-03$ & $1.30 \mathrm{E}+02$ & 2.20E-05 & $2.86 \mathrm{E}+00$ & $0.00 \%$ \\
\hline U-234 & $1.00 \mathrm{E}-03$ & $1.30 E+02$ & $7.50 \mathrm{E}-03$ & $9.75 \mathrm{E}+02$ & $0.35 \%$ \\
\hline U-235 & 1.00E-03 & $1.20 E+02$ & $1.10 \mathrm{E}-04$ & $1.32 E+01$ & $0.00 \%$ \\
\hline U-236 & $1.00 \mathrm{E}-03$ & $1.20 E+02$ & $1.40 E-03$ & $1.68 \mathrm{E}+02$ & $0.06 \%$ \\
\hline U-238 & $1.00 \mathrm{E}-03$ & $1.20 \mathrm{E}+02$ & $2.30 \mathrm{E}-03$ & $2.76 E+02$ & $0.10 \%$ \\
\hline Np-237 & $1.00 \mathrm{E}-03$ & $4.90 \mathrm{E}+02$ & $2.20 \mathrm{E}-05$ & $1.08 E+01$ & $0.00 \%$ \\
\hline $\mathrm{Pu}-238$ & $1.00 E-03$ & $4.60 \mathrm{E}+02$ & $9.10 \mathrm{E}-02$ & $4.19 E+04$ & $14.92 \%$ \\
\hline Pu-239 & $1.00 E-03$ & $5.10 \mathrm{E}+02$ & $9.10 \mathrm{E}-02$ & $4.64 E+04$ & $16.54 \%$ \\
\hline Pu-240 & $1.00 \mathrm{E}-03$ & $5.10 \mathrm{E} \div 02$ & $9.10 \mathrm{E}-02$ & $4.64 E+04$ & $16.54 \%$ \\
\hline Pu-241 & $1.00 \mathrm{E}-03$ & $1.00 \mathrm{E}+01$ & $1.10 \mathrm{E}+00$ & $1.10 E+04$ & $3.92 \%$ \\
\hline $\mathrm{Pu}-242$ & $1.00 \mathrm{E}-03$ & $4.80 \mathrm{E}+02$ & $9.10 \mathrm{E}-02$ & $4.37 E+04$ & $15.57 \%$ \\
\hline Am-241 & $1.00 E-03$ & $5.20 \mathrm{E}+02$ & $9.47 E-02$ & $4.92 E+04$ & $17.55 \%$ \\
\hline
\end{tabular}




\section{ENGINEERING COMPUTATION SHEET}

Title of Project EPHA for THM Reviewed by S. A. Henderson

Subject ' Screening of Radionuclides

Computer E. T. Ketusky
Works Calculation 4

Date $\underline{09 / 95}$

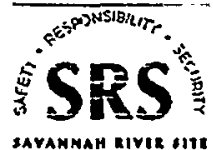

Sheet No. 5 of 19

Container for ILNTV Combustibles (E-Area Vaults)

\begin{tabular}{|l|l|l|l|l|l|}
\hline Radionuclides & RF & $\begin{array}{l}\text { DCF } \\
(\text { rem/uCi })\end{array}$ & $\begin{array}{l}\text { Inventory } \\
(\mathrm{Ci})\end{array}$ & $\begin{array}{l}\text { Total Release } \\
\text { (rem) }\end{array}$ & Percent \\
\hline & $\mathrm{A}$ & $\mathrm{B}$ & $\mathrm{C}$ & $\mathrm{D}=\mathrm{ABC}$ *1E6 & E=D/D TOTAL \\
\hline $\mathrm{H}-3$ & $1.00 \mathrm{E}+00$ & $9.50 \mathrm{E}-05$ & $1.00 \mathrm{E}+01$ & $9.50 \mathrm{E}+02$ & $0.12 \%$ \\
\hline Ni-59 & $1.00 \mathrm{E}-02$ & $2.70 \mathrm{E}-03$ & $2.30 \mathrm{E}+01$ & $6.21 \mathrm{E}+02$ & $0.08 \%$ \\
\hline Co-60 & $1.00 \mathrm{E}-02$ & $1.50 \mathrm{E}-01$ & $3.00 \mathrm{E}+00$ & $4.50 \mathrm{E}+03$ & $0.56 \%$ \\
\hline Se-79 & $1.00 \mathrm{E}-02$ & $8.90 \mathrm{E}-03$ & $2.30 \mathrm{E}-03$ & $2.05 \mathrm{E}-01$ & $0.00 \%$ \\
\hline Sr-90 & $1.00 \mathrm{E}-03$ & $1.30 \mathrm{E}+00$ & $2.30 \mathrm{E}+01$ & $2.99 \mathrm{E}+04$ & $3.73 \%$ \\
\hline Tc-99 & $1.00 \mathrm{E}-02$ & $7.50 \mathrm{E}-03$ & $7.80 \mathrm{E}-04$ & $5.85 \mathrm{E}-02$ & $0.00 \%$ \\
\hline Sn-126 & $1.00 \mathrm{E}-02$ & $8.60 \mathrm{E}-02$ & $2.30 \mathrm{E}-03$ & $1.98 \mathrm{E}+00$ & $0.00 \%$ \\
\hline $\mathrm{I}-129$ & $5.00 \mathrm{E}-01$ & $1.80 \mathrm{E}-01$ & $2.10 \mathrm{E}-07$ & $1.89 \mathrm{E}-02$ & $0.00 \%$ \\
\hline $\mathrm{Cs}-137$ & $1.00 \mathrm{E}-02$ & $3.20 \mathrm{E}-02$ & $2.30 \mathrm{E}+02$ & $7.36 \mathrm{E}+04$ & $9.18 \%$ \\
\hline $\mathrm{U}-233$ & $1.00 \mathrm{E}-03$ & $1.30 \mathrm{E}+02$ & $3.30 \mathrm{E}-05$ & $4.29 \mathrm{E}+00$ & $0.00 \%$ \\
\hline $\mathrm{U}-234$ & $1.00 \mathrm{E}-03$ & $1.30 \mathrm{E}+02$ & $7.50 \mathrm{E}-03$ & $9.75 \mathrm{E}+02$ & $0.12 \%$ \\
\hline $\mathrm{U}-235$ & $1.00 \mathrm{E}-03$ & $1.20 \mathrm{E}+02$ & $1.10 \mathrm{E}-04$ & $1.32 \mathrm{E}+01$ & $0.00 \%$ \\
\hline $\mathrm{U}-236$ & $1.00 \mathrm{E}-03$ & $1.20 \mathrm{E}+02$ & $2.30 \mathrm{E}-03$ & $2.76 \mathrm{E}+02$ & $0.03 \%$ \\
\hline $\mathrm{U}-238$ & $1.00 \mathrm{E}-03$ & $1.20 \mathrm{E}+02$ & $2.30 \mathrm{E}-03$ & $2.76 \mathrm{E}+02$ & $0.03 \%$ \\
\hline Pu-238 & $1.00 \mathrm{E}-03$ & $4.60 \mathrm{E}+02$ & $2.30 \mathrm{E}-01$ & $1.06 \mathrm{E}+05$ & $13.19 \%$ \\
\hline Pu-239 & $1.00 \mathrm{E}-03$ & $5.10 \mathrm{E}+02$ & $2.30 \mathrm{E}-01$ & $1.17 \mathrm{E}+05$ & $14.62 \%$ \\
\hline Pu-240 & $1.00 \mathrm{E}-03$ & $5.10 \mathrm{E}+02$ & $2.30 \mathrm{E}-01$ & $1.17 \mathrm{E}+05$ & $14.62 \%$ \\
\hline Pu-241 & $1.00 \mathrm{E}-03$ & $1.00 \mathrm{E}+01$ & $2.70 \mathrm{E}+00$ & $2.70 \mathrm{E}+04$ & $3.37 \%$ \\
\hline Pu-242 & $1.00 \mathrm{E}-03$ & $4.80 \mathrm{E}+02$ & $2.30 \mathrm{E}-01$ & $1.10 \mathrm{E}+05$ & $13.76 \%$ \\
\hline Am-241 & $1.00 \mathrm{E}-03$ & $5.20 \mathrm{E}+02$ & $4.10 \mathrm{E}-01$ & $2.13 \mathrm{E}+05$ & $26.58 \%$ \\
\hline
\end{tabular}

Container for LNTV Noncombustibles (E-Area Vaults)

\begin{tabular}{|c|c|c|c|c|c|}
\hline Radionuclides & RF & $\begin{array}{l}\text { DCF } \\
\text { (rem/uCi) }\end{array}$ & $\begin{array}{l}\text { Inventory } \\
\text { (Ci) }\end{array}$ & $\begin{array}{l}\text { Total Release } \\
\text { (rem) }\end{array}$ & Percent \\
\hline & A & B & C & $D=A B C^{*} 1 E 6$ & $E=D / D_{T O T A L}$ \\
\hline $\mathrm{H}-3$ & $1.00 \mathrm{E}+00$ & 9.50E-05 & 1.00E+01 & $9.50 E+02$ & $0.01 \%$ \\
\hline $\mathrm{C}-14$ & $1.00 \mathrm{E}-02$ & $2.10 \mathrm{E}-03$ & $2.30 \mathrm{E}-02$ & $4.83 \mathrm{E}-01$ & $0.00 \%$ \\
\hline Ni-59 & $1.00 \mathrm{E}-02$ & $2.70 \mathrm{E}-03$ & $1.80 \mathrm{E}+03$ & $4.86 E+04$ & $0.44 \%$ \\
\hline Co-60 & $1.00 \mathrm{E}-02$ & $1.50 \mathrm{E}-01$ & $1.80 \mathrm{E}+03$ & $2.71 E+06$ & $24.73 \%$ \\
\hline Se-79 & $1.00 \mathrm{E}-02$ & $8.90 E-03$ & 1.80E-02 & $1.60 \mathrm{E}+00$ & $0.00 \%$ \\
\hline Sr-90 & 1.00E-03 & $1.30 E+00$ & $1.80 \mathrm{E}+03$ & $2.34 E+06$ & $21.38 \%$ \\
\hline Tc-99 & $1.00 \mathrm{E}-02$ & $7.50 \mathrm{E}-03$ & $4.70 E-03$ & 3.53E-01 & $0.00 \%$ \\
\hline $5 n-126$ & $1.00 \mathrm{E}-02$ & $8.60 \mathrm{E}-02$ & $1.80 \mathrm{E}-02$ & $1.55 E+01$ & $0.00 \%$ \\
\hline $1-129$ & 5.00E-01 & $1.80 \mathrm{E}-01$ & 1.20E-06 & $1.08 E-01$ & $0.00 \%$ \\
\hline Cs-137 & $1.00 \mathrm{E}-02$ & 3.20E-02 & $1.70 \mathrm{E}+03$ & $5.44 E+05$ & $4.97 \%$ \\
\hline U-233 & 1.00E-03 & $1.30 \mathrm{E}+02$ & 3.30 E-05 & $4.29 E+00$ & $0.00 \%$ \\
\hline $\mathrm{U}-234$ & $1.00 \mathrm{E}-03$ & $1.30 E+02$ & $2.30 \mathrm{E}-02$ & $2.99 E+03$ & $0.03 \%$ \\
\hline U-236 & 1.00E-03 & $1.20 E+02$ & $1.80 \mathrm{E}+00$ & $2.16 \mathrm{E}+05$ & $1.97 \%$ \\
\hline $\mathrm{U}-238$ & $1.00 \mathrm{E}-03$ & $1.20 E+02$ & 1.20E-01 & $1.44 \mathrm{E}+04$ & $0.13 \%$ \\
\hline Np-237 & $1.00 \mathrm{E}-03$ & $4.90 E+02$ & $4.70 \mathrm{E}-04$ & $2.30 E+02$ & $0.00 \%$ \\
\hline $\mathrm{Pu}-238$ & $1.00 \mathrm{E}-03$ & $4.60 E+02$ & $1.80 \mathrm{E}+00$ & $8.28 E+05$ & $7.57 \%$ \\
\hline Pu-239 & 1.00E-03 & $5.10 \mathrm{E}+02$ & $1.80 \mathrm{E}+00$ & $9.18 E+05$ & $8.39 \%$ \\
\hline$P U-240$ & $1.00 \mathrm{E}-03$ & $5.10 \mathrm{E}+02$ & $1.80 E+00$ & $9.18 E+05$ & $8.39 \%$ \\
\hline Pu-241 & $1.00 \mathrm{E}-03$ & $1.00 \mathrm{E}+01$ & $2.20 E+01$ & $2.20 E+05$ & $2.01 \%$ \\
\hline $\mathrm{Pu}-242$ & 1.00E-03 & $4.80 \mathrm{E}+02$ & $1.80 \mathrm{E}+00$ & $8.64 E+05$ & $7.90 \%$ \\
\hline Am-241 & $1.00 \mathrm{E}-03$ & $5.20 \mathrm{E}+02$ & $2.54 E+00$ & $1.32 \mathrm{E}+06$ & $12.07 \%$ \\
\hline
\end{tabular}


Title of Project EPHA for THM

Subject Screening of Radionuclides

Computer E.T. Ketusky
Reviewed by S. A. Henderson Works Calculation 4

Date $09 / 95$

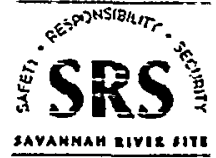

Sheet No. 6 of 19

Container for ILTV Bulk (E Area Vaults)

\begin{tabular}{|c|c|c|c|c|c|}
\hline Radionuclides & RF & $\begin{array}{l}\text { DCF } \\
(\mathrm{rem} / \mathrm{uCi})\end{array}$ & $\begin{array}{l}\text { Inventory } \\
\text { (Ci) }\end{array}$ & $\begin{array}{l}\text { Total Release } \\
(\text { rem) }\end{array}$ & Percent \\
\hline & $A$ & $B$ & c & $D=A B C^{*} 1 E 6$ & $E=D / D_{\text {TOTAL }}$ \\
\hline $\mathrm{H}-3$ & $1.00 \mathrm{E}+00$ & 9.50E-05 & $1.70 \mathrm{E}+02$ & $1.62 E+04$ & $0.44 \%$ \\
\hline Ni-59 & 1.00E-02 & $2.70 \mathrm{E}-03$ & $1.00 \mathrm{E}+00$ & $2.70 \mathrm{E}+01$ & $0.00 \%$ \\
\hline Co-60 & $1.00 \mathrm{E}-02$ & 1.50E-01 & $1.96 \mathrm{E}+03$ & $2.94 E+06$ & $79.91 \%$ \\
\hline Se-79 & 1.00E-02 & 8.90E-03 & $2.30 \mathrm{E}-03$ & 2.05E-01 & $0.00 \%$ \\
\hline Sr-90 & $1.00 \mathrm{E}-03$ & $1.30 \mathrm{E}+00$ & $2.30 \mathrm{E}+01$ & $2.99 E+04$ & $0.81 \%$ \\
\hline Tc-99 & 1.00E-02 & $7.50 \mathrm{E}-03$ & 1.10E-03 & 8.25E-02 & $0.00 \%$ \\
\hline Cs-137 & 1.00E-02 & 3.20E-02 & $2.30 E+02$ & $7.36 \mathrm{E}+04$ & $2.00 \%$ \\
\hline U-233 & $1.00 \mathrm{E}-03$ & $1.30 \mathrm{E}+02$ & $3.30 \mathrm{E}-05$ & $4.29 E+00$ & $0.00 \%$ \\
\hline U-234 & 1.00E-03 & $1.30 \mathrm{E}+02$ & $7.50 \mathrm{E}-02$ & $9.75 E+03$ & $0.27 \%$ \\
\hline U-235 & 1.00E-03 & $1.20 \mathrm{E}+02$ & $1.10 \mathrm{E}-04$ & $1.32 \mathrm{E}+01$ & $0.00 \%$ \\
\hline U-236 & $1.00 \mathrm{E}-03$ & $1.20 \mathrm{E}+02$ & 2.30E-02 & $2.76 E+03$ & $0.08 \%$ \\
\hline U-238 & $1.00 \mathrm{E}-03$ & $1.20 \mathrm{E}+02$ & $2.30 \mathrm{E}-02$ & $2.76 \mathrm{E}+03$ & $0.08 \%$ \\
\hline Np:237 & 1.00 E-03 & $4.90 E+02$ & $1.10 \mathrm{E}-04$ & $5.39 E+01$ & $0.00 \%$ \\
\hline Pu-238 & 1.00E-03 & $4.60 \mathrm{E}+02$ & 2.30E-01 & $1.06 \mathrm{E}+05$ & $2.88 \%$ \\
\hline Pu-239 & 1.00E-03 & $5.10 E+02$ & 2.30E-01 & 1.17E+05 & $3.19 \%$ \\
\hline Pu-240 & 1.00 E-03 & $5.10 E+02$ & 2.30E-01 & $1.17 E+05$ & $3.19 \%$ \\
\hline Pu-241 & $1.00 \mathrm{E}-03$ & $1.00 E+01$ & $2.70 E+00$ & $2.70 E+04$ & $0.73 \%$ \\
\hline $\mathrm{Pu}-242$ & $1.00 E-03$ & $4.80 \mathrm{E}+02$ & $2.30 \mathrm{E}-01$ & $1.10 \mathrm{E}+05$ & $3.00 \%$ \\
\hline Am-241 & $1.00 \mathrm{E}-03$ & $5.20 E+02$ & 2.43E-01 & $1.26 \mathrm{E}+05$ & $3.43 \%$ \\
\hline
\end{tabular}

Container - ILTV Crucibles (E Area Vaults)

\begin{tabular}{|c|c|c|c|c|c|}
\hline Radionuclides & RF & $\begin{array}{l}\text { DCF } \\
\text { (rem/uCi) }\end{array}$ & $\begin{array}{l}\text { Inventory } \\
(\mathrm{Ci})\end{array}$ & $\begin{array}{l}\text { Total Release } \\
\text { (rem) }\end{array}$ & Percent \\
\hline & $A$ & $B$ & c & $D=A B C * 1 E 6$ & $E=D / D_{\text {TOTAL }}$ \\
\hline $\mathrm{H}-3$ & $1.00 \mathrm{E}+00$ & $9.50 E-05$ & $4.00 E+04$ & $3.80 \mathrm{E}+06$ & $73.56 \%$ \\
\hline C-14 & $1.00 \mathrm{E}-02$ & $2.10 \mathrm{E}-03$ & $7.90 \mathrm{E}-03$ & $1.66 E-01$ & $0.00 \%$ \\
\hline Ni-59 & 1.00E-02 & $2.70 \mathrm{E}-03$ & $1.40 \mathrm{E}+00$ & $3.78 \mathrm{E}+01$ & $0.00 \%$ \\
\hline Co-60 & $1.00 \mathrm{E}-02$ & $1.50 E-01$ & $2.70 \mathrm{E}+02$ & $4.05 E+05$ & $7.84 \%$ \\
\hline Se-79 & $1.00 E-02$ & 8.90E-03 & $1.80 \mathrm{E}-03$ & $1.60 \mathrm{E}-01$ & $0.00 \%$ \\
\hline Sr-90 & $1.00 \mathrm{E}-03$ & $1.30 \mathrm{E}+00$ & $1.80 E+02$ & $2.34 E+05$ & $4.53 \%$ \\
\hline TC-99 & $1.00 \mathrm{E}-02$ & $7.50 \mathrm{E}-03$ & $1.60 \mathrm{E}-03$ & $1.20 \mathrm{E}-01$ & $0.00 \%$ \\
\hline Sn-126 & $1.00 \mathrm{E}-02$ & 8.60 E-02 & 1.80E-03 & $1.55 \mathrm{E}+00$ & $0.00 \%$ \\
\hline $1-129$ & 5.00E-01 & $1.80 \mathrm{E}-01$ & $4.20 \mathrm{E}-07$ & $3.78 E-02$ & $0.00 \%$ \\
\hline Cs-137 & 1.00E-02 & $3.20 \mathrm{E}-02$ & $1.80 E+02$ & $5.76 E+04$ & $1.12 \%$ \\
\hline U-234 & $1.00 \mathrm{E}-03$ & $1.30 E+02$ & 7.90E-03 & $1.03 E+03$ & $0.02 \%$ \\
\hline U-235 & $1.00 E-03$ & $1.20 E+02$ & $9.30 \mathrm{E}-06$ & $1.12 \mathrm{E}+00$ & $0.00 \%$ \\
\hline U-236 & 1.00 E-03 & $1.20 E+02$ & $1.80 \mathrm{E}-02$ & $2.16 E+03$ & $0.04 \%$ \\
\hline U-238 & $1.00 \mathrm{E}-03$ & 1.20E+02 & $1.60 \mathrm{E}-04$ & $1.92 E \div 01$ & $0.00 \%$ \\
\hline $\mathrm{Np}-237$ & $1.00 E-03$ & $4.90 E+02$ & $1.80 \mathrm{E}-01$ & $8.82 E+04$ & $1.71 \%$ \\
\hline Pu-238 & 1.00 E-03 & $4.60 E+02$ & $1.80 \mathrm{E}-01$ & $8.28 E+04$ & $1.60 \%$ \\
\hline Pu-239 & $1.00 \mathrm{E}-03$ & $5.10 \mathrm{E}+02$ & $1.80 \mathrm{E}-01$ & $9.18 \mathrm{E}+04$ & $1.78 \%$ \\
\hline Pu-240 & $1.00 \mathrm{E}-03$ & $5.10 E+02$ & 1.80E-01 & $9.18 E+04$ & $1.78 \%$ \\
\hline Pu-241 & $1.00 E-03$ & $1.00 \mathrm{E}+01$ & $2.20 \mathrm{E}+00$ & $2.20 E+04$ & $0.43 \%$ \\
\hline Pu-242 & $1.00 E-03$ & $4.80 E+02$ & $1.80 \mathrm{E}-01$ & $8.64 E+04$ & $1.67 \%$ \\
\hline$A m-241$ & 1.00 E-03 & $5.20 \mathrm{E}+02$ & 3.90E-01 & $2.03 E+05$ & $3.93 \%$ \\
\hline
\end{tabular}


Title of Project EPHA for THM

Subject Screening of Radionuclides

Computer E.T. Ketusky
Reviewed by S. A. Henderson

Works Calculation 4

Date $\underline{09 / 95}$
SPC

saYAMMAM RiviE sire

Sheet No. 7 of 19

Container for LLWSB (E Area Vaults)

\begin{tabular}{|l|l|l|l|l|l|}
\hline Radionuclides & RF & $\begin{array}{l}\text { DCF } \\
(\text { rem/uCi) }\end{array}$ & $\begin{array}{l}\text { Inventory } \\
(\mathrm{Ci})\end{array}$ & $\begin{array}{l}\text { Total Release } \\
(\text { rem })\end{array}$ & Percent \\
\hline & A & B & C & D=ABC*1E6 & E=D/D TOTAL $^{*}$ \\
\hline H-3 & $1.00 \mathrm{E}+00$ & $9.50 \mathrm{E}-05$ & $1.00 \mathrm{E}+04$ & $9.50 \mathrm{E}+05$ & $3.97 \%$ \\
\hline $\mathrm{C}-14$ & $1.00 \mathrm{E}-02$ & $2.10 \mathrm{E}-03$ & $2.30 \mathrm{E}+04$ & $4.83 \mathrm{E}+05$ & $2.02 \%$ \\
\hline Ni-59 & $1.00 \mathrm{E}-02$ & $2.70 \mathrm{E}-03$ & $2.30 \mathrm{E}+00$ & $6.21 \mathrm{E}+01$ & $0.00 \%$ \\
\hline Co-60 & $1.00 \mathrm{E}-02$ & $1.50 \mathrm{E}-01$ & $2.32 \mathrm{E}+02$ & $3.48 \mathrm{E}+05$ & $1.46 \%$ \\
\hline Se-79 & $1.00 \mathrm{E}-02$ & $8.90 \mathrm{E}-03$ & $2.30 \mathrm{E}+00$ & $2.05 \mathrm{E}+02$ & $0.00 \%$ \\
\hline Sr-90 & $1.00 \mathrm{E}-03$ & $1.30 \mathrm{E}+00$ & $2.30 \mathrm{E}+02$ & $2.99 \mathrm{E}+05$ & $1.25 \%$ \\
\hline Tc-99 & $1.00 \mathrm{E}-02$ & $7.50 \mathrm{E}-03$ & $2.30 \mathrm{E}+03$ & $1.73 \mathrm{E}+05$ & $0.72 \%$ \\
\hline Sn-126 & $1.00 \mathrm{E}-02$ & $8.60 \mathrm{E}-02$ & $2.30 \mathrm{E}+00$ & $1.98 \mathrm{E}+03$ & $0.01 \%$ \\
\hline $\mathrm{I}-129$ & $5.00 \mathrm{E}-01$ & $1.80 \mathrm{E}-01$ & $2.30 \mathrm{E}+02$ & $2.07 \mathrm{E}+07$ & $86.49 \%$ \\
\hline $\mathrm{Cs}-137$ & $1.00 \mathrm{E}-02$ & $3.20 \mathrm{E}-02$ & $2.30 \mathrm{E}+02$ & $7.36 \mathrm{E}+04$ & $0.31 \%$ \\
\hline $\mathrm{U}-233$ & $1.00 \mathrm{E}-03$ & $1.30 \mathrm{E}+02$ & $3.30 \mathrm{E}-05$ & $4.29 \mathrm{E}+00$ & $0.00 \%$ \\
\hline $\mathrm{U}-234$ & $1.00 \mathrm{E}-03$ & $1.30 \mathrm{E}+02$ & $2.30 \mathrm{E}-01$ & $2.99 \mathrm{E}+04$ & $0.12 \%$ \\
\hline $\mathrm{U}-235$ & $1.00 \mathrm{E}-03$ & $1.20 \mathrm{E}+02$ & $1.10 \mathrm{E}-04$ & $1.32 \mathrm{E}+01$ & $0.00 \%$ \\
\hline $\mathrm{U}-236$ & $1.00 \mathrm{E}-03$ & $1.20 \mathrm{E}+02$ & $2.30 \mathrm{E}-01$ & $2.76 \mathrm{E}+04$ & $0.12 \%$ \\
\hline $\mathrm{U}-238$ & $1.00 \mathrm{E}-03$ & $1.20 \mathrm{E}+02$ & $2.30 \mathrm{E}-01$ & $2.76 \mathrm{E}+04$ & $0.12 \%$ \\
\hline Np-237 & $1.00 \mathrm{E}-03$ & $4.90 \mathrm{E}+02$ & $2.30 \mathrm{E}-01$ & $1.13 \mathrm{E}+05$ & $0.47 \%$ \\
\hline Pu-238 & $1.00 \mathrm{E}-03$ & $4.60 \mathrm{E}+02$ & $2.30 \mathrm{E}-01$ & $1.06 \mathrm{E}+05$ & $0.44 \%$ \\
\hline Pu-239 & $1.00 \mathrm{E}-03$ & $5.10 \mathrm{E}+02$ & $2.30 \mathrm{E}-01$ & $1.17 \mathrm{E}+05$ & $0.49 \%$ \\
\hline Pu-240 & $1.00 \mathrm{E}-03$ & $5.10 \mathrm{E}+02$ & $2.30 \mathrm{E}-01$ & $1.17 \mathrm{E}+05$ & $0.49 \%$ \\
\hline Pu-241 & $1.00 \mathrm{E}-03$ & $1.00 \mathrm{E}+01$ & $2.70 \mathrm{E}+00$ & $2.70 \mathrm{E}+04$ & $0.11 \%$ \\
\hline Pu-242 & $1.00 \mathrm{E}-03$ & $4.80 \mathrm{E}+02$ & $2.30 \mathrm{E}-01$ & $1.10 \mathrm{E}+05$ & $0.46 \%$ \\
\hline Am-241 & $1.00 \mathrm{E}-03$ & $5.20 \mathrm{E}+02$ & $4.40 \mathrm{E}-01$ & $2.29 \mathrm{E}+05$ & $0.96 \%$ \\
\hline & & & & \\
\hline
\end{tabular}

Pu O2 Container (235-F, 247-F)

\begin{tabular}{|c|c|c|c|c|c|}
\hline Radionuclides & RF & $\begin{array}{l}\text { DCF } \\
\text { (rem/uCi) }\end{array}$ & $\begin{array}{l}\text { Inventory } \\
\text { (Ci) }\end{array}$ & $\begin{array}{l}\text { Total Release } \\
\text { (rem) }\end{array}$ & Percent \\
\hline & $A$ & $B$ & C & $D=A B C^{*} 1 E 6$ & $E=D / D_{\text {TOTAL }}$ \\
\hline Pu-238 & $1.00 E-03$ & $4.60 \mathrm{E}+02$ & $9.16 \mathrm{E}+02$ & 4.21E+08 & $100.00 \%$ \\
\hline
\end{tabular}

Container, Pail, Casket, Charge can, Type A Storage Vault (235-F)

\begin{tabular}{|l|l|l|l|l|l|}
\hline Radionuclides & RF & $\begin{array}{l}\text { DCF } \\
(\text { rem/uCi })\end{array}$ & $\begin{array}{l}\text { Inventory } \\
(\mathrm{Ci})\end{array}$ & $\begin{array}{l}\text { Total Release } \\
(\text { rem })\end{array}$ & Percent \\
\hline & A & B & C & D=ABC*1E6 & E=D/D TOTAL \\
\hline Pu-238 & $1.00 E-03$ & $4.60 E+02$ & $2.74 E+05$ & $1.26 E+11$ & $99.99 \%$ \\
\hline U-235 & $1.00 E-03$ & $1.20 E+02$ & $4.86 E-03$ & $5.83 E+02$ & $0.00 \%$ \\
\hline Np-237 & $1.00 \mathrm{E}-03$ & $4.90 \mathrm{E}+02$ & $1.41 \mathrm{E}+01$ & $6.91 \mathrm{E}+06$ & $0.01 \%$ \\
\hline
\end{tabular}

H Canyon Sample Container to SRTC (221H to $773 \mathrm{~A})$

\begin{tabular}{|l|l|l|l|l|l|}
\hline Radionuclides & RF & $\begin{array}{l}\text { DCF } \\
(\text { rem/uCi })\end{array}$ & $\begin{array}{l}\text { Inventory } \\
(\mathrm{Ci})\end{array}$ & $\begin{array}{l}\text { Total Release } \\
(\mathrm{rem})\end{array}$ & Percent \\
\hline & $\mathrm{A}$ & $\mathrm{B}$ & $\mathrm{C}$ & $\mathrm{D}=\mathrm{ABC}$ 1E6 & E=D/D \\
\hline Pu-238 & $1.00 \mathrm{E}-03$ & $4.60 \mathrm{E}+02$ & $1.54 \mathrm{E}+01$ & $7.08 \mathrm{E}+06$ & $100.00 \%$ \\
\hline
\end{tabular}


Title of Project EPHA for THM

Subject Screening of Radionuclides Works Calculation 4

Computer E. T. Ketusky Date 09/95

H Canyon Sample Container to SRTC (221H to 773A)

\begin{tabular}{|l|l|l|l|l|l|}
\hline Radionuclides & RF & $\begin{array}{l}\text { DCF } \\
(\mathrm{rem} / \mathrm{uCi})\end{array}$ & $\begin{array}{l}\text { Inventory } \\
(\mathrm{Ci})\end{array}$ & $\begin{array}{l}\text { Total Release } \\
(\mathrm{rem})\end{array}$ & Percent \\
\hline & $\mathrm{A}$ & $\mathrm{B}$ & $\mathrm{C}$ & $\mathrm{D}=\mathrm{ABC}{ }^{*} 1 \mathrm{E} 6$ & $\mathrm{E}=\mathrm{D} / \mathrm{D}_{\mathrm{TOTAL}}$ \\
\hline $\mathrm{U}-235$ & $1.00 \mathrm{E}-03$ & $1.20 \mathrm{E}+02$ & $8.64 \mathrm{E}-05$ & $1.04 \mathrm{E}+01$ & $100.00 \%$ \\
\hline
\end{tabular}

H Canyon Sample Container to SRTC (221H to 773A)

\begin{tabular}{|l|l|l|l|l|l|}
\hline Radionuclides & RF & $\begin{array}{l}\text { DCF } \\
(\mathrm{rem} / \mathrm{uCi})\end{array}$ & $\begin{array}{l}\text { Inventory } \\
(\mathrm{Ci})\end{array}$ & $\begin{array}{l}\text { Total Release } \\
(\mathrm{rem})\end{array}$ & Percent \\
\hline & $\mathrm{A}$ & $\mathrm{B}$ & $\mathrm{C}$ & $\mathrm{D}=\mathrm{ABC} \mathrm{CE}^{*} \mathrm{E}$ & $\mathrm{E}=\mathrm{D} / \mathrm{D}_{\mathrm{TOTAL}}$ \\
\hline $\mathrm{Np}-237$ & $1.00 \mathrm{E}-03$ & $4.90 \mathrm{E}+02$ & $7.05 \mathrm{E}-04$ & $3.45 \mathrm{E}+02$ & $100.00 \%$ \\
\hline
\end{tabular}

F Canyon Sample Container to SRTC (221F to 773A)

\begin{tabular}{|l|l|l|l|l|l|}
\hline Radionuclides & RF & $\begin{array}{l}\text { DCF } \\
(\mathrm{rem} / \mathrm{uCi})\end{array}$ & $\begin{array}{l}\text { Inventory } \\
(\mathrm{Ci})\end{array}$ & $\begin{array}{l}\text { Total Release } \\
(\mathrm{rem})\end{array}$ & Percent \\
\hline & $\mathrm{A}$ & $\mathrm{B}$ & $\mathrm{C}$ & $\mathrm{D}=\mathrm{ABC}$ 1E6 & $\mathrm{E}=\mathrm{D} / \mathrm{D}_{\mathrm{TOTAL}}$ \\
\hline PU-238 & $1.00 \mathrm{E}-03$ & $4.60 \mathrm{E}+02$ & $1.20 \mathrm{E}-01$ & $5.51 \mathrm{E}+04$ & $100.00 \%$ \\
\hline
\end{tabular}

F Canyon Sample Container to SRTC (221F to 773A)

\begin{tabular}{|c|c|c|c|c|c|}
\hline Radionuclides & RF & $\begin{array}{l}\text { DCF } \\
(\text { rem/uCi) }\end{array}$ & $\begin{array}{l}\text { Inventory } \\
\text { (Ci) }\end{array}$ & $\begin{array}{l}\text { Total Release } \\
(\mathrm{rem})\end{array}$ & Percent \\
\hline & $\bar{A}$ & $B$ & C & $D=A B C^{*} 1 E 6$ & $E=D / D_{\text {TOTAL }}$ \\
\hline$A m-243$ & $1.00 E-03$ & $5.20 E+02$ & 1.99E-02 & $1.03 E \div 04$ & $100.00 \%$ \\
\hline
\end{tabular}

F Canyon Sample Container to SRTC (221F to 773A)

\begin{tabular}{|l|l|l|l|l|l|}
\hline Radionuclides & RF & $\begin{array}{l}\text { DCF } \\
(\mathrm{rem} / \mathrm{uCi})\end{array}$ & $\begin{array}{l}\text { Inventory } \\
(\mathrm{Ci})\end{array}$ & $\begin{array}{l}\text { Total Release } \\
(\text { rem })\end{array}$ & Percent \\
\hline & $\mathrm{A}$ & $\mathrm{B}$ & $\mathrm{C}$ & $\mathrm{D}=\mathrm{ABC}$ 1E6 & ${\mathrm{E}=\mathrm{D} / \mathrm{D}_{\text {TOTAL }}}$ \\
\hline $\mathrm{Cm}-244$ & $1.00 \mathrm{E}-03$ & $2.70 \mathrm{E}+02$ & $8.09 \mathrm{E}+00$ & $2.18 \mathrm{E}+06$ & $100.00 \%$ \\
\hline
\end{tabular}

HB Line Sample Container (221H\&F to 772-1F)

\begin{tabular}{|l|l|l|l|l|l|}
\hline Radionuclides & RF & $\begin{array}{l}\text { DCF } \\
(\text { rem/uCi })\end{array}$ & $\begin{array}{l}\text { Inventory } \\
(\mathrm{Ci})\end{array}$ & $\begin{array}{l}\text { Total Release } \\
\text { (rem) }\end{array}$ & Percent \\
\hline & A & B & C & D=ABC*1E6 & E=D/D \\
\hline Pu-238 & $1.00 \mathrm{E}-03$ & $4.60 \mathrm{E}+02$ & $8.55 \mathrm{E}-01$ & $3.93 \mathrm{E}+05$ & $100.00 \%$ \\
\hline
\end{tabular}

HB Line Sample Container (221H to 773A)

\begin{tabular}{|c|c|c|c|c|c|}
\hline Radionuclides & RF & $\begin{array}{l}\text { DCF } \\
(\text { rem/uCi) }\end{array}$ & $\begin{array}{l}\text { Inventory } \\
\text { (Ci). }\end{array}$ & $\begin{array}{l}\text { Total Release } \\
\text { (rem) }\end{array}$ & Percent \\
\hline & $A$ & $B$ & C & $D=A B C^{*} 1 E 6$ & $E=D / D_{T O T A L}$ \\
\hline Pu-238 & $1.00 \mathrm{E}-03$ & $4.60 E+02$ & $8.55 E-01$ & $3.93 E+05$ & $100.00 \%$ \\
\hline
\end{tabular}


Title of Project EPHA for THM

Subject Screening of Radionuclides

Computer E.T. Ketusky
Reviewed by S. A. Henderson

Works Calculation 4

Date $09 / 95$

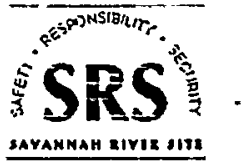

Sheet No. 9 of 19

FB Line Oxide Container (221F to Inter Area)

\begin{tabular}{|l|l|l|l|l|l|}
\hline Radionuclides & RF & $\begin{array}{l}\text { DCF } \\
(\mathrm{rem} / \mathrm{uCi})\end{array}$ & $\begin{array}{l}\text { lnventory } \\
(\mathrm{Ci})\end{array}$ & $\begin{array}{l}\text { Total Release } \\
\text { (rem) }\end{array}$ & Percent \\
\hline & $\mathrm{A}$ & $\mathrm{B}$ & $\mathrm{C}$ & $\mathrm{D}=\mathrm{ABC}$ 1E6 & $\mathrm{E}=\mathrm{D} / \mathrm{D}_{\text {TOTAL }}$ \\
\hline Pu-239 & $1.00 \mathrm{E}-03$ & $5.10 \mathrm{E}+02$ & $5.58 \mathrm{E}+03$ & $2.85 \mathrm{E}+09$ & $100.00 \%$ \\
\hline
\end{tabular}

FB Line Sample Container (221F to $773 \mathrm{~A}$ )

\begin{tabular}{|l|l|l|l|l|l|}
\hline Radionuclides & RF & $\begin{array}{l}\text { DCF } \\
(\mathrm{rem} / \mathrm{uCi})\end{array}$ & $\begin{array}{l}\text { Inventory } \\
(\mathrm{Ci})\end{array}$ & $\begin{array}{l}\text { Total Release } \\
(\mathrm{rem})\end{array}$ & Percent \\
\hline & $\mathrm{A}$ & $\mathrm{B}$ & $\mathrm{C}$ & $\mathrm{D}=\mathrm{ABC}$ 1E6 & $\mathrm{E}=\mathrm{D} / \mathrm{D}_{\mathrm{TOTAL}}$ \\
\hline Pu-239 & $1.00 \mathrm{E}-03$ & $5.10 \mathrm{E}+02$ & $6.20 \mathrm{E}-01$ & $3.16 \mathrm{E}+05$ & $100.00 \%$ \\
\hline
\end{tabular}

235F Material Container to $221 \mathrm{HB}$ Line (235F to $221 \mathrm{H})$

\begin{tabular}{|l|l|l|l|l|l|}
\hline Radionuclides & RF & $\begin{array}{l}\text { DCF } \\
(\mathrm{rem} / \mathrm{UCi})\end{array}$ & $\begin{array}{l}\text { Inventory } \\
(\mathrm{Ci})\end{array}$ & $\begin{array}{l}\text { Total Release } \\
(\mathrm{rem})\end{array}$ & Percent \\
\hline & $\mathrm{A}$ & $\mathrm{B}$ & $\mathrm{C}$ & $\mathrm{D}=\mathrm{ABC} * 1 \mathrm{E6} 6$ & $\mathrm{E}=\mathrm{D} / \mathrm{D}_{\text {TOTAL }}$ \\
\hline Pu-238 & $1.00 \mathrm{E}-03$ & $4.60 \mathrm{E}+02$ & $3.42 \mathrm{E}+04$ & $1.57 \mathrm{E}+10$ & $100.00 \%$ \\
\hline
\end{tabular}

235F Material Container to $221 \mathrm{FB}$ Line (235F to 221F)

\begin{tabular}{|l|l|l|l|l|l|}
\hline Radionuclides & RF & $\begin{array}{l}\text { DCF } \\
(\mathrm{rem} / \mathrm{uCi})\end{array}$ & $\begin{array}{l}\text { Inventory } \\
(\mathrm{Ci})\end{array}$ & $\begin{array}{l}\text { Total Release } \\
(\mathrm{rem})\end{array}$ & Percent \\
\hline & $\mathrm{A}$ & $\mathrm{B}$ & $\mathrm{C}$ & $\mathrm{D}=\mathrm{ABC}$ 1E6 & $\mathrm{E}=\mathrm{D} / \mathrm{D}_{\mathrm{TOTAL}}$ \\
\hline Pu-239 & $1.00 \mathrm{E}-03$ & $5.10 \mathrm{E}+02$ & $1.86 \mathrm{E}+02$ & $9.49 \mathrm{E}+07$ & $100.00 \%$ \\
\hline
\end{tabular}

SRTC Material Container to DWPF (SRTC to DWPF)

\begin{tabular}{|l|l|l|l|l|l|}
\hline Radionuclides & RF & $\begin{array}{l}\text { DCF } \\
(\text { rem/uCi })\end{array}$ & $\begin{array}{l}\text { Inventory } \\
(\mathrm{Ci})\end{array}$ & $\begin{array}{l}\text { Total Release } \\
(\text { rem })\end{array}$ & Percent \\
\hline & A & B & C & D=ABC*1E6 & E=D/D TOTAL \\
\hline Cf-252 & $1.00 E-03$ & $1.30 \mathrm{E}+02$ & $2.69 \mathrm{E}+01$ & $3.50 \mathrm{E}+06$ & $100.00 \%$ \\
\hline
\end{tabular}

A Line Material Container (A Line)

\begin{tabular}{|l|l|l|l|l|l|}
\hline Radionuclides & $\begin{array}{l}\text { RF } \\
0\end{array}$ & $\begin{array}{l}\text { DCF } \\
(\text { rem/uCi })\end{array}$ & $\begin{array}{l}\text { Inventory } \\
(\mathrm{Ci})\end{array}$ & $\begin{array}{l}\text { Total Release } \\
(\mathrm{rem})\end{array}$ & Percent \\
\hline (assumed U235) & $\mathrm{A}$ & $\mathrm{B}$ & $\mathrm{C}$ & $\mathrm{D=ABC} * 1 \mathrm{E} 6$ & $\mathrm{E}=\mathrm{D} / \mathrm{D}_{\text {TOTAL }}$ \\
\hline Depleted UO3 & $1.00 \mathrm{E}-03$ & $1.20 \mathrm{E}+02$ & $3.24 \mathrm{E}-03$ & $3.89 \mathrm{E}+02$ & $100.00 \%$ \\
\hline
\end{tabular}


Title of Project EPHA for THM Reviewed by $S$. A. Henderson

Subject Screening of Radionuclides Works Calculation 4

Computer E.T. Ketusky Date $09 / 95$

Sheet No. 10 of 19

UNH Trailer (H Area Outside Facilities)

\begin{tabular}{|l|l|l|l|l|l|}
\hline Radionuclides & RF & $\begin{array}{l}\text { DCF } \\
(\text { rem/uCi })\end{array}$ & $\begin{array}{l}\text { Inventory } \\
(\mathrm{Ci} /)\end{array}$ & $\begin{array}{l}\text { Total Release } \\
(\mathrm{rem})\end{array}$ & Percent \\
\hline & $\mathrm{A}$ & $\mathrm{B}$ & $\mathrm{C}$ & $\mathrm{D}=\mathrm{ABC}$ 1E6 6 & $\mathrm{E}=\mathrm{D} / \mathrm{D}_{\text {TOTAL }}$ \\
\hline $\mathrm{U}-234$ & $1.00 \mathrm{E}-03$ & $1.30 \mathrm{E}+02$ & $6.55 \mathrm{E}-03$ & $8.52 \mathrm{E}-04$ & $78.06 \%$ \\
\hline $\mathrm{U}-235$ & $1.00 \mathrm{E}-03$ & $1.20 \mathrm{E}+02$ & $2.48 \mathrm{E}-05$ & $2.98 \mathrm{E}-06$ & $0.27 \%$ \\
\hline $\mathrm{U}-236$ & $1.00 \mathrm{E}-03$ & $1.20 \mathrm{E}+02$ & $2.23 \mathrm{E}-04$ & $2.68 \mathrm{E}-05$ & $2.45 \%$ \\
\hline $\mathrm{U}-238$ & $1.00 \mathrm{E}-03$ & $1.20 \mathrm{E}+02$ & $1.64 \mathrm{E}-03$ & $1.97 \mathrm{E}-04$ & $18.04 \%$ \\
\hline Pu-238 & $1.00 \mathrm{E}-03$ & $4.60 \mathrm{E}+02$ & 0.00 & 0.00 & 0.00 \\
\hline PU-239 & $1.00 \mathrm{E}-03$ & $5.10 \mathrm{E}+02$ & $2.50 \mathrm{E}-05$ & $1.28 \mathrm{E}-05$ & $1.17 \%$ \\
\hline
\end{tabular}

RBOF Portable Deionizer Column Transport (RBOF)

\begin{tabular}{|l|l|l|l|l|l|}
\hline Radionuclides & RF & $\begin{array}{l}\text { DCF } \\
(\text { rem/uCi })\end{array}$ & $\begin{array}{l}\text { Inventory } \\
(\mathrm{Ci})\end{array}$ & $\begin{array}{l}\text { Total Release } \\
(\text { rem })\end{array}$ & Percent \\
\hline & A & B & C & D=ABC*1E6 & E=D/D TOTAL \\
\hline $\mathrm{Cr}-51$ & $1.00 \mathrm{E}+00$ & $2.60 \mathrm{E}-04$ & $1.70 \mathrm{E}+01$ & $4.42 \mathrm{E}+03$ & $6 \%$ \\
\hline $\mathrm{Co}-60$ & $1.00 \mathrm{E}-02$ & $1.50 \mathrm{E}-01$ & $3.20 \mathrm{E}-01$ & $4.80 \mathrm{E}+02$ & $1 \%$ \\
\hline $\mathrm{Zn}-65$ & $1.00 \mathrm{E}-02$ & $1.80 \mathrm{E}-02$ & $8.50 \mathrm{E}-01$ & $1.53 \mathrm{E}+02$ & $0 \%$ \\
\hline $\mathrm{Zr}-95$ & $1.00 \mathrm{E}-02$ & $1.90 \mathrm{E}-02$ & $1.40 \mathrm{E}+00$ & $2.66 \mathrm{E}+02$ & $0 \%$ \\
\hline $\mathrm{Nb}-95$ & $1.00 \mathrm{E}-02$ & $4.50 \mathrm{E}-03$ & $7.40 \mathrm{E}-01$ & $3.33 \mathrm{E}+01$ & $0 \%$ \\
\hline $\mathrm{Ru}-106$ & $1.00 \mathrm{E}-03$ & $4.40 \mathrm{E}-01$ & $8.90 \mathrm{E}+00$ & $3.92 \mathrm{E}+03$ & $5 \%$ \\
\hline $\mathrm{Cs}-137$ & $1.00 \mathrm{E}-03$ & $3.20 \mathrm{E}-02$ & $1.00 \mathrm{E}+03$ & $3.20 \mathrm{E}+04$ & $42 \%$ \\
\hline $\mathrm{Ce}-144$ & $1.00 \mathrm{E}-02$ & $3.50 \mathrm{E}-01$ & $1.00 \mathrm{E}+01$ & $3.50 \mathrm{E}+04$ & $46 \%$ \\
\hline
\end{tabular}

RBOF Spent Deionizer Resin Transport (RBOF)

\begin{tabular}{|c|c|c|c|c|c|}
\hline Radionuclides & RF & $\begin{array}{l}\text { DCF } \\
\text { (rem/uCi) }\end{array}$ & $\begin{array}{l}\text { Inventory } \\
\text { (Ci) }\end{array}$ & $\begin{array}{l}\text { Total Release } \\
\text { (rem) }\end{array}$ & Percent \\
\hline & $A$ & $B$ & $c$ & $D=A B C^{\star} 1 E 6$ & $E=D / D_{\text {TOTAL }}$ \\
\hline $\mathrm{Cr}-51$ & $1.00 \mathrm{E}+00$ & 2.61E-04 & $9.60 E+00$ & $2.51 \mathrm{E}+03$ & $0 \%$ \\
\hline Co-60 & $1.00 \mathrm{E}-02$ & $1.50 \mathrm{E}-01$ & $1.80 \mathrm{E}-01$ & $2.70 \mathrm{E} \div 02$ & $0 \%$ \\
\hline $2 n-65$ & $1.00 \mathrm{E}-02$ & $1.80 \mathrm{E}-02$ & $4.70 \mathrm{E}-01$ & $8.46 \mathrm{E}+01$ & $0 \%$ \\
\hline $\mathrm{Zr}-95$ & 1.00E-02 & 1.90E-02 & 7.70E-01 & $1.46 \mathrm{E}+02$ & $0 \%$ \\
\hline $\mathrm{Nb}-95$ & 1.00E-02 & $4.50 \mathrm{E}-03$ & 4.10E-01 & $1.85 \mathrm{E}+01$ & $0 \%$ \\
\hline Ru-106 & $1.00 E-03$ & $4.40 E-01$ & $4.90 \mathrm{E}+00$ & $2.16 \mathrm{E}+03$ & $0 \%$ \\
\hline Cs-134 & $1.00 E-03$ & $4.70 \mathrm{E}-02$ & 4.10E-01 & $1.93 E+01$ & $0 \%$ \\
\hline Cs-137 & 1.00E-02 & $3.20 \mathrm{E}-02$ & $5.70 \mathrm{E}+02$ & $1.82 \mathrm{E}+05$ & $36 \%$ \\
\hline $\mathrm{Ce}-144$ & 1.00E-02 & 3.50E-01 & $5.70 E+00$ & $2.00 E+04$ & $4 \%$ \\
\hline Pu-240 & $1.00 \mathrm{E}-03$ & $5.10 E+02$ & 5.90E-01 & $3.01 E+05$ & $59 \%$ \\
\hline
\end{tabular}

Disassembly Basin Deionizer \& Purif Resin Cask (Reactors, RBOF)

\begin{tabular}{|l|l|l|l|l|l|}
\hline Radionuclides & RF & $\begin{array}{l}\text { DCF } \\
(\mathrm{rem} / \mathrm{uCi})\end{array}$ & $\begin{array}{l}\text { Inventory } \\
(\mathrm{Ci})\end{array}$ & $\begin{array}{l}\text { Total Release } \\
(\mathrm{rem})\end{array}$ & Percent \\
\hline & $\mathrm{A}$ & $\mathrm{B}$ & $\mathrm{C}$ & $\mathrm{D}=\mathrm{ABC}$ 1E6 & E=D/D \\
\hline $\mathrm{Co}-60$ & $1.00 \mathrm{E}-02$ & $1.50 \mathrm{E}-01$ & $1.69 \mathrm{E}+00$ & $2.54 \mathrm{E}+03$ & $100 \%$ \\
\hline
\end{tabular}


Title of Project EPHA for THM Reviewed by S. A. Henderson

Subject Screening of Radionuclides Works Calculation 4

Computer E.T. Ketusky Date $09 / 95$

Container for Reactor Samples to 771-D/F (Reactors to 771D/F)

\begin{tabular}{|l|l|l|l|l|l|}
\hline Radionuclides & RF & $\begin{array}{l}\text { DCF } \\
(\mathrm{rem} / \mathrm{uCi})\end{array}$ & $\begin{array}{l}\text { Inventory } \\
(\mathrm{Ci})\end{array}$ & $\begin{array}{l}\text { Total Release } \\
(\mathrm{rem})\end{array}$ & Percent \\
\hline & $\mathrm{A}$ & $\mathrm{B}$ & $\mathrm{C}$ & $\mathrm{D}=\mathrm{ABC} * 1 \mathrm{E} 6$ & $\mathrm{E}=\mathrm{D} / \mathrm{D}_{\mathrm{TOTAL}}$ \\
\hline $\mathrm{Co} 0-60$ & $1.00 \mathrm{E}-02$ & $1.50 \mathrm{E}-01$ & $1.69 \mathrm{E}+00$ & $2.54 \mathrm{E}+03$ & $100 \%$ \\
\hline
\end{tabular}

Sample Truck (Inter Area to/from SRTC)

\begin{tabular}{|l|l|l|l|l|l|}
\hline Radionuclides & RF & $\begin{array}{l}\text { DCF } \\
(\mathrm{rem} / \mathrm{uCi})\end{array}$ & $\begin{array}{l}\text { Inventory } \\
(\mathrm{Ci})\end{array}$ & $\begin{array}{l}\text { Total Release } \\
(\mathrm{rem})\end{array}$ & Percent \\
\hline & $\mathrm{A}$ & $\mathrm{B}$ & $\mathrm{C}$ & $\mathrm{D}=\mathrm{ABC}$ 1E6 & $\mathrm{E}=\mathrm{D} / \mathrm{D}_{\mathrm{TOTAL}}$ \\
\hline $\mathrm{Cs}-137$ & $1.00 \mathrm{E}-02$ & $3.20 \mathrm{E}-02$ & $8.51 \mathrm{E}+02$ & $2.72 \mathrm{E}+05$ & $100 \%$ \\
\hline
\end{tabular}

Moderator in 55 Gallon Drum (100-400 Areas)

\begin{tabular}{|l|l|l|l|l|l|}
\hline Radionuclides & RF & $\begin{array}{l}\text { DCF } \\
(\mathrm{rem} / \mathrm{uCi})\end{array}$ & $\begin{array}{l}\text { Inventory } \\
(\mathrm{Ci})\end{array}$ & $\begin{array}{l}\text { Total Release } \\
(\mathrm{rem})\end{array}$ & Percent \\
\hline $\mathrm{Am}-241$ & $\mathrm{~A}$ & $\mathrm{~B}$ & $\mathrm{C}$ & $\mathrm{D}=\mathrm{ABC}+1 \mathrm{E} 6$ & $\mathrm{E}=\mathrm{D} / \mathrm{D}_{\mathrm{TOTAL}}$ \\
\hline
\end{tabular}

SRTC 776-A to F Tank Farm 5000 Gallon Truck (SRTC to F Tank Farm)

\begin{tabular}{|c|c|c|c|c|c|}
\hline Radionuclides & RF & $\begin{array}{l}\text { DCF } \\
\text { (rem/uCi) }\end{array}$ & $\begin{array}{l}\text { Inventory } \\
\text { (Ci) }\end{array}$ & $\begin{array}{l}\text { Total Release } \\
\text { (rem) }\end{array}$ & Percent \\
\hline & A & $B$ & C & $D=A B C^{*} 1 E 6$ & $E=D / D_{\text {TOTAL }}$ \\
\hline $\mathrm{Pu}-239$ & $1.00 \mathrm{E}-03$ & $5.10 \mathrm{E}+02$ & 6.23E-02 & 3.17E+04 & $100 \%$ \\
\hline
\end{tabular}

Contaminated Water Transport (100 Area to 211-F)

\begin{tabular}{|l|l|l|l|l|l|}
\hline Radionuclides & RF & $\begin{array}{l}\text { DCF } \\
(\text { rem/uCi })\end{array}$ & $\begin{array}{l}\text { Inventory } \\
(\mathrm{Ci})\end{array}$ & $\begin{array}{l}\text { Total Release } \\
(\mathrm{rem})\end{array}$ & Percent \\
\hline & $\mathrm{A}$ & $\mathrm{B}$ & $\mathrm{C}$ & $\mathrm{D}=\mathrm{ABC}$ 1E6 & $\mathrm{E}=\mathrm{D} / \mathrm{D}_{\mathrm{TOTAL}}$ \\
\hline $\mathrm{Am}-241$ & $1.00 \mathrm{E}-03$ & $5.20 \mathrm{E}+02$ & $2.00 \mathrm{E}+00$ & $1.04 \mathrm{E}+06$ & $100 \%$ \\
\hline
\end{tabular}

100 Area Sludge Transport, 2000 Gallons (100 Areas to 241-F)

\begin{tabular}{|l|l|l|l|l|l|}
\hline Radionuclides & RF & $\begin{array}{l}\text { DCF } \\
(\text { rem/uCi })\end{array}$ & $\begin{array}{l}\text { Inventory } \\
(\mathrm{Ci})\end{array}$ & $\begin{array}{l}\text { Total Release } \\
(\mathrm{rem})\end{array}$ & Percent \\
\hline & $\mathrm{A}$ & $\mathrm{B}$ & $\mathrm{C}$ & $\mathrm{D}=\mathrm{ABC}$ 1E6 & $\mathrm{E}=\mathrm{D} / \mathrm{D}_{\mathrm{TOTAL}}$ \\
\hline $\mathrm{C} 0-60$ & $1.00 \mathrm{E}-02$ & $1.50 \mathrm{E}-01$ & $2.00 \mathrm{E}+00$ & $3.00 \mathrm{E}+03$ & $100 \%$ \\
\hline
\end{tabular}


Title of Project EPHA for THM Reviewed by S. A. Henderson

Subject Screening of Radionuclides Works Calculation 4

Computer E. T. Ketusky

\section{RESULTS} Date 09/95

Table 2 presents the radionuclides inventories determined for the various onsite and offsite shipments to contribute greater than or equal to $95 \%$ of the total dose as required in the EMPP-6Q-001.

Table 2 Screened Radionuclides

Note: Release Fractions (RF) extracted from DOE Standard 1027.92.

Inhalation Dose Conversion Factors (DCF) extracted from DOE/EH-0071.

DCF for $\mathrm{H}-3$ increased by $50 \%$ to account for absorption.

Undesignated alpha radiation considered as Am-241.

Undesignated Beta/Gamma radiation considered as $\mathrm{Co}-60$.

Tank Farm Sludge (25 liters) worst case was from HM-Process.

Depleted Uranium Oxide was considered all U-235 for conservatism.

DTOT(1) refers to the total of column D for that container from Table 1.

Container of LLW SWDF (Waste)

\begin{tabular}{|l|l|l|l|l|l|}
\hline Radionuclides & RF & $\begin{array}{l}\text { DCF } \\
(\mathrm{rem} / \mathrm{uCi})\end{array}$ & $\begin{array}{l}\text { Inventory } \\
(\mathrm{Ci})\end{array}$ & $\begin{array}{l}\text { Total Release } \\
(\mathrm{rem})\end{array}$ & Percent \\
\hline & $\mathrm{A}$ & $\mathrm{B}$ & $\mathrm{C}$ & $\mathrm{D}=\mathrm{ABC}+1 \mathrm{E} 6$ & $\mathrm{E}=\mathrm{D} / \mathrm{D}_{\mathrm{TOT}(1)}$ \\
\hline $\mathrm{U}-234$ & $1.00 \mathrm{E}-03$ & $1.30 \mathrm{E}+02$ & $4.00 \mathrm{E}+04$ & $5.20 \mathrm{E}+09$ & $19.83 \%$ \\
\hline Am-241 & $1.00 \mathrm{E}-03$ & $5.20 \mathrm{E}+02$ & $4.00 \mathrm{E}+04$ & $2.08 \mathrm{E}+10$ & $79.30 \%$ \\
\hline
\end{tabular}

Container for LLW Compactor ( 253-H)

\begin{tabular}{|c|c|c|c|c|c|}
\hline Radionuclides & $\mathrm{RF}$ & $\begin{array}{l}\text { DCF } \\
(\text { rem/uCi) }\end{array}$ & $\begin{array}{l}\text { Inventory } \\
\text { (Ci) }\end{array}$ & $\begin{array}{l}\text { Total Release } \\
\text { (rem) }\end{array}$ & Percent \\
\hline & A & B & C & $D=A B C * 1 E 6$ & $E=D / D_{\text {TOT(1) }}$ \\
\hline $\mathrm{Co}-60$ & $1.00 \mathrm{E}-02$ & $1.50 \mathrm{E}-01$. & $2.09 E+00$ & $3.14 \mathrm{E}+03$ & $13.39 \%$ \\
\hline $\mathrm{Pu}-238$ & $1.00 \mathrm{E}-03$ & $4.60 E+02$ & $7.60 \mathrm{E}-03$ & $3.50 E+03$ & $14.94 \%$ \\
\hline Pu-239 & 1.00E-03 & $5.10 E+02$ & $7.60 \mathrm{E}-03$ & $3.88 E+03$ & $16.56 \%$ \\
\hline $\mathrm{Pu}-240$ & 1.00E-03 & $5.10 E+02$ & 7.60E-03 & $3.88 E+03$ & $16.56 \%$ \\
\hline Pu-242 & $1.00 \mathrm{E}-03$ & $4.80 \mathrm{E}+02$ & 7.60 E-03 & $3.65 \mathrm{E}+03$ & $15.59 \%$ \\
\hline Am-241 & $1.00 E-03$ & $5.20 \mathrm{E}+02$ & 7.91E-03 & $4.11 E+03$ & $17.57 \%$ \\
\hline
\end{tabular}

MW Waste - Tritium Bearing Container (Waste)

\begin{tabular}{|l|l|l|l|l|l|}
\hline Radionuclides & RF & $\begin{array}{l}\text { DCF } \\
(\mathrm{rem} / \mathrm{uCi})\end{array}$ & $\begin{array}{l}\text { Inventory } \\
(\mathrm{Ci})\end{array}$ & $\begin{array}{l}\text { Total Release } \\
(\mathrm{rem})\end{array}$ & Percent \\
\hline & $\mathrm{A}$ & $\mathrm{B}$ & $\mathrm{C}$ & $\mathrm{D}=\mathrm{ABC}$ 1E6 $^{*}$ & $\mathrm{E}=\mathrm{D} / \mathrm{D}_{\text {TOT(1) }}$ \\
\hline $\mathrm{H}-3$ & $1.00 \mathrm{E}+00$ & $9.50 \mathrm{E}-05$ & $5.00 \mathrm{E}+03$ & $4,75 \mathrm{E}+05$ & $100 \%$ \\
\hline
\end{tabular}

Container of MW Waste - Beta/Gamma (Waste)

\begin{tabular}{|l|l|l|l|l|l|}
\hline Radionuclides & RF & $\begin{array}{l}\text { DCF } \\
(\mathrm{rem} / \mathrm{uCi})\end{array}$ & $\begin{array}{l}\text { Inventory } \\
(\mathrm{Ci})\end{array}$ & $\begin{array}{l}\text { Total Release } \\
(\text { rem })\end{array}$ & Percent \\
\hline & A & $\mathrm{B}$ & $\mathrm{C}$ & $\mathrm{D}=\mathrm{ABC}{ }^{*} 1 \mathrm{E} 6$ & $\mathrm{E}=\mathrm{D} / \mathrm{D}_{\text {TOT(1) }}$ \\
\hline $\mathrm{C} 0-60$ & $1.00 \mathrm{E}-02$ & $1.50 \mathrm{E}-01$ & 1 & $1.50 \mathrm{E}+03$ & $24 \%$ \\
\hline $\mathrm{Cs}-137$ & $1.00 \mathrm{E}-02$ & $3.20 \mathrm{E}-02$ & $1.50 \mathrm{E}+01$ & $4.80 \mathrm{E}+03$ & $75.25 \%$ \\
\hline
\end{tabular}


Title of Project EPHA for THM Reviewed by S.A. Henderson

Subject Screening of Radionuclides Works Calculation 4

Computer E.T. Ketusky Date $09 / 95$

Container of MWWASTE - Alpha (Waste)

\begin{tabular}{|l|l|l|l|l|l|}
\hline Radionuclides & RF & $\begin{array}{l}\text { DCF } \\
(\text { rem/uCi })\end{array}$ & $\begin{array}{l}\text { Inventory } \\
(\mathrm{Ci})\end{array}$ & $\begin{array}{l}\text { Total Release } \\
(\mathrm{rem})\end{array}$ & Percent \\
\hline & $\mathrm{A}$ & $\mathrm{B}$ & $\mathrm{C}$ & $\mathrm{D}=\mathrm{ABC}$ 1E6 & $\mathrm{E}=\mathrm{D} / \mathrm{D}_{\text {TOT }(1)}$ \\
\hline Pu-239 & $1.00 \mathrm{E}-03$ & $5.10 \mathrm{E}+02$ & $1.20 \mathrm{E}+00$ & $6.12 \mathrm{E}+05$ & $44 \%$ \\
\hline Pu-240 & $1.00 \mathrm{E}-03$ & $5.10 \mathrm{E}+02$ & $3.00 \mathrm{E}-01$ & $1.53 \mathrm{E}+05$ & $11 \%$ \\
\hline Am-241 & $1.00 \mathrm{E}-03$ & $5.20 \mathrm{E}+02$ & $1.10 \mathrm{E}+00$ & $5.72 \mathrm{E}+05$ & $41 \%$ \\
\hline
\end{tabular}

Container of LAWW (Waste)

\begin{tabular}{|c|c|c|c|c|c|}
\hline Radionuclides & RF & $\begin{array}{l}\text { DCF. } \\
\text { (rem/uCi) }\end{array}$ & $\begin{array}{l}\text { Inventory } \\
\text { (Ci) }\end{array}$ & $\begin{array}{l}\text { Total Release } \\
\text { (rem) }\end{array}$ & Percent \\
\hline & $\bar{A}$ & B & c & $D=A B C * 1 E 6$ & $E=D / D_{\text {TOT }(1)}$ \\
\hline Co-60 & 1.00E-02 & $1.50 \mathrm{E}-01$ & $2.53 E+01$ & $3.80 E+04$ & $13.52 \%$ \\
\hline $\mathrm{Pu}-238$ & $1.00 E-03$ & $4.60 E+02$ & 9.10E-02 & $4.19 E+04$ & $14.92 \%$ \\
\hline Pu-239 & $1.00 \mathrm{E}-03$ & $5.10 \mathrm{E}+02$ & $9.10 \mathrm{E}-02$ & $4.64 \mathrm{E}+04$ & $16.54 \%$ \\
\hline Pu-240 & $1.00 \mathrm{E}-03$ & $5.10 \mathrm{E}+02$ & $9.10 \mathrm{E}-02$ & $4.64 E+04$ & $16.54 \%$ \\
\hline Pu-242 & $1.00 E-03$ & $4.80 E+02$ & $9.10 \mathrm{E}-02$ & $4.37 E+04$ & $15.57 \%$ \\
\hline Am-241 & $1.00 \mathrm{E}-03$ & $5.20 E+02$ & $9.47 E-02$ & $4.92 E+04$ & $17.55 \%$ \\
\hline
\end{tabular}

Container for ILNTV Combustibles (E Area Vaults)

\begin{tabular}{|l|l|l|l|l|l|}
\hline Radionuclides & RF & $\begin{array}{l}\text { DCF } \\
(\text { rem/uCi) }\end{array}$ & $\begin{array}{l}\text { lnventory } \\
(\mathrm{Ci})\end{array}$ & $\begin{array}{l}\text { Total Release } \\
\text { (rem) }\end{array}$ & Percent \\
\hline & A & $B$ & C & D=ABC*1E6 & E=D/D TOT(1) \\
\hline Sr-90 & $1.00 \mathrm{E}-03$ & $1.30 \mathrm{E}+00$ & $2.30 \mathrm{E}+01$ & $2.99 \mathrm{E}+04$ & $3.73 \%$ \\
\hline $\mathrm{Cs}-137$ & $1.00 \mathrm{E}-02$ & $3.20 \mathrm{E}-02$ & $2.30 \mathrm{E}+02$ & $7.36 \mathrm{E}+04$ & $9.18 \%$ \\
\hline Pu-238 & $1.00 \mathrm{E}-03$ & $4.60 \mathrm{E}+02$ & $2.30 \mathrm{E}-01$ & $1.06 \mathrm{E}+05$ & $13.19 \%$ \\
\hline Pu-239 & $1.00 \mathrm{E}-03$ & $5.10 \mathrm{E}+02$ & $2.30 \mathrm{E}-01$ & $1.17 \mathrm{E}+05$ & $14.62 \%$ \\
\hline Pu-240 & $1.00 \mathrm{E}-03$ & $5.10 \mathrm{E}+02$ & $2.30 \mathrm{E}-01$ & $1.17 \mathrm{E}+05$ & $14.62 \%$ \\
\hline Pu-242 & $1.00 \mathrm{E}-03$ & $4.80 \mathrm{E}+02$ & $2.30 \mathrm{E}-01$ & $1.10 \mathrm{E}+05$ & $13.76 \%$ \\
\hline Am-241 & $1.00 \mathrm{E}-03$ & $5.20 \mathrm{E}+02$ & $4.10 \mathrm{E}-01$ & $2.13 \mathrm{E}+05$ & $26.58 \%$ \\
\hline
\end{tabular}

Container for LNTV Noncombustibles (E Area)

\begin{tabular}{|c|c|c|c|c|c|}
\hline Radionuclides & RF & $\begin{array}{l}\text { DCF } \\
(\text { rem/uCi) }\end{array}$ & $\begin{array}{l}\text { Inventory } \\
\text { (Ci) }\end{array}$ & $\begin{array}{l}\text { Total Release } \\
(\mathrm{rem})\end{array}$ & Percent \\
\hline & $A$ & $\bar{B}$ & $\bar{c}$ & $D=A B C^{*} 1 E 6$ & $E=D / D_{T o T(1)}$ \\
\hline Sr-90 & 1.00E-03 & $1.30 E+00$ & $1.80 \mathrm{E}+03$ & $2.34 \mathrm{E}+06$ & $28.58 \%$ \\
\hline Co-60 & $1.00 \mathrm{E}-02$ & $1.50 \mathrm{E}-01$ & $1.80 \mathrm{E}+03$ & $2.7 \overline{1 E+06}$ & $24.73 \%$ \\
\hline PL-238 & 1.00E-03 & $4.60 E+02$ & $1.80 E+00$ & $8.28 \bar{E}+05$ & $7.57 \%$ \\
\hline$\overline{\text { Pu-239 }}$ & 1.00E-03 & $5.10 \mathrm{E}+02$ & $1.80 E+00$ & $9.18 E+05$ & $8.39 \%$ \\
\hline Pu-240 & $1.00 E-03$ & $5.10 E+02$ & $1.80 \mathrm{E}+00$ & $9.18 E+05$ & $8.39 \%$ \\
\hline Pu-242 & 1.00 E-03 & $4.80 \mathrm{E}+02$ & $1.80 \mathrm{E}+00$ & $8.64 E+05$ & $7.90 \%$ \\
\hline Am-241 & $1.00 \mathrm{E}-03$ & $5.20 E+02$ & $2.54 \mathrm{E}+00$ & $1.32 \mathrm{E}+06$ & $12.07 \%$ \\
\hline
\end{tabular}


Title of Project EPHA for THM Reviewed by S. A. Henderson

Subject Screening of Radionuclides Works Calculation 4

SAYAKMAH RIYIR IITE

Computer E.T.Ketusky Date 09/95

ILTV Bulk Container (E Area Vaults)

\begin{tabular}{|l|l|l|l|l|l|}
\hline Radionuclides & RF & $\begin{array}{l}\text { DCF } \\
\text { (rem/uCi) }\end{array}$ & $\begin{array}{l}\text { Inventory } \\
\text { (Ci) }\end{array}$ & $\begin{array}{l}\text { Total Release } \\
(\text { rem })\end{array}$ & Percent \\
\hline & A & B & C & D=ABC*1E6 & E=D/D TOT(1) \\
\hline Co-60 & $1.00 \mathrm{E}-02$ & $1.50 \mathrm{E}-01$ & $1.96 \mathrm{E}+03$ & $2.94 \mathrm{E}+06$ & $79.91 \%$ \\
\hline $\mathrm{Pu}-239$ & $1.00 \mathrm{E}-03$ & $5.10 \mathrm{E}+02$ & $2.30 \mathrm{E}-01$ & $1.17 \mathrm{E}+05$ & $3.19 \%$ \\
\hline $\mathrm{Pu}-240$ & $1.00 \mathrm{E}-03$ & $5.10 \mathrm{E}+02$ & $2.30 \mathrm{E}-01$ & $1.17 \mathrm{E}+05$ & $3.19 \%$ \\
\hline $\mathrm{Pu}-242$ & $1.00 \mathrm{E}-03$ & $4.80 \mathrm{E}+02$ & $2.30 \mathrm{E}-01$ & $1.10 \mathrm{E}+05$ & $3.00 \%$ \\
\hline $\mathrm{Pu}-238$ & $1.00 \mathrm{E}-03$ & $4.60 \mathrm{E}+02$ & $2.30 \mathrm{E}-01$ & $1.06 \mathrm{E}+05$ & $2.88 \%$ \\
\hline Am-241 & $1.00 \mathrm{E}-03$ & $5.20 \mathrm{E}+02$ & $2.43 \mathrm{E}-01$ & $1.26 \mathrm{E}+05$ & $3.43 \%$ \\
\hline
\end{tabular}

ILTV Crucibles Container (E Area Vaults)

\begin{tabular}{|c|c|c|c|c|c|}
\hline Radionuclides & RF & $\begin{array}{l}\text { DCF } \\
\text { (rem/uCi) }\end{array}$ & $\begin{array}{l}\text { Inventory } \\
\text { (Ci) }\end{array}$ & $\begin{array}{l}\text { Total Release } \\
\text { (rem) }\end{array}$ & Percent \\
\hline & $A$ & $\bar{B}$ & C & $D=A B C^{\star} 1 E 6$ & $E=D / D_{\text {TOT }(1)}$ \\
\hline $\mathrm{H}-3$ & $1.00 E+00$ & 9.50E-05 & $4.00 E+04$ & $3.80 E+06$ & $73.56 \%$ \\
\hline Co-60 & $1.00 E-02$ & 1.50E-01 & $2.70 E+02$ & $4.05 E+05$ & $7.84 \%$ \\
\hline Sr-90 & 1.00E-03 & 1.30E+00 & $1.80 \mathrm{E}+02$ & $2.34 E+05$ & $4.53 \%$ \\
\hline Np-237 & $1.00 E-03$ & $4.90 E+02$ & $1.80 \mathrm{E}-01$ & $8.82 E+04$ & $1.71 \%$ \\
\hline Pu-239 & $1.00 E-03$ & $5.10 \mathrm{E}+02$ & $1.80 E-01$ & $9.18 E+04$ & $1.78 \%$ \\
\hline Pu-240 & $1.00 \mathrm{E}-03$ & $5.10 \mathrm{E}+02$ & $1.80 E-01$ & $9.18 E+04$ & $1.78 \%$ \\
\hline$\overline{\mathrm{Pu}-242}$ & $1.00 \mathrm{E}-03$ & $4.80 E+02$ & $1.80 E=01$ & $8.64 E+04$ & $1.67 \%$ \\
\hline Am-241 & $1.00 \mathrm{E}-03$ & $5.20 E+02$ & 3.90E-01 & $2.03 E \div 05$ & $3.93 \%$ \\
\hline
\end{tabular}

LLWSB Container (E Area Vaults)

\begin{tabular}{|l|l|l|l|l|l|}
\hline Radionuclides & RF & $\begin{array}{l}\text { DCF } \\
(\text { rem/uCi) }\end{array}$ & $\begin{array}{l}\text { Inventory } \\
(\mathrm{Ci})\end{array}$ & $\begin{array}{l}\text { Total Release } \\
(\text { rem })\end{array}$ & Percent \\
\hline & A & B & C & D=ABC*1E6 & E=D/D TOT(1) \\
\hline $\mathrm{H}-3$ & $1.00 \mathrm{E}+00$ & $9.50 \mathrm{E}-05$ & $4.00 \mathrm{E}+04$ & $3.80 \mathrm{E}+06$ & $79.82 \%$ \\
\hline $\mathrm{Sr}-90$ & $1.00 \mathrm{E}-03$ & $1.30 \mathrm{E}+00$ & $1.80 \mathrm{E}+02$ & $2.34 \mathrm{E}+05$ & $4.92 \%$ \\
\hline Am-241 & $1.00 \mathrm{E}-03$ & $5.20 \mathrm{E}+02$ & $3.90 \mathrm{E}-01$ & $2.03 \mathrm{E}+05$ & $4.26 \%$ \\
\hline Pu-239 & $1.00 \mathrm{E}-03$ & $5.10 \mathrm{E}+02$ & $1.80 \mathrm{E}-01$ & $9.18 \mathrm{E}+04$ & $1.93 \%$ \\
\hline Pu-240 & $1.00 \mathrm{E}-03$ & $5.10 \mathrm{E}+02$ & $1.80 \mathrm{E}-01$ & $9.18 \mathrm{E}+04$ & $1.93 \%$ \\
\hline Np-237 & $1.00 \mathrm{E}-03$ & $4.90 \mathrm{E}+02$ & $1.80 \mathrm{E}-01$ & $8.82 \mathrm{E}+04$. & $1.85 \%$ \\
\hline
\end{tabular}

Pu 02 Container (235F and 247F)

\begin{tabular}{|l|l|l|l|l|l|}
\hline Radionuclides & RF & $\begin{array}{l}\text { DCF } \\
(\mathrm{rem} / \mathrm{LCi})\end{array}$ & $\begin{array}{l}\text { Inventory } \\
(\mathrm{Ci})\end{array}$ & $\begin{array}{l}\text { Total Release } \\
(\mathrm{rem})\end{array}$ & Percent \\
\hline & $\mathrm{A}$ & $\mathrm{B}$ & $\mathrm{C}$ & $\mathrm{D}=\mathrm{ABC}$ 1E6 & $\mathrm{E}=\mathrm{D} / \mathrm{D}_{\text {TOT(1) }}$ \\
\hline Pu-238 & $1.00 \mathrm{E}-03$ & $4.60 \mathrm{E}+02$ & $9.16 \mathrm{E}+02$ & $4.21 \mathrm{E}+08$ & $100.00 \%$ \\
\hline
\end{tabular}




\section{ENGINEERING COMPUTATION SHEET}

Title of Project EPHA for THM

Reviewed by S. A. Henderson

Subject Screening of Radionuclides

Computer E. T. Ketusky

\section{Works Calculation 4}

Date $09 / 95$

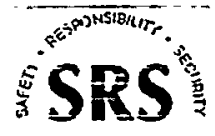

SAYANKAK RIYSE BITE

Container, Pail, Casket, Charge Can, Type A Storage Vault (235-F)

\begin{tabular}{|l|l|l|l|l|l|}
\hline Radionuclides & RF & $\begin{array}{l}\text { DCF } \\
(\mathrm{rem} / \mathrm{uCi})\end{array}$ & $\begin{array}{l}\text { Inventory } \\
(\mathrm{Ci})\end{array}$ & $\begin{array}{l}\text { Total Release } \\
(\mathrm{rem})\end{array}$ & Percent \\
\hline & $\mathrm{A}$ & $\mathrm{B}$ & $\mathrm{C}$ & D=ABC*1E6 & E=D/D $\mathrm{DOT}_{(\mathrm{t})}$ \\
\hline $\mathrm{Pu}-238$ & $1.00 \mathrm{E}-03$ & $1.06 \mathrm{E}-04$ & $2.74 \mathrm{E}+05$ & $2.90 \mathrm{E}+04$ & $99.99 \%$ \\
\hline
\end{tabular}

H Canyon Sample Container (221H to SRTC)

\begin{tabular}{|l|l|l|l|l|l|}
\hline Radionuclides & RF & $\begin{array}{l}\text { DCF } \\
(\text { rem/uCi }) \\
\text { B }\end{array}$ & $\begin{array}{l}\text { Inventory } \\
(\mathrm{Ci}) \\
\mathrm{C}\end{array}$ & $\begin{array}{l}\text { Total Release } \\
(\mathrm{rem}) \\
\mathrm{D}=\mathrm{ABC} \text { 1E6 }\end{array}$ & Percent \\
\hline Pu-238 & $\mathrm{A} / \mathrm{D}_{\mathrm{TOT}(1)}$ & $1.54 \mathrm{E}+01$ & $7.08 \mathrm{E}+06$ & $100.00 \%$ \\
\hline
\end{tabular}

H Canyon Sample Container (221H to SRTC)

\begin{tabular}{|l|l|l|l|l|l|}
\hline Radionuclides & RF & $\begin{array}{l}\text { DCF } \\
(\mathrm{rem} / \mathrm{uCi})\end{array}$ & $\begin{array}{l}\text { Inventory } \\
(\mathrm{Ci})\end{array}$ & $\begin{array}{l}\text { Total Release } \\
(\mathrm{rem})\end{array}$ & Percent \\
\hline & $\mathrm{A}$ & $\mathrm{B}$ & $\mathrm{C}$ & $\mathrm{D}=\mathrm{ABC}$ 1E6 & $\mathrm{E}=\mathrm{D} / \mathrm{D}_{\text {TOT(1) }}$ \\
\hline $\mathrm{U}-235$ & $1.00 \mathrm{E}-03$ & $1.20 \mathrm{E}+02$ & $8.64 \mathrm{E}-05$ & $1.04 \mathrm{E}+01$ & $100.00 \%$ \\
\hline
\end{tabular}

H Canyon Sample Container (221H to SRTC)

\begin{tabular}{|l|l|l|l|l|l|}
\hline Radionuclides & RF & $\begin{array}{l}\text { DCF } \\
(\text { rem/uCi })\end{array}$ & $\begin{array}{l}\text { Inventory } \\
(\mathrm{Ci})\end{array}$ & $\begin{array}{l}\text { Total Release } \\
(\text { rem })\end{array}$ & Percent \\
\hline & $\mathrm{A}$ & $\mathrm{B}$ & $\mathrm{C}$ & $\mathrm{D}=\mathrm{ABC}$ (E6 & E=D/D \\
\hline $\mathrm{Np}-237$ & $1.00 \mathrm{E}-03$ & $4.90 \mathrm{E}+02$ & $7.05 \mathrm{E}-04$ & $3.45 \mathrm{E}+02$ & $100.00 \%$ \\
\hline
\end{tabular}

F Canyon Sample Container (221F to SRTC)

\begin{tabular}{|l|l|l|l|l|l|}
\hline Radionuclides & RF & $\begin{array}{l}\text { DCF } \\
(\text { rem/uCi })\end{array}$ & $\begin{array}{l}\text { Inventory } \\
(\mathrm{Ci})\end{array}$ & $\begin{array}{l}\text { Total Release } \\
(\mathrm{rem})\end{array}$ & Percent \\
\hline & A & B & C & D=ABC1E6 & E=D/D \\
\hline Pu-238 & $1.00 \mathrm{E}-03$ & $4.60 \mathrm{E}+02$ & $1.20 \mathrm{E}-01$ & $5.51 \mathrm{E}+04$ & $100.00 \%$ \\
\hline
\end{tabular}

F Canyon Sample Container (221F to SRTC)

\begin{tabular}{|l|l|l|l|l|l|}
\hline Radionuclides & RF & $\begin{array}{l}\text { DCF } \\
(\text { rem/uCi) }\end{array}$ & $\begin{array}{l}\text { Inventory } \\
(\mathrm{Ci})\end{array}$ & $\begin{array}{l}\text { Total Release } \\
(\mathrm{rem})\end{array}$ & Percent \\
\hline & $\mathrm{A}$ & $\mathrm{B}$ & $\mathrm{C}$ & $\mathrm{D}=\mathrm{ABC}$ 1E6 & $\mathrm{E}=\mathrm{D} / \mathrm{D}_{\mathrm{TOT}(1)}$ \\
\hline $\mathrm{Am}-243$ & $1.00 \mathrm{E}-03$ & $5.20 \mathrm{E}+02$ & $1.99 \mathrm{E}-02$ & $1.03 \mathrm{E}+04$ & $100.00 \%$ \\
\hline
\end{tabular}

F Canyon Sample Container (221F to SRTC)

\begin{tabular}{|l|l|l|l|l|l|}
\hline Radionuclides & RF & $\begin{array}{l}\text { DCF } \\
(\mathrm{rem} / \mathrm{uCi})\end{array}$ & $\begin{array}{l}\text { Inventory } \\
(\mathrm{Ci})\end{array}$ & $\begin{array}{l}\text { Total Release } \\
(\mathrm{rem})\end{array}$ & Percent \\
\hline & $\mathrm{A}$ & $\mathrm{B}$ & $\mathrm{C}$ & $\mathrm{D}=\mathrm{ABC}$ 1E6 & E=D/D \\
\hline $\mathrm{Cm}-244$ & $1.00 \mathrm{E}-03$ & $2.70 \mathrm{E}+02$ & $8.09 \mathrm{E}+00$ & $2.18 \mathrm{E}+06$ & $100.00 \%$ \\
\hline
\end{tabular}


Title of Project EPHA for THM

Subject Screening of Radionuclides

Computer E. T. Ketusky
Reviewed by S. A. Henderson Works Calculation 4 Date $\underline{09 / 95}$

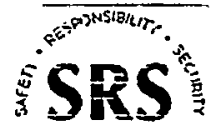

SAYANMAM RIVIE JiYK

HB Line Sample Container (221H to 772-1F)

\begin{tabular}{|l|l|l|l|l|l|}
\hline Radionuclides & RF & $\begin{array}{l}\text { DCF } \\
(\mathrm{rem} / \mathrm{uCi})\end{array}$ & $\begin{array}{l}\text { Inventory } \\
(\mathrm{Ci})\end{array}$ & $\begin{array}{l}\text { Total Release } \\
(\mathrm{rem})\end{array}$ & Percent \\
\hline & $\mathrm{A}$ & $\mathrm{B}$ & $\mathrm{C}$ & $\mathrm{D}=\mathrm{ABC}$ 1E6 & $\mathrm{E}=\mathrm{D} / \mathrm{D}_{\mathrm{TOTAL}}$ \\
\hline $\mathrm{Pu}-238$ & $1.00 \mathrm{E}-03$ & $4.60 \mathrm{E}+02$ & $8.55 \mathrm{E}-01$ & $3.93 \mathrm{E}+05$ & $100.00 \%$ \\
\hline
\end{tabular}

HB Line Sample Container (221H to SRTC)

\begin{tabular}{|l|l|l|l|l|l|}
\hline Radionuclides & RF & $\begin{array}{l}\text { DCF } \\
(\text { rem/uCi })\end{array}$ & $\begin{array}{l}\text { Inventory } \\
(\mathrm{Ci})\end{array}$ & $\begin{array}{l}\text { Total Release } \\
(\mathrm{rem})\end{array}$ & Percent \\
\hline & $\mathrm{A}$ & $\mathrm{B}$ & $\mathrm{C}$ & $\mathrm{D}=\mathrm{ABC}$ 1E6 & ${\mathrm{E}=\mathrm{D} / \mathrm{D}_{\mathrm{TOT}(1)}}$ \\
\hline Pu-238 & $1.00 \mathrm{E}-03$ & $4.60 \mathrm{E}+02$ & $8.55 \mathrm{E}-01$ & $3.93 \mathrm{E}+05$ & $100.00 \%$ \\
\hline
\end{tabular}

FB Line Oxide Container (221F to Inter Area)

\begin{tabular}{|l|l|l|l|l|l|}
\hline $\begin{array}{l}\text { Radionuclides } \\
\cdot\end{array}$ & RF & $\begin{array}{l}\text { DCF } \\
(\mathrm{rem} / \mathrm{uCi})\end{array}$ & $\begin{array}{l}\text { lnventory } \\
(\mathrm{Ci})\end{array}$ & $\begin{array}{l}\text { Total Release } \\
(\mathrm{rem})\end{array}$ & Percent \\
\hline & $\mathrm{A}$ & $\mathrm{B}$ & $\mathrm{C}$ & $\mathrm{D}=\mathrm{ABC}$ 1E6 & E=D/D \\
\hline Pu-239 & $1.00 \mathrm{E}-03$ & $5.10 \mathrm{E}+02$ & $5.58 \mathrm{E}+03$ & $2.85 \mathrm{E}+09$ & $100.00 \%$ \\
\hline
\end{tabular}

FB Line Sample Container (221F to SRTC)

\begin{tabular}{|c|c|c|c|c|c|}
\hline Radionuclides & RF & $\mid \begin{array}{l}\text { DCF } \\
\text { (rem/uCi) }\end{array}$ & $\begin{array}{l}\text { Inventory } \\
\text { (Ci) }\end{array}$ & $\begin{array}{l}\text { Total Release } \\
(\mathrm{rem})\end{array}$ & Percent \\
\hline & $A$ & 8 & $\mathrm{C}$ & $D=A B C * 1 E 6$ & $E=D / D_{\text {TOT(1) }}$ \\
\hline Pu-239 & 1.00E-03 & $5.10 \mathrm{E}+02$ & $6.20 E-01$ & $3.16 E+05$ & $100.00 \%$ \\
\hline
\end{tabular}

235F Material Container to $221 \mathrm{HB}$ Line (235F to $221 \mathrm{H}$ )

\begin{tabular}{|l|l|l|l|l|l|}
\hline Radionuclides & RF & $\begin{array}{l}\text { DCF } \\
(\mathrm{rem} / \mathrm{uCi})\end{array}$ & $\begin{array}{l}\text { Inventory } \\
(\mathrm{Ci})\end{array}$ & $\begin{array}{l}\text { Total Release } \\
(\mathrm{rem})\end{array}$ & Percent \\
\hline & $\mathrm{A}$ & $\mathrm{B}$ & $\mathrm{C}$ & $\mathrm{D}=\mathrm{ABC}$ 1E6 & E=D/D \\
\hline Pu-238 & $1.00 \mathrm{E}-03$ & $4.60 \mathrm{E}+02$ & $3.42 \mathrm{E}+04$ & $1.57 \mathrm{E}+10$ & $100.00 \%$ \\
\hline
\end{tabular}

235F Material Container to $221 \mathrm{FB}$ Line (235F to 221 F)

\begin{tabular}{|l|l|l|l|l|l|}
\hline Radionuclides & RF & $\begin{array}{l}\text { DCF } \\
(\text { rem/uCi })\end{array}$ & $\begin{array}{l}\text { Inventory } \\
(\mathrm{Ci})\end{array}$ & $\begin{array}{l}\text { Total Release } \\
(\mathrm{rem})\end{array}$ & Percent \\
\hline & $\mathrm{A}$ & $\mathrm{B}$ & $\mathrm{C}$ & $\mathrm{D}=\mathrm{ABC} * 1 \mathrm{E} 6$ & $\mathrm{E}=\mathrm{D} / \mathrm{D}_{\mathrm{TOT}(1)}$ \\
\hline $\mathrm{Pu}-239$ & $1.00 \mathrm{E}-03$ & $5.10 \mathrm{E}+02$ & $1.86 \mathrm{E}+02$ & $9.49 \mathrm{E}+07$ & $100.00 \%$ \\
\hline
\end{tabular}

SRTC Material Container (SRTC to DWPF)

\begin{tabular}{|c|c|c|c|c|c|}
\hline Radionuclides & RF & $\begin{array}{l}\text { DCF } \\
(\mathrm{rem} / \mathrm{uCi})\end{array}$ & $\begin{array}{l}\text { Inventory } \\
\text { (Ci) }\end{array}$ & $\begin{array}{l}\text { Total Release } \\
\text { (rem) }\end{array}$ & Percent \\
\hline & $A$ & $B$ & C & $D=A B C * 1 E 6$ & $E=D / D_{\text {TOT(1) }}$ \\
\hline Cf-252 & 1.00E-03 & 4.24E-05 & $2.69 \mathrm{E}+01$ & $1.14 E+00$ & $100.00 \%$ \\
\hline
\end{tabular}


Title of Project EPHA for THM Reviewed by S. A. Henderson

Subject Screening of Radionuclides Works Calculation 4

Computer E.T. Ketusky Date 09/95

A Line Material (A Line)

\begin{tabular}{|l|l|l|l|l|l|}
\hline Radionuclides & RF & $\begin{array}{l}\text { DCF } \\
(\text { rem/uCi) }\end{array}$ & $\begin{array}{l}\text { Inventory } \\
(\mathrm{Ci})\end{array}$ & $\begin{array}{l}\text { Total Release } \\
(\mathrm{rem})\end{array}$ & Percent \\
\hline (assume U-235) & $\mathrm{A}$ & $\mathrm{B}$ & $\mathrm{C}$ & $\mathrm{D}=\mathrm{ABC} * 1 \mathrm{E6}$ & $\mathrm{E}=\mathrm{D} / \mathrm{D}_{\mathrm{TOT}(1)}$ \\
\hline Depleted UO3 & $1.00 \mathrm{E}-03$ & $1.20 \mathrm{E}+02$ & $3.24 \mathrm{E}-03$ & $3.89 \mathrm{E}+02$ & $100.00 \%$ \\
\hline
\end{tabular}

UNH Trailer (H Area Outside)

\begin{tabular}{|c|c|c|c|c|c|}
\hline Radionuclides & RF & $\begin{array}{l}\text { DCF } \\
(\text { rem/uCi) }\end{array}$ & \begin{tabular}{|l} 
Inventory \\
(Ci)
\end{tabular} & $\begin{array}{l}\text { Total Release } \\
\text { (rem) }\end{array}$ & Percent \\
\hline & $A$ & B & $C$ & $D=A B C * 1 E 6$ & $E=D / D_{T O T(1)}$ \\
\hline U-235 & 1.00E-03 & $1.20 E+02$ & $8.20 E+03$ & $9.84 E+08$ & $100 \%$ \\
\hline
\end{tabular}

RBOF Portable Deionizer Column Transport (RBOF)

\begin{tabular}{|l|l|l|l|l|l|}
\hline Radionuclides & RF & $\begin{array}{l}\text { DCF } \\
(\text { rem/uCi })\end{array}$ & $\begin{array}{l}\text { Inventory } \\
(\mathrm{Ci})\end{array}$ & $\begin{array}{l}\text { Total Release } \\
(\mathrm{rem})\end{array}$ & Percent \\
\hline & $\mathrm{A}$ & $\mathrm{B}$ & $\mathrm{C}$ & $\mathrm{D}=\mathrm{ABC}$ 1E6 & $\mathrm{E}=\mathrm{D} / \mathrm{D}_{\mathrm{TOT}(\mathrm{i})}$ \\
\hline $\mathrm{Cr}-51$ & $1.00 \mathrm{E}+00$ & $2.60 \mathrm{E}-04$ & $1.70 \mathrm{E}+01$ & $4.42 \mathrm{E}+03$ & $5.80 \%$ \\
\hline $\mathrm{Ru}-106$ & $1.00 \mathrm{E}-03$ & $4.40 \mathrm{E}-01$ & $8.90 \mathrm{E}+00$ & $3.92 \mathrm{E}+03$ & $5.13 \%$ \\
\hline $\mathrm{Cs}-137$ & $1.00 \mathrm{E}-03$ & $3.20 \mathrm{E}-02$ & $1.00 \mathrm{E}+03$ & $3.20 \mathrm{E}+04$ & $41.96 \%$ \\
\hline $\mathrm{Ce}-144$ & $1.00 \mathrm{E}-02$ & $3.50 \mathrm{E}-01$ & $1.00 \mathrm{E}+01$ & $3.50 \mathrm{E}+04$ & $45.89 \%$ \\
\hline
\end{tabular}

RBOF Spent Deoinizer Resin Transport (RBOF)

\begin{tabular}{|l|l|l|l|l|l|}
\hline Radionuclides & RF & $\begin{array}{l}\text { DCF } \\
(\mathrm{rem} / \mathrm{uCi})\end{array}$ & $\begin{array}{l}\text { Inventory } \\
(\mathrm{Ci})\end{array}$ & $\begin{array}{l}\text { Total Release } \\
(\mathrm{rem})\end{array}$ & Percent \\
\hline & A & B & C & D=ABC*1E6 & E=D/D TOT(1) \\
\hline Cs-137 & $1.00 \mathrm{E}-02$ & $3.20 \mathrm{E}-02$ & $5.70 \mathrm{E}+02$ & $1.82 \mathrm{E}+05$ & $36 \%$ \\
\hline Pu-240 & $1.00 \mathrm{E}-03$ & $5.10 \mathrm{E}+02$ & $5.90 \mathrm{E}-01$ & $3.01 \mathrm{E}+05$ & $59 \%$ \\
\hline
\end{tabular}

Disassembly Basin Deionizer \& Purif Resin Cask (Reactors)

\begin{tabular}{|l|l|l|l|l|l|}
\hline Radionuclides & RF & $\begin{array}{l}\text { DCF } \\
\text { (rem/uCi) }\end{array}$ & $\begin{array}{l}\text { Inventory } \\
(\mathrm{Ci})\end{array}$ & $\begin{array}{l}\text { Total Release } \\
(\mathrm{rem})\end{array}$ & Percent \\
\hline & $\mathrm{A}$ & $\mathrm{B}$ & $\mathrm{C}$ & $\mathrm{D}=\mathrm{ABC}{ }^{*}$ E6 & E=D/D TOT(1) \\
\hline Co-60 & $1.00 \mathrm{E}-02$ & $1.50 \mathrm{E}-01$ & $1.69 \mathrm{E}+00$ & $2.54 \mathrm{E}+03$ & $100 \%$ \\
\hline
\end{tabular}

Container for Reactor Samples to 771-D/F(Reactors to 771-D/F)

\begin{tabular}{|l|l|l|l|l|l|}
\hline Radionuclides & RF & $\begin{array}{l}\text { DCF } \\
(\mathrm{rem} / \mathrm{uCi})\end{array}$ & $\begin{array}{l}\text { Inventory } \\
(\mathrm{Ci})\end{array}$ & $\begin{array}{l}\text { Total Release } \\
(\mathrm{rem})\end{array}$ & Percent \\
\hline & $\mathrm{A}$ & $\mathrm{B}$ & $\mathrm{C}$ & $\mathrm{D}=\mathrm{ABC}$ 1E6 & E=D/D TOT(1) \\
\hline $\mathrm{Co}-60$ & $1.00 \mathrm{E}-02$ & $1.50 \mathrm{E}-01$ & $1.69 \mathrm{E}+00$ & $2.54 \mathrm{E}+03$ & $100 \%$ \\
\hline
\end{tabular}


Subject Screening of Radionuclides Reviewed bv S. A. Henderson

Computer E.T. Ketusky

Sample Truck (Inter Area to/from SRTC)

\begin{tabular}{|l|l|l|l|l|l|}
\hline Radionuclides & RF & $\begin{array}{l}\text { DCF } \\
(\text { rem/uCi })\end{array}$ & $\begin{array}{l}\text { lnventory } \\
(\mathrm{Ci})\end{array}$ & $\begin{array}{l}\text { Total Release } \\
(\mathrm{rem})\end{array}$ & Percent \\
\hline & $\mathrm{A}$ & $\mathrm{B}$ & $\mathrm{C}$ & $\mathrm{D}=\mathrm{ABC}{ }^{*} 1 \mathrm{E} 6$ & $\mathrm{E}=\mathrm{D} / \mathrm{D}_{\mathrm{TOT}(1)}$ \\
\hline $\mathrm{Cs}-137$ & $1.00 \mathrm{E}-02$ & $3.20 \mathrm{E}-02$ & $8.51 \mathrm{E}+02$ & $2.72 \mathrm{E}+05$ & $100 \%$ \\
\hline
\end{tabular}

Moderator Transport in 55 Gallon Drums (100-400 Areas)

\begin{tabular}{|l|l|l|l|l|l|}
\hline Radionuclides & RF & $\begin{array}{l}\text { DCF } \\
(\mathrm{rem} / \mathrm{uCi})\end{array}$ & $\begin{array}{l}\text { Inventory } \\
(\mathrm{Ci})\end{array}$ & $\begin{array}{l}\text { Total Release } \\
(\mathrm{rem})\end{array}$ & Percent \\
\hline & $\mathrm{A}$ & $\mathrm{B}$ & $\mathrm{C}$ & $\mathrm{D}=\mathrm{ABC} \star 1 \mathrm{E} 6$ & $\mathrm{E}=\mathrm{D} / \mathrm{D}_{\mathrm{TOT}(1)}$ \\
\hline $\mathrm{Am}-241$ & $1.00 \mathrm{E}-03$ & $5.20 \mathrm{E}+02$ & $2.00 \mathrm{E}+00$ & $1.04 \mathrm{E}+06$ & $100 \%$ \\
\hline
\end{tabular}

SRTC 776A to F Tank Farm 5000 Gallon Truck (776A to Tank Farm)

\begin{tabular}{|l|l|l|l|l|l|}
\hline Radionuclides & RF & $\begin{array}{l}\text { DCF } \\
(\mathrm{rem} / \mathrm{uCi})\end{array}$ & $\begin{array}{l}\text { Inventory } \\
(\mathrm{Ci})\end{array}$ & $\begin{array}{l}\text { Total Release } \\
(\mathrm{rem})\end{array}$ & Percent \\
\hline & $\mathrm{A}$ & $\mathrm{B}$ & $\mathrm{C}$ & $\mathrm{D}=\mathrm{ABC} * 1 \mathrm{E} 6$ & $\mathrm{E}=\mathrm{D} / \mathrm{D}_{\mathrm{TOT}(1)}$ \\
\hline Pu-239 & $1.00 \mathrm{E}-03$ & $5.10 \mathrm{E}+02$ & $6.23 \mathrm{E}-02$ & $3.17 \mathrm{E}+04$ & $100 \%$ \\
\hline
\end{tabular}

Contaminated Water Transport (100 Area to 211-F)

\begin{tabular}{|l|l|l|l|l|l|}
\hline Radionuclides & RF & $\begin{array}{l}\text { DCF } \\
\text { (rem/uCi) }\end{array}$ & $\begin{array}{l}\text { Inventory } \\
(\mathrm{Ci})\end{array}$ & $\begin{array}{l}\text { Total Release } \\
(\mathrm{rem})\end{array}$ & Percent \\
\hline & $\mathrm{A}$ & $\mathrm{B}$ & $\mathrm{C}$ & $\mathrm{D}=\mathrm{ABC}+1 \mathrm{E} 6$ & $\mathrm{E}={\mathrm{D} / \mathrm{D}_{\text {TOT(1) }}}$ \\
\hline Am-241 & $1.00 \mathrm{E}-03$ & $5.20 \mathrm{E}+02$ & $2.00 \mathrm{E}+00$ & $1.04 \mathrm{E}+06$ & $100 \%$ \\
\hline
\end{tabular}

100 Area Sludge Transport (100 Area to 241-F )(2000 gallons)

\begin{tabular}{|c|c|c|c|c|c|}
\hline Radionuclides & RF & $\begin{array}{l}\text { DCF } \\
\text { (rem/uCi) }\end{array}$ & $\begin{array}{l}\text { Inventory } \\
\text { (Ci) }\end{array}$ & $\begin{array}{l}\text { Total Release } \\
\text { (rem) }\end{array}$ & Percent \\
\hline & $A$ & B & c & $D=A B C * 1 E 6$ & $E=D / D_{\text {TOT(1) }}$ \\
\hline Co-60 & 1.00E-02 & 1.50E-01 & $2.00 E+00$ & $3.00 \mathrm{E}+03$ & $100 \%$ \\
\hline
\end{tabular}

Based on the screening criteria the following radionuclides require accident analysis in the THM EPHA:

$\begin{array}{llllll}\text { Ru-106 } & \text { U-235 } & \text { Pu-238 } & \text { Pu-239 } & \text { Pu-240 } & \text { U-234 } \\ \text { Pu-242 } & \text { Am-241 } & \text { Am-243 } & \text { Cr-51 } & \text { Co-60 } & \text { Sr-90 } \\ \text { Cf-252 } & \text { H-3 } & \text { Cs-137 } & \text { Np-237 } & \text { Cm-244 } & \text { Ce-144 }\end{array}$


Tille of Project EPHA for THM

\section{REFERENCES}

1. Moyer, R.A., SP91-039, SRTS Packagings for Onsite Transportation of Radioactive Materials, March 29, 1991, Savannah River Laboratory, SAIC to Westinghouse Savannah River Company, Aiken, SC

2. Evaluation of Accident Risks in the Transportation of Hazardous Materials by Truck and rail at the Savannah River Site (U), "WSRC-RP-89-715, Revision 1, September 1992, Westinghouse Savannah River Company, Savannah River Site, Aiken SC.

3. Hazards Assessment for HWSF \& MWSF, S-EHA-G-00001, February 10, 1995, Westinghouse Savannah River Company, Savannah River Site, Aiken SC.

4. Emergency Preparedness Hazards Assessment for the Central Laboratory Facility, S-EHA-F00001 , Buildings 772-F, 772-1F, and 772-4F (U), Rev. 0, July 10, 1995, Westinghouse Savannah River Company, Savannah River Site, Aiken SC.

5. Emergency Management Hazards Assessment for the $C, K, L, P$, and R Reactors, SSE-ETS930195, Revision 0, March 30, 1994, Westinghouse Savannah River Company, Savannah River Site, Aiken SC.

6. Emergency Preparedness Hazards Assessment for the RBOF/RRF Facility (U), Draft A, Westinghouse Savannah River Company, Savannah River Site, Aiken SC.

7. Emergency Preparedness Hazards Assessment for the Consolidated Incineration Facility (U), January 24, 1995, Westinghouse Savannah River Company, Savannah River Site, Aiken SC.

8. Emergency Preparedness Hazards Assessment for the Savannah River Site Transportation of Hazardous Materials, Rev. 0, Westinghouse Savannah River Company, Savannah River Site, Aiken SC

9. Emergency Preparedness Hazards Assessment For the ITP/ESP Facility (U), S-EHA-H-00002, Revision 0, Westinghouse Savannah River Company, Savannah River Site, Aiken SC.

10. SRS Intra-Site Transportation Radiological and Toxicological Sabotage Vulnerability Assessment, WSRC-RP-95-624, May 1995, Westinghouse Savannah River Company, SC.

11. Savannah River Site Waste Acceptance Criteria Manual, Procedure Manual 1S, 1/17/95, Westinghouse Savannah River Company, Savannah River Site, Aiken, SC.

12. Westinghouse Savannah River Company Savannah River Site Emergency Plan (U), Procedure Manual 6Q, 6/30/95, Westinghouse Savannah River Company, Savannah River Site, Aiken, SC.

13. DOE Standard 1027.92, Hazard Categorization and Accident Analysis Techniques for Compliance with DOE Order 5480.23, Nuclear Safety Analysis Reports, U.S. Department of Energy, Washington, D.C. 20585, December 1992.

14. Internal Dose Conversion Factors for Calculation of Dose to the Public, DOE/EH-0071, U.S. Department of Energy, July 1988.

15. Transportation Safety Manual, Procedure Manual 19Q, Vol 1, 6/30/93, Westinghouse Savannah River Company, Savannah River Site, Aiken, SC. 


\section{INTRODUCTION}

The radionuclide source terms for fire, impact, and resuspension were calculated for each of the transportation containers as identified in Calculation 4. Calculation 4 determined the radionuclides for each transportation container which contributed greater than or equal to $95 \%$ of the total dose (screened the radionuclides) as required in EMPP-6Q-001. The physical state was also recorded.

In this calculation the results from Calculation 4 are converted to a total PU-238 Material at Risk (MAR) equivalent for each container. The purpose of using a single isotope MAR equivalent is to simplify the calculation for the source term and aide in the dispersion modeling.

Bounding Airborne Release Fractions (ARF) and Airborne Release Rates (ARR) for the recorded physical states, based on Reference 1, were then applied to the Pu-238 equivalent MAR for each of the three release mechanisms (fire, impact, resuspension). For conservatism the fire ARF and resuspension ARR used for solids were the same as those used for powders.

\section{INPUT/ASSUMPTIONS}

The initial quantities of radionuclides per container were based on the maximum inventories of radionuclides transported to and/or across the site based on individual facility EPHAs, SARs, BIOs, JCOs, various transportation documents, and Hazardous materials Shipment Public Access Roads Forms, the Waste Acceptance Manual and appropriate inventories from the Inter-Site Transportation Radiological and Toxicological Sabotage Vulnerability Assessment as identified in Calculation 4.

The radionuclides which do not contribute significantly to the total dose were eliminated in Calculation 4. The radionuclides were screened using quantities listed in 10 CFR 30.72 and employing the methodology identified in DOE STD-1027. Per the analysis in Calculation 4, all of the nuclides failed the screening methodology. The nuclides were further characterized by their contribution to dose upon release. Therefore the radionuclides which did not contribute significantly to the total dose were eliminated. The remaining radionuclides contributed greater or equal to $95 \%$ of the total dose.

When combining isotopes into the Pu-238 equivalent, it was assumed that the contents within the container were homogenous and of one physical form. The physical form of the contents of each container were based on Calculation 4. This was necessary because only isotopes of similar physical characteristics should be combined. The tritium inventory was not combined.

\section{CALCUlation}

The following equation was used to convert multiple isotopes into a single isotopic equivalent. The tritium inventory was not combined.

$$
\mathrm{PU}_{238 \mathrm{eq}}=\frac{1\left(\sum \mathrm{A}_{1} \times \mathrm{DCF}\right)}{\mathrm{DCF} F_{\mathrm{PA} 238}}
$$

where:

$\mathrm{PU}_{238 \text { eq: }}$ Pu-238 is utilized as the base isotope for establishing Curie equivalents.

$D_{\text {Pu 238: }} \quad$ The exposure to dose conversion factor (CEDE) for inhalation per unit

$$
\text { intake for Pu-238 (Ref. 2). }
$$

$\mathrm{A}_{\mathrm{i}}: \quad$ The number of $\mathrm{Ci}$ of the ith isotope.

$\mathrm{DCF}_{\mathrm{i}}$ : $\quad$ The exposure-to dose conversion factor for inhalation for the ith isotope (Ref. 2). 
Title of Project EPHA for THM

Subject Source Terms and Release Designation for Radionuclides

Computer E. T. Ketusky

Date $09 / 03 / 96$
Reviewed by S.A. Henderson

Works Calculation 5

Sheet No. 2 of 20

The isotopes were combined into a Pu-238 equivalent for each of the shipping containers as shown in below in Table 1.

Table 1. PU-238 Equivalent or $\mathrm{H}-3$ for each Shipping Container

Container of LLW SWDF (Waste)

\begin{tabular}{|c|c|c|c|}
\hline Radionuclides & $\begin{array}{l}\text { Inventory } \\
\text { (Ci) }\end{array}$ & $\begin{array}{l}\text { DCF } \\
(\mathrm{rem} / \mathrm{Ci})\end{array}$ & $\mid \begin{array}{l}\mathrm{Pu}- \\
238_{\text {equiv }} \\
(\mathrm{Ci})\end{array}$ \\
\hline $\mathrm{U}-234$ & 4.00E+0 & $1.30 E+08$ & $1.13 \mathrm{E}+03$ \\
\hline Am-241 & $4.00 E+0$ & $5.20 E+08$ & $\frac{4.52 E+03}{5.65 E+03}$ \\
\hline
\end{tabular}

Container of LLW Compactor (253-H)

\begin{tabular}{|c|c|c|c|}
\hline Radionuclides & $\begin{array}{l}\text { inventory } \\
\text { (Ci) }\end{array}$ & $\begin{array}{l}\text { DCF } \\
(\mathrm{rem} / \mathrm{Ci})\end{array}$ & $\mid \begin{array}{l}\mathrm{Pu}- \\
238_{\text {equiv }} \\
(\mathrm{Ci})\end{array}$ \\
\hline$\overline{C o-60}$ & \multicolumn{2}{|c|}{$2.09 E+001.50 E+05$} & $6.82 \mathrm{E}-04$ \\
\hline Pu-238 & \multicolumn{2}{|c|}{$7.60 E-034.60 E+08$} & 7.60E-03 \\
\hline Pu-239 & \multicolumn{2}{|c|}{ 7.60E-03 5.10E+08 } & 8.43E-03 \\
\hline Pu-240 & \multicolumn{2}{|c|}{ 7.60E-03 5.10E+08 } & 8.43E-03 \\
\hline Pu-242 & \multicolumn{2}{|c|}{$7.60 \hat{E}-03 \quad 4.80 E+08$} & $7.93 E-03$ \\
\hline$A m-241$ & \multirow{2}{*}{\multicolumn{2}{|c|}{$7.91 E-035.20 E+08$}} & $8.94 E-03$ \\
\hline & & & \\
\hline
\end{tabular}

MW Waste - Tritium Bearing Container (Waste)

\begin{tabular}{|l|l|l|l|}
\hline Radionuclides & $\begin{array}{l}\text { Inventory } \\
(\mathrm{Ci})\end{array}$ & $\begin{array}{l}\mathrm{DCF} \\
(\mathrm{rem} / \mathrm{Ci})\end{array}$ & $\begin{array}{l}\mathrm{H}-3 \\
(\mathrm{Ci})\end{array}$ \\
\hline $\mathrm{H}-3$ & $5.00 \mathrm{E}+03$ & $9.50 \mathrm{E}+01$ & $\frac{5.00 \mathrm{E}+03}{5.00 \mathrm{E}+03}$
\end{tabular}

Container of MW Waste - Beta/Gamma (Waste)

\begin{tabular}{|c|c|c|c|}
\hline Radionuclides & $\begin{array}{l}\text { Inventory } \\
\text { (Ci) }\end{array}$ & $\begin{array}{l}\text { DCF } \\
(\mathrm{rem} / \mathrm{Ci})\end{array}$ & $\mid \begin{array}{l}\text { Pu- } \\
238_{\text {equiv }} \\
(\mathrm{Ci})\end{array}$ \\
\hline $\begin{array}{l}\text { Co-60 } \\
\text { Cs-137 }\end{array}$ & $\begin{array}{l}1.00 E+01 \\
1.50 E+01\end{array}$ & $\begin{array}{l}1.50 E+05 \\
3.20 E+04\end{array}$ & $\begin{array}{l}3.26 \mathrm{E}-03 \\
1.04 \mathrm{E}-03 \\
\end{array}$ \\
\hline
\end{tabular}

Container of MW WASTE - Alpha (Waste)

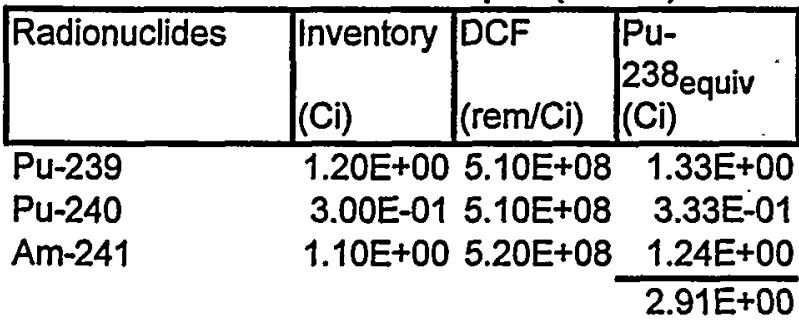


Title of Project EPHA for THM

Subject Source Terms and Release Designation for Radionuclides

Computer E.T. Ketusky
Reviewed by $\underline{\text { S.A. Henderson }}$

Works Calculation 5

Sheet No. 3 of 20

Container of LAWV (Waste)

\begin{tabular}{|c|c|c|c|}
\hline Radionuclides & $\begin{array}{l}\text { Inventory } \\
\text { (Ci) }\end{array}$ & $\begin{array}{l}\text { DCF } \\
(\text { rem/Ci) }\end{array}$ & $\begin{array}{l}\text { Pu- } \\
238_{\text {equiv }} \\
\text { (Ci) }\end{array}$ \\
\hline Co-60 & \multicolumn{2}{|c|}{$2.53 E+011.50 E+05$} & $8.25 E-03$ \\
\hline Pu-238 & \multicolumn{2}{|c|}{$9.10 \mathrm{E}-02 \quad 4.60 \mathrm{E}+08$} & $9.10 \mathrm{E}-02$ \\
\hline Pu-239 & \multicolumn{2}{|c|}{$9.10 \mathrm{E}-025.10 \mathrm{E}+08$} & 1.01E-01 \\
\hline Pu-240 & \multicolumn{2}{|c|}{$9.10 E-02 \quad 5.10 E+08$} & 1.01E-01 \\
\hline Pu-242 & \multicolumn{2}{|c|}{$9.10 E-02 \quad 4.80 E+08$} & $9.50 E-02$ \\
\hline Am-241 & \multirow{2}{*}{\multicolumn{2}{|c|}{ 9.47E-02 5.20E+08 }} & 1.07E-01 \\
\hline & & & $5.03 E-01$ \\
\hline
\end{tabular}

Container for ILNTV Combustibles (E Area Vaults)

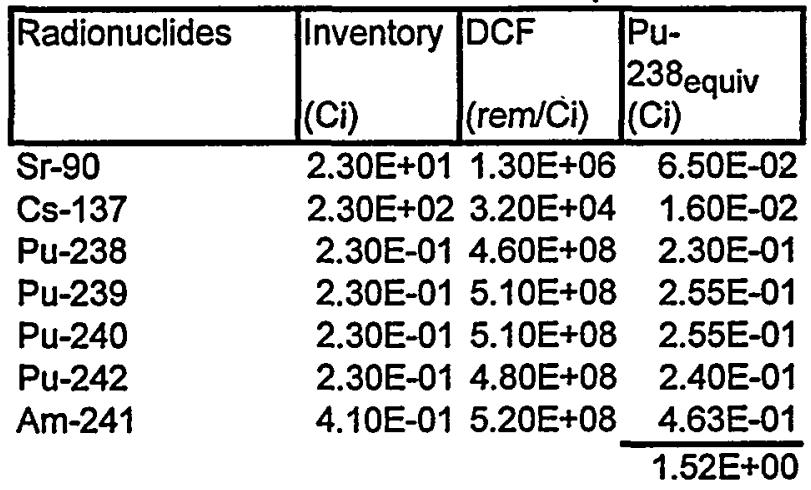

Container for LNTV Noncombustibles (E Area)

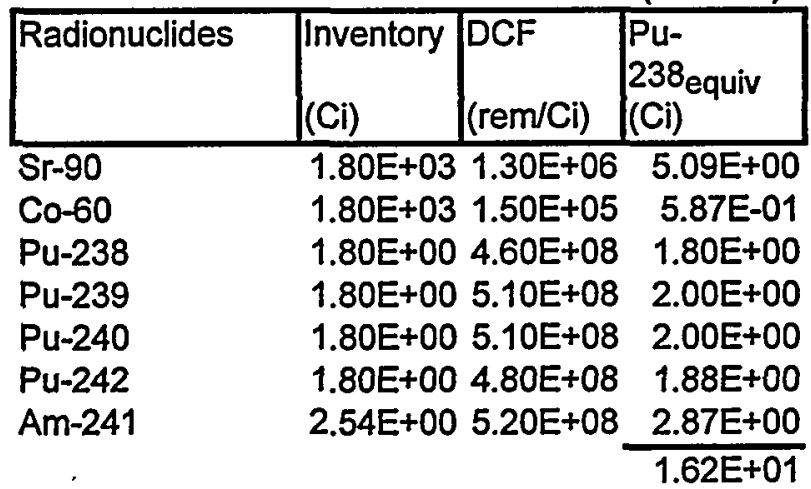

ILTV Bulk Container (E Area Vaults)

\begin{tabular}{l|l|l|l|}
\hline Radionuclides & $\begin{array}{l}\text { Inventory } \\
\text { (Ci) }\end{array}$ & $\begin{array}{l}\text { DCF } \\
\text { (rem/Ci) }\end{array}$ & $\begin{array}{l}\text { Pu- } \\
238 \text { equiv } \\
(\mathrm{Ci})\end{array}$ \\
\hline Co-60 & $1.96 \mathrm{E}+03$ & $1.50 \mathrm{E}+05$ & $6.39 \mathrm{E}-01$ \\
Pu-239 & $2.30 \mathrm{E}-01$ & $5.10 \mathrm{E}+08$ & $2.55 \mathrm{E}-01$ \\
Pu-240 & $2.30 \mathrm{E}-01$ & $5.10 \mathrm{E}+08$ & $2.55 \mathrm{E}-01$ \\
Pu-242 & $2.30 \mathrm{E}-01$ & $4.80 \mathrm{E}+08$ & $2.40 \mathrm{E}-01$ \\
Pu-238 & $2.30 \mathrm{E}-01$ & $4.60 \mathrm{E}+08$ & $2.30 \mathrm{E}-01$.
\end{tabular}


Title of Project EPHA for THM

Reviewed by $\underline{\text { S.A. Henderson }}$

Subject Source Terms and Release Designation for Radionuclides

Works Calculation 5

Computer E. T. Ketusky

Date 09/03/96

Sheet No. 4 of 20

Am-241

2.43E-01 5.20E+08 2.75E-01

$1.89 \mathrm{E}+00$ 
Title of Project EPHA for THM

Subject Source Terms and Release Designation for Radionuclides

Computer E.T. Ketusky
Reviewed by S.A. Henderson Works Calculation 5

Sheet No. $\underline{5 \text { of } 20}$

ILTV Crucibles Container (E Area Vaults)

\begin{tabular}{|c|c|c|c|}
\hline Radionuclides & $\begin{array}{l}\text { Inventory } \\
\text { (Ci) }\end{array}$ & $\begin{array}{l}\mathrm{DCF} \\
(\mathrm{rem} / \mathrm{Ci})\end{array}$ & $\begin{array}{l}\mathrm{H}-3 \\
(\mathrm{Ci})\end{array}$ \\
\hline \multirow[t]{4}{*}{$\mathrm{H}-3$} & \multirow{4}{*}{\multicolumn{2}{|c|}{$4.00 E+049.50 E+01$}} & $4.00 E+04$ \\
\hline & & & $4.00 E+04$ \\
\hline & & & $\begin{array}{l}\text { Pu- } \\
238_{\text {equiv }}\end{array}$ \\
\hline & & & $(\mathrm{Ci})$ \\
\hline Co-60 & $2.70 E+02$ & $1.50 E+05$ & $8.80 E-02$ \\
\hline Sr-90 & $1.80 \mathrm{E}+02$ & $1.30 E+06$ & $5.09 E-01$ \\
\hline $\mathrm{Np}-237$ & $1.80 E-01$ & $4.90 E+08$ & 1.92E-01 \\
\hline Pu-239 & $1.80 E-01$ & $5.10 E+08$ & 2.00E-01 \\
\hline Pu-240 & 1.80E-01 & $5.10 E+08$ & 2.00E-01 \\
\hline Pu-242 & $1.80 E-01$ & $4.80 E+08$ & 1.88E-01 \\
\hline \multirow[t]{2}{*}{ Am-241 } & 3.90E-01 & $5.20 E+08$ & 4.41E-01 \\
\hline & & & $1.82 \mathrm{E}+00$ \\
\hline
\end{tabular}

LLWSB Container (E Area Vaults)

\begin{tabular}{|c|c|c|c|}
\hline Radionuclides & $\begin{array}{l}\text { Inventory } \\
\text { (Ci) }\end{array}$ & $\begin{array}{l}\text { DCF } \\
(\text { rem/Ci) }\end{array}$ & $\mid \begin{array}{l}\mathrm{H}-3 \\
(\mathrm{Ci})\end{array}$ \\
\hline \multirow[t]{3}{*}{$\mathrm{H}-3$} & \multirow[t]{3}{*}{$1.00 \mathrm{E}+04$} & \multirow[t]{3}{*}{$9.50 \mathrm{E}+01$} & $1.00 \mathrm{E}+04$ \\
\hline & & & $1.00 E+04$ \\
\hline & & & $\begin{array}{l}\mathrm{Pu}- \\
238_{\text {equiv }} \\
\text { (Ci) }\end{array}$ \\
\hline Sr-90 & $1.80 E+02$ & $1.30 E+06$ & $5.09 \mathrm{E}-01$ \\
\hline$A m-241$ & $3.90 E-01$ & $5.20 E+08$ & 4.41E-01 \\
\hline Pu-239 & $1.80 \mathrm{E}-01$ & $5.10 E+08$ & 2.00E-01 \\
\hline $\begin{array}{l}\mathrm{Pu}-240 \\
\mathrm{~Np}-237\end{array}$ & $\begin{array}{l}1.80 \mathrm{E}-01 \\
1.80 \mathrm{E}-01\end{array}$ & $\begin{array}{l}5.10 E+08 \\
4.90 E+08\end{array}$ & $\begin{array}{l}2.00 E-01 \\
1.92 E-01\end{array}$ \\
\hline & & & $154 \mathrm{~F}+00$ \\
\hline
\end{tabular}

Pu 02 Container (235F and 247F)

\begin{tabular}{|l|l|l|l|}
\hline Radionuclides & $\begin{array}{l}\text { Inventory } \\
(\mathrm{Ci})\end{array}$ & $\begin{array}{l}\mathrm{DCF} \\
(\mathrm{rem} / \mathrm{Ci})\end{array}$ & $\begin{array}{l}\text { 238 } \\
(\mathrm{Ci})\end{array}$ \\
\hline Pu-238 & $9.16 \mathrm{E}+02$ & $4.60 \mathrm{E}+08$ & $\frac{9.16 \mathrm{E}+02}{9.16 \mathrm{E}+02}$
\end{tabular}

Container, Pail, Casket, Charge Can, Type A Storage Vault (235-F)

\begin{tabular}{|l|l|l|l|}
\hline Radionuclides & Inventory & DCF & $\begin{array}{l}\text { Pu- } \\
238_{\text {equiv }} \\
\end{array}$ \\
& $(\mathrm{Ci})$ & $(\mathrm{rem} / \mathrm{Ci})$ & $(\mathrm{Ci})$ \\
\hline
\end{tabular}

\begin{tabular}{llll}
\hline Pu-238 2.74E+05 4.60E+08 & $2.74 E+05$
\end{tabular} 
Title of Project EPHA for THM

Subject Source Terms and Release Designation for Radionuclides

Computer E. T. Ketusky

Date $\underline{09 / 03 / 96}$
Reviewed by S.A. Henderson

Works Calculation 5

Sheet No. 6 of 20 
Title of Project EPHA for THM

Reviewed by S.A. Henderson

Subject Source Terms and Release Designation for Radionuclides

Works Calculation 5

Date $\underline{09 / 03 / 96}$

Sheet No. 7 of 20

H Canyon Sample Container (221H to SRTC)

\begin{tabular}{|l|l|l|l|}
\hline Radionuclides & Inventory & $\begin{array}{l}\mathrm{DCF} \\
(\mathrm{Ci})\end{array}$ & $\begin{array}{l}\mathrm{Pu}- \\
238_{\text {equiv }} \\
(\mathrm{rem} / \mathrm{Ci})\end{array}$ \\
\hline Pu-238 & $1.54 \mathrm{E}+01$ & $4.60 \mathrm{E}+08$ & $\frac{1.54 \mathrm{E}+01}{1.54 \mathrm{E}+01}$
\end{tabular}

H Canyon Sample Container (221H to SRTC)

\begin{tabular}{|l|l|l|l|}
\hline Radionuclides & $\begin{array}{l}\text { Inventory } \\
\text { DCF }\end{array}$ & $\begin{array}{l}\text { Pu- } \\
238_{\text {equiv }} \\
(\mathrm{Ci})\end{array}$ & $(\mathrm{rem} / \mathrm{Ci})$ \\
\hline $\mathrm{C}-235$
\end{tabular}

H Canyon Sample Container (221H to SRTC)

\begin{tabular}{|l|l|l|l|}
\hline Radionuclides & inventory & $\begin{array}{l}\text { DCF } \\
(\mathrm{Ci})\end{array}$ & $\begin{array}{l}\mathrm{Pu}^{2} \\
238_{\text {equiv }} \\
(\mathrm{rem} / \mathrm{Ci})\end{array}$ \\
\hline $\mathrm{Np}-237$ & $7.05 \mathrm{E}-04$ & $4.90 \mathrm{E}+08$ & $\frac{7.51 \mathrm{E}-04}{7.51 \mathrm{E}-04}$
\end{tabular}

F Canyon Sample Container (221F to SRTC)

\begin{tabular}{|l|l|l|l|}
\hline Radionuclides & Inventory & $\begin{array}{l}\mathrm{DCF} \\
(\mathrm{Cu})\end{array}$ & $\begin{array}{l}\mathrm{Pu} \\
238_{\text {equiv }} \\
(\mathrm{Ci})\end{array}$ \\
\hline Pu-238 & $1.20 \mathrm{E}-01$ & $4.60 \mathrm{E}+08 \frac{1.20 \mathrm{E}-01}{1.20 \mathrm{E}-01}$
\end{tabular}

F Canyon Sample Container (221F to SRTC)

\begin{tabular}{|l|l|l|l|}
\hline Radionuclides & $\begin{array}{l}\text { Inventory } \\
\text { (Ci) }\end{array}$ & $\begin{array}{l}\text { DCF } \\
(\text { rem/Ci) }\end{array}$ & $\begin{array}{l}\text { Pu- } \\
238_{\text {equiv }} \\
(\mathrm{Ci})\end{array}$ \\
\hline Am-241 & $1.99 \mathrm{E}-02$ & $5.20 \mathrm{E}+08 \frac{2.25 \mathrm{E}-02}{2.25 \mathrm{E}-02}$
\end{tabular}

F Canyon Sample Container (221F to SRTC)

\begin{tabular}{|c|c|c|c|}
\hline Radionuclides & $\begin{array}{l}\text { Inventory } \\
\text { (Ci) }\end{array}$ & $\begin{array}{l}\text { DCF } \\
(\mathrm{rem} / \mathrm{Ci})\end{array}$ & $\begin{array}{l}\mathrm{Pu}- \\
238_{\text {equiv }} \\
\text { (Ci) }\end{array}$ \\
\hline $\mathrm{Cm}-244$ & $8.09 E+0$ & $2.70 \mathrm{E}+08$ & $\frac{4.75 \mathrm{E}+00}{4.75 \mathrm{E}+00}$ \\
\hline
\end{tabular}

HB Line Sample Container (221H to 772-1F)

\begin{tabular}{|l|l|l|l|}
\hline Radionuclides & Inventory & DCF & $\begin{array}{l}\text { Pu- } \\
238_{\text {equiv }} \\
\end{array}$ \\
& $(\mathrm{Ci})$ & $(\mathrm{rem} / \mathrm{Ci})$ & $(\mathrm{Ci})$ \\
\hline
\end{tabular}


OSR 25-10 (Rev 6-18-92)

Title of Project EPHA for THM

ENGINEERING COMPUTATION SHEET

Subject Source Terms and Release Designation for Radionuclides
Computer E. T. Ketusky

Date 09/03/96

Pu-238

\subsection{E-01 4.60E+08 8.55E-01

8.55E-01

Reviewed by S.A. Henderson

Works Calculation 5

Sheet No. $\underline{8 \text { of } 20}$ 
Title of Project EPHA for THM

Reviewed by S.A. Henderson

Works Calculation 5

Sheet No. 9 of 20

HB Line Sample Container (221H to SRTC)

\begin{tabular}{|c|c|c|c|}
\hline Radionuclides & $\begin{array}{l}\text { Inventory } \\
\text { (Ci) }\end{array}$ & $\begin{array}{l}\text { DCF } \\
(\mathrm{rem} / \mathrm{Ci})\end{array}$ & $\begin{array}{l}\text { Pu- } \\
238_{\text {equiv }} \\
\text { (Ci) }\end{array}$ \\
\hline Pu-238 & 8.55E-01 & $4.60 \mathrm{E}+08$ & $8.55 \mathrm{E}-01$ \\
\hline
\end{tabular}

FB Line Oxide Container (221F to Inter Area)

\begin{tabular}{|l|l|l|l|}
\hline Radionuclides & $\begin{array}{l}\text { Inventory } \\
\text { (Ci) }\end{array}$ & $\begin{array}{l}\mathrm{Pu}- \\
(\mathrm{rem} / \mathrm{Ci})\end{array}$ & $\begin{array}{l}238_{\text {equiv }} \\
(\mathrm{Ci})\end{array}$ \\
\hline $\mathrm{Pu}-239$ & $5.58 \mathrm{E}+035.10 \mathrm{E}+08$ & $\frac{6.19 \mathrm{E}+03}{6.19 \mathrm{E}+03}$
\end{tabular}

FB Line Sample Container (221F to SRTC)

\begin{tabular}{|l|l|l|l|}
\hline Radionuclides & Inventory & $\begin{array}{l}\mathrm{DCF} \\
\mathrm{Cu} \text { ) }\end{array}$ & $\begin{array}{l}\mathrm{Pu}- \\
238_{\text {equiv }} \\
(\mathrm{rem} / \mathrm{Ci})\end{array}$ \\
\hline Pu-239
\end{tabular}

235F Material Container (235F to 221H)

\begin{tabular}{l|l|l|l|}
\hline Radionuclides & $\begin{array}{l}\text { Inventory } \\
\text { (Ci) }\end{array}$ & $\begin{array}{l}\mathrm{DU} \text { (rem/Ci) } \\
238_{\text {equiv }} \\
(\mathrm{Ci})\end{array}$ \\
\hline Pu-238 $3.42 \mathrm{E}+045.10 \mathrm{E}+08 \frac{3.79 \mathrm{E}+04}{3.42 \mathrm{E}+04}$
\end{tabular}

235F Material Container (235F to 221 F)

\begin{tabular}{|l|l|l|l|}
\hline Radionuclides & $\begin{array}{l}\text { Inventory } \\
\text { (Ci) }\end{array}$ & $\begin{array}{l}\mathrm{Pu}- \\
(\mathrm{rem} / \mathrm{Ci})\end{array}$ & $\begin{array}{l}238_{\text {equiv }} \\
(\mathrm{Ci})\end{array}$ \\
\hline Pu-239 & $1.66 \mathrm{E}+025.10 \mathrm{E}+08$ & $\frac{1.84 \mathrm{E}+02}{1.84 \mathrm{E}+02}$
\end{tabular}

SRTC Material Container (SRTC to DWPF)

\begin{tabular}{l|l|l|l|}
\hline Radionuclides & $\begin{array}{l}\text { Inventory } \\
(\mathrm{Ci})\end{array}$ & $\begin{array}{l}\mathrm{Pu}- \\
238_{\text {equiv }} \\
(\mathrm{rem} / \mathrm{Ci})\end{array}$ \\
\hline $\mathrm{Cf}-252 \mathrm{Ci})$ \\
\hline
\end{tabular}

A Line Material (A Line)

\begin{tabular}{|l|l|l|l|}
\hline Radionuclides & Inventory & DCF & $\begin{array}{l}\text { Pu- } \\
238_{\text {equiv }} \\
\end{array}$ \\
& $(\mathrm{Ci})$ & $(\mathrm{rem} / \mathrm{Ci})$ & $(\mathrm{Ci})$ \\
\hline
\end{tabular}


OSR 25-10 (Rev 6-18-92)

Title of Project EPHA for THM

ENGINEERING COMPUTATION SHEET

Subject Source Terms and Release Designation for Radionuclides

Reviewed by $\underline{\text { S.A. Henderson }}$

Computer E. T. Ketusky

Date 09/03/96

Works Calculation 5

Sheet No. 10 of 20

(assume U-235)

Depleted UO3

$3.24 E-031.20 E+08 \frac{8.45 E-04}{8.45 E-04}$ 
OSR 25-10 (Rev 6-18-92)

Title of Project EPHA for THM

Subject - Source Terms and Release Designation for Radionuclides

Computer E.T. Ketusky
Reviewed by S.A. Henderson

Works Calculation 5

Sheet No. 11 of 20

UNH Trailer (H Area Outside)

\begin{tabular}{|c|c|c|c|}
\hline Radionuclides & $\begin{array}{l}\text { Inventory } \\
\text { (Ci) }\end{array}$ & $\begin{array}{l}\text { DCF } \\
\text { (rem/Ci) }\end{array}$ & $\mid \begin{array}{l}\mathrm{Pu}- \\
238_{\text {equiv }} \\
(\mathrm{Ci})\end{array}$ \\
\hline U-234 & \multirow{3}{*}{\multicolumn{2}{|c|}{$\begin{array}{ll}1.15 E+02 & 1.30 E+08 \\
2.87 E+01 & 1.20 E+08\end{array}$}} & $3.24 E+01$ \\
\hline U-238 & & & $7.49 E+00$ \\
\hline & & & \\
\hline
\end{tabular}

RBOF Portable Deionizer Column Transport (RBOF)

\begin{tabular}{|c|c|c|c|}
\hline Radionuclides ${ }^{\circ}$ & $\begin{array}{l}\text { Inventory } \\
\text { (Ci) }\end{array}$ & $\begin{array}{l}\text { DCF } \\
(\mathrm{rem} / \mathrm{Ci})\end{array}$ & \begin{tabular}{|l} 
Pu- \\
$238_{\text {equiv }}$ \\
(Ci)
\end{tabular} \\
\hline Cr-51 & $1.70 \mathrm{E}+0$ & $2.60 \mathrm{E}+02$ & $9.61 \mathrm{E}-06$ \\
\hline Ru-106 & $8.90 \mathrm{E}+0$ & $4.40 E+05$ & 8.51E-03 \\
\hline Cs-137 & $1.00 E+03$ & $3.20 E+04$ & $6.96 \mathrm{E}-02$ \\
\hline \multirow[t]{2}{*}{ Ce-144 } & $1.00 \mathrm{E}+0$ & $3.50 E+05$ & $7.61 \mathrm{E}-03$ \\
\hline & & & $8.57 \mathrm{E}-02$ \\
\hline
\end{tabular}

RBOF Spent Deoinizer Resin Transport (RBOF)

\begin{tabular}{|l|l|l|l|}
\hline Radionuclides & Inventory & DCF & $\begin{array}{l}\text { Pu- } \\
238_{\text {equiv }}\end{array}$ \\
\hline
\end{tabular}

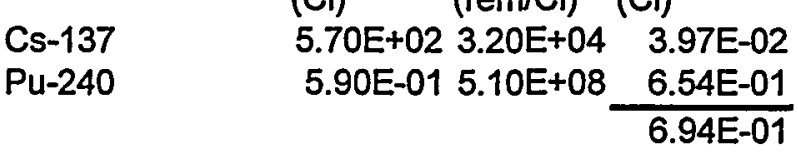

Disassembly Basin Deionizer \& Purif Resin Cask (Reactors)

\begin{tabular}{|l|l|l|l|}
\hline Radionuclides & $\begin{array}{l}\text { Inventory } \\
(\mathrm{Ci})\end{array}$ & $\begin{array}{l}\mathrm{DCF} \\
(\mathrm{rem} / \mathrm{Ci})\end{array}$ & $\begin{array}{l}\text { Pu- } \\
238_{\text {equiv }} \\
(\mathrm{Ci})\end{array}$ \\
\hline Co-60 & $1.69 \mathrm{E}+00$ & $1.50 \mathrm{E}+05 \frac{5.51 \mathrm{E}-04}{5.51 \mathrm{E}-04}$
\end{tabular}

Reactor Samples Container (Reactors to 771-D/F)

\begin{tabular}{l|l|l|l|}
\hline Radionuclides & $\begin{array}{l}\text { Inventory } \\
(\mathrm{Ci})\end{array}$ & $\begin{array}{l}\mathrm{DCF} . \\
(\mathrm{rem} / \mathrm{Ci})\end{array}$ & $\begin{array}{l}\text { Pu- } \\
238 \text { equiv } \\
(\mathrm{Ci})\end{array}$ \\
\hline Co-60 & $1.69 \mathrm{E}+001.50 \mathrm{E}+05 \frac{5.51 \mathrm{E}-04}{5.51 \mathrm{E}-04}$
\end{tabular}

Sample Truck (Inter Area)

\begin{tabular}{|l|l|l|l|}
\hline Radionuclides & $\begin{array}{l}\text { Inventory } \\
\text { (Ci) }\end{array}$ & $\begin{array}{l}\text { Pu- } \\
(\mathrm{rem} / \mathrm{Ci})\end{array}$ & $\begin{array}{l}238_{\text {equiv }} \\
(\mathrm{Ci})\end{array}$ \\
\hline Cs-137 & $8.51 \mathrm{E}+02$ & $3.20 \mathrm{E}+04$ & $\frac{5.92 \mathrm{E}-02}{5.92 \mathrm{E}-02}$
\end{tabular}


Title of Project EPHA for THM

Subject Source Terms and Release Designation for Radionuclides

Computer E. T. Ketusky
Reviewed by S.A. Henderson

Works Calculation 5

Date $\underline{09 / 03 / 96}$ 
Title of Projéct EPHA for THM

Reviewed by $\underline{\text { S.A. Henderson }}$

Subject Source Terms and Release Designation for Radionuclides

Works Calculation 5

Computer E. T. Ketusky

Date 09/03/96

Sheet No. 13 of 20

Moderator Transport in 55 Gallon Drums (100.

400 Areas)

\begin{tabular}{|c|c|c|c|}
\hline Radionuclides & $\begin{array}{l}\text { Inventory } \\
\text { (Ci) }\end{array}$ & $\begin{array}{l}\text { DCF } \\
(\mathrm{rem} / \mathrm{Ci})\end{array}$ & \begin{tabular}{|l} 
Pu- \\
$238_{\text {equiv }}$ \\
(Ci)
\end{tabular} \\
\hline$A m-241$ & $2.00 \mathrm{E}+0$ & $5.20 \mathrm{E}+08$ & $2.26 E+00$ \\
\hline
\end{tabular}

SRTC 776A to F Tank Farm 5000 Gallon Truck (776A to Tank Farm)

\begin{tabular}{|c|c|c|c|}
\hline Radionuclides & $\begin{array}{l}\text { Inventory } \\
\text { (Ci) }\end{array}$ & $\begin{array}{l}\text { DCF } \\
(\mathrm{rem} / \mathrm{Ci})\end{array}$ & $\begin{array}{l}\mathrm{Pu}- \\
238_{\text {equiv }} \\
\text { (Ci) }\end{array}$ \\
\hline & $6.23 E$ & $5.10 \mathrm{E}+08$ & $\frac{6.91 \mathrm{E}-02}{6.91 \mathrm{E}-02}$ \\
\hline
\end{tabular}

Contaminated Water Transport (100 Area to 211-F)

\begin{tabular}{|l|l|l|l|}
\hline Radionuclides & Inventory & $\mathrm{DCF}$ & $\begin{array}{l}\text { Pu- } \\
238_{\text {equiv }} \\
\end{array}$ \\
& $(\mathrm{Ci})$ & $(\mathrm{rem} / \mathrm{Ci})$ & $(\mathrm{Ci})$ \\
\hline
\end{tabular}

$\begin{array}{lll}\text { Am-241 } 2.00 E+00 & 5.20 \mathrm{E}+08 \quad 2.26 \mathrm{E}+00\end{array}$

$2.26 \mathrm{E}+00$

100 Area Sludge Transport (100 Area to 241-F) (2000 gallons)

Radionuclides

\begin{tabular}{|l|l|l|}
\hline Inventory & DCF & $\begin{array}{l}\text { Pu- } \\
238_{\text {equiv }} \\
(\mathrm{Ci})\end{array}$ \\
$(\mathrm{rem} / \mathrm{Ci})$ & $\mathrm{Ci})$ \\
\hline
\end{tabular}

Co-60 2.00E+00 1.50E+05 $\frac{6.52 \mathrm{E}-04}{6.52 \mathrm{E}-04}$ 
Title of Project EPHA for THM

Reviewed by S.A. Henderson

Works Calculation 5

Sheet No. 14 of 20

Table 2 calculates the source terms for the equivalent Pu-238 inventory and tritium inventory using the $A R R$ and $A R F$ for the different release mechanism (fire, impact, resuspension) for the identified different forms (Reference 2). The RF value used for the different mechanisms is equal to one unless otherwise indicated. The time used in resuspension is 2 hours. The DR (damage ratio) was normally conservatively considered to be one (with exceptions as noted). Table 2 also shows the release designations.

Table 2 Source Terms for Shipping Containers

Container of LLW SWDF (Waste)

\begin{tabular}{|c|c|c|c|c|c|c|c|}
\hline \multirow{2}{*}{$\begin{array}{l}\text { Pu-238 equiv } \\
\text { (Ci) }\end{array}$} & \multirow[t]{2}{*}{ Form } & \multicolumn{3}{|c|}{ ARFS $\times$ RF } & \multirow{2}{*}{$\begin{array}{l}\text { time } \\
\text { (hrs) }\end{array}$} & \multicolumn{2}{|c|}{ Source Terms (Ci) } \\
\hline & & Fire* & Impact + & Resuspension (per hour) & & $\begin{array}{c}\text { Fire } \\
\text { 1-RD-67 }\end{array}$ & $\begin{array}{c}\text { Impact }+ \text { Resuspension } \\
\text { 1-RD-68 }\end{array}$ \\
\hline $5.65 \mathrm{E}+03$ & Waste & $5.00 \mathrm{E}-04$ & $1.00 \mathrm{E}-03$ & $4.00 \mathrm{E}-05$ & 2 & $2.83 \mathrm{E}+00$ & $6.10 \mathrm{E}+00$ \\
\hline
\end{tabular}

Container of LLW Compactor (253-H)

\begin{tabular}{|c|c|c|c|c|c|c|c|}
\hline \multirow{2}{*}{$\begin{array}{c}\text { Pu- } 238_{\text {equir }} \\
\text { (Ci) }\end{array}$} & \multirow[t]{2}{*}{ Form } & \multicolumn{3}{|c|}{ ARFS X RF } & \multirow{2}{*}{$\begin{array}{l}\text { time } \\
\text { (hrs) }\end{array}$} & \multicolumn{2}{|c|}{ Source Terms $(\mathbf{C} \mathfrak{i})$} \\
\hline & & Fire* & Impact + & Resuspension (per hour) & & $\begin{array}{c}\text { Fire } \\
\text { 1-RD-69 }\end{array}$ & $\begin{array}{c}\text { Impact + Resuspension } \\
1-R D-70\end{array}$ \\
\hline $4.20 \mathrm{E}-02$ & Waste & $5.00 \mathrm{E}-04$ & $1.00 \mathrm{E}-03$ & $4.00 \mathrm{E}-05$ & 2 & $2.10 \mathrm{E}-05$ & $4.54 \mathrm{E}-05$ \\
\hline
\end{tabular}

MW Waste - Tritium Bearing Container (Waste)

\begin{tabular}{|c|c|c|c|c|c|c|c|}
\hline \multirow{2}{*}{$\begin{array}{l}\mathrm{H}-3 \\
\text { (Ci) }\end{array}$} & \multirow[t]{2}{*}{ Form } & \multicolumn{3}{|c|}{ ARFS $\times$ RF } & \multirow{2}{*}{$\begin{array}{l}\text { time } \\
\text { (hrs) }\end{array}$} & \multicolumn{2}{|c|}{ Source Terms (Ci) } \\
\hline & & Fire* & Impact + & Resuspension (per hour) & & $\begin{array}{c}\text { Fire } \\
\text { 1-RD-71 }\end{array}$ & $\begin{array}{c}\text { Impact + Resuspension } \\
\text { 1-RD-72 }\end{array}$ \\
\hline $5.00 \mathrm{E}+03$ & Waste & $5.00 \mathrm{E}-04$ & $1.00 \mathrm{E}-03$ & $9.00 \mathrm{E}-05$ & 2 & $2.50 \mathrm{E}+00$ & $5.90 \mathrm{E}+00$ \\
\hline
\end{tabular}

Container of MW Waste - Beta/Gamma (Waste)

\begin{tabular}{|c|c|c|c|c|c|c|}
\hline \multirow{2}{*}{$\begin{array}{c}\text { Pu-238 equiv } \\
\text { (Ci) }\end{array}$} & \multirow[t]{2}{*}{ Form } & \multicolumn{2}{|r|}{ ARFS $\times$ RF } & \multirow{2}{*}{$\begin{array}{l}\text { time } \\
\text { (hrs) }\end{array}$} & \multicolumn{2}{|c|}{ Source Terms (Ci) } \\
\hline & & Fire* & Impact $+\quad$ Resuspension (per hour) & & $\begin{array}{c}\text { Fire } \\
\text { 1-RD-73 }\end{array}$ & $\begin{array}{c}\text { Impact + Resuspension } \\
\text { I-RD-74 }\end{array}$ \\
\hline $4.30 \mathrm{E}-03$ & Waste & $5.00 \mathrm{E}-04$ & $9.00 \mathrm{E}-05$ & 2 & $2.15 \mathrm{E}-06$ & $5.08 \mathrm{E}-06$ \\
\hline
\end{tabular}

Container of MW WASTE - Alpha (Waste)

\begin{tabular}{|c|c|c|c|c|c|c|}
\hline \multirow{2}{*}{$\begin{array}{l}\text { Pu-238 oquiv } \\
\text { (Ci) }\end{array}$} & \multirow[t]{2}{*}{ Form } & \multicolumn{2}{|r|}{ ARFS $\times$ RF } & \multirow{2}{*}{$\begin{array}{l}\text { time } \\
\text { (hrs) }\end{array}$} & \multicolumn{2}{|c|}{ Source Terms (Ci) } \\
\hline & & Fire* & Impact $+\quad$ Resuspension (per hour) & & $\begin{array}{c}\text { Fire } \\
\text { 1-RD-75 }\end{array}$ & $\begin{array}{c}\text { Impact + Resuspension } \\
\text { 1-RD-76 }\end{array}$ \\
\hline $2.91 \mathrm{E}+00$ & Waste & $5.00 \mathrm{E}-04$ & $9.00 \mathrm{E}-05$ & 2 & $1.45 \mathrm{E}-03$ & 3.43E-03 \\
\hline
\end{tabular}

Container of TRU (Waste)

\begin{tabular}{|c|c|c|c|c|c|c|c|}
\hline $\begin{array}{c}\text { Pu-238 couv } \\
\text { (Ci) }\end{array}$ & Form & \multicolumn{3}{|c|}{ ARFS x RF } & time & \multicolumn{2}{c|}{ Source Terms (Ci) } \\
\cline { 3 - 8 } & & Fire* & Impact + Resuspension (per hour) & (hrs) & $\begin{array}{c}\text { Fire } \\
1-R D-77\end{array}$ & $\begin{array}{c}\text { Impact + Resuspension } \\
1-R D-78\end{array}$ \\
\hline $5.03 \mathrm{E}-01$ & Waste & $5.00 \mathrm{E}-04$ & $1.00 \mathrm{E}-03$ & $4.00 \mathrm{E}-05$ & .2 & $2.52 \mathrm{E}-04$ & $5.43 \mathrm{E}-04$ \\
\hline
\end{tabular}


Title of Project EPHA for THM

Reviewed by S.A. Henderson

Subject Source Terms and Release Designation for Radionuclides

Works Calculation 5

Computer E.T. Ketusky

Date 09/03/96

Sheet No. 15 of 20

Container for ILNTV Combustibles (E Area Vaults)

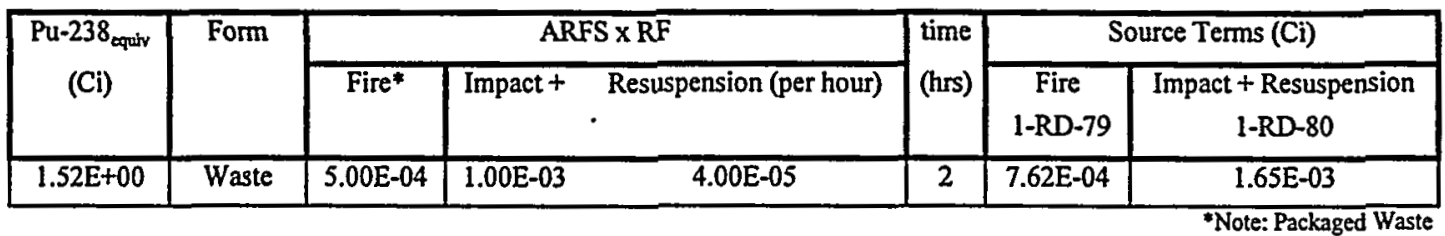

Container for LNTV Noncombustibles (E Area)

\begin{tabular}{|c|c|c|c|c|c|c|c|}
\hline \multirow{2}{*}{$\begin{array}{l}\text { Pu-238 oquv } \\
\text { (Ci) }\end{array}$} & \multirow[t]{2}{*}{ Form } & \multicolumn{3}{|c|}{ ARFS $\times$ RF } & \multirow{2}{*}{$\begin{array}{l}\text { time } \\
\text { (hrs) }\end{array}$} & \multicolumn{2}{|c|}{ Source Terms (Ci) } \\
\hline & & Fire* & Impact + & Resuspension (per hour) & & $\begin{array}{c}\text { Fire } \\
\text { I-RD-81 }\end{array}$ & $\begin{array}{c}\text { Impact + Resuspension } \\
1-R D-82\end{array}$ \\
\hline $1.62 \mathrm{E}+01$ & Waste & $5.00 \mathrm{E}-04$ & $1.00 \mathrm{E}-03$ & $4.00 \mathrm{E}-05$ & 2 & 8.11E-03 & $1.75 \mathrm{E}-02$ \\
\hline
\end{tabular}

ILTV Bulk Container (E Area Vaults)

\begin{tabular}{|c|c|c|c|c|c|c|}
\hline \multirow{2}{*}{$\begin{array}{l}\text { Pu-238 cquiv } \\
\text { (Ci) }\end{array}$} & \multirow[t]{2}{*}{ Form } & \multicolumn{2}{|r|}{ ARFS $\times$ RF } & \multirow{2}{*}{$\begin{array}{l}\text { time } \\
\text { (hrs) }\end{array}$} & \multicolumn{2}{|c|}{ Source Terms (Ci) } \\
\hline & & Fire* & Impact + Resuspension (per hour) & & $\begin{array}{c}\text { Fire } \\
\text { 1-RD-83 }\end{array}$ & $\begin{array}{c}\text { Impact + Resuspension } \\
\text { 1-RD-84 }\end{array}$ \\
\hline $1.89 \mathrm{E}+00$ & Waste & $5.00 \mathrm{E}-04$ & $4.00 \mathrm{E}-05$ & 2 & $9.47 E-04$ & $2.04 \mathrm{E}-03$ \\
\hline
\end{tabular}

ILTV Crucibles Container (E Area Vaults)

\begin{tabular}{|l|c|c|c|c|c|c|c|}
\hline $\begin{array}{l}\text { Pu-238 cautr } \\
\text { (Ci) }\end{array}$ & \multirow{2}{*}{ Form } & \multicolumn{3}{|c|}{ ARFS x RF } & time & \multicolumn{2}{|c|}{ Source Terms (Ci) } \\
\cline { 3 - 8 } & & Fire* & Impact + & Resuspension & (hrs) & $\begin{array}{c}\text { Fire } \\
1-R D-85\end{array}$ & $\begin{array}{c}\text { Impact + Resuspension } \\
\text { 1-RD-86 }\end{array}$ \\
\hline $1.82 \mathrm{E}+00$ & Waste & $5.00 \mathrm{E}-04$ & $1.00 \mathrm{E}-03$ & $4.00 \mathrm{E}-05$ & 2 & $9.10 \mathrm{E}-04$ & $1.97 \mathrm{E}-03$ \\
\hline
\end{tabular}

LLWSB Container (E Area Vaults)

\begin{tabular}{|c|c|c|c|c|c|c|c|}
\hline \multirow{2}{*}{$\begin{array}{l}\mathrm{H}-3 \\
\text { (Ci) }\end{array}$} & \multirow[t]{2}{*}{ Form } & \multicolumn{3}{|c|}{ ARFS $\times$ RF } & \multirow{2}{*}{$\begin{array}{l}\text { time } \\
\text { (hrs) }\end{array}$} & \multicolumn{2}{|c|}{ Source Terms (Ci) } \\
\hline & & Fire* & Impact + & Resuspension & & $\begin{array}{c}\text { Fire } \\
\text { 1-RD-87 }\end{array}$ & $\begin{array}{c}\text { Impact + Resuspension } \\
\text { 1-RD-88 }\end{array}$ \\
\hline $1.54 \mathrm{E}+00$ & Waste & $5.00 \mathrm{E}-04$ & $1.00 \mathrm{E}-03$ & $4.00 \mathrm{E}-05$ & 2 & $7.70 \mathrm{E}-04$ & $1.66 \mathrm{E}-03$ \\
\hline
\end{tabular}

Pu 02 Container (235F and 247F)

\begin{tabular}{|c|c|c|c|c|c|c|c|}
\hline \multirow{2}{*}{$\begin{array}{l}\text { Pu-238 cquv } \\
\text { (Ci) }\end{array}$} & \multirow[t]{2}{*}{ Form } & \multicolumn{3}{|c|}{ ARFS $\times$ RF } & \multirow{2}{*}{$\begin{array}{l}\text { time } \\
\text { (hrs) }\end{array}$} & \multicolumn{2}{|c|}{ Source Terms (Ci) } \\
\hline & & Fire & Impact + & Resuspension & & $\begin{array}{c}\text { Fire } \\
\text { 1-RD-89 }\end{array}$ & $\begin{array}{c}\text { Impact + Resuspension } \\
\text { 1-RD-90 }\end{array}$ \\
\hline $9.16 \mathrm{E}+02$ & $\mathrm{Pu}^{*}$ & $6.00 \mathrm{E}-05$ & $2.00 \mathrm{E}=03$ & $4.00 \mathrm{E}-05$ & 2 & $5.50 \mathrm{E}-02$ & $1.91 E+00$ \\
\hline
\end{tabular}

H Canyon Sample Container (221H to SRTC)

\begin{tabular}{|c|c|c|c|c|c|c|}
\hline \multirow{2}{*}{$\begin{array}{l}\text { Pu-238 } \\
\text { (Cquiv } \\
\text { (Ci) }\end{array}$} & \multirow[t]{2}{*}{ Form } & \multicolumn{2}{|r|}{ ARFS $\times$ RF } & \multirow{2}{*}{$\begin{array}{l}\text { time } \\
\text { (hrs) }\end{array}$} & \multicolumn{2}{|c|}{ Source Terms (Ci) } \\
\hline & & Fire & Impact + Resuspension & & $\begin{array}{c}\text { Fire } \\
\text { 1-RD-91 }\end{array}$ & $\begin{array}{c}\text { Impact + Resuspension } \\
1-R D-92\end{array}$ \\
\hline $1.54 \mathrm{E}+01$ & Liquid & $2.00 \mathrm{E}-03$ & $2.00 \mathrm{E}-03$ & 2 & $3.08 \mathrm{E}-02$ & $3.36 \mathrm{E}-02$ \\
\hline
\end{tabular}


Title of Project EPHA for THM

Reviewed by $\underline{\text { S.A. Henderson }}$

Subject Source Terms and Release Designation for Radionuclides

Works Calculation 5

Computer E. T. Ketusky

Date $\underline{09 / 03 / 96}$

Sheet No. 16 of 20

H Canyon Sample Container (221H to SRTC)

\begin{tabular}{|c|c|c|c|c|c|c|}
\hline \multirow{2}{*}{$\begin{array}{l}\text { Pu-238 } \text { cqulv }_{\text {. }} \\
\text { (Ci) }\end{array}$} & \multirow[t]{2}{*}{ Form } & \multicolumn{2}{|r|}{ ARFS $\times$ RF } & \multirow{2}{*}{$\begin{array}{l}\text { time } \\
\text { (hrs) }\end{array}$} & \multicolumn{2}{|c|}{ Source Terms (Ci) } \\
\hline & & Fire & Impact + Resuspension & & $\begin{array}{c}\text { Fire } \\
\text { 1-RD-93 }\end{array}$ & $\begin{array}{c}\text { Impact + Resuspension } \\
\text { 1-RD-94 }\end{array}$ \\
\hline $2.25 \mathrm{E}-0$ & Liquid & $2.00 \mathrm{E}-03$ & $2.00 \mathrm{E}-03$ & 2 & $4.51 \mathrm{E}-08$ & $4.91 \mathrm{E}-08$ \\
\hline
\end{tabular}

H Canyon Sample Container (221H to SRTC)

\begin{tabular}{|c|c|c|c|c|c|c|}
\hline \multirow{2}{*}{$\begin{array}{l}\text { Pu-238 } \\
\text { (Cqutr } \\
\text { (Ci) }\end{array}$} & \multirow[t]{2}{*}{ Form } & \multicolumn{2}{|r|}{ ARFS $\times$ RF } & \multirow{2}{*}{$\begin{array}{l}\text { time } \\
\text { (hrs) }\end{array}$} & \multicolumn{2}{|c|}{ Source Terms (Ci) } \\
\hline & & Fire & Impact + Resuspension & & $\begin{array}{c}\text { Fire } \\
\text { 1-RD-95 }\end{array}$ & $\begin{array}{c}\text { Impact + Resuspension } \\
\text { 1-RD-96 }\end{array}$ \\
\hline $7.51 \mathrm{E}-04$ & Liquid & $2.00 \mathrm{E}-03$ & $2.00 \mathrm{E}-03$ & 2 & $1.50 \mathrm{E}-06$ & $1.64 \mathrm{E}-06$ \\
\hline
\end{tabular}

F Canyon Sample Container (221F to SRTC)

\begin{tabular}{|c|c|c|c|c|c|c|}
\hline \multirow{2}{*}{$\begin{array}{l}\text { Pu-238 } \text { equiv } \\
\text { (Ci) }\end{array}$} & \multirow[t]{2}{*}{ Form } & \multicolumn{2}{|r|}{ ARFS $\times$ RF } & \multirow{2}{*}{$\begin{array}{l}\text { time } \\
\text { (hrs) }\end{array}$} & \multicolumn{2}{|c|}{ Source Terms (Ci) } \\
\hline & & Fire & Impact + Resuspension & & $\begin{array}{c}\text { Fire } \\
\text { 1-RD-97 }\end{array}$ & $\begin{array}{c}\text { Impact + Resuspension } \\
1-R D-98\end{array}$ \\
\hline $1.20 \mathrm{E}-01$ & Liquid & $2.00 \mathrm{E}-03$ & $2.00 \mathrm{E}-03$ & 2 & $2.40 \mathrm{E}-04$ & $2.62 \mathrm{E}-04$ \\
\hline
\end{tabular}

F Canyon Sample Container (221F to SRTC)

\begin{tabular}{|c|c|c|c|c|c|c|}
\hline \multirow{2}{*}{$\begin{array}{l}\text { Pu-238 } \text { oqulv } \\
\text { (Ci) }\end{array}$} & \multirow[t]{2}{*}{ Form } & \multicolumn{2}{|r|}{ ARFS $\times$ RF } & \multirow{2}{*}{$\begin{array}{l}\text { time } \\
\text { (hrs) }\end{array}$} & \multicolumn{2}{|c|}{ Source Terms (Ci) } \\
\hline & & Fire & Impact + Resuspension & & $\begin{array}{c}\text { Fire } \\
1-R D-99\end{array}$ & $\begin{array}{c}\text { Impact + Resuspension } \\
\text { 1-RD-100 }\end{array}$ \\
\hline $2.25 \mathrm{E}-02$ & Liquid & $2.00 \mathrm{E}-03$ & $2.00 \mathrm{E}-03$ & 2 & $4.50 \mathrm{E}-05$ & $4.90 \mathrm{E}-05$ \\
\hline
\end{tabular}

F Canyon Sample Container (221F to SRTC)

\begin{tabular}{|c|c|c|c|c|c|c|}
\hline \multirow{2}{*}{$\begin{array}{l}\text { Pu-238 } \\
\text { (Ci) }\end{array}$} & \multirow[t]{2}{*}{ Form } & \multicolumn{2}{|r|}{ ARFS $\times$ RF } & \multirow{2}{*}{$\begin{array}{l}\text { time } \\
\text { (hrs) }\end{array}$} & \multicolumn{2}{|c|}{ Source Terms (Ci) } \\
\hline & & Fire & Impact + Resuspension & & $\begin{array}{c}\text { Fire } \\
\text { 1-RD-101 }\end{array}$ & $\begin{array}{c}\text { Impact + Resuspension } \\
\text { 1-RD-102 }\end{array}$ \\
\hline $4.75 E+00$ & Liquid & $2.00 \mathrm{E}-03$ & $2.00 \mathrm{E}-03$ & 2 & $9.50 \mathrm{E}-03$ & $1.04 \mathrm{E}-02$ \\
\hline
\end{tabular}

HB Line Sample Container (221H to 772-1F)

\begin{tabular}{|c|c|c|c|c|c|c|}
\hline \multirow{2}{*}{$\begin{array}{l}\text { Pu-238 } \text { cquiv } \\
\text { (Ci) }\end{array}$} & \multirow[t]{2}{*}{ Form } & \multicolumn{2}{|r|}{ ARFS $\times$ RF } & \multirow{2}{*}{$\begin{array}{l}\text { time } \\
\text { (hrs) }\end{array}$} & \multicolumn{2}{|c|}{ Source Terms (Ci) } \\
\hline & & Fire & Impact + Resuspension & & $\begin{array}{c}\text { Fire } \\
\text { 1-RD-103 }\end{array}$ & $\begin{array}{c}\text { Impact + Resuspension } \\
\text { 1-RD-104 }\end{array}$ \\
\hline $8.55 \mathrm{E}-01$ & Liquid & $2.00 \mathrm{E}-03$ & $2.00 \mathrm{E}-03$ & 2 & $1.71 \mathrm{E}-03$ & $1.86 \mathrm{E}-03$ \\
\hline
\end{tabular}

HB Line Sample Container (221H to SRTC)

\begin{tabular}{|c|c|c|c|c|c|c|}
\hline \multirow{2}{*}{$\begin{array}{l}\text { Pu-238 } \\
\text { (Ci) }\end{array}$} & \multirow[t]{2}{*}{ Form } & \multicolumn{2}{|r|}{ ARFS $\times$ RF } & \multirow{2}{*}{$\begin{array}{l}\text { time } \\
\text { (hrs) }\end{array}$} & \multicolumn{2}{|c|}{ Source Terms (Ci) } \\
\hline & & Fire & Impact + Resuspension & & $\begin{array}{c}\text { Fire } \\
\text { 1-RD-105 }\end{array}$ & $\begin{array}{c}\text { Impact + Resuspension } \\
\text { 1-RD-106 }\end{array}$ \\
\hline $8.55 \mathrm{E}-01$ & Liquid & $2.00 \mathrm{E}-03$ & $2.00 \mathrm{E}-03$ & 2 & $1.71 \mathrm{E}-03$ & $1.86 \mathrm{E}-03$ \\
\hline
\end{tabular}


Title of Project EPHA for THM

Reviewed by $\underline{\text { S.A. Henderson }}$ Works Calculation 5 Sheet No. 17 of 20

FB Line Oxide Container (221F to Inter Area)

\begin{tabular}{|c|c|c|c|c|c|c|}
\hline \multirow{2}{*}{$\begin{array}{l}\text { Pu-238 } \\
\text { (Ci) }\end{array}$} & \multirow[t]{2}{*}{ Form ${ }^{*}$} & \multicolumn{2}{|r|}{ ARFS $\times$ RF } & \multirow{2}{*}{$\begin{array}{l}\text { time } \\
\text { (hrs) }\end{array}$} & \multicolumn{2}{|c|}{ Source Terms $(\mathrm{Ci})$} \\
\hline & & Fire & Impact + Resuspension & & $\begin{array}{c}\text { Fire } \\
\text { 1-RD-107 }\end{array}$ & $\begin{array}{c}\text { Impact + Resuspension } \\
\text { 1-RD-108 }\end{array}$ \\
\hline $6.19 E+03$ & Powder & $6.00 \mathrm{E}-05$ & $4.00 \mathrm{E}-05$ & 2 & $3.71 \mathrm{E}-01$ & $1.11 \mathrm{E}-02$ \\
\hline
\end{tabular}

FB Line Sample Container (221F to SRTC)

\begin{tabular}{|c|c|c|c|c|c|c|}
\hline \multirow{2}{*}{$\begin{array}{l}\text { Pu-238 } \\
\text { (Ci) }\end{array}$} & \multirow[t]{2}{*}{ Form } & \multicolumn{2}{|r|}{ ARFS $\times$ RF } & \multirow{2}{*}{$\begin{array}{l}\text { time } \\
\text { (hrs) }\end{array}$} & \multicolumn{2}{|c|}{ Source Terms (Ci) } \\
\hline & & Fire & Impact + Resuspension & & $\begin{array}{c}\text { Fire } \\
\text { 1-RD-109 }\end{array}$ & $\begin{array}{c}\text { Impact + Resuspension } \\
1-R D-110\end{array}$ \\
\hline $6.87 \mathrm{E}-01$ & Liquid & $2.00 \mathrm{E}-03$ & $2.00 \mathrm{E}-03$ & 2 & $1.37 \mathrm{E}-03$ & $1.50 \mathrm{E}-03$ \\
\hline
\end{tabular}

235F Material Container (235F to 221H)

\begin{tabular}{|c|c|c|c|c|c|c|c|}
\hline \multirow{2}{*}{$\begin{array}{l}\text { Pu-238 equlv } \\
\text { (Ci) }\end{array}$} & \multirow[t]{2}{*}{ Form* } & \multicolumn{3}{|c|}{ ARFS $\times$ RF } & \multirow{2}{*}{$\begin{array}{l}\text { time } \\
\text { (hrs) }\end{array}$} & \multicolumn{2}{|c|}{ Source Terms (Ci) } \\
\hline & & Fire & Impact - & nsion & & $\begin{array}{c}\text { Fire } \\
1-R D-111\end{array}$ & $\begin{array}{c}\text { Impact + Resuspension } \\
\text { 1-RD-112 }\end{array}$ \\
\hline $3.42 \mathrm{E}+04$ & Powder & $6.00 \mathrm{E}-05$ & $1.00 \mathrm{E}-05$ & $4.00 \mathrm{E}-05$ & 2 & $2.27 \mathrm{E}+0$ & $6.08 \mathrm{E}-01$ \\
\hline
\end{tabular}

235F Material Container (235F to $221 \mathrm{~F}$ )

\begin{tabular}{|c|c|c|c|c|c|c|}
\hline \multirow{2}{*}{$\begin{array}{l}\text { Pu-238 } \\
\text { (Ci) }\end{array}$} & \multirow[t]{2}{*}{ Form ${ }^{*}$} & \multicolumn{2}{|r|}{ ARFS $\times$ RF } & \multirow{2}{*}{$\begin{array}{l}\text { time } \\
\text { (hrs) }\end{array}$} & \multicolumn{2}{|c|}{ Source Terms (Ci) } \\
\hline & & Fire & Impact + Resuspension & & $\begin{array}{c}\text { Fire } \\
\text { 1-RD-113 }\end{array}$ & $\begin{array}{c}\text { Impact + Resuspension } \\
\text { 1-RD-114 }\end{array}$ \\
\hline $1.84 E+02$ & Powder & $6.00 \mathrm{E}-05$ & $4.00 \mathrm{E}-05$ & 2 & $1.1 \mathrm{E}-02$ & $3.31 \mathrm{E}-02$ \\
\hline
\end{tabular}

SRTC Material Container (SRTC to DWPF)

\begin{tabular}{|c|c|c|c|c|c|c|}
\hline \multirow{2}{*}{$\begin{array}{l}\text { Pu-238 equatv } \\
\text { (Ci) }\end{array}$} & \multirow[t]{2}{*}{ Form } & \multicolumn{2}{|r|}{ ARFS $\times$ RF } & \multirow{2}{*}{$\begin{array}{l}\text { time } \\
\text { (hrs) }\end{array}$} & \multicolumn{2}{|c|}{ Source Terms (Ci) } \\
\hline & & Fire & Impact + Resuspension & & $\begin{array}{c}\text { Fire } \\
\text { 1-RD-115 }\end{array}$ & $\begin{array}{c}\text { Impact + Resuspension } \\
\text { 1-RD-116 }\end{array}$ \\
\hline $7.60 \mathrm{E}+00$ & Aggr & $6.00 \mathrm{E}-03$ & $4.00 \mathrm{E}-05$ & 2 & $4.56 \mathrm{E}-02$ & $8.21 \mathrm{E}-03$ \\
\hline
\end{tabular}

. A Line Material (A Line)

\begin{tabular}{|c|c|c|c|c|c|c|}
\hline \multirow{2}{*}{$\begin{array}{l}\text { Pu-238 } \text { oquiv }_{\text {oqu }} \\
\text { (Ci) }\end{array}$} & \multirow[t]{2}{*}{ Form } & \multicolumn{2}{|r|}{ ARFS $\times$ RF } & \multirow{2}{*}{$\begin{array}{l}\text { time } \\
\text { (hrs) }\end{array}$} & \multicolumn{2}{|c|}{ Source Terms (Ci) } \\
\hline & & Fire & Impact + Resuspension & & $\begin{array}{c}\text { Fire } \\
1-\mathrm{RD}-117\end{array}$ & $\begin{array}{c}\text { Impact + Resuspension } \\
1-R D-118\end{array}$ \\
\hline $8.45 E-04$ & Liquid & $2.00 \mathrm{E}-03$ & $2.00 \mathrm{E}-03$ & 2 & $1.69 \mathrm{E}-06$ & $1.84 \mathrm{E}-06$ \\
\hline
\end{tabular}

UNH Trailer (H Area Outside)

\begin{tabular}{|c|c|c|c|c|c|c|}
\hline \multirow{2}{*}{$\begin{array}{l}\text { Pu-238 } \\
\text { caqur } \\
\text { (Ci) }\end{array}$} & \multirow[t]{2}{*}{ Form } & \multicolumn{2}{|r|}{ ARFS $\times$ RF } & \multirow{2}{*}{$\begin{array}{l}\text { time } \\
\text { (hrs) }\end{array}$} & \multicolumn{2}{|c|}{ Source Terms (Ci) } \\
\hline & & Fire & Impact + Resuspension & & $\begin{array}{c}\text { Fire } \\
1-R D-119\end{array}$ & $\begin{array}{c}\text { Impact + Resuspension } \\
1-R D-120\end{array}$ \\
\hline $3.99 \mathrm{E}+01$ & Liquid & $2.00 \mathrm{E}-03$ & $2.00 \mathrm{E}-03$ & 2 & $7.98 \mathrm{E}-02$ & $7.98 \mathrm{E}-02$ \\
\hline
\end{tabular}


Title of Project EPHA for THM

Reviewed by $\underline{\text { S.A. Henderson }}$

Subject Source Terms and Release Designation for Radionuclides

Works Calculation 5

Computer E. T. Ketusky

Date $\underline{09 / 03 / 96}$

Sheet No. 18 of 20

Container, Pail, Casket, Charge Can, Type A for Storage Vault (235-F)

\begin{tabular}{|c|c|c|c|c|c|c|c|}
\hline \multirow{2}{*}{$\begin{array}{l}\text { Pu-238 oqutv } \\
\text { (Ci) }\end{array}$} & \multirow[t]{2}{*}{ Form* } & \multicolumn{3}{|c|}{ ARFS $\times$ RF } & \multirow{2}{*}{$\begin{array}{l}\text { time } \\
\text { (hrs) }\end{array}$} & \multicolumn{2}{|c|}{ Source Terms (Ci) } \\
\hline & & Fire & Impact + & nsion & & $\begin{array}{c}\text { Fire } \\
1-R D-121\end{array}$ & $\begin{array}{c}\text { Impact + Resuspension } \\
\text { 1-RD-122 }\end{array}$ \\
\hline $2.74 E+05$ & Surface & $6.00 \mathrm{E}-05$ & $1.00 \mathrm{E}-05$ & $4.00 \mathrm{E}-05$ & 2 & $8.22 \mathrm{E}-01$ & $1.48 \mathrm{E}-02$ \\
\hline
\end{tabular}

RBOF Portable Deionizer Column Transport (RBOF)

\begin{tabular}{|c|c|c|c|c|c|c|}
\hline \multirow{2}{*}{$\begin{array}{l}\text { Pu-238 } \\
\text { (Ci) }\end{array}$} & \multirow[t]{2}{*}{ Form* } & \multicolumn{2}{|r|}{ ARFS $\times$ RF } & \multirow{2}{*}{$\begin{array}{l}\text { time } \\
\text { (hrs) }\end{array}$} & \multicolumn{2}{|c|}{ Source Terms (Ci) } \\
\hline & & Fire & Impact + Resuspension & & $\begin{array}{c}\text { Fire } \\
\text { 1-RD-123 }\end{array}$ & $\begin{array}{c}\text { Impact + Resuspension } \\
\text { l-RD-124 }\end{array}$ \\
\hline $8.57 \mathrm{E}-02$ & L/Resin & $1.00 \mathrm{E}-02$ & $9.00 \mathrm{E}-05$ & 2 & 8.57E-04 & $1.01 \mathrm{E}-04$ \\
\hline
\end{tabular}

RBOF Spent Deoinizer Resin Transport (RBOF)

\begin{tabular}{|c|c|c|c|c|c|c|}
\hline \multirow{2}{*}{$\begin{array}{l}\text { Pu-238 } \\
\text { caquiv } \\
\text { (Ci) }\end{array}$} & \multirow[t]{2}{*}{ Form* } & \multicolumn{2}{|r|}{ ARFS $\times$ RF } & \multirow{2}{*}{$\begin{array}{l}\text { time } \\
\text { (hrs) }\end{array}$} & \multicolumn{2}{|c|}{ Source Terms (Ci) } \\
\hline & & Fire & Impact + Resuspension & & $\begin{array}{c}\text { Fire } \\
\text { 1-RD-125 }\end{array}$ & $\begin{array}{c}\text { Impact }+ \text { Resuspension } \\
1-R D-126\end{array}$ \\
\hline $6.94 \mathrm{E}-01$ & L/Resin & $1.00 \mathrm{E}-02$ & $9.00 \mathrm{E}-05$ & 2 & $6.94 \mathrm{E}-03$ & 8.19E-04 \\
\hline
\end{tabular}

Disassembly Basin Deionizer \& Purif Resin Cask (Reactors)

\begin{tabular}{|c|c|c|c|c|c|c|}
\hline \multirow{2}{*}{$\begin{array}{l}\text { Pu-238 equiv } \\
\text { (Ci) }\end{array}$} & \multirow[t]{2}{*}{ Form* } & \multicolumn{2}{|r|}{ ARFS $\times$ RF } & \multirow{2}{*}{$\begin{array}{l}\text { time } \\
\text { (hrs) }\end{array}$} & \multicolumn{2}{|c|}{ Source Terms (Ci) } \\
\hline & & Fire & Impact + Resuspension & & $\begin{array}{c}\text { Fire } \\
1-R D-127\end{array}$ & $\begin{array}{c}\text { Impact + Resuspension } \\
1-R D-128\end{array}$ \\
\hline $5.51 \mathrm{E}-04$ & L/Resin & $1.00 \mathrm{E}-02$ & $9.00 \mathrm{E}-05$ & 2 & 5.51E-06 & $6.50 \mathrm{E}-07$ \\
\hline
\end{tabular}

Reactor Samples Container (Reactors to 771-D/F)

\begin{tabular}{|c|c|c|c|c|c|c|}
\hline \multirow{2}{*}{$\begin{array}{l}\text { Pu-238 oqutr } \\
\text { (Ci) }\end{array}$} & \multirow[t]{2}{*}{ Form } & \multicolumn{2}{|r|}{ ARFS $\times$ RF } & \multirow{2}{*}{$\begin{array}{l}\text { time } \\
\text { (hrs) }\end{array}$} & \multicolumn{2}{|c|}{ Source Terms (Ci) } \\
\hline & & Fire & Impact + Resuspension & & $\begin{array}{c}\text { Fire } \\
\text { 1-RD-129 }\end{array}$ & $\begin{array}{c}\text { Impact }+ \text { Resuspension } \\
1-R D-130\end{array}$ \\
\hline 5.51E-04 & Liquid & $2.00 \mathrm{E}-03$ & $2.00 \mathrm{E}-03$ & 2 & $1.10 \mathrm{E}-06$ & $1.20 \mathrm{E}-06$ \\
\hline
\end{tabular}

Sample Truck (Inter Area)

\begin{tabular}{|c|c|c|c|c|c|c|}
\hline \multirow{2}{*}{$\begin{array}{l}\text { Pu-238 } \\
\text { (Ci) }\end{array}$} & \multirow[t]{2}{*}{ Form } & \multicolumn{2}{|r|}{ ARFS $\times$ RF } & \multirow{2}{*}{$\begin{array}{l}\text { time } \\
\text { (hrs) }\end{array}$} & \multicolumn{2}{|c|}{ Source Terms (Ci) } \\
\hline & & Fire & Impact + Resuspension & & $\begin{array}{c}\text { Fire } \\
\text { 1-RD-131 }\end{array}$ & $\begin{array}{c}\text { Impact + Resuspension } \\
\text { 1-RD-132 }\end{array}$ \\
\hline $5.92 \mathrm{E}-02$ & Liquid & $2.00 \mathrm{E}-03$ & $2.00 \mathrm{E}-03$ & 2 & $1.18 \mathrm{E}-04$ & $1.29 \mathrm{E}-04$ \\
\hline
\end{tabular}

Moderator Transport in 55 Gallon Drums (100-400 Areas)

\begin{tabular}{|c|c|c|c|c|c|c|}
\hline \multirow{2}{*}{$\begin{array}{l}\text { Pu-238 } \\
\text { (Ci) }\end{array}$} & \multirow[t]{2}{*}{ Form } & \multicolumn{2}{|r|}{ ARFS $\times$ RF } & \multirow{2}{*}{$\begin{array}{l}\text { time } \\
\text { (hrs) }\end{array}$} & \multicolumn{2}{|c|}{ Source Terms (Ci) } \\
\hline & & Fire & Impact + Resuspension & & $\begin{array}{c}\text { Fire } \\
\text { 1-RD-133 }\end{array}$ & $\begin{array}{c}\text { Impact + Resuspension } \\
1-R D-134\end{array}$ \\
\hline $2.26 \mathrm{E}+00$ & Liquid & $2.00 \mathrm{E}-03$ & $2.00 \mathrm{E}-03$ & 2 & $4.52 \mathrm{E}-03$ & $4,93 \mathrm{E}-03$ \\
\hline
\end{tabular}


Title of Project EPHA for THM

Subject Source Terms and Release Designation for Radionuclides

Computer E. T. Ketusky
Reviewed by S.A. Henderson

Works Calculation 5

Sheet No. 19 of 20

SRTC 776A to F Tank Farm 5000 Gallon Truck (776A to Tank Farm)

\begin{tabular}{|c|c|c|c|c|c|c|}
\hline \multirow{2}{*}{$\begin{array}{l}\text { Pu-238 } \\
\text { (Ci) } \\
\text { (Ci) }\end{array}$} & \multirow[t]{2}{*}{ Form } & \multicolumn{2}{|r|}{ ARFS $\times$ RF } & \multirow{2}{*}{$\begin{array}{l}\text { time } \\
\text { (hrs) }\end{array}$} & \multicolumn{2}{|c|}{ Source Terms (Ci) } \\
\hline & & Fire & Impact + Resuspension & & $\begin{array}{c}\text { Fire } \\
\text { 1-RD-135 }\end{array}$ & $\begin{array}{c}\text { Impact + Resuspension } \\
\text { 1-RD-136 }\end{array}$ \\
\hline $6.91 \mathrm{IE}-02$ & Liquid & $2.00 \mathrm{E}-03$ & $2.00 \mathrm{E}-03$ & 2 & $1.38 \mathrm{E}-04$ & $1.51 \mathrm{E}-04$ \\
\hline
\end{tabular}

Contaminated Water Transport (100 Area to 211-F)

\begin{tabular}{|c|c|c|c|c|c|c|}
\hline \multirow{2}{*}{$\begin{array}{l}\text { Pu-238 } \text { oqulv } \\
\text { (Ci) }\end{array}$} & \multirow[t]{2}{*}{ Form } & \multicolumn{2}{|r|}{ ARFS $\times$ RF } & \multirow{2}{*}{$\begin{array}{l}\text { time } \\
\text { (hrs) }\end{array}$} & \multicolumn{2}{|c|}{ Source Terms (Ci) } \\
\hline & & Fire & Impact + Resuspension & & $\begin{array}{c}\text { Fire } \\
\text { 1-RD-137 }\end{array}$ & $\begin{array}{c}\text { Impact + Resuspension } \\
1-R D-138\end{array}$ \\
\hline $2.26 \mathrm{E}+00$ & Liquid & $2.00 \mathrm{E}-03$ & $9.00 \mathrm{E}-05$ & 2 & $4.52 \mathrm{E}-03$ & $4.93 \mathrm{E}-03$ \\
\hline
\end{tabular}

100 Area Sludge Transport (100 Area to 241-F)(2000 gallons)

\begin{tabular}{|c|c|c|c|c|c|c|}
\hline \multirow{2}{*}{$\begin{array}{l}\text { Pu-238 equtv } \\
\text { (Ci) }\end{array}$} & \multirow[t]{2}{*}{ Form* } & \multicolumn{2}{|r|}{ ARFS $\times$ RF } & \multirow{2}{*}{$\begin{array}{l}\text { time } \\
\text { (hrs) }\end{array}$} & \multicolumn{2}{|c|}{ Source Terms (Ci) } \\
\hline & & Fire & Impact + Resuspersion & & $\begin{array}{c}\text { Fire } \\
\text { 1-RD-139 }\end{array}$ & $\begin{array}{c}\text { Impact + Resuspension } \\
\text { 1-RD-140 }\end{array}$ \\
\hline $6.52 \mathrm{E}-04$ & L/Sludge & $2.00 \mathrm{E}-03$ & $9.00 \mathrm{E}-05$ & 2 & $1.30 \mathrm{E}-06$ & $3.78 \mathrm{E}-07$ \\
\hline
\end{tabular}


Title of Project EPHA for THM

Reviewed by $\underline{\text { S.A. Henderson }}$

Subject Source Terms and Release Designation for Radionuclides

Works Calculation 5

Computer E. T. Ketusky

Date 09/03/96

Sheet No. 20 of 20

\section{REFERENCES}

1. DOE Handbook Airborne Release Fractions/Rates and Respirable Fractions for Nonreactor Nuclear Facilities, DOE-HDBK-3010-94, U.S. Department of Energy, October 1994.

2. Internal.Dose Conversion Factors for Calculation of Dose to the Public, DOE/EH-0071, U.S. Department of Energy, Washington, DC, July 1988. 
Title of Project EPHA for THM

Subject Radiological Consequence Assessment

Computer D. J. Richards

Date $\underline{09 / 11 / 96}$

Reviewed by $\underline{S}$. A. Henderson

Works Calculation 6

Sheet No. 1 of 166

\section{INTRODUCTION}

The purpose of this calculation is to determine the downwind consequences of radiological releases that may result from transportation accidents at the Savannah River Site. This calculation utilizes source terms for postulated scenarios involving both fires and impact/re-suspension events. The scenarios include numerous types of packaging and material configurations.

\section{ASSUMPTIONS}

- All source terms have been normalized to a single isotope equivalent, as appropriate.

- A release fraction appropriate to the applicable release mechanism scenario has been applied to all source terms. Accordingly, a release fraction of 1 is utilized as input to the computer dispersion model.

\section{ANALYTICAL METHODS AND CALCULATIONS}

The HOTSPOT computer code is used for all radionuclide dispersion modeling. Dispersion modeling is performed for each scenario identified. Dispersion modeling parameters utilized in this calculation are as follows.

\section{HOTSPOT Settings}

- Complex Geometry

- Wind Height - 2 meters (ground)

- Sample Time - 30 minutes for fires, 10 minutes for impact/re-suspension events

- Source Term Geometry: Area

- Release Elevation - Surface Release

- Release horizontal dimension:3m

- Release vertical dimension: $3 \mathrm{~m}$

Distances to receptor locations

- 30 meters from the release

- 100 meters from the release

- Distance to the 1 rem CEDE point 
ENGINEERING COMPUTATION SHEET

Title of Project EPHA for THM

Subject Radiological Consequence Assessment

Computer D. J. Richards

Date $\underline{09 / 11 / 96}$
Reviewed by S. A. Henderson

Works Calculation 6

Sheet No. 2 of 166

\section{Meteorology}

95\% Adverse Dispersion Conditions - all receptor locations

- Stability Class E

- $1.7 \mathrm{~m} / \mathrm{s}$ wind speed

- Inversion Layer: 200 meters

- $50 \%$ Average Dispersion Conditions - all receptor locations

- Stability Class C

- $2.5 \mathrm{~m} / \mathrm{s}$ wind speed

- Inversion Layer: 500 meters for Pu-238, 200 meters for $\mathrm{H}-3$

\section{RESULTS}

Table $195 \%$ Adverse met Consequence Assessment Results - Radiological

\begin{tabular}{|c|c|c|c|}
\hline $\begin{array}{c}\text { Release } \\
\text { Designation }\end{array}$ & $\begin{array}{l}\text { Total Dose @ } \\
30 \text { meters } \\
\text { (rem) }\end{array}$ & $\begin{array}{l}\text { Total Dose @ } \\
100 \text { meters } \\
\text { (rem) }\end{array}$ & $\begin{array}{c}\text { Maximum Distance } \\
\text { to PAC } \\
\text { (meters) }\end{array}$ \\
\hline 1-RD-67 & $1.40 \mathrm{E}+01$ & $2.40 \mathrm{E}+00$ & $6.00 \mathrm{E}+02$ \\
\hline 1-RD-68 & $2.50 \mathrm{E}+04$ & $4.20 E+03$ & $1.09 \mathrm{E}+04$ \\
\hline 1-RD-69 & $8.00 \mathrm{E}-02$ & $1.20 \mathrm{E}-02$ & $\mathrm{~N} / \mathrm{A}$ \\
\hline $1-R D-70$ & $1.80 \mathrm{E}-01$ & $3.10 \mathrm{E}-02$ & N/A \\
\hline 1-RD-71 & $1.90 \mathrm{E}-03$ & $4.00 \mathrm{E}-04$ & N/A \\
\hline 1-RD-72 & $5.70 \mathrm{E}-03$ & $1.20 \mathrm{E}-03$ & N/A \\
\hline 1-RD-73 & $7.00 \mathrm{E}-02$ & $1.20 \mathrm{E}-03$ & N/A \\
\hline 1-RD-74 & $2.10 \mathrm{E}-02$ & $3.50 \mathrm{E}-03$ & N/A \\
\hline 1-RD-75 & $4.70 \mathrm{E}+00$ & $1.00 \mathrm{E}+00$ & $1.00 \mathrm{E}+02$ \\
\hline 1-RD-76 & $1.40 \mathrm{E}+01$ & $2.40 \mathrm{E}+00$ & $1.60 \mathrm{E}+02$ \\
\hline 1-RD-77 & $8.20 \mathrm{E}-01$ & $1.40 \mathrm{E}-01$ & N/A \\
\hline 1-RD-78 & $2.20 \mathrm{E}+00$ & $3.70 \mathrm{E}-01$ & $5.00 \mathrm{E}+01$ \\
\hline 1-RD-79 & $2.50 \mathrm{E}+00$ & $4.20 \mathrm{E}-01$ & $6.00 \mathrm{E}+01$ \\
\hline 1-RD-80 & $6.70 \mathrm{E}+00$ & $1.10 \mathrm{E}+00$ & $1.10 \mathrm{E}+02$ \\
\hline 1-RD-81 & $2.60 \mathrm{E}+01$ & $4.50 \mathrm{E}+\infty 0$ & $2.30 \mathrm{E}+02$ \\
\hline 1-RD-82 & $7.10 \mathrm{E}+01$ & $1.20 \mathrm{E}+01$ & $3.80 \mathrm{E}+02$ \\
\hline 1-RD-83 & $3.10 \mathrm{E}+00$ & $5.20 \mathrm{E}-01$ & $7.00 \mathrm{E}+01$ \\
\hline 1-RD-84 & $8.20 \mathrm{E}+00$ & $1.40 \mathrm{E}+00$ & $1.20 \mathrm{E}+02$ \\
\hline 1-RD-85 & $7.10 \mathrm{E}-07$ & $1.50 \mathrm{E}-07$ & N/A \\
\hline $1-\mathrm{RD}-86$ & $1.96 \mathrm{E}-06$ & $3.90 \mathrm{E}-07$ & N/A \\
\hline 1-RD-87 & $6.00 \mathrm{E}-07$ & $1.20 \mathrm{E}-07$ & N/A \\
\hline 1-RD-88 & $1.60 \mathrm{E}-06$ & $3.30 \mathrm{E}-07$ & N/A \\
\hline 1-RD-89 & $1.80 \mathrm{E}+02$ & $3.00 \mathrm{E}+01$ & $6.30 \mathrm{E}+02$ \\
\hline 1-RD-90 & $7.70 \mathrm{E}+03$ & $1.30 \mathrm{E}+03$ & $5.16 \mathrm{E}+03$ \\
\hline 1-RD-91 & $1.00 \mathrm{E}+02$ & $1.70 \mathrm{E}+01$ & $4.60 \mathrm{E}+02$ \\
\hline 1-RD-92 & $1.40 \mathrm{E}+02$ & $2.30 \mathrm{E}+01$ & $5.40 \mathrm{E}+02$ \\
\hline 1-RD-93 & $1.50 \mathrm{E}-04$ & $2.50 \mathrm{E}-05$ & $\mathrm{~N} / \mathrm{A}$ \\
\hline l-RD-94 & $2.00 \mathrm{E}-04$ & - $3.40 \mathrm{E}-05$ & N/A \\
\hline 1-RD-95 & $4.90 \mathrm{E}-03$ & $8.30 \mathrm{E}-04$ & N/A \\
\hline 1-RD-96 & $6.60 \mathrm{E}-03$ & $1.10 \mathrm{E}-03$ & N/A \\
\hline l-RD-97 & $7.80 \mathrm{E}-01$ & $1.30 \mathrm{E}-01$ & N/A \\
\hline 1-RD-98 & $1.10 \mathrm{E}+00$ & $1.8 \mathrm{E}-01$ & $3.00 \mathrm{E}+01$ \\
\hline
\end{tabular}


Title of Project EPHA for THM

Subject Radiological Consequence Assessment

Computer D. J. Richards

Date $\underline{09 / 11 / 96}$

Reviewed by $\underline{\text { S. A. Henderson }}$

Works Calculation 6

Sheet No. 3 of 166

Table 1 cont. 95\% Adverse met Consequence Assessment Results - Radiological

\begin{tabular}{|c|c|c|c|}
\hline $\begin{array}{c}\text { Release } \\
\text { Designation }\end{array}$ & $\begin{array}{l}\text { Total Dose @ } \\
30 \text { meters } \\
\text { (rem) }\end{array}$ & $\begin{array}{l}\text { Total Dose @ } \\
100 \text { meters } \\
\text { (rem) }\end{array}$ & $\begin{array}{c}\text { Maximum Distance } \\
\text { to PAC } \\
\text { (meters) }\end{array}$ \\
\hline 1-RD-99 & $1.50 \mathrm{E}-01$ & $2.50 \mathrm{E}-02$ & N/A \\
\hline 1-RD-100 & $2.00 \mathrm{E}-01$ & $3.40 \mathrm{E}-02$ & N/A \\
\hline 1-RD-101 & $3.10 \mathrm{E}+01$ & $5.30 \mathrm{E}+00$ & $2.50 \mathrm{E}+02$ \\
\hline 1-RD-102 & $4.20 \mathrm{E}+01$ & $7.20 E+00$ & $2.90 \mathrm{E}+02$ \\
\hline 1-RD-103 & $5.50 \mathrm{E}+00$ & $9.50 \mathrm{E}-01$ & $9.00 \mathrm{E}+01$ \\
\hline 1-RD-104 & $7.50 \mathrm{E}+00$ & $1.30 \mathrm{E}+00$ & $1.10 \mathrm{E}+02$ \\
\hline 1-RD-105 & $5.50 \mathrm{E}+00$ & $9.50 \mathrm{E}-01$ & $9.00 \mathrm{E}+01$ \\
\hline 1-RD-106 & $7.50 \mathrm{E}+00$ & $1.30 \mathrm{E}+00$ & $1.10 \mathrm{E}+02$ \\
\hline I-RD-107 & $1.20 \mathrm{E}+03$ & $2.10 \mathrm{E}+02$ & $1.74 \mathrm{E}+03$ \\
\hline 1-RD-108 & $4.40 \mathrm{E}+01$ & $7.60 \mathrm{E}+01$ & $3.00 \mathrm{E} \div 02$ \\
\hline 1-RD-109 & $4.40 \mathrm{E}+00$ & $7.60 \mathrm{E}-01$ & $8.00 \mathrm{E}+01$ \\
\hline 1-RD-110 & $6.10 \mathrm{E}+00$ & $1.00 \mathrm{E}+00$ & $1.00 \mathrm{E}+02$ \\
\hline 1-RD-111 & $7.40 \mathrm{E}+03$ & $1.30 \mathrm{E}+03$ & $5.00 \mathrm{E}+03$ \\
\hline 1-RD-112 & $2.50 \mathrm{E}+03$ & $4.20 \mathrm{E}+02$ & $2.70 \mathrm{E}+03$ \\
\hline 1-RD-113 & $3.60 \mathrm{E}+01$ & $6.10 \mathrm{E}+00$ & $2.70 \mathrm{E}+02$ \\
\hline 1-RD-114 & $1.30 \mathrm{E}+02$ & $2.30 \mathrm{E}+01$ & $5.40 \mathrm{E}+02$ \\
\hline 1-RD-115 & $1.50 \mathrm{E}+02$ & $2.50 \mathrm{E}+01$ & $5.70 \mathrm{E}+02$ \\
\hline 1-RD-116 & $3.30 \mathrm{E}+01$ & $5.70 \mathrm{E}+00$ & $2.60 \mathrm{E}+02$ \\
\hline 1-RD-117 & $5.50 \mathrm{E}-03$ & $9.30 \mathrm{E}-04$ & N/A \\
\hline 1-RD-118 & $7.40 \mathrm{E}-03$ & $1.30 \mathrm{E}-03$ & N/A \\
\hline 1-RD-119 & $2.6 \mathrm{E}+02$ & $4.4 \mathrm{E}+01$ & $7.70 \mathrm{E}+02$ \\
\hline $1-R D-120$ & $3.2 \mathrm{E}+02$ & $5.5 E+01$ & $8.60 \mathrm{E}+02$ \\
\hline $1-R D-121$ & $2.70 E+03$ & $4.50 \mathrm{E}+02$ & $2.80 \mathrm{E}+03$ \\
\hline 1-RD-122 & $6.00 \mathrm{E}+01$ & $1.00 \mathrm{E}+01$ & $3.60 \mathrm{E}+02$ \\
\hline 1-RD-123 & $2.80 \mathrm{E}+00$ & $4.70 \mathrm{E}-01$ & $6.00 \mathrm{E}+01$ \\
\hline 1-RD-124 & $4.10 \mathrm{E}-01$ & $7.00 \mathrm{E}-02$ & N/A \\
\hline 1-RD-125 & $2.30 \mathrm{E}+01$ & $3.80 \mathrm{E}+00$ & $2.10 \mathrm{E}+02$ \\
\hline 1-RD-126 & $3.30 \mathrm{E}+00$ & $5.60 \mathrm{E}-01$ & $7.00 \mathrm{E}+01$ \\
\hline 1-RD-127 & $1.80 \mathrm{E}-02$ & $3.00 \mathrm{E}-03$ & N/A \\
\hline 1-RD-128 & $2.60 \mathrm{E}-03$ & $4.50 \mathrm{E}-04$ & N/A \\
\hline 1-RD-129 & $3.60 \mathrm{E}-03$ & $6.10 \mathrm{E}-04$ & N/A \\
\hline 1-RD-130 & $4.90 \mathrm{E}-03$ & $8.30 \mathrm{E}-04$ & N/A \\
\hline 1-RD-131 & $3.80 \mathrm{E}-01$ & $6.50 \mathrm{E}-02$ & N/A \\
\hline 1-RD-132 & $5.20 \mathrm{E}-01$ & $8.90 \mathrm{E}-02$ & N/A \\
\hline l-RD-133 & $1.50 \mathrm{E}+01$ & $2.50 \mathrm{E}+00$ & $1.60 \mathrm{E}+02$ \\
\hline 1-RD-134 & $2.00 \mathrm{E}+01$ & $3.40 \mathrm{E}+00$ & $1.90 \mathrm{E}+02$ \\
\hline 1-RD-135 & $4.50 \mathrm{E}-01$ & $7.60 \mathrm{E}-02$ & N/A \\
\hline 1-RD-136 & $6.10 \mathrm{E}-01$ & $1.00 \mathrm{E}-01$ & N/A \\
\hline 1-RD-137 & $1.50 \mathrm{E}+01$ & $2.50 \mathrm{E}+00$ & $1.60 \mathrm{E}+02$ \\
\hline 1-RD-138 & $2.10 \mathrm{E}+01$ & $3.40 \mathrm{E}+00$ & $1.90 \mathrm{E}+02$ \\
\hline 1-RD-139 & $4.20 \mathrm{E}-03$ & $7.20 \mathrm{E}-04$ & N/A \\
\hline $1-R D-140$ & $1.50 \mathrm{E}-03$ & $2.60 \mathrm{E}-04$ & N/A \\
\hline
\end{tabular}


ENGINEERING COMPUTATION SHEET

Title of Project EPHA for THM

Subject Radiological Consequence Assessment

Computer D. J. Richards

Date $\underline{09 / 11 / 96}$
Reviewed by S. A. Henderson

Works Calculation 6

Sheet No. 4 of 166

Table $250 \%$ Average met Consequence Assessment Results - Radiological

\begin{tabular}{|c|c|c|c|}
\hline $\begin{array}{c}\text { Release } \\
\text { Designation }\end{array}$ & $\begin{array}{c}\text { Total Dose @ } \\
30 \text { meters } \\
\text { (rem) }\end{array}$ & $\begin{array}{c}\text { Total Dose @ } \\
100 \text { meters } \\
\text { (rem) }\end{array}$ & $\begin{array}{c}\begin{array}{c}\text { Maximum Distance } \\
\text { to PAC } \\
\text { (meters) }\end{array} \\
\end{array}$ \\
\hline 1-RD-67 & $1.40 \mathrm{E}+00$ & $1.00 \mathrm{E}-01$ & $1.00 \mathrm{E}+02$ \\
\hline 1-RD-68 & $7.23 E+03$ & $9.90 \mathrm{E}+02$ & $3.98 \mathrm{E}+03$ \\
\hline 1-RD-69 & $2.00 \mathrm{E}-02$ & $2.70 \mathrm{E}-03$ & $\mathrm{~N} / \mathrm{A}$ \\
\hline 1-RD-70 & $5.40 \mathrm{E}-02$ & $7.73 \mathrm{E}-03$ & $\mathrm{~N} / \mathrm{A}$ \\
\hline 1-RD-71 & $5.00 \mathrm{E}-04$ & $7.20 \mathrm{E}-05$ & N/A \\
\hline 1-RD-72 & $1.50 \mathrm{E}-03$ & $2.10 \mathrm{E}-04$ & N/A \\
\hline I-RD-73 & $2.00 \mathrm{E}-03$ & $2.80 \mathrm{E}-04$ & N/A \\
\hline 1-RD-74 & $6.00 \mathrm{E}-03$ & $8.20 \mathrm{E}-04$ & N/A \\
\hline 1-RD-75 & $1.40 E+00$ & $1.00 \mathrm{E}+00$ & $1.00 \mathrm{E}+03$ \\
\hline 1-RD-76 & $4.10 \mathrm{E}+00$ & $1.00 \mathrm{E}+00$ & $1.00 \mathrm{E}+02$ \\
\hline 1-RD-77 & $2.40 \mathrm{E}-01$ & 3.30E-02 & N/A \\
\hline 1-RD-78 & $6.40 \mathrm{E}-01$ & $8.80 \mathrm{E}-02$ & N/A \\
\hline I-RD-79 & $7.20 \mathrm{E}-01$ & $9.90 \mathrm{E}-02$ & N/A \\
\hline 1-RD-80 & $2.00 \mathrm{E}+00$ & $1.00 \mathrm{E}+00$ & $1.00 \mathrm{E}+02$ \\
\hline - 1-RD-81 & $7.70 \mathrm{E}+00$ & $1.10 \mathrm{E}+00$ & $1.00 \mathrm{E}+02$ \\
\hline 1-RD-82 & $2.10 \mathrm{E}+01$ & $2.80 \mathrm{E}+01$ & $1.70 \mathrm{E}+02$ \\
\hline 1-RD-83 & $9.00 \mathrm{E}-01$ & $1.20 \mathrm{E}-01$ & N/A \\
\hline 1-RD-84 & $2.40 \mathrm{E}+00$ & $3.30 \mathrm{E}-01$ & $5.00 \mathrm{E}+1$ \\
\hline 1-RD-85 & $1.80 \mathrm{E}-07$ & $2.60 \mathrm{E}-08$ & N/A \\
\hline l-RD-86 & $5.00 \mathrm{E}-07$ & $7.10 \mathrm{E}-08$ & N/A \\
\hline 1-RD-87 & $1.60 \mathrm{E}-07$ & $2.20 \mathrm{E}-08$ & N/A \\
\hline 1-RD-88 & $4.20 \mathrm{E}-07$ & $6.00 \mathrm{E}-08$ & N/A \\
\hline 1-RD-89 & $5.20 \mathrm{E}+01$ & $7.10 \mathrm{E}+00$ & $2.80 \mathrm{E}+02$ \\
\hline 1-RD-90 & $2.30 \mathrm{E}+03$ & $3.10 \mathrm{E}+02$ & $2.05 \mathrm{E}+03$ \\
\hline 1-RD-91 & $2.90 \mathrm{E}+01$ & $4.00 \mathrm{E}+00$ & $2.10 \mathrm{E}+02$ \\
\hline 1-RD-92 & $4.00 \mathrm{E}+01$ & $5.40 \mathrm{E}+00$ & $2.50 \mathrm{E}+02$ \\
\hline 1-RD-93 & $4.30 \mathrm{E}-05$ & $5.90 \mathrm{E}-06$ & N/A \\
\hline 1-RD-94 & $5.80 \mathrm{E}-05$ & $7.90 \mathrm{E}-06$ & N/A \\
\hline 1-RD-95 & $1.40 \mathrm{E}-03$ & $1.90 \mathrm{E}-04$ & N/A \\
\hline 1-RD-96 & $1.90 \mathrm{E}-03$ & $2.70 \mathrm{E}-04$ & N/A \\
\hline 1-RD-97 & $2.30 \mathrm{E}-01$ & $3.10 \mathrm{E}-02$ & N/A \\
\hline 1-RD-98 & $3.10 \mathrm{E}-01$ & $4.20 \mathrm{E}-02$ & N/A \\
\hline I-RD-99 & $4.20 \mathrm{E}-02$ & $5.80 \mathrm{E}-03$ & N/A \\
\hline
\end{tabular}




\section{ENGINEERING COMPUTATION SHEET}

Title of Project EPHA for THM

Subject Radiological Consequence Assessment

Computer D. J. Richards
Reviewed by S. A. Henderson

Works Calculation 6

Date $\underline{09 / 11 / 96}$

Sheet No. 5 of 166

Table2 cont. 50\% Average met Consequence Assessment Results - Radiological

\begin{tabular}{|c|c|c|c|}
\hline $\begin{array}{c}\text { Release } \\
\text { Designation }\end{array}$ & $\begin{array}{c}\text { Total Dose @ } \\
30 \text { meters } \\
\text { (rem) }\end{array}$ & $\begin{array}{c}\text { Total Dose @ } \\
100 \text { meters } \\
\text { (rem) }\end{array}$ & \begin{tabular}{|c|}
$\begin{array}{c}\text { Maximum Distance } \\
\text { to PAC } \\
\text { (meters) }\end{array}$ \\
\end{tabular} \\
\hline 1-RD-100 & $5.80 \mathrm{E}-02$ & $7.90 \mathrm{E}-03$ & N/A \\
\hline l-RD-101 & $9.00 \mathrm{E}+00$ & $1.20 \mathrm{E}+00$ & $1.10 \mathrm{E}+02$ \\
\hline 1-RD-102 & $1.20 \mathrm{E}+01$ & $1.70 \mathrm{E}+00$ & $1.30 \mathrm{E}+02$ \\
\hline 1-RD-103 & $1.60 \mathrm{E}+00$ & $1.00 \mathrm{E}+00$ & $1.00 \mathrm{E}+02$ \\
\hline 1-RD-104 & $2.20 \mathrm{E}+00$ & $1.00 \mathrm{E}+00$ & $1.00 \mathrm{E}+02$ \\
\hline 1-RD-105 & $1.60 \mathrm{E}+00$ & $1.00 \mathrm{E}+00$ & $1.00 \mathrm{E}+02$ \\
\hline 1-RD-106 & $2.20 \mathrm{E}+00$ & $1.00 \mathrm{E}+00$ & $1.00 \mathrm{E}+02$ \\
\hline 1-RD-107 & $3.50 \mathrm{E}+02$ & $4.80 \mathrm{E}+01$ & $7.60 \mathrm{E}+02$ \\
\hline 1-RD-108 & $1.30 \mathrm{E}+01$ & $1.80 \mathrm{E}+00$ & $1.40 \mathrm{E}+02$ \\
\hline 1-RD-109 & $1.30 \mathrm{E}+00$ & $1.00 \mathrm{E}+00$ & $1.00 \mathrm{E}+02$ \\
\hline 1-RD-110 & $1.80 \mathrm{E}+00$ & $1.00 \mathrm{E}+00$ & $1.00 \mathrm{E}+02$ \\
\hline 1-RD-111 & $2.20 E+03$ & $2.90 \mathrm{E}+02$ & $1.99 \mathrm{E}+03$ \\
\hline 1-RD-112 & $7.20 \mathrm{E}+02$ & $9.80 \mathrm{E}+01$ & $1.10 \mathrm{E}+03$ \\
\hline 1-RD-113 & $1.00 \mathrm{E}+01$ & $1.40 \mathrm{E}+00$ & $1.20 \mathrm{E}+02$ \\
\hline 1-RD-114 & $3.90 \mathrm{E}+01$ & $5.40 \mathrm{E}+00$ & $2.40 \mathrm{E}+02$ \\
\hline 1-RD-115 & $4.30 \mathrm{E}+01$ & $5.90 \mathrm{E}+00$ & $2.50 \mathrm{E}+02$ \\
\hline $1-R D-116$ & $9.70 \mathrm{E}+00$ & $1.30 \mathrm{E}+00$ & $1.10 \mathrm{E}+02$ \\
\hline 1-RD-117 & $1.60 \mathrm{E}-03$ & $2.20 \mathrm{E}-04$ & $\mathrm{~N} / \mathrm{A}$ \\
\hline 1-RD-118 & $2.20 \mathrm{E}-03$ & $3.00 \mathrm{E}-04$ & N/A \\
\hline 1-RD-119 & $7.6 \mathrm{E}+01$ & $1.0 \mathrm{E}+01$ & $3.40 \mathrm{E}+02$ \\
\hline 1-RD-120 & $9.5 \mathrm{E}+01$ & $1.3 \mathrm{E}+01$ & $3.80 \mathrm{E}+02$ \\
\hline 1-RD-121 & $7.80 \mathrm{E}+02$ & $1.10 \mathrm{E}+02$ & $1.15 \mathrm{E}+03$ \\
\hline 1-RD-122 & $1.80 \mathrm{E} \div 01$ & $2.40 \mathrm{E}+00$ & $1.60 \mathrm{E}+02$ \\
\hline 1-RD-123 & $8.10 \mathrm{E}-01$ & $1.10 \mathrm{E}-01$ & N/A \\
\hline 1-RD-124 & $1.20 \mathrm{E}-01$ & $1.60 \mathrm{E}-02$ & N/A \\
\hline 1-RD-125 & $6.60 \mathrm{E}+00$ & $1.00 \mathrm{E}+00$ & $1.00 \mathrm{E}+02$ \\
\hline 1-RD-126 & $9.70 \mathrm{E}-01$ & $1.30 \mathrm{E}-01$ & N/A \\
\hline 1-RD-127 & $5.20 \mathrm{E}-03$ & $7.20 \mathrm{E}-04$ & N/A \\
\hline 1-RD-128 & $7.70 \mathrm{E}-04$ & $1.10 \mathrm{E}-04$ & N/A \\
\hline 1-RD-129 & $1.00 \mathrm{E}-03$ & $1.40 \mathrm{E}-04$ & N/A \\
\hline 1-RD-130 & $1.40 \mathrm{E}-03$ & $1.90 \mathrm{E}-04$ & N/A \\
\hline 1-RD-131 & $1.10 \mathrm{E}-01$ & $1.50 \mathrm{E}-02$ & N/A \\
\hline 1-RD-132 & $1.50 \mathrm{E}-01$ & $2.10 \mathrm{E}-02$ & N/A \\
\hline 1-RD-133 & $4.30 \mathrm{E}+00$ & $1.00 \mathrm{E}+00$ & $1.00 \mathrm{E}+02$ \\
\hline 1-RD-134 & $5.80 \mathrm{E}+00$ & $1.00 \mathrm{E}+00$ & $1.00 \mathrm{E}+02$ \\
\hline 1-RD-135 & $1.30 \mathrm{E}-01$ & $1.80 \mathrm{E}-02$ & N/A \\
\hline 1-RD-136 & $1.80 \mathrm{E}-01$ & $2.40 \mathrm{E}-02$ & N/A \\
\hline 1-RD-137 & $4.30 \mathrm{E}+00$ & $1.00 \mathrm{E}+00$ & $1.00 \mathrm{E}+02$ \\
\hline 1-RD-138 & $5.80 \mathrm{E}+00$ & $1.00 \mathrm{E}+00$ & $1.00 \mathrm{E}+02$ \\
\hline 1-RD-139 & $1.20 \mathrm{E}-03$ & $1.70 \mathrm{E}-04$ & N/A \\
\hline l-RD-140 & $4.50 \mathrm{E}-04$ & $6.10 \mathrm{E}-05$ & N/A \\
\hline
\end{tabular}


Title of Project EPHA for THM

Subject Radiological Consequence Assessment

Computer D.J. Richards
Date $\underline{09 / 11 / 96}$
Reviewed by $\underline{S}$. A. Henderson

Works Calculation 6

Sheet No. 6 of 166

\section{REFERENCES}

1. Hadlock, D.J., Consequence Assessment For Emergency Preparedness Hazards Assessments. TP-95-002, Rev. 2. Westinghouse Savannah River Company, Savannah River Site, Aiken, SC, November 9, 1995.

2. Hadlock, D.J., Emergency Preparedness Hazards Assessments. WSRC-RP-95-1001, Guideline 2.1.3, Rev. 0. Westinghouse Savannah River Company, Savannah River Site, Aiken, SC, November 30, 1995.

3. Hadlock, D.J., Emergency Preparedness Hazards Assessment Style Guide. ECS-EST-95-0018, Rev. 0. Westinghouse Savannah River Company, Savannah River Site, Aiken, SC, February 28, 1995.

4. Standards for Development and Maintenance of an Emergency Preparedness Hazards Assessment.6Q, Vol. II, EMPP 6Q-001, Rev. 1. Westinghouse Savannah River Company, Savannah River Site, Aiken, SC, November 1, 1995. 
Title of Project EPHA for THM

Subject Radiological Consequence Assessment

Computer D. J. Richards

Date $\underline{09 / 11 / 96}$

Reviewed by $\underline{\text { S.A. Henderson }}$

Works Calculation 6

Sheet No. 7 of 166

Release Designation: 1-RD-67, Adverse

HOTSPOT 7.0 GENERAL PLUME 9-10-1996 20:46

RADIONUCLIDE : PU-238 Inhalation Class : W

HALFLIFE : 87.740 years

SOURCE TERM : $3.4 \mathrm{E}-03 \mathrm{Ci}$

Source Term Width: $\quad 3 \mathrm{~m}$ Source Term Height: $3 \mathrm{~m}$

RELEASE FRACTION : $1.00 \mathrm{E}+00$

FILTER EFFICIENCY: $0.000000 \%$

EFFECTIVE RELEASE HEIGHT : $\quad 0.00 \mathrm{~m}$

WIND SPEED $(\mathrm{h}=2 \mathrm{~m}): 1.7 \mathrm{~m} / \mathrm{s}$

STABILITY CLASS :E DEPOSITION VELOCITY $: 1.00 \mathrm{~cm} / \mathrm{s}$

RECEPTOR HEIGHT $\quad: 0.0 \mathrm{~m}$ INVERSION LAYER HEIGHT $: 500.0 \mathrm{~m}$

SAMPLE TIME $\quad: 10.000 \mathrm{~min}$

MAXIMUM DOSE DISTANCE $:<0.10 \mathrm{~km}$ MAXIMUM CEDE $:>2.4 \mathrm{rem}$

$D=0.03 \mathrm{~km}$

$\mathrm{DEP}=9.1 \mathrm{E}-01 \mathrm{uCi} / \mathrm{m}^{\wedge} 2$

$\mathrm{CHI}=9.1 \mathrm{E}-05(\mathrm{Ci}-\mathrm{s}) / \mathrm{m}^{\wedge} 3$

50-YR DOSE COMMITMENT:

SURFACE BONE 2.4E+02 rem RED MARROW 2.0E+01 rem LIVER 5.4E $+01 \mathrm{rem}$ GONADS 3.0E+00 rem EFFECTIVE DOSE

EQUIVALENT 1.4E+01 rem
$\mathrm{D}=0.10 \mathrm{~km}$

$\mathrm{DEP}=1.5 \mathrm{E}-01 \mathrm{uCi} / \mathrm{m}^{\wedge} 2$

$\mathrm{CHI}=1.5 \mathrm{E}-05(\mathrm{Ci}-\mathrm{s}) / \mathrm{m}^{\wedge} 3$

50-YR DOSE COMMITMENT:

SURFACE BONE 4.2E+01 rem RED MARROW $3.4 \mathrm{E}+00 \mathrm{rem}$ LIVER $\quad 9.2 \mathrm{E}+00 \mathrm{rem}$ GONADS 5.1E-01 rem EFFECTIVE DOSE EQUIVALENT $\quad 2.4 \mathrm{E}+00 \mathrm{rem}$
$\mathrm{D}=0.16 \mathrm{~km}$

$\mathrm{DEP}=6.8 \mathrm{E}-02 \mathrm{uCi} / \mathrm{m}^{\wedge} 2$ $\mathrm{CHI}=6.8 \mathrm{E}-06(\mathrm{Ci}-\mathrm{s}) / \mathrm{m}^{\wedge} 3$ 50-YR DOSE COMMITMENT:

SURFACE BONE 1.8E+01 rem RED MARROW $1.5 \mathrm{E}+00 \mathrm{rem}$ LIVER 4.1E+00 rem GONADS 2.3E-01 rem EFFECTIVE DOSE EQUIVALENT $1.0 \mathrm{E}+00 \mathrm{rem}$ 
Title of Projèct EPHA for THM

Subject Radiological Consequence Assessment

Computer D. J. Richards

Release Designation: 1-RD-67, Average

HOTSPOT 7.0 GENERAL PLUME 9-09-1996 10:54

RADIONUCLIDE : PU-238 Inhalation Class : W

HALFLIFE : 87.740 years

SOURCE TERM : $3.4 \mathrm{E}-03 \mathrm{Ci}$

Source Term Width: $3 \mathrm{~m}$ Source Term Height: $\quad 3 \mathrm{~m}$

RELEASE FRACTION : $1.00 \mathrm{E}+00$

FILTER EFFICIENCY: $0.000000 \%$

EFFECTIVE RELEASE HEIGHT : $\quad 0.00 \mathrm{~m}$

WIND SPEED $(\mathrm{h}=2 \mathrm{~m}): 2.5 \mathrm{~m} / \mathrm{s}$

STABILITY CLASS :C DEPOSITION VELOCITY $: 1.00 \mathrm{~cm} / \mathrm{s}$

RECEPTOR HEIGHT $\quad: 0.0 \mathrm{~m}$ INVERSION LAYER HEIGHT $: 500.0 \mathrm{~m}$

SAMPLE TIME $\quad: 30.000 \mathrm{~min}$

MAXIMUM DOSE DISTANCE $:<0.10 \mathrm{~km}$ MAXIMUM CEDE $:>0.188 \mathrm{rem}$

$\mathrm{D}=0.03 \mathrm{~km}$

$\mathrm{DEP}=9.0 \mathrm{E}-02 \mathrm{uCi} / \mathrm{m}^{\wedge} 2$

$\mathrm{CHI}=9.0 \mathrm{E}-06(\mathrm{Ci}-\mathrm{s}) / \mathrm{m}^{\wedge} 3$

50-YR DOSE COMMITMENT:

SURFACE BONE $2.4 \mathrm{E}+01 \mathrm{rem}$ RED MARROW $2.0 \mathrm{E}+00 \mathrm{rem}$ LIVER $\quad 5.4 \mathrm{E}+00 \mathrm{rem}$ GONADS 3.0E-01 rem EFFECTIVE DOSE

EQUIVALENT $1.4 \mathrm{E}+00 \mathrm{rem}$
$\mathrm{D}=0.04 \mathrm{~km}$

$\mathrm{DEP}=6.8 \mathrm{E}-02 \mathrm{uCi} / \mathrm{m}^{\wedge} 2$

$\mathrm{CHI}=6.8 \mathrm{E}-06(\mathrm{Ci}-\mathrm{s}) / \mathrm{m}^{\wedge} 3$

50-YR DOSE COMMITMENT:

SURFACE BONE 1.8E+01 rem RED MARROW $1.5 \mathrm{E}+00 \mathrm{rem}$ LIVER 4.1E+00 rem GONADS 2.3E-01 rem EFFECTIVE DOSE

EQUIVALENT $\quad 1.0 \mathrm{E}+00 \mathrm{rem}$
$\mathrm{D}=0.10 \mathrm{~km}$

$\mathrm{DEP}=1.2 \mathrm{E}-02 \mathrm{uCi} / \mathrm{m}^{\wedge} 2$

$\mathrm{CHI}=1.2 \mathrm{E}-06(\mathrm{Ci}-\mathrm{s}) / \mathrm{m}^{\wedge} 3$

50-YR DOSE COMMITMENT:

SURFACE BONE $3.3 \mathrm{E}+00 \mathrm{rem}$ RED MARROW 2.7E-01 rem LIVER 7.4E-01 rem GONADS 4.1E-02 rem EFFECTIVE DOSE EQUIVALENT 1.9E-01 rem 
Title of Projèct EPHA for THM

Subject Radiological Consequence Assessment

Computer D. J. Richards

Date $\underline{09 / 11 / 96}$

Reviewed by $\underline{S . A . ~ H e n d e r s o n ~}$

Works Calculation 6

Sheet No. 9 of 166

Release Designation: 1-RD-68, Adverse

HOTSPOT 7.0 GENERAL PLUME 9-10-1996 20:35

RADIONUCLIDE : PU-238 Inhalation Class : W

HALFLIFE : 87.740 years

SOURCE TERM : $6.1 \mathrm{E}+00 \mathrm{Ci}$

Source Term Width: $\quad 3 \mathrm{~m}$ Source Term Height: $\quad 3 \mathrm{~m}$

RELEASE FRACTION : $1.00 \mathrm{E}+00$

FILTER EFFICIENCY: $0.000000 \%$

EFFECTIVE RELEASE HEIGHT : $\quad 0.00 \mathrm{~m}$

WIND SPEED ( $\mathrm{h}=2 \mathrm{~m}): 1.7 \mathrm{~m} / \mathrm{s}$

STABILITY CLASS :E DEPOSITION VELOCITY $: 1.00 \mathrm{~cm} / \mathrm{s}$

RECEPTOR HEIGHT $\quad: 0.0 \mathrm{~m}$ INVERSION LAYER HEIGHT $: 200.0 \mathrm{~m}$

SAMPLE TIME $\quad: 10.000 \mathrm{~min}$

MAXIMUM DOSE DISTANCE $:<0.10 \mathrm{~km}$ MAXIMUM CEDE $:>4.2 \mathrm{E}+03 \mathrm{rem}$

$\mathrm{D}=0.03 \mathrm{~km}$

$\mathrm{DEP}=1.6 \mathrm{E}+03 \mathrm{uCi} / \mathrm{m}^{\wedge} 2$

$\mathrm{CHI}=1.6 \mathrm{E}-01(\mathrm{Ci}-\mathrm{s}) / \mathrm{m}^{\wedge} 3$

50-YR DOSE COMMITMENT:

SURFACE BONE $4.3 \mathrm{E}+05 \mathrm{rem}$

RED MARROW $\quad 3.6 \mathrm{E}+04 \mathrm{rem}$

LIVER $\quad 9.6 \mathrm{E}+04 \mathrm{rem}$

GONADS 5.4E+03 rem

EFFECTIVE DOSE

EQUIVALENT 2.5E+04 rem
$\mathrm{D}=0.10 \mathrm{~km}$

$\mathrm{DEP}=2.7 \mathrm{E}+02 \mathrm{uCi} / \mathrm{m}^{\wedge} 2$

$\mathrm{CHI}=2.7 \mathrm{E}-02(\mathrm{Ci}-\mathrm{s}) / \mathrm{m}^{\wedge} 3$

50-YR DOSE COMMITMENT:

SURFACE BONE 7.4E+04 rem RED MARROW $6.1 \mathrm{E}+03 \mathrm{rem}$ LIVER $\quad 1.6 \mathrm{E}+04 \mathrm{rem}$ GONADS 9.1E+02 rem EFFECTIVE DOSE

EQUIVALENT $4.2 \mathrm{E}+03 \mathrm{rem}$
$\mathrm{D}=10.88 \mathrm{~km}$

$\mathrm{DEP}=6.8 \mathrm{E}-02 \mathrm{uCi} / \mathrm{m}^{\wedge} 2$ $\mathrm{CHI}=6.8 \mathrm{E}-06(\mathrm{Ci}-\mathrm{s}) / \mathrm{m}^{\wedge} 3$

50-YR DOSE COMMITMENT:

SURFACE BONE $1.8 \mathrm{E}+01 \mathrm{rem}$ RED MARROW $1.5 \mathrm{E}+00 \mathrm{rem}$ LIVER $\quad 4.1 \mathrm{E}+00 \mathrm{rem}$ GONADS 2.3E-01 rem EFFECTIVE DOSE EQUIVALENT $\quad 1.0 \mathrm{E}+00 \mathrm{rem}$ 
Title of Project EPHA for THM

Subject Radiological Consequence Assessment

Computer D. J. Richards

Date $\underline{09 / 11 / 96}$
Reviewed by $\underline{S}$. A. Henderson Works Calculation 6

Sheet No. 10 of 166

Release Designation: 1-RD-68, Average

HOTSPOT 7.0 GENERAL PLUME 9-10-1996 20:37

RADIONUCLIDE : PU-238 Inhalation Class : W

HALFLIFE $: 87.740$ years

SOURCE TERM $: 6.1 \mathrm{E}+00 \mathrm{Ci}$.

Source Term Width: $\quad 3 \mathrm{~m}$ Source Term Height: $3 \mathrm{~m}$

RELEASE FRACTION : $1.00 \mathrm{E}+00$

FILTER EFFICIENCY: $0.000000 \%$

EFFECTIVE RELEASE HEIGHT : $\quad 0.00 \mathrm{~m}$

WIND SPEED $(\mathrm{h}=2 \mathrm{~m}): 2.5 \mathrm{~m} / \mathrm{s}$

STABILITY CLASS :C DEPOSITION VELOCITY : $1.00 \mathrm{~cm} / \mathrm{s}$

RECEPTOR HEIGHT $\quad: 0.0 \mathrm{~m}$ INVERSION LAYER HEIGHT $: 500.0 \mathrm{~m}$

SAMPLE TIME $\quad: 10.000 \mathrm{~min}$

MAXIMUM DOSE DISTANCE $:<0.10 \mathrm{~km}$ MAXIMUM CEDE $:>9.9 \mathrm{E}+02 \mathrm{rem}$

$D=0.03 \mathrm{~km}$

$\mathrm{DEP}=4.7 \mathrm{E}+02 \mathrm{uCi} / \mathrm{m}^{\wedge} 2$

$\mathrm{CHI}=4.7 \mathrm{E}-02(\mathrm{Ci}-\mathrm{s}) / \mathrm{m}^{\wedge} 3$

50-YR DOSE COMMITMENT:

SURFACE BONE $1.3 \mathrm{E}+05 \mathrm{rem}$ RED MARROW $1.1 \mathrm{E}+04 \mathrm{rem}$ LIVER 2.8E+04 rem GONADS $1.6 \mathrm{E}+03 \mathrm{rem}$ EFFECTIVE DOSE

EQUIVALENT 7.2E+03 rem
$\mathrm{D}=0.10 \mathrm{~km}$

$\mathrm{DEP}=6.4 \mathrm{E}+01 \mathrm{uCi} / \mathrm{m}^{\wedge} 2$

$\mathrm{CHI}=6.4 \mathrm{E}-03(\mathrm{Ci}-\mathrm{s}) / \mathrm{m}^{\wedge} 3$

50-YR DOSE COMMITMENT:

SURFACE BONE 1.7E+04 rem RED MARROW $1.4 \mathrm{E}+03 \mathrm{rem}$

LIVER 3.9E+03 rem GONADS 2.1E+02 rem EFFECTIVE DOSE EQUIVALENT $9.9 \mathrm{E}+02 \mathrm{rem}$
$\mathrm{D}=3.98 \mathrm{~km}$

$\mathrm{DEP}=6.8 \mathrm{E}-02 \mathrm{uCi} / \mathrm{m}^{\wedge} 2$

$\mathrm{CHI}=6.8 \mathrm{E}-06(\mathrm{Ci}-\mathrm{s}) / \mathrm{m}^{\wedge} 3$

50-YR DOSE COMMITMENT:

SURFACE BONE 1.8E+01 rem RED MARROW $1.5 \mathrm{E}+00 \mathrm{rem}$ LIVER 4.1E+00 rem GONADS 2.3E-01 rem EFFECTIVE DOSE EQUTVALENT $1.0 \mathrm{E}+00 \mathrm{rem}$ 
Title of Project EPHA for THM

Subject Radiological Consequence Assessment

Computer D. J. Richards

Date $\underline{09 / 11 / 96}$

Reviewed by S. A. Henderson

Works Calculation 6

Sheet No. 11 of 166

Release Designation: 1-RD-69, Adverse

HOTSPOT 7.0 GENERAL PLUME 9-09-1996 11:16

RADIONUCLIDE : PU-238 Inhalation Class : W

HALFLIFE $: 87.740$ years

SOURCE TERM : $2.1 \mathrm{E}-05 \mathrm{Ci}$

Source Term Width: $3 \mathrm{~m}$ Source Term Height: $3 \mathrm{~m}$

RELEASE FRACTION : $1.00 \mathrm{E}+00$

FILTER EFFICIENCY: $0.000000 \%$

EFFECTTVE RELEASE HEIGHT : $\quad 0.00 \mathrm{~m}$

WIND SPEED $(\mathrm{h}=2 \mathrm{~m}): 1.7 \mathrm{~m} / \mathrm{s}$

STABILITY CLASS :E DEPOSITION VELOCITY $: 1.00 \mathrm{~cm} / \mathrm{s}$

RECEPTOR HEIGHT $: 0.0 \mathrm{~m}$ INVERSION LAYER HEIGHT $: 200.0 \mathrm{~m}$

SAMPLE TIME $: 30.000 \mathrm{~min}$

MAXIMUM DOSE DISTANCE $:<0.10 \mathrm{~km}$ MAXIMUM CEDE $:>0.012 \mathrm{rem}$

$\mathrm{D}=0.03 \mathrm{~km}$

$\mathrm{DEP}=4.4 \mathrm{E}-03 \mathrm{uCi} / \mathrm{m}^{\wedge} 2$

$\mathrm{CHI}=4.4 \mathrm{E}-07(\mathrm{Ci}-\mathrm{s}) / \mathrm{m}^{\wedge} 3$

50-YR DOSE COMMITMENT:

SURFACE BONE $1.2 \mathrm{E}+00 \mathrm{rem}$ RED MARROW 9.9E-02 rem

LIVER 2.7E-01 rem GONADS 1.5E-02 rem EFFECTIVE DOSE

EQUIVALENT 6.8E-02 rem
$\mathrm{D}=0.10 \mathrm{~km}$
$\mathrm{DEP}=7.6 \mathrm{E}-04 \mathrm{uCi} / \mathrm{m}^{\wedge} 2$
$\mathrm{CHI}=7.6 \mathrm{E}-08(\mathrm{Ci}-\mathrm{s}) / \mathrm{m}^{\wedge} 3$
50-YR DOSE COMMITMENT:
SURFACE BONE 2.0E-01 rem RED MARROW 1.7E-02 rem
LIVER 4.5E-02 rem
GONADS 2.5E-03 rem
EFFECTIVE DOSE
EQUTVALENT $1.2 \mathrm{E}-02 \mathrm{rem}$ 
Title of Project EPHA for THM

Subject Radiological Consequence Assessment

Computer D. J. Richards

Date $\underline{09 / 11 / 96}$
Reviewed by S. A. Henderson

Works Calculation 6

Sheet No. 12 of 166

Release Designation: 1-RD-69, Average

HOTSPOT 7.0 GENERAL PLUME • 9-09-1996 11:17

RADIONUCLIDE : PU-238 Inhalation Class : W

HALFLIFE : 87.740 years

SOURCE TERM : $2.1 \mathrm{E}-05 \mathrm{Ci}$

Source Term Width: $\quad 3 \mathrm{~m}$ Source Term Height: $3 \mathrm{~m}$

RELEASE FRACTION : $1.00 \mathrm{E}+00$

FILTER EFFICIENCY: $0.000000 \%$

EFFECTIVE RELEASE HEIGHT : $\quad 0.00 \mathrm{~m}$

WIND SPEED $(\mathrm{h}=2 \mathrm{~m}): 2.5 \mathrm{~m} / \mathrm{s}$

STABILITY CLASS :C DEPOSITION VELOCITY : $1.00 \mathrm{~cm} / \mathrm{s}$

RECEPTOR HEIGHT $\quad: 0.0 \mathrm{~m}$ INVERSION LAYER HEIGHT $: 500.0 \mathrm{~m}$

SAMPLE TIME $: 30.000 \mathrm{~min}$

MAXIMUM DOSE DISTANCE $:<0.10 \mathrm{~km}$ MAXIMUM CEDE $:>2.7 \mathrm{E}-03 \mathrm{rem}$.

$\mathrm{D}=0.03 \mathrm{~km}$

$\mathrm{DEP}=1.3 \mathrm{E}-03 \mathrm{uCi} / \mathrm{m}^{\wedge} 2$

$\mathrm{CHI}=1.3 \mathrm{E}-07(\mathrm{Ci}-\mathrm{s}) / \mathrm{m}^{\wedge} 3$

50-YR DOSE COMMITMENT:

SURFACE BONE 3.5E-01 rem RED MARROW 2.9E-02 rem LIVER 7.8E-02 rem GONADS 4.3E-03 rem EFFECTIVE DOSE

EQUTVALENT $2.0 \mathrm{E}-02 \mathrm{rem}$
$\mathrm{D}=0.10 \mathrm{~km}$

$\mathrm{DEP}=1.8 \mathrm{E}-04 \mathrm{uCi} / \mathrm{m}^{\wedge} 2$

$\mathrm{CHI}=1.8 \mathrm{E}-08(\mathrm{Ci}-\mathrm{s}) / \mathrm{m}^{\wedge} 3$

50-YR DOSE COMMITMENT:

SURFACE BONE 4.8E-02 rem RED MARROW 4.0E-03 rem

LIVER $1.1 \mathrm{E}-02$ rem GONADS 5.9E-04 rem EFFECTIVE DOSE

EQUIVALENT 2.7E-03 rem 
Title of Project EPHA for THM

Subject Radiological Consequence Assessment

Computer D. J. Richards
Reviewed by $\mathrm{S}$. A. Henderson

Works Calculation 6

Sheet No. 13 of 166

Release Designation: 1-RD-70, Adverse

HOTSPOT 7.0 GENERAL PLUME 9-10-1996 20:39

RADIONUCLIDE : PU-238 Inhalation Class : W

HALFLIFE $: 87.740$ years

SOURCE TERM : $4.5 \mathrm{E}-05 \mathrm{Ci}$

Source Term Width: $3 \mathrm{~m}$ Source Term Height: $3 \mathrm{~m}$

RELEASE FRACTION : $1.00 \mathrm{E}+00$

FILTER EFFICIENCY: $0.000000 \%$

EFFECTIVE RELEASE HEIGHT : $\quad 0.00 \mathrm{~m}$

WIND SPEED $(\mathrm{h}=2 \mathrm{~m}): 1.7 \mathrm{~m} / \mathrm{s}$

STABILITY CLASS :E DEPOSITION VELOCITY : $1.00 \mathrm{~cm} / \mathrm{s}$

RECEPTOR HEIGHT $: 0.0 \mathrm{~m}$ INVERSION LAYER HEIGHT $: 200.0 \mathrm{~m}$

SAMPLE TIME $\quad: 10.000 \mathrm{~min}$

MAXIMUM DOSE DISTANCE $:<0.10 \mathrm{~km}$ MAXIMUM CEDE $:>0.031 \mathrm{rem}$

$\mathrm{D}=0.03 \mathrm{~km}$

$\mathrm{DEP}=1.2 \mathrm{E}-02 \mathrm{uCi} / \mathrm{m}^{\wedge} 2$

$\mathrm{CHI}=1.2 \mathrm{E}-06(\mathrm{Ci}-\mathrm{s}) / \mathrm{m}^{\wedge} 3$

50-YR DOSE COMMITMENT:

SURFACE BONE 3.2E+00 rem RED MARROW 2.7E-01 rem

LIVER 7.2E-01 rem

GONADS 4.0E-02 rem

EFFECTIVE DOSE

EQUIVALENT $1.8 \mathrm{E}-01 \mathrm{rem}$
$\mathrm{D}=0.10 \mathrm{~km}$

$\mathrm{DEP}=2.0 \mathrm{E}-03 \mathrm{uCi} / \mathrm{m}^{\wedge} 2$

$\mathrm{CHI}=2.0 \mathrm{E}-07(\mathrm{Ci}-\mathrm{s}) / \mathrm{m}^{\wedge} 3$

50-YR DOSE COMMITMENT:

SURFACE BONE 5.5E-01 rem

RED MARROW $\quad 4.6 \mathrm{E}-02 \mathrm{rem}$

LIVER $\quad 1.2 \mathrm{E}-01 \mathrm{rem}$

GONADS 6.8E-03 rem

EFFECTIVE DOSE

EQUIVALENT $3.1 \mathrm{E}-02 \mathrm{rem}$ 
Title of Project EPHA for THM

Subject Radiological Consequence Assessment

Computer D. J. Richards
Date $\underline{09 / 11 / 96}$
Reviewed by $\underline{\text { S. A. Henderson }}$

Works Calculation 6

Sheet No. 14 of 166

Release Designation: 1-RD-70, Average

HOTSPOT 7.0 GENERAL PLUME 9-10-1996 20:39

RADIONUCLIDE : PU-238 Inhalation Class : W

HALFLIFE $: 87.740$ years

SOURCE TERM : $4.5 \mathrm{E}-05 \mathrm{Ci}$

Source Term Width: $3 \mathrm{~m}$ Source Term Height: $3 \mathrm{~m}$

RELEASE FRACTION : $1.00 \mathrm{E}+00$

FILTER EFFICIENCY: $0.000000 \%$

EFFECTIVE RELEASE HEIGHT : $\quad 0.00 \mathrm{~m}$

WIND SPEED $(\mathrm{h}=2 \mathrm{~m}): 2.5 \mathrm{~m} / \mathrm{s}$

STABILITY CLASS :C DEPOSITION VELOCITY : $1.00 \mathrm{~cm} / \mathrm{s}$

RECEPTOR HEIGHT $: 0.0 \mathrm{~m}$ INVERSION LAYER HEIGHT $: 500.0 \mathrm{~m}$

SAMPLE TIME $\quad: 10.000 \mathrm{~min}$

MAXIMUM DOSE DISTANCE $:<0.10 \mathrm{~km}$ MAXIMUM CEDE $:>7.3 \mathrm{E}-03 \mathrm{rem}$
$\mathrm{D}=0.03 \mathrm{~km}$
$\mathrm{DEP}=3.5 \mathrm{E}-03 \mathrm{uCi} / \mathrm{m}^{\wedge} 2$
$\mathrm{D}=0.10 \mathrm{~km}$
$\mathrm{CHI}=3.5 \mathrm{E}-07(\mathrm{Ci}-\mathrm{s}) / \mathrm{m}^{\wedge} 3$
$\mathrm{DEP}=4.8 \mathrm{E}-04 \mathrm{uCi} / \mathrm{m}^{\wedge} 2$
$\mathrm{CHI}=4.8 \mathrm{E}-08(\mathrm{Ci}-\mathrm{s}) / \mathrm{m}^{\wedge} 3$
50-YR DOSE COMMITMENT:
50-YR DOSE COMMITMENT:
SURFACE BONE 9.5E-01 rem
SURFACE BONE 1.3E-01 rem
RED MARROW 7.8E-02 rem
LIVER 2.1E-01 rem
GONADS 1.2E-02 rem
EFFECTIVE DOSE
EQUIVALENT 5.4E-02 rem
RED MARROW 1.1E-02 rem
LIVER 2.9E-02 rem
GONADS 1.6E-03 rem
EFFECTIVE DOSE
EQUTVALENT 7.3E-03 rem 
Title of Project EPHA for THM

Subject Radiological Consequence Assessment

Computer D. J. Richards

Date $\underline{09 / 11 / 96}$
Reviewed by S. A. Henderson Works Calculation 6

Sheet No. 15 of 166

Release Designation: 1-RD-71, Adverse

HOTSPOT 7.0 TRITIUM RELEASE 9-09-1996 12:49

TOTAL TRITIUM RELEASE : $2.5 \mathrm{E}+00$ curies $100.000 \%$ TRITIUM OXIDE

Dose Results Include Skin Absorption

Source Term Width: $3 \mathrm{~m}$ Source Term Height: $3 \mathrm{~m}$

EFFECTIVE RELEASE HEIGHT : $\quad 0.00 \mathrm{~m}$

WIND SPEED $(\mathrm{h}=2 \mathrm{~m}): 1.7 \mathrm{~m} / \mathrm{s}$

STABILITY CLASS :E DEPOSITION VELOCITY $: 0.0 \mathrm{E}+00 \mathrm{~cm} / \mathrm{s}$

RECEPTOR HEIGHT $: 0.0 \mathrm{~m}$ INVERSION LAYER HEIGHT $: 200.0 \mathrm{~m}$

SAMPLE TIME $: 30.000 \mathrm{~min}$

MAXIMUM DOSE DISTANCE $:<0.10 \mathrm{~km}$ MAXIMUM CEDE $:>4.0 \mathrm{E}-04 \mathrm{rem}$

$\mathrm{D}=0.03 \mathrm{~km}$

$\mathrm{CHI}=6.3 \mathrm{E}-02(\mathrm{Ci}-\mathrm{s}) / \mathrm{m}^{\wedge} 3$

50-YR DOSE COMMITMENT:

EFFECTIVE DOSE

EQUIVALENT 1.9E-03 rem
$\mathrm{D}=0.10 \mathrm{~km}$

$\mathrm{CHI}=1.3 \mathrm{E}-02(\mathrm{Ci}-\mathrm{s}) / \mathrm{m}^{\wedge} 3$

50-YR DOSE COMMITMENT:

EFFECTIVE DOSE

EQUIVALENT 4.0E-04 rem 
Title of Project EPHA for THM

Subject Radiological Consequence Assessment

Computer D. J. Richards
Reviewed by $\mathrm{S}$. A. Henderson Works Calculation 6

Sheet No. 16 of 166

Release Designation: 1-RD-71, Average

HOTSPOT 7.0 TRITIUM RELEASE 9-09-1996 12:50

TOTAL TRITIUM RELEASE : $2.5 E+00$ curies $100.000 \%$ TRITIUM OXIDE

Dose Results Include Skin Absorption

Source Term Width: $3 \mathrm{~m}$ Source Term Height: $3 \mathrm{~m}$

EFFECTIVE RELEASE HEIGHT : $\quad 0.00 \mathrm{~m}$

WIND SPEED $(\mathrm{h}=2 \mathrm{~m}): 2.5 \mathrm{~m} / \mathrm{s}$

STABILITY CLASS :C DEPOSITION VELOCITY $\quad: 0.0 \mathrm{E}+00 \mathrm{~cm} / \mathrm{s}$

RECEPTOR HEIGHT $\quad: 0.0 \mathrm{~m}$ INVERSION LAYER HEIGHT $: 200.0 \mathrm{~m}$

SAMPLE TIME $\quad: 30.000 \mathrm{~min}$

MAXIMUM DOSE DISTANCE $:<0.10 \mathrm{~km}$ MAXIMUM CEDE $:>7.2 \mathrm{E}-05 \mathrm{rem}$

$\mathrm{D}=0.03 \mathrm{~km}$

$\mathrm{CHI}=1.6 \mathrm{E}-02(\mathrm{Ci}-\mathrm{s}) / \mathrm{m}^{\wedge} 3$

50-YR DOSE COMMITMENT:

EFFECTIVE DOSE

EQUIVALENT 5.0E-04 rem
$\mathrm{D}=0.10 \mathrm{~km}$

$\mathrm{CHI}=2.3 \mathrm{E}-03(\mathrm{Ci}-\mathrm{s}) / \mathrm{m}^{\wedge} 3$

50-YR DOSE COMMITMENT:

EFFECTIVE DOSE

EQUTVALENT 7.2E-05 rem 
Title of Project EPHA for THM

Subject Radiological Consequence Assessment

Computer D. J. Richards

Release Designation: 1-RD-72, Adverse

HOTSPOT 7.0 TRITIUM RELEASE 9-10-1996 20:41

TOTAL TRITIUM RELEASE : $5.9 E+00$ curies $100.000 \%$ TRITIUM OXIDE

Dose Results Include Skin Absorption

Source Term Width: $3 \mathrm{~m}$ Source Term Height:, $3 \mathrm{~m}$

EFFECTIVE RELEASE HEIGHT : $\quad 0.00 \mathrm{~m}$

WIND SPEED ( $\mathrm{h}=2 \mathrm{~m}): 1.7 \mathrm{~m} / \mathrm{s}$

STABILITY CLASS :E DEPOSITION VELOCITY $: 0.0 \mathrm{E}+00 \mathrm{~cm} / \mathrm{s}$

RECEPTOR HEIGHT $\quad: 0.0 \mathrm{~m}$ INVERSION LAYER HEIGHT $: 200.0 \mathrm{~m}$

SAMPLE TIME $\quad: 10.000 \mathrm{~min}$

MAXIMUM DOSE DISTANCE $:<0.10 \mathrm{~km}$ MAXIMUM CEDE $:>1.2 \mathrm{E}-03 \mathrm{rem}$

$\mathrm{D}=0.03 \mathrm{~km}$

$\mathrm{CHI}=1.8 \mathrm{E}-01(\mathrm{Ci}-\mathrm{s}) / \mathrm{m}^{\wedge} 3$

50-YR DOSE COMMITMENT:

EFFECTIVE DOSE

EQUIVALENT 5.7E-03 rem
$\mathrm{D}=0.10 \mathrm{~km}$

$\mathrm{CHI}=3.8 \mathrm{E}-02(\mathrm{Ci}-\mathrm{s}) / \mathrm{m}^{\wedge} 3$

50-YR DOSE COMMITMENT:

EFFECTIVE DOSE

EQUIVALENT 1.2E-03 rem 
Title of Project EPHA for THM

Subject Radiological Consequence Assessment

Computer D. J. Richards

Release Designation: 1-RD-72, Average

HOTSPOT 7.0 TRITIUM RELEASE 9-10-1996 20:42

TOTAL TRITIUM RELEASE : $5.9 \mathrm{E}+00$ curies $100.000 \%$ TRITIUM OXIDE

Dose Results Include Skin Absorption

Source Term Width: $3 \mathrm{~m}$ Source Term Height: $3 \mathrm{~m}$

EFFECTIVE RELEASE HEIGHT : $\quad 0.00 \mathrm{~m}$

WIND SPEED $(\mathrm{h}=2 \mathrm{~m}): 2.5 \mathrm{~m} / \mathrm{s}$

STABILITY CLASS :C DEPOSITION VELOCITY : $0.0 \mathrm{E}+00 \mathrm{~cm} / \mathrm{s}$

RECEPTOR HEIGHT $\quad: 0.0 \mathrm{~m}$ INVERSION LAYER HEIGHT $: 200.0 \mathrm{~m}$

SAMPLE TIME $\quad: 10.000 \mathrm{~min}$

MAXIMUM DOSE DISTANCE $:<0.10 \mathrm{~km}$ MAXIMUM CEDE $:>2.1 \mathrm{E}-04$ rem

$\mathrm{D}=0.03 \mathrm{~km}$

$\mathrm{CHI}=4.8 \mathrm{E}-02(\mathrm{Ci}-\mathrm{s}) / \mathrm{m}^{\wedge} 3$

50-YR DOSE COMMITMENT:

EFFECTIVE DOSE

EQUIVALENT $1.5 \mathrm{E}-03 \mathrm{rem}$
$\mathrm{D}=0.10 \mathrm{~km}$.

$\mathrm{CHI}=6.8 \mathrm{E}-03(\mathrm{Ci}-\mathrm{s}) / \mathrm{m}^{\wedge} 3$

50-YR DOSE COMMITMENT:

EFFECTIVE DOSE

EQUIVALENT 2.1E-04 rem 
Title of Project EPHA for THM

Reviewed by S. A. Henderson

Subject Radiological Consequence Assessment

Works Calculation 6

Computer D. J. Richards

Date $09 / 11 / 96$

Sheet No. 19 of 166

Release Designation: 1-RD-73, Adverse

HOTSPOT 7.0 GENERAL PLUME 9-09-1996 11:24

RADIONUCLIDE : PU-238 Inhalation Class : W

HALFLIFE : 87.740 years

SOURCE TERM : $2.1 \mathrm{E}-06 \mathrm{Ci}$

Source Term Width: $3 \mathrm{~m}$ Source Term Height: $3 \mathrm{~m}$

RELEASE FRACTION : $1.00 \mathrm{E}+00$

FILTER EFFICIENCY: $0.000000 \%$

EFFECTIVE RELEASE HEIGHT : $\quad 0.00 \mathrm{~m}$

WIND SPEED $(\mathrm{h}=2 \mathrm{~m}): 1.7 \mathrm{~m} / \mathrm{s}$

STABILITY CLASS :E DEPOSITION VELOCITY $: 1.00 \mathrm{~cm} / \mathrm{s}$

RECEPTOR HEIGHT $\quad: 0.0 \mathrm{~m}$ INVERSION LAYER HEIGHT $: 200.0 \mathrm{~m}$

SAMPLE TIME $\quad: 30.000 \mathrm{~min}$

MAXIMUM DOSE DISTANCE $:<0.10 \mathrm{~km}$ MAXIMUM CEDE $:>1.2 \mathrm{E}-03 \mathrm{rem}$

$\mathrm{D}=0.03 \mathrm{~km}$

$\mathrm{DEP}=4.6 \mathrm{E}-04 \mathrm{uCi} / \mathrm{m}^{\wedge} 2$

$\mathrm{CHI}=4.6 \mathrm{E}-08(\mathrm{Ci}-\mathrm{s}) / \mathrm{m}^{\wedge} 3$

50-YR DOSE COMMITMENT:

SURFACE BONE 1.2E-01 rem RED MARROW 1.0E-02 rem

LIVER 2.7E-02 rem GONADS 1.5E-03 rem EFFECTIVE DOSE

EQUIVALENT $\quad 7.0 \mathrm{E}-03 \mathrm{rem}$
$\mathrm{D}=0.10 \mathrm{~km}$

$\mathrm{DEP}=7.8 \mathrm{E}-05 \mathrm{uCi} / \mathrm{m}^{\wedge} 2$

$\mathrm{CHI}=7.8 \mathrm{E}-09(\mathrm{Ci}-\mathrm{s}) / \mathrm{m}^{\wedge} 3$

50-YR DOSE COMMITMENT:

SURFACE BONE 2.1E-02 rem

RED MARROW 1.7E-03 rem

LIVER 4.7E-03 rem

GONADS 2.6E-04 rem

EFFECTIVE DOSE

EQUIVALENT 1.2E-03 rem 
Title of Project EPHA for THM

Subject Radiological Consequence Assessment

Computer D. J. Richards

Date $\underline{09 / 11 / 96}$

Reviewed by S. A. Henderson Works Calculation 6

Sheet No. 20 of 166

Release Designation: 1-RD-73, Average

HOTSPOT 7.0 GENERAL PLUME 9-09-1996 11:25

RADIONUCLIDE : PU-238 Inhalation Class : W

HALFLIFE $\quad: 87.740$ years

SOURCE TERM : $2.1 \mathrm{E}-06 \mathrm{Ci}$

Source Term Width: $\quad 3 \mathrm{~m}$ Source Term Height: $3 \mathrm{~m}$

RELEASE FRACTION : $1.00 \mathrm{E}+00$

FILTER EFFICIENCY: $0.000000 \%$

EFFECTIVE RELEASE HEIGHT : $\quad 0.00 \mathrm{~m}$

WIND SPEED $(\mathrm{h}=2 \mathrm{~m}): 2.5 \mathrm{~m} / \mathrm{s}$

STABILITY CLASS :C DEPOSITION VELOCITY $: 1.00 \mathrm{~cm} / \mathrm{s}$

RECEPTOR HEIGHT $\quad: 0.0 \mathrm{~m}$ INVERSION LAYER HEIGHT $: 500.0 \mathrm{~m}$

SAMPLE TIME $\quad: 30.000 \mathrm{~min}$

MAXIMUM DOSE DISTANCE $:<0.10 \mathrm{~km}$ MAXIMUM CEDE $:>2.8 \mathrm{E}-04 \mathrm{rem}$

$D=0.03 \mathrm{~km}$

$\mathrm{DEP}=1.3 \mathrm{E}-04 \mathrm{uCi} / \mathrm{m}^{\wedge} 2$

$\mathrm{CHI}=1.3 \mathrm{E}-08(\mathrm{Ci}-\mathrm{s}) / \mathrm{m}^{\wedge} 3$

50-YR DOSE COMMITMENT:

SURFACE BONE 3.6E-02 rem RED MARROW 3.0E-03 rem LIVER $\quad 8.0 \mathrm{E}-03 \mathrm{rem}$ GONADS 4.4E-04 rem EFFECTIVE DOSE EQUIVALENT 2.0E-03 rem
$\mathrm{D}=0.10 \mathrm{~km}$

$\mathrm{DEP}=1.8 \mathrm{E}-05 \mathrm{uCi} / \mathrm{m}^{\wedge} 2$

$\mathrm{CHI}=1.8 \mathrm{E}-09(\mathrm{Ci}-\mathrm{s}) / \mathrm{m}^{\wedge} 3$

50-YR DOSE COMMITMENT:

SURFACE BONE 4.9E-03 rem RED MARROW 4.1E-04 rem LIVER 1.1E-03 rem GONADS 6.1E-05 rem EFFECTIVE DOSE

EQUTVALENT $2.8 \mathrm{E}-04 \mathrm{rem}$ 
Title of Project EPHA for THM

Subject Radiological Consequence Assessment

Computer D. J. Richards
Reviewed by S. A. Henderson Works Calculation 6

Sheet No. 21 of 166

Release Designation: 1-RD-74, Adverse

HOTSPOT 7.0 GENERAL PLUME 9-10-1996 20:43

RADIONUCLIDE : PU-238 Inhalation Class : W

HALFLIFE : 87.740 years

SOURCE TERM : $5.1 \mathrm{E}-06 \mathrm{Ci}$

Source Term Width: $3 \mathrm{~m}$ Source Term Height: $3 \mathrm{~m}$

RELEASE FRACTION : $1.00 \mathrm{E}+00$

FILTER EFFICIENCY: $0.000000 \%$

EFFECTIVE RELEASE HEIGHT : $\quad 0.00 \mathrm{~m}$

WIND SPEED ( $\mathrm{h}=2 \mathrm{~m}): 1.7 \mathrm{~m} / \mathrm{s}$

STABILITY CLASS :E DEPOSITION VELOCITY $: 1.00 \mathrm{~cm} / \mathrm{s}$

RECEPTOR HEIGHT $: 0.0 \mathrm{~m}$ INVERSION LAYER HEIGHT $: 200.0 \mathrm{~m}$

SAMPLE TIME $\quad: 10.000 \mathrm{~min}$

MAXIMUM DOSE DISTANCE $:<0.10 \mathrm{~km}$ MAXIMUM CEDE $:>3.5 \mathrm{E}-03 \mathrm{rem}$

$\mathrm{D}=0.03 \mathrm{~km}$

$\mathrm{DEP}=1.3 \mathrm{E}-03 \mathrm{uCi} / \mathrm{m}^{\wedge} 2$

$\mathrm{CHI}=1.3 \mathrm{E}-07(\mathrm{Ci}-\mathrm{s}) / \mathrm{m}^{\wedge} 3$

50-YR DOSE COMMITMENT:

SURFACE BONE 3.6E-01 rem RED MARROW $3.0 \mathrm{E}-02 \mathrm{rem}$ LIVER 8.0E-02 rem GONADS 4.5E-03 rem EFFECTIVE DOSE EQUIVALENT $2.1 \mathrm{E}-02 \mathrm{rem}$
$\mathrm{D}=0.10 \mathrm{~km}$

$\mathrm{DEP}=2.3 \mathrm{E}-04 \mathrm{uCi} / \mathrm{m}^{\wedge} 2$

$\mathrm{CHI}=2.3 \mathrm{E}-08(\mathrm{Ci}-\mathrm{s}) / \mathrm{m}^{\wedge} 3$

50-YR DOSE COMMITMENT:

SURFACE BONE 6.2E-02 rem

RED MARROW $\quad 5.1 \mathrm{E}-03 \mathrm{rem}$

LIVER 1.4E-02 rem .

GONADS 7.6E-04 rem

EFFECTIVE DOSE

EQUIVALENT $3.5 \mathrm{E}-03 \mathrm{rem}$ 
Title of Project EPHA for THM

Subject Radiological Consequence Assessment

Computer D. J. Richards

Date $\underline{09 / 11 / 96}$

Reviewed by $\underline{\text { S. A. Hènderson }}$

Works Calculation 6

Sheet No. 22 of 166

Release Designation: 1-RD-74, Average

HOTSPOT 7.0 GENERAL PLUME 9-10-1996 20:44

RADIONUCLIDE: PU-238 Inhalation Class : W

HALFLIFE : 87.740 years

SOURCE TERM : $5.1 \mathrm{E}-06 \mathrm{Ci}$

Source Term Width: $3 \mathrm{~m}$ Source Term Height: $3 \mathrm{~m}$

RELEASE FRACTION : $1.00 \mathrm{E}+00$

FILTER EFFICIENCY: $0.000000 \%$

EFFECTIVE RELEASE HEIGHT : $\quad 0.00 \mathrm{~m}$

WIND SPEED $(\mathrm{h}=2 \mathrm{~m}): 2.5 \mathrm{~m} / \mathrm{s}$

STABILITY CLASS :C DEPOSITION VELOCITY $: 1.00 \mathrm{~cm} / \mathrm{s}$

RECEPTOR HEIGHT $: 0.0 \mathrm{~m}$ INVERSION LAYER HEIGHT $: 500.0 \mathrm{~m}$

SAMPLE TIME $\quad: 10.000 \mathrm{~min}$

MAXIMUM DOSE DISTANCE $:<0.10 \mathrm{~km}$ MAXIMUM CEDE $:>8.2 \mathrm{E}-04 \mathrm{rem}$

$\mathrm{D}=0.03 \mathrm{~km}$

$\mathrm{DEP}=3.9 \mathrm{E}-04 \mathrm{uCi} / \mathrm{m}^{\wedge} 2$

$\mathrm{CHI}=3.9 \mathrm{E}-08(\mathrm{Ci}-\mathrm{s}) / \mathrm{m}^{\wedge} 3$

50-YR DOSE COMMITMENT:

SURFACE BONE 1.1E-01 rem RED MARROW $8.8 \mathrm{E}-03 \mathrm{rem}$ LIVER 2.4E-02 rem GONADS 1.3E-03 rem EFFECTIVE DOSE

EQUTVALENT 6.0E-03 rem

\author{
$\mathrm{D}=0.10 \mathrm{~km}$ \\ $\mathrm{DEP}=5.4 \mathrm{E}-05 \mathrm{uCi} / \mathrm{m}^{\wedge} 2$ \\ $\mathrm{CHI}=5.4 \mathrm{E}-09(\mathrm{Ci}-\mathrm{s}) / \mathrm{m}^{\wedge} 3$ \\ 50-YR DOSE COMMITMENT: \\ SURFACE BONE 1.4E-02 rem \\ RED MARROW 1.2E-03 rem \\ LIVER 3.2E-03 rem \\ GONADS 1.8E-04 rem \\ EFFECTIVE DOSE \\ EQUIVALENT 8.2E-04 rem
}


Title of Project EPHA for THM

Subject Radiological Consequence Assessment

Computer D. J. Richards

Release Designation: 1-RD-75, Adverse

HOTSPOT 7.0 GENERAL PLUME 9-09-1996 11:29

RADIONUCLIDE : PU-238 Inhalation Class : W

HALFLIFE : 87.740 years

SOURCE TERM $: 1.5 \mathrm{E}-03 \mathrm{Ci}$

Source Term Width: $3 \mathrm{~m}$ Source Term Height: $3 \mathrm{~m}$

RELEASE FRACTION : $1.00 E+00$

FILTER EFFICIENCY: $0.000000 \%$

EFFECTIVE RELEASE HEIGHT : $\quad 0.00 \mathrm{~m}$

WIND SPEED $(\mathrm{h}=2 \mathrm{~m}): 1.7 \mathrm{~m} / \mathrm{s}$

STABILITY CLASS :E DEPOSITION VELOCITY $: 1.00 \mathrm{~cm} / \mathrm{s}$

RECEPTOR HEIGHT $\quad: 0.0 \mathrm{~m}$ INVERSION LAYER HEIGHT $: 200.0 \mathrm{~m}$

SAMPLE TIME $\quad: 30.000 \mathrm{~min}$

MAXIMUM DOSE DISTANCE $:<0.10 \mathrm{~km}$ MAXIMUM CEDE $:>0.802 \mathrm{rem}$

$\mathrm{D}=0.03 \mathrm{~km}$

$\mathrm{DEP}=3.1 \mathrm{E}-01 \mathrm{uCi} / \mathrm{m}^{\wedge} 2$

$\mathrm{CHI}=3.1 \mathrm{E}-05(\mathrm{Ci}-\mathrm{s}) / \mathrm{m}^{\wedge} 3$

50-YR DOSE COMMITMENT:

SURFACE BONE 8.3E+01 rem RED MARROW 6.9E+00 rem LIVER $1.8 \mathrm{E}+01 \mathrm{rem}$ GONADS $1.0 \mathrm{E}+00 \mathrm{rem}$ EFFECTIVE DOSE

EQUIVALENT 4.7E+00 rem

\author{
$\mathrm{D}=0.09 \mathrm{~km}$ \\ $\mathrm{DEP}=6.9 \mathrm{E}-02 \mathrm{uCi} / \mathrm{m}^{\wedge} 2$ \\ $\mathrm{CHI}=6.9 \mathrm{E}-06(\mathrm{Ci}-\mathrm{s}) / \mathrm{m}^{\wedge} 3$ \\ 50-YR DOSE COMMITMENT: \\ SURFACE BONE $1.8 \mathrm{E}+01 \mathrm{rem}$ \\ RED MARROW $1.5 \mathrm{E}+00 \mathrm{rem}$ \\ LIVER $\quad 4.1 \mathrm{E}+00 \mathrm{rem}$ \\ GONADS 2.3E-01 rem \\ EFFECTIVE DOSE \\ EQUIVALENT $1.0 \mathrm{E}+00 \mathrm{rem}$
}

$\mathrm{DEP}=5.2 \mathrm{E}-02 \mathrm{uCi} / \mathrm{m}^{\wedge} 2$ $\mathrm{CHI}=5.2 \mathrm{E}-06(\mathrm{Ci}-\mathrm{s}) / \mathrm{m}^{\wedge} 3$ 50-YR DOSE COMMITMENT:

SURFACE BONE $1.4 \mathrm{E}+01 \mathrm{rem}$ RED MARROW 1.2E+00 rem LIVER 3.1E+00 rem GONADS 1.7E-01 rem EFFECTIVE DOSE EQUTVALENT 8.0E-01 rem 
Title of Project EPHA for THM

Subject Radiological Consequence Assessment

Computer D. J. Richards

Date 09/11/96

Reviewed by S. A. Henderson Works Calculation 6

Sheet No. 24 of 166

Release Designation: 1-RD-75, Average

HOTSPOT 7.0 GENERAL PLUME 9-09-1996 11:30

RADIONUCLIDE : PU-238 Inhalation Class : W

HALFLIFE $: 87.740$ years

SOURCE TERM : $1.5 \mathrm{E}-03 \mathrm{Ci}$

Source Term Width: $\quad 3 \mathrm{~m}$ Source Term Height: $3 \mathrm{~m}$

RELEASE FRACTION : $1.00 \mathrm{E}+00$

FILTER EFFICIENCY: $0.000000 \%$

EFFECTIVE RELEASE HEIGHT : $\quad 0.00 \mathrm{~m}$

WIND SPEED $(\mathrm{h}=2 \mathrm{~m}): 2.5 \mathrm{~m} / \mathrm{s}$

STABILITY CLASS :C DEPOSITION VELOCITY $: 1.00 \mathrm{~cm} / \mathrm{s}$

RECEPTOR HEIGHT $\quad: 0.0 \mathrm{~m}$ INVERSION LAYER HEIGHT $: 500.0 \mathrm{~m}$

SAMPLE TIME $\quad: 30.000 \mathrm{~min}$

MAXIMUM DOSE DISTANCE $:<0.10 \mathrm{~km}$ MAXIMUM CEDE $:>0.188 \mathrm{rem}$

$\mathrm{D}=0.03 \mathrm{~km}$

$\mathrm{DEP}=9.0 \mathrm{E}-02 \mathrm{uCi} / \mathrm{m}^{\wedge} 2$

$\mathrm{CHI}=9.0 \mathrm{E}-06(\mathrm{Ci}-\mathrm{s}) / \mathrm{m}^{\wedge} 3$

50-YR DOSE COMMITMENT:

SURFACE BONE 2.4E+01 rem RED MARROW $2.0 \mathrm{E}+00 \mathrm{rem}$ LIVER $\quad 5.4 \mathrm{E}+00 \mathrm{rem}$ GONADS 3.0E-01 rem EFFECTIVE DOSE

EQUIVALENT $1.4 \mathrm{E}+00$ rem

$$
\begin{aligned}
& \mathrm{D}=0.04 \mathrm{~km} \\
& \mathrm{DEP}=6.8 \mathrm{E}-02 \mathrm{uCi} / \mathrm{m}^{\wedge} 2 \\
& \mathrm{CHI}=6.8 \mathrm{E}-06(\mathrm{Ci}-\mathrm{s}) / \mathrm{m}^{\wedge} 3 \\
& \text { 50-YR DOSE COMMTTMENT: } \\
& \text { SURFACE BONE } 1.8 \mathrm{E}+01 \mathrm{rem} \\
& \text { RED MARROW } 1.5 \mathrm{E}+00 \mathrm{rem} \\
& \text { LIVER } \quad 4.1 \mathrm{E}+00 \mathrm{rem} \\
& \text { GONADS } \quad 2.3 \mathrm{E}-01 \mathrm{rem} \\
& \text { EFFECTIVE DOSE } \\
& \text { EQUIVALENT } \quad 1.0 \mathrm{E}+00 \mathrm{rem}
\end{aligned}
$$

\author{
$\mathrm{D}=0.10 \mathrm{~km}$ \\ $\mathrm{DEP}=1.2 \mathrm{E}-02 \mathrm{uCi} / \mathrm{m}^{\wedge} 2$ \\ $\mathrm{CHI}=1.2 \mathrm{E}-06(\mathrm{Ci}-\mathrm{s}) / \mathrm{m}^{\wedge} 3$ \\ 50-YR DOSE COMMITMENT: \\ SURFACE BONE 3.3E+00 rem \\ RED MARROW 2.7E-01 rem \\ LIVER 7.4E-01 rem \\ GONADS 4.1E-02 rem \\ EFFECTIVE DOSE \\ EQUIVALENT 1.9E-01 rem
}


Title of Project EPHA for THM

Subject Radiological Consequence Assessment

Computer D. J. Richards

Date $\underline{09 / 11 / 96}$

Reviewed by S. A. Henderson

Works Calculation 6

Sheet No. 25 of 166

Release Designation: 1-RD-76, Adverse

HOTSPOT 7.0 GENERAL PLUME 9-10-1996 20:46

RADIONUCLIDE : PU-238 Inhalation Class : W

HALFLIFE : : 87.740 years

SOURCE TERM : $3.4 \mathrm{E}-03 \mathrm{Ci}$

Source Term Width: $\quad 3 \mathrm{~m}$ Source Term Height: $3 \mathrm{~m}$

RELEASE FRACTION : $1.00 \mathrm{E}+00$

FILTER EFFICIENCY: $0.000000 \%$

EFFECTIVE RELEASE HEIGHT : $0.00 \mathrm{~m}$

WIND SPEED $(\mathrm{h}=2 \mathrm{~m}): 1.7 \mathrm{~m} / \mathrm{s}$

STABILITY CLASS :E DEPOSITION VELOCITY $: 1.00 \mathrm{~cm} / \mathrm{s}$

RECEPTOR HEIGHT $\quad: 0.0 \mathrm{~m}$ INVERSION LAYER HEIGHT $: 500.0 \mathrm{~m}$

SAMPLE TIME $\quad: 10.000 \mathrm{~min}$

MAXIMUM DOSE DISTANCE $:<0.10 \mathrm{~km}$ MAXIMUM CEDE $:>2.4 \mathrm{rem}$

$\mathrm{D}=0.03 \mathrm{~km}$

$\mathrm{DEP}=9.1 \mathrm{E}-01 \mathrm{uCi} / \mathrm{m}^{\wedge} 2$

$\mathrm{CHI}=9.1 \mathrm{E}-05(\mathrm{Ci}-\mathrm{s}) / \mathrm{m}^{\wedge} 3$

50-YR DOSE COMMITMENT:

SURFACE BONE 2.4E+02 rem RED MARROW 2.0E+01 rem LIVER 5.4E+01 rem GONADS $3.0 \mathrm{E}+00 \mathrm{rem}$ EFFECTIVE DOSE

EQUTVALENT 1.4E+01 rem

\author{
$\mathrm{D}=0.10 \mathrm{~km}$ \\ $\mathrm{DEP}=1.5 \mathrm{E}-01 \mathrm{uCi} / \mathrm{m}^{\wedge} 2$ \\ $\mathrm{CHI}=1.5 \mathrm{E}-05(\mathrm{Ci}-\mathrm{s}) / \mathrm{m}^{\wedge} 3$ \\ 50-YR DOSE COMMITMENT: \\ SURFACE BONE 4.2E+01' rem \\ RED MARROW $3.4 \mathrm{E}+00 \mathrm{rem}$ \\ LIVER $\quad 9.2 \mathrm{E}+00 \mathrm{rem}$ \\ GONADS 5.1E-01 rem \\ EFFECTIVE DOSE \\ EQUIVALENT $2.4 \mathrm{E}+00 \mathrm{rem}$
}
$\mathrm{D}=0.16 \mathrm{~km}$
$\mathrm{DEP}=6.8 \mathrm{E}-02 \mathrm{uCi} / \mathrm{m}^{\wedge} 2$
$\mathrm{CHI}=6.8 \mathrm{E}-06(\mathrm{Ci}-\mathrm{s}) / \mathrm{m}^{\wedge} 3$
50-YR DOSE COMMITMENT:
SURFACE BONE 1.8E+01 rem RED MARROW 1.5E+00 rem LIVER 4.1E+00 rem GONADS 2.3E-01 rem EFFECTIVE DOSE
EQUIVALENT $1.0 \mathrm{E}+00 \mathrm{rem}$ 
Title of Project EPHA for THM

Subject Radiological Consequence Assessment

Computer D. J. Richards

Release Designation: 1-RD-76, Average

HOTSPOT 7.0 GENERAL PLUME 9-10-1996 20:47

RADIONUCLIDE : PU-238 Inhalation Class : W

HALFLIFE $: 87.740$ years

SOURCE TERM : $3.4 \mathrm{E}-03 \mathrm{Ci}$

Source Term Width: $\quad 3 \mathrm{~m}$ Source Term Height: $3 \mathrm{~m}$

RELEASE FRACTION : $1.00 \mathrm{E}+00$

FILTER EFFICIENCY: $0.000000 \%$

EFFECTIVE RELEASE HEIGHT : $\quad 0.00 \mathrm{~m}$

WIND SPEED $(\mathrm{h}=2 \mathrm{~m}): 2.5 \mathrm{~m} / \mathrm{s}$

STABILITY CLASS :C DEPOSITION VELOCITY : $1.00 \mathrm{~cm} / \mathrm{s}$

RECEPTOR HEIGHT $: 0.0 \mathrm{~m}$ INVERSION LAYER HEIGHT $: 500.0 \mathrm{~m}$

SAMPLE TIME $: 10.000 \mathrm{~min}$

MAXIMUM DOSE DISTANCE $:<0.10 \mathrm{~km}$ MAXIMUM CEDE $:>0.555 \mathrm{rem}$

$\mathrm{D}=0.03 \mathrm{~km}$

$\mathrm{DEP}=2.7 \mathrm{E}-01 \mathrm{uCi} / \mathrm{m}^{\wedge} 2$

$\mathrm{CHI}=2.7 \mathrm{E}-05(\mathrm{Ci}-\mathrm{s}) / \mathrm{m}^{\wedge} 3$

50-YR DOSE COMMTTMENT:

SURFACE BONE 7.2E+01 rem RED MARROW $5.9 \mathrm{E}+00 \mathrm{rem}$ LIVER $1.6 \mathrm{E}+01 \mathrm{rem}$ GONADS 8.8E-01 rem EFFECTIVE DOSE EQUIVALENT 4.1E+00 rem

$$
\begin{aligned}
& \mathrm{D}=0.07 \mathrm{~km} \\
& \mathrm{DEP}=6.8 \mathrm{E}-02 \mathrm{uCi} / \mathrm{m}^{\wedge} 2 \\
& \mathrm{CHI}=6.8 \mathrm{E}-06(\mathrm{Ci}-\mathrm{s}) / \mathrm{m}^{\wedge} 3 \\
& \text { 50-YR DOSE COMMITMENT: } \\
& \text { SURFACE BONE } 1.8 \mathrm{E}+01 \mathrm{rem} \\
& \text { RED MARROW. } 1.5 \mathrm{E}+00 \mathrm{rem} \\
& \text { LIVER } \quad 4.1 \mathrm{E}+00 \mathrm{rem} \\
& \text { GONADS } \quad 2.3 \mathrm{E}-01 \mathrm{rem} \\
& \text { EFFECTIVE DOSE } \\
& \text { EQUTVALENT } \quad 1.0 \mathrm{E}+00 \mathrm{rem}
\end{aligned}
$$

\author{
$\mathrm{D}=0.10 \mathrm{~km}$ \\ $\mathrm{DEP}=3.6 \mathrm{E}-02 \mathrm{uCi} / \mathrm{m}^{\wedge} 2$ \\ $\mathrm{CHI}=3.6 \mathrm{E}-06(\mathrm{Ci}-\mathrm{s}) / \mathrm{m}^{\wedge} 3$ \\ 50-YR DOSE COMMITMENT: \\ SURFACE BONE $9.8 \mathrm{E}+00 \mathrm{rem}$ \\ RED MARROW 8.1E-01 rem \\ LIVER $2.2 \mathrm{E}+00 \mathrm{rem}$ \\ GONADS 1.2E-01 rem \\ EFFECTIVE DOSE \\ EQUTVALENT 5.5E-01 rem
}


Title of Project EPHA for THM

Subject Radiological Consequence Assessment

Computer D. J. Richards

Release Designation: 1-RD-77, Adverse

HOTSPOT 7.0 GENERAL PLUME 9-09-1996 11:32

RADIONUCLIDE: PU-238 Inhalation Class : W

HALFLIFE : 87.740 years

SOURCE TERM : $2.5 \mathrm{E}-04 \mathrm{Ci}$

Source Term Width: $\quad 3 \mathrm{~m}$ Source Term Height: $3 \mathrm{~m}$

RELEASE FRACTION : $1.00 \mathrm{E}+00$

FILTER EFFICIENCY: $0.000000 \%$

EFFECTIVE RELEASE HEIGHT : $\quad 0.00 \mathrm{~m}$

WIND SPEED $(\mathrm{h}=2 \mathrm{~m}): 1.7 \mathrm{~m} / \mathrm{s}$

STABILITY CLASS :E DEPOSITION VELOCITY $: 1.00 \mathrm{~cm} / \mathrm{s}$

RECEPTOR HEIGHT $: 0.0 \mathrm{~m}$ INVERSION LAYER HEIGHT $: 200.0 \mathrm{~m}$

SAMPLE TIME $\quad: 30.000 \mathrm{~min}$

MAXIMUM DOSE DISTANCE $:<0.10 \mathrm{~km}$ MAXIMUM CEDE $:>0.139 \mathrm{rem}$

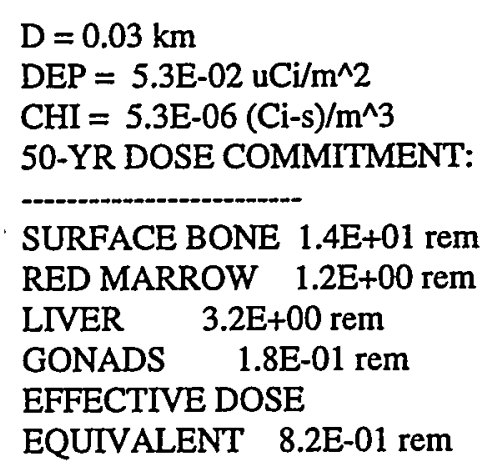

\author{
$\mathrm{D}=0.10 \mathrm{~km}$ \\ $\mathrm{DEP}=9.1 \mathrm{E}-03 \mathrm{uCi} / \mathrm{m}^{\wedge} 2$ \\ $\mathrm{CHI}=9.1 \mathrm{E}-07(\mathrm{Ci}-\mathrm{s}) / \mathrm{m}^{\wedge} 3$ \\ 50-YR DOSE COMMITMENT: \\ SURFACE BONE $2.5 \mathrm{E}+00 \mathrm{rem}$ \\ RED MARROW 2.0E-01 rem \\ LIVER 5.5E-01 rem \\ GONADS 3.0E-02 rem \\ EFFECTIVE DOSE \\ EQUIVALENT 1.4E-01 rem
}


Title of Project EPHA for THM

Reviewed by S. A. Henderson

Subject Radiological Consequence Assessment

Computer D. J. Richards

Date $\underline{09 / 11 / 96}$
Works Calculation 6

Sheet No. 28 of 166

Release Designation: 1-RD-77, Average

HOTSPOT 7.0 GENERAL PLUME 9-09-1996 11:33

RADIONUCLIDE : PU-238 Inhalation Class : W

HALFLIFE : 87.740 years

SOURCE TERM : $2.5 \mathrm{E}-04 \mathrm{Ci}$

Source Term Width: $3 \mathrm{~m}$ Source Term Height: $3 \mathrm{~m}$

RELEASE FRACTION : $1.00 \mathrm{E}+00$

FILTER EFFICIENCY: $0.000000 \%$

EFFECTIVE RELEASE HEIGHT : $\quad 0.00 \mathrm{~m}$

WIND SPEED $(\mathrm{h}=2 \mathrm{~m}): 2.5 \mathrm{~m} / \mathrm{s}$

STABILITY CLASS :C DEPOSITION VELOCITY $\quad: 1.00 \mathrm{~cm} / \mathrm{s}$

RECEPTOR HEIGHT $\quad: 0.0 \mathrm{~m}$ INVERSION LAYER HEIGHT $: 500.0 \mathrm{~m}$

SAMPLE TIME $\quad: 30.000 \mathrm{~min}$

MAXIMUM DOSE DISTANCE $:<0.10 \mathrm{~km}$ MAXIMUM CEDE $:>0.033 \mathrm{rem}$

$\mathrm{D}=0.03 \mathrm{~km}$

$\mathrm{DEP}=1.6 \mathrm{E}-02 \mathrm{uCi} / \mathrm{m}^{\wedge} 2$

$\mathrm{CHI}=1.6 \mathrm{E}-06(\mathrm{Ci}-\mathrm{s}) / \mathrm{m}^{\wedge} 3$

50-YR DOSE COMMITMENT:

SURFACE BONE $4.2 \mathrm{E}+00 \mathrm{rem}$ RED MARROW 3.5E-01 rem

LIVER 9.4E-01 rem GONADS 5.2E-02 rem EFFECTIVE DOSE

EQUIVALENT 2.4E-01 rem

$$
\begin{aligned}
& \mathrm{D}=0.10 \mathrm{~km} \\
& \mathrm{DEP}=2.1 \mathrm{E}-03 \mathrm{uCi} / \mathrm{m}^{\wedge} 2 \\
& \mathrm{CHI}=2.1 \mathrm{E}-07(\mathrm{Ci} \mathrm{-s}) / \mathrm{m}^{\wedge} 3 \\
& \text { 50-YR DOSE COMMITMENT: }
\end{aligned}
$$

SURFACE BONE 5.8E-01 rem RED MARROW 4.8E-02 rem LIVER 1.3E-01 rem GONADS 7.1E-03 rem EFFECTIVE DOSE

EQUIVALENT 3.3E-02 rem 
Title of Project EPHA for THM

Reviewed by $\underline{\text { S. A. Henderson }}$

Subject Radiological Consequence Assessment

Works Calculation 6

Computer D. J. Richards

Date $\underline{09 / 11 / 96}$

Sheet No. 29 of 166

Release Designation: 1-RD-78, Adverse

HOTSPOT 7.0 GENERAL PLUME 9-10-1996 20:49

RADIONUCLIDE : PU-238 Inhalation Class : W

HALFLIFE $: 87.740$ years

SOURCE TERM : $5.4 \mathrm{E}-04 \mathrm{Ci}$

Source Term Width: $\quad 3 \mathrm{~m}$ Source Term Height: $3 \mathrm{~m}$

RELEASE FRACTION : $1.00 E+00$

FILTER EFFICIENCY: $0.000000 \%$

EFFECTIVE RELEASE HEIGHT : $\quad 0.00 \mathrm{~m}$

WIND SPEED $(\mathrm{h}=2 \mathrm{~m}): 1.7 \mathrm{~m} / \mathrm{s}$

STABILITY CLASS :E DEPOSITION VELOCITY $: 1.00 \mathrm{~cm} / \mathrm{s}$

RECEPTOR HEIGHT $: 0.0 \mathrm{~m}$ INVERSION LAYER HEIGHT $: 200.0 \mathrm{~m}$

SAMPLE TIME $\quad: 10.000 \mathrm{~min}$

MAXIMUM DOSE DISTANCE $:<0.10 \mathrm{~km}$ MAXIMUM CEDE $:>0.374 \mathrm{rem}$

$\mathrm{D}=0.03 \mathrm{~km}$.

$\mathrm{DEP}=1.4 \mathrm{E}-01 \mathrm{uCi} / \mathrm{m}^{\wedge} 2$

$\mathrm{CHI}=1.4 \mathrm{E}-05(\mathrm{Ci}-\mathrm{s}) / \mathrm{m}^{\wedge} 3$

50-YR DOSE COMMITMENT:

SURFACE BONE 3.9E+01 rem RED MARROW 3.2E+00 rem . LIVER $\quad 8.6 \mathrm{E}+00 \mathrm{rem}$ GONADS 4.8E-01 rem EFFECTIVE DOSE

EQUIVALENT $2.2 \mathrm{E}+00 \mathrm{rem}$
$\mathrm{D}=0.05 \mathrm{~km}$

$\mathrm{DEP}=6.8 \mathrm{E}-02 \mathrm{uCi} / \mathrm{m}^{\wedge} 2$

$\mathrm{CHI}=6.8 \mathrm{E}-06(\mathrm{Ci}-\mathrm{s}) / \mathrm{m}^{\wedge} 3$

50-YR DOSE COMMITMENT:

SURFACE BONE 1.8E+01 rem RED MARROW $1.5 \mathrm{E}+00 \mathrm{rem}$ LIVER $\quad 4.1 \mathrm{E} \div 00 \mathrm{rem}$ GONADS 2.3E-01 rem EFFECTIVE DOSE EQUIVALENT $1.0 \mathrm{E}+00 \mathrm{rem}$
$\mathrm{D}=0.10 \mathrm{~km}$ $\mathrm{DEP}=2.4 \mathrm{E}-02 \mathrm{uCi} / \mathrm{m}^{\wedge} 2$ $\mathrm{CHI}=2.4 \mathrm{E}-06(\mathrm{Ci}-\mathrm{s}) / \mathrm{m}^{\wedge} 3$ 50-YR DOSE COMMITMENT:

SURFACE BONE $6.6 \mathrm{E}+00 \mathrm{rem}$ RED MARROW 5.4E-01 rem LIVER $1.5 \mathrm{E}+00 \mathrm{rem}$ GONADS 8.1E-02 rem EFFECTIVE DOSE EQUIVALENT 3.7E-01 rem 
Title of Project EPHA for THM

Reviewed by S. A. Henderson

Subject Radiological Consequence Assessment

Works Calculation 6

Computer D. J. Richards

Date 09/11/96

Sheet No. 30 of 166

Release Designation: 1-RD-78, Average

HOTSPOT 7.0 GENERAL PLUME 9-10-1996 20:50

RADIONUCLIDE : PU-238 Inhalation Class : W

HALFLIFE : 87.740 years

SOURCE TERM : $5.4 \mathrm{E}-04 \mathrm{Ci}$

Source Term Width: $3 \mathrm{~m}$ Source Term Height: $3 \mathrm{~m}$

RELEASE FRACTION : $1.00 \mathrm{E}+00$

FILTER EFFICIENCY: $0.000000 \%$

EFFECTIVE RELEASE HEIGHT : $\quad 0.00 \mathrm{~m}$

WIND SPEED $(\mathrm{h}=2 \mathrm{~m}): 2.5 \mathrm{~m} / \mathrm{s}$

STABILITY CLASS :C DEPOSITION VELOCITY $: 1.00 \mathrm{~cm} / \mathrm{s}$

RECEPTOR HEIGHT $\quad: 0.0 \mathrm{~m}$ INVERSION LAYER HEIGHT $: 500.0 \mathrm{~m}$

SAMPLE TIME $\quad: 10.000 \mathrm{~min}$

MAXIMUM DOSE DISTANCE $:<0.10 \mathrm{~km}$ MAXIMUM CEDE $:>0.088 \mathrm{rem}$

$\mathrm{D}=0.03 \mathrm{~km}$

$\mathrm{DEP}=4.2 \mathrm{E}-02 \mathrm{uCi} / \mathrm{m}^{\wedge} 2$

$\mathrm{CHI}=4.2 \mathrm{E}-06(\mathrm{Ci}-\mathrm{s}) / \mathrm{m}^{\wedge} 3$

50-YR DOSE COMMITMENT:

SURFACE BONE 1.1E+01 rem RED MARROW 9.4E-01 rem LIVER $2.5 \mathrm{E}+00 \mathrm{rem}$ GONADS 1.4E-01 rem EFFECTIVE DOSE

EQUIVALENT 6.4E-01 rem
$\mathrm{D}=0.10 \mathrm{~km}$
$\mathrm{DEP}=5.7 \mathrm{E}-03 \mathrm{uCi} / \mathrm{m}^{\wedge} \mathrm{C}$
$\mathrm{CHI}=5.7 \mathrm{E}-07(\mathrm{Ci}-\mathrm{s}) / \mathrm{m}^{\wedge} 3$
50-YR DOSE COMMITMENT:
SURFACE BONE $1.5 \mathrm{E}+00 \mathrm{rem}$
RED MARROW 1.3E-01 rem
LIVER 3.4E-01 rem
GONADS 1.9E-02 rem
EFFECTIVE DOSE
EQUIVALENT $8.8 \mathrm{E}-02 \mathrm{rem}$ 
Title of Project EPHA for THM

Subject Radiological Consequence Assessment

Computer D. J. Richards

Date $09 / 11 / 96$
Reviewed by S. A. Henderson

Works Calculation 6

Sheet No. 31 of 166

Release Designation: 1-RD-79, Adverse

HOTSPOT 7.0 GENERAL PLUME 9-09-1996 11:35

RADIONUCLIDE : PU-238 Inhalation Class : W

HALFLIFE : 87.740 years

SOURCE TERM : $7.6 \mathrm{E}-04 \mathrm{Ci}$

Source Term Width: $\quad 3 \mathrm{~m}$ Source Term Height: $3 \mathrm{~m}$

RELEASE FRACTION : $1.00 \mathrm{E}+00$

FILTER EFFICIENCY: $0.000000 \%$

EFFECTIVE RELEASE HEIGHT : $\quad 0.00 \mathrm{~m}$

WIND SPEED $(\mathrm{h}=2 \mathrm{~m}): 1.7 \mathrm{~m} / \mathrm{s}$

STABILITY CLASS :E DEPOSITION VELOCITY : $1.00 \mathrm{~cm} / \mathrm{s}$

RECEPTOR HEIGHT $\quad: 0.0 \mathrm{~m}$ INVERSION LAYER HEIGHT $: 200.0 \mathrm{~m}$

SAMPLE TIME $\quad: 30.000 \mathrm{~min}$

MAXIMUM DOSE DISTANCE $:<0.10 \mathrm{~km}$ MAXIMUM CEDE $:>0.421 \mathrm{rem}$

$\mathrm{D}=0.03 \mathrm{~km}$

$\mathrm{DEP}=1.6 \mathrm{E}-01 \mathrm{uCi} / \mathrm{m}^{\wedge} 2$

$\mathrm{CHI}=1.6 \mathrm{E}-05(\mathrm{Ci}-\mathrm{s}) / \mathrm{m}^{\wedge} 3$

50-YR DOSE COMMITMENT:

SURFACE BONE 4.4E+01 rem RED MARROW $3.6 \mathrm{E}+00 \mathrm{rem}$

LIVER $\quad 9.7 \mathrm{E}+00 \mathrm{rem}$

GONADS 5.4E-01 rem

EFFECTIVE DOSE

EQUIVALENT $2.5 \mathrm{E}+00 \mathrm{rem}$

$\mathrm{D}=0.06 \mathrm{~km}$
$\mathrm{DEP}=6.7 \mathrm{E}-02 \mathrm{uCi} / \mathrm{m}^{\wedge} 2$
$\mathrm{CHI}=6.7 \mathrm{E}-06(\mathrm{Ci}-\mathrm{s}) / \mathrm{m}^{\wedge} 3$
50-YR DOSE COMMITMENT:
SURFACE BONE $1.8 \mathrm{E}+01 \mathrm{rem}$
RED MARROW $1.5 \mathrm{E}+00 \mathrm{rem}$
LIVER $\quad 4.0 \mathrm{E}+00 \mathrm{rem}$
GONADS $\quad 2.2 \mathrm{E}-01 \mathrm{rem}$
EFFECTIVE DOSE
EQUIVALENT $\quad 1.0 \mathrm{E}+00 \mathrm{rem}$

$\mathrm{D}=0: 10 \mathrm{~km}$ $\mathrm{DEP}=2.8 \mathrm{E}-02 \mathrm{uCi} / \mathrm{m}^{\wedge} 2$ $\mathrm{CHI}=2.8 \mathrm{E}-06(\mathrm{Ci}-\mathrm{s}) / \mathrm{m}^{\wedge} 3$ 50-YR DOSE COMMITMENT:

SURFACE BONE 7.4E +00 rem RED MARROW 6.1E-01 rem LIVER $1.6 \mathrm{E}+00 \mathrm{rem}$ GONADS 9.2E-02 rem EFFECTIVE DOSE

EQUIVALENT 4.2E-01 rem 
Title of Project EPHA for THM

Subject Radiological Consequence Assessment

Computer D. J. Richards

Date $\underline{09 / 11 / 96}$

Reviewed by $\underline{S}$. A. Henderson

Works Calculation 6

Sheet No. 32 of 166

Release Designation: 1-RD-79, Average

HOTSPOT 7.0 GENERAL PLUME 9-09-1996 11:36

RADIONUCLIDE : PU-238 Inhalation Class : W

HALFLIFE : 87.740 years

SOURCE TERM : $7.6 \mathrm{E}-04 \mathrm{Ci}$

Source Term Width: $\quad 3 \mathrm{~m}$ Source Term Height: $3 \mathrm{~m}$

RELEASE FRACTION : $1.00 \mathrm{E}+00$

FILTER EFFICIENCY: $0.000000 \%$

EFFECTIVE RELEASE HEIGHT : $\quad 0.00 \mathrm{~m}$

WIND SPEED ( $\mathrm{h}=2 \mathrm{~m}): 2.5 \mathrm{~m} / \mathrm{s}$

STABILITY CLASS :C DEPOSITION VELOCITY $: 1.00 \mathrm{~cm} / \mathrm{s}$

RECEPTOR HEIGHT $\quad: 0.0 \mathrm{~m}$ INVERSION LAYER HEIGHT $: 500.0 \mathrm{~m}$

SAMPLE TIME $: 30.000 \mathrm{~min}$

MAXIMUM DOSE DISTANCE $:<0.10 \mathrm{~km}$ MAXIMUM CEDE $:>0.099 \mathrm{rem}$

$\mathrm{D}=0.03 \mathrm{~km}$

$\mathrm{DEP}=4.7 \mathrm{E}-02 \mathrm{uCi} / \mathrm{m}^{\wedge} 2$

$\mathrm{CHI}=4.7 \mathrm{E}-06(\mathrm{Ci}-\mathrm{s}) / \mathrm{m}^{\wedge} 3$

50-YR DOSE COMMITMENT:

SURFACE BONE 1.3E+01 rem RED MARROW $1.1 \mathrm{E}+00 \mathrm{rem}$ LIVER 2.8E+00 rem GONADS 1.6E-01 rem EFFECTIVE DOSE

EQUIVALENT 7.2E-01 rem
$\mathrm{D}=0.10 \mathrm{~km}$
$\mathrm{DEP}=6.5 \mathrm{E}-03 \mathrm{uCi} / \mathrm{m}^{\wedge} 2$
$\mathrm{CHI}=6.5 \mathrm{E}-07(\mathrm{Ci}-\mathrm{s}) / \mathrm{m}^{\wedge} 3$
50-YR DOSE COMMITMENT:
SURFACE BONE $1.7 \mathrm{E}+00 \mathrm{rem}$
RED MARROW 1.4E-01 rem
LIVER 3.9E-01 rem
GONADS 2.2E-02 rem
EFFECTIVE DOSE
EQUTVALENT 9.9E-02 rem 
Title of Project EPHA for THM

Subject Radiological Consequence Assessment

Computer D. J. Richards

Date $\underline{09 / 11 / 96}$

Reviewed by $\underline{\text { S. A. Henderson }}$ Works Calculation 6 Sheet No. 33 of 166

Release Designation: 1-RD-80, Adverse

HOTSPOT 7.0 GENERAL PLUME 9-10-1996 20:52

RADIONUCLIDE : PU-238 Inhalation Class : W

HALFLIFE $: 87.740$ years

SOURCE TERM : $1.6 \mathrm{E}-03 \mathrm{Ci}$

Source Term Width: $\quad 3 \mathrm{~m}$ Source Term Height: $3 \mathrm{~m}$

RELEASE FRACTION : $1,00 \mathrm{E}+00$

FILTER EFFICIENCY: $0.000000 \%$

EFFECTIVE RELEASE HEIGHT : $\quad 0.00 \mathrm{~m}$

WIND SPEED $(\mathrm{h}=2 \mathrm{~m}): 1.7 \mathrm{~m} / \mathrm{s}$

STABILITY CLASS :E DEPOSITION VELOCITY $: 1.00 \mathrm{~cm} / \mathrm{s}$

RECEPTOR HEIGHT $: 0.0 \mathrm{~m}$ INVERSION LAYER HEIGHT $: 200.0 \mathrm{~m}$

SAMPLE TIME $\quad: 10.000 \mathrm{~min}$

MAXIMUM DOSE DISTANCE $:<0.10 \mathrm{~km}$ MAXIMUM CEDE $:>1.1 \mathrm{rem}$

$\mathrm{D}=0.03 \mathrm{~km}$

$\mathrm{DEP}=4.4 \mathrm{E}-01 \mathrm{uCi} / \mathrm{m}^{\wedge} 2$

$\mathrm{CHI}=4.4 \mathrm{E}-05(\mathrm{Ci}-\mathrm{s}) / \mathrm{m}^{\wedge} 3$

50-YR DOSE COMMITMENT:

SURFACE BONE $1.2 \mathrm{E}+02 \mathrm{rem}$ RED MARROW $9.7 \mathrm{E}+00 \mathrm{rem}$ LIVER 2.6E+01 rem GONADS 1.4E+00 rem EFFECTIVE DOSE EQUTVALENT $6.7 \mathrm{E}+00 \mathrm{rem}$

\author{
$\mathrm{D}=0.10 \mathrm{~km}$ \\ $\mathrm{DEP}=7.4 \mathrm{E}-02 \mathrm{uCi} / \mathrm{m}^{\wedge} 2$ \\ $\mathrm{CHI}=7.4 \mathrm{E}-06(\mathrm{Ci}-\mathrm{s}) / \mathrm{m}^{\wedge} 3$ \\ 50-YR DOSE COMMITMENT: \\ SURFACE BONE 2.0E+01 rem \\ RED MARROW $1.7 \mathrm{E}+00 \mathrm{rem}$ \\ LIVER $\quad 4.4 \mathrm{E}+00 \mathrm{rem}$ \\ GONADS 2.5E-01 rem \\ EFFECTIVE DOSE \\ EQUIVALENT $1.1 \mathrm{E}+00 \mathrm{rem}$
}

\author{
$\mathrm{D}=0.11 \mathrm{~km}$ \\ $\mathrm{DEP}=6.8 \mathrm{E}-02 \mathrm{uCi} / \mathrm{m}^{\wedge} 2$ \\ $\mathrm{CHI}=6.8 \mathrm{E}-06(\mathrm{Ci}-\mathrm{s}) / \mathrm{m}^{\wedge} 3$ \\ 50-YR DOSE COMMITMENT: \\ SURFACE BONE $1.8 \mathrm{E}+01 \mathrm{rem}$ \\ RED MARROW $1.5 E+00 \mathrm{rem}$ \\ LIVER 4.1E+00 rem \\ GONADS 2.3E-01 rem \\ EFFECTIVE DOSE \\ EQUIVALENT $1.0 \mathrm{E}+00 \mathrm{rem}$
}


Title of Project EPHA for THM

Subject Radiological Consequence Assessment

Computer D. J. Richards

Date $\underline{09 / 11 / 96}$

Reviewed by S. A. Hénderson

Works Calculation 6

Sheet No. 34 of 166

Release Designation: 1-RD-80, Average

HOTSPOT 7.0 GENERAL PLUME 9-10-1996 20:53

RADIONUCLIDE : PU-238 Inhalation Class : W

HALFLIFE : 87.740 years

SOUTRCE TERM $: 1.6 \mathrm{E}-03 \mathrm{Ci}$

Source Term Width: $3 \mathrm{~m}$ Source Term Height: $3 \mathrm{~m}$

RELEASE FRACTION : $1.00 \mathrm{E}+00$

FILTER EFFICIENCY: $0.000000 \%$

EFFECTIVE RELEASE HEIGHT : $\quad 0.00 \mathrm{~m}$

WIND SPEED $(\mathrm{h}=2 \mathrm{~m}): 2.5 \mathrm{~m} / \mathrm{s}$

STABILITY CLASS :C DEPOSITION VELOCITY $: 1.00 \mathrm{~cm} / \mathrm{s}$

RECEPTOR HEIGHT $\quad: 0.0 \mathrm{~m}$ INVERSION LAYER HEIGHT $: 500.0 \mathrm{~m}$

SAMPLE TIME $\quad: 10.000 \mathrm{~min}$

MAXIMUM DOSE DISTANCE $:<0.10 \mathrm{~km}$ MAXIMUM CEDE $:>0.267 \mathrm{rem}$

$\mathrm{D}=0.03 \mathrm{~km}$

$\mathrm{DEP}=1.3 \mathrm{E}-01 \mathrm{uCi} / \mathrm{m}^{\wedge} 2$

$\mathrm{CHI}=1.3 \mathrm{E}-05(\mathrm{Ci}-\mathrm{s}) / \mathrm{m}^{\wedge} 3$

50-YR DOSE COMMITMENT:

SURFACE BONE 3.4E+01 rem RED MARROW $2.8 \mathrm{E}+00 \mathrm{rem}$

LIVER $\quad 7.6 \mathrm{E}+00 \mathrm{rem}$

GONADS 4.2E-01 rem

EFFECTIVE DOSE

EQUTVALENT $2.0 \mathrm{E}+00 \mathrm{rem}$

\author{
$\mathrm{D}=0.05 \mathrm{~km}$ \\ $\mathrm{DEP}=6.8 \mathrm{E}-02 \mathrm{uCi} / \mathrm{m}^{\wedge} 2$ \\ $\mathrm{CHI}=6.8 \mathrm{E}-06(\mathrm{Ci}-\mathrm{s}) / \mathrm{m}^{\wedge} 3$ \\ 50-YR DOSE COMMITMENT: \\ SURFACE BONE 1.8E+01 rem \\ RED MARROW $1.5 \mathrm{E}+00 \mathrm{rem}$ \\ LIVER \\ 4.1E+00 rem \\ GONADS 2.3E-01 rem \\ EFFECTIVE DOSE \\ EQUTVALENT $1.0 \mathrm{E}+00 \mathrm{rem}$
}

$\mathrm{D}=0.10 \mathrm{~km}$
$\mathrm{DEP}=1.7 \mathrm{E}-02 \mathrm{uCi} / \mathrm{m}^{\wedge} 2$
$\mathrm{CHI}=1.7 \mathrm{E}-06(\mathrm{Ci}-\mathrm{s}) / \mathrm{m}^{\wedge} 3$
50-YR DOSE COMMITMENT:

SURFACE BONE 4.7E+00 rem RED MARROW 3.9E-01 rem LIVER $1.0 \mathrm{E}+00 \mathrm{rem}$ GONADS 5.8E-02 rem EFFECTIVE DOSE ${ }^{\circ}$ EQUIVALENT 2.7E-01 rem 
Title of Project EPHA for THM

Subject Radiological Consequence Assessment

Computer D. J. Richards

Release Designation: 1-RD-81, Adverse

HOTSPOT 7.0 GENERAL PLUME

9-09-1996 20:13

RADIONUCLIDE : PU-238 Inhalation Class : W

HALFLIFE : 87.740 years

SOURCE TERM : $8.1 \mathrm{E}-03 \mathrm{Ci}$

Source Term Width: $3 \mathrm{~m}$ Source Term Height: $3 \mathrm{~m}$

RELEASE FRACTION : $1.00 \mathrm{E}+00$

FILTER EFFICIENCY: $0.000000 \%$

EFFECTIVE RELEASE HEIGHT : $\quad 0.00 \mathrm{~m}$

WIND SPEED $(\mathrm{h}=2 \mathrm{~m}): 1.7 \mathrm{~m} / \mathrm{s}$

STABILITY CLASS :E DEPOSITION VELOCITY : $1.00 \mathrm{~cm} / \mathrm{s}$

RECEPTOR HEIGHT $\quad: 0.0 \mathrm{~m}$ INVERSION LAYER HEIGHT $: 200.0 \mathrm{~m}$

SAMPLE TIME $\quad: 30.000 \mathrm{~min}$

MAXIMUM DOSE DISTANCE : $<0.10 \mathrm{~km}$ MAXIMUM CEDE $:>4.5 \mathrm{rem}$

$\mathrm{D}=0.03 \mathrm{~km}$

$\mathrm{DEP}=1.7 \mathrm{E}+00 \mathrm{uCi} / \mathrm{m}^{\wedge} 2$

$\mathrm{CHI}=1.7 \mathrm{E}-04(\mathrm{Ci}-\mathrm{s}) / \mathrm{m}^{\wedge} 3$

50-YR DOSE COMMITMENT:

SURFACE BONE $4.6 \mathrm{E}+02 \mathrm{rem}$ RED MARROW $3.8 \mathrm{E}+01 \mathrm{rem}$ LIVER $1.0 \mathrm{E}+02$ rem GONADS 5.7E+00 rem EFFECTIVE DOSE EQUIVALENT 2.6E+01 rem

\author{
$\mathrm{D}=0.10 \mathrm{~km}$ \\ $\mathrm{DEP}=2.9 \mathrm{E}-01 \mathrm{uCi} / \mathrm{m}^{\wedge} 2$ \\ $\mathrm{CHI}=2.9 \mathrm{E}-05(\mathrm{Ci}-\mathrm{s}) / \mathrm{m}^{\wedge} 3$ \\ 50-YR DOSE COMMITMENT: \\ SURFACE BONE 7.9E+01 rem \\ RED MARROW $\quad 6.5 \mathrm{E}+00 \mathrm{rem}$ \\ LIVER $1.8 \mathrm{E}+01 \mathrm{rem}$ \\ GONADS 9.7E-01 rem \\ EFFECTIVE DOSE \\ EQUIVALENT $4.5 \mathrm{E}+00 \mathrm{rem}$
}

\author{
$\mathrm{D}=0.23 \mathrm{~km}$ \\ $\mathrm{DEP}=6.8 \mathrm{E}-02 \mathrm{uCi} / \mathrm{m}^{\wedge} 2$ \\ $\mathrm{CHI}=6.8 \mathrm{E}-06(\mathrm{Ci}-\mathrm{s}) / \mathrm{m}^{\wedge} 3$ \\ 50-YR DOSE COMMITMENT: \\ SURFACE BONE $1.8 \mathrm{E}+01 \mathrm{rem}$ \\ RED MARROW 1.5E+00 rem \\ LIVER 4.1E+00 rem \\ GONADS 2.3E-01 rem \\ EFFECTIVE DOSE \\ EQUIVALENT $1.0 \mathrm{E}+00 \mathrm{rem}$
}


Title of Project EPHA for THM

Subject Radiological Consequence Assessment

Computer D. J. Richards

Date $\underline{09 / 11 / 96}$

Reviewed by $\underline{S}$. A. Henderson

Works Calculation 6

Sheet No. 36 of 166

Release Designation: 1-RD-81, Average

HOTSPOT 7.0 GENERAL PLUME 9-09-1996 20:15

RADIONUCLIDE : PU-238 Inhalation Class : W

HALFLIFE : 87.740 years

SOURCE TERM $: 8.1 \mathrm{E}-03 \mathrm{Ci}$

Source Term Width: $\quad 3 \mathrm{~m}$ Source Term Height: $3 \mathrm{~m}$

RELEASE FRACTION : $1.00 \mathrm{E}+00$

FILTER EFFICIENCY: $0.000000 \%$

EFFECTIVE RELEASE HEIGHT : $\quad 0.00 \mathrm{~m}$

WIND SPEED $(\mathrm{h}=2 \mathrm{~m}): 2.5 \mathrm{~m} / \mathrm{s}$

STABILITY CLASS :C DEPOSITION VELOCITY : $1.00 \mathrm{~cm} / \mathrm{s}$

RECEPTOR HEIGHT $\quad: 0.0 \mathrm{~m}$ INVERSION LAYER HEIGHT $: 500.0 \mathrm{~m}$

SAMPLE TIME $: 30.000 \mathrm{~min}$

MAXIMUM DOSE DISTANCE $:<0.10 \mathrm{~km}$ MAXIMUM CEDE $:>1.1 \mathrm{rem}$

$\mathrm{D}=0.03 \mathrm{~km}$

$\mathrm{DEP}=5.0 \mathrm{E}-01 \mathrm{uCi} / \mathrm{m}^{\wedge} 2$

$\mathrm{CHI}=5.0 \mathrm{E}-05(\mathrm{Ci}-\mathrm{s}) / \mathrm{m}^{\wedge} 3$

50-YR DOSE COMMITMENT:

SURFACE BONE $1.4 \mathrm{E}+02 \mathrm{rem}$ RED MARROW $1.1 \mathrm{E}+01 \mathrm{rem}$ LIVER $\quad 3.0 \mathrm{E}+01 \mathrm{rem}$ GONADS 1.7E+00 rem EFFECTIVE DOSE EQUTVALENT 7.7E+00 rem

\author{
$\mathrm{D}=0.10 \mathrm{~km}$ \\ $\mathrm{DEP}=6.9 \mathrm{E}-02 \mathrm{uCi} / \mathrm{m}^{\wedge} 2$ \\ $\mathrm{CHI}=6.9 \mathrm{E}-06(\mathrm{Ci}-\mathrm{s}) / \mathrm{m}^{\wedge} 3$ \\ 50-YR DOSE COMMITMENT: \\ SURFACE BONE 1.9E+01 rem \\ RED MARROW $1.5 \mathrm{E}+00 \mathrm{rem}$ \\ LIVER $\quad 4.1 \mathrm{E}+00 \mathrm{rem}$ \\ GONADS 2.3E-01 rem \\ EFFECTIVE DOSE \\ EQUIVALENT $1.1 \mathrm{E}+00 \mathrm{rem}$
}

\author{
$\mathrm{D}=0.10 \mathrm{~km}$ \\ $\mathrm{DEP}=6.7 \mathrm{E}-02 \mathrm{uCi} / \mathrm{m}^{\wedge} 2$ \\ $\mathrm{CHI}=6.7 \mathrm{E}-06(\mathrm{Ci}-\mathrm{s}) / \mathrm{m}^{\wedge} 3$ \\ 50-YR DOSE COMMITMENT: \\ SURFACE BONE $1.8 \mathrm{E}+01 \mathrm{rem}$ \\ RED MARROW 1.5E+00 rem \\ LIVER $\quad 4.0 \mathrm{E}+00 \mathrm{rem}$ \\ GONADS 2.2E-01 rem \\ EFFECTIVE DOSE \\ EQUIVALENT $1.0 \mathrm{E}+00 \mathrm{rem}$
}


Title of Project EPHA for THM

Subject Radiological Consequence Assessment

Computer D. J. Richards

Date $\underline{09 / 11 / 96}$

Reviewed by $\underline{S}$. A. Henderson

Works Calculation 6

Sheet No. 37 of 166

Release Designation: 1-RD-82, Adverse

HOTSPOT 7.0 GENERAL PLUME 9-10-1996 20:55

RADIONUCLIDE : PU-238 Inhalation Class : W

HALFLIFE $: 87.740$ years

SOURCE TERM $: 1.8 \mathrm{E}-02 \mathrm{Ci}$

Source Term Width: $\quad 3 \mathrm{~m}$ Source Term Height: $3 \mathrm{~m}$

RELEASE FRACTION : $1.00 \mathrm{E}+00$

FILTER EFFICIENCY: $0.000000 \%$

EFFECTIVE RELEASE HEIGHT : $\quad 0.00 \mathrm{~m}$

WIND SPEED $(\mathrm{h}=2 \mathrm{~m}): 1.7 \mathrm{~m} / \mathrm{s}$

STABILITY CLASS :E DEPOSITION VELOCITY : $1.00 \mathrm{~cm} / \mathrm{s}$

RECEPTOR HEIGHT $\quad: 0.0 \mathrm{~m}$ INVERSION LAYER HEIGHT $: 200.0 \mathrm{~m}$

SAMPLE TIME $\quad: 10.000 \mathrm{~min}$

MAXIMUM DOSE DISTANCE $:<0.10 \mathrm{~km}$ MAXIMUM CEDE $:>12 \mathrm{rem}$

$\mathrm{D}=0.03 \mathrm{~km}$

$\mathrm{DEP}=4.6 \mathrm{E}+00 \mathrm{uCi} / \mathrm{m}^{\wedge} 2$

$\mathrm{CHI}=4.6 \mathrm{E}-04(\mathrm{Ci}-\mathrm{s}) / \mathrm{m}^{\wedge} 3$

50-YR DOSE COMMITMENT:

SURFACE BONE $1.2 \mathrm{E}+03 \mathrm{rem}$ RED MARROW 1:0E+02 rem LIVER 2.8E+02 rem GONADS $1.5 \mathrm{E}+01 \mathrm{rem}$ EFFECTIVE DOSE EQUIVALENT $7.1 \mathrm{E}+01 \mathrm{rem}$
$\mathrm{D}=0.10 \mathrm{~km}$

$\mathrm{DEP}=7.9 \mathrm{E}-01 \mathrm{uCi} / \mathrm{m}^{\wedge} 2$

$\mathrm{CHI}=7.9 \mathrm{E}-05(\mathrm{Ci}-\mathrm{s}) / \mathrm{m}^{\wedge} 3$

50-YR DOSE COMMITMENT:

SURFACE BONE 2.1E+02 rem RED MARROW 1.8E+01 rem

LIVER 4.7E+01 rem GONADS $2.6 \mathrm{E}+00 \mathrm{rem}$ EFFECTIVE DOSE EQUTVALENT $1.2 \mathrm{E}+01 \mathrm{rem}$
$\mathrm{D}=0.38 \mathrm{~km}$

$\mathrm{DEP}=6.8 \mathrm{E}-02 \mathrm{uCi} / \mathrm{m}^{\wedge} 2$

$\mathrm{CHI}=6.8 \mathrm{E}-06(\mathrm{Ci}-\mathrm{s}) / \mathrm{m}^{\wedge} 3$

50-YR DOSE COMMITMENT:

SURFACE BONE $1.8 \mathrm{E}+01$ rem RED MARROW $1.5 \mathrm{E}+00 \mathrm{rem}$ LIVER $\quad 4.1 \mathrm{E}+00 \mathrm{rem}$ GONADS 2.3E-01 rem EFFECTIVE DOSE EQUTVALENT $1.0 \mathrm{E}+00 \mathrm{rem}$ 
Title of Project EPHA for THM

Subject Radiological Consequence Assessment

Computer D. J. Richards

Date $\underline{09 / 11 / 96}$

Reviewed by $\underline{\text { S. A. Hènderson }}$

Works Calculation 6

Sheet No. 38 of 166

Release Designation: 1-RD-82, Average

HOTSPOT 7.0 GENERAL PLUME 9-10-1996 20:56

RADIONUCLIDE : PU-238 Inhalation Class : W

HALFLIFE : 87.740 years

SOURCE TERM : $1.8 \mathrm{E}-02 \mathrm{Ci}$

Source Term Width: $\quad 3 \mathrm{~m}$ Source Term Height: $3 \mathrm{~m}$

RELEASE FRACTION : $1.00 \mathrm{E}+00$

FILTER EFFICIENCY: $0.000000 \%$

EFFECTIVE RELEASE HEIGHT : $\quad 0.00 \mathrm{~m}$

WIND SPEED $(\mathrm{h}=2 \mathrm{~m}): 2.5 \mathrm{~m} / \mathrm{s}$

STABILITY CLASS :C DEPOSITION VELOCITY $\quad: 1.00 \mathrm{~cm} / \mathrm{s}$

RECEPTOR HEIGHT $: 0.0 \mathrm{~m}$ INVERSION LAYER HEIGHT $: 500.0 \mathrm{~m}$

SAMPLE TIME $: 10.000 \mathrm{~min}$

MAXIMUM DOSE DISTANCE $:<0.10 \mathrm{~km}$ MAXIMUM CEDE $:>2.8 \mathrm{rem}$

$\mathrm{D}=0.03 \mathrm{~km}$

$\mathrm{DEP}=1.4 \mathrm{E}+00 \mathrm{uCi} / \mathrm{m}^{\wedge} 2$

$\mathrm{CHI}=1.4 \mathrm{E}-04(\mathrm{Ci}-\mathrm{s}) / \mathrm{m}^{\wedge} 3$

50-YR DOSE COMMITMENT:

SURFACE BONE 3.7E+02 rem RED MARROW $3.0 \mathrm{E}+01 \mathrm{rem}$ LIVER 8.1E+01 rem GONADS 4.5E+00 rem EFFECTIVE DOSE EQUIVALENT $2.1 \mathrm{E}+01 \mathrm{rem}$
$\mathrm{D}=0.10 \mathrm{~km}$
$\mathrm{DEP}=1.8 \mathrm{E}-01 \mathrm{uCi} / \mathrm{m}^{\wedge} 2$
$\mathrm{CHI}=1.8 \mathrm{E}-05(\mathrm{Ci}-\mathrm{s}) / \mathrm{m}^{\wedge} 3$
50-YR DOSE COMMITMENT:
SURFACE BONE 5.0E+01 rem RED MARROW 4.1E+00 rem LIVER 1.1E+01 rem GONADS 6.2E-01 rem EFFECTIVE DOSE EQUTVALENT $2.8 \mathrm{E}+00 \mathrm{rem}$

\author{
$\mathrm{D}=0.17 \mathrm{~km}$ \\ $\mathrm{DEP}=6.8 \mathrm{E}-02 \mathrm{uCi} / \mathrm{m}^{\wedge} 2$ \\ $\mathrm{CHI}=6.8 \mathrm{E}-06(\mathrm{Ci}-\mathrm{s}) / \mathrm{m}^{\wedge} 3$ \\ 50-YR DOSE COMMITMENT: \\ SURFACE BONE $1.8 \mathrm{E}+01 \mathrm{rem}$ \\ RED MARROW $1.5 \mathrm{E}+00 \mathrm{rem}$ \\ LIVER $\quad 4.1 \mathrm{E}+00 \mathrm{rem}$ \\ GONADS 2.3E-01 rem \\ EFFECTIVE DOSE \\ EQUTVALENT $\quad 1.0 \mathrm{E}+00 \mathrm{rem}$
}


Title of Project EPHA for THM

Subject Radiological Consequence Assessment

Computer D. J. Richards

Date $\underline{09 / 11 / 96}$

Reviewed by $\underline{\text { S. A. Henderson }}$

Works Calculation 6

Sheet No. 39 of 166

Release Designation: 1-RD-83, Adverse

HOTSPOT 7.0 GENERAL PLUME 9-09-1996 20:18

RADIONUCLIDE : PU-238 Inhalation Class : W

HALFLIFE $: 87.740$ years

SOURCE TERM $: 9.5 \mathrm{E}-04 \mathrm{Ci}$

Source Term Width: $3 \mathrm{~m}$ Source Term Height: $3 \mathrm{~m}$

RELEASE FRACTION : $1.00 \mathrm{E}+00$

FILTER EFFICIENCY: $0.000000 \%$

EFFECTIVE RELEASE HEIGHT : $\quad 0.00 \mathrm{~m}$

WIND SPEED $(\mathrm{h}=2 \mathrm{~m}): 1.7 \mathrm{~m} / \mathrm{s}$

STABILITY CLASS :E DEPOSITION VELOCITY $: 1.00 \mathrm{~cm} / \mathrm{s}$

RECEPTOR HEIGHT $\quad: 0.0 \mathrm{~m}$ INVERSION LAYER HEIGHT $: 200.0 \mathrm{~m}$

SAMPLE TIME $\quad: 30.000 \mathrm{~min}$

MAXIMUM DOSE DISTANCE $:<0.10 \mathrm{~km}$ MAXIMUM CEDE $:>0.524 \mathrm{rem}$

$\mathrm{D}=0.03 \mathrm{~km}$

$\mathrm{DEP}=2.0 \mathrm{E}-01 \mathrm{uCi} / \mathrm{m}^{\wedge} 2$

$\mathrm{CHI}=2.0 \mathrm{E}-05(\mathrm{Ci}-\mathrm{s}) / \mathrm{m}^{\wedge} 3$

50-YR DOSE COMMITMENT:

SURFACE BONE 5.4E+01 rem RED MARROW $4.5 \mathrm{E}+00 \mathrm{rem}$

LIVER $1.2 \mathrm{E}+01 \mathrm{rem}$

GONADS 6.7E-01 rem

EFFECTIVE DOSE

EQUIVALENT $3.1 \mathrm{E}+00 \mathrm{rem}$

$$
\begin{aligned}
& \mathrm{D}=0.07 \mathrm{~km} \\
& \mathrm{DEP}=6.9 \mathrm{E}-02 \mathrm{uCi} / \mathrm{m}^{\wedge} 2 \\
& \mathrm{CHI}=6.9 \mathrm{E}-06(\mathrm{Ci}-\mathrm{s}) / \mathrm{m}^{\wedge} 3 \\
& \text { 50-YR DOSE COMMITMENT: } \\
& \text { SURFACE BONE } 1.8 \mathrm{E}+01 \mathrm{rem} \\
& \text { RED MARROW } 1.5 \mathrm{E}+00 \mathrm{rem} \\
& \text { LIVER } \quad 4.1 \mathrm{E}+00 \mathrm{rem} \\
& \text { GONADS } \quad 2.3 \mathrm{E}-01 \mathrm{rem} \\
& \text { EFFECTIVE DOSE } \\
& \text { EQUTVALENT } \quad 1.0 \mathrm{E}+00 \mathrm{rem}
\end{aligned}
$$

$\mathrm{D}=0.10 \mathrm{~km}$

$\mathrm{DEP}=3.4 \mathrm{E}-02 \mathrm{uCi} / \mathrm{m}^{\wedge} 2$

$\mathrm{CHI}=3.4 \mathrm{E}-06(\mathrm{Ci}-\mathrm{s}) / \mathrm{m}^{\wedge} 3$

50-YR DOSE COMMITMENT:

SURFACE BONE 9.2E+00 rem RED MARROW 7.6E-01 rem LIVER $\quad 2.0 \mathrm{E}+00 \mathrm{rem}$ GONADS 1.1E-01 rem EFFECTIVE DOSE EQUIVALENT 5.2E-01 rem 
Title of Project EPHA for THM

Subject Radiological Consequence Assessment

Computer D. J. Richards

Release Designation: 1-RD-83, Average

HOTSPOT 7.0 GENERAL PLUME 9-09-1996 20:18

RADIONUCLIDE : PU-238 Inhalation Class : W

HALFLIFE : 87.740 years

SOURCE TERM : $9.5 \mathrm{E}-04 \mathrm{Ci}$

Source Term Width: $3 \mathrm{~m}$ Source Term Height: $3 \mathrm{~m}$

RELEASE FRACTION : $1.00 \mathrm{E}+00$

FILTER EFFICIENCY: $0.000000 \%$

EFFECTIVE RELEASE HEIGHT : $\quad 0.00 \mathrm{~m}$

WIND SPEED $(\mathrm{h}=2 \mathrm{~m}): 2.5 \mathrm{~m} / \mathrm{s}$

STABILITY CLASS :C DEPOSITION VELOCITY : $1.00 \mathrm{~cm} / \mathrm{s}$

RECEPTOR HEIGHT $\quad: 0.0 \mathrm{~m}$ INVERSION LAYER HEIGHT $: 500.0 \mathrm{~m}$

SAMPLE TIME $\quad: 30.000 \mathrm{~min}$

MAXIMUM DOSE DISTANCE $:<0.10 \mathrm{~km}$ MAXIMUM CEDE $:>0.123 \mathrm{rem}$

$\mathrm{D}=0.03 \mathrm{~km}$

$\mathrm{DEP}=5.9 \mathrm{E}-02 \mathrm{uCi} / \mathrm{m}^{\wedge} 2$

$\mathrm{CHI}=5.9 \mathrm{E}-06(\mathrm{Ci}-\mathrm{s}) / \mathrm{m}^{\wedge} 3$

50-YR DOSE COMMITMENT:

SURFACE BONE $1.6 \mathrm{E}+01 \mathrm{rem}$ RED MARROW $1.3 E+00$ rem LIVER $\quad 3.5 \mathrm{E}+00 \mathrm{rem}$ GONADS 2.0E-01 rem EFFECTIVE DOSE

EQUIVALENT 9.0E-01 rem
$D=0.10 \mathrm{~km}$

$\mathrm{DEP}=8.0 \mathrm{E}-03 \mathrm{uCi} / \mathrm{m}^{\wedge} 2$

$\mathrm{CHI}=8.0 \mathrm{E}-07(\mathrm{Ci}-\mathrm{s}) / \mathrm{m}^{\wedge} 3$

50-YR DOSE COMMITMENT:

SURFACE BONE $2.2 \mathrm{E}+00 \mathrm{rem}$ RED MARROW $1.8 \mathrm{E}-01 \mathrm{rem}$ LIVER 4.8E-01 rem GONADS 2.7E-02 rem EFFECTIVE DOSE EQUIVALENT $1.2 \mathrm{E}-01 \mathrm{rem}$ 
Title of Project EPHA for THM

Reviewed by S. A. Henderson

Subject Radiological Consequence Assessment

Sheet No. 41 of 166

Release Designation: 1-RD-84, Adverse

HOTSPOT 7.0 GENERAL PLUME 9-27-1996 19:08

RADIONUCLIDE : PU-238 Inhalation Class : W

HALFLIFE : 87.740 years

SOURCE TERM : $2.0 \mathrm{E}-03 \mathrm{Ci}$

Source Term Width: $3 \mathrm{~m}$ Source Term Height: $3 \mathrm{~m}$

RELEASE FRACTION : $1.00 \mathrm{E}+00$

FILTER EFFICIENCY: $0.000000 \%$

EFFECTIVE RELEASE HEIGHT : $\quad 0.00 \mathrm{~m}$

WIND SPEED $(\mathrm{h}=2 \mathrm{~m}): 1.7 \mathrm{~m} / \mathrm{s}$

STABILITY CLASS :E DEPOSITION VELOCITY $\quad: 1.00 \mathrm{~cm} / \mathrm{s}$

RECEPTOR HEIGHT $\quad: 0.0 \mathrm{~m}$ INVERSION LAYER HEIGHT $: 200.0 \mathrm{~m}$

SAMPLE TIME $\quad: 10.000 \mathrm{~min}$

MAXIMUM DOSE DISTANCE $:<0.10 \mathrm{~km}$. MAXIMUM CEDE $:>1.4 \mathrm{rem}$

$\mathrm{D}=0.03 \mathrm{~km}$

$\mathrm{DEP}=5.4 \mathrm{E}-01 \mathrm{uCi} / \mathrm{m}^{\wedge} 2$

$\mathrm{CHI}=5.4 \mathrm{E}-05(\mathrm{Ci}-\mathrm{s}) / \mathrm{m}^{\wedge} 3$

50-YR DOSE COMMITMENT:

SURFACE BONE $1.5 \mathrm{E}+02$ rem RED MARROW 1.2E+01 rem

LIVER 3.2E+01 rem

GONADS $1.8 \mathrm{E}+00 \mathrm{rem}$

EFFECTIVE DOSE

EQUIVALENT $8.2 \mathrm{E}+00 \mathrm{rem}$

\author{
$\mathrm{D}=0.10 \mathrm{~km}$ \\ $\mathrm{DEP}=9.2 \mathrm{E}-02 \mathrm{uCi} / \mathrm{m}^{\wedge} 2$ \\ $\mathrm{CHI}=9.2 \mathrm{E}-06(\mathrm{Ci}-\mathrm{s}) / \mathrm{m}^{\wedge} 3$ \\ 50-YR DOSE COMMITMENT: \\ SURFACE BONE 2.5E+01 rem \\ RED MARROW $2.0 \mathrm{E}+00 \mathrm{rem}$ \\ LIVER $\quad 5.5 \mathrm{E}+00 \mathrm{rem}$ \\ GONADS 3.1E-01 rem \\ EFFECTTVE DOSE \\ EQUTVALENT $1.4 \mathrm{E}+00 \mathrm{rem}$
}
$\mathrm{D}=0.12 \mathrm{~km}$
$\mathrm{DEP}=6.7 \mathrm{E}-02 \mathrm{uCi} / \mathrm{m}^{\wedge} 2$
$\mathrm{CHI}=6.7 \mathrm{E}-06(\mathrm{Ci}-\mathrm{s}) / \mathrm{m}^{\wedge} 3$
50-YR DOSE COMMITMENT:
SURFACE BONE $1.8 \mathrm{E}+01 \mathrm{rem}$ RED MARROW $1.5 \mathrm{E}+00 \mathrm{rem}$ LIVER 4.0E $+00 \mathrm{rem}$ GONADS 2.2E-01 rem EFFECTIVE DOSE
EQUTVALENT $1.0 \mathrm{E}+00 \mathrm{rem}$ 
Title of Project EPHA for THM

Subject Radiological Consequence Assessment

Computer D. J. Richards

Date $\underline{09 / 11 / 96}$

Reviewed by $\underline{\text { S. A. Henderson }}$ Works Calculation 6

Sheet No. 42 of 166

Release Designation: 1-RD-84, Average

HOTSPOT 7.0 GENERAL PLUME 9-27-1996 19:11

RADIONUCLIDE: PU-238 Inhalation Class : W

HALFLIFE $: 87.740$ years

SOURCE TERM : 2.0E-03 Ci

Source Term Width: $3 \mathrm{~m}$ Source Term Height: $3 \mathrm{~m}$

RELEASE FRACTION : $1.00 \mathrm{E}+00$

FILTER EFFICIENCY: $0.000000 \%$

EFFECTIVE RELEASE HEIGHT : $\quad 0.00 \mathrm{~m}$

WIND SPEED $(\mathrm{h}=2 \mathrm{~m}): 2.5 \mathrm{~m} / \mathrm{s}$

STABILITY CLASS :C DEPOSITION VELOCITY : $1.00 \mathrm{~cm} / \mathrm{s}$

RECEPTOR HEIGHT $\quad: 0.0 \mathrm{~m}$ INVERSION LAYER HEIGHT $: 500.0 \mathrm{~m}$

SAMPLE TIME $\quad: 10.000 \mathrm{~min}$

MAXIMUM DOSE DISTANCE $:<0.10 \mathrm{~km}$ MAXIMUM CEDE $:>0.330 \mathrm{rem}$

$\mathrm{D}=0.03 \mathrm{~km}$

$\mathrm{DEP}=1.6 \mathrm{E}-01 \mathrm{uCi} / \mathrm{m}^{\wedge} 2$

$\mathrm{CHI}=1.6 \mathrm{E}-05(\mathrm{Ci}-\mathrm{s}) / \mathrm{m}^{\wedge} 3$

50-YR DOSE COMMITMENT:

SURFACE BONE 4.3E+01 rem RED MARROW $3.5 E+00$ rem

LIVER $\quad 9.5 \mathrm{E}+00 \mathrm{rem}$ GONADS 5.3E-01 rem EFFECTIVE DOSE EQUIVALENT $2.4 \mathrm{E}+00$ rem
$\mathrm{D}=0.05 \mathrm{~km}$

$\mathrm{DEP}=6.7 \mathrm{E}-02 \mathrm{uCi} / \mathrm{m}^{\wedge} 2$

$\mathrm{CHI}=6.7 \mathrm{E}-06(\mathrm{Ci}-\mathrm{s}) / \mathrm{m}^{\wedge} 3$

50-YR DOSE COMMITMENT:

SURFACE BONE 1.8E+01 rem RED MARROW $1.5 E+00$ rem LIVER 4.0E+00 rem GONADS 2.2E-01 rem EFFECTIVE DOSE EQUIVALENT 1.0E+00 rem
$\mathrm{D}=0.10 \mathrm{~km}$

$\mathrm{DEP}=2.2 \mathrm{E}-02 \mathrm{uCi} / \mathrm{m}^{\wedge} 2$

$\mathrm{CHI}=2.2 \mathrm{E}-06(\mathrm{Ci}-\mathrm{s}) / \mathrm{m}^{\wedge} 3$

50-YR DOSE COMMITMENT:

SURFACE BONE 5.8E+00 rem RED MARROW 4.8E-01 rem LIVER 1.3E+00 rem GONADS 7.2E-02 rem EFFECTIVE DOSE

EQUIVALENT 3.3E-01 rem 
Title of Project EPHA for THM

Subject Radiological Consequence Assessment

Computer D. J. Richards

Date $09 / 11 / 96$

Reviewed by S. A. Henderson

Works Calculation 6

Sheet No. 43 of 166

Release Designation: 1-RD-85, Adverse

HOTSPOT 7.0 TRITIUM RELEASE 9-28-1996 20:30

TOTAL TRITIUM RELEASE : $9.1 \mathrm{E}-04$ curies $100.000 \%$ TRITIUM OXIDE

Dose Results Include Skin Absorption

Source Term Width: $3 \mathrm{~m}$ Source Term Height: $3 \mathrm{~m}$ EFFECTIVE RELEASE HEIGHT : $\quad 0.00 \mathrm{~m}$

WIND SPEED $(\mathrm{h}=2 \mathrm{~m}): 1.7 \mathrm{~m} / \mathrm{s}$

STABILITY CLASS :E DEPOSITION VELOCITY : $0.0 \mathrm{E}+00 \mathrm{~cm} / \mathrm{s}$

RECEPTOR HEIGHT $\quad: 0.0 \mathrm{~m}$ INVERSION LAYER HEIGHT $: 200.0 \mathrm{~m}$

SAMPLE TIME $\quad: 30.000 \mathrm{~min}$

MAXIMUM DOSE DISTANCE $:<0.10 \mathrm{~km}$ MAXIMUM CEDE $:>1.5 E-07 \mathrm{rem}$

$\mathrm{D}=0.03 \mathrm{~km}$

$\mathrm{CHI}=2.3 \mathrm{E}-05(\mathrm{Ci}-\mathrm{s}) / \mathrm{m}^{\wedge} 3$

50-YR DOSE COMMITMENT:

EFFECTIVE DOSE

EQUIVALENT 7.1E-07 rem
$\mathrm{D}=0.10 \mathrm{~km}$

$\mathrm{CHI}=4.7 \mathrm{E}-06(\mathrm{Ci}-\mathrm{s}) / \mathrm{m}^{\wedge} 3$

50-YR DOSE COMMITMENT:

EFFECTIVE DOSE

- EQUTVALENT 1.5E-07 rem 
Title of Project EPHA for THM

Subject Radiological Consequence Assessment

Computer D. J. Richards
Reviewed by S. A. Henderson Works Calculation 6

Sheet No. 44 of 166

Release Designation: 1-RD-85, Average

HOTSPOT 7.0 TRITIUM RELEASE $\quad$ 9-28-1996 20:31

TOTAL TRITIUM RELEASE : $9.1 \mathrm{E}-04$ curies $100.000 \%$ TRITIUM OXIDE

Dose Results Include Skin Absorption

Source Term Width: $\quad 3 \mathrm{~m}$ Source Term Height: $3 \mathrm{~m}$

EFFECTIVE RELEASE HEIGHT : $\quad 0.00 \mathrm{~m}$

WIND SPEED $(\mathrm{h}=2 \mathrm{~m}): 2.5 \mathrm{~m} / \mathrm{s}$

STABILITY CLASS :C DEPOSITION VELOCITY : $0.0 \mathrm{E}+00 \mathrm{~cm} / \mathrm{s}$

RECEPTOR HEIGHT $\quad: 0.0 \mathrm{~m}$ INVERSION LAYER HEIGHT $: 200.0 \mathrm{~m}$

SAMPLE TIME $: 30.000 \mathrm{~min}$

MAXIMUM DOSE DISTANCE $:<0.10 \mathrm{~km}$ MAXIMUM CEDE $:>2.6 \mathrm{E}-08 \mathrm{rem}$

$\mathrm{D}=0.03 \mathrm{~km}$

$\mathrm{CHI}=5.9 \mathrm{E}-06(\mathrm{Ci}-\mathrm{s}) / \mathrm{m}^{\wedge} 3$

50-YR DOSE COMMITMENT:

EFFECTIVE DOSE

EQUIVALENT $1.8 \mathrm{E}-07$ rem
$\mathrm{D}=0.10 \mathrm{~km}$

$\mathrm{CHI}=8.5 \mathrm{E}-07(\mathrm{Ci}-\mathrm{s}) / \mathrm{m}^{\wedge} 3$

50-YR DOSE COMMITMENT:

EFFECTIVE DOSE

EQUIVALENT 2.6E-08 rem 
Title of Project EPHA for THM

Subject Radiological Consequence Assessment

Computer D. J. Richards

Date $\underline{09 / 11 / 96}$

Reviewed by $\mathrm{S}$. A. Henderson

Works Calculation 6

Sheet No. 45 of 166

Release Designation: 1-RD-86, Adverse

HOTSPOT 7.0 TRITIUM RELEASE 9-28-1996 20:32

TOTAL TRITIUM RELEASE : $2.0 \mathrm{E}-03$ curies $100.000 \%$ TRITIUM OXIDE

Dose Results Include Skin Absorption

Source Term Width: $3 \mathrm{~m}$ Source Term Height: $3 \mathrm{~m}$

EFFECTIVE RELEASE HEIGHT : $\quad 0.00 \mathrm{~m}$

WIND SPEED $(\mathrm{h}=2 \mathrm{~m}): 1.7 \mathrm{~m} / \mathrm{s}$

STABILITY CLASS :E DEPOSITION VELOCITY $: 0.0 \mathrm{E}+00 \mathrm{~cm} / \mathrm{s}$

RECEPTOR HEIGHT $: 0.0 \mathrm{~m}$ INVERSION LAYER HEIGHT $: 200.0 \mathrm{~m}$

SAMPLE TIME $: 10.000 \mathrm{~min}$

MAXIMUM DOSE DISTANCE $:<0.10 \mathrm{~km}$ MAXIMUM CEDE $:>3.9 \mathrm{E}-07 \mathrm{rem}$

$\mathrm{D}=0.03 \mathrm{~km}$

$\mathrm{CHI}=6.2 \mathrm{E}-05(\mathrm{Ci}-\mathrm{s}) / \mathrm{m}^{\wedge} 3$

50-YR DOSE COMMITMENT:

EFFECTIVE DOSE

EQUIVALENT 1.9E-06 rem
$\mathrm{D}=0.10 \mathrm{~km}$

$\mathrm{CHI}=1.3 \mathrm{E}-05(\mathrm{Ci}-\mathrm{s}) / \mathrm{m}^{\wedge} 3$

50-YR DOSE COMMITMENT:

EFFECTIVE DOSE

EQUTVALENT 3.9E-07 rem 
Title of Project EPHA for THM

-

Subject Radiological Consequence Assessment

Computer D. J. Richards

Release Designation: 1-RD-86, Average

HOTSPOT 7.0 TRITIUM RELEASE 9-28-1996 20:32

TOTAL TRITIUM RELEASE : $2.0 \mathrm{E}-03$ curies $100.000 \%$ TRITIUM OXIDE

Dose Results Include Skin Absorption

Source Term Width: $\quad 3 \mathrm{~m}$ Source Term Height: $3 \mathrm{~m}$

EFFECTIVE RELEASE HEIGHT : $\quad 0.00 \mathrm{~m}$

WIND SPEED $(\mathrm{h}=2 \mathrm{~m}): 2.5 \mathrm{~m} / \mathrm{s}$

STABILITY CLASS :C DEPOSITION VELOCITY $: 0.0 \mathrm{E}+00 \mathrm{~cm} / \mathrm{s}$

RECEPTOR HEIGHT $: 0.0 \mathrm{~m}$ INVERSION LAYER HEIGHT $: 200.0 \mathrm{~m}$

SAMPLE TIME $\quad: 10.000 \mathrm{~min}$

MAXIMUM DOSE DISTANCE $:<0.10 \mathrm{~km}$ MAXIMUM CEDE $:>7.1 \mathrm{E}-08 \mathrm{rem}$

$\mathrm{D}=0.03 \mathrm{~km}$

$\mathrm{CHI}=1.6 \mathrm{E}-05(\mathrm{Ci}-\mathrm{s}) / \mathrm{m}^{\wedge} 3$

50-YR DOSE COMMITMENT:

EFFECTIVE DOSE

EQUTVALENT 5.0E-07 rem
$\mathrm{D}=0.10 \mathrm{~km}$

$\mathrm{CHI}=2.3 \mathrm{E}-06(\mathrm{Ci}-\mathrm{s}) / \mathrm{m}^{\wedge} 3$

50-YR DOSE COMMITMENT:

EFFECTIVE DOSE

EQUIVALENT $7.1 \mathrm{E}-08 \mathrm{rem}$ 
Title of Project EPHA for THM

Subject Radiological Consequence Assessment

Computer D. J. Richards
Date $\underline{09 / 11 / 96}$
Reviewed by S. A. Henderson Works Calculation 6

Sheet No. 47 of 166

Release Designation: 1-RD-87, Adverse

HOTSPOT 7.0 TRITIUM RELEASE 9-28-1996 20:38

TOTAL TRITIUM RELEASE : $7.7 \mathrm{E}-04$ curies $100.000 \%$ TRITIUM OXIDE

Dose Results Include Skin Absorption

Source Term Width: $3 \mathrm{~m}$ Source Term Height: $3 \mathrm{~m}$

EFFECTIVE RELEASE HEIGHT : $\quad 0.00 \mathrm{~m}$

WIND SPEED ( $\mathrm{h}=2 \mathrm{~m}): 1.7 \mathrm{~m} / \mathrm{s}$

STABILITY CLASS :E DEPOSITION VELOCITY $: 0.0 \mathrm{E}+00 \mathrm{~cm} / \mathrm{s}$

RECEPTOR HEIGHT $: 0.0 \mathrm{~m}$ INVERSION LAYER HEIGHT $: 200.0 \mathrm{~m}$

SAMPLE TIME $\quad: 30.000 \mathrm{~min}$

MAXIMUM DOSE DISTANCE $:<0.10 \mathrm{~km}$ MAXIMUM CEDE :> $1.2 \mathrm{E}-07 \mathrm{rem}$

$\mathrm{D}=0.03 \mathrm{~km}$

$\mathrm{D}=0.10 \mathrm{~km}$

$\mathrm{CHI}=1.9 \mathrm{E}-05(\mathrm{Ci}-\mathrm{s}) / \mathrm{m}^{\wedge} 3$

$\mathrm{CHI}=4.0 \mathrm{E}-06(\mathrm{Ci}-\mathrm{s}) / \mathrm{m}^{\wedge} 3$

50-YR DOSE COMMITMENT:

50-YR DOSE COMMITMENT:

EFFECTIVE DOSE

EFFECTIVE DOSE

EQUIVALENT 6.0E-07 rem

EQUIVALENT 1.2E-07 rem 
Title of Project EPHA for THM

Subject Radiological Consequence Assessment

Computer D. J. Richards

Release Designation: 1-RD-87, Average

HOTSPOT 7.0 TRITIUM RELEASE 9-28-1996 20:38

TOTAL TRITIUM RELEASE : $7.7 \mathrm{E}-04$ curies $100.000 \%$ TRITIUM OXIDE

Dose Results Include Skin Absorption

Source Term Width: $3 \mathrm{~m}$ Source Term Height: $3 \mathrm{~m}$ EFFECTIVE RELEASE HEIGHT : $\quad 0.00 \mathrm{~m}$

WIND SPEED $(\mathrm{h}=2 \mathrm{~m}): 2.5 \mathrm{~m} / \mathrm{s}$

$$
\begin{array}{lll}
\text { STABILITY CLASS } & : \text { C. DEPOSITION VELOCITY }: 0.0 \mathrm{E}+00 \mathrm{~cm} / \mathrm{s} \\
\text { RECEPTOR HEIGHT } & : 0.0 \mathrm{~m} \text { INVERSION LAYER HEIGHT }: 200.0 \mathrm{~m} \\
\text { SAMPLE TIME } & : 30.000 \mathrm{~min} \\
\text { MAXIMUM DOSE DISTANCE }:<0.10 \mathrm{~km} \text { MAXIMUM CEDE }:>2.2 \mathrm{E}-08 \mathrm{rem}
\end{array}
$$

$\mathrm{D}=0.03 \mathrm{~km}$

$\mathrm{CHI}=5.0 \mathrm{E}-06(\mathrm{Ci}-\mathrm{s}) / \mathrm{m}^{\wedge} 3$

50-YR DOSE COMMITMENT:

EFFECTIVE DOSE

EQUTVALENT $1.6 \mathrm{E}-07 \mathrm{rem}$
$\mathrm{D}=0.10 \mathrm{~km}$

$\mathrm{CHI}=7.2 \mathrm{E}-07(\mathrm{Ci}-\mathrm{s}) / \mathrm{m}^{\wedge} 3$

50-YR DOSE COMMITMENT:

EFFECTIVE DOSE

EQUTVALENT 2.2E-08 rem 
Title of Project EPHA for THM

Subject Radiological Consequence Assessment

Computer D. J. Richards

Release Designation: 1-RD-88, Adverse

HOTSPOT 7.0 TRITIUM RELEASE $\quad$ 9-28-1996 20:36

TOTAL TRITIUM RELEASE : 1.7 E-03 curies $100.000 \%$ TRITIUM OXIDE

Dose Results Include Skin Absorption

Source Term Width: $3 \mathrm{~m}$ Source Term Height: $3 \mathrm{~m}$

EFFECTIVE RELEASE HEIGHT : $\quad 0.00 \mathrm{~m}$

WIND SPEED $(\mathrm{h}=2 \mathrm{~m}): 1.7 \mathrm{~m} / \mathrm{s}$

STABILITY CLASS :E DEPOSITION VELOCITY $: 0.0 \mathrm{E}+00 \mathrm{~cm} / \mathrm{s}$

RECEPTOR HEIGHT $\quad: 0.0 \mathrm{~m}$ INVERSION LAYER HEIGHT $: 200.0 \mathrm{~m}$

SAMPLE TIME $\quad: 10.000 \mathrm{~min}$

MAXIMUM DOSE DISTANCE $:<0.10 \mathrm{~km}$ MAXIMUM CEDE $:>3.3 \mathrm{E}-07 \mathrm{rem}$

$\mathrm{D}=0.03 \mathrm{~km}$

$\mathrm{CHI}=5.2 \mathrm{E}-05(\mathrm{Ci}-\mathrm{s}) / \mathrm{m}^{\wedge} 3$

50-YR DOSE COMMITMENT:

EFFECTIVE DOSE

EQUTVALENT 1.6E-06 rem
$\mathrm{D}=0.10 \mathrm{~km}$

$\mathrm{CHI}=1.1 \mathrm{E}-05(\mathrm{Ci}-\mathrm{s}) / \mathrm{m}^{\wedge} 3$

50-YR DOSE COMMITMENT:

EFFECTIVE DOSE

EQUTVALENT 3.3E-07 rem 
Title of Project EPHA for THM

Subject Radiological Consequence Assessment

Computer D. J. Richards

Date $\underline{09 / 11 / 96}$

Reviewed by S. A. Henderson

Works Calculation 6

Sheet No. 50 of 166

Release Designation: 1-RD-88, Average

HOTSPOT 7.0 TRITIUM RELEASE 9-28-1996 20:37

TOTAL TRITIUM RELEASE : $1.7 \mathrm{E}-03$ curies $100.000 \%$ TRITIUM OXIDE

Dose Results Include Skin Absorption

Source Term Width: $3 \mathrm{~m}$ Source Term Height: $3 \mathrm{~m}$

EFFECTIVE RELEASE HEIGHT : $\quad 0.00 \mathrm{~m}$

WIND SPEED $(\mathrm{h}=2 \mathrm{~m}): 2.5 \mathrm{~m} / \mathrm{s}$

STABILITY CLASS :C DEPOSITION VELOCITY $: 0.0 \mathrm{E}+00 \mathrm{~cm} / \mathrm{s}$

RECEPTOR HEIGHT $\quad: 0.0 \mathrm{~m}$ INVERSION LAYER HEIGHT $: 200.0 \mathrm{~m}$

SAMPLE TIME $\quad: 10.000 \mathrm{~min}$

MAXIMUM DOSE DISTANCE $:<0.10 \mathrm{~km}$ MAXIMUM CEDE $:>6.0 \mathrm{E}-08 \mathrm{rem}$
$\mathrm{D}=0.03 \mathrm{~km}$
$\mathrm{D}=0.10 \mathrm{~km}$
$\mathrm{CHI}=1.3 \mathrm{E}-05(\mathrm{Ci}-\mathrm{s}) / \mathrm{m}^{\wedge} 3$
$\mathrm{CHI}=1.9 \mathrm{E}-06(\mathrm{Ci}-\mathrm{s}) / \mathrm{m}^{\wedge} 3$
50-YR DOSE COMMTTMENT:
50-YR DOSE COMMITMENT:
EFFECTIVE DOSE
EFFECTIVE DOSE
EQUIVALENT 4.2E-07 rem
EQUTVALENT $6.0 \mathrm{E}-08 \mathrm{rem}$ 
Title of Project EPHA for THM

Subject Radiological Consequence Assessment

Computer D. J. Richards
Reviewed by $\underline{\text { S. A. Henderson }}$

Works Calculation 6

Sheet No. 51 of 166

Release Designation: 1-RD-89, Adverse

HOTSPOT 7.0 GEṆERAL PLUME 9-09-1996 20:45

RADIONUCLIDE : PU-238 Inhalation Class : W

HALFLIFE $: 87.740$ years

SOURCE TERM : $5.5 \mathrm{E}-02 \mathrm{Ci}$

Source Term Width: $3 \mathrm{~m}$ Source Term Height: $3 \mathrm{~m}$

RELEASE FRACTION : $1.00 \mathrm{E}+00$

FILTER EFFICIENCY: $0.000000 \%$

EFFECTIVE RELEASE HEIGHT : $\quad 0.00 \mathrm{~m}$

WIND SPEED $(\mathrm{h}=2 \mathrm{~m}): 1.7 \mathrm{~m} / \mathrm{s}$

STABILITY CLASS :E DEPOSITION VELOCITY $: 1.00 \mathrm{~cm} / \mathrm{s}$

RECEPTOR HEIGHT $: 0.0 \mathrm{~m}$ INVERSION LAYER HEIGHT $: 200.0 \mathrm{~m}$

SAMPLE TIME $\quad: \quad 30.000 \mathrm{~min}$

MAXIMUM DOSE DISTANCE $:<0.10 \mathrm{~km}$ MAXIMUM CEDE $:>\quad 30 \mathrm{rem}$

$\mathrm{D}=0.03 \mathrm{~km}$

$\mathrm{DEP}=1.2 \mathrm{E}+01 \mathrm{uCi} / \mathrm{m}^{\wedge} 2$

$\mathrm{CHI}=1.2 \mathrm{E}-03(\mathrm{Ci}-\mathrm{s}) / \mathrm{m}^{\wedge} 3$

50-YR DOSE COMMITMENT:

SURFACE BONE 3.1E+03 rem

RED MARROW $2.6 \mathrm{E}+02 \mathrm{rem}$

LIVER 7.0E+02 rem

GONADS 3.9E+01 rem

EFFECTIVE DOSE

EQUIVALENT $1.8 \mathrm{E}+02$ rem

\author{
$\mathrm{D}=0.10 \mathrm{~km}$ \\ $\mathrm{DEP}=2.0 \mathrm{E}+00 \mathrm{uCi} / \mathrm{m}^{\wedge} 2$ \\ $\mathrm{CHI}=2.0 \mathrm{E}-04(\mathrm{Ci}-\mathrm{s}) / \mathrm{m}^{\wedge} 3$ \\ 50-YR DOSE COMMITMENT: \\ SURFACE BONE 5.4E+02 rem \\ RED MARROW $4.4 \mathrm{E}+01 \mathrm{rem}$ \\ LIVER 1.2E+02 rem \\ GONADS $6.6 \mathrm{E}+00 \mathrm{rem}$ \\ EFFECTIVE DOSE \\ EQUIVALENT $3.0 \mathrm{E}+01 \mathrm{rem}$
}

$\mathrm{D}=0.63 \mathrm{~km}$

$\mathrm{DEP}=6.7 \mathrm{E}-02 \mathrm{uCi} / \mathrm{m}^{\wedge} 2$

$\mathrm{CHI}=6.7 \mathrm{E}-06(\mathrm{Ci}-\mathrm{s}) / \mathrm{m}^{\wedge} 3$

50-YR DOSE COMMITMENT:

SURFACE BONE 1.8E+01 rem RED MARROW $1.5 \mathrm{E}+00 \mathrm{rem}$ LIVER $\quad 4.0 \mathrm{E}+00 \mathrm{rem}$ GONADS 2.2E-01 rem EFFECTIVE DOSE EQUTVALENT $1.0 \mathrm{E}+00 \mathrm{rem}$ 
Title of Project EPHA for THM

Subject Radiological Consequence Assessment

Computer D. J. Richards

Date $\underline{09 / 11 / 96}$

Reviewed by $\underline{\text { S. A. Henderson }}$

Works Calculation 6

Sheet No. 52 of 166

Release Designation: 1-RD-89, Average

HOTSPOT 7.0 GENERAL PLUME 9-09-1996 20:44

RADIONUCLIDE : PU-238 Inhalation Class : W

HALFLIFE : 87.740 years

SOURCE TERM : $5.5 \mathrm{E}-02 \mathrm{Ci}$

Source Term Width: $\quad 3 \mathrm{~m}$ Source Term Height: $3 \mathrm{~m}$

RELEASE FRACTION : $1.00 \mathrm{E}+00$

FILTER EFFICIENCY: $0.000000 \%$

EFFECTIVE RELEASE HEIGHT : $0.00 \mathrm{~m}$

WIND SPEED $(\mathrm{h}=2 \mathrm{~m}): 2.5 \mathrm{~m} / \mathrm{s}$

STABILITY CLASS :C DEPOSITION VELOCITY $: 1.00 \mathrm{~cm} / \mathrm{s}$

RECEPTOR HEIGHT $\quad: 0.0 \mathrm{~m}$ INVERSION LAYER HEIGHT $: 500.0 \mathrm{~m}$

SAMPLE TIME $\quad: 30.000 \mathrm{~min}$

MAXIMUM DOSE DISTANCE $:<0.10 \mathrm{~km}$ MAXIMUM CEDE $:>7.1 \mathrm{rem}$

$\mathrm{D}=0.03 \mathrm{~km}$

$\mathrm{DEP}=3.4 \mathrm{E}+00 \mathrm{uCi} / \mathrm{m}^{\wedge} 2$

$\mathrm{CHI}=3.4 \mathrm{E}-04(\mathrm{Ci}-\mathrm{s}) / \mathrm{m}^{\wedge} 3$

50-YR DOSE COMMITMENT:

SURFACE BONE 9.2E+02 rem RED MARROW 7.6E+01 rem

LIVER 2.0E+02 rem GONADS 1.1E+01 rem EFFECTIVE DOSE

EQUTVALENT 5.2E+01 rem

\author{
$D=0.10 \mathrm{~km}$ \\ $\mathrm{DEP}=4.7 \mathrm{E}-01 \mathrm{uCi} / \mathrm{m}^{\wedge} 2$ \\ $\mathrm{CHI}=4.7 \mathrm{E}-05(\mathrm{Ci}-\mathrm{s}) / \mathrm{m}^{\wedge} 3$ \\ 50-YR DOSE COMMTTMENT: \\ SURFACE BONE $1.3 \mathrm{E}+02 \mathrm{rem}$ \\ RED MARROW $1.0 \mathrm{E}+01 \mathrm{rem}$ \\ LIVER $\quad 2.8 \mathrm{E}+01 \mathrm{rem}$ \\ GONADS $1.6 \mathrm{E}+00 \mathrm{rem}$ \\ EFFECTIVE DOSE \\ EQUIVALENT $7.1 \mathrm{E}+00 \mathrm{rem}$
}

\author{
$\mathrm{D}=0.28 \mathrm{~km}$ \\ $\mathrm{DEP}=6.8 \mathrm{E}-02 \mathrm{uCi} / \mathrm{m}^{\wedge} 2$ \\ $\mathrm{CHI}=6.8 \mathrm{E}-06(\mathrm{Ci}-\mathrm{s}) / \mathrm{m}^{\wedge} 3$ \\ 50-YR DOSE COMMITMENT: \\ SURFACE BONE 1.8E+01 rem \\ RED MARROW $1.5 \mathrm{E}+00 \mathrm{rem}$ \\ LIVER 4.1E+00 rem \\ GONADS 2.3E-01 rem \\ EFFECTIVE DOSE \\ EQUIVALENT $\quad 1.0 \mathrm{E}+00 \mathrm{rem}$
}


Title of Project EPHA for THM

Subject Radiological Consequence Assessment

Computer D. J. Richards

Date $\underline{09 / 11 / 96}$

Reviewed by $\underline{S}$. A. Henderson

Works Calculation 6

Sheet No. 53 of 166

Release Designation: 1-RD-90, Adverse

HOTSPOT 7.0 GENERAL PLUME 9-10-1996 21:52

RADIONUCLIDE : PU-238 Inhalation Class : W

HALFLIFE $: 87.740$ years

SOURCE TERM : $1.9 \mathrm{E}+00 \mathrm{Ci}$

Source Term Width: $3 \mathrm{~m}$ Source Term Height: $3 \mathrm{~m}$

RELEASE FRACTION : $1.00 \mathrm{E}+00$

FILTER EFFICIENCY: $0.000000 \%$

EFFECTIVE RELEASE HEIGHT : $\quad 0.00 \mathrm{~m}$

WIND SPEED $(\mathrm{h}=2 \mathrm{~m}): 1.7 \mathrm{~m} / \mathrm{s}$

STABILITY CLASS :E DEPOSITION VELOCITY $\quad: 1.00 \mathrm{~cm} / \mathrm{s}$

RECEPTOR HEIGHT $\quad: 0.0 \mathrm{~m}$ INVERSION LAYER HEIGHT $: 200.0 \mathrm{~m}$

SAMPLE TIME $\quad: 10.000 \mathrm{~min}$

MAXIMUM DOSE DISTANCE $:<0.10 \mathrm{~km}$ MAXIMUM CEDE $:>1.3 \mathrm{E}+03 \mathrm{rem}$

$D=0.03 \mathrm{~km}$

$\mathrm{DEP}=5.0 \mathrm{E}+02 \mathrm{uCi} / \mathrm{m}^{\wedge} 2$

$\mathrm{CHI}=5.0 \mathrm{E}-02(\mathrm{Ci}-\mathrm{s}) / \mathrm{m}^{\wedge} 3$

50-YR DOSE COMMITMENT:

SURFACE BONE $1.4 \mathrm{E}+05 \mathrm{rem}$

RED MARROW $1.1 \mathrm{E}+04 \mathrm{rem}$

LIVER $3.0 \mathrm{E}+04 \mathrm{rem}$

GONADS 1.7E+03 rem

EFFECTIVE DOSE .

EQUIVALENT 7.7E+03 rem

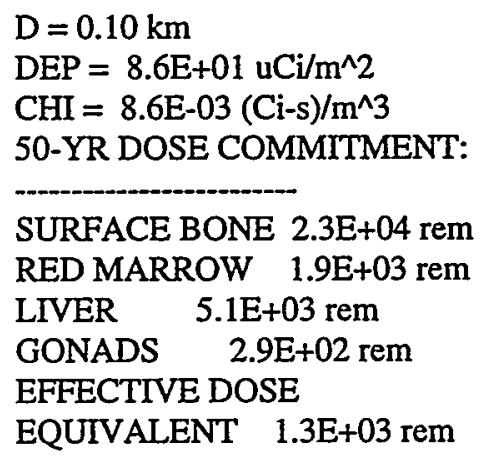

$\mathrm{D}=0.10 \mathrm{~km}$

$\mathrm{DEP}=8.6 \mathrm{E}+01 \mathrm{uCi} / \mathrm{m}^{\wedge} 2$

$\mathrm{CHI}=8.6 \mathrm{E}-03(\mathrm{Ci}-\mathrm{s}) / \mathrm{m}^{\wedge} 3$

50-YR DOSE COMMITMENT:

SURFACE BONE 2.3E+04 rem RED MARROW $1.9 \mathrm{E}+03 \mathrm{rem}$

LIVER $\quad 5.1 \mathrm{E}+03 \mathrm{rem}$ GONADS 2.9E+02 rem EFFECTIVE DOSE

EQUIVALENT $1.3 \mathrm{E}+03 \mathrm{rem}$

$\mathrm{D}=5.16 \mathrm{~km}$

$\mathrm{DEP}=6.8 \mathrm{E}-02 \mathrm{uCi} / \mathrm{m}^{\wedge} 2$

$\mathrm{CHI}=6.8 \mathrm{E}-06(\mathrm{Ci}-\mathrm{s}) / \mathrm{m}^{\wedge} 3$

50-YR DOSE COMMITMENT:

SURFACE BONE 1.8E+01 rem RED MARROW $1.5 \mathrm{E}+00 \mathrm{rem}$ LIVER 4.1E+00 rem GONADS 2.3E-01 rem EFFECTIVE DOSE EQUTVALENT $1.0 \mathrm{E}+00 \mathrm{rem}$ 
Title of Project EPHA for THM

Subject Radiological Consequence Assessment

Computer D. J. Richards
Reviewed by $\mathrm{S}$. A. Henderson

Works Calculation 6

Sheet No. 54 of 166

Release Designation: 1-RD-90, Average

HOTSPOT 7.0 GENERAL PLUME 9-10-1996 21:53

RADIONUCLIDE : PU-238 Inhalation Class : W

HALFLIFE : 87.740 years

SOURCE TERM : $1.9 \mathrm{E}+00 \mathrm{Ci}$

Source Term Width: $3 \mathrm{~m}$ Source Term Height: $3 \mathrm{~m}$

RELEASE FRACTION : $1.00 \mathrm{E}+00$

FILTER EFFICIENCY: $0.000000 \%$

EFFECTIVE RELEASE HEIGHT : $\quad 0.00 \mathrm{~m}$

WIND SPEED $(h=2 \mathrm{~m}): 2.5 \mathrm{~m} / \mathrm{s}$

STABILITY CLASS :C DEPOSITION VELOCITY $: 1.00 \mathrm{~cm} / \mathrm{s}$

RECEPTOR HEIGHT $: 0.0 \mathrm{~m}$ INVERSION LAYER HEIGHT $: 500.0 \mathrm{~m}$

SAMPLE TIME $: 10.000 \mathrm{~min}$

MAXIMUM DOSE DISTANCE $:<0.10 \mathrm{~km}$ MAXIMUM CEDE $:>3.1 E+02 \mathrm{rem}$

$\mathrm{D}=0.03 \mathrm{~km}$

$\mathrm{DEP}=1.5 \mathrm{E}+02 \mathrm{uCi} / \mathrm{m}^{\wedge} 2$

$\mathrm{CHI}=1.5 \mathrm{E}-02(\mathrm{Ci}-\mathrm{s}) / \mathrm{m}^{\wedge} 3$

50-YR DOSE COMMITMENT:

SURFACE BONE 4.0E+04 rem RED MARROW $3.3 \mathrm{E}+03 \mathrm{rem}$ LIVER $\quad 8.9 \mathrm{E}+03 \mathrm{rem}$ GONADS $4.9 \mathrm{E}+02$ rem EFFECTIVE DOSE

EQUIVALENT 2.3E+03 rem
$\mathrm{D}=0.10 \mathrm{~km}$

$\mathrm{DEP}=2.0 \mathrm{E}+01 \mathrm{uCi} / \mathrm{m}^{\wedge} 2$

$\mathrm{CHI}=2.0 \mathrm{E}-03(\mathrm{Ci}-\mathrm{s}) / \mathrm{m}^{\wedge} 3$

50-YR DOSE COMMITMENT:

SURFACE BONE 5.4E+03 rem RED MARROW 4.5E+02 rem LIVER 1.2E+03 rem GONADS 6.7E+01 rem EFFECTTVE DOSE EQUTVALENT 3.1E+02 rem
$\mathrm{D}=2.05 \mathrm{~km}$ $\mathrm{DEP}=6.8 \mathrm{E}-02 \mathrm{uCi} / \mathrm{m}^{\wedge} 2$ $\mathrm{CHI}=6.8 \mathrm{E}-06(\mathrm{Ci}-\mathrm{s}) / \mathrm{m}^{\wedge} 3$ 50-YR DOSE COMMITMENT:

SURFACE BONE $1.8 \mathrm{E}+01 \mathrm{rem}$ RED MARROW $1.5 \mathrm{E}+00 \mathrm{rem}$ LIVER $\quad 4.1 \mathrm{E}+00 \mathrm{rem}$ GONADS 2.3E-01 rem EFFECTIVE DOSE EQUIVALENT $1.0 \mathrm{E}+00 \mathrm{rem}$ 
Title of Project EPHA for THM

Subject Radiological Consequence Assessment

Computer D. J. Richards
Reviewed by S. A. Henderson

Works Calculation 6

Sheet No. 55 of 166

Release Designation: 1-RD-91, Adverse

HOTSPOT 7.0 GENERAL PLUME 9-09-1996 21:00

RADIONUCLIDE : PU-238 Inhalation Class : W

HALFLIFE $: 87.740$ years

SOURCE TERM $\quad: 3.1 \mathrm{E}-02 \mathrm{Ci}$

Source Term Width: $\quad 3 \mathrm{~m}$ Source Term Height: $3 \mathrm{~m}$

RELEASE FRACTION : $1.00 \mathrm{E}+00$

FILTER EFFICIENCY: $0.000000 \%$

EFFECTIVE RELEASE HEIGHT : $\quad 0.00 \mathrm{~m}$

WIND SPEED $(\mathrm{h}=2 \mathrm{~m}): 1.7 \mathrm{~m} / \mathrm{s}$

STABILITY CLASS :E DEPOSITION VELOCITY $: 1.00 \mathrm{~cm} / \mathrm{s}$

RECEPTOR HEIGHT $\quad: 0.0 \mathrm{~m}$ INVERSION LAYER HEIGHT $: 200.0 \mathrm{~m}$

SAMPLE TIME $\quad: 30.000 \mathrm{~min}$

MAXIMUM DOSE DISTANCE $:<0.10 \mathrm{~km} \mathrm{MAXIMUM} \mathrm{CEDE}:>17 \mathrm{rem}$

$\mathrm{D}=0.03 \mathrm{~km}$

$\mathrm{DEP}=6.5 \mathrm{E}+00 \mathrm{uCi} / \mathrm{m}^{\wedge} 2$

$\mathrm{CHI}=6.5 \mathrm{E}-04(\mathrm{Ci}-\mathrm{s}) / \mathrm{m}^{\wedge} 3$

50-YR DOSE COMMITMENT:

SURFACE BONE 1.8E+03 rem RED MARROW $1.5 \mathrm{E}+02$ rem LIVER 3.9E+02 rem GONADS 2.2E+01 rem EFFECTIVE DOSE

EQUIVALENT $1.0 \mathrm{E}+02 \mathrm{rem}$
$D=0.10 \mathrm{~km}$

$\mathrm{DEP}=1.1 \mathrm{E}+00 \mathrm{uCi} / \mathrm{m}^{\wedge} 2$

$\mathrm{CHI}=1.1 \mathrm{E}-04(\mathrm{Ci}-\mathrm{s}) / \mathrm{m}^{\wedge} 3$

50-YR DOSE COMMITMENT:

SURFACE BONE 3.0E+02 rem RED MARROW $2.5 \mathrm{E}+01 \mathrm{rem}$

LIVER 6.7E +01 rem GONADS 3.7E+00 rem EFFECTIVE DOSE

EQUIVALENT $1.7 \mathrm{E}+01 \mathrm{rem}$
$\mathrm{D}=0.46 \mathrm{~km}$ $\mathrm{DEP}=6.8 \mathrm{E}-02 \mathrm{uCi} / \mathrm{m}^{\wedge} 2$

$\mathrm{CHI}=6.8 \mathrm{E}-06(\mathrm{Ci}-\mathrm{s}) / \mathrm{m}^{\wedge} 3$

50-YR DOSE COMMITMENT:

SURFACE BONE 1.8E+01 rem RED MARROW $1.5 \mathrm{E}+00 \mathrm{rem}$ LIVER 4.1E $+00 \mathrm{rem}$ GONADS 2.3E-01 rem EFFECTIVE DOSE EQUIVALENT $1.0 \mathrm{E}+00 \mathrm{rem}$ 
Title of Project EPHA for THM

Subject Radiological Consequence Assessment

Computer D. J. Richards

Date $\underline{09 / 11 / 96}$

Reviewed by S. A. Henderson

Release Designation: 1-RD-91, Average

HOTSPOT 7.0 GENERAL PLUME 9-09-1996 21:01

RADIONUCLIDE : PU-238 Inhalation Class : W

HALFLIFE $: 87.740$ years

SOURCE TERM : $3.1 \mathrm{E}-02 \mathrm{Ci}$

Source Term Width: $3 \mathrm{~m}$ Source Term Height: $3 \mathrm{~m}$

RELEASE FRACTION : $1.00 \mathrm{E}+00$

FILTER EFFICIENCY: $0.000000 \%$

EFFECTIVE RELEASE HEIGHT : $\quad 0.00 \mathrm{~m}$

WIND SPEED $(\mathrm{h}=2 \mathrm{~m}): 2.5 \mathrm{~m} / \mathrm{s}$

STABILITY CLASS :C DEPOSITION VELOCITY : $1.00 \mathrm{~cm} / \mathrm{s}$

RECEPTOR HEIGHT $\quad: 0.0 \mathrm{~m}$ INVERSION LAYER HEIGHT $: 500.0 \mathrm{~m}$

SAMPLE TIME $\quad: 30.000 \mathrm{~min}$

MAXIMUM DOSE DISTANCE $:<0.10 \mathrm{~km}$ MAXIMUM CEDE $:>4.0 \mathrm{rem}$

$\mathrm{D}=0.03 \mathrm{~km}$

$\mathrm{DEP}=1.9 \mathrm{E}+00 \mathrm{uCi} / \mathrm{m}^{\wedge} 2$

$\mathrm{CHI}=1.9 \mathrm{E}-04(\mathrm{Ci}-\mathrm{s}) / \mathrm{m}^{\wedge} 3$

50-YR DOSE COMMITMENT:

SURFACE BONE 5.2E+02 rem RED MARROW 4.3E+01 rem LIVER 1.1E+02 rem GONADS 6.4E+00 rem EFFECTIVE DOSE

EQUIVALENT $2.9 \mathrm{E}+01 \mathrm{rem}$

\author{
$\mathrm{D}=0.10 \mathrm{~km}$ \\ $\mathrm{DEP}=2.6 \mathrm{E}-01 \mathrm{uCi} / \mathrm{m}^{\wedge} 2$ \\ $\mathrm{CHI}=2.6 \mathrm{E}-05(\mathrm{Ci}-\mathrm{s}) / \mathrm{m}^{\wedge} 3$ \\ 50-YR DOSE COMMITMENT: \\ SURFACE BONE 7.0E+01 rem \\ RED MARROW $\quad 5.8 \mathrm{E}+00 \mathrm{rem}$ \\ LIVER $1.6 \mathrm{E}+01 \mathrm{rem}$ \\ GONADS 8.7E-01 rem \\ EFFECTIVE DOSE \\ EQUTVALENT $4.0 \mathrm{E}+00 \mathrm{rem}$
}

\author{
$\mathrm{D}=0.21 \mathrm{~km}$ \\ $\mathrm{DEP}=6.6 \mathrm{E}-02 \mathrm{uCi} / \mathrm{m}^{\wedge} 2$ \\ $\mathrm{CHI}=6.6 \mathrm{E}-06(\mathrm{Ci}-\mathrm{s}) / \mathrm{m}^{\wedge} 3$ \\ 50-YR DOSE COMMITMENT: \\ SURFACE BONE 1.8E+01 rem \\ RED MARROW $1.5 \mathrm{E}+00 \mathrm{rem}$ \\ LIVER 4.0E $+00 \mathrm{rem}$ \\ GONADS 2.2E-01 rem \\ EFFECTIVE DOSE \\ EQUTVALENT $1.0 \mathrm{E}+00 \mathrm{rem}$
}


Title of Project EPHA for THM

Subject Radiological Consequence Assessment

Computer D. J. Richards

Date $\underline{09 / 11 / 96}$

Reviewed by $\mathrm{S}$. A. Henderson

Works Calculation 6

Sheet No. $\underline{57 \text { of } 166}$

Release Designation: 1-RD-92, Adverse

HOTSPOT 7.0 GENERAL PLUME 9-10-1996 22:05

RADIONUCLIDE : PU-238 Inhalation Class : W

HALFLIFE : 87.740 years

SOURCE TERM : $3.4 \mathrm{E}-02 \mathrm{Ci}$

Source Term Width: $\quad 3 \mathrm{~m}$ Source Term Height: $3 \mathrm{~m}$

RELEASE FRACTION : $1.00 \mathrm{E}+00$

FILTER EFFICIENCY: $0.000000 \%$

EFFECTIVE RELEASE HEIGHT : $\quad 0.00 \mathrm{~m}$

WIND SPEED $(\mathrm{h}=2 \mathrm{~m}): 1.7 \mathrm{~m} / \mathrm{s}$

STABILITY CLASS :E DEPOSITION VELOCITY $: 1.00 \mathrm{~cm} / \mathrm{s}$

RECEPTOR HEIGHT $\quad: 0.0 \mathrm{~m}$ INVERSION LAYER HEIGHT $: 200.0 \mathrm{~m}$

SAMPLE TIME $\quad: 10.000 \mathrm{~min}$

MAXIMUM DOSE DISTANCE $:<0.10 \mathrm{~km}$ MAXIMUM CEDE $:>23 \mathrm{rem}$

$\mathrm{D}=0.03 \mathrm{~km}$

$\mathrm{DEP}=8.9 \mathrm{E}+00 \mathrm{uCi} / \mathrm{m}^{\wedge} 2$

$\mathrm{CHI}=8.9 \mathrm{E}-04(\mathrm{Ci}-\mathrm{s}) / \mathrm{m}^{\wedge} 3$

50-YR DOSE COMMITMENT:

SURFACE BONE $2.4 \mathrm{E}+03 \mathrm{rem}$ RED MARROW 2.0E+02 rem LIVER $5.3 \mathrm{E}+02 \mathrm{rem}$ GONADS 3.0E+01 rem EFFECTIVE DOSE

EQUIVALENT $\quad 1.4 \mathrm{E}+02 \mathrm{rem}$
$\mathrm{D}=0.10 \mathrm{~km}$

$\mathrm{DEP}=1.5 \mathrm{E}+00 \mathrm{uCi} / \mathrm{m}^{\wedge} 2$

$\mathrm{CHI}=1.5 \mathrm{E}-04(\mathrm{Ci}-\mathrm{s}) / \mathrm{m}^{\wedge} 3$

50-YR DOSE COMMITMENT:

SURFACE BONE 4.1E+02 rem RED MARROW $\quad 3.4 \mathrm{E}+01$ rem

LIVER 9.1E+01 rem GONADS 5.0E+00 rem EFFECTIVE DOSE

EQUTVALENT 2.3E+01 rem
$\mathrm{D}=0.54 \mathrm{~km}$

$\mathrm{DEP}=6.8 \mathrm{E}-02 \mathrm{uCi} / \mathrm{m}^{\wedge} 2$

$\mathrm{CHI}=6.8 \mathrm{E}-06(\mathrm{Ci}-\mathrm{s}) / \mathrm{m}^{\wedge} 3$

50-YR DOSE COMMITMENT:

SURFACE BONE 1.8E+01 rem RED MARROW $1.5 \mathrm{E}+00 \mathrm{rem}$ LIVER 4.1E+00 rem GONADS 2.3E-01 rem EFFECTIVE DOSE

EQUIVALENT $1.0 \mathrm{E}+00 \mathrm{rem}$ 
Title of Project EPHA for THM

Subject Radiological Consequence Assessment

Computer D. J. Richards

Date $\underline{09 / 11 / 96}$

Reviewed by S. A. Henderson Works Calculation 6

Sheet No. 58 of 166

Release Designation: 1-RD-92, Average

HOTSPOT 7.0 GENERAL PLUME 9-10-1996 22:06

RADIONUCLIDE : PU-238 Inhalation Class : W

HALFLIFE $: 87.740$ years

SOURCE TERM : $3.4 \mathrm{E}-02 \mathrm{Ci}$

Source Term Width: $3 \mathrm{~m}$. Source Term Height: $3 \mathrm{~m}$

RELEASE FRACTION : $1.00 \mathrm{E}+00$

FILTER EFFICIENCY: $0.000000 \%$

EFFECTIVE RELEASE HEIGHT : $\quad 0.00 \mathrm{~m}$

WIND SPEED $(\mathrm{h}=2 \mathrm{~m}): 2.5 \mathrm{~m} / \mathrm{s}$

STABILITY CLASS :C DEPOSITION VELOCITY $: 1.00 \mathrm{~cm} / \mathrm{s}$

RECEPTOR HEIGHT $\quad: 0.0 \mathrm{~m}$ INVERSION LAYER HEIGHT $: 500.0 \mathrm{~m}$

SAMPLE TIME $\quad: 10.000 \mathrm{~min}$

MAXIMUM DOSE DISTANCE $:<0.10 \mathrm{~km}$ MAXIMUM CEDE $:>5.4 \mathrm{rem}$

$\mathrm{D}=0.03 \mathrm{~km}$

$\mathrm{DEP}=2.6 \mathrm{E}+00 \mathrm{uCi} / \mathrm{m}^{\wedge} 2$

$\mathrm{CHI}=2.6 \mathrm{E}-04(\mathrm{Ci}-\mathrm{s}) / \mathrm{m}^{\wedge} 3$

50-YR DOSE COMMITMENT:

SURFACE BONE 7.0E $+02 \mathrm{rem}$ RED MARROW $5.8 \mathrm{E}+01 \mathrm{rem}$ LIVER $\quad 1.6 \mathrm{E}+02 \mathrm{rem}$ GONADS 8.7E+00 rem EFFECTIVE DOSE EQUTVALENT $4.0 \mathrm{E}+01 \mathrm{rem}$
$\mathrm{D}=0.10 \mathrm{~km}$

$\mathrm{DEP}=3.5 \mathrm{E}-01 \mathrm{uCi} / \mathrm{m}^{\wedge} 2$

$\mathrm{CHI}=3.5 \mathrm{E}-05(\mathrm{Ci}-\mathrm{s}) / \mathrm{m}^{\wedge} 3$

50-YR DOSE COMMITMENT:

SURFACE BONE 9.6E+01 rem RED MARROW $7.9 \mathrm{E}+00 \mathrm{rem}$

LIVER 2.1E+01 rem GONADS 1.2E+00 rem

EFFECTIVE DOSE

EQUIVALENT $5.4 \mathrm{E}+00 \mathrm{rem}$
$\mathrm{D}=0.25 \mathrm{~km}$

$\mathrm{DEP}=6.7 \mathrm{E}-02 \mathrm{uCi} / \mathrm{m}^{\wedge} 2$

$\mathrm{CHI}=6.7 \mathrm{E}-06(\mathrm{Ci}-\mathrm{s}) / \mathrm{m}^{\wedge} 3$

50-YR DOSE COMMITMENT:

SURFACE BONE $1.8 \mathrm{E}+01 \mathrm{rem}$ RED MARROW $1.5 \mathrm{E}+00$ rem LIVER $\quad 4.0 \mathrm{E}+00 \mathrm{rem}$ GONADS 2.2E-01 rem EFFECTIVE DOSE EQUIVALENT $1.0 \mathrm{E}+00 \mathrm{rem}$ 
Title of Project EPHA for THM

Subject Radiological Consequence Assessment

Computer D. J. Richards

Date $\underline{09 / 11 / 96}$

Reviewed by $\underline{\text { S. A. Henderson }}$

Works Calculation 6

Sheet No. 59 of 166.

Release Designation: 1-RD-93, Adverse

HOTSPOT 7.0 GENERAL PLUME 9-09-1996 21:06

RADIONUCLIDE : PU-238 Inhalation Class : W

HALFLIFE : 87.740 years

SOURCE TERM : $4.5 \mathrm{E}-08 \mathrm{Ci}$

Source Term Width: $\quad 3 \mathrm{~m}$ Source Term Height: $3 \mathrm{~m}$

RELEASE FRACTION : $1.00 \mathrm{E}+00$

FILTER EFFICIENCY: $0.000000 \%$

EFFECTIVE RELEASE HEIGHT : $\quad 0.00 \mathrm{~m}$

WIND SPEED ( $\mathrm{h}=2 \mathrm{~m}): 1.7 \mathrm{~m} / \mathrm{s}$

STABILITY CLASS :E DEPOSITION VELOCITY : $1.00 \mathrm{~cm} / \mathrm{s}$

RECEPTOR HEIGHT $\quad: 0.0 \mathrm{~m}$ INVERSION LAYER HEIGHT $: 200.0 \mathrm{~m}$

SAMPLE TIME $\quad: 30.000 \mathrm{~min}$

MAXIMUM DOSE DISTANCE $:<0.10 \mathrm{~km}$ MAXIMUM CEDE $:>2.5 \mathrm{E}-05 \mathrm{rem}$

$\mathrm{D}=0.03 \mathrm{~km}$

$\mathrm{DEP}=9.6 \mathrm{E}-06 \mathrm{uCi} / \mathrm{m}^{\wedge} 2$

$\mathrm{CHI}=9.6 \mathrm{E}-10(\mathrm{Ci}-\mathrm{s}) / \mathrm{m}^{\wedge} 3$

50-YR DOSE COMMITMENT:

SURFACE BONE 2.6E-03 rem RED MARROW 2.1E-04 rem

LIVER 5.7E-04 rem GONADS 3.2E-05 rem EFFECTIVE DOSE

EQUTVALENT $1.5 \mathrm{E}-04 \mathrm{rem}$
$\mathrm{D}=0.10 \mathrm{~km}$

$\mathrm{DEP}=1.6 \mathrm{E}-06 \mathrm{uCi} / \mathrm{m}^{\wedge} 2$

$\mathrm{CHI}=1.6 \mathrm{E}-10(\mathrm{Ci}-\mathrm{s}) / \mathrm{m}^{\wedge} 3$

50-YR DOSE COMMITMENT:

SURFACE BONE 4.4E-04 rem RED MARROW 3.6E-05 rem

LIVER 9.8E-05 rem

GONADS 5.4E-06 rem

EFFECTIVE DOSE

EQUTVALENT $2.5 \mathrm{E}-05 \mathrm{rem}$ 
Title of Project EPHA for THM

Subject Radiological Consequence Assessment

Computer D. J. Richards

Date $\underline{09 / 11 / 96}$

Reviewed by S. A. Henderson

Works Calculation 6

Sheet No. 60 of 166

Release Designation: 1-RD-93, Average

HOTSPOT 7.0 GENERAL PLUME 9-09-1996 21:08

RADIONUCLIDE : PU-238 Inhalation Class : W

HALFLIFE : 87.740 years

SOURCE TERM : 4.5E-08 Ci

Source Term Width: $\quad 3 \mathrm{~m}$ Source Term Height: $3 \mathrm{~m}$

RELEASE FRACTION : $1.00 \mathrm{E}+00$

FILTER EFFICIENCY: $0.000000 \%$

EFFECTIVE RELEASE HEIGHT : $\quad 0.00 \mathrm{~m}$

WIND SPEED ( $\mathrm{h}=2 \mathrm{~m}): 2.5 \mathrm{~m} / \mathrm{s}$

STABILITY CLASS $:$ C DEPOSITION VELOCITY $: 100 \mathrm{~cm} / \mathrm{s}$

RECEPTOR HEIGHT $\quad: 0.0 \mathrm{~m}$ INVERSION LAYER HEIGHT $: 500.0 \mathrm{~m}$

SAMPLE TIME $\quad: 30.000 \mathrm{~min}$

MAXIMUM DOSE DISTANCE $:<0.10 \mathrm{~km}$ MAXIMUM CEDE $:>5.9 \mathrm{E}-06 \mathrm{rem}$

$\mathrm{D}=0.03 \mathrm{~km}$

$\mathrm{DEP}=2.8 \mathrm{E}-06 \mathrm{uCi} / \mathrm{m}^{\wedge} 2$

$\mathrm{CHI}=2.8 \mathrm{E}-10(\mathrm{Ci}-\mathrm{s}) / \mathrm{m}^{\wedge} 3$

50-YR DOSE COMMITMENT:

SURFACE BONE 7.6E-04 rem RED MARROW 6.2E-05 rem

LIVER 1.7E-04 rem

GONADS 9.3E-06 rem

EFFECTIVE DOSE

EQUIVALENT $4.3 \mathrm{E}-05 \mathrm{rem}$
$\mathrm{D}=0.10 \mathrm{~km}$

$\mathrm{DEP}=3.8 \mathrm{E}-07 \mathrm{uCi} / \mathrm{m}^{\wedge} 2$

$\mathrm{CHI}=3.8 \mathrm{E}-11(\mathrm{Ci}-\mathrm{s}) / \mathrm{m}^{\wedge} 3$

50-YR DOSE COMMITMENT:

SURFACE BONE 1.0E-04 rem RED MARROW $8.5 \mathrm{E}-06 \mathrm{rem}$ LIVER 2.3E-05 rem GONADS 1.3E-06 rem EFFECTIVE DOSE

EQUTVALENT $5.9 \mathrm{E}-06 \mathrm{rem}$ 
Title of Project EPHA for THM

Subject Radiological Consequence Assessment

Computer D. J. Richards

Release Designation: 1-RD-94, Adverse

HOTSPOT 7.0 GENERAL PLUME 9-10-1996 22:07

RADIONUCLIDE : PU-238 Inhalation Class : $\mathrm{W}$

HALFLIFE : 87.740 years

SOURCE TERM : $4.9 \mathrm{E}-08 \mathrm{Ci}$

Source Term Width: $\quad 3 \mathrm{~m}$ Source Term Height: $3 \mathrm{~m}$

RELEASE FRACTION : $1.00 \mathrm{E}+00$

FILTER EFFICIENCY: $0.000000 \%$

EFFECTIVE RELEASE HEIGHT : $\quad 0.00 \mathrm{~m}$

WIND SPEED $(\mathrm{h}=2 \mathrm{~m}): 1.7 \mathrm{~m} / \mathrm{s}$

STABILITY CLASS :E DEPOSITION VELOCITY $: 1.00 \mathrm{~cm} / \mathrm{s}$

RECEPTOR HEIGHT $: 0.0 \mathrm{~m}$ INVERSION LAYER HEIGHT $: 200.0 \mathrm{~m}$

SAMPLE TIME $\quad: 10.000 \mathrm{~min}$

MAXIMUM DOSE DISTANCE $:<0.10 \mathrm{~km}$ MAXIMUM CEDE $:>3.4 \mathrm{E}-05 \mathrm{rem}$

$D=0.03 \mathrm{~km}$

$\mathrm{DEP}=1.3 \mathrm{E}-05 \mathrm{uCi} / \mathrm{m}^{\wedge} 2$

$\mathrm{CHI}=1.3 \mathrm{E}-09(\mathrm{Ci}-\mathrm{s}) / \mathrm{m}^{\wedge} 3$

50-YR DOSE COMMITMENT:

SURFACE BONE 3.5E-03 rem RED MARROW 2.9E-04 rem

LIVER 7.8E-04 rem GONADS 4.3E-05 rem EFFECTIVE DOSE

EQUIVALENT 2.0E-04 rem
$\mathrm{D}=0.10 \mathrm{~km}$

$\mathrm{DEP}=2.2 \mathrm{E}-06 \mathrm{uCi} / \mathrm{m}^{\wedge} 2$

$\mathrm{CHI}=2.2 \mathrm{E}-10(\mathrm{Ci}-\mathrm{s}) / \mathrm{m}^{\wedge} 3$

50-YR DOSE COMMITMÉNT:

SURFACE BONE 6.0E-04 rem

RED MARROW $\quad 4.9 \mathrm{E}-05 \mathrm{rem}$

LIVER 1.3E-04 rem

GONADS 7.4E-06 rem

EFFECTIVE DOSE

EQUIVALENT 3.4E-05 rem 
Title of Project EPHA for THM

Subject Radiological Consequence Assessment

Computer D. J. Richards

Date $\underline{09 / 11 / 96}$

Reviewed by $\underline{\text { S. A. Henderson }}$

Works Calculation 6

Sheet No. 62 of 166

Release Designation: 1-RD-94, Average

HOTSPOT 7.0 GENERAL PLUME 9-10-1996 22:08

RADIONUCLIDE : PU-238 Inhalation Class : W

HALFLIFE $: 87.740$ years

SOURCE TERM : $4.9 \mathrm{E}-08 \mathrm{Ci}$

Source Term Width: $3 \mathrm{~m}$ Source Term Height: $3 \mathrm{~m}$

RELEASE FRACTION : $1.00 \mathrm{E}+00$

FILTER EFFICIENCY: $0.000000 \%$

EFFECTIVE RELEASE HEIGHT : $\quad 0.00 \mathrm{~m}$

WIND SPEED $(\mathrm{h}=2 \mathrm{~m}): 2.5 \mathrm{~m} / \mathrm{s}$

STABILITY CLASS $\quad$ :C DEPOSITION VELOCITY $\quad: 1.00 \mathrm{~cm} / \mathrm{s}$

RECEPTOR HEIGHT $\quad: 0.0 \mathrm{~m}$ INVERSION LAYER HEIGHT $: 500.0 \mathrm{~m}$

SAMPLE TIME $\quad: 10.000 \mathrm{~min}$

MAXIMUM DOSE DISTANCE $:<0.10 \mathrm{~km}$ MAXIMUM CEDE $:>7.9 \mathrm{E}-06 \mathrm{rem}$

$\mathrm{D}=0.03 \mathrm{~km}$

$\mathrm{DEP}=3.8 \mathrm{E}-06 \mathrm{uCi} / \mathrm{m}^{\wedge} 2$

$\mathrm{CHI}=3.8 \mathrm{E}-10(\mathrm{Ci}-\mathrm{s}) / \mathrm{m}^{\wedge} 3$

50-YR DOSE COMMITMENT:

SURFACE BONE 1.0E-03 rem RED MARROW $8.5 \mathrm{E}-05 \mathrm{rem}$

LIVER 2.3E-04 rem GONADS 1.3E-05 rem EFFECTIVE DOSE

EQUTVALENT 5.8E-05 rem
$\mathrm{D}=0.10 \mathrm{~km}$

$\mathrm{DEP}=5.2 \mathrm{E}-07 \mathrm{uCi} / \mathrm{m}^{\wedge} 2$

$\mathrm{CHI}=5.2 \mathrm{E}-11(\mathrm{Ci}-\mathrm{s}) / \mathrm{m}^{\wedge} 3$

50-YR DOSE COMMITMENT:

SURFACE BONE 1.4E-04 rem

RED MARROW $1.2 \mathrm{E}-05 \mathrm{rem}$

LIVER 3.1E-05 rem

GONADS 1.7E-06 rem

EFFECTIVE DOSE

EQUIVALENT 7.9E-06 rem 
Title of Project EPHA for THM

Subject Radiological Consequence Assessment

Computer D. J. Richards

Date $\underline{09 / 11 / 96}$

Reviewed by $\underline{S}$. A. Henderson

Works Calculation 6

Sheet No. 63 of 166

Release Designation: 1-RD-95, Adverse

HOTSPOT 7.0 GENERAL PLUME 9-09-1996 21:09

RADIONUCLIDE : PU-238 Inhalation Class : W

HALFLIFE $: 87.740$ years

SOURCE TERM $: 1.5 \mathrm{E}-06 \mathrm{Ci}$

Source Term Width: $3 \mathrm{~m}$ Source Term Height: $3 \mathrm{~m}$

RELEASE FRACTION : $1.00 \mathrm{E}+00$

FILTER EFFICIENCY: $0.000000 \%$

EFFECTIVE RELEASE HEIGHT : $\quad 0.00 \mathrm{~m}$

WIND SPEED $(\mathrm{h}=2 \mathrm{~m}): 1.7 \mathrm{~m} / \mathrm{s}$

STABILITY CLASS $\quad$ E DEPOSITION VELOCITY $: 1.00 \mathrm{~cm} / \mathrm{s}$

RECEPTOR HEIGHT $\quad: 0.0 \mathrm{~m}$ INVERSION LAYER HEIGHT $: 200.0 \mathrm{~m}$

SAMPLE TIME $: 30.000 \mathrm{~min}$

MAXIMUM DOSE DISTANCE $:<0.10 \mathrm{~km}$ MAXIMUM CEDE $:>8.3 \mathrm{E}-04 \mathrm{rem}$

$\mathrm{D}=0.03 \mathrm{~km}$

$\mathrm{DEP}=3.2 \mathrm{E}-04 \mathrm{uCi} / \mathrm{m}^{\wedge} 2$

$\mathrm{CHI}=3.2 \mathrm{E}-08(\mathrm{Ci}-\mathrm{s}) / \mathrm{m}^{\wedge} 3$

50-YR DOSE COMMITMENT:

SURFACE BONE 8.6E-02 rem RED MARROW 7.1E-03 rem

LIVER 1.9E-02 rem

GONADS 1.1E-03 rem

EFFECTIVE DOSE

EQUIVALENT 4.9E-03 rem
$\mathrm{D}=0.10 \mathrm{~km}$

$\mathrm{DEP}=5.4 \mathrm{E}-05 \mathrm{uCi} / \mathrm{m}^{\wedge} 2$

$\mathrm{CHI}=5.4 \mathrm{E}-09(\mathrm{Ci}-\mathrm{s}) / \mathrm{m}^{\wedge} 3$

50-YR DOSE COMMITMENT:

SURFACE BONE 1.5E-02 rem

RED MARROW $1.2 \mathrm{E}-03 \mathrm{rem}$

LIVER 3.2E-03 rem

GONADS 1.8E-04 rem

EFFECTIVE DOSE

EQUTVALENT 8.3E-04 rem 
Title of Project EPHA for THM

Reviewed by $\underline{\text { S. A. Henderson }}$

Subject Radiological Consequence Assessment

Works Calculation 6

Computer D. J. Richards

Date 09/11/96

Sheet No. 64 of 166

Release Designation: 1-RD-95, Average

HOTSPOT 7.0 GENERAL PLUME 9-09-1996 21:10

RADIONUCLIDE : PU-238 Inhalation Class : W

HALFLIFE $: 87.740$ years

SOURCE TERM : $1.5 \mathrm{E}-06 \mathrm{Ci}$

Source Term Width: $3 \mathrm{~m}$ Source Term Height: $3 \mathrm{~m}$

RELEASE FRACTION : $1.00 \mathrm{E}+00$

FILTER EFFICIENCY: $0.000000 \%$

EFFECTIVE RELEASE HEIGHT : $\quad 0.00 \mathrm{~m}$

WIND SPEED $(\mathrm{h}=2 \mathrm{~m}): 2.5 \mathrm{~m} / \mathrm{s}$

STABILITY CLASS :C DEPOSITION VELOCITY : $1.00 \mathrm{~cm} / \mathrm{s}$

RECEPTOR HEIGHT $: 0.0 \mathrm{~m}$ INVERSION LAYER HEIGHT $: 500.0 \mathrm{~m}$

SAMPLE TIME $\quad: 30.000 \mathrm{~min}$

MAXIMUM DOSE DISTANCE $:<0.10 \mathrm{~km}$ MAXIMUM CEDE $:>1.9 \mathrm{E}-04 \mathrm{rem}$

$\mathrm{D}=0.03 \mathrm{~km}$

$\mathrm{DEP}=9.3 \mathrm{E}-05 \mathrm{uCi} / \mathrm{m}^{\wedge} 2$

$\mathrm{CHI}=9.3 \mathrm{E}-09(\mathrm{Ci}-\mathrm{s}) / \mathrm{m}^{\wedge} 3$

50-YR DOSE COMMITMENT:

SURFACE BONE 2.5E-02 rem RED MARROW 2.1E-03 rem LIVER $\quad 5.6 \mathrm{E}-03 \mathrm{rem}$ GONADS 3.1E-04 rem EFFECTIVE DOSE EQUTVALENT 1.4E-03 rem
$\mathrm{D}=0.10 \mathrm{~km}$

$\mathrm{DEP}=1.3 \mathrm{E}-05 \mathrm{uCi} / \mathrm{m}^{\wedge} 2$

$\mathrm{CHI}=1.3 \mathrm{E}-09(\mathrm{Ci}-\mathrm{s}) / \mathrm{m}^{\wedge} 3$

50-YR DOSE COMMITMENT:

SURFACE BONE 3.4E-03 rem

RED MARROW 2.8E-04 rem

LIVER 7.6E-04 rem

GONADS 4.2E-05 rem

EFFECTIVE DOSE

EQUIVALENT $1.9 \mathrm{E}-04 \mathrm{rem}$ 
Title of Project EPHA for THM

Subject Radiological Consequence Assessment

Computer D. J. Richards
Reviewed by $\underline{S}$. A. Hènderson

Works Calculation 6

Sheet No. 65 of 166

Release Designation: 1-RD-96, Adverse

HOTSPOT 7.0 GENERAL PLUME 9-10-1996 22:09

RADIONUCLIDE : PU-238 Inhalation Class : W

HALFLIFE $: 87.740$ years

SOURCE TERM : $1.6 \mathrm{E}-06 \mathrm{Ci}$

Source Term Width: $3 \mathrm{~m}$ Source Term Height: $3 \mathrm{~m}$

RELEASE FRACTION : $1.00 \mathrm{E}+00$

FILTER EFFICIENCY: $0.000000 \%$

EFFECTIVE RELEASE HEIGHT : $\quad 0.00 \mathrm{~m}$

WIND SPEED $(\mathrm{h}=2 \mathrm{~m}): 1.7 \mathrm{~m} / \mathrm{s}$

STABILITY CLASS :E DEPOSITION VELOCITY $: 1.00 \mathrm{~cm} / \mathrm{s}$

RECEPTOR HEIGHT $\quad: 0.0 \mathrm{~m}$ INVERSION LAYER HEIGHT $: 200.0 \mathrm{~m}$

SAMPLE TIME $\quad: 10.000 \mathrm{~min}$

MAXIMUM DOSE DISTANCE $:<0.10 \mathrm{~km}$ MAXIMUM CEDE $:>1.1 \mathrm{E}-03 \mathrm{rem}$

$\mathrm{D}=0.03 \mathrm{~km}$

$\mathrm{DEP}=4.3 \mathrm{E}-04 \mathrm{uCi} / \mathrm{m}^{\wedge} 2$

$\mathrm{CHI}=4.3 \mathrm{E}-08(\mathrm{Ci}-\mathrm{s}) / \mathrm{m}^{\wedge} 3$

50-YR DOSE COMMITMENT:

SURFACE BONE 1.2E-01 rem RED MARROW 9.7E-03 rem

LIVER $\quad 2.6 \mathrm{E}-02$ rem GONADS 1.4E-03 rem EFFECTIVE DOSE

EQUIVALENT $6.6 \mathrm{E}-03 \mathrm{rem}$
$\mathrm{D}=0.10 \mathrm{~km}$

$\mathrm{DEP}=-7.4 \mathrm{E}-05 \mathrm{uCi} / \mathrm{m}^{\wedge} 2$

$\mathrm{CHI}=7.4 \mathrm{E}-09(\mathrm{Ci}-\mathrm{s}) / \mathrm{m}^{\wedge} 3$

50-YR DOSE COMMITMENT:

SURFACE BONE 2.0E-02 rem

RED MARROW $1.6 \mathrm{E}-03 \mathrm{rem}$

LIVER 4.4E-03 rem

GONADS 2.5E-04 rem

EFFECTIVE DOSE

EQUIVALENT $1.1 \mathrm{E}-03 \mathrm{rem}$ 
Title of Project EPHA for THM

Subject Radiological Consequence Assessment

Computer D. J. Richards
Date $\underline{09 / 11 / 96}$
Reviewed by S. A. Henderson

Works Calculation 6

Sheet No. 66 of 166

Release Designation: 1-RD-96, Average

HOTSPOT 7.0 GENERAL PLUME 9-10-1996 22:09

RADIONUCLIDE: : PU-238 Inhalation Class : W

HALFLIFE $: 87.740$ years

SOURCE TERM : $1.6 \mathrm{E}-06 \mathrm{Ci}$

Source Term Width: $3 \mathrm{~m}$ Source Term Height: $3 \mathrm{~m}$

RELEASE FRACTION : $1.00 \mathrm{E}+00$

FILTER EFFICIENCY: $0.000000 \%$

EFFECTIVE RELEASE HEIGHT : $\quad 0.00 \mathrm{~m}$

WIND SPEED $(\mathrm{h}=2 \mathrm{~m}): 2.5 \mathrm{~m} / \mathrm{s}$

STABILITY CLASS :C DEPOSITION VELOCITY $: 1.00 \mathrm{~cm} / \mathrm{s}$

RECEPTOR HEIGHT $\quad: 0.0 \mathrm{~m}$ INVERSION LAYER HEIGHT $: 500.0 \mathrm{~m}$

SAMPLE TIME $\quad: 10.000 \mathrm{~min}$

MAXIMUM DOSE DISTANCE $:<0.10 \mathrm{~km}$ MAXIMUM CEDE $:>2.7 \mathrm{E}-04 \mathrm{rem}$

$\mathrm{D}=0.03 \mathrm{~km}$

$\mathrm{DEP}=1.3 \mathrm{E}-04 \mathrm{uCi} / \mathrm{m}^{\wedge} 2$

$\mathrm{CHI}=1.3 \mathrm{E}-08(\mathrm{Ci}-\mathrm{s}) / \mathrm{m}^{\wedge} 3$

50-YR DOSE COMMITMENT:

SURFACE BONE 3.4E-02 rem RED MARROW 2.8E-03 rem LIVER 7.6E-03 rem GONADS 4.2E-04 rem EFFECTIVE DOSE EQUIVALENT $1.9 \mathrm{E}-03 \mathrm{rem}$

\author{
$\mathrm{D}=0.10 \mathrm{~km}$ \\ $\mathrm{DEP}=1.7 \mathrm{E}-05 \mathrm{uCi} / \mathrm{m}^{\wedge} 2$ \\ $\mathrm{CHI}=1.7 \mathrm{E}-09(\mathrm{Ci}-\mathrm{s}) / \mathrm{m}^{\wedge} 3$ \\ 50-YR DOSE COMMITMENT: \\ SURFACE BONE 4.7E-03 rem \\ RED MARROW 3.9E-04 rem \\ LIVER $\quad 1.0 \mathrm{E}-03 \mathrm{rem}$ \\ GONADS 5.8E-05 rem \\ EFFECTIVE DOSE \\ EQUIVALENT 2.7E-04 rem
}


Title of Project EPHA for THM

Subject Radiological Consequence Assessment

Computer D. J. Richards

Date $\underline{09 / 11 / 96}$

Reviewed by $\underline{\text { S. A. Henderson }}$

Works Calculation 6

Sheet No. 67 of 166

Release Designation: 1-RD-97, Adverse

HOTSPOT 7.0 GENERAL PLUME 9-09-1996 21:11

RADIONUCLIDE : PU-238 Inhalation Class : W

HALFLIFE $: 87.740$ years

SOURCE TERM : $2.4 \mathrm{E}-04 \mathrm{Ci}$

Source Term Width: $\quad 3 \mathrm{~m}$ Source Term Height: $3 \mathrm{~m}$

RELEASE FRACTION : $1.00 \mathrm{E}+00$

FILTER EFFICIENCY: $0.000000 \%$

EFFECTIVE RELEASE HEIGHT : $\quad 0.00 \mathrm{~m}$

WIND SPEED $(\mathrm{h}=2 \mathrm{~m}): 1.7 \mathrm{~m} / \mathrm{s}$

STABILITY CLASS :E DEPOSITION VELOCITY : $1.00 \mathrm{~cm} / \mathrm{s}$

RECEPTOR HEIGHT $: 0.0 \mathrm{~m}$ INVERSION LAYER HEIGHT $: 200.0 \mathrm{~m}$

SAMPLE TIME $\quad: 30.000 \mathrm{~min}$

MAXIMUM DOSE DISTANCE $:<0.10 \mathrm{~km}$ MAXIMUM CEDE $:>0.133 \mathrm{rem}$

$\mathrm{D}=0.03 \mathrm{~km}$

$\mathrm{DEP}=5.1 \mathrm{E}-02 \mathrm{uCi} / \mathrm{m}^{\wedge} 2$

$\mathrm{CHI}=5.1 \mathrm{E}-06(\mathrm{Ci}-\mathrm{s}) / \mathrm{m}^{\wedge} 3$

50-YR DOSE COMMITMENT:

SURFACE BONE $1.4 \mathrm{E}+01 \mathrm{rem}$ RED MARROW $1.1 \mathrm{E}+00 \mathrm{rem}$ LIVER $\quad 3.0 \mathrm{E}+00 \mathrm{rem}$ GONADS 1.7E-01 rem EFFECTIVE DOSE EQUTVALENT 7.8E-01 rem
$\mathrm{D}=0.10 \mathrm{~km}$

$\mathrm{DEP}=8.7 \mathrm{E}-03 \mathrm{uCi} / \mathrm{m}^{\wedge} 2$

$\mathrm{CHI}=8.7 \mathrm{E}-07(\mathrm{Ci}-\mathrm{s}) / \mathrm{m}^{\wedge} 3$

50-YR DOSE COMMITMENT:

SURFACE BONE 2.3E+00 rem RED MARROW 1.9E-01 rem LIVER 5.2E-01 rem GONADS 2.9E-02 rem EFFECTTVE DOSE EQUIVALENT $1.3 E-01 \mathrm{rem}$ 
Title of Project EPHA for THM

Subject Radiological Consequence Assessment

Computer D. J. Richards

Date $\underline{09 / 11 / 96}$
Reviewed by S. A. Henderson

Works Calculation 6

Sheet No. 68 of 166

Release Designation: 1-RD-97, Average

HOTSPOT 7.0 GENERAL PLUME 9-09-1996 21:12

RADIONUCLIDE : PU-238 Inhalation Class : W

HALFLIFE : 87.740 years

SOURCE TERM : $2.4 \mathrm{E}-04 \mathrm{Ci}$

Source Term Width: $\quad 3 \mathrm{~m}$ Source Term Height: $3 \mathrm{~m}$

RELEASE FRACTION : $1.00 \mathrm{E}+00$

FILTER EFFICIENCY: $0.000000 \%$

EFFECTIVE RELEASE HEIGHT : $\quad 0.00 \mathrm{~m}$

WIND SPEED $(\mathrm{h}=2 \mathrm{~m}): 2.5 \mathrm{~m} / \mathrm{s}$

STABILITY CLASS :C DEPOSITION VELOCITY $: 1.00 \mathrm{~cm} / \mathrm{s}$

RECEPTOR HEIGHT $: 0.0 \mathrm{~m}$ INVERSION LAYER HEIGHT $: 500.0 \mathrm{~m}$

SAMPLE TIME $: 30.000 \mathrm{~min}$

MAXIMUM DOSE DISTANCE $:<0.10 \mathrm{~km}$ MAXIMUM CEDE $:>0.031 \mathrm{rem}$

\begin{tabular}{l}
$D=0.03 \mathrm{~km}$ \\
$\mathrm{DEP}=1.5 \mathrm{E}-02 \mathrm{uCi} / \mathrm{m}^{\wedge} 2$ \\
$\mathrm{CHI}=1.5 \mathrm{E}-06(\mathrm{Ci}-\mathrm{s}) / \mathrm{m}^{\wedge} 3$ \\
50-YR DOSE COMMITMENT: \\
\hdashline SURFACE BONE $4.0 \mathrm{E}+00 \mathrm{rem}$ \\
RED MARROW $3.3 \mathrm{E}-01 \mathrm{rem}$ \\
LIVER $\quad 8.9 \mathrm{E}-01 \mathrm{rem}$ \\
GONADS $\quad 5.0 \mathrm{E}-02 \mathrm{rem}$ \\
EFFECTIVE DOSE
\end{tabular}

EQUTVALENT 2.3E-01 rem

\author{
$\mathrm{D}=0.10 \mathrm{~km}$ \\ $\mathrm{DEP}=2.0 \mathrm{E}-03 \mathrm{uCi} / \mathrm{m}^{\wedge} 2$ \\ $\mathrm{CHI}=2.0 \mathrm{E}-07(\mathrm{Ci}-\mathrm{s}) / \mathrm{m}^{\wedge} 3$ \\ 50-YR DOSE COMMITMENT: \\ SURFACE BONE 5.5E-01 rem \\ RED MARROW $4.5 E-02$ rem \\ LIVER 1.2E-01 rem \\ GONADS 6.8E-03 rem \\ EFFECTIVE DOSE \\ EQUIVALENT 3.1E-02 rem
}


Title of Project EPHA for THM

Subject Radiological Consequence Assessment

Computer D. J. Richards

Date $\underline{09 / 11 / 96}$

Reviewed by $\underline{\text { S. A. Henderson }}$

Works Calculation 6

Sheet No. 69 of 166

Release Designation: 1-RD-98, Adverse

HOTSPOT 7.0 GENERAL PLUME 9-10-1996 22:11

RADIONUCLIDE : PU-238 Inhalation Class : W

HALFLIFE : 87.740 years

SOURCE TERM : $2.6 \mathrm{E}-04 \mathrm{Ci}$

Source Term Width: $\quad 3 \mathrm{~m}$ Source Term Height: $\quad 3 \mathrm{~m}$

RELEASE FRACTION : $1.00 \mathrm{E}+00$

FILTER EFFICIENCY: $0.000000 \%$

EFFECTIVE RELEASE HEIGHT : $\quad 0.00 \mathrm{~m}$

WIND SPEED ( $h=2 \mathrm{~m}): 1.7 \mathrm{~m} / \mathrm{s}$

STABILITY CLASS :E DEPOSITION VELOCITY : $1.00 \mathrm{~cm} / \mathrm{s}$

RECEPTOR HEIGHT $\quad: 0.0 \mathrm{~m}$ INVERSION LAYER HEIGHT $: 200.0 \mathrm{~m}$

SAMPLE TIME $\quad: 10.000 \mathrm{~min}$

MAXIMUM DOSE DISTANCE $:<0.10 \mathrm{~km}$ MAXIMUM CEDE $:>0.180 \mathrm{rem}$

$\mathrm{D}=0.03 \mathrm{~km}$

$\mathrm{DEP}=6.9 \mathrm{E}-02 \mathrm{uCi} / \mathrm{m}^{\wedge} 2$

$\mathrm{CHI}=6.9 \mathrm{E}-06(\mathrm{Ci}-\mathrm{s}) / \mathrm{m}^{\wedge} 3$

50-YR DOSE COMMITMENT:

SURFACE BONE $1.9 E+01 \mathrm{rem}$ RED MARROW $1.5 \mathrm{E}+00 \mathrm{rem}$

LIVER 4.1E+00 rem

GONADS 2.3E-01 rem

EFFECTIVE DOSE

EQUIVALENT $1.1 \mathrm{E}+00 \mathrm{rem}$
$\mathrm{D}=0.03 \mathrm{~km}$
$\mathrm{DEP}=6.6 \mathrm{E}-02 \mathrm{uCi} / \mathrm{m}^{\wedge} 2$
$\mathrm{CHI}=6.6 \mathrm{E}-06(\mathrm{Ci}-\mathrm{s}) / \mathrm{m}^{\wedge} 3$
$50-\mathrm{YR}$ DOSE COMMTMENT:
SURFACE BONE $1.8 \mathrm{E}+01 \mathrm{rem}$
RED MARROW $\cdot 1.5 \mathrm{E}+00 \mathrm{rem}$
LIVER $\quad 4.0 \mathrm{E}+00 \mathrm{rem}$
GONADS $\quad 2.2 \mathrm{E}-01 \mathrm{rem}$
EFFECTIVE DOSE
EQUTVALENT $1.0 \mathrm{E}+00 \mathrm{rem}$

$\mathrm{D}=0.10 \mathrm{~km}$

$\mathrm{DEP}=1.2 \mathrm{E}-02 \mathrm{uCi} / \mathrm{m}^{\wedge} 2$

$\mathrm{CHI}=1.2 \mathrm{E}-06(\mathrm{Ci}-\mathrm{s}) / \mathrm{m}^{\wedge} 3$

50-YR DOSE COMMITMENT:

SURFACE BONE 3.2E+00 rem RED MARROW 2.6E-01 rem

LIVER 7.1E-01 rem GONADS 3.9E-02 rem EFFECTIVE DOSE EQUIVALENT $1.8 \mathrm{E}-01 \mathrm{rem}$ 
Title of Project EPHA for THM

Subject Radiological Consequence Assessment

Computer D. J. Richards
Reviewed by S. A. Hënderson

Works Calculation 6

Sheet No. 70 of 166

Release Designation: 1-RD-98, Average

HOTSPOT 7.0 GENERAL PLUME 9-10-1996 22:12

RADIONUCLIDE : PU-238 Inhalation Class : W

HALFLIFE $: 87.740$ years

SOURCE TERM : $2.6 \mathrm{E}-04 \mathrm{Ci}$

Source Term Width: $3 \mathrm{~m}$ Source Term Height: $3 \mathrm{~m}$

RELEASE FRACTION : $1.00 \mathrm{E}+00$

FILTER EFFICIENCY: $0.000000 \%$

EFFECTIVE RELEASE HEIGHT : $\quad 0.00 \mathrm{~m}$

WIND SPEED $(\mathrm{h}=2 \mathrm{~m}): 2.5 \mathrm{~m} / \mathrm{s}$

STABILITY CLASS :C DEPOSITION VELOCITY : $1.00 \mathrm{~cm} / \mathrm{s}$

RECEPTOR HEIGHT $\quad: 0.0 \mathrm{~m}$ INVERSION LAYER HEIGHT $: 500.0 \mathrm{~m}$

SAMPLE TIME $\quad: 10.000 \mathrm{~min}$

MAXIMUM DOSE DISTANCE $:<0.10 \mathrm{~km}$ MAXIMUM CEDE $:>0.042 \mathrm{rem}$

$D=0.03 \mathrm{~km}$

$\mathrm{DEP}=2.0 \mathrm{E}-02 \mathrm{uCi} / \mathrm{m}^{\wedge} 2$

$\mathrm{CHI}=2.0 \mathrm{E}-06(\mathrm{Ci}-\mathrm{s}) / \mathrm{m}^{\wedge} 3$

50-YR DOSE COMMITMENT:

SURFACE BONE 5.5E+00 rem RED MARROW 4.5E-01 rem LIVER 1.2E+00 rem GONADS 6.7E-02 rem EFFECTIVE DOSE

EQUIVALENT 3.1E-ò1 rem
$\mathrm{D}=0.10 \mathrm{~km}$

$\mathrm{DEP}=2.8 \mathrm{E}-03 \mathrm{uCi} / \mathrm{m}^{\wedge} 2$

$\mathrm{CHI}=2.8 \mathrm{E}-07(\mathrm{Ci}-\mathrm{s}) / \mathrm{m}^{\wedge} 3$

50-YR DOSE COMMITMENT:

SURFACE BONE 7.5E-01 rem RED MARROW 6.2E-02 rem

LIVER 1.7E-01 rem GONADS 9.2E-03 rem EFFECTTVE DOSE

EQUTVALENT 4.2E-02 rem 
Title of Project EPHA for THM

Reviewed by $\underline{\text { S. A. Henderson }}$

Subject Radiological Consequence Assessment

Sheet No. 71 of 166

Release Designation: 1-RD-99, Adverse

HOTSPOT 7.0 GEṆERAL PLUME 9-09-1996 21:13

RADIONUCLIDE : PU-238 Inhalation Class : W

HALFLIFE $: 87.740$ years

SOURCE TERM : $4.5 \mathrm{E}-05 \mathrm{Ci}$

Source Term Width: $3 \mathrm{~m}$ Source Term Height: $3 \mathrm{~m}$

RELEASE FRACTION : $1.00 \mathrm{E}+00$

FILTER EFFICIENCY: $0.000000 \%$

EFFECTIVE RELEASE HEIGHT : $\quad 0.00 \mathrm{~m}$

WIND SPEED $(h=2 \mathrm{~m}): 1.7 \mathrm{~m} / \mathrm{s}$

STABILITY CLASS :E DEPOSITION VELOCITY $: 1.00 \mathrm{~cm} / \mathrm{s}$

RECEPTOR HEIGHT $: 0.0 \mathrm{~m}$ INVERSION LAYER HEIGHT $: 200.0 \mathrm{~m}$

SAMPLE TIME $\quad: 30.000 \mathrm{~min}$

MAXIMUM DOSE DISTANCE $:<0.10 \mathrm{~km}$ MAXIMUM CEDE $:>0.025 \mathrm{rem}$

$\mathrm{D}=0.03 \mathrm{~km}$

$\mathrm{DEP}=9.5 \mathrm{E}-03 \mathrm{uCi} / \mathrm{m}^{\wedge} 2$

$\mathrm{CHI}=9.5 \mathrm{E}-07(\mathrm{Ci}-\mathrm{s}) / \mathrm{m}^{\wedge} 3$

50-YR DOSE COMMITMENT:

SURFACE BONE $2.6 \mathrm{E}+00 \mathrm{rem}$ RED MARROW 2.1E-01 rem LIVER 5.7E-01 rem GONADS 3.2E-02 rem EFFECTIVE DOSE

EQUIVALENT $1.5 \mathrm{E}-01 \mathrm{rem}$
$\mathrm{D}=0.10 \mathrm{~km}$
$\mathrm{DEP}=1.6 \mathrm{E}-03 \mathrm{uCi} / \mathrm{m}^{\wedge} 2$
$\mathrm{CHI}=1.6 \mathrm{E}-07(\mathrm{Ci}-\mathrm{s}) / \mathrm{m}^{\wedge} 3$
50-YR DOSE COMMITMENT:
SURFACE BONE 4.4E-01 rem RED MARROW 3.6E-02 rem LIVER 9.7E-02 rem GONADS 5.4E-03 rem EFFECTIVE DOSE
EQUIVALENT - 2.5E-02 rem 
Title of Project EPHA for THM

Subject Radiological Consequence Assessment

Computer D. J. Richards
Reviewed by $\mathbf{S . A . ~ H e n d e r s o n}$ Works Calculation 6

Sheet No. 72 of 166

Release Designation: 1-RD-99, Average

HOTSPOT 7.0 GENERAL PLUME 9-09-1996 21:14

RADIONUCLIDE : PU-238 Inhalation Class : W

HALFLIFE : 87.740 years

SOURCE TERM : 4.5E-05 Ci

Source Term Width: $\quad 3 \mathrm{~m}$ Source Term Height: $3 \mathrm{~m}$

RELEASE FRACTION : $1.00 \mathrm{E}+00$

FILTER EFFICIENCY: $0.000000 \%$

EFFECTIVE RELEASE HEIGHT : $\quad 0.00 \mathrm{~m}$

WIND SPEED $(\mathrm{h}=2 \mathrm{~m}): 2.5 \mathrm{~m} / \mathrm{s}$

STABILITY CLASS :C DEPOSITION VELOCITY $\quad: 1.00 \mathrm{~cm} / \mathrm{s}$

RECEPTOR HEIGHT $: 0.0 \mathrm{~m}$ INVERSION LAYER HEIGHT $: 500.0 \mathrm{~m}$

SAMPLE TIME $: 30.000 \mathrm{~min}$

MAXIMUM DOSE DISTANCE $:<0.10 \mathrm{~km}$ MAXIMUM CEDE $:>5.8 \mathrm{E}-03 \mathrm{rem}$

$\mathrm{D}=0.03 \mathrm{~km}$

$\mathrm{DEP}=2.8 \mathrm{E}-03 \mathrm{uCi} / \mathrm{m}^{\wedge} 2$

$\mathrm{CHI}=2.8 \mathrm{E}-07(\mathrm{Ci}-\mathrm{s}) / \mathrm{m}^{\wedge} 3$

50-YR DOSE COMMITMENT:

SURFACE BONE 7.5E-01 rem RED MARROW 6.2E-02 rem LIVER 1.7E-01 rem GONADS 9.3E-03 rem EFFECTIVE DOSE

EQUIVALENT 4.3E-02 rem
$\mathrm{D}=0.10 \mathrm{~km}$

$\mathrm{DEP}=3.8 \mathrm{E}-04 \mathrm{uCi} / \mathrm{m}^{\wedge} 2$

$\mathrm{CHI}=3.8 \mathrm{E}-08(\mathrm{Ci}-\mathrm{s}) / \mathrm{m}^{\wedge} 3$

50-YR DOSE COMMITMENT:

SURFACE BONE 1.0E-01 rem

RED MARROW $8.5 \mathrm{E}-03 \mathrm{rem}$

LIVER 2.3E-02 rem

GONADS 1.3E-03 rem

EFFECTIVE DOSE

EQUTVALENT $5.8 \mathrm{E}-03 \mathrm{rem}$ 
Title of Project EPHA for THM

Subject Radiological Consequence Assessment

Computer D. J. Richards

Date $09 / 11 / 96$

Reviewed by S.A. Henderson

Works Calculation 6

Sheet No. 73 of 166

Release Designation: 1-RD-100, Adverse

HOTSPOT 7.0 GENERAL PLUME 9-10-1996 22:13

RADIONUCLIDE : PU-238 Inhalation Class : W

HALFLIFE : 87.740 years

SOURCE TERM : 4.9E-05 Ci

Source Term Width: $3 \mathrm{~m}$ Source Term Height: $3 \mathrm{~m}$

RELEASE FRACTION : $1.00 \mathrm{E}+00$

FILTER EFFICIENCY: $0.000000 \%$

EFFECTIVE RELEASE HEIGHT : $\quad 0.00 \mathrm{~m}$

WIND SPEED ( $h=2 \mathrm{~m}): 1.7 \mathrm{~m} / \mathrm{s}$

STABILITY CLASS :E DEPOSITION VELOCITY $: 1.00 \mathrm{~cm} / \mathrm{s}$

RECEPTOR HEIGHT $: 0.0 \mathrm{~m}$ INVERSION LAYER HEIGHT $: 200.0 \mathrm{~m}$

SAMPLE TIME $\quad: 10.000 \mathrm{~min}$

MAXIMUM DOSE DISTANCE $:<0.10 \mathrm{~km}$ MAXIMUM CEDE $:>0.034 \mathrm{rem}$

$\mathrm{D}=0.03 \mathrm{~km}$

$\mathrm{DEP}=1.3 \mathrm{E}-02 \mathrm{uCi} / \mathrm{m}^{\wedge} 2$

$\mathrm{CHI}=1.3 \mathrm{E}-06(\mathrm{Ci}-\mathrm{s}) / \mathrm{m}^{\wedge} 3$

50-YR DOSE COMMITMENT:

SURFACE BONE 3.5E+00 rem RED MARROW 2.9E-01 rem

LIVER 7.8E-01 rem GONADS 4.3E-02 rem EFFECTIVE DOSE

EQUIVALENT 2.0E-01 rem

$$
\begin{aligned}
& \mathrm{D}=0.10 \mathrm{~km} \\
& \mathrm{DEP}=2.2 \mathrm{E}-03 \mathrm{uCi} / \mathrm{m}^{\wedge} 2 \\
& \mathrm{CHI}=2.2 \mathrm{E}-07(\mathrm{Ci}) / \mathrm{m}^{\wedge} 3 \\
& \text { 50-YR DOSE COMMITMENT: }
\end{aligned}
$$

SURFACE BONE 5.9E-01 rem RED MARROW 4.9E-02 rem LIVER 1.3E-01 rem GONADS 7.3E-03 rem EFFECTIVE DOSE EQUIVALENT 3.4E-02 rem 
Title of Project EPHA for THM

Subject Radiological Consequence Assessment

Computer D. J. Richards
Reviewed by S. A. Henderson Works Calculation 6 Sheet No. 74 of 166

Release Designation: 1-RD-100, Average

HOTSPOT 7.0 GENERAL PLUME 9-10-1996 22:13

RADIONUCLIDE : PU-238 Inhalation Class : W

HALFLIFE : 87.740 years

SOURCE TERM : 4.9E-05 Ci

Source Term Width: $\quad 3 \mathrm{~m}$ Source Term Height: $3 \mathrm{~m}$

RELEASE FRACTION : $1.00 \mathrm{E}+00$

FILTER EFFICIENCY: $0.000000 \%$

EFFECTIVE RELEASE HEIGHT : $\quad 0.00 \mathrm{~m}$

WIND SPEED ( $\mathrm{h}=2 \mathrm{~m}): 2.5 \mathrm{~m} / \mathrm{s}$

STABILITY CLASS :C DEPOSITION VELOCITY $: 1.00 \mathrm{~cm} / \mathrm{s}$

RECEPTOR HEIGHT $\quad: 0.0 \mathrm{~m}$ INVERSION LAYER HEIGHT $: 500.0 \mathrm{~m}$

SAMPLE TIME $\quad: 10.000 \mathrm{~min}$

MAXIMUM DOSE DISTANCE $:<0.10 \mathrm{~km}$ MAXIMUM CEDE $:>7.9 \mathrm{E}-03 \mathrm{rem}$

$\mathrm{D}=0.03 \mathrm{~km}$

$\mathrm{DEP}=3.8 \mathrm{E}-03 \mathrm{uCi} / \mathrm{m}^{\wedge} 2$

$\mathrm{CHI}=3.8 \mathrm{E}-07(\mathrm{Ci}-\mathrm{s}) / \mathrm{m}^{\wedge} 3$

50-YR DOSE COMMITMENT:

SURFACE BONE $1.0 \mathrm{E}+00 \mathrm{rem}$ RED MARROW 8.5E-02 rem LIVER 2.3E-01 rem GONADS 1.3E-02 rem EFFECTIVE DOSE

EQUIVALENT 5.8E-02 rem
$\mathrm{D}=0.10 \mathrm{~km}$
$\mathrm{DEP}=5.2 \mathrm{E}-04 \mathrm{uCi} / \mathrm{m}^{\wedge} 2$
$\mathrm{CHI}=5.2 \mathrm{E}-08(\mathrm{Ci}-\mathrm{s}) / \mathrm{m}^{\wedge} 3$
50-YR DOSE COMMITMENT:
SURFACE BONE 1.4E-01 rem
RED MARROW 1.2E-02 rem
LIVER 3.1E-02 rem
GONADS 1.7E-03 rem
EFFECTIVE DOSE
EQUIVALENT 7.9E-03 rem 
Title of Project EPHA for THM

Subject Radiological Consequence Assessment

Computer D. J. Richards

Date $\underline{09 / 11 / 96}$

Reviewed by $\underline{S}$. A. Hènderson

Works Calculation 6

Sheet No. 75 of 166

Release Designation: 1-RD-101, Adverse

HOTSPOT 7.0 GENERAL PLUME 9-09-1996 21:16

RADIONUCLIDE : PU-238 Inhalation Class : W

HALFLIFE : 87.740 years

SOURCE TERM : $9.5 \mathrm{E}-03 \mathrm{Ci}$

Source Term Width: $\quad 3 \mathrm{~m}$ Source Term Height: $3 \mathrm{~m}$

RELEASE FRACTION : $1.00 \mathrm{E}+00$

FILTER EFFICIENCY: $0.000000 \%$

EFFECTIVE RELEASE HEIGHT : $\quad 0.00 \mathrm{~m}$

WIND SPEED $(\mathrm{h}=2 \mathrm{~m}): 1.7 \mathrm{~m} / \mathrm{s}$

STABILITY CLASS :E DEPOSITION VELOCITY $: 1.00 \mathrm{~cm} / \mathrm{s}$

RECEPTOR HEIGHT $\quad: 0.0 \mathrm{~m}$ INVERSION LAYER HEIGHT $: 200.0 \mathrm{~m}$

SAMPLE TIME $\quad: 30.000 \mathrm{~min}$

MAXIMUM DOSE DISTANCE $:<0.10 \mathrm{~km}$ MAXIMUM CEDE $:>5.3 \mathrm{rem}$

$\mathrm{D}=0.03 \mathrm{~km}$

$\mathrm{DEP}=2.0 \mathrm{E}+00 \mathrm{uCi} / \mathrm{m}^{\wedge} 2$

$\mathrm{CHI}=2.0 \mathrm{E}-04(\mathrm{Ci}-\mathrm{s}) / \mathrm{m}^{\wedge} 3$

50-YR DOSE COMMITMENT:

SURFACE BONE 5.4E+02 rem RED MARROW 4.5E+01 rem LIVER $\quad 1.2 \mathrm{E}+02 \mathrm{rem}$ GONADS 6.7E+00 rem EFFECTIVE DOSE EQUIVALENT $3.1 \mathrm{E}+01 \mathrm{rem}$
$\mathrm{D}=0.10 \mathrm{~km}$

$\mathrm{DEP}=3.4 \mathrm{E}-01 \mathrm{uCi} / \mathrm{m}^{\wedge} 2$

$\mathrm{CHI}=3.4 \mathrm{E}-05(\mathrm{Ci}-\mathrm{s}) / \mathrm{m}^{\wedge} 3$

50-YR DOSE COMMITMENT:

SURFACE BONE 9.3E+01 rem RED MARROW 7.7E+00 rem LIVER 2.1E+01 rem GONADS $1.1 \mathrm{E}+00 \mathrm{rem}$ EFFECTIVE DOSE EQUTVALENT $5.3 \mathrm{E}+00 \mathrm{rem}$
$\mathrm{D}=0.25 \mathrm{~km}$ $\mathrm{DEP}=6.7 \mathrm{E}-02 \mathrm{uCi} / \mathrm{m}^{\wedge} 2$

$\mathrm{CHI}=6.7 \mathrm{E}-06(\mathrm{Ci}-\mathrm{s}) / \mathrm{m}^{\wedge} 3$ 50-YR DOSE COMMITMENT:

SURFACE BONE 1.8E+01 rem RED MARROW $1.5 \mathrm{E}+00 \mathrm{rem}$ LIVER $\quad 4.0 \mathrm{E}+00 \mathrm{rem}$ GONADS 2.2E-01 rem EFFECTIVE DOSE EQUIVALENT $1.0 \mathrm{E}+00 \mathrm{rem}$ 
Title of Project EPHA for THM

Subject Radiological Consequence Assessment

Computer D. J. Richards

Date $\underline{09 / 11 / 96}$

Reviewed by S. A. Henderson

Works Calculation 6

Sheet No. 76 of 166

Release Designation: 1-RD-101, Average

HOTSPOT 7.0 GENERAL PLUME 9-09-1996 21:29

RADIONUCLIDE: PU-238 Inhalation Class : W

HALFLIFE : 87.740 years

SOURCE TERM $\quad: 9.5 \mathrm{E}-03 \mathrm{Ci}$

Source Term Width: $3 \mathrm{~m}$ Source Term Height: $3 \mathrm{~m}$

RELEASE FRACTION : $1.00 E+00$

FILTER EFFICIENCY: $0.000000 \%$

EFFECTIVE RELEASE HEIGHT : $\quad 0.00 \mathrm{~m}$

WIND SPEED $(\mathrm{h}=2 \mathrm{~m}): 2.5 \mathrm{~m} / \mathrm{s}$

STABILITY CLASS $\quad$ :C DEPOSITION VELOCITY $: 1.00 \mathrm{~cm} / \mathrm{s}$

RECEPTOR HEIGHT $: 0.0 \mathrm{~m}$ INVERSION LAYER HEIGHT $: 500.0 \mathrm{~m}$

SAMPLE TIME $\quad: 30.000 \mathrm{~min}$

MAXIMUM DOSE DISTANCE $:<0.10 \mathrm{~km}$ MAXIMUM CEDE $:>1.2 \mathrm{rem}$

\begin{abstract}
$\mathrm{D}=0.03 \mathrm{~km}$
$\mathrm{DEP}=5.9 \mathrm{E}-01 \mathrm{uCi} / \mathrm{m}^{\wedge} 2$

$\mathrm{CHI}=5.9 \mathrm{E}-05(\mathrm{Ci}-\mathrm{s}) / \mathrm{m}^{\wedge} 3$

50-YR DOSE COMMTTMENT:

SURFACE BONE $1.6 \mathrm{E}+02 \mathrm{rem}$ RED MARROW 1.3E $+01 \mathrm{rem}$ LIVER $3.5 \mathrm{E}+01 \mathrm{rem}$ GONADS 2.0E+00 rem EFFECTIVE DOSE

EQUIVALENT $\quad 9.0 \mathrm{E}+00 \mathrm{rem}$
\end{abstract}

\author{
$\mathrm{D}=0.10 \mathrm{~km}$ \\ $\mathrm{DEP}=8.0 \mathrm{E}-02 \mathrm{uCi} / \mathrm{m}^{\wedge} 2$ \\ $\mathrm{CHI}=8.0 \mathrm{E}-06(\mathrm{Ci}-\mathrm{s}) / \mathrm{m}^{\wedge} 3$ \\ 50-YR DOSE COMMITMENT: \\ SURFACE BONE 2.2E+01 rem \\ RED MARROW $1.8 \mathrm{E}+00 \mathrm{rem}$ \\ LIVER $\quad 4.8 \mathrm{E}+00 \mathrm{rem}$ \\ GONADS 2.7E-01 rem \\ EFFECTIVE DOSE \\ EQUIVALENT $1.2 \mathrm{E}+00 \mathrm{rem}$
}

$$
\begin{aligned}
& \mathrm{D}=0.11 \mathrm{~km} \\
& \mathrm{DEP}=6.8 \mathrm{E}-02 \mathrm{uCi} / \mathrm{m}^{\wedge} 2 \\
& \mathrm{CHI}=6.8 \mathrm{E}-06(\mathrm{Ci}-\mathrm{s}) / \mathrm{m}^{\wedge} 3 \\
& \text { 50-YR DOSE COMMITMENT: }
\end{aligned}
$$

SURFACE BONE $1.8 \mathrm{E}+01 \mathrm{rem}$ RED MARROW $1.5 \mathrm{E}+00 \mathrm{rem}$ LIVER 4.1E $+00 \mathrm{rem}$ GONADS 2.3E-01 rem EFFECTIVE DOSE EQUIVALENT $1.0 \mathrm{E}+00 \mathrm{rem}$ 
Title of Project EPHA for THM

Reviewed by $\underline{\text { S. A. Henderson }}$

Subject Radiological Consequence Assessment

Sheet No. 77 of 166

Release Designation: 1-RD-102, Adverse

HOTSPOT 7.0 GENERAL PLUME 9-10-1996 22:16

RADIONUCLIDE : PU-238 Inhalation Class : W

HALFLIFE : 87.740 years

SOURCE TERM $: 1.0 \mathrm{E}-02 \mathrm{Ci}$

Source Term Width: $3 \mathrm{~m}$ Source Term Height: $3 \mathrm{~m}$

RELEASE FRACTION : $1.00 \mathrm{E}+00$

FILTER EFFICIENCY: $0.000000 \%$

EFFECTIVE RELEASE HEIGHT : $\quad 0.00 \mathrm{~m}$

WIND SPEED ( $\mathrm{h}=2 \mathrm{~m}): 1.7 \mathrm{~m} / \mathrm{s}$

STABILITY CLASS :E DEPOSITION VELOCITY $: 1.00 \mathrm{~cm} / \mathrm{s}$

RECEPTOR HEIGHT $\quad: 0.0 \mathrm{~m}$ INVERSION LAYER HEIGHT $: 200.0 \mathrm{~m}$

SAMPLE TIME $\quad: 10.000 \mathrm{~min}$

MAXIMUM DOSE DISTANCE $:<0.10 \mathrm{~km}$ MAXIMUM CEDE $:>7.2 \mathrm{rem}$

$D=0.03 \mathrm{~km}$

$\mathrm{DEP}=2.7 \mathrm{E}+00 \mathrm{uCi} / \mathrm{m}^{\wedge} 2$

$\mathrm{CHI}=2.7 \mathrm{E}-04(\mathrm{Ci}-\mathrm{s}) / \mathrm{m}^{\wedge} 3$

50-YR DOSE COMMITMENT:

SURFACE BONE 7.4E+02 rem RED MARROW $6.1 \mathrm{E}+01 \mathrm{rem}$ LIVER $1.6 \mathrm{E}+02$ rem GONADS 9.1E+00 rem EFFECTIVE DOSE EQUIVALENT 4.2E+01 rem
$\mathrm{D}=0.10 \mathrm{~km}$

$\mathrm{DEP}=4.7 \mathrm{E}-01 \mathrm{uCi} / \mathrm{m}^{\wedge} 2$

$\mathrm{CHI}=4.7 \mathrm{E}-05(\mathrm{Ci}-\mathrm{s}) / \mathrm{m}^{\wedge} 3$

50-YR DOSE COMMITMENT:

SURFACE BONE 1.3E+02 rem RED MARROW $1.0 \mathrm{E}+01 \mathrm{rem}$ LIVER 2.8E+01 rem GONADS $1.6 \mathrm{E}+00 \mathrm{rem}$ ' EFFECTIVE DOSE EQUIVALENT $7.2 \mathrm{E}+00 \mathrm{rem}$
$\mathrm{D}=0.29 \mathrm{~km}$

$\mathrm{DEP}=6.8 \mathrm{E}-02 \mathrm{uCi} / \mathrm{m}^{\wedge} 2$

$\mathrm{CHI}=6.8 \mathrm{E}-06(\mathrm{Ci}-\mathrm{s}) / \mathrm{m}^{\wedge} 3$

50-YR DOSE COMMITMENT:

SURFACE BONE 1.8E+01 rem RED MARROW $1.5 \mathrm{E}+00 \mathrm{rem}$ LIVER $\quad 4.1 \mathrm{E}+00 \mathrm{rem}$ GONADS 2.3E-01 rem EFFECTIVE DOSE EQUTVALENT $1.0 \mathrm{E}+00 \mathrm{rem}$ 
Title of Project EPHA for THM

Subject Radiological Consequence Assessment

Computer D. J. Richards

Date $\underline{09 / 11 / 96}$

Reviewed by S. A. Henderson

Works Calculation 6

Sheet No. 78 of 166

Release Designation: 1-RD-102, Average

HOTSPOT 7.0 GENERAL PLUME 9-10-1996 22:17

RADIONUCLIDE : PU-238 Inhalation Class : W

HALFLIFE $: 87.740$ years

SOURCE TERM : $1.0 \mathrm{E}-02 \mathrm{Ci}$

Source Term Width: $\quad 3 \mathrm{~m}$ Source Term Height: $3 \mathrm{~m}$

RELEASE FRACTION : $1.00 \mathrm{E}+00$

FILTER EFFICIENCY: $0.000000 \%$

EFFECTIVE RELEASE HEIGHT : $\quad 0.00 \mathrm{~m}$

WIND SPEED $(\mathrm{h}=2 \mathrm{~m}): 2.5 \mathrm{~m} / \mathrm{s}$

STABILITY CLASS :C DEPOSITION VELOCITY $: 1.00 \mathrm{~cm} / \mathrm{s}$

RECEPTOR HEIGHT $\quad: 0.0 \mathrm{~m}$ INVERSION LAYER HEIGHT $: 500.0 \mathrm{~m}$

SAMPLE TIME $\quad: 10.000 \mathrm{~min}$

MAXIMUM DOSE DISTANCE $:<0.10 \mathrm{~km}$ MAXIMUM CEDE $:>1.7 \mathrm{rem}$

$\mathrm{D}=0.03 \mathrm{~km}$

$\mathrm{DEP}=8.0 \mathrm{E}-01 \mathrm{uCi} / \mathrm{m}^{\wedge} 2$

$\mathrm{CHI}=8.0 \mathrm{E}-05(\mathrm{Ci}-\mathrm{s}) / \mathrm{m}^{\wedge} 3$

50-YR DOSE COMMITMENT:

SURFACE BONE 2.2E+02 rem RED MARROW $1.8 \mathrm{E}+01 \mathrm{rem}$ LIVER 4.8E+01 rem GONADS 2.7E+00 rem EFFECTIVE DOSE

EQUIVALENT $1.2 \mathrm{E}+01 \mathrm{rem}$
$\mathrm{D}=0.10 \mathrm{~km}$

$\mathrm{DEP}=1.1 \mathrm{E}-01 \mathrm{uCi} / \mathrm{m}^{\wedge} 2$

$\mathrm{CHI}=1.1 \mathrm{E}-05(\mathrm{Ci}-\mathrm{s}) / \mathrm{m}^{\wedge} 3$

50-YR DOSE COMMITMENT:

SURFACE BONE 3.0E+01 rem RED MARROW $2.4 \mathrm{E}+00 \mathrm{rem}$ LIVER $\quad 6.6 \mathrm{E}+00 \mathrm{rem}$ GONADS 3.7E-01 rem EFFECTIVE DOSE EQUIVALENT 1.7E+00 rem
$\mathrm{D}=0.13 \mathrm{~km}$

$\mathrm{DEP}=6.8 \mathrm{E}-02 \mathrm{uCi} / \mathrm{m}^{\wedge} 2$

$\mathrm{CHI}=6.8 \mathrm{E}-06(\mathrm{Ci}-\mathrm{s}) / \mathrm{m}^{\wedge} 3$

50-YR DOSE COMMITMENT:

SURFACE BONE $1.8 \mathrm{E}+01 \mathrm{rem}$ RED MARROW $1.5 \mathrm{E}+00 \mathrm{rem}$ LIVER 4.1E+00 rem GONADS 2.3E-01 rem EFFECTIVE DOSE EQUTVALENT $\quad 1.0 \mathrm{E}+00 \mathrm{rem}$ 
Title of Project EPHA for THM

Subject Radiological Consequence Assessment

Computer D. J. Richards
Reviewed by $\mathrm{S}$. A. Henderson

Works Calculation 6

Sheet No. 79 of 166

Release Designation: 1-RD-103, Adverse

HOTSPOT 7.0 GENERAL PLUME 9-09-1996 21:31

RADIONUCLIDE : PU-238 Inhalation Class : W

HALFLIFE $: 87.740$ years

SOURCE TERM : 1.7E-03 Ci

Source Term Width: $\quad 3 \mathrm{~m}$ Source Term Height: $3 \mathrm{~m}$

RELEASE FRACTION : $1.00 \mathrm{E}+00$

FILTER EFFICIENCY: $0.000000 \%$

EFFECTTVE RELEASE HEIGHT : $\quad 0.00 \mathrm{~m}$

WIND SPEED ( $\mathrm{h}=2 \mathrm{~m}): 1.7 \mathrm{~m} / \mathrm{s}$

STABILITY CLASS :E DEPOSITION VELOCITY $\quad: 1.00 \mathrm{~cm} / \mathrm{s}$

RECEPTOR HEIGHT $\quad: 0.0 \mathrm{~m}$ INVERSION LAYER HEIGHT $: 200.0 \mathrm{~m}$

SAMPLE TIME $\quad: 30.000 \mathrm{~min}$

MAXIMUM DOSE DISTANCE $:<0.10 \mathrm{~km}$ MAXIMUM CEDE $:>0.946 \mathrm{rem}$

$\mathrm{D}=0.03 \mathrm{~km}$

$\mathrm{DEP}=3.6 \mathrm{E}-01 \mathrm{uCi} / \mathrm{m}^{\wedge} 2$

$\mathrm{CHI}=3.6 \mathrm{E}-05(\mathrm{Ci}-\mathrm{s}) / \mathrm{m}^{\wedge} 3$

50-YR DOSE COMMITMENT:

SURFACE BONE 9.8E+01 rem RED MARROW $\quad 8.1 \mathrm{E}+00 \mathrm{rem}$ LIVER 2.2E+01 rem GONADS $1.2 \mathrm{E}+00 \mathrm{rem}$ EFFECTIVE DOSE

EQUIVALENT $5.5 \mathrm{E}+00 \mathrm{rem}$

\author{
$\mathrm{D}=0.09 \mathrm{~km}$ \\ $\mathrm{DEP}=6.8 \mathrm{E}-02 \mathrm{uCi} / \mathrm{m}^{\wedge} 2$ \\ $\mathrm{CHI}=6.8 \mathrm{E}-06(\mathrm{Ci}-\mathrm{s}) / \mathrm{m}^{\wedge} 3$ \\ 50-YR DOSE COMMITMENT: \\ SURFACE BONE $1.8 \mathrm{E}+01 \mathrm{rem}$ \\ RED MARROW $1.5 \mathrm{E}+00 \mathrm{rem}$ \\ LIVER 4.1E+00 rem \\ GONADS 2.3E-01 rem \\ EFFECTIVE DOSE \\ EQUTVALENT $1.0 \mathrm{E}+00 \mathrm{rem}$
}

\author{
$\mathrm{D}=0.10 \mathrm{~km}$ \\ $\mathrm{DEP}=6.2 \mathrm{E}-02 \mathrm{uCi} / \mathrm{m}^{\wedge} 2$ \\ $\mathrm{CHI}=6.2 \mathrm{E}-06(\mathrm{Ci}-\mathrm{s}) / \mathrm{m}^{\wedge} 3$ \\ 50-YR DOSE COMMITMENT: \\ SURFACE BONE 1.7E+01 rem \\ RED MARROW $1.4 \mathrm{E}+00 \mathrm{rem}$ \\ LIVER 3.7E+00 rem \\ GONADS 2.1E-01 rem \\ EFFECTIVE DOSE \\ EQUIVALENT 9.5E-01 rem
}


Title of Project EPHA for THM

Subject Radiological Consequence Assessment

Computer D. J. Richards

Date 09/11/96
Reviewed by $\underline{\text { S. A. Henderson }}$

Works Calculation 6

Sheet No. 80 of 166

Release Designation: 1-RD-103, Average

HOTSPOT 7.0 GENERAL PLUME 9-09-1996 21:33

RADIONUCLIDE : PU-238 Inhalation Class : W

HALFLIFE : 87.740 years

SOURCE TERM : $1.7 \mathrm{E}-03 \mathrm{Ci}$

Source Term Width: $\quad 3 \mathrm{~m}$ Source Term Height: $\quad 3 \mathrm{~m}$

RELEASE FRACTION : $1.00 E+00$

FILTER EFFICIENCY: $0.000000 \%$

EFFECTIVE RELEASE HEIGHT : $\quad 0.00 \mathrm{~m}$

WIND SPEED $(\mathrm{h}=2 \mathrm{~m}): 2.5 \mathrm{~m} / \mathrm{s}$

STABILITY CLASS :C DEPOSITION VELOCITY $: 1.00 \mathrm{~cm} / \mathrm{s}$

RECEPTOR HEIGHT $: 0.0 \mathrm{~m}$ INVERSION LAYER HEIGHT $: 500.0 \mathrm{~m}$

SAMPLE TIME $\quad: 30.000 \mathrm{~min}$

MAXIMUM DOSE DISTANCE $:<0.10 \mathrm{~km}$ MAXIMUM CEDE $:>0.222 \mathrm{rem}$

$\mathrm{D}=0.03 \mathrm{~km}$

$\mathrm{DEP}=1.1 \mathrm{E}-01 \mathrm{uCi} / \mathrm{m}^{\wedge} 2$

$\mathrm{CHI}=1.1 \mathrm{E}-05(\mathrm{Ci}-\mathrm{s}) / \mathrm{m}^{\wedge} 3$

50-YR DOSE COMMITMENT:

SURFACE BONE 2.9E+01 rem RED MARROW $2.4 \mathrm{E}+00 \mathrm{rem}$

LIVER $\quad 6.4 \mathrm{E}+00 \mathrm{rem}$ GONADS 3.5E-01 rem EFFECTIVE DOSE EQUIVALENT $1.6 \mathrm{E}+00 \mathrm{rem}$
$\mathrm{D}=0.04 \mathrm{~km}$

$\mathrm{DEP}=6.6 \mathrm{E}-02 \mathrm{uCi} / \mathrm{m}^{\wedge} 2$

$\mathrm{CHI}=6.6 \mathrm{E}-06(\mathrm{Ci}-\mathrm{s}) / \mathrm{m}^{\wedge} 3$

50-YR DOSE COMMITMENT:

SURFACE BONE $1.8 \mathrm{E}+01$ rem RED MARROW $1.5 \mathrm{E}+00 \mathrm{rem}$ LIVER $\quad 4.0 \mathrm{E}+00 \mathrm{rem}$ GONADS 2.2E-01 rem EFFECTIVE DOSE EQUIVALENT $1.0 \mathrm{E}+00 \mathrm{rem}$
$\mathrm{D}=0.10 \mathrm{~km}$

$\mathrm{DEP}=1.4 \mathrm{E}-02 \mathrm{uCi} / \mathrm{m}^{\wedge} 2$

$\mathrm{CHI}=1.4 \mathrm{E}-06(\mathrm{Ci}-\mathrm{s}) / \mathrm{m}^{\wedge} 3$

50-YR DOSE COMMITMENT:

SURFACE BONE 3.9E+00 rem RED MARROW 3.2E-01 rem LIVER $\quad 8.7 \mathrm{E}-01 \mathrm{rem}$ GONADS 4.8E-02 rem EFFECTIVE DOSE EQUIVALENT 2.2E-01 rem 
Title of Project EPHA for THM

Subject Radiological Consequence Assessment

Computer D. J. Richards

Release Designation: 1-RD-104, Adverse

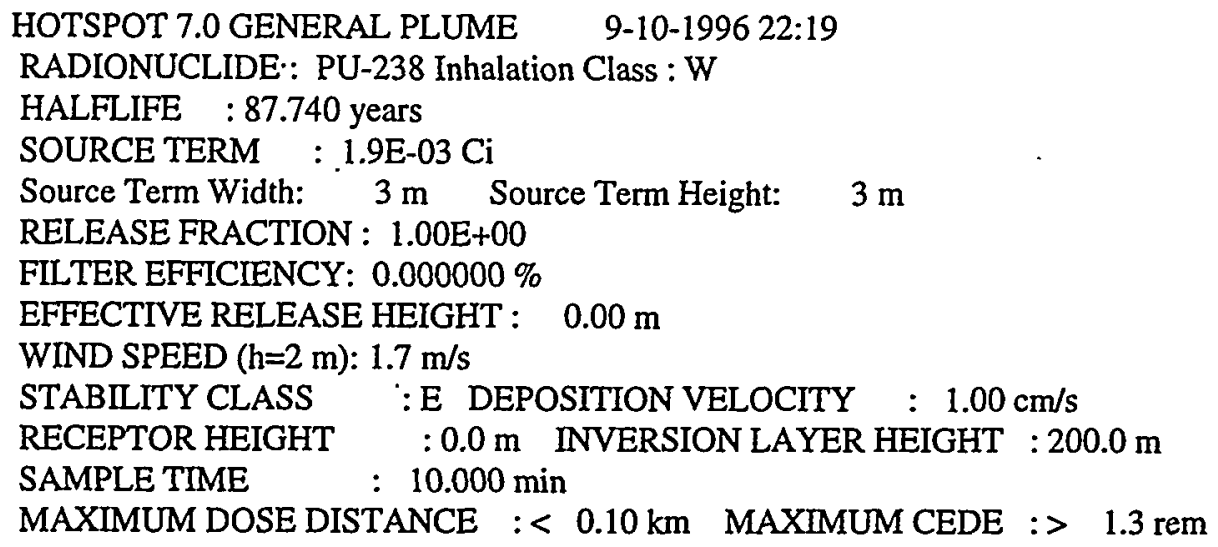

$\mathrm{D}=0.03 \mathrm{~km}$

$\mathrm{DEP}=4.9 \mathrm{E}-01 \mathrm{uCi} / \mathrm{m}^{\wedge} 2$

$\mathrm{CHI}=4.9 \mathrm{E}-05(\mathrm{Ci}-\mathrm{s}) / \mathrm{m}^{\wedge} 3$

50-YR DOSE COMMITMENT:

SURFACE BONE 1.3E+02 rem RED MARROW $1.1 \mathrm{E}+01 \mathrm{rem}$ LIVER 2.9E+01 rem GONADS $1.6 \mathrm{E}+00 \mathrm{rem}$ EFFECTIVE DOSE EQUIVALENT $7.5 \mathrm{E}+00 \mathrm{rem}$

$$
\begin{aligned}
& \mathrm{D}=0.10 \mathrm{~km} \\
& \mathrm{DEP}=8.4 \mathrm{E}-02 \mathrm{uCi} / \mathrm{m}^{\wedge} 2 \\
& \mathrm{CHI}=8.4 \mathrm{E}-06(\mathrm{Ci}-\mathrm{s}) / \mathrm{m}^{\wedge} 3 \\
& 50-\mathrm{Y} \text { DOSE COMMITMENT: } \\
& \hdashline \text { SURFACE BONE } 2.3 \mathrm{E}+01 \mathrm{rem} \\
& \text { RED MARROW } 1.9 \mathrm{E}+00 \mathrm{rem} \\
& \text { LIVER } \quad 5.0 \mathrm{E}+00 \mathrm{rem} \\
& \text { GONADS } 2.8 \mathrm{E}-01 \mathrm{rem} \\
& \text { EFFECTIVE DOSE } \\
& \text { EQUTVALENT } 1.3 \mathrm{E}+00 \mathrm{rem}
\end{aligned}
$$

$\mathrm{D}=0.11 \mathrm{~km}$

$\mathrm{DEP}=6.8 \mathrm{E}-02 \mathrm{uCi} / \mathrm{m}^{\wedge} 2$ $\mathrm{CHI}=6.8 \mathrm{E}-06(\mathrm{Ci}-\mathrm{s}) / \mathrm{m}^{\wedge} 3$ 50-YR DOSE COMMITMENT:

SURFACE BONE 1.8E+01 rem RED MARROW $1.5 \mathrm{E}+00 \mathrm{rem}$ LIVER . 4.1E+00 rem GONADS 2.3E-01 rem EFFECTIVE DOSE EQUTVALENT $1.0 \mathrm{E}+00 \mathrm{rem}$ 
Title of Project EPHA for THM

Reviewed by $\underline{\text { S. A. Henderson }}$

Subject Radiological Consequence Assessment

Computer D. J. Richards

Release Designation: 1-RD-104, Average

HOTSPOT 7.0 GENERAL PLUME 9-10-1996 22:20

RADIONUCLIDE : PU-238 Inhalation Class : W

HALFLIFE $: 87.740$ years

SOURCE TERM : $1.9 \mathrm{E}-03 \mathrm{Ci}$

Source Term Width: $\quad 3 \mathrm{~m}$ Source Term Height: $3 \mathrm{~m}$

RELEASE FRACTION : $1.00 \mathrm{E}+00$

FILTER EFFICIENCY: $0.000000 \%$

EFFECTIVE RELEASE HEIGHT : $\quad 0.00 \mathrm{~m}$

WIND SPEED $(\mathrm{h}=2 \mathrm{~m}): 2.5 \mathrm{~m} / \mathrm{s}$

STABILITY CLASS :C DEPOSITION VELOCITY $: 1.00 \mathrm{~cm} / \mathrm{s}$

RECEPTOR HEIGHT $\quad: 0.0 \mathrm{~m}$ INVERSION LAYER HEIGHT $: 500.0 \mathrm{~m}$

SAMPLE TIME $\quad: 10.000 \mathrm{~min}$

MAXIMUM DOSE DISTANCE $:<0.10 \mathrm{~km}$ MAXIMUM CEDE $:>0.301 \mathrm{rem}$

$D=0.03 \mathrm{~km}$

$\mathrm{DEP}=1.4 \mathrm{E}-01 \mathrm{uCi} / \mathrm{m}^{\wedge} 2$

$\mathrm{CHI}=1.4 \mathrm{E}-05(\mathrm{Ci}-\mathrm{s}) / \mathrm{m}^{\wedge} 3$

50-YR DOSE COMMITMENT:

SURFACE BONE $3.9 \mathrm{E}+01$ rem RED MARROW $3.2 \mathrm{E}+00 \mathrm{rem}$ LIVER $\quad 8.6 \mathrm{E}+00 \mathrm{rem}$ GONADS 4.8E-01 rem EFFECTIVE DOSE

EQUIVALENT $2.2 \mathrm{E}+00 \mathrm{rem}$
$\mathrm{D}=0.05 \mathrm{~km}$

$\mathrm{DEP}=6.7 \mathrm{E}-02 \mathrm{uCi} / \mathrm{m}^{\wedge} 2$

$\mathrm{CHI}=6.7 \mathrm{E}-06(\mathrm{Ci}-\mathrm{s}) / \mathrm{m}^{\wedge} 3$

50-YR DOSE COMMITMENT:

SURFACE BONE 1.8E+01 rem

RED MARROW $1.5 \mathrm{E}+00 \mathrm{rem}$

LIVER $\quad 4.0 \mathrm{E}+00 \mathrm{rem}$

GONADS 2.2E-01 rem

EFFECTIVE DOSE

EQUIVALENT $\quad 1.0 \mathrm{E}+00 \mathrm{rem}$
$\mathrm{D}=0.10 \mathrm{~km}$

$\mathrm{DEP}=2.0 \mathrm{E}-02 \mathrm{uCi} / \mathrm{m}^{\wedge} 2$

$\mathrm{CHI}=2.0 \mathrm{E}-06(\mathrm{Ci}-\mathrm{s}) / \mathrm{m}^{\wedge} 3$

50-YR DOSE COMMITMENT:

SURFACE BONE 5.3E+00 rem RED MARROW 4.4E-01 rem LIVER . 1.2E+00 rem GONADS 6.5E-02 rem EFFECTIVE DOSE

EQUTVALENT 3.0E-01 rem 
Title of Project EPHA for THM

Subject Radiological Consequence Assessment

Computer D. J. Richards

Date 09/11/96

Reviewed by $\mathrm{S}$. A. Henderson

Works Calculation 6

Sheet No. 83 of 166

Release Designation: 1-RD-105, Adverse

HOTSPOT 7.0 GENERAL PLUME 9-09-1996 21:35

RADIONUCLIDE : PU-238 Inhalation Class : W

HALFLIFE $: 87.740$ years

SOURCE TERM : $1.7 \mathrm{E}-03 \mathrm{Ci}$

Source Term Width: $\quad 3 \mathrm{~m}$ Source Term Height: $3 \mathrm{~m}$

RELEASE FRACTION : $1.00 \mathrm{E}+00$

FILTER EFFICIENCY: $0.000000 \%$

EFFECTIVE RELEASE HEIGHT : $\quad 0.00 \mathrm{~m}$

WIND SPEED $(\mathrm{h}=2 \mathrm{~m}): 1.7 \mathrm{~m} / \mathrm{s}$

STABILITY CLASS :E DEPOSITION VELOCITY $: 1.00 \mathrm{~cm} / \mathrm{s}$

RECEPTOR HEIGHT $: 0.0 \mathrm{~m}$ INVERSION LAYER HEIGHT $: 200.0 \mathrm{~m}$

SAMPLE TIME $\quad: 30.000 \mathrm{~min}$

MAXIMUM DOSE DISTANCE $: \quad<0.10 \mathrm{~km}$ MAXIMUM CEDE $:>0.946 \mathrm{rem}$

$D=0.03 \mathrm{~km}$

$\mathrm{DEP}=3.6 \mathrm{E}-01 \mathrm{uCi} / \mathrm{m}^{\wedge} 2$

$\mathrm{CHI}=3.6 \mathrm{E}-05(\mathrm{Ci}-\mathrm{s}) / \mathrm{m}^{\wedge} 3$

50-YR DOSE COMMITMENT:

SURFACE BONE 9.8E+01 rem RED MARROW $\quad 8.1 \mathrm{E}+00 \mathrm{rem}$ LIVER 2.2E+01 rem GONADS 1.2E+00 rem EFFECTIVE DOSE

EQUTVALENT $5.5 \mathrm{E}+00 \mathrm{rem}$
$D=0.09 \mathrm{~km}$
$\mathrm{DEP}=6.8 \mathrm{E}-02 \mathrm{uCi} / \mathrm{m}^{\wedge} 2$
$\mathrm{CHI}=6.8 \mathrm{E}-06(\mathrm{Ci}-\mathrm{s}) / \mathrm{m}^{\wedge} 3$
50-YR DOSE COMMITMENT:
SURFACE BONE 1.8E+01 rem RED MARROW $1.5 \mathrm{E}+00 \mathrm{rem}$
LIVER 4.1E+00 rem GONADS 2.3E-01 rem EFFECTIVE DOSE
EQUIVALENT $1.0 \mathrm{E}+00 \mathrm{rem}$

$\mathrm{D}=0.10 \mathrm{~km}$

$\mathrm{DEP}=6.2 \mathrm{E}-02 \mathrm{uCi} / \mathrm{m}^{\wedge} 2$
50-YR DOSE COMMITMENT:

SURFACE BONE 1.7E+01 rem RED MARROW $1.4 \mathrm{E}+00 \mathrm{rem}$ LIVER $\quad 3.7 \mathrm{E}+00 \mathrm{rem}$ GONADS 2.1E-01 rem EFFECTIVE DOSE

EQUTVALENT 9.5E-01 rem 
Title of Project EPHA for THM

Subject Radiological Consequence Assessment

Computer D. J. Richards

Date $\underline{09 / 11 / 96}$

Reviewed by $\underline{\text { S. A. Henderson }}$

Works Calculation 6

Sheet No. 84 of 166

Release Designation: 1-RD-105, Average

HOTSPOT 7.0 GENERAL PLUME 9-09-1996 21:36

RADIONUCLIDE : PU-238 Inhalation Class : W

HALFLIFE $: 87.740$ years

SOURCE TERM : $1.7 \mathrm{E}-03 \mathrm{Ci}$

Source Term Width: $\quad 3 \mathrm{~m}$ Source Term Height: $3 \mathrm{~m}$

RELEASE FRACTION : $1.00 \mathrm{E}+00$

FILTER EFFICIENCY: $0.000000 \%$

EFFECTIVE RELEASE HEIGHT : $\quad 0.00 \mathrm{~m}$

WIND SPEED $(\mathrm{h}=2 \mathrm{~m}): 2.5 \mathrm{~m} / \mathrm{s}$

STABILITY CLASS :C DEPOSITION VELOCITY $: 1.00 \mathrm{~cm} / \mathrm{s}$

RECEPTOR HEIGHT $\quad: 0.0 \mathrm{~m}$ INVERSION LAYER HEIGHT $: 500.0 \mathrm{~m}$

SAMPLE TIME $\quad: 30.000 \mathrm{~min}$

MAXIMUM DOSE DISTANCE $:<0.10 \mathrm{~km}$ MAXIMUM CEDE $:>0.222 \mathrm{rem}$

$\mathrm{D}=0.03 \mathrm{~km}$

$\mathrm{DEP}=1.1 \mathrm{E}-01 \mathrm{uCi} / \mathrm{m}^{\wedge} 2$

$\mathrm{CHI}=1.1 \mathrm{E}-05(\mathrm{Ci}-\mathrm{s}) / \mathrm{m}^{\wedge} 3$

50-YR DOSE COMMITMENT:

SURFACE BONE 2.9E +01 rem RED MARROW $2.4 \mathrm{E}+00 \mathrm{rem}$ LIVER 6.4E $+00 \mathrm{rem}$ GONADS 3.5E-01 rem EFFECTIVE DOSE EQUIVALENT $1.6 \mathrm{E}+00$ rem
$\mathrm{D}=0.04 \mathrm{~km}$

$\mathrm{DEP}=6.6 \mathrm{E}-02 \mathrm{uCi} / \mathrm{m}^{\wedge} 2$

$\mathrm{CHI}=6.6 \mathrm{E}-06(\mathrm{Ci}-\mathrm{s}) / \mathrm{m}^{\wedge} 3$

50-YR DOSE COMMITMENT:

SURFACE BONE $1.8 \mathrm{E}+01 \mathrm{rem}$ RED MARROW $1.5 \mathrm{E}+00 \mathrm{rem}$ LIVER $\quad 4.0 \mathrm{E}+00 \mathrm{rem}$ GONADS 2.2E-01 rem EFFECTIVE DOSE EQUTVALENT $\quad 1.0 \mathrm{E}+00 \mathrm{rem}$
$\mathrm{D}=0.10 \mathrm{~km}$

$\mathrm{DEP}=1.4 \mathrm{E}-02 \mathrm{uCi} / \mathrm{m}^{\wedge} 2$

$\mathrm{CHI}=1.4 \mathrm{E}-06(\mathrm{Ci}-\mathrm{s}) / \mathrm{m}^{\wedge} 3$

50-YR DOSE COMMITMENT:

SURFACE BONE 3.9E+00 rem RED MARROW 3.2E-01 rem LIVER 8.7E-01 rem GONADS 4.8E-02 rem EFFECTIVE DOSE

EQUTVALENT 2.2E-01 rem 
Title of Project EPHA for THM

Reviewed by S. A. Henderson

Subject Radiological Consequence Assessment

Computer D. J. Richards

Release Designation: 1-RD-106, Adverse

HOTSPOT 7.0 GENERAL PLUME 9-10-1996 22:21

RADIONUCLIDE : PU-238 Inhalation Class : W

HALFLIFE $: 87.740$ years

SOURCE TERM : $1.9 \mathrm{E}-03 \mathrm{Ci}$

Source Term Width: $\quad 3 \mathrm{~m}$ Source Term Height: $3 \mathrm{~m}$

RELEASE FRACTION : $1.00 \mathrm{E}+00$

FILTER EFFICIENCY: $0.000000 \%$

EFFECTIVE RELEASE HEIGHT : $\quad 0.00 \mathrm{~m}$

WIND SPEED $(\mathrm{h}=2 \mathrm{~m}): 1.7 \mathrm{~m} / \mathrm{s}$

STABILITY CLASS :E DEPOSITION VELOCITY $: 1.00 \mathrm{~cm} / \mathrm{s}$

RECEPTOR HEIGHT $\quad: 0.0 \mathrm{~m}$ INVERSION LAYER HEIGHT $: 200.0 \mathrm{~m}$

SAMPLE TIME $\quad: 10.000 \mathrm{~min}$

MAXIMUM DOSE DISTANCE $:<0.10 \mathrm{~km}$ MAXIMUM CEDE $:>1.3 \mathrm{rem}$

$\mathrm{D}=0.03 \mathrm{~km}$

$\mathrm{DEP}=4.9 \mathrm{E}-01 \mathrm{uCi} / \mathrm{m}^{\wedge} 2$

$\mathrm{CHI}=4.9 \mathrm{E}-05(\mathrm{Ci}-\mathrm{s}) / \mathrm{m}^{\wedge} 3$

50-YR DOSE COMMITMENT:

SURFACE BONE $1.3 \mathrm{E}+02 \mathrm{rem}$ RED MARROW $1.1 \mathrm{E}+01 \mathrm{rem}$ LIVER 2.9E+01 rem GONADS $1.6 \mathrm{E}+00 \mathrm{rem}$ EFFECTIVE DOSE EQUTVALENT $7.5 \mathrm{E}+00 \mathrm{rem}$
$\mathrm{D}=0.10 \mathrm{~km}$

$\mathrm{DEP}=8.4 \mathrm{E}-02 \mathrm{uCi} / \mathrm{m}^{\wedge} 2$

$\mathrm{CHI}=8.4 \mathrm{E}-06(\mathrm{Ci}-\mathrm{s}) / \mathrm{m}^{\wedge} 3$

50-YR DOSE COMMITMENT:

SURFACE BONE 2.3E+01 rem RED MARROW $1.9 \mathrm{E}+00 \mathrm{rem}$ LIVER $\quad 5.0 \mathrm{E}+00 \mathrm{rem}$ GONADS 2.8E-01 rem EFFECTIVE DOSE EQUIVALENT $1.3 \mathrm{E}+00 \mathrm{rem}$
$\mathrm{D}=0.11 \mathrm{~km}$

$\mathrm{DEP}=6.8 \mathrm{E}-02 \mathrm{uCi} / \mathrm{m}^{\wedge} 2$

$\mathrm{CHI}=6.8 \mathrm{E}-06(\mathrm{Ci}-\mathrm{s}) / \mathrm{m}^{\wedge} 3$

50-YR DOSE COMMITMENT:

SURFACE BONE $1.8 \mathrm{E}+01 \mathrm{rem}$ RED MARROW $1.5 \mathrm{E}+00$ rem LIVER 4.1E+00 rem GONADS 2.3E-01 rem EFFECTIVE DOSE EQUTVALENT $1.0 \mathrm{E}+00 \mathrm{rem}$ 
Title of Project EPHA for THM

Subject Radiological Consequence Assessment

Computer D. J. Richards

Date 09/11/96
Reviewed by $\underline{\text { S. A. Henderson }}$

Works Calculation 6

Sheet No. 86 of 166

Release Designation: 1-RD-106, Average

HOTSPOT 7.0 GENERAL PLUME 9-10-1996 22:21

RADIONUCLIDE: : PU-238 Inhalation Class : W

HALFLIFE : 87.740 years

SOURCE TERM $: 1.9 \mathrm{E}-03 \mathrm{Ci}$

Source Term Width: $3 \mathrm{~m}$ Source Term Height: $3 \mathrm{~m}$

RELEASE FRACTION : $1.00 \mathrm{E}+00$

FILTER EFFICIENCY: $0.000000 \%$

EFFECTIVE RELEASE HEIGHT : $\quad 0.00 \mathrm{~m}$

WIND SPEED $(\mathrm{h}=2 \mathrm{~m}): 2.5 \mathrm{~m} / \mathrm{s}$

STABILITY CLASS :C DEPOSITION VELOCITY $: 1.00 \mathrm{~cm} / \mathrm{s}$

RECEPTOR HEIGHT $\quad: 0.0 \mathrm{~m}$ INVERSION LAYER HEIGHT $: 500.0 \mathrm{~m}$

SAMPLE TIME $: 10.000 \mathrm{~min}$

MAXIMUM DOSE DISTANCE $:<0.10 \mathrm{~km}$ MAXIMUM CEDE $:>0.301 \mathrm{rem}$

$\mathrm{D}=0.03 \mathrm{~km}$

$\mathrm{DEP}=1.4 \mathrm{E}-01 \mathrm{uCi} / \mathrm{m}^{\wedge} 2$

$\mathrm{CHI}=1.4 \mathrm{E}-05(\mathrm{Ci}-\mathrm{s}) / \mathrm{m}^{\wedge} 3$

50-YR DOSE COMMITMENT:

SURFACE BONE $3.9 E+01$ rem RED MARROW $3.2 \mathrm{E}+00 \mathrm{rem}$ LIVER $\quad 8.6 \mathrm{E}+00 \mathrm{rem}$ GONADS 4.8E-01 rem EFFECTIVE DOSE

EQUIVALENT $\quad 2.2 \mathrm{E}+00 \mathrm{rem}$

$$
\begin{aligned}
& D=0.05 \mathrm{~km} \\
& \mathrm{DEP}=6.7 \mathrm{E}-02 \mathrm{uCi} / \mathrm{m}^{\wedge} 2 \\
& \mathrm{CHI}=6.7 \mathrm{E}-06(\mathrm{Ci}-\mathrm{s}) / \mathrm{m}^{\wedge} 3 \\
& \text { 50-YR DOSE COMMITMENT: } \\
& - \text { SURFACE BONE } 1.8 \mathrm{E}+01 \mathrm{rem} \\
& \text { RED MARROW } 1.5 \mathrm{E}+00 \mathrm{rem} \\
& \text { LIVER } \quad 4.0 \mathrm{E}+00 \mathrm{rem} \\
& \text { GONADS } \quad 2.2 \mathrm{E}-01 \mathrm{rem} \\
& \text { EFFECTIVE DOSE } \\
& \text { EQUIVALENT } \quad 1.0 \mathrm{E}+00 \mathrm{rem}
\end{aligned}
$$

$\mathrm{D}=0.10 \mathrm{~km}$

$\mathrm{DEP}=2.0 \mathrm{E}-02 \mathrm{uCi} / \mathrm{m}^{\wedge} 2$

$\mathrm{CHI}=2.0 \mathrm{E}-06(\mathrm{Ci}-\mathrm{s}) / \mathrm{m}^{\wedge} 3$

50-YR DOSE COMMITMENT:

SURFACE BONE 5.3E+00 rem RED MARROW 4.4E-01 rem LIVER 1.2E+00 rem GONADS 6.5E-02 rem EFFECTIVE DOSE EQUIVALENT 3.0E-01 rem 
Title of Project EPHA for THM

Subject Radiological Consequence Assessment

Computer D. J. Richards

Date 09/11/96

Reviewed by S. A. Henderson

Works Calculation 6

Sheet No. 87 of 166

Release Designation: 1-RD-107, Adverse

HOTSPOT 7.0 GENERAL PLUME 9-28-1996 10:31

RADIONUCLIDE : PU-238 Inhalation Class : W

HALFLIFE $: 87.740$ years

SOURCE TERM : $3.7 \mathrm{E}-01 \mathrm{Ci}$

Source Term Width: $3 \mathrm{~m}$ Source Term Height: $3 \mathrm{~m}$

RELEASE FRACTION : $1.00 \mathrm{E}+00$

FILTER EFFICIENCY: $0.000000 \%$

EFFECTIVE RELEASE HEIGHT : $\quad 0.00 \mathrm{~m}$

WIND SPEED $(\mathrm{h}=2 \mathrm{~m}): 1.7 \mathrm{~m} / \mathrm{s}$

STABILITY CLASS :E DEPOSITION VELOCITY $: 1.00 \mathrm{~cm} / \mathrm{s}$

RECEPTOR HEIGHT $\quad: 0.0 \mathrm{~m}$ INVERSION LAYER HEIGHT $: 200.0 \mathrm{~m}$

SAMPLE TIME $\quad: \quad 30.000 \mathrm{~min}$

MAXIMUM DOSE DISTANCE $:<0.10 \mathrm{~km}$ MAXIMUM CEDE $:>2.1 \mathrm{E}+02 \mathrm{rem}$

$\mathrm{D}=0.03 \mathrm{~km}$

$\mathrm{DEP}=7.9 \mathrm{E}+01 \mathrm{uCi} / \mathrm{m}^{\wedge} 2$

$\mathrm{CHI}=7.9 \mathrm{E}-03(\mathrm{Ci}-\mathrm{s}) / \mathrm{m}^{\wedge} 3$

50-YR DOSE COMMITMENT:

SURFACE BONE 2.1E+04 rem RED MARROW $1.8 \mathrm{E}+03 \mathrm{rem}$ LIVER $\quad 4.7 \mathrm{E}+03 \mathrm{rem}$ GONADS 2.6E+02 rem EFFECTIVE DOSE

EQUTVALENT $1.2 \mathrm{E}+03 \mathrm{rem}$
$\mathrm{D}=0.10 \mathrm{~km}$
$\mathrm{DEP}=1.3 \mathrm{E}+01 \mathrm{uCi} / \mathrm{m}^{\wedge} 2$
$\mathrm{CHI}=1.3 \mathrm{E}-03(\mathrm{Ci}-\mathrm{s}) / \mathrm{m}^{\wedge} 3$
50-YR DOSE COMMITMENT:
SURFACE BONE $3.6 \mathrm{E}+03 \mathrm{rem}$ RED MARROW $3.0 \mathrm{E}+02 \mathrm{rem}$ LIVER $\quad 8.0 \mathrm{E}+02 \mathrm{rem}$ GONADS 4.5E+01 rem EFFECTTVE DOSE EQUTVALENT $2.1 \mathrm{E}+02$ rem

\author{
$\mathrm{D}=1.74 \mathrm{~km}$ \\ $\mathrm{DEP}=6.8 \mathrm{E}-02 \mathrm{uCi} / \mathrm{m}^{\wedge} 2$ \\ $\mathrm{CHI}=6.8 \mathrm{E}-06(\mathrm{Ci}-\mathrm{s}) / \mathrm{m}^{\wedge} 3$ \\ 50-YR DOSE COMMITMENT: \\ SURFACE BONE 1.8E+01 rem \\ RED MARROW $1.5 \mathrm{E}+00 \mathrm{rem}$ \\ LIVER 4.1E+00 rem \\ GONADS 2.3E-01 rem \\ EFFECTIVE DOSE \\ EQUTVALENT $\quad 1.0 \mathrm{E}+00 \mathrm{rem}$
}


Title of Project EPHA for THM

Reviewed by S. A. Henderson

Subject Radiological Consequence Assessment

Works Calculation 6

Computer D. J. Richards

Date $\underline{09 / 11 / 96}$

Sheet No. 88 of 166

Release Designation: 1-RD-107, Average

HOTSPOT 7.0 GENERAL PLUME

9-28-1996 10:30

RADIONUCLIDE : PU-238 Inhalation Class : W

HALFLIFE : 87.740 years

SOURCE TERM : $3.7 \mathrm{E}-01 \mathrm{Ci}$

Source Term Width: $3 \mathrm{~m}$ Source Term Height: $3 \mathrm{~m}$

RELEASE FRACTION : $1.00 \mathrm{E}+00$

FILTER EFFICIENCY: $0.000000 \%$

EFFECTIVE RELEASE HEIGHT : $\quad 0.00 \mathrm{~m}$

WIND SPEED $(\mathrm{h}=2 \mathrm{~m}): 2.5 \mathrm{~m} / \mathrm{s}$

STABILITY CLASS : C DEPOSITION VELOCITY $\quad: 1.00 \mathrm{~cm} / \mathrm{s}$

RECEPTOR HEIGHT $\quad: 0.0 \mathrm{~m}$ INVERSION LAYER HEIGHT $: 500.0 \mathrm{~m}$

SAMPLE TIME $\quad: 30.000 \mathrm{~min}$

MAXIMUM DOSE DISTANCE $:<0.10 \mathrm{~km}$ MAXIMUM CEDE $:>48 \mathrm{rem}$

$\mathrm{D}=0.03 \mathrm{~km}$

$\mathrm{DEP}=2.3 \mathrm{E}+01 \mathrm{uCi} / \mathrm{m}^{\wedge} 2$

$\mathrm{CHI}=2.3 \mathrm{E}-03(\mathrm{Ci}-\mathrm{s}) / \mathrm{m}^{\wedge} 3$

50-YR DOSE COMMITMENT:

SURFACE BONE 6.2E+03 rem RED MARROW 5.1E+02 rem LIVER $1.4 \mathrm{E}+03 \mathrm{rem}$ GONADS 7.7E+01 rem EFFECTIVE DOSE

EQUTVALENT $3.5 \mathrm{E}+02 \mathrm{rem}$
$\mathrm{D}=0.10 \mathrm{~km}$

$\mathrm{DEP}=3.1 \mathrm{E}+00 \mathrm{uCi} / \mathrm{m}^{\wedge} 2$

$\mathrm{CHI}=3.1 \mathrm{E}-04(\mathrm{Ci}-\mathrm{s}) / \mathrm{m}^{\wedge} 3$

50-YR DOSE COMMITMENT:

SURFACE BONE $8.5 \mathrm{E}+02 \mathrm{rem}$ RED MARROW 7.0E+01 rem LIVER 1.9E+02 rem GONADS $1.0 \mathrm{E}+01 \mathrm{rem}$ EFFECTIVE DOSE EQUIVALENT $4.8 \mathrm{E}+01 \mathrm{rem}$
$\mathrm{D}=0.76 \mathrm{~km}$

$\mathrm{DEP}=6.8 \mathrm{E}-02 \mathrm{uCi} / \mathrm{m}^{\wedge} 2$

$\mathrm{CHI}=6.8 \mathrm{E}-06(\mathrm{Ci}-\mathrm{s}) / \mathrm{m}^{\wedge} 3$

50-YR DOSE COMMITMENT:

SURFACE BONE 1.8E+01 rem RED MARROW $1.5 \mathrm{E}+00 \mathrm{rem}$ LIVER $\quad 4.1 \mathrm{E}+00 \mathrm{rem}$ GONADS 2.3E-01 rem EFFECTIVE DOSE EQUTVALENT $\quad 1.0 \mathrm{E}+00 \mathrm{rem}$ 
Title of Project EPHA for THM

Reviewed by S. A. Henderson

Subject Radiological Consequence Assessment

Computer D. J. Richards

Date 09/11/96
Sheet No. 89 of 166
Works Calculation 6

Release Designation 1-RD-108, Adverse

HOTSPOT 7.0 GENERAL PLUME 10-02-1996 10:37

RADIONUCLIDE : PU-238 Inhalation Class : W

HALFLIFE $: 87.740$ years

SOURCE TERM : $1.1 \mathrm{E}-02 \mathrm{Ci}$

Source Term Width: $3 \mathrm{~m}$ Source Term Height: $3 \mathrm{~m}$

RELEASE FRACTION : $1.00 \mathrm{E}+00$

FILTER EFFICIENCY: $0.000000 \%$

EFFECTIVE RELEASE HEIGHT : $\quad 0.00 \mathrm{~m}$

WIND SPEED $(\mathrm{h}=2 \mathrm{~m}): 1.7 \mathrm{~m} / \mathrm{s}$

STABILITY CLASS :E DEPOSITION VELOCITY $: 1.00 \mathrm{~cm} / \mathrm{s}$

RECEPTOR HEIGHT $: 0.0 \mathrm{~m}$ INVERSION LAYER HEIGHT $: 200.0 \mathrm{~m}$

SAMPLE TIME $: 10.000 \mathrm{~min}$

MAXIMUM DOSE DISTANCE $:<0.10 \mathrm{~km}$ MAXIMUM CEDE $:>7.6 \mathrm{rem}$

$D=0.03 \mathrm{~km}$

$\mathrm{DEP}=2.9 \mathrm{E}+00 \mathrm{uCi} / \mathrm{m}^{\wedge} 2$

$\mathrm{CHI}=2.9 \mathrm{E}-04(\mathrm{Ci}-\mathrm{s}) / \mathrm{m}^{\wedge} 3$

50-YR DOSE COMMITMENT:

SURFACE BONE 7.8E+02 rem RED MARROW $6.5 \mathrm{E}+01 \mathrm{rem}$ LIVER $1.7 \mathrm{E}+02 \mathrm{rem}$ GONADS 9.7E+00 rem EFFECTIVE DOSE

EQUIVALENT $4.4 \mathrm{E}+01 \mathrm{rem}$

$\mathrm{D}=0.10 \mathrm{~km}$

$\mathrm{DEP}=4.9 \mathrm{E}-01 \mathrm{uCi} / \mathrm{m}^{\wedge} 2$

$\mathrm{CHI}=4.9 \mathrm{E}-05(\mathrm{Ci}-\mathrm{s}) / \mathrm{m}^{\wedge} 3$

50-YR DOSE COMMITMENT:

SURFACE BONE $1.3 \mathrm{E}+02$ rem RED MARROW 1.1E+01 rem LIVER $\quad 3.0 \mathrm{E}+01 \mathrm{rem}$ GONADS $1.6 \mathrm{E}+00 \mathrm{rem}$ EFFECTIVE DOSE EQUIVALENT $7.6 \mathrm{E}+00 \mathrm{rem}$

$\mathrm{D}=0.30 \mathrm{~km}$

$\mathrm{DEP}=6.8 \mathrm{E}-02 \mathrm{uCi} / \mathrm{m}^{\wedge} 2$

$\mathrm{CHI}=6.8 \mathrm{E}-06(\mathrm{Ci}-\mathrm{s}) / \mathrm{m}^{\wedge} 3$

50-YR DOSE COMMITMENT:

SURFACE BONE $1.8 \mathrm{E}+01 \mathrm{rem}$ RED MARROW $1.5 \mathrm{E}+00 \mathrm{rem}$ LIVER 4.1E+00 rem GONADS 2.3E-01 rem EFFECTIVE DOSE EQUIVALENT $1.0 \mathrm{E}+00 \mathrm{rem}$ 
Title of Project EPHA for THM

Reviewed by $\mathrm{S}$. A. Henderson

Subject Radiological Consequence Assessment

Computer D. J. Richards
Works Calculation 6

Sheet No. 90 of 166 
Title of Project EPHA for THM

Subject Radiological Consequence Assessment

Computer D. J. Richards

Date $\underline{09 / 11 / 96}$

Reviewed by S. A. Hènderson

Works Calculation 6

Sheet No. 91 of 166

Release Designation 1-RD-108, Average

HOTSPOT 7.0 GENERAL PLUME 10-02-1996 9:53

RADIONUCLIDE : PU-238 Inhalation Class : W

HALFLIFE $: 87.740$ years

SOURCE TERM : $1.1 \mathrm{E}-02 \mathrm{Ci}$

Source Term Width: $3 \mathrm{~m}$ Source Term Height: $3 \mathrm{~m}$

RELEASE FRACTION : $1.00 \mathrm{E}+00$

FILTER EFFICIENCY: $0.000000 \%$

EFFECTIVE RELEASE HEIGHT : $\quad 0.00 \mathrm{~m}$

WIND SPEED $(\mathrm{h}=2 \mathrm{~m}): 2.5 \mathrm{~m} / \mathrm{s}$

STABILITY CLASS :C DEPOSITION VELOCITY $: 1.00 \mathrm{~cm} / \mathrm{s}$

RECEPTOR HEIGHT $\quad: 0.0 \mathrm{~m}$ INVERSION LAYER HEIGHT :NONE

SAMPLE TIME $\quad: 10.000 \mathrm{~min}$

MAXIMUM DOSE DISTANCE $:<0.10 \mathrm{~km}$ MAXIMUM CEDE $:>1.8 \mathrm{rem}$

$\mathrm{D}=0.03 \mathrm{~km}$

$\mathrm{DEP}=8.6 \mathrm{E}-01 \mathrm{uCi} / \mathrm{m}^{\wedge} 2$

$\mathrm{CHI}=8.6 \mathrm{E}-05(\mathrm{Ci}-\mathrm{s}) / \mathrm{m}^{\wedge} 3$

50-YR DOSE COMMITMENT:

SURFACE BONE $2.3 E+02$ rem

RED MARROW $1.9 \mathrm{E}+01 \mathrm{rem}$

LIVER 5.1E+01 rem

GONADS 2.9E+00 rem

EFFECTIVE DOSE

EQUIVALENT $1.3 \mathrm{E}+01$ rem

$\mathrm{D}=0.10 \mathrm{~km}$

$\mathrm{DEP}=1.2 \mathrm{E}-01 \mathrm{uCi} / \mathrm{m}^{\wedge} 2$

$\mathrm{CHI}=1.2 \mathrm{E}-05(\mathrm{Ci}-\mathrm{s}) / \mathrm{m}^{\wedge} 3$

50-YR DOSE COMMITMENT:

SURFACE BONE 3.2E+01 rem

RED MARROW $2.6 \mathrm{E}+00 \mathrm{rem}$

LIVER $\quad 7.0 \mathrm{E}+00 \mathrm{rem}$

GONADS 3.9E-01 rem

EFFECTIVE DOSE

EQUTVALENT $1.8 \mathrm{E}+00 \mathrm{rem}$

$D=0.14 \mathrm{~km}$

$\mathrm{DEP}=6.8 \mathrm{E}-02 \mathrm{uCi} / \mathrm{m}^{\wedge} 2$

$\mathrm{CHI}=6.8 \mathrm{E}-06(\mathrm{Ci}-\mathrm{s}) / \mathrm{m}^{\wedge} 3$

50-YR DOSE COMMITMENT:

SURFACE BONE $1.8 \mathrm{E}+01 \mathrm{rem}$

RED MARROW $1.5 \mathrm{E}+00 \mathrm{rem}$

LIVER 4.1E+00 rem

GONADS 2.3E-01 rem

EFFECTIVE DOSE

EQUIVALENT $1.0 \mathrm{E}+00 \mathrm{rem}$ 
Title of Project EPHA for THM

Subject Radiological Consequence Assessment

Computer D. J. Richards

Date $\underline{09 / 11 / 96}$

Reviewed by S. A. Henderson

Works Calculation 6

Sheet No. $\underline{.92 \text { of } 166}$

Release Designation: 1-RD-109, Adverse

HOTSPOT 7.0 GENERAL PLUME

RADIONUCLIDE : PU-238 Inhalation Class : W

9-09-1996 21:40

HALFLIFE $\quad: 87.740$ years

SOURCE TERM : $1.4 \mathrm{E}-03 \mathrm{Ci}$

Source Term Width: $\quad 3 \mathrm{~m}$ Source Term Height: $3 \mathrm{~m}$

RELEASE FRACTION : $1.00 \mathrm{E}+00$

FILTER EFFICIENCY: $0.000000 \%$

EFFECTIVE RELEASE HEIGHT : $\quad 0.00 \mathrm{~m}$

WIND SPEED $(\mathrm{h}=2 \mathrm{~m}): 1.7 \mathrm{~m} / \mathrm{s}$

STABILITY CLASS :E DEPOSITION VELOCITY : $1.00 \mathrm{~cm} / \mathrm{s}$

RECEPTOR HEIGHT $\quad: 0.0 \mathrm{~m}$ INVERSION LAYER HEIGHT $: 200.0 \mathrm{~m}$

SAMPLE TIME $\quad: 30.000 \mathrm{~min}$

MAXIMUM DOSE DISTANCE $:<0.10 \mathrm{~km}$ MAXIMUM CEDE $:>0.758 \mathrm{rem}$

$\mathrm{D}=0.03 \mathrm{~km}$

$\mathrm{DEP}=2.9 \mathrm{E}-01 \mathrm{uCi} / \mathrm{m}^{\wedge} 2$

$\mathrm{CHI}=2.9 \mathrm{E}-05(\mathrm{Ci}-\mathrm{s}) / \mathrm{m}^{\wedge} 3$

50-YR DOSE COMMITMENT:

SURFACE BONE 7.8E+01 rem RED MARROW $6.5 \mathrm{E}+00 \mathrm{rem}$ LIVER $\quad 1.7 \mathrm{E}+01 \mathrm{rem}$ GONADS 9.7E-01 rem EFFECTIVE DOSE EQUIVALENT $4.4 \mathrm{E}+00 \mathrm{rem}$

$$
\begin{aligned}
& \mathrm{D}=0.08 \mathrm{~km} \\
& \mathrm{DEP}=6.6 \mathrm{E}-02 \mathrm{uCi} / \mathrm{m}^{\wedge} 2 \\
& \mathrm{CHI}=6.6 \mathrm{E}-06(\mathrm{Ci}-\mathrm{s}) / \mathrm{m}^{\wedge} 3 \\
& \text { 50-YR DOSE COMMIMENT: } \\
& \text { SURFACE BONE } 1.8 \mathrm{E}+01 \mathrm{rem} \\
& \text { RED MARROW } 1.5 \mathrm{E}+00 \mathrm{rem} \\
& \text { LIVER } \quad 4.0 \mathrm{E}+00 \mathrm{rem} \\
& \text { GONADS } \quad 2.2 \mathrm{E}-01 \mathrm{rem} \\
& \text { EFFECTIVE DOSE } \\
& \text { EQUIVALENT } \quad 1.0 \mathrm{E}+00 \mathrm{rem}
\end{aligned}
$$

\author{
$\mathrm{D}=0.10 \mathrm{~km}$ \\ $\mathrm{DEP}=4.9 \mathrm{E}-02 \mathrm{uCi} / \mathrm{m}^{\wedge} 2$ \\ $\mathrm{CHI}=4.9 \mathrm{E}-06(\mathrm{Ci}-\mathrm{s}) / \mathrm{m}^{\wedge} 3$ \\ 50-YR DOSE COMMITMENT: \\ SURFACE BONE $1.3 E+01 \mathrm{rem}$ \\ RED MARROW $1.1 \mathrm{E}+00 \mathrm{rem}$ \\ LIVER $3.0 \mathrm{E}+00 \mathrm{rem}$ \\ GONADS 1.6E-01 rem \\ EFFECTIVE DOSE \\ EQUTVALENT 7.6E-01 rem
}


Title of Project EPHA for THM

Subject Radiological Consequence Assessment

Computer D. J. Richards
Reviewed by $\mathrm{S}$. A. Henderson

Works Calculation 6

Sheet No. 93 of 166

Release Designation: 1-RD-109, Average

HOTSPOT 7.0 GENERAL PLUME 9-09-1996 21:42

RADIONUCLIDE : PU-238 Inhalation Class : W

HALFLIFE $: 87.740$ years

SOURCE TERM $: 1.4 \mathrm{E}-03 \mathrm{Ci}$

Source Term Width: $\quad 3 \mathrm{~m}$ Source Term Height: $3 \mathrm{~m}$

RELEASE FRACTION : $1.00 \mathrm{E}+00$

FILTER EFFICIENCY: $0.000000 \%$

EFFECTIVE RELEASE HEIGHT : $\quad 0.00 \mathrm{~m}$

WIND SPEED $(\mathrm{h}=2 \mathrm{~m}): 2.5 \mathrm{~m} / \mathrm{s}$

STABILITY CLASS :C DEPOSITION VELOCITY $: 1.00 \mathrm{~cm} / \mathrm{s}$

RECEPTOR HEIGHT $\quad: 0.0 \mathrm{~m}$ INVERSION LAYER HEIGHT $: 500.0 \mathrm{~m}$

SAMPLE TIME $\quad: 30.000 \mathrm{~min}$

MAXIMUM DOSE DISTANCE $:<0.10 \mathrm{~km}$ MAXIMUM CEDE $:>0.178 \mathrm{rem}$

$\mathrm{D}=0.03 \mathrm{~km}$

$\mathrm{DEP}=8.5 \mathrm{E}-02 \mathrm{uCi} / \mathrm{m}^{\wedge} 2$

$\mathrm{CHI}=8.5 \mathrm{E}-06(\mathrm{Ci}-\mathrm{s}) / \mathrm{m}^{\wedge} 3$

50-YR DOSE COMMITMENT:

SURFACE BONE 2.3E+01 rem RED MARROW $1.9 \mathrm{E}+00 \mathrm{rem}$ LIVER $5.1 \mathrm{E}+00 \mathrm{rem}$ GONADS 2.8E-01 rem EFFECTIVE DOSE

EQUIVALENT $1.3 \mathrm{E}+00 \mathrm{rem}$
$\mathrm{D}=0.04 \mathrm{~km}$

$\mathrm{DEP}=6.8 \mathrm{E}-02 \mathrm{uCi} / \mathrm{m}^{\wedge} 2$

$\mathrm{CHI}=6.8 \mathrm{E}-06(\mathrm{Ci}-\mathrm{s}) / \mathrm{m}^{\wedge} 3$

50-YR DOSE COMMITMENT:

SURFACE BONE $1.8 \mathrm{E}+01 \mathrm{rem}$ RED MARROW $1.5 \mathrm{E}+00 \mathrm{rem}$ LIVER $\quad 4.0 \mathrm{E}+00 \mathrm{rem}$ GONADS 2.2E-01 rem EFFECTIVE DOSE EQUTVALENT $1.0 \mathrm{E}+00 \mathrm{rem}$
$\mathrm{D}=0.10 \mathrm{~km}$

$\mathrm{DEP}=1.2 \mathrm{E}-02 \mathrm{uCi} / \mathrm{m}^{\wedge} 2$

$\mathrm{CHI}=1.2 \mathrm{E}-06(\mathrm{Ci}-\mathrm{s}) / \mathrm{m}^{\wedge} 3$

50-YR DOSE COMMITMENT:

SURFACE BONE 3.1E+00 rem RED MARROW 2.6E-01 rem LIVER 7.0E-01 rem GONADS 3.9E-02 rem EFFECTIVE DOSE EQUTVALENT $1.8 \mathrm{E}-01 \mathrm{rem}$ 
Title of Project EPHA for THM

Subject Radiological Consequence Assessment

Computer D. J. Richards

Date 09/11/96

Reviewed by $\underline{\text { S. A. Henderson }}$

Works Calculation 6

Sheet No. 94 of 166

Release Designation: 1-RD-110, Adverse

HOTSPOT 7.0 GENERAL PLUME 9-10-1996 22:28

RADIONUCLIDE : PU-238 Inhalation Class : W

HALFLIFE : 87.740 years

SOURCE TERM : $1.5 \mathrm{E}-03 \mathrm{Ci}$

Source Term Width: $\quad 3 \mathrm{~m}$ Source Term Height: $3 \mathrm{~m}$

RELEASE FRACTION : $1.00 \mathrm{E}+00$

FILTER EFFICIENCY: $0.000000 \%$

EFFECTIVE RELEASE HEIGHT : $\quad 0.00 \mathrm{~m}$

WIND SPEED $(\mathrm{h}=2 \mathrm{~m}): 1.7 \mathrm{~m} / \mathrm{s}$

STABILITY CLASS :E DEPOSITION VELOCITY $: 1.00 \mathrm{~cm} / \mathrm{s}$

RECEPTOR HEIGHT $\quad: 0.0 \mathrm{~m}$ INVERSION LAYER HEIGHT $: 200.0 \mathrm{~m}$

SAMPLE TIME $\quad: 10.000 \mathrm{~min}$

MAXIMUM DOSE DISTANCE $:<0.10 \mathrm{~km}$ MAXIMUM CEDE $:>1.0 \mathrm{rem}$

$\mathrm{D}=0.03 \mathrm{~km}$

$\mathrm{DEP}=4.0 \mathrm{E}-01 \mathrm{uCi} / \mathrm{m}^{\wedge} 2$

$\mathrm{CHI}=4.0 \mathrm{E}-05(\mathrm{Ci}-\mathrm{s}) / \mathrm{m}^{\wedge} 3$

50-YR DOSE COMMITMENT:

SURFACE BONE $1.1 \mathrm{E}+02$ rem RED MARROW $8.8 \mathrm{E}+00 \mathrm{rem}$

LIVER

$2.4 \mathrm{E}+01 \mathrm{rem}$

GONADS $1.3 E+00 \mathrm{rem}$

EFFECTTVE DOSE

EQUIVALENT $6.1 \mathrm{E}+00 \mathrm{rem}$
$\mathrm{D}=0.10 \mathrm{~km}$

$\mathrm{DEP}=6.7 \mathrm{E}-02 \mathrm{uCi} / \mathrm{m}^{\wedge} 2$

$\mathrm{CHI}=6.7 \mathrm{E}-06(\mathrm{Ci}-\mathrm{s}) / \mathrm{m}^{\wedge} 3$

50-YR DOSE COMMITMENT:

SURFACE BONE $1.8 \mathrm{E}+01 \mathrm{rem}$

RED MARROW $1.5 \mathrm{E}+00 \mathrm{rem}$

LIVER $\quad 4.0 \mathrm{E}+00 \mathrm{rem}$

GONADS 2.2E-01 rem

EFFECTIVE DOSE

EQUIVALENT $1.0 \mathrm{E}+00 \mathrm{rem}$ 
Title of Project EPHA for THM

Subject Radiological Consequence Assessment

Computer D. J. Richards

Date $\underline{09 / 11 / 96}$

Reviewed by $\underline{S}$. A. Henderson

Works Calculation 6

Sheet No. 95 of 166

Release Designation: 1-RD-110, Average

HOTSPOT 7.0 GENERAL PLUME 9-10-1996 22:28

RADIONUCLIDE : PU-238 Inhalation Class : W

HALFLIFE : 87.740 years

SOURCE TERM : $1.5 \mathrm{E}-03 \mathrm{Ci}$

Source Term Width: $3 \mathrm{~m}$ Source Term Height: $3 \mathrm{~m}$

RELEASE FRACTION : $1.00 \mathrm{E}+00$

FILTER EFFICIENCY: $0.000000 \%$

EFFECTIVE RELEASE HEIGHT : $\quad 0.00 \mathrm{~m}$

WIND SPEED $(\mathrm{h}=2 \mathrm{~m}): 2.5 \mathrm{~m} / \mathrm{s}$

STABILITY CLASS :C DEPOSITION VELOCITY : $1.00 \mathrm{~cm} / \mathrm{s}$

RECEPTOR HEIGHT $\quad: 0.0 \mathrm{~m}$ INVERSION LAYER HEIGHT $: 500.0 \mathrm{~m}$

SAMPLE TIME $\quad: 10.000 \mathrm{~min}$

MAXIMUM DOSE DISTANCE $:<0.10 \mathrm{~km}$ MAXIMUM CEDE $:>0.243 \mathrm{rem}$

$\mathrm{D}=0.03 \mathrm{~km}$

$\mathrm{DEP}=1.2 \mathrm{E}-01 \mathrm{uCi} / \mathrm{m}^{\wedge} 2$

$\mathrm{CHI}=1.2 \mathrm{E}-05(\mathrm{Ci}-\mathrm{s}) / \mathrm{m}^{\wedge} 3$

50-YR DOSE COMMITMENT:

SURFACE BONE 3.1E+01 rem RED MARROW $2.6 \mathrm{E}+00 \mathrm{rem}$ LIVER 7.0E+00 rem GONADS 3.9E-01 rem EFFECTIVE DOSE EQUIVALENT $1.8 \mathrm{E}+00 \mathrm{rem}$

$$
\begin{aligned}
& \mathrm{D}=0.04 \mathrm{~km} \\
& \mathrm{DEP}=6.7 \mathrm{E}-02 \mathrm{uCi} / \mathrm{m}^{\wedge} 2 \\
& \mathrm{CHI}=6.7 \mathrm{E}-06(\mathrm{Ci}-\mathrm{s}) / \mathrm{m}^{\wedge} 3 \\
& \text { 50-YR DOSE COMMITMENT: } \\
& \text { SURFACE BONE } 1.8 \mathrm{E}+01 \mathrm{rem} \\
& \text { RED MARROW } 1.5 \mathrm{E}+00 \mathrm{rem} \\
& \text { LIVER } \quad 4.0 \mathrm{E}+00 \mathrm{rem} \\
& \text { GONADS } \quad 2.2 \mathrm{E}-01 \mathrm{rem} \\
& \text { EFFECTIVE DOSE } \\
& \text { EQUTVALENT } \quad 1.0 \mathrm{E}+00 \mathrm{rem}
\end{aligned}
$$

\author{
$\mathrm{D}=0.10 \mathrm{~km}$ \\ $\mathrm{DEP}=1.6 \mathrm{E}-02 \mathrm{uCi} / \mathrm{m}^{\wedge} 2$ \\ $\mathrm{CHI}=1.6 \mathrm{E}-06(\mathrm{Ci}-\mathrm{s}) / \mathrm{m}^{\wedge} 3$ \\ 50-YR DOSE COMMITMENT: \\ SURFACE BONE $4.3 \mathrm{E}+00 \mathrm{rem}$ \\ RED MARROW 3.5E-01 rem \\ LIVER $9.5 \mathrm{E}-01 \mathrm{rem}$ \\ GONADS 5.3E-02 rem \\ EFFECTIVE DOSE \\ EQUIVALENT 2.4E-01 rem
}


Title of Project EPHA for THM

Subject Radiological Consequence Assessment

Computer D. J. Richards

Date $\underline{09 / 11 / 96}$

Reviewed by $\underline{S}$. A. Henderson

Works Calculation 6

Sheet No. 96 of 166

Release Designation: 1-RD-111, Adverse

HOTSPOT 7.0 GENERAL PLUME 9-28-1996 10:38

RADIONUCLIDE: PU-238 Inhalation Class : W

HALFLIFE $: 87.740$ years

SOURCE TERM : $2.3 \mathrm{E}+00 \mathrm{Ci}$

Source Term Width: $3 \mathrm{~m}$ Source Term Height: $3 \mathrm{~m}$

RELEASE FRACTION : $1.00 \mathrm{E}+00$

FILTER EFFICIENCY: $0.000000 \%$

EFFECTIVE RELEASE HEIGHT : $\quad 0.00 \mathrm{~m}$

WIND SPEED $(\mathrm{h}=2 \mathrm{~m}): 1.7 \mathrm{~m} / \mathrm{s}$

STABILITY CLASS :E DEPOSITION VELOCITY $: 1.00 \mathrm{~cm} / \mathrm{s}$

RECEPTOR HEIGHT $\quad: 0.0 \mathrm{~m}$ INVERSION LAYER HEIGHT $: 200.0 \mathrm{~m}$

SAMPLE TIME $\quad: 30.000 \mathrm{~min}$

MAXIMUM DOSE DISTANCE $:<0.10 \mathrm{~km}$ MAXIMUM CEDE $:>1.3 \mathrm{E}+03 \mathrm{rem}$

$\mathrm{D}=0.03 \mathrm{~km}$

$\mathrm{DEP}=4.8 \mathrm{E}+02 \mathrm{uCi} / \mathrm{m}^{\wedge} 2$

$\mathrm{CHI}=4.8 \mathrm{E}-02(\mathrm{Ci}-\mathrm{s}) / \mathrm{m}^{\wedge} 3$

50-YR DOSE COMMITMENT:

SURFACE BONE $1.3 E+05$ rem RED MARROW $1.1 \mathrm{E}+04 \mathrm{rem}$

LIVER $2.9 \mathrm{E}+04 \mathrm{rem}$ GONADS $1.6 \mathrm{E}+03 \mathrm{rem}$ EFFECTIVE DOSE

EQUTVALENT 7.4E+03 rem

\author{
$\mathrm{D}=0.10 \mathrm{~km}$ \\ $\mathrm{DEP}=8.2 \mathrm{E}+01 \mathrm{uCi} / \mathrm{m}^{\wedge} 2$ \\ $\mathrm{CHI}=8.2 \mathrm{E}-03(\mathrm{Ci}-\mathrm{s}) / \mathrm{m}^{\wedge} 3$ \\ 50-YR DOSE COMMITMENT: \\ SURFACE BONE $2.2 \mathrm{E}+04 \mathrm{rem}$ \\ RED MARROW $1.8 \mathrm{E}+03 \mathrm{rem}$ \\ LIVER 4.9E+03 rem \\ GONADS 2.7E+02 rem \\ EFFECTIVE DOSE \\ EQUIVALENT $1.3 \mathrm{E}+03 \mathrm{rem}$
}

$\mathrm{D}=5.01 \mathrm{~km}$

$\mathrm{DEP}=6.9 \mathrm{E}-02 \mathrm{uCi} / \mathrm{m}^{\wedge} 2$

$\mathrm{CHI}=6.9 \mathrm{E}-06(\mathrm{Ci}-\mathrm{s}) / \mathrm{m}^{\wedge} 3$

50-YR DOSE COMMITMENT:

SURFACE BONE $1.8 E+01$ rem RED MARROW $1.5 \mathrm{E}+00 \mathrm{rem}$ LIVER 4.1E+00 rem GONADS 2.3E-01 rem EFFECTIVE DOSE EQUIVALENT $1.0 \mathrm{E}+00 \mathrm{rem}$ 
Title of Project EPHA for THM Reviewed by S. A. Henderson

Subject Radiological Consequence Assessment

Works Calculation 6

Computer D. J. Richards

Date $\underline{09 / 11 / 96}$

Sheet No. 97 of 166

Release Designation: 1-RD-111, Average

HOTSPOT 7.0 GENERAL PLUME 9-28-1996 10:37

RADIONUCLIDE : PU-238 Inhalation Class : W

HALFLIFE : 87.740 years

SOURCE TERM $: 2.3 \mathrm{E}+00 \mathrm{Ci}$

Source Term Width: $3 \mathrm{~m}$ Source Term Height: $3 \mathrm{~m}$

RELEASE FRACTION : $1.00 E+00$

FILTER EFFICIENCY: $0.000000 \%$

EFFECTIVE RELEASE HEIGHT : $\quad 0.00 \mathrm{~m}$

WIND SPEED $(\mathrm{h}=2 \mathrm{~m}): 2.5 \mathrm{~m} / \mathrm{s}$

STABILITY CLASS :C DEPOSITION VELOCITY $: 1.00 \mathrm{~cm} / \mathrm{s}$

RECEPTOR HEIGHT $: 0.0 \mathrm{~m}$ INVERSION LAYER HEIGHT $: 500.0 \mathrm{~m}$

SAMPLE TIME $\quad: 30.000 \mathrm{~min}$

MAXIMUM DOSE DISTANCE $:<0.10 \mathrm{~km}$ MAXIMUM CEDE $:>2.9 \mathrm{E}+02 \mathrm{rem}$

$\mathrm{D}=0.03 \mathrm{~km}$

$\mathrm{DEP}=1.4 \mathrm{E}+02 \mathrm{uCi} / \mathrm{m}^{\wedge} 2$

$\mathrm{CHI}=1.4 \mathrm{E}-02(\mathrm{Ci}-\mathrm{s}) / \mathrm{m}^{\wedge} 3$

50-YR DOSE COMMITMENT:

SURFACE BONE $3.8 E+04$ rem RED MARROW $3.1 \mathrm{E}+03 \mathrm{rem}$ LIVER $\quad 8.4 \mathrm{E}+03 \mathrm{rem}$ GONADS 4.7E+02 rem EFFECTIVE DOSE

EQUIVALENT $2.2 \mathrm{E}+03 \mathrm{rem}$
$\mathrm{D}=0.10 \mathrm{~km}$

$\mathrm{DEP}=1.9 \mathrm{E}+01 \mathrm{uCi} / \mathrm{m}^{\wedge} 2$

$\mathrm{CHI}=1.9 \mathrm{E}-03(\mathrm{Ci}-\mathrm{s}) / \mathrm{m}^{\wedge} 3$

50-YR DOSE COMMITMENT:

SURFACE BONE 5.2E+03 rem RED MARROW $4.3 \mathrm{E}+02 \mathrm{rem}$ LIVER $1.2 \mathrm{E}+03 \mathrm{rem}$ GONADS 6.4E+01 rem EFFECTIVE DOSE EQUTVALENT $2.9 \mathrm{E}+02 \mathrm{rem}$
$\mathrm{D}=1.99 \mathrm{~km}$

$\mathrm{DEP}=6.8 \mathrm{E}-02 \mathrm{uCi} / \mathrm{m}^{\wedge} 2$

$\mathrm{CHI}=6.8 \mathrm{E}-06(\mathrm{Ci}-\mathrm{s}) / \mathrm{m}^{\wedge} 3$

50-YR DOSE COMMITMENT:

SURFACE BONE $1.8 \mathrm{E}+01 \mathrm{rem}$ RED MARROW $1.5 \mathrm{E}+00 \mathrm{rem}$ LIVER $\quad 4.1 \mathrm{E}+00 \mathrm{rem}$ GONADS 2.3E-01 rem EFFECTIVE DOSE EQUIVALENT $\quad 1.0 \mathrm{E}+00 \mathrm{rem}$ 
Title of Project EPHA for THM

Subject Radiological Consequence Assessment

Computer D. J. Richards
Reviewed by $\underline{\text { S. A. Henderson }}$

Works Calculation 6

Sheet No. 98 of 166

Release Designation 1-RD-112, Adverse

HOTSPOT 7.0 GENERAL PLUME 10-02-1996 9:56

RADIONUCLIDE : PU-238 Inhalation Class : W

HALFLIFE $: 87.740$ years

SOURCE TERM : $6.1 \mathrm{E}-01 \mathrm{Ci}$

Source Term Width: $\quad 3 \mathrm{~m}$ Source Term Height: $3 \mathrm{~m}$

RELEASE FRACTION : $1.00 \mathrm{E}+00$

FILTER EFFICIENCY: $0.000000 \%$

EFFECTIVE RELEASE HEIGHT : $\quad 0.00 \mathrm{~m}$

WIND SPEED $(\mathrm{h}=2 \mathrm{~m}): 1.7 \mathrm{~m} / \mathrm{s}$

STABILITY CLASS :E DEPOSITION VELOCITY $: 1.00 \mathrm{~cm} / \mathrm{s}$

RECEPTOR HEIGHT $: 0.0 \mathrm{~m}$ INVERSION LAYER HEIGHT : NONE

SAMPLE TIME $\quad: 10.000 \mathrm{~min}$

MAXIMUM DOSE DISTANCE $:<0.10 \mathrm{~km}$ MAXIMUM CEDE $:>4.2 \mathrm{E}+02 \mathrm{rem}$

$\mathrm{D}=0.03 \mathrm{~km}$

$\mathrm{DEP}=1.6 \mathrm{E}+02 \mathrm{uCi} / \mathrm{m}^{\wedge} 2$

$\mathrm{CHI}=1.6 \mathrm{E}-02(\mathrm{Ci}-\mathrm{s}) / \mathrm{m}^{\wedge} 3$

50-YR DOSE COMMITMENT:

SURFACE BONE 4.3E+04 rem

RED MARROW $3.6 \mathrm{E}+03 \mathrm{rem}$

LIVER $9.6 \mathrm{E}+03 \mathrm{rem}$

GONADS 5.3E+02 rem

EFFECTIVE DOSE

EQUIVALENT $2.5 \mathrm{E}+03 \mathrm{rem}$

$\mathrm{D}=0.10 \mathrm{~km}$

$\mathrm{DEP}=2.7 \mathrm{E}+01 \mathrm{uCi} / \mathrm{m}^{\wedge} 2$

$\mathrm{CHI}=2.7 \mathrm{E}-03(\mathrm{Ci}-\mathrm{s}) / \mathrm{m}^{\wedge} 3$

50-YR DOSE COMMITMENT:

SURFACE BONE 7.4E+03 rem

RED MARROW $\quad 6.1 \mathrm{E}+02 \mathrm{rem}$

LIVER $1.6 \mathrm{E}+03 \mathrm{rem}$

GONADS $9.1 E+01 \mathrm{rem}$

EFFECTIVE DOSE

EQUIVALENT $4.2 \mathrm{E}+02 \mathrm{rem}$

$\mathrm{D}=2.70 \mathrm{~km}$

$\mathrm{DEP}=6.5 \mathrm{E}-02 \mathrm{uCi} / \mathrm{m}^{\wedge} 2$

$\mathrm{CHI}=6.5 \mathrm{E}-06(\mathrm{Ci}-\mathrm{s}) / \mathrm{m}^{\wedge} 3$

50-YR DOSE COMMITMENT:

SURFACE BONE $1.8 \mathrm{E}+01 \mathrm{rem}$

RED MARROW $1.5 \mathrm{E}+00 \mathrm{rem}$

LIVER $3.9 \mathrm{E}+00 \mathrm{rem}$

GONADS 2.2E-01 rem

EFFECTIVE DOSE

EQUTVALENT $\quad 1.0 \mathrm{E}+00 \mathrm{rem}$ 
Title of Project EPHA for THM

Subject Radiological Consequence Assessment

Computer D. J. Richards

Date $\underline{09 / 11 / 96}$

Reviewed by $\mathrm{S}$. A. Henderson

Works Calculation 6

Sheet No. 99 of 166

Release Designation 1-RD-112, Average

HOTSPOT 7.0 GENERAL PLUME 10-02-1996 9:57

RADIONUCLIDE : PU-238 Inhalation Class : W

HALFLIFE $: 87.740$ years

SOURCE TERM : $6.1 \mathrm{E}-01 \mathrm{Ci}$

Source Term Width: $3 \mathrm{~m}$ Source Term Height: $3 \mathrm{~m}$

RELEASE FRACTION : $1.00 \mathrm{E}+00$

FILTER EFFICIENCY: $0.000000 \%$

EFFECTIVE RELEASE HEIGHT : $\quad 0.00 \mathrm{~m}$

WIND SPEED $(\mathrm{h}=2 \mathrm{~m}): 2.5 \mathrm{~m} / \mathrm{s}$

STABILITY CLASS :C DEPOSITION VELOCITY : $1.00 \mathrm{~cm} / \mathrm{s}$

RECEPTOR HEIGHT $: 0.0 \mathrm{~m}$ INVERSION LAYER HEIGHT : NONE

SAMPLE TIME $\quad: 10.000 \mathrm{~min}$

MAXIMUM DOSE DISTANCE $:<0.10 \mathrm{~km}$ MAXIMUM CEDE $:>98 \mathrm{rem}$

$\mathrm{D}=0.03 \mathrm{~km}$

$\mathrm{DEP}=4.7 \mathrm{E}+01 \mathrm{uCi} / \mathrm{m}^{\wedge} 2$

$\mathrm{CHI}=4.7 \mathrm{E}-03(\mathrm{Ci}-\mathrm{s}) / \mathrm{m}^{\wedge} 3$

50-YR DOSE COMMITMENT:

SURFACE BONE $1.3 \mathrm{E}+04 \mathrm{rem}$

RED MARROW $1.0 \mathrm{E}+03 \mathrm{rem}$

LIVER 2.8E+03 rem

GONADS 1.6E+02 rem

EFFECTIVE DOSE

EQUIVALENT $\quad 7.2 \mathrm{E}+02 \mathrm{rem}$

$\mathrm{D}=0.10 \mathrm{~km}$

$\mathrm{DEP}=6.4 \mathrm{E}+00 \mathrm{uCi} / \mathrm{m}^{\wedge} 2$

$\mathrm{CHI}=6.4 \mathrm{E}-04(\mathrm{Ci}-\mathrm{s}) / \mathrm{m}^{\wedge} 3$

50-YR DOSE COMMITMENT:

SURFACE BONE $1.7 \mathrm{E}+03 \mathrm{rem}$

RED MARROW $1.4 \mathrm{E}+02$ rem

LIVER 3.8E+02 rem

GONADS 2.1E+01 rem

EFFECTIVE DOSE

EQUTVALENT $9.8 \mathrm{E}+01 \mathrm{rem}$

$\mathrm{D}=1.10 \mathrm{~km}$

$\mathrm{DEP}=6.9 \mathrm{E}-02 \mathrm{uCi} / \mathrm{m}^{\wedge} 2$

$\mathrm{CHI}=6.9 \mathrm{E}-06(\mathrm{Ci}-\mathrm{s}) / \mathrm{m}^{\wedge} 3$

50-YR DOSE COMMITMENT:

SURFACE BONE $1.8 \mathrm{E}+01 \mathrm{rem}$

RED MARROW $1.5 \mathrm{E}+00 \mathrm{rem}$

LIVER 4.1E+00 rem

GONADS 2.3E-01 rem

EFFECTIVE DOSE

EQUIVALENT $1.0 \mathrm{E}+00 \mathrm{rem}$ 
Title of Project EPHA for THM

Subject Radiological Consequence Assessment

Computer D. J. Richards

Date 09/11/96

Reviewed by S. A. Henderson

Works Calculation 6

Sheet No. 100 of 166

Release Designation: 1-RD-113, Adverse

HOTSPOT 7.0 GENERAL PLUME 9-28-1996 10:40

RADIONUCLIDE : PU-238 Inhalation Class : W

HALFLIFE $: 87.740$ years

SOURCE TERM : $1.1 \mathrm{E}-02 \mathrm{Ci}$

Source Term Width: $\quad 3 \mathrm{~m}$ Source Term Height: $3 \mathrm{~m}$

RELEASE FRACTION : $1.00 \mathrm{E}+00$

FILTER EFFICIENCY: $0.000000 \%$

EFFECTIVE RELEASE HEIGHT : $\quad 0.00 \mathrm{~m}$

WIND SPEED $(\mathrm{h}=2 \mathrm{~m}): 1.7 \mathrm{~m} / \mathrm{s}$

STABILITY CLASS :E DEPOSITION VELOCITY $: 1.00 \mathrm{~cm} / \mathrm{s}$

RECEPTOR HEIGHT $\quad: 0.0 \mathrm{~m}$ INVERSION LAYER HEIGHT $: 200.0 \mathrm{~m}$

SAMPLE TIME $\quad: 30.000 \mathrm{~min}$

MAXIMUM DOSE DISTANCE $:<0.10 \mathrm{~km}$ MAXIMUM CEDE $:>6.1 \mathrm{rem}$

$\mathrm{D}=0.03 \mathrm{~km}$

$\mathrm{DEP}=2.3 \mathrm{E}+00 \mathrm{uCi} / \mathrm{m}^{\wedge} 2$

$\mathrm{CHI}=2.3 \mathrm{E}-04(\mathrm{Ci}-\mathrm{s}) / \mathrm{m}^{\wedge} 3$

50-YR DOSE COMMITMENT:

SURFACE BONE $6.3 E+02$ rem RED MARROW -5.2E+01 rem LIVER 1.4E+02 rem GONADS $\quad 7.8 \mathrm{E}+00 \mathrm{rem}$ EFFECTIVE DOSE

EQUIVALENT $3.6 \mathrm{E}+01 \mathrm{rem}$
$\mathrm{D}=0.10 \mathrm{~km}$

$\mathrm{DEP}=4.0 \mathrm{E}-01 \mathrm{uCi} / \mathrm{m}^{\wedge} 2$

$\mathrm{CHI}=4.0 \mathrm{E}-05(\mathrm{Ci}-\mathrm{s}) / \mathrm{m}^{\wedge} 3$

50-YR DOSE COMMITMENT:

SURFACE BONE 1.1E+02 rem RED MARROW $8.9 \mathrm{E}+00 \mathrm{rem}$ LIVER 2.4E+01 rem GONADS 1.3E+00 rem EFFECTIVE DOSE EQUIVALENT $6.1 \mathrm{E}+00 \mathrm{rem}$
$\mathrm{D}=0.27 \mathrm{~km}$

$\mathrm{DEP}=6.7 \mathrm{E}-02 \mathrm{uCi} / \mathrm{m}^{\wedge} 2$

$\mathrm{CHI}=6.7 \mathrm{E}-06(\mathrm{Ci}-\mathrm{s}) / \mathrm{m}^{\wedge} 3$

50-YR DOSE COMMITMENT:

SURFACE BONE $1.8 \mathrm{E}+01 \mathrm{rem}$ RED MARROW $1.5 \mathrm{E}+00 \mathrm{rem}$ LIVER $\quad 4.0 \mathrm{E}+00 \mathrm{rem}$ GONADS 2.2E-01 rem EFFECTIVE DOSE EQUTVALENT $1.0 \mathrm{E}+00 \mathrm{rem}$ 
Title of Project EPHA for THM

Subject Radiological Consequence Assessment

Computer D.J. Richards

Date $\underline{09 / 11 / 96}$

Reviewed by. S. A. Henderson

Works Calculation 6

Sheet No. 101 of 166

Release Designation: 1-RD-113, Average

HOTSPOT 7.0 GENYERAL PLUME 9-28-1996 10:41

RADIONUCLIDE : PU-238 Inhalation Class : W

HALFLIFE : 87.740 years

SOURCE TERM : $1.1 \mathrm{E}-02 \mathrm{Ci}$

Source Term Width: $\quad 3 \mathrm{~m}$ Source Term Height: $3 \mathrm{~m}$

RELEASE FRACTION : $1.00 \mathrm{E}+00$

FILTER EFFICIENCY: $0.000000 \%$

EFFECTIVE RELEASE HEIGHT : $\quad 0.00 \mathrm{~m}$

WIND SPEED $(\mathrm{h}=2 \mathrm{~m}): 2.5 \mathrm{~m} / \mathrm{s}$

STABILITY CLASS :C DEPOSITION VELOCITY $: 1.00 \mathrm{~cm} / \mathrm{s}$

RECEPTOR HEIGHT $\quad: 0.0 \mathrm{~m}$ INVERSION LAYER HEIGHT $: 500.0 \mathrm{~m}$

SAMPLE TIME $\quad: \quad 30.000 \mathrm{~min}$

MAXIMUM DOSE DISTANCE $:<0.10 \mathrm{~km}$ MAXIMUM CEDE $:>1.4 \mathrm{rem}$

$\mathrm{D}=0.03 \mathrm{~km}$

$\mathrm{DEP}=6.8 \mathrm{E}-01 \mathrm{uCi} / \mathrm{m}^{\wedge} 2$

$\mathrm{CHI}=6.8 \mathrm{E}-05(\mathrm{Ci}-\mathrm{s}) / \mathrm{m}^{\wedge} 3$

50-YR DOSE COMMITMENT:

SURFACE BONE $1.8 \mathrm{E}+02 \mathrm{rem}$ RED MARROW $1.5 \mathrm{E}+01 \mathrm{rem}$

LIVER 4.1E+01 rem GONADS 2.3E+00 rem EFFECTIVE DOSE EQUTVALENT $1.0 \mathrm{E}+01 \mathrm{rem}$
$\mathrm{D}=0.10 \mathrm{~km}$

$\mathrm{DEP}=9.3 \mathrm{E}-02 \mathrm{uCi} / \mathrm{m}^{\wedge} 2$

$\mathrm{CHI}=9.3 \mathrm{E}-06(\mathrm{Ci}-\mathrm{s}) / \mathrm{m}^{\wedge} 3$

50-YR DOSE COMMITMENT:

SURFACE BONE $2.5 \mathrm{E}+01 \mathrm{rem}$ RED MARROW $2.1 \mathrm{E}+00 \mathrm{rem}$ LIVER $\quad 5.6 \mathrm{E}+00 \mathrm{rem}$ GONADS 3.1E-01 rem EFFECTIVE DOSE EQUIVALENT $1.4 \mathrm{E}+00 \mathrm{rem}$
$\mathrm{D}=0.12 \mathrm{~km}$

$\mathrm{DEP}=6.7 \mathrm{E}-02 \mathrm{uCi} / \mathrm{m}^{\wedge} 2$

$\mathrm{CHI}=6.7 \mathrm{E}-06(\mathrm{Ci}-\mathrm{s}) / \mathrm{m}^{\wedge} 3$

50-YR DOSE COMMITMENT:

SURFACE BONE $1.8 E+01$ rem RED MARROW $1.5 \mathrm{E}+00 \mathrm{rem}$ LIVER $\quad 4.0 \mathrm{E}+00 \mathrm{rem}$ GONADS 2.2E-01 rem EFFECTTVE DOSE EQUTVALENT $1.0 \mathrm{E}+00 \mathrm{rem}$ 
Title of Project EPHA for THM

Subject Radiological Consequence Assessment

Computer D. J. Richards

Release Designation: 1-RD-114, Adverse

HOTSPOT 7.0 GENERAL PLUME 9-28-1996 10:14

RADIONUCLIDE : PU-238 Inhalation Class : W

HALFLIFE : 87.740 years

SOURCE TERM : 3.3E-02 Ci

Source Term Width: $\quad 3 \mathrm{~m}$ Source Term Height: $3 \mathrm{~m}$

RELEASE FRACTION : $1.00 \mathrm{E}+00$

FILTER EFFICIENCY: $0.000000 \%$

EFFECTIVE RELEASE HEIGHT : $\quad 0.00 \mathrm{~m}$

WIND SPEED $(\mathrm{h}=2 \mathrm{~m}): 1.7 \mathrm{~m} / \mathrm{s}$

STABILITY CLASS :E DEPOSITION VELOCITY $\quad: 1.00 \mathrm{~cm} / \mathrm{s}$

RECEPTOR HEIGHT $\quad: 0.0 \mathrm{~m}$ INVERSION LAYER HEIGHT $: 200.0 \mathrm{~m}$

SAMPLE TIME $\quad: 10.000 \mathrm{~min}$

MAXIMUM DOSE DISTANCE $:<0.10 \mathrm{~km}$ MAXIMUM CEDE :> $23 \mathrm{rem}$

$\mathrm{D}=0.03 \mathrm{~km}$

$\mathrm{DEP}=8.7 \mathrm{E}+00 \mathrm{uCi} / \mathrm{m}^{\wedge} 2$

$\mathrm{CHI}=8.7 \mathrm{E}-04(\mathrm{Ci}-\mathrm{s}) / \mathrm{m}^{\wedge} 3$

50-YR DOSE COMMITMENT:

SURFACE BONE 2.4E+03 rem RED MARROW 1.9E+02 rem LIVER 5.2E+02 rem GONADS 2.9E+01 rem EFFECTIVE DOSE EQUIVALENT 1.3E+02 rem
$\mathrm{D}=0.10 \mathrm{~km}$

$\mathrm{DEP}=1.5 \mathrm{E}+00 \mathrm{uCi} / \mathrm{m}^{\wedge} 2$

$\mathrm{CHI}=1.5 \mathrm{E}-04(\mathrm{Ci}-\mathrm{s}) / \mathrm{m}^{\wedge} 3$

50-YR DOSE COMMITMENT:

SURFACE BONE 4.0E+02 rem RED MARROW 3.3E+01 rem LIVER 8.9E+01 rem GONADS 5.0E+00 rem EFFECTIVE DOSE EQUIVALENT 2.3E+01 rem
$D=0.54 \mathrm{~km}$

$\mathrm{DEP}=6.7 \mathrm{E}-02 \mathrm{uCi} / \mathrm{m}^{\wedge} 2$

$\mathrm{CHI}=6.7 \mathrm{E}-06(\mathrm{Ci}-\mathrm{s}) / \mathrm{m}^{\wedge} 3$

50-YR DOSE COMMITMENT:

SURFACE BONE $1.8 \mathrm{E}+01 \mathrm{rem}$ RED MARROW $1.5 \mathrm{E}+00 \mathrm{rem}$ LIVER $\quad 4.0 \mathrm{E}+00 \mathrm{rem}$ GONADS 2.2E-01 rem EFFECTIVE DOSE EQUIVALENT $1.0 \mathrm{E}+00 \mathrm{rem}$ 
Title of Project EPHA for THM

Subject Radiological Consequence Assessment

Computer D. J. Richards
Reviewed by $\underline{\text { S. A. Henderson }}$ Works Calculation 6 Sheet No. 103 of 166

Release Designation: 1-RD-114, Average

HOTSPOT 7.0 GENERAL PLUME 9-28-1996 10:16

RADIONUCLIDE : PU-238 Inhalation Class : W

HALFLIFE $: 87.740$ years

SOURCE TERM : 3.3E-02 Ci

Source Term Width: $3 \mathrm{~m}$ Source Term Height: $3 \mathrm{~m}$

RELEASE FRACTION : $1.00 \mathrm{E}+00$

FILTER EFFICIENCY: $0.000000 \%$

EFFECTIVE RELEASE HEIGHT : $\quad 0.00 \mathrm{~m}$

WIND SPEED $(\mathrm{h}=2 \mathrm{~m}): 2.5 \mathrm{~m} / \mathrm{s}$

STABILITY CLASS :C DEPOSITION VELOCITY $: 1.00 \mathrm{~cm} / \mathrm{s}$

RECEPTOR HEIGHT $\quad: 0.0 \mathrm{~m}$ INVERSION LAYER HEIGHT $: 500.0 \mathrm{~m}$

SAMPLE TIME $\quad: 10.000 \mathrm{~min}$

MAXIMUM DOSE DISTANCE $:<0.10 \mathrm{~km}$ MAXIMUM CEDE $:>5.4 \mathrm{rem}$

$D=0.03 \mathrm{~km}$

$\mathrm{DEP}=2.6 \mathrm{E}+00 \mathrm{uCi} / \mathrm{m}^{\wedge} 2$

$\mathrm{CHI}=2.6 \mathrm{E}-04(\mathrm{Ci}-\mathrm{s}) / \mathrm{m}^{\wedge} 3$

50-YR DOSE COMMITMENT:

SURFACE BONE $6.9 \mathrm{E}+02 \mathrm{rem}$ RED MARROW 5.7E+01 rem LIVER - $1.5 \mathrm{E}+02 \mathrm{rem}$ GONADS 8.5E+00 rem EFFECTIVE DOSE EQUTVALENT 3.9E+01 rem
$\mathrm{D}=0.10 \mathrm{~km}$

$\mathrm{DEP}=3.5 \mathrm{E}-01 \mathrm{uCi} / \mathrm{m}^{\wedge} 2$

$\mathrm{CHI}=3.5 \mathrm{E}-05(\mathrm{Ci}-\mathrm{s}) / \mathrm{m}^{\wedge} 3$

50-YR DOSE COMMITMENT:

SURFACE BONE 9.4E+01 rem RED MARROW $7.8 \mathrm{E}+00 \mathrm{rem}$ LIVER 2.1E+01 rem GONADS 1.2E+00 rem EFFECTIVE DOSE EQUIVALENT $5.4 \mathrm{E}+00 \mathrm{rem}$
$\mathrm{D}=0.24 \mathrm{~km}$

$\mathrm{DEP}=6.8 \mathrm{E}-02 \mathrm{uCi} / \mathrm{m}^{\wedge} 2$

$\mathrm{CHI}=6.8 \mathrm{E}-06(\mathrm{Ci}-\mathrm{s}) / \mathrm{m}^{\wedge} 3$

50-YR DOSE COMMITMENT:

SURFACE BONE $1.8 \mathrm{E}+01 \mathrm{rem}$ RED MARROW $1.5 \mathrm{E}+00 \mathrm{rem}$ LIVER 4.1E+00 rem GONADS 2.3E-01 rem EFFECTIVE DOSE EQUTVALENT $1.0 \mathrm{E}+00 \mathrm{rem}$ 
Title of Project EPHA for THM

Subject Radiological Consequence Assessment

Computer D. J. Richards

Release Designation: 1-RD-115, Adverse

HOTSPOT 7.0 GENERAL PLUME 9-09-1996 22:00

RADIONUCLIDE : PU-238 Inhalation Class : W

HALFLIFE : 87.740 years

SOURCE TERM : $4.6 \mathrm{E}-02 \mathrm{Ci}$

Source Term Width: $3 \mathrm{~m}$ Source Term Height: $3 \mathrm{~m}$

RELEASE FRACTION : $1.00 \mathrm{E}+00^{\circ}$

FILTER EFFICIENCY: $0.000000 \%$

EFFECTIVE RELEASE HEIGHT : $\quad 0.00 \mathrm{~m}$

WIND SPEED $(\mathrm{h}=2 \mathrm{~m}): 1.7 \mathrm{~m} / \mathrm{s}$

STABILITY CLASS :E DEPOSITION VELOCITY $: 1.00 \mathrm{~cm} / \mathrm{s}$

RECEPTOR HEIGHT $\quad: 0.0 \mathrm{~m}$ INVERSION LAYER HEIGHT $: 200.0 \mathrm{~m}$

SAMPLE TIME $\quad: \quad 30.000 \mathrm{~min}$

MAXIMUM DOSE DISTANCE $:<0.10 \mathrm{~km}$ MAXIMUM CEDE $:>\quad 25 \mathrm{rem}$

$\mathrm{D}=0.03 \mathrm{~km}$

$\mathrm{DEP}=9.7 \mathrm{E}+00 \mathrm{uCi} / \mathrm{m}^{\wedge} 2$

$\mathrm{CHI}=9.7 \mathrm{E}-04(\mathrm{Ci}-\mathrm{s}) / \mathrm{m}^{\wedge} 3$

50-YR DOSE COMMITMENT:

SURFACE BONE $2.6 \mathrm{E}+03$ rem RED MARROW $2.2 \mathrm{E}+02$ rem LIVER 5.8E+02 rem GONADS 3.2E+01 rem EFFECTIVE DOSE

EQUIVALENT $1.5 \mathrm{E}+02$ rem

\author{
$\mathrm{D}=0.10 \mathrm{~km}$ \\ $\mathrm{DEP}=1.6 \mathrm{E}+00 \mathrm{uCi} / \mathrm{m}^{\wedge} 2$ \\ $\mathrm{CHI}=1.6 \mathrm{E}-04(\mathrm{Ci}-\mathrm{s}) / \mathrm{m}^{\wedge} 3$ \\ 50-YR DOSE COMMITMENT: \\ SURFACE BONE 4.4E+02 rem \\ RED MARROW $3.7 \mathrm{E}+01 \mathrm{rem}$ \\ LIVER 9.9E+01 rem \\ GONADS 5.5E+00 rem \\ EFFECTIVE DOSE . \\ EQUIVALENT $2.5 \mathrm{E}+01 \mathrm{rem}$
}

\author{
$\mathrm{D}=0.57 \mathrm{~km}$ \\ $\mathrm{DEP}=6.7 \mathrm{E}-02 \mathrm{uCi} / \mathrm{m}^{\wedge} 2$ \\ $\mathrm{CHI}=6.7 \mathrm{E}-06(\mathrm{Ci}-\mathrm{s}) / \mathrm{m}^{\wedge} 3$ \\ 50-YR DOSE COMMITMENT: \\ SURFACE BONE 1.8E+01 rem \\ RED MARROW $1.5 \mathrm{E}+00 \mathrm{rem}$ \\ LIVER . 4.0E+00 rem \\ GONADS 2.2E-01 rem \\ EFFECTIVE DOSE \\ EQUIVALENT $\quad 1.0 \mathrm{E}+00 \mathrm{rem}$
}


Title of Project EPHA for THM

Subject Radiological Consequence Assessment

Computer D. J. Richards

Date $\underline{09 / 11 / 96}$

Reviewed by S. A. Henderson

Works Calculation 6

Sheet No. 105 of 166

Release Designation: 1-RD-115, Average

HOTSPOT 7.0 GENERAL PLUME 9-09-1996 22:01

RADIONUCLIDE : PU-238 Inhalation Class : W

HALFLIFE : 87.740 years

SOURCE TERM : $4.6 \mathrm{E}-02 \mathrm{Ci}$

Source Term Width: $3 \mathrm{~m}$ Source Term Height: $3 \mathrm{~m}$

RELEASE FRACTION : $1.00 \mathrm{E}+00$

FILTER EFFICIENCY: $0.000000 \%$

EFFECTIVE RELEASE HEIGHT : $\quad 0.00 \mathrm{~m}$

WIND SPEED $(\mathrm{h}=2 \mathrm{~m}): 2.5 \mathrm{~m} / \mathrm{s}$

STABILITY CLASS :C DEPOSITION VELOCITY $: 1.00 \mathrm{~cm} / \mathrm{s}$

RECEPTOR HEIGHT $\quad: 0.0 \mathrm{~m}$ INVERSION LAYER HEIGHT $: 500.0 \mathrm{~m}$

SAMPLE TIME $\quad: 30.000 \mathrm{~min}$

MAXIMUM DOSE DISTANCE $:<0.10 \mathrm{~km}$ MAXIMUM CEDE $:>5.9 \mathrm{rem}$

$\mathrm{D}=0.03 \mathrm{~km}$

$\mathrm{DEP}=2.8 \mathrm{E}+00 \mathrm{uCi} / \mathrm{m}^{\wedge} 2$

$\mathrm{CHI}=2.8 \mathrm{E}-04(\mathrm{Ci}-\mathrm{s}) / \mathrm{m}^{\wedge} 3$

50-YR DOSE COMMITMENT:

SURFACE BONE 7.6E+02 rem

RED MARROW $6.3 \mathrm{E}+01 \mathrm{rem}$

LIVER 1.7E+02 rem

GONADS $9.4 \mathrm{E}+00 \mathrm{rem}$

EFFECTIVE DOSE

EQUIVALENT $4.3 E+01 \mathrm{rem}$

\author{
$\mathrm{D}=0.10 \mathrm{~km}$ \\ $\mathrm{DEP}=3.9 \mathrm{E}-01 \mathrm{uCi} / \mathrm{m}^{\wedge} 2$ \\ $\mathrm{CHI}=3.9 \mathrm{E}-05(\mathrm{Ci}-\mathrm{s}) / \mathrm{m}^{\wedge} 3$ \\ 50-YR DOSE COMMITMENT: \\ SURFACE BONE $1.0 \mathrm{E}+02 \mathrm{rem}$ \\ RED MARROW $8.6 \mathrm{E}+00$ rem \\ LIVER 2.3E+01 rem \\ GONADS 1.3E+00 rem \\ EFFECTIVE DOSE \\ EQUTVALENT $5.9 \mathrm{E}+00 \mathrm{rem}$
}

\author{
$\mathrm{D}=0.25 \mathrm{~km}$ \\ $\mathrm{DEP}=6.8 \mathrm{E}-02 \mathrm{uCi} / \mathrm{m}^{\wedge} 2$ \\ $\mathrm{CHI}=6.8 \mathrm{E}-06(\mathrm{Ci}-\mathrm{s}) / \mathrm{m}^{\wedge} 3$ \\ 50-YR DOSE COMMITMENT: \\ SURFACE BONE 1.8E+01 rem \\ RED MARROW $1.5 \mathrm{E}+00 \mathrm{rem}$ \\ LIVER 4.1E+00 rem \\ GONADS 2.3E-01 rem \\ EFFECTIVE DOSE \\ EQUIVALENT $1.0 \mathrm{E}+00 \mathrm{rem}$
}


Title of Project EPHA for THM

Subject Radiological Consequence Assessment

Computer D. J. Richards

Date $\underline{09 / 11 / 96}$

Reviewed by $\underline{\mathrm{S}}$. A. Henderson

Works Calculation 6 . Sheet No. 106 of 166

Release Designation: 1-RD-116, Adverse

HOTSPOT 7.0 GEṆERAL PLUME 9-11-1996 11:04

RADIONUCLIDE : PU-238 Inhalation Class : W

HALFLIFE : 87.740 years

SOURCE TERM : $8.2 \mathrm{E}-03 \mathrm{Ci}$

Source Term Width: $3 \mathrm{~m}$ Source Term Height: $3 \mathrm{~m}$

RELEASE FRACTION : $1.00 \mathrm{E}+00$

FILTER EFFICIENCY: $0.000000 \%$

EFFECTIVE RELEASE HEIGHT : $\quad 0.00 \mathrm{~m}$

WIND SPEED $(\mathrm{h}=2 \mathrm{~m}): 1.7 \mathrm{~m} / \mathrm{s}$

STABILITY CLASS :E DEPOSITION VELOCITY $: 1.00 \mathrm{~cm} / \mathrm{s}$

RECEPTOR HEIGHT $\quad: 0.0 \mathrm{~m}$ INVERSION LAYER HEIGHT $: 200.0 \mathrm{~m}$

SAMPLE TIME $\quad: 10.000 \mathrm{~min}$

MAXIMUM DOSE DISTANCE $:<0.10 \mathrm{~km}$ MAXIMUM CEDE $:>5.7 \mathrm{rem}$

$D=0.03 \mathrm{~km}$

$\mathrm{DEP}=2.2 \mathrm{E}+00 \mathrm{uCi} / \mathrm{m}^{\wedge} 2$

$\mathrm{CHI}=2.2 \mathrm{E}-04(\mathrm{Ci}-\mathrm{s}) / \mathrm{m}^{\wedge} 3$

50-YR DOSE COMMITMENT:

SURFACE BONE 5.8E+02 rem RED MARROW 4.8E+01 rem LIVER 1.3E+02 rem GONADS 7.2E+00 rem EFFECTIVE DOSE EQUIVALENT $3.3 \mathrm{E}+01 \mathrm{rem}$

$\mathrm{D}=0.10 \mathrm{~km}$
$\mathrm{DEP}=3.7 \mathrm{E}-01 \mathrm{uCi} / \mathrm{m}^{\wedge} 2$
$\mathrm{CHI}=3.7 \mathrm{E}-05(\mathrm{Ci}-\mathrm{s}) / \mathrm{m}^{\wedge} 3$
50-YR DOSE COMMITMENT:
SURFACE BONE $1.0 \mathrm{E}+02 \mathrm{rem}$
RED MARROW $8.2 \mathrm{E}+00 \mathrm{rem}$
LIVER $\quad 2.2 \mathrm{E}+01 \mathrm{rem}$
GONADS $\quad 1.2 \mathrm{E}+00 \mathrm{rem}$
EFFECTIVE DOSE
EQUTVALENT $\quad 5.7 \mathrm{E}+00 \mathrm{rem}$

$\mathrm{D}=0.26 \mathrm{~km}$ $\mathrm{DEP}=6.7 \mathrm{E}-02 \mathrm{uCi} / \mathrm{m}^{\wedge} 2$ $\mathrm{CHI}=6.7 \mathrm{E}-06(\mathrm{Ci}-\mathrm{s}) / \mathrm{m}^{\wedge} 3$ 50-YR DOSE COMMITMENT:

SURFACE BONE 1.8E+01 rem RED MARROW $1.5 \mathrm{E}+00 \mathrm{rem}$ LIVER $\quad 4.0 \mathrm{E}+00 \mathrm{rem}$ GONADS 2.2E-01 rem EFFECTIVE DOSE EQUIVALENT $1.0 \mathrm{E}+00 \mathrm{rem}$ 
Title of Project EPHA for THM

Subject Radiological Consequence Assessment

Computer D. J. Richards

Date $\underline{09 / 11 / 96}$

Reviewed by $\underline{\text { S. A. Henderson }}$

Works Calculation 6

Sheet No. 107 of 166

Release Designation: 1-RD-116, Average

HOTSPOT 7.0 GENERAL PLUME 9-11-1996 11:05

RADIONUCLIDE : PU-238 Inhalation Class : W

HALFLIFE $: 87.740$ years

SOURCE TERM $\quad: 8.2 \mathrm{E}-03 \mathrm{Ci}$

Source Term Width: $\quad 3 \mathrm{~m}$ Source Term Height: $\quad 3 \mathrm{~m}$

RELEASE FRACTION : $1.00 \mathrm{E}+00$

FILTER EFFICIENCY: $0.000000 \%$

EFFECTIVE RELEASE HEIGHT : $0.00 \mathrm{~m}$

WIND SPEED $(\mathrm{h}=2 \mathrm{~m}): 2.5 \mathrm{~m} / \mathrm{s}$

STABILITY CLASS :C DEPOSITION VELOCITY $: 1.00 \mathrm{~cm} / \mathrm{s}$

RECEPTOR HEIGHT $: 0.0 \mathrm{~m}$ INVERSION LAYER HEIGHT $: 500.0 \mathrm{~m}$

SAMPLE TIME $\quad: 10.000 \mathrm{~min}$

MAXIMUM DOSE DISTANCE $:<0.10 \mathrm{~km}$ MAXIMUM CEDE $:>1.3 \mathrm{rem}$

$D=0.03 \mathrm{~km}$

$\mathrm{DEP}=6.3 \mathrm{E}-01 \mathrm{uCi} / \mathrm{m}^{\wedge} 2$

$\mathrm{CHI}=6.3 \mathrm{E}-05(\mathrm{Ci}-\mathrm{s}) / \mathrm{m}^{\wedge} 3$

50-YR DOSE COMMITMENT:

SURFACE BONE $1.7 \mathrm{E}+02 \mathrm{rem}$ RED MARROW $1.4 \mathrm{E}+01$ rem

LIVER 3.8E+01 rem GONADS 2.1E+00 rem EFFECTIVE DOSE

EQUTVALENT $\quad 9.7 \mathrm{E}+00 \mathrm{rem}$

\author{
$D=0.10 \mathrm{~km}$ \\ $\mathrm{DEP}=8.7 \mathrm{E}-02 \mathrm{uCi} / \mathrm{m}^{\wedge} 2$ \\ $\mathrm{CHI}=8.7 \mathrm{E}-06(\mathrm{Ci}-\mathrm{s}) / \mathrm{m}^{\wedge} 3$ \\ 50-YR DOSE COMMITMENT: \\ SURFACE BONE 2.3E +01 rem \\ RED MARROW $1.9 \mathrm{E}+00 \mathrm{rem}$ \\ LIVER 5.2E+00 rem \\ GONADS 2.9E-01 rem \\ EFFECTIVE DOSE \\ EQUIVALENT $\quad 1.3 \mathrm{E}+00 \mathrm{rem}$
}
$\mathrm{D}=0.11 \mathrm{~km}$
$\mathrm{DEP}=6.8 \mathrm{E}-02 \mathrm{uCi} / \mathrm{m}^{\wedge} 2$
$\mathrm{CHI}=6.8 \mathrm{E}-06(\mathrm{Ci}-\mathrm{s}) / \mathrm{m}^{\wedge} 3$
50-YR DOSE COMMITMENT:
SURFACE BONE 1.8E+01 rem RED MARROW $1.5 \mathrm{E}+00 \mathrm{rem}$ LIVER 4.1E+00 rem GONADS 2.3E-01 rem EFFECTIVE DOSE
EQUIVALENT $\quad 1.0 \mathrm{E}+00 \mathrm{rem}$ 
Title of Project EPHA for THM

Reviewed by S. A. Henderson

Subject Radiological Consequence Assessment

Computer D. J. Richards

Release Designation: 1-RD-117, Adverse

HOTSPOT 7.0 GENERAL PLUME 9-09-1996 22:02

RADIONUCLIDE : PU-238 Inhalation Class : W

HALFLIFE $: 87.740$ years

SOURCE TERM : $1.7 \mathrm{E}-06 \mathrm{Ci}$

Source Term Width: $3 \mathrm{~m}$ Source Term Height: $3 \mathrm{~m}$ 、

RELEASE FRACTION : $1.00 \mathrm{E}+00$

FILTER EFFICIENCY: $0.000000 \%$

EFFECTIVE RELEASE HEIGHT : $\quad 0.00 \mathrm{~m}$

WIND SPEED $(\mathrm{h}=2 \mathrm{~m}): 1.7 \mathrm{~m} / \mathrm{s}$

STABILITY CLASS :E DEPOSITION VELOCITY $\quad: 1.00 \mathrm{~cm} / \mathrm{s}$

RECEPTOR HEIGHT $: 0.0 \mathrm{~m}$ INVERSION LAYER HEIGHT $: 200.0 \mathrm{~m}$

SAMPLE TIME $\quad: 30.000 \mathrm{~min}$

MAXIMUM DOSE DISTANCE $:<0.10 \mathrm{~km} \quad$ MAXIMUM CEDE $:>9.3 \mathrm{E}-04 \mathrm{rem}$

$\mathrm{D}=0.03 \mathrm{~km}$

$\mathrm{DEP}=3.6 \mathrm{E}-04 \mathrm{uCi} / \mathrm{m}^{\wedge} 2$

$\mathrm{CHI}=3.6 \mathrm{E}-08(\mathrm{Ci}-\mathrm{s}) / \mathrm{m}^{\wedge} 3$

50-YR DOSE COMMITMENT:

SURFACE BONE 9.7E-02 rem RED MARROW $8.0 \mathrm{E}-03 \mathrm{rem}$ LIVER 2.1E-02 rem GONADS 1.2E-03 rem EFFECTIVE DOSE

EQUIVALENT 5.5E-03 rem

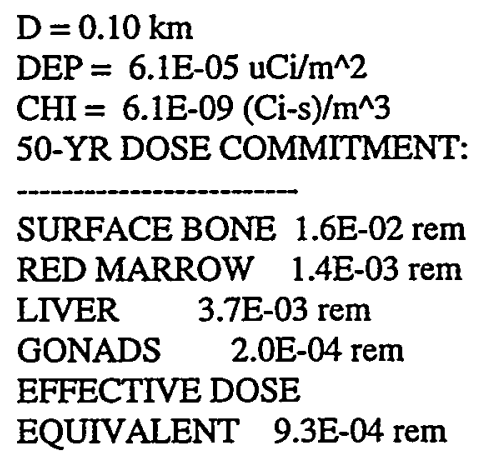


Title of Project EPHA for THM

Subject Radiological Consequence Assessment

Computer D. J. Richards
Reviewed by S. A. Henderson

Works Calculation 6

Sheet No. 109 of 166

Release Designation: 1-RD-117, Average

HOTSPOT 7.0 GENERAL PLUME 9-09-1996 22:03

RADIONUCLIDE : PU-238 Inhalation Class : W

HALFLIFE $: 87.740$ years

SOURCE TERM : $1.7 \mathrm{E}-06 \mathrm{Ci}$

Source Term Width: $\quad 3 \mathrm{~m}$ Source Term Height: $3 \mathrm{~m}$

RELEASE FRACTION : $1.00 \mathrm{E}+00$

FILTER EFFICIENCY: $0.000000 \%$

EFFECTIVE RELEASE HEIGHT : $\quad 0.00 \mathrm{~m}$

WIND SPEED ( $\mathrm{h}=2 \mathrm{~m}): 2.5 \mathrm{~m} / \mathrm{s}$

STABILITY CLASS :C DEPOSITION VELOCITY $: 1.00 \mathrm{~cm} / \mathrm{s}$

RECEPTOR HEIGHT $\quad: 0.0 \mathrm{~m}$ INVERSION LAYER HEIGHT $: 500.0 \mathrm{~m}$

SAMPLE TIME $\quad: 30.000 \mathrm{~min}$

MAXIMUM DOSE DISTANCE $:<0.10 \mathrm{~km}$ MAXIMUM CEDE $:>2.2 \mathrm{E}-04 \mathrm{rem}$

$\mathrm{D}=0.03 \mathrm{~km}$

$\mathrm{DEP}=1.0 \mathrm{E}-04 \mathrm{uCi} / \mathrm{m}^{\wedge} 2$

$\mathrm{CHI}=1.0 \mathrm{E}-08(\mathrm{Ci}-\mathrm{s}) / \mathrm{m}^{\wedge} 3$

50-YR DOSE COMMITMENT:

SURFACE BONE 2.8E-02 rem RED MARROW 2.3E-03 rem LIVER 6.3E-03 rem GONADS 3.5E-04 rem EFFECTIVE DOSE EQUTVALENT $1.6 \mathrm{E}-03 \mathrm{rem}$
$\mathrm{D}=0.10 \mathrm{~km}$

$\mathrm{DEP}=1.4 \mathrm{E}-05 \mathrm{uCi} / \mathrm{m}^{\wedge} 2$

$\mathrm{CHI}=1.4 \mathrm{E}-09(\mathrm{Ci}-\mathrm{s}) / \mathrm{m}^{\wedge} 3$

50-YR DOSE COMMITMENT:

SURFACE BONE 3.9E-03 rem RED MARROW 3.2E-04 rem LIVER $\quad 8.6 \mathrm{E}-04 \mathrm{rem}$ GONADS 4.8E-05 rem EFFECTIVE DOSE

EQUIVALENT 2.2E-04 rem 
Title of Project EPHA for THM

Subject Radiological Consequence Assessment

Computer D. J. Richards

Date $\underline{09 / 11 / 96}$

Reviewed by S. A. Henderson

Release Designation: 1-RD-118, Adverse

HOTSPOT 7.0 GENERAL PLUME 9-11-1996 11:07

RADIONUCLIDE : PU-238 Inhalation Class : W

HALFLIFE $: 87.740$ years

SOURCE TERM $: 1.8 \mathrm{E}-06 \mathrm{Ci}$

Source Term Width: $3 \mathrm{~m}$ Source Term Height: $3 \mathrm{~m}$

RELEASE FRACTION : $1.00 \mathrm{E}+00$

FILTER EFFICIENCY: $0.000000 \%$

EFFECTIVE RELEASE HEIGHT : $\quad 0.00 \mathrm{~m}$

WIND SPEED $(\mathrm{h}=2 \mathrm{~m}): 1.7 \mathrm{~m} / \mathrm{s}$

STABILITY CLASS :E DEPOSITION VELOCITY $\quad \vdots 1.00 \mathrm{~cm} / \mathrm{s}$

RECEPTOR HEIGHT $: 0.0 \mathrm{~m}$ INVERSION LAYER HEIGHT $: 200.0 \mathrm{~m}$

SAMPLE TIME $: 10.000 \mathrm{~min}$

MAXIMUM DOSE DISTANCE $:<0.10 \mathrm{~km}$ MAXIMUM CEDE $:>1.3 E-03 \mathrm{rem}$

$D=0.03 \mathrm{~km}$

$\mathrm{DEP}=4.9 \mathrm{E}-04 \mathrm{uCi} / \mathrm{m}^{\wedge} 2$

$\mathrm{CHI}=4.9 \mathrm{E}-08(\mathrm{Ci}-\mathrm{s}) / \mathrm{m}^{\wedge} 3$

50-YR DOSE COMMITMENT:

SURFACE BONE 1.3E-01 rem

RED MARROW 1.1E-02 rem

LIVER 2.9E-02 rem

GONADS 1.6E-03 rem

EFFECTIVE DOSE

EQUIVALENT ${ }^{\prime}$ 7.4E-03 rem
$\mathrm{D}=0.10 \mathrm{~km}$

$\mathrm{DEP}=8.3 \mathrm{E}-0.5 \mathrm{uCi} / \mathrm{m}^{\wedge} 2$

$\mathrm{CHI}=8.3 \mathrm{E}-09(\mathrm{Ci}-\mathrm{s}) / \mathrm{m}^{\wedge} 3$

50-YR DOSE COMMITMENT:

SURFACE BONE 2.2E-02 rem

RED MARROW 1.8E-03 rem

LIVER 5.0E-03 rem

GONADS 2.8E-04 rem

EFFECTIVE DOSE

EQUIVALENT $1.3 \mathrm{E}-03 \mathrm{rem}$ 
Title of Project EPHA for THM

Reviewed by $\underline{\text { S. A. Henderson }}$

Subject Radiological Consequence Assessment

Computer D. J. Richards

Date 09/11/96
Works Calculation 6

Sheet No. 111 of 166

Release Designation: 1-RD-118, Average

HOTSPOT 7.0 GENERAL PLUME 9-11-1996 11:08

RADIONUCLIDE : PU-238 Inhalation Class : W

HALFLIFE $: 87.740$ years

SOURCE TERM $: 1.8 \mathrm{E}-06 \mathrm{Ci}$

Source Term Width: $\quad 3 \mathrm{~m}$ Source Term Height: $3 \mathrm{~m}$

RELEASE FRACTION : $1.00 \mathrm{E}+00$

FILTER EFFICIENCY: $0.000000 \%$

EFFECTIVE RELEASE HEIGHT : $\quad 0.00 \mathrm{~m}$

WIND SPEED $(\mathrm{h}=2 \mathrm{~m}): 2.5 \mathrm{~m} / \mathrm{s}$

STABILITY CLASS :C DEPOSITION VELOCITY $: 1.00 \mathrm{~cm} / \mathrm{s}$

RECEPTOR HEIGHT $\quad: 0.0 \mathrm{~m}$ INVERSION LAYER HEIGHT $: 500.0 \mathrm{~m}$

SAMPLE TIME $\quad: 10.000 \mathrm{~min}$

MAXIMUM DOSE DISTANCE $:<0.10 \mathrm{~km}$ MAXIMUM CEDE $:>3.0 \mathrm{E}-04 \mathrm{rem}$

$D=0.03 \mathrm{~km}$

$\mathrm{DEP}=1.4 \mathrm{E}-04 \mathrm{uCi} / \mathrm{m}^{\wedge} 2$

$\mathrm{CHI}=1.4 \mathrm{E}-08(\mathrm{Ci}-\mathrm{s}) / \mathrm{m}^{\wedge} 3$

50-YR DOSE COMMTTMENT:

SURFACE BONE 3.8E-02 rem RED MARROW 3.2E-03 rem

LIVER 8.5E-03 rem GONADS 4.7E-04 rem EFFECTIVE DOSE

EQUIVALENT 2.2E-03 rem
$D=0.10 \mathrm{~km}$

$\mathrm{DEP}=1.9 \mathrm{E}-05 \mathrm{uCi} / \mathrm{m}^{\wedge} 2$

$\mathrm{CHI}=1.9 \mathrm{E}-09(\mathrm{Ci}-\mathrm{s}) / \mathrm{m}^{\wedge} 3$

50-YR DOSE COMMITMENT:

SURFACE BONE 5.2E-03 rem

RED MARROW 4.3E-04 rem

LIVER $1.2 \mathrm{E}-03 \mathrm{rem}$

GONADS 6.5E-05 rem

EFFECTIVE DOSE

EQUTVALENT 3.0E-04 rem 
- Title of Project EPHA for THM

Subject Radiological Consequence Assessment

Computer D. J. Richards

Date $\underline{09 / 11 / 96}$

Reviewed by $\underline{\text { S. A. Henderson }}$ Works Calculation 6

Sheet No. 112 of 166

Release Designation: 1-RD-119, Adverse

HOTSPOT 7.0 GENERAL PLUME 10-08-1996 14:53 RADIONUCLIDE : PU-238 Inhalation Class : W HALFLIFE : 87.740 years

SOURCE TERM $\quad: 8.0 \mathrm{E}-02 \mathrm{Ci}$

Source Term Width: $\quad 3 \mathrm{~m}$ Source Term Height: $\quad 3 \mathrm{~m}$

RELEASE FRACTION : $1.00 \mathrm{E}+00$

FILTER EFFICIENCY: $0.000000 \%$

EFFECTIVE RELEASE HEIGHT : $\quad 0.00 \mathrm{~m}$

WIND SPEED ( $\mathrm{h}=2 \mathrm{~m}): 1.7 \mathrm{~m} / \mathrm{s}$

STABILITY CLASS $\quad$ E DEPOSITION VELOCITY $\quad: 1.00 \mathrm{~cm} / \mathrm{s}$

RECEPTOR HEIGHT $: 0.0 \mathrm{~m}$ INVERSION LAYER HEIGHT $: 200.0 \mathrm{~m}$

SAMPLE TIME $\quad: 30.000 \mathrm{~min}$

MAXIMUM DOSE DISTANCE $:<0.10 \mathrm{~km}$ MAXIMUM CEDE $:>\quad 44 \mathrm{rem}$

$\mathrm{D}=0.03 \mathrm{~km}$

$\mathrm{DEP}=1.7 \mathrm{E}+01 \mathrm{uCi} / \mathrm{m}^{\wedge} 2$

$\mathrm{CHI}=1.7 \mathrm{E}-03(\mathrm{Ci}-\mathrm{s}) / \mathrm{m}^{\wedge} 3$

50-YR DOSE COMMITMENT:

SURFACE BONE 4.6E +03 rem

RED MARROW $3.8 \mathrm{E}+02 \mathrm{rem}$

LIVER $\quad 1.0 \mathrm{E}+03 \mathrm{rem}$

GONADS 5.6E+01 rem

EFFECTIVE DOSE

EQUIVALENT $2.6 \mathrm{E}+02 \mathrm{rem}$

$\mathrm{D}=0.10 \mathrm{~km}$

$\mathrm{DEP}=2.9 \mathrm{E}+00 \mathrm{uCi} / \mathrm{m}^{\wedge} 2$

$\mathrm{CHI}=2.9 \mathrm{E}-04(\mathrm{Ci}-\mathrm{s}) / \mathrm{m}^{\wedge} 3$

50-YR DOSE COMMITMENT:

SURFACE BONE 7.8E+02 rem

RED MARROW $6.4 \mathrm{E}+01$ rem

LIVER 1.7E+02 rem

GONADS $\quad 9.6 \mathrm{E}+00 \mathrm{rem}$

EFFECTIVE DOSE

EQUIVALENT 4.4E+01 rem

$\mathrm{D}=0.77 \mathrm{~km}$

$\mathrm{DEP}=6.6 \mathrm{E}-02 \mathrm{uCi} / \mathrm{m}^{\wedge} 2$

$\mathrm{CHI}=6.6 \mathrm{E}-06(\mathrm{Ci}-\mathrm{s}) / \mathrm{m}^{\wedge} 3$

50-YR DOSE COMMITMENT:

SURFACE BONE 1.8E+01 rem

RED MARROW $1.5 \mathrm{E}+00 \mathrm{rem}$

LIVER $\quad 4.0 \mathrm{E}+00 \mathrm{rem}$

GONADS 2.2E-01 rem

EFFECTIVE DOSE

EQUTVALENT $1.0 \mathrm{E}+00 \mathrm{rem}$ 
Title of Project EPHA for THM

Subject Radiological Consequence Assessment

Computer D. J. Richards

Date $\underline{09 / 11 / 96}$

Reviewed by S. A. Henderson

Works Calculation 6

Sheet No. 113 of 166

Release Designation: 1-RD-119, Average

HOTSPOT 7.0 GENERAL PLUME 10-08-1996 14:43

RADIONUCLIDE : PU-238 Inhalation Class : W

HALFLIFE $: 87.740$ years

SOURCE TERM $: 8.0 \mathrm{E}-02 \mathrm{Ci}$

Source Term Width: $3 \mathrm{~m}$ Source Term Hèight: $3 \mathrm{~m}$

RELEASE FRACTION : $1.00 \mathrm{E}+00$

FILTER EFFICIENCY: $0.000000 \%$

EFFECTIVE RELEASE HEIGHT : $\quad 0.00 \mathrm{~m}$

WIND SPEED $(\mathrm{h}=2 \mathrm{~m}): 2.5 \mathrm{~m} / \mathrm{s}$

$\begin{array}{lcl}\text { STABILITY CLASS } & \text { C DEPOSITION VELOCITY }: 1.00 \mathrm{~cm} / \mathrm{s} \\ \text { RECEPTOR HEIGHT } & : 0.0 \mathrm{~m} \text { INVERSION LAYER HEIGHT }: 500.0 \mathrm{~m}\end{array}$

SAMPLE TIME $\quad: 30.000 \mathrm{~min}$

MAXIMUM DOSE DISTANCE $:<0.10 \mathrm{~km}$ MAXIMUM CEDE $:>10 \mathrm{rem}$

$\mathrm{D}=0.03 \mathrm{~km}$

$\mathrm{DEP}=5.0 \mathrm{E}+00 \mathrm{uCi} / \mathrm{m}^{\wedge} 2$

$\mathrm{CHI}=5.0 \mathrm{E}-04(\mathrm{Ci}-\mathrm{s}) / \mathrm{m}^{\wedge} 3$

50-YR DOSE COMMITMENT:

SURFACE BONE 1.3E+03 rem

RED MARROW 1.1E+02 rem

LIVER $3.0 \mathrm{E}+02 \mathrm{rem}$

GONADS $1.6 \mathrm{E}+01 \mathrm{rem}$

EFFECTIVE DOSE

EQUIVALENT 7.6E+01 rem

$D=0.10 \mathrm{~km}$

$\mathrm{DEP}=6.8 \mathrm{E}-01 \mathrm{uCi} / \mathrm{m}^{\wedge} 2$

$\mathrm{CHI}=6.8 \mathrm{E}-05(\mathrm{Ci}-\mathrm{s}) / \mathrm{m}^{\wedge} 3$

50-YR DOSE COMMITMENT:

SURFACE BONE $1.8 \mathrm{E}+02 \mathrm{rem}$

RED MARROW $1.5 \mathrm{E}+01 \mathrm{rem}$

LIVER 4.1E+01 rem

GONADS 2.3E+00 rem

EFFECTIVE DOSE

EQUIVALENT $1.0 \mathrm{E}+01 \mathrm{rem}$

$\mathrm{D}=0.34 \mathrm{~km}$

$\mathrm{DEP}=6.8 \mathrm{E}-02 \mathrm{uCi} / \mathrm{m}^{\wedge} 2$

$\mathrm{CHI}=6.8 \mathrm{E}-06(\mathrm{Ci}-\mathrm{s}) / \mathrm{m}^{\wedge} 3$

50-YR DOSE COMMITMENT:

SURFACE BONE 1.8E+01 rem

RED MARROW $1.5 \mathrm{E}+00 \mathrm{rem}$

LIVER $\quad 4.1 \mathrm{E}+00 \mathrm{rem}$

GONADS 2.3E-01 rem

EFFECTIVE DOSE

EQUIVALENT $1.0 \mathrm{E}+00 \mathrm{rem}$ 
Title of Project EPHA for THM

Subject Radiological Consequence Assessment

Computer $\underline{\text { D. J. Richards }}$

Date $\underline{09 / 11 / 96}$

Reviewed by S. A. Henderson

Works Calculation 6

Sheet No. 114 of 166

Release Designation: 1-RD-120, Adverse

HOTSPOT 7.0 GENERAL PLUME 10-08-1996 14:47

RADIONUCLIDE : PU-238 Inhalation Class : W

HALFLIFE $: 87.740$ years

SOURCE TERM $: 8.0 \mathrm{E}-02 \mathrm{Ci}$

Source Term Width: $3 \mathrm{~m}$ Source Term Height: $3 \mathrm{~m}$

RELEASE FRACTION : $1.00 \mathrm{E}+00$

FILTER EFFICIENCY: $0.000000 \%$

EFFECTIVE RELEASE HEIGHT : $\quad 0.00 \mathrm{~m}$

WIND SPEED $(\mathrm{h}=2 \mathrm{~m}): 1.7 \mathrm{~m} / \mathrm{s}$

$\begin{array}{lcc}\text { STABILITY CLASS } & : \text { E DEPOSITION VELOCITY }: 1.00 \mathrm{~cm} / \mathrm{s} \\ \text { RECEPTOR HEIGHT } & : 0.0 \mathrm{~m} \text { INVERSION LAYER HEIGHT }: 200.0 \mathrm{~m} \\ \text { SAMPLE TIME } & : 10.000 \mathrm{~min}\end{array}$

MAXIMUM DOSE DISTANCE $:<0.10 \mathrm{~km}$ MAXIMUM CEDE $:>55 \mathrm{rem}$

$D=0.03 \mathrm{~km}$

$\mathrm{DEP}=2.1 \mathrm{E}+01 \mathrm{uCi} / \mathrm{m}^{\wedge} 2$

$\mathrm{CHI}=2.1 \mathrm{E}-03(\mathrm{Ci}-\mathrm{s}) / \mathrm{m}^{\wedge} 3$

50-YR DOSE COMMITMENT:

SURFACE BONE 5.7E+03 rem

RED MARROW $4.7 \mathrm{E}+02 \mathrm{rem}$

LIVER $\quad 1.3 \mathrm{E}+03 \mathrm{rem}$

GONADS 7.0E+01 rem

EFFECTIVE DOSE

EQUIVALENT $3.2 \mathrm{E}+02 \mathrm{rem}$

$\mathrm{D}=0.10 \mathrm{~km}$

$\mathrm{DEP}=3.6 \mathrm{E}+00 \mathrm{uCi} / \mathrm{m}^{\wedge} 2$

$\mathrm{CHI}=3.6 \mathrm{E}-04(\mathrm{Ci}-\mathrm{s}) / \mathrm{m}^{\wedge} 3$

50-YR DOSE COMMITMENT:

SURFACE BONE 9.7E+02 rem

RED MARROW $8.0 \mathrm{E}+01 \mathrm{rem}$

LIVER 2.2E+02 rem

GONADS 1.2E+01 rem

EFFECTIVE DOSE

EQUIVALENT $\quad 5.5 \mathrm{E}+01 \mathrm{rem}$

$\mathrm{D}=0.86 \mathrm{~km}$

$\mathrm{DEP}=6.7 \mathrm{E}-02 \mathrm{uCi} / \mathrm{m}^{\wedge} 2$

$\mathrm{CHI}=6.7 \mathrm{E}-06(\mathrm{Ci}-\mathrm{s}) / \mathrm{m}^{\wedge} 3$

50-YR DOSE COMMITMENT:

SURFACE BONE 1.8E+01 rem

RED MARROW $1.5 \mathrm{E}+00 \mathrm{rem}$

LIVER $\quad 4.0 \mathrm{E}+00 \mathrm{rem}$

GONADS 2.2E-01 rem

EFFECTIVE DOSE

EQUIVALENT $1.0 \mathrm{E}+00 \mathrm{rem}$ 
Title of Project EPHA for THM

Subject Radiological Consequence Assessment

Computer D. J. Richards

Date $\underline{09 / 11 / 96}$

Reviewed by S. A. Hènderson

Works Calculation 6

Sheet No. 115 of 166

Release Designation: 1-RD-120, Average

OTSPOT 7.0 GENERAL PLUME 10-08-1996 14:48

RADIONUCLIDE : PU-238 Inhalation Class : W

HALFLIFE : 87.740 years

SOURCE TERM $: 8.0 \mathrm{E}-02 \mathrm{Ci}$

Source Term Width: $3 \mathrm{~m}$ Source Term Height: $3 \mathrm{~m}$

RELEASE FRACTION : $1.00 \mathrm{E}+00$

FILTER EFFICIENCY: $0.000000 \%$

EFFECTIVE RELEASE HEIGHT : $\quad 0.00 \mathrm{~m}$

WIND SPEED $(\mathrm{h}=2 \mathrm{~m}): 2.5 \mathrm{~m} / \mathrm{s}$

STABILITY CLASS :C DEPOSITION VELOCITY $: 1.00 \mathrm{~cm} / \mathrm{s}$

RECEPTOR HEIGHT $\quad: 0.0 \mathrm{~m}$ INVERSION LAYER HEIGHT $: 500.0 \mathrm{~m}$

SAMPLE TIME $\quad: 10.000 \mathrm{~min}$

MAXIMUM DOSE DISTANCE $:<0.10 \mathrm{~km}$ MAXIMUM CEDE $:>13 \mathrm{rem}$

$\mathrm{D}=0.03 \mathrm{~km}$

$\mathrm{DEP}=6.2 \mathrm{E}+00 \mathrm{uCi} / \mathrm{m}^{\wedge} 2$

$\mathrm{CHI}=6.2 \mathrm{E}-04(\mathrm{Ci}-\mathrm{s}) / \mathrm{m}^{\wedge} 3$

50-YR DOSE COMMITMENT:

SURFACE BONE $1.7 \mathrm{E}+03$ rem

RED MARROW $1.4 \mathrm{E}+02 \mathrm{rem}$

LIVER 3.7E+02 rem

GONADS 2.1E+01 rem

EFFECTIVE DOSE

EQUIVALENT $9.5 \mathrm{E}+01 \mathrm{rem}$

$\mathrm{D}=0.10 \mathrm{~km}$

$\mathrm{DEP}=8.4 \mathrm{E}-01 \mathrm{uCi} / \mathrm{m}^{\wedge} 2$

$\mathrm{CHI}=8.4 \mathrm{E}-05(\mathrm{Ci}-\mathrm{s}) / \mathrm{m}^{\wedge} 3$

50-YR DOSE COMMITMENT:

SURFACE BONE 2.3E+02 rem

RED MARROW $1.9 \mathrm{E}+01 \mathrm{rem}$

LIVER $\quad 5.0 \mathrm{E}+01 \mathrm{rem}$

GONADS 2.8E+00 rem

EFFECTIVE DOSE

EQUIVALENT 1.3E+01 rem

$\mathrm{D}=0.38 \mathrm{~km}$

$\mathrm{DEP}=6.8 \mathrm{E}-02 \mathrm{uCi} / \mathrm{m}^{\wedge} 2$

$\mathrm{CHI}=6.8 \mathrm{E}-06(\mathrm{Ci}-\mathrm{s}) / \mathrm{m}^{\wedge} 3$

50-YR DOSE COMMITMENT:

SURFACE BONE 1.8E +01 rem RED MARROW $1.5 \mathrm{E}+00$ rem

LIVER $\quad 4.1 \mathrm{E}+00 \mathrm{rem}$

GONADS 2.3E-01 rem

EFFECTIVE DOSE

EQUIVALENT $1.0 \mathrm{E}+00 \mathrm{rem}$ 
Title of Project EPHA for THM

Subject Radiological Consequence Assessment

Computer D. J. Richards
Reviewed by $\underline{S}$. A. Hènderson

Works Calculation 6

Sheet No. 116 of 166

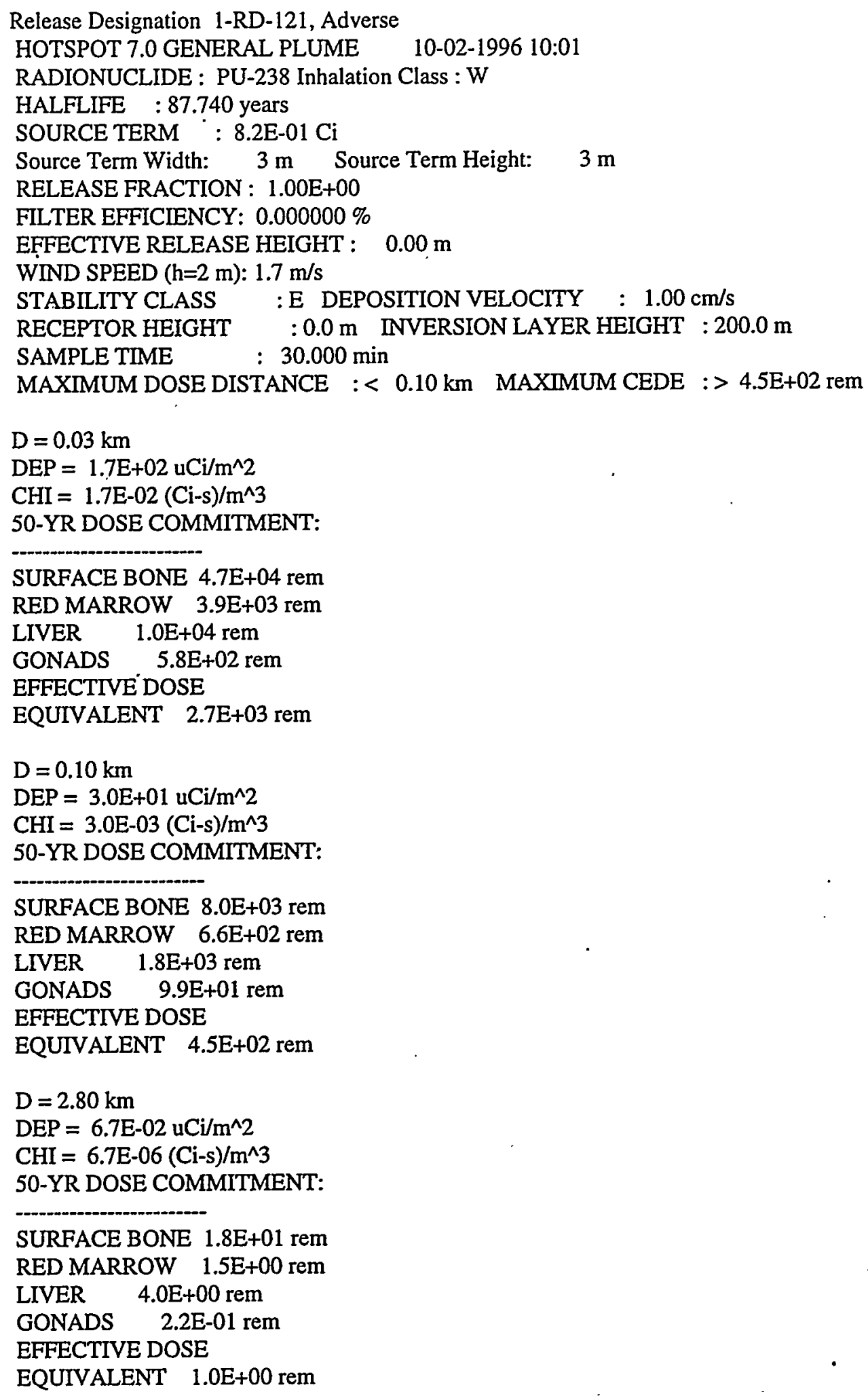


Title of Project EPHA for THM

Subject Radiological Consequence Assessment

Computer D. J. Richards

Date $\underline{09 / 11 / 96}$

Reviewed by $\underline{\text { S. A. Henderson }}$ Works Calculation 6

Sheet No. 117 of 166

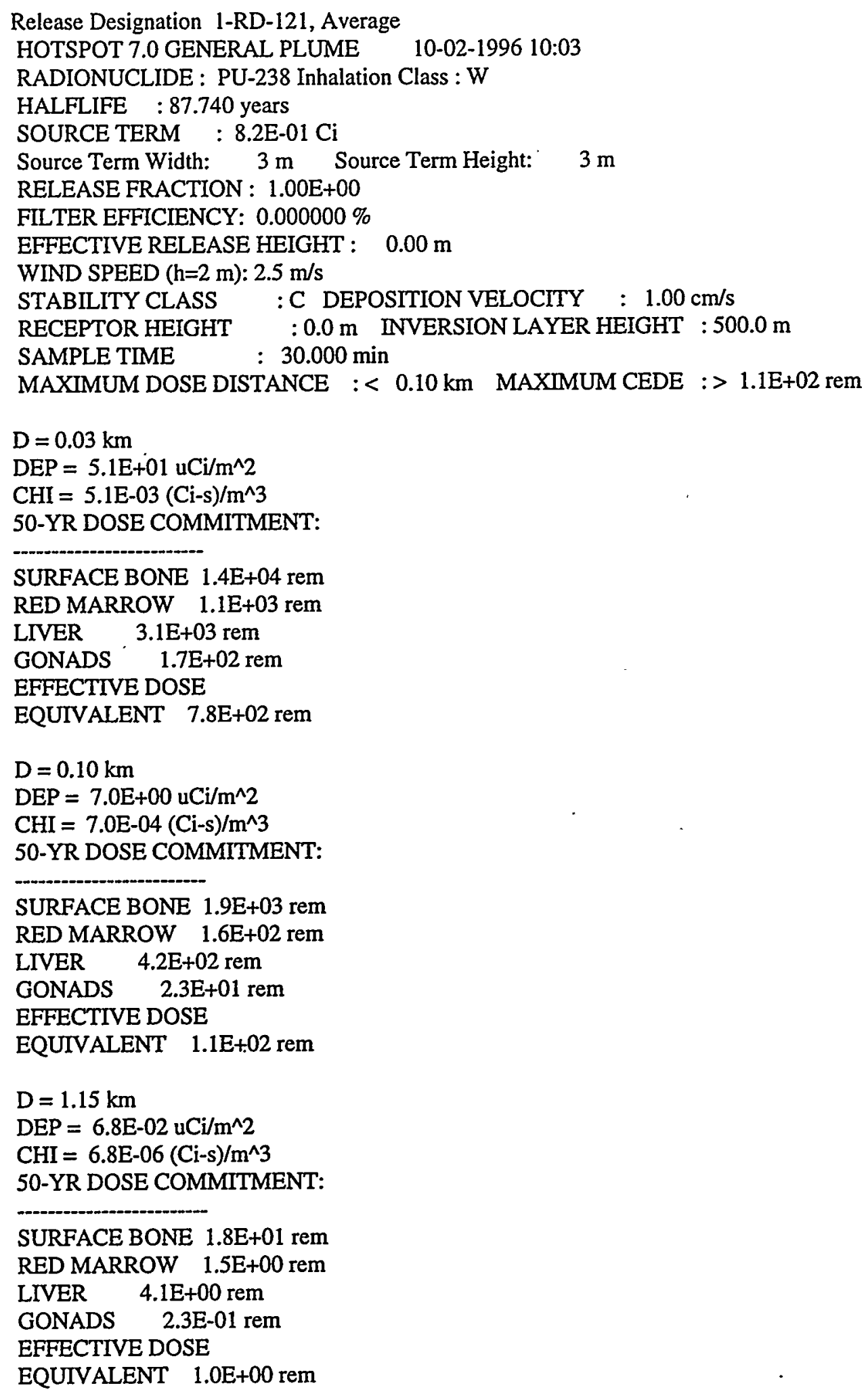


Title of Project EPHA for THM

Subject Radiological Consequence Assessment

Computer D. J. Richards
Reviewed by S. A. Henderson

Works Calculation 6

Sheet No. 118 of 166

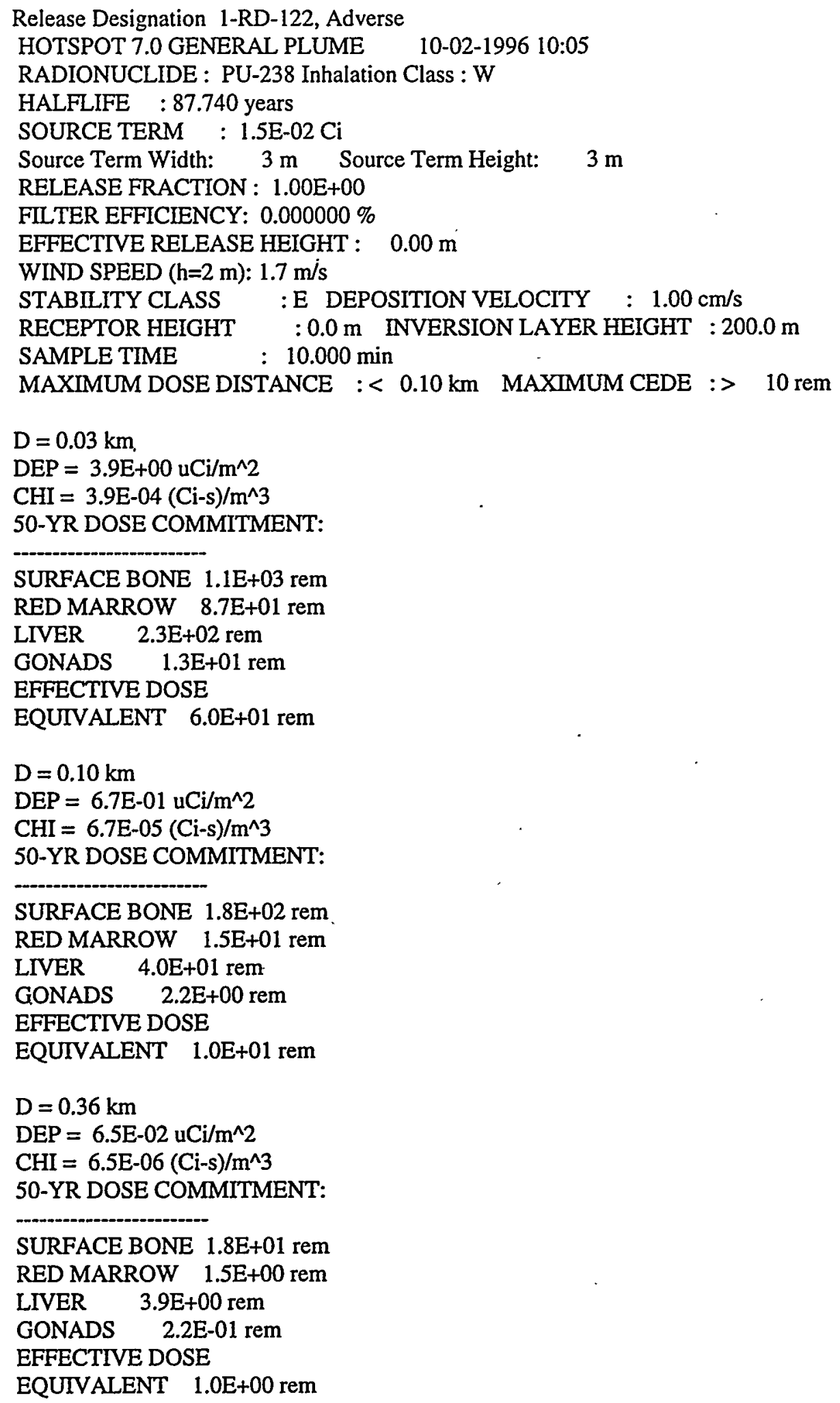


Title of Project EPHA for THM

Subject Radiological Consequence Assessment

Computer D. J. Richards

Date $\underline{09 / 11 / 96}$

Reviewed by $\underline{S}$. A. Henderson

Works Calculation 6

Sheet No. 119 of 166

Release Designation 1-RD-122, Average

HOTSPOT 7.0 GENERAL PLUME 10-02-1996 10:06

RADIONUCLIDE : PU-238 Inhalation Class : W

HALFLIFE : 87.740 years

SOURCE TERM : $1.5 \mathrm{E}-02 \mathrm{Ci}$

Source Term Width: $3 \mathrm{~m}$ Source Term Height: $3 \mathrm{~m}$

RELEASE FRACTION : $1.00 \mathrm{E}+00$

FILTER EFFICIENCY: $0.000000 \%$

EFFECTIVE RELEASE HEIGHT : $\quad 0.00 \mathrm{~m}$

WIND SPEED $(\mathrm{h}=2 \mathrm{~m}): 2.5 \mathrm{~m} / \mathrm{s}$

STABILITY CLASS $\quad: C$ DEPOSITION VELOCITY $: 1.00 \mathrm{~cm} / \mathrm{s}$

$\begin{array}{lcc}\text { RECEPTOR HEIGHT } & : 0.0 \mathrm{~m} \text { INVERSION LAYER HEIGHT }: 500.0 \mathrm{~m} \\ \text { SAMPLE TIME } & : 10.000 \mathrm{~min}\end{array}$

MAXIMUM DOSE DISTANCE $:<0.10 \mathrm{~km}$ MAXIMUM CEDE $:>2.4 \mathrm{rem}$

$\mathrm{D}=0.03 \mathrm{~km}$

$\mathrm{DEP}=1.1 \mathrm{E}+00 \mathrm{uCi} / \mathrm{m}^{\wedge} 2$

$\mathrm{CHI}=1.1 \mathrm{E}-04(\mathrm{Ci}-\mathrm{s}) / \mathrm{m}^{\wedge} 3$

50-YR DOSE COMMITMENT:

SURFACE BONE 3.1E+02 rem

RED MARROW 2.6E+01 rem

LIVER $6.9 \mathrm{E}+01 \mathrm{rem}$

GONADS $3.8 \mathrm{E}+00 \mathrm{rem}$

EFFECTIVE DOSE

EQUTVALENT 1.8E+01 rem

$\mathrm{D}=0.10 \mathrm{~km}$

$\mathrm{DEP}=1.6 \mathrm{E}-01 \mathrm{uCi} / \mathrm{m}^{\wedge} 2$

$\mathrm{CHI}=1.6 \mathrm{E}-05(\mathrm{Ci}-\mathrm{s}) / \mathrm{m}^{\wedge} 3$

50-YR DOSE COMMITMENT:

SURFACE BONE 4.2E+01 rem RED MARROW $3.5 \mathrm{E}+00 \mathrm{rem}$

LIVER $\quad 9.4 \mathrm{E}+00 \mathrm{rem}$

GONADS 5.2E-01 rem

EFFECTIVE DOSE

EQUTVALENT $2.4 \mathrm{E}+00 \mathrm{rem}$

$\mathrm{D}=0.16 \mathrm{~km}$

$\mathrm{DEP}=6.6 \mathrm{E}-02 \mathrm{uCi} / \mathrm{m}^{\wedge} 2$

$\mathrm{CHI}=6.6 \mathrm{E}-06(\mathrm{Ci}-\mathrm{s}) / \mathrm{m}^{\wedge} 3$

50-YR DOSE COMMITMENT:

SURFACE BONE $1.8 \mathrm{E}+01 \mathrm{rem}$

RED MARROW $1.5 \mathrm{E}+00 \mathrm{rem}$

LIVER $\quad 3.9 \mathrm{E}+00 \mathrm{rem}$

GONADS 2.2E-01 rem

EFFECTIVE DOSE

EQUIVALENT $1.0 \mathrm{E}+00 \mathrm{rem}$ 
Title of Project EPHA for THM

Subject Radiological Consequence Assessment

Computer D. J. Richards

Date 09/11/96

Reviewed by S. A. Henderson

Works Calculation 6

Sheet No. 120 of 166

Release Designation: 1-RD-123, Adverse

HOTSPOT 7.0 GENERAL PLUME 9-09-1996 22:12

RADIONUCLIDE : PU-238 Inhalation Class : W

HALFLIFE $: 87.740$ years

SOURCE TERM $: 8.6 \mathrm{E}-04 \mathrm{Ci}$

Source Term Width: $\quad 3 \mathrm{~m}$ Source Term Height: $3 \mathrm{~m}$

RELEASE FRACTION : $1.00 \mathrm{E}+00$

FILTER EFFICIENCY: $0.000000 \%$

EFFECTIVE RELEASE HEIGHT : $\quad 0.00 \mathrm{~m}$

WIND SPEED $(\mathrm{h}=2 \mathrm{~m}): 1.7 \mathrm{~m} / \mathrm{s}$

STABILITY CLASS :E DEPOSITION VELOCITY : $1.00 \mathrm{~cm} / \mathrm{s}$

RECEPTOR HEIGHT $\quad: 0.0 \mathrm{~m}$ INVERSION LAYER HEIGHT $: 200.0 \mathrm{~m}$

SAMPLE TIME $: 30.000 \mathrm{~min}$

MAXIMUM DOSE DISTANCE $:<0.10 \mathrm{~km}$ MAXIMUM CEDE $:>0.474 \mathrm{rem}$

$\mathrm{D}=0.03 \mathrm{~km}$

$\mathrm{DEP}=1.8 \mathrm{E}-01 \mathrm{uCi} / \mathrm{m}^{\wedge} 2$

$\mathrm{CHI}=1.8 \mathrm{E}-05(\mathrm{Ci}-\mathrm{s}) / \mathrm{m}^{\wedge} 3$

50-YR DOSE COMMITMENT:

SURFACE BONE 4.9E+01 rem RED MARROW $4.1 \mathrm{E}+00 \mathrm{rem}$ LIVER 1.1E+01 rem GONADS 6.0E-01 rem EFFECTIVE DOSE

EQUIVALENT $2.8 \mathrm{E}+00 \mathrm{rem}$
$\mathrm{D}=0.06 \mathrm{~km}$

$\mathrm{DEP}=6.8 \mathrm{E}-02 \mathrm{uCi} / \mathrm{m}^{\wedge} 2$

$\mathrm{CHI}=6.8 \mathrm{E}-06(\mathrm{Ci}-\mathrm{s}) / \mathrm{m}^{\wedge} 3$

50-YR DOSE COMMITMENT:

SURFACE BONE 1.8E+01 rem RED MARROW $1.5 \mathrm{E}+00 \mathrm{rem}$ LIVER $\quad 4.1 \mathrm{E}+00 \mathrm{rem}$ GONADS 2.3E-01 rem EFFECTIVE DOSE EQUTVALENT $1.0 \mathrm{E}+00 \mathrm{rem}$
$\mathrm{D}=0.10 \mathrm{~km}$

$\mathrm{DEP}=3.1 \mathrm{E}-02 \mathrm{uCi} / \mathrm{m}^{\wedge} 2$

$\mathrm{CHI}=3.1 \mathrm{E}-06(\mathrm{Ci}-\mathrm{s}) / \mathrm{m}^{\wedge} 3$

50-YR DOSE COMMITMENT:

SURFACE BONE 8.3E+00 rem RED MARROW 6.9E-01 rem LIVER $1.9 \mathrm{E}+00 \mathrm{rem}$ GONADS 1.0E-01 rem EFFECTIVE DOSE EQUIVALENT 4.7E-01 rem 
Title of Project EPHA for THM

Subject Radiological Consequence Assessment

Computer D. J. Richards

Date $\underline{09 / 11 / 96}$

Reviewed by $\underline{\text { S. A. Henderson }}$

Works Calculation 6

Sheet No. 121 of 166

Release Designation: 1-RD-123, Average

HOTSPOT 7.0 GENERAL PLUME 9-09-1996 22:12

RADIONUCLIDE : PU-238 Inhalation Class : W

HALFLIFE $: 87.740$ years

SOURCE TERM : $8.6 \mathrm{E}-04 \mathrm{Ci}$

Source Term Width: $\quad 3 \mathrm{~m}$ Source Term Height: $\quad 3 \mathrm{~m}$

RELEASE FRACTION : $1.00 \mathrm{E}+00$

FILTER EFFICIENCY: $0.000000 \%$

EFFECTIVE RELEASE HEIGHT : $\quad 0.00 \mathrm{~m}$

WIND SPEED $(\mathrm{h}=2 \mathrm{~m}): 2.5 \mathrm{~m} / \mathrm{s}$

STABILITY CLASS :C DEPOSITION VELOCITY $: 1.00 \mathrm{~cm} / \mathrm{s}$

RECEPTOR HEIGHT $\quad: 0.0 \mathrm{~m}$ INVERSION LAYER HEIGHT $: 500.0 \mathrm{~m}$

SAMPLE TIME $: 30.000 \mathrm{~min}$

MAXIMUM DOSE DISTANCE $:<0.10 \mathrm{~km}$ MAXIMUM CEDE $:>0.111 \mathrm{rem}$

$D=0.03 \mathrm{~km}$

$\mathrm{DEP}=5.3 \mathrm{E}-02 \mathrm{uCi} / \mathrm{m}^{\wedge} 2$

$\mathrm{CHI}=5.3 \mathrm{E}-06(\mathrm{Ci}-\mathrm{s}) / \mathrm{m}^{\wedge} 3$

50-YR DOSE COMMITMENT:

SURFACE BONE $1.4 \mathrm{E}+01 \mathrm{rem}$ RED MARROW $1.2 \mathrm{E}+00 \mathrm{rem}$ LIVER $\quad 3.2 \mathrm{E}+00 \mathrm{rem}$ GONADS 1.8E-01 rem EFFECTIVE DOSE

EQUIVALENT 8.1E-01 rem
$\mathrm{D}=0.10 \mathrm{~km}$

$\mathrm{DEP}=7.3 \mathrm{E}-03 \mathrm{uCi} / \mathrm{m}^{\wedge} 2$

$\mathrm{CHI}=7.3 \mathrm{E}-07(\mathrm{Ci}-\mathrm{s}) / \mathrm{m}^{\wedge} 3$

50-YR DOSE COMMTTMENT:

SURFACE BONE 2.0E+00 rem

RED MARROW 1.6E-01 rem

LIVER 4.4E-01 rem

GONADS 2.4E-02 rem

EFFECTIVE DOSE

EQUIVALENT 1.1E-01 rem 
Title of Project EPHA for THM

Subject Radiological Consequence Assessment

Computer D. J. Richards

Date $\underline{09 / 11 / 96}$

Reviewed by $\underline{\text { S. A. Henderson }}$

Works Calculation 6

Sheet No. 122 of 166

Release Designation: 1-RD-124, Adverse

HOTSPOT 7.0 GENERAL PLUME 9-11-1996 11:16

RADIONUCLIDE : PU-238 Inhalation Class : W

HALFLIFE : 87.740 years

SOURCE TERM : $1.0 \mathrm{E}-04 \mathrm{Ci}$

Source Term Width: $3 \mathrm{~m}$ Source Term Height: $3 \mathrm{~m}$

RELEASE FRACTION : $1.00 \mathrm{E}+00$

FILTER EFFICIENCY: $0.000000 \%$

EFFECTIVE RELEASE HEIGHT : $\quad 0.00 \mathrm{~m}$

WIND SPEED $(\mathrm{h}=2 \mathrm{~m}): 1.7 \mathrm{~m} / \mathrm{s}$

STABILITY CLASS :E DEPOSITION VELOCITY $: 1.00 \mathrm{~cm} / \mathrm{s}$

RECEPTOR HEIGHT $\quad: 0.0 \mathrm{~m}$ INVERSION LAYER HEIGHT $: 200.0 \mathrm{~m}$

SAMPLE TIME $\quad: 10.000 \mathrm{~min}$

MAXIMUM DOSE DISTANCE $:<0.10 \mathrm{~km}$ MAXIMUM CEDE $:>0.070 \mathrm{rem}$

$\mathrm{D}=0.03 \mathrm{~km}$

$\mathrm{DEP}=2.7 \mathrm{E}-02 \mathrm{uCi} / \mathrm{m}^{\wedge} 2$

$\mathrm{CHI}=2.7 \mathrm{E}-06(\mathrm{Ci}-\mathrm{s}) / \mathrm{m}^{\wedge} 3$

50-YR DOSE COMMTTMENT:

SURFACE BONE 7.2E+00 rem RED MARROW 5.9E-01 rem LIVER $\quad 1.6 \mathrm{E}+00 \mathrm{rem}$ GONADS 8.9E-02 rem EFFECTIVE DOSE

EQUIVALENT 4.1E-01 rem
$\mathrm{D}=0.10 \mathrm{~km}$
$\mathrm{DEP}=4.5 \mathrm{E}-03 \mathrm{uCi} / \mathrm{m}^{\wedge} 2$
$\mathrm{CHI}=4.5 \mathrm{E}-07(\mathrm{Ci}-\mathrm{s}) / \mathrm{m}^{\wedge} 3$
50-YR DOSE COMMITMENT:
SURFACE BONE $1.2 \mathrm{E}+00 \mathrm{rem}$
RED MARROW $1.0 \mathrm{E}-01 \mathrm{rem}$
LIVER 2.7E-01 rem
GONADS 1.5E-02 rem
EFFECTIVE DOSE
EQUIVALENT 7.0E-02 rem 
Title of Project EPHA for THM

Subject Radiological Consequence Assessment

Computer D. J. Richards

Date 09/11/96

Reviewed by S. A. Henderson

Works Calculation 6

Sheet No. 123 of 166

Release Designation: 1-RD-124, Average

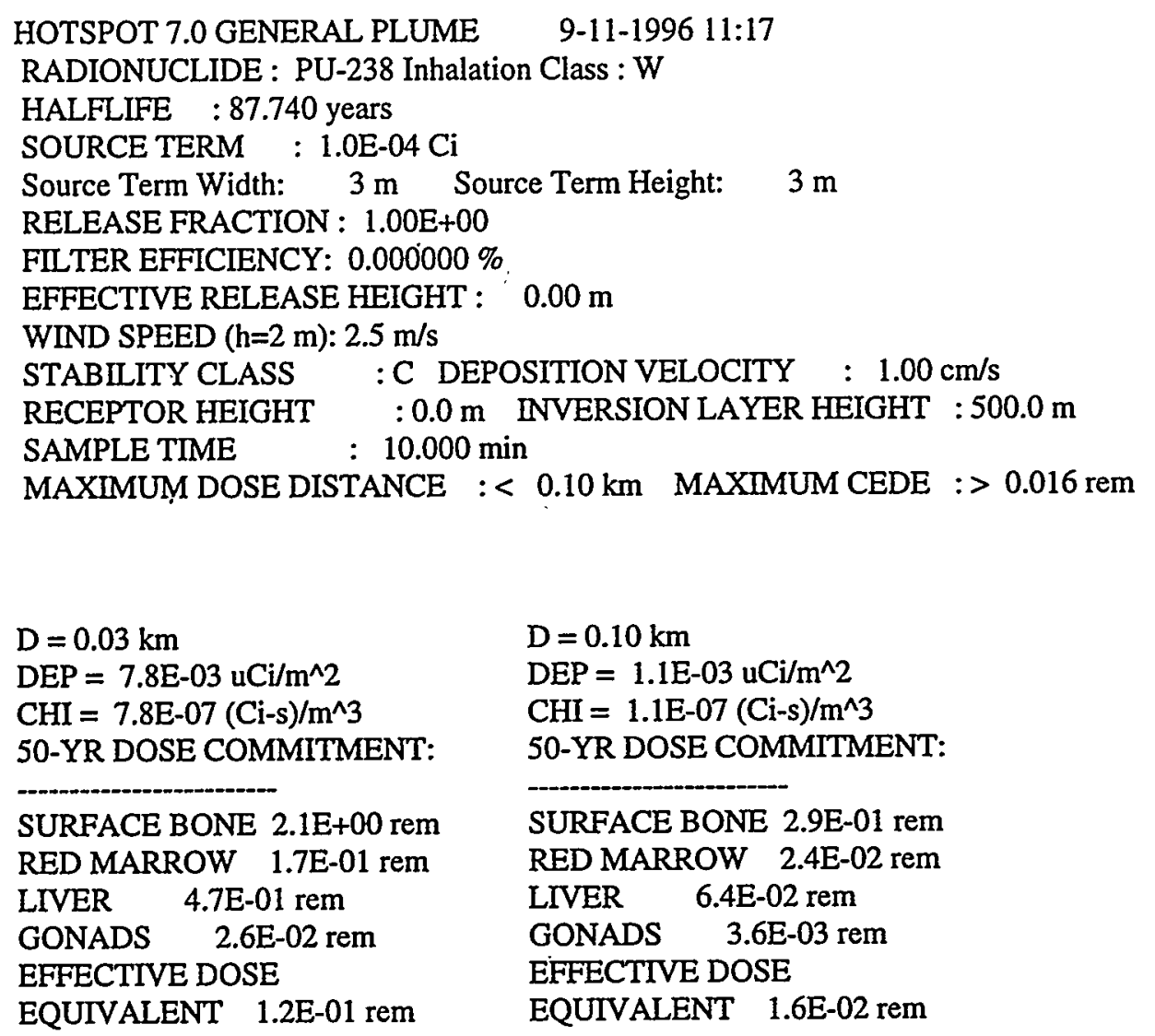


Títle of Project EPHA for THM

Subject Radiological Consequence Assessment

Computer D. J. Richards
Reviewed by S. A. Henderson

Works Calculation 6

Sheet No. 124 of 166

Release Designation: 1-RD-125, Adverse

HOTSPOT 7.0 GENERAL PLUME 9-09-1996 22:14

RADIONUCLIDE : PU-238 Inhalation Class : W

HALFLIFE $: 87.740$ years

SOURCE TERM $: 6.9 \mathrm{E}-03 \mathrm{Ci}$

Source Term Width: $\quad 3 \mathrm{~m}$ Source Term Height: $3 \mathrm{~m}$

RELEASE FRACTION : $1.00 \mathrm{E}+00$

FILTER EFFICIENCY: $0.000000 \%$

EFFECTIVE RELEASE HEIGHT : $\quad 0.00 \mathrm{~m}$

WIND SPEED $(\mathrm{h}=2 \mathrm{~m}): 1.7 \mathrm{~m} / \mathrm{s}$

STABILITY CLASS :E DEPOSITION VELOCITY : $1.00 \mathrm{~cm} / \mathrm{s}$

RECEPTOR HEIGHT $\quad: 0.0 \mathrm{~m}$ INVERSION LAYER HEIGHT $: 200.0 \mathrm{~m}$

SAMPLE TIME : $30.000 \mathrm{~min}$

MAXIMUM DOSE DISTANCE $:<0.10 \mathrm{~km}$ MAXIMUM CEDE $:>3.8 \mathrm{rem}$

$D=0.03 \mathrm{~km}$

$\mathrm{DEP}=1.5 \mathrm{E}+00 \mathrm{uCi} / \mathrm{m}^{\wedge} 2$

$\mathrm{CHI}=1.5 \mathrm{E}-04(\mathrm{Ci}-\mathrm{s}) / \mathrm{m}^{\wedge} 3$

50-YR DOSE COMMITMENT:

SURFACE BONE 4.0E+02 rem RED MARROW $3.3 \mathrm{E}+01 \mathrm{rem}$

LIVER 8.8E+01 rem GONADS $\quad 4.9 \mathrm{E}+00 \mathrm{rem}$ EFFECTIVE DOSE

EQUIVALENT $\quad 2.3 E+01$ rem

$\mathrm{D}=0.10 \mathrm{~km}$
$\mathrm{DEP}=2.5 \mathrm{E}-01 \mathrm{uCi} / \mathrm{m}^{\wedge} 2$
$\mathrm{CHI}=2.5 \mathrm{E}-05(\mathrm{Ci}-\mathrm{s}) / \mathrm{m}^{\wedge} 3$
$50-\mathrm{YR}$ DOSE COMMITMENT:
- SURFACE BONE $6.8 \mathrm{E}+01 \mathrm{rem}$
RED MARROW $5.6 \mathrm{E}+00 \mathrm{rem}$
LIVER $1.5 \mathrm{E}+01 \mathrm{rem}$
GONADS $8.3 \mathrm{E}-01 \mathrm{rem}$
EFFECTIVE DOSE
EQUIVALENT $3.8 \mathrm{E}+00 \mathrm{rem}$

$\mathrm{D}=0.21 \mathrm{~km}$ $\mathrm{DEP}=6.7 \mathrm{E}-02 \mathrm{uCi} / \mathrm{m}^{\wedge} 2$

$\mathrm{CHI}=6.7 \mathrm{E}-06(\mathrm{Ci}-\mathrm{s}) / \mathrm{m}^{\wedge} 3$

50-YR DOSE COMMITMENT:

SURFACE BONE $1.8 \mathrm{E}+01 \mathrm{rem}$ RED MARROW $1.5 \mathrm{E}+00 \mathrm{rem}$ LIVER $\quad 4.0 \mathrm{E}+00 \mathrm{rem}$ GONADS 2.2E-01 rem EFFECTIVE DOSE EQUIVALENT $1.0 \mathrm{E}+00 \mathrm{rem}$ 
Title of Project EPHA for THM

Subject Radiological Consequence Assessment

Computer D. J. Richards

Date $\underline{09 / 11 / 96}$

Reviewed by S.A. Henderson

Works Calculation 6

Sheet No. 125 of 166

Release Designation: 1-RD-125, Average

HOTSPOT 7.0 GENERAL PLUME 9-09-1996 22:15

RADIONUCLIDE : PU-238 Inhalation Class : W

HALFLIFE : 87.740 years

SOURCE TERM : 6.9E-03 Ci

Source Term Width: $3 \mathrm{~m}$ Source Term Height: $3 \mathrm{~m}$

RELEASE FRACTION : $1.00 \mathrm{E}+00$

FILTER EFFICIENCY: $0.000000 \%$

EFFECTIVE RELEASE HEIGHT : $\quad 0.00 \mathrm{~m}$

WIND SPEED $(\mathrm{h}=2 \mathrm{~m}): 2.5 \mathrm{~m} / \mathrm{s}$

STABILITY CLASS :C DEPOSITION VELOCITY $: 1.00 \mathrm{~cm} / \mathrm{s}$

RECEPTOR HEIGHT $: 0.0 \mathrm{~m}$ INVERSION LAYER HEIGHT $: 500.0 \mathrm{~m}$

SAMPLE TIME $\quad: 30.000 \mathrm{~min}$

MAXIMUM DOSE DISTANCE $:<0.10 \mathrm{~km}$ MAXIMUM CEDE $:>0.901 \mathrm{rem}$

$\mathrm{D}=0.03 \mathrm{~km}$

$\mathrm{DEP}=4.3 \mathrm{E}-01 \mathrm{uCi} / \mathrm{m}^{\wedge} 2$

$\mathrm{CHI}=4.3 \mathrm{E}-05(\mathrm{Ci}-\mathrm{s}) / \mathrm{m}^{\wedge} 3$

50-YR DOSE COMMITMENT:

SURFACE BONE $1.2 \mathrm{E}+02 \mathrm{rem}$ RED MARROW $9.6 \mathrm{E}+00 \mathrm{rem}$ LIVER 2.6E+01 rem GONADS 1.4E+00 rem EFFECTIVE DOSE EQUTVALENT $\quad 6.6 \mathrm{E}+00 \mathrm{rem}$

\author{
$\mathrm{D}=0.09 \mathrm{~km}$ \\ $\mathrm{DEP}=6.8 \mathrm{E}-02 \mathrm{uCi} / \mathrm{m}^{\wedge} 2$ \\ $\mathrm{CHI}=6.8 \mathrm{E}-06(\mathrm{Ci}-\mathrm{s}) / \mathrm{m}^{\wedge} 3$ \\ 50-YR DOSE COMMITMENT: \\ SURFACE BONE 1.8E+01 rem \\ RED MARROW $1.5 \mathrm{E}+00 \mathrm{rem}$ \\ LIVER 4.1E+00 rem \\ GONADS 2.3E-01 rem \\ EFFECTTVE DOSE \\ EQUTVALENT $\quad 1.0 \mathrm{E}+00 \mathrm{rem}$
}

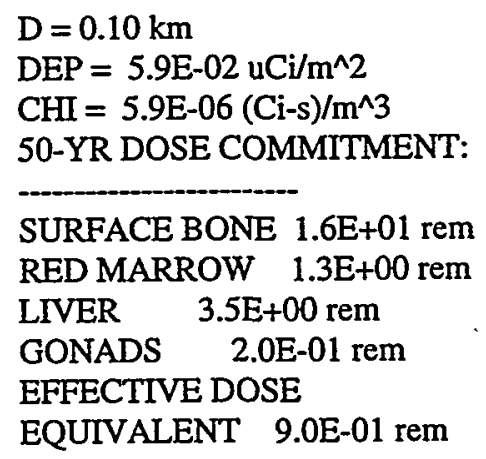


Title of Project EPHA for THM

Subject Radiological Consequence Assessment

Computer D. J. Richards
Reviewed by S. A. Henderson

Works Calculation 6

Sheet No. 126 of 166

Release Designation: 1-RD-126, Adverse

HOTSPOT 7.0 GENERAL PLUME 9-11-1996 17:41

RADIONUCLIDE : PU-238 Inhalation Class : W

HALFLIFE : 87.740 years

SOURCE TERM $\quad: 8.2 \mathrm{E}-04 \mathrm{Ci}$

Source Term Width: $3 \mathrm{~m}$ Source Term Height: $3 . \mathrm{m}$

RELEASE FRACTION : $1.00 \mathrm{E}+00$

FILTER EFFICIENCY: $0.000000 \%$

EFFECTIVE RELEASE HEIGHT : $\quad 0.00 \mathrm{~m}$

WIND SPEED $(\mathrm{h}=2 \mathrm{~m}): 1.7 \mathrm{~m} / \mathrm{s}$

STABILITY CLASS :E DEPOSITION VELOCITY $: 1.00 \mathrm{~cm} / \mathrm{s}$

RECEPTOR HEIGHT $\quad: 0.0 \mathrm{~m}$ INVERSION LAYER HEIGHT $: 200.0 \mathrm{~m}$

SAMPLE TIME $\quad: 10.000 \mathrm{~min}$

MAXIMUM DOSE DISTANCE $:<0.10 \mathrm{~km}$ MAXIMUM CEDE $:>0.564 \mathrm{rem}$

$D=0.03 \mathrm{~km}$

$\mathrm{DEP}=2.2 \mathrm{E}-01 \mathrm{uCi} / \mathrm{m}^{\wedge} 2$

$\mathrm{CHI}=2.2 \mathrm{E}-05(\mathrm{Ci}-\mathrm{s}) / \mathrm{m}^{\wedge} 3$

50-YR DOSE COMMITMENT:

SURFACE BONE 5.8E+01 rem RED MARROW $4.8 \mathrm{E}+00 \mathrm{rem}$

LIVER $1.3 \mathrm{E}+01 \mathrm{rem}$ GONADS 7.2E-01 rem EFFECTIVE DOSE EQUIVALENT $3.3 \mathrm{E}+00 \mathrm{rem}$

\author{
$\mathrm{D}=0.07 \mathrm{~km}$ \\ $\mathrm{DEP}=6.6 \mathrm{E}-02 \mathrm{uCi} / \mathrm{m}^{\wedge} 2$ \\ $\mathrm{CHI}=6.6 \mathrm{E}-06(\mathrm{Ci}-\mathrm{s}) / \mathrm{m}^{\wedge} 3$ \\ 50-YR DOSE COMMITMENT: \\ SURFACE BONE $1.8 \mathrm{E}+01 \mathrm{rem}$ \\ RED MARROW $1.5 \mathrm{E}+00 \mathrm{rem}$ \\ LIVER $3.9 \mathrm{E}+00 \mathrm{rem}$ \\ GONADS 2.2E-01 rem \\ EFFECTIVE DOSE \\ EQUIVALENT $1.0 \mathrm{E}+00 \mathrm{rem}$
}

\author{
$\mathrm{D}=0.10 \mathrm{~km}$ \\ $\mathrm{DEP}=3.7 \mathrm{E}-02 \mathrm{uCi} / \mathrm{m}^{\wedge} 2$ \\ $\mathrm{CHI}=3.7 \mathrm{E}-06(\mathrm{Ci}-\mathrm{s}) / \mathrm{m}^{\wedge} 3$ \\ 50-YR DOSE COMMITMENT: \\ SURFACE BONE $9.9 \mathrm{E}+00 \mathrm{rem}$ \\ RED MARROW 8.2E-01 rem \\ LIVER 2.2E+00 rem \\ GONADS 1.2E-01 rem \\ EFFECTIVE DOSE \\ EQUTVALENT 5.6E-01 rem
}


Title of Project EPHA for THM

Reviewed by $\mathrm{S}$. A. Henderson

Works Calculation 6

Subject Radiological Consequence Assessment

Computer D. J. Richards

Date $\underline{09 / 11 / 96}$

Release Designation: 1-RD-126, Average

HOTSPOT 7.0 GENERAL PLUME 9-11-1996 17:12

RADIONUCLIDE : PU-238 Inhalation Class : W

HALFLIFE $: 87.740$ years

SOURCE TERM $: 8.2 \mathrm{E}-04 \mathrm{Ci}$

Source Term Width: $3 \mathrm{~m}$ Source Term Height: $3 \mathrm{~m}$

RELEASE FRACTION : $1.00 \mathrm{E}+00$

FILTER EFFICIENCY: $0.000000 \%$

EFFECTIVE RELEASE HEIGHT : $\quad 0.00 \mathrm{~m}$

WIND SPEED ( $\mathrm{h}=2 \mathrm{~m}): 2.5 \mathrm{~m} / \mathrm{s}$

STABILITY CLASS :C DEPOSITION VELOCITY $: 1.00 \mathrm{~cm} / \mathrm{s}$

RECEPTOR HEIGHT $\quad: 0.0 \mathrm{~m}$ INVERSION LAYER HEIGHT $: 500.0 \mathrm{~m}$

SAMPLE TIME $\quad: 10.000 \mathrm{~min}$

MAXIMUM DOSE DISTANCE $:<0.10 \mathrm{~km}$ MAXIMUM CEDE $:>0.132 \mathrm{rem}$

$\mathrm{D}=0.03 \mathrm{~km}$

$\mathrm{DEP}=6.3 \mathrm{E}-02 \mathrm{uCi} / \mathrm{m}^{\wedge} 2$

$\mathrm{CHI}=6.3 \mathrm{E}-06(\mathrm{Ci}-\mathrm{s}) / \mathrm{m}^{\wedge} 3$

50-YR DOSE COMMITMENT:

SURFACE BONE $1.7 \mathrm{E}+01 \mathrm{rem}$ RED MARROW $1.4 \mathrm{E}+00 \mathrm{rem}$

LIVER $3.8 \mathrm{E}+00 \mathrm{rem}$

GONADS 2.1E-01 rem

EFFECTIVE DOSE

EQUIVALENT 9.7E-01 rem
$\mathrm{D}=0.10 \mathrm{~km}$

$\mathrm{DEP}=8.6 \mathrm{E}-03 \mathrm{uCi} / \mathrm{m}^{\wedge} 2$

$\mathrm{CHI}=8.6 \mathrm{E}-07(\mathrm{Ci}-\mathrm{s}) / \mathrm{m}^{\wedge} 3$

50-YR DOSE COMMTTMENT:

SURFACE BONE 2.3E+00 rem

RED MARROW 1.9E-01 rem

LIVER 5.2E-01 rem

GONADS 2.9E-02 rem

EFFECTIVE DOSE

EQUTVALENT 1.3E-01 rem 
Title of Project EPHA for THM

Subject Radiological Consequence Assessment

Computer D. J. Richards

Date $\underline{09 / 11 / 96}$

Reviewed by $\underline{\text { S. A. Henderson }}$

Works Calculation 6

Sheet No. 128 of 166

Release Designation: 1-RD-127, Adverse

HOTSPOT 7.0 GENERAL PLUME 9-09-1996 22:25

RADIONUCLIDE : PU-238 Inhalation Class : W

HALFLIFE $: 87.740$ years

SOURCE TERM : $5.5 \mathrm{E}-06 \mathrm{Ci}$

Source Term Width: $3 \mathrm{~m}$ Source Term Height: $3 \mathrm{~m}$

RELEASE FRACTION : $1.00 \mathrm{E}+00$

FILTER EFFICIENCY: $0.000000 \%$

EFFECTIVE RELEASE HEIGHT : $\quad 0.00 \mathrm{~m}$

WIND SPEED $(\mathrm{h}=2 \mathrm{~m}): 1.7 \mathrm{~m} / \mathrm{s}$

STABILITY CLASS :E DEPOSITION VELOCITY : $1.00 \mathrm{~cm} / \mathrm{s}$

RECEPTOR HEIGHT $: 0.0 \mathrm{~m}$ INVERSION LAYER HEIGHT $: 200.0 \mathrm{~m}$

SAMPLE TIME $\quad: 30.000 \mathrm{~min}$

MAXIMUM DOSE DISTANCE $:<0.10 \mathrm{~km}$ MAXIMUM CEDE $:>3.0 \mathrm{E}-03 \mathrm{rem}$

$\mathrm{D}=0.03 \mathrm{~km}$

$\mathrm{DEP}=1.2 \mathrm{E}-03 \mathrm{uCi} / \mathrm{m}^{\wedge} 2$

$\mathrm{CHI}=1.2 \mathrm{E}-07(\mathrm{Ci}-\mathrm{s}) / \mathrm{m}^{\wedge} 3$

50-YR DOSE COMMITMENT:

SURFACE BONE 3.1E-01 rem RED MARROW 2.6E-02 rem LIVER 7.0E-02 rem GONADS 3.9E-03 rem EFFECTIVE DOSE

EQUIVALENT $1.8 \mathrm{E}-02 \mathrm{rem}$
$\mathrm{D}=0.10 \mathrm{~km}$

$\mathrm{DEP}=2.0 \mathrm{E}-04 \mathrm{uCi} / \mathrm{m}^{\wedge} 2$

$\mathrm{CHI}=2.0 \mathrm{E}-08(\mathrm{Ci}-\mathrm{s}) / \mathrm{m}^{\wedge} 3$

50-YR DOSE COMMITMENT:

SURFACE BONE 5.4E-02 rem RED MARROW 4.4E-03 rem

LIVER $1.2 \mathrm{E}-02 \mathrm{rem}$

GONADS 6.6E-04 rem

EFFECTIVE DOSE

EQUIVALENT 3.0E-03 rem 
Title of Project EPHA for THM

Subject Radiological Consequence Assessment

Computer D. J. Richards

Date $\underline{09 / 11 / 96}$

Reviewed by S. A. Henderson

Works Calculation 6

Sheet No. 129 of 166

Release Designation: 1-RD-127, Average

HOTSPOT 7.0 GENERAL PLUME 9-09-1996 22:26

RADIONUCLIDE : PU-238 Inhalation Class : W

HALFLIFE $: 87.740$ years

SOURCE TERM $: 5.5 \mathrm{E}-06 \mathrm{Ci}$

Source Term Width: $\quad 3 \mathrm{~m}$ Source Term Height: $3 \mathrm{~m}$

RELEASE FRACTION : $1.00 \mathrm{E}+00$

FILTER EFFICIENCY: $0.000000 \%$

EFFECTIVE RELEASE HEIGHT : $\quad 0.00 \mathrm{~m}$

WIND SPEED $(\mathrm{h}=2 \mathrm{~m}): 2.5 \mathrm{~m} / \mathrm{s}$

STABILITY CLASS :C DEPOSITION VELOCITY $: 1.00 \mathrm{~cm} / \mathrm{s}$

RECEPTOR HEIGHT $\quad: 0.0 \mathrm{~m}$ INVERSION LAYER HEIGHT $: 500.0 \mathrm{~m}$

SAMPLE TIME $\quad: 30.000 \mathrm{~min}$

MAXIMUM DOSE DISTANCE $:<0.10 \mathrm{~km}$ MAXIMUM CEDE $:>7.2 \mathrm{E}-04 \mathrm{rem}$

$\mathrm{D}=0.03 \mathrm{~km}$

$\mathrm{D}=0.10 \mathrm{~km}$

$\mathrm{DEP}=3.4 \mathrm{E}-04 \mathrm{uCi} / \mathrm{m}^{\wedge} 2$

$\mathrm{DEP}=4.7 \mathrm{E}-05 \mathrm{uCi} / \mathrm{m}^{\wedge} 2$

$\mathrm{CHI}=3.4 \mathrm{E}-08(\mathrm{Ci}-\mathrm{s}) / \mathrm{m}^{\wedge} 3$

$\mathrm{CHI}=4.7 \mathrm{E}-09(\mathrm{Ci}-\mathrm{s}) / \mathrm{m}^{\wedge} 3$

50-YR DOSE COMMITMENT:

50-YR DOSE COMMITMENT:

SURFACE BONE 9.2E-02 rem

SURFACE BONE 1.3E-02 rem

RED MARROW 7.6E-03 rem

LIVER 2.1E-02 rem

GONADS 1.1E-03 rem

EFFECTIVE DOSE

RED MARROW 1.0E-03 rem

LIVER $2.8 \mathrm{E}-03 \mathrm{rem}$

GONADS 1.6E-04 rem

EQUTVALENT 5.2E-03 rem

EFFECTIVE DOSE

EQUIVALENT 7.2E-04 rem 
Title of Project EPHA for THM

Subject Radiological Consequence Assessment

Computer D. J. Richards

Date $\underline{09 / 11 / 96}$

Reviewed by $\underline{\text { S. A. Henderson }}$

Works Calculation 6

Sheet No. 130 of 166

Release Designation: 1-RD-128, Adverse

HOTSPOT 7.0 GENERAL PLUME 9-11-1996 11:26

RADIONUCLIDE : PU-238 Inhalation Class : W

HALFLIFE : 87.740 years

SOURCE TERM : $6.5 \mathrm{E}-07 \mathrm{Ci}$

Source Term Width: $3 \mathrm{~m}$ Source Term Height: $3 \mathrm{~m}$

RELEASE FRACTION : $1.00 \mathrm{E}+00$

FILTER EFFICIENCY: $0.000000 \%$

EFFECTIVE RELEASE HEIGHT : $\quad 0.00 \mathrm{~m}$

WIND SPEED $(\mathrm{h}=2 \mathrm{~m}): 1.7 \mathrm{~m} / \mathrm{s}$

STABILITY CLASS :E DEPOSITION VELOCITY $\quad: 1.00 \mathrm{~cm} / \mathrm{s}$

RECEPTOR HEIGHT $\quad: 0.0 \mathrm{~m}$ INVERSION LAYER HEIGHT $: 200.0 \mathrm{~m}$

SAMPLE TIME $\quad: 10.000 \mathrm{~min}$

MAXIMUM DOSE DISTANCE $:<0.10 \mathrm{~km}$ MAXIMUM CEDE $:>4.5 \mathrm{E}-04 \mathrm{rem}$

$\mathrm{D}=0.03 \mathrm{~km}$

$\mathrm{DEP}=1.7 \mathrm{E}-04 \mathrm{uCi} / \mathrm{m}^{\wedge} 2$

$\mathrm{CHI}=1.7 \mathrm{E}-08(\mathrm{Ci}-\mathrm{s}) / \mathrm{m}^{\wedge} 3$

50-YR DOSE COMMITMENT:

SURFACE BONE $4.6 \mathrm{E}-02 \mathrm{rem}$ RED MARROW 3.8E-03 rem

LIVER $\quad 1.0 \mathrm{E}-02$ rem GONADS 5.7E-04 rem EFFECTIVE DOSE

EQUIVALENT 2.6E-03 rem
$\mathrm{D}=0.10 \mathrm{~km}$

$\mathrm{DEP}=2.9 \mathrm{E}-05 \mathrm{uCi} / \mathrm{m}^{\wedge} 2$

$\mathrm{CHI}=2.9 \mathrm{E}-09(\mathrm{Ci}-\mathrm{s}) / \mathrm{m}^{\wedge} 3$

50-YR DOSE COMMITMENT:

SURFACE BONE 7.9E-03 rem RED MARROW $6.5 \mathrm{E}-04 \mathrm{rem}$

LIVER $1.8 \mathrm{E}-03 \mathrm{rem}$

GONADS 9.7E-05 rem

EFFECTIVE DOSE

EQUIVALENT 4.5E-04 rem 
Title of Project EPHA for THM

Reviewed by S. A. Henderson

Subject Radiological Consequence Assessment

Sheet No. 131 of 166

Computer D. J. Richards

Date 09/11/96

Release Designation: 1-RD-128, Average

HOTSPOT 7.0 GENERAL PLUME 9-11-1996 11:26

RADIONUCLIDE : PU-238 Inhalation Class : W

HALFLIFE : 87.740 years

SOURCE TERM : $6.5 \mathrm{E}-07 \mathrm{Ci}$

Source Term Width: $\quad 3 \mathrm{~m}$ Source Term Height: $3 \mathrm{~m}$

RELEASE FRACTION : $1.00 \mathrm{E}+00$

FILTER EFFICIENCY: $0.000000 \%$

EFFECTIVE RELEASE HEIGHT : $0.00 \mathrm{~m}$

WIND SPEED $(\mathrm{h}=2 \mathrm{~m}): 2.5 \mathrm{~m} / \mathrm{s}$

STABILITY CLASS :C DEPOSITION VELOCITY : $1.00 \mathrm{~cm} / \mathrm{s}$

RECEPTOR HEIGHT $\quad: 0.0 \mathrm{~m}$ INVERSION LAYER HEIGHT $: 500.0 \mathrm{~m}$

SAMPLE TIME $\quad: 10.000 \mathrm{~min}$

MAXIMUM DOSE DISTANCE $:<0.10 \mathrm{~km}$ MAXIMUM CEDE $:>1.1 \mathrm{E}-04 \mathrm{rem}$

$\mathrm{D}=0.03 \mathrm{~km}$

$\mathrm{DEP}=5.0 \mathrm{E}-05 \mathrm{uCi} / \mathrm{m}^{\wedge} 2$

$\mathrm{CHI}=5.0 \mathrm{E}-09(\mathrm{Ci}-\mathrm{s}) / \mathrm{m}^{\wedge} 3$

50-YR DOSE COMMITMENT:

SURFACE BONE 1.4E-02 rem RED MARROW 1.1E-03 rem LIVER 3.0E-03 rem GONADS 1.7E-04 rem EFFECTIYE DOSE EQUIVALENT 7.7E-04 rem
$\mathrm{D}=0.10 \mathrm{~km}$

$\mathrm{DEP}=6.9 \mathrm{E}-06 \mathrm{uCi} / \mathrm{m}^{\wedge} 2$

$\mathrm{CHI}=6.9 \mathrm{E}-10(\mathrm{Ci}-\mathrm{s}) / \mathrm{m}^{\wedge} 3$

50-YR DOSE COMMITMENT:

SURFACE BONE 1.9E-03 rem

RED MARROW 1.5E-04 rem

LIVER 4.1E-04 rem

GONADS 2.3E-05 rem

EFFECTIVE DOSE

EQUIVALENT 1.1E-04 rem 
Title of Project EPHA for THM

Subject Radiological Consequence Assessment

Computer D. J. Richards
Reviewed by S. A. Henderson Works Calculation 6

- Sheet No. 132 of 166

Release Designation: 1-RD-129, Adverse

HOTSPOT 7.0 GENERAL PLUME 9-09-1996 22:27

RADIONUCLIDE : PU-238 Inhalation Class : W

HALFLIFE : 87.740 years

SOURCE TERM : $1.1 \mathrm{E}-06 \mathrm{Ci}$

Source Term Width: $3 \mathrm{~m}$ Source Term Height: $3 \mathrm{~m}$

RELEASE FRACTION : $1.00 \mathrm{E}+00$

FILTER EFFICIENCY: $0.000000 \%$

EFFECTIVE RELEASE HEIGHT : $\quad 0.00 \mathrm{~m}$

WIND SPEED $(\mathrm{h}=2 \mathrm{~m}): 1.7 \mathrm{~m} / \mathrm{s}$

STABILITY CLASS :E DEPOSITION VELOCITY $\quad: 1.00 \mathrm{~cm} / \mathrm{s}$

RECEPTOR HEIGHT $\quad: 0.0 \mathrm{~m}$ INVERSION LAYER HEIGHT $: 200.0 \mathrm{~m}$

SAMPLE TIME $\quad: 30.000 \mathrm{~min}$

MAXIMUM DOSE DISTANCE $:<0.10 \mathrm{~km}$ MAXIMUM CEDE $:>6.1 \mathrm{e}-04 \mathrm{rem}$

$\mathrm{D}=0.03 \mathrm{~km}$

$\mathrm{DEP}=2.3 \mathrm{E}-04 \mathrm{uCi} / \mathrm{m}^{\wedge} 2$

$\mathrm{CHI}=2.3 \mathrm{E}-08(\mathrm{Ci}-\mathrm{s}) / \mathrm{m}^{\wedge} 3$

50-YR DOSE COMMITMENT:

SURFACE BONE 6.3E-02 rem RED MARROW $5.2 \mathrm{E}-03 \mathrm{rem}$

LIVER 1.4E-02 rem GONADS 7.8E-04 rem EFFECTIVE DOSE

EQUIVALENT 3.6E-03 rem

\author{
$\mathrm{D}=0.10 \mathrm{~km}$ \\ $\mathrm{DEP}=4.0 \mathrm{E}-05 \mathrm{uCi} / \mathrm{m}^{\wedge} 2$ \\ $\mathrm{CHI}=4.0 \mathrm{E}-09(\mathrm{Ci}-\mathrm{s}) / \mathrm{m}^{\wedge} 3$ \\ 50-YR DOSE COMMITMENT: \\ SURFACE BONE 1.1E-02 rem \\ RED MARROW $8.9 \mathrm{E}-04 \mathrm{rem}$ \\ LIVER 2.4E-03 rem \\ GONADS 1.3E-04 rem \\ EFFECTIVE DOSE \\ EQUIVALENT 6.1E-04 rem
}


OSR 25-10 (Rev 6-18.92)

Title of Project EPHA for THM

Reviewed by S. A. Henderson

Subject Radiological Consequence Assessment

Sheet No. 133 of 166

Release Designation: 1-RD-129, Average

HOTSPOT 7.0 GENERAL PLUME

9-09-1996 22:28

RADIONUCLIDE : PU-238 Inhalation Class : W

HALFLIFE $: 87.740$ years

SOURCE TERM : $1.1 \mathrm{E}-06 \mathrm{Ci}$

Source Term Width: $3 \mathrm{~m}$ Source Term Height: $3 \mathrm{~m}$

RELEASE FRACTION : $1.00 \mathrm{E}+00$

FILTER EFFICIENCY: $0.000000 \%$

EFFECTIVE RELEASE HEIGHT : $\quad 0.00 \mathrm{~m}$

WIND SPEED $(\mathrm{h}=2 \mathrm{~m}): 2.5 \mathrm{~m} / \mathrm{s}$

STABILITY CLASS $\quad$ :C DEPOSITION VELOCITY $\quad: 1.00 \mathrm{~cm} / \mathrm{s}$

RECEPTOR HEIGHT $\quad: 0.0 \mathrm{~m}$ INVERSION LAYER HEIGHT $: 500.0 \mathrm{~m}$

SAMPLE TIME $\quad: 30.000 \mathrm{~min}$

MAXIMUM DOSE DISTANCE $:<0.10 \mathrm{~km}$ MAXIMUM CEDE $:>1.4 \mathrm{E}-04 \mathrm{rem}$

$\mathrm{D}=0.03 \mathrm{~km}$

$\mathrm{DEP}=6.8 \mathrm{E}-05 \mathrm{uCi} / \mathrm{m}^{\wedge} 2$

$D=0.10 \mathrm{~km}$

$\mathrm{CHI}=6.8 \mathrm{E}-09(\mathrm{Ci}-\mathrm{s}) / \mathrm{m}^{\wedge} 3$

$\mathrm{DEP}=9.3 \mathrm{E}-06 \mathrm{uCi} / \mathrm{m}^{\wedge} 2$

$\mathrm{CHI}=9.3 \mathrm{E}-10(\mathrm{Ci}-\mathrm{s}) / \mathrm{m}^{\wedge} 3$

50-YR DOSE COMMTTMENT:

50-YR DOSE COMMITMENT:

SURFACE BONE 1.8E-02 rem

SURFACE BONE 2.5E-03 rem

RED MARROW $1.5 \mathrm{E}-03 \mathrm{rem}$

LIVER 4.1E-03 rem

GONADS 2.3E-04 rem

RED MARROW 2.1E-04 rem

LIVER 5.6E-04 rem

GONADS 3.1E-05 rem

EFFECTIVE DOSE

EFFECTIVE DOSE

EQUTVALENT $1.0 \mathrm{E}-03 \mathrm{rem}$

EQUIVALENT 1.4E-04 rem 
Title of Project EPHA for THM

Reviewed by $\underline{S}$. A. Henderson

Subject Radiological Consequence Assessment

Works Calculation 6

Computer D. J. Richards

Date 09/11/96

Sheet No. 134 of 166

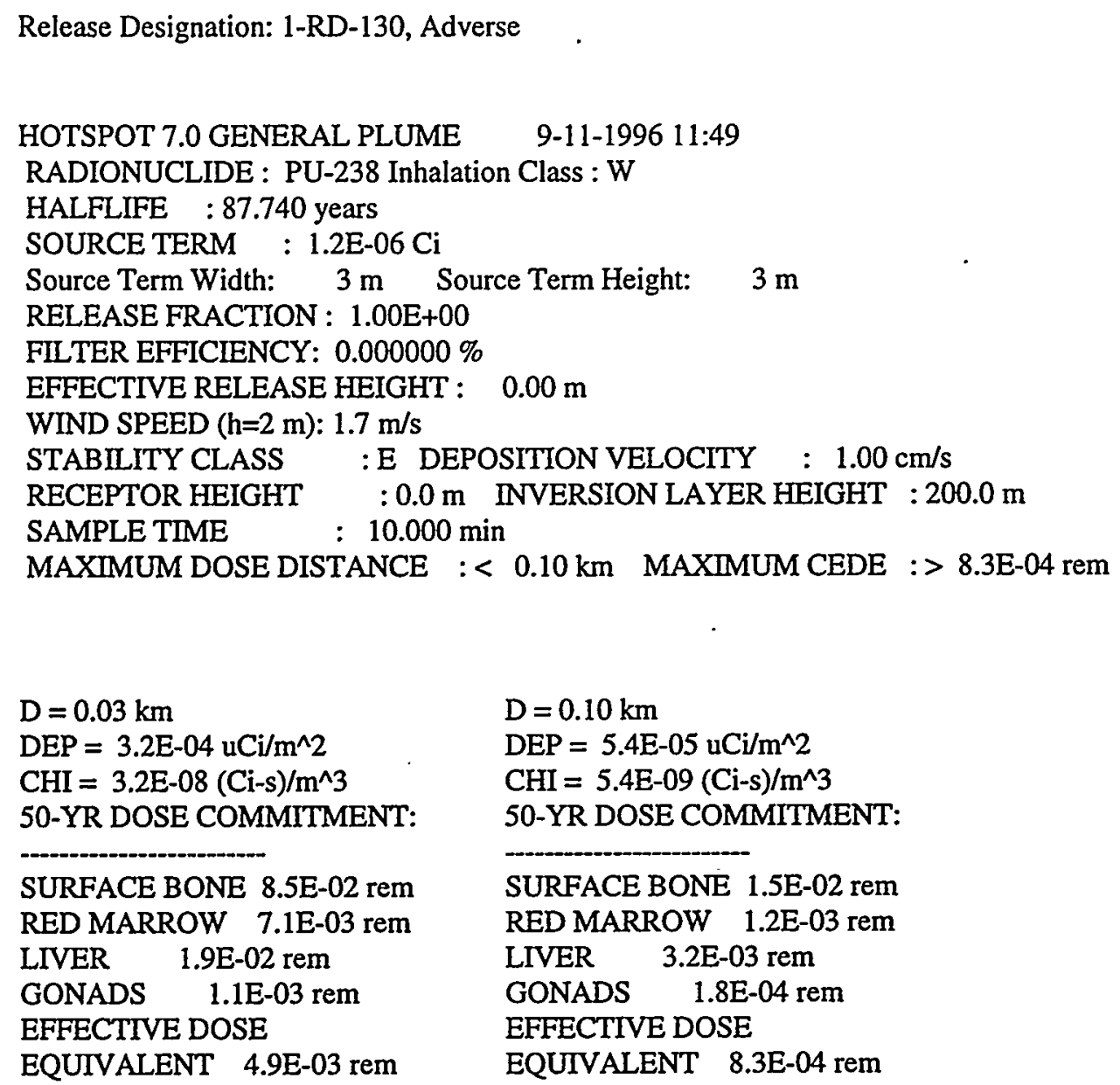

Release Designation: 1-RD-130, Adverse

HOTSPOT 7.0 GENERAL PLUME 9-11-1996 11:49

RADIONUCLIDE : PU-238 Inhalation Class : W

HALFLIFE $: 87.740$ years

SOURCE TERM : $1.2 \mathrm{E}-06 \mathrm{Ci}$

Source Term Width: $\quad 3 \mathrm{~m}$ Source Term Height: $3 \mathrm{~m}$

RELEASE FRACTION : $1.00 \mathrm{E}+00$

FILTER EFFICIENCY: $0.000000 \%$

EFFECTIVE RELEASE HEIGHT : $\quad 0.00 \mathrm{~m}$

WIND SPEED $(\mathrm{h}=2 \mathrm{~m}): 1.7 \mathrm{~m} / \mathrm{s}$

STABILITY CLASS :E DEPOSITION VELOCITY $\quad: 1.00 \mathrm{~cm} / \mathrm{s}$

RECEPTOR HEIGHT $\quad: 0.0 \mathrm{~m}$ INVERSION LAYER HEIGHT $: 200.0 \mathrm{~m}$

SAMPLE TIME $\quad: 10.000 \mathrm{~min}$

MAXIMUM DOSE DISTANCE $:<0.10 \mathrm{~km}$ MAXIMUM CEDE $:>8.3 \mathrm{E}-04 \mathrm{rem}$

$\mathrm{D}=0.03 \mathrm{~km}$

$\mathrm{DEP}=3.2 \mathrm{E}-04 \mathrm{uCi} / \mathrm{m}^{\wedge} 2$

$\mathrm{CHI}=3.2 \mathrm{E}-08(\mathrm{Ci}-\mathrm{s}) / \mathrm{m}^{\wedge} 3$

50-YR DOSE COMMITMENT:

SURFACE BONE 8.5E-02 rem RED MARROW 7.1E-03 rem LIVER 1.9E-02 rem GONADS 1.1E-03 rem EFFECTIVE DOSE EQUIVALENT $4.9 \mathrm{E}-03 \mathrm{rem}$

$$
\begin{aligned}
& \mathrm{D}=0.10 \mathrm{~km} \\
& \mathrm{DEP}=5.4 \mathrm{E}-05 \mathrm{uCi} / \mathrm{m}^{\wedge} 2 \\
& \mathrm{CHI}=5.4 \mathrm{E}-09(\mathrm{Ci}-\mathrm{s}) / \mathrm{m}^{\wedge} 3 \\
& \text { 50-YR DOSE COMMITMENT: }
\end{aligned}
$$

SURFACE BONE 1.5E-02 rem RED MARROW 1.2E-03 rem LIVER 3.2E-03 rem GONADS 1.8E-04 rem EFFECTIVE DOSE EQUIVALENT $\quad 8.3 \mathrm{E}-04 \mathrm{rem}$ 
Title of Project EPHA for THM

Subject Radiological Consequence Assessment

Computer D. J. Richards

Date $09 / 11 / 96$

Reviewed by $\underline{S}$. A. Henderson

Works Calculation 6

Sheet No. 135 of 166

Release Designation: 1-RD-130, Average

HOTSPOT 7.0 GENERAL PLUME 9-11-1996 12:13

RADIONUCLIDE : PU-238 Inhalation Class : W

HALFLIFE : 87.740 years

SOURCE TERM : $1.2 \mathrm{E}-06 \mathrm{Ci}$

Source Term Width: $3 \mathrm{~m}$ Source Term Height: $3 \mathrm{~m}$

RELEASE FRACTION : $1.00 \mathrm{E}+00$

FILTER EFFICIENCY: $0.000000 \%$

EFFECTIVE RELEASE HEIGHT : $\quad 0.00 \mathrm{~m}$

WIND SPEED ( $\mathrm{h}=2 \mathrm{~m}): 2.5 \mathrm{~m} / \mathrm{s}$

STABILITY CLASS :C DEPOSITION VELOCITY $\quad: 1.00 \mathrm{~cm} / \mathrm{s}$

RECEPTOR HEIGHT $\quad: 0.0 \mathrm{~m}$ INVERSION LAYER HEIGHT $: 500.0 \mathrm{~m}$

SAMPLE TIME $\quad: 10.000 \mathrm{~min}$

MAXIMUM DOSE DISTANCE $:<0.10 \mathrm{~km}$ MAXIMUM CEDE $:>1.9 \mathrm{E}-04 \mathrm{rem}$

$\mathrm{D}=0.03 \mathrm{~km}$

$\mathrm{DEP}=9.3 \mathrm{E}-05 \mathrm{uCi} / \mathrm{m}^{\wedge} 2$

$\mathrm{CHI}=9.3 \mathrm{E}-09(\mathrm{Ci}-\mathrm{s}) / \mathrm{m}^{\wedge} 3$

50-YR DOSE COMMITMENT:

SURFACE BONE 2.5E-02 rem RED MARROW 2.1E-03 rem LIVER 5.6E-03 rem GONADS 3.1E-04 rem EFFECTIVE DOSE

EQUTVALENT 1.4E-03 rem

\author{
$\mathrm{D}=0.10 \mathrm{~km}$ \\ $\mathrm{DEP}=1.3 \mathrm{E}-05 \mathrm{uCi} / \mathrm{m}^{\wedge} 2$ \\ $\mathrm{CHI}=1.3 \mathrm{E}-09(\mathrm{Ci}-\mathrm{s}) / \mathrm{m}^{\wedge} 3$ \\ 50-YR DOSE COMMITMENT: \\ SURFACE BONE 3.4E-03 rem \\ RED MARROW 2.8E-04 rem \\ LIVER 7.6E-04 rem \\ GONADS 4.2E-05 rem \\ EFFECTIVE DOSE \\ EQUIVALENT 1.9E-04 rem
}


Title of Project EPHA for THM

Subject Radiological Consequence Assessment

Computer D. J. Richards
Date $\underline{09 / 11 / 96}$
Reviewed by S. A. Henderson

Works Calculation 6

Sheet No. 136 of 166

Release Designation: 1-RD-131, Adverse

HOTSPOT 7.0 GENERAL PLUME 9-09-1996 22:30

RADIONUCLIDE : PU-238 Inhalation Class : W

HALFLIFE : 87.740 years

SOURCE TERM : $1.2 \mathrm{E}-04 \mathrm{Ci}$

Source Term Width: $\quad 3 \mathrm{~m}$ Source Term Height: $\quad 3 \mathrm{~m}$

RELEASE FRACTION : $1.00 \mathrm{E}+00$

FILTER EFFICIENCY: $0.000000 \%$

EFFECTIVE RELEASE HEIGHT : $\quad 0.00 \mathrm{~m}$

WIND SPEED $(\mathrm{h}=2 \mathrm{~m}): 1.7 \mathrm{~m} / \mathrm{s}$

STABILITY CLASS :E DEPOSITION VELOCITY $: 1.00 \mathrm{~cm} / \mathrm{s}$

RECEPTOR HEIGHT $: 0.0 \mathrm{~m}$ INVERSION LAYER HEIGHT $: 200.0 \mathrm{~m}$

SAMPLE TIME $\quad: 30.000 \mathrm{~min}$

MAXIMUM DOSE DISTANCE $:<0.10 \mathrm{~km}$ MAXIMUM CEDE $:>0.065 \mathrm{rem}$

$\mathrm{D}=0.03 \mathrm{~km}$

$\mathrm{DEP}=2.5 \mathrm{E}-02 \mathrm{uCi} / \mathrm{m}^{\wedge} 2$

$\mathrm{CHI}=2.5 \mathrm{E}-06(\mathrm{Ci}-\mathrm{s}) / \mathrm{m}^{\wedge} 3$

50-YR DOSE COMMITMENT:

SURFACE BONE 6.7E+00 rem

RED MARROW 5.6E-01 rem

LIVER $\quad 1.5 \mathrm{E}+00 \mathrm{rem}$

GONADS 8.3E-02 rem

EFFECTIVE DOSE

EQUTVALENT 3.8E-01 rem
$\mathrm{D}=0.10 \mathrm{~km}$

$\mathrm{DEP}=4.3 \mathrm{E}-03 \mathrm{uCi} / \mathrm{m}^{\wedge} 2$

$\mathrm{CHI}=4.3 \mathrm{E}-07(\mathrm{Ci}-\mathrm{s}) / \mathrm{m}^{\wedge} 3$

50-YR DOSE COMMITMENT:

SURFACE BONE $1.1 \mathrm{E}+00 \mathrm{rem}$

RED MARROW 9.5E-02 rem

LIVER 2.6E-01 rem

GONADS 1.4E-02 rem

EFFECTIVE DOSE

EQUIVALENT 6.5E-02 rem 
Title of Project EPHA for THM

Subject Radiological Consequence Assessment

Computer D. J. Richards

Date $\underline{09 / 11 / 96}$

Reviewed by S. A. Henderson

Works Calculation 6

Sheet No. 137 of 166

Release Designation: 1-RD-131, Average

HOTSPOT 7.0 GENERAL PLUME 9-09-1996 22:31

RADIONUCLIDE : PU-238 Inhalation Class : W

HALFLIFE $: 87.740$ years

SOURCE TERM : $1.2 \mathrm{E}-04 \mathrm{Ci}$

Source Term Width: $3 \mathrm{~m}$ Source Term Height: $3 \mathrm{~m}$

RELEASE FRACTION : $1.00 \mathrm{E}+00$

FILTER EFFICIENCY: $0.000000 \%$

EFFECTIYE RELEASE HEIGHT : $\quad 0.00 \mathrm{~m}$

WIND SPEED $(\mathrm{h}=2 \mathrm{~m}): 2.5 \mathrm{~m} / \mathrm{s}$

STABILITY CLASS :C DEPOSITION VELOCITY : $1.00 \mathrm{~cm} / \mathrm{s}$

RECEPTOR HEIGHT $\quad: 0.0 \mathrm{~m}$ INVERSION LAYER HEIGHT $: 500.0 \mathrm{~m}$

SAMPLE TIME $\quad: 30.000 \mathrm{~min}$

MAXIMUM DOSE DISTANCE $:<0.10 \mathrm{~km}$ MAXIMUM CEDE $:>0.015 \mathrm{rem}$

$\mathrm{D}=0.03 \mathrm{~km}$

$\mathrm{DEP}=7.3 \mathrm{E}-03 \mathrm{uCi} / \mathrm{m}^{\wedge} 2$

$\mathrm{CHI}=7.3 \mathrm{E}-07(\mathrm{Ci}-\mathrm{s}) / \mathrm{m}^{\wedge} 3$

50-YR DOSE COMMITMENT:

SURFACE BONE $2.0 \mathrm{E}+00 \mathrm{rem}$ RED MARROW 1.6E-01 rem

LIVER 4.4E-01 rem GONADS 2.4E-02 rem EFFECTIVE DOSE EQUIVALENT 1.1E-01 rem
$\mathrm{D}=0.10 \mathrm{~km}$

$\mathrm{DEP}=1.0 \mathrm{E}-03 \mathrm{uCi} / \mathrm{m}^{\wedge} 2$

$\mathrm{CHI}=\cdot 1.0 \mathrm{E}-07(\mathrm{Ci}-\mathrm{s}) / \mathrm{m}^{\wedge} 3$

50-YR DOSE COMMITMENT:

SURFACE BONE 2.7E-01 rem

RED MARROW 2.2E-02 rem

LIVER 6.0E-02 rem

GONADS 3.3E-03 rem .

EFFECTIVE DOSE

EQUIVALENT $1.5 \mathrm{E}-02 \mathrm{rem}$ 
Title of Project EPHA for THM

Reviewed by $\underline{\text { S. A. Henderson }}$

Subject Radiological Consequence Assessment

Works Calculation 6

Computer D. J. Richards

Date $\underline{09 / 11 / 96}$

Sheet No. 138 of 166

Release Designation: 1-RD-132, Adverse

HOTSPOT 7.0 GENERAL PLUME 9-11-1996 11:54

RADIONUCLIDE : PU-238 Inhalation Class : W

HALFLIFE : 87.740 years

SOURCE TERM : 1.3E-04 Ci

Source Term Width: $3 \mathrm{~m}$ Source Term Height: $3 \mathrm{~m}$

RELEASE FRACTION : $1.00 \mathrm{E}+00$

FILTER EFFICIENCY: $0.000000 \%$

EFFECTIVE RELEASE HEIGHT : $\quad 0.00 \mathrm{~m}$

WIND SPEED $(\mathrm{h}=2 \mathrm{~m}): 1.7 \mathrm{~m} / \mathrm{s}$

STABILITY CLASS :E DEPOSITION VELOCITY $\quad: 1.00 \mathrm{~cm} / \mathrm{s}$

RECEPTOR HEIGHT $\quad: 0.0 \mathrm{~m}$ INVERSION LAYER HEIGHT $: 200.0 \mathrm{~m}$

SAMPLE TIME $\quad: 10.000 \mathrm{~min}$

MAXIMUM DOSE DISTANCE $:<0.10 \mathrm{~km}$ MAXIMUM CEDE $:>0.089 \mathrm{rem}$

$\mathrm{D}=0.03 \mathrm{~km}$

$\mathrm{DEP}=3.4 \mathrm{E}-02 \mathrm{uCi} / \mathrm{m}^{\wedge} 2$

$\mathrm{CHI}=3.4 \mathrm{E}-06(\mathrm{Ci}-\mathrm{s}) / \mathrm{m}^{\wedge} 3$

50-YR DOSE COMMITMENT:

SURFACE BONE 9.2E+00 rem

RED MARROW 7.6E-01 rem

LIVER $2.0 \mathrm{E}+00 \mathrm{rem}$

GONADS 1.1E-01 rem

EFFECTIVE DOSE

EQUIVALENT 5.2E-01 rem
$\mathrm{D}=0.10 \mathrm{~km}$
$\mathrm{DEP}=5.8 \mathrm{E}-03 \mathrm{uCi} / \mathrm{m}^{\wedge} 2$
$\mathrm{CHI}=5.8 \mathrm{E}-07(\mathrm{Ci}-\mathrm{s}) / \mathrm{m}^{\wedge} 3$
50-YR DOSE COMMITMENT:
SURFACE BONE $1.6 \mathrm{E}+00 \mathrm{rem}$
RED MARROW 1.3E-01 rem
LIVER 3.5E-01 rem
GONADS 1.9E-02 rem
EFFECTIVE DOSE
EQUIVALENT 8.9E-02 rem 
Title of Project EPHA for THM

Subject Radiological Consequence Assessment

Computer D. J. Richards
Reviewed by S. A. Henderson

Works' Calculation 6

Sheet No. 139 of 166

Release Designation: 1-RD-132, Average

HOTSPOT 7.0 GENERAL PLUME 9-11-1996 11:55

RADIONUCLIDE : PU-238 Inhalation Class : W

HALFLIFE : 87.740 years

SOURCE TERM : $1.3 \mathrm{E}-04 \mathrm{Ci}$

Source Term Width: $\quad 3 \mathrm{~m}$ Source Term Height: $3 \mathrm{~m}$

RELEASE FRACTION : $1.00 \mathrm{E}+00$

FILTER EFFICIENCY: $0.000000 \%$

EFFECTIVE RELEASE HEIGHT : $\quad 0.00 \mathrm{~m}$

WIND SPEED $(\mathrm{h}=2 \mathrm{~m}): 2.5 \mathrm{~m} / \mathrm{s}$

STABILITY CLASS $\quad$ :C DEPOSITION VELOCITY $: 1.00 \mathrm{~cm} / \mathrm{s}$

RECEPTOR HEIGHT $\quad: 0.0 \mathrm{~m}$ INVERSION LAYER HEIGHT $: 500.0 \mathrm{~m}$

SAMPLE TIME $\quad: 10.000 \mathrm{~min}$

MAXIMUM DOSE DISTANCE $:<0.10 \mathrm{~km}$ MAXIMUM CEDE $:>0.021 \mathrm{rem}$

$\mathrm{D}=0.03 \mathrm{~km}$

$\mathrm{DEP}=1.0 \mathrm{E}-02 \mathrm{uCi} / \mathrm{m}^{\wedge} 2$

$\mathrm{CHI}=1.0 \mathrm{E}-06(\mathrm{Ci}-\mathrm{s}) / \mathrm{m}^{\wedge} 3$

50-YR DOSE COMMITMENT:

SURFACE BONE 2.7E+00 rem

RED MARROW 2.2E-01 rem

LIVER 6.0E-01 rem

GONADS 3.3E-02 rem

EFFECTIVE DOSE

EQUIVALENT $1.5 \mathrm{E}-01 \mathrm{rem}$
$\mathrm{D}=0.10 \mathrm{~km}$

$\mathrm{DEP}=1.4 \mathrm{E}-03 \mathrm{uCi} / \mathrm{m}^{\wedge} 2$

$\mathrm{CHI}=1.4 \mathrm{E}-07(\mathrm{Ci}-\mathrm{s}) / \mathrm{m}^{\wedge} 3$

50-YR DOSE COMMITMENT:

SURFACE BONE 3.7E-01 rem

RED MARROW 3.0E-02 rem

LIVER 8.2E-02 rem

GONADS 4.5E-03 rem

EFFECTIVE DOSE

EQUTVALENT 2.1E-02 rem 
Title of Project EPHA for THM

Subject Radiological Consequence Assessment

Computer D. J. Richards
Reviewed by $S$. A. Henderson Works Calculation 6 Sheet No. 140 of 166

Release Designation: 1-RD-133, Adverse

HOTSPOT 7.0 GENERAL PLUME 9-09-1996 22:36

RADIONUCLIDE : PU-238 Inhalation Class : W

HALFLIFE : 87.740 years

SOURCE TERM : $4.5 \mathrm{E}-03 \mathrm{Ci}$

Source Term Width: $3 \mathrm{~m} \quad$ Source Term Height: $3 \mathrm{~m}$

RELEASE FRACTION : $1.00 \mathrm{E}+00$

FILTER EFFICIENCY: $0.000000 \%$

EFFECTIVE RELEASE HEIGHT : $0.00 \mathrm{~m}$

WIND SPEED $(\mathrm{h}=2 \mathrm{~m}): 1.7 \mathrm{~m} / \mathrm{s}$

STABILITY CLASS :E DEPOSITION VELOCITY $\quad: 1.00 \mathrm{~cm} / \mathrm{s}$

RECEPTOR HEIGHT $\quad: 0.0 \mathrm{~m}$ INVERSION LAYER HEIGHT : $200.0 \mathrm{~m}$

SAMPLE TIME : $30.000 \mathrm{~min}$

MAXIMUM DOSE DISTANCE $:<0.10 \mathrm{~km}$ MAXIMUM CEDE : $>2.5 \mathrm{rem}$

$D=0.03 \mathrm{~km}$

$\mathrm{DEP}=9.6 \mathrm{E}-01 \mathrm{uCi} / \mathrm{m}^{\wedge} 2$

$\mathrm{CHI}=9.6 \mathrm{E}-05(\mathrm{Ci}-\mathrm{s}) / \mathrm{m}^{\wedge} 3$

50.YR DOSE COMMITMENT:

SURFACE BONE $2.6 \mathrm{E}+02 \mathrm{rem}$ RED MARROW 2.1E+01 rem LIVER 5.7E+01 rem GONADS $3.2 \mathrm{E}+00 \mathrm{rem}$ EFFECTIVE DOSE

EQUIVALENT 1.5E+01 rem

\author{
$\mathrm{D}=0.10 \mathrm{~km}$ \\ $\mathrm{DEP}=1.6 \mathrm{E}-01 \mathrm{uCi} / \mathrm{m}^{\wedge} 2$ \\ $\mathrm{CHI}=1.6 \mathrm{E}-05(\mathrm{Ci}-\mathrm{s}) / \mathrm{m}^{\wedge} 3$ \\ 50-YR DOSE COMMITMENT: \\ SURFACE BONE 4.4E+01 rem \\ RED MARROW $3.6 \mathrm{E}+00 \mathrm{rem}$ \\ LIVER $\quad 9.8 \mathrm{E}+00 \mathrm{rem}$ \\ GONADS 5.4E-01 rem \\ EFFECTIVE DOSE \\ EQUTVALENT $2.5 \mathrm{E}+00 \mathrm{rem}$
}

\author{
$D=0.16 \mathrm{~km}$ \\ $\mathrm{DEP}=6.8 \mathrm{E}-02 \mathrm{uCi} / \mathrm{m}^{\wedge} 2$ \\ $\mathrm{CHI}=6.8 \mathrm{E}-06(\mathrm{Ci}-\mathrm{s}) / \mathrm{m}^{\wedge} 3$ \\ 50-YR DOSE COMMITMENT: \\ SURFACE BONE $1.8 E+01$ rem \\ RED MARROW $1.5 \mathrm{E}+00 \mathrm{rem}$ \\ LIVER 4.1E+00 rem \\ GONADS 2.3E-01 rem \\ EFFECTIVE DOSE \\ EQUIVALENT $1.0 \mathrm{E}+00 \mathrm{rem}$
}


Title of Project EPHA for THM

Reviewed by S. A. Henderson

Subject Radiological Consequence Assessment

Computer D. J. Richards

Date $\underline{09 / 11 / 96}$
Works Calculation 6

Sheet No. 141 of 166

Release Designation: 1-RD-133, Average

HOTSPOT 7.0 GENERAL PLUME 9-09-1996 22:35

RADIONUCLIDE : PU-238 Inhalation Class : W

HALFLIFE : 87.740 years

SOURCE TERM : $4.5 \mathrm{E}-03 \mathrm{Ci}$

Source Term Width: $\quad 3 \mathrm{~m}$ Source Term Height: $3 \mathrm{~m}$

RELEASE FRACTION : $1.00 \mathrm{E}+00$

FILTER EFFICIENCY: $0.000000 \%$

EFFECTIVE RELEASE HEIGHT : $\quad 0.00 \mathrm{~m}$

WIND SPEED $(\mathrm{h}=2 \mathrm{~m}): 2.5 \mathrm{~m} / \mathrm{s}$

STABILITY CLASS :C DEPOSITION VELOCITY $: 1.00 \mathrm{~cm} / \mathrm{s}$

RECEPTOR HEIGHT $\quad: 0.0 \mathrm{~m}$ INVERSION LAYER HEIGHT $: 500.0 \mathrm{~m}$

SAMPLE TIME $\quad: 30.000 \mathrm{~min}$

MAXIMUM DOSE DISTANCE $:<0.10 \mathrm{~km}$ MAXIMUM CEDE $:>0.587 \mathrm{rem}$

$D=0.03 \mathrm{~km}$

$\mathrm{DEP}=2.8 \mathrm{E}-01 \mathrm{uCi} / \mathrm{m}^{\wedge} 2$

$\mathrm{CHI}=2.8 \mathrm{E}-05(\mathrm{Ci}-\mathrm{s}) / \mathrm{m}^{\wedge} 3$

50-YR DOSE COMMITMENT:

SURFACE BONE 7.6E+01 rem RED MARROW $6.3 E+00 \mathrm{rem}$

LIVER 1.7E+01 rem GONADS 9.3E-01 rem EFFECTIVE DOSE

EQUTVALENT $\quad 4.3 \mathrm{E}+00 \mathrm{rem}$
$\mathrm{D}=0.07 \mathrm{~km}$

$\mathrm{DEP}=6.9 \mathrm{E}-02 \mathrm{uCi} / \mathrm{m}^{\wedge} 2$

$\mathrm{CHI}=6.9 \mathrm{E}-06(\mathrm{Ci}-\mathrm{s}) / \mathrm{m}^{\wedge} 3$

50-YR DOSE COMMITMENT:

SURFACE BONE 1.8E+01 rem RED MARROW $1.5 \mathrm{E}+00 \mathrm{rem}$

LIVER $\quad 4.1 \mathrm{E}+00 \mathrm{rem}$ GONADS 2.3E-01 rem EFFECTIVE DOSE

EQUIVALENT $1.0 \mathrm{E}+00 \mathrm{rem}$
$\mathrm{D}=0.10 \mathrm{~km}$

$\mathrm{DEP}=3.8 \mathrm{E}-02 \mathrm{uCi} / \mathrm{m}^{\wedge} 2$

$\mathrm{CHI}=3.8 \mathrm{E}-06(\mathrm{Ci}-\mathrm{s}) / \mathrm{m}^{\wedge} 3$

50-YR DOSE COMMITMENT:

SURFACE BONE 1.0E+01 rem RED MARROW $\quad 8.5 \mathrm{E}-01 \mathrm{rem}$ LIVER 2.3E+00 rem GONADS 1.3E-01 rem EFFECTIVE DOSE

EQUIVALENT 5.9E-01 rem 
Title of Project EPHA for THM

Subject Radiological Consequence Assessment

Computer D. J. Richards

Date 09/11/96

Reviewed by S. A. Hénderson

Works Calculation 6

Sheet No. 142 of 166

Release Designation: 1-RD-134, Adverse

HOTSPOT 7.0 GENERAL PLUME 9-11-1996 17:14

RADIONUCLIDE : PU-238 Inhalation Class : W

HALFLIFE $: 87.740$ years

SOURCE TERM : 4.9E-03 Ci

Source Term Width: $\quad 3 \mathrm{~m}$ Source Term Height: $3 \mathrm{~m}$

RELEASE FRACTION : $1.00 \mathrm{E}+00$

FILTER EFFICIENCY: $0.000000 \%$

EFFECTIVE RELEASE HEIGHT : $\quad 0.00 \mathrm{~m}$

WIND SPEED $(\mathrm{h}=2 \mathrm{~m}): 1.7 \mathrm{~m} / \mathrm{s}$

STABILITY CLASS :E DEPOSITION VELOCITY : $1.00 \mathrm{~cm} / \mathrm{s}$

RECEPTOR HEIGHT $\quad: 0.0 \mathrm{~m}$ INVERSION LAYER HEIGHT $: 200.0 \mathrm{~m}$

SAMPLE TIME $\quad: 10.000 \mathrm{~min}$

MAXIMUM DOSE DISTANCE $:<0.10 \mathrm{~km}$ MAXIMUM CEDE $:>3.4 \mathrm{rem}$.

$\mathrm{D}=0.03 \mathrm{~km}$

$\mathrm{DEP}=1.3 \mathrm{E}+00 \mathrm{uCi} / \mathrm{m}^{\wedge} 2$

$\mathrm{CHI}=1.3 \mathrm{E}-04(\mathrm{Ci}-\mathrm{s}) / \mathrm{m}^{\wedge} 3$

50-YR DOSE COMMITMENT:

SURFACE BONE 3.5E+02 rem RED MARROW $2.9 \mathrm{E}+01 \mathrm{rem}$ LIVER 7.8E+01 rem GONADS 4.3E+00 rem EFFECTIVE DOSE EQUIVALENT $2.0 \mathrm{E}+0 \dot{1} \mathrm{rem}$
$\mathrm{D}=0.10 \mathrm{~km}$

$\mathrm{DEP}=2.2 \mathrm{E}-01 \mathrm{uCi} / \mathrm{m}^{\wedge} 2$

$\mathrm{CHI}=2.2 \mathrm{E}-05(\mathrm{Ci}-\mathrm{s}) / \mathrm{m}^{\wedge} 3$

50-YR DOSE COMMITMENT:

SURFACE BONE 6.0E+01 rem RED MARROW $\quad 4.9 \mathrm{E}+00 \mathrm{rem}$

LIVER $1.3 \mathrm{E} \div 01 \mathrm{rem}$ GONADS 7.4E-01 rem EFFECTIVE DOSE

EQUIVALENT $3.4 \mathrm{E}+00 \mathrm{rem}$
$\mathrm{D}=0.19 \mathrm{~km}$

$\mathrm{DEP}=6.8 \mathrm{E}-02 \mathrm{uCi} / \mathrm{m}^{\wedge} 2$

$\mathrm{CHI}=6.8 \mathrm{E}-06(\mathrm{Ci}-\mathrm{s}) / \mathrm{m}^{\wedge} 3$

50-YR DOSE COMMITMENT:

SURFACE BONE 1.8E+01 rem RED MARROW $1.5 \mathrm{E}+00 \mathrm{rem}$ LIVER 4.1E +00 rem GONADS 2.3E-01 rem EFFECTIVE DOSE EQUTVALENT $1.0 \mathrm{E}+00 \mathrm{rem}$ 
Title of Project EPHA for THM

Subject Radiological Consequence Assessment

Computer D. J. Richards

Release Designation: 1-RD-134, Average

HOTSPOT 7.0 GENERAL PLUME 9-11-1996 12:17

RADIONUCLIDE : PU-238 Inhalation Class : W

HALFLIFE $: 87.740$ years

SOURCE TERM : 4.9E-03 Ci

Source Term Width: $\quad 3 \mathrm{~m}$ Source Term Height: $3 \mathrm{~m}$

RELEASE FRACTION : $1.00 \mathrm{E}+00$

FILTER EFFICIENCY: $0.000000 \%$

EFFECTIVE RELEASE HEIGHT : $\quad 0.00 \mathrm{~m}$

WIND SPEED $(\mathrm{h}=2 \mathrm{~m}): 2.5 \mathrm{~m} / \mathrm{s}$

STABILITY CLASS :C DEPOSITION VELOCITY $: 1.00 \mathrm{~cm} / \mathrm{s}$

RECEPTOR HEIGHT $\quad: 0.0 \mathrm{~m}$ INVERSION LAYER HEIGHT $: 500.0 \mathrm{~m}$

SAMPLE TIME $\quad: 10.000 \mathrm{~min}$

MAXIMUM DOSE DISTANCE $:<0.10 \mathrm{~km}$ MAXIMUM CEDE $:>0.797 \mathrm{rem}$

$\mathrm{D}=0.03 \mathrm{~km}$

$\mathrm{DEP}=3.8 \mathrm{E}-01 \mathrm{uCi} / \mathrm{m}^{\wedge} 2$

$\mathrm{CHI}=3.8 \mathrm{E}-05(\mathrm{Ci}-\mathrm{s}) / \mathrm{m}^{\wedge} 3$

50-YR DOSE COMMITMENT:

SURFACE BONE $1.0 \mathrm{E}+02$ rem RED MARROW $\quad 8.5 \mathrm{E}+00 \mathrm{rem}$ LIVER 2.3E+01 rem GONADS $1.3 E+00$ rem EFFECTIVE DOSE EQUIVALENT $5.8 \mathrm{E}+00$ rem
$\mathrm{D}=0.09 \mathrm{~km}$

$\mathrm{DEP}=6.8 \mathrm{E}-02 \mathrm{uCi} / \mathrm{m}^{\wedge} 2$

$\mathrm{CHI}=6.8 \mathrm{E}-06(\mathrm{Ci}-\mathrm{s}) / \mathrm{m}^{\wedge} 3$

50-YR DOSE COMMITMENT:

SURFACE BONE 1.8E+01 rem RED MARROW $1.5 \mathrm{E}+00 \mathrm{rem}$ LIVER 4.1E+00 rem GONADS 2.3E-01 rem EFFECTIVE DOSE EQUTVALENT $1.0 \mathrm{E}+00 \mathrm{rem}$
$\mathrm{D}=0.10 \mathrm{~km}$

$\mathrm{DEP}=5.2 \mathrm{E}-02 \mathrm{uCi} / \mathrm{m}^{\wedge} 2$

$\mathrm{CHI}=5.2 \mathrm{E}-06(\mathrm{Ci}-\mathrm{s}) / \mathrm{m}^{\wedge} 3$

50-YR DOSE COMMITMENT:

SURFACE BONE $1.4 \mathrm{E}+01 \mathrm{rem}$ RED MARROW $1.2 \mathrm{E}+00 \mathrm{rem}$ LIVER $3.1 \mathrm{E}+00 \mathrm{rem}$ GONADS 1.7E-01 rem EFFECTIVE DOSE EQUIVALENT 8.0E-01 rem 
Title of Project EPHA for THM

Subject Radiological Consequence Assessment

Computer D. J. Richards

Date $\underline{09 / 11 / 96}$

Reviewed by $\underline{S}$. A. Henderson

Works Calculation 6

Sheet No. 144 of 166

Release Designation: 1-RD-135, Adverse

HOTSPOT 7.0 GENERAL PLUME 9-09-1996 22:38

RADIONUCLIDE : PU-238 Inhalation Class : W

HALFLIFE : 87.740 years

SOURCE TERM : $1.4 \mathrm{E}-04 \mathrm{Ci}$

Source Term Width: $3 \mathrm{~m}$ Source Term Height: $3 \mathrm{~m}$

RELEASE FRACTION : $1.00 \mathrm{E}+00$

FILTER EFFICIENCY: $0.000000 \%$

EFFECTIVE RELEASE HEIGHT : $\quad 0.00 \mathrm{~m}$

WIND SPEED $(\mathrm{h}=2 . \mathrm{m}): 1.7 \mathrm{~m} / \mathrm{s}$

STABILITY CLASS :E DEPOSITION VELOCITY $: 1.00 \mathrm{~cm} / \mathrm{s}$

RECEPTOR HEIGHT $\quad: 0.0 \mathrm{~m}$ INVERSION LAYER HEIGHT $: 200.0 \mathrm{~m}$

SAMPLE TIME $\quad: 30.000 \mathrm{~min}$

MAXIMUM DOSE DISTANCE $:<0.10 \mathrm{~km}$ MAXIMUM CEDE $:>0.076 \mathrm{rem}$

\begin{tabular}{ll}
$\mathrm{D}=0.03 \mathrm{~km}$ & $\mathrm{D}=0.10 \mathrm{~km}$ \\
$\mathrm{DEP}=2.9 \mathrm{E}-02 \mathrm{uCi} / \mathrm{m}^{\wedge} 2$ & $\mathrm{DEP}=5.0 \mathrm{E}-03 \mathrm{uCi} / \mathrm{m}^{\wedge} 2$ \\
$\mathrm{CHI}=2.9 \mathrm{E}-06(\mathrm{Ci}-\mathrm{s}) / \mathrm{m}^{\wedge} 3$ & $\mathrm{CHI}=5.0 \mathrm{E}-07(\mathrm{Ci}-\mathrm{s}) / \mathrm{m}^{\wedge} 3$ \\
$50-\mathrm{YR}$ DOSE COMMITMENT: & $50-\mathrm{YR}$ DOSE COMMITMENT: \\
\hline SURFACE BONE 7.9E+00 rem & SURFACE BONE $1.3 \mathrm{E}+00 \mathrm{rem}$ \\
RED MARROW 6.5E-01 rem & RED MARROW $1.1 \mathrm{E}-01 \mathrm{rem}$ \\
LIVER 1.8E+00 rem & LIVER 3.0E-01 rem \\
GONADS 9.7E-02 rem & GONADS 1.7E-02 rem \\
EFFECTIVE DOSE & EFFECTIVE DOSE \\
EQUIVALENT 4.5E-01 rem & EQUIVALENT 7.6E-02 rem
\end{tabular}


Title of Project EPHA for THM $\cdot$

Subject Radiological Consequence Assessment

Computer D. J. Richards

Date $\underline{09 / 11 / 96}$
Reviewed by S. A. Henderson

Works Calculation 6

Sheet No. 145 of 166

Release Designation: 1-RD-135, Average

HOTSPOT 7.0 GENERAL PLUME 9-09-1996 22:38

RADIONUCLIDE : PU-238 Inhalation Class : W

HALFLIFE $: 87.740$ years

SOURCE TERM : $1.4 \mathrm{E}-04 \mathrm{Ci}$

Source Term Width: $\quad 3 \mathrm{~m}$ Source Term Height: $3 \mathrm{~m}$

RELEASE FRACTION : $1.00 \mathrm{E}+00$

FILTER EFFICIENCY: $0.000000 \%$

EFFECTIVE RELEASE HEIGHT : $\quad 0.00 \mathrm{~m}$

WIND SPEED $(\mathrm{h}=2 \mathrm{~m}): 2.5 \mathrm{~m} / \mathrm{s}$

STABILITY CLASS :C DEPOSITION VELOCITY : $1.00 \mathrm{~cm} / \mathrm{s}$

RECEPTOR HEIGHT $\quad: 0.0 \mathrm{~m}$ INVERSION LAYER HEIGHT $: 500.0 \mathrm{~m}$

SAMPLE TIME $\quad: 30.000 \mathrm{~min}$

MAXIMUM DOSE DISTANCE $:<0.10 \mathrm{~km}$ MAXIMUM CEDE $:>0.018 \mathrm{rem}$

$\mathrm{D}=0.03 \mathrm{~km}$

$\mathrm{DEP}=8.6 \mathrm{E}-03 \mathrm{uCi} / \mathrm{m}^{\wedge} 2$

$\mathrm{CHI}=8.6 \mathrm{E}-07(\mathrm{Ci}-\mathrm{s}) / \mathrm{m}^{\wedge} 3$

50-YR DOSE COMMITMENT:

SURFACE BONE 2.3E+00 rem RED MARROW 1.9E-01 rem

LIVER 5.1E-01 rem

GONADS 2.9E-02 rem

EFFECTIVE DOSE

EQUIVALENT 1.3E-01 rem
$\mathrm{D}=0.10 \mathrm{~km}$

$\mathrm{DEP}=1.2 \mathrm{E}-03 \mathrm{uCi} / \mathrm{m}^{\wedge} 2$

$\mathrm{CHI}=1.2 \mathrm{E}-07(\mathrm{Ci}-\mathrm{s}) / \mathrm{m}^{\wedge} 3$

50-YR DOSE COMMITMENT:

SURFACE BONE 3.2E-01 rem

RED MARROW 2.6E-02 rem

LIVER - 7.0E-02 rem

GONADS 3.9E-03 rem

EFFECTIVE DOSE

EQUIVALENT $1.8 \mathrm{E}-02$ rem 
Title of Project EPHA for THM

Subject Radiological Consequence Assessment

Computer D. J. Richards

Date $\underline{09 / 11 / 96}$

Reviewed by $\mathrm{S}$. A. Henderson Works Calculation 6 Sheet No. 146 of 166

Release Designation: 1-RD-136, Adverse

HOTSPOT 7.0 GENERAL PLUME 9-11-1996 12:03

RADIONUCLIDE : PU-238 Inhalation Class : W

HALFLIFE : 87.740 years

SOURCE TERM : $1.5 \mathrm{E}-04 \mathrm{Ci}$

Source Term Width: $3 \mathrm{~m}$ Source Term Height: $3 \mathrm{~m}$

RELEASE FRACTION : $1.00 \mathrm{E}+00$

FILTER EFFICIENCY: $0.000000 \%$

EFFECTIVE RELEASE HEIGHT : $\quad 0.00 \mathrm{~m}$

WIND SPEED $(\mathrm{h}=2 \mathrm{~m}): 1.7 \mathrm{~m} / \mathrm{s}$

STABILITY CLASS :E DEPOSITION VELOCITY $: 1.00 \mathrm{~cm} / \mathrm{s}$

RECEPTOR HEIGHT $: 0.0 \mathrm{~m}$ INVERSION LAYER HEIGHT $: 200.0 \mathrm{~m}$

SAMPLE TIME $\quad: 10.000 \mathrm{~min}$

MAXIMUM DOSE DISTANCE $:<0.10 \mathrm{~km}$ MAXIMUM CEDE $:>0.104 \mathrm{rem}$

$\mathrm{D}=0.03 \mathrm{~km}$

$\mathrm{DEP}=4.0 \mathrm{E}-02 \mathrm{uCi} / \mathrm{m}^{\wedge} 2$

$\mathrm{CHI}=4.0 \mathrm{E}-06(\mathrm{Ci}-\mathrm{s}) / \mathrm{m}^{\wedge} 3$

50-YR DOSE COMMITMENT:

SURFACE BONE $1.1 \mathrm{E}+01 \mathrm{rem}$ RED MARROW $8.9 \mathrm{E}-01 \mathrm{rem}$ LIVER 2.4E+00 rem GONADS 1.3E-01 rem EFFECTIVE DOSE

EQUTVALENT 6.1E-01 rem
$\mathrm{D}=0.10 \mathrm{~km}$
$\mathrm{DEP}=6.8 \mathrm{E}-03 \mathrm{uCi} / \mathrm{m}^{\wedge} 2$
$\mathrm{CHI}=6.8 \mathrm{E}-07(\mathrm{Ci}-\mathrm{s}) / \mathrm{m}^{\wedge} 3$
50-YR DOSE COMMITMENT:
SURFACE BONE $1.8 \mathrm{E}+00 \mathrm{rem}$
RED MARROW 1.5E-01 rem
LIVER 4.1E-01 rem
GONADS 2.3E-02 rem
EFFECTTVE DOSE
EQUIVALENT 1.0E-01 rem 
Title of Project EPHA for THM

Reviewed by S. A. Henderson

Subject Radiological Consequence Assessment

Works_Calculation 6

Computer D. J. Richards

Date $\underline{09 / 11 / 96}$

Sheet No. 147 of 166

Release Designation: 1-RD-136, Average

HOTSPOT 7.0 GENERAL PLUME 9-11-1996 12:04

RADIONUCLIDE : PU-238 Inhalation Class : W

HALFLIFE $: 87.740$ years

SOURCE TERM : $1.5 \mathrm{E}-04 \mathrm{Ci}$

Source Term Width: $3 \mathrm{~m}$ Source Term Height: $3 \mathrm{~m}$

RELEASE FRACTION : $1.00 \mathrm{E}+00$

FILTER EFFICIENCY: $0.000000 \%$

EFFECTIVE RELEASE HEIGHT : $\quad 0.00 \mathrm{~m}$

WIND SPEED $(\mathrm{h}=2 \mathrm{~m}): 2.5 \mathrm{~m} / \mathrm{s}$

STABILITY CLASS :C DEPOSITION VELOCITY $: 1.00 \mathrm{~cm} / \mathrm{s}$

RECEPTOR HEIGHT $\quad: 0.0 \mathrm{~m}$ INVERSION LAYER HEIGHT $: 500.0 \mathrm{~m}$

SAMPLE TIME $\quad: 10.000 \mathrm{~min}$

MAXIMUM DOSE DISTANCE $:<0.10 \mathrm{~km}$ MAXIMUM CEDE $:>0.024 \mathrm{rem}$

$\mathrm{D}=0.03 \mathrm{~km}$

$\mathrm{DEP}=1.2 \mathrm{E}-02 \mathrm{uCi} / \mathrm{m}^{\wedge} 2$

$\mathrm{CHI}=1.2 \mathrm{E}-06(\mathrm{Ci}-\mathrm{s}) / \mathrm{m}^{\wedge} 3$

50-YR DOSE COMMITMENT:

SURFACE BONE $3.1 \mathrm{E}+00 \mathrm{rem}$ RED MARROW 2.6E-01 rem LIVER 7.0E-01 rem GONADS 3.9E-02 rem EFFECTIVE DOSE EQUIVALENT $\quad 1.8 \mathrm{E}-0 \mathrm{i} \mathrm{rem}$
$\mathrm{D}=0.10 \mathrm{~km}$

$\mathrm{DEP}=1.6 \mathrm{E}-03 \mathrm{uCi} / \mathrm{m}^{\wedge} 2$

$\mathrm{CHI}=1.6 \mathrm{E}-07(\mathrm{Ci}-\mathrm{s}) / \mathrm{m}^{\wedge} 3$

50-YR DOSE COMMITMENT:

SURFACE BONE 4.3E-01 rem

RED MARROW 3.6E-02 rem

LIVER 9.6E-02 rem

GONADS 5.3E-03 rem

EFFECTIVE DOSE

EQUTVALENT 2.4E-02 rem 
Title of Project EPHA for THM

Subject Radiological Consequence Assessment

Computer D. J. Richards
Reviewed by S.A. Henderson

Works Calculation 6

Sheet No. 148 of 166

Release Designation: 1-RD-137, Adverse

HOTSPOT 7.0 GENERAL PLUME 9-09-1996 22:40

RADIONUCLIDE : PU-238 Inhalation Class : W

HALFLIFE : 87.740 years

SOURCE TERM $\quad: 4.5 \mathrm{E}-03 \mathrm{Ci}$

Source Term Width: $3 \mathrm{~m}$ Source Term Height: $3 \mathrm{~m}$

RELEASE FRACTION : $1.00 \mathrm{E}+00$

FILTER EFFICIENCY: $0.000000 \%$

EFFECTIVE RELEASE HEIGHT : $\quad 0.00 \mathrm{~m}$

WIND SPEED $(\mathrm{h}=2 \mathrm{~m}): 1.7 \mathrm{~m} / \mathrm{s}$

STABILITY CLASS :E DEPOSITION VELOCITY $: 1.00 \mathrm{~cm} / \mathrm{s}$

RECEPTOR HEIGHT $\quad: 0.0 \mathrm{~m}$ INVERSION LAYER HEIGHT $: 200.0 \mathrm{~m}$

SAMPLE TIME : $30.000 \mathrm{~min}$

MAXIMUM DOSE DISTANCE $:<0.10 \mathrm{~km}$ MAXIMUM CEDE $:>2.5 \mathrm{rem}$

$\mathrm{D}=0.03 \mathrm{~km}$

$\mathrm{DEP}=9.6 \mathrm{E}-01 \mathrm{uCi} / \mathrm{m}^{\wedge} 2$

$\mathrm{CHI}=9.6 \mathrm{E}-05(\mathrm{Ci}-\mathrm{s}) / \mathrm{m}^{\wedge} 3$

50-YR DOSE COMMITMENT:

SURFACE BONE $2.6 E+02$ rem RED MARROW $2.1 \mathrm{E}+01 \mathrm{rem}$ LIVER 5.7E+01 rem GONADS 3.2E+00 rem EFFECTIVE DOSE

EQUIVALENT $1.5 \mathrm{E}+01 \mathrm{rem}$

\author{
$\mathrm{D}=0.10 \mathrm{~km}$ \\ $\mathrm{DEP}=1.6 \mathrm{E}-01 \mathrm{uCi} / \mathrm{m}^{\wedge} 2$ \\ $\mathrm{CHI}=1.6 \mathrm{E}-05(\mathrm{Ci}-\mathrm{s}) / \mathrm{m}^{\wedge} 3$ \\ 50-YR DOSE COMMITMENT: \\ SURFACE BONE 4.4E+01 rem \\ RED MARROW $3.6 \mathrm{E}+00 \mathrm{rem}$ \\ LIVER $9.8 \mathrm{E}+00 \mathrm{rem}$ \\ GONADS 5.4E-01 rem \\ EFFECTIVE DOSE \\ EQUIVALENT $2.5 \mathrm{E}+00 \mathrm{rem}$
}

\author{
$\mathrm{D}=0.16 \mathrm{~km}$ \\ $\mathrm{DEP}=6.8 \mathrm{E}-02 \mathrm{uCi} / \mathrm{m}^{\wedge} 2$ \\ $\mathrm{CHI}=6.8 \mathrm{E}-06(\mathrm{Ci}-\mathrm{s}) / \mathrm{m}^{\wedge} 3$ \\ 50-YR DOSE COMMITMENT: \\ SURFACE BONE 1.8E+01 rem \\ RED MARROW $1.5 \mathrm{E}+00 \mathrm{rem}$ \\ LIVER 4.1E+00 rem \\ GONADS 2.3E-01 rem \\ EFFECTIVE DOSE \\ EQUIVALENT $1.0 \mathrm{E}+00 \mathrm{rem}$
}


Title of Project EPHA for THM

Subject Radiological Consequence Assessment

Computer D. J. Richards

Date 09/11/96
Reviewed by $\mathrm{S}$. A. Hènderson

Works Calculation 6

Sheet No. 149 of 166

Release Designation: 1-RD-137, Average

HOTSPOT 7.0 GENERAL PLUME 9-09-1996 22:41

RADIONUCLIDE : PU-238 Inhalation Class : W

HALFLIFE : 87.740 years

SOURCE TERM $: 4.5 \mathrm{E}-03 \mathrm{Ci}$

Source Term Width: $\quad 3 \mathrm{~m}$ Source Term Height: $3 \mathrm{~m}$

RELEASE FRACTION : $1.00 \mathrm{E}+00$

FILTER EFFICIENCY: $0.000000 \%$

EFFECTIVE RELEASE HEIGHT : $\quad 0.00 \mathrm{~m}$

WIND SPEED $(\mathrm{h}=2 \mathrm{~m}): 2.5 \mathrm{~m} / \mathrm{s}$

STABILITY CLASS :C DEPOSITION VELOCITY $: \quad: 1.00 \mathrm{~cm} / \mathrm{s}$

RECEPTOR HEIGHT $\quad: 0.0 \mathrm{~m}$ INVERSION LAYER HEIGHT $: 500.0 \mathrm{~m}$

SAMPLE TIME $\quad: 30.000 \mathrm{~min}$

MAXIMUM DOSE DISTANCE $:<0.10 \mathrm{~km}$ MAXIMUM CEDE $:>0.587 \mathrm{rem}$

$\mathrm{D}=0.03 \mathrm{~km}$

$\mathrm{DEP}=2.8 \mathrm{E}-01 \mathrm{uCi} / \mathrm{m}^{\wedge} 2$

$\mathrm{CHI}=2.8 \mathrm{E}-05(\mathrm{Ci}-\mathrm{s}) / \mathrm{m}^{\wedge} 3$

50-YR DOSE COMMITMENT:

SURFACE BONE 7.6E+01 rem RED MARROW $6.3 \mathrm{E}+00 \mathrm{rem}$ LIVER $1.7 \mathrm{E}+01 \mathrm{rem}$ GONADS 9.3E-01 rem EFFECTIVE DOSE

EQUIVALENT $\quad 4.3 \mathrm{E}+00 \mathrm{rem}$
$\mathrm{D}=0.07 \mathrm{~km}$

$\mathrm{DEP}=6.9 \mathrm{E}-02 \mathrm{uCi} / \mathrm{m}^{\wedge} 2$

$\mathrm{CHI}=6.9 \mathrm{E}-06(\mathrm{Ci}-\mathrm{s}) / \mathrm{m}^{\wedge} 3$

50-YR DOSE COMMITMENT:

SURFACE BONE 1.8E+01 rem RED MARROW $1.5 \mathrm{E}+00 \mathrm{rem}$ LIVER $\quad 4.1 \mathrm{E}+00 \mathrm{rem}$ GONADS 2.3E-01 rem EFFECTIVE DOSE

EQUIVALENT $1.0 \mathrm{E}+00 \mathrm{rem}$
$\mathrm{D}=0.10 \mathrm{~km}$

$\mathrm{DEP}=3.8 \mathrm{E}-02 \mathrm{uCi} / \mathrm{m}^{\wedge} 2$

$\mathrm{CHI}=3.8 \mathrm{E}-06(\mathrm{Ci}-\mathrm{s}) / \mathrm{m}^{\wedge} 3$

50-YR DOSE COMMITMENT:

SURFACE BONE $1.0 \mathrm{E}+01 \mathrm{rem}$ RED MARROW $\quad 8.5 \mathrm{E}-01 \mathrm{rem}$ LIVER $\quad 2.3 \mathrm{E}+00 \mathrm{rem}$ GONADS 1.3E-01 rem EFFECTIVE DOSE

EQUIVALENT $5.9 \mathrm{E}-01 \mathrm{rem}$ 
Title of Project EPHA for THM

Subject Radiological Consequence Assessment

Computer D. J. Richards

Date $\underline{09 / 11 / 96}$

Reviewed by $\underline{\text { S. A. Henderson }}$

Works Calculation 6

Sheet No. 150 of 166

Release Designation: 1-RD-138, Adverse

HOTSPOT 7.0 GENERAL PLUME 9-11-1996 12:06

RADIONUCLIDE : PU-238 Inhalation Class : W

HALFLIFE $: 87.740$ years

SOURCE TERM : $4.9 \mathrm{E}-03 \mathrm{Ci}$

Source Term Width: $3 \mathrm{~m}$ Source Term Height: $3 \mathrm{~m}$

RELEASE FRACTION : $1.00 \mathrm{E}+00$

FILTER EFFICIENCY: $0.000000 \%$

EFFECTIVE RELEASE HEIGHT : $\quad 0.00 \mathrm{~m}$

WIND SPEED $(\mathrm{h}=2 \mathrm{~m}): 1.7 \mathrm{~m} / \mathrm{s}$

STABILITY CLASS : E DEPOSITION VELOCITY $: 1.00 \mathrm{~cm} / \mathrm{s}$

RECEPTOR HEIGHT $\quad: 0.0 \mathrm{~m}$ INVERSION LAYER HEIGHT $: 200.0 \mathrm{~m}$

SAMPLE TIME $\quad: 10.000 \mathrm{~min}$

MAXIMUM DOSE DISTANCE $:<0.10 \mathrm{~km}$ MAXIMUM CEDE $:>3.4 \mathrm{rem}$

$D=0.03 \mathrm{~km}$

$\mathrm{DEP}=1.3 \mathrm{E}+00 \mathrm{uCi} / \mathrm{m}^{\wedge} 2$

$\mathrm{CHI}=1.3 \mathrm{E}-04(\mathrm{Ci}-\mathrm{s}) / \mathrm{m}^{\wedge} 3$

50-YR DOSE COMMITMENT:

SURFACE BONE $3.5 \mathrm{E}+02 \mathrm{rem}$ RED MARROW $2.9 \mathrm{E}+01$ rem LIVER 7.8E+01 rem GONADS 4.3E+00 rem EFFECTIVE DOSE EQUTVALENT 2.0E+01 rem
$\mathrm{D}=0.10 \mathrm{~km}$

$\mathrm{DEP}=2.2 \mathrm{E}-01 \mathrm{uCi} / \mathrm{m}^{\wedge} 2$ $\mathrm{CHI}=2.2 \mathrm{E}-05(\mathrm{Ci}-\mathrm{s}) / \mathrm{m}^{\wedge} 3$

50-YR DOSE COMMITMENT:

SURFACE BONE 6.0E+01 rem RED MARROW $4.9 \mathrm{E}+00 \mathrm{rem}$

LIVER 1.3E+01 rem GONADS 7.4E-01 rem EFFECTIVE DOSE

EQUIVALENT $3.4 \mathrm{E}+00 \mathrm{rem}$
$\mathrm{D}=0.19 \mathrm{~km}$

$\mathrm{DEP}=6.8 \mathrm{E}-02 \mathrm{uCi} / \mathrm{m}^{\wedge} 2$

$\mathrm{CHI}=6.8 \mathrm{E}-06(\mathrm{Ci}-\mathrm{s}) / \mathrm{m}^{\wedge} 3$

50-YR DOSE COMMITMENT:

SURFACE BONE $1.8 \mathrm{E}+01 \mathrm{rem}$ RED MARROW $1.5 \mathrm{E}+00 \mathrm{rem}$ LIVER 4.1E+00 rem GONADS 2.3E-01 rem EFFECTIVE DOSE EQUTVALENT $1.0 \mathrm{E}+00 \mathrm{rem}$ 
Title of Project EPHA for THM

Subject Radiological Consequence Assessment

Computer D. J. Richards

Date 09/11/96

Reviewed by S. A. Henderson Works Calculation 6 Sheet No. 151 of 166

Release Designation: 1-RD-138, Average

HOTSPOT 7.0 GENERAL PLUME 9-11-1996 12:08

RADIONUCLIDE : PU-238 Inhalation Class : W

HALFLIFE : 87.740 years

SOURCE TERM : 4.9E-03 Ci

Source Term Width: $\quad 3 \mathrm{~m}$ Source Term Height: $3 \mathrm{~m}$

RELEASE FRACTION : $1.00 \mathrm{E}+00$

FILTER EFFICIENCY: $0.000000 \%$

EFFECTIVE RELEASE HEIGHT : $0.00 \mathrm{~m}$

WIND SPEED $(\mathrm{h}=2 \mathrm{~m}): 2.5 \mathrm{~m} / \mathrm{s}$

STABILITY CLASS $\quad$ :C DEPOSITION VELOCITY $: 1.00 \mathrm{~cm} / \mathrm{s}$

RECEPTOR HEIGHT $: 0.0 \mathrm{~m}$ INVERSION LAYER HEIGHT $: 500.0 \mathrm{~m}$

SAMPLE TIME $\quad: 10.000 \mathrm{~min}$

MAXIMUM DOSE DISTANCE : $<0.10 \mathrm{~km}$ MAXIMUM CEDE $:>0.797 \mathrm{rem}$

$\mathrm{D}=0.03 \mathrm{~km}$

$\mathrm{DEP}=3.8 \mathrm{E}-01 \mathrm{uCi} / \mathrm{m}^{\wedge} 2$

$\mathrm{CHI}=3.8 \mathrm{E}-05(\mathrm{Ci}-\mathrm{s}) / \mathrm{m}^{\wedge} 3$

50-YR DOSE COMMITMENT:

SURFACE BONE $1.0 \mathrm{E}+02 \mathrm{rem}$ RED MARROW $8.5 \mathrm{E}+00 \mathrm{rem}$ LIVER 2.3E+01 rem GONADS $1.3 \mathrm{E}+00 \mathrm{rem}$ EFFECTIVE DOSE EQUIVALENT $5.8 \mathrm{E}+00 \mathrm{rem}$

$\mathrm{D}=0.09 \mathrm{~km}$
$\mathrm{DEP}=6.8 \mathrm{E}-02 \mathrm{uCi} / \mathrm{m}^{\wedge} 2$
$\mathrm{CHI}=6.8 \mathrm{E}-06(\mathrm{Ci}-\mathrm{s}) / \mathrm{m}^{\wedge} 3$
50-YR DOSE COMMITMENT:
SURFACE BONE $1.8 \mathrm{E}+01 \mathrm{rem}$
RED MARROW $1.5 \mathrm{E}+00 \mathrm{rem}$
LIVER $\quad 4.1 \mathrm{E}+00 \mathrm{rem}$
GONADS $\quad 2.3 \mathrm{E}-01 \mathrm{rem}$
EFFECTIVE DOSE
EQUIVALENT $\quad 1.0 \mathrm{E}+00 \mathrm{rem}$

$D=0.10 \mathrm{~km}$

$\mathrm{DEP}=5.2 \mathrm{E}-02 \mathrm{uCi} / \mathrm{m}^{\wedge} 2$

$\mathrm{CHI}=5.2 \mathrm{E}-06(\mathrm{Ci}-\mathrm{s}) / \mathrm{m}^{\wedge} 3$

50-YR DOSE COMMITMENT:

SURFACE BONE $1.4 \mathrm{E}+01 \mathrm{rem}$ RED MARROW 1.2E+00 rem LIVER 3.1E $+00 \mathrm{rem}$ GONADS 1.7E-01 rem EFFECTIVE DOSE EQUIVALENT $8.0 \mathrm{E}-01 \mathrm{rem}$ 
Title of Project EPHA for THM

Subject Radiological Consequence Assessment

Computer D. J. Richards

Date 09/11/96

Reviewed by $\underline{S}$. A. Hènderson

Works Calculation 6

Sheet No. 152 of 166

Release Designation: 1-RD-139, Adverse

HOTSPOT 7.0 GENERAL PLUME 9-09-1996 22:43

RADIONUCLIDE : PU-238 Inhalation Class : W

HALFLIFE : 87.740 years

SOURCE TERM : $1.3 \mathrm{E}-06 \mathrm{Ci}$

Source Term Width: $\quad 3 \mathrm{~m}$ Source Term Height: $3 \mathrm{~m}$

RELEASE FRACTION : $1.00 \mathrm{E}+00$

FILTER EFFICIENCY: $0.000000 \%$

EFFECTIVE RELEASE HEIGHT : $\quad 0.00 \mathrm{~m}$

WIND SPEED $(\mathrm{h}=2 \mathrm{~m}): 1.7 \mathrm{~m} / \mathrm{s}$

STABILITY CLASS :E DEPOSITION VELOCITY : $1.00 \mathrm{~cm} / \mathrm{s}$

RECEPTOR HEIGHT $: 0.0 \mathrm{~m}$ INVERSION LAYER HEIGHT $: 200.0 \mathrm{~m}$

SAMPLE TIME $\quad: 30.000 \mathrm{~min}$

MAXIMUM DOSE DISTANCE $: 0.10 \mathrm{~km}$ MAXIMUM CEDE $:>7.2 \mathrm{E}-04 \mathrm{rem}$

$\mathrm{D}=0.03 \mathrm{~km}$

$\mathrm{DEP}=2.8 \mathrm{E}-04 \mathrm{uCi} / \mathrm{m}^{\wedge} 2$

$\mathrm{CHI}=2.8 \mathrm{E}-08(\mathrm{Ci}-\mathrm{s}) / \mathrm{m}^{\wedge} 3$

50-YR DOSE COMMITMENT:

SURFACE BONE 7.4E-02 rem RED MARROW 6.1E-03 rem LIVER $\quad 1.7 \mathrm{E}-02$ rem GONADS 9.2E-04 rem EFFECTIVE DOSE

EQUIVALENT 4.2E-03 rem
$\mathrm{D}=0.10 \mathrm{~km}$
$\mathrm{DEP}=4.7 \mathrm{E}-05 \mathrm{uCi} / \mathrm{m}^{\wedge} 2$
$\mathrm{CHI}=4.7 \mathrm{E}-09(\mathrm{Ci}-\mathrm{s}) / \mathrm{m}^{\wedge} 3$
50-YR DOSE COMMITMENT:
SURFACE BONE 1.3E-02 rem
RED MARROW $1.0 \mathrm{E}-03 \mathrm{rem}$
LIVER 2.8E-03 rem
GONADS 1.6E-04 rem
EFFECTIVE DOSE
EQUTVALENT 7.2E-04 rem 
Title of Project EPHA for THM

Reviewed by $\underline{S}$. A. Hënderson

Subject Radiological Consequence Assessment

Works Calculation 6

Computer D. J. Richards

Date $\underline{09 / 11 / 96}$

Sheet No. 153 of 166

Release Designation: 1-RD-139, Average

HOTSPOT 7.0 GENERAL PLUME 9-09-1996 22:43

RADIONUCLIDE : PU-238 Inhalation Class : W

HALFLIFE : 87.740 years

SOURCE TERM : $1.3 \mathrm{E}-06 \mathrm{Ci}$

Source Term Width: $\quad 3 \mathrm{~m}$ Source Term Height: $3 \mathrm{~m}$

RELEASE FRACTION : $1.00 \mathrm{E}+00$

FILTER EFFICIENCY: $0.000000 \%$

EFFECTIVE RELEASE HEIGHT : ${ }^{\prime} 0.00 \mathrm{~m}$

WIND SPEED $(\mathrm{h}=2 \mathrm{~m}): 2.5 \mathrm{~m} / \mathrm{s}$

STABILITY CLASS $\quad$ :C DEPOSITION VELOCITY $\quad: 1.00 \mathrm{~cm} / \mathrm{s}$

RECEPTOR HEIGHT $\quad: 0.0 \mathrm{~m}$ INVERSION LAYER HEIGHT $: 500.0 \mathrm{~m}$

SAMPLE TIME $\quad: 30.000 \mathrm{~min}$

MAXIMUM DOSE DISTANCE $:<0.10 \mathrm{~km}$ MAXIMUM CEDE $:>1.7 \mathrm{E}-04 \mathrm{rem}$
$\mathrm{D}=0.03 \mathrm{~km}$
$\mathrm{DEP}=8.1 \mathrm{E}-05 \mathrm{uCi} / \mathrm{m}^{\wedge} 2$
$\mathrm{D}=0.10 \mathrm{~km}$
$\mathrm{CHI}=8.1 \mathrm{E}-09(\mathrm{Ci}-\mathrm{s}) / \mathrm{m}^{\wedge} 3$
50-YR DOSE COMMITMENT
$\mathrm{DEP}=1.1 \mathrm{E}-05 \mathrm{uCi} / \mathrm{m}^{\wedge} 2$
$\mathrm{CHI}=1.1 \mathrm{E}-09(\mathrm{Ci}-\mathrm{s}) / \mathrm{m}^{\wedge} 3$
SURFACE BONE 2.2E-02 rem
50-YR DOSE COMMITMENT:
RED MARROW $1.8 \mathrm{E}-03 \mathrm{rem}$
LIVER 4.8E-03 rem
GONADS 2.7E-04 rem
EFFECTIVE DOSE
EQUIVALENT $1.2 \mathrm{E}-03 \mathrm{rem}$
SURFACE BONE 3.0E-03 rem
RED MARROW 2.5E-04 rem
LIVER 6.6E-04 rem
GONADS 3.7E-05 rem
EFFECTIVE DOSE
EQUIVALENT $1.7 \mathrm{E}-04 \mathrm{rem}$ 
Title of Project EPHA for THM

Subject Radiological Consequence Assessment

Computer D. J. Richards
Reviewed by $\mathrm{S}$. A. Hënderson Works Calculation 6 Sheet No. 154 of 166

Release Designation: 1-RD-140, Adverse

HOTSPOT 7.0 GENERAL PLUME 9-11-1996 12:09

RADIONUCLIDE : PU-238 Inhalation Class : W

HALFLIFE : 87.740 years

SOURCE TERM : $3.8 \mathrm{E}-07 \mathrm{Ci}$

Source Term Width: $3 \mathrm{~m}$ Source Term Height: $3 \mathrm{~m}$

RELEASE FRACTION : $1.00 \mathrm{E}+00$

FILTER EFFICIENCY: $0.000000 \%$

EFFECTIVE RELEASE HEIGHT : $\quad 0.00 \mathrm{~m}$

WIND SPEED $(\mathrm{h}=2 \mathrm{~m}): 1.7 \mathrm{~m} / \mathrm{s}$

STABILITY CLASS :E DEPOSITION VELOCITY $: 1.00 \mathrm{~cm} / \mathrm{s}$

RECEPTOR HEIGHT $\quad: 0.0 \mathrm{~m}$ INVERSION LAYER HEIGHT $: 200.0 \mathrm{~m}$

SAMPLE TIME $\quad: 10.000 \mathrm{~min}$

MAXIMUM DOSE DISTANCE $:<0.10 \mathrm{~km}$ MAXIMUM CEDE $:>2.6 \mathrm{E}-04 \mathrm{rem}$

$\mathrm{D}=0.03 \mathrm{~km}$

$\mathrm{DEP}=1.0 \mathrm{E}-04 \mathrm{uCi} / \mathrm{m}^{\wedge} 2$

$\mathrm{CHI}=1.0 \mathrm{E}-08(\mathrm{Ci}-\mathrm{s}) / \mathrm{m}^{\wedge} 3$

50-YR DOSE COMMITMENT:

SURFACE BONE 2.7E-02 rem RED MARROW 2.2E-03 rem LIVER 6.0E-03 rem GONADS 3.3E-04 rem EFFECTIVE DOSE EQUIVALENT $1.5 \mathrm{E}-03 \mathrm{rem} \cdot$
$\mathrm{D}=0.10 \mathrm{~km}$

$\mathrm{DEP}=1.7 \mathrm{E}-05 \mathrm{uCi} / \mathrm{m}^{\wedge} 2$

$\mathrm{CHI}=1.7 \mathrm{E}-09(\mathrm{Ci}-\mathrm{s}) / \mathrm{m}^{\wedge} 3$

50-YR DOSE COMMITMENT:

SURFACE BONE 4.6E-03 rem RED MARROW $3.8 \mathrm{E}-04 \mathrm{rem}$ LIVER $\quad 1.0 \mathrm{E}-03 \mathrm{rem}$ GONADS 5.7E-05 rem EFFECTIVE DOSE EQUTVALENT 2.6E-04 rem 
Title of Project EPHA for THM

Subject Radiological Consequence Assessment

Computer D. J. Richards

Date $\underline{09 / 11 / 96}$

Reviewed by $\underline{\text { S. A. Henderson }}$

Works Calculation 6

Sheet No. 155 of 166

Release Designation: 1-RD-140, Average

HOTSPOT 7.0 GENERAL PLUME 9-11-1996 12:10

RADIONUCLIDE : PU-238 Inhalation Class : W

HALFLIFE $: 87.740$ years

SOURCE TERM : $3.8 \mathrm{E}-07 \mathrm{Ci}$

Source Term Width: $\quad 3 \mathrm{~m}$ Source Term Height: $3 \mathrm{~m}$

RELEASE FRACTION : $1.00 \mathrm{E}+00$

FILTER EFFICIENCY: $0.000000 \%$

EFFECTIVE RELEASE HEIGHT : $\quad 0.00 \mathrm{~m}$

WIND SPEED $(\mathrm{h}=2 \mathrm{~m}): 2.5 \mathrm{~m} / \mathrm{s}$

STABILITY CLASS :C DEPOSITION VELOCITY $: 1.00 \mathrm{~cm} / \mathrm{s}$

RECEPTOR HEIGHT $\quad: 0.0 \mathrm{~m}$ INVERSION LAYER HEIGHT $: 500.0 \mathrm{~m}$

SAMPLE TIME $\quad: 10.000 \mathrm{~min}$

MAXIMUM DOSE DISTANCE $:<0.10 \mathrm{~km}$ MAXIMUM CEDE $:>6.1 \mathrm{E}-05 \mathrm{rem}$

$\mathrm{D}=0.03 \mathrm{~km}$

$\mathrm{DEP}=2.9 \mathrm{E}-05 \mathrm{uCi} / \mathrm{m}^{\wedge} 2$

$\mathrm{CHI}=2.9 \mathrm{E}-09(\mathrm{Ci}-\mathrm{s}) / \mathrm{m}^{\wedge} 3$

50-YR DOSE COMMITMENT:

SURFACE BONE 7.9E-03 rem RED MARROW 6.5E-04 rem

LIVER $1.8 \mathrm{E}-03 \mathrm{rem}$ GONADS 9.7E-05 rem EFFECTIVE DOSE EQUTVALENT 4.5E-04 rem
$\mathrm{D}=0.10 \mathrm{~km}$

$\mathrm{DEP}=4.0 \mathrm{E}-06 \mathrm{uCi} / \mathrm{m}^{\wedge} 2$

$\mathrm{CHI}=4.0 \mathrm{E}-10(\mathrm{Ci}-\mathrm{s}) / \mathrm{m}^{\wedge} 3$

50-YR DOSE COMMITMENT:

SURFACE BONE 1.1E-03 rem

RED MARROW $8.9 \mathrm{E}-05 \mathrm{rem}$

LIVER 2.4E-04 rem

GONADS 1.3E-05 rem

EFFECTIVE DOSE

EQUIVALENT 6.1E-05 rem 
Title of Project EPHA for THM

Subject Concentration Analysis for THM Hazardous Chemicals

Computer T. M. Franey

Date $\underline{09 / 16 / 96}$
Reviewed by $\mathrm{S}$. A. Henderson

Works Calculation 7

Sheet No. 1 of 577

\section{INTRODUCTION}

This calculation estimates the vapor concentrations of chemicals requiring full hazards analysis for the transportation EPHA, at 30 and 100 meter receptor points and the maximum distance to ERPG-2 concentration for each of the THM EPHA segments for adverse and average meteorological conditions. Concentrations for ALOHA default distances for maximum segment source terms are also analyzed.

\section{ASSUMPTIONS}

Releases are ground level and immediately spread to $1 \mathrm{~cm}$ depth. (Ref 1 )

SRS established $95 \%$ adverse meteorological conditions: (Ref 2)

E-stability, $1.7 \mathrm{~m} / \mathrm{s}$ wind, $200 \mathrm{~m}$ inversion and $29^{\circ} \mathrm{C}$ ambient temperature.

SRS established $50 \%$ average meteorological conditions: (Ref 2)

C-stability, $2.5 \mathrm{~m} / \mathrm{s}$ wind, $500 \mathrm{~m}$ inversion and $25^{\circ} \mathrm{C}$ ambient temperature.

Receptor points are at ground level. (Ref 2)

\section{CALCULATION}

The Areal Locations of Hazardous Atmospheres (ALOHA), dispersion model, Version 5.1, (Ref. 3) is utilized to calculate and/or back calculate, as appropriate, concentration information for the following chemicals and associated ERPG-2 values (Ref. 4).

Table 1 Chemicals, Associated ERPG, and State

\begin{tabular}{|c|c|c|c|}
\hline $\begin{array}{l}\text { Chemical Name / } \\
\text { Molecular Weight }\end{array}$ & $\begin{array}{c}\text { ERPG or } \\
\text { Equiv } \\
\text { ppm }\end{array}$ & $\begin{array}{l}\text { State } \\
\text { (S)olid } \\
\text { (L)iquidd } \\
\text { (G)as }\end{array}$ & $\begin{array}{c}\text { RELEASE } \\
\text { DESIGNATION }\end{array}$ \\
\hline Acetaldehyde / 44.1 & 200 & L & 1-RD-1 \\
\hline Acetic Acid & SEE CALC 8 & $\mathbf{L}$ & 1-RD-2 \\
\hline Acetonitrile /41.1 & SEE CALC 8 & $\mathbf{L}$ & 1-RD-3 \\
\hline Alpha-Pinene / 136.3 & SEE CALC 8 & $\mathbf{L}$ & 1-RD-4 \\
\hline Ammonium Hydroxide & 200 & $\mathbf{L}$ & $1-\mathrm{RD}-5$ \\
\hline Benzene /78.1 & 150 & $\mathbf{L}$ & $1-\mathrm{RD}-6$ \\
\hline Benzyl Alcohol & SEE CALC 8 & $\mathbf{L}$ & 1-RD-7 \\
\hline Benzyl Chloride & 10 & $\mathbf{L}$ & $1-R D-8$ \\
\hline Butyraldehyde & SEE CALC 8 & $\mathbf{L}$ & 1-RD-9 \\
\hline Carbon disulfide /76.1 & 50 & $\mathrm{~L}$ & 1-RD-10 \\
\hline Chloracetone / 92.5 & - SEE CALC 8 & $\mathbf{L}$ & $1-\mathrm{RD}-11$ \\
\hline Chlorosulfonic Acid / 116,52 & 10 & $\bar{L}$ & $1-R D-12$ \\
\hline Cyclohexane / 84.2 & 1300 & $\mathrm{~L}$ & 1-RD-13 \\
\hline Cyclohexanone /98.2 & SEE CALC 8 & L & 1-RD-14 \\
\hline Diethylene Glycol / 106.1 & SEE CAIC 8 & $\mathrm{~L}$ & 1-RD-15 \\
\hline Dioxane / 88.1 & SEE CALC 8 & $\mathbf{L}$ & $1-R D-16$ \\
\hline $\begin{array}{l}\text { Diphenyimethane-4,4-Diisocyanate } / \\
250.3\end{array}$ & SEE CALC 8 & L/S & 1-RD-17 \\
\hline Ethyl Acetate / 88.1 & 2000 & $\mathbf{L}$ & 1-RD-18 \\
\hline Ethyl benzene / 106.2 & SEE CALC 8 & $\bar{L}$ & 1-RD-19 \\
\hline Ethylene Glycol Monobutyl Ether /118.2 & 150 & $\mathbf{L}$ & $1-R D-20$ \\
\hline Fuorosilicic Acid / 144.1 & 20 & L & 1-RD-21 \\
\hline Formic Acid / 46.0 & SEE CALC 8 & $\mathbf{L}$ & 1-RD-22 \\
\hline Glycol Ether DE / 134.2 & SEE CALC 8 & $\bar{L}$ & $1-\mathrm{RD}-23$ \\
\hline Hydrochloric Acid 31\% / 36.5 & 20 & L & $1-R D-24$ \\
\hline
\end{tabular}




\section{ENGINEERING COMPUTATION SHEET}

Title of Project EPHA for THM

Subject Concentration Analysis for THM Hazardous Chemicals

Computer T. M. Franey
Date $\underline{09 / 16 / 96}$
Reviewed by S. A. Henderson

Works Calculation 7

Sheet No. 2 of 577

Table 1 cont. Chemicals, Associated ERPG, and State

\begin{tabular}{|c|c|c|c|}
\hline $\begin{array}{l}\text { Chemical Name / } \\
\text { Molecular Weight } \\
\text {. }\end{array}$ & $\begin{array}{c}\text { ERPG or } \\
\text { Equiv } \\
\text { ppm }\end{array}$ & $\begin{array}{l}\text { State } \\
\text { (S)olid } \\
\text { (L)iquid } \\
\text { (G)as }\end{array}$ & $\begin{array}{c}\text { RELEASE } \\
\text { DESIGNATION }\end{array}$ \\
\hline Hydrofluoric Acid $70 \% / 20.0$ & $20^{\circ}$ & $\mathrm{L}$ & 1-RD-25 \\
\hline Hydrogen Peroxide $/ 34.0$ & SEE CALC 8 & $\mathrm{~L}$ & 1-RD-26 \\
\hline Isopropyl Alcohol 60.1 & 1400 & L & I-RD-27 \\
\hline Methyl Alcohol / 32.0 & 1000 & $\mathbf{L}$ & 1-RD-28 \\
\hline Methyl Ethyl Ketone / 72.1 & SEE CALC 8 & $\mathbf{L}$ & 1-RD-29 \\
\hline Methyl Isobutyl Ketone / 100.2 & SEE CALC 8 & $\mathbf{L}$ & $1-R D-30$ \\
\hline Methylene Chloride / 84.9 & 750 & $\mathrm{~L}$ & $1-\mathrm{RD}-31$ \\
\hline Methyol Cellosolve /76.1 & SEE CALC 8 & $\mathbf{L}$ & $1-R D-32$ \\
\hline Monoethanolamine $/ 61.1$ & SEE CALC 8 & $\mathrm{~L}$ & 1-RD-33 \\
\hline Morpholine / 87.1 & SEE CALC 8 & $\mathrm{~L}$ & 1-RD-34 \\
\hline N Butyl Alcohol /74.1 & 50 & $\mathbf{L}$ & 1-RD-35 \\
\hline N Methyl Pyrrolidone / 99.15 & SEE CALC 8 & $\mathrm{~L}$ & 1-RD-36 \\
\hline Nitric Acid / 63.0 & 15 & $\mathbf{L}$ & 1-RD-37 \\
\hline Onyxide & SEE CALC 8 & - & 1-RD-38 \\
\hline Perchloroethylene / 165.8 & 200 & L & $1-\mathrm{RD}-39$ \\
\hline Phenol, Molten /94.1 & 50 & LS & $1-R D-40$ \\
\hline Propagyl Alcohol / 56.1 & 5 & $\mathbf{L}$ & 1-RD-41 \\
\hline Propionic Acid / 74.1 & SEE CALC 8 & $\mathbf{L}$ & 1-RD-42 \\
\hline Rodine 50 (formaldehyde) $/ 30.0$ & 10 & $\mathrm{~L}$ & $1-R D-43$ \\
\hline Sulfuric Acid / 98.1 & 10 & $\mathbf{L}$ & $1-\mathrm{RD}-44$ \\
\hline Sulfuric Acid, Fuming / 98.1 & 10 & $\mathbf{L}$ & 1-RD-45 \\
\hline Tetrahydrofuran / 72.1 & SEE CALC 8 & L & 1-RD-46 \\
\hline Toluene / 92.1 & 300 & $\mathbf{L}$ & $1-\mathrm{RD}-47$ \\
\hline Toluidine-o / 107.2 & 10 & $\mathrm{~L}$ & 1-RD-48 \\
\hline Trichloroethane-1,1,I / 133.4 & SEE CALC 8 & $L$ & 1-RD-49 \\
\hline Trichloroethylene / 131.4 & 500 & $\mathrm{~L}$ & 1-RD-50 \\
\hline Triethylamine / 101.22 & SEE CALC 8 & $\mathbf{L}$ & 1-RD-51 \\
\hline Vinyl Acetate / 86.1 & 75 & $\bar{L}$ & 1-RD-52 \\
\hline Xylenes / 106.2 & 200 & $\mathbf{L}$ & 1-RD-53 \\
\hline Benzoic Acid & SEE CALC 8 & $\mathbf{s}$ & 1-RD-54 \\
\hline Magnesium Oxide / 40.3 & 50 & $s$ & 1-RD-55 \\
\hline Silica (as respirable dust) & SEE CALC 8 & 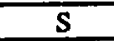 & 1-RD-56 \\
\hline Sodium Sulfhydrate $/ 56.06$ & SEE CALC 8 & $\mathbf{s}$ & 1-RD-57 \\
\hline Titanium Dioxide 179.9 & 50 & s & 1-RD-58 \\
\hline Ammonia / 17.0 & 200 & $\mathbf{G}^{*}$ & 1-RD-59 \\
\hline Chlorine / 70.9 & 3 & $G^{*}$ & $1-R D-60$ \\
\hline Trimethylamine & 25 & $G^{*}$ & $1-R D-61$ \\
\hline
\end{tabular}

\section{* Liquefied Gas}

The ALOHA computer code was utilized to develop ground level concentrations at specified receptor points. Liquids (including liquefied gases) are input as puddles, solids are input as direct releases after applying appropriate release fractions. The ALOHA code is then allowed to calculate the ground level concentration at the specified receptor point and these results are compared with the target concentration of ERPG-2. If the ALOHA projected concentration is greater than the target concentration the source term (puddle area, release rate as applicable) is reduced and the concentration re-calculated by ALOHA. If the ALOHA projected concentration is less than the target concentration the source term (puddle area, release rate as applicable) is increased and the concentration recalculated by ALOHA. This process continues until the source term producing the target concentration at the target receptor point is established.

Additional ALOHA calculations were performed to determine the footprint of the ERPG-2 concentration for the maximum allowed source term per segment for each of the materials at risk. The segment maximums were also 
Title of Project EPHA for THM

Subject Concentration Analysis for THM Hazardous Chemicals

Computer T. M. Franey
Reviewed by S. A. Henderson

Works Calculation 7

Sheet No. 3 of 577

utilized to allow ALOHA to calculate the maximum concentration at 6,000 meters for $95 \%$ meteorological conditions (ALOHA default distance based on wind speed for one hour) and 10,000 meters for $50 \%$ meteorological conditions (ALOHA default distance).

For releases lasting less than fifteen minutes, a fifteen minute peak average is calculated by:

(concentration) $\left(\frac{\text { release time }}{15 \mathrm{~min} .}\right)=$ min. avg.

The computational preference in The ALOHA Code was allowed (by selecting "Let Model Decide" option) to have the code select the spreading algorithm (Gaussian or Heavy Gas). The algorithm selected is displayed in the data output for the particular analysis.

The following formulas are utilized to convert various results to the various quantities presented.

Equation 1.

- $\mathrm{G}=\mathrm{SM} \times 2.642$

where:

G=gallons

SM= square meters at one centimeter depth

$2.642=$ gallons per square meter at one centimeter depth.

Equation 2.

- $\quad$ LBM $=\mathrm{G} \times$ SG X 8.328

where:

$\mathrm{LBM}=$ pounds mass

$G=$ gallons

SG = specific gravity

8.328 = density of water (pounds per gallon)

Data output beginning on sheet 8 shows key input values and the calculated values of interest. 


\section{ENGINEERING COMPUTATION SHEET}

Title of Project EPHA for THM

Subject Concentration Analysis for THM Hazardous Chemicals

Computer T. M. Franey

Date 09/16/96
Reviewed by S. A. Henderson

Works Calculation 7

Sheet No. 4 of 577

\section{RESULTS All results shown are centerline distance downwind.}

Table $2 \quad 95 \%$ Adverse met Consequence Assessment Results - Chemical

\begin{tabular}{|c|c|c|c|c|c|}
\hline & $\begin{array}{c}\text { Puddle Size Required } \\
\text { to produce ERPG-2 } \\
\text { Concentration @ } \\
30 \text { meters (ALERT) } \\
\left(\mathrm{ft}^{2}\right)\end{array}$ & $\begin{array}{l}\text { Puddle Size Required } \\
\text { to produce ERPG-2 } \\
\text { Concentration @ } 100 \\
\text { meters (SAE) } \\
\left(\mathrm{ft}^{2}\right)\end{array}$ & $\begin{array}{c}\text { Maximum Distance to } \\
\text { PAC (GE) for Segment } \\
1 \text { Maximum Source } \\
\text { Term }\left(24,420 \mathrm{ft}^{2}\right) \\
\text { (meters) }\end{array}$ & $\begin{array}{c}\text { Maximum Distance to } \\
\text { PAC (GE) for } \\
\text { Segment } 2 \text { Maximum } \\
\text { Source Term }(40,700 \\
\left.\mathrm{ft}^{2}\right) \\
\text { (meters) }\end{array}$ & $\begin{array}{l}\text { Maximum Distance to } \\
\text { PAC (GE) for Segment } \\
3 \text { Maximum Source } \\
\text { Term }\left(170,940, \mathrm{ft}^{2}\right) \\
\text { (meters) }\end{array}$ \\
\hline 1-RD-1 & 60 & 672 & 635 & 824 & 1400 \\
\hline 1-RD-2 & 223 & 2820 & 299 & 393 & 770 \\
\hline 1-RD-3 & 14 & 172 & 1500 & 1900 & 3000 \\
\hline 1-RD-4 & $>30000$ & $>100000$ & 27 & 34 & 56 \\
\hline 1-RD-5 & 655 & 3500 & 259 & Runtime Default & Runtime Default \\
\hline 1-RD-6 & 68 & 695 & 549 & 708 & 1200 \\
\hline 1-RD-7 & $>30000$ & $>100000$ & 27 & 34 & 56 \\
\hline 1-RD-8 & 730 & 9200 & 163 & 213 & 360 \\
\hline 1-RD-9 & 17 & 202 & 1200 & 1600 & 2500 \\
\hline 1-RD-10 & 4.4 & 31 & 2200 & 2800 & 4500 \\
\hline 1-RD-11 & 0.5 & 7 & 8900 & $>10000$ & $>10000$ \\
\hline 1-RD-12 & $>30000$ & $>100000$ & 27 & 34 & 56 \\
\hline 1-RD-13 & 1045 & 11000 & 135 & 196 & 332 \\
\hline 1-RD-14 & $>30000$ & $>100000$ & 27 & 34 & 56 \\
\hline 1-RD-15 & $>30000$ & $>100000$ & 27 & 34 & 56 \\
\hline 1-RD-16 & 200 & 1700 & 324 & 457 & 751 \\
\hline 1-RD-17 & WIP & WIP & WIP & WIP & WIP \\
\hline 1-RD-18 & 1885 & 18400 & 103 & 150 & 255 \\
\hline 1-RD-19 & 3450 & $>14182$ & 134 & 172 & 283 \\
\hline $1-R D-20$ & $>30000$ & $>100000$ & 27 & 34 & 56 \\
\hline 1-RD-21 & 24600 & $>100000$ & 21 & 38 & 61 \\
\hline $1-R D-22$ & 26 & 319 & 975 & 1400 & 2300 \\
\hline 1-RD-23 & 2650 & 31400 & 88 & 114 & 186 \\
\hline 1-RD-24 & 3230 & 39400 & 79 & 102 & 165 \\
\hline 1-RD-25 & 24600 & $>100000$ & 2.18 & 38 & 61 \\
\hline 1-RD-26 & 555 & 7500 & 179 & 232 & 390 \\
\hline 1-RD-27 & 848 & 4930 & 217 & 282 & 468 \\
\hline 1-RD-28 & 1115 & 14000 & 132 & 253 & 442 \\
\hline 1-RD-29 & 1030 & 11100 & 148 & 193 & 324 \\
\hline 1-RD-30 & 2510 & 9750 & 155 & 200 & 333 \\
\hline 1-RD-31 & 96 & 1110 & 450 & 592 & 1000 \\
\hline 1-RD-32 & $>30000$ & $>100000$ & 27 & 34 & 56 \\
\hline 1-RD-33 & $>30000$ & $>100000$ & 27 & 34 & 56 \\
\hline 1-RD-34 & $>30000$ & $>100000$ & 27 & 26 & 42 \\
\hline 1-RD-35 & 288 & 3630 & 262 & 436 & 701 \\
\hline 1-RD-36 & $>30000$ & $>100000$ & 27 & 34 & 56 \\
\hline $1-R D-37$ & 12 & 141 & 1700 & 2200 & 3500 \\
\hline 1-RD-38 & insf info for ERPG & insf info for ERPG & insf info for ERPG & insf info for ERPG & insf info for ERPG \\
\hline 1-RD-39 & 1175 & 8575 & 166 & 217 & 363 \\
\hline 1-RD-40 & 4550 & 53500 & 68 & 88 & 142 \\
\hline $1-R D-41$ & 6 & 71 & 2700 & 3700 & 5900 \\
\hline I-RD-42 & 2850 & 34300 & 85 & 109 & 177 \\
\hline 1-RD-43 & 18 & 226 & 1200 & 1600 & 2900 \\
\hline 1-RD-44 & $>30000$ & $>100000$ & 27 & 34 & 56 \\
\hline 1-RD-45 & 37 & 437 & 936 & 1200 & 1900 \\
\hline $1-R D-46$ & 425 & 4738 & 228 & 298 & 504 \\
\hline 1-RD-47 & 1810 & 7730 & 174 & 226 & 376 \\
\hline 1-RD-48 & 6260 & 73000 & 58 & 75 & 122 \\
\hline $1-R D-49$ & 690 & 7080 & 188 & 248 & 421 \\
\hline
\end{tabular}




\section{ENGINEERING COMPUTATION SHEET}

Title of Project EPHA for THM

Subject Concentration Analysis for THM Hazardous Chemicals

Computer T. M. Franey

Date 09/16/96
Reviewed by S. A. Henderson

Works Calculation 7

Sheet No. $\underline{5 \text { of } 577}$

Table 2 cont. 95\% Adverse met Consequence Assessment Results - Chemical

\begin{tabular}{|c|c|c|c|c|c|}
\hline 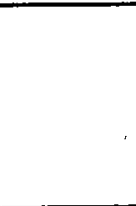 & $\begin{array}{l}\text { Puddle Size Required } \\
\text { to produce ERPG-2 } \\
\text { Concentration @ } \\
30 \text { meters (ALERT) } \\
\left(\mathrm{ft}^{2}\right)\end{array}$ & $\begin{array}{l}\text { Puddle Size Required } \\
\text { to produce ERPG-2 } \\
\text { Concentration @ } 100 \\
\text { meters (SAE) } \\
\left(\mathrm{ft}^{2}\right)\end{array}$ & $\begin{array}{l}\text { Maximum Distance to } \\
\text { PAC (GE) for Segment } \\
1 \text { Maximum Source } \\
\text { Term }\left(24,420 \mathrm{ft}^{2}\right) \\
\text { (meters) }\end{array}$ & $\begin{array}{c}\text { Maximum Distance to } \\
\text { PAC (GE) for } \\
\text { Segment } 2 \text { Maximum } \\
\text { Source Term }(40,700 \\
\mathrm{ft}^{2} \text { ) } \\
\text { (meters) }\end{array}$ & $\begin{array}{l}\text { Maximum Distance to } \\
\text { PAC (GE) for Segment } \\
3 \text { Maximum Source } \\
\text { Term (170,940 } \mathrm{ft}^{2} \text { ) } \\
\text { (meters) }\end{array}$ \\
\hline 1-RD-50 & 522 & 5760 & 206 & 271 & 456 \\
\hline 1-RD-51 & 65 & 630 & 568 & 734 & 1200 \\
\hline 1-RD-52 & 25.6 & 225 & 914 & 1200 & 1900 \\
\hline 1-RD-53 & 6720 & $>32140$ & 56 & 111 & 182 \\
\hline 1-RD-54 & 490 & 5100 & 260 & 437 & 567 \\
\hline 1-RD-55 & 490 & 5100 & 260 & 437 & 567 \\
\hline 1-RD-56 & 490 & 5100 & 260 & 437 & 567 \\
\hline 1-RD-57 & 490 & 5100 & 260 & 437 & 567 \\
\hline 1-RD-58 & 490 & 5100 & 260 & 437 & 567 \\
\hline & $\begin{array}{c}\text { Pounds Required to } \\
\text { produce ERPG-2 } \\
\text { Concentration @ } \\
30 \text { meters (ALERT) } \\
\left(\mathrm{b}_{\mathfrak{m}}\right) \\
\end{array}$ & $\begin{array}{l}\text { Pounds Required to } \\
\text { produce ERPG-2 } \\
\text { Concentration @ 100 } \\
\text { meters (SAE) } \\
\left(\mathrm{b}_{\mathrm{m}}\right) \\
\end{array}$ & $\begin{array}{c}\text { Maximum Distance to } \\
\text { PAC (GE) for Segment } \\
1 \text { Maximum Source } \\
\text { Term (1 ton) ** } \\
\text { (meters) } \\
\end{array}$ & $\begin{array}{l}\text { Maximum Distance to } \\
\text { PAC (GE) for } \\
\text { Segment } 2 \text { Maximum } \\
\text { Source Term } \\
\text { (10 tons)* (meters) }\end{array}$ & $\begin{array}{l}\text { Maximum Distance to } \\
\text { PAC (GE) for Segment } \\
3 \text { Maximum Source } \\
\text { Term (17.5 tons)** } \\
\text { (meters) } \\
\end{array}$ \\
\hline 1-RD-59 & 7 & 77 & 550 & 1800 & 2400 \\
\hline $1-R D-60$ & 0.3 & 3 & 4000 & 6300 & 6400 \\
\hline 1-RD-61 & 1 & 6 & 1200 & 3200 & 4000 \\
\hline
\end{tabular}

* ALOHA default maximum (see section 6.3.1.1)

Table $3 \quad 50 \%$ Average met Consequence Assessment Results - Chemical

\begin{tabular}{|c|c|c|c|c|c|}
\hline & $\begin{array}{c}\text { Puddle Size Required } \\
\text { to produce ERPG-2 } \\
\text { Concentration Q } \\
30 \text { meters (ALERT) } \\
\left(\mathrm{ft}^{2}\right)\end{array}$ & $\begin{array}{c}\text { Puddle Size Required } \\
\text { to produce ERPG-2 } \\
\text { Concentration @ } 100 \\
\text { meters (SAE) } \\
\left(\mathrm{ft}^{2}\right)\end{array}$ & $\begin{array}{l}\text { Maximum Distance to } \\
\text { PAC (GE) for Segment } \\
1 \text { Maximum Source } \\
\text { Term }\left(24,420 \mathrm{n}^{3}\right) \\
\text { (meters) }\end{array}$ & $\begin{array}{c}\text { Maximum Distance to } \\
\text { PAC (GE) for } \\
\text { Segment } 2 \text { Maximum } \\
\text { Source Term (40,700 } \\
\mathrm{n}^{2} \text { ) } \\
\text { (meters) }\end{array}$ & $\begin{array}{l}\text { Maximum Distance to } \\
\text { PAC (GE) for Segrment } \\
3 \text { Maximum Source } \\
\text { Term (170,940 } \mathrm{ft}^{\text {) }} \\
\text { (meters) }\end{array}$ \\
\hline 1-RD-1 & 410 & 4600 & 601 & 780 & 1300 \\
\hline 1-RD-2 & 1587 & 21400 & 107 & 135 & 211 \\
\hline 1-RD-3 & 103 & 1297 & 408 & 522 & 2200 \\
\hline 1-RD-4 & $>30000$ & $>100000$ & 27 & 34 & 56 \\
\hline 1-RD-5 & 57 & 714 & 548 & 703 & 1100 \\
\hline 1-RD-6 & 535 & $>4000$ & 499 & 641 & 1000 \\
\hline 1-RD-7 & $>30000$ & $>100000$ & 27 & 34 & 56 \\
\hline 1-RD-8 & 3700 & 50000 & 72 & 91 & 143 \\
\hline 1-RD-9 & 119 & 1485 & 997 & 1300 & 2000 \\
\hline 1-RD-10 & 32.3 & $>260$ & 1800 & 2400 & 3900 \\
\hline 1-RD-11 & 4 & 50 & 5400 & 6900 & $>10000$ \\
\hline 1-RD-12 & $>30000$ & $>100000$ & 27 & 34 & 56 \\
\hline 1-RD-13 & $>2950$ & 18700 & 115 & 149 & 247 \\
\hline 1-RD-14 & $>30000$ & $>100000$ & 27 & 34 & 56 \\
\hline 1-RD-15 & $>30000$ & $>100000$ & 27 & 34 & 56 \\
\hline 1-RD-16 & 1750 & $>17150$ & $257^{\circ}$ & 312 & 435 \\
\hline 1-RD-17 & WIP & WIP & WIP & WIP & WIP \\
\hline 1-RD-18 & 3140 & 35500 & 82 & 107 & 180 \\
\hline 1-RD-19 & 16700 & $>100000$ & 36 & 46 & 74 \\
\hline
\end{tabular}




\section{ENGINEERING COMPUTATION SHEET}

Title of Project EPHA for THM

Subject Concentration Analysis for THM Hazardous Chemicals

Reviewed by $\underline{\text { S. A. Henderson }}$

Works Calculation 7

Sheet No. $\underline{6 \text { of } 577}$

Computer T. M. Franey

Date $\underline{09 / 16 / 96}$

Table 3 cont. $\quad 50 \%$ Average met Consequence Assessment Results - Chemical

\begin{tabular}{|c|c|c|c|c|c|}
\hline & $\begin{array}{c}\text { Puddle Size Required } \\
\text { to produce ERPG-2 } \\
\text { Concentration @ } \\
\text { 30 meters (ALERT) } \\
\left(\mathrm{ft}^{2}\right)\end{array}$ & $\begin{array}{l}\text { Puddle Size Required } \\
\text { to produce ERPG-2 } \\
\text { Concentration @ } 100 \\
\text { meters (SAE) } \\
\left(\mathrm{ft}^{2}\right)\end{array}$ & $\begin{array}{l}\text { Maximum Distance to } \\
\text { PAC (GE) for Segment } \\
1 \text { Maximum Source } \\
\text { Term (24,420 } \mathrm{ft}^{2} \text { ) } \\
\text { (meters) }\end{array}$ & $\begin{array}{c}\text { Maximum Distance to } \\
\text { PAC (GE) for } \\
\text { Segment } 2 \text { Maximum } \\
\text { Source Term (40,700 } \\
\left.\mathrm{ft}^{2}\right) \\
\text { (meters) }\end{array}$ & $\begin{array}{l}\text { Maximum Distance to } \\
\text { PAC (GE) for Segment } \\
3 \text { Maximum Source } \\
\text { Term (170,940 } \mathrm{ft}^{2} \text { ) } \\
\text { (meters) }\end{array}$ \\
\hline 1-RD-20 & $>30000$ & $>100000$ & 27 & 34 & 56 \\
\hline I-RD-21 & $>30000$ & $>100000$ & 27 & 34 & 56 \\
\hline $1-R D-22$ & 168 & 2090 & 326 & 417 & 669 \\
\hline 1-RD-23 & 11180 & $>100000$ & 44 & 56 & 89 \\
\hline 1-RD-24 & 13080 & $>100000$ & 41 & 52 & 82 \\
\hline 1-RD-25 & $>30000$ & $>100000$ & 27 & 34 & 56 \\
\hline I-RD-26 & 3307 & 46580 & 74 & 94 & 147 \\
\hline 1-RD-27 & 9600 & $>36000$ & 47 & 248 & 408 \\
\hline $1-R D-28$ & 12200 & $\geq 100000$ & 42 & 53 & 85 \\
\hline 1-RD-29 & $>6250$ & 19000 & 113 & 147 & 242 \\
\hline 1-RD-30 & 10750 & $>34500$ & 39 & 112 & 148 \\
\hline 1-RD-31 & 132 & 1712 & 380 & 494 & 819 \\
\hline $1-\mathrm{RD}-32$ & $>30000$ & $>100000$ & 27 & 34 & 56 \\
\hline 1-RD-33 & $>30000$ & $>100000$ & 27 & 34 & 56 \\
\hline 1-RD-34 & $>30000$ & $>100000$ & 27 & 34 & 56 \\
\hline 1-RD-35 & 1995 & 26900 & 96 & 121 & 191 \\
\hline I-RD-36 & $>30000$ & $>100000$ & 27 & 34 & 56 \\
\hline 1-RD-37 & 82 & 1015 & 469 & 603 & 2200 \\
\hline $1-R D-38$ & insf info for ERPG & insf info for ERPG & insf info for ERPG & insf info for ERPG & insf info for ERPG \\
\hline 1-RD-39 & 9850 & 12900 & 113 & 133 & 177 \\
\hline 1-RD-40 & 14550 & $>100000$ & 39 & 49 & 78 \\
\hline $1-R D-41$ & 37 & 447 & 696 & 895 & 1500 \\
\hline $1-R D-42$ & 12850 & $>100000$ & 41 & 52 & 83 \\
\hline $1-R D-43$ & 116 & 1440 & 392 & 501 & 805 \\
\hline 1-RD-44 & $>30000$ & $>100000$ & 27 & 34 & 56 \\
\hline 1-RD-45 & 189 & 2300 & 313 & 402 & 647 \\
\hline 1-RD-46 & $>1850$ & 7500 & 181 & 236 & 392 \\
\hline $1-\mathrm{RD}-47$ & 11950 & $>22350$ & 118 & 144 & 191 \\
\hline $1-R D-48$ & 23880 & $>100000$ & 30 & 39 & \\
\hline $1-R D-49$ & 955 & 11300 & 150 & 196 & 329 \\
\hline 1-RD-50 & $>1700$ & 8200 & 174 & 225 & 375 \\
\hline $1-R D-51$ & 485 & $>3670$ & 522 & 691 & 1100 \\
\hline 1-RD-52 & 181 & $>2130$ & 786 & 1000 & 1800 \\
\hline $1-\mathrm{RD}-53$ & 30300 & $>100000$ & 27 & 34 & 56 \\
\hline 1-RD-54 & 3400 & 37200 & 100 & 191 & 267 \\
\hline 1-RD-55 & 3400 & 37200 & 100 & 191 & 267 \\
\hline 1-RD-56 & 3400 & 37200 & 100 & 191 & 267 \\
\hline 1-RD-57 & 3400 & 37200 & 100 & 191 & 267 \\
\hline 1-RD-58 & 3400 & 37200 & 100 & 191 & 267 \\
\hline , & $\begin{array}{l}\text { Pounds Required to } \\
\text { produce ERPG-2 } \\
\text { Concentration Q } \\
30 \text { meters (ALERT) }\end{array}$ & $\begin{array}{l}\text { Pounds Required to } \\
\text { produce ERPG-2 } \\
\text { Concentration @ } 100 \\
\text { meters (SAE) }\end{array}$ & $\begin{array}{c}\text { Maximum Distance to } \\
\text { PAC (GE) for Segment } \\
1 \text { Maximum Source } \\
\text { Term (1 ton) } * * \\
\text { (meters) }\end{array}$ & $\begin{array}{l}\text { Maximum Distance to } \\
\text { PAC (GE) for } \\
\text { Segment } 2 \text { Marimum } \\
\text { Source Term } \\
\text { (10 tons)** (meters) } \\
\end{array}$ & $\begin{array}{l}\text { Maximum Distance to } \\
\text { PAC (GE) for Segment } \\
3 \text { Maximum Source } \\
\text { Term (17.5 tons)** } \\
\text { (meters) }\end{array}$ \\
\hline 1-RD-59 & 47 & 558 & 190 & 570 & 750 \\
\hline 1-RD-60 & 2 & 10 & 2700 & 7500 & 9300 \\
\hline $1-R D-61$ & 9 & 110 & 1000 & 2800 & 3600 \\
\hline
\end{tabular}

- ALOHA default maximum (see section 6.3.1.1) 


\section{ENGINEERING COMPUTATION SHEET}

Title of Project EPHA for THM

Reviewed by S. A. Henderson

Works Calculation 7

Sheet No. 7 of 577

Date $\underline{09 / 16 / 96}$

Subject Concentration Analysis for THM Hazardous Chemicals

Computer T. M. Franey

\section{REFERENCES}

1. Hadlock, D. J., Standards for Development and Maintenance of a Facility Hazards Assessment, $6 Q$ Vol. 2, EMPP 6Q-001, Rev. 1, Westinghouse Savannah River Company, Savannah River Site, Aiken, SC, November 1, 1995.

2. Hadlock, D. J., Consequence Assessment for Emergency Preparedness Hazards Assessments, TP-95002, Rev. 1, May 26, 1995.

3. ALOHA, Areal Locations Of Hazardous Atmospheres, Version 5.1, User's Manuel, National Oceanic and Atmospheric Administration and U.S. Environmental Protection Agency, October, 1992.

4. Craig, D. K., ERPGs and TEELs for Chemicals of Concern at SRS, ECS-CAT-96-0071, Rev. 10, August, 1996. 
Title of Project EPHA for THM

Reviewed by $\underline{\text { S. A. Henderson }}$

Subject Concentration Analysis for THM Hazardous Chemicals

Works Calculation 7

Computer T.M. Franey

Date $\underline{09 / 16 / 96}$

Sheet No. $\underline{8 \text { of } 577}$

95\% Adverse Meteorological Conditions

ALOHA Runs 
Title of Project EPHA for THM

Subject Concentration Analysis for THM Hazardous Chemicals

Computer T.M. Franey
Date $\underline{09 / 16 / 96}$

SITE DATA INFORMATION: $\quad 1-R D-1$
Location: AIKEN, SOUTH CAROLINA

Building Air Exchanges Per Hour: 60 (User specified)

Date and Time: Fixed at July 1, 19960000 hours

CHEMICAL INFORMATION:

Chemical Name: ACETALDEHYDE

Molecular Weight: $44.05 \mathrm{~kg} / \mathrm{kmol}$

TLV-TWA: $100.00 \mathrm{ppm}$ IDLH: $10000.00 \mathrm{ppm}$

Note: Potential or confirmed human carcinogen.

Footprint Level of Concern: $200 \mathrm{ppm}$

Boiling Point: $20.40^{\circ} \mathrm{C}$

Vapor Pressure at Ambient Temperature: greater than 1 atm

Ambient Saturation Concentration: $1,000,000$ ppm or $100.0 \%$

ATMOSPHERIC INFORMATION: (MANUAL INPUT OF DATA)

Wind: 1.7 meters $/ \mathrm{sec}$ from $90^{\circ}$ true

Inversion Height: 200 meters

Stability Class: E Air Temperature: $29^{\circ} \mathrm{C}$

Relative Humidity: 50\% Ground Roughness: 100 centimeters

Cloud Cover: 1 tenths

\section{SOURCE STRENGTH INFORMATION:}

Puddle Area: 60 square feet

Average Puddle Depth: 1 centimeters

Soil Type: Default $\quad$ Ground Temperature: $29^{\circ} \mathrm{C}$

Initial Puddle Temperature: boiling point

Release Duration: ALOHA limited the duration to 1 hour

Max Computed Release Rate: 8.41 kilograms/min

Max Average Sustained Release Rate: 552 grams/min

(averaged over a minute or more)

Total Amount Released: 17.4 kilograms

FOOTPRINT INFORMATION:

Dispersion Module: Gaussian

User specified LOC: $200 \mathrm{ppm}$

Max Threat Zone for LOC: 30 meters

Max Threat Zone for IDLH: less than 10 meters(10.9 yards)

Note: Footprint was not drawn because

effects of near-field patchiness make plume

presentation unreliable for short distances.

TIME DEPENDENT INFORMATION:

Concentration Estimates at the point:

Downwind: 30 meters

Off Centerline: 0 meters

Max Concentration:

Outdoor: $201 \mathrm{ppm}$

Indoor: $201 \mathrm{ppm}$

Note: Indoor graph is shown with a dotted line.
Reviewed by S. A. Henderson

Works Calculation 7

Sheet No. 9 of 577 
Title of Project EPHA for THM

Subject Concentration Analysis for THM Hazardous Chemicals

Computer T. M. Franey

Date 09/16/96
Reviewed by $\mathrm{S}$. A. Henderson

Works Calculation 7

Sheet No. 10 of 577

SITE DATA INFORMATION: 1-RD-1

Location: AIKEN, SOUTH CAROLINA

Building Air Exchanges Per Hour: 60 (User specified)

Date and Time: Fixed at July 1, 19960000 hours

CHEMICAL INFORMATION:

Chemical Name: ACETALDEHYDE

Molecular Weight: $44.05 \mathrm{~kg} / \mathrm{kmol}$

TLV-TWA: $100.00 \mathrm{ppm}$ IDLH: $10000.00 \mathrm{ppm}$

Note: Potential or confirmed human carcinogen.

Footprint Level of Concern: $200 \mathrm{ppm}$

Boiling Point: $20.40^{\circ} \mathrm{C}$

Vapor Pressure at Ambient Temperature: greater than $1 \mathrm{~atm}$

Ambient Saturation Concentration: $1,000,000 \mathrm{ppm}$ or $100.0 \%$

ATMOSPHERIC INFORMATION: (MANUAL INPUT OF DATA)

Wind: 1.7 meters $/ \mathrm{sec}$ from $90^{\circ}$ true

Inversion Height: 200 meters

Stability Class: E Air Temperature: $29^{\circ} \mathrm{C}$

Relative Humidity: 50\% Ground Roughness: 100 centimeters

Cloud Cover: 1 tenths

SOURCE STRENGTH INFORMATTON:

Puddle Area: 672 square feet

Average Puddle Depth: 1 centimeters

Soil Type: Default Ground Temperature: $29^{\circ} \mathrm{C}$

Initial Puddle Temperature: boiling point

Release Duration: ALOHA limited the duration to 1 hour

Max Computed Release Rate: 87.3 kilograms/min

Max Average Sustained Release Rate: 5.8 kilograms/min (averaged over a minute or more)

Total Amount Released: 179 kilograms

\section{FOOTPRINT INFORMATION:}

Dispersion Module: Gaussian

User specified LOC: $200 \mathrm{ppm}$

Max Threat Zone for LOC: 100 meters

Max Threat Zone for IDLH: less than 10 meters(10.9 yards)

Note: Footprint was not drawn because

effects of near-field patchiness make plume

presentation unreliable for short distances.

\section{TIME DEPENDENT INFORMATION:}

Concentration Estimates at the point:

Downwind: 100 meters

Off Centerline: 0 meters

Max Concentration:

Outdoor: $200 \mathrm{ppm}$

Indoor: $200 \mathrm{ppm}$

Note: Indoor graph is shown with a dotted line. 
Title of Project EPHA for THM

Subject Concentration Analysis for THM Hazardous Chemicals

Computer T. M. Franey

SITE DATA INFORMATION: 1-RD-1

Location: AIKEN, SOUTH CAROLINA

Building Air Exchanges Per Hour: 60 (User specified)

Date and Time: Fixed at July 1, 19960000 hours

CHEMICAL INFORMATION:

Chemical Name: ACETALDEHYDE

Molecular Weight: $44.05 \mathrm{~kg} / \mathrm{kmol}$

TLV-TWA: 100.00 ppm IDLH: $10000.00 \mathrm{ppm}$

Note: Potential or confirmed human carcinogen.

Footprint Level of Concern: 200 ppm

Boiling Point: $20.40^{\circ} \mathrm{C}$

Vapor Pressure at Ambient Temperature: greater than $1 \mathrm{~atm}$

Ambient Saturation Concentration: $1,000,000$ ppm or $100.0 \%$

ATMOSPHERIC INFORMATION: (MANUAL INPUT OF DATA)

Wind: 1.7 meters $/ \mathrm{sec}$ from $90^{\circ}$ true

Inversion Height: 200 meters

Stability Class: $\mathrm{E} \quad$ Air Temperature: $29^{\circ} \mathrm{C}$

Relative Humidity: $50 \%$ Ground Roughness: 100 centimeters

Cloud Cover: 1 tenths

\section{SOURCE STRENGTH INFORMATION:}

Puddle Area: 24420 square feet

Average Puddle Depth: 1 centimeters

Soil Type: Default Ground Temperature: $29^{\circ} \mathrm{C}$

Initial Puddle Temperature: boiling point

Release Duration: ALOHA limited the duration to 1 hour

Max Computed Release Rate: 2,880 kilograms/min

Max Average Sustained Release Rate: 197 kilograms/min

(averaged over a minute or more)

Total Amount Released: 5,886 kilograms

\section{FOOTPRINT INFORMATION:}

Model Run: Heavy Gas

User specified LOC: $200 \mathrm{ppm}$

Max Threat Zone for LOC: 635 meters

Max Threat Zone for IDLH: 53 meters

Note: The Heavy Gas footprint is an initial screening.

For short releases it may be an overestimation.

Be sure to check concentration information at specific locations.

\section{TIME DEPENDENT INFORMATION:}

Concentration Estimates at the point:

Downwind: 100 meters

Off Centerline: 0 meters

Max Concentration:

Outdoor: $4,160 \mathrm{ppm}$

Indoor: $4,160 \mathrm{ppm}$

Note: Indoor graph is shown with a dotted line.
Reviewed by S. A. Henderson

Works Calculation 7

Sheet No. 11 of 577 
Title of Project EPHA for THM

Subject Concentration Analysis for THM Hazardous Chemicals

Computer T. M. Franey

Date $09 / 16 / 96$
.Reviewed by S. A. Henderson

Works Calculation 7

Sheet No. 12 of 577

\section{SITE DATA INFORMATION: I-RD-1}

Location: AIKEN, SOUTH CAROLINA

Building Air Exchanges Per Hour: 60 (User specified)

Date and Time: Fixed at July 1, 19960000 hours

CHEMICAL INFORMATION:

Chemical Name: ACETALDEHYDE

Molecular Weight: $44.05 \mathrm{~kg} / \mathrm{kmol}$

TLV-TWA: $100.00 \mathrm{ppm}$ IDLH: $10000.00 \mathrm{ppm}$

Note: Potential or confirmed human carcinogen.

Footprint Level of Concern: 200 ppm

Boiling Point: $20.40^{\circ} \mathrm{C}$

Vapor Pressure at Ambient Temperature: greater than 1 atm

Ambient Saturation Concentration: $1,000,000$ ppm or $100.0 \%$

ATMOSPHERIC INFORMATION: (MANUAL INPUT OF DATA)

Wind: 1.7 meters $/ \mathrm{sec}$ from $90^{\circ}$ true

Inversion Height: 200 meters

Stability Class: E Air Temperature: $29^{\circ} \mathrm{C}$

Relative Humidity: $50 \%$ Ground Roughness: 100 centimeters

Cloud Cover: 1 tenths

SOURCE STRENGTH INFORMATION:

Puddle Area: 40700 square feet

Average Puddle Depth: 1 centimeters

Soil Type: Default Ground Temperature: $29^{\circ} \mathrm{C}$

Initial Puddle Temperature: boiling point

Release Duration: ALOHA limited the duration to 1 hour

Max Computed Release Rate: 4,740 kilograms/min

Max Average Sustained Release Rate: 325 kilograms/min

(averaged over a minute or more)

Total Amount Released: 9,693 kilograms

FOOTPRINT INFORMATION:

Model Run: Heavy Gas

User specified LOC: $200 \mathrm{ppm}$

Max Threat Zone for LOC: 824 meters

Max Threat Zone for IDLH: 71 meters

Note: The Heavy Gas footprint is an initial screening.

For short releases it may be an overestimation.

Be sure to check concentration information at specific locations.

TIME DEPENDENT INFORMATION:

Concentration Estimates at the point:

Downwind: 100 meters

Off Centerline: 0 meters

Max Concentration:

Outdoor: $6,410 \mathrm{ppm}$

Indoor: $6,410 \mathrm{ppm}$

Note: Indoor graph is shown with a dotted line. 
Title of Project EPHA for THM

Subject Concentration Analysis for THM Hazardous Chemicals

Computer T. M. Franey

Date 09/16/96
Reviewed by $\mathrm{S}$. A. Henderson

Works Calculation 7

Sheet No. 13 of 577

SITE DATA INFORMATION: 1-RD-1

Location: AIKEN, SOUTH CAROLINA

Building Air Exchanges Per Hour: 60 (User specified)

Date and Time: Fixed at July 1, 19960000 hours

CHEMICAL INFORMATION:

Chemical Name: ACETALDEHYDE

- Molecular Weight: $44.05 \mathrm{~kg} / \mathrm{kmol}$

TLV-TWA: $100.00 \mathrm{ppm}$ IDLH: $10000.00 \mathrm{ppm}$

Note: Potential or confirmed human carcinogen.

Footprint Level of Concern: 200 ppm

Boiling Point: $20.40^{\circ} \mathrm{C}$

Vapor Pressure at Ambient Temperature: greater than $1 \mathrm{~atm}$

Ambient Saturation Concentration: $1,000,000$ ppm or $100.0 \%$

\section{ATMOSPHERIC INFORMATION: (MANUAL INPUT OF DATA)}

Wind: 1.7 meters $/ \mathrm{sec}$ from $90^{\circ}$ true

Inversion Height: 200 meters

Stability Class: E Air Temperature: $29^{\circ} \mathrm{C}$

Relative Humidity: 50\% Ground Roughness: 100 centimeters

Cloud Cover: 1 tenths

SOURCE STRENGTH INFORMATION:

Puddle Area: 10000 square meters

Average Puddle Depth: 1 centimeters

Soil Type: Default Ground Temperature: $29^{\circ} \mathrm{C}$

Initial Puddle Temperature: boiling point

Release Duration: ALOHA limited the duration to 1 hour

Max Computed Release Rate: 12,300 kilograms/min

Max Average Sustained Release Rate: 847 kilograms/min

(averaged over a minute or more)

Total Amount Released: 25,079 kilograms

FOOTPRINT INFORMATION:

Model Run: Heavy Gas

User specified LOC: $200 \mathrm{ppm}$

Max Threat Zone for LOC: 1.4 kilometers

Max Threat Zone for IDLH: 125 meters

Note: The Heavy Gas footprint is an initial screening.

For short releases it may be an overestimation.

Be sure to check concentration information at specific locations.

\section{TIME DEPENDENT INFORMATION:}

Concentration Estimates at the point:

Downwind: 100 meters

Off Centerline: 0 meters

Max Concentration:

Outdoor: $14,000 \mathrm{ppm}$

Indoor: $14,000 \mathrm{ppm}$

Note: Indoor graph is shown with a dotted line. 
Title of Project EPHA for THM

Subject Concentration Analysis for THM Hazardous Chemicals

Computer T. M. Franey
Reviewed by S.A. Henderson

Works Calculation 7

Sheet No. 14 of 577

\section{SITE DATA INFORMATION: 1-RD-2}

Location: AIKEN, SOUTH CAROLINA

Building Air Exchanges Per Hour: 60 (User specified)

Date and Time: Fixed at July 1, 19960000 hours

CHEMICAL INFORMATION:

Chemical Name: ACETIC ACID

Molecular Weight: $60.05 \mathrm{~kg} / \mathrm{kmol}$

TLV-TWA: $10.00 \mathrm{ppm}$ DLH: $1000.00 \mathrm{ppm}$

Footprint Level of Concern: $50 \mathrm{ppm}$

Boiling Point: $117.90^{\circ} \mathrm{C}$

Vapor Pressure at Ambient Temperature: $0.026 \mathrm{~atm}$

Ambient Saturation Concentration: 26,293 ppm or $2.63 \%$

ATMOSPHERIC INFORMATION: (MANUAL INPUT OF DATA)

Wind: 1.7 meters $/ \mathrm{sec}$ from $90^{\circ}$ true

Inversion Height: 200 meters

Stability Class: E Air Temperature: $29^{\circ} \mathrm{C}$

Relative Humidity: $50 \%$ Ground Roughness: 100 centimeters

Cloud Cover: 1 tenths

\section{SOURCE STRENGTH INFORMATION:}

Puddle Area: 223 square feet

Average Puddle Depth: 1 centimeters

Soil Type: Default $\quad$ Ground Temperature: $29^{\circ} \mathrm{C}$

Initial Puddle Temperature: Ground temperature

Release Duration: ALOHA limited the duration to 1 hour

Max Computed Release Rate: 269 grams/min

Max Average Sustained Release Rate: 250 grams $/ \mathrm{min}$

(averaged over a minute or more)

Total Amount Released: 13.8 kilograms

\section{FOOTPRINT INFORMATION:}

Dispersion Module: Gaussian

User specified LOC: $50 \mathrm{ppm}$

Max Threat Zone for LOC: 30 meters

Max Threat Zone for IDLH: less than 10 meters(10.9 yards)

Note: Footprint was not drawn because effects of near-field patchiness make plume presentation unreliable for short distances.

\section{TIME DEPENDENT INFORMATION:}

Concentration Estimates at the point:

Downwind: 30 meters

Off Centerline: 0 meters

Max Concentration:

Outdoor: $50 \mathrm{ppm}$

Indoor: $50 \mathrm{ppm}$

Note: Indoor graph is shown with a dotted line. 
Title of Project EPHA for THM

Subject Concentration Analysis for THM Hazardous Chemicals

Computer T. M. Franey
Reviewed by S. A. Henderson

Works Calculation 7

Sheet No. 15 of 577

SITE DATA INFORMATION: 1-RD-2

Location: AIKEN, SOUTH CAROLINA

Building Air Exchanges Per Hour: 60 (User specified)

Date and Time: Fixed at July 1, 19960000 hours

CHEMICAL INFORMATION:

Chemical Name: ACETIC ACID

Molecular Weight: $60.05 \mathrm{~kg} / \mathrm{kmol}$

TLV-TWA: $10.00 \mathrm{ppm}$ IDLH: $1000.00 \mathrm{ppm}$

Footprint Level of Concern: 50 ppm

Boiling Point: $117.90^{\circ} \mathrm{C}$

Vapor Pressure at Ambient Temperature: $0.026 \mathrm{~atm}$

Ambient Saturation Concentration: 26,293 ppm or $2.63 \%$

ATMOSPHERIC INFORMATION: (MANUAL INPUT OF DATA)

Wind: 1.7 meters $/ \mathrm{sec}$ from $90^{\circ}$ true

Inversion Height: 200 meters

Stability Class: E Air Temperature: $29^{\circ} \mathrm{C}$

Relative Humidity: $50 \%$ Ground Roughness: 100 centimeters

Cloud Cover: 1 tenths

SOURCE STRENGTH INFORMATION:

Puddle Area: 2820 square feet

Average Puddle Depth: 1 centimeters

Soil Type: Default Ground Temperature: $29^{\circ} \mathrm{C}$

Initial Puddle Temperature: Ground temperature

Release Duration: ALOHA limited the duration to 1 hour

Max Computed Release Rate: 2.97 kilograms/min

Max Average Sustained Release Rate: 2.78 kilograms/min

(averaged over a minute or more)

Total Amount Released: 154 kilograms

FOOTPRINT INFORMATION:

Dispersion Module: Gaussian

User specified LOC: $50 \mathrm{ppm}$

Max Threat Zone for LOC: 100 meters

Max Threat Zone for IDLH: 13 meters

Note: Footprint was not drawn because effects of near-field patchiness make plume presentation unreliable for short distances.

\section{TIME DEPENDENT INFORMATION:}

Concentration Estimates at the point:

Downwind: 100 meters

Off Centerline: 0 meters

Max Concentration:

Outdoor: $50 \mathrm{ppm}$

Indoor: $50 \mathrm{ppm}$

Note: Indoor graph is shown with a dotted line. 


\section{ENGINEERING COMPUTATION SHEET}

Title of Project EPHA for THM

Subject Concentration Analysis for THM Hazardous Chemicals

Computer T. M. Franey

Date $\underline{09 / 16 / 96}$
Reviewed by S. A. Henderson

Works Calculation 7

Sheet No. 16 of 577

SITE DATA INFORMATION: 1-RD-2

Location: AIKEN, SOUTH CAROLINA

Building Air Exchanges Per Hour: 60 (User specified)

Date and Time: Fixed at July 1, 19960000 hours

CHEMICAL INFORMATION:

Chemical Name: ACETIC ACID

Molecular Weight: $60.05 \mathrm{~kg} / \mathrm{kmol}$

TLV-TWA: $10.00 \mathrm{ppm}$ IDLH: $1000.00 \mathrm{ppm}$

Footprint Level of Concern: $50 \mathrm{ppm}$

Boiling Point: $117.90^{\circ} \mathrm{C}$

Vapor Pressure at Ambient Temperature: 0.026 atm

Ambient Saturation Concentration: 26,293 ppm or $2.63 \%$

ATMOSPHERIC INFORMATION: (MANUAL INPUT OF DATA)

Wind: 1.7 meters $/ \mathrm{sec}$ from $90^{\circ}$ true

Inversion Height: 200 meters

Stability Class: E Air Temperature: $29^{\circ} \mathrm{C}$

Relative Humidity: 50\% Ground Roughness: 100 centimeters

Cloud Cover: 1 tenths

SOURCE STRENGTH INFORMATION:

Puddle Area: 24420 square feet

Average Puddle Depth: 1 centimeters

Soil Type: Default Ground Temperature: $29^{\circ} \mathrm{C}$

Initial Puddle Temperature: Ground temperature

Release Duration: ALOHA limited the duration to 1 hour

Max Computed Release Rate: 23.2 kilograms/min

Max Average Sustained Release Rate: 21.8 kilograms/min

(averaged over a minute or more)

Total Amount Released: 1,213 kilograms

FOOTPRINT INFORMATION:

Dispersion Module: Gaussian

User specified LOC: $50 \mathrm{ppm}$

Max Threat Zone for LOC: 299 meters

Max Threat Zone for IDLH: 34 meters

TIME DEPENDENT INFORMATION:

Concentration Estimates at the point:

Downwind: 100 meters

Off Centerline: 0 meters

Max, Concentration:

Outdoor: $146 \mathrm{ppm}$

Indoor: $146 \mathrm{ppm}$

Note: Indoor graph is shown with a dotted line. 
Title of Project EPHA for THM

Subject Concentration Analysis for THM Hazardous Chemicals .

Computer T. M. Franey
Date $\underline{09 / 16 / 96}$
Reviewed by S. A. Henderson

Works Calculation 7

Sheet No. 17 of 577

\section{SITE DATA INFORMATION: 1-RD-2}

Location: AIKEN, SOUTH CAROLINA

Building Air Exchanges Per Hour: 60 (User specified)

Date and Time: Fixed at July 1, 19960000 hours

CHEMICAL INFORMATION:

Chemical Name: ACETIC ACID

Molecular Weight: $60.05 \mathrm{~kg} / \mathrm{kmol}$

TLV-TWA: $10.00 \mathrm{ppm}$ IDLH: $1000.00 \mathrm{ppm}$

Footprint Level of Concern: 50 ppm

Boiling Point: $117.90^{\circ} \mathrm{C}$

Vapor Pressure at Ambient Temperature: $0.026 \mathrm{~atm}$

Ambient Saturation Concentration: 26,293 ppm or $2.63 \%$

\section{ATMOSPHERIC INFORMATION: (MANUAL INPUT OF DATA)}

Wind: 1.7 meters $/ \mathrm{sec}$ from $90^{\circ}$ true

Inversion Height: 200 meters

Stability Class: E Air Temperature: $29^{\circ} \mathrm{C}$

Relative Humidity: 50\% Ground Roughness: 100 centimeters

Cloud Cover: 1 tenths

\section{SOURCE STRENGTH INFORMATION:}

Puddle Area: 40700 square feet

Average Puddle Depth: 1 centimeters

Soil Type: Default Ground Temperature: $29^{\circ} \mathrm{C}$

Initial Puddle Temperature: Ground temperature

Release Duration: ALOHA limited the duration to 1 hour

Max Computed Release Rate: 37.7 kilograms $/ \mathrm{min}$

Max Average Sustained Release Rate: 35.5 kilograms/min

(averaged over a minute or more)

Total Amount Released: 1,979 kilograms

FOOTPRINT INFORMATION:

Dispersion Module: Gaussian .

User specified LOC: $50 \mathrm{ppm}$

Max Threat Zone for LOC: 393 meters

Max Threat Zone for IDLH: 44 meters

TIME DEPENDENT INFORMATION:

Concentration Estimates at the point:

Downwind: 100 meters

Off Centerline: 0 meters

Max Concentration:

Outdoor: $211 \mathrm{ppm}$

Indoor: $211 \mathrm{ppm}$

Note: Indoor graph is shown with a dotted line. 
Title of Project EPHA for THM

Subject Concentration Analysis for THM Hazardous Chemicals

Computer T. M. Franey
Date $\underline{09 / 16 / 96}$
Reviewed by S. A. Henderson

Works Calculation 7

Sheet No. 18 of 577

SITE DATA INFORMATION: 1-RD-2

Location: AIKEN, SOUTH CAROLINA

Building Air Exchanges Per Hour: 60 (User specified)

Date and Time: Fixed at July 1, 19960000 hours

CHEMICAL INFORMATION:

Chemical Name: ACETIC ACID

Molecular Weight: $60.05 \mathrm{~kg} / \mathrm{kmol}$

TLV-TWA: $10.00 \mathrm{ppm}$ IDLH: $1000.00 \mathrm{ppm}$

Footprint Level of Concern: $50 \mathrm{ppm}$

Boiling Point: $117.90^{\circ} \mathrm{C}$

Vapor Pressure at Ambient Temperature: $0.026 \mathrm{~atm}$

Ambient Saturation Concentration: 26,293 ppm or $2.63 \%$

ATMOSPHERIC INFORMATION: (MANUAL INPUT OF DATA)

Wind: 1.7 meters $/ \mathrm{sec}$ from $90^{\circ}$ true

Inversion Height: 200 meters

Stability Class: E Air Temperature: $29^{\circ} \mathrm{C}$

Relative Humidity: $50 \%$ Ground Roughness: 100 centimeters

Cloud Cover: 1 tenths

SOURCE STRENGTH INFORMATION:

Puddle Area: 10000 square meters

Average Puddle Depth: 1 centimeters

Soil Type: Default Ground Temperature: $29^{\circ} \mathrm{C}$

Initial Puddle Temperature: Ground temperature

Release Duration: ALOHA limited the duration to 1 hour

Max Computed Release Rate: 95.6 kilograms/min

Max Average Sustained Release Rate: 90 kilograms/min

(averaged over a minute or more)

Total Amount Released: 5,029 kilograms

\section{FOOTPRINT INFORMATION:}

Model Run: Heavy Gas

User specified LOC: $50 \mathrm{ppm}$

Max Threat Zone for LOC: 770 meters

Max Threat Zone for IDLH: 79 meters

Note: The Heavy Gas footprint is an initial screening.

For short releases it may be an overestimation.

Be sure to check concentration information at specific locations.

TIME DEPENDENT INFORMATION:

Concentration Estimates at the point:

Downwind: 100 meters

Off Centerline: 0 meters

Max Concentration:

Outdoor: 804 ppm

Indoor: $804 \mathrm{ppm}$

Note: Indoor graph is shown with a dotted line. 
Title of Project EPHA for THM

Subject Concentration Analysis for THM Hazardous Chemicals

Computer T. M. Franey
Reviewed by $\underline{\text { S. A. Henderson }}$

Works Calculation 7

Sheet No. 19 of 577

\title{
SITE DATA INFORMATION: 1-RD-3 \\ Location: AIKEN, SOUTH CAROLINA \\ Building Air Exchanges Per Hour: 60 (User specified) \\ Date and Time: Fixed at July 1, 19960000 hours
}

CHEMICAL INFORMATION:

Chemical Name: ACETONTTRILE

Molecular Weight: $41.05 \mathrm{~kg} / \mathrm{kmol}$

TLV-TWA: $40.00 \mathrm{ppm}$ IDLH: $4000.00 \mathrm{ppm}$

Footprint Level of Concern: 35 ppm

Boiling Point: $81.60^{\circ} \mathrm{C}$

Vapor Pressure at Ambient Temperature: $0.14 \mathrm{~atm}$

Ambient Saturation Concentration: $145,909 \mathrm{ppm}$ or $14.6 \%$
ATMOSPHERIC INFORMATION: (MANUAL INPUT OF DATA)
Wind: 1.7 meters $/ \mathrm{sec}$ from $90^{\circ}$ true
Inversion Height: 200 meters
Stability Class: E Air Temperature: $29^{\circ} \mathrm{C}$
Relative Humidity: 50\% Ground Roughness: 100 centimeters
Cloud Cover: 1 tenths

\section{SOURCE STRENGTH INFORMATION:}

Puddle Area: 14 square feet

Average Puddle Depth: 1 centimeters

Soil Type: Default Ground Temperature: $29^{\circ} \mathrm{C}$

Initial Puddle Temperature: Ground temperature

Release Duration: ALOHA limited the duration to 1 hour

Max Computed Release Rate: 103 grams/min

Max Average Sustained Release Rate: $80.7 \mathrm{grams} / \mathrm{min}$ (averaged over a minute or more)

Total Amount Released: 3.92 kilograms

\author{
FOOTPRINT INFORMATTON: \\ Dispersion Module: Gaussian \\ User specified LOC: $35 \mathrm{ppm}$ \\ Max Threat Zone for LOC: 30 meters \\ Max Threat Zone for IDLH: less than 10 meters(10.9 yards) \\ Note: Footprint was not drawn because \\ effects of near-field patchiness make plume \\ presentation unreliable for short distances.
}

\section{TIME DEPENDENT INFORMATION:}

Concentration Estimates at the point:

Downwind: 30 meters

Off Centerline: 0 meters

Max Concentration:

Outdoor: $34.9 \mathrm{ppm}$

Indoor: $34.9 \mathrm{ppm}$

Note: Indoor graph is shown with a dotted line. 
Title of Project EPHA for THM

Subject Concentration Analysis for THM Hazardous Chemicals

Computer T. M. Franey

Date $\underline{09 / 16 / 96}$

Reviewed by $\mathrm{S}$. A. Henderson

Works Calculation 7

Sheet No. 20 of 577

SITE DATA INFORMATION: 1-RD-3

Location: AIKEN, SOUTH CAROLINA

Building Air Exchanges Per Hour: 60 (User specified)

Date and Time: Fixed at July 1, 19960000 hours

\section{CHEMICAL INFORMATION:}

Chemical Name: ACETONITRIE

Molecular Weight: $41.05 \mathrm{~kg} / \mathrm{kmol}$

TLV-TWA: $40.00 \mathrm{ppm}$ IDLH: $4000.00 \mathrm{ppm}$

Footprint Level of Concern: $35 \mathrm{ppm}$

Boiling Point: $81.60^{\circ} \mathrm{C}$

Vapor Pressure at Ambient Temperature: 0.14 atm

Ambient Saturation Concentration: $145,909 \mathrm{ppm}$ or $14.6 \%$

\section{ATMOSPHERIC INFORMATTON: (MANUAL INPUT OF DATA)}

Wind: 1.7 meters $/ \mathrm{sec}$ from $90^{\circ}$ true

Inversion Height: 200 meters

Stability Class: E Air Temperature: $29^{\circ} \mathrm{C}$

Relative Humidity: 50\% Ground Roughness: 100 centimeters

Cloud Cover: 1 tenths

\section{SOURCE STRENGTH INFORMATION:}

Puddle Area: 172 square feet

Average Puddle Depth: 1 centimeters

Soil Type: Default Ground Temperature: $29^{\circ} \mathrm{C}$

Initial Puddle Temperature: Ground temperature

Release Duration: ALOHA limited the duration to 1 hour

Max Computed Release Rate: 1.07 kilograms/min

Max Average Sustained Release Rate: 858 grams/min

(averaged over a minute or more)

Total Amount Released: 42.4 kilograms

FOOTPRINT INFORMATION:

Dispersion Module: Gaussian

User specified LOC: $35 \mathrm{ppm}$

Max Threat Zone for LOC: 100 meters

Max Threat Zone for IDLH: less than 10 meters(10.9 yards)

\section{TIME DEPENDENT INFORMATION:}

Concentration Estimates at the point:

Downwind: 100 meters

Off Centerline: 0 meters

Max Concentration:

Outdoor: $35.1 \mathrm{ppm}$

Indoor: $35.1 \mathrm{ppm}$

Note: Indoor graph is shown with a dotted line. 
Title of Project EPHA for THM

Subject Concentration Analysis for THM Hazardous Chemicals

Computer T. M. Franey

Date 09/16/96

Reviewed by $\mathrm{S}$. A. Henderson

Works Calculation 7

Sheet No. 21 of 577

SITE DATA INFORMATION: $\quad$ 1-RD-3

Location: AIKEN, SOUTH CAROLINA

Building Air Exchanges Per Hour: 60 (User specified)

Date and Time: Fixed at July 1, 19960000 hours

CHEMICAL INFORMATION:

Chemical Name: ACETONITRIE

Molecular Weight: $41.05 \mathrm{~kg} / \mathrm{kmol}$

TLV-TWA: $40.00 \mathrm{ppm}$ IDLH: $4000.00 \mathrm{ppm}$

Footprint Level of Concern: 35 ppm

Boiling Point: $81.60^{\circ} \mathrm{C}$

Vapor Pressure at Ambient Temperature: $0.14 \mathrm{~atm}$

Ambient Saturation Concentration: $145,909 \mathrm{ppm}$ or $14.6 \%$

ATMOSPHERIC INFORMATION: (MANUAL INPUT OF DATA)

Wind: 1.7 meters $/ \mathrm{sec}$ from $90^{\circ}$ true

Inversion Height: 200 meters

Stability Class: E Air Temperature: $29^{\circ} \mathrm{C}$

Relative Humidity: $50 \%$ Ground Roughness: 100 centimeters

Cloud Cover: 1 tenths

SOURCE STRENGTH INFORMATION:

Puddle Area: 24420 square feet

Average Puddle Depth: 1 centimeters

Soil Type: Default Ground Temperature: $29^{\circ} \mathrm{C}$

Initial Puddle Temperature: Ground temperature

Release Duration: ALOHA limited the duration to 1 hour

Max Computed Release Rate: 115 kilograms/min

Max Average Sustained Release Rate: 96.4 kilograms/min

(averaged over a minute or more)

Total Amount Released: 4,876 kilograms

FOOTPRINT INFORMATION:

Model Run: Heavy Gas

User specified LOC: $35 \mathrm{ppm}$

Max Threat Zone for LOC: 1.5 kilometers

Max Threat Zone for IDLH: 60 meters

Note: The Heavy Gas footprint is an initial screening.

For short releases it may be an overestimation.

Be sure to check concentration information at specific locations.

\section{TIME DEPENDENT INFORMATION:}

Concentration Estimates at the point:

Downwind: 100 meters

Off Centerline: 0 meters

Max Concentration:

Outdoor: $2,070 \mathrm{ppm}$

Indoor: $2,070 \mathrm{ppm}$

Note: Indoor graph is shown with a dotted line. 
Title of Project EPHA for THM

Subject Concentration Analysis for THM Hazardous Chemicals

Computer T. M. Franey
Reviewed by S. A. Henderson

Works Calculation 7

Sheet No. 22 of 577

SITE DATA INFORMATION: 1-RD-3

Location: AIKEN, SOUTH CAROLINA

Building Air Exchanges Per Hour: 60 (User specified)

Date and Time: Fixed at July 1, 19960000 hours

CHEMICAL INFORMATION:

Chemical Name: ACETONITRILE

Molecular Weight: $41.05 \mathrm{~kg} / \mathrm{kmol}$

TLV-TWA: $40.00 \mathrm{ppm}$ IDLH: $4000.00 \mathrm{ppm}$

Footprint Level of Concern: $35 \mathrm{ppm}$

Boiling Point: $81.60^{\circ} \mathrm{C}$

Vapor Pressure at Ambient Temperature: 0.14 atm

Ambient Saturation Concentration: 145,909 ppm or $14.6 \%$

ATMOSPHERIC INFORMATION: (MANUAL INPUT OF DATA)

Wind: 1.7 meters $/ \mathrm{sec}$ from $90^{\circ}$ true

Inversion Height: 200 meters

Stability Class: E Air Temperature: $29^{\circ} \mathrm{C}$

Relative Humidity: 50\% Ground Roughness: 100 centimeters

Cloud Cover: 1 tenths

SOURCE STRENGTH INFORMATION:

Puddle Area: 40700 square feet

Average Puddle Depth: 1 centimeters

Soil Type: Default Ground Temperature: $29^{\circ} \mathrm{C}$

Initial Puddle Temperature: Ground temperature

Release Duration: ALOHA limited the duration to 1 hour

Max Computed Release Rate: 188 kilograms/min

Max Average Sustained Release Rate: 157 kilograms/min

(averaged over a minute or more)

Total Amount Released: 7,970 kilograms

FOOTPRINT INFORMATION:

Model Run: Heavy Gas

User specified LOC: $35 \mathrm{ppm}$

Max Threat Zone for LOC: 1.9 kilometers

Max Threat Zone for IDLH: 78 meters

Note: The Heavy Gas footprint is an initial screening.

For short releases it may be an overestimation.

Be sure to check concentration information at specific locations.

TIME DEPENDENT INFORMATION:

Concentration Estimates at the point:

Downwind: 100 meters

Off Centerline: 0 meters

Max Concentration:

Outdoor: $3,020 \mathrm{ppm}$

Indoor: $3,020 \mathrm{ppm}$

Note: Indoor graph is shown with a dotted line. 
Title of Project EPHA for THM

Subject Concentration Analysis for THM Hazardous Chemicals

Computer T. M. Franey
Reviewed by S. A. Henderson

Works Calculation 7

Sheet No. 23 of 577

SITE DATA INFORMATION: 1-RD-3

Location: AIKEN, SOUTH CAROLINA

Building Air Exchanges Per Hour: 60 (User specified)

Date and Time: Fixed at July 1, 19960000 hours

CHEMICAL INFORMATION:

Chemical Name: ACETONITRIE

Molecular Weight: $41.05 \mathrm{~kg} / \mathrm{kmol}$

TLV-TWA: $40.00 \mathrm{ppm}$ IDLH: $4000.00 \mathrm{ppm}$

Footprint Level of Concern: 35 ppm

Boiling Point: $81.60^{\circ} \mathrm{C}$

Vapor Pressure at Ambient Temperature: $0.14 \mathrm{~atm}$

Ambient Saturation Concentration: 145,909 ppm or $14.6 \%$

ATMOSPHERIC INFORMATION: (MANUAL INPUT OF DATA)

Wind: 1.7 meters $/ \mathrm{sec}$ from $90^{\circ}$ true

Inversion Height: 200 meters

Stability Class: E Air Temperature: $29^{\circ} \mathrm{C}$

Relative Humidity: 50\% Ground Roughness: 100 centimeters

Cloud Cover: 1 tenths

\section{SOURCE STRENGTH INFORMATION:}

Puddle Area: 10000 square meters

Average Puddle Depth: 1 centimeters

Soil Type: Default Ground Temperature: $29^{\circ} \mathrm{C}$

Initial Puddle Temperature: Ground temperature

Release Duration: ALOHA limited the duration to 1 hour

Max Computed Release Rate: 474 kilograms/min

Max Average Sustained Release Rate: 400 kilograms/min

(averaged over a minute or more)

Total Amount Released: 20,338 kilograms

FOOTPRINT INFORMATION:

Model Run: Heavy Gas

User specified LOC: $35 \mathrm{ppm}$

Max Threat Zone for LOC: 3.0 kilometers

Max Threat Zone for IDLH: 134 meters

Note: The Heavy Gas footprint is an initial screening.

For short releases it may be an overestimation.

Be sure to check concentration information at specific locations.

\section{TIME DEPENDENT INFORMATION:}

Concentration Estimates at the point:

Downwind: 100 meters

Off Centerline: 0 meters

Max Concentration:

Outdoor: $5,960 \mathrm{ppm}$

Indoor: $5,960 \mathrm{ppm}$

Note: Indoor graph is shown with a dotted line. 
Title of Project EPHA for THM

Subject Concentration Analysis for THM Hazardous Chemicals

Computer T. M. Franey

Date $\underline{09 / 16 / 96}$
Reviewed by S. A. Henderson

Works Calculation 7

Sheet No. 24 of 577

\section{$1-\mathrm{RD}-4$}

SITE DATA INFORMATION:

Location: AIKEN, SOUTH CAROLINA

Building Air Exchanges Per Hour: 60 (User specified)

Date and Time: Fixed at July 1, 19960000 hours

\section{CHEMICAL INFORMATION:}

Chemical Name: TERPINOLENE / ALPHA-PINENE / TURP

Molecular Weight: $136.24 \mathrm{~kg} / \mathrm{kmol}$

TLV-TWA: -unavail- IDLH: -unavail-

Footprint Level of Concern: 500 ppm

Boiling Point: $185.00^{\circ} \mathrm{C}$

Vapor Pressure at Ambient Temperature: $0.0010 \mathrm{~atm}$

Ambient Saturation Concentration: $1,056 \mathrm{ppm}$ or $0.11 \%$

ATMOSPHERIC INFORMATION: (MANUAL INPUT OF DATA)

Wind: 1.7 meters $/ \mathrm{sec}$ from $90^{\circ}$ true

Inversion Height: 200 feet

\section{Stability Class: E Air Temperature: $29^{\circ} \mathrm{C}$}

Relative Humidity: 50\% Ground Roughness: Urban or forest

Cloud Cover: 1 tenths

\section{SOURCE STRENGTH INFORMATION:}

Puddle Area: 30000 square feet

Average Puddle Depth: 1 centimeters

Soil Type: Default Ground Temperature: $29^{\circ} \mathrm{C}$

Initial Puddle Temperature: Ground temperature

Release Duration: ALOHA limited the duration to 1 hour

Max Computed Release Rate: 7.12 kilograms/min

Max Average Sustained Release Rate: 6.91 kilograms/min (averaged over a minute or more)

Total Amount Released: 400 kilograms

\section{TIME DEPENDENT INFORMATION:}

Concentration Estimates at the point:

Downwind: 30 meters

Off Centerline: 0 meters

Max Concentration:

Outdoor: 186 ppm 


\section{ENGINEERING COMPUTATION SHEET}

Title of Project EPHA for THM

Subject Concentration Analysis for THM Hazardous Chemicals

Computer T. M. Franey

Date $09 / 16 / 96$

1-RD-4 SITE DATA INFORMATION:

Location: AIKEN, SOUTH CAROLINA

Building Air Exchanges Per Hour: 60 (User specified)

Date and Time: Fixed at July 1, 19960000 hours

\section{CHEMICAL INFORMATION:}

Chemical Name: TERPINOLENE / ALPHA-PINENE / TURP

Molecular Weight: $136.24 \mathrm{~kg} / \mathrm{kmol}$

TLV-TWA: -unavail- IDLH: -unavail-

Footprint Level of Concern: 500 ppm

Boiling Point: $185.00^{\circ} \mathrm{C}$

Vapor Pressure at Ambient Temperature: $0.0010 \mathrm{~atm}$

Ambient Saturation Concentration: $1,056 \mathrm{ppm}$ or $0.11 \%$

\section{ATMOSPHERIC INFORMATION: (MANUAL INPUT OF DATA)}

Wind: 1.7 meters $/ \mathrm{sec}$ from $90^{\circ}$ true

Inversion Height: 200 feet

Stability Class: E Air Temperature: $29^{\circ} \mathrm{C}$

Relative Humidity: 50\% Ground Roughness: Urban or forest

Cloud Cover: 1 tenths

\section{SOURCE STRENGTH INFORMATION:}

Puddle Area: 100000 square feet

Average Puddle Depth: 1 centimeters

Soil Type: Default Ground Temperature: $29^{\circ} \mathrm{C}$

Initial Puddle Temperature: Ground temperature

Release Duration: ALOHA limited the duration to 1 hour

Max Computed Release Rate: 22.7 kilograms/min

Max Average Sustained Release Rate: 22 kilograms/min (averaged over a minute or more)

Total Amount Released: 1,274 kilograms

\section{TIME DEPENDENT INFORMATION:}

Concentration Estimates at the point:

Downwind: 100 meters

Off Centerline: 0 meters

Max Concentration:

Outdoor: $56.1 \mathrm{ppm}$
Reviewed by S. A. Henderson

Works Calculation 7

Sheet No. 25 of 577 
Title of Project EPHA for THM

Subject Concentration Analysis for THM Hazardous Chemicals

Computer T. M. Franey

Date $\underline{09 / 16 / 96}$

Reviewed by S. A. Henderson

Works Calculation 7

Sheet No. 26 of 577

1-RD-4 SITE DATA INFORMATION: Location: AIKEN, SOUTH CAROLINA Building Air Exchanges Per Hour: 60 (User specified)

Date and Time: Fixed at July 1, 19960000 hours

\section{CHEMICAL INFORMATION:}

Chemical Name: TERPINOLENE / ALPHA-PINENE / TURP.

Molecular Weight: $136.24 \mathrm{~kg} / \mathrm{kmol}$

TLV-TWA: -unavail- IDLH: -unavail-

Footprint Level of Concern: 500 ppm

Boiling Point: $185.00^{\circ} \mathrm{C}$

Vapor Pressure at Ambient Temperature: $0.0010 \mathrm{~atm}$

Ambient Saturation Concentration: 1,056 ppm or $0.11 \%$

\section{ATMOSPHERIC INFORMATION: (MANUAL INPUT OF DATA)}

Wind: 1.7 meters $/ \mathrm{sec}$ from $90^{\circ}$ true

Inversion Height: 200 feet

Stability Class: $\mathrm{E} \quad$ Air Temperature: $29^{\circ} \mathrm{C}$

Relative Humidity: $50 \%$ Ground Roughness: Urban or forest

Cloud Cover: 1 tenths

\section{SOURCE STRENGTH INFORMATION:}

Puddle Area: 24420 square feet

Average Puddle Depth: 1 centimeters

Soil Type: Default Ground Temperature: $29^{\circ} \mathrm{C}$

Initial Puddle Temperature: Ground temperature

Release Duration: ALOHA limited the duration to 1 hour

Max Computed Release Rate: 5.84 kilograms/min

Max Average Sustained Release Rate: 5.67 kilograms/min

(averaged over a minute or more)

Total Amount Released: 328 kilograms

FOOTPRINT INFORMATION:

Dispersion Module: Gaussian

User specified LOC: $500 \mathrm{ppm}$

Max Threat Zone for LOC: 27 meters

Note: Footprint was not drawn because effects of near-field patchiness make plume presentation unreliable for short distances.

TIME DEPENDENT INFORMATION:

Concentration Estimates at the point:

Downwind: 100 meters

Off Centerline: 0 meters

Max Concentration:

Outdoor: $16.7 \mathrm{ppm}$ 
Title of Project EPHA for THM

Subject Concentration Analysis for THM Hazardous Chemicals

Computer T. M. Franey
Reviewed by S. A. Henderson

Works Calculation 7

Sheet No. 27 of 577

\section{1-RD-4 SITE DATA INFORMATION:}

Location: AIKEN, SOUTH CAROLINA

Building Air Exchanges Per Hour: 60 (User specified)

Date and Time: Fixed at July 1, 19960000 hours

\section{CHEMICAL INFORMATION:}

Chemical Name: TERPINOLENE / ALPHA-PINENE / TURP

Molecular Weight: $136.24 \mathrm{~kg} / \mathrm{kmol}$

TLV-TWA: -unavail- IDLH: -unavail-

- Footprint Level of Concern: 500 ppm

Boiling Point: $185.00^{\circ} \mathrm{C}$

Vapor Pressure at Ambient Temperature: $0.0010 \mathrm{~atm}$

Ambient Saturation Concentration: $1,056 \mathrm{ppm}$ or $0.11 \%$

ATMOSPHERIC INFORMATION: (MANUAL INPUT OF DATA)

Wind: 1.7 meters $/ \mathrm{sec}$ from $90^{\circ}$ true

Inversion Hëight: 200 feet

Stability Class: E Air Temperature: $29^{\circ} \mathrm{C}$

Relative Humidity: 50\% Ground Roughness: Urban or forest

Cloud Cover: 1 tenths

\section{SOURCE STRENGTH INFORMATION:}

Puddle Area: 40700 square feet

Average Puddle Depth: 1 centimeters

Soil Type: Default Ground Temperature: $29^{\circ} \mathrm{C}$

Initial Puddle Temperature: Ground temperature

Release Duration: ALOHA limited the duration to 1 hour

Max Computed Release Rate: 9.55 kilograms/min

Max Average Sustained Release Rate: 9.26 kilograms/min (averaged over a minute or more)

Total Amount Released: 536 kilograms

\section{FOOTPRINT INFORMATION:}

Dispersion Module: Gaussian

User specified LOC: $500 \mathrm{ppm}$

Max Threat Zone for LOC: 34 meters

Note: Footprint was not drawn because effects of near-field patchiness make plume presentation unreliable for short distances.

\section{TIME DEPENDENT INFORMATION:}

Concentration Estimates at the point:

Downwind: 100 meters

- Off Centerline: 0 meters

Max Concentration:

Outdoor: $24.3 \mathrm{ppm}$ 
Title of Project EPHA for THM

Subject Concentration Analysis for THM Hazardous Chemicals

Computer T. M. Franey

Date $\underline{09 / 16 / 96}$

Reviewed by S.A. Henderson

Works Calculation 7

Sheet No. $\underline{28 \text { of } 577}$

1-RD-4 SITE DATA INFORMATION:

Location: AIKEN, SOUTH CAROLINA

Building Air Exchanges Per Hour: 60 (User specified)

Date and Time: Fixed at July 1, 19960000 hours

\section{CHEMICAL INFORMATION:}

Chemical Name: TERPINOLENE / ALPHA-PINENE / TURP

Molecular Weight: $136.24 \mathrm{~kg} / \mathrm{kmol}$

TLV-TWA: -unavail- IDLH: -unavail-

Footprint Level of Concern: 500 ppm

Boiling Point: $185.00^{\circ} \mathrm{C}$

Vapor Pressure at Ambient Temperature: $0.0010 \mathrm{~atm}$

Ambient Saturation Concentration: $1,056 \mathrm{ppm}$ or $0.11 \%$

ATMOSPHERIC INFORMATION: (MANUAL INPUT OF DATA)

.Wind: 1.7 meters $/ \mathrm{sec}$ from $90^{\circ}$ true

Inversion Height: 200 feet

Stability Class: E Air Temperature: $29^{\circ} \mathrm{C}$

Relative Humidity: 50\% Ground Roughness: Urban or forest

Cloud Cover: 1 tenths

\section{SOURCE STRENGTH INFORMATION:}

Puddle Area: 10000 square meters

Average Puddle Depth: 1 centimeters

Soil Type: Default Ground Temperature: $29^{\circ} \mathrm{C}$

Initial Puddle Temperature: Ground temperature

Release Duration: ALOHA limited the duration to 1 hour

Max Computed Release Rate: 24.3 kilograms/min

Max Average Sustained Release Rate: 23.6 kilograms/min

(averaged over a minute or more)

Total Amount Released: 1,367 kilograms

FOOTPRINT INFORMATION:

Dispersion Module: Gaussian

User specified LOC: $500 \mathrm{ppm}$

Max Threat Zone for LOC: 56 meters

Note: Footprint was not drawn because effects of near-field patchiness make plume presentation unreliable for short distances.

\section{TIME DEPENDENT INFORMATION:}

Concentration Estimates at the point:

Downwind: 100 meters

Off Centerline: 0 meters

Max Concentration:

Outdoor: $60.2 \mathrm{ppm}$ 
Title of Project EPHA for THM

Subject Concentration Analysis for THM Hazardous Chemicals

Computer T. M. Franey Date 09/16/96
Reviewed by S. A. Henderson

Works Calculation 7

Sheet No. 29 of 577

\section{SITE DATA INFORMATION: 1-RD-5}

Location: AIKEN, SOUTH CAROLINA

Building Air Exchanges Per Hour: 60 (User specified)

Date and Time: Fixed at July 1, 19960000 hours

CHEMICAL INFORMATION:

Chemical Name: AMMONIA - 29\% - SAH

Molecular Weight: $74.00 \mathrm{~kg} / \mathrm{kmol}$

TLV-TWA: -unavail- IDLH: -unavail-

Footprint Level of Concern: $200 \mathrm{ppm}$

Boiling Point: $27.66^{\circ} \mathrm{C}$

Vapor Pressure at Ambient Temperature: greater than $1 \mathrm{~atm}$

Ambient Saturation Concentration: $1,000,000 \mathrm{ppm}$ or $100.0 \%$

ATMOSPHERIC INFORMATION: (MANUAL INPUT OF DATA)

Wind: 1.7 meters $/ \mathrm{sec}$ from $90^{\circ}$ true

Inversion Height: 200 meters

Stability Class: E Air Temperature: $29^{\circ} \mathrm{C}$

Relative Humidity: $50 \%$ Ground Roughness: 100 centimeters

Cloud Cover: 1 tenths

\section{SOURCE STRENGTH INFORMATION:}

Puddle Area: 655 square feet

Average Puddle Depth: 1 centimeters

Soil Type: Default Ground Temperature: $29^{\circ} \mathrm{C}$

Initial Puddle Temperature: boiling point

Release Duration: ALOHA limited the duration to 1 hour

Max Computed Release Rate: 15.7 kilograms/min

Max Average Sustained Release Rate: 1.97 kilograms/min

(averaged over a minute or more)

Total Amount Released: 32.3 kilograms

\section{FOOTPRINT INFORMATION:}

Dispersion Module: Gaussian

User specified LOC: $200 \mathrm{ppm}$

Max Threat Zone for LOC: 30 meters

Note: Footprint was not drawn because effects of near-field patchiness make plume presentation unreliable for short distances.

\section{TIME DEPENDENT INFORMATION:}

Concentration Estimates at the point:

Downwind: 30 meters

Off Centerline: 0 meters

Max Concentration:

Outdoor: $200 \mathrm{ppm}$

Indoor: $200 \mathrm{ppm}$

Note: Indoor graph is shown with a dotted line. 
Title of Project EPHA for THM

Subject Concentration Analysis for THM Hazardous Chemicals

Computer T.M. Franey

Date $\underline{09 / 16 / 96}$
Reviewed by S.A. Henderson

Works Calculation 7

Sheet No. 30 of 577
SITE DATA INFORMATION: 1-RD-5

Location: AIKEN, SOUTH CAROLINA

Building Air Exchanges Per Hour: 60 (User specified)

Date and Time: Fixed at July 1, 19960000 hours

\section{CHEMICAL INFORMATION:}

Chemical Name: AMMONIA - 29\% - SAH

Molecular Weight: $74.00 \mathrm{~kg} / \mathrm{kmol}$

TLV-TWA: -unavail- IDLH: -unavail-

Footprint Level of Concern: 200 ppm

Boiling Point: $27.66^{\circ} \mathrm{C}$

Vapor Pressure at Ambient Temperature: greater than $1 \mathrm{~atm}$

Ambient Saturation Concentration: $1,000,000 \mathrm{ppm}$ or $100.0 \%$

\section{ATMOSPHERIC INFORMATION: (MANUAL INPUT OF DATA)}

Wind: 1.7 meters $/ \mathrm{sec}$ from $90^{\circ}$ true

Inversion Height: 200 meters

Stability Class: E Air Temperature: $29^{\circ} \mathrm{C}$

Relative Humidity: 50\% Ground Roughness: 100 centimeters

Cloud Cover: 1 tenths

\section{SOURCE STRENGTH INFORMATION:}

Puddle Area: 3500 square feet

Average Puddle Depth: 1 centimeters

Soil Type: Default $\quad$ Ground Temperature: $29^{\circ} \mathrm{C}$

Initial Puddle Temperature: boiling point

Release Duration: ALOHA limited the duration to 1 hour

Max Computed Release Rate: 80.1 kilograms/min

Max Average Sustained Release Rate: 10.3 kilograms/min (averaged over a minute or more)

Total Amount Released: 159 kilograms

\section{FOOTPRINT INFORMATION:}

Model Run: Heavy Gas

User specified LOC: $200 \mathrm{ppm}$

Max Threat Zone for LOC: 97 meters

Note: Footprint wasn't drawn because effects of near-field patchiness make plume presentation unreliable for short distances.

\section{TIME DEPENDENT INFORMATION:}

Concentration Estimates at the point:

Downwind: 100 meters

Off Centerline: 0 meters

Max Concentration:

Outdoor: $200 \mathrm{ppm}$

Indoor: $200 \mathrm{ppm}$

Note: Indoor graph is shown with a dotted line. 
Title of Project EPHA for THM

Subject Concentration Analysis for THM Hazardous Chemicals

Computer T. M. Franey

Date $\underline{09 / 16 / 96}$
Reviewed by S. A. Henderson

Works Calculation 7

Sheet No. $\underline{31 \text { of } 577}$

SITE DATA INFORMATION: 1-RD-5

Location: AIKEN, SOUTH CAROLINA

Building Air Exchanges Per Hour: 60 (User specified)

Date and Time: Fixed at July 1, 19960000 hours

CHEMICAL INFORMATION:

Chemical Name: AMMONLA - 29\% - SAH

Molecular Weight: $74.00 \mathrm{~kg} / \mathrm{kmol}$

TLV-TWA: -unavail- IDLH: -unavail-

Footprint Level of Concern: 200 ppm

Boiling Point: $27.66^{\circ} \mathrm{C}$

Vapor Pressure at Ambient Temperature: greater than $1 \mathrm{~atm}$

Ambient Saturation Concentration: $1,000,000 \mathrm{ppm}$ or $100.0 \%$

ATMOSPHERIC INFORMATION: (MANUAL INPUT OF DATA)

Wind: 1.7 meters $/ \mathrm{sec}$ from $90^{\circ}$ true

Inversion Height: 200 meters

Stability Class: E Air Temperature: $29^{\circ} \mathrm{C}$

Relative Humidity: 50\% Ground Roughness: 100 centimeters

Cloud Cover: 1 tenths

SOURCE STRENGTH INFORMATION:

Puddle Area: 24420 square feet

Average Puddle Depth: 1 centimeters

Soil Type: Default Ground Temperature: $29^{\circ} \mathrm{C}$

Initial Puddle Temperature: boiling point

Release Duration: ALOHA limited the duration to 1 hour

Max Computed Release Rate: 533 kilograms/min

Max Average Sustained Release Rate: 69.8 kilograms/min

(averaged over a minute or more)

Total Amount Released: 1,022 kilograms

FOOTPRINT INFORMATION:

Model Run: Heavy Gas

User specified LOC: $200 \mathrm{ppm}$

Max Threat Zone for LOC: 259 meters

Note: The Heavy Gas footprint is an initial screening.

For short releases it may be an overestimation.

Be sure to check concentration information at specific locations.

TIME DEPENDENT INFORMATION:

Concentration Estimates at the point:

Downwind: 100 meters

Off Centerline: 0 meters

Max Concentration:

Outdoor: $855 \mathrm{ppm}$

Indoor: $855 \mathrm{ppm}$

Note: Indoor graph is shown with a dotted line. 
Title of Project EPHA for THM

Subject Concentration Analysis for THM Hazardous Chemicals

Computer T. M. Franey
Reviewed by $\underline{\text { S. A. Henderson }}$

Works Calculation 7

Sheet No. $\underline{32 \text { of } 577}$

SITE DATA INFORMATION: 1-RD-6 Location: AIKEN, SOUTH CAROLINA

Building Air Exchanges Per Hour: 60 (User specified)

Date and Time: Fixed at July 1, 19960000 hours

CHEMICAL INFORMATION:

Chemical Name: BENZENE

TLV-TWA: $0.10 \mathrm{ppm}$ IDLH: $3000.00 \mathrm{ppm}$

Note: Potential or confirmed human carcinogen.

Footprint Level of Concern: $150 \mathrm{ppm}$

Boiling Point: $80.09^{\circ} \mathrm{C}$

Vapor Pressure at Ambient Temperature: $0.15 \mathrm{~atm}$

Ambient Saturation Concentration: $152,425 \mathrm{ppm}$ or $15.2 \%$

ATMOSPHERIC INFORMATION: (MANUAL INPUT OF DATA)

Wind: 1.7 meters $/ \mathrm{sec}$ from $90^{\circ}$ true

Inversion Height: 200 meters

Stability Class: E Air Temperature: $29^{\circ} \mathrm{C}$

Relative Humidity: 50\% Ground Roughness: 100 centimeters

Cloud Cover: 1 tenths

SOURCE STRENGTH INFORMATION:

Puddle Area: 68 square feet

Average Puddle Depth: 1 centimeters

Soil Type: Default Ground Temperature: $29^{\circ} \mathrm{C}$

Initial Puddle Temperature: Ground temperature

Release Duration: ALOHA limited the duration to 1 hour

Max Computed Release Rate: 909 grams/min

Max Average Sustained Release Rate: 743 grams/min

(averaged over a minute or more)

Total Amount Released: 36.7 kilograms

\section{FOOTPRINT INFORMATION:}

Dispersion Module: Gaussian

User specified LOC: $150 \mathrm{ppm}$

Max Threat Zone for LOC: 30 meters

Max Threat Zone for IDLH: less than 10 meters(10.9 yards)

Note: Footprint was not drawn because effects of near-field patchiness make plume presentation unreliable for short distances.

\section{TIME DEPENDENT INFORMATION:}

Concentration Estimates at the point:

Downwind: 30 meters

Off Centerline: 0 meters

Max Concentration:

Outdoor: $151 \mathrm{ppm}$

Indoor: $151 \mathrm{ppm}$

Note: Indoor graph is shown with a dotted line. 


\section{ENGINEERING COMPUTATION SHEET}

Title of Project EPHA for THM

Subject Concentration Analysis for THM Hazardous Chemicals

Computer T. M. Franey Date $\underline{09 / 16 / 96}$

\section{SITE DATA INFORMATION: 1-RD-6}

Location: AIKEN, SOUTH CAROLINA

Building Air Exchanges Per Hour: 60 (User specified)

Date and Time: Fixed at July 1, 19960000 hours

CHEMICAL INFORMATION:

Chemical Name: BENZENE

TLV-TWA: $0.10 \mathrm{ppm}$

Molecular Weight: $78.11 \mathrm{~kg} / \mathrm{kmol}$

Footprint Level of Concern: $150 \mathrm{ppm}$

Boiling Point: $80.09^{\circ} \mathrm{C}$

Vapor Pressure at Ambient Temperature: $0.15 \mathrm{~atm}$

Ambient Saturation Concentration: 152,425 ppm or $15.2 \%$

ATMOSPHERIC INFORMATION: (MANUAL INPUT OF DATA)

Wind: 1.7 meters $/ \mathrm{sec}$ from $90^{\circ}$ true

Inversion Height: 200 meters

Stability Class: E Air Temperature: $29^{\circ} \mathrm{C}$

Relative Humidity: 50\% Ground Roughness: 100 centimeters

Cloud Cover: 1 tenths'

\section{SOURCE STRENGTH INFORMATION:}

Puddle Area: 695 square feet

Average Puddle Depth: 1 centimeters

Soil Type: Default Ground Temperature: $29^{\circ} \mathrm{C}$

Initial Puddle Temperature: Ground temperature

Release Duration: ALOHA limited the duration to 1 hour

Max Computed Release Rate: 8.19 kilograms/min

Max Average Sustained Release Rate: 6.8 kilograms/min (averaged over a minute or more)

Total Amount Released: 340 kilograms

\section{FOOTPRINT INFORMATION:}

Model Run: Heavy Gas

User specified LOC: $150 \mathrm{ppm}$

Max Threat Zone for LOC: 97 meters

Max Threat Zone for IDLH: 14 meters

Note: Footprint wasn't drawn because effects of near-field patchiness make plume presentation unreliable for short distances.

TIME DEPENDENT INFORMATION:

Concentration Estimates at the point:

Downwind: 100 meters

Off Centerline: 0 meters

Max Concentration:

Outdoor: $150 \mathrm{ppm}$

Indoor: $150 \mathrm{ppm}$

Note: Indoor graph is shown with a dotted line.
Reviewed by $\mathrm{S}$. A. Henderson

Works Calculation 7

Sheet No. $\underline{33 \text { of } 577}$ 
Title of Project EPHA for THM

Subject Concentration Analysis for THM Hazardous Chemicals

Computer T. M. Franey Date $\underline{09 / 16 / 96}$
Reviewed by $\underline{S}$. A. Henderson

Works Calculation 7

Sheet No. $\underline{34 \text { of } 577}$

\section{SITE DATA INFORMATION: $\quad$ 1-RD-6}

Location: AIKEN, SOUTH CAROLINA

Building Air Exchanges Per Hour: 60 (User specified)

Date and Time: Fixed at July 1, 19960000 hours

\section{CHEMICAL INFORMATION:}

Chemical Name: BENZENE Molecular Weight: $78.11 \mathrm{~kg} / \mathrm{kmol}$

TLV-TWA: $0.10 \mathrm{ppm}$ IDLH: $3000.00 \mathrm{ppm}$

Note: Potential or confirmed human carcinogen.

Footprint Level of Concern: $150 \mathrm{ppm}$

Boiling Point: $80.09^{\circ} \mathrm{C}$

Vapor Pressure at Ambient Temperature: $0.15 \mathrm{~atm}$

Ambient Saturation Concentration: 152,425 ppm or $15.2 \%$

\section{ATMOSPHERIC INFORMATION: (MANUAL INPUT OF DATA)}

Wind: 1.7 meters $/ \mathrm{sec}$ from $90^{\circ}$ true

Inversion Height: 200 meters

Stability Class: E Air Temperature: $29^{\circ} \mathrm{C}$

Relative Humidity: $50 \% \quad$ Ground Roughness: 100 centimeters

Cloud Cover: 1 tenths

\section{SOURCE STRENGTH INFORMATION:}

Puddle Area: 24420 square feet

Average Puddle Depth: 1 centimeters

Soil Type: Default Ground Temperature: $29^{\circ} \mathrm{C}$.

Initial Puddle Temperature: Ground temperature

Release Duration: ALOHA limited the duration to 1 hour

Max Computed Release Rate: 243 kilograms/min

Max Average Sustained Release Rate: 207 kilograms/min

(averaged over a minute or more)

Total Amount Released: 10,465 kilograms

\section{FOOTPRINT INFORMATION:}

Model Run:'Heavy Gas

User specified LOC: $150 \mathrm{ppm}$

Max Threat Zone for LOC: 549 meters

Max Threat Zone for IDLH: 89 meters

Note: The Heavy Gas footprint is an initial screening.

For short releases it may be an overestimation.

Be sure to check concentration information at specific locations.

TIME DEPENDENT INFORMATION:

Concentration Estimates at the point:

Downwind: 100 meters

Off Centerline: 0 meters

Max Concentration:

Outdoor: $2,600 \mathrm{ppm}$

Indoor: $2,600 \mathrm{ppm}$

Note: Indoor graph is shown with a dotted line. 
Title of Project EPHA for THM

Subject Concentration Analysis for THM Hazardous Chemicals

Computer T. M. Franey
Reviewed by S. A. Henderson

Works Calculation 7.

Sheet No. $\underline{35 \text { of } 577}$

\section{SITE DATA INFORMATION: $\quad$ 1-RD-6 \\ Location: AIKEN, SOUTH CAROLINA \\ Building Air Exchanges Per Hour: 60 (User specified) \\ Date and Time: Fixed at July 1, 19960000 hours}

\section{CHEMICAL INFORMATION:}

Chemical Name: BENZENE

TLV-TWA: $0.10 \mathrm{ppm}$

Molecular Weight: $78.11 \mathrm{~kg} / \mathrm{kmol}$

Note: Potential or confirmed human carcinogen.

Footprint Level of Concern: 150 ppm

Boiling Point: $80.09^{\circ} \mathrm{C}$

Vapor Pressure at Ambient Temperature: $0.15 \mathrm{~atm}$

Ambient Saturation Concentration: $152,425 \mathrm{ppm}$ or $15.2 \%$

ATMOSPHERIC INFORMATION: (MANUAL INPUT OF DATA)

Wind: 1.7 meters $/ \mathrm{sec}$ from $90^{\circ}$ true

Inversion Height: 200 meters

Stability Class: E Air Temperature: $29^{\circ} \mathrm{C}$

Relative Humidity: $50 \%$ Ground Roughness: 100 centimeters

Cloud Cover: 1 tenths

\section{SOURCE STRENGTH INFORMATION:}

Puddle Area: 40700 square feet

Average Puddle Depth: 1 centimeters

Soil Type: Default Ground Temperature: $29^{\circ} \mathrm{C}$

Initial Puddle Temperature: Ground temperature

Release Duration: ALOHA limited the duration to 1 hour

Max Computed Release Rate: 397 kilograms/min

Max Average Sustained Release Rate: 337 kilograms/min

(averaged over a minute or more)

Total Amount Released: 17,140 kilograms

FOOTPRINT INFORMATION:

Model Run: Heavy Gas

User specified LOC: $150 \mathrm{ppm}$

Max Threat Zone for LOC: 708 meters

Max Threat Zone for IDLH: 117 meters

- Note: The Heavy Gas footprint is an initial screening.

For short releases it may be an overestimation.

Be sure to check concentration information at specific locations.

\section{TIME DEPENDENT INFORMATION:}

Concentration Estimates at the point:

Downwind: 100 meters

Off Centerline: 0 meters

Max Concentration:

Outdoor: $4,060 \mathrm{ppm}$

Indoor: 4,060 ppm

Note: Indoor graph is shown with a dotted line. 
Title of Project EPHA for THM

Subject Concentration Analysis for THM Hazardous Chemicals

Computer T. M. Franey
Reviewed by S. A. Henderson

Works Calculation 7

Sheet No. 36 of 577

SITE DATA INFORMATION: $\quad$ 1-RD-6

Location: AIKEN, SOUTH CAROLINA

Building Air Exchanges Per Hour: 60 (User specified)

Date and Time: Fixed at July 1, 19960000 hours

CHEMICAL INFORMATION:

Chemical Name: BENZENE Molecular Weight: $78.11 \mathrm{~kg} / \mathrm{kmol}$

TLV-TWA: $0.10 \mathrm{ppm}$.IDLH: $3000.00 \mathrm{ppm}$

Note: Potential or confirmed human carcinogen.

Footprint Level of Concern: $150 \mathrm{ppm}$

Boiling Point: $80.09^{\circ} \mathrm{C}$

Vapor Pressure at Ambient Temperature: 0.15 atm

Ambient Saturation Concentration: 152,425 ppm or $15.2 \%$

ATMOSPHERIC INFORMATION: (MANUAL INPUT OF DATA)

Wind: 1.7 meters $/ \mathrm{sec}$ from $90^{\circ}$ true

Inversion Height: 200 meters

Stability Class: E Air Temperature: $29^{\circ} \mathrm{C}$

Relative Humidity: 50\% Ground Roughness: 100 centimeters

Cloud Cover: 1 tenths

SOURCE STRENGTH INFORMATION:

Puddle Area: 10000 square meters

Average Puddle Depth: 1 centimeters

Soil Type: Default Ground Temperature: $29^{\circ} \mathrm{C}$

Initial Puddle Temperature: Ground temperature

Release Duration: ALOHA limited the duration to 1 hour

Max Computed Release Rate: 1,010 kilograms/min

Max Average Sustained Release Rate: 858 kilograms/min

(averaged over a minute or more)

Total Amount Released: 43,878 kilograms

FOOTPRINT INFORMATION:

Model Run: Heavy Gas

User specified LOC: $150 \mathrm{ppm}$

Max Threat Zone for LOC: 1.2 kilometers

Max Threat Zone for IDLH: 200 meters

Note: The Heavy Gas footprint is an initial screening.

For short releases it may be an overestimation.

Be sure to check concentration information at specific locations.

\section{TIME DEPENDENT INFORMATION:}

Concentration Estimates at the point:

Downwind: 100 meters

Off Centerline:, 0 meters

Max Concentration:

Outdoor: $9,300 \mathrm{ppm}$

Indoor: $9,300 \mathrm{ppm}$

Note: Indoor graph is shown with a dotted line. 
Title of Project EPHA for THM

Subject Concentration Analysis for THM Hazardous Chemicals

Computer T. M. Franey Date $\underline{09 / 16 / 96}$

SITE DATA INFORMATION: 1-RD-7 Location: AIKEN, SOUTH CAROLINA Building Air Exchanges Per Hour: 60 (User specified)

Date and Time: Fixed at July 1, 19960000 hours

CHEMICAL INFORMATION:

Chemical Name: BENZYL ALCOHOL

Molecular Weight: $108.14 \mathrm{~kg} / \mathrm{kmol}$

TLV-TWA: -unavail- IDLH: -unavail-

Footprint Level of Concern: 200 ppm

Boiling Point: $204.70^{\circ} \mathrm{C}$

Vapor Pressure at Ambient Temperature: $1.54 \mathrm{e}-004 \mathrm{~atm}$

Ambient Saturation Concentration: 157 ppm or $0.016 \%$

ATMOSPHERIC INFORMATION: (MANUAL INPUT OF DATA)

Wind: 1.7 meters $/ \mathrm{sec}$ from $90^{\circ}$ true

Inversion Height: 200 meters

Stability Class: E Air Temperature: $29^{\circ} \mathrm{C}$

Relative Humidity: 50\% Ground Roughness: 100 centimeters

Cloud Cover: 1 tenths

\section{SOURCE STRENGTH INFORMATION:}

Puddle Area: 30430 square feet

Average Puddle Depth: 1 centimeters

Soil Type: Default Ground Temperature: $29^{\circ} \mathrm{C}$

Initial Puddle Temperature: Ground temperature

Release Duration: ALOHA limited the duration to 1 hour

Max Computed Release Rate: 308 grams/min

Max Average Sustained Release Rate: 298 grams/min

(averaged over a minute or more)

Total Amount Released: 17.1 kilograms

\section{FOOTPRINT INFORMATION:}

Dispersion Module: Gaussian

User specified LOC: $200 \mathrm{ppm}$

Max Threat Zone for LOC: 30 meters

Note: Footprint was not drawn because effects of near-field patchiness make plume presentation unreliable for short distances.

TIME DEPENDENT INFORMATION:

Concentration Estimates at the point:

Downwind: 30 meters

Off Centerline: 0 meters

Max Concentration:

Outdoor: $10.1 \mathrm{ppm}$

Indoor: $10.1 \mathrm{ppm}$

Note: Indoor graph is shown with a dotted line.
Reviewed by S. A. Henderson

Works Calculation 7

Sheet No. 37 of 577 
Title of Project EPHA for THM

Subject Concentration Analysis for THM Hazardous Chemicals

Computer T. M. Franey

Date $\underline{09 / 16 / 96}$
Reviewed by S. A. Henderson

Works Calculation 7

Sheet No. $\underline{38 \text { of } 577}$

\section{SITE DATA INFORMATION: 1-RD-7}

Location: AIKEN, SOUTH CAROLINA

Building Air Exchanges Per Hour: 60 (User specified)

Date and Time: Fixed at July 1, 19960000 hours

CHEMICAL INFORMATION:

Chemical Name: BENZYL ALCOHOL

Molecular Weight: $108.14 \mathrm{~kg} / \mathrm{kmol}$

TLV-TWA: -unavail- IDLH: -unavail-

Footprint Level of Concern: 200 ppm

Boiling Point: $204.70^{\circ} \mathrm{C}$

Vapor Pressure at Ambient Temperature: $1.54 \mathrm{e}-004 \mathrm{~atm}$

Ambient Saturation Concentration: 157 ppm or $0.016 \%$

ATMOSPHERIC INFORMATION: (MANUAL INPUT OF DATA)

Wind: 1.7 meters $/ \mathrm{sec}$ from $90^{\circ}$ true

Inversion Height: 200 meters

Stability Class: E Air Temperature: $29^{\circ} \mathrm{C}$

Relative Humidity: $50 \%$ Ground Roughness: 100 centimeters

Cloud Cover: 1 tenths

SOURCE STRENGTH INFORMATION:

Puddle Area: 99999 square feet

Average Puddle Depth: 1 centimeters

Soil Type: Default Ground Temperature: $29^{\circ} \mathrm{C}$

Initial Puddle Temperature: Ground temperature

Release Duration: ALOHA limited the duration to 1 hour

Max Computed Release Rate: 967 grams/min

Max Average Sustained Release Rate: 933 grams/min

(averaged over a minute or more)

Total Amount Released: 53.5 kilograms

FOOTPRINT INFORMATION:

Dispersion Module: Gaussian

User specified LOC: 200 ppm

Max Threat Zone for LOC: 55 meters

Note: Footprint was not drawn because effects of near-field patchiness make plume presentation unreliable for short distances.

TIME DEPENDENT INFORMATION:

Concentration Estimates at the point:

Downwind: 100 meters

Off Centerline: 0 meters

Max Concentration:

Outdoor: 3 ppm

Indoor: $3 \mathrm{ppm}$

Note: Indoor graph is shown with a dotted line. 


\section{ENGINEERING COMPUTATION SHEET}

Title of Project EPHA for THM

Subject Concentration Analysis for THM Hazardous Chemicals

Computer T. M. Franey Date $09 / 16 / 96$
Reviewed by $\underline{\text { S. A. Henderson }}$

Works Calculation 7

Sheet No. $\underline{39 \text { of } 577}$

SITE DATA INFORMATTON: 1-RD-7

Location: AIKEN, SOUTH CAROLINA

Building Air Exchanges Per Hour: 60 (User specified)

Date and Time: Fixed at July 1, 19960000 hours

CHEMICAL INFORMATION:

Chemical Name: BENZYL ALCOHOL

Molecular Weight: $108.14 \mathrm{~kg} / \mathrm{kmol}$

TLV-TWA: -unavail- IDLH: -unavail-

Footprint Level of Concern: 200 ppm

Boiling Point: $204.70^{\circ} \mathrm{C}$

Vapor Pressure at Ambient Temperature: 1.54e-004 atm

Ambient Saturation Concentration: $157 \mathrm{ppm}$ or $0.016 \%$

\section{ATMOSPHERIC INFORMATION: (MANUAL INPUT OF DATA)}

Wind: 1.7 meters $/ \mathrm{sec}$ from $90^{\circ}$ true

Inversion Height: 200 meters

Stability Class: E Air Temperature: $29^{\circ} \mathrm{C}$

Relative Humidity: $50 \%$ Ground Roughness: 100 centimeters

Cloud Cover: 1 tenths

\section{SOURCE STRENGTH INFORMATION:}

Puddle Area: 24420 square feet

Average Puddle Depth: 1 centimeters

Soil Type: Default Ground Temperature: $29^{\circ} \mathrm{C}$

Initial Puddle Temperature: Ground temperature

Release Duration: ALOHA limited the duration to 1 hour

Max Computed Release Rate: 250 grams/min

Max Average Sustained Release Rate: 241 grams/min

(averaged over a minute or more)

Total Amount Released: 13.8 kilograms

\section{FOOTPRINT INFORMATION:}

Dispersion Module: Gaussian

User specified LOC: $200 \mathrm{ppm}$

Max Threat Zone for LOC: 27 meters

Note: Footprint was not drawn because effects of near-field patchiness make plume presentation unreliable for short distances.

\section{TIME DEPENDENT INFORMATION:}

Concentration Estimates at the point:

Downwind: 100 meters

Off Centerline: 0 meters

Max Concentration:

Outdoor: $0.897 \mathrm{ppm}$

Indoor: $0.897 \mathrm{ppm}$

Note: Indoor graph is shown with a dotted line. 


\section{ENGINEERING COMPUTATION SHEET}

Title of Project EPHA for THM

Reviewed by $\mathrm{S}$. A. Henderson

Subject Concentration Analysis for THM Hazardous Chemicals

Computer T. M. Franey

Date 09/16/96
Works Calculation 7

Sheet No. 40 of 577

SITE DATA INFORMATION: 1-RD-7

Location: AIKEN, SOUTH CAROLINA

Building Air Exchanges Per Hour: 60 (User specified)

Date and Time: Fixed at July 1, 19960000 hours

CHEMICAL INFORMATION:

Chemical Name: BENZYL ALCOHOL

Molecular Weight: $108.14 \mathrm{~kg} / \mathrm{kmol}$

TLV-TWA: -unavail- IDLH: -unavail-

Footprint Level of Concern: 200 ppm

Boiling Point: $204.70^{\circ} \mathrm{C}$

Vapor Pressure at Ambient Temperature: $1.54 \mathrm{e}-004 \mathrm{~atm}$

Ambient Saturation Concentration: $157 \mathrm{ppm}$ or $0.016 \%$

\section{ATMOSPHERIC INFORMATION: (MANUAL INPUT OF DATA)}

Wind: 1.7 meters $/ \mathrm{sec}$ from $90^{\circ}$ true

Inversion Height: 200 meters

Stability Class: E Air Temperature: $29^{\circ} \mathrm{C}$

Relative Humidity: 50\% Ground Roughness: 100 centimeters

Cloud Cover: 1 tenths

\section{SOURCE STRENGTH INFORMATION:}

Puddle Area: 40700 square feet

Average Puddle Depth: 1 centimeters

Soil Type: Default Ground Temperature: $29^{\circ} \mathrm{C}$

Initial Puddle Temperature: Ground temperature

Release Duration: ALOHA limited the duration to 1 hour

Max Computed Release Rate: 408 grams/min

Max Average Sustained Release Rate: 393 grams/min

(averaged over a minute or more)

Total Amount Released: 22.6 kilograms

FOOTPRINT INFORMATION:

Dispersion Module: Gaussian

User specified LOC: $200 \mathrm{ppm}$

Max Threat Zone for LOC: 34 meters

Note: Footprint was not drawn because effects of near-field patchiness make plume presentation unreliable for short distances.

TIME DEPENDENT INFORMATION:

Concentration Estimates at the point:

Downwind: 100 meters

Off Centerline: 0 meters

Max Concentration:

Outdoor: $1.3 \mathrm{ppm}$

Indoor: $1.3 \mathrm{ppm}$

Note: Indoor graph is shown with a dotted line. 
Title of Project EPHA for THM

Subject Concentration Analysis for THM Hazardous Chemicals

Computer T. M. Franey Date $\underline{09 / 16 / 96}$
Reviewed by $\underline{\text { S. A. Henderson }}$

Works Calculation 7

Sheet No. 41 of 577
SITE DATA INFORMATION: 1-RD-7

Location: AIKEN, SOUTH CAROLINA

Building Air Exchanges Per Hour: 60 (User specified)

Date and Time: Fixed at July 1, 19960000 hours

CHEMICAL INFORMATION:

Chemical Name: BENZYL ALCOHOL

Molecular Weight: $108.14 \mathrm{~kg} / \mathrm{kmol}$

-TLV-TWA: -unavail- IDLH: -unavail-

Footprint Level of Concern: $200 \mathrm{ppm}$

Boiling Point: $204.70^{\circ} \mathrm{C}$

Vapor Pressure at Ambient Temperature: $1.54 \mathrm{e}-004 \mathrm{~atm}$

Ambient Saturation Concentration: 157 ppm or $0.016 \%$

ATMOSPHERIC INFORMATION: (MANUAL INPUT OF DATA)

Wind: 1.7 meters $/ \mathrm{sec}$ from $90^{\circ}$ true

Inversion Height: 200 meters

Stability Class: E Air Temperature: $29^{\circ} \mathrm{C}$

Relative Humidity: $50 \%$ Ground Roughness: 100 centimeters

Cloud Cover: 1 tenths

\section{SOURCE STRENGTH INFORMATION:}

Puddle Area: 10000 square meters

Average Puddle Depth: 1 centimeters

Soil Type: Default Ground Temperature: $29^{\circ} \mathrm{C}$

Initial Puddle Temperature: Ground temperature

Release Duration: ALOHA limited the duration to 1 hour

Max Computed Release Rate: 1.04 kilograms/min

Max Average Sustained Release Rate: 1 kilograms/min

(averaged over a minute or more)

Total Amount Released: 57.4 kilograms

FOOTPRINT INFORMATION:

Dispersion Module: Gaussian

User specified LOC: $200 \mathrm{ppm}$

Max Threat Zone for LOC: 56 meters

Note: Footprint was not drawn because effects of near-field patchiness make plume presentation unreliable for short distances.

\section{TIME DEPENDENT INFORMATION:}

Concentration Estimates at the point:

Downwind: 100 meters

Off Centerline: 0 meters

Max Concentration:

Outdoor: $3.22 \mathrm{ppm}$

Indoor: $3.22 \mathrm{ppm}$

Note: Indoor graph is shown with a dotted line. 


\section{ENGINEERING COMPUTATION SHEET}

Title of Project EPHA for THM

Reviewed by S.A. Henderson

Subject Concentration Analysis for THM Hazardous Chemicals

Computer T. M. Franey

Date $\underline{09 / 16 / 96}$
Works Calculation 7

Sheet No. 42 of 577

\section{SITE DATA INFORMATION: $\quad$ 1-RD-8}

Location: AIKEN, SOUTH CAROLINA

Building Air Exchanges Per Hour: 60 (User specified)

Date and Time: Fixed at July 1, 19960000 hours

\section{CHEMICAL INFORMATION:}

Chemical Name: BENZYL CHLORIDE

Molecular. Weight: $126.58 \mathrm{~kg} / \mathrm{kmol}$

TLV-TWA: $1.00 \mathrm{ppm}$ IDLH: $10.00 \mathrm{ppm}$.

Footprint Level of Concern: 10 ppm

Boiling Point: $179.40^{\circ} \mathrm{C}$

Vapor Pressure at Ambient Temperature: $0.0022 \mathrm{~atm}$

Ambient Saturation Concentration: 2,263 ppm or $0.23 \%$

\section{ATMOSPHERIC INFORMATION: (MANUAL INPUT OF DATA)}

Wind: 1.7 meters $/ \mathrm{sec}$ from $90^{\circ}$ true

Inversion Height: 200 meters

Stability Class: E Air Temperature: $29^{\circ} \mathrm{C}$

Relative Humidity: $50 \%$ Ground Roughness: 100 centimeters

Cloud Cover: 1 tenths

\section{SOURCE STRENGTH INFORMATION:}

Puddle Area: 730 square feet

Average Puddle Depth: 1 centimeters

Soil Type: Default Ground Temperature: $29^{\circ} \mathrm{C}$

Initial Puddle Temperature: Ground temperature

Release Duration: ALOHA limited the duration to 1 hour

Max Computed Release Rate: 184 grams/min

Max Average Sustained Release Rate: 179 grams/min

(averaged over a minute or more)

Total Amount Released: 10.3 kilograms

\section{FOOTPRINT INFORMATION:}

Dispersion Module: Gaussian

User specified LOC: $10 \mathrm{ppm}$

Max Threat Zone for LOC: 30 meters

Max Threat Zone for IDLH: $\mathbf{3 0}$ meters

Note: Footprint was not drawn because effects of near-field patchiness make plume

presentation unreliable for short distances.

\section{TIME DEPENDENT INFORMATION:}

Concentration Estimates at the point:

Downwind: 30 meters

Off Centerline: 0 meters

Max Concentration:

Outdoor: $10 \mathrm{ppm}$

Indoor: $10 \mathrm{ppm}$

Note: Indoor graph is shown with a dotted line. 
Title of Project EPHA for THM

Subject Concentration Analysis for THM Hazardous Chemicals

Computer T. M. Franey

Date $09 / 16 / 96$
Reviewed by S. A. Henderson

Works Calculation 7

Sheet No. $\underline{43 \text { of } 577}$

\section{SITE DATA INFORMATION: $\quad$ 1-RD-8}

Location: AIKEN, SOUTH CAROLINA

Building Air Exchanges Per Hour: 60 (User specified)

Date and Time: Fixed at July 1, 19960000 hours

\section{CHEMICAL INFORMATION:}

Chemical Name: BENZYL CHLORIDE

Molecular Weight: $126.58 \mathrm{~kg} / \mathrm{kmol}$

TLV-TWA: $1.00 \mathrm{ppm}$ IDLH: $10.00 \mathrm{ppm}$

Footprint Level of Concern: $10 \mathrm{ppm}$

Boiling Point: $179.40^{\circ} \mathrm{C}$

Vapor Pressure at Ambient Temperature: $0.0022 \mathrm{~atm}$

Ambient Saturation Concentration: $2,263 \mathrm{ppm}$ or $0.23 \%$

ATMOSPHERIC INFORMATION: (MANUAL INPUT OF DATA)

Wind: 1.7 meters $/ \mathrm{sec}$ from $90^{\circ}$ true

Inversion Height: 200 meters

Stability Class: E Air Temperature: $29^{\circ} \mathrm{C}$

Relative Humidity: 50\% Ground Roughness: 100 centimeters

Cloud Cover: 1 tenths

\section{SOURCE STRENGTH INFORMATION:}

Puddle Area: 9200 square feet

Average Puddle Depth: 1 centimeters

Soil Type: Default Ground Temperature: $29^{\circ} \mathrm{C}$

Initial Puddle Temperature: Ground temperature

Release Duration: ALOHA limited the duration to 1 hour

Max Computed Release Rate: 2.08 kilograms/min

Max Average Sustained Release Rate: 2.01 kilograms/min

(averaged over a minute or more)

Total Amount Released: 117 kilograms

\section{FOOTPRINT INFORMATTON:}

Dispersion Module: Gaussian

User specified LOC: $10 \mathrm{ppm}$

Max Threat Zone for LOC: 100 meters

Max Threat Zone for IDLH: 100 meters

\section{TIME DEPENDENT INFORMATION:}

Concentration Estimates at the point:

Downwind: 100 meters

Off Centerline: 0 meters

Max Concentration:

Outdoor: $10 \mathrm{ppm}$

Indoor: $10 \mathrm{ppm}$

Note: Indoor graph is shown with a dotted line. 
Title of Project EPHA for THM

Subject Concentration Analysis for THM Hazardous Chemicals

Computer T. M. Franey
Date $09 / 16 / 96$
Reviewed by $\underline{\text { S. A. Henderson }}$

Works Calculation 7

Sheet No. 44 of 577

\section{SITE DATA INFORMATION: 1-RD-8 \\ Location: AIKEN, SOUTH CAROLINA \\ Building Air Exchanges Per Hour: 60 (User specified) \\ Date and Time: Fixed at July 1, 19960000 hours}

\section{CHEMICAL INFORMATION:}

Chemical Name: BENZYL CHLORIDE

Molecular Weight: $126.58 \mathrm{~kg} / \mathrm{kmol}$

TLV-TWA: $1.00 \mathrm{ppm}$ IDLH: $10.00 \mathrm{ppm}$

Footprint Level of Concern: 10 ppm

Boiling Point: $179.40^{\circ} \mathrm{C}$

Vapor Pressure at Ambient Temperature: $0.0022 \mathrm{~atm}$

Ambient Saturation Concentration: 2,263 ppm or $0.23 \%$

\section{ATMOSPHERIC INFORMATION: (MANUAL INPUT OF DATA)}

Wind: 1.7 meters $/ \mathrm{sec}$ from $90^{\circ}$ true

Inversion Height: 200 meters

Stability Class: E Air Temperature: $29^{\circ} \mathrm{C}$

Relative Humidity: 50\% Ground Roughness: 100 centimeters

Cloud Cover: 1 tenths

\section{SOURCE STRENGTH INFORMATION:}

Puddle Area: 24420 square feet

Average Puddle Depth: 1 centimeters

Soil Type: Default Ground Temperature: $29^{\circ} \mathrm{C}$

Initial Puddle Temperature: Ground temperature

Release Duration: ALOHA limited the duration to 1 hour

Max Computed Release Rate: 5.31 kilograms/min

Max Average Sustained Release Rate: 5.14 kilograms/min

(averaged over a minute or more)

Total Amount Released: 297 kilograms

\section{FOOTPRINT INFORMATION:}

Dispersion Module: Gaussian

User specified LOC: $10 \mathrm{ppm}$

Max Threat Zone for LOC: 163 meters

Max Threat Zone for IDLH: 163 meters

\section{TIME DEPENDENT INFORMATION:}

Concentration Estimates at the point:

Downwind: 100 meters

Off Centerline: 0 meters

Max Concentration:

Outdoor: $16.4 \mathrm{ppm}$

Indoor: $16.4 \mathrm{ppm}$

Note: Indoor graph is shown with a dotted line. 


\section{ENGINEERING COMPUTATION SHEET}

Title of Project EPHA for THM

Subject Concentration Analysis for THM Hazardous Chemicals

Computer T. M. Franey Date 09/16/96
Reviewed by S. A. Henderson

Works Calculation 7

Sheet No. 45 of 577

\section{SITE DATA INFORMATION: 1-RD-8}

Location: AIKEN, SOUTH CAROLINA

Building Air Exchanges Per Hour: 60 (User specified)

Date and Time: Fixed at July 1, 19960000 hours

CHEMICAL INFORMATION:

Chemical Name: BENZYL CHLORIDE

Molecular Weight: $126.58 \mathrm{~kg} / \mathrm{kmol}$

TLV-TWA: $1.00 \mathrm{ppm}$ IDLH: $10.00 \mathrm{ppm}$

Footprint Level of Concern: $10 \mathrm{ppm}$

Boiling Point: $179.40^{\circ} \mathrm{C}$

Vapor Pressure at Ambient Temperature: $0.0022 \mathrm{~atm}$

Ambient Saturation Concentration: 2,263 ppm or $0.23 \%$

ATMOSPHERIC INFORMATION: (MANUAL INPUT OF DATA)

Wind: 1.7 meters $/ \mathrm{sec}$ from $90^{\circ}$ true

Inversion Height: 200 meters

Stability Class: E Air Temperature: $29^{\circ} \mathrm{C}$

Relative Humidity: 50\% Ground Roughness: 100 centimeters

Cloud Cover: 1 tenths

SOURCE STRENGTH INFORMATION:

Puddle Area: 40700 square feet

Average Puddle Depth: 1 centimeters

Soil Type: Default Ground Temperature: $29^{\circ} \mathrm{C}$

Initial Puddle Temperature: Ground temperature

Release Duration: ALOHA limited the duration to 1 hour

Max Computed Release Rate: 8.67 kilograms/min

Max Average Sustained Release Rate: 8.4 kilograms/min (averaged over a minute or more)

Total Amount Released: 486 kilograms

FOOTPRINT INFORMATION:

Dispersion Module: Gaussian

User specified LOC: $10 \mathrm{ppm}$

Max Threat Zone for LOC: 213 meters

Max Threat Zone for IDLH: 213 meters

TIME DEPENDENT INFORMATION:

Concentration Estimates at the point:

Downwind: 100 meters

Off Centerline: 0 meters

Max Concentration:

Outdoor: $23.7 \mathrm{ppm}$

Indoor: $23.7 \mathrm{ppm}$

Note: Indoor graph is shown with a dotted line. 
Title of Project EPHA for THM

Subject Concentration Analysis for THM Hazardous Chemicals

Computer T. M. Franey
Reviewed by S. A. Henderson

Works Calculation 7

Sheet No. 46 of 577
SITE DATA INFORMATION: $\quad$ 1-RD-8

Location: AIKEN, SOUTH CAROLINA

Building Air Exchanges Per Hour: 60 (User specified)

Date and Time: Fixed at July 1, 19960000 hours

CHEMICAL INFORMATION:

Chemical Name: BENZYL CHLORIDE

Molecular Weight: $126.58 \mathrm{~kg} / \mathrm{kmol}$

TLV-TWA: $1.00 \mathrm{ppm}$ IDLH: $10.00 \mathrm{ppm}$

Footprint Level of Concern: 10 ppm

Boiling Point: $179.40^{\circ} \mathrm{C}$

Vapor Pressure at Ambient Temperature: $0.0022 \mathrm{~atm}$

Ambient Saturation Concentration: $2,263 \mathrm{ppm}$ or $0.23 \%$

ATMOSPHERIC INFORMATION: (MANUAL INPUT OF DATA)

Wind: 1.7 meters $/ \mathrm{sec}$ from $90^{\circ}$ true

Inversion Height: 200 meters.

Stability Class: E Air Temperature: $29^{\circ} \mathrm{C}$

Relative Humidity: 50\% Ground Roughness: 100 centimeters

Cloud Cover: 1 tenths

\section{SOURCE STRENGTH INFORMATION:}

Puddle Area: 10000 square meters

Average Puddle Depth: 1 centimeters

Soil Type: Default Ground Temperature: $29^{\circ} \mathrm{C}$

Initial Puddle Temperature: Ground temperature

Release Duration: ALOHA limited the duration to 1 hour

Max Computed Release Rate: 22.1 kilograms/min

Max Average Sustained Release Rate: 21.4 kilograms/min

(averaged over a minute or more)

Total Amount Released: 1,238 kilograms

FOOTPRINT INFORMATION:

Dispersion Module: Gaussian

User specified LOC: $10 \mathrm{ppm}$

Max Threat Zone for LOC: 360 meters

Max Threat Zone for IDLH: 360 meters

TIME DEPENDENT INFORMATION:

Concentration Estimates at the point:

Downwind: 100 meters

Off Centerline: 0 meters

Max Concentration:

Outdoor: $58.7 \mathrm{ppm}$

Indoor: $58.7 \mathrm{ppm}$

Note: Indoor graph is shown with a dotted line. 


\section{ENGINEERING COMPUTATION SHEET}

Title of Project EPHA for THM

Subject Concentration Analysis for THM Hazardous Chemicals

Computer T.M. Franey Date $\underline{09 / 16 / 96}$
Reviewed by S.A. Henderson

Works Calculation 7

Sheet No. 47 of 577

SITE DATA INFORMATION: 1-RD-9

Location: AIKEN, SOUTH CAROLINA

Building Air Exchanges Per Hour: 60 (User specified)

Date and Time: Fixed at July 1, 19960000 hours

CHEMICAL INFORMATION:

Chemical Name: BUTYRALDEHYDE

Molecular Weight: $72.11 \mathrm{~kg} / \mathrm{kmol}$

TLV-TWA: -unavail- IDLH: -unavail-

Footprint Level of Concern: 50 ppm

Boiling Point: $74.80^{\circ} \mathrm{C}$

Vapor Pressure at Ambient Temperature: $0.18 \mathrm{~atm}$

Ambient Saturation Concentration: $179,016 \mathrm{ppm}$ or $17.9 \%$

ATMOSPHERIC INFORMATION: (MANUAL INPUTT OF DATA)

Wind: 1.7 meters $/ \mathrm{sec}$ from $90^{\circ}$ true

Inversion Height: 200 meters

Stability Class: E Air Temperature: $29^{\circ} \mathrm{C}$

Relative Humidity: 50\% Ground Roughness: 100 centimeters

Cloud Cover: 1 tenths

\section{SOURCE STRENGTH INFORMATION:}

Puddle Area: 17 square feet

Average Puddle Depth: 1 centimeters

Soil Type: Default Ground Temperature: $29^{\circ} \mathrm{C}$

Initial Puddle Temperature: Ground temperature

Release Duration: ALOHA limited the duration to 1 hour

Max Computed Release Rate: 266 grams/min

Max Average Sustained Release Rate: 208 grams/min

(averaged over a minute or more)

Total Amount Released: 9.82 kilograms

\section{FOOTPRINT INFORMATION:}

Dispersion Module: Gaussian

User specified LOC: $50 \mathrm{ppm}$

Max Threat Zone for LOC: 30 meters

Note: Footprint was not drawn because effects of near-field patchiness make plume

presentation unreliable for short distances.

\section{TIME DEPENDENT INFORMATION:}

Concentration Estimates at the point:

Downwind: 30 meters

Off Centerline: 0 meters

Max Concentration:

Outdoor: $50.8 \mathrm{ppm}$

Indoor: $50.8 \mathrm{ppm}$

Note: Indoor graph is shown with a dotted line. 


\section{ENGINEERING COMPUTATION SHEET}

Title of Project EPHA for THM

Subject Concentration Analysis for THM Hazardous Chemicals

Computer T. M. Franey

Date 09/16/96

SITE DATA INFORMATION: 1-RD-9

Location: AIKEN, SOUTH CAROLINA

Building Air Exchanges Per Hour: 60 (User specified)

Date and Time: Fixed at July 1, 19960000 hours

CHEMICAL INFORMATION:

Chemical Name: BUTYRALDEHYDE

Molecular Weight: $72.11 \mathrm{~kg} / \mathrm{kmol}$

TLV-TWA: -unavail- IDLH: -unavail-

Footprint Level of Concern: $50 \mathrm{ppm}$

Boiling Point: $74.80^{\circ} \mathrm{C}$

Vapor Pressure at Ambient Temperature: $0.18 \mathrm{~atm}$

Ambient Saturation Concentration: $179,016 \mathrm{ppm}$ or $17.9 \%$

ATMOSPHERIC INFORMATION: (MANUAL INPUT OF DATA)

Wind: 1.7 meters $/ \mathrm{sec}$ from $90^{\circ}$ true

Inversion Height: 200 meters

Stability Class: E Air Temperature: $29^{\circ} \mathrm{C}$

Relative Humidity: 50\% Ground Roughness: 100 centimeters

Cloud Cover: 1 tenths

SOURCE STRENGTH INFORMATION:

Puddle Area: 202 square feet

Average Puddle Depth: 1 centimeters

Soil Type: Default Ground Temperature: $29^{\circ} \mathrm{C}$

Initial Puddle Temperature: Ground temperature

Release Duration: ALOHA limited the duration to 1 hour

Max Computed Release Rate: 2.73 kilograms/min

Max Average Sustained Release Rate: 2.17 kilograms/min

(averaged over a minute or more)

Total Amount Released: 105 kilograms

FOOTPRINT INFORMATION:

Dispersion Module: Gaussian

User specified LOC: $50 \mathrm{ppm}$

Max Threat Zone for LOC: 100 meters

TIME DEPENDENT INFORMATION:

Concentration Estimates at the point:

Downwind: 100 meters

Off Centerline: 0 meters

Max Concentration:

Outdoor: $50.1 \mathrm{ppm}$

Indoor: $50.1 \mathrm{ppm}$

Note: Indoor graph is shown with a dotted line.
Reviewed by S. A. Henderson

Works Calculation 7

Sheet No. 48 of 577 


\section{ENGINEERING COMPUTATION SHEET}

Title of Project EPHA for THM

Subject Concentration Analysis for THM Hazardous Chemicals

Computer T.M. Franey

Date 09/16/96
Reviewed by S. A. Henderson

Works Calculation 7

Sheet No. 49 of 577

SITE DATA INFORMATION: $\quad$ 1-RD-9

Location: AIKEN, SOUTH CAROLINA

Building Air Exchanges Per Hour: 60 (User specified)

Date and Time: Fixed at July 1, 19960000 hours

CHEMICAL INFORMATION:

Chemical Name: BUTYRALDEHYDE

Molecular Weight: $72.11 \mathrm{~kg} / \mathrm{kmol}$

TLV-TWA: -unavail- IDLH: -unavail-

Footprint Level of Concern: $50 \mathrm{ppm}$

Boiling Point: $74.80^{\circ} \mathrm{C}$

Vapor Pressure at Ambient Temperature: $0.18 \mathrm{~atm}$

Ambient Saturation Concentration: 179,016 ppm or $17.9 \%$

ATMOSPHERIC INFORMATION: (MANUAL INPUT OF DATA)

Wind: 1.7 meters $/ \mathrm{sec}$ from $90^{\circ}$ true

Inversion Height: 200 meters

Stability Class: E Air Temperature: $29^{\circ} \mathrm{C}$

Relative Humidity: 50\% Ground Roughness: 100 centimeters

Cloud Cover: 1 tenths

SOURCE STRENGTH INFORMATION:

Puddle Area: 24420 square feet

Average Puddle Depth: 1 centimeters

Soil Type: Default Ground Temperature: $29^{\circ} \mathrm{C}$

Initial Puddle Temperature: Ground temperature

Release Duration: ALOHA limited the duration to 1 hour

Max Computed Release Rate: 260 kilograms/min

Max Average Sustained Release Rate: 215 kilograms/min

(averaged over a minute or more)

Total Amount Released: 10,585 kilograms

FOOTPRINT INFORMATION:

Model Run: Heavy Gas

User specified LOC: $50 \mathrm{ppm}$

Max Threat Zone for LOC: 1.2 kilometers

Note: The Heavy Gas footprint is an initial screening.

For short releases it,may be an overestimation.

Be sure to check concentration information at specific locations.

TIME DEPENDENT INFORMATION:

Concentration Estimates at the point:

Downwind: 100 meters

Off Centerline: 0 meters

Max Concentration:

Outdoor: $2,920 \mathrm{ppm}$

Indoor: $2,920 \mathrm{ppm}$

Note: Indoor graph is shown with a dotted line. 


\section{ENGINEERING COMPUTATION SHEET}

Title of Project EPHA for THM

Subject Concentration Analysis for THM Hazardous Chemicals

Computer T. M. Franey

Date $\underline{09 / 16 / 96}$
Reviewed by S. A. Henderson

Works Calculation 7

Sheet No. $\underline{50 \text { of } 577}$
CHEMICAL INFORMATION:

Chemical Name: BUTYRALDEHYDE

Molecular Weight: $72.11 \mathrm{~kg} / \mathrm{kmol}$

TLV-TWA: -unavail- IDLH: -unavail-

Footprint Level of Concern: $50 \mathrm{ppm}$

Boiling Point: $74.80^{\circ} \mathrm{C}$

Vapor Pressure at Ambient Temperature: $0.18 \mathrm{~atm}$

Ambient Saturation Concentration: $179,016 \mathrm{ppm}$ or $17.9 \%$

ATMOSPHERIC INFORMATION: (MANUAL INPUT OF DATA)

Wind: 1.7 meters $/ \mathrm{sec}$ from $90^{\circ}$ true

Inversion Height: 200 meters

Stability Class: E Air Temperature: $29^{\circ} \mathrm{C}$

Relative Humidity: 50\% Ground Roughness: 100 centimeters

Cloud Cover: 1 tenths

SOURCE STRENGTH INFORMATION:

Puddle Area: 40700 square feet

Average Puddle Depth: 1 centimeters

Soil Type: Default Ground Temperature: $29^{\circ} \mathrm{C}$

Initial Puddle Temperature: Ground temperature

Release Duration: ALOHA limited the duration to 1 hour

Max Computed Release Rate: 424 kilograms/min

Max Average Sustained Release Rate: 351 kilograms/min

(averaged over a minute or more)

Total Amount Released: 17,342 kilograms

FOOTPRINT INFORMATION:

Model Run: Heavy Gas

User specified LOC: $50 \mathrm{ppm}$

Max Threat Zone for LOC: 1.6 kilometers

Note: The Heavy Gas footprint is an initial screening.

For short releases it may be an overestimation.

Be sure to check concentration information at specific locations.

TIME DEPENDENT INFORMATION:

Concentration Estimates at the point:

Downwind: 100 meters

Off Centerline: 0 meters

Max Concentration:

Outdoor: $4,570 \mathrm{ppm}$

Indoor: 4,570 ppm

Note: Indoor graph is shown with a dotted line. 
Title of Project EPHA for THM

Subject Concentration Analysis for THM Hazardous Chemicals

Computer T. M. Franey

Date $\underline{09 / 16 / 96}$

Reviewed by S. A. Henderson

Works Calculation 7

Sheet No. 51 of 577

\section{SITE DATA INFORMATION: 1-RD-9 \\ Location: AIKEN, SOUTH CAROLINA \\ Building Air Exchanges Per Hour: 60 (User specified) \\ Date and Time: Fixed at July 1, 19960000 hours}

\section{CHEMICAL INFORMATION:}

Chemical Name: BUTYRALDEHYDE

Molecular Weight: $72.11 \mathrm{~kg} / \mathrm{kmol}$

TLV-TWA: -unavail- IDLH: -unavail-

Footprint Level of Concern: 50 ppm

Boiling Point: $74.80^{\circ} \mathrm{C}$

Vapor Pressure at Ambient Temperature: $0.18 \mathrm{~atm}$

Ambient Saturation Concentration: $179,016 \mathrm{ppm}$ or $17.9 \%$

\section{ATMOSPHERIC INFORMATION: (MANUAL INPUT OF DATA)}

Wind: 1.7 meters $/ \mathrm{sec}$ from $90^{\circ}$ true

Inversion Height: 200 meters

Stability Class: E Air Temperature: $29^{\circ} \mathrm{C}$

Relative Humidity: 50\% Ground Roughness: 100 centimeters

Cloud Cover: 1 tenths

\section{SOURCE STRENGTH INFORMATION:}

Puddle Area: 10000 square meters

Average Puddle Depth: 1 centimeters

Soil Type: Default Ground Temperature: $29^{\circ} \mathrm{C}$

Initial Puddle Temperature: Ground temperature

Release Duration: ALOHA limited the duration to 1 hour

Max Computed Release Rate: 1,080 kilograms/min

Max Average Sustained Release Rate: 892 kilograms/min

(averaged over a minute or more)

Total Amount Released: 44,423 kilograms

\section{FOOTPRINT INFORMATION:}

Model Run: Heavy Gas

User specified LOC: $50 \mathrm{ppm}$

Max Threat Zone for LOC: 2.5 kilometers

Note: The Heavy Gas footprint is an initial screening.

For short releases it may be an overestimation.

Be sure to check concentration information at specific locations.

\section{TIME DEPENDENT INFORMATION:}

Concentration Estimates at the point:

Downwind: 100 meters

Off Centerline: 0 meters

Max Concentration:

Outdoor: $10,500 \mathrm{ppm}$

Indoor: $10,500 \mathrm{ppm}$

Note: Indoor graph is shown with a dotted line. 
Title of Project EPHA for THM

Subject Concentration Analysis for THM Hazardous Chemicals

Computer T.M. Franey

Date $\underline{09 / 16 / 96}$
Reviewed by S. A. Henderson

Works Calculation 7

Sheet No. $\underline{52 \text { of } 577}$

SITE DATA INFORMATION: 1-RD-10

Location: AIKEN, SOUTH CAROLINA

Building Air Exchanges Per Hour: 60 (User specified)

Date and Time: Fixed at July 1, 19960000 hours

CHEMICAL INFORMATION:

Chemical Name: CARBON BISULFIDE

Molecular Weight: $76.14 \mathrm{~kg} / \mathrm{kmol}$

TLV-TWA: $10.00 \mathrm{ppm}$ IDLH: $500.00 \mathrm{ppm}$

Footprint Level of Concern: $50 \mathrm{ppm}$

Boiling Point: $46.23^{\circ} \mathrm{C}$

Vapor Pressure at Ambient Temperature: $0.55 \mathrm{~atm}$

Ambient Saturation Concentration: 559,378 ppm or $55.9 \%$

ATMOSPHERIC INFORMATION: (MANUAL INPUT OF DATA)

Wind: 1.7 meters $/ \mathrm{sec}$ from $90^{\circ}$ true

Inversion Height: 200 meters

Stability Class: E Air Temperature: $29^{\circ} \mathrm{C}$

Relative Humidity: $50 \%$ Ground Roughness: 100 centimeters

Cloud Cover: 1 tenths

SOURCE STRENGTH INFORMATION:

Puddle Area: 4.4 square feet

Average Puddle Depth: 1 centimeters

Soil Type: Default Ground Temperature: $29^{\circ} \mathrm{C}$

Initial Puddle Temperature: Ground temperature

Release Duration: 35 minutes

Max Computed Release Rate: 322 grams/min

Max Average Sustained Release Rate: $210 \mathrm{grams} / \mathrm{min}$ (averaged over a minute or more)

Total Amount Released: 5.11 kilograms

FOOTPRINT INFORMATION:

Dispersion Module: Gaussian .

User specified LOC: 50 ppm

Max Threat Zone for LOC: 30 meters

Max Threat Zone for IDLH: less than 10 meters(10.9 yards)

Note: Footprint was not drawn because effects of near-field patchiness make plume presentation unreliable for short distances.

\section{TIME DEPENDENT INFORMATION:}

Concentration Estimates at the point:

Downwind: 30 meters

Off Centerline: 0 meters

Max Concentration:

Outdoor: $50 \mathrm{ppm}$

Indoor: $49.5 \mathrm{ppm}$

Note: Indoor graph is shown with a dotted line. 
Title of Project EPHA for THM

Subject Concentration Analysis for THM Hazardous Chemicals

$$
\text { Computer T. M. Franey }
$$

Reviewed by S.A. Henderson

Works Calculation 7

Sheet No. $\underline{53 \text { of } 577}$

SITE DATA INFORMATION: 1-RD-10

Location: AIKEN, SOUTH CAROLINA

Building Air Exchanges Per Hour: 60 (User specified)

Date and Time: Fixed at July 1, 19960000 hours

CHEMICAL INFORMATION:

Chemical Name: CARBON BISULFIDE

Molecular Weight: $76.14 \mathrm{~kg} / \mathrm{kmol}$

TLV-TWA: $10.00 \mathrm{ppm}$ IDLH: $500.00 \mathrm{ppm}$

Footprint Level of Concern: 50 ppm

Boiling Point: $46.23^{\circ} \mathrm{C}$

Vapor Pressure at Ambient Temperature: $0.55 \mathrm{~atm}$

Ambient Saturation Concentration: 559,378 ppm or 55.9\%

ATMOSPHERIC INFORMATION: (MANUAL INPUT OF DATA)

Wind: 1.7 meters $/ \mathrm{sec}$ from $90^{\circ}$ true

Inversion Height: 200 meters

Stability Class: E Air Temperature: $29^{\circ} \mathrm{C}$

Relative Humidity: $50 \%$ Ground Roughness: 100 centimeters

Cloud Cover: 1 tenths

SOURCE STRENGTH INFORMATTON:

Puddle Area: 31 square feet

Average Puddle Depth: 1 centimeters

Soil Type: Default Ground Temperature: $29^{\circ} \mathrm{C}$

Initial Puddle Temperature: Ground temperature

Release Duration: 39 minutes

Max Computed Release Rate: 2 kilograms/min

Max Average Sustained Release Rate: 1.33 kilograms/min

(averaged over a minute or more)

Total Amount Released: 36.0 kilograms

FOOTPRINT INFORMATION:

Model Run: Heavy Gas

User specified LOC: $50 \mathrm{ppm}$

Max Threat Zone for LOC: 97 meters

Max Threat Zone for IDLH: 21 meters

Note: Footprint wasn't drawn because effects of near-field patchiness make plume presentation unreliable for short distances.

\section{TIME DEPENDENT INFORMATION:}

Concentration Estimates at the point:

Downwind: 100 meters

Off Centerline: 0 meters

Max Concentration:

Outdoor: $49.7 \mathrm{ppm}$

Indoor: $48.8 \mathrm{ppm}$

Note: Indoor graph is shown with a dotted line. 
Title of Project EPHA for THM

Subject Concentration Analysis for THM Hazardous Chemicals

Computer T. M. Franey

Date $\underline{09 / 16 / 96}$

SITE DATA INFORMATION: 1-RD-10

Location: AIKEN, SOUTH CAROLINA

Building Air Exchanges Per Hour: 60 (User specified)

Date and Time: Fixed at July 1, 19960000 hours

\section{CHEMICAL INFORMATION:}

Chemical Name: CARBON BISULFIDE

Molecular Weight: $76.14 \mathrm{~kg} / \mathrm{kmol}$

TLV-TWA: $10.00 \mathrm{ppm}$ IDLH: $500.00 \mathrm{ppm}$

Footprint Level of Concern: 50 ppm

Boiling Point: $46.23^{\circ} \mathrm{C}$

Vapor Pressure at Ambient Temperature: $0.55 \mathrm{~atm}$

Ambient Saturation Concentration: 559,378 ppm or $55.9 \%$

\section{ATMOSPHERIC INFORMATION: (MANUAL INPUT OF DATA)}

Wind: 1.7 meters $/ \mathrm{sec}$ from $90^{\circ}$ true

Inversion Height: 200 meters

Stability Class: E Air Temperature: $29^{\circ} \mathrm{C}$

Relative Humidity: $50 \%$ Ground Roughness: 100 centimeters

Cloud Cover: 1 tenths

- SOURCE STRENGTH INFORMATION:

Puddle Area: 24420 square feet

Average Puddle Depth: 1 centimeters

Soil Type: Default Ground Temperature: $29^{\circ} \mathrm{C}$

Initial Puddle Temperature: Ground temperature

Release Duration: 51 minutes

Max Computed Release Rate: 1,120 kilograms/min

Max Average Sustained Release Rate: 775 kilograms/min

(averaged over a minute or more)

Total Amount Released: 28,347 kilograms

FOOTPRINT INFORMATION:

Model Run: Heavy Gas

User specified LOC: $50 \mathrm{ppm}$

Max Threat Zone for LOC: 2.2 kilometers

Max Threat Zone for IDLH: 524 meters

Note: The Heavy Gas footprint is an initial screening.

For short releases it may be an overestimation.

Be sure to check concentration information at specific locations.

\section{TIME DEPENDENT INFORMATION:}

Concentration Estimates at the point:

Downwind: 100 meters

Off Centerline: 0 meters

Max Concentration:

Outdoor: $11,600 \mathrm{ppm}$

Indoor: $11,600 \mathrm{ppm}$

Note: Indoor graph is shown with a dotted line.
Reviewed by $\underline{\text { S.A. Aenderson }}$

Works Calculation 7

Sheet No. 54 of 577 
Title of Project EPHA for THM

Subject Concentration Analysis for THM Hazardous Chemicals

Computer T. M. Franey

Date $\underline{09 / 16 / 96}$
Reviewed by $\mathrm{S}$. A. Henderson

Works Calculation 7

Sheet No. $\underline{55 \text { of } 577}$

SITE DATA INFORMATION: I-RD-10

Location: AIKEN, SOUTH CAROLINA

Building Air Exchanges Per Hour: 60 (User specified)

Date and Time: Fixed at July 1, 19960000 hours

CHEMICAL INFORMATION:

Chemical Name: CARBON BISULFIDE

Molecular Weight: $76.14 \mathrm{~kg} / \mathrm{kmol}$

TLV-TWA: $10.00 \mathrm{ppm}$ IDLH: $500.00 \mathrm{ppm}$

Footprint Level of Concern: 50 ppm

Boiling Point: $46.23^{\circ} \mathrm{C}$

Vapor Pressure at Ambient Temperature: $0.55 \mathrm{~atm}$

Ambient Saturation Concentration: 559,378 ppm or 55.9\%

ATMOSPHERIC INFORMATION: (MANUAL INPUT OF DATA)

Wind: 1.7 meters $/ \mathrm{sec}$ from $90^{\circ}$ true

Inversion Height: 200 meters

Stability Class: E Air Temperature: $29^{\circ} \mathrm{C}$

Relative Humidity: $50 \%$ Ground Roughness: 100 centimeters

Cloud Cover: 1 tenths

SOURCE STRENGTH INFORMATION:

Puddle Area: 40700 square feet

Average Puddle Depth: 1 centimeters

Soil Type: Default Ground Temperature: $29^{\circ} \mathrm{C}$

Initial Puddle Temperature: Ground temperature

Release Duration: 51 minutes

Max Computed Release Rate: 1,820 kịlograms/min

Max Average Sustained Release Rate: 1,270 kilograms/min

(averaged over a minute or more)

Total Amount Released: 47,244 kilograms

FOOTPRINT INFORMATION:

Model Run: Heavy Gas

User specified LOC: 50 ppm

Max Threat Zone for LOC: 2.8 kilometers

Max Threat Zone for ILH: 684 meters

Note: The Heavy Gas footprint is an initial screening.

For short releases it may be an overestimation.

Be sure to check concentration information at specific locations.

TIME DEPENDENT INFORMATTON:

Concentration Estimates at the point:

Downwind: 100 meters

Off Centerline: 0 meters

Max Concentration:

Outdoor: $19,400 \mathrm{ppm}$

Indoor: $19,300 \mathrm{ppm}$

Note: Indoor graph is shown with a dotted line. 


\section{ENGINEERING COMPUTATION SHEET}

Title of Project EPHA for THM

Subject Concentration Analysis for THM Hazardous Chemicals

Computer T.M. Franey

Date 09/16/96
Reviewed by $\underline{\text { S. A. Henderson }}$

Works Calculation 7

Sheet No. $\underline{56 \text { of } 577}$

SITE DATA INFORMATION: 1-RD-10

Location: AIKEN, SOUTH CAROLINA

Building Air Exchanges Per'Hour: 60 (User specified)

Date and Time: Fixed at July 1, 19960000 hours

CHEMICAL INFORMATION:

Chemical Name: CARBON BISULFIDE

Molecular Weight: $76.14 \mathrm{~kg} / \mathrm{kmol}$

TLV-TWA: $10.00 \mathrm{ppm}$ IDLH: $500.00 \mathrm{ppm}$

Footprint Level of Concern: $50 \mathrm{ppm}$

Boiling Point: $46.23^{\circ} \mathrm{C}$

Vapor Pressure at Ambient Temperature: $0.55 \mathrm{~atm}$

Ambient Saturation Concentration: $559,378 \mathrm{ppm}$ or $55.9 \%$

ATMOSPHERIC INFORMATION: (MANUAL INPUT OF DATA)

Wind: 1.7 meters $/ \mathrm{sec}$ from $90^{\circ}$ true .

Inversion Height: 200 meters

Stability Class: E Air Temperature: $29^{\circ} \mathrm{C}$

Relative Humidity: 50\% Ground Roughness: 100 centimeters

Cloud Cover: 1 tenths

SOURCE STRENGTH INFORMATION:

Puddle Area: 10000 square meters

Average Puddle Depth: 1 centimeters

Soil Type: Default Ground Temperature: $29^{\circ} \mathrm{C}$

Initial Puddle Temperature: Ground temperature

Release Duration: 53 minutes

Max Computed Release Rate: 4,630 kilograms/min

Max Average Sustained Release Rate: 3,230 kilograms/min

(averaged over a minute or more)

Total Amount Released: 124,947 kilograms

FOOTPRINT INFORMATION:

Model Run: Heavy Gas .

User specified LOC: $50 \mathrm{ppm}$

Max Threat Zone for LOC: 4.5 kilometers

Max Threat Zone for IDLH: 1.1 kilometers

Note: The Heavy Gas footprint is an initial screening.

For short releases it may be an overestimation.

Be sure to check concentration information at specific locations.

TIME DEPENDENT INFORMATION:

Conçentration Estimates at the point:

Downwind: 100 meters

Off Centerline: 0 meters

Max Concentration:

Outdoor: $101,000 \mathrm{ppm}$

Indoor: $101,000 \mathrm{ppm}$

Note: Indoor graph is shown with a dotted line. 
Title of Project EPHA for THM

Subject Concentration Analysis for THM Hazardous Chemicals

Computer T. M. Franey
Reviewed by S.A. Henderson

Works Calculation 7

Sheet No. 57 of 577

\section{1-RD-11}

SITE DATA INFORMATION:

Location: AIKEN, SOUTH CAROLINA

Building Air Exchanges Per Hour: 60 (User specified)

Date and Time: Fixed at July 1, 19960000 hours

\section{CHEMICAL INFORMATION:}

Chemical Name: ACETYL CHLORIDE

Molecular Weight: $78.50 \mathrm{~kg} / \mathrm{kmol}$

TLV-TWA: -unavail- IDLH: -unavail-

Footprint Level of Concern: 5 ppm

Boiling Point: $50.75^{\circ} \mathrm{C}$

Vapor Pressure at Ambient Temperature: $0.45 \mathrm{~atm}$

Ambient Saturation Concentration: 453,066 ppm or $45.3 \%$

ATMOSPHERIC INFORMATION: (MANUAL INPUT OF DATA)

Wind: 1.7 meters $/ \mathrm{sec}$ from $90^{\circ}$ true

Inversion Height: 200 meters

Stability Class: E Air Temperature: $29^{\circ} \mathrm{C}$

Relative Humidity: $50 \% \quad$ Ground Roughness: Urban or forest

Cloud Cover: 1 tenths

\section{SOURCE STRENGTH INFORMATION:}

Puddle Area: 0.5 square feet

Average Puddle Depth: 1 centimeters

Soil Type: Default Ground Temperature: $29^{\circ} \mathrm{C}$

Initial Puddle Temperature: Ground temperature

Release Duration: 38 minutes

Max Computed Release Rate: 30.7 grams/min

Max Average Sustained Release Rate: 19.9 grams/min (averaged over a minute or more)

Total Amount Released: 509 grams

\section{TIME DEPENDENT INFORMATION:}

Concentration Estimates at the point:

Downwind: 30 meters

Off Centerline: 0 meters

Max Concentration:

Outdoor: $4.65 \mathrm{ppm}$

Indoor: $4.61 \mathrm{ppm}$

Note: Indoor graph is shown with a dotted line. 


\section{ENGINEERING COMPUTATION SHEET}

Title of Project EPHA for THM

Subject Concentration Analysis for THM Hazardous Chemicals

Computer T.M. Franey

Date $\underline{09 / 16 / 96}$
Reviewed by S. A. Henderson

Works Calculation 7

Sheet No. $\underline{58 \text { of } 577}$

1-RD-11

SITE DATA INFORMATION:

Location: AIKEN, SOUTH CAROLINA

Building Air Exchanges Per Hour: 60 (User specified)

Date and Time: Fixed at July 1, 19960000 hours

CHEMICAL INFORMATION:

Chemical Name: ACETYL CHLORIDE

Molecular Weight: $78.50 \mathrm{~kg} / \mathrm{kmol}$

TLV-TWA: -unavail- IDLH: -unavail-

Footprint Level of Concern: 5 ppm

Boiling Point: $50.75^{\circ} \mathrm{C}$

Vapor Pressure at Ambient Temperature: 0.45 atm

Ambient Saturation Concentration: 453,066 ppm or $45.3 \%$

ATMOSPHERIC INFORMATION: (MANUAL INPUT OF DATA)

Wind: 1.7 meters $/ \mathrm{sec}$ from $90^{\circ}$ true

Inversion Height: 200 meters

Stability Class: E Air Temperature: $29^{\circ} \mathrm{C}$

Relative Humidity: 50\% Ground Roughness: Urban or forest

Cloud Cover: I tenths

SOURCE STRENGTH INFORMATION:

Puddle Area: 7 square feet

Average Puddle Depth: 1 centimeters

Soil Type: Default Ground Temperature: $29^{\circ} \mathrm{C}$

Initial Puddle Temperature: Ground temperature

Release Duration: 43 minutes

Max Computed Release Rate: 357 grams/min

Max Average Sustained Release Rate: 237 grams/min

(averaged over a minute or more)

Total Amount Released: 7.13 kilograms

TIME DEPENDENT INFORMATION:

Concentration Estimates at the point:

Downwind: 100 meters

Off Centerline: 0 meters

Max Concentration:

Outdoor: $5.24 \mathrm{ppm}$

Indoor: $5.21 \mathrm{ppm}$

Note: Indoor graph is shown with a dotted line. 


\section{ENGINEERING COMPUTATION SHEET}

Title of Project EPHA for THM

Subject Concentration Analysis for THM Hazardous Chemicals

Computer T.M. Franey
Reviewed by $\underline{\text { S. A. Henderson }}$

Works Calculation 7

Sheet No. $\underline{59 \text { of } 577}$

1-RD-11

SITE DATA INFORMATION:

Location: AIKEN, SOUTH CAROLINA

Building Air Exchanges Per Hour: 60 (User specified)

Date and Time: Fixed at July 1, 19960000 hours

CHEMICAL INFORMATION:

Chemical Name: ACETYL CHLORIDE

Molecular Weight: $78.50 \mathrm{~kg} / \mathrm{kmol}$

TLV-TWA: -unavail- IDLH: -unavail-

Footprint Level of Concern: 5 ppm

Boiling Point: $50.75^{\circ} \mathrm{C}$

Vapor Pressure at Ambient Temperature: $0.45 \mathrm{~atm}$

Ambient Saturation Concentration: 453,066 ppm or $45.3 \%$

ATMOSPHERIC INFORMATION: (MANUAL INPUT OF DATA)

Wind: 1.7 meters $/ \mathrm{sec}$ from $90^{\circ}$ true

Inversion Height: 200 meters

Stability Class: E Air Temperature: $29^{\circ} \mathrm{C}$

Relative Humidity: 50\% Ground Roughness: Urban or forest

Cloud Cover: 1 tenths

SOURCE STRENGTH INFORMATION:

Puddle Area: 24420 square feet

Average Puddle Depth: 1 centimeters

Soil Type: Default Ground Temperature: $29^{\circ} \mathrm{C}$

Initial Puddle Temperature: Ground temperature

Release Duration: ALOHA limited the duration to 1 hour

Max Computed Release Rate: 808 kilograms/min

Max Average Sustained Release Rate: 565 kilograms/min

(averaged over a minute or more)

Total Amount Released: 24,523 kilograms

FOOTPRINT INFORMATION:

Model Run: Heavy Gas

User specified LOC: 5 ppm

Max Threat Zone for LOC: 8.9 kilometers

Note: The Heavy Gas footprint is an initial screening.

For short releases it may be an overestimation.

Be sure to check concentration information at specific locations.

TIME DEPENDENT INFORMATION:

Concentration Estimates at the point:

Downwind: 100 meters

Off Centerline: 0 meters

Max Concentration:

Outdoor: $7,910 \mathrm{ppm}$ 


\section{ENGINEERING COMPUTATION SHEET}

Title of Project EPHA for THM

Reviewed by $S$. A. Henderson

Subject Concentration Analysis for THM Hazardous Chemicals

Works Calculation 7

Computer T.M. Franey

Date $\underline{09 / 16 / 96}$

Sheet No. 60 of 577

\section{1-RD-11 SITE DATA INFORMATION:}

Location: AIKEN, SOUTH CAROLINA

Building Air Exchanges Per Hour: 60 (User specified)

Date and Time: Fixed at July 1, 19960000 hours

CHEMICAL INFORMATION:

Chemical Name: ACETYL CHLORIDE

Molecular Weight: $78.50 \mathrm{~kg} / \mathrm{kmol}$

TLV-TWA: -unavail- IDLH: -unavail-

Footprint Level of Concern: 5 ppm

Boiling Point: $50.75^{\circ} \mathrm{C}$

Vapor Pressure at Ambient Temperature: $0.45 \mathrm{~atm}$

Ambient Saturation Concentration: $453,066 \mathrm{ppm}$ or $45.3 \%$

\section{ATMOSPHERIC INFORMATION: (MANUAL INPUT OF DATA) \\ Wind: 1.7 meters $/ \mathrm{sec}$ from $90^{\circ}$ true \\ Inversion Height: 200 meters \\ Stability Class: E Air Temperature: $29^{\circ} \mathrm{C}$ \\ Relative Humidity: $50 \%$ Ground Roughness: Urban or forest \\ Cloud Cover: 1 tenths}

\section{SOURCE STRENGTH INFORMATION:}

Puddle Area: 40700 square feet

Average Puddle Depth: 1 centimeters

Soil Type: Default Ground Temperature: $29^{\circ} \mathrm{C}$

Initial Puddle Temperature: Ground temperature

Release Duration: ALOHA limited the duration to 1 hour

Max Computed Release Rate: 1,320 kilograms/min

Max Average Sustained Release Rate: 923 kilograms/min

(averaged over a minute or more)

Total Amount Released: 40,292 kilograms

FOOTPRINT INFORMATION:

Model Run: Heavy Gas

User specified LOC: 5 ppm

Max Threat Zone for LOC: greater than 10 kilometers

Note: The Heavy Gas footprint is an initial screening.

For short releases it may be an overestimation.

Be sure to check concentration information at specific locations.

TIME DEPENDENT INFORMATION:

Concentration Estimates at the point:

Downwind: 100 meters

Off Centerline: 0 meters

Max Concentration:

Outdoor: $13,100 \mathrm{ppm}$ 
Title of Project EPHA for THM

Subject Concentration Analysis for THM Hazardous Chemicals

Computer T. M. Franey

Date $\underline{09 / 16 / 96}$
Reviewed by $\mathbf{S}$. A. Henderson

Works Calculation 7

Sheet No. 61 of 577

\section{1-RD-11 SITE DATA INFORMATION:}

Location: AIKEN, SOUTH CAROLINA

Building Air Exchanges Per Hour: 60 (User specified)

Date and Time: Fixed at July 1, 19960000 hours

CHEMICAL INFORMATION:

Chemical Name: ACETYL CHLORIDE

Molecular Weight: $78.50 \mathrm{~kg} / \mathrm{kmol}$

TLV-TWA: -unavail- IDLH: -unavail-

Footprint Level of Concern: 5 ppm

Boiling Point: $50.75^{\circ} \mathrm{C}$

Vapor Pressure at Ambient Temperature: $0.45 \mathrm{~atm}$

Ambient Saturation Concentration: $453,066 \mathrm{ppm}$ or $45.3 \%$

ATMOSPHERIC INFORMATION: (MANUAL INPUT OF DATA)

Wind: 1.7 meters $/ \mathrm{sec}$ from $90^{\circ}$ true

Inversion Height: 200 meters

Stability Class: E Air Temperature: $29^{\circ} \mathrm{C}$

Relative Humidity: 50\% Ground Roughness: Urban or forest

Cloud Cover: 1 tenths

SOURCE STRENGTH INFORMATION:

Puddle Area: 10000 square meters

Average Puddle Depth: 1 centimeters

Soil Type: Default Ground Temperature: $29^{\circ} \mathrm{C}$

Initial Puddle Temperature: Ground temperature

Release Duration: ALOHA limited the duration to 1 hour

Max Computed Release Rate: 3,340 kilograms/min

Max Average Sustained Release Rate: 2,360 kilograms/min (averaged over a minute or more)

Total Amount Released: 103,802 kilograms

\section{FOOTPRINT INFORMATION:}

Model Run: Heavy Gas

User specified LOC: 5 ppm

Max Threat Zone for LOC: greater than 10 kilometers

Note: The Heavy Gas footprint is an initial screening:

For short releases it may be an overestimation.

$\mathrm{Be}$ sure to check concentration information at specific locations.

TIME DEPENDENT INFORMATION:

Concentration Estimates at the point:

Downwind: 100 meters

Off Centerline: 0 meters

Max Concentration:

Outdoor: $33,600 \mathrm{ppm}$ 
Title of Project EPHA for THM

Subject Concentration Analysis for THM Hazardous Chemicals

Computer T. M. Franey

Date $\underline{09 / 16 / 96}$
Reviewed by $\underline{\text { S. A. Henderson }}$

Works Calculation 7

Sheet No. $\underline{62 \text { of } 577}$

1-RD-12

SITE DATA INFORMATION:

Location: AIKEN, SOUTH CAROLINA

Building Air Exchanges Per Hour: 60 (User specified)

Date and Time: Fixed at July 1, 19960000 hours

\section{CHEMICAL INFORMATION:}

Chemical Name: CHLOROSULFONIC ACID

Molecular Weight: $116.52 \mathrm{~kg} / \mathrm{kmol}$

TLV-TWA: unk ppm IDLH: not available ppm

Footprint Level of Concern: $10 \mathrm{ppm}$

Boiling Point: $330.00^{\circ} \mathrm{C}$

Vapor Pressure at Ambient Temperature: 7.51e-006 atm

Ambient Saturation Concentration: $7.64 \mathrm{ppm}$ or $7.64 \mathrm{e}-004 \%$

ATMOSPHERIC INFORMATION: (MANUAL INPUT OF DATA)

Wind: 1.7 meters $/ \mathrm{sec}$ from $90^{\circ}$ true

Inversion Height: 200 meters

Stability Class: E Air Temperature: $29^{\circ} \mathrm{C}$

Relative Humidity: 50\% Ground Roughness: 100 centimeters

Cloud Cover: 1 tenths

SOURCE STRENGTH INFORMATION:

Puddle Area: 30430 square feet

Average Puddle Depth: 1 centimeters

Soil Type: Default Ground Temperature: $29^{\circ} \mathrm{C}$

Initial Puddle Temperature: Ground temperature

Release Duration: ALOHA limited the duration to 1 hour

Max Computed Release Rate: 17.2 grams/min

Mảx Average Sustained Release Rate: $16.6 \mathrm{grams} / \mathrm{min}$

(averaged over a minute or more)

Total Amount Released: 954 grams

\section{TIME DEPENDENT INFORMATION:}

Concentration Estimates at the point:

Downwind: 30 meters

Off Centerline: 0 meters

Max Concentration:

Outdoor: $0.62 \mathrm{ppm}$

Indoor: $0.62 \mathrm{ppm}$

Note: Indoor graph is shown with a dotted line. 


\section{ENGINEERING COMPUTATION SHEET}

Title of Project EPHA for THM

Reviewed by $\mathrm{S}$. A. Henderson

Subject Concentration Analysis for THM Hazardous Chemicals

Works Calculation 7

Computer T. M. Franey

Date $\underline{09 / 16 / 96}$

Sheet No. 63 of 577

1-RD-12

SITE DATA INFORMATION:

Location: AIKEN, SOUTH CAROLINA

1 Building Air Exchanges Per Hour: 60 (User specified)

Date and Time: Fixed at July 1, 19960000 hours

CHEMICAL INFORMATION:

Chemical Name: CHLOROSULFONIC ACID

Molecular Weight: $116.52 \mathrm{~kg} / \mathrm{kmol}$

TLV-TWA: unk ppm IDLH: not available ppm

Footprint Level of Concern: $10 \mathrm{ppm}$

Boiling Point: $330.00^{\circ} \mathrm{C}$

Vapor Pressure at Ambient Temperature: 7.51e-006 atm

Ambient Saturation Concentration: $7.64 \mathrm{ppm}$ or $7.64 \mathrm{e}-004 \%$

ATMOSPHERIC INFORMATION: (MANUAL INPUT OF DATA)

Wind: 1.7 meters $/ \mathrm{sec}$ from $90^{\circ}$ true

Inversion Height: 200 meters

Stability Class: E Air Temperature: $29^{\circ} \mathrm{C}$

Relative Humidity: 50\% Ground Roughness: 100 centimeters

Cloud Cover: 1 tenths

SOURCE STRENGTH INFORMATION:

Puddle Area: 99999 square feet

Average Puddle Depth: 1 centimeters

Soil Type: Default Ground Temperature: $29^{\circ} \mathrm{C}$

Initial Puddle Temperature: Ground temperature

Release Duration: ALOHA limited the duration to 1 hour

Max Computed Release Rate: $53.8 \mathrm{grams} / \mathrm{min}$

Max Average Sustained Release Rate: 51.9 grams/min

(averaged over a minute or more)

Total Amount Released: 2.99 kilograms

FOOTPRINT INFORMATION:

Dispersion Module: Gaussian

User specified LOC: $10 \mathrm{ppm}$

Max Threat Zone for LOC: 55 meters

Max Threat Zone for IDLH: 54 meters

Note: Footprint was not drawn because effects of near-field patchiness make plume presentation unreliable for short distances.

TIME DEPENDENT INFORMATION:

Concentration Estimates at the point:

Downwind: 100 meters

Off Centerline: 0 meters

Max Concentration:

Outdoor: $0.184 \mathrm{ppm}$

Indoor: $0.184 \mathrm{ppm}$

Note: Indoor graph is shown with a dotted line. 
Title of Project EPHA for THM

Subject Concentration Analysis for THM Hazardous Chemicals

Computer T. M. Franey
Reviewed by S. A. Henderson

Works Calculation 7

Sheet No. 64 of 577

1-RD-12

SITE DATA INFORMATION:

Location: AIKEN, SOUTH CAROLINA

Building Air Exchanges Per Hour: 60 (User specified).

Date and Time: Fixed at July 1, 19960000 hours

CHEMICAL INFORMATTON:

Chemical Name: CHLOROSULFONIC ACID

Molecular Weight: $116.52 \mathrm{~kg} / \mathrm{kmol}$

TLV-TWA: unk ppm IDLH: not available ppm

Footprint Level of Concern: $10 \mathrm{ppm}$

Boiling Point: $330.00^{\circ} \mathrm{C}$

Vapor Pressure at Ambient Temperature: 7.51e-006 atm

Ambient Saturation Concentration: 7.64 ppm or $7.64 \mathrm{e}-004 \%$

ATMOSPHERIC INFORMATION: (MANUAL INPUT OF DATA)

Wind: 1.7 meters $/ \mathrm{sec}$ from $90^{\circ}$ true

Inversion Height: 200 meters

Stability Class: E Air Temperature: $29^{\circ} \mathrm{C}$

Relative Humidity: 50\% Ground Roughness: 100 centimeters

Cloud Cover: 1 tenths

\section{SOURCE STRENGTH INFORMATION:}

Puddle Area: 24420 square feet

Average Puddle Depth: 1 centimeters

Soil Type: Default Ground Temperature: $29^{\circ} \mathrm{C}$

Initial Puddle Temperature: Ground temperature

Release Duration: ALOHA limited the duration to 1 hour

Max Computed Release Rate: 13.9 grams/min

Max Average Sustained Release Rate: $13.4 \mathrm{grams} / \mathrm{min}$

(averaged over a minute or more)

Total Amount Released: 773 grams

\section{FOOTPRINT INFORMATION:}

Dispersion Module: Gaussian

User specified LOC: $10 \mathrm{ppm}$

Max Threat Zone for LOC: 27 meters

Max Threat Zone for IDLH: 27 meters

Note: Footprint was not drawn because effects of near-field patchiness make plume presentation unreliable for short distances.

\section{TIME DEPENDENT INFORMATION:}

Concentration Estimates at the point:

Downwind: 100 meters

Off Centerline: 0 meters

Max Concentration:

Outdoor: $0.0551 \mathrm{ppm}$

Indoor: $0.0551 \mathrm{ppm}$

Note: Indoor graph is shown with a dotted line. 
Title of Project EPHA for THM

Subject Concentration Analysis for THM Hazardous Chemicals

Computer T.M. Franey
Reviewed by S.A. Henderson

Works Calculation 7

Sheet No. 65 of 577

1-RD-12

SITE DATA INFORMATION:

Location: AIKEN, SOUTH CAROLINA

Building Air Exchanges Per Hour: 60 (User specified)

Date and Time: Fixed at July 1, 19960000 hours

CHEMICAL INFORMATION:

Chemical Name: CHLOROSULFONIC ACID

Molecular Weight: $116.52 \mathrm{~kg} / \mathrm{kmol}$

TLV-TWA: unk ppm IDH: not available ppm

Footprint Level of Concern: $10 \mathrm{ppm}$

Boiling Point: $330.00^{\circ} \mathrm{C}$

Vapor Pressure at Ambient Temperature: 7.51e-006 atm

Ambient Saturation Concentration: $7.64 \mathrm{ppm}$ or $7.64 \mathrm{e}-004 \%$

ATMOSPHERIC INFORMATION: (MANUAL INPUT OF DATA)

Wind: 1.7 meters $/ \mathrm{sec}$ from $90^{\circ}$ true

Inversion Height: 200 meters

Stability Class: E Air Temperature: $29^{\circ} \mathrm{C}$

Relative Humidity: 50\% Ground Roughness: 100 centimeters

Cloud Cover: 1 tenths

SOURCE STRENGTH INFORMATION:

Puddle Area: 40700 square feet

Average Puddle Depth: 1 centimeters

Soil Type: Default Ground Temperature: $29^{\circ} \mathrm{C}$

Initial Puddle Temperature: Ground temperature

- Release Duration: ALOHA limited the duration to 1 hour

Max Computed Release Rate: 22.7 grams/min

Max Average Sustained Release Rate: 21.9 grams/min

(averaged over a minute or more)

Total Amount Released: 1.26 kilograms

FOOTPRINT INFORMATION:

Dispersion Module: Gaussian

User specified LOC: $10 \mathrm{ppm}$

Max Threat Zone for LOC: 34 meters

Max Threat Zone for IDLH: 35 meters

Note: Footprint was not drawn because effects of near-field patchiness make plume presentation unreliable for short distances.

TIME DEPENDENT INFORMATION:

Concentration Estimates at the point:

Downwind: 100 meters

Off Centerline: 0 meters

Max Concentration:

Outdoor: $0.0799 \mathrm{ppm}$

Indoor: $0.0799 \mathrm{ppm}$

Note: Indoor graph is shown with a dotted line. 
Title of Project EPHA for THM

Subject Concentration Analysis for THM Hazardous Chemicals

Computer T. M. Franey

Date $\underline{09 / 16 / 96}$

1-RD-12

SITE DATA INFORMATION

Location: AIKEN, SOUTH CAROLINA

Building Air Exchanges Per Hour: 60 (User specified)

Date and Time: Fixed at July 1, 19960000 hours

CHEMICAL INFORMATION:

Chemical Name: CHLOROSULFONIC ACID

Molecular Weight: $116.52 \mathrm{~kg} / \mathrm{kmol}$

TLV-TWA: unk ppm IDLH: not available ppm

Footprint Level of Concern: 10 ppm

Boiling Point: $330.00^{\circ} \mathrm{C}$

Vapor Pressure at Ambient Temperature: 7.51e-006 atm

Ambient Saturation Concentration: 7.64 ppm or $7.64 \mathrm{e}-004 \%$

\section{ATMOSPHERIC INFORMATION: (MANUAL INPUT OF DATA)}

Wind: 1.7 meters $/ \mathrm{sec}$ from $90^{\circ}$ true

Inversion Height: 200 meters

Stability Class: E Air Temperature: $29^{\circ} \mathrm{C}$

Relative Humidity: $50 \%$ Ground Roughness: 100 centimeters

Cloud Cover: 1 tenths

\section{SOURCE STRENGTH INFORMATION:}

Puddle Area: 10000 square meters

Average Puddle Depth: 1 centimeters

Soil Type: Default Ground Temperature: $29^{\circ} \mathrm{C}$

Initial Puddle Temperature: Ground temperature

Release Duration: ALOHA limited the duration to 1 hour

Max Computed Release Rate: 57.7 grams/min

Max Average Sustained Release Rate: 55.7 grams/min

(averaged over a minute or more)

Total Amount Released: 3.21 kilograms

\section{FOOTPRINT INFORMATION:}

Dispersion Module: Gaussian

User specified LOC: $10 \mathrm{ppm}$

Max Threat Zone for LOC: 56 meters

Max Threat Zone for IDLH: 56 meters

Note: Footprint was not drawn because effects of near-field patchiness make plume presentation unreliable for short distances.

\section{TIME DEPENDENT INFORMATION:}

Concentration Estimates at the point:

Downwind: 100 meters

Off Centerline: 0 meters

Max Concentration:

Outdoor: $0.197 \mathrm{ppm}$

Indoor: $0.197 \mathrm{ppm}$

Note: Indoor graph is shown with a dotted line.
Reviewed by $\underline{S}$. A. Henderson

Works Calculation 7

Sheet No. 66 of 577 


\section{ENGINEERING COMPUTATION SHEET}

Title of Project EPHA for THM

Subject Concentration Analysis for THM Hazardous Chemicals

Computer T. M. Franey Date $\underline{09 / 16 / 96}$

SITE DATA INFORMATION: 1-RD-13

Location: AIKEN, SOUTH CAROLINA

Building Air Exchanges Per Hour: 60 (User specified)

Date and Time: Fixed at July 1, 19960000 hours

CHEMICAL INFORMATION:

Chemical Name: CYCLOHEXANE

Molecular Weight: $84.16 \mathrm{~kg} / \mathrm{kmol}$

TLV-TWA: $300.00 \mathrm{ppm}$ IDLH: $10000.00 \mathrm{ppm}$

Footprint Level of Concern: 1300 ppm

Boiling Point: $80.72^{\circ} \mathrm{C}$

Vapor Pressure at Ambient Temperature: $0.15 \mathrm{~atm}$

Ambient Saturation Concentration: 157,328 ppm or $15.7 \%$

ATMOSPHERIC INFORMATION: (MANUAL INPUT OF DATA)

Wind: 1.7 meters $/ \mathrm{sec}$ from $90^{\circ}$ true

Inversion Height: 200 meters

Stability Class: E Air Temperature: $29^{\circ} \mathrm{C}$

Relative Humidity: 50\% Ground Roughness: 100 centimeters

Cloud Cover: 1 tenths

\section{SOURCE STRENGTH INFORMATION:}

Puddle Area: 1045 square feet

Average Puddle Depth: 1 centimeters

Soil Type: Default Ground Temperature: $29^{\circ} \mathrm{C}$

Initial Puddle Temperature: Ground temperature

Release Duration: ALOHA limited the duration to 1 hour

Max Computed Release Rate: 13.2 kilograms/min

Max Average Sustained Release Rate: 11 kilograms/min

(averaged over a minute or more)

Total Amount Released: 555 kilograms

\section{FOOTPRINT INFORMATION:}

Model Run: Heavy Gas

User specified LOC: $1300 \mathrm{ppm}$

Max Threat Zone for LOC: 30 meters

Max Threat Zone for IDLH: less than 10 meters(10.9 yards)

Note: Footprint wasn't drawn because effects of near-field patchiness make plume presentation unreliable for short distances.

TIME DEPENDENT INFORMATION:

Concentration Estimates at the point:

Downwind: 30 meters

Off Centerline: 0 meters

Max Concentration:

Outdoor: $1,300 \mathrm{ppm}$

Indoor: $1,300 \mathrm{ppm}$

Note: Indoor graph is shown with a dotted line.
Reviewed by S. A. Henderson

Works Calculation 7

Sheet No. 67 of 577 
Title of Project EPHA for THM

Subject Concentration Analysis for THM Hazardous Chemicals

Computer T. M. Franey

Date $\underline{09 / 16 / 96}$
Reviewed by $\underline{\text { S. A. Henderson }}$

Works Calculation 7

Sheet No. 68 of 577

SITE DATA INFORMATION: 1-RD-13

Location: AIKEN, SOUTH CAROLINA

Building Air Exchanges Per Hour: 60 (User specified)

Date and Time: Fixed at July 1, 19960000 hours

CHEMICAL INFORMATION:

Chemical Name: CYCLOHEXANE

Molecular Weight: $84.16 \mathrm{~kg} / \mathrm{kmol}$

TLV-TWA: $300.00 \mathrm{ppm}$ IDLH: $10000.00 \mathrm{ppm}$

Footprint Level of Concern: 1300 ppm

Boiling Point: $80.72^{\circ} \mathrm{C}$

Vapor Pressure at Ambient Temperature: $0.15 \mathrm{~atm}$

Ambient Saturation Concentration: 157,328 ppm or $15.7 \%$

ATMOSPHERIC INFORMATION: (MANUAL INPUT OF DATA)

Wind: 1.7 meters $/ \mathrm{sec}$ from $90^{\circ}$ true

Inversion Height: 200 meters

Stability Class: E . Air Temperature: $29^{\circ} \mathrm{C}$

Relative Humidity: $50 \%$ Ground Roughness: 100 centimeters

Cloud Cover: 1 tenths

SOURCE STRENGTH INFORMATION:

Puddle Area: 11000 square feet

Average Puddle Depth: 1 centimeters

Soil Type: Default Ground Temperature: $29^{\circ} \mathrm{C}$

Initial Puddle Temperature: Ground temperature

Release Duration: ALOHA limited the duration to 1 hour

Max Computed Release Rate: 125 kilograms/min

Max Average Sustained Release Rate: 106 kilograms/min

(averaged over a minute or more)

Total Amount Released: 5,356 kilograms

FOOTPRINT INFORMATION:

Model Run: Heavy Gas

User specified LOC: $1300 \mathrm{ppm}$

Max Threat Zone for LOC: 97 meters

Max Threat Zone for IDLH: 26 meters

Note: Footprint wasn't drawn because effects of near-field patchiness make plume presentation unreliable for short distances.

TLME DEPENDENT INFORMATION:

Concentration Estimates at the point:

Downwind: 100 meters

Off Centerline: 0 meters

Max Concentration:

Outdoor: $1,300 \mathrm{ppm}$

Indoor: $1,300 \mathrm{ppm}$

Note: Indoor graph is shown with a dotted line. 
Title of Project EPHA for THM

Subject Concentration Analysis for THM Hazardous Chemicals

Computer T.M. Franey
Date 09/16/96
Reviewed by S. A. Henderson

Works Calculation 7

Sheet No. 69 of 577

\section{SITE DATA INFORMATION: 1-RD-13}

Location: AIKEN, SOUTH CAROLINA

Building Air Exchanges Per Hour: 60 (User specified)

Date and Time: Fixed at July 1, 19960000 hours

CHEMICAL INFORMATION:

Chemical Name: CYCLOHEXANE

Molecular Weight: $84.16 \mathrm{~kg} / \mathrm{kmol}$

TLV-TWA: $300.00 \mathrm{ppm}$ IDLH: $10000.00 \mathrm{ppm}$

Footprint Level of Concern: 1300 ppm

Boiling Point: $80.72^{\circ} \mathrm{C}$

Vapor Pressure at Ambient Temperature: $0.15 \mathrm{~atm}$

Ambient Saturation Concentration: 157,328 ppm or $15.7 \%$

ATMOSPHERIC INFORMATION: (MANUAL INPUT OF DATA)

Wind: 1.7 meters $/ \mathrm{sec}$ from $90^{\circ}$ true

Inversion Height: 200 meters

Stability Class: E Air Temperature: $29^{\circ} \mathrm{C}$

Relative Humidity: 50\% Ground Roughness: 100 centimeters

Cloud Cover: 1 tenths

\section{SOURCE STRENGTH INFORMATION:}

Puddle Area: 20420 square feet

Average Puddle Depth: 1 centimeters

Soil Type: Default Ground Temperature: $29^{\circ} \mathrm{C}$

Initial Puddle Temperature: Ground temperature

Release Duration: ALOHA limited the duration to 1 hour

Max Computed Release Rate: 225 kilograms/min

Max Average Sustained Release Rate: 191 kilograms/min

(averaged over a minute or more)

Total Amount Released: 9,730 kilograms

\section{FOOTPRINT INFORMATION:}

Model Run: Heavy Gas

User specified LOC: $1300 \mathrm{ppm}$

Max Threat Zone for LOC: 135 meters

Max Threat Zone for IDLH: 36 meters

Note: The Heavy Gas footprint is an initial screening.

For short releases it may be an overestimation.

Be sure to check concentration information at specific locations.

\section{TIME DEPENDENT INFORMATION:}

Concentration Estimates at the point:

Downwind: 100 meters

Off Centerline: 0 meters

Max Concentration:

Outdoor: 2,270 ppm

Indoor: $2,270 \mathrm{ppm}$

Note: Indoor graph is shown with a dotted line. 


\section{ENGINEERING COMPUTATION SHEET}

Title of Project EPHA for THM

Subject Concentration Analysis for THM Hazardous Chemicals

Computer T. M. Franey
Reviewed by $\underline{S . A . H e n d e r s o n}$

Works Calculation 7

Sheet No. 70 of 577

\section{SITE DATA INFORMATION: 1-RD-13}

Location: AIKEN, SOUTH CAROLINA

Building Air Exchanges Per Hour: 60 (User specified)

Date and Time: Fixed at July 1, 19960000 hours

CHEMICAL INFORMATION:

Chemical Name: CYCLOHEXANE

Molecular Weight: $84.16 \mathrm{~kg} / \mathrm{kmol}$

TLV-TWA: $300.00 \mathrm{ppm}$ IDLH: $10000.00 \mathrm{ppm}$

Footprint Level of Concern: 1300 ppm

Boiling Point: $80.72^{\circ} \mathrm{C}$

Vapor Pressure at Ambient Temperature: 0.15 atm

Ambient Saturation Concentration: 157,328 ppm or $15.7 \%$

\section{ATMOSPHERIC INFORMATION: (MANUAL INPUT OF DATA)}

Wind: 1.7 meters $/ \mathrm{sec}$ from $90^{\circ}$ true

Inversion Height: 200 meters

Stability Class: E Air Temperature: $29^{\circ} \mathrm{C}$

Relative Humidity: $50 \%$ Ground Roughness: 100 centimeters

Cloud Cover: 1 tenths

\section{SOURCE STRENGTH INFORMATION:}

Puddle Area: 40700 square feet

Average Puddle Depth: 1 centimeters

Soil Type: Default Ground Temperature: $29^{\circ} \mathrm{C}$

Initial Puddle Temperature: Ground temperature

Release Duration: ALOHA limited the duration to 1 hour

Max Computed Release Rate: 436 kilograms/min

Max Average Sustained Release Rate: 371 kilograms/min

(averaged over a minute or more)

Total Amount Released: 18,941 kilograms

\section{FOOTPRINT INFORMATION:}

Model Run: Heavy Gas

User specified LOC: 1300 ppm

Max Threat Zone for LOC: 196 meters

- Max Threat Zone for IDLH: 55 meters

Note: The Heavy Gas footprint is an initial screening.

For short releases it may be an overestimation.

Be sure to check concentration information at specific locations.

\section{TIME DEPENDENT INFORMATION:}

Concentration Estimates at the point:

Downwind: 100 meters

Off Centerline: 0 meters

Max Concentration:

Outdoor: $4,230 \mathrm{ppm}$

Indoor: 4,230 ppm

Note: Indoor graph is shown with a dotted line. 
Title of Project EPHA for THM

Subject Concentration Analysis for THM Hazardous Chemicals

Computer T.M. Franey

Date $\underline{09 / 16 / 96}$
Reviewed by $\underline{\text { S. A. Henderson }}$

Works Calculation 7

Sheet No. 71 of 577

\section{SITE DATA INFORMATION: 1-RD-13}

Location: AIKEN, SOUTH CAROLINA

Building Air Exchanges Per Hour: 60 (User specified)

Date and Time: Fixed at July 1, 19960000 hours

\section{CHEMICAL INFORMATION:}

Chemical Name: CYCLOHEXANE

Molecular Weight: $84.16 \mathrm{~kg} / \mathrm{kmol}$

TLV-TWA: $300.00 \mathrm{ppm}$ IDLH: $10000.00 \mathrm{ppm}$

Footprint Level of Concern: $1300 \mathrm{ppm}$

Boiling Point: $80.72^{\circ} \mathrm{C}$

Vapor Pressure at Ambient Temperature: $0.15 \mathrm{~atm}$

Ambient Saturation Concentration: $157,328 \mathrm{ppm}$ or $15.7 \%$

\section{ATMOSPHERIC INFORMATION: (MANUAL INPUT OF DATA)}

Wind: 1.7 meters $/ \mathrm{sec}$ from $90^{\circ}$ true

Inversion Height: 200 meters

Stability Class: E Air Temperature: $29^{\circ} \mathrm{C}$

Relative Humidity: $50 \%$ Ground Roughness: 100 centimeters

Cloud Cover: 1 tenths

\section{SOURCE STRENGTH INFORMATION:}

Puddle Area: 10000 square meters

Average Puddle Depth: 1 centimeters

Soil Type: Default Ground Temperature: $29^{\circ} \mathrm{C}$

Initial Puddle Temperature: Ground temperature

Release Duration: ALOHA limited the duration to 1 hour

Max Computed Release Rate: 1,110 kilograms/min

Max Average Sustained Release Rate: 949 kilograms/min

(averaged over a minute or more)

Total Amount Released: 48,500 kilograms

\section{FOOTPRINT INFORMATION:}

Model Run: Heavy Gas

User specified LOC: $1300 \mathrm{ppm}$

Max Threat Zone for LOC: 332 meters

Max Threat Zone for IDLH: 96 meters

Note: The Heavy Gas footprint is an initial screening.

For short releases it may be an overestimation.

Be sure to check concentration information at specific locations.

TIME DEPENDENT INFORMATION:

Concentration Estimates at the point:

Downwind: 100 meters

Off Centerline: 0 meters

Max Concentration:

Outdoor: $9,940 \mathrm{ppm}$

Indoor: $9,940 \mathrm{ppm}$

Note: Indoor graph is shown with a dotted line. 


\section{ENGINEERING COMPUTATION SHEET}

Title of Project EPHA for THM

Subject Concentration Analysis for THM Hazardous Chemicals

Computer T. M. Franey Date 09/16/96

SITE DATA INFORMATION: $\quad$ 1-RD-14

Location: AIKEN, SOUTH CAROLINA

Building Air Exchanges Per Hour: 60 (User specified)

Date and Time: Fixed at July 1, 19960000 hours

\section{CHEMICAL INFORMATION:}

Chemical Name: CYCLOHEXANONE

Molecular Weight: $98.14 \mathrm{~kg} / \mathrm{kmol}$

TLV-TWA: $25.00 \mathrm{ppm}$ IDLH: $5000.00 \mathrm{ppm}$

Footprint Level of Concern: 1300 ppm

Boiling Point: $155.75^{\circ} \mathrm{C}$

Vapor Pressure at Ambient Temperature: $0.0072 \mathrm{~atm}$

Ambient Saturation Concentration: 7,361 ppm or $0.74 \%$

\section{ATMOSPHERIC INFORMATION: (MANUAL INPUT OF DATA)}

Wind: 1.7 meters $/ \mathrm{sec}$ from $90^{\circ}$ true

Inversion Height: 200 meters

Stability Class: E

Air Temperature: $29^{\circ} \mathrm{C}$

Relative Humidity: 50\% Ground Roughness: 100 centimeters

Cloud Cover: 1 tenths

\section{SOURCE STRENGTH INFORMATION:}

Puddle Area: 30430 square feet

Average Puddle Depth: 1 centimeters

Soil Type: Default Ground Temperature: $29^{\circ} \mathrm{C}$

Initial Puddle Temperature: Ground temperature

Release Duration: ALOHA limited the duration to 1 hour

Max Computed Release Rate: 9.7 kilograms/min

Max Average Sustained Release Rate: 9.35 kilograms/min

(averaged over a minute or more)

Total Amount Released: 537 kilograms

\section{FOOTPRINT INFORMATION:}

Dispersion Module: Gaussian

User specified LOC: $1300 \mathrm{ppm}$

Max Threat Zone for LOC: 30 meters

Max Threat Zone for IDLH: 30 meters

Note: Footprint was not drawn because effects of near-field patchiness make plume presentation unreliable for short distances.

\section{TIME DEPENDENT INFORMATION:}

Concentration Estimates at the point:

Downwind: 30 meters .

Off Centerline: 0 meters

Max Concentration:

Outdoor: $349 \mathrm{ppm}$

Indoor: $349 \mathrm{ppm}$

Note: Indoor graph is shown with a dotted line.
Reviewed by S. A. Henderson

Works Calculation 7

Sheet No. 72 of 577 


\section{ENGINEERING COMPUTATION SHEET}

Title of Project EPHA for THM

Subject Concentration Analysis for THM Hazardous Chemicals

Computer T. M. Franey

Date $\underline{09 / 16 / 96}$

SITE DATA INFORMATION: 1-RD-14

Location: AIKEN, SOUTH CAROLINA

Building Air Exchanges Per Hour: 60 (User specified)

Date and Time: Fixed at July 1, 19960000 hours

CHEMICAL INFORMATION:

Chemical Name: CYCLOHEXANONE

Molecular Weight: $98.14 \mathrm{~kg} / \mathrm{kmol}$

TLV-TWA: $25.00 \mathrm{ppm}$ IDLH: $5000.00 \mathrm{ppm}$

Footprint Level of Concern: 1300 ppm

Boiling Point: $155.75^{\circ} \mathrm{C}$

Vapor Pressure at Ambient Temperature: 0.0072 atm

Ambient Saturation Concentration: 7,361 ppm or $0.74 \%$

ATMOSPHERIC INFORMATION: (MANUAL INPUT OF DATA)

Wind: 1.7 meters $/ \mathrm{sec}$ from $90^{\circ}$ true

Inversion Height: 200 meters

Stability Class: E Air Temperature: $29^{\circ} \mathrm{C}$

Relative Humidity: 50\% Ground Roughness: 100 centimeters

Cloud Cover: 1 tenths

SOURCE STRENGTH INFORMATION:

Puddle Area: 99999 square feet

Average Puddle Depth: 1 centimeters

Soil Type: Default Ground Temperature: $29^{\circ} \mathrm{C}$

Initial Puddle Temperature: Ground temperature

Release Duration: ALOHA limited the duration to 1 hour

Max Computed Release Rate: 30.4 kilograms/min

Max Average Sustained Release Rate: 29.3 kilograms/min

(averaged over a minute or more)

Total Amount Released: 1,684 kilograms

FOOTPRINT INFORMATION:

Dispersion Module: Gaussian

User specified LOC: $1300 \mathrm{ppm}$

Max Threat Zone for LOC: 55 meters

Max Threat Zone for IDLH: 54 meters

Note: Footprint was not drawn because

effects of near-field patchiness make plume

presentation unreliable for short distances.

\section{TIME DEPENDENT INFORMATION:}

Concentration Estimates at the point:

Downwind: 100 meters

Off Centerline: 0 meters

Max Concentration:

Outdoor: $104 \mathrm{ppm}$

Indoor: $104 \mathrm{ppm}$

Note: Indoor graph is shown with a dotted line.
Reviewed by S. A. Henderson

Works Calculation 7

Sheet No. 73 of 577 


\section{ENGINEERING COMPUTATION SHEET}

Title of Project EPHA for THM

Subject Concentration Analysis for THM Hazardous Chemicals

Computer T. M. Franey

Date 09/16/96

Reviewed by S. A. Henderson

Works Calculation 7

Sheet No. 74 of 577

SITE DATA INFORMATION: 1-RD-14

Location: AIKEN, SOUTH CAROLINA

Building Air Exchanges Per Hour: 60 (User specified)

Date and Time: Fixed at July 1, 19960000 hours

CHEMICAL INFORMATION:

Chemical Name: CYCLOHEXANONE

Molecular Weight: $98.14 \mathrm{~kg} / \mathrm{kmol}$

TLV-TWA: $25.00 \mathrm{ppm}$. IDLH: $5000.00 \mathrm{ppm}$

Footprint Level of Concern: 1300 ppm

Boiling Point: $155.75^{\circ} \mathrm{C}$

Vapor Pressure at Ambient Temperature: $0.0072 \mathrm{~atm}$

Ambient Saturation Concentration: 7,361 ppm or $0.74 \%$

ATMOSPHERIC INFORMATION: (MANUAL INPUT OF DATA)

Wind: 1.7 meters $/ \mathrm{sec}$ from $90^{\circ}$ true

Inversion Height: 200 meters

Stability Class: E Air Temperature: $29^{\circ} \mathrm{C}$

Relative Humidity: 50\% Ground Roughness: 100 centimeters

Cloud Cover: 1 tenths

SOURCE STRENGTH INFORMATION:

Puddle Area: 24420 square feet

Average Puddle Depth: 1 centimeters

Soil Type: Default Ground Temperature: $29^{\circ} \mathrm{C}$

Initial Puddle Temperature: Ground temperature

Release Duration: ALOHA limited the duration to 1 hour

Max Computed Release Rate: 7.85 kilograms/min

Max Average Sustained Release Rate: 7.57 kilograms/min

(averaged over a minute or more)

Total Amount Released: 435 kilograms

FOOTPRINT INFORMATION:

Dispersion Module: Gaussian

User specified LOC: $1300 \mathrm{ppm}$

Max Threat Zone for LOC: 27 meters

Max Threat Zone for IDLH: 27 meters

Note: Footprint was not drawn because

effects of near-field patchiness make plume

presentation unreliable for short distances.

TIME DEPENDENT INFORMATION:

Concentration Estimates at the point:

Downwind: 100 meters

Off Centerline: 0 meters

Max Concentration:

Outdoor: $31.1 \mathrm{ppm}$

Indoor: $31.1 \mathrm{ppm}$

Note: Indoor graph is shown with a dotted line. 
Title of Project EPHA for THM

Subject Concentration Analysis for THM Hazardous Chemicals

Computer T.M. Franey Date $\underline{09 / 16 / 96}$
Reviewed by S. A. Henderson

Works Calculation 7

Sheet No. 75 of 577

\section{SITE DATA INFORMATION: 1-RD-14 \\ Location: AIKEN, SOUTH CAROLINA \\ Building Air Exchanges Per Hour: 60 (User specified) \\ Date and Time: Fixed at July 1, 19960000 hours}

\section{CHEMICAL INFORMATION:}

Chemical Name: CYCLOHEXANONE

Molecular Weight: $98.14 \mathrm{~kg} / \mathrm{kmol}$

TLV-TWA: $25.00 \mathrm{ppm}$ IDLH: $5000.00 \mathrm{ppm}$

Footprint Level of Concern: 1300 ppm

Boiling Point: $155.75^{\circ} \mathrm{C}$

Vapor Pressure at Ambient Temperature: $0.0072 \mathrm{~atm}$

Ambient Saturation Concentration: 7,361 ppm or $0.74 \%$

ATMOSPHERIC INFORMATION: (MANUAL INPUT OF DATA)

Wind: 1.7 meters $/ \mathrm{sec}$ from $90^{\circ}$ true

Inversion Height: 200 meters

Stability Class: E Air Temperature: $29^{\circ} \mathrm{C}$

Relative Humidity: $50 \%$ Ground Roughness: 100 centimeters

Cloud Cover: 1 tenths

\section{SOURCE STRENGTH INFORMATION:}

Puddle Area: 40700 square feet

Average Puddle Depth: 1 centimeters

Soil Type: Default Ground Temperature: $29^{\circ} \mathrm{C}$

Initial Puddle Temperature: Ground temperature

Release Duration: ALOHA limited the duration to 1 hour

Max Computed Release Rate: 12.8 kilograms/min

Max Average Sustained Release Rate: 12.4 kilograms/min (averaged over a minute or more)

Total Amount Released: 710 kilograms

\section{FOOTPRINT INFORMATION:}

Dispersion Module: Gaussian

User specified LOC: $1300 \mathrm{ppm}$

Max Threat Zone for LOC: 34 meters

Max Threat Zone for IDLH: 35 meters

Note: Footprint was not drawn because effects of near-field patchiness make plume presentation unreliable for short distances.

\section{TIME DEPENDENT INFORMATION:}

Concentration Estimates at the point:

Downwind: 100 meters

Off Centerline: 0 meters

Max Concentration:

Outdoor: $45 \mathrm{ppm}$

Indoor: $45 \mathrm{ppm}$

Note: Indoor graph is shown with a dotted line. 


\section{ENGINEERING COMPUTATION SHEET}

Title of Project EPHA for THM

Reviewed by S. A. Henderson

Subject Concentration Analysis for THM Hazardous Chemicals

Computer T. M. Franey

Date 09/16/96

SITE DATA INFORMATION: 1-RD-14

Location: AIKEN, SOUTH CAROLINA

Building Air Exchanges Per Hour: 60 (User specified)

Date and Time: Fixed at July 1, 19960000 hours

CHEMICAL INFORMATION:

Chemical Name: CYCLOHEXANONE

Molecular Weight: $98.14 \mathrm{~kg} / \mathrm{kmol}$

TLV-TWA: $25.00 \mathrm{ppm}$ IDLH: $5000.00 \mathrm{ppm}$

Footprint Level of Concern: 1300 ppm

Boiling Point: $155.75^{\circ} \mathrm{C}$

Vapor Pressure at Ambient Temperature: $0.0072 \mathrm{~atm}$

Ambient Saturation Concentration: 7,361 ppm or $0.74 \%$

ATMOSPHERIC INFORMATION: (MANUAL INPUT OF DATA)

Wind: 1.7 meters $/ \mathrm{sec}$ from $90^{\circ}$ true

Inversion Height: 200 meters

Stability Class: E Air Temperature: $29^{\circ} \mathrm{C}$

Relative Humidity: 50\% Ground Roughness: 100 centimeters

Cloud Cover: 1 tenths

\section{SOURCE STRENGTH INFORMATION:}

Puddle Area: 10000 square meters

Average Puddle Depth: 1 centimeters

Soil Type: Default Ground Temperature: $29^{\circ} \mathrm{C}$

Initial Puddle Temperature: Ground temperature

Release Duration: ALOHA limited the duration to 1 hour

Max Computed Release Rate: 32.6 kilograms/min

Max Average Sustained Release Rate: 31.4 kilograms/min (averaged over a minute or more)

Total Amount Released: 1,808 kilograms

FOOTPRINT INFORMATION:

Dispersion Module: Gaussian

User specified LOC: 1300 ppm

Max Threat Zone for LOC: 56 meters

Max Threat Zone for IDLH: 56 meters

Note: Footprint was not drawn because effects of near-field patchiness make plume presentation unreliable for short distances.

\section{TIME DEPENDENT INFORMATION:}

Concentration Estimates at the point:

Downwind: 100 meters

Off Centerline: 0 meters

Max Concentration:

Outdoor: $111 \mathrm{ppm}$

Indoor: $111 \mathrm{ppm}$

Note: Indoor graph is shown with a dotted line.
Works Calculation 7

Sheet No. 76 of 577 


\section{ENGINEERING COMPUTATION SHEET}

Title of Project EPHA for THM

Reviewed by $\underline{\text { S. A. Henderson }}$

Works Calculation 7

Sheet No. $\underline{77 \text { of } 577}$

SITE DATA INFORMATION:

$$
\text { 1-RD-15 }
$$

Location: AIKEN, SOUTH CAROLINA

Building Air Exchanges Per Hour: 60 (User specified)

Date and Time: Fixed at July 1, 19960000 hours

\section{CHEMICAL INFORMATION:}

Chemical Name: DIETHYLENE GLYCOL MONOETHYL ETHER

Molecular Weight: $134.18 \mathrm{~kg} / \mathrm{kmol}$

TLV-TWA: -unavail- IDLH: -unavail-

Footprint Level of Concern: 125 ppm

Boiling Point: $202.00^{\circ} \mathrm{C}$

Vapor Pressure at Ambient Temperature: 2.38e-004 atm

Ambient Saturation Concentration: 242 ppm or $0.024 \%$

ATMOSPHERIC INFORMATION: (MANUAL INPUT OF DATA)

Wind: 1.7 meters $/ \mathrm{sec}$ from $90^{\circ}$ true

Inversion Height: 200 meters

Stability Class: E Air Temperature: $29^{\circ} \mathrm{C}$

Relative Humidity: 50\% Ground Roughness: 100 centimeters

Cloud Cover: 1 tenths

\section{SOURCE STRENGTH INFORMATION:}

Puddle Area: 30430 square feet

Average Puddle Depth: 1 centimeters

Soil Type: Default Ground Temperature: $29^{\circ} \mathrm{C}$

Initial Puddle Temperature: Ground temperature

Release Duration: ALOHA limited the duration to 1 hour

Max Computed Release Rate: 255 grams/min

Max Average Sustained Release Rate: 245 grams/min

(averaged over a minute or more)

Total Amount Released: 14.1 kilograms

\section{TIME DEPENDENT INFORMATION:}

\section{Concentration Estimates at the point:}

Downwind: 30 meters

Off Centerline: 0 meters

Max Concentration:

Outdoor: $6.71 \mathrm{ppm}$

Indoor: $6.71 \mathrm{ppm}$

Note: Indoor graph is shown with a dotted line. 
Title of Project EPHA for THM

Subject Concentration Analysis for THM Hazardous Chemicals

Computer T. M. Franey

Date $\underline{09 / 16 / 96}$

SITE DATA INFORMATION:

$$
\text { 1-RD-15 }
$$

Location: AIKEN, SOUTH CAROLINA

Building Air Exchanges Per Hour: 60 (User specified)

Date and Time: Fixed at July 1, 19960000 hours

\section{CHEMICAL INFORMATION:}

Chemical Name: DIETHYLENE GLYCOL MONOETHYL ETHER

Molecular Weight: $134.18 \mathrm{~kg} / \mathrm{kmol}$

TLV-TWA: -unavail- IDLH: -unavail-

Footprint Level of Concern: 125 ppm

Boiling Point: $202.00^{\circ} \mathrm{C}$

Vapor Pressure at Ambient Temperature: 2.38e-004 atm

Ambient Saturation Concentration: 242 ppm or $0.024 \%$

\section{ATMOSPHERIC INFORMATION: (MANUAL INPUT OF DATA)}

Wind: 1.7 meters $/ \mathrm{sec}$ from $90^{\circ}$ true

Inversion Height: 200 meters

Stability Class: E Air Temperature: $29^{\circ} \mathrm{C}$

Relative Humidity: $50 \%$ Ground Roughness: 100 centimeters

Cloud Cover: 1 tenths

\section{SOURCE STRENGTH INFORMATION:}

Puddle Area: 99999 square feet

Average Puddle Depth: 1 centimeters

Soil Type: Default Ground Temperature: $29^{\circ} \mathrm{C}$

Initial Puddle Temperature: Ground temperature

Release Duration: ALOHA limited the duration to 1 hour

Max Computed Release Rate: 801 grams/min

Max Average Sustained Release Rate: 771 grams/min

(averaged over a minute or more)

Total Amount Released: 44.1 kilograms

\section{TIME DEPENDENT INFORMATION:}

\section{Concentration Estimates at the point:}

Downwind: 100 meters

Off Centerline: 0 meters

Max Concentration:

Outdoor: 2 ppm

Indoor: $2 \mathrm{ppm}$

Note: Indoor graph is shown with a dotted line. 


\section{ENGINEERING COMPUTATION SHEET}

Title of Project EPHA for THM

Subject Concentration Analysis for THM Hazardous Chemicals

Computer T. M. Franey

Date $09 / 16 / 96$

1-RD-15

SITE DATA INFORMATION:

Location: AIKEN, SOUTH CAROLINA

Building Air Exchanges Per Hour: 60 (User specified)

Date and Time: Fixed at July 1, 19960000 hours

CHEMICAL INFORMATION:

Chemical Name: DIETHYLENE GLYCOL MONOETHYL ETHER

Molecular Weight: $134.18 \mathrm{~kg} / \mathrm{kmol}$

TLV-TWA: -unavail- IDLH: -unavail-

Footprint Level of Concern: 125 ppm

Boiling Point: $202.00^{\circ} \mathrm{C}$

Vapor Pressure at Ambient Temperature: 2.38e-004 atm

Ambient Saturation Concentration: 242 ppm or 0.024\%

ATMOSPHERIC INFORMATION: (MANUAL INPUT OF DATA)

Wind: 1.7 meters $/ \mathrm{sec}$ from $90^{\circ}$ true

Inversion Height: 200 meters

Stability Class: E Air Temperature: $29^{\circ} \mathrm{C}$

Relative Humidity: 50\% Ground Roughness: 100 centimeters

Cloud Cover: 1 tenths

\section{SOURCE STRENGTH INFORMATION:}

Puddle Area: 24420 square feet

Average Puddle Depth: 1 centimeters

Soil Type: Default Ground Temperature: $29^{\circ} \mathrm{C}$

Initial Puddle Temperature: Ground temperature

Release Duration: ALOHA limited the duration to 1 hour

Max Computed Release Rate: 207 grams/min

Max Average Sustained Release Rate: 199 grams/min

(averaged over a minute or more)

Total Amount Released: 11.4 kilograms

FOOTPRINT INFORMATION:

Dispersion Module: Gaussian

User specified LOC: $125 \mathrm{ppm}$

Max Threat Zone for LOC: 27 meters

Note: Footprint was not drawn because

effects of near-field patchiness make plume

presentation unreliable for short distances.

\section{TIME DEPENDENT INFORMATION:}

Concentration Estimates at the point:

Downwind: 100 meters

Off Centerline: 0 meters

Max Concentration:

Outdoor: $0.596 \mathrm{ppm}$

Indoor: $0.596 \mathrm{ppm}$

Note: Indoor graph is shown with a dotted line.
Reviewed by $\underline{S}$. A. Henderson

Works Calculation 7

Sheet No. 79 of 577 
Title of Project EPHA for THM

Subject Concentration Analysis for THM Hazardous Chemicals

Computer T. M. Franey
Reviewed by S. A. Henderson

Works Calculation 7

Sheet No. 80 of 577

SITE DATA INFORMATION:

1-RD-15

Location: AIKEN, SOUTH CAROLINA

Building Air Exchanges Per Hour: 60 (User specified)

Date and Time: Fixed at July 1, 19960000 hours

CHEMICAL INFORMATION:

Chemical Name: DIETHYLENE GLYCOL MONOETHYL ETHER

Molecular Weight: $134.18 \mathrm{~kg} / \mathrm{kmol}$

TLV-TWA: -unavail- IDLH: -unavail-

Footprint Level of Concern: 125 ppm

Boiling Point: $202.00^{\circ} \mathrm{C}$

Vapor Pressure at Ambient Temperature: 2.38e-004 atm

Ambient Saturation Concentration: 242 ppm or $0.024 \%$

ATMOSPHERIC INFORMATION: (MANUAL INPUT OF DATA)

Wind: 1.7 meters $/ \mathrm{sec}$ from $90^{\circ}$ true

Inversion Height: 200 meters

Stability Class: E Air Temperature: $29^{\circ} \mathrm{C}$

Relative Humidity: $50 \%$ Ground Roughness: 100 centimeters

Cloud Cover: 1 tenths

SOURCE STRENGTH INFORMATION:

Puddle Area: 40700 square feet

Average Puddle Depth: 1 centimeters

Soil Type: Default Ground Temperature: $29^{\circ} \mathrm{C}$

Initial Puddle Temperature: Ground temperature

Release Duration: ALOHA limited the duration to 1 hour

Max Computed Release Rate: 338 grams/min

Max Average Sustained Release Rate: 325 grams/min

(averaged over a minute or more)

Total Amount Released: 18.6 kilograms

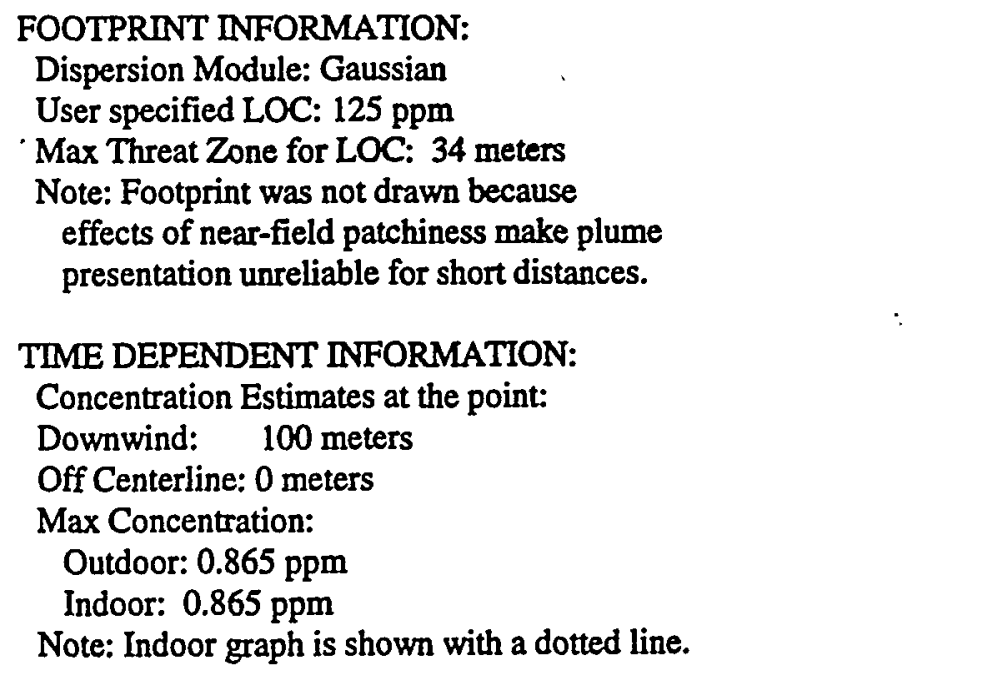


Title of Project EPHA for THM

Subject Concentration Analysis for THM Hazardous Chemicals

Computer T. M. Franey

Date $09 / 16 / 96$

$$
1-R D-15
$$

SITE DATA INFORMATION:

Location: AIKEN, SOUTH CAROLINA

Building Air Exchanges Per Hour: 60 (User specified)

Date and Time: Fixed at July 1, 19960000 hours

CHEMICAL INFORMATION:

Chemical Name: DIETHYLENE GLYCOL MONOETHYL ETHER Molecular Weight: $134.18 \mathrm{~kg} / \mathrm{kmol}$

TLV-TWA: -unavail- IDLH: -unavail-

Footprint Level of Concern: 125 ppm

Boiling Point: $202.00^{\circ} \mathrm{C}$

Vapor Pressure at Ambient Temperature: 2.38e-004 atm

Ambient Saturation Concentration: 242 ppm or $0.024 \%$

\section{ATMOSPHERIC INFORMATION: (MANUAL INPUT OF DATA)}

Wind: 1.7 meters $/ \mathrm{sec}$ from $90^{\circ}$ true

Inversion Height: 200 meters

Stability Class: E Air Temperature: $29^{\circ} \mathrm{C}$

Relative Humidity: $50 \%$ Ground Roughness: 100 centimeters

Cloud Cover: 1 tenths

\section{SOURCE STRENGTH INFORMATION:}

Puddle Area: 10000 square meters

Average Puddle Depth: 1 centimeters

Soil Type: Default Ground Temperature: $29^{\circ} \mathrm{C}$

Initial Puddle Temperature: Ground temperature

Release Duration: ALOHA limited the duration to 1 hour

Max Computed Release Rate: 860 grams/min

Max Average Sustained Release Rate: 827 grams/min

(averaged over a minute or more)

Total Amount Released: 47.4 kilograms

\section{FOOTPRINT INFORMATION:}

Dispersion Module: Gaussian

User specified LOC: 125 ppm

Max Threat Zone for LOC: 56 meters

Note: Footprint was not drawn because effects of near-field patchiness make plume presentation unreliable for short distances.

\section{TIME DEPENDENT INFORMATION:}

Concentration Estimates at the point:

Downwind: 100 meters

Off Centerline: 0 meters

Max Concentration:

Outdoor: $2.14 \mathrm{ppm}$

Indoor: $2.14 \mathrm{ppm}$

Note: Indoor graph is shown with a dotted line.
Reviewed by $\mathrm{S}$. A. Henderson

Works Calculation 7

Sheet No. 81 of 577

Works Calculation 7
- Sheet No. $\underline{81 \text { of } 577}$


ENGINEERING COMPUTATION SHEET

Title of Project EPHA for THM

Subject Concentration Analysis for THM Hazardous Chemicals

Computer T. M. Franey

Date $\underline{09 / 16 / 96}$

SITE DATA INFORMATION: 1-RD-16

Location: AIKEN, SOUTH CAROLINA

Building Air Exchanges Per Hour: 60 (User specified)

Date and Time: Fixed at July 1, 19960000 hours

CHEMICAL INFORMATION:

Chemical Name: DIOXANE

TLV-TWA: $25.00 \mathrm{ppm}$

Molecular Weight: $88.11 \mathrm{~kg} / \mathrm{kmol}$

Footprint Level of Concern: 125 ppm

Boiling Point: $101.32^{\circ} \mathrm{C}$

Vapor Pressure at Ambient Temperature: $0.061 \mathrm{~atm}$

Ambient Saturation Concentration: 62,381 ppm or $6.24 \%$

ATMOSPHERIC INFORMATION: (MANUAL INPUT OF DATA)

Wind: 1.7 meters $/ \mathrm{sec}$ from $90^{\circ}$ true

Inversion Height: 200 meters

Stability Class: E Air Temperature: $29^{\circ} \mathrm{C}$

Relative Humidity: 50\% Ground Roughness: 100 centimeters

Cloud Cover: 1 tenths

SOURCE STRENGTH INFORMATION:

Puddle Area: 200 square feet

Average Puddle Depth: 1 centimeters

Soil Type: Default Ground Temperature: $29^{\circ} \mathrm{C}$

Initial Puddle Temperature: Ground temperature

Release Duration: ALOHA limited the duration to 1 hour

Max Computed Release Rate: 994 grams/min

Max Average Sustained Release Rate: 887 grams/min

(averaged over a minute or more)

Total Amount Released: 47.5 kilograms

FOOTPRINT INFORMATION:

Dispersion Module: Gaussian

User specified LOC: $125 \mathrm{ppm}$

Max Threat Zone for LOC: 30 meters

Max Threat Zone for IDLH: less than 10 meters(10.9 yards)

Note: Footprint was not drawn because

effects of near-field patchiness make plume

presentation unreliable for short distances.

TIME DEPENDENT INFORMATION:

Concentration Estimates at the point:

Downwind: 30 meters

Off Centerline: 0 meters

Max Concentration:

Outdoor: $125 \mathrm{ppm}$

Indoor: $125 \mathrm{ppm}$

Note; Indoor graph is shown with a dotted line.
Reviewed by S. A. Henderson

Works Calculation 7

Sheet No. $\underline{82 \text { of } 577}$ 


\section{ENGINEERING COMPUTATION SHEET}

Title of Project EPHA for THM

Subject Concentration Analysis for THM Hazardous Chemicals

Computer T. M. Franey
Reviewed by $\underline{\text { S. A. Henderson }}$

Works Calculation 7

Sheet No. 83 of 577

\section{SITE DATA INFORMATION: 1-RD-16 \\ Location: AIKEN, SOUTH CAROLINA \\ Building Air Exchanges Per Hour: 60 (User specified) \\ Date and Time: Fixed at July 1, 19960000 hours}

\section{CHEMICAL INFORMATION:}

\section{Chemical Name: DIOXANE}

TLV-TWA: $25.00 \mathrm{ppm}$

Molecular Weight: $88.11 \mathrm{~kg} / \mathrm{kmol}$

Footprint Level of Concern: $125 \mathrm{ppm}$

Boiling Point: $101.32^{\circ} \mathrm{C}$

Vapor Pressure at Ambient Temperature: 0.061 atm

Ambient Saturation Concentration: 62,381 ppm or $6.24 \%$

\section{ATMOSPHERIC INFORMATION: (MANUAL INPUT OF DATA)}

Wind: 1.7 meters $/ \mathrm{sec}$ from $90^{\circ}$ true

Inversion Height: 200 meters

Stability Class: E Air Temperature: $29^{\circ} \mathrm{C}$

Relative Humidity: $50 \%$ Ground Roughness: 100 centimeters

Cloud Cover: 1 tenths

\section{SOURCE STRENGTH INFORMATION:}

Puddle Area: 1700 square feet

Average Puddle Depth: 1 centimeters

Soil Type: Default Ground Temperature: $29^{\circ} \mathrm{C}$

Initial Puddle Temperature: Ground temperature

Release Duration: ALOHA limited the duration to 1 hour

Max Computed Release Rate: 7.58 kilograms/min

Max Average Sustained Release Rate: 6.82 kilograms/min (averaged over a minute or more)

Total Amount Released: 368 kilograms

\section{FOOTPRINT INFORMATION:}

Model Run: Heavy Gas

User specified LOC: $125 \mathrm{ppm}$

Max Threat Zone for LOC: 97 meters

Max Threat Zone for IDLH: 15 meters

Note: Footprint wasn't drawn because effects of near-field patchiness make plume presentation unreliable for short distances.

TIME DEPENDENT INFORMATION:

Concentration Estimates at the point:

Downwind: 100 meters

Off Centerline: 0 meters

Max Concentration:

Outdoor: $125 \mathrm{ppm}$

Indoor: $125 \mathrm{ppm}$

Note: Indoor graph is shown with a dotted line. 


\section{ENGINEERING COMPUTATION SHEET}

Title of Project EPHA for THM

Subject Concentration Analysis for THM Hazardous Chemicals

Computer T. M. Franey

Date $\underline{09 / 16 / 96}$
Reviewed by S. A. Henderson

Works Calculation 7

Sheet No. 84 of 577

SITE DATA INFORMATION: 1-RD-16

Location: AIKEN, SOUTH CAROLINA

Building Air Exchanges Per Hour: 60 (User specified)

Date and Time: Fixed at July 1, 19960000 hours

CHEMICAL INFORMATION:

Chemical Name: DIOXANE Molecular Weight: $88.11 \mathrm{~kg} / \mathrm{kmol}$

TLV-TWA: $25.00 \mathrm{ppm}$ IDLH: $2000.00 \mathrm{ppm}$

Note: Potential or confirmed human carcinogen.

Footprint Level of Concern: 125 ppm

Boiling Point: $101.32^{\circ} \mathrm{C}$

Vapor Pressure at Ambient Temperature: $0.061 \mathrm{~atm}$

Ambient Saturation Concentration: $62,381 \mathrm{ppm}$ or $6.24 \%$

ATMOSPHERIC INFORMATION: (MANUAL INPUT OF DATA)

Wind: 1.7 meters $/ \mathrm{sec}$ from $90^{\circ}$ true

Inversion Height: 200 meters

Stability Class: E Air Temperature: $29^{\circ} \mathrm{C}$

Relative Humidity: $50 \%$ Ground Roughness: 100 centimeters

Cloud Cover: 1 tenths

\section{SOURCE STRENGTH INFORMATION:}

Puddle Area: 20420 square feet

Average Puddle Depth: 1 centimeters

Soil Type: Default Ground Temperature: $29^{\circ} \mathrm{C}$

Initial Puddle Temperature: Ground temperature

Release Duration: ALOHA limited the duration to 1 hour

Max Computed Release Rate: 81.4 kilograms/min

Max Average Sustained Release Rate: 73.8 kilograms/min ·

(averaged over a minute or more)

Total Amount Released: 4,002 kilograms

FOOTPRINT INFORMATION:

Model Run: Heavy Gas

User specified LOC: $125 \mathrm{ppm}$

Max Threat Zone for LOC: 324 meters

Max Threat Zone for IDLH: 50 meters

Note: The Heavy Gas footprint is an initial screening.

For short releases it may be an overestimation.

Be sure to check concentration information at specific locations.

TIME DEPENDENT INFORMATION:

Concentration Estimates at the point:

Downwind: 100 meters

Off Centerline: 0 meters

Max Concentration:

Outdoor: 792 ppm

Indoor: $792 \mathrm{ppm}$

Note: Indoor graph is shown with a dotted line. 


\section{ENGINEERING COMPUTATION SHEET}

Title of Project EPHA for THM

Reviewed by $\underline{S}$. A. Henderson

Subject Concentration Analysis for THM Hazardous Chemicals

Computer T. M. Franey

Date $\underline{09 / 16 / 96}$

SITE DATA INFORMATION: 1-RD-16

Location: AIKEN, SOUTH CAROLINA

Building Air Exchanges Per Hour: 60 (User specified)

Date and Time: Fixed at July 1, 19960000 hours

CHEMICAL INFORMATION:

Chemical Name: DIOXANE Molecular Weight: $88.11 \mathrm{~kg} / \mathrm{kmol}$

TLV-TWA: $25.00 \mathrm{ppm}$ IDLH: $2000.00 \mathrm{ppm}$

Note: Potential or confirmed human carcinogen.

Footprint Level of Concern: 125 ppm

Boiling Point: $101.32^{\circ} \mathrm{C}$

Vapor Pressure at Ambient Temperature: $0.061 \mathrm{~atm}$

Ambient Saturation Concentration: 62,381 ppm or $6.24 \%$

\section{ATMOSPHERIC INFORMATION: (MANUAL INPUT OF DATA)}

Wind: 1.7 meters $/ \mathrm{sec}$ from $90^{\circ}$ true

Inversion Height: 200 meters

Stability Class: E Air Temperature: $29^{\circ} \mathrm{C}$

Relative Humidity: 50\% Ground Roughness: 100 centimeters

Cloud Cover: 1 tenths

\section{SOURCE STRENGTH INFORMATION:}

Puddle Area: 40700 square feet

Average Puddle Depth: 1 centimeters

Soil Type: Default Ground Temperature: $29^{\circ} \mathrm{C}$

Initial Puddle Temperature: Ground temperature

Release Duration: ALOHA limited the duration to 1 hour

Max Computed Release Rate: 158 kilograms/min

Max Average Sustained Release Rate: 143 kilograms/min

(averaged over a minute or more)

Total Amount Released: 7,773 kilograms

\section{FOOTPRINT INFORMATION:}

Model Run: Heavy Gas

User specified LOC: $125 \mathrm{ppm}$

Max Threat Zone for LOC: 457 meters

.Max Threat Zone for IDLH: 74 meters

Note: The Heavy Gas footprint is an initial screening.

For short releases it may be an overestimation.

Be sure to check concentration information at specific locations.

\section{TIME DEPENDENT INFORMATION:}

Concentration Estimates at the point:

Downwind: 100 meters

Off Centerline: 0 meters

Max Concentration:

Outdoor: $1,360 \mathrm{ppm}$

Indoor: $1,360 \mathrm{ppm}$

Note: Indoor graph is shown with a dotted line.
Works Calculation 7

Sheet No. 85 of 577 
Title of Project EPHA for THM

Subject Concentration Analysis for THM Hazardous Chemicals

Computer T. M. Franey Date $09 / 16 / 96$
Reviewed by S. A. Henderson

Works Calculation 7

Sheet No. 86 of 577

\section{SITE DATA INFORMATION: 1-RD-16}

Location: AIKEN, SOUTH CAROLINA

Building Air Exchanges Per Hour: 60 (User specified)

Date and Time: Fixed at July 1, 19960000 hours

CHEMICAL INFORMATION:

Chemical Name: DIOXANE Molecular Weight: $88.11 \mathrm{~kg} / \mathrm{kmol}$ TLV-TWA: $25.00 \mathrm{ppm}$ IDLH: $2000.00 \mathrm{ppm}$

Note: Potential or confirmed human carcinogen.

Footprint Level of Concern: 125 ppm

Boiling Point: $101.32^{\circ} \mathrm{C}$

Vapor Pressure at Ambient Temperature: $0.061 \mathrm{~atm}$

Ambient Saturation Concentration: 62,381 ppm or $6.24 \%$

ATMOSPHERIC INFORMATION: (MANUAL INPUT OF DATA)

Wind: 1.7 meters $/ \mathrm{sec}$ from $90^{\circ}$ true

Inversion Height: 200 meters

Stability Class: E Air Temperature: $29^{\circ} \mathrm{C}$

Relative Humidity: 50\% Ground Roughness: 100 centimeters

Cloud Cover: 1 tenths

\section{SOURCE STRENGTH INFORMATION:}

Puddle Area: 10000 square meters

Average Puddle Depth: 1 centimeters

Soil Type: Default Ground Temperature: $29^{\circ} \mathrm{C}$

Initial Puddle Temperature: Ground temperature

Release Duration: AlOHA limited the duration to 1 hour

Max Computed Release Rate: 400 kilograms/min

Max Average Sustained Release Rate: 365 kilograms/min

(averaged over a minute or more)

Total Amount Released: 19,846 kilograms

\section{FOOTPRINT INFORMATION:}

Model Run: Heavy Gas

User specified LOC: $125 \mathrm{ppm}$

Max Threat Zone for LOC: 751 meters

Max Threat Zone for IDLH: 128 meters

Note: The Heavy Gas footprint is an initial screening.

For short releases it may be an overestimation.

Be sure to check concentration information at specific locations.

\section{TIME DEPENDENT INFORMATION:}

Concentration Estimates at the point:

Downwind: 100 meters

Off Centerline: 0 meters

Max Concentration:

Outdoor: $2,900 \mathrm{ppm}$

Indoor: $2,900 \mathrm{ppm}$

Note: Indoor graph is shown with a dotted line. 


\section{ENGINEERING COMPUTATION SHEET}

Title of Project EPHA for THM

Subject Concentration Analysis for THM Hazardous Chemicals

Computer T. M. Franey
Date $\underline{09 / 16 / 96}$

1-RD-17

SITE DATA INFORMATION:

Location: AIKEN, SOUTH CAROLINA

Building Air Exchanges Per Hour: 60 (User specified)

Date and Time: Fixed at July 1, 19960000 hours

\section{CHEMICAL INFORMATION:}

Chemical Name: METHYLANILINE (DIPHEYNLMETHANE DIISOCYANATE)

Molecular Weight: $250.3 \mathrm{~kg} / \mathrm{kmol}$

TLV-TWA: $0.50 \mathrm{ppm}$ IDLH: $100.00 \mathrm{ppm}$

Footprint Level of Concern: $.5 \mathrm{ppm}$

Boiling Point: $195.87^{\circ} \mathrm{C}$

Vapor Pressure at Ambient Temperature: 7.96e-004 atm

Ambient Saturation Concentration: 810 ppm or $0.081 \%$

\section{ATMOSPHERIC INFORMATION: (MANUAL INPUT OF DATA)}

Wind: 1.7 meters $/ \mathrm{sec}$ from $90^{\circ}$ true

Inversion Height: 200 meters

Stability Class: E Air Temperature: $29^{\circ} \mathrm{C}$

Relative Humidity: 50\% Ground Roughness: Urban or forest

Cloud Cover: 1 tenths

\section{SOURCE STRENGTH INFORMATION:}

Puddle Area: 75 square feet

Average Puddle Depth: 1 inches

Soil Type: Default Ground Temperature: $29^{\circ} \mathrm{C}$

Initial Puddle Temperature: Ground temperature

Release Duration: ALOHA limited the duration to 1 hour

Max Computed Release Rate: 3.49 grams/min

Max Average Sustained Release Rate: 3.4 grams/min

(averaged over a minute or more)

Total Amount Released: 196 grams

\section{TIME DEPENDENT INFORMATION:}

Concentration Estimates at the point:

Downwind: 30 meters

Off Centerline: 0 meters

Max Concentration:

Outdoor: $0.496 \mathrm{ppm}$

Indoor: $0.496 \mathrm{ppm}$ 


\section{ENGINEERING COMPUTATION SHEET}

Title of Project EPHA for THM

Reviewed by $\underline{\text { S. A. Henderson }}$

Subject Concentration Analysis for THM Hazardous Chemicals

Computer T. M. Franey

Date 09/16/96
Works Calculation 7
Sheet No. 88 of 577

\section{1-RD-17 SITE DATA INFORMATION:}

Location: AIKEN, SOUTH CAROLINA

Building Air Exchanges Per Hour: 60 (User specified)'

Date and Time: Fixed at July 1, 19960000 hours

\section{CHEMICAL INFORMATION:}

Chemical Name: METHYLANILINE (DIPHEYNLMETHANE DISOCYANATE)

Molecular Weight: $250.3 \mathrm{~kg} / \mathrm{kmol}$

TLV-TWA: $0.50 \mathrm{ppm}$ IDLH: $100.00 \mathrm{ppm}$

Footprint Level of Concern: $.5 \mathrm{ppm}$

Boiling Point: $195.87^{\circ} \mathrm{C}$

Vapor Pressure at Ambient Temperature: 7.96e-004 atm

Ambient Saturation Concentration: 810 ppm or $0.081 \%$

ATMOSPHERIC INFORMATION: (MANUAL INPUT OF DATA)

Wind: 1.7 meters $/ \mathrm{sec}$ from $90^{\circ}$ true

Inversion Height: 200 meters

Stability Class: E Air Temperature: $29^{\circ} \mathrm{C}$

Relative Humidity: 50\% Ground Roughness: Urban or forest

Cloud Cover: 1 tenths.

\section{SOURCE STRENGTH INFORMATION:}

Puddle Area: 920 square feet

Average Puddle Depth: 1 inches

Soil Type: Default $\quad$ Ground Temperature: $29^{\circ} \mathrm{C}$

Initial Puddle Temperature: Ground temperature

Release Duration: ALOHA limited the duration to 1 hour

Max Computed Release Rate: $37.8 \mathrm{grams} / \mathrm{min}$

Max Average Sustained Release Rate: 36.8 grams/min (averaged over a minute or more)

Total Amount Released: 2.12 kilograms

\section{TIME DEPENDENT INFORMATION:}

Concentration Estimates at the point:

Downwind: 100 meters

Off Centerline: 0 meters

Max Concentration:

Outdoor: $0.5 \mathrm{ppm}$

Indoor: $0.5 \mathrm{ppm}$ 


\section{ENGINEERING COMPUTATION SHEET}

Title of Project EPHA for THM

Reviewed by $\underline{\text { S. A. Henderson }}$

Subject Concentration Analysis for THM Hazardous Chemicals

Computer T. M. Franey
Works Calculation ?

Sheet No. 89 of 577

1-RD-17 SITE DATA INFORMATION:

Location: AIKEN, SOUTH CAROLINA

Building Air Exchanges Per Hour: 60 (User specified)

Date and Time: Fixed at July 1, 19960000 hours

CHEMICAL INFORMATION:

Chemical Name: METHYLANILINE (DIPHEYNLMETHANE DIISOCYANATE)

Molecular Weight: $250.3 \mathrm{~kg} / \mathrm{kmol}$

TLV-TWA: $0.50 \mathrm{ppm}$ IDLH: $100.00 \mathrm{ppm}$

Footprint Level of Concern: .5 ppm

Boiling Point: $195.87^{\circ} \mathrm{C}$

Vapor Pressure at Ambient Temperature: 7.96e-004 atm

Ambient Saturation Concentration: 810 ppm or $0.081 \%$

ATMOSPHERIC INFORMATION: (MANUAL INPUT OF DATA)

Wind: 1.7 meters $/ \mathrm{sec}$ from $90^{\circ}$ true

Inversion Height: 200 meters

Stability Class: E Air Temperature: $29^{\circ} \mathrm{C}$

Relative Humidity: 50\% Ground Roughness: Urban or forest

Cloud Cover: 1 tenths

\section{SOURCE STRENGTH INFORMATION:}

Puddle Area: 24420 square feet

Average Puddle Depth: 1 inches

Soil Type: Default Ground Temperature: $29^{\circ} \mathrm{C}$

Initial Puddle Temperature: Ground temperature

Release Duration: ALOHA limited the duration to 1 hour

Max Computed Release Rate: 867 grams/min

Max Average Sustained Release Rate: 846 grams/min

(averaged over a minute or more)

Total Amount Released: 48.8 kilograms

FOOTPRINT INFORMATION:

Dispersion Module: Gaussian

User specified LOC: $.5 \mathrm{ppm}$

Max Threat Zone for LOC: 547 meters

Max Threat Zone for IDLH: 27 meters

TIME DEPENDENT INFORMATION:

Concentration Estimates at the point:

Downwind: 100 meters

Off Centerline: 0 meters

Max Concentration:

Outdoor: $3.18 \mathrm{ppm}$ 


\section{ENGINEERING COMPUTATION SHEET}

Title of Project EPHA for THM

Subject Concentration Analysis for THM Hazardous Chemicals

Computer T. M. Franey

Date 09/16/96
Reviewed by $\underline{S}$. A. Henderson

Works Calculation 7

Sheet No. 90 of 577

\section{1-RD-17 SITE DATA INFORMATION:}

Location: AIKEN, SOUTH CAROLINA

Building Air Exchanges Per Hour: 60 (User specified)

Date and Time: Fixed at July 1, 19960000 hours

\section{CHEMICAL INFORMATION:}

Chemical Name: METHYLANILINE (DIPHEYNLMETHANE DIISOCYANATE)

Molecular Weight: $250.3 \mathrm{~kg} / \mathrm{kmol}$

TLV-TWA: $0.50 \mathrm{ppm}$ IDLH: $100.00 \mathrm{ppm}$

Footprint Level of Concern: .5 .ppm

Boiling Point: $195.87^{\circ} \mathrm{C}$

Vapor Pressure at Ambient Temperature: 7.96e-004 atm

Ambient Saturation Concentration: 810 ppm or $0.081 \%$

\section{ATMOSPHERIC INFORMATION: (MANUAL INPUT OF DATA) \\ Wind: 1.7 meters $/ \mathrm{sec}$ from $90^{\circ}$ true \\ Inversion Height: 200 meters \\ Stability Class: E Air Temperature: $29^{\circ} \mathrm{C}$ \\ Relative Humidity: 50\% Ground Roughness: Urban or forest \\ Cloud Cover: 1 tenths}

\section{SOURCE STRENGTH INFORMATION:}

Puddle Area: 40700 square feet

Average Puddle Depth: 1 inches

Soil Type: Default Ground Temperature: $29^{\circ} \mathrm{C}$

Initial Puddle Temperature: Ground temperature

Release Duration: ALOHA limited the duration to 1 hour

Max Computed Release Rate: 1.42 kilograms/min

Max Average Sustained Release Rate: 1.38 kilograms/min

(averaged over a minute or more)

Total Amount Released: 79.6 kilograms

FOOTPRINT INFORMATION:

Dispersion Module: Gaussian

- User specified LOC: $.5 \mathrm{ppm}$

Max Threat Zone for LOC: 729 meters

Max Threat Zone for IDLH: 34 meters

TIME DEPENDENT INFORMATION:

Concentration Estimates at the point:

Downwind: 100 meters

Off Centerline: 0 meters

Max Concentration:

Outdoor: $4.61 \mathrm{ppm}$ 
Title of Project EPHA for THM

Subject Concentration Analysis for THM Hazardous Chemicals

Computer T. M. Franey

\section{1-RD-17 SITE DATA INFORMATION:}

Location: AIKEN, SOUTH CAROLINA

Building Air Exchanges Per Hour: 60 (User specified)

Date and Time: Fixed at July 1, 19960000 hours

\section{CHEMICAL INFORMATION:}

Chemical Name: METHYLANILINE (DIPHEYNLMETHANE DIISOCYANATE)

Molecular Weight: $250.3 \mathrm{~kg} / \mathrm{kmol}$

TLV-TWA: $0.50 \mathrm{ppm}$ IDLH: $100.00 \mathrm{ppm}$

Footprint Level of Concern: $.5 \mathrm{ppm}$

Boiling Point: $195.87^{\circ} \mathrm{C}$

Vapor Pressure at Ambient Temperature: 7.96e-004 atm

Ambient Saturation Concentration: 810 ppm or $0.081 \%$

\section{ATMOSPHERIC INFORMATION: (MANUAL INPUT OF DATA)}

Wind: 1.7 meters $/ \mathrm{sec}$ from $90^{\circ}$ true

Inversion Height: 200 meters

Stability Class: E Air Temperature: $29^{\circ} \mathrm{C}$

Relative Humidity: 50\% Ground Roughness: Urban or forest

Cloud Cover: 1 tenths

\section{SOURCE STRENGTH INFORMATION:}

Puddle Area: 10000 square meters

Average Puddle Depth: 1 inches

Soil Type: Default Ground Temperature: $29^{\circ} \mathrm{C}$

Initial Puddle Temperature: Ground temperature

Release Duration: ALOHA limited the duration to 1 hour

Max Computed Release Rate: 3.6 kilograms/min

Max Average Sustained Release Rate: 3.52 kilograms/min

(averaged over a minute or more)

Total Amount Released: 203 kilograms

\section{FOOTPRINT INFORMATION:}

Dispersion Module: Gaussian

User specified LOC: $.5 \mathrm{ppm}$

Max Threat Zone for LOC: 1.3 kilometers

Max Threat Zone for IDLH: 56 meters

\section{TIME DEPENDENT INFORMATION:}

Concentration Estimates at the point:

Downwind: ' 100 meters

Off Centerline: 0 meters

Max Concentration:

Outdoor: $11.4 \mathrm{ppm}$ 


\section{ENGINEERING COMPUTATION SHEET}

Title of Project EPHA for THM

Reviewed by $\underline{\text { S. A. Henderson }}$

Subject Concentration Analysis for THM Hazardous Chemicals

Computer T. M. Franey Date $\underline{09 / 16 / 96}$

SITE DATA INFORMATION: 1-RD-18

Location: AIKEN, SOUTH CAROLINA

Building Air Exchanges Per Hour: 60 (User specified)

Date and Time: Fixed at July 1, 19960000 hours

CHEMICAL INFORMATION:

Chemical Name: ETHYL ACETATE

Molecular Weight: $88.11 \mathrm{~kg} / \mathrm{kmol}$

TLV-TWA: $400.00 \mathrm{ppm}$ IDLH: $10000.00 \mathrm{ppm}$

Footprint Level of Concern: 2000 ppm

Boiling Point: $77.06^{\circ} \mathrm{C}$

Vapor Pressure at Ambient Temperature: $0.15 \mathrm{~atm}$

Ambient Saturation Concentration: 151,884 ppm or $15.2 \%$

\section{ATMOSPHERIC INFORMATION: (MANUAL INPUT OF DATA)}

Wind: 1.7 meters $/ \mathrm{sec}$ from $90^{\circ}$ true

Inversion Height: 200 meters

Stability Class: E Air Temperature: $29^{\circ} \mathrm{C}$

Relative Humidity: $50 \%$ Ground Roughness: 100 centimeters

Cloud Cover: 1 tenths

\section{SOURCE STRENGTH INFORMATION:}

Puddle Area: 1885 square feet

Average Puddle Depth: 1 centimeters

Soil Type: Default Ground Temperature: $29^{\circ} \mathrm{C}$

Initial Puddle Temperature: Ground temperature

Release Duration: ALOHA limited the duration to 1 hour

Max Computed Release Rate: 23.7 kilograms/min

Max Average Sustained Release Rate: 19.5 kilograms/min

(averaged over a minute or more)

Total Amount Released: 967 kilograms

FOOTPRINT INFORMATION:

Model Run:.Heavy Gas

User specified LOC: $2000 \mathrm{ppm}$

Max Threat Zone for LOC: 30 meters

Max Threat Zone for IDLH: less than 10 meters(10.9 yards)

Note: Footprint wasn't drawn because effects of near-field patchiness make plume presentation unreliable for short distances.

\section{TIME DEPENDENT INFORMATION:}

Concentration Estimates at the point:

Downwind: 30 meters

Off Centerline: 0 meters

Max Concentration:

Outdoor: 2,000 ppm

Indoor: $2,000 \mathrm{ppm}$

Note: Indoor graph is shown with a dotted line.
Works Calculation 7

Sheet No. 92 of 577 


\section{ENGINEERING COMPUTATION SHEET}

Title of Project EPHA for THM

Subject Concentration Analysis for THM Hazardous Chemicals

Computer T. M. Franey

Date $\underline{09 / 16 / 96}$

SITE DATA INFORMATION: 1-RD-18

Location: AIKEN, SOUTH CAROLINA

Building Air Exchanges Per Hour: 60 (User specified)

Date and Time: Fixed at July 1, 19960000 hours

\section{CHEMICAL INFORMATION:}

Chemical Name: ETHYL ACETATE

Molecular Weight: $88.11 \mathrm{~kg} / \mathrm{kmol}$

TLV-TWA: $400.00 \mathrm{ppm}$ IDLH: $10000.00 \mathrm{ppm}$

Footprint Level of Concern: 2000 ppm

Boiling Point: $77.06^{\circ} \mathrm{C}$

Vapor Pressure at Ambient Temperature: $0.15 \mathrm{~atm}$

Ambient Saturation Concentration: 151,884 ppm or $15.2 \%$

ATMOSPHERIC INFORMATION: (MANUAL INPUT OF DATA)

Wind: 1.7 meters $/ \mathrm{sec}$ from $90^{\circ}$ true

Inversion Height: 200 meters

Stability Class: E Air Temperature: $29^{\circ} \mathrm{C}$

Relative Humidity: 50\% Ground Roughness: 100 centimeters

Cloud Cover: 1 tenths

\section{SOURCE STRENGTH INFORMATION:}

Puddle Area: 18400 square feet

Average Puddle Depth: 1 centimeters

Soil Type: Default Ground Temperature: $29^{\circ} \mathrm{C}$

Initial Puddle Temperature: Ground temperature

Release Duration: ALOHA limited the duration to 1 hour

Max Computed Release Rate: 209 kilograms/min

Max Average Sustained Release Rate: 175 kilograms/min (averaged over a minute or more)

Total Amount Released: 8,721 kilograms

\section{FOOTPRINT INFORMATION:}

Model Run: Heavy Gas

User specified LOC: 2000 ppm

Max Threat Zone for LOC: 97 meters

Max Threat Zone for IDLH: 34 meters

Note: Footprint wasn't drawn because effects of near-field patchiness make plume presentation unreliable for short distances.

\section{TIME DEPENDENT INFORMATION:}

Concentration Estimates at the point:

Downwind: 100 meters

Off Centerline: 0 meters

Max Concentration:

Outdoor: $2,000 \mathrm{ppm}$

Indoor: $2,000 \mathrm{ppm}$

Note: Indoor graph is shown with a dotted line.
Reviewed by $\underline{S}$. A. Henderson

Works Calculation 7

Sheet No: 93 of 577 
Title of Project. EPHA for THM

Subject Concentration Analysis for THM Hazardous Chemicals

Computer T. M. Franey
Reviewed by S. A. Henderson

Works Calculation 7

Sheet No. 94 of 577

SITE DATA INFORMATION: 1-RD-18

Location: AIKEN, SOUTH CAROLINA

Building Air Exchanges Per Hour: 60 (User specified)

Date and Time: Fixed at July 1, 19960000 hours

CHEMICAL INFORMATION:

Chemical Name: ETHYL ACETATE

Molecular Weight: $88.11 \mathrm{~kg} / \mathrm{kmol}$

TLV-TWA: $400.00 \mathrm{ppm}$ IDLH: $10000.00 \mathrm{ppm}$

Footprint Level of Concern: 2000 ppm

Boiling Point: $77.06^{\circ} \mathrm{C}$

Vapor Pressure at Ambient Temperature: $0.15 \mathrm{~atm}$

Ambient Saturation Concentration: 151,884 ppm or $15.2 \%$

ATMOSPHERIC INFORMATION: (MANUAL INPUT OF DATA)

Wind: 1.7 meters $/ \mathrm{sec}$ from $90^{\circ}$ true

Inversion Height: 200 meters

Stability Class: E Air Temperature: $29^{\circ} \mathrm{C}$

Relative Humidity: 50\% Ground Roughness: 100 centimeters

Cloud Cover: 1 tenths

SOURCE STRENGTH INFORMATION:

Puddle Area: 20420 square feet

Average Puddle Depth: 1 centimeters

Soil Type: Default Ground Temperature: $29^{\circ} \mathrm{C}$

Initial Puddle Temperature: Ground temperature

Release Duration: ALOHA limited the duration to 1 hour

Max Computed Release Rate: 231 kilograms/min

Max Average Sustained Release Rate: 194 kilograms/min (averaged over a minute or more)

Total Amount Released: 9,644 kilograms

\section{FOOTPRINT INFORMATION:}

Model Run: Heavy Gas

User specified LOC: $2000 \mathrm{ppm}$

Max Threat Zone for LOC: 103 meters

Max Threat Zone for IDLH: 36 meters

Note: The Heavy Gas footprint is an initial screening.

For short releases it may be an overestimation.

Be sure to check concentration information at specific locations.

\section{TIME DEPENDENT INFORMATION:}

Concentration Estimates at the point:

Downwind: 100 meters

Off Centerline: 0 meters

Max Concentration:

Outdoor: $2,200 \mathrm{ppm}$

Indoor: $2,200 \mathrm{ppm}$

Note: Indoor graph is shown with a dotted line. 
Title of Project EPHA for THM

Subject Concentration Analysis for THM Hazardous Chemicals

Computer T. M. Franey

Date 09/16/96
Reviewed by S. A. Henderson

Works Calculation 7

Sheet No. 95 of 577

SITE DATA INFORMATION: 1-RD-18

Location: AIKEN, SOUTH CAROLINA

Building Air Exchanges Per Hour: 60 (User specified)

Date and Time: Fixed at July 1, 19960000 hours

CHEMICAL INFORMATION:

Chemical Name: ETHYL ACETATE

Molecular Weight: $88.11 \mathrm{~kg} / \mathrm{kmol}$

TLV-TWA: $400.00 \mathrm{ppm}$ IDLH: $10000.00 \mathrm{ppm}$

Footprint Level of Concern: 2000 ppm

Boiling Point: $77.06^{\circ} \mathrm{C}$

Vapor Pressure at Ambient Temperature: $0.15 \mathrm{~atm}$

Ambient Saturation Concentration: 151,884 ppm or $15.2 \%$

ATMOSPHERIC INFORMATION: (MANUAL INPUT OF DATA)

Wind: 1.7 meters $/ \mathrm{sec}$ from $90^{\circ}$ true

Inversion Height: 200 meters

Stability Class: E Air Temperature: $29^{\circ} \mathrm{C}$

Relative Humidity: $50 \%$ Ground Roughness: 100 centimeters

Cloud Cover: 1 tenths

SOURCE STRENGTH INFORMATION:

Puddle Area: 40700 square feet

Average Puddle Depth: 1 centimeters

Soil Type: Default Ground Temperature: $29^{\circ} \mathrm{C}$

Initial Puddle Temperature: Ground temperature

Release Duration: ALOHA limited the duration to 1 hour

Max Computed Release Rate: 447 kilograms/min

Max Average Sustained Release Rate: 375 kilograms/min

(averaged over a minute or more)

Total Amount Released: 18,791 kilograms

\section{FOOTPRINT INFORMATION:}

Model Run: Heavy Gas

User specified LOC: $2000 \mathrm{ppm}$

Max Threat Zone for LOC: 150 meters

Max Threat Zone for IDLH: 54 meters

Note: The Heavy Gas footprint is an initial screening.

For short releases it may be an overestimation.

Be sure to check concentration information at specific locations.

\section{TIME DEPENDENT INFORMATION:}

Concentration Estimates at the point:

Downwind: 100 meters

Off Centerline: 0 meters

Max Concentration:

Outdoor: $4,120 \mathrm{ppm}$

Indoor: 4,120 ppm

Note: Indoor graph is shown with a dotted line. 
Title of Project EPHA for THM

Subject Concentration Analysis for THM Hazardous Chemicals

Computer T. M. Franey

Date 09/16/96

SITE DATA INFORMATION: 1-RD-18

Location: AIKEN, SOUTH CAROLINA

Building Air Exchanges Per Hour: 60 (User specified)

Date and Time: Fixed at July 1, 19960000 hours

\section{CHEMICAL INFORMATION:}

Chemical Name: ETHYL ACETATE

Molecular Weight: $88.11 \mathrm{~kg} / \mathrm{kmol}$

TLV-TWA: $400.00 \mathrm{ppm}$ IDLH: $10000.00 \mathrm{ppm}$

Footprint Level of Concern: 2000 ppm

Boiling Point: $77.06^{\circ} \mathrm{C}$

Vapor Pressure at Ambient Temperature: 0.15 atm

Ambient Saturation Concentration: 151,884 ppm or $15.2 \%$

ATMOSPHERIC INFORMATION: (MANUAL INPUT OF DATA)

Wind: 1.7 meters $/ \mathrm{sec}$ from $90^{\circ}$ true

Inversion Height: 200 meters

Stability Class: E Air Temperature: $29^{\circ} \mathrm{C}$

Relative Humidity: 50\% Ground Roughness: 100 centimeters

Cloud Cover: 1 tenths

\section{SOURCE STRENGTH INFORMATTON:}

Puddle Area: 10000 square meters

Average Puddle Depth: 1 centimeters

Soil Type: Default Ground Temperature: $29^{\circ} \mathrm{C}$

Initial Puddle Temperature: Ground temperature

Release Duration: ALOHA limited the duration to 1 hour

Max Computed Release Rate: 1,140 kilograms/min

Max Average Sustained Release Rate: 956 kilograms/min

(averaged over a minute or more)

Total Amount Released: 48,160 kilograms

\section{FOOTPRINT INFORMATION:}

Model Run: Heavy Gas

User specified LOC: 2000 ppm

Max Threat Zone for LOC: 255 meters

Max Threat Zone for IDLH: 94 meters

Note: The Heavy Gas footprint is an initial screening.

For short releases it may be an overestimation.

Be sure to check concentration information at specific locations.

\section{TIME DEPENDENT INFORMATION:}

Concentration Estimates at the point:

Downwind: 100 meters

Off Centerline: 0 meters

Max Concentration:

Outdoor: $9,630 \mathrm{ppm}$

Indoor: $9,630 \mathrm{ppm}$

Note: Indoor graph is shown with a dotted line.
Reviewed by $\underline{S}$. A. Henderson

Works Calculation 7

Sheet No. 96 of 577 
Title of Project EPHA for THM

Subject Concentration Analysis for THM Hazardous Chemicals

Computer T.M. Franey
Reviewed by S. A. Henderson

Works Calculation 7

Sheet No. 97 of 577

\section{CHEMICAL INFORMATION:}

Chemical Name: ETHYL BENZENE

Molecular Weight: $106.17 \mathrm{~kg} / \mathrm{kmol}$

TLV-TWA: $100.00 \mathrm{ppm}$ IDLH: $2000.00 \mathrm{ppm}$

Footprint Level of Concern: $160 \mathrm{ppm}$

Boiling Point: $136.20^{\circ} \mathrm{C}$

Vapor Pressure at Ambient Temperature: 0.016 atm

Ambient Saturation Concentration: $16,157 \mathrm{ppm}$ or $1.62 \%$

\section{ATMOSPHERIC INFORMATTON: (MANUAL INPUT OF DATA)}

Wind: 1.7 meters $/ \mathrm{sec}$ from $90^{\circ}$ true

Inversion Height: 200 meters

Stability Class: E Air Temperature: $29^{\circ} \mathrm{C}$

Relative Humidity: 50\% Ground Roughness: 100 centimeters

Cloud Cover: 1 tenths

\section{SOURCE STRENGTH INFORMATION:}

Puddle Area: 3450 square feet

Average Puddle Depth: 1 centimeters

Soil Type: Default Ground Temperature: $29^{\circ} \mathrm{C}$

Initial Puddle Temperature: Ground temperature

Release Duration: ALOHA limited the duration to 1 hour

Max Computed Release Rate: 4.68 kilograms/min

Max Average Sustained Release Rate: 4.45 kilograms/min (averaged over a minute or more)

Total Amount Released: 252 kilograms

\section{FOOTPRINT INFORMATION:}

Dispersion Module: Gaussian

User specified LOC: $160 \mathrm{ppm}$

Max Threat Zone for LOC: 30 meters

Max Threat Zone for IDLH: 10 meters

Note: Footprint was not drawn because effects of near-field patchiness make plume presentation unreliable for short distances.

\section{TIME DEPENDENT INFORMATION:}

\section{Concentration Estimates at the point:}

Downwind: 30 meters

Off Centerline: 0 meters

Max Concentration:

Outdoor: $160 \mathrm{ppm}$

Indoor: $160 \mathrm{ppm}$

Note: Indoor graph is shown with a dotted line. 


\section{ENGINEERING COMPUTATION SHEET}

Title of Project EPHA for THM

Reviewed by $\underline{\text { S. A. Henderson }}$

Subject Concentration Analysis for THM Hazardous Chemicals

Computer T. M. Franey

Date $\underline{09 / 16 / 96}$

\section{Works Calculation 7}

Sheet No. 98 of 577

\section{SITE DATA INFORMATION: 1-RD-19}

Location: AIKEN, SOUTH CAROLINA

Building Air Exchanges Per Hour: 60 (User specified)

Date and Time: Fixed at July 1, 19960000 hours

CHEMICAL INFORMATION:

Chemical Name: ETHYL BENZENE

Molecular Weight: $106.17 \mathrm{~kg} / \mathrm{kmol}$

TLV-TWA: $100.00 \mathrm{ppm}$ IDLH: $2000.00 \mathrm{ppm}$

Footprint Level of Concern: 160 ppm

Boiling Point: $136.20^{\circ} \mathrm{C}$

Vapor Pressure at Ambient Temperature: $0.016 \mathrm{~atm}$

Ambient Saturation Concentration: 16,157 ppm or $1.62 \%$

ATMOSPHERIC INFORMATION: (MANUAL INPUT OF DATA)

Wind: 1.7 meters $/ \mathrm{sec}$ from $90^{\circ}$ true

Inversion Height: 200 meters

Stability Class: E Air Temperature: $29^{\circ} \mathrm{C}$

Relative Humidity: $50 \%$ Ground Roughness: 100 centimeters

Cloud Cover: 1 tenths

SOURCE STRENGTH INFORMATION:

Puddle Area: 14182 square feet

Average Puddle Depth: 1 centimeters

Soil Type: Default Ground Temperature: $29^{\circ} \mathrm{C}$

Initial Puddle Temperature: Ground temperature

Release Duration: ALOHA limited the duration to 1 hour

Max Computed Release Rate: 18.1 kilograms/min

Max Average Sustained Release Rate: 17.2 kilograms/min

(averaged over a minute or more)

Total Amount Released: 978 kilograms

FOOTPRINT INFORMATION:

Dispersion Module: Gaussian

User specified LOC: $160 \mathrm{ppm}$

Max Threat Zone for LOC: 60 meters

Max Threat Zone for IDLH: 20 meters

Note: Footprint was not drawn because effects of near-field patchiness make plume presentation unreliable for short distances.

\section{TIME DEPENDENT INFORMATION:}

Concentration Estimates at the point:

Downwind: 100 meters

Off Centerline: 0 meters

Max Concentration:

Outdoor: $82.6 \mathrm{ppm}$

Indoor: $82.6 \mathrm{ppm}$

Note: Indoor graph is shown with a dotted line. 


\section{ENGINEERING COMPUTATION SHEET}

Title of Project EPHA for THM

Reviewed by S. A. Henderson

Subject Concentration Analysis for THM Hazardous Chemicals

Computer T. M. Franey

Date $09 / 16 / 96$
Works Calculation 7

Sheet No: 99 of 577

SITE DATA INFORMATION: 1-RD-19

Location: AIKEN, SOUTH CAROLINA

Building Air Exchanges Per Hour: 60 (User specified)

Date and Time: Fixed at July 1, 19960000 hours

CHEMICAL INFORMATION:

Chemical Name: ETHYL BENZENE

Molecular Weight: $106.17 \mathrm{~kg} / \mathrm{kmol}$

TLV-TWA: $100.00 \mathrm{ppm}$ IDLH: $2000.00 \mathrm{ppm}$

Footprint Level of Concern: 160 ppm

Boiling Point: $136.20^{\circ} \mathrm{C}$

Vapor Pressure at Ambient Temperature: $0.016 \mathrm{~atm}$

Ambient Saturation Concentration: 16,157 ppm or $1.62 \%$

ATMOSPHERIC INFORMATION: (MANUAL INPUT OF DATA)

. Wind: 1.7 meters $/ \mathrm{sec}$ from $90^{\circ}$ true

Inversion Height: 200 meters

Stability Class: E Air Temperature: $29^{\circ} \mathrm{C}$

Relative Humidity: 50\% Ground Roughness: 100 centimeters

Cloud Cover: 1 tenths

\section{SOURCE STRENGTH INFORMATION:}

Puddle Area: 24420 square feet

Average Puddle Depth: 1 centimeters

Soil Type: Default Ground Temperature: $29^{\circ} \mathrm{C}$

Initial Puddle Temperature: Ground temperature

Release Duration: ALOHA limited the duration to 1 hour

Max Computed Release Rate: 30.4 kilograms/min

Max Average Sustained Release Rate: 29 kilograms/min

(averaged over a minute or more)

Total Amount Released: 1,648 kilograms

FOOTPRINT INFORMATION:

Model Run: Heavy Gas

User specified LOC: $160 \mathrm{ppm}$

Max Threat Zone for LOC: 134 meters

Max Threat Zone for IDLH: IDLH level was never exceeded

Note: The Heavy Gas footprint is an initial screening.

For short releases it may be an overestimation.

$\mathrm{Be}$ sure to check concentration information at specific locations.

TIME DEPENDENT INFORMATION:

Concentration Estimates at the point:

Downwind: 100 meters

Off Centerline: 0 meters

Max Concentration:

Outdoor: $246 \mathrm{ppm}$

Indoor: $246 \mathrm{ppm}$

Note: Indoor graph is shown with a dotted line. 
Title of Project EPHA for THM

Subject Concentration Analysis for THM Hazardous Chemicals

Computer T.M. Franey
Reviewed by S. A. Henderson

Works Calculation 7

Sheet No. 100 of 577

\section{SITE DATA INFORMATION: 1-RD-19}

Location: AIKEN, SOUTH CAROLINA

Building Air Exchanges Per Hour: 60 (User specified)

Date and Time: Fixed at July 1, 19960000 hours

CHEMICAL INFORMATION:

Chemical Name: ETHYL BENZENE

Molecular Weight: $106.17 \mathrm{~kg} / \mathrm{kmol}$

TLV-TWA: $100.00 \mathrm{ppm}$ IDLH: $2000.00 \mathrm{ppm}$

Footprint Level of Concern: 160 ppm

Boiling Point: $136.20^{\circ} \mathrm{C}$

Vapor Pressure at Ambient Temperature: $0.016 \mathrm{~atm}$

Ambient Saturation Concentration: $16,157 \mathrm{ppm}$ or $1.62 \%$

ATMOSPHERIC INFORMATION: (MANUAL INPUT OF DATA)

Wind: 1.7 meters $/ \mathrm{sec}$ from $90^{\circ}$ true

Inversion Height: 200 meters

Stability Class: E Air Temperature: $29^{\circ} \mathrm{C}$

Relative Humidity: $50 \%$ Ground Roughness: 100 centimeters

Cloud Cover: 1 tenths

\section{SOURCE STRENGTH INFORMATION:}

Puddle Area: 40700 square feet

Average Puddle Depth: 1 centimeters

Soil Type: Default Ground Temperature: $29^{\circ} \mathrm{C}$

Initial Puddle Temperature: Ground temperature

Release Duration: ALOHA limited the duration to 1 hour

Max Computed Release Rate: 49.7 kilograms/min

Max Average Sustained Release Rate: 47.4 kilograms/min

(averaged over a minute or more)

Total Amount Released: 2,692 kilograms

\section{FOOTPRINT INFORMATION:}

Model Run: Heavy Gas

User specified LOC: $160 \mathrm{ppm}$

Max Threat Zone for LOC: 172 meters

Max Threat Zone for IDLH: IDLH level was never exceeded .

Note: The Heavy Gas footprint is an initial screening.

For short releases it may be an overestimation.

Be sure to check concentration information at specific locations.

TIME DEPENDENT INFORMATION:

Concentration Estimates at the point:

Downwind: 100 meters

Off Centerline: 0 meters

Max Concentration:

Outdoor: $346 \mathrm{ppm}$

Indoor: $346 \mathrm{ppm}$

Note: Indoor graph is shown with a dotted line. 
Title of Project EPHA for THM

Subject Concentration Analysis for THM Hazardous Chemicals

Computer T. M. Franey Date $09 / 16 / 96$
Reviewed by $\underline{\text { S. A. Henderson }}$

Works Calculation 7

Sheet No. 101 of 577
SITE DATA INFORMATION: 1-RD-19

Location: AIKEN, SOUTH CAROLINA

Building Air Exchanges Per Hour: 60 (User specified)

Date and Time: Fixed at July 1, 19960000 hours

\section{CHEMICAL INFORMATION:}

Chemical Name: ETHYL BENZENE

Molecular Weight: $106.17 \mathrm{~kg} / \mathrm{kmol}$

TLV-TWA: $100.00 \mathrm{ppm}$ IDLH: $2000.00 \mathrm{ppm}$

Footprint Level of Concern: 160 ppm

Boiling Point: $136.20^{\circ} \mathrm{C}$

Vapor Pressure at Ambient Temperature: $0.016 \mathrm{~atm}$

Ambient Saturation Concentration: 16,157 ppm or $1.62 \%$

\section{ATMOSPHERIC INFORMATION: (MANUAL INPUT OF DATA)}

Wind: 1.7 meters $/ \mathrm{sec}$ from $90^{\circ}$ true

Inversion Height: 200 meters

Stability Class: E Air Temperature: $29^{\circ} \mathrm{C}$

Relative Humidity: $50 \%$ Ground Roughness: 100 centimeters

Cloud Cover: 1 tenths

\section{SOURCE STRENGTH INFORMATION:}

Puddle Area: 10000 square meters

Average Puddle Depth: 1 centimeters

Soil Type: Default Ground Temperature: $29^{\circ} \mathrm{C}$

Initial Puddle Temperature: Ground temperature

Release Duration: ALOHA limited the duration to 1 hour

Max Computed Release Rate: 126 kilograms/min

Max Average Sustained Release Rate: 121 kilograms/min (averaged over a minute or more)

Total Amount Released: 6,862 kilograms

\section{FOOTPRINT INFORMATION:}

Model Run: Heavy Gas

User specified LOC: 160 ppm

Max Threat Zone for LOC: 283 meters

Max Threat Zone for IDLH: IDLH level was never exceeded

Note: The Heavy Gas footprint is an initial screening.

For short releases it may be an overestimation.

Be sure to check concentration information at specific locations.

\section{TIME DEPENDENT INFORMATION:}

Concentration Estimates at the point:

Downwind: 100 meters

Off Centerline: 0 meters

Max Concentration:

Outdoor: 653 ppm

Indoor: $653 \mathrm{ppm}$

Note: Indoor graph is shown with a dotted line. 


\section{ENGINEERING COMPUTATION SHEET}

Title of Project EPHA for THM

Reviewed by S. A. Henderson

Subject Concentration Analysis for THM Hazardous Chemicals

Computer T. M. Franey Date $\underline{09 / 16 / 96}$

1-RD-20

SITE DATA INFORMATION:

Location: AIKEN, SOUTH CAROLINA

Building Air Exchanges Per Hour: 60 (User specified)

Date and Time: Fixed at July 1, 19960000 hours

\section{CHEMICAL INFORMATION:}

Chemical Name: ETHYLENE GLYCOL MONOBUTYL ETHER

Molecular Weight: $118.18 \mathrm{~kg} / \mathrm{kmol}$

TLV-TWA: $25.00 \mathrm{ppm}$

IDLH: $700.00 \mathrm{ppm}$

Footprint Level of Concern: 150 ppm

Boiling Point: $171.32^{\circ} \mathrm{C}$

Vapor Pressure at Ambient Temperature: $0.0015 \mathrm{~atm}$

Ambient Saturation Concentration: $1,570 \mathrm{ppm}$ or $0.16 \%$

\section{ATMOSPHERIC INFORMATION: (MANUAL INPUT OF DATA) \\ Wind: 1.7 meters $/ \mathrm{sec}$ from $90^{\circ}$ true \\ Inversion Height: 200 meters \\ Stability Class: E Air Temperature: $29^{\circ} \mathrm{C}$ \\ Relative Humidity: 50\% Ground Roughness: Urban or forest \\ Cloud Cover: 1 tenths}

\section{SOURCE STRENGTH INFORMATION:}

Puddle Area: 30000 square feet

Average Puddle Depth: 1 inches

Soil Type: Default Ground Temperature: $29^{\circ} \mathrm{C}$

Initial Puddle Temperature: Ground temperature

Release Duration: ALOHA limited the duration to 1 hour

Max Computed Release Rate: 1.81 kilograms/min

Max Average Sustained Release Rate: 1.76 kilograms/min

(averaged over a minute or more)

Total Amount Released: 101 kilograms

\section{TIME DEPENDENT INFORMATION:}

Concentration Estimates at the point:

Downwind: 30 meters

Off Centerline: 0 meters

Max Concentration:

Outdoor: $54.8 \mathrm{ppm}$
Works Calculation 7

Sheet No. 102 of 577 


\section{ENGINEERING COMPUTATION SHEET}

Title of Project EPHA for THM

Subject Concentration Analysis for THM Hazardous Chemicals

Computer T. M. Franey Date 09/16/96

1-RD-20

SITE DATA INFORMATION:

Location: AIKEN, SOUTH CAROLINA

Building Air Exchanges Per Hour: 60 (User specified)

Date and Time: Fixed at July 1, 19960000 hours

CHEMICAL INFORMATION:

Chemical Name: ETHYLENE GLYCOL MONOBUTYL ETHER

Molecular Weight: $118.18 \mathrm{~kg} / \mathrm{kmol}$

TLV-TWA: $25.00 \mathrm{ppm}$ IDLH: $700.00 \mathrm{ppm}$

Footprint Level of Concern: $150 \mathrm{ppm}$

Boiling Point: $171.32^{\circ} \mathrm{C}$

Vapor Pressure at Ambient Temperature: 0.0015 atm

Ambient Saturation Concentration: 1,570 ppm or $0.16 \%$

ATMOSPHERIC INFORMATION: (MANUAL INPUT OF DATA)

Wind: 1.7 meters $/ \mathrm{sec}$ from $90^{\circ}$ true

Inversion Height: 200 meters

Stability Class: E Air Temperature: $29^{\circ} \mathrm{C}$

Relative Humidity: $50 \%$ Ground Roughness: Urban or forest

Cloud Cover: 1 tenths

\section{SOURCE STRENGTH INFORMATION:}

Puddle Area: 100000 square feet

Average Puddle Depth: 1 centimeters

Soil Type: Default Ground Temperature: $29^{\circ} \mathrm{C}$

Initial Puddle Temperature: Ground temperature

Release Duration: ALOHA limited the duration to 1 hour

Max Computed Release Rate: 5.75 kilograms/min

Max Average Sustained Release Rate: 5.56 kilograms/min

(averaged over a minute or more)

Total Amount Released: 319 kilograms

TIME DEPENDENT INFORMATION:

Concentration Estimates at the point:

Downwind: 100 meters

Off Centerline: 0 meters

Max Concentration:

Outdoor: $16.3 \mathrm{ppm}$
Reviewed by S. A. Henderson

Works Calculation 7

Sheet No. 103 of 577 


\section{ENGINEERING COMPUTATION SHEET}

Title of Project EPHA for THM

Reviewed by $\underline{\text { S. A. Henderson }}$

Subject Concentration Analysis for THM Hazardous Chemicals

Computer T. M. Franey Date $\underline{09 / 16 / 96}$

\section{1-RD-20}

SITE DATA INFORMATION:

Location: AIKEN, SOUTH CAROLINA

Building Air Exchanges Per Hour: 60 (User specified)

Date and Time: Fixed at July 1, 19960000 hours

CHEMICAL INFORMATION:

Chemical Name: ETHYLENE GLYCOL MONOBUTYL ETHER

Molecular Weight: $118.18 \mathrm{~kg} / \mathrm{kmol}$

TLV-TWA: $25.00 \mathrm{ppm}$

IDLH: $700.00 \mathrm{ppm}$

Footprint Level of Concern: $150 \mathrm{ppm}$

Boiling Point: $171.32^{\circ} \mathrm{C}$

Vapor Pressure at Ambient Temperature: 0.0015 atm

Ambient Saturation Concentration: 1,570 ppm or $0.16 \%$

ATMOSPHERIC INFORMATION: (MANUAL INPUT OF DATA)

Wind: 1.7 meters $/ \mathrm{sec}$ from $90^{\circ}$ true

Inversion Height: 200 meters

Stability Class: E Air Temperature: $29^{\circ} \mathrm{C}$

Relative Humidity: 50\% Ground Roughness: Urban or forest

Cloud Cover: 1 tenths

\section{SOURCE STRENGTH INFORMATION:}

Puddle Area: 24420 square feet

Average Puddle Depth: 1 centimeters

Soil Type: Default Ground Temperature: $29^{\circ} \mathrm{C}$

Initial Puddle Temperature: Ground temperature

Release Duration: ALOHA limited the duration to 1 hour

Max Computed Release Rate: 1.49 kilograms/min

Max Average Sustained Release Rate: 1.43 kilograms/min

(averaged over a minute or more)

Total Amount Released: 82.3 kilograms

\section{FOOTPRINT INFORMATION:}

Dispersion Module: Gaussian

User specified LOC: $150 \mathrm{ppm}$

Max Threat Zone for LOC: 27 meters

Max Threat Zone for IDLH: 27 meters

Note: Footprint was not drawn because

effects of near-field patchiness make plume

presentation unreliable for short distances.

\section{TIME DEPENDENT INFORMATION:}

Concentration Estimates at the point:

Downwind: 100 meters

Off Centerline: 0 meters

Max Concentration:

Outdoor: $4.89 \mathrm{ppm}$
Works Calculation 7

Sheet No. 104 of 577 
Title of Project EPHA for THM

Subject Concentration Analysis for THM Hazardous Chemicals

Computer T. M. Franey
Reviewed by S. A. Henderson

Works Calculation 7

Sheet No. 105 of 577

\section{1-RD-20}

SITE DATA INFORMATION:

Location: AIKEN, SOUTH CAROLINA

Building Air Exchanges Per Hour: 60 (User specified)

Date and Time: Fixed at July 1, 19960000 hours

\section{CHEMICAL INFORMATION:}

Chemical Name: ETHYLENE GLYCOL MONOBUTYL ETHER

Molecular Weight: $118.18 \mathrm{~kg} / \mathrm{kmol}$

TLV-TWA: $25.00 \mathrm{ppm}$ IDLH: $700.00 \mathrm{ppm}$

Footprint Level of Concern: $150 \mathrm{ppm}$

Boiling Point: $171.32^{\circ} \mathrm{C}$

Vapor Pressure at Ambient Temperature: 0.0015 atm

Ambient Saturation Concentration: 1,570 ppm or $0.16 \%$

ATMOSPHERIC INFORMATION: (MANUAL INPUT OF DATA)

Wind: 1.7 meters/sec from $90^{\circ}$ true

Inversion Height: 200 meters

Stability Class: E Air Temperature: $29^{\circ} \mathrm{C}$

Relative Humidity: 50\% Ground Roughness: Urban or forest

Cloud Cover: 1 tenths

\section{SOURCE STRENGTH INFORMATION:}

Puddle Area: 40700 square feet

Average Puddle Depth: 1 centimeters

Soil Type: Default Ground Temperature: $29^{\circ} \mathrm{C}$

Initial Puddle Temperature: Ground temperature

Release Duration: ALOHA limited the duration to 1 hour

Max Computed Release Rate: 2.43 kilograms/min

Max Average Sustained Release Rate: 2.34 kilograms/min

(averaged over a minute or more)

Total Amount Released: 134 kilograms

\section{FOOTPRINT INFORMATION:}

Dispersion Module: Gaussian

User specified LOC: $150 \mathrm{ppm}$

Max Threat Zone for LOC: 34 meters

Max Threat Zone for IDLH: 35 meters

Note: Footprint was not drawn because effects of near-field patchiness make plume presentation unreliable for short distances.

\section{TIME DEPENDENT INFORMATION:}

Concentration Estimates at the point:

Downwind: 100 meters

Off Centerline: 0 meters

Max Concentration:

Outdoor: 7.09 ppm 
Title of Project EPHA for THM

Subject Concentration Analysis for THM Hazardous Chemicals

Computer T.M. Franey

Date $09 / 16 / 96$
Reviewed by S.A. Henderson

Works Calculation 7

Sheet No. 106 of 577

1-RD-20

SITE DATA INFORMATION:

Location: AIKEN, SOUTH CAROLINA

Building Air Exchanges Per Hour: 60 (User specified)

Date and Time: Fixed at July I, 19960000 hours

CHEMICAL INFORMATION:

Chemical Name: ETHYLENE GLYCOL MONOBUTYL ETHER

Molecular Weight: $118,18 \mathrm{~kg} / \mathrm{kmol}$

TLV-TWA: $25.00 \mathrm{ppm}$

IDLH: $700.00 \mathrm{ppm}$

Footprint Level of Concern: 150 ppm

Boiling Point: $171.32^{\circ} \mathrm{C}$

Vapor Pressure at Ambient Temperature: 0.0015 atm

Ambient Saturation Concentration: 1,570 ppm or $0.16 \%$

ATMOSPHERIC INFORMATION: (MANUAL INPUT OF DATA)

Wind: 1.7 meters $/ \mathrm{sec}$ from $90^{\circ}$ true

Inversion Height: 200 meters

Stability Class: E Air Temperature: $29^{\circ} \mathrm{C}$

Relative Humidity: 50\% Ground Roughness: Urban or forest

Cloud Cover: 1 tenths

SOURCE STRENGTH INFORMATION:

Puddle Area: 10000 square mieters

Average Puddle Depth: 1 centimeters

Soil Type: Default Ground Temperature: $29^{\circ} \mathrm{C}$

Initial Puddle Temperature: Ground temperature

Release Duration: ALOHA limited the duration to 1 hour

Max Computed Release Rate: 6.18 kilograms/min

Max Average Sustained Release Rate: 5.96 kilograms/min

(averaged over a minute or more)

Total Amount Released: 342 kilograms

FOOTPRINT INFORMATION:

Dispersion Module: Gaussian

User specified LOC: $150 \mathrm{ppm}$

Max Threat Zone for LOC: 56 meters

Max Threat Zone for IDLH: 56 meters

Note: Footprint was not drawn because

effects of near-field patchiness make plume

presentation unreliable for short distances.

TIME DEPENDENT INFORMATION:

Concentration Estimates at the point:

Downwind: 100 meters

Off Centerline: 0 meters

Max Concentration:

Outdoor: 17.5 ppm 
Title of Project EPHA for THM

Subject Concentration Analysis for THM Hazardous Chemicals

Computer T. M. Franey

\section{1-RD-21}

SITE DATA INFORMATION:

Location: AIKEN, SOUTH CAROLINA

Building Air Exchanges Per Hour: 60 (User specified)

Date and Time: Fixed at July 1, 19960000 hours

\section{CHEMICAL INFORMATION:}

Chemical Name: Fluorosilicic acid as HYDDUFLOURIC ACID - 70 WT\% - SAH

Molecular Weight: $20.01 \mathrm{~kg} / \mathrm{kmol}$

TLV-TWA: -unavail- IDLH: -unavail-

Footprint Level of Concern: $20 \mathrm{ppm}$

Boiling Point: $110.35^{\circ} \mathrm{C}$

Vapor Pressure at Ambient Temperature: 2.36e-004 atm

Ambient Saturation Concentration: 240 ppm or $0.024 \%$

\section{ATMOSPHERIC INFORMATION: (MANUAL INPUT OF DATA)}

Wind: 1.7 meters $/ \mathrm{sec}$ from $90^{\circ}$ true

Inversion Height: 200 meters

Stability Class: E Air Temperature: $29^{\circ} \mathrm{C}$

Relative Humidity: $50 \%$ Ground Roughness: 100 centimeters

Cloud Cover: 1 tenths

\section{SOURCE STRENGTH INFORMATION:}

Puddle Area: 24600 square feet

Average Puddle Depth: 1 centimeters

Soil Type: Default Ground Temperature: $29^{\circ} \mathrm{C}$

Initial Puddle Temperature: Ground temperature

Release Duration: ALOHA limited the duration to 1 hour

Max Computed Release Rate: 117 grams/min

Max Average Sustained Release Rate: 109 grams/min

(averaged over a minute or more)

Total Amount Released: 6.01 kilograms

FOOTPRINT INFORMATION:

Dispersion Module: Gaussian

User specified LOC: 20 ppm

Max Threat Zone for LOC: 30 meters

Note: Footprint was not drawn because effects of near-field patchiness make plume presentation unreliable for short distances.

TIME DEPENDENT INFORMATION:

Concentration Estimates at the point:

Downwind: 30 meters

Off Centerline: 0 meters

Max Concentration:

Outdoor: $20 \mathrm{ppm}$

Indoor: $20 \mathrm{ppm}$

Note: Indoor graph is shown with a dotted line. 


\section{ENGLNEERING COMPUTATION SHEET}

Title of Project EPHA for THM

Subject Concentration Analysis for THM Hazardous Chemicals

Computer T. M. Franey

Date $\underline{09 / 16 / 96}$
Reviewed by $\mathrm{S}$. A. Henderson

Works Calculation 7

Sheet No. 108 of 577

\section{1-RD-21}

SITE DATA INFORMATION:

Location: AIKEN, SOUTH CAROLINA

Building Air Exchanges Per Hour: 60 (User specified)

Date and Time: Fixed at July 1, 19960000 hours

\section{CHEMICAL INFORMATION:}

Chemical Name: Fluorosilicic acid as HYDDUFLOURIC ACID - 70 WT\% - SAH

Molecular Weight: $20.01 \mathrm{~kg} / \mathrm{kmol}$

TLV-TWA: -unavail- IDLH: -unavail-

Footprint Level of Concern: 20 ppm

Boiling Point: $110.35^{\circ} \mathrm{C}$

Vapor Pressure at Ambient Temperature: 2.36e-004 atm

Ambient Saturation Concentration: 240 ppm or $0.024 \%$

ATMOSPHERIC INFORMATION: (MANUAL INPUT OF DATA)

Wind: 1.7 meters $/ \mathrm{sec}$ from $90^{\circ}$ true

Inversion Height: 200 meters

Stability Class: E Air Temperature: $29^{\circ} \mathrm{C}$

Relative Humidity: 50\% Ground Roughness: 100 centimeters

Cloud Cover: 1 tenths

SOURCE STRENGTH INFORMATION:

Puddle Area: 107639 square feet

Average Puddle Depth: 1 centimeters

Soil Type: Default Ground Temperature: $29^{\circ} \mathrm{C}$

Initial Puddle Temperature: Ground temperature

Release Duration: ALOHA limited the duration to 1 hour

Max Computed Release Rate: 476 grams/min

Max Average Sustained Release Rate: 443 grams/min

(averaged over a minute or more)

Total Amount Released: 24.3 kilograms

\section{FOOTPRINT INFORMATION:}

Dispersion Module: Gaussian

User specified LOC: $20 \mathrm{ppm}$

.Max Threat Zone for LOC: 61 meters

Note: Footprint was not drawn because

effects of near-field patchiness make plume

presentation unreliable for short distances.

\section{TIME DEPENDENT INFORMATION:}

Concentration Estimates at the point:

Downwind: 100 meters

Off Centerline: 0 meters

Max Concentration:

Outdoor: $7.69 \mathrm{ppm}$

Indoor: $7.69 \mathrm{ppm}$

Note: Indoor graph is shown with a dotted line. 


\section{ENGINEERING COMPUTATION SHEET}

Title of Project EPHA for THM

Reviewed by $\mathrm{S}$. A. Henderson

Subject Concentration Analysis for THM Hazardous Chemicals

Computer T. M. Franey

Date 09/16/96
Works Calculation 7

Sheet No. 109 of 577

1-RD-21

SITE DATA INFORMATION:

Location: AIKEN, SOUTH CAROLINA

Building Air Exchanges Per Hour: 60 (User specified)

Date and Time: Fixed at July 1, 19960000 hours

\section{CHEMICAL INFORMATION:}

Chemical Name: Fluorosilicic acid as HYDDUFLOURIC ACID - 70 WT\% - SAH

Molecular Weight: $20.01 \mathrm{~kg} / \mathrm{kmol}$

TLV-TWA: -unavail- IDLH: -unavail-

Footprint Level of Concern: $20 \mathrm{ppm}$

Boiling Point: $110.35^{\circ} \mathrm{C}$

Vapor Pressure at Ambient Temperature: 2.36e-004 atm

Ambient Saturation Concentration: 240 ppm or $0.024 \%$

ATMOSPHERIC INFORMATION: (MANUAL INPUT OF DATA)

Wind: 1.7 meters $/ \mathrm{sec}$ from $90^{\circ}$ true

Inversion Height: 200 meters

Stability Class: E Air Temperature: $29^{\circ} \mathrm{C}$

Relative Humidity: $50 \%$ Ground Roughness: 100 centimeters

Cloud Cover: 1 tenths

SOURCE STRENGTH INFORMATION:

Puddle Area: 24420 square feet

Average Puddle Depth: 1 centimeters

Soil Type: Default Ground Temperature: $29^{\circ} \mathrm{C}$

Initial Puddle Temperature: Ground temperature

Release Duration: ALOHA limited the duration to 1 hour

Max Computed Release Rate: 117 grams/min

Max Average Sustained Release Rate: 109 grams/min

(averaged over a minute or more)

Total Amount Released: 5.97 kilograms

FOOTPRINT INFORMATION:

Dispersion Module: Gaussian

User specified LOC: $20 \mathrm{ppm}$

Max Threat Zone for LOC: 30 meters

Note: Footprint was not drawn because effects of near-field patchiness make plume presentation unreliable for short distances.

TIME DEPENDENT INFORMATION:

Concentration Estimates at the point:

Downwind: 100 meters

Off Centerline: 0 meters

Max Concentration:

Outdoor: $2.18 \mathrm{ppm}$

Indoor: $2.18 \mathrm{ppm}$

Note: Indoor graph is shown with a dotted line. 
Title of Project EPHA for THM

Subject Concentration Analysis for THM Hazardous Chemicals

Computer T. M. Franey
Reviewed by $\mathrm{S}$. A. Henderson

Works Calculation 7

Sheet No. 110 of 577

\section{1-RD-21}

SITE DATA INFORMATION:

Location: AIKEN, SOUTH CAROLINA

Building Air Exchanges Per Hour: 60 (User specified)

Date and Time: Fixed at July 1, 19960000 hours

\section{CHEMICAL INFORMATION:}

Chemical Name: Fluorosilicic acid as HYDDUFLOURIC ACID - 70 WT\% - SAH

Molecular Weight: $20.01 \mathrm{~kg} / \mathrm{kmol}$

TLV-TWA: -unavail- IDLH: -unavail-

Footprint Level of Concern: 20 ppm

Boiling Point: $110.35^{\circ} \mathrm{C}$

Vapor Pressure at Ambient Temperature: 2.36e-004 atm

Ambient Saturation Concentration: 240 ppm or $0.024 \%$

ATMOSPHERIC INFORMATION: (MANUAL INPUT OF DATA)

Wind: 1.7 meters $/ \mathrm{sec}$ from $90^{\circ}$ true

Inversion Height: 200 meters

Stability Class: E Air Temperature: $29^{\circ} \mathrm{C}$

Relative Humidity: 50\% Ground Roughness: 100 centimeters

Cloud Cover: 1 tenths

\section{SOURCE STRENGTH INFORMATION:}

Puddle Area: 40700 square feet

Average Puddle Depth: 1 centimeters

Soil Type: Default Ground Temperature: $29^{\circ} \mathrm{C}$

Initial Puddle Temperature: Ground temperature

Release Duration: ALOHA limited the duration to 1 hour

Max Computed Release Rate: 189 grams/min

Max Average Sustained Release Rate: 176 grams/min

(averaged over a minute or more)

Total Amount Released: 9.68 kilograms

\section{FOOTPRINT INFORMATION:}

Dispersion Module: Gaussian

User specified LOC: $20 \mathrm{ppm}$

Max Threat Zone for LOC: 38 meters

Note: Footprint was not drawn because effects of near-field patchiness make plume presentation unreliable for short distances.

\section{TIME DEPENDENT INFORMATION:}

Concentration Estimates at the point:

Downwind: 100 meters

Off Centerline: 0 meters

Max Concentration:

Outdoor: $3.15 \mathrm{ppm}$

Indoor: $3.15 \mathrm{ppm}$

Note: Indoor graph is shown with a dotted line. 
Title of Project EPHA for THM

Subject Concentration Analysis for THM Hazardous Chemicals

Computer T. M. Franey

Date $\underline{09 / 16 / 96}$
Reviewed by $\underline{\text { S. A. Henderson }}$

Works Calculation?

Sheet No. 111 of 577

\section{1-RD-21}

\section{SITE DATA INFORMATION}

Location: AIKEN, SOUTH CAROLINA

Building Air Exchanges Per Hour: 60 (User specified)

Date and Time: Fixed at July 1, 19960000 hours

\section{CHEMICAL INFORMATION:}

Chemical Name: Fluorosilicic acid as HYDDUFLOURIC ACID - 70 WT\% - SAH

Molecular Weight: $20.01 \mathrm{~kg} / \mathrm{kmol}$

TLV-TWA: -unavail- IDLH: -unavail-

Footprint Level of Concern: 20 ppm

Boiling Point: $110.35^{\circ} \mathrm{C}$

Vapor Pressure at Ambient Temperature: 2.36e-004 atm

Ambient Saturation Concentration: 240 ppm or $0.024 \%$

\section{ATMOSPHERIC INFORMATION: (MANUAL INPUT OF DATA)}

Wind: 1.7 meters $/ \mathrm{sec}$ from $90^{\circ}$ true

Inversion Height: 200 meters

Stability Class: E Air Temperature: $29^{\circ} \mathrm{C}$

Relative Humidity: $50 \%$ Ground Roughness: 100 centimeters

Cloud Cover: 1 tenths

\section{SOURCE STRENGTH INFORMATION:}

Puddle Area: 10000 square meters

Average Puddle Depth: 1 centimeters

Soil Type: Default Ground Temperature: $29^{\circ} \mathrm{C}$

Initial Puddle Temperature: Ground temperature

Release Duration: ALOHA limited the duration to 1 hour

Max Computed Release Rate: 476 grams/min

Max Average Sustained Release Rate: 443 grams/min

(averaged over a minute or more)

Total Amount Released: 24.3 kilograms

\section{FOOTPRINT INFORMATION:}

Dispersion Module: Gaussian

User specified LOC: 20 ppm

Max Threat Zone for LOC: 61 meters

Note: Footprint was not drawn because

effects of near-field patchiness make plume

presentation unreliable for short distances.

\section{TIME DEPENDENT INFORMATION:}

Concentration Estimates at the point:

Downwind: 100 meters

Off Centerline: 0 meters

Max Concentration:

Outdoor: 7.69 ppm

Indoor: $7.69 \mathrm{ppm}$

Note: Indoor graph is shown with a dotted line. 
Title of Projéct EPHA for THM

Subject Concentration Analysis for THM Hazardous Chemicals

Computer T. M. Franey
Reviewed by $\mathrm{S}$. A. Henderson

Works Calculation 7

Sheet No. 112 of 577

SITE DATA INFORMATION: 1-RD-22

Location: AIKEN, SOUTH CAROLINA

Building Air Exchanges Per Hour: 60 (User specified)

Date and Time: Fixed at July 1, 19960000 hours

CHEMICAL INFORMATION:

Chemical Name: FORMIC ACID

Molecular Weight: $46.03 \mathrm{~kg} / \mathrm{kmol}$

TLV-TWA: $5.00 \mathrm{ppm}$ IDLH: $30.00 \mathrm{ppm}$

Footprint Level of Concern: 20 ppm

Boiling Point: $100.56^{\circ} \mathrm{C}$

Vapor Pressure at Ambient Temperature: 0.068 atm

Ambient Saturation Concentration: $68,996 \mathrm{ppm}$ or $6.90 \%$

ATMOSPHERIC INFORMATION: (MANUAL INPUT OF DATA)

Wind: 1.7 meters $/ \mathrm{sec}$ from $90^{\circ}$ true

Inversion Height: 200 meters

Stability Class: E Air Temperature: $29^{\circ} \mathrm{C}$

Relative Humidity: 50\% Ground Roughness: 100 centimeters

Cloud Cover: 1 tenths

\section{SOURCE STRENGTH INFORMATION:}

Puddle Area: 26 square feet

Average Puddle Depth: 1 centimeters

Soil Type: Default Ground Temperature: $29^{\circ} \mathrm{C}$

Initial Puddle Temperature: Ground temperature

Release Duration: ALOHA limited the duration to 1 hour

Max Computed Release Rate: 60.5 grams/min

Max Average Sustained Release Rate: 53.3 grams/min

(averaged over a minute or more)

Total Amount Released: 2.82 kilograms

FOOTPRINT INFORMATION:

Dispersion Module: Gaussian

User specified LOC: $20 \mathrm{ppm}$

Max Threat Zone for LOC: 30 meters

Max Threat Zone for IDLH: 24 meters

Note: Footprint was not drawn because effects of near-field patchiness make plume presentation unreliable for short distances.

TIME DEPENDENT INFORMATION:

Concentration Estimates at the point:

Downwind: 30 meters

Off Centerline: 0 meters

Max Concentration:

Outdoor: $20 \mathrm{ppm}$

Indoor: $20 \mathrm{ppm}$

Note: Indoor graph is shown with a dotted line. 


\section{ENGINEERING COMPUTATION SHEET}

Title of Project EPHA for THM

Subject Concentration Analysis for THM Hazardous Chemicals

Computer T. M. Franey

Date $\underline{09 / 16 / 96}$

\section{SITE DATA INFORMATION: 1-RD-22}

Location: AIKEN, SOUTH CAROLINA

Building Air Exchanges Per Hour: 60 (User specified)

Date and Time: Fixed at July 1, 19960000 hours

\section{CHEMICAL INFORMATION:}

Chemical Name: FORMIC ACID

Molecular Weight: $46.03 \mathrm{~kg} / \mathrm{kmol}$.

TLV-TWA: $5.00 \mathrm{ppm}$

IDLH: $30.00 \mathrm{ppm}$

Footprint Level of Concern: $20 \mathrm{ppm}$

Boiling Point: $100.56^{\circ} \mathrm{C}$

Vapor Pressure at Ambient Temperature: $0.068 \mathrm{~atm}$

Ambient Saturation Concentration: 68,996 ppm or $6.90 \%$

\section{ATMOSPHERIC INFORMATION: (MANUAL INPUT OF DATA) \\ Wind: 1.7 meters $/ \mathrm{sec}$ from $90^{\circ}$ true \\ Inversion Height: 200 meters \\ Stability Class: E Air Temperature: $29^{\circ} \mathrm{C}$ \\ Relative Humidity: $50 \%$ Ground Roughness: 100 centimeters \\ Cloud Cover: 1 tenths}

\section{SOURCE STRENGTH INFORMATION:}

Puddle Area: 319 square feet

Average Puddle Depth: 1 centimeters

Soil Type: Default Ground Temperature: $29^{\circ} \mathrm{C}$

Initial Puddle Temperature: Ground temperature

Release Duration: ALOHA limited the duration to 1 hour

Max Computed Release Rate: 633 grams/min

Max Average Sustained Release Rate: 565 grams/min (averaged over a minute or more)

Total Amount Released: 30.2 kilograms

\section{FOOTPRINT INFORMATION:}

Dispersion Module: Gaussian

User specified LOC: 20 ppm

Max Threat Zone for LOC: 100 meters

Max Threat Zone for IDLH: 80 meters

Note: Footprint was not drawn because effects of near-field patchiness make plume

presentation unreliable for short distances.

\section{TIME DEPENDENT INFORMATION:}

Concentration Estimates at the point:

Downwind: 100 meters

Off Centerline: 0 meters

Max Concentration:

Outdoor: $20 \mathrm{ppm}$

Indoor: $20 \mathrm{ppm}$

Note: Indoor graph is shown with a dotted line.
Reviewed by S. A. Henderson

Works Calculation 7

Sheet No. 113 of 577 


\section{ENGINEERING COMPUTATION SHEET}

Title of Project EPHA for THM

Reviewed by S. A. Henderson

Works Calculation 7

Sheet No. 114 of 577

\section{SITE DATA INFORMATION: 1-RD-22}

Location: AIKEN, SOUTH CAROLINA

Building Air Exchanges Per Hour: 60 (User specified)

Date and Time: Fixed at July 1, 19960000 hours

CHEMICAL INFORMATION:

Chemical Name: FORMIC ACID

Molecular Weight: $46.03 \mathrm{~kg} / \mathrm{kmol}$

TLV-TWA: $5.00 \mathrm{ppm}$ IDLH: $30.00 \mathrm{ppm}$

Footprint Level of Concern: 20 ppm

Boiling Point: $100.56^{\circ} \mathrm{C}$

Vapor Pressure at Ambient Temperature: $0.068 \mathrm{~atm}$

Ambient Saturation Concentration: 68,996 ppm or $6.90 \%$

\section{ATMOSPHERIC INFORMATION: (MANUAL INPUT OF DATA)}

Wind: 1.7 meters $/ \mathrm{sec}$ from $90^{\circ}$ true

Inversion Height: 200 meters

Stability Class: E Air Temperature: $29^{\circ} \mathrm{C}$

Relative Humidity: 50\% Ground Roughness: 100 centimeters

Cloud Cover: 1 tenths

\section{SOURCE STRENGTH INFORMATION:}

Puddle Area: 24420 square feet

Average Puddle Depth: 1 centimeters

Soil Type: Default Ground Temperature: $29^{\circ} \mathrm{C}$

Initial Puddle Temperature: Ground temperature

Release Duration: ALOHA limited the duration to 1 hour

Max Computed Release Rate: 38.5 kilograms/min

Max Average Sustained Release Rate: 34.9 kilograms/min (averaged over a minute or more)

Total Amount Released: 1,887 kilograms

\section{FOOTPRINT INFORMATION:}

Dispersion Module: Gaussian

User specified LOC: $20 \mathrm{ppm}$

Max Threat Zone for LOC: 975 meters

Max Threat Zone for IDLH: 750 meters

\section{TIME DEPENDENT INFORMATION:}

Concentration Estimates at the point:

Downwind: 100 meters

Off Centerline: 0 meters

Max Concentration:

Outdoor: $306 \mathrm{ppm}$

Indoor: $306 \mathrm{ppm}$

Note: Indoor graph is shown with a dotted line. 
Title of Project EPHA for THM

Subject Concentration Analysis for THM Hazardous Chemicals

Computer T. M. Franey Date 09/16/96

SITE DATA INFORMATION: 1-RD-22

Location: AIKEN, SOUTH CAROLINA

Building Air Exchanges Per Hour: 60 (User specified)

Date and Time: Fixed at July 1, 19960000 hours

CHEMICAL INFORMATION:

Chemical Name: FORMIC ACID

Molecular Weight: $46.03 \mathrm{~kg} / \mathrm{kmol}$

TLV-TWA: $5.00 \mathrm{ppm}$ IDLH: $30.00 \mathrm{ppm}$

Footprint Level of Concern: $20 \mathrm{ppm}$

Boiling Point: $100.56^{\circ} \mathrm{C}$

Vapor Pressure at Ambient Temperature: 0.068 atm

Ambient Saturation Concentration: $68,996 \mathrm{ppm}$ or $6.90 \%$

ATMOSPHERIC INFORMATION: (MANUAL INPUT OF DATA)

Wind: 1.7 meters $/ \mathrm{sec}$ from $90^{\circ}$ true

Inversion Height: 200 meters

Stability Class: E Air Temperature: $29^{\circ} \mathrm{C}$

Relative Humidity: 50\% Ground Roughness: 100 centimeters

Cloud Cover: 1 tenths

SOURCE STRENGTH INFORMATION:

Puddle Area: 40700 square feet

Average Puddle Depth: 1 centimeters

Soil Type: Default Ground Temperature: $29^{\circ} \mathrm{C}$

Initial Puddle Temperature: Ground temperature

Release Duration: ALOHA limited the duration to 1 hour

Max Computed Release Rate: 62.6 kilograms/min

Max Average Sustained Release Rate: 56.9 kilograms/min

(averaged over a minute or more)

Total Amount Released: 3,079 kilograms

FOOTPRINT INFORMATION:

Model Run: Heavy Gas

User specified LOC: $20 \mathrm{ppm}$

Max Threat Zone for LOC: 1.4 kilometers

Max Threat Zone for IDLH: 1.1 kilometers

Note: The Heavy Gas footprint is an initial screening.

For short releases it may be an overestimation.

$\mathrm{Be}$ sure to check concentration information at specific locations.

\section{TIME DEPENDENT INFORMATION:}

Concentration Estimates at the point:

Downwind: 100 meters

Off Centerline: 0 meters

Max Concentration:

Outdoor: $930 \mathrm{ppm}$

Indoor: $930 \mathrm{ppm}$

Note: Indoor graph is shown with a dotted line.
Reviewed by S.A. Henderson

Works Calculation 7

Sheet No. 115 of 577 


\section{ENGINEERING COMPUTATION SHEET}

Title of Project EPHA for THM

Subject Concentration Analysis for THM Hazardous Chemicals

Computer T. M. Franey

Date $\underline{09 / 16 / 96}$

SITE DATA INFORMATION: 1-RD-22

Location: AIKEN, SOUTH CAROLINA

Building Air Exchanges Per Hour: 60 (User specified)

Date and Time: Fixed at July 1, 19960000 hours

CHEMICAL INFORMATION:

Chemical Name: FORMIC ACID

Molecular Weight: $46.03 \mathrm{~kg} / \mathrm{kmol}$

TLV-TWA: $5.00 \mathrm{ppm}$ IDLH: $30.00 \mathrm{ppm}$

Footprint Level of Concern: $20 \mathrm{ppm}$

Boiling Point: $100.56^{\circ} \mathrm{C}$

Vapor Pressure at Ambient Temperature: $0.068 \mathrm{~atm}$

Ambient Saturation Concentration: 68,996 ppm or $6.90 \%$

ATMOSPHERIC INFORMATION: (MANUAL INPUT OF DATA)

Wind: 1.7 meters $/ \mathrm{sec}$ from $90^{\circ}$ true

Inversion Height: 200 meters

Stability Class: E Air Temperature: $29^{\circ} \mathrm{C}$

Relative Humidity: $50 \%$ Ground Roughness: 100 centimeters

Cloud Cover: 1 tenths

\section{SOURCE STRENGTH INFORMATION:}

Puddle Area: 10000 square meters

Average Puddle Depth: 1 centimeters

Soil Type: Default Ground Temperature: $29^{\circ} \mathrm{C}$

Initial Puddle Temperature: Ground temperature

Release Duration: ALOHA limited the duration to 1 hour

Max Computed Release Rate: 158 kilograms/min

Max Average Sustained Release Rate: 144 kilograms/min (averaged over a minute or more)

Total Amount Released: 7,828 kilograms

\section{FOOTPRINT INFORMATION:}

Model Run: Heavy Gas

User specified LOC: $20 \mathrm{ppm}$

Max Threat Zone for LOC: 2.3 kilometers

Max Threat Zone for IDLH: 1.7 kilometers

Note: The Heavy Gas footprint is an initial screening.

For short releases it may be an overestimation.

Be sure to check concentration information at specific locations.

TIME DEPENDENT INFORMATION:

Concentration Estimates at the point:

Downwind: 100 meters

Off Centerline: 0 meters

Max Concentration:

Outdoor: $1,710 \mathrm{ppm}$

Indoor: $1,710 \mathrm{ppm}$

Note: Indoor graph is shown with a dotted line.
Reviewed by S. A. Henderson

Works Calculation 7

Sheet No. 116 of 577 
Title of Project EPHA for THM

Subject Concentration Analysis for THM Hazardous Chemicals

Computer T. M. Franey

Date $\underline{09 / 16 / 96}$
Reviewed by $\mathrm{S}$. A. Henderson

Works Calculation 7

Sheet No. 117 of 577

SITE DATA INFORMATION: 1-RD-23

Location: AIKEN, SOUTH CAROLINA

Building Air Exchanges Per Hour: 60 (User specified)

Date and Time: Fixed at July 1, 19960000 hours

\section{CHEMICAL INFORMATION:}

Chemical Name: DIETHYLENE GLYCOL DIMETHYL ETHER

Molecular Weight: $134.18 \mathrm{~kg} / \mathrm{kmol}$

TLV-TWA: -unavail- IDLH: -unavail-

Footprint Level of Concern: 25 ppm

Boiling Point: $159.76^{\circ} \mathrm{C}$

Vapor Pressure at Ambient Temperature: $0.0050 \mathrm{~atm}$

Ambient Saturation Concentration: $5,107 \mathrm{ppm}$ or $0.51 \%$

\section{ATMOSPHERIC INFORMATION: (MANUAL INPUT OF DATA)}

Wind: 1.7 meters $/ \mathrm{sec}$ from $90^{\circ}$ true

Inversion Height: 200 meters

Stability Class: E Air Temperature: $29^{\circ} \mathrm{C}$

Relative Humidity: 50\% Ground Roughness: 100 centimeters

Cloud Cover: 1 tenths

\section{SOURCE STRENGTH INFORMATION:}

Puddle Area: 2650 square feet

Average Puddle Depth: 1 centimeters

Soil Type: Default Ground Temperature: $29^{\circ} \mathrm{C}$

Initial Puddle Temperature: Ground temperature

Release Duration: ALOHA limited the duration to 1 hour

Max Computed Release Rate: 865 grams/min

Max Average Sustained Release Rate: 835 grams/min

(averaged over a minute or more)

Total Amount Released: 48.0 kilograms

\section{FOOTPRINT INFORMATION:}

Dispersion Module: Gaussian

User specified LOC: $25 \mathrm{ppm}$

Max Threat Zone for LOC: 30 meters

Note: Footprint was not drawn because effects of near-field patchiness make plume presentation unreliable for short distances.

\section{TIME DEPENDENT INFORMATION:}

Concentration Estimates at the point:

Downwind: 30 meters

Off Centerline: 0 meters

Max Concentration:

Outdoor: 25 ppm

Indoor: $25 \mathrm{ppm}$

Note: Indoor graph is shown with a dotted line. 


\section{ENGINEERING COMPUTATION SHEET}

Title of Project EPHA for THM

Subject Concentration Analysis for THM Hazardous Chemicals

Computer T.M. Franey Date $\underline{09 / 16 / 96}$

SITE DATA INFORMATION: 1-RD-23

Location: AIKEN, SOUTH CAROLINA

Building Air Exchanges Per Hour: 60 (User specified)

Date and Time: Fixed at July 1, 19960000 hours

CHEMICAL INFORMATION:

Chemical Name: DIETHYLENE GLYCOL DIMETHYL ETHER

Molecular Weight: $134.18 \mathrm{~kg} / \mathrm{kmol}$

TLV-TWA: -unavail- IDLH: -unavail-

Footprint Level of Concern: 25 ppm

Boiling Point: $159.76^{\circ} \mathrm{C}$

Vapor Pressure at Ambient Temperature: $0.0050 \mathrm{~atm}$

Ambient Saturation Concentration: 5,107 ppm or $0.51 \%$

\section{ATMOSPHERIC INFORMATION: (MANUAL INPUT OF DATA)}

Wind: 1.7 meters $/ \mathrm{sec}$ from $90^{\circ}$ true

Inversion Height: 200 meters

Stability Class: E Air Temperature: $29^{\circ} \mathrm{C}$

Relative Humidity: 50\% Ground Roughness: 100 centimeters

Cloud Cover: 1 tenths

\section{SOURCE STRENGTH INFORMATION:}

Puddle Area: 31400 square feet

Average Puddle Depth: 1 centimeters

Soil Type: Default Ground Temperature: $29^{\circ} \mathrm{C}$

Initial Puddle Temperature: Ground temperature

Release Duration: ALOHA limited the duration to 1 hour

Max Computed Release Rate: 9.27 kilograms/min

Max Average Sustained Release Rate: 8.95 kilograms/min

(averaged over a minute or more)

Total Amount Released: 515 kilograms

\section{FOOTPRINT INFORMATION:}

Dispersion Module: Gaussian

User specified LOC: $25 \mathrm{ppm}$

Max Threat Zone for LOC: 100 meters

Note: Footprint was not drawn because effects of near-field patchiness make plume

presentation unreliable for short distances.

\section{TIME DEPENDENT INFORMATION:}

Concentration Estimates at the point:

Downwind: 100 meters

Off Centerline: 0 meters

Max Concentration:

Outdoor: $25 \mathrm{ppm}$

Indoor: $25 \mathrm{ppm}$

Note: Indoor graph is shown with a dotted line.
Reviewed by S. A. Henderson

Works Calculation 7

Sheet No. 118 of 577 


\section{ENGINEERING COMPUTATION SHEET}

Title of Project EPHA for THM

Subject Concentration Analysis for THM Hazardous Chemicals

Computer T. M. Franey

Date $09 / 16 / 96$
Reviewed by $\mathrm{S}$. A. Henderson

Works Calculation 7

Sheet No. 119 of 577

SITE DATA INFORMATION: 1-RD-23

Location: AIKEN, SOUTH CAROLINA

Building Air Exchanges Per Hour: 60 (User specified)

Date and Time: Fixed at July 1, 19960000 hours

CHEMICAL INFORMATION:

Chemical Name: DIETHYLENE GLYCOL DIMETHYL ETHER

Molecular Weight: $134.18 \mathrm{~kg} / \mathrm{kmol}$

TLV-TWA: -unavail- IDLH: -unavail-

Footprint Level of Concern: 25 ppm

Boiling Point: $159.76^{\circ} \mathrm{C}$

Vapor Pressure at Ambient Temperature: $0.0050 \mathrm{~atm}$

Ambient Saturation Concentration: 5,107 ppm or $0.51 \%$

ATMOSPHERIC INFORMATION: (MANUAL INPUT OF DATA)

Wind: 1.7 meters $/ \mathrm{sec}$ from $90^{\circ}$ true

Inversion Height: 200 meters

Stability Class: E Air Temperature: $29^{\circ} \mathrm{C}$

Relative Humidity: 50\% Ground Roughness: 100 centimeters

Cloud Cover: 1 tenths

\section{SOURCE STRENGTH INFORMATION:}

Puddle Area: 24420 square feet

Average Puddle Depth: 1 centimeters

Soil Type: Default Ground Temperature: $29^{\circ} \mathrm{C}$

Initial Puddle Temperature: Ground temperature

Release Duration: ALOHA limited the duration to 1 hour

Max Computed Release Rate: 7.28 kilograms/min

Max Average Sustained Release Rate: 7.03 kilograms/min

(averaged over a minute or more)

Total Amount Released: 404 kilograms

FOOTPRINT INFORMATION:

Dispersion Module: Gaussian

User specified LOC: $25 \mathrm{ppm}$

Max Threat Zone for LOC: 88 meters

Note: Footprint was not drawn because effects of near-field patchiness make plume

presentation unreliable for short distances.

TIME DEPENDENT INFORMATION:

Concentration Estimates at the point:

Downwind: 100 meters

Off Centerline: 0 meters

Max Concentration:

Outdoor: $21.1 \mathrm{ppm}$

Indoor: $21.1 \mathrm{ppm}$

Note: Indoor graph is shown with a dotted line. 
Title of Project EPHA for THM

Subject Concentration Analysis for THM Hazardous Chemicals

Computer T. M. Franey Date 09/16/96

SITE DATA INFORMATION: 1-RD-23

Location: AIKEN, SOUTH CAROLINA

Building Air Exchanges Per Hour: 60 (User specified)

Date and Time: Fixed at July 1, 19960000 hours

CHEMICAL INFORMATION:

Chemical Name: DIETHYLENE GLYCOL DIMETHYL ETHER

Molecular Weight: $134.18 \mathrm{~kg} / \mathrm{kmol}$

TLV-TWA: -unavail-

IDLH: -unavail-

Footprint Level of Concern: 25 ppm

Boiling Point: $159.76^{\circ} \mathrm{C}$

Vapor Pressure at Ambient Temperature: $0.0050 \mathrm{~atm}$

Ambient Saturation Concentration: 5,107 ppm or $0.51 \%$

ATMOSPHERIC INFORMATION: (MANUAL INPUT OF DATA)

Wind: 1.7 meters $/ \mathrm{sec}$ from $90^{\circ}$ true

Inversion Height: 200 meters

Stability Class: E Air Temperature: $29^{\circ} \mathrm{C}$

Relative Humidity: $50 \%$ Ground Roughness: 100 centimeters

Cloud Cover: 1 tenths

\section{SOURCE STRENGTH INFORMATION:}

Puddle Area: 40700 square feet

Average Puddle Depth: 1 centimeters

Soil Type: Default Ground Temperature: $29^{\circ} \mathrm{C}$

Initial Puddle Temperature: Ground temperature

Release Duration: ALOHA limited the duration to 1 hour

Max Computed Release Rate: 11.9 kilograms/min

Max Average Sustained Release Rate: 11.5 kilograms/min

(averaged over a minute or more)

Total Amount Released: 661 kilograms

FOOTPRINT INFORMATION:

Dispersion Module: Gaussian

User specified LOC: $25 \mathrm{ppm}$

Max Threat Zone for LOC: 114 meters

TIME DEPENDENT INFORMATION:

Concentration Estimates at the point: .

Downwind: 100 meters

Off Centerline: 0 meters

Max Concentration:

Outdoor: $30.6 \mathrm{ppm}$

Indoor: $30.6 \mathrm{ppm}$

Note: Indoor graph is shown with a dotted line.
Reviewed by S.A. Henderson

Works Calculation 7

Sheet No. 120 of 577 
Title of Project EPHA for THM

Subject Concentration Analysis for THM Hazardous Chemicals

Computer T.M. Franey

Date $\underline{09 / 16 / 96}$

SITE DATA INFORMATION: 1-RD-24

Location: AIKEN, SOUTH CAROLINA

Building Air Exchanges Per Hour: 60 (User specified)

Date and Time: Fixed at July 1, 19960000 hours

CHEMICAL INFORMATION:

Chemical Name: HYDROGEN CHLORIDE 33-40\% / MSDS 6966

Molecular Weight: $36.46 \mathrm{~kg} / \mathrm{kmol}$

TLV-TWA: -unavail- IDLH: -unavail-

Footprint Level of Concern: 20 ppm

Boiling Point: $148.89^{\circ} \mathrm{C}$

Vapor Pressure at Ambient Temperature: $0.0016 \mathrm{~atm}$

Ambient Saturation Concentration: $1,670 \mathrm{ppm}$ or $0.17 \%$

ATMOSPHERIC INFORMATION: (MANUAL INPUT OF DATA)

Wind: 1.7 meters $/ \mathrm{sec}$ from $90^{\circ}$ true

Inversion Height: 200 meters

Stability Class: E Air Temperature: $29^{\circ} \mathrm{C}$

Relative Humidity: $50 \%$ Ground Roughness: 100 centimeters

Cloud Cover: 1 tenths

SOURCE STRENGTH INFORMATION:

Puddle Area: 3230 square feet

Average Puddle Depth: 1 centimeters

Soil Type: Default Ground Temperature: $29^{\circ} \mathrm{C}$

Initial Puddle Temperature: Ground temperature

Release Duration: ALOHA limited the duration to 1 hour

Max Computed Release Rate: 197 grams/min

Max Average Sustained Release Rate: 189 grams/min

(averaged over a minute or more)

Total Amount Released: 10.8 kilograms

\section{FOOTPRINT INFORMATION:}

Dispersion Module: Gaussian

User specified LOC: $20 \mathrm{ppm}$

Max Threat Zone for LOC: 30 meters

Note: Footprint was not drawn because

effects of near-field patchiness make plume

presentation unreliable for short distances.

\section{TIME DEPENDENT INFORMATION:}

Concentration Estimates at the point:

Downwind: 30 meters

Off Centerline: 0 meters

Max Concentration:

Outdoor: $20 \mathrm{ppm}$

Indoor: $20 \mathrm{ppm}$

Note: Indoor graph is shown with a dotted line.
Reviewed by S. A. Henderson

Works Calculation 7

Sheet No. 121 of 577 
Title of Project EPHA for THM

Subject Concentration Analysis for THM Hazardous Chemicals

Computer T. M. Franey

Date $\underline{09 / 16 / 96}$
Reviewed by S. A. Henderson

Works Calculation 7

Sheet No. 122 of 577

SITE DATA INFORMATION: $\quad$ 1-RD-24

Location: AIKEN, SOUTH CAROLINA

Building Air Exchanges Per Hour: 60 (User specified)

Date and Time: Fixed at July 1, 19960000 hours

CHEMICAL INFORMATION:

Chemical Name: HYDROGEN CHLORIDE 33-40\% / MSDS 6966

Molecular Weight: $36.46 \mathrm{~kg} / \mathrm{kmol}$

TLV-TWA: -unavail- . IDLH: -unavail-

Footprint Level of Concern: $20 \mathrm{ppm}$

Boiling Point: $148.89^{\circ} \mathrm{C}$

Vapor Pressure at Ambient Temperature: $0.0016 \mathrm{~atm}$

Ambient Saturation Concentration: $1,670 \mathrm{ppm}$ or $0.17 \%$

ATMOSPHERIC INFORMATION: (MANUAL INPUT OF DATA)

Wind: 1.7 meters $/ \mathrm{sec}$ from $90^{\circ}$ true

Inversion Height: 200 meters

Stability Class: E Air Temperature: $29^{\circ} \mathrm{C}$

Relative Humidity: 50\% Ground Roughness: 100 centimeters

Cloud Cover: 1 tenths

\section{SOURCE STRENGTH INFORMATION:}

Puddle Area: 39400 square feet

Average Puddle Depth: 1 centimeters

Soil Type: Default Ground Temperature: $29^{\circ} \mathrm{C}$

Initial Puddle Temperature: Ground temperature

Release Duration: ALOHA limited the duration to 1 hour

Max Computed Release Rate: 2.11 kilograms/min

Max Average Sustained Release Rate: 2.03 kilograms/min

(averaged over a minute or more)

Total Amount Released: 116 kilograms

\section{FOOTPRINT INFORMATION:}

Dispersion Module: Gaussian

User specified LOC: $20 \mathrm{ppm}$

Max Threat Zone for LOC: 100 meters

Note: Footprint was not drawn because

effects of near-field patchiness make plume

presentation unreliable for short distances.

\section{TIME DEPENDENT INFORMATION:}

Concentration Estimates at the point:

Downwind: 100 meters

Off Centerline: 0 meters

Max Concentration:

Outdoor: $20 \mathrm{ppm}$

Indoor: $20 \mathrm{ppm}$

Note: Indoor graph is shown with a dotted line. 


\section{ENGINEERING COMPUTATION SHEET}

Title of Project EPHA for THM

Subject Concentration Analysis for THM Hazardous Chemicals

Computer T. M. Franey Date $\underline{09 / 16 / 96}$
Reviewed by S. A. Henderson

Works Calculation 7

Sheet No. 123 of 577

\section{SITE DATA INFORMATION: 1-RD-24 \\ Location: AIKEN, SOUTH CAROLINA \\ Building Air Exchanges Per Hour: 60 (User specified) \\ Date and Time: Fixed at July 1, 19960000 hours}

\section{CHEMICAL INFORMATION:}

Chemical Name: HYDROGEN CHLORIDE 33-40\% / MSDS 6966

Molecular Weight: $36.46 \mathrm{~kg} / \mathrm{kmol}$

TLV-TWA: -unavail- IDLH: -unavail-

Footprint Level of Concern: $20 \mathrm{ppm}$

Boiling Point: $148.89^{\circ} \mathrm{C}$

Vapor Pressure at Ambient Temperature: $0.0016 \mathrm{~atm}$

Ambient Saturation Concentration: $1,670 \mathrm{ppm}$ or $0.17 \%$

ATMOSPHERIC INFORMATION: (MANUAL INPU்T OF DATA)

Wind: 1.7 meters $/ \mathrm{sec}$ from $90^{\circ}$ true

Inversion Height: 200 meters

Stability Class: E Air Temperature: $29^{\circ} \mathrm{C}$

Relative Humidity: 50\% . Ground Roughness: 100 centimeters

Cloud Cover: 1 tenths

\section{SOURCE STRENGTH INFORMATION:}

Puddle Area: 24420 square feet

Average Puddle Depth: 1 centimeters

Soil Type: Default Ground Temperature: $29^{\circ} \mathrm{C}$

Initial Puddle Temperature: Ground temperature

Release Duration: ALOHA limited the duration to 1 hour

Max Computed Release Rate: 1.34 kilograms/min

Max Average Sustained Release Rate: 1.29 kilograms/min

(averaged over a minute or more)

Total Amount Released: 73.5 kilograms

\section{FOOTPRINT INFORMATION:}

Dispersion Module: Gaussian

User specified LOC: $20 \mathrm{ppm}$

Max Threat Zone for LOC: 79 meters

Note: Footprint was not drawn because effects of near-field patchiness make plume presentation unreliable for short distances.

\section{TIME DEPENDENT INFORMATION:}

Concentration Estimates at the point:

Downwind: 100 meters

Off Centerline: 0 meters

Max Concentration:

Outdoor: $14.2 \mathrm{ppm}$

Indoor: $14.2 \mathrm{ppm}$

Note: Indoor graph is shown with a dotted line. 
Title of Project EPHA for THM

Subject Concentration Analysis for THM Hazardous Chemicals

Computer T. M. Franey

Date $\underline{09 / 16 / 96}$

Reviewed by S. A. Henderson

Works Calculation 7

Sheet No. 124 of 577

SITE DATA INFORMATION: 1-RD-24

Location: AIKEN, SOUTH CAROLINA

Building Air Exchanges Per Hour: 60 (User specified)

Date and Time: Fixed at July 1, 19960000 hours

CHEMICAL INFORMATION:

Chemical Name: HYDROGEN CHLORIDE 33-40\% / MSDS 6966

Molecular Weight: $36.46 \mathrm{~kg} / \mathrm{kmol}$

TLV-TWA: -unavail- IDLH: -unavail-

Footprint Level of Concern: 20 ppm

Boiling Point: $148.89^{\circ} \mathrm{C}$

Vapor Pressure at Ambient Temperature: $0.0016 \mathrm{~atm}$

Ambient Saturation Concentration: $1,670 \mathrm{ppm}$ or $0.17 \%$

ATMOSPHERIC INFORMATION: (MANUAL INPUT OF DATA)

Wind: 1.7 meters $/ \mathrm{sec}$ from $90^{\circ}$ true

Inversion Height: 200 meters

Stability Class: E Air Temperature: $29^{\circ} \mathrm{C}$

Relative Humidity: 50\% Ground Roughness: 100 centimeters

Cloud Cover: 1 tenths

\section{SOURCE STRENGTH INFORMATION:}

Puddle Area: 40700 square feet

Average Puddle Depth: 1 centimeters

Soil Type: Default Ground Temperature: $29^{\circ} \mathrm{C}$

Initial Puddle Temperature: Ground temperature

Release Duration: ALOHA limited the duration to 1 hour

Max Computed Release Rate: 2.18 kilograms/min

Max Average Sustained Release Rate: 2.09 kilograms/min

(averaged over a minute or more)

Total Amount Released: 120 kilograms

\section{FOOTPRINT INFORMATION:}

Dispersion Module: Gaussian

User specified LOC: $20 \mathrm{ppm}$

Max Threat Zone for LOC: 102 meters

\section{TIME DEPENDENT INFORMATION:}

Concentration Estimates at the point:

Downwind: 100 meters

Off Centerline: 0 meters

Max Concentration:

Outdoor: $20.5 \mathrm{ppm}$

Indoor: $20.5 \mathrm{ppm}$

Note: Indoor graph is shown with a dotted line. 


\section{ENGINEERING COMPUTATION SHEET}

Title of Project EPHA for THM

Subject Concentration Analysis for THM Hazardous Chemicals

Computer T. M. Franey

Date $\underline{09 / 16 / 96}$
Reviewed by $\underline{\text { S. A. Henderson }}$

Works Calculation 7

Sheet No: 125 of 577

SITE DATA INFORMATION: 1-RD-24

Location: AIKEN, SOUTH CAROLINA

Building Air Exchanges Per Hour: 60 (User specified)

Date and Time: Fixed at July 1, 19960000 hours

\section{CHEMICAL INFORMATION:}

Chemical Name: HYDROGEN CHLORIDE 33-40\% / MSDS 6966

Molecular Weight: $36.46 \mathrm{~kg} / \mathrm{kmol}$

TLV-TWA: -unavail- IDLH: -unavail-

Footprint Level of Concern: $20 \mathrm{ppm}$

Boiling Point: $148.89^{\circ} \mathrm{C}$

Vapor Pressure at Ambient Temperature: 0.0016 atm

Ambient Saturation Concentration: 1,670 ppm or 0.17\%

ATMOSPHERIC INFORMATION: (MANUAL INPUT OF DATA)

Wind: 1.7 meters $/ \mathrm{sec}$ from $90^{\circ}$ true

Inversion Height: 200 meters

Stability Class: E Air Temperature: $29^{\circ} \mathrm{C}$

Relative Humidity: $50 \%$ Ground Roughness: 100 centimeters

Cloud Cover: 1 tenths

SOURCE STRENGTH INFORMATION:

Puddle Area: 10000 square meters

Average Puddle Depth: 1 centimeters

Soil Type: Default Ground Temperature: $29^{\circ} \mathrm{C}$

Initial Puddle Temperature: Ground temperature

Release Duration: ALOHA limited the duration to 1 hour

Max Computed Release Rate: 5.51 kilograms/min

Max Average Sustained Release Rate: 5.29 kilograms/min (averaged over a minute or more)

Total Amount Released: 302 kilograms

FOOTPRINT INFORMATION:

Dispersion Module: Gaussian

User specified LOC: $20 \mathrm{ppm}$

Max Threat Zone for LOC: 165 meters

\section{TIME DEPENDENT INFORMATION:}

Concentration Estimates at the point:

Downwind: 100 meters

Off Centerline: 0 meters

Max Concentration:

Outdoor: $50.4 \mathrm{ppm}$

Indoor: $50.4 \mathrm{ppm}$

Note: Indoor graph is shown with a dotted line. 
Title of Project EPHA for THM

Subject Concentration Analysis for THM Hazardous Chemicals

Computer T. M. Franey

Date $\underline{09 / 16 / 96}$
Reviewed by $\mathrm{S}$. A. Henderson

Works Calculation 7

Sheet No. 126 of 577

SITE DATA INFORMATION: 1-RD-25

Location: AIKEN, SOUTH CAROLINA

Building Air Exchanges Per Hour: 60 (User specified)

Date and Time: Fixed at July 1, 19960000 hours

\section{CHEMICAL INFORMATION:}

Chemical Name: HYDDUFLOURIC ACID - 70 WT\% - SAH

Molecular Weight: $20.01 \mathrm{~kg} / \mathrm{kmol}$

TLV-TWA: -unavail- IDLH: -unavail-

Footprint Level of Concern: $20 \mathrm{ppm}$

Boiling Point: $110.35^{\circ} \mathrm{C}$

Vapor Pressure at Ambient Temperature: 2.36e-004 atm

Ambient Saturation Concentration: 240 ppm or $0.024 \%$

ATMOSPHERIC INFORMATION: (MANUAL INPUT OF DATA)

Wind: 1.7 meters $/ \mathrm{sec}$ from $90^{\circ}$ true

Inversion Height: 200 meters

Stability Class: E Air Temperature: $29^{\circ} \mathrm{C}$

Relative Humidity: $50 \%$ Ground Roughness: 100 centimeters

Cloud Cover: 1 tenths

\section{SOURCE STRENGTH INFORMATION:}

Puddle Area: 24600 square feet

Average Puddle Depth: 1 centimeters

Soil Type: Default Ground Temperature: $29^{\circ} \mathrm{C}$

Initial Puddle Temperature: Ground temperature

Release Duration: ALOHA limited the duration to 1 hour

Max Computed Release Rate: 117 grams/min

Max Average Sustained Release Rate: 109 grams/min

(averaged over a minute or more)

Total Amount Released: 6.01 kilograms

\section{FOOTPRINT INFORMATION:}

Dispersion Module: Gaussian

User specified LOC: $20 \mathrm{ppm}$

Max Threat Zone for LOC: $\mathbf{3 0}$ meters

Note: Footprint was not drawn because effects of near-field patchiness make plume presentation unreliable for short distances.

\section{TIME DEPENDENT INFORMATION:}

Concentration Estimates at the point:

Downwind: 30 meters

Off Centerline: 0 metẹs

Max Concentration:

Outdoor: $20 \mathrm{ppm}$

Indoor: $20 \mathrm{ppm}$

Note: Indoor graph is shown with a dotted line. 
Title of Project EPHA for THM

Subject Concentration Analysis for THM Hazardous Chemicals

Computer T. M. Franey

Date $\underline{09 / 16 / 96}$
Reviewed by $\underline{\text { S. A. Henderson }}$

Works Calculation 7

Sheet No. 127 of 577

SITE DATA INFORMATION: 1-RD-25

Location: AIKEN, SOUTH CAROLINA

Building Air Exchanges Per Hour: 60 (User specified)

Date and Time: Fixed at July 1, 19960000 hours

\section{CHEMICAL INFORMATION:}

Chemical Name: HYDDUFLOURIC ACID - 70 WT\% - SAH

Molecular Weight: $20.01 \mathrm{~kg} / \mathrm{kmol}$

TLV-TWA: -unavail- IDLH: -unavail-

Footprint Level of Concern: 20 ppm

Boiling Point: $110.35^{\circ} \mathrm{C}$

Vapor Pressure at Ambient Temperature: 2.36e-004 atm

Ambient Saturation Concentration: 240 ppm or $0.024 \%$

\section{ATMOSPHERIC INFORMATION: (MANUAL INPUT OF DATA)}

Wind: 1.7 meters $/ \mathrm{sec}$ from $90^{\circ}$ true

Inversion Height: 200 meters

Stability Class: E Air Temperature: $29^{\circ} \mathrm{C}$

Relative Humidity: 50\% Ground Roughness: 100 centimeters

Cloud Cover: 1 tenths

\section{SOURCE STRENGTH INFORMATION:}

Puddle Area: 107639 square feet

Average Puddle Depth: 1 centimeters

Soil Type: Default . Ground Temperature: $29^{\circ} \mathrm{C}$

Initial Puddle Temperature: Ground temperature

Release Duration: ALOHA limited the duration to 1 hour

Max Computed Release Rate: 476 grams/min

Max Average Sustained Release Rate: 443 grams/min

(averaged over a minute or more)

Total Amount Released: 24.3 kilograms

\section{FOOTPRINT INFORMATION:}

Dispersion Module: Gaussian

User specified LOC: $20 \mathrm{ppm}$

Max Threat Zone for LOC: 61 meters

Note: Footprint was not drawn because effects of near-field patchiness make plume presentation unreliable for short distances.

\section{TIME DEPENDENT INFORMATION:}

Concentration Estimates at the point:

Downwind: 100 meters

Off Centerline: 0 meters

Max Concentration:

Outdoor: $7.69 \mathrm{ppm}$

Indoor: $7.69 \mathrm{ppm}$

Note: Indoor graph is shown with a dotted line. 


\section{ENGINEERING COMPUTATION SHEET}

Title of Project EPHA for THM

Subject Concentration Analysis for THM Hazardous Chemicals

Computer T. M. Franey

Date 09/16/96

SITE DATA INFORMATTON: 1-RD-25

Location: AIKEN, SOUTH CAROLINA

Building Air Exchanges Per Hour: 60 (User specified)

Date and Time: Fixed at July 1, 19960000 hours

CHEMICAL INFORMATION:

Chemical Name: HYDDUFLOURIC ACID - 70 WT\% - SAH

Molecular Weight: $20.01 \mathrm{~kg} / \mathrm{kmol}$

TLV-TWA: -unavail-

IDLH: -unavail-

Footprint Level of Concern: 20 ppm

Boiling Point: $110.35^{\circ} \mathrm{C}$

Vapor Pressure at Ambient Temperature: $2.36 \mathrm{e}-004 \mathrm{~atm}$

Ambient Saturation Concentration: 240 ppm or $0.024 \%$

ATMOSPHERIC INFORMATION: (MANUAL INPUT OF DATA)

Wind: 1.7 meters $/ \mathrm{sec}$ from $90^{\circ}$ true

Inversion Height: 200 meters

Stability Class: E Air Temperature: $29^{\circ} \mathrm{C}$

Relative Humidity: 50\% Ground Roughness: 100 centimeters

Cloud Cover: 1 tenths

SOURCE STRENGTH INFORMATION:

Puddle Area: 24420 square feet

Average Puddle Depth: 1 centimeters

Soil Type: Default Ground Temperature: $29^{\circ} \mathrm{C}$

Initial Puddle Temperature: Ground temperature

Release Duration: ALOHA limited the duration to 1 hour

Max Computed Release Rate: 117 grams/min

Max Average Sustained Release Rate: 109 grams/min (averaged over a minute or more)

Total Amount Released: 5.97 kilograms

FOOTPRINT INFORMATION:

Dispersion Module: Gaussian

User specified LOC: $20 \mathrm{ppm}$

Max Threat Zone for LOC: 30 meters

Note: Footprint was not drawn because effects of near-field patchiness make plume presentation unreliable for short distances.

TIME DEPENDENT INFORMATION:

Concentration Estimates at the point:

Downwind: 100 meters

Off Centerline: 0 meters

Max Concentration:

Outdoor: $2.18 \mathrm{ppm}$

Indoor: $2.18 \mathrm{ppm}$

Note: Indoor graph is shown with a dotted line.
Reviewed by S. A. Henderson

Works Calculation 7

Sheet No. 128 of 577 


\section{ENGINEERING COMPUTATION SHEET}

Title of Project EPHA for THM

Reviewed by $\underline{\text { S. A. Henderson }}$

Subject Concentration Analysis for THM Hazardous Chemicals

Computer T. M. Franey

Date $\underline{09 / 16 / 96}$

SITE DATA INFORMATION: 1-RD-25

Location: AIKEN, SOUTH CAROLINA

Building Air Exchanges Per Hour: 60 (User specified)

Date and Time: Fixed at July 1, 19960000 hours

CHEMICAL INFORMATION:

Chemical Name: HYDDUFLOURIC ACID - 70 WT\% - SAH

Molecular Weight: $20.01 \mathrm{~kg} / \mathrm{kmol}$

TLV-TWA: -unavail- IDLH: -unavail-

Footprint Level of Concern: 20 ppm

Boiling Point: $110.35^{\circ} \mathrm{C}$

Vapor Pressure at Ambient Temperature: 2.36e-004 atm

Ambient Saturation Concentration: 240 ppm or $0.024 \%$

ATMOSPHERIC INFORMATION: (MANUAL INPUT OF DATA)

Wind: 1.7 meters $/ \mathrm{sec}$ from $90^{\circ}$ true

Inversion Height: 200 meters

Stability Class: E Air Temperature: $29^{\circ} \mathrm{C}$

Relative Humidity: $50 \%$ Ground Roughness: 100 centimeters

Cloud Cover: 1 tenths

SOURCE STRENGTH INFORMATION:

Puddle Area: 40700 square feet

Average Puddle Depth: 1 centimeters

Soil Type: Default Ground Temperature: $29^{\circ} \mathrm{C}$

Initial Puddle Temperature: Ground temperature

Release Duration: ALOHA limited the duration to 1 hour

Max Computed Release Rate: 189 grams/min

Max Average Sustained Release Rate: 176 grams/min (averaged over a minute or more)

Total Amount Released: 9.68 kilograms

FOOTPRINT INFORMATION:

Dispersion Module: Gaussian

User specified LOC: $20 \mathrm{ppm}$

Max Threat Zone for LOC: 38 meters

Note: Footprint was not drawn because effects of near-field patchiness make plume presentation unreliable for short distances.

TIME DEPENDENT INFORMATION:

Concentration Estimates at the point:

Downwind: 100 meters

Off Centerline: 0 meters

Max Concentration:

Outdoor: $3.15 \mathrm{ppm}$

Indoor: $3.15 \mathrm{ppm}$

Note: Indoor graph is shown with a dotted line.
Works Calculation 7

Sheet No. 129 of 577 
Title of Project EPHA for THM

Subject Concentration Analysis for THM Hazardous Chemicals

Computer T. M. Franey Date $\underline{09 / 16 / 96}$
Reviewed by $\mathrm{S}$. A. Henderson

Works Calculation 7

Sheet No. 130 of 577

SITE DATA INFORMATION: 1-RD-25

Location: AIKEN, SOUTH CAROLINA

Building Air Exchanges Per Hour: 60 (User specified)

Date and Time: Fixed at July 1, 19960000 hours

CHEMICAL INFORMATION:

Chemical Name: HYDDUFLOURIC ACID - 70 WT\% - SAH

Molecular Weight: $20.01 \mathrm{~kg} / \mathrm{kmol}$

TLV-TWA: -unavail- IDLH: -unavail-

Footprint Level of Concern: $20 \mathrm{ppm}$

Boiling Point: $110.35^{\circ} \mathrm{C}$

Vapor Pressure at Ambient Temperature: 2.36e-004 atm

Ambient Saturation Concentration: 240 ppm or $0.024 \%$

ATMOSPHERIC INFORMATION: (MANUAL INPUT OF DATA)

Wind: 1.7 meters $/ \mathrm{sec}$ from $90^{\circ}$ true

Inversion Height: 200 meters

Stability Class: E Air Temperature: $29^{\circ} \mathrm{C}$

Relative Humidity: $50 \%$ Ground Roughness: 100 centimeters

Cloud Cover: 1 tenths

SOURCE STRENGTH INFORMATION:

Puddle Area: 10000 square meters

Average Puddle Depth: 1 centimeters

Soil Type: Default Ground Temperature: $29^{\circ} \mathrm{C}$

Initial Puddle Temperature: Ground temperature

Release Duration: ALOHA limited the duration to 1 hour

Max Computed Release Rate: 476 grams/min

Max Average Sustained Release Rate: 443 grams/min

(averaged over a minute or more)

Total Amount Released: 24.3 kilograms

FOOTPRINT INFORMATION:

Dispersion Module: Gaussian

User specified LOC: $20 \mathrm{ppm}$

Max Threat Zone for LOC: 61 meters

Note: Footprint was not drawn because effects of near-field patchiness make plume

presentation unreliable for short distances.

TIME DEPENDENT INFORMATION:

Concentration Estimates at the point:

Downwind: 100 meters

Off Centerline: 0 meters

Max Concentration:

Outdoor: $7.69 \mathrm{ppm}$

Indoor: $7.69 \mathrm{ppm}$

Note: Indoor graph is shown with a dotted line. 
Title of Project EPHA for THM

Subject Concentration Analysis for THM Hazardous Chemicals

Computer T. M. Franey

Date $09 / 16 / 96$
Reviewed by S. A. Henderson

Works Calculation 7

Sheet No. 131 of 577

SITE DATA INFORMATION: 1-RD-26

Location: AIKEN, SOUTH CAROLINA

Building Air Exchanges Per Hour: 60 (User specified)

Date and Time: Fixed at July 1, 19960000 hours

CHEMICAL INFORMATION:

Chemical Name: HYDROGEN PEROXIDE

Molecular Weight: $34.01 \mathrm{~kg} / \mathrm{kmol}$

TLV-TWA: $1.00 \mathrm{ppm}$ IDLH: $75.00 \mathrm{ppm}$

Footprint Level of Concern: $10 \mathrm{ppm}$

Boiling Point: $150.20^{\circ} \mathrm{C}$

Vapor Pressure at Ambient Temperature: $0.0034 \mathrm{~atm}$

Ambient Saturation Concentration: 3,488 ppm or $0.35 \%$

ATMOSPHERIC INFORMATION: (MANUAL INPUT OF DATA)

Wind: 1.7 meters $/ \mathrm{sec}$ from $90^{\circ}$ true

Inversion Height: 200 meters

Stability Class: E Air Temperature: $29^{\circ} \mathrm{C}$

Relative Humidity: 50\% Ground Roughness: 100 centimeters

Cloud Cover: 1 tenths

\section{SOURCE STRENGTH INFORMATION:}

Puddle Area: 555 square feet

Average Puddle Depth: 1 centimeters

Soil Type: Default Ground Temperature: $29^{\circ} \mathrm{C}$

Initial Puddle Temperature: Ground temperature

Release Duration: ALOHA limited the duration to 1 hour

Max Computed Release Rate: 43.2 grams/min

Max Average Sustained Release Rate: 41.8 grams/min

(averaged over a minute or more)

Total Amount Released: 2.40 kilograms

FOOTPRINT INFORMATION:

Dispersion Module: Gaussian

User specified LOC: $10 \mathrm{ppm}$

Max Threat Zone for LOC: 30 meters

Max Threat Zone for IDLH: less than 10 meters(10.9 yards)

Note: Footprint was not drawn because effects of near-field patchiness make plume

presentation unreliable for short distances.

\section{TIME DEPENDENT INFORMATION:}

Concentration Estimates at the point:

Downwind: 30 meters

Off Centerline: 0 meters

Max Concentration:

Outdoor: $10 \mathrm{ppm}$

Indoor: $10 \mathrm{ppm}$

Note: Indoor graph is shown with a dotted line. 


\section{ENGINEERING COMPUTATION SHEET}

Title of Project EPHA for THM

Subject Concentration Analysis for THM Hazardous Chemicals

Computer T.M. Franey Date $\underline{09 / 16 / 96}$

SITE DATA INFORMATION: 1-RD-26

Location: AIKEN, SOUTH CAROLINA

Building Air Exchanges Per Hour: 60 (User specified)

Date and Time: Fixed at July 1, 19960000 hours

\section{CHEMICAL INFORMATION:}

Chemical Name: HYDROGEN PEROXIDE

Molecular Weight: $34.01 \mathrm{~kg} / \mathrm{kmol}$

TLV-TWA: $1.00 \mathrm{ppm}$ IDLH: $75.00 \mathrm{ppm}$

Footprint Level of Concern: $10 \mathrm{ppm}$

Boiling Point: $150.20^{\circ} \mathrm{C}$

Vapor Pressure at Ambient Temperature: $0.0034 \mathrm{~atm}$

Ambient Saturation Concentration: $3,488 \mathrm{ppm}$ or $0.35 \%$

\section{ATMOSPHERIC INFORMATION: (MANUAL INPUT OF DATA)}

Wind: 1.7 meters $/ \mathrm{sec}$ from $90^{\circ}$ true

Inversion Height: 200 meters

Stability Class: E Air Temperature: $29^{\circ} \mathrm{C}$

Relative Humidity: 50\% Ground Roughness: 100 centimeters

Cloud Cover: 1 tenths

\section{SOURCE STRENGTH INFORMATION;}

Puddle Area: 7500 square feet

Average Puddle Depth: 1 centimeters

Soil Type: Default Ground Temperature: $29^{\circ} \mathrm{C}$

Initial Puddle Temperature: Ground temperature

Release Duration: ALOHA limited the duration to 1 hour

Max Computed Release Rate: 503 grams/min

Max Average Sustained Release Rate: 488 grams/min

(averaged over a minute or more)

Total Amount Released: 28.0 kilograms

\section{FOOTPRINT INFORMATION:}

Dispersion Module: Gaussian

User specified LOC: $10 \mathrm{ppm}$

Max Threat Zone for LOC: 100 meters

Max Threat Zone for IDLH: 25 meters

Note: Footprint was not drawn because effects of near-field patchiness make plume presentation unreliable for short distances.

\section{TIME DEPENDENT INFORMATION:}

Concentration Estimates at the point:

Downwind: 100 meters

Off Centerline: 0 meters

Max Concentration:

Outdoor: $10 \mathrm{ppm}$

Indoor: $10 \mathrm{ppm}$

Note: Indoor graph is shown with a dotted line.
Reviewed by S. A. Henderson

Works Calculation 7

Sheet No. 132 of 577 


\section{ENGINEERING COMPUTATION SHEET}

Title of Project EPHA for THM

Reviewed by $\mathrm{S}$. A. Henderson

Subject Concentration Analysis for THM Hazardous Chemicals

Computer T.M. Franey

Date $\underline{09 / 16 / 96}$
Works Calculation 7

Sheet Nò. 133 of 577
SITE DATA INFORMATION: 1-RD-26
Location: AIKEN, SOUTH CAROLINA
Building Air Exchanges Per Hour: 60 (User specified)
Date and Time: Fixed at July 1, 19960000 hours

CHEMICAL INFORMATION:

Chemical Name: HYDROGEN PEROXIDE

Molecular Weight: $34.01 \mathrm{~kg} / \mathrm{kmol}$

TLV-TWA: $1.00 \mathrm{ppm}$

IDLH: $75.00 \mathrm{ppm}$

Footprint Level of Concern: $10 \mathrm{ppm}$

Boiling Point: $150.20^{\circ} \mathrm{C}$

Vapor Pressure at Ambient Temperature: 0.0034 atm

Ambient Saturation Concentration: 3,488 ppm or $0.35 \%$

ATMOSPHERIC INFORMATION: (MANUAL INPUT OF DATA)

Wind: 1.7 meters $/ \mathrm{sec}$ from $90^{\circ}$ true

Inversion Height: 200 meters

Stability Class: E Air Temperature: $29^{\circ} \mathrm{C}$

Relative Humidity: $50 \%$ Ground Roughness: 100 centimeters

Cloud Cover: 1 tenths

SOURCE STRENGTH INFORMATION:

Puddle Area: 24420 square feet

Average Puddle Depth: 1 centimeters

Soil Type: Default Ground Temperature: $29^{\circ} \mathrm{C}$

Initial Puddle Temperature: Ground temperature

Release Duration: ALOHA limited the duration to 1 hour

Max Computed Release Rate: 1.54 kilograms/min

Max Average Sustained Release Rate: 1.49 kilograms/min

(averaged over a minute or more)

Total Amount Released: 85.8 kilograms

FOOTPRINT INFORMATION:

Dispersion Module: Gaussian

User specified LOC: $10 \mathrm{ppm}$

Max Threat Zone for LOC: 179 meters

Max Threat Zone for IDLH: 44 meters

\section{TIME DEPENDENT INFORMATION:}

Concentration Estimates at the point:

Downwind: 100 meters

Off Centerline: 0 meters

Max Concentration:

Outdoor: $17.7 \mathrm{ppm}$

Indoor: $17.7 \mathrm{ppm}$

Note: Indoor graph is shown with a dotted line. 
Title of Project EPHA for THM

Subject Concentration Analysis for THM Hazardous Chemicals

Computer T. M. Franey

Date $09 / 16 / 96$

SITE DATA INFORMATION: 1-RD-26

Location: AIKEN, SOUTH CAROLINA

Building Air Exchanges Per Hour: 60 (User specified)

Date and Time: Fixed at July 1, 19960000 hours

CHEMICAL INFORMATION:

Chemical Name: HYDROGEN PEROXIDE

Molecular Weight: $34.01 \mathrm{~kg} / \mathrm{kmol}$

TLV-TWA: $1.00 \mathrm{ppm}$ IDLH: $75.00 \mathrm{ppm}$

Footprint Level of Concern: $10 \mathrm{ppm}$

Boiling Point: $150.20^{\circ} \mathrm{C}$

Vapor Pressure at Ambient Temperature: $0.0034 \mathrm{~atm}$

Ambient Saturation Concentration: 3,488 ppm or $0.35 \%$

ATMOSPHERIC INFORMATION: (MANUAL INPUT OF DATA)

Wind: 1.7 meters $/ \mathrm{sec}$ from $90^{\circ}$ true

Inversion Height: 200 meters

Stability Class: E Air Temperature: $29^{\circ} \mathrm{C}$

Relative Humidity: 50\% Ground Roughness: 100 centimeters

Cloud Cover: 1 tenths

SOURCE STRENGTH INFORMATION:

Puddle Area: 40700 square feet

Average Puddle Depth: 1 centimeters

Soil Type: Default Ground Temperature: $29^{\circ} \mathrm{C}$

Initial Puddle Temperature: Ground temperature

Release Duration: ALOHA limited the duration to 1 hour

Max Computed Release Rate: 2.51 kilograms/min

Max Average Sustained Release Rate: 2.43 kilograms/min

(averaged over a minute or more)

Total Amount Released: 139 kilograms

FOOTPRINT INFORMATION:

Dispersion Module: Gaussian

User specified LOC: $10 \mathrm{ppm}$

Max Threat Zone for LOC: 232 meters

Max Threat Zone for IDLH: 56 meters

TIME DEPENDENT INFORMATION:

Concentration Estimates at the point:

Downwind: 100 meters

Off Centerline: 0 meters

Max Concentration:

Outdoor: $25.5 \mathrm{ppm}$

Indoor: $25.5 \mathrm{ppm}$

Note: Indoor graph is shown with a dotted line.
Reviewed by $\underline{\text { S. A. Henderson }}$

Works Calculation 7

Sheet No. 134 of 577 
Title of Project EPHA for THM

Subject Concentration Analysis for THM Hazardous Chemicals

Computer T.M. Franey

Date $\underline{09 / 16 / 96}$

\section{SITE DATA INFORMATION: 1-RD-26}

Location: AIKEN, SOUTH CAROLINA

Building Air Exchanges Per Hour: 60 (User specified)

Date and Time: Fixed at July 1, 19960000 hours

\section{CHEMICAL INFORMATION:}

Chemical Name: HYDROGEN PEROXIDE

Molecular Weight: $34.01 \mathrm{~kg} / \mathrm{kmol}$

TLV-TWA: $1.00 \mathrm{ppm}$ IDLH: $75.00 \mathrm{ppm}$

Footprint Level of Concern: $10 \mathrm{ppm}$

Boiling Point: $150.20^{\circ} \mathrm{C}$

Vapor Pressure at Ambient Temperature: $0.0034 \mathrm{~atm}$

Ambient Saturation Concentration: 3,488 ppm or $0.35 \%$

\section{ATMOSPHERIC INFORMATION: (MANUAL INPUT OF DATA)}

Wind: 1.7 meters $/ \mathrm{sec}$ from $90^{\circ}$ true

Inversion Height: 200 meters

Stability Class: E Air Temperature: $29^{\circ} \mathrm{C}$

Relative Humidity: 50\% Ground Roughness: 100 centimeters

Cloud Cover: 1 tenths

\section{SOURCE STRENGTH INFORMATION:}

Puddle Area: 10000 square meters

Average Puddle Depth: 1 centimeters

Soil Type: Defauit Ground Temperature: $29^{\circ} \mathrm{C}$

Initial Puddle Temperature: Ground temperature

Release Duration: ALOHA limited the duration to 1 hour

Max Computed Release Rate: 6.33 kilograms/min

Max Average Sustained Release Rate: 6.13 kilograms/min

(averaged over a minute or more)

Total Amount Released: 352 kilograms

\section{FOOTPRINT INFORMATION:}

Dispersion Module: Gaussian

User specified LOC: $10 \mathrm{ppm}$

Max Threat Zone for LOC: 390 meters

Max Threat Zone for IDLH: 91 meters

\section{TIME DEPENDENT INFORMATION:}

Concentration Estimates at the point:

Downwind: 100 meters

Off Centerline: 0 meters

Max Concentration:

Outdoor: $62.7 \mathrm{ppm}$

Indoor: $62.7 \mathrm{ppm}$

Note: Indoor graph is shown with a dotted line.
Reviewed by S. A. Henderson

Works Calculation 7

Sheet No. $\underline{135 \text { of } 577}$ 


\section{ENGINEERING COMPUTATION SHEET}

Title of Project EPHA for THM

Subject Concentration Analysis for THM Hazardous Chemicals

Computer T. M. Franey Date $\underline{09 / 16 / 96}$
Reviewed by $\mathrm{S}$. A. Henderson

Works Calculation 7

Sheet No. 136 of 577

SITE DATA INFORMATION: 1-RD-27

Location: AIKEN, SOUTH CAROLINA

Building Air Exchanges Per Hour: 60 (User specified)

Date and Time: Fixed at July 1, 19960000 hours

CHEMICAL INFORMATION:

Chemical Name: ISOPROPANOL

Molecular Weight: $60.10 \mathrm{~kg} / \mathrm{kmol}$

TLV-TWA: $400.00 \mathrm{ppm}$ IDLH: $12000.00 \mathrm{ppm}$

Footprint Level of Concern: 400 ppm

Boiling Point: $82.26^{\circ} \mathrm{C}$

Vapor Pressure at Ambient Temperature: $0.076 \mathrm{~atm}$

Ambient Saturation Concentration: $77,490 \mathrm{ppm}$ or $7.75 \%$

ATMOSPHERIC INFORMATION: (MANUAL INPUT OF DATA)

Wind: 1.7 meters $/ \mathrm{sec}$ from $90^{\circ}$ true

Inversion Height: 200 meters

Stability Class: E Air Temperature: $29^{\circ} \mathrm{C}$

Relative Humidity: 50\% Ground Roughness: 100 centimeters

Cloud Cover: 1 tenths

\section{SOURCE STRENGTH INFORMATION:}

Puddle Area: 848 square feet

Average Puddle Depth: 1 centimeters

Soil Type: Default Ground Temperature: $29^{\circ} \mathrm{C}$

Initial Puddle Temperature: Ground temperature

Release Duration: ALOHA limited the duration to 1 hour

Max Computed Release Rate: 4.43 kilograms/min

Max Average Sustained Release Rate: 3.64 kilograms/min

(averaged over a minute or more)

Total Amount Released: 182 kilograms

\section{FOOTPRINT INFORMATION:}

Dispersion Module: Gaussian

User specified LOC: $400 \mathrm{ppm}$

Max Threat Zone for LOC: 30 meters

Max Threat Zone for IDLH: less than 10 meters(10.9 yards)

Note: Footprint was not drawn because

effects of near-field patchiness make plume

presentation unreliable for short distances.

\section{TIME DEPENDENT INFORMATION:}

Concentration Estimates at the point:

Downwind: 30 meters

Off Centerline: 0 meters

Max Concentration:

Outdoor: $400 \mathrm{ppm}$

Indoor: $400 \mathrm{ppm}$

Note: Indoor graph is shown with a dotted line. 


\section{ENGINEERING COMPUTATION SHEET}

Title of Project EPHA for THM

Subject Concentration Analysis for THM Hazardous Chemicals

Computer T. M. Franey

Date 09/16/96

SITE DATA INFORMATION: 1-RD-27

Location: AIKEN, SOUTH CAROLINA

Building Air Exchanges Per Hour: 60 (User specified)

Date and Time: Fixed at July 1, 19960000 hours

CHEMICAL INFORMATION:

Chemical Name: ISOPROPANOL

Molecular Weight: $60.10 \mathrm{~kg} / \mathrm{kmol}$

TLV-TWA: $400.00 \mathrm{ppm}$ IDLH: $12000.00 \mathrm{ppm}$

Footprint Level of Concern: 400 ppm

Boiling Point: $82.26^{\circ} \mathrm{C}$

Vapor Pressure at Ambient Temperature: $0.076 \mathrm{~atm}$

Ambient Saturation Concentration: $77,490 \mathrm{ppm}$ or $7.75 \%$

ATMOSPHERIC INFORMATION: (MANUAL INPUT OF DATA)

Wind: 1.7 meters $/ \mathrm{sec}$ from $90^{\circ}$ true

Inversion Height: 200 meters

Stability Class: E Air Temperature: $29^{\circ} \mathrm{C}$

Relative Humidity: 50\% Ground Roughness: 100 centimeters

Cloud Cover: 1 tenths

SOURCE STRENGTH INFORMATION:

Puddle Area: 4930 square feet

Average Puddle Depth: 1 centimeters

Soil Type: Default Ground Temperature: $29^{\circ} \mathrm{C}$

Initial Puddle Temperature: Ground temperature

Release Duration: ALOHA limited the duration to 1 hour

Max Computed Release Rate: 23.5 kilograms/min

Max Average Sustained Release Rate: 19.6 kilograms/min

(averaged over a minute or more)

Total Amount Released: 988 kilograms

FOOTPRINT INFORMATION:

Model Run: Heavy Gas

User specified LOC: 400 ppm

Max Threat Zone for LOC: 97 meters

Max Threat Zone for IDLH: IDLH level was never exceeded

Note: Footprint wasn't drawn because effects of near-field patchiness make plume presentation unreliable for short distances.

\section{TIME DEPENDENT INFORMATION:}

Concentration Estimates at the point:

Downwind: 100 meters

Off Centerline: 0 meters

Max Concentration:

Outdoor: $400 \mathrm{ppm}$

Indoor: $400 \mathrm{ppm}$

Note: Indoor graph is shown with a dotted line.
Reviewed by $\underline{S}$. A. Henderson

Works Calculation 7

Sheet No. 137 of 577 


\section{ENGINEERING COMPUTATION SHEET}

Title of Project EPHA for THM

Reviewed by S. A. Henderson

Subject Concentration Analysis for THM Hazardous Chemicals

Computer T.M. Franey

Date $\underline{09 / 16 / 96}$

SITE DATA INFORMATION: 1-RD-27

Location: AIKEN, SOUTH CAROLINA

Building Air Exchanges Per Hour: 60 (User specified)

Date and Time: Fixed at July 1, 19960000 hours

CHEMICAL INFORMATION:

Chemical Name: ISOPROPANOL

Molecular Weight: $60.10 \mathrm{~kg} / \mathrm{kmol}$

TLV-TWA: $400.00 \mathrm{ppm}$ IDLH: $12000.00 \mathrm{ppm}$

Footprint Level of Concern: 400 ppm

Boiling Point: $82.26^{\circ} \mathrm{C}$

Vapor Pressure at Ambient Temperature: $0.076 \mathrm{~atm}$

Ambient Saturation Concentration: 77,490 ppm or $7.75 \%$

\section{ATMOSPHERIC INFORMATION: (MANUAL INPUT OF DATA)}

Wind: 1.7 meters $/ \mathrm{sec}$ from $90^{\circ}$ true

Inversion Height: 200 meters

Stability Class: E Air Temperature: $29^{\circ} \mathrm{C}$

Relative Humidity: 50\% Ground Roughness: 100 centimeters

Cloud Cover: 1 tenths

\section{SOURCE STRENGTH INFORMATION:}

Puddle Area: 24420 square feet

Average Puddle Depth: 1 centimeters

Soil Type: Default Ground Temperature: $29^{\circ} \mathrm{C}$

Initial Puddle Temperature: Ground temperature

Release Duration: ALOHA limited the duration to 1 hour

Max Computed Release Rate: 108 kilograms/min

Max Average Sustained Release Rate: 90.9 kilograms/min

(averaged over a minute or more)

Total Amount Released: 4,610 kilograms

FOOTPRINT INFORMATION:

Model Run: Heavy Gas

User specified LOC: $400 \mathrm{ppm}$

Max Threat Zone for LOC: 217 meters

Max Threat Zone for IDLH: IDLH level was never exceeded

Note: The Heavy Gas footprint is an initial screening.

For short releases it may be an overestimation.

Be sure to check concentration information at specific locations.

\section{TIME DEPENDENT INFORMATION:}

Concentration Estimates at the point:

Downwind: 100 meters

Off Centerline: 0 meters

Max Concentration:

Outdoor: $1,370 \mathrm{ppm}$

Indoor: $1,370 \mathrm{ppm}$

Note: Indoor graph is shown with a dotted line.
Works Calculation 7

Sheet No. 138 of 577 


\section{ENGINEERING COMPUTATION SHEET}

Title of Project EPHA for THM

Subject Concentration Analysis for THM Hazardous Chemicals

Computer T. M. Franey

Date 09/16/96
Reviewed by S.A. Henderson

Works Calculation 7

Sheet No. 139 of 577

SITE DATA INFORMATION: 1-RD-27

Location: AIKEN, SOUTH CAROLINA

Building Air Exchanges Per Hour: 60 (User specified)

Date and Time: Fixed at July 1, 19960000 hours

CHEMICAL INFORMATION:

Chemical Name: ISOPROPANOL

Molecular Weight: $60.10 \mathrm{~kg} / \mathrm{kmol}$

TLV-TWA: $400.00 \mathrm{ppm}$ IDLH: $12000.00 \mathrm{ppm}$

Footprint Level of Concern: 400 ppm

Boiling Point: $82.26^{\circ} \mathrm{C}$

Vapor Pressure at Ambient Temperature: $0.076 \mathrm{~atm}$

Ambient Saturation Concentration: 77,490 ppm or $7.75 \%$

ATMOSPHERIC INFORMATION: (MANUAL INPUT OF DATA)

Wind: 1.7 meters $/ \mathrm{sec}$ from $90^{\circ}$ true

Inversion Height: 200 meters

Stability Class: E Air Temperature: $29^{\circ} \mathrm{C}$

Relative Humidity: 50\% Ground Roughness: 100 centimeters

Cloud Cover: 1 tenths

\section{SOURCE STRENGTH INFORMATION:}

Puddle Area: 40700 square feet

Average Puddle Depth: 1 centimeters

Soil Type: Default Ground Temperature: $29^{\circ} \mathrm{C}$

Initial Puddle Temperature: Ground temperature

Release Duration: ALOHA limited the duration to 1 hour

Max Computed Release Rate: 176 kilograms/min

Max Average Sustained Release Rate: 148 kilograms/min

(averaged over a minute or more)

Total Amount Released: 7,543 kilograms

FOOTPRINT INFORMATION:

Model Run: Heavy Gas

User specified LOC: $400 \mathrm{ppm}$

Max Threat Zone for LOC: 282 meters

Max Threat Zone for IDLH: IDLH level was never exceeded

Note: The Heavy Gas footprint is an initial screening.

For short releases it may be an overestimation.

Be sure to check concentration information at specific locations.

\section{TIME DEPENDENT INFORMATION:}

Concentration Estimates at the point:

Downwind: 100 meters

Off Centerline: 0 meters

Max Concentration:

Outdoor: $2,030 \mathrm{ppm}$

Indoor: $2,030 \mathrm{ppm}$

Note: Indoor graph is shown with a dotted line. 
Title of Project EPHA for THM

Subject Concentration Analysis for THM Hazardous Chemicals

Computer T. M. Franey
Date $\underline{09 / 16 / 96}$
Reviewed by $\underline{S}$. A. Henderson

Works Calculation 7

Sheet No. 140 of 577

SITE DATA INFORMATION: I-RD-27

Location: AIKEN, SOUTH CAROLINA

Building Air Exchanges Per Hour: 60 (User specified)

Date and Time: Fixed at July 1, 19960000 hours

\section{CHEMICAL INFORMATION:}

Chemical Name: ISOPROPANOL

Molecular Weight: $60.10 \mathrm{~kg} / \mathrm{kmol}$

TLV-TWA: $400.00 \mathrm{ppm}$ IDLH: $12000.00 \mathrm{ppm}$

Footprint Level of Concern: $400 \mathrm{ppm}$

Boiling Point: $82.26^{\circ} \mathrm{C}$

Vapor Pressure at Ambient Temperature: $0.076 \mathrm{~atm}$

Ambient Saturation Concentration: 77,490 ppm or $7.75 \%$

ATMOSPHERIC INFORMATION: (MANUAL INPUT OF DATA)

Wind: 1.7 meters $/ \mathrm{sec}$ from $90^{\circ}$ true

Inversion Height: 200 meters

Stability Class: E Air Temperature: $29^{\circ} \mathrm{C}$

Relative Humidity: 50\% Ground Roughness: 100 centimeters

Cloud Cover: 1 tenths

\section{SOURCE STRENGTH INFORMATION:}

Puddle Area: 10000 square meters

Average Puddle Depth: 1 centimeters

Soil Type: Default Ground Temperature: $29^{\circ} \mathrm{C}$

Initial Puddle Temperature: Ground temperature

Release Duration: ALOHA limited the duration to 1 hour

Max Computed Release Rate: 446 kilograms/min

Max Average Sustained Release Rate: 378 kilograms/min

(averaged over a minute or more)

Total Amount Released: 19,280 kilograms

\section{FOOTPRINT INFORMATION:}

Model Run: Heavy Gas

User specified LOC: $400 \mathrm{ppm}$

Max Threat Zone for LOC: 468 meters

Max Threat Zone for IDLH: IDLH level was never exceeded

Note: The Heavy Gas footprint is an initial screening.

For short releases it may be an overestimation.

Be sure to check concentration information at specific locations.

\section{TIME DEPENDENT INFORMATION:}

Concentration Estimates at the point:

Downwind: 100 meters

Off Centerline: 0 meters

Max Concentration:

Outdoor: $4,210 \mathrm{ppm}$

Indoor: $4,210 \mathrm{ppm}$

Note: Indoor graph is shown with a dotted line. 
Title of Project EPHA for THM

Subject Concentration Analysis for THM Hazardous Chemicals

Computer T. M. Franey Date $\underline{09 / 16 / 96}$
Reviewed by $\underline{\text { S. A. Henderson }}$

Works Calculation 7

Sheet No. 141 of 577

SITE DATA INFORMATION: 1-RD-28

Location: AIKEN, SOUTH CAROLINA

Building Air Exchanges Per Hour: 60 (User specified)

Date and Time: Fixed at July 1, 19960000 hours

CHEMICAL INFORMATION:

Chemical Name: METHYL ALCOHOL

Molecular Weight: $32.04 \mathrm{~kg} / \mathrm{kmol}$

TLV-TWA: $200.00 \mathrm{ppm}$ IDLH: $25000.00 \mathrm{ppm}$

Footprint Level of Concern: 1000 ppm

Boiling Point: $64.70^{\circ} \mathrm{C}$

Vapor Pressure at Ambient Temperature: $0.20 \mathrm{~atm}$

Ambient Saturation Concentration: 207,481 ppm or $20.7 \%$

ATMOSPHERIC INFORMATION: (MANUAL INPUT OF DATA)

Wind: 1.7 meters $/ \mathrm{sec}$ from $90^{\circ}$ true

Inversion Height: 200 meters

Stability Class: E Air Temperature: $29^{\circ} \mathrm{C}$

Relative Humidity: 50\% Ground Roughness: 100 centimeters

Cloud Cover: 1 tenths

SOURCE STRENGTH INFORMATION:

Puddle Area: 1115 square feet

Average Puddle Depth: 1 centimeters

Soil Type: Default Ground Temperature: $29^{\circ} \mathrm{C}$

Initial Puddle Temperature: Ground temperature

Release Duration: ALOHA limited the duration to 1 hour

Max Computed Release Rate: 7.8 kilograms/min

Max Average Sustained Release Rate: 5.56 kilograms/min

(averaged over a minute or more)

Total Amount Released: 253 kilograms

FOOTPRINT INFORMATION:

Dispersion Module: Gaussian

User specified LOC: $1000 \mathrm{ppm}$

Max Threat Zone for LOC: 30 meters

Max Threat Zone for IDLH: less than 10 meters(10.9 yards)

Note: Footprint was not drawn because effects of near-field patchiness make plume presentation unreliable for short distances.

TIME DEPENDENT INFORMATION:

Concentration Estimates at the point:

Downwind: 30 meters

Off Centerline: 0 meters

Max Concentration:

Outdoor: $1,000 \mathrm{ppm}$

Indoor: $1,000 \mathrm{ppm}$

Note: Indoor graph is shown with a dotted line. 
Title of Project EPHA for THM

Subject Concentration Analysis for THM Hazardous Chemicals

Computer T. M. Franey Date $\underline{09 / 16 / 96}$

SITE DATA INFORMATION: 1-RD-28

Location: AIKEN, SOUTH CAROLINA

Building Air Exchanges Per Hour: 60 (User specified)

Date and Time: Fixed at July 1, 19960000 hours

CHEMICAL INFORMATION:

Chemical Name: METHYL ALCOHOL

Molecular Weight: $32.04 \mathrm{~kg} / \mathrm{kmol}$

TLV-TWA: $200.00 \mathrm{ppm}$ IDLH: $25000.00 \mathrm{ppm}$

Footprint Level of Concern: 1000 ppm

Boiling Point: $64.70^{\circ} \mathrm{C}$

Vapor Pressure at Ambient Temperature: $0.20 \mathrm{~atm}$

Ambient Saturation Concentration: 207,481 ppm or $20.7 \%$

ATMOSPHERIC INFORMATION: (MANUAL INPUT OF DATA)

Wind: 1.7 meters $/ \mathrm{sec}$ from $90^{\circ}$ true

Inversion Height: 200 meters

Stability Class: E Air Temperature: $29^{\circ} \mathrm{C}$

Relative Humidity: 50\% Ground Roughness: 100 centimeters

Cloud Cover: 1 tenths

\section{SOURCE STRENGTH INFORMATION:}

Puddle Area: 14000 square feet

Average Puddle Depth: 1 centimeters

Soil Type: Default Ground Temperature: $29^{\circ} \mathrm{C}$

Initial Puddle Temperature: Ground temperature

Release Duration: ALOHA limited the duration to 1 hour

Max Computed Release Rate: 85.1 kilograms/min

Max Average Sustained Release Rate: 62.5 kilograms/min

(averaged over a minute or more)

Total Amount Released: 2,890 kilograms

\section{FOOTPRINT INFORMATION:}

Dispersion Module: Gaussian

User specified LOC: $1000 \mathrm{ppm}$

Max Threat Zone for LOC: 100 meters

Max Threat Zone for IDLH: 20 meters

Note: Footprint was not drawn because effects of near-field patchiness make plume presentation unreliable for short distances.

\section{TIME DEPENDENT INFORMATION:}

Concentration Estimates at the point:

Downwind: 100 meters

Off Centerline: 0 meters

Max Concentration:

Outdoor: 1,000 ppm

Indoor: $1,000 \mathrm{ppm}$

Note: Indoor graph is shown with a dotted line. 
Title of Project EPHA for THM

Subject Concentration Analysis for THM Hazardous Chemicals

Computer T.M. Franey

Date $09 / 16 / 96$
Reviewed by $\underline{S}$. A. Henderson

Works Calculation 7

Sheet No. 143 of 577

SITE DATA INFORMATION: 1-RD-28

Location: AIKEN, SOUTH CAROLINA

Building Air Exchanges Per Hour: 60 (User specified)

Date and Time: Fixed at July 1, 19960000 hours

\section{CHEMICAL INFORMATION:}

Chemical Name: METHYL ALCOHOL

Molecular Weight: $32.04 \mathrm{~kg} / \mathrm{kmol}$

TLV-TWA: $200.00 \mathrm{ppm}$ IDLH: $25000.00 \mathrm{ppm}$

Footprint Level of Concern: $1000 \mathrm{ppm}$

Boiling Point: $64.70^{\circ} \mathrm{C}$

Vapor Pressure at Ambient Temperature: $0.20 \mathrm{~atm}$

Ambient Saturation Concentration: 207,481 ppm or 20.7\%

ATMOSPHERIC INFORMATION: (MANUAL INPUT OF DATA)

Wind: 1.7 meters $/ \mathrm{sec}$ from $90^{\circ}$ true

Inversion Height: 200 meters

Stability Class: E Air Temperature: $29^{\circ} \mathrm{C}$

Relative Humidity: $50 \%$ Ground Roughness: 100 centimeters

Cloud Cover: 1 tenths

\section{SOURCE STRENGTH INFORMATION:}

Puddle Area: 24420 square feet

Average Puddle Depth: 1 centimeters

Soil Type: Default Ground Temperature: $29^{\circ} \mathrm{C}$

Initial Puddle Temperature: Ground temperature

Release Duration: ALOHA limited the duration to 1 hour

Max Computed Release Rate: 144 kilograms/min

Max Average Sustained Release Rate: 107 kilograms/min

(averaged over a minute or more)

Total Amount Released: 4,944 kilograms

\section{FOOTPRINT INFORMATION:}

Dispersion Module: Gaussian

User specified LOC: $1000 \mathrm{ppm}$

Max Threat Zone for LOC: 132 meters

Max Threat Zone for IDLH: 27 meters

\section{TIME DEPENDENT INFORMATION:}

\section{Concentration Estimates at the point:}

Downwind: 100 meters

Off Centerline: 0 meters

Max Concentration:

Outdoor: 1,340 ppm

Indoor: $1,340 \mathrm{ppm}$

Note: Indoor graph is shown with a dotted line. 


\section{ENGINEERING COMPUTATION SHEET}

Title of Project EPHA for THM

Reviewed by $\underline{\text { S. A. Henderson }}$

Subject Concentration Analysis for THM Hazardous Chemicals

Computer T.M. Franey

Date 09/16/96

SITE DATA INFORMATION: 1-RD-28

Location: AIKEN, SOUTH CAROLINA

Building Air Exchanges Per Hour: 60 (User specified)

Date and Time: Fixed at July 1, 19960000 hours

CHEMICAL INFORMATION:

Chemical Name: METHYL ALCOHOL

Molecular Weight: $32.04 \mathrm{~kg} / \mathrm{kmol}$

TLV-TWA: $200.00 \mathrm{ppm}$ IDLH: $25000.00 \mathrm{ppm}$

Footprint Level of Concern: $1000 \mathrm{ppm}$

Boiling Point: $64.70^{\circ} \mathrm{C}$

Vapor Pressure at Ambient Temperature: 0.20 atm

Ambient Saturation Concentration: 207,481 ppm or 20.7\%

ATMOSPHERIC INFORMATION: (MANUAL INPUT OF DATA)

Wind: 1.7 meters $/ \mathrm{sec}$ from $90^{\circ}$ true

Inversion Height: 200 meters

Stability Class: E Air Temperature: $29^{\circ} \mathrm{C}$

Relative Humidity: 50\% Ground Roughness: 100 centimeters

Cloud Cover: 1 tenths

\section{SOURCE STRENGTH INFORMATION:}

Puddle Area: 40700 square feet

Average Puddle Depth: 1 centimeters

Soil Type: Default Ground Temperature: $29^{\circ} \mathrm{C}$

Initial Puddle Temperature: Ground temperature

Release Duration: ALOHA limited the duration to 1 hour

Max Computed Release Rate: 234 kilograms/min

Max Average Sustained Release Rate: 174 kilograms/min (averaged over a minute or more)

Total Amount Released: 8,096 kilograms

FOOTPRINT INFORMATION:

Model Run: Heavy Gas

User specified LOC: $1000 \mathrm{ppm}$

Max Threat Zone for LOC: 253 meters

Max Threat Zone for IDLH: IDLH level was never exceeded

Note: The Heavy Gas footprint is an initial screening.

For short releases it may be an overestimation.

Be sure to check concentration information at specific locations.

\section{TIME DEPENDENT INFORMATION:}

Concentration Estimates at the point:

Downwind: 100 meters

Off Centerline: 0 meters

Max Concentration:

Outdoor: 4,080 ppm

Indoor: $4,080 \mathrm{ppm}$

Note: Indoor graph is shown with a dotted line.
Works Calculation 7

Sheet No. 144 of 577 


\section{ENGINEERING COMPUTATION SHEET}

Title of Project EPHA for THM

Reviewed by $\mathrm{S}$. A. Henderson

Subject Concentration Analysis for THM Hazardous Chemicals

Computer T. M. Franey

Date $\underline{09 / 16 / 96}$
Works Calculation 7

Sheet No. 145 of 577

\section{SITE DATA INFORMATION: $\quad$ 1-RD-28}

Location: AIKEN, SOUTH CAROLINA

Building Air Exchanges Per Hour: 60 (User specified)

Date and Time: Fixed at July 1, 19960000 hours

CHEMICAL INFORMATION:

Chemical Name: METHYL ALCOHOL

Molecular Weight: $32.04 \mathrm{~kg} / \mathrm{kmol}$

TLV-TWA: $200.00 \mathrm{ppm}$ IDLH: $25000.00 \mathrm{ppm}$

Footprint Level of Concern: $1000 \mathrm{ppm}$

Boiling Point: $64.70^{\circ} \mathrm{C}$

Vapor Pressure at Ambient Temperature: $0.20 \mathrm{~atm}$

Ambient Saturation Concentration: 207,481 ppm or 20.7\%

ATMOSPHERIC INFORMATION: (MANUAL INPUT OF DATA)

Wind: 1.7 meters $/ \mathrm{sec}$ from $90^{\circ}$ true

Inversion Height: 200 meters

Stability Class: E Air Temperature: $29^{\circ} \mathrm{C}$

Relative Humidity: 50\% Ground Roughness: 100 centimeters

Cloud Cover: 1 tenths

\section{SOURCE STRENGTH INFORMATION:}

Puddle Area: 10000 square meters

Average Puddle Depth: 1 centimeters

Soil Type: Default Ground Temperature: $29^{\circ} \mathrm{C}$

Initial Puddle Temperature: Ground temperature

Release Duration: ALOHA limited the duration to 1 hour

Max Computed Release Rate: 591 kilograms/min

Max Average Sustained Release Rate: 444 kilograms/min (averaged over a minute or more)

Total Amount Released: 20,724 kilograms

\section{FOOTPRINT INFORMATION:}

Model Run: Heavy Gas

User specified LOC: $1000 \mathrm{ppm}$

Max Threat Zone for LOC: 422 meters

Max Threat Zone for IDLH: IDLH level was never exceeded

Note: The Heavy Gas footprint is an initial screening.

For short releases it may be an overestimation.

Be sure to check concentration information at specific locations.

\section{TIME DEPENDENT INFORMATION:}

Concentration Estimates at the point:

Downwind: 100 meters

Off Centerline: 0 meters

Max Concentration:

Outdoor: 7,640 ppm

Indoor: 7,640 ppm

Note: Indoor graph is shown with a dotted line. 
Title of Project EPHA for THM

Subject Concentration Analysis for THM Hazardous Chemicals

Computer T. M. Franey
Reviewed by S. A. Henderson

Works Calculation 7

Sheet No. 146 of 577

\section{SITE DATA INFORMATION: 1-RD-29 \\ Location: AIKEN, SOUTH CAROLINA \\ Building Air Exchanges Per Hour: 60 (User specified) \\ Date and Time: Fixed at July 1, 19960000 hours}

\section{CHEMICAL INFORMATION:}

Chemical Name: METHYL ETHYL KETONE

Molecular Weight: $72.11 \mathrm{~kg} / \mathrm{kmol}$

TLV-TWA: $200.00 \mathrm{ppm}$ IDLH: $3000.00 \mathrm{ppm}$ •

Footprint Level of Concern: 1200 ppm

Boiling Point: $79.64^{\circ} \mathrm{C}$

Vapor Pressure at Ambient Temperature: 0.15 atm

Ambient Saturation Concentration: 153,614 ppm or $15.4 \%$

ATMOSPHERIC INFORMATION: (MANUAL INPUT OF DATA)

Wind: 1.7 meters $/ \mathrm{sec}$ from $90^{\circ}$ true

Inversion Height: 200 meters

Stability Class: E Air Temperature: $29^{\circ} \mathrm{C}$

Relative Humidity: 50\% Ground Roughness: 100 centimeters

Cloud Cover: 1 tenths

\section{SOURCE STRENGTH INFORMATION:}

Puddle Area: 1030 square feet

Average Puddle Depth: 1 centimeters

Soil Type: Default Ground Temperature: $29^{\circ} \mathrm{C}$

Initial Puddle Temperature: Ground temperature

Release Duration: ALOHA limited the duration to 1 hour

Max Computed Release Rate: 10.3 kilograms/min

Max Average Sustained Release Rate: 8.56 kilograms/min

(averaged over a minute or more)

Total Amount Released: 425 kilograms

\section{FOOTPRINT INFORMATION:}

Model Run: Heavy Gas

User specified LOC: $1200 \mathrm{ppm}$

Max Threat Zone for LOC: 30 meters

Max Threat Zone for IDLH: 18 meters

Note: Footprint wasn't drawn because effects of near-field patchiness make plume presentation unreliable for short distances.

\section{TIME DEPENDENT INFORMATION:}

Concentration Estimates at the point:

Downwind: 30 meters

Off Centerline: 0 meters

Max Concentration:

Outdoor: $1,200 \mathrm{ppm}$

Indoor: $1,200 \mathrm{ppm}$

Note: Indoor graph is shown with a dotted line. 


\section{ENGINEERING COMPUTATION SHEET}

Title of Project EPHA for THM

Subject Concentration Analysis for THM Hazardous Chemicals

Computer T. M. Franey

Date 09/16/96
Reviewed by $\underline{\text { S. A. Henderson }}$

Works Calculation 7

Sheet No. 147 of 577

SITE DATA INFORMATION: 1-RD-29

Location: AIKEN, SOUTH CAROLINA

Building Air Exchanges Per Hour: 60 (User specified)

Date and Time: Fixed at July 1, 19960000 hours

CHEMICAL INFORMATION:

Chemical Name: METHYL ETHYL KETONE

Molecular Weight: $72.11 \mathrm{~kg} / \mathrm{kmol}$

TLV-TWA: $200.00 \mathrm{ppm}$ IDLH: $3000.00 \mathrm{ppm}$

Footprint Level of Concern: 1200 ppm

Boiling Point: $79.64^{\circ} \mathrm{C}$

Vapor Pressure at Ambient Temperature: 0.15 atm

Ambient Saturation Concentration: 153,614 ppm or $15.4 \%$

ATMOSPHERIC INFORMATION: (MANUAL INPUT OF DATA)

Wind: 1.7 meters $/ \mathrm{sec}$ from $90^{\circ}$ true

Inversion Height: 200 meters

Stability Class: E Air Temperature: $29^{\circ} \mathrm{C}$

Relative Humidity: $50 \%$ Ground Roughness: 100 centimeters

Cloud Cover: 1 tenths

SOURCE STRENGTH INFORMATION:

Puddle Area: 11100 square feet

Average Puddle Depth: 1 centimeters

Soil Type: Default Ground Temperature: $29^{\circ} \mathrm{C}$

Initial Puddle Temperature: Ground temperature

Release Duration: ALOHA limited the duration to 1 hour

Max Computed Release Rate: 99.2 kilograms/min

Max Average Sustained Release Rate: 83.4 kilograms/min

(averaged over a minute or more)

Total Amount Released: 4,183 kilograms

\section{FOOTPRINT INFORMATION:}

Model Run: Heavy Gas

User specified LOC: $1200 \mathrm{ppm}$

Max Threat Zone for LOC: 97 meters

Max Threat Zone for IDLH: 54 meters

Note: Footprint wasn't drawn because effects of near-field patchiness make plume presentation unreliable for short distances.

\section{TIME DEPENDENT INFORMATION:}

Concentration Estimates at the point:

Downwind: 100 meters

Off Centerline: 0 meters

Max Concentration:

Outdoor: $1,200 \mathrm{ppm}$

Indoor: $1,200 \mathrm{ppm}$

Note: Indoor graph is shown with a dotted line. 
Title of Project EPHA for THM

Subject Concentration Analysis for THM Hazardous Chemicals

Computer T.M. Franey

Date 09/16/96
Reviewed by $\underline{\text { S. A. Henderson }}$

Works Calculation 7

Sheet No. 148 of 577

SITE DATA INFORMATION: 1-RD-29

Location: AIKEN, SOUTH CAROLINA

Building Air Exchanges Per Hour: 60 (User specified)

Date and Time: Fixed at July 1, 19960000 hours

CHEMICAL INFORMATION:

Chemical Name: METHYL ETHYL KETONE

Molecular Weight: $72.11 \mathrm{~kg} / \mathrm{kmol}$

TLV-TWA: $200.00 \mathrm{ppm}$ IDLH: $3000.00 \mathrm{ppm}$

Footprint Level of Concern: 1200 ppm

Boiling Point: $79.64^{\circ} \mathrm{C}$

Vapor Pressure at Ambient Temperature: $0.15 \mathrm{~atm}$

Ambient Saturation Concentration: 153,614 ppm or $15.4 \%$

ATMOSPHERIC INFORMATION: (MANUAL INPUT OF DATA)

Wind: 1.7 meters $/ \mathrm{sec}$ from $90^{\circ}$ true

Inversion Height: 200 meters

Stability Class: E Air Temperature: $29^{\circ} \mathrm{C}$

Relative Humidity: 50\% Ground Roughness: 100 centimeters

Cloud Cover: 1 tenths

\section{SOURCE STRENGTH INFORMATION:}

Puddle Area: 24420 square feet

Average Puddle Depth: 1 centimeters

Soil Type: Default Ground Temperature: $29^{\circ} \mathrm{C}$

Initial Puddle Temperature: Ground temperature

Release Duration: ALOHA limited the duration to 1 hour

Max Computed Release Rate: 211 kilograms/min

Max Average Sustained Release Rate: 177 kilograms/min

(averaged over a minute or more)

Total Amount Released: 8,949 kilograms

FOOTPRINT INFORMATION:

Model Run: Heavy Gas

User specified LOC: $1200 \mathrm{ppm}$

Max Threat Zone for LOC: 148 meters

Max Threat Zone for IDLH: 83 meters

Note: The Heavy Gas footprint is an initial screening.

For short releases it may be an overestimation.

Be sure to check concentration information at specific locations.

TIME DEPENDENT INFORMATION:

Concentration Estimates at the point:

Downwind: 100 meters

Off Centerline: 0 meters

Max Concentration:

Outdoor: $2,370 \mathrm{ppm}$

Indoor: $2,370 \mathrm{ppm}$

Note: Indoor graph is shown with a dotted line. 
Title of Project EPHA for THM

Subject Concentration Analysis for THM Hazardous Chemicals

Computer T. M. Franey
Date 09/16/96
Reviewed by $\underline{\text { S. A. Henderson }}$

Works Calculation 7

Sheet No. 149 of 577

SITE DATA INFORMATION: 1-RD-29

Location: AIKEN, SOUTH CAROLINA

Building Air Exchanges Per Hour: 60 (User specified)

Date and Time: Fixed at July 1, 19960000 hours

CHEMICAL INFORMATION:

Chemical Name: METHYL ETHYL KETONE

Molecular Weight: $72.11 \mathrm{~kg} / \mathrm{kmol}$

TLV-TWA: $200.00 \mathrm{ppm}$ IDLH: $3000.00 \mathrm{ppm}$

Footprint Level of Concern: 1200 ppm

Boiling Point: $79.64^{\circ} \mathrm{C}$

Vapor Pressure at Ambient Temperature: $0.15 \mathrm{~atm}$

Ambient Saturation Concentration: 153,614 ppm or $15.4 \%$

ATMOSPHERIC INFORMATION: (MANUAL INPUT OF DATA)

Wind: 1.7 meters $/ \mathrm{sec}$ from $90^{\circ}$ true

Inversion Height: 200 meters

Stability Class: E Air Temperature: $29^{\circ} \mathrm{C}$

Relative Humidity: 50\% Ground Roughness: 100 centimeters

Cloud Cover: 1 tenths

\section{SOURCE STRENGTH INFORMATION:}

Puddle Area: 40700 square feet

Average Puddle Depth: 1 centimeters

Soil Type: Default Ground Temperature: $29^{\circ} \mathrm{C}$

Initial Puddle Temperature: Ground temperature

Release Duration: ALOHA limited the duration to 1 hour

Max Computed Release Rate: 343 kilograms/min

Max Average Sustained Release Rate: 289 kilograms/min

(averaged over a minute or more)

Total Amount Released: 14,653 kilograms

FOOTPRINT INFORMATION:

Model Run: Heavy Gas

User specified LOC: $1200 \mathrm{ppm}$

Max Threat Zone for LOC: 193 meters

Max Threat Zone for IDLH: 109 meters

Note: The Heavy Gas footprint is an initial screening.

For short releases it may be an overestimation.

Be sure to check concentration information at specific locations.

TIME DEPENDENT INFORMATION:

Concentration Estimates at the point:

Downwind: 100 meters

Off Centerline: 0 meters

Max Concentration:

Outdoor: $3,650 \mathrm{ppm}$

Indoor: $3,650 \mathrm{ppm}$

Note: Indoor graph is shown with a dotted line. 


\section{ENGINEERING COMPUTATION SHEET}

Title of Project EPHA for THM

Subject Concentration Analysis for THM Hazardous Chemicals

Computer T. M. Franey

Date $\underline{09 / 16 / 96}$
Reviewed by S. A. Henderson

Works Calculation 7

Sheet No. $\underline{150 \text { of } 577}$

SITE DATA INFORMATION: 1-RD-29

Location: AIKEN, SOUTH CAROLINA

Building Air Exchanges Per Hour: 60 (User specified)

Date and Time: Fixed at July 1, 19960000 hours

CHEMICAL INFORMATION:

Chemical Name: METHYL ETHYL KETONE

Molecular Weight: $72.11 \mathrm{~kg} / \mathrm{kmol}$

TLV-TWA: $200.00 \mathrm{ppm}$ IDLH: $3000.00 \mathrm{ppm}$

Footprint Level of Concern: 1200 ppm

Boiling Point: $79.64^{\circ} \mathrm{C}$

Vapor Pressure at Ambient Temperature: $0.15 \mathrm{~atm}$

Ambient Saturation Concentration: 153,614 ppm or $15.4 \%$

ATMOSPHERIC INFORMATION: (MANUAL INPUT OF DATA)

Wind: 1.7 meters $/ \mathrm{sec}$ from $90^{\circ}$ true

Inversion Height: 200 meters

Stability Class: E Air Temperature: $29^{\circ} \mathrm{C}$

Relative Humidity: 50\% Ground Roughness: 100 centimeters

Cloud Cover: 1 tenths

\section{SOURCE STRENGTH INFORMATION:}

Puddle Area: 10000 square meters

Average Puddle Depth: 1 centimeters

Soil Type: Default Ground Temperature: $29^{\circ} \mathrm{C}$

Initial Puddle Temperature: Ground temperature

Release Duration: ALOHA limited the duration to 1 hour

Max Computed Release Rate: 871 kilograms/min

Max Average Sustained Release Rate: 736 kilograms/min

(averaged over a minute or more)

Total Amount Released: 37,509 kilograms

FOOTPRINT INFORMATION:

Model Run: Heavy Gas

User specified LOC: $1200 \mathrm{ppm}$

Max Threat Zone for LOC: 324 meters

Max Threat Zone for IDLH: 188 meters

Note: The Heavy Gas footprint is an initial screening.

For short releases it may be an overestimation.

Be sure to check concentration information at specific locations.

\section{TIME DEPENDENT INFORMATION:}

Concentration Estimates at the point:

Downwind: 100 meters

Off Centerline: 0 meters

Max Concentration:

Outdoor: $8,180 \mathrm{ppm}$

Indoor: $8,180 \mathrm{ppm}$

Note: Indoor graph is shown with a dotted line. 


\section{ENGINEERING COMPUTATION SHEET}

Title of Project EPHA for THM

Subject Concentration Analysis for THM Hazardous Chemicals

Computer T. M. Franey

Date $\underline{09 / 16 / 96}$

SITE DATA INFORMATION: $\quad$ 1-RD-30

Location: AIKEN, SOUTH CAROLINA

Building Air Exchanges Per Hour: 60 (User specified)

Date and Time: Fixed at July 1, 19960000 hours

CHEMICAL INFORMATION:

Chemical Name: METHYL ISOBUTYL KETONE

Molecular Weight: $100.16 \mathrm{~kg} / \mathrm{kmol}$

TLV-TWA: $50.00 \mathrm{ppm}$ IDLH: $3000.00 \mathrm{ppm}$

Footprint Level of Concern: $250 \mathrm{ppm}$

Boiling Point: $116.50^{\circ} \mathrm{C}$

Vapor Pressure at Ambient Temperature: $0.033 \mathrm{~atm}$

Ambient Saturation Concentration: 33,167 ppm or $3.32 \%$

ATMOSPHERIC INFORMATION: (MANUAL INPUT OF DATA)

Wind: 1.7 meters $/ \mathrm{sec}$ from $90^{\circ}$ true

Inversion Height: 200 meters

Stability Class: E Air Temperature: $29^{\circ} \mathrm{C}$

Relative Humidity: 50\% Ground Roughness: 100 centimeters

Cloud Cover: 1 tenths

\section{SOURCE STRENGTH INFORMATION:}

Puddle Area: 2510 square feet

Average Puddle Depth: 1 centimeters

Soil Type: Default Ground Temperature: $29^{\circ} \mathrm{C}$

Initial Puddle Temperature: Ground temperature

Release Duration: ALOHA limited the duration to 1 hour

Max Computed Release Rate: 6.61 kilograms/min

Max Average Sustained Release Rate: 6.13 kilograms/min

(averaged over a minute or more)

Total Amount Released: 340 kilograms

FOOTPRINT INFORMATTON:

Dispersion Module: Gaussian

User specified LOC: $250 \mathrm{ppm}$

Max Threat Zone for LOC: 30 meters

Max Threat Zone for IDLH: less than 10 meters(10.9 yards)

Note: Footprint was not drawn becauise

effects of near-field patchiness make plume

presentation unreliable for short distances.

TIME DEPENDENT INFORMATION:

Concentration Estimates at the point:

Dowinwind: 30 meters

Off Centerline: 0 meters

Max Concentration:

Outdoor: $250 \mathrm{ppm}$

Indoor: $250 \mathrm{ppm}$

Note: Indoor graph is shown with a dotted line.
Reviewed by S. A. Henderson

Works Calculation 7

Sheet No. 151 of 577 


\section{ENGINEERING COMPUTATION SHEET}

Title of Project EPHA for THM

Subject Concentration Analysis for THM Hazardous Chemicals

Computer T. M. Franey

Date $\underline{09 / 16 / 96}$

SITE DATA INFORMATION: $\quad$ 1-RD-30

Location: AIKEN, SOUTH CAROLINA

Building Air Exchanges Per Hour: 60 (User specified)

Date and Time: Fixed at July 1, 19960000 hours

CHEMICAL INFORMATION:

Chemical Name: METHYL ISOBUTYL KETONE

Molecular Weight: $100.16 \mathrm{~kg} / \mathrm{kmol}$

TLV-TWA: $50.00 \mathrm{ppm}$ IDLH: $3000.00 \mathrm{ppm}$

Footprint Level of Concern: 250 ppm

Boiling Point: $116.50^{\circ} \mathrm{C}$

Vapor Pressure at Ambient Temperature: $0.033 \mathrm{~atm}$

Ambient Saturation Concentration: 33,167 ppm or $3.32 \%$

ATMOSPHERIC INFORMATION: (MANUAL INPUT OF DATA)

Wind: 1.7 meters $/ \mathrm{sec}$ from $90^{\circ}$ true

Inversion Height: 200 meters

Stability Class: E Air Temperature: $29^{\circ} \mathrm{C}$

Relative Humidity: 50\% Ground Roughness: 100 centimeters

Cloud Cover: 1 tenths

\section{SOURCE STRENGTH INFORMATION:}

Puddle Area: 9750 square feet

Average Puddle Depth: 1 centimeters

Soil Type: Default Ground Temperature: $29^{\circ} \mathrm{C}$

Initial Puddle Temperature: Ground temperature

Release Duration: ALOHA limited the duration to 1 hour

Max Computed Release Rate: 24.2 kilograms/min

Max Average.Sustained Release Rate: 22.5 kilograms/min

(averaged over a minute or more)

Total Amount Released: 1,250 kilograms

FOOTPRINT INFORMATION:

Model Run: Heavy Gas

User specified LOC: $250 \mathrm{ppm}$

Max Threat Zone for LOC: 97 meters

Max Threat Zone for IDLH: 13 meters

Note: Footprint wasn't drawn because effects of near-field patchiness make plume presentation unreliable for short distances.

\section{TIME DEPENDENT INFORMATION:}

Concentration Estimates at the point:

Downwind: 100 meters

Off Centerline: 0 meters

Max Concentration:

Outdoor: $250 \mathrm{ppm}$

Indoor: $250 \mathrm{ppm}$

Note: Indoor graph is shown with a dotted line.
Reviewed by S. A. Henderson

Works Calculation 7

Sheet No. 152 of 577 


\section{ENGINEERING COMPUTATION SHEET}

Title of Project EPHA for THM

Subject Concentration Analysis for THM Hazardous Chemicals

Computer T. M. Franey

Date $\underline{09 / 16 / 96}$

SITE DATA INFORMATION: 1-RD-30

Location: AIKEN, SOUTH CAROLINA

Building Air Exchanges Per Hour: 60 (User specified)

Date and Time: Fixed at July 1, 19960000 hours

CHEMICAL INFORMATION:

Chemical Name: METHYL ISOBUTYL KETONE

Molecular Weight: $100.16 \mathrm{~kg} / \mathrm{kmol}$

TLV-TWA: $50.00 \mathrm{ppm}$ IDLH: $3000.00 \mathrm{ppm}$

Footprint Level of Concern: 250 ppm

Boiling Point: $116.50^{\circ} \mathrm{C}$

Vapor Pressure at Ambient Temperature: 0.033 atm

Ambient Saturation Concentration: 33,167 ppm or 3.32\%

ATMOSPHERIC INFORMATION: (MANUAL INPUT OF DATA)

Wind: 1.7 meters $/ \mathrm{sec}$ from $90^{\circ}$ true

Inversion Height: 200 meters

Stability Class: E Air Temperature: $29^{\circ} \mathrm{C}$

Relative Humidity: 50\% Ground Roughness: 100 centimeters

Cloud Cover: 1 tenths

\section{SOURCE STRENGTH INFORMATION:}

Puddle Area: 24420 square feet

Average Puddle Depth: 1 centimeters

Soil Type: Default Ground Temperature: $29^{\circ} \mathrm{C}$

Initial Puddle Temperature: Ground temperature

Release Duration: ALOHA limited the duration to 1 hour

Max Computed Release Rate: 58.2 kilograms/min

Max Average Sustained Release Rate: 54.3 kilograms $/ \mathrm{min}$ (averaged over a minute or more)

Total Amount Released: 3,019 kilograms

\section{FOOTPRINT INFORMATION:}

Model Run: Heavy Gas

User specified LOC: $250 \mathrm{ppm}$

Max Threat Zone for LOC: 155 meters

Max Threat Zone for IDLH: 18 meters

Note: The Heavy Gas footprint is an initial screening.

For short releases it may be an overestimation.

Be sure to check concentration information at specific locations.

\section{TIME DEPENDENT INFORMATION:}

Concentration Estimates at the point:

Downwind: 100 meters

Off Centerline: 0 meters

Max Concentration:

Outdoor: 491 ppm

Indoor: $491 \mathrm{ppm}$

Note: Indoor graph is shown with a dotted line.
Reviewed by S.A. Henderson

Works Calculation 7

Sheet No. 153 of 577 
Title of Project EPHA for THM

Subject Concentration Analysis for THM Hazardous Chemicals

Computer T.M. Franey
Reviewed by $S$. A. Henderson

Works Calculation 7

Sheet No. 154 of 577

SITE DATA INFORMATION: I-RD-30

Location: AIKEN, SOUTH CAROLINA

Building Air Exchanges Per Hour: 60 (User specified)

Date and Time: Fixed at July 1, 19960000 hours

\section{CHEMICAL INFORMATION:}

Chemical Name: METHYL ISOBUTYL KETONE

Molecular Weight: $100.16 \mathrm{~kg} / \mathrm{kmol}$

TLV-TWA: $50.00 \mathrm{ppm}$ IDLH: $3000.00 \mathrm{ppm}$

Footprint Level of Concern: 250 ppm

Boiling Point: $116.50^{\circ} \mathrm{C}$

Vapor Pressure at Ambient Temperature: $0.033 \mathrm{~atm}$

Ambient Saturation Concentration: 33,167 ppm or $3.32 \%$

\section{ATMOSPHERIC INFORMATION: (MANUAL INPUT OF DATA)}

Wind: 1.7 meters $/ \mathrm{sec}$ from $90^{\circ}$ true

Inversion Height: 200 meters

Stability Class: E Air Temperature: $29^{\circ} \mathrm{C}$

Relative Humidity: 50\% Ground Roughness: 100 centimeters

Cloud Cover: 1 tenths

\section{SOURCE STRENGTH INFORMATION:}

Puddle Area: 40700 square feet

Average Puddle Depth: 1 centimeters

Soil Type: Default Ground Temperature: $29^{\circ} \mathrm{C}$

Initial Puddle Temperature: Ground temperature

Release Duration: ALOHA limited the duration to 1 hour

Max Computed Release Rate: 95 kilograms/min

Max Average Sustained Release Rate: 88.7 kilograms/min

(averaged over a minute or more)

Total Amount Released: 4,935 kilograms

\section{FOOTPRINT INFORMATION:}

Model Run: Heavy Gas

User specified LOC: $250 \mathrm{ppm}$

Max Threat Zone for LOC: 200 meters

Max Threat Zone for IDLH: 27 meters

Note: The Heavy Gas footprint is an initial screening.

For short releases it may be an overestimation.

Be sure to check concentration information at specific locations.

\section{TIME DEPENDENT INFORMATION:}

Concentration Estimates at the point:

Downwind: 100 meters

Off Centerline: 0 meters

Max Concentration:

Outdoor: $711 \mathrm{ppm}$

Indoor: $711 \mathrm{ppm}$

Note: Indoor graph is shown with a dotted line. 


\section{ENGINEERING COMPUTATION SHEET}

Title of Project EPHA for THM

Subject Concentration Analysis for THM Hazardous Chemicals

Computer T. M. Franey

Date $\underline{09 / 16 / 96}$

SITE DATA INFORMATION: 1-RD-31

Location: AIKEN, SOUTH CAROLINA

Building Air Exchanges Per Hour: 60 (User specified)

Date and Time: Fixed at July 1, 19960000 hours

CHEMICAL INFORMATION:

Chemical Name: METHYLENE CHLORIDE

Molecular Weight: $84.90 \mathrm{~kg} / \mathrm{kmol}$

TLV-TWA: -unavail- IDLH: -unavail-

Footprint Level of Concern: $7.50 \mathrm{ppm}$

Boiling Point: $40.00^{\circ} \mathrm{C}$

Vapor Pressure at Ambient Temperature: $0.65 \mathrm{~atm}$

Ambient Saturation Concentration: 656,830 ppm or $65.7 \%$

ATMOSPHERIC INFORMATION: (MANUAL INPUT OF DATA)

Wind: 1.7 meters $/ \mathrm{sec}$ from $90^{\circ}$ true

Inversion Height: 200 meters

Stability Class: E Air Temperature: $29^{\circ} \mathrm{C}$

Relative Humidity: $50 \%$ Ground Roughness: 100 centimeters

Cloud Cover: 1 tenths

SOURCE STRENGTH INFORMATION:

Puddle Area: 96 square feet

Average Puddle Depth: 1 centimeters

Soil Type: Default Ground Temperature: $29^{\circ} \mathrm{C}$

Initial Puddle Temperature: Ground temperature

Release Duration: 28 minutes

Max Computed Release Rate: 8.71 kilograms/min

Max Average Sustained Release Rate: 5.53 kilograms/min

(averaged over a minute or more)

Total Amount Released: 109 kilograms

FOOTPRINT INFORMATION:

Model Run: Heavy Gas

User specified LOC: $750 \mathrm{ppm}$

Max Threat Zone for LOC: 30 meters

- Note: Footprint wasn't drawn because effects of near-field patchiness make plume presentation unreliable for short distances.

TIME DEPENDENT INFORMATION:

Concentration Estimates at the point:

Downwind: 30 meters

Off Centerline: 0 meters

Max Concentration:

Outdoor: $751 \mathrm{ppm}$

Indoor: $710 \mathrm{ppm}$

Note: Indoor graph is shown with a dotted line.
Reviewed by S. A. Henderson

Works Calculation 7

Sheet No. 156 of 577 
Title of Project EPHA for THM

Subject Concentration Analysis for THM Hazardous Chemicals

Computer T. M. Franey
Reviewed by S. A. Henderson

Works Calculation 7

Sheet No. 157 of 577

SITE DATA INFORMATION: 1-RD-31

Location: AIKEN, SOUTH CAROLINA

Building Air Exchanges Per Hour: 60 (User specified)

Date and Time: Fixed at July 1, 19960000 hours

CHEMICAL INFORMATION:

Chemical Name: METHYLENE CHLORIDE

Molecular Weight: $84.90 \mathrm{~kg} / \mathrm{kmol}$

TLV-TWA: -unavail- IDLH: -unavail-

Footprint Level of Concern: 750 ppm

Boiling Point: $40.00^{\circ} \mathrm{C}$

Vapor Pressure at Ambient Temperature: $0.65 \mathrm{~atm}$

Ambient Saturation Concentration: $656,830 \mathrm{ppm}$ or $65.7 \%$

ATMOSPHERIC INFORMATION: (MANUAL INPUT OF DATA)

Wind: 1.7 meters $/ \mathrm{sec}$ from $90^{\circ}$ true

Inversion Height: 200 meters

Stability Class: E Air Temperature: $29^{\circ} \mathrm{C}$

Relative Humidity: 50\% Ground Roughness: 100 centimeters

Cloud Cover: 1 tenths

\section{SOURCE STRENGTH INFORMATION:}

Puddle Area: 1110 square feet

Average Puddle Depth: 1 centimeters

Soil Type: Default Ground Temperature: $29^{\circ} \mathrm{C}$

Initial Puddle Temperature: Ground temperature

Release Duration: 31 minutes

Max Computed Release Rate: 88.6 kilograms/min

Max Average Sustained Release Rate: $\mathbf{5 7 . 4}$ kilograms/min

(averaged over a minute or more)

Total Amount Released: 1,256 kilograms

FOOTPRINT INFORMATION:

Model Run: Heavy Gas

User specified LOC: $750 \mathrm{ppm}$

Max Threat Zone for LOC: 97 meters

Note: Footprint wasn't drawn because effects of near-field patchiness make plume presentation unreliable for short distances.

\section{TIME DEPENDENT INFORMATION:}

Concentration Estimates at the point:

Downwind: 100 meters

Off Centerline: 0 meters

Max Concentration:

Outdoor: $750 \mathrm{ppm}$

Indoor: $715 \mathrm{ppm}$

Note: Indoor graph is shown with a dotted line. 
Title of Project EPHA for THM

Subject Concentration Analysis for THM Hazardous Chemicals

Computer T.M. Franey Date $09 / 16 / 96$

\section{SITE DATA INFORMATION: 1-RD-31}

Location: AIKEN, SOUTH CAROLINA

Building Air Exchanges Per Hour: 60 (User specified) .

Date and Time: Fixed at July 1, 19960000 hours

\section{CHEMICAL INFORMATION:}

Chemical Name: METHYLENE CHLORIDE

Molecular Weight: $84.90 \mathrm{~kg} / \mathrm{kmol}$

TLV-TWA: -unavail- IDLH: -unavail-

Footprint Level of Concern: 750 ppm

Boiling Point: $40.00^{\circ} \mathrm{C}$

Vapor Pressure at Ambient Temperature: $0.65 \mathrm{~atm}$

Ambient Saturation Concentration: 656,830 ppm or $65.7 \%$

ATMOSPHERIC INFORMATION: (MANUAL INPUT OF DATA)

Wind: 1.7 meters $/ \mathrm{sec}$ from $90^{\circ}$ true

Inversion Height: 200 meters

Stability Cläss: E Air Temperature: $29^{\circ} \mathrm{C}$

Relative Humidity: 50\% Ground Roughness: 100 centimeters

Cloud Cover: 1 tenths

\section{SOURCE STRENGTH INFORMATION:}

Puddle Area: 24420 square feet

Average Puddle Depth: 1 centimeters

Soil Type: Default Ground Temperature: $29^{\circ} \mathrm{C}$

Initial Puddle Temperature: Ground temperature

Release Duration: 35 minutes

Max Computed Release Rate: 1,690 kilograms/min

Max Average Sustained Release Rate: 1,120 kilograms/min

(averaged over a minute or more)

Total Amount Released: 27,638 kilograms

FOOTPRINT INFORMATION:

Model Run: Heavy Gas

User specified LOC: $750 \mathrm{ppm}$

Max Threat Zone for LOC: 450 meters

Note: The Heavy Gas footprint is an initial screening.

For short releases it may be an overestimation.

Be sure to check concentration information at specific locations.

\section{TIME DEPENDENT INFORMATION:}

Concentration Estimates at the point:

Downwind: 100 meters

Off Centerline: 0 meters

Max Concentration:

Outdoor: $17,000 \mathrm{ppm}$

Indoor: $16,700 \mathrm{ppm}$

Note: Indoor graph is shown with a dotted line.
Reviewed by S. A. Henderson

Works Calculation 7

Sheet No. 158 of 577 


\section{ENGINEERING COMPUTATION SHEET}

Title of Project EPHA for THM

Subject Concentration Analysis for THM Hazardous Chemicals

Computer T. M. Franey

Date $\underline{09 / 16 / 96}$
Reviewed by $\underline{\text { S. A. Henderson }}$

Works Calculation 7

Sheet No: 159 of 577

SITE DATA INFORMATION: 1-RD-31

Location: AIKEN, SOUTH CAROLINA

Building Air Exchanges Per Hour: 60 (User specified)

Date and Time: Fixed at July 1, 19960000 hours

\title{
CHEMICAL INFORMATTON:
}

Chemical Name: METHYLENE CHLORIDE

Molecular Weight: $84.90 \mathrm{~kg} / \mathrm{kmol}$

TLV-TWA: -unavail- IDLH: -unavail-

Footprint Level of Concern: 750 ppm

Boiling Point: $40.00^{\circ} \mathrm{C}$

Vapor Pressure at Ambient Temperature: $0.65 \mathrm{~atm}$

Ambient Saturation Concentration: 656,830 ppm or $65.7 \%$

\author{
ATMOSPHERIC INFORMATION: (MANUAL INPUT OF DATA) \\ Wind: 1.7 meters $/ \mathrm{sec}$ from $90^{\circ}$ true \\ Inversion Height: 200 meters \\ Stability Class: E Air Temperature: $29^{\circ} \mathrm{C}$ \\ Relative Humidity: $50 \%$ Ground Roughness: 100 centimeters \\ Cloud Cover: 1 tenths
}

\section{SOURCE STRENGTH INFORMATION:}

Puddle Area: 40700 square feet

Average Puddle Depth: 1 centimeters

Soil Type: Default Ground Temperature: $29^{\circ} \mathrm{C}$

Initial Puddle Temperature: Ground temperature

Release Duration: 35 minutes

Max Computed Release Rate: 2,760 kilograms/min

Max Average Sustained Release Rate: 1,840 kilograms/min

(averaged over a minute or more)

Total Amount Released: 46,063 kilograms

\section{FOOTPRINT INFORMATION:}

Model Run: Heavy Gas

User specified LOC: $750 \mathrm{ppm}$

Max Threat Zone for LOC: 592 meters

Note: The Heavy Gas footprint is an initial screening.

For short releases it may be an overestimation.

Be sure to check concentration information at specific locations.

\section{TIME DEPENDENT INFORMATION:}

Concentration Estimates at the point:

\section{Downwind: 100 meters}

Off Centerline: 0 meters

Max Concentration:

Outdoor: $35,800 \mathrm{ppm}$

Indoor: $35,100 \mathrm{ppm}$

Note: Indoor graph is shown with a dotted line. 


\section{ENGINEERING COMPUTATION SHEET}

Title of Project EPHA for THM

Subject Concentration Analysis for THM Hazardous Chemicals

Computer T. M. Franey

Date $\underline{09 / 16 / 96}$
Reviewed by S. A. Henderson

Works Calculation 7

Sheet No. 160 of 577

SITE DATA INFORMATION: 1-RD-31

Location: AIKEN, SOUTH CAROLINA

Building Air Exchanges Per Hour: 60 (User specified)

Date and Time: Fixed at July 1, 19960000 hours

CHEMICAL INFORMATION:

Chemical Name: METHYLENE CHLORIDE

Molecular Weight: $84.90 \mathrm{~kg} / \mathrm{kmol}$

TLV-TWA: -unavail- IDLH: -unavail-

Footprint Level of Concern: 750 ppm

Boiling Point: $40.00^{\circ} \mathrm{C}$

Vapor Pressure at Ambient Temperature: 0.65 atm

Ambient Saturation Concentration: 656,830 ppm or $65.7 \%$

ATMOSPHERIC INFORMATION: (MANUAL INPUT OF DATA)

Wind: 1.7 meters $/ \mathrm{sec}$ from $90^{\circ}$ true

Inversion Height: 200 meters

Stability Class: E Air Temperature: $29^{\circ} \mathrm{C}$

Relative Humidity: 50\% Ground Roughness: 100 centimeters

Cloud Cover: 1 tenths

SOURCE STRENGTH INFORMATION:

Puddle Area: 10000 square meters

Average Puddle Depth: 1 centimeters

Soil Type: Default Ground Temperature: $29^{\circ} \mathrm{C}$

Initial Puddle Temperature: Ground temperature

Release Duration: 37 minutes

Max Computed Release Rate: 7,010 kilograms/min

Max Average Sustained Release Rate: 4,690 kilograms/min (averaged over a minute or more)

Total Amount Released: 121,824 kilograms

\section{FOOTPRINT INFORMATION:}

Model Run: Heavy Gas

User specified LOC: $750 \mathrm{ppm}$

Max Threat Zone for LOC: 1.0 kilometers

Note: The Heavy Gas footprint is an initial screening.

For short releases it may be an overestimation.

Be sure to check concentration information at specific locations.

\section{TIME DEPENDENT INFORMATION:}

Concentration Estimates at the point:

Downwind: 100 meters

Off Centerline: 0 meters

Max Concentration:

Outdoor: $187,000 \mathrm{ppm}$

Indoor: $184,000 \mathrm{ppm}$

Note: Indoor graph is shown with a dotted line. 


\section{ENGINEERING COMPUTATION SHEET}

Title of Project EPHA for THM

Subject Concentration Analysis for THM Hazardous Chemicals

Computer T. M. Franey

Date $\underline{09 / 16 / 96}$

SITE DATA INFORMATION: 1-RD-32

Location: AIKEN, SOUTH CAROLINA

Building Air Exchanges Per Hour: 60 (User specified)

Date and Time: Fixed at July 1, 19960000 hours

CHEMICAL INFORMATION:

Chemical Name: METHL CELLOSOLVE

Molecular Weight: $76.1 \mathrm{~kg} / \mathrm{kmol}$

TLV-TWA: not available

IDLH: not available

Footprint Level of Concern: $250 \mathrm{ppm}$

Boiling Point: $171.00^{\circ} \mathrm{C}$

Vapor Pressure at Ambient Temperature: 6.82e-004 atm

Ambient Saturation Concentration: 694 ppm or $0.069 \%$

ATMOSPHERIC INFORMATION: (MANUAL INPUT OF DATA)

Wind: 1.7 meters $/ \mathrm{sec}$ from $90^{\circ}$ true

Inversion Height: 200 meters

Stability Class: E Air Temperature: $29^{\circ} \mathrm{C}$

Relative Humidity: $50 \%$ Ground Roughness: 100 centimeters

Cloud Cover: 1 tenths

\section{SOURCE STRENGTH INFORMATION:}

Puddle Area: 30430 square feet

Average Puddle Depth: 1 centimeters

Soil Type: Default Ground Temperature: $29^{\circ} \mathrm{C}$

Initial Puddle Temperature: Ground temperature

Release Duration: ALOHA limited the duration to 1 hour

Max Computed Release Rate: 637 grams/min

Max Average Sustained Release Rate: $616 \mathrm{grams} / \mathrm{min}$

(averaged over a minute or more)

Total Amount Released: 35.3 kilograms

\section{FOOTPRINT INFORMATION:}

Dispersion Module: Gaussian

User specified LOC: $250 \mathrm{ppm}$

Max Threat Zone for LOC: 30 meters

Max Threat Zone for IDLH: 30 meters

Note: Footprint was not drawn because effects of near-field patchiness make plume presentation unreliable for short distances.

\section{TIME DEPENDENT INFORMATION:}

Concentration Estimates at the point:

Downwind: 30 meters

Off Centerline: 0 meters

Max Concentration:

Outdoor: $37 \mathrm{ppm}$

Indoor: $37 \mathrm{ppm}$

Note: Indoor graph is shown with a dotted line.
Reviewed by S. A. Henderson

Works Calculation 7

Sheet No. 161 of 577 


\section{ENGINEERING COMPUTATION SHEET}

Title of Project EPHA for THM

Subject Concentration Analysis for THM Hazardous Chemicals

Computer T. M. Franey

Date 09/16/96
Reviewed by $\underline{\text { S. A. Henderson }}$

Works Calculation 7

Sheet No. 162 of 577

SITE DATA INFORMATION: 1-RD-32

Location: AIKEN, SOUTH CAROLINA

Building Air Exchanges Per Hour: 60 (User specified)

Date and Time: Fixed at July 1, 19960000 hours

CHEMICAL INFORMATION:

Chemical Name: METHL CELLOSOLVE

Molecular Weight: $76.1 \mathrm{~kg} / \mathrm{kmol}$

TLV-TWA: not available

IDLH: not available

Footprint Level of Concern: $250 \mathrm{ppm}$

Boiling Point: $171.00^{\circ} \mathrm{C}$

Vapor Pressure at Ambient Temperature: 6.82e-004 atm

Ambient Saturation Concentration: 694 ppm or $0.069 \%$

\section{ATMOSPHERIC INFORMATION: (MANUAL INPUT OF DATA)}

Wind: 1.7 meters $/ \mathrm{sec}$ from $90^{\circ}$ true

Inversion Height: 200 meters

Stability Class: E Air Temperature: $29^{\circ} \mathrm{C}$

Relative Humidity: 50\% Ground Roughness: 100 centimeters.

Cloud Cover: 1 tenths

\section{SOURCE STRENGTH INFORMATION:}

Puddle Area: 107639 square feet

Average Puddle Depth: 1 centimeters

Soil Type: Default Ground Temperature: $29^{\circ} \mathrm{C}$

Initial Puddle Temperature: Ground temperature

Release Duration: ALOHA limited the duration to 1 hour

Max Computed Release Rate: 2.13 kilograms/min

Max Average Sustained Releáse Rate: 2.06 kilograms/min

(averaged over a minute or more)

Total Amount Released: 118 kilograms

FOOTPRINT INFORMATION:

Dispersion Module: Gaussian

User specified LOC: $250 \mathrm{ppm}$

Max Threat Zone for LOC: 56 meters

Max Threat Zone for IDLH: 56 meters

Note: Footprint was not drawn because effects of near-field patchiness make plume presentation unreliable for short distances.

\section{TIME DEPENDENT INFORMATION:}

Concentration Estimates at the point:

Downwind: 100 meters

Off Centerline: 0 meters

Max Concentration:

Outdoor: $11.7 \mathrm{ppm}$

Indoor: $11.7 \mathrm{ppm}$

Note: Indoor graph is shown with a dotted line. 


\section{ENGINEERING COMPUTATION SHEET}

Title of Project EPHA for THM

Subject Concentration Analysis for THM Hazardous Chemicals

Computer T. M. Franey

Date $\underline{09 / 16 / 96}$
Reviewed by S.A. Henderson

Works Calculation 7

Sheet No. 163 of 577

SITE DATA INFORMATION: 1-RD-32

Location: AIKEN, SOUTH CAROLINA

Building Air Exchanges Per Hour: 60 (User specified)

Date and Time: Fixed at July 1, 19960000 hours

CHEMICAL INFORMATION:

Chemical Name: METHL CELLOSOLVE

Molecular Weight: $76.1 \mathrm{~kg} / \mathrm{kmol}$

TLV-TWA: not available

IDLH: not available

Footprint Level of Concern: $250 \mathrm{ppm}$

Boiling Point: $171.00^{\circ} \mathrm{C}$

Vapor Pressure at Ambient Temperature: 6.82e-004 atm

Ambient Saturation Concentration: 694 ppm or 0.069\%

ATMOSPHERIC INFORMATION: (MANUAL INPUT OF DATA)

Wind: 1.7 meters $/ \mathrm{sec}$ from $90^{\circ}$ true

Inversion Height: 200 meters

Stability Class: E Air Temperature: $29^{\circ} \mathrm{C}$

Relative Humidity: 50\% Ground Roughness: 100 centimeters

Cloud Cover: 1 tenths

SOURCE STRENGTH INFORMATION:

Puddle Area: 24420 square feet

Average Puddle Depth: 1 centimeters

Soil Type: Default Ground Temperature: $29^{\circ} \mathrm{C}$

Initial Puddle Temperature: Ground temperature

Release Duration: ALOHA limited the duration to 1 hour

Max Computed Release Rate: 516 grams/min

Max Average.Sustained Release Rate: 500 grams/min

(averaged over a minute or more)

Total Amount Released: 28.6 kilograms

FOOTPRINT INFORMATION:

Dispersion Module: Gaussian

User specified LOC: $250 \mathrm{ppm}$

Max Threat Zone for LOC: 27 meters

Max Threat Zone for IDLH: 27 meters

Note: Footprint was not drawn because

effects of near-field patchiness make plume

presentation unreliable for short distances.

\section{TIME DEPENDENT INFORMATION:}

Concentration Estimates at the point:

Downwind: 100 meters

Off Centerline: 0 meters

Max Concentration:

Outdoor: $3.29 \mathrm{ppm}$

Indoor: $3.29 \mathrm{ppm}$

Note: Indoor graph is shown with a dotted line. 
Title of Project EPHA for THM

Subject Concentration Analysis for THM Hazardous Chemicals

Computer T. M. Franey

Date $\underline{09 / 16 / 96}$
Reviewed by $\underline{\text { S. A. Henderson }}$

Works Calculation 7

Sheet No: 164 of 577

SITE DATA INFORMATION: 1-RD-32

Location: AIKEN, SOUTH CAROLINA

Building Air Exchanges Per Hour: 60 (User specified)

Date and Time: Fixed at July 1, 19960000 hours

\section{CHEMICAL INFORMATION:}

Chemical Name: METHL CELLOSOLVE

Molecular Weight: $76.1 \mathrm{~kg} / \mathrm{kmol}$

TLV-TWA: not available IDLH: not available

Footprint Level of Concern: 250 ppm

Boiling Point: $171.00^{\circ} \mathrm{C}$

Vapor Pressure at Ambient Temperature: 6.82e-004 atm

Ambient Saturation Concentration: 694 ppm or $0.069 \%$

ATMOSPHERIC INFORMATION: (MANUAL INPUT OF DATA)

Wind: 1.7 meters $/ \mathrm{sec}$ from $90^{\circ}$ true

Inversion Height: 200 meters

Stability Class: E Air Temperature: $29^{\circ} \mathrm{C}$

Relative Humidity: 50\% Ground Roughness: 100 centimeters

Cloud Cover: 1 tenths

SOURCE STRENGTH INFORMATION:

Puddle Area: 40700 square feet

Average Puddle Depth: 1 centimeters

Soil Type: Default Ground Temperature: $29^{\circ} \mathrm{C}$

Initial Puddle Temperature: Ground temperature

Release Duration: ALOHA limited the duration to 1 hour

Max Computed Release Rate: 841 grams/min

Max Average Sustained Release Rate: 814 grams/min

(averaged over a minute or more)

Total Amount Released: 46.6 kilograms

FOOTPRINT INFORMATION:

Dispersion Module: Gaussian

User specified LOC: $250 \mathrm{ppm}$

Max Threat Zone for LOC: 34 meters

Max Threat Zone for IDLH: 35 meters

Note: Footprint was not drawn because effects of near-field patchiness make plume

presentation unreliable for short distances.

\section{TIME DEPENDENT INFORMATION:}

Concentration Estimates at the point:

Downwind: 100 meters

Off Centerline: 0 meters

Max Concentration:

Outdoor: $4.76 \mathrm{ppm}$

Indoor: $4.76 \mathrm{ppm}$

Note: Indoor graph is shown with a dotted line. 
Title of Project EPHA for THM

Subject Concentration Analysis for THM Hazardous Chemicals

Computer T. M. Franey

\section{SITE DATA INFORMATION: 1-RD-32}

Location: AIKEN, SOUTH CAROLINA

Building Air Exchanges Per Hour: 60 (User specified)

Date and Time: Fixed at July 1, 19960000 hours

\section{CHEMICAL INFORMATION:}

Chemical Name: METHL CELLOSOLVE

Molecular Weight: $76.1 \mathrm{~kg} / \mathrm{kmol}$

TLV-TWA: not available IDLH: not available

Footprint Level of Concern: $250 \mathrm{ppm}$

Boiling Point: $171.00^{\circ} \mathrm{C}$

Vapor Pressure at Ambient Temperature: 6.82e-004 atm

Ambient Saturation Concentration: 694 ppm or 0.069\%

ATMOSPHERIC INFORMATION: (MANUAL INPUT OF DATA)

Wind: 1.7 meters $/ \mathrm{sec}$ from $90^{\circ}$ true

Inversion Height: 200 meters

Stability Class: E Air Temperature: $29^{\circ} \mathrm{C}$

Relative Humidity: 50\% Ground Roughness: 100 centimeters

Cloud Cover: 1 tenths

\section{SOURCE STRENGTH INFORMATION:}

Puddle Area: 10000 square meters

Average Puddle Depth: 1 centimeters

Soil Type: Default Ground Temperature: $29^{\circ} \mathrm{C}$

Initial Puddle Temperature: Ground temperature

Release Duration: ALOHA limited the duration to 1 hour

Max Computed Release Rate: 2.13 kilograms/min

Max Average Sustained Release Rate: 2.06 kilograms/min

(averaged over a minute or more)

Total Amount Released: 118 kilograms

\section{FOOTPRINT INFORMATION:}

Dispersion Module: Gaussian

User specified LOC: $250 \mathrm{ppm}$

Max Threat Zone for LOC: 56 meters

Max Threat Zone for IDLH: 56 meters

Note: Footprint was not drawn because effects of near-field patchiness make plume

presentation unreliable for short distances.

\section{TIME DEPENDENT INFORMATION:}

Concentration Estimates at the point:

Downwind: $\quad 100$ meters

Off Centerline: 0 meters

Max Concentration:

Outdoor: $11.7 \mathrm{ppm}$

Indoor: $11.7 \mathrm{ppm}$

Note: Indoor graph is shown with a dotted line. 
Title of Project EPHA for THM

Subject Concentration Analysis for THM Hazardous Chemicals

Computer T. M. Franey

Date $\underline{09 / 16 / 96}$
Reviewed by S. A. Henderson

Works Calculation 7

Sheet No. 166 of 577

SITE DATA INFORMATION: 1-RD-33

Location: AIKEN, SOUTH CAROLINA

Building Air Exchanges Per Hour: 60 (User specified)

Date and Time: Fixed at July 1, 19960000 hours

CHEMICAL INFORMATION:

Chemical Name: MONOETHANOLAMINE

Molecular Weight: $61.08 \mathrm{~kg} / \mathrm{kmol}$

TLV-TWA: $3.00 \mathrm{ppm}$ IDLH: $1000.00 \mathrm{ppm}$

Footprint Level of Concern: 100 ppm

Boiling Point: $171.00^{\circ} \mathrm{C}$

Vapor Pressure at Ambient Temperature: $6.82 \mathrm{e}-004 \mathrm{~atm}$

Ambient Saturation Concentration: 694 ppm or $0.069 \%$

ATMOSPHERIC INFORMATION: (MANUAL INPUT OF DATA)

Wind: 1.7 meters $/ \mathrm{sec}$ from $90^{\circ}$ true

Inversion Height: 200 meters

Stability Class: E Air Temperature: $29^{\circ} \mathrm{C}$

Relative Humidity: $50 \%$ Ground Roughness: 100 centimeters

Cloud Cover: 1 tenths

SOURCE STRENGTH INFORMATION:

Puddle Area: 30430 square feet

Average Puddle Depth: 1 centimeters

Soil Type: Default Ground Temperature: $29^{\circ} \mathrm{C}$

Initial Puddle Temperature: Ground temperature

Release Duration: ALOHA limited the duration to 1 hour

Max Computed Release Rate: 637 grams/min

Max Average Sustained Release Rate: 616 grams/min

(averaged over a minute or more)

Total Amount Released: 35.3 kilograms

FOOTPRINT INFORMATION:

Dispersion Module: Gaussian

User specified LOC: 100 ppm

Max Threat Zone for LOC: 30 meters

Max Threat Zone for IDLH: 30 meters

Note: Footprint was not drawn because

effects of near-field patchiness make plume

presentation unreliable for short distances.

TIME DEPENDENT INFORMATION:

Concentration Estimates at the point:

Downwind: 30 meters

Off Centerline: 0 meters

Max Concentration:

Outdoor: $37 \mathrm{ppm}$

Indoor: $37 \mathrm{ppm}$

Note: Indoor graph is shown with a dotted line. 


\section{ENGINEERING COMPUTATION SHEET}

Title of Project EPHA for THM

Subject Concentration Analysis for THM Hazardous Chemicals

Computer T. M. Franey

Date $\underline{09 / 16 / 96}$
Reviewed by S. A. Henderson

Works Calculation 7

Sheet No. 167 of 577

SITE DATA INFORMATION: 1-RD-33

Location: AIKEN, SOUTH CAROLINA

Building Air Exchanges Per Hour: 60 (User specified)

Date and Time: Fixed at July 1, 19960000 hours

CHEMICAL INFORMATION:

Chemical Name: MONOETHANOLAMINE

Molecular Weight: $61.08 \mathrm{~kg} / \mathrm{kmol}$

TLV-TWA: $3.00 \mathrm{ppm}$ IDLH: $1000.00 \mathrm{ppm}$

Footprint Level of Concern: 100 ppm

Boiling Point: $171.00^{\circ} \mathrm{C}$

Vapor Pressure at Ambient Temperature: 6.82e-004 atm

Ambient Saturation Concentration: 694 ppm or $0.069 \%$

ATMOSPHERIC INFORMATION: (MANUAL INPUT OF DATA)

Wind: 1.7 meters $/ \mathrm{sec}$ from $90^{\circ}$ true

Inversion Height: 200 meters

Stability Class: E Air Temperature: $29^{\circ} \mathrm{C}$

Relative Humidity: 50\% Ground Roughness: 100 centimeters

Cloud Cover: 1 tenths

SOURCE STRENGTH INFORMATION:

Puddle Area: 107639 square feet

Average Puddle Depth: 1 centimeters

Soil Type: Default Ground Temperature: $29^{\circ} \mathrm{C}$

Initial Puddle Temperature: Ground temperature

Release Duration: ALOHA limited the duration to 1 hour

Max Computed Release Rate: 2.13 kilograms/min

Max Average Sustained Release Rate: 2.06 kilograms/min (averaged over a minute or more)

Total Amount Released: 118 kilograms

FOOTPRINT INFORMATION:

Dispersion Module: Gaussian

User specified LOC: 100 ppm

Max Threat Zone for LOC: 56 meters

Max Threat Zone for IDLH: 56 meters

Note: Footprint was not drawn because effects of near-field patchiness make plume presentation unreliable for short distances.

TIME DEPENDENT INFORMATION:

Concentration Estimates at the point:

Downwind: 100 meters

Off Centerline: 0 meters

Max Concentration:

Outdoor: $11.7 \mathrm{ppm}$

Indoor: $11.7 \mathrm{ppm}$

Note: Indoor graph is shown with a dotted line. 


\section{ENGINEERING COMPUTATION SHEET}

Title of Project EPHA for THM

Subject Concentration Analysis for THM Hazardous Chemicals

Computer T. M. Franey

Date $\underline{09 / 16 / 96}$
Reviewed by $\mathrm{S}$. A. Henderson

Works Calculation 7

Sheet No. 168 of 577

SITE DATA INFORMATION: 1-RD-33

Location: AIKEN, SOUTH CAROLINA

Building Air Exchanges Per Hour: 60 (User specified)

Date and Time: Fixed at July 1, 19960000 hours

CHEMICAL INFORMATION:

Chemical Name: MONOETHANOLAMINE

Molecular Weight: $61.08 \mathrm{~kg} / \mathrm{kmol}$

TLV-TWA: $3.00 \mathrm{ppm}$ IDLH: $1000.00 \mathrm{ppm}$

Footprint Level of Concern: 100 ppm

Boiling Point: $171.00^{\circ} \mathrm{C}$

Vapor Pressure at Ambient Temperature: 6.82e-004 atm

Ambient Saturation Concentration: 694 ppm or $0.069 \%$

ATMOSPHERIC INFORMATION: (MANUAL INPUT OF DATA)

Wind: 1.7 meters $/ \mathrm{sec}$ from $90^{\circ}$ true

Inversion Height: 200 meters

Stability Class: E Air Temperature: $29^{\circ} \mathrm{C}$

Relative Humidity: 50\% Ground Roughness: 100 centimeters

Cloud Cover: 1 tenths

SOURCE STRENGTH INFORMATION:

Puddle Area: 24420 square feet

Average Puddle Depth: 1 centimeters

Soil Type: Default Ground Temperature: $29^{\circ} \mathrm{C}$

Initial Puddle Temperature: Ground temperature

Release Duration: ALOHA limited the duration to 1 hour

Max Computed Release Rate: 516 grams/min

Max Average Sustained Release Rate: 500 grams/min

(averaged over a minute or more)

Total Amount Released: 28.6 kilograms

FOOTPRINT INFORMATION:

Dispersion Module: Gaussian

User specified LOC: $100 \mathrm{ppm}$

Max Threat Zone for LOC: 27 meters

Max Threat Zone for IDLH: 27 meters

Note: Footprint was not drawn because

effects of near-field patchiness make plume

presentation unreliable for short distances.

TIME DEPENDENT INFORMATION:

Concentration Estimates at the point:

Downwind: 100 meters

Off Centerline: 0 meters

Max Concentration:

Outdoor: $3.29 \mathrm{ppm}$

Indoor: $3.29 \mathrm{ppm}$

Note: Indoor graph is shown with a dotted line. 
Title of Project EPHA for THM

Subject Concentration Analysis for THM Hazardous Chemicals

Computer T. M. Franey

Date $\underline{09 / 16 / 96}$

Reviewed by S. A. Henderson

Works Calculation 7

Sheet No. 169 of 577

SITE DATA INFORMATION: 1-RD-33

Location: AIKEN, SOUTH CAROLINA

Building Air Exchanges Per Hour: 60 (User specified)

Date and Time: Fixed at July 1, 19960000 hours

CHEMICAL INFORMATION:

Chemical Name: MONOETHANOLAMINE

Molecular Weight: $61.08 \mathrm{~kg} / \mathrm{kmol}$

TLV-TWA: $3.00 \mathrm{ppm}$ IDLH: $1000.00 \mathrm{ppm}$

Footprint Level of Concern: 100 ppm

Boiling Point: $171.00^{\circ} \mathrm{C}$

Vapor Pressure at Ambient Temperature: 6.82e-004 atm

Ambient Saturation Concentration: 694 ppm or $0.069 \%$

ATMOSPHERIC INFORMATION: (MANUAL INPUT OF DATA)

Wind: 1.7 meters $/ \mathrm{sec}$ from $90^{\circ}$ true

Inversion Height: 200 meters

Stability Class: E Air Temperature: $29^{\circ} \mathrm{C}$

Relative Humidity: $50 \%$ Ground Roughness: 100 centimeters

Cloud Cover: 1 tenths

\section{SOURCE STRENGTH INFORMATTON:}

Puddle Area: 40700 square feet

Average Puddle Depth: 1 centimeters

Soil Type: Default Ground Temperature: $29^{\circ} \mathrm{C}$

Initial Puddle Temperature: Ground temperature

Release Duration: ALOHA limited the duration to 1 hour

Max Computed Release Rate: 841 grams/min

Max Average Sustained Release Rate: 814 grams/min

(averaged over a minute or more)

Total Amount Released: 46.6 kilograms

FOOTPRINT INFORMATION:

Dispersion Module: Gaussian

User specified LOC: 100 ppm

Max Threat Zone for LOC: 34 meters

Max Threat Zone for IDLH: 35 meters

Note: Footprint was not drawn because effects of near-field patchiness make plume presentation unreliable for short distances.

TIME DEPENDENT INFORMATION:

Concentration Estimates at the point:

Downwind: 100 meters

Off Centerline: 0 meters

Max Concentration:

Outdoor: $4.76 \mathrm{ppm}$

Indoor: $4.76 \mathrm{ppm}$

Note: Indoor graph is shown with a dotted line. 


\section{ENGINEERING COMPUTATION SHEET}

Title of Project EPHA for THM

Subject Concentration Analysis for THM Hazardous Chemicals

Computer T.M. Franey

Date $\underline{09 / 16 / 96}$

SITE DATA INFORMATION: 1-RD-33

Location: AIKEN, SOUTH CAROLINA

Building Air Exchanges Per Hour: 60 (User specified)

Date and Time: Fixed at July 1, 19960000 hours

CHEMICAL INFORMATION:

Chemical Name: MONOETHANOLAMINE

Molecular Weight: $61.08 \mathrm{~kg} / \mathrm{kmol}$

TLV-TWA: $3.00 \mathrm{ppm}$ IDLH: $1000.00 \mathrm{ppm}$

Footprint Level of Concern: 100 ppm

Boiling Point: $171.00^{\circ} \mathrm{C}$

Vapor Pressure at Ambient Temperature: 6.82e-004 atm

Ambient Saturation Concentration: 694 ppm or $0.069 \%$

ATMOSPHERIC INFORMATION: (MANUAL INPUT OF DATA)

Wind: 1.7 meters $/ \mathrm{sec}$ from $90^{\circ}$ true

Inversion Height: 200 meters

Stability Class: E Air Temperature: $29^{\circ} \mathrm{C}$

Relative Humidity: $50 \%$ Ground Roughness: 100 centimeters

Cloud Cover: 1 tenths

SOURCE STRENGTH INFORMATION:

Puddle Area: 10000 square meters

Average Puddle Depth: 1 centimeters

Soil Type: Default Ground Temperature: $29^{\circ} \mathrm{C}$

Initial Puddle Temperature: Ground temperature

Release Duration: ALOHA limited the duration to 1 hour

Max Computed Release Rate: 2.13 kilograms/min

Max Average Sustained Release Rate: 2.06 kilograms/min

(averaged over a minute or more)

Total Amount Released: 118 kilograms

FOOTPRINT INFORMATION:

Dispersion Module: Gaussian

User specified LOC: $100 \mathrm{ppm}$

Max Threat Zone for LOC: 56 meters

Max Threat Zone for IDLH: 56 meters

Note: Footprint was not drawn because

effects of near-field patchiness make plume

presentation unreliable for short distances.

TIME DEPENDENT INFORMATION:

Concentration Estimates at the point:

Downwind: 100 meters

Off Centerline: 0 meters

Max Concentration:

Outdoor: $11.7 \mathrm{ppm}$

Indoor: $11.7 \mathrm{ppm}$

Note: Indoor graph is shown with a dotted line.
Reviewed by S. A. Henderson

Works Calculation 7

Sheet No. 170 of 577 
Title of Project EPHA for THM

Subject Concentration Analysis for THM Hazardous Chemicals

Computer T. M. Franey

Date $\underline{09 / 16 / 96}$

Reviewed by S. A. Henderson

Works Calculation 7

Sheet No. 171 of 577

SITE DATA INFORMATION: 1-RD-34

Location: AIKEN, SOUTH CAROLINA

Building Air Exchanges Per Hour: 60 (User specified)

Date and Time: Fixed at July 1, 19960000 hours

CHEMICAL INFORMATION:

Chemical Name: MORPHOLINE Molecular Weight: $87.12 \mathrm{~kg} / \mathrm{kmol}$

TLV-TWA: $20.00 \mathrm{ppm}$ IDLH: $8000.00 \mathrm{ppm}$

Footprint Level of Concern: $1400 \mathrm{ppm}$

Boiling Point: $128.00^{\circ} \mathrm{C}$

Vapor Pressure at Ambient Temperature: $0.017 \mathrm{~atm}$

Ambient Saturation Concentration: $16,979 \mathrm{ppm}$ or $1.70 \%$

ATMOSPHERIC INFORMATION: (MANUAL INPUT OF DATA)

Wind: 1.7 meters $/ \mathrm{sec}$ from $90^{\circ}$ true

Inversion Height: 200 meters

Stability Class: E Air Temperature: $29^{\circ} \mathrm{C}$

Relative Humidity: 50\% Ground Roughness: 100 centimeters

Cloud Cover: 1 tenths

SOURCE STRENGTH INFORMATION:

Puddle Area: 30430 square feet

Average Puddle Depth: 1 centimeters

Soil Type: Default Ground Temperature: $29^{\circ} \mathrm{C}$

Initial Puddle Temperature: Ground temperature

Release Duration: ALOHA limited the duration to 1 hour

Max Computed Release Rate: 31.5 kilograms/min

Max Average Sustained Release Rate: 29.9 kilograms/min

(averaged over a minute or more)

Total Amount Released: 1,692 kilograms

FOOTPRINT INFORMATION:

Model Run: Heavy Gas

User specified LOC: 1400 ppm

Max Threat Zone for LOC: 19 meters

Max Threat Zone for IDLH: IDLH level was never exceeded

Note: Footprint wasn't drawn because effects of near-field patchiness make plume presentation unreliable for short distances.

\section{TIME DEPENDENT INFORMATION:}

Concentration Estimates at the point:

Downwind: 30 meters

Off Centerline: 0 meters

Max Concentration:

Outdoor: $1,130 \mathrm{ppm}$

Indoor: $1,130 \mathrm{ppm}$

Note: Indoor graph is shown with a dotted line. 


\section{ENGINEERING COMPUTATION SHEET}

Title of Project EPHA for THM

Reviewed by $\underline{\text { S. A. Henderson }}$

Subject Concentration Analysis for THM Hazardous Chemicals

Computer T. M. Franey

Date 09/16/96

SITE DATA INFORMATION: 1-RD-34

Location: AIKEN, SOUTH CAROLINA

Building Air Exchanges Per Hour: 60 (User specified)

Date and Time: Fixed at July 1, 19960000 hours

CHEMICAL INFORMATION:

Chemical Name: MORPHOLINE Molecular Weight: $87.12 \mathrm{~kg} / \mathrm{kmol}$

TLV-TWA: $20.00 \mathrm{ppm}$ IDLH: $8000.00 \mathrm{ppm}$

Footprint Level of Concern: 1400 ppm

Boiling Point: $128.00^{\circ} \mathrm{C}$

Vapor Pressure at Ambient Temperature: $0.017 \mathrm{~atm}$

Ambient Saturation Concentration: 16,979 ppm or $1.70 \%$

ATMOSPHERIC INFORMATION: (MANUAL INPUT OF DATA)

Wind: 1.7 meters $/ \mathrm{sec}$ from $90^{\circ}$ true

Inversion Height: 200 meters

Stability Class: E Air Temperature: $29^{\circ} \mathrm{C}$

Relative Humidity: 50\% Ground Roughness: 100 centimeters

Cloud Cover: 1 tenths

\section{SOURCE STRENGTH INFORMATION:}

Puddle Area: 107639 square feet

Average Puddle Depth: 1 centimeters

Soil Type: Default Ground Temperature: $29^{\circ} \mathrm{C}$

Initial Puddle Temperature: Ground temperature

Release Duration: ALOHA limited the duration to 1 hour

Max Computed Release Rate: 106 kilograms/min

Max Average Sustained Release Rate: 101 kilograms/min

(averaged over a minute or more)

Total Amount Released: 5,693 kilograms

\section{FOOTPRINT INFORMATION:}

Model Run: Heavy Gas

User specified LOC: $1400 \mathrm{ppm}$

Max Threat Zone for LOC: 41 meters

Max Threat Zone for IDLH: IDLH level was never exceeded

Note: Footprint wasn't drawn because effects of near-field patchiness make plume presentation unreliable for short distances.

\section{TIME DEPENDENT INFORMATION:}

Concentration Estimates at the point:

Downwind: 100 meters

Off Centerline: 0 meters

Max Concentration:

Outdoor: $638 \mathrm{ppm}$

Indoor: $638 \mathrm{ppm}$

Note: Indoor graph is shown with a dotted line.
Works Calculation 7

Sheet No. 172 of 577 


\section{ENGINEERING COMPUTATION SHEET}

Title of Project EPHA for THM

Subject Concentration Analysis for THM Hazardous Chemicals

Computer T. M. Franey

Date $\underline{09 / 16 / 96}$

SITE DATA INFORMATION: 1-RD-34

Location: AIKEN, SOUTH CAROLINA

Building Air Exchanges Per Hour: 60 (User specified)

Date and Time: Fixed at July 1, 19960000 hours

\section{CHEMICAL INFORMATION:}

Chemical Name: MORPHOLINE Molecular Weight: $87.12 \mathrm{~kg} / \mathrm{kmol}$ TLV-TWA: $20.00 \mathrm{ppm}$ IDLH: $8000.00 \mathrm{ppm}$

Footprint Level of Concern: $1400 \mathrm{ppm}$

Boiling Point: $128.00^{\circ} \mathrm{C}$

Vapor Pressure at Ambient Temperature: $0.017 \mathrm{~atm}$

Ambient Saturation Concentration: $16,979 \mathrm{ppm}$ or $1.70 \%$

ATMOSPHERIC INFORMATION: (MANUAL INPUT OF DATA)

Wind: 1.7 meters $/ \mathrm{sec}$ from $90^{\circ}$ true

Inversion Height: 200 meters

Stability Class: E Air Temperature: $29^{\circ} \mathrm{C}$

Relative Humidity: 50\% Ground Roughness: 100 centimeters

Cloud Cover: 1 tenths

\section{SOURCE STRENGTH INFORMATION:}

Puddle Area: 24420 square feet

Average Puddle Depth: 1 centimeters

Soil Type: Default Ground Temperature: $29^{\circ} \mathrm{C}$

Initial Puddle Temperature: Ground temperature

Release Duration: ALOHA limited the duration to 1 hour

Max Computed Release Rate: 25.5 kilograms/min

Max Average Sustained Release Rate: 24.2 kilograms/min

(averaged over a minute or more)

Total Amount Released: 1,370 kilograms

FOOTPRINT INFORMATION:

Dispersion Module: Gaussian

User specified LOC: $1400 \mathrm{ppm}$

Max Threat Zone for LOC: 27 meters

Max Threat Zone for IDLH: 27 meters

Note: Footprint was not drawn because effects of near-field patchiness make plume presentation unreliable for short distances.

TIME DEPENDENT INFORMATION:

Concentration Estimates at the point:

Downwind: 100 meters

Off Centerline: 0 meters

Max Concentration:

Outdoor: $112 \mathrm{ppm}$

Indoor: $112 \mathrm{ppm}$

Note: Indoor graph is shown with a dotted line.
Reviewed by S. A. Henderson

Works Calculation 7

Sheet No. 173 of 577 


\section{ENGINEERING COMPUTATION SHEET}

Title of Project EPHA for THM

Subject Concentration Analysis for THM Hazardous Chemicals

Computer T.M. Franey

Date 09/16/96

SITE DATA INFORMATION: 1-RD-34

Location: AIKEN, SOUTH CAROLINA

Building Air Exchanges Per Hour: 60 (User specified)

Date and Time: Fixed at July 1, 19960000 hours

CHEMICAL INFORMATION:

Chemical Name: MORPHOLINE Molecular Weight: $87.12 \mathrm{~kg} / \mathrm{kmol}$

TLV-TWA: 20.00 ppm IDLH: 8000.00 ppm

Footprint Level of Concern: 1400 ppm

Boiling Point: $128.00^{\circ} \mathrm{C}$

Vapor Pressure at Ambient Temperature: $0.017 \mathrm{~atm}$

Ambient Saturation Concentration: $16,979 \mathrm{ppm}$ or $1.70 \%$

ATMOSPHERIC INFORMATION: (MANUAL INPUT OF DATA)

Wind: 1.7 meters $/ \mathrm{sec}$ from $90^{\circ}$ true

Inversion Height: 200 meters

Stability Class: E Air Temperature: $29^{\circ} \mathrm{C}$

Relative Humidity: $50 \%$ Ground Roughness: 100 centimeters

Cloud Cover: 1 tenths

SOURCE STRENGTH INFORMATION:

Puddle Area: 40700 square feet

Average Puddle Depth: 1 centimeters

Soil Type: Default Ground Temperature: $29^{\circ} \mathrm{C}$

Initial Puddle Temperature: Ground temperature

Release Duration: ALOHA limited the duration to 1 hour

Max Computed Release Rate: 41.6 kilograms/min

Max Average Sustained Release Rate: 39.6 kilograms/min

(averaged over a minute or more)

Total Amount Released: 2,236 kilograms

FOOTPRINT INFORMATION:

Model Run: Heavy Gas

User specified LOC: $1400 \mathrm{ppm}$

Max Threat Zone for LOC: 26 meters

Max Threat Zone for IDLH: IDLH level was never exceeded

Note: Footprint wasn't drawn because effects of near-field patchiness make plume presentation unreliable for short distances.

TIME DEPENDENT INFORMATION:

Concentration Estimates at the point:

Downwind: 100 meters

Off Centerline: 0 meters

Max Concentration:

Outdoor: 348 ppm

Indoor: $348 \mathrm{ppm}$

Note: Indoor graph is shown with a dotted line.
Reviewed by $\underline{S}$. A. Henderson

Works Calculation 7

Sheet No. 174 of 577 


\section{ENGINEERING COMPUTATION SHEET}

Title of Project EPHA for THM

Reviewed by S. A. Henderson

Subject Concentration Analysis for THM Hazardous Chemicals

Computer T. M. Franey

Date $\underline{09 / 16 / 96}$

SITE DATA INFORMATION: 1-RD-34

Location: AIKEN, SOUTH CAROLINA

Building Air Exchanges Per Hour: 60 (User specified)

Date and Time: Fixed at July 1, 19960000 hours

CHEMICAL INFORMATION:

Chemical Name: MORPHOLINE Molecular Weight: $87.12 \mathrm{~kg} / \mathrm{kmol}$

TLV-TWA: $20.00 \mathrm{ppm}$

IDLH: $8000.00 \mathrm{ppm}$

Footprint Level of Concern: 1400 ppm

Boiling Point: $128.00^{\circ} \mathrm{C}$

Vapor Pressure at Ambient Temperature: $0.017 \mathrm{~atm}$

Ambient Saturation Concentration: 16,979 ppm or $1.70 \%$

ATMOSPHERIC INFORMATION: (MANUAL INPUT OF DATA)

Wind: 1.7 meters $/ \mathrm{sec}$ from $90^{\circ}$ true

Inversion Height: 200 meters

Stability Class: E Air Temperature: $29^{\circ} \mathrm{C}$

Relative Humidity: 50\% Ground Roughness: 100 centimeters

Cloud Cover: 1 tenths

SOURCE STRENGTH INFORMATION:

Puddle Area: 10000 square meters

Average Puddle Depth: 1 centimeters

Soil Type: Default $\quad$ Ground Temperature: $29^{\circ} \mathrm{C}$

Initial Puddle Temperature: Ground temperature

Release Duration: ALOHA limited the duration to 1 hour

Max Computed Release Rate: 106 kilograms/min

Max Average Sustained Release Rate: 101 kilograms/min

(averaged over a minute or more)

Total Amount Released: 5,693 kilograms

FOOTPRINT INFORMATION:

Model Run: Heavy Gas

User specified LOC: $1400 \mathrm{ppm}$

Max Threat Zone for LOC: 42 meters

Max Threat Zone for IDLH: IDLH level was never exceeded

Note: Footprint wasn't drawn because effects of

near-field patchiness make plume presentation

unreliable for short distances.

\section{TIME DEPENDENT INFORMATION:}

Concentration Estimates at the point:

Downwind: 100 meters

Off Centerline: 0 meters

Max Concentration:

Outdoor: 642 ppm

Indoor: $642 \mathrm{ppm}$

Note: Indoor graph is shown with a dotted line.
Works Calculation 7

Sheet No. 175 of 577 
Title of Project EPHA for THM

Subject Concentration Analysis for THM Hazardous Chemicals

Computer T. M. Franey

SITE DATA INFORMATION: 1-RD-35

Location: AIKEN, SOUTH CAROLINA

Building Air Exchanges Per Hour: 60 (User specified)

Date and Time: Fixed at July 1, 19960000 hours

\section{CHEMICAL INFORMATION:}

Chemical Name: BUTYL ALCOHOL

Molecular Weight: $74.12 \mathrm{~kg} / \mathrm{kmol}$

TLV-TWA: $50.00 \mathrm{ppm}$ IDLH: $8000.00 \mathrm{ppm}$

Footprint Level of Concern: 50 ppm

Boiling Point: $117.66^{\circ} \mathrm{C}$

Vapor Pressure at Ambient Temperature: $0.012 \mathrm{~atm}$

Ambient Saturation Concentration: 12,452 ppm or $1.25 \%$

ATMOSPHERIC INFORMATION: (MANUAL INPUT OF DATA)

Wind: 1.7 meters $/ \mathrm{sec}$ from $90^{\circ}$ true

Inversion Height: 200 meters

Stability Class: E Air Temperature: $29^{\circ} \mathrm{C}$

Relative Humidity: 50\% Ground Roughness: 100 centimeters

Cloud Cover: 1 tenths

\section{SOURCE STRENGTH INFORMATION:}

Puddle Area: 288 square feet

Average Puddle Depth: 1 centimeters

Soil Type: Default Ground Temperature: $29^{\circ} \mathrm{C}$

Initial Puddle Temperature: Ground temperature

Release Duration: ALOHA limited the duration to 1 hour

Max Computed Release Rate: 365 grams/min

Max Average Sustained Release Rate: 340 grams/min

(averaged over a minute or more)

Total Amount Released: 18.8 kilograms

FOOTPRINT INFORMATION:

Dispersion Module: Gaussian

User specified LOC: $50 \mathrm{ppm}$

Max Threat Zone for LOC: 30 meters

Max Threat Zone for IDLH: less than 10 meters(10.9 yards)

Note: Footprint was not drawn because effects of near-field patchiness make plume presentation unreliable for short distances.

\section{TIME DEPENDENT INFORMATION:}

Concentration Estimates at the point:

Downwind: 30 meters

Off Centerline: 0 meters

Max Concentration:

Outdoor: $50 \mathrm{ppm}$

Indoor: $50 \mathrm{ppm}$

Note: Indoor graph is shown with a dotted line. . 
Title of Project EPHA for THM

Subject Concentration Analysis for THM Hazardous Chemicals

Computer T. M. Franey

Date $\underline{09 / 16 / 96}$
Reviewed by $\mathrm{S}$. A. Henderson

Works Calculation 7

Sheet No. 177 of 577

\section{SITE DATA INFORMATION: 1-RD-35}

Location: AIKEN, SOUTH CAROLINA

Building Air Exchanges Per Hour: 60 (User specified)

Date and Time: Fixed at July 1, 19960000 hours

CHEMICAL INFORMATION:

Chemical Name: BUTYL ALCOHOL

Molecular Weight: $74.12 \mathrm{~kg} / \mathrm{kmol}$

TLV-TWA: $50.00 \mathrm{ppm}$ IDLH: $8000.00 \mathrm{ppm}$

Footprint Level of Concern: $50 \mathrm{ppm}$

Boiling Point: $117.66^{\circ} \mathrm{C}$

Vapor Pressure at Ambient Temperature: $0.012 \mathrm{~atm}$

Ambient Saturation Concentration: 12,452 ppm or $1.25 \%$

ATMOSPHERIC INFORMATION: (MANUAL INPUT OF DATA)

Wind: 1.7 meters $/ \mathrm{sec}$ from $90^{\circ}$ true

Inversion Height: 200 meters

Stability Class: E Air Temperature: $29^{\circ} \mathrm{C}$

Relative Humidity: $50 \%$ Ground Roughness: 100 centimeters

Cloud Cover: 1 tenths

\section{SOURCE STRENGTH INFORMATION:}

Puddle Area: 3630 square feet

Average Puddle Depth: 1 centimeters

Soil Type: Default Ground Temperature: $29^{\circ} \mathrm{C}$

Initial Puddle Temperature: Ground temperature

Release Duration: ALOHA limited the duration to 1 hour

Max Computed Release Rate: 4.05 kilograms/min

Max Average Sustained Release Rate: 3.79 kilograms/min

(averaged over a minute or more)

Total Amount Released: 211 kilograms

FOOTPRINT INFORMATION:

Dispersion Module: Gaussian

User specified LOC: $50 \mathrm{ppm}$

Max Threat Zone for LOC: 100 meters

Max Threat Zone for IDLH: 10 meters

Note: Footprint was not drawn because effects of near-field patchiness make plume presentation unreliable for short distances.

\section{TIME DEPENDENT INFORMATION:}

Concentration Estimates at the point:

Downwind: 100 meters

Off Centerline: 0 meters

Max Concentration:

Outdoor: $50 \mathrm{ppm}$

Indoor: $50 \mathrm{ppm}$

Note: Indoor graph is shown with a dotted line. 


\section{ENGINEERING COMPUTATION SHEET}

Title of Project EPHA for THM

Subject Concentration Analysis for THM Hazardous Chemicals

Computer T. M. Franey

Date 09/16/96

SITE DATA INFORMATION: 1-RD-35

Location: AIKEN, SOUTH CAROLINA

Building Air Exchanges Per Hour: 60 (User specified)

Date and Time: Fixed at July 1, 19960000 hours

CHEMICAL INFORMATION:

Chemical Name: BUTYL ALCOHOL

Molecular Weight: $74.12 \mathrm{~kg} / \mathrm{kmol}$

TLV-TWA: $50.00 \mathrm{ppm}$ IDLH: $8000.00 \mathrm{ppm}$

Footprint Level of Concern: $50 \mathrm{ppm}$

Boiling Point: $117.66^{\circ} \mathrm{C}$

Vapor Pressure at Ambient Temperature: 0.012 atm

Ambient Saturation Concentration: $12,452 \mathrm{ppm}$ or $1.25 \%$

ATMOSPHERIC INFORMATION: (MANUAL INPUT OF DATA)

Wind: 1.7 meters $/ \mathrm{sec}$ from $90^{\circ}$ true

Inversion Height: 200 meters

Stability Class: E Air Temperature: $29^{\circ} \mathrm{C}$

Relative Humidity: 50\% Ground Roughness: 100 centimeters

Cloud Cover: 1 tenths

\section{SOURCE STRENGTH INFORMATION:}

Puddle Area: 24420 square feet

Average Puddle Depth: 1 centimeters

Soil Type: Default Ground Temperature: $29^{\circ} \mathrm{C}$

Initial Puddle Temperature: Ground temperature

Release Duration: ALOHA limited the duration to 1 hour

Max Computed Release Rate: 25 kilograms/min

Max Average Sustained Release Rate: 23.4 kilograms/min

(averaged over a minute or more)

Total Amount Released: 1,306 kilograms

FOOTPRINT INFORMATION:

Dispersion Module: Gaussian

User specified LOC: $50 \mathrm{ppm}$

Max Threat Zone for LOC: 262 meters

Max Threat Zone for IDLH: 27 meters

TIME DEPENDENT INFORMATION:

Concentration Estimates at the point:

Downwind: 100 meters

Off Centerline: 0 meters

Max Concentration:

Outdoor: $127 \mathrm{ppm}$

Indoor: $127 \mathrm{ppm}$

Note: Indoor graph is shown with a dotted line.
Reviewed by $\mathrm{S}$. A. Henderson

Works Calculation 7

Sheet No. 178 of 577

Sheet No. 178 of 577


Title of Project EPHA for THM

Subject Concentration Analysis for THM Hazardous Chemicals

Computer T. M. Franey Date $\underline{09 / 16 / 96}$
Reviewed by $\underline{\text { S. A. Henderson }}$

Works Calculation 7

Sheet No. 179 of 577

\section{SITE DATA INFORMATION: 1-RD-35}

Location: AIKEN, SOUTH CAROLINA

Building Air Exchanges Per Hour: 60 (User specified)

Date and Time: Fixed at July 1, 19960000 hours

\section{CHEMICAL INFORMATION:}

Chemical Name: BUTYL ALCOHOL

Molecular Weight: $74.12 \mathrm{~kg} / \mathrm{kmol}$

TLV-TWA: $50.00 \mathrm{ppm}$ IDLH: $8000.00 \mathrm{ppm}$

Footprint Level of Concern: $50 \mathrm{ppm}$

Boiling Point: $117.66^{\circ} \mathrm{C}$

Vapor Pressure at Ambient Temperature: $0.012 \mathrm{~atm}$

Ambient Saturation Concentration: $12,452 \mathrm{ppm}$ or $1.25 \%$

\section{ATMOSPHERIC INFORMATION: (MANUAL INPUT OF DATA) \\ Wind: 1.7 meters $/ \mathrm{sec}$ from $90^{\circ}$ true \\ Inversion Height: 200 meters \\ Stability Class: E Air Temperature: $29^{\circ} \mathrm{C}$ \\ Relative Humidity: 50\% Ground Roughness: 100 centimeters \\ Cloud Cover: 1 tenths}

\section{SOURCE STRENGTH INFORMATION:}

Puddle Area: 40700 square feet

Average Puddle Depth: 1 centimeters

Soil Type: Default Ground Temperature: $29^{\circ} \mathrm{C}$

Initial Puddle Temperature: Ground temperature

Release Duration: ALOHA limited the duration to 1 hour

Max Computed Release Rate: 40.7 kilograms/min

Max Average Sustained Release Rate: 38.2 kilograms $/ \mathrm{min}$

(averaged over a minute or more)

Total Amount Released: 2,133 kilograms

\section{FOOTPRINT INFORMATION:}

Model Run: Heavy Gas

User specified LOC: $50 \mathrm{ppm}$

Max Threat Zone for LOC: 436 meters

Max Threat Zone for IDLH: IDLH level was never exceeded

Note: The Heavy Gas footprint is an initial screening.

For short releases it may be an overestimation.

Be sure to check concentration information at specific locations.

\section{TIME DEPENDENT INFORMATION:}

Concentration Estimates at the point:

Downwind: 100 meters

Off Centerline: 0 meters

Max Concentration:

Outdoor: $392 \mathrm{ppm}$

Indoor: $392 \mathrm{ppm}$

Note: Indoor graph is shown with a dotted line. 


\section{ENGINEERING COMPUTATION SHEET}

Title of Project EPHA for THM

Subject Concentration Analysis for THM Hazardous Chemicals

Computer T. M. Franey
Reviewed by S.A. Henderson

Works Calculation 7

Sheet No. 180 of 577

SITE DATA INFORMATION: 1-RD-35

Location: AIKEN, SOUTH CAROLINA

Building Air Exchanges Per Hour: 60 (User specified)

Date and Time: Fixed at July 1, 19960000 hours

CHEMICAL INFORMATION:

Chemical Name: BUTYL ALCOHOL

Molecular Weight: $74.12 \mathrm{~kg} / \mathrm{kmol}$

TLV-TWA: $50.00 \mathrm{ppm}$ IDLH: $8000.00 \mathrm{ppm}$

Footprint Level of Concern: $50 \mathrm{ppm}$

Boiling Point: $117.66^{\circ} \mathrm{C}$

Vapor Pressure at Ambient Temperature: 0.012 atm

Ambient Saturation Concentration: 12,452 ppm or $1.25 \%$

\section{ATMOSPHERIC INFORMATION: (MANUAL INPUT OF DATA)}

Wind: 1.7 meters $/ \mathrm{sec}$ from $90^{\circ}$ true

Inversion Height: 200 meters

Stability Class: E Air Temperature: $29^{\circ} \mathrm{C}$

Relative Humidity: 50\% Ground Roughness: 100 centimeters

Cloud Cover: 1 tenths

\section{SOURCE STRENGTH INFORMATION:}

Puddle Area: 10000 square meters

Average Puddle Depth: 1 centimeters

Soil Type: Default Ground Temperature: $29^{\circ} \mathrm{C}$

Initial Puddle Temperature: Ground temperature

Release Duration: ALOHA limited the duration to 1 hour

Max Computed Release Rate: 103 kilograms/min

Max Average Sustained Release Rate: 97.1 kilograms/min

(averaged over a minute or more)

Total Amount Released: 5,427 kilograms

\section{FOOTPRINT INFORMATION:}

Model Run: Heavy Gas

User specified LOC: $50 \mathrm{ppm}$

Max Threat Zone for LOC: 701 meters

Max Threat Zone for IDLH: IDLH level was never exceeded

Note: The Heavy Gas footprint is an initial screening.

For short releases it may be an overestimation.

Be sure to check concentration information at specific locations.

TIME DEPENDENT INFORMATION:

Concentration Estimates at the point:

Downwind: 100 meters

Off Centerline: 0 meters

Max Concentration:

Outdoor: $719 \mathrm{ppm}$

Indoor: $719 \mathrm{ppm}$

Note: Indoor graph is shown with a dotted line. 


\section{ENGINEERING COMPUTATION SHEET}

Title of Project EPHA for THM

Subject Concentration Analysis for THM Hazardous Chemicals

Computer T. M. Franey

Date $\underline{09 / 16 / 96}$
Reviewed by S.A. Henderson

Works Calculation 7

Sheet No. 181 of 577

1-RD-36 SITE DATA INFORMATION:

Location: AIKEN, SOUTH CAROLINA

Building Air Exchanges Per Hour: 60 (User specified)

Date and Time: Fixed at July 1, 19960000 hours

CHEMICAL INFORMATION:

Chemical Name: 1-METHYLPYRROLIDONE

Molecular Weight: $99.13 \mathrm{~kg} / \mathrm{kmol}$

TLV-TWA: -unavail- IDLH: -unavail-

Footprint Level of Concern: $1000 \mathrm{ppm}$

Boiling Point: $202.00^{\circ} \mathrm{C}$

Vapor Pressure at Ambient Temperature: 6.02e-004 atm

Ambient Saturation Concentration: 613 ppm or $0.061 \%$

ATMOSPHERIC INFORMATION: (MANUAL INPUT OF DATA)

Wind: 1.7 meters $/ \mathrm{sec}$ from $90^{\circ}$ true

Inversion Height: 200 feet

Stability Class: E Air Temperature: $29^{\circ} \mathrm{C}$

Relative Humidity: 50\% Ground Roughness: Urban or forest

Cloud Cover: 1 tenths

SOURCE STRENGTH INFORMATION:

Puddle Area: 30000 square feet

Average Puddle Depth: 1 centimeters

Soil Type: Default Ground Temperature: $29^{\circ} \mathrm{C}$

Initial Puddle Temperature: Ground temperature

Release Duration: ALOHA limited the duration to 1 hour

Max Computed Release Rate: 1.31 kilograms/min

Max Average Sustained Release Rate: 1.27 kilograms/min

(averaged over a minute or more)

Total Amount Released: 73.3 kilograms

TIME DEPENDENT INFORMATION:

Concentration Estimates at the point:

Downwind: 30 meters

Off Centerline: 0 meters

Max Concentration:

Outdoor: 46.9 ppm 
Title of Ptoject EPHA for THM

Subject Concentration Analysis for THM Hazardous Chemicals

Computer T. M. Franey
Reviewed by $\underline{S . \dot{A} \text {. Henderson }}$

Works Calculation 7

Sheet No. 182 of 577

\section{1-RD-36 SITE DATA INFORMATION:}

Location: AIKEN, SOUTH CAROLINA

Building Air Exchanges Per Hour: 60 (User specified)

Date and Time: Fixed at July 1, 19960000 hours

CHEMICAL INFORMATION:

Chemical Name: 1-METHYLPYRROLIDONE

Molecular Weight: $99.13 \mathrm{~kg} / \mathrm{kmol}$

TLV-TWA: -unavail- IDLH: -unavail-

Footprint Level of Concern: 1000 ppm

Boiling Point: $202.00^{\circ} \mathrm{C}$

Vapor Pressure at Ambient Temperature: 6.02e-004 atm

Ambient Saturation Concentration: 613 ppm or $0.061 \%$

ATMOSPHERIC INFORMATION: (MANUAL INPUT OF DATA)

Wind: 1.7 meters $/ \mathrm{sec}$ from $90^{\circ}$ true

Inversion Height: 200 feet

Stability Class: E Air Temperature: $29^{\circ} \mathrm{C}$

Relative Humidity: 50\% Ground Roughness: Urban or forest

Cloud Cover: 1 tenths

\section{SOURCE STRENGTH INFORMATION:}

Puddle Area: 100000 square feet

Average Puddle Depth: 1 centimeters

Soil Type: Default Ground Temperature: $29^{\circ} \mathrm{C}$

Initial Puddle Temperature: Ground temperature

Release Duration: ALOHA limited the duration to 1 hour

Max Computed Release Rate: 4.15 kilograms/min

Max Average Sustained Release Rate: 4.02 kilograms/min

(averaged over a minute or more)

Total Amount Released: 233 kilograms

\section{TIME DEPENDENT INFORMATION:}

Concentration Estimates at the point:

Downwind: 100 meters

Off Centerline: 0 meters

Max Concentration:

Outdoor: $14.1 \mathrm{ppm}$ 
Title of Project EPHA for THM

Subject Concentration Analysis for THM Hazardous Chemicals

Computer T. M. Franey

Date $\underline{09 / 16 / 96}$

\section{1-RD-36 SITE DATA INFORMATION:}

Location: AIKEN, SOUTH CAROLINA

Building Air Exchanges Per Hour; 60 (User specified)

Date and Time: Fixed at July 1, 19960000 hours.

CHEMICAL INFORMATION:

Chemical Name: 1-METHYLPYRROLIDONE

Molecular Weight: $99.13 \mathrm{~kg} / \mathrm{kmol}$

TLV-TWA: -unavail- IDLH: -unavail-

Footprint Level of Concern: $1000 \mathrm{ppm}$

Boiling Point: $202.00^{\circ} \mathrm{C}$

Vapor Pressure at Ambient Temperature: 6.02e-004 atm

Ambient Saturation Concentration: 613 ppm or $0.061 \%$

ATMOSPHERIC INFORMATION: (MANUAL INPUT OF DATA)

Wind: 1.7. meters $/ \mathrm{sec}$ from $90^{\circ}$ true

Inversion Height: 200 feet

Stability Class: E Air Temperature: $29^{\circ} \mathrm{C}$

Relative Humidity: 50\% Ground Roughness: Urban or forest

Cloud Cover: 1 tenths

\section{SOURCE STRENGTH INFORMATION:}

Puddle Area: 24420 square feet

Average Puddle Depth: 1 centimeters

Soil Type: Default Ground Temperature: $29^{\circ} \mathrm{C}$

Initial Puddle Temperature: Ground temperature

Release Duration: ALOHA limited the duration to 1 hour

Max Computed Release Rate: 1.07 kilograms/min

Max Average Sustained Release Rate: 1.04 kilograms/min

(averaged over a minute or more)

Total Amount Released: 60.2 kilograms

FOOTPRINT INFORMATION:

Dispersion Module: Gaussian

User specified LOC: $1000 \mathrm{ppm}$

Max Threat Zone for LOC: 27 meters

Note: Footprint was not drawn because effects of near-field patchiness make plume presentation unreliable for short distances.

TIME DEPENDENT INFORMATION:

Concentration Estimates at the point:

Downwind: 100 meters

Off Centerline: 0 meters

Max Concentration:

Outdoor: $4.23 \mathrm{ppm}$
Reviewed by S. A. Henderson

Works Calculation 7

Sheet No. 183 of 577 
Title of Project EPHA for THM

Subject Concentration Analysis for THM Hazardous Chemicals

Computer T. M. Franey

\section{1-RD-36 SITE DATA INFORMATION:}

Location: AIKEN, SOUTH CAROLINA

Building Air Exchanges Per Hour: 60 (User specified)

Date and Time: Fixed at July 1, 19960000 hours

\section{CHEMICAL INFORMATION:}

Chemical Name: 1-METHYLPYRROLIDONE

Molecular Weight: $99.13 \mathrm{~kg} / \mathrm{kmol}$

TLV-TWA: -unavail- IDLH: -unavail-

Footprint Level of Concern: 1000 ppm

Boiling Point: $202.00^{\circ} \mathrm{C}$

Vapor Pressure at Ambient Temperature: 6.02e-004 atm

Ambient Saturation Concentration: 613 ppm or $0.061 \%$

ATMOSPHERIC INFORMATION: (MANUAL INPUT OF DATA)

Wind: 1.7 meters $/ \mathrm{sec}$ from $90^{\circ}$ true

Inversion Height: 200 feet

Stability Class: E Air Temperature: $29^{\circ} \mathrm{C}$

Relative Humidity: 50\% Ground Roughness: Urban or forest

Cloud Cover: 1 tenths

\section{SOURCE STRENGTH INFORMATION:}

Puddle Area: 40700 square feet

Average Puddle Depth: 1 centimeters

Soil Type: Default Ground Temperature: $29^{\circ} \mathrm{C}$

Initial Puddle Temperature: Ground temperature

Release Duration: ALOHA limited the duration to 1 hour

Max Computed Release Rate: 1.75 kilograms $/ \mathrm{min}$

Max Average Sustained Release Rate: 1.7 kilograms/min (averaged over a minute or more)

Total Amount Released: 98.2 kilograms

FOOTPRINT INFORMATION:

Dispersion Module: Gaussian

User specified LOC: $1000 \mathrm{ppm}$

Max Threat Zone for LOC: 34 meters

Note: Footprint was not drawn because effects of near-field patchiness make plume presentation unreliable for short distances.

TIME DEPENDENT INFORMATION:

Concentration Estimates at the point:

Downwind: 100 meters

Off Centerline: 0 meters

Max Concentration:

Outdoor: $6.13 \mathrm{ppm}$ 
Title of Project EPHA for THM

Subject Concentration Analysis for THM Hazardous Chemicals

Computer T.M. Franey

Date 09/16/96
Reviewed by $\underline{S}$. A. Hénderson

Works Calculation 7

Sheet No. 185 of 577

1-RD-36 SITE DATA INFORMATTON:

Location: AIKEN, SOUTH CAROLINA

Building Air Exchanges Per Hour: 60 (User specified)

Date and Time: Fixed at July 1, 19960000 hours

CHEMICAL INFORMATION:

Chemical Name: 1-METHYLPYRROLIDONE

Molecular Weight: $99.13 \mathrm{~kg} / \mathrm{kmol}$

TLV-TWA: -unavail- - IDLH: -unavail-

Footprint Level of Concern: $1000 \mathrm{ppm}$

Boiling Point: $202.00^{\circ} \mathrm{C}$

Vapor Pressure at Ambient Temperature: 6.02e-004 atm

Ambient Saturation Concentration: 613 ppm or $0.061 \%$

ATMOSPHERIC INFORMATION: (MANUAL INPUT OF DATA)

Wind: 1.7 meters $/ \mathrm{sec}$ from $90^{\circ}$ true

Inversion Height: 200 feet

Stability Class: E Air Temperature: $29^{\circ} \mathrm{C}$

Relative Humidity: 50\% Ground Roughness: Urban or forest

Cloud Cover: 1 tenths

\section{SOURCE STRENGTH INFORMATION:}

Puddle Area: 10000 square meters

Average Puddle Depth: 1 centimeters

Soil Type: Default Ground Temperature: $29^{\circ} \mathrm{C}$

Initial Puddle Temperature: Ground temperature

Release Duration: ALOHA limited the duration to 1 hour

Max Computed Release Rate: 4.46 kilograms/min

Max Average Sustained Release Rate: 4.32 kilograms/min

(averaged over a minute or more)

Total Amount Released: 250 kilograms

\section{FOOTPRINT INFORMATION:}

Dispersion Module: Gaussian

User specified LOC: 1000 ppm

Max Threat Zone for LOC: 56 meters

Note: Footprint was not drawn becaise effects of near-field patchiness make plume

presentation unreliable for short distances.

TIME DEPENDENT INFORMATION:

Concentration Estimates at the point:

Downwind: 100 meters

Off Centerline: 0 meters

Max Concentration:

Outdoor: $15.1 \mathrm{ppm}$ 
Title of Project EPHA for THM

Subject Concentration Analysis for THM Hazardous Chemicals

Computer T. M. Franey

Date 09/16/96
Reviewed by S. A. Henderson

Works Calculation 7

Sheet No. 186 of 577

SITE DATA INFORMATION: $\quad$ 1-RD-37

Location: AIKEN, SOUTH CAROLINA

Building Air Exchanges Per Hour: 60 (User specified)

Date and Time: Fixed at July 1, 19960000 hours

CHEMICAL INFORMATION:

Chemical Name: NITRIC ACID; FUMING OR CONCENTRATED

Molecular Weight: $63.01 \mathrm{~kg} / \mathrm{kmol}$

TLV-TWA: $2.00 \mathrm{ppm}$

IDLH: $100.00 \mathrm{ppm}$

Footprint Level of Concern: 15 ppm

Boiling Point: $83.00^{\circ} \mathrm{C}$

Vapor Pressure at Ambient Temperature: $0.10 \mathrm{~atm}$

Ambient Saturation Concentration: 104,109 ppm or $10.4 \%$

ATMOSPHERIC INFORMATION: (MANUAL INPUT OF DATA)

Wind: 1.7 meters $/ \mathrm{sec}$ from $90^{\circ}$ true

Inversion Height: 200 meters

Stability Class: E Air Temperature: $29^{\circ} \mathrm{C}$

Relative Humidity: 50\% Ground Roughness: 100 centimeters

Cloud Cover: 1 tenths

\section{SOURCE STRENGTH INFORMATION:}

Puddle Area: 12 square feet

Average Puddle Depth: 1 centimeters

Soil Type: Default Ground Temperature: $29^{\circ} \mathrm{C}$

Initial Puddle Temperature: Ground temperature

Release Duration: ALOHA limited the duration to 1 hour

Max Computed Release Rate: 67.3 grams/min

Max Average Sustained Release Rate: $54.2 \mathrm{grams} / \mathrm{min}$

(averaged over a minute or more)

Total Amount Released: 2.70 kilograms

TIME DEPENDENT INFORMATION:

Concentration Estimates at the point:

Downwind: 30 meters

Off Centerline: 0 meters

. Max Concentration:

Outdoor: $15.4 \mathrm{ppm}$

Indoor: $15.4 \mathrm{ppm}$

Note: Indoor graph is shown with a dotted line. 
Title of Project EPHA for THM

Subject Concentration Analysis for THM Hazardous Chemicals

Computer T. M. Franey

Date 09/16/96

Reviewed by S. A. Henderson

Works Calculation 7

Sheet No. 187 of 577

\section{SITE DATA INFORMATION: $\quad$ 1-RD-37 \\ Location: AIKEN, SOUTH CAROLINA \\ Building Air Exchanges Per Hour: 60 (User specified) \\ Date and Time: Fixed at July 1, 19960000 hours}

\section{CHEMICAL INFORMATION:}

Chemical Name: NITRIC ACID; FUMING OR CONCENTRATED

Molecular Weight: $63.01 \mathrm{~kg} / \mathrm{kmol}$

TLV-TWA: $2.00 \mathrm{ppm}$ IDLH: $100.00 \mathrm{ppm}$
Footprint Level of Concern: $15 \mathrm{ppm}$

Boiling Point: $83.00^{\circ} \mathrm{C}$

Vapor Pressure at Ambient Temperature: $0.10 \mathrm{~atm}$

Ambient Saturation Concentration: $104,109 \mathrm{ppm}$ or $10.4 \%$

\section{ATMOSPHERIC INFORMATION: (MANUAL INPUT OF DATA)}

Wind: 1.7 meters $/ \mathrm{sec}$ from $90^{\circ}$ true

Inversion Height: 200 meters

Stability Class: E Air Temperature: $29^{\circ} \mathrm{C}$

Relative Humidity: 50\% Ground Roughness: 100 centimeters

Cloud Cover: 1 tenths

\section{SOURCE STRENGTH INFORMATION:}

Puddle Area: 141 square feet

Average Puddle Depth: 1 centimeters

Soil Type: Default Ground Temperature: $29^{\circ} \mathrm{C}$

Initial Puddle Temperature: Ground temperature

Release Duration: ALOHA limited the duration to 1 hour

Max Computed Release Rate: 679 grams/min

Max Average.Sustained Release Rate: 559 grams/min

(averaged over a minute or more)

Total Amount Released: 28.1 kilograms

\section{FOOTPRINT INFORMATION:}

Dispersion Module: Gaussian

User specified LOC: $15 \mathrm{ppm}$

Max Threat Zone for LOC: 100 meters

Max Threat Zone for IDLH: 34 meters

Note: Footprint was not drawn because effects of near-field patchiness make plume presentation unreliable for short distances.

\section{TIME DEPENDENT INFORMATION:}

Concentration Estimates at the point:

Downwind: 100 meters

Off Centerline: 0 meters

Max Concentration:

Outdoor: $15 \mathrm{ppm}$

Indoor: $15 \mathrm{ppm}$

Note: Indoor graph is shown with a dotted line. 


\section{ENGINEERING COMPUTATION SHEET}

Title of Project EPHA for THM

Subject Concentration Analysis for THM Hazardous Chemicals

Computer T. M. Franey Date 09/16/96

\section{SITE DATA INFORMATION: 1-RD-37}

Location: AIKEN, SOUTH CAROLINA

Building Air Exchanges Per Hour: 60 (User specified)

Date and Time: Fixed at July 1, 19960000 hours

\section{CHEMICAL INFORMATION:}

Chemical Name: NITRIC ACID; FUMING OR CONCENTRATED

Molecular Weight: $63.01 \mathrm{~kg} / \mathrm{kmol}$

TLV-TWA: $2.00 \mathrm{ppm}$ IDLH: $100.00 \mathrm{ppm}$

Footprint Level of Concern: $15 \mathrm{ppm}$

Boiling Point: $83.00^{\circ} \mathrm{C}$

Vapor Pressure at Ambient Temperature: $0.10 \mathrm{~atm}$

Ambient Saturation Concentration: 104,109 ppm or $10.4 \%$

ATMOSPHERIC INFORMATION: (MANUAL INPUT OF DATA)

Wind: 1.7 meters $/ \mathrm{sec}$ from $90^{\circ}$ true

Inversion Height: 200 meters

Stability Class: E Air Temperature: $29^{\circ} \mathrm{C}$

Relative Humidity: 50\% Ground Roughness: 100 centimeters

Cloud Cover: 1 tenths

SOURCE STRENGTH INFORMATION:

Puddle Area: 24420 square feet

Average Puddle Depth: 1 centimeters

Soil Type: Default Ground Temperature: $29^{\circ} \mathrm{C}$

Initial Puddle Temperature: Ground temperature

Release Duration: ALOHA limited the duration to 1 hour

Max Computed Release Rate: 90.3 kilograms/min

Max Average Sustained Release Rate: 76.9 kilograms/min

(averaged over a minute or more)

Total Amount Released:'3,947 kilograms

FOOTPRINT INFORMATION:

Model Run: Heavy Gas

User specified LOC: $15 \mathrm{ppm}$

Max Threat Zone for LOC: 1.7 kilometers

Max Threat Zone for IDLH: 467 meters

Note: The Heavy Gas footprint is an initial screening.

For short releases it may be an overestimation.

Be sure to check concentration information at specific locations.

\section{TIME DEPENDENT INFORMATION:}

Concentration Estimates at the point:

Downwind: 100 meters

Off Centerline: 0 meters

Max Concentration:

Outdoor: $1,100 \mathrm{ppm}$

Indoor: $1,100 \mathrm{ppm}$

Note: Indoor graph is shown with a dotted line. .
Reviewed by S. A. Henderson

Works Calculation 7

Sheet No. 188 of 577 
Title of Project EPHA for THM

Subject Concentration Analysis for THM Hazardous Chemicals

Computer T. M. Franey
Reviewed by S. A. Henderson

Works Calculation 7

Sheet No. 189 of 577

\section{SITE DATA INFORMATION: 1-RD-37 \\ Location: AIKEN, SOUTH CAROLINA \\ Building Air Exchanges Per Hour: 60 (User specified) \\ Date and Time: Fixed at July 1, 19960000 hours}

\section{CHEMICAL INFORMATION:}

Chemical Name: NITRIC ACID; FUMING OR CONCENTRATED

Molecular Weight: $63.01 \mathrm{~kg} / \mathrm{kmol}$

TLV-TWA: $2.00 \mathrm{ppm}$

Footprint Level of Concern: 15 ppm

Boiling Point: $83.00^{\circ} \mathrm{C}$

Vapor Pressure at Ambient Temperature: $0.10 \mathrm{~atm}$

Ambient Saturation Concentration: 104,109 ppm or $10.4 \%$

\section{ATMOSPHERIC INFORMATION: (MANUAL INPUT OF DATA)}

Wind: 1.7 meters $/ \mathrm{sec}$ from $90^{\circ}$ true

Inversion Height: 200 meters

Stability Class: E Air Temperature: $29^{\circ} \mathrm{C}$

Relative Humidity: 50\% Ground Roughness: 100 centimeters

Cloud Cover: 1 tenths

\section{SOURCE STRENGTH INFORMATION:}

Puddle Area: 40700 square feet

Average Puddle Depth: 1 centimeters

Soil Type: Default Ground Temperature: $29^{\circ} \mathrm{C}$

Initial Puddle Temperature: Ground temperature

Release Duration: ALOHA limited the duration to 1 hour

Max Computed Release Rate: 147 kilograms/min

Max Average Sustained Release Rate: 126 kilograms/min

(averaged over a minute or more)

Total Amount Released: 6,457 kilograms

\section{FOOTPRINT INFORMATION:}

Model Run: Heavy Gas

User specified LOC: $15 \mathrm{ppm}$

Max Threat Zone for LOC: 2.2 kilometers

Max Threat Zone for IDLH: 601 meters

Note: The Heavy Gas footprint is an initial screening.

For short releases it may be an overestimation.

Be sure to check concentration information at specific locations.

\section{TIME DEPENDENT INFORMATION:}

\section{Concentration Estimates at the point:}

Downwind: 100 meters

Off Centerline: 0 meters

Max Concentration:

Outdoor: 1,620 ppm

Indoor: $1,620 \mathrm{ppm}$

Note: Indoor graph is shown with a dotted line. 


\section{ENGINEERING COMPUTATION SHEET}

Title of Project EPHA for THM

Subject Concentration Analysis for THM Hazardous Chemicals

Computer T.M. Franey

Date 09/16/96
Reviewed by $\underline{\text { S. A. Henderson }}$

Works Calculation 7

Sheet No. 190 of 577

SITE DATA INFORMATION: 1-RD-37

Location: AIKEN, SOUTH CAROLINA

Building Air Exchanges Per Hour: 60 (User specified)

Date and Time: Fixed at July 1, 19960000 hours

CHEMICAL INFORMATION:

Chemical Name: NITRIC ACID; FUMING OR CONCENTRATED

Molecular Weight: $63.01 \mathrm{~kg} / \mathrm{kmol}$

TLV-TWA: $2.00 \mathrm{ppm}$ IDLH: $100.00 \mathrm{ppm}$

Footprint Level of Concern: 15 ppm

Boiling Point: $83.00^{\circ} \mathrm{C}$

Vapor Pressure at Ambient Temperature: $0.10 \mathrm{~atm}$

Ambient Saturation Concentration: 104,109 ppm or $10.4 \%$

ATMOSPHERIC INFORMATION: (MANUAL INPUT OF DATA)

Wind: 1.7 meters $/ \mathrm{sec}$ from $90^{\circ}$ true

Inversion Height: 200 meters

Stability Class: E Air Temperature: $29^{\circ} \mathrm{C}$

Relative Humidity: $50 \%$ Ground Roughness: 100 centimeters

Cloud Cover: 1 tenths

SOURCE STRENGTH INFORMATION:

Puddle Area: 10000 square meters

Average Puddle Depth: 1 centimeters

Soil Type: Default Ground Temperature: $29^{\circ} \mathrm{C}$

Initial Puddle Temperature: Ground temperature

Release Duration: ALOHA limited the duration to 1 hour

Max Computed Release Rate: 373 kilograms/min

Max Average Sustained Release Rate: 320 kilograms/min

(averaged over a minute or more)

Total Amount Released: 16,500 kilograms

FOOTPRINT INFORMATION:

Model Run: Heavy Gas

User specified LOC: $15 \mathrm{ppm}$

Max Threat Zone for LOC: 3.5 kilometers

Max Threat Zone for IDLH: 982 meters

Note: The Heavy Gas footprint is an initial screening.

For short releases it may be an overestimation.

Be sure to check concentration information at specific locations.

TIME DEPENDENT INFORMATION:

Concentration Estimates at the point:

Downwind: 100 meters

Off Centerline: 0 meters

Max Concentration:

Outdoor: $3,300 \mathrm{ppm}$

Indoor: $3,300 \mathrm{ppm}$

Note: Indoor graph is shown with a dotted line. 
OSR $\geq S-10(\operatorname{Rev} 6-18-42)$

\section{ENGINEERING COMPUTATION SHEET}

Title of Project EPHA for THM

Subject Concentration Analysis for THM Hazardous Chemicals

Reviewed by $\mathrm{S}$. A. Henderson

Works Calculation 7

Computer T. M. Franey

Date $\underline{09 / 16 / 96}$

Sheet No. 191 of 577

\section{1-RD-38}

This page intentionally left blank 


\section{ENGINEERING COMPUTATION SHEET}

Title of Project EPHA for THM

Subject Concentration Analysis for THM Hazardous Chemicals

Reviewed by S. A. Henderson

Works Calculation 7

Computer T. M. Franey

Date 09/16/96

1-RD-38

This page intentionally left blank

Sheet No. 192 of 577
$5 R S$ ingaman arrie... 
Title of Project EPHA for THM

Subject Concentration Analysis for THM Hazardous Chemicals

Computer T. M. Franey

Date $\underline{09 / 16 / 96}$
Reviewed by S. A. Henderson

Works Calculation 7

Sheet No. 193 of 577

1-RD-38

This page intentionally left blank 


\section{ENGINEERING COMPUTATION SHEET}

Title of Project EPHA for THM

Reviewed by $\underline{S}$. A. Henderson

Subject Concentration Analysis for THM Hazardous Chemicals

Computer I. M. Franey

Date 09/16/96

1-RD-38

This page intentionally left blank 
ENGINEERING COMPUTATION SHEET

Title of Project EPHA for THM

Reviewed by S. A. Henderson

Subject Concentration Analysis for THM Hazardous Chemicals

Computer T.M. Franey

Date 09/16/96
Works Calculation 7

Sheet No. 195 of 577

1-RD-38

This page intentionally left blank 


\section{ENGINEERING COMPUTATION SHEET}

Title of Project EPHA for THM

Subject Concentration Analysis for THM Hazardous Chemicals

Computer T.M. Franey Date $\underline{09 / 16 / 96}$

1-RD-39

SITE DATA INFORMATION:

Location: AIKEN, SOUTH CAROLINA

Building Air Exchanges Per Hour: 60 (User specified)

Date and Time: Fixed at July 1, 19960000 hours

CHEMICAL INFORMATION:

Chemical Name: TETRACHLOROETHYLENE

Molecular Weight: $165.83 \mathrm{~kg} / \mathrm{kmol}$

TLV-TWA: 50.00 ppm IDLH: $500.00 \mathrm{ppm}$

Note: Potential or confirmed human carcinogen.

Footprint Level of Concern: 200 ppm

Boiling Point: $121.25^{\circ} \mathrm{C}$

Vapor Pressure at Ambient Temperature: $0.030 \mathrm{~atm}$

Ambient Saturation Concentration: 30,601 ppm or $3.06 \%$

ATMOSPHERIC INFORMATION: (MANUAL INPUT OF DATA)

Wind: 1.7 meters $/ \mathrm{sec}$ from $90^{\circ}$ true

Inversion Height: 200 meters

Stability Class: E Air Temperature: $29^{\circ} \mathrm{C}$

Relative Humidity: $50 \%$ Ground Roughness: 100 centimeters

Cloud Cover: 1 tenths

\section{SOURCE STRENGTH INFORMATION:}

Puddle Area: 1175 square feet

Average Puddle Depth: 1 centimeters

Soil Type: Default Ground Temperature: $29^{\circ} \mathrm{C}$

Initial Puddle Temperature: Ground temperature

Release Duration: ALOHA limited the duration to 1 hour

Max Computed Release Rate: 4.7 kilograms/min

Max Average Sustained Release Rate: 4.4 kilograms/min (averaged over a minute or more)

Total Amount Released: 247 kilograms

\section{TIME DEPENDENT INFORMATION:}

Concentration Estimates at the point:

Downwind: 30 meters

Off Centerline: 0 meters

Max Concentration:

Outdoor: $149 \mathrm{ppm}$

Indoor: $149 \mathrm{ppm}$

Note: Indoor graph is shown with a dotted line.
Reviewed by $\underline{\text { S. A. Henderson }}$

Works Calculation 7

Sheet No. 196 of 577 
Title of Project EPHA for THM

Subject Concentration Analysis for THM Hazardous Chemicals

Computer T. M. Franey

SITE DATA INFORMATION:

$$
1-R D-39
$$

Location: AIKEN, SOUTH CAROLINA

Building Air Exchanges Per Hour: 60 (User specified)

Date and Time: Fixed at July 1, 19960000 hours

\section{CHEMICAL INFORMATION:}

Chemical Name: TETRACHLOROETHYLENE

Molecular Weight: $165.83 \mathrm{~kg} / \mathrm{kmol}$

TLV-TWA: $50.00 \mathrm{ppm}$ IDLH: $500.00 \mathrm{ppm}$

Note: Potential or confirmed human carcinogen.

Footprint Level of Concern: $200 \mathrm{ppm}$

Boiling Point: $121.25^{\circ} \mathrm{C}$

Vapor Pressure at Ambient Temperature: 0.030 atm

Ambient Saturation Concentration: $30,601 \mathrm{ppm}$ or $3.06 \%$

\section{ATMOSPHERIC INFORMATION: (MANUAL INPUT OF DATA)}

Wind: 1.7 meters $/ \mathrm{sec}$ from $90^{\circ}$ true

Inversion Height: 200 meters

Stability Class: E Air Temperature: $29^{\circ} \mathrm{C}$

Relative Humidity: 50\% Ground Roughness: 100 centimeters

Cloud Cover: 1 tenths

\section{SOURCE STRENGTH INFORMATION:}

Puddle Area: 8575 square feet

Average Puddle Depth: 1 centimeters

Soil Type: Default Ground Temperature: $29^{\circ} \mathrm{C}$

Initial Puddle Temperature: Ground temperature

Release Duration: ALOHA limited the duration to 1 hour

Max Computed Release Rate: 31.6 kilograms/min

Max Average Sustained Release Rate: 29.7 kilograms/min

(averaged over a minute or more)

Total Amount Released: 1,669 kilograms

\section{FOOTPRINT INFORMATION:}

Model Run: Heavy Gas

User specified LOC: $200 \mathrm{ppm}$

Max Threat Zone for LOC: 97 meters

Max Threat Zone for IDLH: 52 meters

Note: Footprint wasn't drawn because effects of near-field patchiness make plume presentation unreliable for short distances.

TIME DEPENDENT INFORMATION:

Concentration Estimates at the point:

Downwind: 100 meters

Off Centerline: 0 meters

Max Concentration:

Outdoor: $200 \mathrm{ppm}$

Indoor: $200 \mathrm{ppm}$ 
Title of Project EPHA for THM

Subject Concentration Analysis for THM Hazardous Chemicals

Computer T. M. Franey Date $\underline{09 / 16 / 96}$
Reviewed by S. A. Henderson

Works Calculation 7

Sheet No. 198 of 577

1-RD-39

SITE DATA INFORMATION:

Location: AIKEN, SOUTH CAROLINA

Building Air Exchanges Per Hour: 60 (User specified)

Date and Time: Fixed at July 1, 19960000 hours

CHEMICAL INFORMATION:

Chemical Name: TETRACHIOROETHYLENE

Molecular Weight: $165.83 \mathrm{~kg} / \mathrm{kmol}$

TLV-TWA: $50.00 \mathrm{ppm}$ IDLH: $500.00 \mathrm{ppm}$

Note: Potential or confirmed human carcinogen.

Footprint Level of Concern: 200 ppm

Boiling Point: $121.25^{\circ} \mathrm{C}$

Vapor Pressure at Ambient Temperature: $0.030 \mathrm{~atm}$

Ambient Saturation Concentration: 30,601 ppm or 3.06\%

ATMOSPHERIC INFORMATION: (MANUAL INPUT OF DATA)

Wind: 1.7 meters $/ \mathrm{sec}$ from $90^{\circ}$ true

Inversion Height: 200 meters

Stability Class: E Air Temperature: $29^{\circ} \mathrm{C}$

Relative Humidity: $50 \%$ Ground Roughness: 100 centimeters

Cloud Cover: 1 tenths

SOURCE STRENGTH INFORMATION:

Puddle Area: 24420 square feet

Average Puddle Depth: 1 centimeters

Soil Type: Default Ground Temperature: $29^{\circ} \mathrm{C}$

Initial Puddle Temperature: Ground temperature

Release Duration: ALOHA limited the duration to 1 hour

Max Computed Release Rate: 86.5 kilograms/min

Max Average Sustained Release Rate: 81.4 kilograms/min

(averaged over a minute or more)

Total Amount Released: 4,577 kilograms

FOOTPRINT INFORMATION:

Model Run: Heavy Gas

User specified LOC: $200 \mathrm{ppm}$

Max Threat Zone for LOC: 166 meters

Max Threat Zone for IDLH: 91 meters

Note: The Heavy Gas footprint is an initial screening.

For short releases it may be an overestimation.

Be sure to check concentration information at specific locations.

TIME DEPENDENT INFORMATION:

Concentration Estimates at the point:

Downwind: 100 meters

Off Centerline: 0 meters

Max Concentration:

Outdoor: $458 \mathrm{ppm}$

Indoor: $458 \mathrm{ppm}$

Note: Indoor graph is shown with a dotted line. 


\section{ENGINEERING COMPUTATION SHEET}

Title of Project EPHA for THM

Subject Concentration Analysis for THM Hazardous Chemicals

Computer T. M. Franey

Date $\underline{09 / 16 / 96}$

SITE DATA INFORMATION: 1-RD-39

Location: AIKEN, SOUTH CAROLINA

Building Air Exchanges Per Hour: 60 (User specified)

Date and Time: Fixed at July 1, 19960000 hours

CHEMICAL INFORMATION:

Chemical Name: TETRACHLOROETHYLENE

Molecular Weight: $165.83 \mathrm{~kg} / \mathrm{kmol}$

TLV-TWA: $50.00 \mathrm{ppm}$ IDLH: $500.00 \mathrm{ppm}$

Note: Potential or confirmed human carcinogen.

Footprint Level of Concern: 200 ppm

Boiling Point: $121.25^{\circ} \mathrm{C}$

Vapor Pressure at Ambient Temperature: $0.030 \mathrm{~atm}$

Ambient Saturation Concentration: $30,601 \mathrm{ppm}$ or $3.06 \%$

ATMOSPHERIC INFORMATION: (MANUAL INPUT OF DATA)

Wind: 1.7 meters $/ \mathrm{sec}$ from $90^{\circ}$ true

Inversion Height: 200 meters

Stability Class: E Air Temperature: $29^{\circ} \mathrm{C}$

Relative Humidity: 50\% Ground Roughness: 100 centimeters

Cloud Cover: 1 tenths

\section{SOURCE STRENGTH INFORMATION:}

Puddle Area: 40700 square feet

Average Puddle Depth: 1 centimeters

Soil Type: Default Ground Temperature: $29^{\circ} \mathrm{C}$

Initial Puddle Temperature: Ground temperature

Release Duration: ALOHA limited the duration to 1 hour

Max Computed Release Rate: 141 kilograms/min

Max Average Sustained Release Rate: 133 kilograms/min

(averaged over a minute or more)

Total Amount Released: 7,493 kilograms

FOOTPRINT INFORMATION:

Model Run: Heavy Gas

User specified LOC: 200 ppm

Max Threat Zone for LOC: 217 meters

Max Threat Zone for IDLH: 120 meters

Note: The Heavy Gas footprint is an initial screening.

For short releases it may be an overestimation.

Be sure to check concentration information at specific locations.

\section{TIME DEPENDENT INFORMATION:}

Concentration Estimates at the point:

Downwind: 100 meters

Off Centerline: 0 meters

Max Concentration:

Outdoor: $685 \mathrm{ppm}$

Indoor: $685 \mathrm{ppm}$

Note: Indoor graph is shown with a dotted line. 


\section{ENGINEERING COMPUTATION SHEET}

Title of Project EPHA for THM

Subject Concentration Analysis for THM Hazardous Chemicals

Computer T.M. Franey Date $09 / 16 / 96$

1-RD-39

SITE DATA INFORMATION:

Location: AIKEN, SOUTH CAROLINA

Building Air Exchanges Per Hour: 60 (User specified)

Date and Time: Fixed at July 1, 19960000 hours

CHEMICAL INFORMATION:

Chemical Name: TETRACHLOROETHYLENE

Molecular Weight: $165.83 \mathrm{~kg} / \mathrm{kmol}$

TLV-TWA: $50.00 \mathrm{ppm}$ IDLH: $500.00 \mathrm{ppm}$

Note: Potential or confirmed human carcinogen.

Footprint Level of Concern: 200 ppm

Boiling Point: $121.25^{\circ} \mathrm{C}$

Vapor Pressure at Ambient Temperature: $0.030 \mathrm{~atm}$

Ambient Saturation Concentration: 30,601 ppm or $3.06 \%$

ATMOSPHERIC INFORMATION: (MANUAL INPUT OF DATA)

Wind: 1.7 meters $/ \mathrm{sec}$ from $90^{\circ}$ true

Inversion Height: 200 meters

Stability Class: E Air Temperature: $29^{\circ} \mathrm{C}$

Relative Humidity: 50\% Ground Roughness: 100 centimeters

Cloud Cover: 1 tenths

SOURCE STRENGTH INFORMATION:

Puddle Area: 10000 square meters

Average Puddle Depth: 1 centimeters

Soil Type: Default Ground Temperature: $29^{\circ} \mathrm{C}$

Initial Puddle Temperature: Ground temperature

Release Duration: ALOHA limited the duration to 1 hour

Max Computed Release Rate: 361 kilograms/min

Max Average Sustained Release Rate: 340 kilograms/min

(averaged over a minute or more)

Total Amount Released: 19,169 kilograms

FOOTPRINT INFORMATION:

Model Run: Heavy Gas

User specified LOC: $200 \mathrm{ppm}$

Max Threat Zone for LOC: 363 meters

Max Threat Zone for IDLH: 204 meters

Note: The Heavy Gas footprint is an initial screening.

For short releases it may be an overestimation.

Be sure to check concentration information at specific locations.

TIME.DEPENDENT INFORMATION:

Concentration Estimates at the point:

Downwind: 100 meters

Off Centerline: 0 meters

Max Concentration:

Outdoor: $1,490 \mathrm{ppm}$

Indoor: $1,490 \mathrm{ppm}$

Note: Indoor graph is shown with a dotted line.
Reviewed by $\underline{S}$. A. Henderson

Works Calculation 7

Sheet No. 200 of 577 
Title of Project EPHA for THM

Subject Concentration Analysis for THM Hazardous Chemicals

Computer T. M. Franey

Date $\underline{09 / 16 / 96}$
Reviewed by S. A. Henderson

Works Calculation 7

Sheet No. 201 of 577

\section{SITE DATA INFORMATION: $\quad$ 1-RD-40 \\ Location: AIKEN, SOUTH CAROLINA \\ Building Air Exchanges Per Hour: 60 (User specified) \\ Date and Time: Fixed at July 1, 19960000 hours}

\section{CHEMICAL INFORMATION:}

Chemical Name: PHENOL

TLV-TWA: $5.00 \mathrm{ppm}$

Molecular Weight: $94.11 \mathrm{~kg} / \mathrm{kmol}$

Footprint Level of Concern: 50 ppm

Boiling Point: $181.84^{\circ} \mathrm{C}$

Vapor Pressure at Ambient Temperature: $0.0038 \mathrm{~atm}$

Ambient Saturation Concentration: 3,865 ppm or $0.39 \%$

\section{ATMOSPHERIC INFORMATION: (MANUAL INPUT OF DATA)}

Wind: 1.7 meters $/ \mathrm{sec}$ from $90^{\circ}$ true

Inversion Height: 200 meters

Stability Class: E Air Temperature: $50^{\circ} \mathrm{C}$

Relative Humidity: 50\% Ground Roughness: 100 centimeters

Cloud Cover: 1 tenths

\section{SOURCE STRENGTH INFORMATION:}

Puddle Area: 4550 square feet

Average Puddle Depth: 1 centimeters

Soil Type: Default Ground Temperature: $50^{\circ} \mathrm{C}$

Initial Puddle Temperature: Ground temperature

Release Duration: ALOHA limited the duration to 1 hour

Max Computed Release Rate: 1.19 kilograms/min

Max Average Sustained Release Rate: 1.19 kilograms/min (averaged over a minute or more)

Total Amount Released: 71.0 kilograms

TIME DEPENDENT INFORMATION:

Concentration Estimates at the point:

Downwind: 30 meters

Off Centerline: 0 meters

Max Concentration:

. Outdoor: 50 ppm

Indoor: $50 \mathrm{ppm}$

Note: Indoor graph is shown with a dotted line. 
Title of Project EPHA for THM

Subject Concentration Analysis for THM Hazardous Chemicals

Computer T. M. Franey Date $\underline{09 / 16 / 96}$
Reviewed by S. A. Henderson

Works Calculation 7

Sheet No. 202 of 577

SITE DATA INFORMATION: $\quad$ 1-RD-40

Location: AIKEN, SOUTH CAROLINA

Building Air Exchanges Per Hour: 60 (User specified)

Date and Time: Fixed at July 1, 19960000 hours

CHEMICAL INFORMATION:

Chemical Name: PHENOL Molecular Weight: $94.11 \mathrm{~kg} / \mathrm{kmol}$

TLV-TWA: $5.00 \mathrm{ppm}$ IDLH: $250.00 \mathrm{ppm}$

Footprint Level of Concern: $50 \mathrm{ppm}$

Boiling Point: $181.84^{\circ} \mathrm{C}$

Vapor Pressure at Ambient Temperature: $0.0038 \mathrm{~atm}$.

Ambient Saturation Concentration: 3,865 ppm or 0.39\%

ATMOSPHERIC INFORMATION: (MANUAL INPUT OF DATA)

Wind: 1.7 meters $/ \mathrm{sec}$ from $90^{\circ}$ true

Inversion Height: 200 meters

Stability Class: E Air Temperature: $50^{\circ} \mathrm{C}$

Relative Humidity: 50\% Ground Roughness: 100 centimeters

Cloud Cover: 1 tenths

\section{SOURCE STRENGTH INFORMATION:}

Puddle Area: 53500 square feet

Average Puddle Depth: 1 centimeters

Soil Type: Default Ground Temperature: $50^{\circ} \mathrm{C}$

Initial Puddle Temperature: Ground temperature

Release Duration: ALOHA limited the duration to 1 hour

Max Computed Release Rate: 12.6 kilograms/min

Max Average Sustained Release Rate: 12.6 kilograms/min

(averaged over a minute or more)

Total Amount Released: 753 kilograms

\section{TIME DEPENDENT INFORMATION:}

Concentration Estimates at the point:

Downwind: 100 meters

Off Centerline: 0 meters

Max Concentration:

Outdoor: $50 \mathrm{ppm}$

Indoor: $50 \mathrm{ppm}$

Note: Indoor graph is shown with a dotted line. 


\section{ENGINEERING COMPUTATION SHEET}

Title of Project EPHA for THM

Subject Concentration Analysis for THM Hazardous Chemicals

Computer T. M. Franey

SITE DATA INFORMATION:

$$
\text { 1-RD-40 }
$$

Location: AIKEN, SOUTH CAROLINA

Building Air Exchanges Per Hour: 60 (User specified)

Date and Time: Fixed at July 1, 19960000 hours

\section{CHEMICAL INFORMATION:}

Chemical Name: PHENOL Molecular Weight: $94.11 \mathrm{~kg} / \mathrm{kmol}$ TLV-TWA: $5.00 \mathrm{ppm}$ IDLH: $250.00 \mathrm{ppm}$

Footprint Level of Concern: $50 \mathrm{ppm}$

Boiling Point: $181.84^{\circ} \mathrm{C}$

Vapor Pressure at Ambient Temperature: 0.0038 atm

Ambient Saturation Concentration: 3,865 ppm or $0.39 \%$

\section{ATMOSPHERIC INFORMATION: (MANUAL INPUT OF DATA)}

Wind: 1.7 meters $/ \mathrm{sec}$ from $90^{\circ}$ true

Inversion Height: 200 meters

Stability Class: E Air Temperature: $50^{\circ} \mathrm{C}$

Relative Humidity: 50\% Ground Roughness: 100 centimeters

Cloud Cover: 1 tenths

\section{SOURCE STRENGTH INFORMATION:}

Puddle Area: 24420 square feet

Average Puddle Depth: 1 centimeters

Soil Type: Default Ground Temperature: $50^{\circ} \mathrm{C}$

Initial Puddle Temperature: Ground temperature

Release Duration: ALOHA limited the duration to 1 hour

Max Computed Release Rate: 5.94 kilograms/min

Max Average Sustained Release Rate: 5.92 kilograms $/ \mathrm{min}$ (averaged over a minute or more)

Total Amount Released: 355 kilograms

\section{FOOTPRINT INFORMATION:}

Dispersion Module: Gaussian

User specified LOC: $50 \mathrm{ppm}$

Max Threat Zone for LOC: 68 meters

Max Threat Zone for IDLH: 30 meters

Note: Footprint was not drawn because

effects of near-field patchiness make plume

presentation unreliable for short distances. 
Title of Project EPHA for THM

Subject Concentration Analysis for THM Hazardous Chemicals

Computer T. M. Franey

Date $\underline{09 / 16 / 96}$

$1-\mathrm{RD}-40$

SITE DATA INFORMATION:

Location: AIKEN, SOUTH CAROLINA

Building Air Exchanges Per Hour: 60 (User specified)

Date and Time: Fixed at July 1, 19960000 hours

CHEMICAL INFORMATION:

Chemical Name: PHENOL

TLV-TWA: 5.00 ppm

Molecular Weight: $94.11 \mathrm{~kg} / \mathrm{kmol}$

Footprint Level of Concern: 50 ppm

Boiling Point: $181.84^{\circ} \mathrm{C}$

Vapor Pressure at Ambient Temperature: 0.0038 atm

Ambient Saturation Concentration: 3,865 ppm or $0.39 \%$

\section{ATMOSPHERIC INFORMATION: (MANUAL INPUT OF DATA)}

Wind: 1.7 meters/sec from $90^{\circ}$ true

Inversion Height: 200 meters

Stability Class: E Air Temperature: $50^{\circ} \mathrm{C}$

Relative Humidity: $50 \%$ Ground Roughness: 100 centimeters

Cloud Cover: 1 tenths

SOURCE STRENGTH INFORMATION:

Puddle Area: 40700 square feet

Average Puddle Depth: 1 centimeters

Soil Type: Default Ground Temperature: $50^{\circ} \mathrm{C}$

Initial Puddle Temperature: Ground temperature

Release Duration: ALOHA limited the duration to 1 hour

Max Computed Release Rate: 9.68 kilograms/min

Max Average Sustained Release Rate: 9.67 kilograms/min

(averaged over a minute or more)

Total Amount Released: 579 kilograms

\section{FOOTPRINT INFORMATION:}

Dispersion Module: Gaussian

User specified LOC: 50 ppm

Max Threat Zone for LOC: 88 meters

Max Threat Zone for IDLH: 38 meters

Note: Footprint was not drawn because effects of near-field patchiness make plume presentation unreliable for short distances.
Reviewed by S. A. Henderson

Works Calculation 7

Sheet No. 204 of 577 
Title of Project EPHA for THM

Subject Concentration Analysis for THM Hazardous Chemicals

Computer T. M. Franey
Reviewed by S. A. Henderson

Works Calculation 7

Sheet No. 205 of 577

SITE DATA INFORMATION:

$$
1-\mathrm{RD}-40
$$

Location: AIKEN, SOUTH CAROLINA

Building Air Exchanges Per Hour: 60 (User specified)

Date and Time: Fixed at July 1, 19960000 hours

\section{CHEMICAL INFORMATION:}

Chemical Name: PHENOL Molecular Weight: $94.11 \mathrm{~kg} / \mathrm{kmol}$

TLV-TWA: $5.00 \mathrm{ppm}$ IDLH: $250.00 \mathrm{ppm}$

Footprint Level of Concern: $50 \mathrm{ppm}$

Boiling Point: $181.84^{\circ} \mathrm{C}$

Vapor Pressure at Ambient Temperature: $0.0038 \mathrm{~atm}$

Ambient Saturation Concentration: 3,865 ppm or $0.39 \%$

\section{ATMOSPHERIC INFORMATION: (MANUAL INPUT OF DATA) \\ Wind: 1.7 meters $/ \mathrm{sec}$ from $90^{\circ}$ true \\ Inversion Height: 200 meters \\ Stability Class: E Air Temperature: $50^{\circ} \mathrm{C}$ \\ Relative Humidity: 50\% Ground Roughness: 100 centimeters \\ Cloud Cover: 1 tenths}

\section{SOURCE STRENGTH INFORMATION:}

Puddle Area: 10000 square meters Average Puddle Depth: 1 centimeters

Soil Type: Default Ground Temperature: $50^{\circ} \mathrm{C}$

Initial Puddle Temperature: Ground temperature

Release Duration: ALOHA limited the duration to 1 hour

Max Computed Release Rate: 24.6 kilograms/min

Max Average Sustained Release Rate: 24.6 kilograms/min

(averaged over a minute or more)

Total Amount Released: 1,473 kilograms

FOOTPRINT INFORMATION:

Dispersion Module: Gaussian

User specified LOC: $50 \mathrm{ppm}$

Max Threat Zone for LOC: 142 meters

Max Threat Zone for IDLH: 61 meters 
This page cannot be converted.

Please view the native document

for the original page. 


\section{ENGINEERING COMPUTATION SHEET}

Title of Project EPHA for THM

Reviewed by $\underline{\text { S. A. Henderson }}$

Subject Concentration Analysis for THM Hazardous Chemicals

Computer T. M. Franey

Date $\underline{09 / 16 / 96}$

$$
1-R D-41
$$

SITE DATA INFORMATION:

Location: AIKEN, SOUTH CAROLINA

Building Air Exchanges Per Hour: 60 (User specified)

Date and Time: Fixed at July 1, 19960000 hours

CHEMICAL INFORMATION:

Chemical Name: PROPYL ALCOHOL

Molecular Weight: $60.10 \mathrm{~kg} / \mathrm{kmol}$

TLV-TWA: $200.00 \mathrm{ppm}$ IDLH: $4000.00 \mathrm{ppm}$

Footprint Level of Concern: 5 ppm

Boiling Point: $97.20^{\circ} \mathrm{C}$

Vapor Pressure at Ambient Temperature: $0.035 \mathrm{~atm}$

Ambient Saturation Concentration: $35,735 \mathrm{ppm}$ or $3.57 \%$

ATMOSPHERIC INFORMATION: (MANUAL INPUT OF DATA)

Wind: 1.7 meters $/ \mathrm{sec}$ from $90^{\circ}$ true

Inversion Height: 200 meters

Stability Class: E Air Temperature: $29^{\circ} \mathrm{C}$

Relative Humidity: 50\% Ground Roughness: 100 centimeters

Cloud Cover: 1 tenths

\section{SOURCE STRENGTH INFORMATION:}

Puddle Area: 71 square feet

Average Puddle Depth: 1 centimeters

Soil Type: Default Ground Temperature: $29^{\circ} \mathrm{C}$

Initial Puddle Temperature: Ground temperature

Release Duration: ALOHA limited the duration to 1 hour

Max Computed Release Rate: 202 grams/min

Max Average Sustained Release Rate: 176 grams/min

(averaged over a minute or more)

Total Amount Released: 9.26 kilograms

\section{TIME DEPENDENT INFORMATION:}

Concentration Estimates at the point:

Downwind: 100 meters

Off Centerline: 0 meters

Max Concentration:

Outdoor: $5.02 \mathrm{ppm}$

Indoor: $5.02 \mathrm{ppm}$

Note: Indoor graph is shown with a dotted line.
Works Calculation 7

Sheet No. 207 of 577 


\section{ENGINEERING COMPUTATION SHEET}

Title of Project EPHA for THM

Subject Concentration Analysis for THM Hazardous Chemicals

Computer T. M. Franey

Date 09/16/96
Reviewed by S. A. Henderson

Works Calculation 7

Sheet No. 208 of 577

SITE DATA INFORMATION:

$$
1-R D-41
$$

Location: AIKEN, SOUTH CAROLINA

Building Air Exchanges Per Hour: 60 (User specified)

Date and Time: Fixed at July 1, 19960000 hours

\section{CHEMICAL INFORMATION:}

Chemical Name: PROPYL ALCOHOL

Molecular Weight: $60.10 \mathrm{~kg} / \mathrm{kmol}$

TLV-TWA: $200.00 \mathrm{ppm}$ IDLH: $4000.00 \mathrm{ppm}$

Footprint Level of Concern: 5 ppm

Boiling Point: $97.20^{\circ} \mathrm{C}$

Vapor Pressure at Ambient Temperature: $0.035 \mathrm{~atm}$

Ambient Saturation Concentration: 35,735 ppm or $3.57 \%$

\section{ATMOSPHERIC INFORMATION: (MANUAL INPUT OF DATA)}

Wind: 1.7 meters $/ \mathrm{sec}$ from $90^{\circ}$ true

Inversion Height: 200 meters

Stability Class: E Air Temperature: $29^{\circ} \mathrm{C}$

Relative Humidity: 50\% Ground Roughness: 100 centimeters

Cloud Cover: 1 tenths

\section{SOURCE STRENGTH INFORMATION:}

Puddle Area: 24420 square feet

Average Puddle Depth: 1 centimeters

Soil Type: Default Ground Temperature: $29^{\circ} \mathrm{C}$

Initial Puddle Temperature: Ground temperature

Release Duration: ALOHA limited the duration to 1 hour

Max Computed Release Rate: 51.1 kilograms/min

Max Average Sustained.Release Rate: 45.8 kilograms/min

(averaged over a minute or more)

Total Amount Released: 2,447 kilograms

\section{FOOTPRINT INFORMATION:}

Model Run: Heavy Gas

User specified LOC: $5 \mathrm{ppm}$

Max Threat Zone for LOC: 2.7 kilometers

Max Threat Zone for IDLH: 15 meters

Note: The Heavy Gas footprint is an initial screening.

For short releases it may be an overestimation.

Be sure to check concentration information at specific locations.

\section{TIME DEPENDENT INFORMATION:}

Concentration Estimates at the point:

Downwind: 100 meters

Off Centerline: 0 meters

Max Concentration:

Outdoor: $675 \mathrm{ppm}$

Indoor: $675 \mathrm{ppm}$

Note: Indoor graph is shown with a dotted line. 
Title of Project EPHA for THM

Subject Concentration Analysis for THM Hazardous Chemicals

Computer T. M. Franey

\section{SITE DATA INFORMATION:}

$$
1-R D-41
$$

Location: AIKEN, SOUTH CAROLINA

Building Air Exchanges Per Hour: 60 (User specified)

Date and Time: Fixed at July 1, 19960000 hours

\section{CHEMICAL INFORMATION:}

Chemical Name: PROPYL ALCOHOL

Molecular Weight: $60.10 \mathrm{~kg} / \mathrm{kmol}$

TLV-TWA: $200.00 \mathrm{ppm}$ IDLH: $4000.00 \mathrm{ppm}$

Footprint Level of Concern: 5 ppm

Boiling Point: $97.20^{\circ} \mathrm{C}$

Vapor Pressure at Ambient Temperature: 0.035 atm

Ambient Saturation Concentration: 35,735 ppm or 3.57\%

\section{ATMOSPHERIC INFORMATION: (MANUAL INPUT OF DATA)}

Wind: 1.7 meters $/ \mathrm{sec}$ from $90^{\circ}$ true

Inversion Height: 200 meters

Stability Class: E Air Temperature: $29^{\circ} \mathrm{C}$

Relative Humidity: 50\% Ground Roughness: 100 centimeters

Cloud Cover: 1 tenths

\section{SOURCE STRENGTH INFORMATION:}

Puddle Area: 40700 square feet

Average Puddle Depth: 1 centimeters

Soil Type: Default Ground Temperature: $29^{\circ} \mathrm{C}$

Initial Puddle Temperature: Ground temperature

Release Duration: ALOHA limited the duration to 1 hour

Max Computed Release Rate: 83.2 kilograms/min

Max Average Sustained Release Rate: 74.7 kilograms/min

(averaged over a minute or more)

Total Amount Released: 3,997 kilograms

\section{FOOTPRINT INFORMATION:}

\section{Model Run: Heavy Gas}

User specified LOC: 5 ppm

Max Threat Zone for LOC: 3.7 kilometers

Max Threat Zone for IDLH: 25 meters

Note: The Heavy Gas footprint is an initial screening.

For short releases it may be an overestimation.

Be sure to check concentration information at specific locations.

\section{TIME DEPENDENT INFORMATION:}

Concentration Estimates at the point:

Downwind: 100 meters

Off Centerline: 0 meters

Max Concentration:

Outdoor: $963 \mathrm{ppm}$

Indoor: $963 \mathrm{ppm}$

Note: Indoor graph is shown with a dotted line. 
Title of Project EPHA for THM

Subject Concentration Analysis for THM Hazardous Chemicals

Computer T. M. Franey

Date $\underline{09 / 16 / 96}$

$$
\text { 1-RD-41 }
$$

SITE DATA INFORMATION:

Location: AIKEN, SOUTH CAROLINA

Building Air Exchanges Per Hour: 60 (User specified)

Date and Time: Fixed at July 1, 19960000 hours

\section{CHEMICAL INFORMATION:}

Chemical Name: PROPYL ALCOHOL

Molecular Weight: $60.10 \mathrm{~kg} / \mathrm{kmol}$

TLV-TWA: 200.00 ppm IDLH: 4000.00 ppm

Footprint Level of Concern: 5 ppm

Boiling Point: $97.20^{\circ} \mathrm{C}$

Vapor Pressure at Ambient Temperature: $0.035 \mathrm{~atm}$

Ambient Saturation Concentration: 35,735 ppm or $3.57 \%$

\section{ATMOSPHERIC INFORMATION: (MANUAL INPUT OF DATA)}

Wind: 1.7 meters $/ \mathrm{sec}$ from $90^{\circ}$ true

Inversion Height: 200 meters

Stability Class: E Air Temperature: $29^{\circ} \mathrm{C}$

Relative Humidity: $50 \%$ Ground Roughness: 100 centimeters

Cloud Cover: 1 tenths

\section{SOURCE STRENGTH INFORMATION:}

Puddle Area: 10000 square meters

Average Puddle Depth: 1 centimeters

Soil Type: Default Ground Temperature: $29^{\circ} \mathrm{C}$

Initial Puddle Temperature: Ground temperature

Release Duration: ALOHA limited the duration to 1 hour

Max Computed Release Rate: 211 kilograms/min

Max Average Sustained Release Rate: 190 kilograms/min

(averaged over a minute or more)

Total Amount Released: 10,186 kilograms

\section{FOOTPRINT INFORMATION:}

Model Run: Heavy Gas

User specified LOC: 5 ppm

Max Threat Zone for LOC: 5.9 kilometers

Max Threat Zone for IDLH: 42 meters

Note: The Heavy Gas footprint is an initial screening.

For short releases it may be an overestimation.

Be sure to check concentration information at specific locations.

TIME DEPENDENT INFORMATION:

Concentration Estimates at the point:

Downwind: 100 meters

Off Centerline: 0 meters

Max Concentration:

Outdoor: $1,850 \mathrm{ppm}$

Indoor: $1,850 \mathrm{ppm}$

Note: Indoor graph is shown with a dotted line.
Reviewed by S. A. Henderson

Works Calculation 7

Sheet No. 210 of 577 
Title of Project EPHA for THM

Subject Concentration Analysis for THM Hazardous Chemicals

Computer T. M. Franey Date $\underline{09 / 16 / 96}$
Reviewed by $\mathbf{S}$. A. Henderson

Works Calculation 7

Sheet No. 211 of 577

\section{SITE DATA INFORMATION: 1-RD-42}

Location: AIKEN, SOUTH CAROLINA

Building Air Exchanges Per Hour: 60 (User specified)

Date and Time: Fixed at July 1, 19960000 hours

CHEMICAL INFORMATION:

Chemical Name: PROPIONIC ACID

Molecular Weight: $74.08 \mathrm{~kg} / \mathrm{kmol}$

TLV-TWA: $10.00 \mathrm{ppm}$ IDLH: -unavail-

Footprint Level of Concern: $50 \mathrm{ppm}$

Boiling Point: $141.17^{\circ} \mathrm{C}$

Vapor Pressure at Ambient Temperature: $0.0055 \mathrm{~atm}$

Ambient Saturation Concentration: $5,590 \mathrm{ppm}$ or $0.56 \%$

ATMOSPHERIC INFORMATION: (MANUAL INPUT OF DATA)

Wind: 1.7 meters $/ \mathrm{sec}$ from $90^{\circ}$ true

Inversion Height: 200 meters

Stability Class: E Air Temperature: $29^{\circ} \mathrm{C}$

Relative Humidity: 50\% Ground Roughness: 100 centimeters

Cloud Cover: 1 tenths

\section{SOURCE STRENGTH INFORMATION:}

Puddle Area: 2850 square feet

Average Puddle Depth: 1 centimeters

Soil Type: Default Ground Temperature: $29^{\circ} \mathrm{C}$

Initial Puddle Temperature: Ground temperature ?

Release Duration: ALOHA limited the duration to 1 hour

Max Computed Release Rate: 978 grams/min

Max Average Sustained Release Rate: 938 grams/min

(averaged over a minute or more)

Total Amount Released: 53.5 kilograms

FOOTPRINT INFORMATION:

Dispersion Module: Gaussian

User specified LOC: $50 \mathrm{ppm}$

Max Threat Zone for LOC: 30 meters

Note: Footprint was not drawn because

effects of near-field patchiness make plume

presentation unreliable for short distances.

\section{TIME DEPENDENT INFORMATION:}

Concentration Estimates at the point:

Downwind: 30 meters

Off Centerline: 0 meters

Max Concentration:

Outdoor: $50 \mathrm{ppm}$

Indoor: $50 \mathrm{ppm}$

Note: Indoor graph is shown with a dotted line. 


\section{ENGINEERING COMPUTATION SHEET}

Title of Project EPHA for THM

Subject Concentration Analysis for THM Hazardous Chemicals

Computer T.M. Franey Date 09/16/96
Reviewed by S. A. Henderson

Works Calculation 7

Sheet No. 212 of 577

SITE DATA INFORMATION: 1-RD-42

Location: AIKEN, SOUTH CAROLINA

Building Air Exchanges Per Hour: 60 (User specified)

Date and Time: Fixed at July 1, 19960000 hours

\section{CHEMICAL INFORMATION:}

Chemical Name: PROPIONIC ACID

Molecular Weight: $74.08 \mathrm{~kg} / \mathrm{kmol}$

TLV-TWA: $10.00 \mathrm{ppm}$ IDLH: -unavail-

Footprint Level of Concern: 50 ppm

Boiling Point: $141.17^{\circ} \mathrm{C}$

Vapor Pressure at Ambient Temperature: $0.0055 \mathrm{~atm}$

Ambient Saturation Concentration: $5,590 \mathrm{ppm}$ or $0.56 \%$

\section{ATMOSPHERIC INFORMATION: (MANUAL INPUT OF DATA)}

Wind: 1.7 meters $/ \mathrm{sec}$ from $90^{\circ}$ true

Inversion Height: 200 meters

Stability Class: E Air Temperature: $29^{\circ} \mathrm{C}$

Relative Humidity: 50\% Ground Roughnéss: 100 centimeters

Cloud Cover: 1 tenths

\section{SOURCE STRENGTH INFORMATION:}

Puddle Area: 34300 square feet

Average Puddle Depth: 1 centimeters

Soil Type: Default Ground Temperature: $29^{\circ} \mathrm{C}$

Initial Puddle Temperature: Ground temperature

Release Duration: ALOHA limited the duration to 1 hour

Max Computed Release Rate: 10.5 kilograms/min

Max Average Sustained Release Rate: 10.1 kilograms/min (averaged over a minute or more)

Total Amount Released: 576 kilograms

\section{FOOTPRINT INFORMATION:}

Dispersion Module: Gaussian

User specified LOC: $50 \mathrm{ppm}$

Max Threat Zone for LOC: 100 meters

Note: Footprint was not drawn because effects of near-field patchiness make plume presentation unreliable for short distances.

\section{TIME DEPENDENT INFORMATION:}

- Concentration Estimates at the point:

Downwind: 100 meters

Off Centerline: 0 meters

Max Concentration:

Outdoor: $50 \mathrm{ppm}$

Indoor: $50 \mathrm{ppm}$

Note: Indoor graph is shown with a dotted line. 
Title of Project EPHA for THM

Subject Concentration Analysis for THM Hazardous Chemicals

Computer T. M. Franey Date $\underline{09 / 16 / 96}$
Reviewed by S. A. Henderson

Works Calculation 7

Sheet No. 213 of 577

SITE DATA INFORMATION: 1-RD-42

Location: AIKEN, SOUTH CAROLINA

Building Air Exchanges Per Hour: 60 (User specified)

Date and Time: Fixed at July 1, 19960000 hours

CHEMICAL INFORMATION:

Chemical Name: PROPIONIC ACID

Molecular Weight: $74.08 \mathrm{~kg} / \mathrm{kmol}$

TLV-TWA: $10.00 \mathrm{ppm}$ IDLH: -unavail-

Footprint Level of Concern: $50 \mathrm{ppm}$

Boiling Point: $141.17^{\circ} \mathrm{C}$

Vapor Pressure at Ambient Temperature: 0.0055 atm

Ambient Saturation Concentration: 5,590 ppm or $0.56 \%$

ATMOSPHERIC INFORMATION: (MANUAL INPUT OF DATA)

Wind: 1.7 meters $/ \mathrm{sec}$ from $90^{\circ}$ true

Inversion Height: 200 meters

Stability Class: E Air Temperature: $29^{\circ} \mathrm{C}$

Relative Humidity: 50\% Ground Roughness: 100 centimeters

Cloud Cover: 1 tenths

SOURCE STRENGTH INFORMATION:

Puddle Area: 24420 square feet

Average Puddle Depth: 1 centimeters

Soil Type: Default Ground Temperature: $29^{\circ} \mathrm{C}$

Initial Puddle Temperature: Ground temperature

Release Duration: ALOHA limited the duration to 1 hour

Max Computed Release Rate: 7.59 kilograms/min

Max Average.Sustained Release Rate: 7.29 kilograms/min

(averaged over a minute or more)

Total Amount Released: 416 kilograms

FOOTPRINT INFORMATION:

Dispersion Module: Gaussian

User specified LOC: $50 \mathrm{ppm}$

Max Threat Zone for LOC: 85 meters

Note: Footprint was not drawn because

effects of near-field patchiness make plume

presentation unreliable for short distances.

TIME DEPENDENT INFORMATION:

Concentration Estimates at the point:

Downwind: 100 meters

Off Centerline: 0 meters

Max Concentration:

Outdoor: $39.6 \mathrm{ppm}$

Indoor: $39.6 \mathrm{ppm}$

Note: Indoor graph is shown with a dotted line. 


\section{ENGINEERING COMPUTATION SHEET}

Title of Project EPHA for THM

Reviewed by $\underline{\text { S. A. Henderson }}$

Subject Concentration Analysis for THM Hazardous Chemicals

Computer T. M. Franey

Date $\underline{09 / 16 / 96}$

SITE DATA INFORMATION: 1-RD-42

Location: AIKEN, SOUTH CAROLINA

Building Air Exchanges Per Hour: 60 (User specified)

Date and Time: Fixed at July'1, 19960000 hours

\section{CHEMICAL INFORMATION:}

Chemical Name: PROPIONIC ACID

Molecular Weight: $74.08 \mathrm{~kg} / \mathrm{kmol}$

TLV-TWA: $10.00 \mathrm{ppm}$ IDLH: -unavail-

Footprint Level of Concern: 50 ppm

Boiling Point: $141.17^{\circ} \mathrm{C}$

Vapor Pressure at Ambient Temperature: $0.0055 \mathrm{~atm}$

Ambient Saturation Concentration: $5,590 \mathrm{ppm}$ or $0.56 \%$

ATMOSPHERIC INFORMATION: (MANUAL INPUT OF DATA)

Wind: 1.7 meters $/ \mathrm{sec}$ from $90^{\circ}$ true

Inversion Height: 200 meters

Stability Class: E Air Temperature: $29^{\circ} \mathrm{C}$

Relative Humidity: $50 \%$ Ground Roughness: 100 centimeters

Cloud Cover: 1 tenths

\section{SOURCE STRENGTH INFORMATION:}

Puddle Area: 40700 square feet

Average Puddle Depth: 1 centimeters

Soil Type: Default Ground Temperature: $29^{\circ} \mathrm{C}$

Initial Puddle Temperature: Ground temperature

Release Duration: ALOHA limited the duration to 1 hour

Max Computed Release Rate: 12.4 kilograms/min

Max Average Sustained Release Rate: 11.9 kilograms/min

(averaged over a minute or more)

Total Amount Released: 678 kilograms

\section{FOOTPRINT INFORMATION:}

Dispersion Module: Gaussian

User specified LOC: $50 \mathrm{ppm}$

Max Threat Zone for LOC: 109 meters

TIME DEPENDENT INFORMATION:

Concentration Estimates at the point:

Downwind: $\quad 100$ meters

Off Centerline: 0 meters

Max Concentration:

Outdoor: $57.4 \mathrm{ppm}$

Indoor: $57.4 \mathrm{ppm}$

Note: Indoor graph is shown with a dotted line.
Works Calculation 7

Sheet No. 214 of 577 


\section{ENGINEERING COMPUTATION SHEET}

Title of Project EPHA for THM

Subject Concentration Analysis for THM Hazardous Chemicals

Computer T. M. Franey

Date 09/16/96
Reviewed by $\underline{\text { S. A. Henderson }}$

Works Calculation 7

Sheet No. 215 of 577

CHEMICAL INFORMATION:

Chemical Name: PROPIONIC ACID

Molecular Weight: $74.08 \mathrm{~kg} / \mathrm{kmol}$

TLV-TWA: $10.00 \mathrm{ppm}$ IDLH: -unavail-

Footprint Level of Concern: $50 \mathrm{ppm}$

Boiling Point: $141.17^{\circ} \mathrm{C}$

Vapor Pressure at Ambient Temperature: $0.0055 \mathrm{~atm}$

Ambient Saturation Concentration: $5,590 \mathrm{ppm}$ or $0.56 \%$

ATMOSPHERIC INFORMATION: (MANUAL INPUT OF DATA)

Wind: 1.7 meters $/ \mathrm{sec}$ from $90^{\circ}$ true

Inversion Height: 200 meters

Stability Class: E Air Temperature: $29^{\circ} \mathrm{C}$

Relative Humidity: $50 \%$ Ground Roughness: 100 centimeters

Cloud Cover: 1 tenths

SOURCE STRENGTH INFORMATION:

Puddle Area: 10000 square meters

Average Puddle Depth: 1 centimeters

Soil Type: Default Ground Temperature: $29^{\circ} \mathrm{C}$

Initial Puddle Temperature: Ground temperature

Release Duration: ALOHA limited the duration to 1 hour

Max Computed Release Rate: 31.4 kilograms/min

Max Average Sustained Release Rate: 30.2 kilograms/min

(averaged over a minute or more)

Total Amount Released: 1,723 kilograms

FOOTPRINT INFORMATION:

Dispersion Module: Gaussian

User specified LOC: $50 \mathrm{ppm}$

Max Threat Zone for LOC: 177 meters

TIME DEPENDENT INFORMATION:

Concentration Estimates at the point:

Downwind: 100 meters

Off Centerline: 0 meters

Max Concentration:

Outdoor: $142 \mathrm{ppm}$

Indoor: $142 \mathrm{ppm}$

Note: Indoor graph is shown with a dotted line. 
Title of Project EPHA for THM

Subject Concentration Analysis for THM Hazardous Chemicals

Computer T. M. Franey Date 09/16/96
Reviewed by S. A. Henderson

Works Calculation 7

Sheet No. 216 of 577

SITE DATA INFORMATION: 1-RD-43

Location: AIKEN, SOUTH CAROLINA

Building Air Exchanges Per Hour: 60 (User specified)

Date and Time: Fixed at July 1, 19960000 hours

CHEMICAL INFORMATION:

Chemical Name: FORMALDEHYDE-56\%-SAH

Molecular Weight: $24.78 \mathrm{~kg} / \mathrm{kmol}$

TLV-TWA: -unavail- IDLH: -unavail-

Footprint Level of Concern: $10 \mathrm{ppm}$

Boiling Point: $100.10^{\circ} \mathrm{C}$

Vapor Pressure at Ambient Temperature: $0.023 \mathrm{~atm}$

Ambient Saturation Concentration: 23,824 ppm or $2.38 \%$

ATMOSPHERIC INFORMATION: (MANUAL INPUT OF DATA)

Wind: 1.7 meters $/ \mathrm{sec}$ from $90^{\circ}$ true

Inversion Height: 200 meters

Stability Class: E Air Temperature: $29^{\circ} \mathrm{C}$

Relative Humidity: 50\% Ground Roughness: 100 centimeters

Cloud Cover: 1 tenths

SOURCE STRENGTH INFORMATION:

Puddle Area: 18 square feet

Average Puddle Depth: 1 centimeters

Soil Type: Default Ground Temperature: $29^{\circ} \mathrm{C}$

Initial Puddle Temperature: Ground temperature

Release Duration: ALOHA limited the duration to 1 hour

Max Computed Release Rate: 16.5 grams/min

Max Average Sustained Release Rate: 14.1 grams/min

(averaged over a minute or more)

Total Amount Released: 731 grams

FOOTPRINT INFORMATION:

Dispersion Module: Gaussian

User specified LOC: $10 \mathrm{ppm}$

Max Threat Zone for LOC: 30 meters

Note: Footprint was not drawn because

effects of near-field patchiness make plume

presentation unreliable for short distances.

TIME DEPENDENT INFORMATION:

Concentration Estimates at the point:

Downwind: 30 meters

Off Centerline: 0 meters

Max Concentration:

Outdoor: $10 \mathrm{ppm}$

Indoor: $10 \mathrm{ppm}$

Note: Indoor graph is shown with a dotted line. 
Title of Project EPHA for THM

Subject Concentration Analysis for THM Hazardous Chemicals

Computer T. M. Franey Date $\underline{09 / 16 / 96}$
Reviewed by $\underline{S}$. A. Henderson

Works Calculation 7

Sheet No. 217 of 577

SITE DATA INFORMATION: 1-RD-43

Location: AIKEN, SOUTH CAROLINA

Building Air Exchanges Per Hour: 60 (User specified)

Date and Time: Fixed at July 1, 19960000 hours

CHEMICAL INFORMATION:

Chemical Name: FORMALDEHYDE-56\%-SAH

Molecular Weight: $24.78 \mathrm{~kg} / \mathrm{kmol}$

TLV-TWA: -unavail- IDLH: -unavail-

Footprint Level of Concern: $10 \mathrm{ppm}$

Boiling Point: $100.10^{\circ} \mathrm{C}$

Vapor Pressure at Ambient Temperature: 0.023 atm

Ambient Saturation Concentration: 23,824 ppm or $2.38 \%$

ATMOSPHERIC INFORMATION: (MANUAL INPUT OF DATA)

Wind: 1.7 meters $/ \mathrm{sec}$ from $90^{\circ}$ true

Inversion Height: 200 meters

Stability Class: E Air Temperature: $29^{\circ} \mathrm{C}$

Relative Humidity: $50 \%$ Ground Roughness: 100 centimeters

Cloud Cover: 1 tenths

\section{SOURCE STRENGTH INFORMATION:}

Puddle Area: 226 square feet

Average Puddle Depth: 1 centimeters

Soil Type: Default Ground Temperature: $29^{\circ} \mathrm{C}$

Initial Puddle Temperature: Ground temperature

Release Duration: ALOHA limited the duration to 1 hour

Max Computed Release Rate: 171 grams/min

Max Average Sustained Release Rate: 150 grams/min (averaged over a minute or more)

Total Amount Released: 7.83 kilograms

\section{FOOTPRINT INFORMATION:}

Dispersion Module: Gaussian

User specified LOC: $10 \mathrm{ppm}$

Max Threat Zone for LOC: 100 meters

\section{TIME DEPENDENT INFORMATION:}

Concentration Estimates at the point:

Downwind: 100 meters

Off Centerline: 0 meters

Max Concentration:

Outdoor: $10 \mathrm{ppm}$

Indoor: $10 \mathrm{ppm}$

Note: Indoor graph is shown with a dotted line. 


\section{ENGINEERING COMPUTATION SHEET}

Title of Project EPHA for THM

Subject Concentration Analysis for THM Hazardous Chemicals

Computer T. M. Franey

Date 09/16/96

SITE DATA INFORMATION: $\quad$ 1-RD-43

Location: AIKEN, SOUTH CAROLINA

Building Air Exchanges Per Hour: 60 (User specified)

Date and Time: Fixed at July 1, 19960000 hours

\section{CHEMICAL INFORMATION:}

Chemical Name: FORMALDEHYDE-56\%-SAH

Molecular Weight: $24.78 \mathrm{~kg} / \mathrm{kmol}$

TLV-TWA: -unavail- IDLH: -unavail-

Footprint Level of Concern: $10 \mathrm{ppm}$

Boiling Point: $100.10^{\circ} \mathrm{C}$

Vapor Pressure at Ambient Temperature: $0.023 \mathrm{~atm}$

Ambient Saturation Concentration: 23,824 ppm or $2.38 \%$

\section{ATMOSPHERIC INFORMATION: (MANUAL INPUT OF DATA)}

Wind: 1.7 meters $/ \mathrm{sec}$ from $90^{\circ}$ true

Inversion Height: 200 meters

Stability Class: E Air Temperature: $29^{\circ} \mathrm{C}$

Relative Humidity: 50\% Ground Roughness: 100 centimeters

Cloud Cover: 1 tenths

\section{SOURCE STRENGTH INFORMATION:}

Puddle Area: 24420 square feet

Average Puddle Depth: 1 centimeters

Soil Type: Default Ground Temperature: $29^{\circ} \mathrm{C}$

Initial Puddle Temperature: Ground temperature

Release Duration: ALOHA limited the duration to 1 hour

Max Computed Release Rate: 14 kilograms/min

Max Average Sustained Release Rate: 12.5 kilograms/min

(averaged over a minute or more)

Total Amount Released: 665 kilograms

\section{FOOTPRINT INFORMATION:}

Dispersion Module: Gaussian

User specified LOC: $10 \mathrm{ppm}$

Max Threat Zone for LOC: 1.2 kilometers

TIME DEPENDENT INFORMATION:

Concentration Estimates at the point:

Downwind: 100 meters

Off Centerline: 0 meters.

Max Concentration:

Outdoor: $203 \mathrm{ppm}$

Indoor: $203 \mathrm{ppm}$

Note: Indoor graph is shown with a dotted line.
Reviewed by S. A. Henderson

Works Calculation 7

Sheet No. 218 of 577 
Title of Project EPHA for THM

Subject Concentration Analysis for THM Hazardous Chemicals

Computer T. M. Franey

Date $\underline{09 / 16 / 96}$
Reviewed by $\underline{\text { S. A. Henderson }}$

Works Calculation 7

Sheet No. $\underline{219 \text { of } 577}$

\section{SITE DATA INFORMATION: 1-RD-43}

Location: AIKEN, SOUTH CAROLINA

Building Air Exchanges Per Hour: 60 (User specified)

Date and Time: Fixed at July 1, 19960000 hours

\section{CHEMICAL INFORMATION:}

Chemical Name: FORMALDEHYDE-56\%-SAH

Molecular Weight: $24.78 \mathrm{~kg} / \mathrm{kmol}$

TLV-TWA: -unavail- IDLH: -unavail-

Footprint Level of Concern: $10 \mathrm{ppm}$

Boiling Point: $100.10^{\circ} \mathrm{C}$

Vapor Pressure at Ambient Temperature: 0.023 atm

Ambient Saturation Concentration: 23,824 ppm or $2.38 \%$

\section{ATMOSPHERIC INFORMATION: (MANUAL INPUT OF DATA)}

Wind: 1.7 meters $/ \mathrm{sec}$ from $90^{\circ}$ true

Inversion Height: 200 meters

Stability Class: E Air Temperature: $29^{\circ} \mathrm{C}$

Relative Humidity: 50\% Ground Roughness: 100 centimeters

Cloud Cover: 1 tenths

\section{SOURCE STRENGTH INFORMATION:}

Puddle Area: 40700 square feet

Average Puddle Depth: 1 centimeters

Soil Type: Default Ground Temperature: $29^{\circ} \mathrm{C}$

Initial Puddle Temperature: Ground temperature

Release Duration: ALOHA limited the duration to 1 hour

Max Computed Release Rate: 22.7 kilograms/min

Max Average Sustained Release Rate: 20.3 kilograms/min (averaged over a minute or more)

Total Amount Released: 1,082 kilograms

\section{FOOTPRINT INFORMATION:}

Dispersion Module: Gaussian

User specified LOC: $10 \mathrm{ppm}$

Max Threat Zone for LOC: 1.6 kilometers

\section{TIME DEPENDENT INFORMATION:}

Concentration Estimates at the point:

- Downwind: 100 meters

Off Centerline: 0 meters

Max Concentration:

- Outdoor: 293 ppm

Indoor: $293 \mathrm{ppm}$

Note: Indoor graph is shown with a dotted line. 


\section{ENGINEERING COMPUTATION SHEET}

Title of Project EPHA for THM

Subject Concentration Analysis for THM Hazardous Chemicals

Computer T. M. Franey

Date $\underline{09 / 16 / 96}$

SITE DATA INFORMATION: 1-RD-43

Location: AIKEN, SOUTH CAROLINA

Building Air Exchanges Per Hour: 60 (User specified)

Date and Time: Fixed at July 1, 19960000 hours

CHEMICAL INFORMATION:

Chemical Name: FORMALDEHYDE-56\%-SAH

Molecular Weight: $24.78 \mathrm{~kg} / \mathrm{kmol}$

TLV-TWA: -unavail- IDLH: -unavail-

Footprint Level of Concern: 10 ppm

Boiling Point: $100.10^{\circ} \mathrm{C}$

Vapor Pressure at Ambient Temperature: $0.023 \mathrm{~atm}$

Ambient Saturation Concentration: $23,824 \mathrm{ppm}$ or $2.38 \%$

ATMOSPHERIC INFORMATION: (MANUAL INPUT OF DATA)

Wind: 1.7 meters $/ \mathrm{sec}$ from $90^{\circ}$ true

Inversion Height: 200 meters

Stability Class: E Air Temperature: $29^{\circ} \mathrm{C}$

Relative Humidity: 50\% Ground Roughness: 100 centimeters

Cloud Cover: 1 tenths

SOURCE STRENGTH INFORMATION:

Puddle Area: 10000 square meters

Average Puddle Depth: 1 centimeters

Soil Type: Default Ground Temperature: $29^{\circ} \mathrm{C}$

Initial Puddle Temperature: Ground temperature

Release Duration: ALOHA limited the duration to 1 hour

Max Computed Release Rate: 57.2 kilograms/min

Max Average.Sustained Release Rate: 51.4 kilograms/min

(averaged over a minute or more)

Total Amount Released: 2,742 kilograms

FOOTPRINT INFORMATION:

Dispersion Module: Gaussian

User specified LOC: $10 \mathrm{ppm}$

Max Threat Zone for LOC: 2.9 kilometers

TIME DEPENDENT INFORMATION:

Concentration. Estimates at the point:

Downwind: 100 meters

Off Centerline: 0 meters

Max Concentration:

Outdoor: $721 \mathrm{ppm}$

Indoor: $721 \mathrm{ppm}$

Note: Indoor graph is shown with a dotted line.
Reviewed by S.A. Henderson

Works Calculation 7

Sheet No. 220 of 577 


\section{ENGINEERING COMPUTATION SHEET}

Title of Project EPHA for THM

Subject Concentration Analysis for THM Hazardous Chemicals

Computer T. M. Franey

Date 09/16/96
Reviewed by S. A. Henderson

Works Calculation 7

Sheet No. 221 of 577

\section{SITE DATA INFORMATION: 1-RD-44 \\ Location: AIKEN, SOUTH CAROLINA \\ Building Air Exchanges Per Hour: 60 (User specified) \\ Date and Time: Fixed at July 1, 19960000 hours}

\section{CHEMICAL INFORMATION:}

Chemical Name: SULFURIC ACID

Molecular Weight: $98.08 \mathrm{~kg} / \mathrm{kmol}$

TLV-TWA: $0.25 \mathrm{ppm}$ IDLH: $19.60 \mathrm{ppm}$

Footprint Level of Concern: $10 \mathrm{ppm}$

Boiling Point: $330.00^{\circ} \mathrm{C}$

Vapor Pressure at Ambient Temperature: 7.51e-006 atm

Ambient Saturation Concentration: 7.64 ppm or $7.64 \mathrm{e}-004 \%$

ATMOSPHERIC INFORMATION: (MANUAL INPUT OF DATA)

Wind: 1.7 meters $/ \mathrm{sec}$ from $90^{\circ}$ true

Inversion Height: 200 meters

Stability Class: E Air Temperature: $29^{\circ} \mathrm{C}$

Relative Humidity: 50\% Ground Roughness: 100 centimeters

Cloud Cover: 1 tenths

\section{SOURCE STRENGTH INFORMATION:}

Puddle Area: 30430 square feet

Average Puddle Depth: 1 centimeters

Soil Type: Default Ground Temperature: $29^{\circ} \mathrm{C}$

Initial Puddle Temperature: Ground temperature

Release Duration: ALOHA limited the duration to 1 hour

Max Computed Release Rate: 17.2 grams/min

Max Average Sustained Release Rate: 16.6 grams/min (averaged over a minute or more)

Total Amount Released: 954 grams

TIME DEPENDENT INFORMATION:

Concentration Estimates at the point:

Downwind: 30 meters

Off Centerline: 0 meters

Max Concentration:

Outdoor: $0.62 \mathrm{ppm}$

Indoor: $0.62 \mathrm{ppm}$

Note: Indoor graph is shown with a dotted line. 
Title of Project EPHA for THM

Subject Concentration Analysis for THM Hazardous Chemicals

Computer T. M. Franey

Date $09 / 16 / 96$
Reviewed by $\underline{\text { S. A. Henderson }}$

Works Calculation 7

Sheet No. 222 of 577

SITE DATA INFORMATION: 1-RD-44

Location: AIKEN, SOUTH CAROLINA

Building Air Exchanges Per Hour: 60 (User specified)

Date and Time; Fixed at July 1, 19960000 hours

CHEMICAL INFORMATION:

Chemical Name: SULFURIC ACID

Molecular Weight: $98.08 \mathrm{~kg} / \mathrm{kmol}$

TLV-TWA: $0.25 \mathrm{ppm}$ IDLH: $19.60 \mathrm{ppm}$

Footprint Level of Concern: 10 ppm

Boiling Point: $330.00^{\circ} \mathrm{C}$

Vapor Pressure at Ambient Temperature: 7.51e-006 atm

Ambient Saturation Concentration: 7.64 ppm or $7.64 \mathrm{e}-004 \%$

ATMOSPHERIC INFORMATION: (MANUAL INPUT OF DATA)

Wind: 1.7 meters $/ \mathrm{sec}$ from $90^{\circ}$ true

Inversion Height: 200 meters

Stability Class: E Air Temperature: $29^{\circ} \mathrm{C}$

Relative Humidity: 50\% Ground Roughness: 100 centimeters

Cloud Cover: 1 tenths

SOURCE STRENGTH INFORMATION:

Puddle Area: 99999 square feet

Average Puddle Depth: 1 centimeters

Soil Type: Default Ground Temperature: $29^{\circ} \mathrm{C}$

Initial Puddle Temperature: Ground temperature

Release Duration: ALOHA limited the duration to 1 hour

Max Computed Release Rate: 53.8 grams/min

Max Average Sustained Release Rate: 51.9 grams/min

(averaged over a minute or more)

Total Amount Released: 2.99 kilograms

\section{FOOTPRINT INFORMATION:}

Dispersion Module: Gaussian

User specified LOC: $10 \mathrm{ppm}$

Max Threat Zone for LOC: 55 meters

Max Threat Zone for IDLH: 54 meters

Note: Footprint was not drawn because

effects of near-field patchiness make plume

presentation unreliable for short distances.

\section{TIME DEPENDENT INFORMATION:}

Concentration Estimates at the point:

Downwind: 100 meters

Off Centerline: 0 meters

Max Concentration:

Outdoor: $0.184 \mathrm{ppm}$

Indoor: $0.184 \mathrm{ppm}$

Note: Indoor graph is shown with a dotted line. 


\section{ENGINEERING COMPUTATION SHEET}

Title of Project EPHA for THM

Reviewed by S. A. Henderson

Subject Concentration Analysis for THM Hazardous Chemicals

Computer T. M. Franey

Date 09/16/96
Works Calculation 7

Sheet No. $\underline{223 \text { of } 577}$

\section{SITE DATA INFORMATION: 1-RD-44 \\ Location: AIKEN, SOUTH CAROLINA \\ Building Air Exchanges Per Hour: 60 (User specified) \\ Date and Time: Fixed at July 1, 19960000 hours}

\section{CHEMICAL INFORMATION:}

Chemical Name: SULFURIC ACID

Molecular Weight: $98.08 \mathrm{~kg} / \mathrm{kmol}$

TLV-TWA: $0.25 \mathrm{ppm}$ IDLH: $19.60 \mathrm{ppm}$

Footprint Level of Concern: $10 \mathrm{ppm}$

Boiling Point: $330.00^{\circ} \mathrm{C}$

Vapor Pressure at Ambient Temperature: 7.51e-006 atm

Ambient Saturation Concentration: 7.64 ppm or $7.64 \mathrm{e}-004 \%$

\section{ATMOSPHERIC INFORMATION: (MANUAL INPUT OF DATA)}

Wind: 1.7 meters $/ \mathrm{sec}$ from $90^{\circ}$ true

Inversion Height: 200 meters

Stability Class: E Air Temperature: $29^{\circ} \mathrm{C}$

Relative Humidity: 50\% Ground Roughness: 100 centimeters

Cloud Cover: 1 tenths

\section{SOURCE STRENGTH INFORMATION:}

Puddle Area: 24420 square feet

Average Puddle Depth: 1 centimeters

Soil Type: Default Ground Temperature: $29^{\circ} \mathrm{C}$

Initial Puddle Temperature: Ground temperature

Release Duration: ALOHA limited the duration to 1 hour

Max Computed Release Rate: 13.9 grams/min

Max Average Sustained Release Rate: 13.4 grams/min (averaged over a minute or more)

Total Amount Released: 773 grams

FOOTPRINT INFORMATION:

Dispersion Module: Gaussian

User specified LOC: $10 \mathrm{ppm}$

Max Threat Zone for LOC: 27 meters

Max Threat Zone for IDLH: 27 meters

Note: Footprint was not drawn because effects of near-field patchiness make plume presentation unreliable for short distances.

\section{TIME DEPENDENT INFORMATION:}

Concentration Estimates at the point:

Downwind: 100 meters

Off Centerline: 0 meters

Max Concentration: Outdoor: $0.0551 \mathrm{ppm}$

Indoor: $0.0551 \mathrm{ppm}$

Note: Indoor graph is shown with a dotted line. 
Title of Project EPHA for THM

Subject Concentration Analysis for THM Hazardous Chemicals

Computer T. M. Franey

Date $\underline{09 / 16 / 96}$

SITE DATA INFORMATION: 1-RD-44

Location: AIKEN, SOUTH CAROLINA

Building Air Exchanges Per Hour: 60 (User specified)

Date and Time: Fixed at July 1, 19960000 hours

CHEMICAL INFORMATION:

Chemical Name: SULFURIC ACID

Molecular Weight: $98.08 \mathrm{~kg} / \mathrm{kmol}$

TLV-TWA: $0.25 \mathrm{ppm}$ IDLH: $19.60 \mathrm{ppm}$

Footprint Level of Concern: $10 \mathrm{ppm}$

Boiling Point: $330.00^{\circ} \mathrm{C}$

Vapor Pressure at Ambient Temperature: $7.51 \mathrm{e}-006 \mathrm{~atm}$

Ambient Saturation Concentration: 7.64 ppm or 7.64 e-004\%

ATMOSPHERIC INFORMATION: (MANUAL INPUT OF DATA)

Wind: 1.7 meters $/ \mathrm{sec}$ from $90^{\circ}$ true

Inversion Height: 200 meters

Stability Class: E Air Temperature: $29^{\circ} \mathrm{C}$

Relative Humidity: 50\% Ground Roughness: 100 centimeters

Cloud Cover: 1 tenths

\section{SOURCE STRENGTH INFORMATION:}

Puddle Area: 40700 square feet

Average Puddle Depth: 1 centimeters

Soil Type: Default Ground Temperature: $29^{\circ} \mathrm{C}$

Initial Puddle Temperature: Ground temperature

Release Duration: ALOHA limited the duration to 1 hour

Max Computed Release Rate: 22.7 grams/min

Max Average Sustained Release Rate: $21.9 \mathrm{grams} / \mathrm{min}$ (averaged over a minute or more)

Total Amount Released: 1.26 kilograms

\section{FOOTPRINT INFORMATION:}

Dispersion Module: Gaussian

User specified LOC: $10 \mathrm{ppm}$

Max Threat Zone for LOC: 34 meters

Max Threat Zone for IDLH: 35 meters

Note: Footprint was not drawn because effects of near-field patchiness make plume presentation unreliable for short distances.

\section{TIME DEPENDENT INFORMATION:}

Concentration Estimates at the point:

Downwind: 100 meters

Off Centerline: 0 meters

Max Concentration:

Outdoor: $0.0799 \mathrm{ppm}$

Indoor: $0.0799 \mathrm{ppm}$

Note: Indoor graph is shown with a dotted line.
Reviewed by $\mathrm{S}$. A. Henderson

Works Calculation 7

Sheet No. $\underline{224 \text { of } 577}$ 
Title of Project EPHA for THM

Subject Concentration Analysis for THM Hazardous Chemicals

Computer T. M. Franey
Reviewed by $\underline{\text { S. A. Henderson }}$

Works Calculation 7

Sheet No. 225 of 577
CHEMICAL INFORMATION:

Chemical Name: SULFURIC ACID

Molecular Weight: $98.08 \mathrm{~kg} / \mathrm{kmol}$

TLV-TWA: $0.25 \mathrm{ppm}$ IDLH:-19.60 ppm

Footprint Level of Concern: $10 \mathrm{ppm}$

Boiling Point: $330.00^{\circ} \mathrm{C}$

Vapor Pressure at Ambient Temperature: 7.51e-006 atm

Ambient Saturation Concentration: 7.64 ppm or $7.64 \mathrm{e}-004 \%$

\section{ATMOSPHERIC INFORMATION: (MANUAL INPUT OF DATA)}

Wind: 1.7 meters $/ \mathrm{sec}$ from $90^{\circ}$ true

Inversion Height: 200 meters

Stability Class: E Air Temperature: $29^{\circ} \mathrm{C}$

Relative Humidity: 50\% Ground Roughness: 100 centimeters

Cloud Cover: 1 tenths

\section{SOURCE STRENGTH INFORMATION:}

Puddle Area: 10000 square meters

Average Puddle Depth: 1 centimeters

Soil Type: Default Ground Temperature: $29^{\circ} \mathrm{C}$

Initial Puddle Temperature: Ground temperature

Release Duration: ALOHA limited the duration to 1 hour

Max Computed Release Rate: 57.7 grams/min

Max Average:Sustained Release Rate: 55.7 grams/min

(averaged over a minute or more)

Total Amount Released: 3.21 kilograms

\section{FOOTPRINT INFORMATION:}

Dispersion Module: Gaussian

User specified LOC: $10 \mathrm{ppm}$

Max Threat Zone for LOC: 56 meters

Max Threat Zone for IDLH: 56 meters

Note: Footprint was not drawn because

effects of near-field patchiness make plume

presentation unreliable for short distances.

\section{TIME DEPENDENT INFORMATION:}

Concentration Estimates at the point:

Downwind: 100 meters

Off Centerline: 0 meters

Max Concentration:

Outdoor: $0.197 \mathrm{ppm}$

Indoor: $0.197 \mathrm{ppm}$

Note: Indoor graph is shown with a dotted line. 


\section{ENGINEERING COMPUTATION SHEET}

Title of Project EPHA for THM

Subject Concentration Analysis for THM Hazardous Chemicals

Computer T. M. Franey Date $\underline{09 / 16 / 96}$

SITE DATA INFORMATION: 1-RD-45

Location: AIKEN, SOUTH CAROLINA

Building Air Exchanges Per Hour: 60 (User specified)

Date and Time: Fixed at July 1, 19960000 hours

CHEMICAL INFORMATION:

Chemical Name: SULFURIC ACID, FUMING -

Molecular Weight: $98.00 \mathrm{~kg} / \mathrm{kmol}$

TLV-TWA: $0.25 \mathrm{ppm}$ IDLH: $19.60 \mathrm{ppm}$

Footprint Level of Concern: 10 ppm

Boiling Point: $93.50^{\circ} \mathrm{C}$

Vapor Pressure at Ambient Temperature: 0.018 atm

Ambient Saturation Concentration: 17,855 ppm or $1.79 \%$

ATMOSPHERIC INFORMATION: (MANUAL INPUT OF DATA)

Wind: 1.7 meters $/ \mathrm{sec}$ from $90^{\circ}$ true

Inversion Height: 200 meters

Stability Class: E Air Temperature: $29^{\circ} \mathrm{C}$

Relative Humidity: $50 \%$ Ground Roughness: 100 centimeters

Cloud Cover: 1 tenths

\section{SOURCE STRENGTH INFORMATION:}

Puddle Area: 37 square feet

Average Puddle Depth: 1 centimeters

Soil Type: Default Ground Temperature: $29^{\circ} \mathrm{C}$

Initial Puddle Temperature: Ground temperature

Release Duration: ALOHA limited the duration to 1 hour

Max Computed Release Rate: 67.3 grams/min

Max Average Sustained Release Rate: $59 \mathrm{grams} / \mathrm{min}$

(averaged over a minute or more)

Total Amount Released: 3.11 kilograms

\section{FOOTPRINT INFORMATION:}

Dispersion Module: Gaussian

User specified LOC: $10 \mathrm{ppm}$

Max Threat Zone for LOC: 30 meters

Max Threat Zone for IDLH: 20 meters

Note: Footprint was not drawn because effects of near-field patchiness make plume presentation unreliable for short distances.

\section{TIME DEPENDENT INFORMATION:}

Concentration Estimates at the point:

Downwind: 30 meters

Off Centerline: 0 meters

Max Concentration:

Outdoor: $10.2 \mathrm{ppm}$

Indoor: $10.2 \mathrm{ppm}$

Note: Indoor graph is shown with a dotted line.
Reviewed by S. A. Henderson

Works Calculation 7

Sheet No., 226 of 577 


\section{ENGINEERING COMPUTATION SHEET}

Title of Project EPHA for THM

Reviewed by $\underline{\text { S. A. Henderson }}$

Subject Concentration Analysis for THM Hazardous Chemicals

Computer T. M. Franey

Date $\underline{09 / 16 / 96}$

SITE DATA INFORMATION: 1-RD-45

Location: AIKEN, SOUTH CAROLINA

Building Air Exchanges Per Hour: 60 (User specified)

Date and Time: Fixed at July 1, 19960000 hours

CHEMICAL INFORMATION:

Chemical Name: SULFURIC ACID, FUMING -

Molecular Weight: $98.00 \mathrm{~kg} / \mathrm{kmol}$

TLV-TWA: $0.25 \mathrm{ppm}$ IDLH: $19.60 \mathrm{ppm}$

Footprint Level of Concern: $10 \mathrm{ppm}$

Boiling Point: $93.50^{\circ} \mathrm{C}$

Vapor Pressure at Ambient Temperature: 0.018 atm

Ambient Saturation Concentration: $17,855 \mathrm{ppm}$ or $1.79 \%$

ATMOSPHERIC INFORMATION: (MANUAL INPUT OF DATA)

Wind: 1.7 meters $/ \mathrm{sec}$ from $90^{\circ}$ true

Inversion Height: 200 meters

Stability Class: E Air Temperature: $29^{\circ} \mathrm{C}$

Relative Humidity: 50\% Ground Roughness: 100 centimeters

Cloud Cover: 1 tenths

\section{SOURCE STRENGTH INFORMATION:}

Puddle Area: 437 square feet

Average Puddle Depth: 1 centimeters

Soil Type: Default Ground Temperature: $29^{\circ} \mathrm{C}$

Initial Puddle Temperature: Ground temperature

Release Duration: ALOHA limited the duration to 1 hour

Max Computed Release Rate: 697 grams/min

Max Average Sustained Release Rate: 618 grams/min

(averaged over a minute or more)

Total Amount Released: 32.8 kilograms

FOOTPRINT INFORMATION:

Dispersion Module: Gaussian

User specified LOC: $10 \mathrm{ppm}$

Max Threat Zone for LOC: 100 meters

Max Threat Zone for IDLH: 67 meters

TIME DEPENDENT INFORMATION:

Concentration Estimates at the point:

Downwind: 100 meters

Off Centerline: 0 meters

Max Concentration:

Outdoor: $10 \mathrm{ppm}$

Indoor: $10 \mathrm{ppm}$

Note: Indoor graph is shown with a dotted line.
Works Calculation 7

Sheet No. $\underline{227 \text { of } 577}$ 
Title of Project EPHA for THM

Subject Concentration Analysis for THM Hazardous Chemicals

Computer T. M. Franey

Date 09/16/96
Reviewed by $\mathrm{S}$. A. Henderson

Works Calculation 7

Sheet No. 228 of 577
SITE DATA INFORMATION: 1-RD-45

Location: AIKEN, SOUTH CAROLINA

Building Air Exchanges Per Hour: 60 (User specified)

Date and Time: Fixed at July 1, 19960000 hours

CHEMICAL INFORMATION:

Chemical Name: SULFURIC ACID, FUMING -

Molecular Weight: $98.00 \mathrm{~kg} / \mathrm{kmol}$

TLV-TWA: $0.25 \mathrm{ppm}$ IDLH: $19.60 \mathrm{ppm}$

Footprint Level of Concern: 10 ppm

Boiling Point: $93.50^{\circ} \mathrm{C}$

Vapor Pressure at Ambient Temperature: $0.018 \mathrm{~atm}$

Ambient Saturation Concentration: 17,855 ppm or $1.79 \%$

ATMOSPHERIC INFORMATION: (MANUAL INPUT OF DATA)

Wind: 1.7 meters $/ \mathrm{sec}$ from $90^{\circ}$ true

Inversion Height: 200 meters

Stability Class: E Air Temperature: $29^{\circ} \mathrm{C}$

Relative Humidity: 50\% Ground Roughness: 100 centimeters

Cloud Cover: 1 tenths

\section{SOURCE STRENGTH INFORMATION:}

Puddle Area: 24420 square feet

Average Puddle Depth: 1 centimeters

Soil Type: Default Ground Temperature: $29^{\circ} \mathrm{C}$

Initial Puddle Temperature: Ground temperature

Release Duration: ALOHA limited the duration to 1 hour

Max Computed Release Rate: 32.4 kilograms/min

Max Average Sustained Release Rate: 29.1 kilograms/min

(averaged over a minute or more)

Total Amount Released: 1,556 kilograms

\section{FOOTPRINT INFORMATION:}

Model Run: Heavy Gas

User specified LOC: $10 \mathrm{ppm}$

Max Threat Zone for LOC: 936 meters

-Max Threat Zone for IDLH: 584 meters

Note: The Heavy Gas footprint is an initial screening.

For short releases it may be an overestimation.

Be sure to check concentration information at specific locations.

\section{TIME DEPENDENT INFORMATION:}

Concentration Estimates at the point:

Downwind: 100 meters

Off Centerline: 0 meters

Max Concentration:

Outdoor: $265 \mathrm{ppm}$

Indoor: $265 \mathrm{ppm}$

Note: Indoor graph is shown with a dotted line. 
Title of Project EPHA for THM

Subject Concentration Analysis for THM Hazardous Chemicals

Computer T. M. Franey
Reviewed by $\underline{\text { S. A. Henderson }}$

Works Calculation 7

Sheet No. $\underline{229 \text { of } 577}$

\section{SITE DATA INFORMATION: 1-RD-45 \\ Location: AIKEN, SOUTH CAROLINA \\ Building Air Exchanges Per Hour: 60 (User specified) \\ Date and Time: Fixed at July 1, 19960000 hours}

\section{CHEMICAL INFORMATION:}

Chemical Name: SULFURIC ACID, FUMING -

Molecular Weight: $98.00 \mathrm{~kg} / \mathrm{kmol}$

TLV-TWA: $0.25 \mathrm{ppm}$ IDLH: $19.60 \mathrm{ppm}$

Footprint Level of Concern: $10 \mathrm{ppm}$

Boiling Point: $93.50^{\circ} \mathrm{C}$

Vapor Pressure at Ambient Temperature: $0.018 \mathrm{~atm}$

Ambient Saturation Concentration: 17,855 ppm or $1.79 \%$

ATMOSPHERIC INFORMATION: (MANUAL INPUT OF DATA)

Wind: 1.7 meters $/ \mathrm{sec}$ from $90^{\circ}$ true

Inversion Height: 200 meters

Stability Class: E Air Temperature: $29^{\circ} \mathrm{C}$

Relative Humidity: 50\% Ground Roughness: 100 centimeters

Cloud Cover: 1 tenths

\section{SOURCE STRENGTH INFORMATION:}

Puddle Area: 40700 square feet

Average Puddle Depth: 1 centimeters

Soil Type: Default Ground Temperature: $29^{\circ} \mathrm{C}$

Initial Puddle Temperature: Ground temperature

Release Duration: ALOHA limited the duration to 1 hour

Max Computed Release Rate: 52.9 kilograms/min

Max Average Sustained Release Rate: 47.6 kilograms/min (averaged over a minute or more)

Total Amount Released: 2,546 kilograms

\section{FOOTPRINT INFORMATION:}

Model Run: Heavy Gas

User specified LOC: $10 \mathrm{ppm}$

Max Threat Zone for LOC: 1.2 kilometers

Max Threat Zone for IDLH: 748 meters

Note: The Heavy Gas footprint is an initial screening.

For short releases it may be an overestimation.

Be sure to check concentration information at specific locations.

\section{TIME DEPENDENT INFORMATION:}

Concentration Estimates at the point:

Downwind: 100 meters

Off Centerline: 0 meters

Max Concentration:

Outdoor: 375 ppm

Indoor: $375 \mathrm{ppm}$

Note: Indoor graph is shown with a dotted line. 
Title of Project EPHA for THM

Subject Concentration Analysis for THM Hazardous Chemicals

Computer T.M. Franey Date $\underline{09 / 16 / 96}$
Reviewed by $\mathrm{S}$. A. Henderson

Works Calculation 7

Sheet No. 230 of 577

SITE DATA INFORMATION: 1-RD-45

Location: AIKEN, SOUTH CAROLINA

Building Air Exchanges Per Hour: 60 (User specified)

Date and Time: Fixed at July 1, 19960000 hours

CHEMICAL INFORMATION:

Chemical Name: SULFURIC ACID, FUMING -

Molecular Weight: $98.00 \mathrm{~kg} / \mathrm{kmol}$

TLV-TWA: $0.25 \mathrm{ppm}$ IDLH: $19.60 \mathrm{ppm}$

Footprint Level of Concern: $10 \mathrm{ppm}$

Boiling Point: $93.50^{\circ} \mathrm{C}$

Vapor Pressure at Ambient Temperature: $0.018 \mathrm{~atm}$

Ambient Saturation Concentration: 17,855 ppm or $1.79 \%$

ATMOSPHERIC INFORMATION: (MANUAL INPUT OF DATA)

Wind: 1.7 meters $/ \mathrm{sec}$ from $90^{\circ}$ true

Inversion Height: 200 meters

Stability Class: E Air Temperature: $29^{\circ} \mathrm{C}$

Relative Humidity: 50\% Ground Roughness: 100 centimeters

Cloud Cover: 1 tenths

SOURCE STRENGTH INFORMATION:

Puddle Area: 10000 square meters

Average Puddle Depth: 1 centimeters

Soil Type: Default Ground Temperature: $29^{\circ} \mathrm{C}$

Initial Puddle Temperature: Ground temperature

Release Duration: ALOHA limited the duration to 1 hour

Max Computed Release Rate: 135 kilograms/min

Max Average Sustained Release Rate: 121 kilograms/min

(averaged over a minute or more)

Total Amount Released: 6,501 kilograms

FOOTPRINT INFORMATION:

Model Run:' Heavy Gas

User specified LOC: $10 \mathrm{ppm}$

Max Threat Zone for LOC: 1.9 kilometers

Max Threat Zone for IDLH: 1.2 kilometers

Note: The Heavy Gas footprint is an initial screening.

For short releases it may be an overestimation.

Be sure to check concentration information at specific locations.

\section{TIME DEPENDENT INFORMATION:}

Concentration Estimates at the point:

Downwind: 100 meters

Off Centerline: 0 meters

Max Concentration:

Outdoor: $713 \mathrm{ppm}$

Indoor: $713 \mathrm{ppm}$

Note: Indoor graph is shown with a dotted line. 
Title of Project EPHA for THM

Subject Concentration Analysis for THM Hazardous Chemicals

Computer T. M. Franey

Date $\underline{09 / 16 / 96}$

SITE DATA INFORMATION: I-RD-46

Location: AIKEN, SOUTH CAROLINA

Building Air Exchanges Per Hour: 60 (User specified)

Date and Time: Fixed at July 1, 19960000 hours

CHEMICAL INFORMATION:

Chemical Name: TETRAHYDROFURAN

Molecular Weight: $72.11 \mathrm{~kg} / \mathrm{kmol}$

TLV-TWA: $200.00 \mathrm{ppm}$ IDLH: $20000.00 \mathrm{ppm}$

Footprint Level of Concern: 1000 ppm

Boiling Point: $65.97^{\circ} \mathrm{C}$

Vapor Pressure at Ambient Temperature: $0.25 \mathrm{~atm}$

Ambient Saturation Concentration: 257,246 ppm or $25.7 \%$

ATMOSPHERIC INFORMATION: (MANUAL INPUT OF DATA)

Wind: 1.7 meters $/ \mathrm{sec}$ from $90^{\circ}$ true

Inversion Height: 200 meters

Stability Class: E Air Temperature: $29^{\circ} \mathrm{C}$

Relative Humidity: $50 \%$ Ground Roughness: 100 centimeters

Cloud Cover: 1 tenths

SOURCE STRENGTH INFORMATION:

Puddle Area: 425 square feet

Average Puddle Depth: 1 centimeters

Soil Type: Default Ground Temperature: $29^{\circ} \mathrm{C}$

Initial Puddle Temperature: Ground temperature

Release Duration: ALOHA limited the duration to 1 hour

Max Computed Release Rate: 8.48 kilograms/min

Max Average Sustained Release Rate: 6.47 kilograms/min

(averaged over a minute or more)

Total Amount Released: 302 kilograms

FOOTPRINT INFORMATION:

Model Run: Heavy Gas

User specified LOC: 1000 ppm

Max Threat Zone for LOC: 30 meters

Max Threat Zone for IDLH: less than 10 meters(10.9 yards)

Note: Footprint wasn't drawn because effects of near-field patchiness make plume presentation unreliable for short distances.

TIME DEPENDENT INFORMATION:

Concentration Estimates at the point:

Downwind: 30 meters

Off Centerline: 0 meters

Max Concentration:

Outdoor: $1,000 \mathrm{ppm}$

Indoor: $1,000 \mathrm{ppm}$

Note: Indoor graph is shown with a dotted line.
Reviewed by S. A. Henderson

Works Calculation 7

Sheet No. 231 of 577 
Title of Project EPHA for THM

Subject Concentration Analysis for THM Hazardous Chemicals

Computer T. M. Franey

Date $\underline{09 / 16 / 96}$
Reviewed by $\underline{S}$. A. Henderson

Works Calculation 7

Sheet No. 232 of 577

SITE DATA INFORMATION: $\quad$ 1-RD-46

Location: AIKEN, SOUTH CAROLINA

Building Air Exchanges Per Hour: 60 (User specified)

Date and Time: Fixed at July 1, 19960000 hours

CHEMICAL INFORMATION:

Chemical Name: TETRAHYDROFURAN

Molecular Weight: $72.11 \mathrm{~kg} / \mathrm{kmol}$

TLV-TWA: $200.00 \mathrm{ppm}$ IDLH: $20000.00 \mathrm{ppm}$

Footprint Level of Concern: 1000 ppm

Boiling Point: $65.97^{\circ} \mathrm{C}$

Vapor Pressure at Ambient Temperature: $0.25 \mathrm{~atm}$

Ambient Saturation Concentration: 257,246 ppm or $25.7 \%$

ATMOSPHERIC INFORMATION: (MANUAL INPUT OF DATA)

Wind: 1.7 meters $/ \mathrm{sec}$ from $90^{\circ}$ true

Inversion Height: 200 meters

Stability Class: E Air Temperature: $29^{\circ} \mathrm{C}$

Relative Humidity: $50 \%$ Ground Roughness: 100 centimeters

Cloud Cover: 1 tenths

SOURCE STRENGTH INFORMATION:

Puddle Area: 4738 square feet

Average Puddle Depth: 1 centimeters

Soil Type: Default Ground Temperature: $29^{\circ} \mathrm{C}$

Initial Puddle Temperature: Ground temperature

Release Duration: ALOHA limited the duration to 1 hour

Max Computed Release Rate: 83.7 kilograms/min

Max Average Sustained Release Rate: 65 kilograms/min

(averaged over a minute or more)

Total Amount Released: 3,091 kilograms

\section{FOOTPRINT INFORMATION:}

Model Run: Heavy Gas

User specified LOC: $1000 \mathrm{ppm}$

Max Threat Zone for LOC: 97 meters

Max Threat Zone for IDLH: 16 meters

Note: Footprint wasn't drawn because effects of near-field patchiness make plume presentation unreliable for short distances.

TIME DEPENDENT INFORMATION:

Concentration Estimates at the point:

Downwind: 100 meters

Off Centerline: 0 meters

Max Concentration:

Outdoor: $1,000 \mathrm{ppm}$

Indoor: $1,000 \mathrm{ppm}$

Note: Indoor graph is shown with a dotted line. 
Title of Project EPHA for THM

Subject Concentration Analysis for THM Hazardous Chemicals

Computer T. M. Franey

Date $09 / 16 / 96$

\section{SITE DATA INFORMATION: $\quad$ 1-RD-46}

Location: AIKEN, SOUTH CAROLINA

Building Air Exchanges Per Hour: 60 (User specified)

Date and Time: Fixed at July 1, 19960000 hours

CHEMICAL INFORMATION:

Chemical Name: TETRAHYDROFURAN

Molecular Weight: $72.11 \mathrm{~kg} / \mathrm{kmol}$

TLV-TWA: $200.00 \mathrm{ppm}$ IDLH: $20000.00 \mathrm{ppm}$

Footprint Level of Concern: $1000 \mathrm{ppm}$

Boiling Point: $65.97^{\circ} \mathrm{C}$

Vapor Pressure at Ambient Temperature: 0.25 atm

Ambient Saturation Concentration: 257,246 ppm or 25.7\%

ATMOSPHERIC INFORMATION: (MANUAL INPUT OF DATA)

Wind: 1.7 meters $/ \mathrm{sec}$ from $90^{\circ}$ true

Inversion Height: 200 meters

Stability Class: E Air Temperature: $29^{\circ} \mathrm{C}$

Relative Humidity: 50\% Ground Roughness: 100 centimeters

Cloud Cover: 1 tenths

\section{SOURCE STRENGTH INFORMATION:}

Puddle Area: 24420 square feet

Average Puddle Depth: 1 centimeters

Soil Type: Default Ground Temperature: $29^{\circ} \mathrm{C}$

Initial Puddle Temperature: Ground temperature

Release Duration: ALOHA limited the duration to 1 hour

Max Computed Release Rate: 400 kilograms/min

Max Average Sustained Release Rate: 315 kilograms/min

(averaged over a minute or more)

Total Amount Released: 15,088 kilograms

\section{FOOTPRINT INFORMATION:}

Model Run: Heavy Gas

User specified LOC: $1000 \mathrm{ppm}$

Max Threat Zone for LOC: 228 meters

Max Threat Zone for IDLH: 36 meters

Note: The Heavy Gas footprint is an initial screening.

For short releases it may be an overestimation.

Be sure to check concentration information at specific locations.

\section{TIME DEPENDENT INFORMATION:}

Concentration Estimates at the point:

Downwind: 100 meters

Off Centerline: 0 meters

Max Concentration:

Outdoor: $4,410 \mathrm{ppm}$

Indoor: $4,410 \mathrm{ppm}$

Note: Indoor graph is shown with a dotted line.
Reviewed by S. A. Henderson

Works Calculation 7

Sheet No. 233 of 577 
Title of Project EPHA for THM

Subject Concentration Analysis for THM Hazardous Chemicals

Computer T. M. Franey

Date $\underline{09 / 16 / 96}$
Reviewed by S. A. Henderson

Works Calculation 7

Sheet No. 234 of 577

SITE DATA INFORMATION: 1-RD-46

Location: AIKEN, SOUTH CAROLINA

Building Air Exchanges Per Hour: 60 (User specified)

Date and Time: Fixed at July 1, 19960000 hours

CHEMICAL INFORMATION:

Chemical Name: TETRAHYDROFURAN

Molecular Weight: $72.11 \mathrm{~kg} / \mathrm{kmol}$

TLV-TWA: $200.00 \mathrm{ppm}$ IDLH: $20000.00 \mathrm{ppm}$

Footprint Level of Concern: $1000 \mathrm{ppm}$

Boiling Point: $65.97^{\circ} \mathrm{C}$

Vapor Pressure at Ambient Temperature: $0.25 \mathrm{~atm}$

Ambient Saturation Concentration: 257,246 ppm or $25.7 \%$

ATMOSPHERIC INFORMATION: (MANUAL INPUT OF DATA)

Wind: 1.7 meters $/ \mathrm{sec}$ from $90^{\circ}$ true

Inversion Height: 200 meters

Stability Class: E Air Temperature: $29^{\circ} \mathrm{C}$

Relative Humidity: 50\% Ground Roughness: 100 centimeters

Cloud Cover: 1 tenths

SOURCE STRENGTH INFORMATION:

Puddle Area: 40700 square feet

Average Puddle Depth: 1 centimeters

Soil Type: Default Ground Temperature: $29^{\circ} \mathrm{C}$

Initial Puddle Temperature: Ground temperature

Release Duration: ALOHA limited the duration to 1 hour

Max Computed Release Rate: 653 kilograms/min

Max Average Sustained Release Rate: 516 kilograms/min

(averaged over a minute or more)

Total Amount Released: 24,763 kilograms

FOOTPRINT INFORMATION:

Model Run: Heavy Gas

User specified LOC: $1000 \mathrm{ppm}$

Max Threat Zone for LOC: 298 meters

Max Threat Zone for IDLH: 49 meters

Note: The Heavy Gas footprint is an initial screening.

For short releases it may be an overestimation.

Be sure to check concentration information at specific locations.

TIME DEPENDENT INFORMATION:

Concentration Estimates at the point:

Downwind: 100 meters

Off Centerline: 0 meters

Max Concentration:

Outdoor: 7,170 ppm

Indoor: $7,170 \mathrm{ppm}$

Note: Indoor graph is shown with a dotted line. 


\section{ENGINEERING COMPUTATION SHEET}

Title of Project EPHA for THM

Subject Concentration Analysis for THM Hazardous Chemicals

Computer T. M. Franey

Date $09 / 16 / 96$

SITE DATA INFORMATION: 1-RD-46

Location: AIKEN, SOUTH CAROLINA

Building Air Exchanges Per Hour: 60 (User specified)

Date and Time: Fixed at July 1, 19960000 hours

CHEMICAL INFORMATION:

Chemical Name: TETRAHYDROFURAN

Molecular Weight: $72.11 \mathrm{~kg} / \mathrm{kmol}$

TLV-TWA: $200.00 \mathrm{ppm}$ IDLH: $20000.00 \mathrm{ppm}$

Footprint Level of Concern: $1000 \mathrm{ppm}$

Boiling Point: $65.97^{\circ} \mathrm{C}$

Vapor Pressure at Ambient Temperature: $0.25 \mathrm{~atm}$

Ambient Saturation Concentration: 257,246 ppm or $25.7 \%$

\section{ATMOSPHERIC INFORMATION: (MANUAL INPUT OF DATA)}

Wind: 1.7 meters $/ \mathrm{sec}$ from $90^{\circ}$ true

Inversion Height: 200 meters

Stability Class: E Air Temperature: $29^{\circ} \mathrm{C}$

Relative Humidity: 50\% Ground Roughness: 100 centimeters

Cloud Cover: 1 tenths

\section{SOURCE STRENGTH INFORMATION:}

Puddle Area: 10000 square meters

Average Puddle Depth: 1 centimeters

Soil Type: Default Ground Temperature: $29^{\circ} \mathrm{C}$

Initial Puddle Temperature: Ground temperature

Release Duration: ALOHA limited the duration to 1 hour

Max Computed Release Rate: 1,660 kilograms/min

Max Average Sustained Release Rate: 1,320 kilograms/min

(averaged over a minute or more)

Total Amount Released:"63,532 kilograms

\section{FOOTPRINT INFORMATION:}

Model Run: Heavy Gas

User specified LOC: $1000 \mathrm{ppm}$

Max Threat Zone for LOC: 504 meters

Max Threat Zone for IDLH: 87 meters

Note: The Heavy Gas footprint is an initial screening.

For short releases it may be an overestimation.

Be sure to check concentration information at specific locations.

TIME DEPENDENT INFORMATION:

Concentration Estimates at the point:

Downwind: 100 meters

Off Centerline: 0 meters

Max Concentration:

Outdoor: $17,200 \mathrm{ppm}$

Indoor: $17,200 \mathrm{ppm}$

Note: Indoor graph is shown with a dotted line.
Reviewed by $\underline{\mathrm{S}}$. A. Henderson

Works Calculation 7

Sheet No. 235 of 577 


\section{ENGINEERING COMPUTATION SHEET}

Title of Project EPHA for THM

Reviewed by S. A. Henderson

Subject Conicentration Analysis for THM Hazardous Chemicals

Computer T. M. Franey

Date $\underline{09 / 16 / 96}$
Works Calculation 7

Sheet No. 236 of 577

\title{
SITE DATA INFORMATION: 1-RD-47
}

Location: AIKEN, SOUTH CAROLINA

Building Air Exchanges Per Hour: 60 (User specified)

Date and Time: Fixed at July 1, 19960000 hours

\section{CHEMICAL INFORMATION:}

Chemical Name: TOLUENE Molecular Weight: $92.14 \mathrm{~kg} / \mathrm{kmol}$ TLV-TWA: $50.00 \mathrm{ppm}$ IDLH: $2000.00 \mathrm{ppm}$

Footprint Level of Concern: 300 ppm

Boiling Point: $110.63^{\circ} \mathrm{C}$

Vapor Pressure at Ambient Temperature: $0.046 \mathrm{~atm}$

Ambient Saturation Concentration: $46,708 \mathrm{ppm}$ or $4.67 \%$

\author{
ATMOSPHERIC INFORMATION: (MANUAL INPUT OF DATA) \\ Wind: 1.7 meters $/ \mathrm{sec}$ from $90^{\circ}$ true \\ Inversion Height: 200 meters \\ Stability Class: E Air Temperature: $29^{\circ} \mathrm{C}$ \\ Relative Humidity: 50\% Ground Roughness: 100 centimeters \\ Cloud Cover: 1 tenths
}

\section{SOURCE STRENGTH INFORMATION:}

Puddle Area: 1810 square feet

Average Puddle Depth: 1 centimeters

Soil Type: Default Ground Temperature: $29^{\circ} \mathrm{C}$

Initial Puddle Temperature: Ground temperature

Release Duration: ALOHA limited the duration to 1 hour

Max Computed Release Rate: 6.56 kilograms/min

Max Average Sustained Release Rate: 6.01 kilograms/min

(averaged over a minute or more)

Total Amount Released: 329 kilograms

\section{FOOTPRINT INFORMATION: \\ Dispersion Module: Gaussian \\ User specified LOC: $300 \mathrm{ppm}$ \\ Max Threat Zone for LOC: 30 meters \\ Max Threat Zone for IDLH: 10 meters \\ Note: Footprint was not drawn because effects of near-field patchiness make plume presentation unreliable for short distances.}

\section{TIME DEPENDENT INFORMATION:}

Concentration Estimates at the point:

Downwind: . 30 meters

Off Centerline: 0 meters

Max Concentration:

Outdoor: $300 \mathrm{ppm}$

Indoor: $300 \mathrm{ppm}$

Note: Indoor graph is shown with a dotted line. 
Title of Project EPHA for THM

Subject Concentration Analysis for THM Hazardous Chemicals

Computer T.M. Franey Date $\underline{09 / 16 / 96}$

SITE DATA INFORMATION: $\quad$ 1-RD-47

Location: AIKEN, SOUTH CAROLINA

Building Air Exchanges Per Hour: 60 (User specified)

Date and Time: Fixed at July 1, 19960000 hours

CHEMICAL INFORMATION:

Chemical Name: TOLUENE Molecular Weight: $92.14 \mathrm{~kg} / \mathrm{kmol}$

TLV-TWA: 50.00 ppm IDLH: 2000.00 ppm

Footprint Level of Concern: 300 ppm

Boiling Point: $110.63^{\circ} \mathrm{C}$

Vapor Pressure at Ambient Temperature: $0.046 \mathrm{~atm}$

Ambient Saturation Concentration: 46,708 ppm or $4.67 \%$

ATMOSPHERIC INFORMATION: (MANUAL INPUT OF DATA)

Wind: 1.7 meters $/ \mathrm{sec}$ from $90^{\circ}$ true

Inversion Height: 200 meters

Stability Class: E Air Temperature: $29^{\circ} \mathrm{C}$

Relative Humidity: $50 \%$ Ground Roughness: 100 centimeters

Cloud Cover: 1 tenths

\section{SOURCE STRENGTH INFORMATION:}

Puddle Area: 7730 square feet

Average Puddle Depth: 1 centimeters

Soil Type: Default Ground Temperature: $29^{\circ} \mathrm{C}$

Initial Puddle Temperature: Ground temperature

Release Duration: ALOHA limited the duration to 1 hour

Max Computed Release Rate: 26.2 kilograms/min

Max Average Sustained Release Rate: 24.1 kilograms/min (averaged over a minute or more)

Total Amount Released: 1,325 kilograms

\section{FOOTPRINT INFORMATION:}

Model Run: Heavy Gas

User specified LOC: $300 \mathrm{ppm}$

Max Threat Zone for LOC: 97 meters

Max Threat Zone for IDLH: 25 meters

Note: Footprint wasn't drawn because effects of near-field patchiness make plume presentation unreliable for short distances.

\section{TIME DEPENDENT INFORMATION:}

Concentration Estimates at the point:

Downwind: 100 meters

Off Centerline: 0 meters

Max Concentration:

Outdoor: $300 \mathrm{ppm}$

Indoor: $300 \mathrm{ppm}$

Note: Indoor graph is shown with a dotted line. 
Title of Project EPHA for THM

Subject Concentration Analysis for THM Hazardous Chemicals

Computer T.M. Franey

Date $09 / 16 / 96$
Reviewed by S. A. Henderson

Works Calculation 7

Sheet No. 238 of 577
SITE DATA INFORMATION: 1-RD-47

Location: AIKEN, SOUTH CAROLINA

Building Air Exchanges Per Hour: 60 (User specified)

Date and Time: Fixed at July 1, 19960000 hours

\section{CHEMICAL INFORMATION:}

Chemical Name: TOLUENE Molecular Weight: $92.14 \mathrm{~kg} / \mathrm{kmol}$

TLV-TWA: $50.00 \mathrm{ppm}$ IDLH: $2000.00 \mathrm{ppm}$

Footprint Level of Concern: 300 ppm

Boiling Point: $110.63^{\circ} \mathrm{C}$

Vapor Pressure at Ambient Temperature: $0.046 \mathrm{~atm}$

Ambient Saturation Concentration: 46,708 ppm or $4.67 \%$

\section{ATMOSPHERIC INFORMATION: (MANUAL INPUT OF DATA)}

Wind: 1.7 meters $/ \mathrm{sec}$ from $90^{\circ}$ true

Inversion Height: 200 meters

Stability Class: E Air Temperature: $29^{\circ} \mathrm{C}$

Relative Humidity: 50\% Ground Roughness: 100 centimeters

Cloud Cover: 1 tenths

\section{SOURCE STRENGTH INFORMATION:}

Puddle Area: 24420 square feet

Average Puddle Depth: 1 centimeters

Soil Type: Default Ground Temperature: $29^{\circ} \mathrm{C}$

Initial Puddle Temperature: Ground temperature

Release Duration: ALOHA limited the duration to 1 hour

Max Computed Release Rate: 78.8 kilograms/min

Max Average Sustained Release Rate: 72.7 kilograms/min

(averaged over a minute or more)

Total Amount Released: 4,001 kilograms

\section{FOOTPRINT INFORMATION:}

Model Run: Heavy Gas

User specified LOC: $300 \mathrm{ppm}$

Max Threat Zone for LOC: 174 meters

Max Threat Zone for IDLH: 46 meters

- Note: The Heavy Gas footprint is an initial screening.

For short releases it may be an overestimation.

Be sure to check concentration information at specific locations.

\section{TIME DEPENDENT INFORMATION:}

Concentration Estimates at the point:

Downwind: 100 meters

Off Centerline: 0 meters

Max Concentration:

Outdoor: $721 \mathrm{ppm}$

Indoor: $721 \mathrm{ppm}$

Note: Indoor graph is shown with a dotted line. 
Title of Project EPHA for THM

Subject Concentration Analysis for THM Hazardous Chemicals

Computer T. M. Franey Date 09/16/96
Reviewed by $\underline{\text { S. A. Henderson }}$

Works Calculation 7

Sheet No. 239 of 577

SITE DATA INFORMATION: $\quad$ 1-RD-47

Location: AIKEN, SOUTH CAROLINA

Building Air Exchanges Per Hour: 60 (User specified)

Date and Time: Fixed'at July 1, 19960000 hours

CHEMICAL INFORMATION:

Chemical Name: TOLUENE Molecular Weight: $92.14 \mathrm{~kg} / \mathrm{kmol}$

TLV-TWA: $50.00 \mathrm{ppm}$ IDLH: $2000.00 \mathrm{ppm}$

Footprint Level of Concern: 300 ppm

Boiling Point: $110.63^{\circ} \mathrm{C}$

Vapor Pressure at Ambient Temperature: $0.046 \mathrm{~atm}$

Ambient Saturation Concentration: 46,708 ppm or $4.67 \%$

ATMOSPHERIC INFORMATION: (MANUAL INPUT OF DATA)

Wind: 1.7 meters $/ \mathrm{sec}$ from $90^{\circ}$ true

Inversion Height: 200 meters

Stability Class: E Air Temperature: $29^{\circ} \mathrm{C}$

Relative Humidity: 50\% Ground Roughness: 100 centimeters

Cloud Cover: 1 tenths

SOURCE STRENGTH INFORMATION:

Puddle Area: 40700 square feet

Average Puddle Depth: 1 centimeters

Soil Type: Default Ground Temperature: $29^{\circ} \mathrm{C}$

Initial Puddle Temperature: Ground temperature

Release Duration: ALOHA limited the duration to 1 hour

Max Computed Release Rate: 129 kilograms/min

Max Average Sustained Release Rate: 119 kilograms/min

(averaged over a minute or more)

Total Amount Released: 6,541 kilograms

FOOTPRINT INFORMATION:

Model Run: Heavy Gas

User specified LOC: $300 \mathrm{ppm}$

Max Threat Zone, for LOC: 226 meters

Max Threat Zone for IDLH: 61 meters

Note: The Heavy Gas footprint is an initial screening.

For short releases it may be an overestimation.

Be sure to check concentration information at specific locations.

TIME DEPENDENT INFORMATION:

Concentration Estimates at the point:

Downwind: 100 meters

Off Centerline: 0 meters

Max Concentration:

Outdoor: $1,060 \mathrm{ppm}$

Indoor: $1,060 \mathrm{ppm}$

Note: Indoor graph is shown with a dotted line. 
Title of Project EPHA for THM

Subject Concentration Analysis for THM Hazardous Chemicals

Computer T.M. Franey
Date $\underline{09 / 16 / 96}$
Reviewed by S. A. Henderson

Works Calculation 7

Sheet No. 240 of 577
SITE DATA INFORMATION: 1-RD-47
Location: AIKEN, SOUTH CAROLINA
Building Air Exchanges Per Hour: 60 (User specified)
Date and Time: Fixed at July 1, 19960000 hours

CHEMICAL INFORMATION:

Chemical Name: TOLUENE

TLV-TWA: $50.00 \mathrm{ppm}$

Molecular Weight: $92.14 \mathrm{~kg} / \mathrm{kmol}$

Footprint Level of Concern: 300 ppm

Boiling Point: $110.63^{\circ} \mathrm{C}$

Vapor Pressure at Ambient Temperature: $0.046 \mathrm{~atm}$

Ambient Saturation Concentration: $46,708 \mathrm{ppm}$ or $4.67 \%$

ATMOSPHERIC INFORMATION: (MANUAL INPUT OF DATA)

Wind: 1.7 meters $/ \mathrm{sec}$ from $90^{\circ}$ true

Inversion Height: 200 meters

Stability Class: E Air Temperature: $29^{\circ} \mathrm{C}$

Relative Humidity: 50\% Ground Roughness: 100 centimeters

Cloud Cover: 1 tenths

SOURCE STRENGTH INFORMATION:

Puddle Area: 10000 square meters

Average Puddle Depth: 1 centimeters

Soil Type: Default Ground Temperature: $29^{\circ} \mathrm{C}$

Initial Puddle Temperature: Ground temperature

Release Duration: ALOHA limited the duration to 1 hour

Max Computed Release Rate: 327 kilograms/min

Max Average Sustained Release Rate: 302 kilograms/min (averaged over a minute or more)

Total Amount Released: 16,690 kilograms

\section{FOOTPRINT INFORMATION:}

Model Run: Heavy Gas

User specified LOC: $300 \mathrm{ppm}$

Max Threat Zone for LOC: 376 meters

Max Threat Zone for IDLH: 105 meters

Note: The Heavy Gas footprint is an initial screening.

For short releases it may be an overestimation.

Be sure to check concentration information at specific locations.

\section{TIME DEPENDENT INFORMATION:}

Concentration Estimates at the point:

Downwind: 100 meters

Off Centerline: 0 meters

Max Concentration:

Outdoor: $2,210 \mathrm{ppm}$

Indoor: $2,210 \mathrm{ppm}$

Note: Indoor graph is shown with a dotted line. 


\section{ENGINEERING COMPUTATION SHEET}

Title of Project EPHA for THM

Reviewed by $\underline{\text { S. A. Henderson }}$

Subject Concentration Analysis for THM Hazardous Chemicals

Computer T. M. Franey

Date $\underline{09 / 16 / 96}$

SITE DATA INFORMATION: $\quad$ 1-RD-48

Location: AIKEN, SOUTH CAROLINA

Building Air Exchanges Per Hour: 60 (User specified)

Date and Time: Fixed at July 1, 19960000 hours

CHEMICAL INFORMATION:

Chemical Name: O-TOLUIDINE

Molecular Weight: $107.15 \mathrm{~kg} / \mathrm{kmol}$

TLV-TWA: $2.00 \mathrm{ppm}$ IDLH: $100.00 \mathrm{ppm}$

Note: Potential or confirmed human carcinogen.

Footprint Level of Concern: 10 ppm

Boiling Point: $200.40^{\circ} \mathrm{C}$

Vapor Pressure at Ambient Temperature: $4.69 \mathrm{e}-004 \mathrm{~atm}$

Ambient Saturation Concentration: 477 ppm or $0.048 \%$

ATMOSPHERIC INFORMATION: (MANUAL INPUT OF DATA)

Wind: 1.7 meters $/ \mathrm{sec}$ from $90^{\circ}$ true

Inversion Height: 200 meters

Stability Class: E Air Temperature: $29^{\circ} \mathrm{C}$

Relative Humidity: $50 \%$ Ground Roughness: 100 centimeters

Cloud Cover: 1 tenths

\section{SOURCE STRENGTH INFORMATION:}

Puddle Area: 6260 square feet

Average Puddle Depth: 1 centimeters

Soil Type::Default Ground Temperature: $29^{\circ} \mathrm{C}$

Initial Puddle Temperature: Ground temperature

Release Duration: ALOHA limited the duration to 1 hour

Max Computed Release Rate: 302 grams/min

Max Average Sustained Release Rate: 293 grams/min

(averaged over a minute or more)

Total Amount Released: 16.9 kilograms

FOOTPRINT INFORMATION:

Dispersion Module: Gaussian

User specified LOC: $10 \mathrm{ppm}$

Max Threat Zone for LOC: 30 meters

Max Threat Zone for IDLH: 14 meters

Note: Footprint was not drawn because

effects of near-field patchiness make plume

presentation unreliable for short distances.

TIME DEPENDENT INFORMATION:

Concentration Estimates at the point:

Downwind: 30 meters

Off Centerline: 0 meters

Max Concentration:

Outdoor: $10 \mathrm{ppm}$

Indoor: $10 \mathrm{ppm}$

Note: Indoor graph is shown with a dotted line.
Works Calculation 7

Sheet No. 241 of 577 
Title of Project EPHA for THM

Subject Concentration Analysis for THM Hazardous Chemicals

Computer T. M. Franey
Reviewed by $\mathbf{S}$. A. Henderson

Works Calculation 7

Sheet No. 242 of 577

SITE DATA INFORMATION: 1-RD-48

Location: AIKEN, SOUTH CAROLINA

Building Air Exchanges Per Hour: 60 (User specified)

Date and Time: Fixed at July 1, 19960000 hours

CHEMICAL INFORMATION:

Chemical Name: O-TOLUIDINE

Molecular Weight: $107.15 \mathrm{~kg} / \mathrm{kmol}$

TLV-TWA: $2.00 \mathrm{ppm}$ IDLH: $100.00 \mathrm{ppm}$

Note: Potential or confirmed human carcinogen.

Footprint Level of Concern: $10 \mathrm{ppm}$

Boiling Point: $200.40^{\circ} \mathrm{C}$

Vapor Pressure at Ambient Temperature: $4.69 \mathrm{e}-004 \mathrm{~atm}$

Ambient Saturation Concentration: 477 ppm or $0.048 \%$

ATMOSPHERIC INFORMATION: (MANUAL INPUT OF DATA)

Wind: 1.7 meters $/ \mathrm{sec}$ from $90^{\circ}$ true

Inversion Height: 200 meters

Stability Class: E Air Temperature: $29^{\circ} \mathrm{C}$

Relative Humidity: 50\% Ground Roughness: 100 centimeters

Cloud Cover: 1 tenths

SOURCE STRENGTH INFORMATION:

Puddle Area: 73000 square feet

Average Puddle Depth: 1 centimeters

Soil Type: Default Ground Temperature: $29^{\circ} \mathrm{C}$

Initial Puddle Temperature: Ground temperature

Release Duration: ALOHA limited the duration to 1 hour

Max Computed Release Rate: 3.18 kilograms/min

Max Average Sustained Release Rate: 3.08 kilograms/min

(averaged over a minute or more)

Total Amount Released: 178 kilograms

FOOTPRINT INFORMATION:

Dispersion Module: Gaussian

User specified LOC: $10 \mathrm{ppm}$

Max Threat Zone for LOC: 100 meters

Max Threat Zone for IDLH: 47 meters

Note: Footprint was not drawn because effects of near-field patchiness make plume presentation unreliable for short distances.

TIME DEPENDENT INFORMATION:

Concentration Estimates at the point:

Downwind: 100 meters

Off Centerline: 0 meters

Max Concentration:

Outdoor: $10 \mathrm{ppm}$

Indoor: $10 \mathrm{ppm}$

Note: Indoor graph is shown with a dotted line. 


\section{ENGINEERING COMPUTATION SHEET}

Title of Project EPHA for THM

Subject Concentration Analysis for THM Hazardous Chemicals

Computer T. M. Franey

Date $\underline{09 / 16 / 96}$
Reviewed by $\underline{\text { S. A. Henderson }}$

Works Calculation 7

Sheet No. 243 of 577

SITE DATA INFORMATION: 1-RD-48

Location: AIKEN, SOUTH CAROLINA

Building Air Exchanges Per Hour: 60 (User specified)

Date and Time: Fixed at July 1, 19960000 hours

CHEMICAL INFORMATION:

Chemical Name: O-TOLUIDINE

Molecular Weight: $107.15 \mathrm{~kg} / \mathrm{kmol}$

TLV-TWA: $2.00 \mathrm{ppm}$ IDLH: $100.00 \mathrm{ppm}$

Note: Potential or confirmed human carcinogen.

Footprint Level of Concern: $10 \mathrm{ppm}$

Boiling Point: $200.40^{\circ} \mathrm{C}$

Vapor Pressure at Ambient Temperature: $4.69 \mathrm{e}-004 \mathrm{~atm}$

Ambient Saturation Concentration: 477 ppm or $0.048 \%$

ATMOSPHERIC INFORMATION: (MANUAL INPUT OF DATA)

Wind: 1.7 meters $/ \mathrm{sec}$ from $90^{\circ}$ true

Inversion Height: 200 meters

Stability Class: E Air Temperature: $29^{\circ} \mathrm{C}$

Relative Humidity: 50\% Ground Roughness: 100 centimeters

Cloud Cover: 1 tenths

SOURCE STRENGTH INFORMATION:

Puddle Area: 24420 square feet

Average Puddle Depth: 1 centimeters

Soil Type: Default Ground Temperature: $29^{\circ} \mathrm{C}$

Initial Puddle Temperature: Ground temperature

Release Duration: ALOHA limited the duration to 1 hour

Max Computed Release Rate: 1.11 kilograms/min

Max Average Sustained Release Rate: 1.08 kilograms/min

(averaged over a minute or more)

Total Amount Released: 62.3 kilograms

FOOTPRINT INFORMATION:

Dispersion Module: Gaussian

User specified LOC: $10 \mathrm{ppm}$

Max Threat Zone for LOC: 58 meters

Max Threat Zone for IDLH: 27 meters

Note: Footprint was not drawn because

effects of near-field patchiness make plume

presentation unreliable for short distances.

TIME DEPENDENT INFORMATION:

Concentration Estimates at the point:

Downwind: 100 meters

Off Centerline: 0 meters

Max Concentration:

Outdoor: $4.05 \mathrm{ppm}$

Indoor: $4.05 \mathrm{ppm}$

Note: Indoor graph is shown with a dotted line. 


\section{ENGINEERING COMPUTATION SHEET}

Title of Project EPHA for THM

Subject Concentration Analysis for THM Hazardous Chemicals

Computer T. M. Franey

Date $\underline{09 / 16 / 96}$
Reviewed by $\mathrm{S}$. A. Henderson

Works Calculation 7

Sheet No. 244 of 577

SITE DATA INFORMATION: I-RD-48

Location: AIKEN, SOUTH CAROLINA

Building Air Exchanges Per Hour: 60 (User specified)

Date and Time: Fixed at July 1, 19960000 hours

CHEMICAL INFORMATION:

Chemical Name: O-TOLUIDINE

Molecular Weight: $107.15 \mathrm{~kg} / \mathrm{kmol}$

TLV-TWA: $2.00 \mathrm{ppm}$ IDLH: $100.00 \mathrm{ppm}$

Note: Potential or confirmed human carcinogen.

Footprint Level of Concern: $10 \mathrm{ppm}$

Boiling Point: $200.40^{\circ} \mathrm{C}$

Vapor Pressure at Ambient Temperature: $4.69 \mathrm{e}-004 \mathrm{~atm}$

Ambient Saturation Concentration: 477 ppm or $0.048 \%$

ATMOSPHERIC INFORMATION: (MANUAL INPUT OF DATA)

Wind: 1.7 meters $/ \mathrm{sec}$ from $90^{\circ}$ true

Inversion Height: 200 meters

Stability Class: E Air Temperature: $29^{\circ} \mathrm{C}$

Relative Humidity: 50\% Ground Roughness: 100 centimeters

Cloud Cover: 1 tenths

SOURCE STRENGTH INFORMATION:

Puddle Area: 40700 square feet

Average Puddle Depth: 1 centimeters

Soil Type: Default Ground Temperature: $29^{\circ} \mathrm{C}$

Initial Puddle Temperature: Ground temperature

Release Duration: ALOHA limited the duration to 1 hour

Max Computed Release Rate: 1.82 kilograms/min

Max Average Sustained Release Rate: 1.76 kilograms/min

(averaged over a minute or more)

Total Amount Released: 102 kilograms

FOOTPRINT INFORMATION:

Dispersion Module: Gaussian

User specified LOC: $10 \mathrm{ppm}$

Max Threat Zone for LOC: 75 meters

Max Threat Zone for IDLH: 34 meters

Note: Footprint was not drawn because effects of near-field patchiness make plume presentation unreliable for short distances.

TIME DEPENDENT INFORMATION:

Concentration Estimates at the point:

Downwind: 100 meters

Off Centerline: 0 meters

Max Concentration:

Outdoor: $5.88 \mathrm{ppm}$

Indoor: $5.88 \mathrm{ppm}$

Note: Indoor graph is shown with a dotted line. 
Title of Project EPHA for THM

Subject Concentration Analysis for THM Hazardous Chemicals

Computer T. M. Franey Date $09 / 16 / 96$
Reviewed by $\mathrm{S}$. A. Henderson

Works Calculation 7

Sheet No. 245 of 577
SITE DATA INFORMATION: $\quad$ 1-RD-48

Location: AIKEN, SOUTH CAROLINA

Building Air Exchanges Per Hour: 60 (User specified)

Date and Time: Fixed at July 1, 19960000 hours

\section{CHEMICAL INFORMATION:}

Chemical Name: O-TOLUIDINE

Molecular Weight: $107.15 \mathrm{~kg} / \mathrm{kmol}$

TLV-TWA: $2.00 \mathrm{ppm}$ IDLH: $100.00 \mathrm{ppm}$

Note: Potential or confirmed human carcinogen.

Footprint Level of Concern: 10 ppm

Boiling Point: $200.40^{\circ} \mathrm{C}$

Vapor Pressure at Ambient Temperature: $4.69 \mathrm{e}-004 \mathrm{~atm}$

Ambient Saturation Concentration: 477 ppm or $0.048 \%$

\section{ATMOSPHERIC INFORMATION: (MANUAL INPUT OF DATA)}

Wind: 1.7 meters $/ \mathrm{sec}$ from $90^{\circ}$ true

Inversion Height: 200 meters

Stability Class: E Air Temperature: $29^{\circ} \mathrm{C}$

Relative Humidity: $50 \%$ Ground Roughness: 100 centimeters

Cloud Cover: 1 tenths

\section{SOURCE STRENGTH INFORMATION:}

Puddle Area: 10000 square meters

Average Puddle Depth: 1 centimeters

Soil Type: Default Ground Temperature: $29^{\circ} \mathrm{C}$

Initial Puddle Temperature: Ground temperature

Release Duration: ALOHA limited the duration to 1 hour

Max Computed Release Rate: 4.62 kilograms/min

Max Average Sustained Release Rate: 4.48 kilograms/min

(averaged over a minute or more)

Total Amount Released: 259 kilograms

FOOTPRINT INFORMATION:

Dispersion Module: Gaussian

User specified LOC: $10 \mathrm{ppm}$

Max Threat Zone for LOC: 122 meters

Max Threat Zone for IDLH: 56 meters

TIME DEPENDENT INFORMATION:

Concentration Estimates at the point:

Downwind: 100 meters

Off Centerline: 0 meters

Max Concentration:

Outdoor: $14.5 \mathrm{ppm}$

Indoor: $14.5 \mathrm{ppm}$

Note: Indoor graph is shown with a dotted line. 


\section{ENGINEERING COMPUTATION SHEET}

Title of Project EPHA for THM

Subject Concentration Analysis for THM Hazardous Chemicals

Computer T.M. Franey Date $\underline{09 / 16 / 96}$
Reviewed by S. A. Henderson

Works Calculation 7

Sheet No. 246 of 577

\section{SITE DATA INFORMATION: 1-RD-49}

Location: AIKEN, SOUTH CAROLINA

Building Air Exchanges Per Hour: 60 (User specified)

Date and Time: Fixed at July 1, 19960000 hours

CHEMICAL INFORMATION:

Chemical Name: 1,1,1-TRICHLOROETHANE

Molecular Weight: $133.40 \mathrm{~kg} / \mathrm{kmol}$

TLV-TWA: $350.00 \mathrm{ppm}$ IDLH: $1000.00 \mathrm{ppm}$

Footprint Level of Concern: 1000 ppm

Boiling Point: $74.08^{\circ} \mathrm{C}$

Vapor Pressure at Ambient Temperature: $0.19 \mathrm{~atm}$

Ambient Saturation Concentration: 197,357 ppm or $19.7 \%$

ATMOSPHERIC INFORMATION: (MANUAL INPUT OF DATA)

- Wind: 1.7 meters $/ \mathrm{sec}$ from $90^{\circ}$ true

Inversion Height: 200 meters

Stability Class: E Air Temperature: $29^{\circ} \mathrm{C}$

Relative Humidity: 50\% Ground Roughness: 100 centimeters

Cloud Cover: 1 tenths

SOURCE STRENGTH INFORMATION:

Puddle Area: 690 square feet

Average Puddle Depth: 1 centimeters

Soil Type: Default Ground Temperature: $29^{\circ} \mathrm{C}$

Initial Puddle Temperature: Ground temperature

Release Duration: ALOHA limited the duration to 1 hour

Max Computed Release Rate: 16.3 kilograms/min

Max Average Sustained Release Rate: 13.4 kilograms/min

(averaged over a minute or more)

Total Amount Released: 664 kilograms

FOOTPRINT INFORMATION:

Model Run: Heavy Gas

User specified LOC: $1000 \mathrm{ppm}$

Max Threat Zone for LOC: 30 meters

Max Threat Zone for IDLH: 30 meters

Note: Footprint wasn't drawn because effects of near-field patchiness make plume presentation unreliable for short distances.

\section{TIME DEPENDENT INFORMATION:}

Concentration Estimates at the point:

Downwind: 30 meters

Off Centerline: 0 meters

Max Concentration:

Outdoor: $1,000 \mathrm{ppm}$

Indoor: $1,000 \mathrm{ppm}$

Note: Indoor graph is shown with a dotted line. 
ENGINEERING COMPUTATION SHEET

Title of Project EPHA for THM

Subject Concentration Analysis for THM Hazardous Chemicals

Computer T. M. Franey

Date $\underline{09 / 16 / 96}$

SITE DATA INFORMATION: $\quad$ 1-RD-49

Location: AIKEN, SOUTH CAROLINA

Building Air Exchanges Per Hour: 60 (User specified)

Date and Time: Fixed at July 1, 19960000 hours

CHEMICAL INFORMATTON:

Chemical Name: 1,1,1-TRICHLOROETHANE

Molecular Weight: $133.40 \mathrm{~kg} / \mathrm{kmol}$

TLV-TWA: $350.00 \mathrm{ppm}$, IDLH: $1000.00 \mathrm{ppm}$

Footprint Level of Concern: 1000 ppm

Boiling Point: $74.08^{\circ} \mathrm{C}$

Vapor Pressure at Ambient Temperature: $0.19 \mathrm{~atm}$

Ambient Saturation Concentration: 197,357 ppm or $19.7 \%$

ATMOSPHERIC INFORMATION: (MANUAL INPUT OF DATA)

Wind: 1.7 meters $/ \mathrm{sec}$ from $90^{\circ}$ true

Inversion Height: 200 meters

Stability Class: E Air Temperature: $29^{\circ} \mathrm{C}$

Relative Humidity: 50\% Ground Roughness: 100 centimeters

Cloud Cover: 1 tenths

\section{SOURCE STRENGTH INFORMATION:}

Puddle Area: 7080 square feet

Average Puddle Depth: 1 centimeters

Soil Type: Default Ground Temperature: $29^{\circ} \mathrm{C}$

Initial Puddle Temperature: Ground temperature

Release Duration: ALOHA limited the duration to 1 hour

Max Computed Release Rate: 151 kilograms/min

Max Average Sustained Release Rate: 126 kilograms/min

(averaged over a minute or more)

Total Amount Released: 6,302 kilograms

\section{FOOTPRINT INFORMATION:}

Model Run: Heavy Gas

User specified LOC: $1000 \mathrm{ppm}$

Max Threat Zone for LOC: 97 meters

Max Threat Zone for IDLH: 96 meters

Note: Footprint wasn't drawn because effects of near-field patchiness make plume presentation unreliable for short distances.

\section{TIME DEPENDENT INFORMATION:}

Concentration Estimates at the point:

Downwind: 100 meters

Off Centerline: 0 meters

Max Concentration:

Outdoor: $1,000 \mathrm{ppm}$

Indoor: $1,000 \mathrm{ppm}$

Note: Indoor graph is shown with a dotted line.
Reviewed by $\underline{S}$. A. Henderson

Works Calculation 7

Sheet No. 247 of 577 
Title of Project EPHA for THM

Subject Concentration Analysis for THM Hazardous Chemicals

Computer T. M. Franey Date $\underline{09 / 16 / 96}$
Reviewed by S. A. Henderson

Works Calculation 7

Sheet No. 248 of 577

SITE DATA INFORMATION: 1-RD-49

Location: AIKEN, SOUTH CAROLINA

Building Air Exchanges Per Hour: 60 (User specified)

Date and Time: Fixed at July 1, 19960000 hours

CHEMICAL INFORMATION:

Chemical Name: 1,1,1-TRICHLOROETHANE

Molecular Weight: $133.40 \mathrm{~kg} / \mathrm{kmol}$

TLV-TWA: $350.00 \mathrm{ppm}$ IDLH: $1000.00 \mathrm{ppm}$

Footprint Level of Concern: 1000 ppm

Boiling Point: $74.08^{\circ} \mathrm{C}$

Vapor Pressure at Ambient Temperature: $0.19 \mathrm{~atm}$

Ambient Saturation Concentration: 197,357 ppm or $19.7 \%$

ATMOSPHERIC INFORMATION: (MANUAL INPUT OF DATA)

Wind: 1.7 meters $/ \mathrm{sec}$ from $90^{\circ}$ true

Inversion Height: 200 meters

Stability Class: E Air Temperature: $29^{\circ} \mathrm{C}$

Relative Humidity: 50\% Ground Roughness: 100 centimeters

Cloud Cover: 1 tenths

\section{SOURCE STRENGTH INFORMATION:}

Puddle Area: 24420 square feet

Average Puddle Depth: 1 centimeters

Soil Type: Default Ground Temperature: $29^{\circ} \mathrm{C}$

Initial Puddle Temperature: Ground temperature

Release Duration: ALOHA limited the duration to 1 hour

Max Computed Release Rate: 496 kilograms/min

Max Average Sustained Release Rate: 415 kilograms/min

(averaged over a minute or more)

Total Amount Released: 20,901 kilograms

FOOTPRINT INFORMATION:

Model Run: Heavy Gas

User specified LOC: $1000 \mathrm{ppm}$

Max Threat Zone for LOC: 188 meters

Max Threat Zone for IDLH: 187 meters

Note: The Heavy Gas footprint is an initial screening.

For short releases it may be an overestimation.

Be sure to check concentration information at specific locations.

TIME DEPENDENT INFORMATION:

Concentration Estimates at the point:

Downwind: 100 meters

Off Centerline: 0 meters

Max Concentration:

Outdoor: $3,350 \mathrm{ppm}$

Indoor: $3,350 \mathrm{ppm}$

Note: Indoor graph is shown with a dotted line. 
ENGINEERING COMPUTATION SHEET

Title of Project EPHA for THM

Subject Concentration Analysis for THM Hazardous Chemicals

Computer T. M. Franey

Date $\underline{09 / 16 / 96}$

SITE DATA INFORMATION: 1-RD-49

Location: AIKEN, SOUTH CAROLINA

Building Air Exchanges Per Hour: 60 (User specified)

Date and Time: Fixed at July 1, 19960000 hours

CHEMICAL INFORMATION:

Chemical Name: 1,1,1-TRICHLOROETHANE

Molecular Weight: $133.40 \mathrm{~kg} / \mathrm{kmol}$

TLV-TWA: $350.00 \mathrm{ppm}$ IDLH: $1000.00 \mathrm{ppm}$

Footprint Level of Concern: $1000 \mathrm{ppm}$

Boiling Point: $74.08^{\circ} \mathrm{C}$

Vapor Pressure at Ambient Temperature: 0.19 atm

Ambient Saturation Concentration: 197,357 ppm or $19.7 \%$

ATMOSPHERIC INFORMATION: (MANUAL INPUT OF DATA)

Wind: 1.7 meters $/ \mathrm{sec}$ from $90^{\circ}$ true

Inversion Height: 200 meters

Stability Class: E Air Temperature: $29^{\circ} \mathrm{C}$

Relative Humidity: 50\% Ground Roughness: 100 centimeters

Cloud Cover: 1 tenths

SOURCE STRENGTH INFORMATION:

Puddle Area: 40700 square feet

Average Puddle Depth: 1 centimeters

Soil Type: Default Ground Temperature: $29^{\circ} \mathrm{C}$

Initial Puddle Temperature: Ground temperature

Release Duration: ALOHA limited the duration to 1 hour

Max Computed Release Rate: 811 kilograms/min

Max Average Sustained Release Rate: 682 kilograms/min

(averaged over a minute or more)

Total Amount Released: 34,290 kilograms

\section{FOOTPRINT INFORMATION:}

Model Run: Heavy Gas

User specified LOC: $1000 \mathrm{ppm}$

Max Threat Zone for LOC: 248 meters

Max Threat Zone for IDLH: 246 meters

Note: The Heavy Gas footprint is an initial screening.

For short releases it may be an overestimation.

Be sure to check concentration information at specific locations.

TIME DEPENDENT INFORMATION:

Concentration Estimates at the point:

Downwind: 100 meters

Off Centerline: 0 meters

Max Concentration:

Outdoor: 5,610 ppm

Indoor: $5,610 \mathrm{ppm}$

Note: Indoor graph is shown with a dotted line.
Reviewed by $\underline{S}$. A. Henderson

Works Calculation 7

Sheet No. $\underline{249 \text { of } 577}$ 
Title of Project EPHA for THM

Subject Concentration Analysis for THM Hazardous Chemicals

Computer T. M. Franey

Date $\underline{09 / 16 / 96}$
Reviewed by $\underline{\text { S. A. Henderson }}$

Works Calculation 7

Sheet No. 250 of 577

SITE DATA INFORMATION: 1-RD-49

Location: AIKEN, SOUTH CAROLINA

Building Air Exchanges Per Hour: 60 (User specified)

Date and Time: Fixed at July 1, 19960000 hours

CHEMICAL INFORMATION:

Chemical Name: 1,1,1-TRICHLOROETHANE

Molecular Weight: $133.40 \mathrm{~kg} / \mathrm{kmol}$

TLV-TWA: $350.00 \mathrm{ppm}$ IDLH: $1000.00 \mathrm{ppm}$

Footprint Level of Concern: 1000 ppm

Boiling Point: $74.08^{\circ} \mathrm{C}$

Vapor Pressure at Ambient Temperature: 0.19 atm

Ambient Saturation Concentration: 197,357 ppm or $19.7 \%$

ATMOSPHERIC INFORMATION: (MANUAL INPUT OF DATA)

Wind: 1.7 meters $/ \mathrm{sec}$ from $90^{\circ}$ true

Inversion Height: 200 meters

Stability Class: E Air Temperature: $29^{\circ} \mathrm{C}$

Relative Humidity: 50\% Ground Roughness: 100 centimeters

Cloud Cover: 1 tenths

SOURCE STRENGTH INFORMATION:

Puddle Area: 10000 square meters

Average Puddle Depth: 1 centimeters

Soil Type: Default Ground Temperature: $29^{\circ} \mathrm{C}$

Initial Puddle Temperature: Ground temperature

Release Duration: ALOHA limited the duration to 1 hour

Max Computed Release Rate: 2,070 kilograms/min

Max Average Sustained Release Rate: 1,740 kilograms/min

(averaged over a minute or more)

Total Amount Released: 88,085 kilograms

FOOTPRINT INFORMATION:

Model Run: Heavy Gas

User specified LOC: 1000 ppm

Max Threat Zone for LOC: 421 meters

Max Threat Zone for IDLH: 419 meters

Note: The Heavy Gas footprint is an initial screening.

For short releases it may be an overestimation.

Be sure to check concentration information at specific locations.

TIME DEPENDENT INFORMATION:

Concentration Estimates at the point:

Downwind: 100 meters

Off Centerline: 0 meters

Max Concentration:

Outdoor: $13,900 \mathrm{ppm}$

Indoor: $13,900 \mathrm{ppm}$

Note: Indoor graph is shown with a dotted line. 
Title of Project EPHA for THM

Subject Concentration Analysis for THM Hazardous Chemicals

Computer T. M. Franey Date $\underline{09 / 16 / 96}$
Reviewed by S. A. Henderson

Works Calculation 7

Sheet No. 251 of 577

\section{SITE DATA INFORMATION: 1-RD-50 \\ Location: AIKEN, SOUTH CAROLINA \\ Building Air Exchanges Per Hour: 60 (User specified) \\ Date and Time: Fixed at July 1, 19960000 hours}

\section{CHEMICAL INFORMATION:}

Chemical Name: TRICHLOROETHYLENE

Molecular Weight: $131.39 \mathrm{~kg} / \mathrm{kmol}$

TLV-TWA: $50.00 \mathrm{ppm}$ ' IDLH: $1000.00 \mathrm{ppm}$

Note: Potential or confirmed human carcinogen.

Footprint Level of Concern: 500 ppm

Boiling Point: $86.95^{\circ} \mathrm{C}$

Vapor Pressure at Ambient Temperature: $0.12 \mathrm{~atm}$

Ambient Saturation Concentration: 118,599 ppm or $11.9 \%$

\section{ATMOSPHERIC INFORMATION: (MANUAL INPUT OF DATA)}

Wind: 1.7 meters $/ \mathrm{sec}$ from $90^{\circ}$ true

Inversion Height: 200 meters

Stability Class: E Air Temperature: $29^{\circ} \mathrm{C}$

Relative Humidity: $50 \%$ Ground Roughness: 100 centimeters

Cloud Cover: 1 tenths

\section{SOURCE STRENGTH INFORMATION:}

Puddle Area: 522 square feet

Average Puddle Depth: 1 centimeters

Soil Type: Default Ground Temperature: $29^{\circ} \mathrm{C}$

Initial Puddle Temperature: Ground temperature

Release Duration: ALOHA limited the duration to 1 hour

Max Computed Release Rate: 6.9 kilograms/min

Max Average Sustained Release Rate: 5.95 kilograms/min

(averaged over a minute or more)

Total Amount Released: 309 kilograms

\section{FOOTPRINT INFORMATION:}

Model Run: Heavy Gas

User specified LOC: 500 ppm

Max Threat Zone for LOC: 30 meters

Max Threat Zone for IDLH: 20 meters

Note: Footprint wasn't drawn because effects of near-field patchiness make plume presentation unreliable for short distances.

\section{TIME DEPENDENT INFORMATION:}

Concentration Estimates at the point:

Downwind: 30 meters

Off Centerline: 0 meters

Max Concentration:

Outdoor: $500 \mathrm{ppm}$

Indoor: $499 \mathrm{ppm}$

Note: Indoor graph is shown with a dotted line. 
Title of Project EPHA for THM

Subject Concentration Analysis for THM Hazardous Chemicals

Computer T.M. Franey
Reviewed by $\underline{\text { S. A. Henderson }}$

Works Calculation 7

Sheet No. 252 of 577

SITE DATA INFORMATION: 1-RD-50

Location: AIKEN, SOUTH CAROLINA

Building Air Exchanges Per Hour: 60 (User specified)

Date and Time: Fixed at July 1, 19960000 hours

\section{CHEMICAL INFORMATION:}

Chemical Name: TRICHLOROETHYLENE

Molecular Weight: $131.39 \mathrm{~kg} / \mathrm{kmol}$

TLV-TWA: $50.00 \mathrm{ppm}$ IDLH: $1000.00 \mathrm{ppm}$

Note: Potential or confirmed human carcinogen.

Footprint Level of Concern: 500 ppm

Boiling Point: $86.95^{\circ} \mathrm{C}$

Vapor Pressure at Ambient Temperature: 0.12 atm

Ambient Saturation Concentration: 118,599 ppm or $11.9 \%$

\section{ATMOSPHERIC INFORMATION: (MANUAL INPUT OF DATA)}

Wind: 1.7 meters $/ \mathrm{sec}$ from $90^{\circ}$ true

Inversion Height: 200 meters

Stability Class: E Air Temperature: $29^{\circ} \mathrm{C}$

Relative Humidity: 50\% Ground Roughness: 100 centimeters

Cloud Cover: 1 tenths

\section{SOURCE STRENGTH INFORMATION:}

Puddle Area: 5760 square feet

Average Puddle Depth: 1 centimeters

Soil Type: Default Ground Temperature: $29^{\circ} \mathrm{C}$

Initial Puddle Temperature: Ground temperature

Release Duration: ALOHA limited the duration to 1 hour

Max Computed Release Rate: 68.5 kilograms/min

Max Average Sustained Release Rate: 59.6 kilograms/min

(averaged over a minute or more)

Total Amount Released: 3,126 kilograms

FOOTPRINT INFORMATION:

Model Run: Heavy Gas

User specified LOC: 500 ppm

Max Threat Zone for LOC: 97 meters

Max Threat Zone for IDLH: 63 meters

Note: Footprint wasn't drawn because effects of near-field patchiness make plume presentation unreliable for short distances.

\section{TIME DEPENDENT INFORMATION:}

Concentration Estimates at the point:

Downwind: 100 meters

Off Centerline: 0 meters.

Max Concentration:

Outdoor: $500 \mathrm{ppm}$

Indoor: $500 \mathrm{ppm}$

Note: Indoor graph is shown with a dotted line. 
Title of Project EPHA for THM

Subject Concentration Analysis for THM Hazardous Chemicals

Computer T.M. Franey

Date $\underline{09 / 16 / 96}$

SITE DATA INFORMATION: $\quad$ 1-RD-50

Location: AIKEN, SOUTH CAROLINA

Building Air Exchanges Per Hour: 60 (User specified)

Date and Time: Fixed at July 1, 19960000 hours

CHEMICAL INFORMATION:

Chemical Name: TRICHLOROETHYLENE

Molecular Weight: $131.39 \mathrm{~kg} / \mathrm{kmol}$

TLV-TWA: $50.00 \mathrm{ppm}$ IDLH: $1000.00 \mathrm{ppm}$

Note: Potential or confirmed human carcinogen.

Footprint Level of Concern: 500 ppm

Boiling Point: $86.95^{\circ} \mathrm{C}$

Vapor Pressure at Ambient Temperature: $0.12 \mathrm{~atm}$

Ambient Saturation Concentration: 118,599 ppm or 11.9\%

ATMOSPHERIC INFORMATION: (MANUAL INPUT OF DATA)

Wind: 1.7 meters $/ \mathrm{sec}$ from $90^{\circ}$ true

Inversion Height: 200 meters

Stability Class: E Air Temperature: $29^{\circ} \mathrm{C}$

Relative Humidity: 50\% Ground Roughness: 100 centimeters

Cloud Cover: 1 tenths

\section{SOURCE STRENGTH INFORMATION:}

Puddle Area: 24420 square feet

Average Puddle Depth: 1 centimeters

Soil Type: Default Ground Temperature: $29^{\circ} \mathrm{C}$

Initial Puddle Temperature: Ground temperature

Release Duration: ALOHA limited the duration to 1 hour

Max Computed Release Rate: 274 kilograms/min

Max Average Sustained Release Rate: 240 kilograms/min

(averaged over a minute or more)

Total Amount Released: 12,617 kilograms

\section{FOOTPRINT INFORMATION:}

Model Run: Heavy Gas

User specified LOC: $500 \mathrm{ppm}$

Max Threat Zone for LOC: 206 meters

Max Threat Zone for IDLH: 138 meters

Note: The Heavy Gas footprint is an initial screening.

For short releases it may be an overestimation.

Be sure to check concentration information at specific locations.

\section{TIME DEPENDENT INFORMATION:}

Concentration Estimates at the point:

Downwind: 100 meters

Off Centerline: 0 meters

Max Concentration:

Outdoor: $1,870 \mathrm{ppm}$

Indoor: $1,870 \mathrm{ppm}$

Note: Indoor graph is shown with a dotted line.
Reviewed by S.A. Henderson

Works Calculation 7

Sheet No. 253 of 577 


\section{ENGINEERING COMPUTATION SHEET}

Title of Project EPHA for THM

Reviewed by S. A. Hènderson

Subject Concentration Analysis for THM Hazardous Chemicals

Computer T. M. Franey Date $\underline{09 / 16 / 96}$

SITE DATA INFORMATION: 1-RD-50

Location: AIKEN, SOUTH CAROLINA

Building Air Exchanges Per Hour: 60 (User specified)

Date and Time: Fixed at July 1, 19960000 hours

\section{CHEMICAL INFORMATION:}

Chemical Name: TRICHLOROETHYLENE

Molecular Weight: $131.39 \mathrm{~kg} / \mathrm{kmol}$

TLV-TWA: $50.00 \mathrm{ppm}$ IDLH: $1000.00 \mathrm{ppm}$

Note: Potential or confirmed human carcinogen.

Footprint Level of Concern: 500 ppm

Boiling Point: $86.95^{\circ} \mathrm{C}$

Vapor Pressure at Ambient Temperature: $0.12 \mathrm{~atm}$

Ambient Saturation Concentration: 118,599 ppm or $11.9 \%$

\section{ATMOSPHERIC INFORMATION: (MANUAL INPUT OF DATA)}

Wind: 1.7 meters $/ \mathrm{sec}$ from $90^{\circ}$ true

Inversion Height: 200 meters

Stability Class: E Air Temperature: $29^{\circ} \mathrm{C}$

Relative Humidity: 50\% Ground Roughness: 100 centimeters

Cloud Cover: 1 tenths

\section{SOURCE STRENGTH INFORMATION:}

Puddle Area: 40700 square feet

Average Puddle Depth: 1 centimeters

Soil Type: Default Ground Temperature: $29^{\circ} \mathrm{C}$

Initial Puddle Temperature: Ground temperature

Release Duration: ALOHA limited the duration to 1 hour

Max Computed Release Rate: 447 kilograms/min

Max Average Sustained Release Rate: 392 kilograms/min

(averaged over a minute or more)

Total Amount Released: 20,678 kilograms

\section{FOOTPRINT INFORMATION:}

Model Run: Heavy Gas

User specified LOC: $500 \mathrm{ppm}$

Max Threat Zone for LOC: 271 meters

Max Threat Zone for IDLH: 181 meters

Note: The Heavy Gas footprint is an initial screening.

For short releases it may be an overestimation.

Be sure to check concentration information at specific locations.

\section{TIME DEPENDENT INFORMATION:}

Concentration Estimates at the point:

Downwind: 100 meters

Off Centerline: 0 meters

Max Concentration:

Outdoor: $2,980 \mathrm{ppm}$

Indoor: $2,980 \mathrm{ppm}$

Note: Indoor graph is shown with a dotted line.
Works Calculation 7

Sheet No. $\underline{254 \text { of } 577}$ 


\section{ENGINEERING COMPUTATION SHEET}

Title of Project EPHA for THM

Reviewed by S. A. Henderson

Works Calculation 7

Sheet No. 255 of 577

Computer T. M. Franey
SITE DATA INFORMATION: 1-RD-50

Location: AIKEN, SOUTH CAROLINA

Building Air Exchanges Per Hour: 60 (User specified)

Date and Time: Fixed at July 1, 19960000 hours

\section{CHEMICAL INFORMATION:}

Chemical Name: TRICHILOROETHYLENE

Molecular Weight: $131.39 \mathrm{~kg} / \mathrm{kmol}$

TLV-TWA: 50.00 ppm IDLH: $1000.00 \mathrm{ppm}$

Note: Potential or confirmed human carcinogen.

Footprint Level of Concern: 500 ppm

Boiling Point: $86.95^{\circ} \mathrm{C}$

Vapor Pressure at Ambient Temperature: $0.12 \mathrm{~atm}$

Ambient Saturation Concentration: 118,599 ppm or $11.9 \%$

\section{ATMOSPHERIC INFORMATION: (MANUAL INPUT OF DATA) \\ Wind: 1.7 meters/sec from $90^{\circ}$ true \\ Inversion Height: 200 meters \\ Stability Class: E Air Temperature: $29^{\circ} \mathrm{C}$ \\ Relative Humidity: $50 \%$ Ground Roughness: 100 centimeters \\ Cloud Cover: 1 tenths}

\section{SOURCE STRENGTH INFORMATION:}

Puddle Area: 10000 square meters

Average Puddle Depth: 1 centimeters

Soil Type: Default Ground Temperature: $29^{\circ} \mathrm{C}$

Initial Puddle Temperature: Ground temperature

Release Duration: ALOHA limited the duration to 1 hour

Max Computed Release Rate: 1,140 kilograms/min

Max Average Sustained Release Rate: 1,000 kilograms/min

(averaged over a minute or more)

Total Amount Released: 53,010 kilograms

\section{FOOTPRINT INFORMATION:}

Model Run: Heavy Gas

User specified LOC: $500 \mathrm{ppm}$

Max Threat Zone for LOC: 456 meters

Max Threat Zone for IDLH: 311 meters

Note: The Heavy Gas footprint is an initial screening.

- For short releases it may be an overestimation.

Be sure to check concentration information at specific locations.

\section{TIME DEPENDENT INFORMATION:}

Concentration Estimates at the point:

Downwind: 100 meters

Off Centerline: 0 meters

Max Concentration:

Outdoor: 7,370 ppm

Indoor: $7,360 \mathrm{ppm}$

Note: Indoor graph is shown with a dotted line. 
Title of Project EPHA for THM

Subject Concentration Analysis for THM Hazardous Chemicals

Computer T. M. Franey

Date 09/16/96
Reviewed by S. A. Henderson

Works Calculation 7

Sheet No. $\underline{256 \text { of } 577}$

SITE DATA INFORMATION: 1-RD-51

Location: AIKEN, SOUTH CAROLINA

Building Air Exchanges Per Hour: 60 (User specified)

Date and Time: Fixed at July 1, 19960000 hours

CHEMICAL INFORMATION:

Chemical Name: TRIETHYLAMINE

Molecular Weight: $101.19 \mathrm{~kg} / \mathrm{kmol}$

TLV-TWA: $10.00 \mathrm{ppm}$ IDLH: $1000.00 \mathrm{ppm}$

Footprint Level of Concern: $100 \mathrm{ppm}$

Boiling Point: $88.77^{\circ} \mathrm{C}$

Vapor Pressure at Ambient Temperature: $0.11 \mathrm{~atm}$

Ambient Saturation Concentration: 110,492 ppm or $11.0 \%$

ATMOSPHERIC INFORMATION: (MANUAL INPUT OF DATA)

Wind: 1.7 meters $/ \mathrm{sec}$ from $90^{\circ}$ true

Inversion Height: 200 meters

Stability Class: E Air Temperature: $29^{\circ} \mathrm{C}$

Relative Humidity: $50 \%$ Ground Roughness: 100 centimeters

Cloud Cover: 1 tenths

\section{SOURCE STRENGTH INFORMATION:}

Puddle Area: 65 square feet

Average Puddle Depth: 1 centimeters

Soil Type: Default Ground Temperature: $29^{\circ} \mathrm{C}$

Initial Puddle Temperature: Ground temperature

Release Duration: ALOHA limited the duration to 1 hour

Max Computed Release Rate: 751 grams/min

Max Average Sustained Release Rate: 639 grams/min

(averaged over a minute or more)

Total Amount Released: 32.7 kilograms

FOOTPRINT INFORMATION:

Dispersion Module: Gaussian

User specified LOC: $100 \mathrm{ppm}$

Max Threat Zone for LOC: 30 meters

Max Threat Zone for IDLH: less than 10 meters(10.9 yards)

Note: Footprint was not drawn because effects of near-field patchiness make plume presentation unreliable for short distances.

TIME DEPENDENT INFORMATION:

Concentration Estimates at the point:

Downwind: 30 meters

Off Centerline: 0 meters

Max Concentration:

Outdoor: $101 \mathrm{ppm}$

Indoor: $101 \mathrm{ppm}$

Note: Indoor graph is shown with a dotted line. 
Title of Project EPHA for THM

Subject Concentration Analysis for THM Hazardous Chemicals

Computer T. M. Franey
Reviewed by $\mathrm{S}$. A. Henderson

Works Calculation 7

Sheet No. 257 of 577
SITE DATA INFORMATION: 1-RD-51

Location: AIKEN, SOUTH CAROLINA

Building Air Exchanges Per Hour: 60 (User specified)

Date and Time: Fixed at July 1, 19960000 hours

CHEMICAL INFORMATION:

Chemical Name: TRIETHYLAMINE

Molecular Weight: $101.19 \mathrm{~kg} / \mathrm{kmol}$

TLV-TWA: $10.00 \mathrm{ppm}$ IDLH: $1000.00 \mathrm{ppm}$

Footprint Level of Concern: 100 ppm

Boiling Point: $88.77^{\circ} \mathrm{C}$

Vapor Pressure at Ambient Temperature: $0.11 \mathrm{~atm}$

Ambient Saturation Concentration: 110,492 ppm or $11.0 \%$

ATMOSPHERIC INFORMATION: (MANUAL INPUT OF DATA)

Wind: 1.7 meters $/ \mathrm{sec}$ from $90^{\circ}$ true

Inversion Height: 200 meters

Stability Class: E Air Temperature: $29^{\circ} \mathrm{C}$

Relative Humidity: $50 \%$ Ground Roughness: 100 centimeters

Cloud Cover: 1 tenths

\section{SOURCE STRENGTH INFORMATION:}

Puddle Area: 630 square feet

Average Puddle Depth: 1 centimeters

Soil Type: Default Ground Temperature: $29^{\circ} \mathrm{C}$

Initial Puddle Temperature: Ground temperature

Release Duration: ALOHA limited the duration to 1 hour

Max Computed Release Rate: 6.48 kilograms/min

Max Average Sustained Release Rate: 5.6 kilograms/min (averaged over a minute or more)

Total Amount Released: 288 kilograms

\section{FOOTPRINT INFORMATION:}

Model Run: Heavy Gas

User specified LOC: $100 \mathrm{ppm}$

Max Threat Zone for LOC: 97 meters

Max Threat Zone for IDLH: 22 meters

Note: Footprint wasn't drawn because effects of near-field patchiness make plume presentation unreliable for short distances.

\section{TMME DEPENDENT INFORMATION:}

Concentration Estimates at the point:

Downwind: 100 meters

Off Centerline: 0 meters

Max Concentration:

Outdoor: $100 \mathrm{ppm}$

Indoor: $100 \mathrm{ppm}$

Note: Indoor graph is shown with a dotted line. 


\section{ENGINEERING COMPUTATION SHEET}

Title of Project EPHA for THM

Reviewed by $\mathrm{S}$. A. Henderson

Works Calculation 7

Sheet No. $\underline{258 \text { of } 577}$

SITE DATA INFORMATION: I-RD-51

Location: AIKEN, SOUTH CAROLINA

Building Air Exchanges Per Hour: 60 (User specified)

Date and Time: Fixed at July 1, 19960000 hours

CHEMICAL INFORMATION:

Chemical Name: TRIETHYLAMINE

Molecular Weight: $101.19 \mathrm{~kg} / \mathrm{kmol}$

TLV-TWA: $10.00 \mathrm{ppm}$ IDLH: $1000.00 \mathrm{ppm}$

Footprint Level of Concern: 100 ppm

Boiling Point: $88.77^{\circ} \mathrm{C}$

Vapor Pressure at Ambient Temperature: 0.11 atm

Ambient Saturation Concentration: 110,492 ppm or $11.0 \%$

ATMOSPHERIC INFORMATION: (MANUAL INPUT OF DATA)

Wind: 1.7 meters $/ \mathrm{sec}$ from $90^{\circ}$ true

Inversion Height: 200 meters

Stability Class: E Air Temperature: $29^{\circ} \mathrm{C}$

Relative Humidity: 50\% Ground Roughness: 100 centimeters

Cloud Cover: 1 tenths

SOURCE STRENGTH INFORMATION:

Puddle Area: 24420 square feet

Average Puddle Depth: 1 centimeters

Soil Type: Default Ground Temperature: $29^{\circ} \mathrm{C}$

Initial Puddle Temperature: Ground temperature

Release Duration: ALOHA limited the duration to 1 hour

Max Computed Release Rate: 213 kilograms/min

Max Average Sustained Release Rate: 186 kilograms/min

(averaged over a minute or more)

Total Amount Released: 9,745 kilograms

FOOTPRINT INFORMATION:

Model Run: Heavy Gas

User specified LOC: $100 \mathrm{ppm}$

Max Threat Zone for LOC: 568 meters

Max Threat Zone for IDLH: 138 meters

Note: The Heavy Gas footprint is an initial screening.

For short releases it may be an overestimation.

Be sure to check concentration information at specific locations.

TIME DEPENDENT INFORMATION:

Concentration Estimates at the point:

Downwind: 100 meters

Off Centerline: 0 meters

Max Concentration:

Outdoor: $1,810 \mathrm{ppm}$

Indoor: $1,810 \mathrm{ppm}$

Note: Indoor graph is shown with a dotted line. 
Title of Project EPHA for THM

Subject Concentration Analysis for THM Hazardous Chemicals

Computer T. M. Franey Date 09/16/96

\section{SITE DATA INFORMATION: 1-RD-51}

Location: AIKEN, SOUTH CAROLINA

Building Air Exchanges Per Hour: 60 (User specified)

Date and Time: Fixed at July 1, 19960000 hours

\section{CHEMICAL INFORMATION:}

Chemical Name: TRIETHYLAMINE

Molecular Weight: $101.19 \mathrm{~kg} / \mathrm{kmol}$

TLV-TWA: $10.00 \mathrm{ppm}$ IDLH: $1000.00 \mathrm{ppm}$

Footprint Level of Concern: $100 \mathrm{ppm}$

Boiling Point: $88.77^{\circ} \mathrm{C}$

Vapor Pressure at Ambient Temperature: 0.11 atm

Ambient Saturation Concentration: 110,492 ppm or $11.0 \%$

\section{ATMOSPHERIC INFORMATION: (MANUAL INPUT OF DATA)}

Wind: 1.7 meters $/ \mathrm{sec}$ from $90^{\circ}$ true

Inversion Height: 200 meters

Stability Class: E Air Temperature: $29^{\circ} \mathrm{C}$

Relative Humidity: 50\% Ground Roughness: 100 centimeters

Cloud Cover: 1 tenths

\section{SOURCE STRENGTH INFORMATION:}

Puddle Area: 40700 square feet

Average Puddle Depth: 1 centimeters

Soil Type: Default Ground Temperature: $29^{\circ} \mathrm{C}$

Initial Puddle Temperature: Ground temperature

Release Duration: ALOHA limited the duration to 1 hour

Max Computed Release Rate: 348 kilograms/min

Max Average:Sustained Release Rate: 306 kilograms/min (averaged over a minute or more)

Total Amount Released: 15,958 kilograms

\section{FOOTPRINT INFORMATION:}

\section{Model Run: Heavy Gas}

User specified LOC: $100 \mathrm{ppm}$

Max Threat Zone for LOC: 734 meters

Max Threat Zone for IDLH: 181 meters

Note: The Heavy Gas footprint is an initial screening.

For short releases it may be an overestimation.

Be sure to check concentration information at specific locations.

\section{TIME DEPENDENT INFORMATION:}

Concentration Estimates at the point:

Downwind: 100 meters

Off Centerline: 0 meters

Max Concentration:

Outdoor: $2,850 \mathrm{ppm}$

Indoor: $2,850 \mathrm{ppm}$

Note: Indoor graph is shown with a dotted line.
Reviewed by $\underline{S}$. A. Henderson

Works Calculation 7

Sheet No. 259 of 577 


\section{ENGINEERING COMPUTATION SHEET}

Title of Project EPHA for THM

Reviewed by $\underline{S}$. A. Henderson

Subject Concentration Analysis for THM Hazardous Chemicals

Computer T. M. Franey

Date $\underline{09 / 16 / 96}$
Works Calculation 7

Sheet No. 260 of 577
SITE DATA INFORMATION: 1-RD-51

Location: AIKEN, SOUTH CAROLINA

Building Air Exchanges Per Hour: 60 (User specified)

Date and Time: Fixed at July 1, 19960000 hours

CHEMICAL INFORMATION:

Chemical Name: TRIETHYLAMINE

Molecular Weight: $101.19 \mathrm{~kg} / \mathrm{kmol}$

TLV-TWA: $10.00 \mathrm{ppm}$ IDLH: $1000.00 \mathrm{ppm}$

Footprint Level of Concern: $100 \mathrm{ppm}$

Boiling Point: $88.77^{\circ} \mathrm{C}$

Vapor Pressure at Ambient Temperature: $0.11 \mathrm{~atm}$

Ambient Saturation Concentration: 110,492 ppm or $11.0 \%$

ATMOSPHERIC INFORMATION: (MANUAL INPUT OF DATA)

Wind: 1.7 meters $/ \mathrm{sec}$ from $90^{\circ}$ true

Inversion Height: 200 meters

Stability Class: E Air Temperature: $29^{\circ} \mathrm{C}$

Relative Humidity: $50 \%$ Ground Roughness: 100 centimeters

Cloud Cover: 1 tenths

\section{SOURCE STRENGTH INFORMATION:}

Puddle Area: 10000 square meters

Average Puddle Depth: 1 centimeters

Soil Type: Default Ground Temperature: $29^{\circ} \mathrm{C}$

Initial Puddle Temperature: Ground temperature

Release Duration: ALOHA limited the duration to 1 hour

Max Computed Release Rate: 884 kilograms/min

Max Average Sustained Release Rate: 779 kilograms/min

(averaged over a minute or more)

Total Amount Released: 40,857 kilograms

FOOTPRINT INFORMATION:

Model Run: Heavy Gas

User specified LOC: $100 \mathrm{ppm}$

Max Threat Zone for LOC: 1.2 kilometers

Max Threat Zone for IDLH: 307 meters

Note: The Heavy Gas footprint is an initial screening.

For short releases it may be an overestimation.

Be sure to check concentration information at specific locations.

\section{TIME DEPENDENT INFORMATION:}

Concentration Estimates at the point:

Downwind: 100 meters

Off Centerline: 0 meters

Max Concentration:

Outdoor: 6,590 ppm

Indoor: $6,590 \mathrm{ppm}$

Note: Indoor graph is shown with a dotted line. 
Title of Project EPHA for THM

Subject Concentration Analysis for THM Hazardous Chemicals

Computer T. M. Franey

Date $\underline{09 / 16 / 96}$

Reviewed by $\underline{\text { S. A. Henderson }}$

Works Calculation 7

Sheet No. 261 of 577

SITE DATA INFORMATION: 1-RD-52

Location: AIKEN, SOUTH CAROLINA

Building Air Exchanges Per Hour: 60 (User specified)

Date and Time: Fixed at July 1, 19960000 hours

CHEMICAL INFORMATION:

Chemical Name: VINYL ACETATE

Molecular Weight: $86.09 \mathrm{~kg} / \mathrm{kmol}$

TLV-TWA: $10.00 \mathrm{ppm}$ ' IDLH: -unavail-

Note: Potential or confirmed human carcinogen.

Footprint Level of Concern: 75 ppm

Boiling Point: $72.50^{\circ} \mathrm{C}$

Vapor Pressure at Ambient Temperature: $0.18 \mathrm{~atm}$

Ambient Saturation Concentration: 184,394 ppm or $18.4 \%$

ATMOSPHERIC INFORMATION: (MANUAL INPUT OF DATA)

Wind: 1.7 meters $/ \mathrm{sec}$ from $90^{\circ}$ true

Inversion Height: 200 meters

Stability Class: E Air Temperature: $29^{\circ} \mathrm{C}$

Relative Humidity: $50 \%$ Ground Roughness: 100 centimeters

Cloud Cover: 1 tenths

SOURCE STRENGTH INFORMATION:

Puddle Area: 25.6 square feet

Average Puddle Depth: 1 centimeters

Soil Type: Default Ground Temperature: $29^{\circ} \mathrm{C}$

Initial Puddle Temperature: Ground temperature

Release Duration: ALOHA limited the duration to 1 hour

Max Computed Release Rate: 482 grams/min

Max Average Sustained Release Rate: 374 grams/min

(averaged over a minute or more)

Total Amount Released: 17.7 kilograms

FOOTPRINT INFORMATION:

Dispersion Module: Gaussian

User specified LOC: 75 ppm

Max Threat Zone for LOC: 30 meters

Note: Footprint was not drawn because effects of near-field patchiness make plume presentation unreliable for short distances.

TIME DEPENDENT INFORMATION:

Concentration Estimates at the point:

Downwind: 30 meters

Off Centerline: 0 meters

Max Concentration:

Outdoor: $75.1 \mathrm{ppm}$

Indoor: $75.1 \mathrm{ppm}$

Note: Indoor graph is shown with a dotted line. 


\section{ENGINEERING COMPUTATION SHEET}

Title of Project EPHA for THM

Subject Concentration Analysis for THM Hazardous Chemicals

Computer T.M. Franey

Date $\underline{09 / 16 / 96}$

SITE DATA INFORMATION: 1-RD-52

Location: AIKEN, SOUTH CAROLINA

Building Air Exchanges Per Hour: 60 (User specified)

Date and Time: Fixed at July 1, 19960000 hours

\section{CHEMICAL INFORMATION:}

Chemical Name: VINYL ACETATE

Molecular Weight: $86.09 \mathrm{~kg} / \mathrm{kmol}$

TLV-TWA: 10.00 ppm IDLH: -unavail-

Note: Potential or confirmed human carcinogen.

Footprint Level of Concern: 75 ppm

Boiling Point: $72.50^{\circ} \mathrm{C}$

Vapor Pressure at Ambient Temperature: $0.18 \mathrm{~atm}$

Ambient Saturation Concentration: 184,394 ppm or $18.4 \%$

\section{ATMOSPHERIC INFORMATION: (MANUAL INPUT OF DATA) \\ Wind: 1.7 meters $/ \mathrm{sec}$ from $90^{\circ}$ true \\ Inversion Height: 200 meters \\ Stability Class: E Air Temperature: $29^{\circ} \mathrm{C}$ \\ Relative Humidity: $50 \%$ Ground Roughness: 100 centimeters \\ Cloud Cover: 1 tenths}

\section{SOURCE STRENGTH INFORMATION:}

Puddle Area: 225 square feet

Average Puddle Depth: 1 centimeters

Soil Type: Default Ground Temperature: $29^{\circ} \mathrm{C}$

Initial Puddle Temperature: Ground temperature

Release Duration: ALOHA limited the duration to 1 hour

Max Computed Release Rate: 3.75 kilograms/min

Max Average Sustained Release Rate: 2.95 kilograms/min

(averaged over a minute or more)

Total Amount Released: 143 kilograms

\section{FOOTPRINT INFORMATION:}

Model Run: Heavy Gas

User specified LOC: $75 \mathrm{ppm}$

Max Threat Zone for LOC: 97 meters

Note: Footprint wasn't drawn because effects of near-field patchiness make plume presentation unreliable for short distances.

\section{TIME DEPENDENT INFORMATION:}

Concentration Estimates at the point:

Downwind: 100 meters

Off Centerline: 0 meters

Max Concentration:

Outdoor: $75.2 \mathrm{ppm}$

Indoor: $75.1 \mathrm{ppm}$

Note: Indoor graph is shown with a dotted line.
Reviewed by S. A. Henderson

Works Calculation 7

Sheet No. 262 of 577 
Title of Project EPHA for THM

Subject Concentration Analysis for THM Hazardous Chemicals

Computer T.M. Franey
Reviewed by S. A. Henderson

Works Calculation 7

Sheet No. 263 of 577

SITE DATA INFORMATION: 1-RD-52

Location: AIKEN, SOUTH CAROLINA

Building Air Exchanges Per Hour: 60 (User specified)

Date and Time: Fixed at July 1, 19960000 hours

CHEMICAL INFORMATION:

Chemical Name: VINYL ACETATE

Molecular Weight: $86.09 \mathrm{~kg} / \mathrm{kmol}$

TLV-TWA: $10.00 \mathrm{ppm}$ IDLH: -unavail-

Note: Potential or confirmed human carcinogen.

Footprint Level of Concern: 75 ppm

Boiling Point: $72.50^{\circ} \mathrm{C}$

Vapor Pressure at Ambient Temperature: $0.18 \mathrm{~atm}$

Ambient Saturation Concentration: 184,394 ppm or $18.4 \%$

ATMOSPHERIC INFORMATION: (MANUAL INPUT OF DATA)

Wind: 1.7 meters $/ \mathrm{sec}$ from $90^{\circ}$ true

Inversion Height: 200 meters

Stability Class: E Air Temperature: $29^{\circ} \mathrm{C}$

Relative Humidity: 50\% Ground Roughness: 100 centimeters

Cloud Cover: 1 tenths

SOURCE STRENGTH INFORMATION:

Puddle Area: 24420 square feet

Average Puddle Depth: 1 centimeters

Soil Type: Default Ground Temperature: $29^{\circ} \mathrm{C}$

Initial Puddle Temperature: Ground temperature

Release Duration: ALOHA limited the duration to 1 hour

Max Computed Release Rate: 326 kilograms/min

Max Average Sustained Release Rate: 266 kilograms/min

(averaged over a minute or more)

Total Amount Released: 13,083 kilograms

FOOTPRINT INFORMATION:

Model Run: Heavy Gas

User specified LOC: 75 ppm

Max Threat Zone for LOC: 914 meters

Note: The Heavy Gas footprint is an initial screening.

For short releases it may be an overestimation.

Be sure to check concentration information at specific locations.

TIME DEPENDENT INFORMATION:

Concentration Estimates at the point:

Downwind: 100 meters

Off Centerline: 0 meters

Max Concentration:

Outdoor: $3,140 \mathrm{ppm}$

Indoor: $3,140 \mathrm{ppm}$

Note: Indoor graph is shown with a dotted line. 


\section{ENGINEERING COMPUTATION SHEET}

Title of Project EPHA for THM

Subject Concentration Analysis for THM Hazardous Chemicals

Computer T. M. Franey Date $\underline{09 / 16 / 96}$

SITE DATA INFORMATION: 1-RD-52

Location: AIKEN, SOUTH CAROLINA

Building Air Exchanges Per Hour: 60 (User specified)

Date and Time: Fixed at July 1, 19960000 hours

CHEMICAL INFORMATION:

Chemical Name: VINYL ACETATE

Molecular Weight: $86.09 \mathrm{~kg} / \mathrm{kmol}$

TLV-TWA: $10.00 \mathrm{ppm}$ IDLH: -unavail-

Note: Potential or confirmed human carcinogen.

Footprint Level of Concern: 75 ppm

Boiling Point: $72.50^{\circ} \mathrm{C}$

Vapor Pressure at Ambient Temperature: $0.18 \mathrm{~atm}$

Ambient Saturation Concentration: $184,394 \mathrm{ppm}$ or $18.4 \%$

ATMOSPHERIC INFORMATION: (MANUAL INPUT OF DATA)

Wind: 1.7 meters $/ \mathrm{sec}$ from $90^{\circ}$ true

Inversion Height: 200 meters

Stability Class: E Air Temperature: $29^{\circ} \mathrm{C}$

Relative Humidity: 50\% Ground Roughness: 100 centimeters

Cloud Cover: 1 tenths

SOURCE STRENGTH INFORMATION:

Puddle Area: 40700 square feet

Average Puddle Depth: 1 centimeters

Soil Type: Default Ground Temperature: $29^{\circ} \mathrm{C}$

Initial Puddle Temperature: Ground temperature

Release Duration: ALOHA limited the duration to 1 hour

Max Computéd Release Rate: 531 kilograms/min

Max Average Sustained Release Rate: 435 kilograms/min

(averaged over a minute or more)

Total Amount Released: 21,449 kilograms

FOOTPRINT INFORMATION:

Model Run: Heavy Gas

User specified LOC: $75 \mathrm{ppm}$

Max Threat Zone for LOC: 1.2 kilometers

Note: The Heavy Gas footprint is an initial screening.

For short releases it may be an overestimation.

$\mathrm{Be}$ sure to check concentration information at specific locations.

TIME DEPENDENT INFORMATION:

Concentration Estimates at the point:

Downwind: 100 meters

Off Centerline: 0 meters

Max Concentration:

Outdoor: $5,000 \mathrm{ppm}$

Indoor: $5,000 \mathrm{ppm}$

Note: Indoor graph is shown with a dotted line.
Reviewed by S. A. Henderson

Works Calculation 7

Sheet No. 264 of 577 
Title of Project EPHA for THM

Subject Concentration Analysis for THM Hazardous Chemicals

Computer T. M. Franey

Date $09 / 16 / 96$
Reviewed by S. A. Henderson

Works Calculation 7

Sheet No. 265 of 577

\section{CHEMICAL INFORMATION:}

Chemical Name: VINYL ACETATE

Molecular Weight: $86.09 \mathrm{~kg} / \mathrm{kmol}$

TLV-TWA: $10.00 \mathrm{ppm}$ IDLH: -unavail-

Note: Potential or confirmed human carcinogen.

Footprint Level of Concern: 75 ppm

Boiling Point: $72.50^{\circ} \mathrm{C}$

Vapor Pressure at Ambient Temperature: 0.18 atm

Ambient Saturation Concentration: 184,394 ppm or $18.4 \%$

ATMOSPHERIC INFORMATION: (MANUAL INPUT OF DATA)

Wind: 1.7 meters $/ \mathrm{sec}$ from $90^{\circ}$ true

Inversion Height: 200 meters

Stability Class: E Air Temperature: $29^{\circ} \mathrm{C}$

Relative Humidity: $50 \%$ Ground Roughness: 100 centimeters

Cloud Cover: 1 tenths

SOURCE STRENGTH INFORMATION:

Puddle Area: 10000 square meters

Average Puddle Depth: 1 centimeters

Soil Type: Default Ground Temperature: $29^{\circ} \mathrm{C}$

Initial Puddle Temperature: Ground temperature

Release Duration: ALOHA limited the duration to 1 hour

Max Computed Release Rate: 1,350 kilograms/min

Max Average Sustained Release Rate: 1,110 kilograms/min

(averaged over a minute or more)

Total Amount Released: 55,081 kilograms

FOOTPRINT INFORMATION:

Model Run: Heavy Gas

User specified LOC: 75 ppm

Max Threat Zone for LOC: 1.9 kilometers

Note: The Heavy Gas footprint is an initial screening.

For short releases it may be an overestimation.

Be sure to check concentration information at specific locations.

\section{TIME DEPENDENT INFORMATION:}

Concentration Estimates at the point:

Downwind: 100 meters

Off Centerline: 0 meters

Max Concentration:

Outdoor: $11,900 \mathrm{ppm}$

Indoor: $11,900 \mathrm{ppm}$

Note: Indoor graph is shown with a dotted line. 
Title of Project EPHA for THM

Subject Concentration Analysis for THM Hazardous Chemicals

Computer T. M. Franey
Reviewed by S. A. Henderson

Works Calculation 7

Sheet No. 266 of 577

SITE DATA INFORMATION: 1-RD-53

Location: AIKEN, SOUTH CAROLINA

Building Air Exchanges Per Hour: 60 (User specified)

Date and Time: Fixed at July 1, 19960000 hours

CHEMICAL INFORMATION:

Chemical Name: O-XYLENE Molecular Weight: $106.17 \mathrm{~kg} / \mathrm{kmol}$ TLV-TWA: $100.00 \mathrm{ppm}$ IDLH: $1000.00 \mathrm{ppm}$

Footprint Level of Concern: $200 \mathrm{ppm}$

Boiling Point: $144.43^{\circ} \mathrm{C}$

Vapor Pressure at Ambient Temperature: 0.011 atm

Ambient Saturation Concentration: $11,207 \mathrm{ppm}$ or $1.12 \%$

ATMOSPHERIC INFORMATION: (MANUAL INPUT OF DATA)

Wind: 1.7 meters $/ \mathrm{sec}$ from $90^{\circ}$ true

Inversion Height: 200 meters

Stability Class: E Air Temperature: $29^{\circ} \mathrm{C}$

Relative Humidity: 50\% Ground Roughness: 100 centimeters

Cloud Cover: 1 tenths

\section{SOURCE STRENGTH INFORMATION:}

Puddle Area: 6720 square feet

Average Puddle Depth: 1 centimeters

Soil Type: Default Ground Temperature: $29^{\circ} \mathrm{C}$

Initial Puddle Temperature: Ground temperature

Release Duration: ALOHA limited the duration to 1 hour

Max Computed Release Rate: 6.04 kilograms/min

Max Average Sustained Release Rate: 5.78 kilograms/min (averaged over a minute or more)

Total Amount Released: 330 kilograms

FOOTPRINT INFORMATION:

Dispersion Module: Gaussian

User specified LOC: $200 \mathrm{ppm}$

Max Threat Zone for LOC: 30 meters

Max Threat Zone for IDLH: 14 meters

Note: Footprint was not drawn because effects of near-field patchiness make plume

presentation unreliable for short distances.

TIME DEPENDENT INFORMATION:

Concentration Estimates at the point:

Downwind: 30 meters

Off Centerline: 0 meters

Max Concentration:

Outdoor: $200 \mathrm{ppm}$

Indoor: $200 \mathrm{ppm}$

Note: Indoor graph is shown with a dotted line. 


\section{ENGINEERING COMPUTATION SHEET}

Title of Project EPHA for THM

Reviewed by S. A. Henderson

Works Calculation 7

Sheet No. 267 of 577

SITE DATA INFORMATION: 1-RD-53

Location: AIKEN, SOUTH CAROLINA

Building Air Exchanges Per Hour: 60 (User specified)

Date and Time: Fixed at July 1, 19960000 hours

CHEMICAL INFORMATION:

Chemical Name: O-XYLENE Molecular Weight: $106.17 \mathrm{~kg} / \mathrm{kmol}$

TLV-TWA: $100.00 \mathrm{ppm}$ IDLH: $1000.00 \mathrm{ppm}$

Footprint Level of Concern: 200 ppm

Boiling Point: $144.43^{\circ} \mathrm{C}$

Vapor Pressure at Ambient Temperature: 0.011 atm

Ambient Saturation Concentration: 11,207 ppm or $1.12 \%$

ATMOSPHERIC INFORMATION: (MANUAL INPUT OF DATA)

Wind: 1.7 meters $/ \mathrm{sec}$ from $90^{\circ}$ true

Inversion Height: 200 meters

Stability Class: E Air Temperature: $29^{\circ} \mathrm{C}$

Relative Humidity: 50\% Ground Roughness: 100 centimeters

Cloud Cover: 1 tenths

SOURCE STRENGTH INFORMATION:

Puddle Area: 32140 square feet

Average Puddle Depth: 1 centimeters

Soil Type: Default Ground Temperature: $29^{\circ} \mathrm{C}$

Initial Puddle Temperature: Ground temperature

Release Duration: ALOHA limited the duration to 1 hour

Max Computed Release Rate: 27 kilograms/min

Max Average Sustained Release Rate: 25.9 kilograms/min

(averaged over a minute or more)

Total Amount Released: 1,483 kilograms

\section{FOOTPRINT INFORMATION:}

Dispersion Module: Gaussian

User specified LOC: $200 \mathrm{ppm}$

Max Threat Zone for LOC: 64 meters

Max Threat Zone for IDLH: 31 meters

Note: Footprint was not drawn because effects of near-field patchiness make plume presentation unreliable for short distances.

\section{TIME DEPENDENT INFORMATION:}

Concentration Estimates at the point:

Downwind: 100 meters

Off Centerline: 0 meters

Max Concentration:

Outdoor: $90.9 \mathrm{ppm}$

Indoor: $90.9 \mathrm{ppm}$

Note: Indoor graph is shown with a dotted line. 


\section{ENGINEERING COMPUTATION SHEET}

Title of Project EPHA for THM

Subject Concentration Analysis for THM Hazardous Chemicals

Computer T. M. Franey

Date $\underline{09 / 16 / 96}$

SITE DATA INFORMATION: 1-RD-53

Location: AIKEN, SOUTH CAROLINA

Building Air Exchanges Per Hour: 60 (User specified)

Date and Time: Fixed at July 1, 19960000 hours

CHEMICAL INFORMATION:

Chemical Name: O-XYLENE Molecular Weight: $106.17 \mathrm{~kg} / \mathrm{kmol}$

TLV-TWA: $100.00 \mathrm{ppm}$ IDLH: $1000.00 \mathrm{ppm}$

Footprint Level of Concern: 200 ppm

Boiling Point: $144.43^{\circ} \mathrm{C}$

Vapor Pressure at Ambient Temperature: $0.011 \mathrm{~atm}$

Ambient Saturation Concentration: 11,207 ppm or $1.12 \%$

ATMOSPHERIC INFORMATION: (MANUAL INPUT OF DATA)

Wind: 1.7 meters $/ \mathrm{sec}$ from $90^{\circ}$ true

Inversion Height: 200 meters

Stability Class: E Air Temperature: $29^{\circ} \mathrm{C}$

Relative Humidity: 50\% Ground Roughness: 100 centimeters

Cloud Cover: 1 tenths

\section{SOURCE STRENGTH INFORMATION:}

Puddle Area: 24420 square feet

Average Puddle Depth: 1 centimeters

Soil Type: Default Ground Temperature: $29^{\circ} \mathrm{C}$

Initial Puddle Temperature: Ground temperature

Release Duration: ALOHA limited the duration to 1 hour

Max Computed Release Rate: 20.8 kilograms/min

Max Average Sustained Release Rate: 19.9 kilograms/min

(averaged over a minute or more)

Total Amount Released: 1,139 kilograms

FOOTPRINT INFORMATION:

Dispersion Module: Gaussian

User specified LOC: $200 \mathrm{ppm}$

Max Threat Zone for LOC: 56 meters

Max Threat Zone for IDLH: 27 meters

Note: Footprint was not drawn because effects of near-field patchiness make plume presentation unreliable for short distances.

\section{TIME DEPENDENT INFORMATION:}

Concentration Estimates at the point:

Downwind: 100 meters

Off Centerline: 0 meters

Max Concentration:

Outdoor: $75.6 \mathrm{ppm}$

Indoor: $75.6 \mathrm{ppm}$

Note: Indoor graph is shown with a dotted line.

(2)
Reviewed by S. A. Henderson

Works Calculation 7

Sheet No. 268 of 577 


\section{ENGINEERING COMPUTATION SHEET}

Title of Project EPHA for THM

Subject Concentration Analysis for THM Hazardous Chemicals

Computer T. M. Franey

Date 09/16/96

SITE DATA INFORMATION: 1-RD-53

Location: AIKEN, SOUTH CAROLINA

Building Air Exchanges Per Hour: 60 (User specified)

Date and Time: Fixed at July 1, 19960000 hours

\section{CHEMICAL INFORMATION:}

Chemical Name: O-XYLENE

TLV-TWA: $100.00 \mathrm{ppm}$

Molecular Weight: $106.17 \mathrm{~kg} / \mathrm{kmol}$

Footprint Level of Concern: 200 ppm

Boiling Point: $144.43^{\circ} \mathrm{C}$

Vapor Pressure at Ambient Temperature: $0.011 \mathrm{~atm}$

Ambient Saturation Concentration: $11,207 \mathrm{ppm}$ or $1.12 \%$

ATMOSPHERIC INFORMATION: (MANUAL INPUT OF DATA)

Wind: 1.7 meters $/ \mathrm{sec}$ from $90^{\circ}$ true

Inversion Height: 200 meters

Stability Class: E Air Temperature: $29^{\circ} \mathrm{C}$

Relative Humidity: 50\% Ground Roughness: 100 centimeters

Cloud Cover: 1 tenths

\section{SOURCE STRENGTH INFORMATION:}

Puddle Area: 40700 square feet

Average Puddle Depth: 1 centimeters

Soil Type: Default Ground Temperature: $29^{\circ} \mathrm{C}$

Initial Puddle Temperature: Ground temperature

Release Duration: ALOHA limited the duration to 1 hour

Max Computed Release Rate: 33.9 kilograms/min

Max Average Sustained Release Rate: 32.5 kilograms/min

(averaged over a minute or more)

Total Amount Released: 1,860 kilograms

\section{FOOTPRINT INFORMATION:}

Model Run: Heavy Gas

User specified LOC: $200 \mathrm{ppm}$

Max Threat Zone for LOC: 111 meters

Max Threat Zone for IDLH: IDLH level was never exceeded

Note: The Heavy Gas footprint is an initial screening.

For short releases it may be an overestimation.

Be sure to check concentration information at specific locations.

\section{TIME DEPENDENT INFORMATION:}

\section{Concentration Estimates at the point:}

Downwind: 100 meters

Off Centerline: 0 meters

Max Concentration:

Outdoor: 235 ppm

Indoor: $235 \mathrm{ppm}$

Note: Indoor graph is shown with a dotted line.
Reviewed by S.A. Henderson

Works Calculation 7

Sheet No. 269 of 577 
Title of Project EPHA for THM

Subject Concentration Analysis for THM Hazardous Chemicals

Computer T. M. Franey
Reviewed by $\underline{S . A . ~ H e n d e r s o n ~}$

Works Calculation 7

Sheet No. 270 of 577

SITE DATA INFORMATION: 1-RD-53

Location: AIKEN, SOUTH CAROLINA

Building Air Exchanges Per Hour: 60 (User specified)

Date and Time: Fixed at July 1, 19960000 hours

CHEMICAL INFORMATION:

Chemical Name: O-XYLENE Molecular Weight: $106.17 \mathrm{~kg} / \mathrm{kmol}$ TLV-TWA: $100.00 \mathrm{ppm}$ IDLH: $1000.00 \mathrm{ppm}$

Footprint Level of Concern: 200 ppm

Boiling Point: $144.43^{\circ} \mathrm{C}$

Vapor Pressure at Ambient Temperature: 0.011 atm

Ambient Saturation Concentration: 11,207 ppm or $1.12 \%$

\section{ATMOSPHERIC INFORMATION: (MANUAL INPUT OF DATA)}

Wind: 1.7 meters $/ \mathrm{sec}$ from $90^{\circ}$ true

Inversion Height: 200 meters

Stability Class: E Air Temperature: $29^{\circ} \mathrm{C}$

Relative Humidity: 50\% Ground Roughness: 100 centimeters

Cloud Cover: 1 tenths

\section{SOURCE STRENGTH INFORMATION:}

Puddle Area: 10000 square meters

Average Puddle Depth: 1 centimeters

Soil Type: Default Ground Temperature: $29^{\circ} \mathrm{C}$

Initial Puddle Temperature: Ground temperature

Release Duration: ALOHA limited the duration to 1 hour

Max Computed Release Rate: 86.3 kilograms/min

Max Average Sustained Release Rate: 82.8 kilograms/min

(averaged over a minute or more)

Total Amount Released: 4,738 kilograms

\section{FOOTPRINT INFORMATION: \\ Model Run: Heavy Gas \\ User specified LOC: $200 \mathrm{ppm}$ \\ Max Threat Zone for LOC: 182 meters \\ Max Threat Zone for IDLH: IDLH level was never exceeded \\ Note: The Heavy Gas footprint is an initial screening. \\ For short releases it may be an overestimation. \\ Be sure to check concentration information at specific locations.}

\section{TIME DEPENDENT INFORMATION:}

Concentration Estimates at the point:

Downwind: 100 meters

Off Centerline: 0 meters

Max Concentration:

Outdoor: 429 ppm

Indoor: $429 \mathrm{ppm}$

Note: Indoor graph is shown with a dotted line. 


\section{ENGINEERING COMPUTATION SHEET}

Title of Project EPHA for THM

Subject Concentration Analysis for THM Hazardous Chemicals

Computer T. M. Franey

Date $\underline{09 / 16 / 96}$

SITE DATA INFORMATION: 1-RD-54 - 1-RD-58

Location: AIKEN, SOUTH CAROLINA

Building Air Exchanges Per Hour: 60 (User specified)

Date and Time: Fixed at July 1, 19960000 hours

CHEMICAL INFORMATION:

Chemical Name: RESPIRABLE DUST

Molecular Weight: $80.00 \mathrm{~kg} / \mathrm{kmol}$

TLV-TWA: -unavail- IDLH: -unavail-

Footprint Level of Concern: $50 \mathrm{ppm}$

Boiling Point: -unavail-

Note: Not enough chemical data to use Heavy Gas option

ATMOSPHERIC INFORMATION: (MANUAL INPUT OF DATA)

Wind: 1.7 meters $/ \mathrm{sec}$ from $90^{\circ}$ true

Inversion Height: 200 meters

Stability Class: E Air Temperature: $29^{\circ} \mathrm{C}$

Relative Humidity: 50\% Ground Roughness: 100 centimeters

Cloud Cover: 1 tenths

\section{SOURCE STRENGTH INFORMATION:}

Direct Source: 49 pounds Source Height: 0

Release Duration: 1 minute

Release Rate: 3.7 grams/sec

Total Amount Released: 222 grams

FOOTPRINT INFORMATION:

Dispersion Module: Gaussian

User specified LOC: $50 \mathrm{ppm}$

Max Threat Zone for LOC: 30 meters

Note: Footprint was not drawn because effects of near-field patchiness make plume presentation unreliable for short distances.

TIME DEPENDENT INFORMATION:

Concentration Estimates at the point:

Downwind: 30 meters

Off Centerline: 0 meters

Max Concentration:

Outdoor: $50.9 \mathrm{ppm}$

Indoor: $30.7 \mathrm{ppm}$

Note: Indoor graph is shown with a dotted line.
Reviewed by S. A. Henderson

Works Calculation 7

Sheet No. 271 of 577 


\section{ENGINEERING COMPUTATION SHEET}

Title of Project EPHA for THM

Reviewed by $\underline{\text { S. A. Henderson }}$

Subject Concentration Analysis for THM Hazardous Chemicals

Computer T.M. Franey

Date $\underline{09 / 16 / 96}$

SITE DATA INFORMATION: 1-RD-54 - 1-RD-58

Location: AIKEN, SOUTH CAROLINA

Building Air Exchanges Per Hour: 60 (User specified)

Date and Time: Fixed at July 1, 19960000 hours

\section{CHEMICAL INFORMATION:}

Chemical Name: RESPIRABLE DUST

Molecular Weight: $80.00 \mathrm{~kg} / \mathrm{kmol}$

TLV-TWA: -unavail- IDLH: -unavail-

Footprint Level of Concern: $50 \mathrm{ppm}$

Boiling Point: -unavail-

Note: Not enough chemical data to use Heavy Gas option

ATMOSPHERIC INFORMATION: (MANUAL INPUT OF DATA)

Wind: 1.7 meters $/ \mathrm{sec}$ from $90^{\circ}$ true

Inversion Height: 200 meters

Stability Class: E Air Temperature: $29^{\circ} \mathrm{C}$

Relative Humidity: $50 \%$ Ground Roughness: 100 centimeters

Cloud Cover: 1 tenths

\section{SOURCE STRENGTH INFORMATION:}

Direct Source: 5.1 pounds Source Height: 0

Release Duration: 1 minute

Release Rate: 38.6 grams $/ \mathrm{sec}$

Total Amount Released: 2.31 kilograms

\section{FOOTPRINT INFORMATION:}

Dispersion Module: Gaussian

User specified LOC: $50 \mathrm{ppm}$

Max Threat Zone for LOC: 100 meters

TIME DEPENDENT INFORMATION:

Concentration Estimates at the point:

Downwind: 100 meters

Off Centerline: 0 meters

Max Concentration:

Outdoor: $50.2 \mathrm{ppm}$

Indoor: $28.3 \mathrm{ppm}$

Note: Indoor graph is shown with a dotted line.
Works Calculation 7

\section{Sheet No. 272 of 577}


Title of Project EPHA for THM

Reviewed by $\underline{\text { S.A. Henderson }}$

Subject Concentration Analysis for THM Hazardous Chemicals

Computer T. M. Franey

Date 09/16/96
Works Calculation 7

Sheet No. 273 of 577

SITE DATA INFORMATION: 1-RD-54 - 1-RD-58

Location: AIKEN, SOUTH CAROLINA

Building Air Exchanges Per Hour: 60 (User specified)

Date and Time: Fixed at July 1, 19960000 hours

CHEMICAL INFORMATION:

Chemical Name: RESPIRABLE DUST

Molecular Weight: $80.00 \mathrm{~kg} / \mathrm{kmol}$

TLV-TWA: -unavail- IDLH: -unavail-

Footprint Level of Concern: $50 \mathrm{ppm}$

Boiling Point: -unavail-

Note: Not enough chemical data to use Heavy Gas option

ATMOSPHERIC INFORMATION: (MANUAL INPUT OF DATA)

Wind: 1.7 meters $/ \mathrm{sec}$ from $90^{\circ}$ true

Inversion Height: 200 meters

Stability Class: E Air Temperature: $29^{\circ} \mathrm{C}$

Relative Humidity: 50\% Ground Roughness: 100 centimeters

Cloud Cover: 1 tenths

SOURCE STRENGTH INFORMATION:

Direct Source: 37.6 pounds

Source Height: 0

Release Duration: 1 minute

Release Rate: 284 grams/sec

Total Amount Released: 17.1 kilograms

FOOTPRINT INFORMATION:

Dispersion Module: Gaussian

User specified LOC: $50 \mathrm{ppm}$

Max Threat Zone for LOC: 260 meters

TIME DEPENDENT INFORMATION:

Concentration Estimates at the point:

Downwind: 100 meters

Off Centerline: 0 meters

Max Concentration:

Outdoor: 370 ppm

Indoor: $209 \mathrm{ppm}$

Note: Indoor graph is shown with a dotted line. 
Title of Project EPHA for THM

Subject Concentration Analysis for THM Hazardous Chemicals

Computer T. M. Franey Date 09/16/96

SITE DATA INFORMATION: 1-RD-54 - 1-RD-58

Location: AIKEN, SOUTH CAROLINA

Building Air Exchanges Per Hour: 60 (User specified)

Date and Time: Fixed at July 1, 19960000 hours

\section{CHEMICAL INFORMATION:}

Chemical Name: RESPIRABLE DUST

Molecular Weight: $80.00 \mathrm{~kg} / \mathrm{kmol}$

TLV-TWA: -unavail- IDLH: -unavail-

Footprint Level of Concern: 50 ppm

Boiling Point: -unavail-

Note: Not enough chemical data to use Heavy Gas option

ATMOSPHERIC INFORMATION: (MANUAL INPUT OF DATA)

Wind: 1.7 meters $/ \mathrm{sec}$ from $90^{\circ}$ true

Inversion Height: 200 meters

Stability Class: E Air Temperature: $29^{\circ} \mathrm{C}$

Relative Humidity: 50\% Ground Roughness: 100 centimeters

Cloud Cover: 1 tenths

\section{SOURCE STRENGTH INFORMATION:}

Direct Source: 135 pounds Source Height: 0

Release Duration: 1 minute

Release Rate: 1.02 kilograms/sec

Total Amount Released: 61.2 kilograms

FOOTPRINT INFORMATION:

Dispersion Module: Gaussian

User specified LOC: $50 \mathrm{ppm}$

Max Threat Zone for LOC: 437 meters

\section{TIME DEPENDENT INFORMATION:}

Concentration Estimates at the point:

Downwind: 100 meters

Off Centerline: 0 meters

Max Concentration:

Outdoor: $1,330 \mathrm{ppm}$

Indoor: $749 \mathrm{ppm}$

Note: Indoor graph is shown with a dotted line.
Reviewed by $\underline{S}$. A. Henderson

Works Calculation 7

Sheet No. 274 of 577 
Title of Project EPHA for THM

Subject Concentration Analysis for THM Hazardous Chemicals

Computer T. M. Franey Date $09 / 16 / 96$
Reviewed by $\underline{S}$. A. Henderson

Works Calculation ?

Sheet No. 275 of 577

SITE DATA INFORMATION: 1-RD-54 - 1-RD-58

Location: AIKEN, SOUTH CAROLINA

Building Air Exchanges Per Hour: 60 (User specified)

Date and Time: Fixed at July 1, 19960000 hours

CHEMICAL INFOQRMATION:

Chemical Name: RESPIRABLE DUST

Molecular Weight: $80.00 \mathrm{~kg} / \mathrm{kmol}$

TLV-TWA: -unavail- IDLH: -unavail-

Footprint Level of Concern: 50 ppm

Boiling Point: -unavail-

Note: Not enough chemical data to use Heavy Gas option

ATMOSPHERIC INFORMATION: (MANUAL INPUT OF DATA)

Wind: 1.7 meters $/ \mathrm{sec}$ from $90^{\circ}$ true

Inversion Height: 200 meters

Stability Class: E Air Temperature: $29^{\circ} \mathrm{C}$

Relative Humidity: $50 \%$ Ground Roughness: 100 centimeters

Cloud Cover: 1 tenths

SOURCE STRENGTH INFORMATION:

Direct Source: 263 pounds Source Height: 0

Release Duration: I minute

Release Rate: 1.99 kilograms/sec

Total Amount Released: 119 kilograms

FOOTPRINT INFORMATION:

Dispersion Module: Gaussian

User specified LOC: $50 \mathrm{ppm}$

Max Threat Zone for LOC: 567 meters

TIME DEPENDENT INFORMATION:

Concentration Estimates at the point:

Downwind: 100 meters

Off Centerline: 0 meters

Max Concentration:

Outdoor: $2,590 \mathrm{ppm}$

Indoor: $1,460 \mathrm{ppm}$

Note: Indoor graph is shown with a dotted line. 


\section{ENGINEERING COMPUTATION SHEET}

Title of Project EPHA for THM

Reviewed by $\underline{S}$. A. Henderson

Subject Concentration Analysis for THM Hazardous Chemicals

Computer T. M. Franey

Date $\underline{09 / 16 / 96}$
Works Calculation 7

Sheet No. 276 of 577

1-RD-59

SITE DATA INFORMATION:

Location: AIKEN, SOUTH CAROLINA

Building Air Exchanges Per Hour: 60 (User specified)

Date and Time: Fixed at July 1, 19960000 hours

\section{CHEMICAL INFORMATION:}

Chemical Name: AMMONIA, ANHYDROUS

Molecular Weight: $17.03 \mathrm{~kg} / \mathrm{kmol}$

TLV-TWA: $25.00 \mathrm{ppm}$ IDLH: $500.00 \mathrm{ppm}$

Footprint Level of Concern: 200 ppm

Boiling Point: $-33.43^{\circ} \mathrm{C}$

Vapor Pressure at Ambient Temperature: greater than $1 \mathrm{~atm}$

Ambient Saturation Concentration: $1,000,000 \mathrm{ppm}$ or $100.0 \%$

ATMOSPHERIC INFORMATION: (MANUAL INPUT OF DATA)

Wind: 1.7 meters $/ \mathrm{sec}$ from $90^{\circ}$ true

Inversion Height: 200 meters

Stability Class: E Air Temperature: $29^{\circ} \mathrm{C}$

Relative Humidity: 50\% Ground Roughness: Urban or forest

Cloud Cover: 1 tenths

\section{SOURCE STRENGTH INFORMATION:}

Puddle Area: 5 square feet

Average Puddle Depth: 1 centimeters

Soil Type: Default Ground Temperature: $29^{\circ} \mathrm{C}$

Initial Puddle Temperature: boiling point

Release Duration: 32 minutes

Max Computed Release Rate: 2.01 kilograms/min

Max Average Sustained Release Rate: 203 grams/min (averaged over a minute or more)

Total Amount Released: 3.17 kilograms

\section{TIME DEPENDENT INFORMATION:}

Concentration Estimates at the point:

Downwind: 30 meters

Off Centerline: 0 meters

Max Concentration:

Outdoor: 217 ppm 


\section{ENGINEERING COMPUTATION SHEET}

Title of Project EPHA for THM

Subject Concentration Analysis for THM Hazardous Chemicals

Computer T. M. Franey

Date $\underline{09 / 16 / 96}$
Reviewed by $\underline{\text { S. A. Henderson }}$

Works Calculation 7

Sheet No. $\underline{277 \text { of } 577}$

1-RD-59

SITE DATA INFORMATION:

Location: AIKEN, SOUTH CAROLINA

Building Air Exchanges Per Hour: 60 (User specified)

Date and Time: Fixed at July 1, 19960000 hours

CHEMICAL INFORMATION:

Chemical Name: AMMONLA, ANHYDROUS

Molecular Weight: $17.03 \mathrm{~kg} / \mathrm{kmol}$

TLV-TWA: $25.00 \mathrm{ppm}$ IDLH: $500.00 \mathrm{ppm}$

Footprint Level of Concern: 200 ppm

Boiling Point: $-33.43^{\circ} \mathrm{C}$

Vapor Pressure at Ambient Temperature: greater than 1 atm

Ambient Saturation Concentration: $1,000,000 \mathrm{ppm}$ or $100.0 \%$

ATMOSPHERIC INFORMATION: (MANUAL INPUT OF DATA)

Wind: 1.7 meters $/ \mathrm{sec}$ from $90^{\circ}$ true

Inversion Height: 200 meters

Stability Class: E Air Temperature: $29^{\circ} \mathrm{C}$

Relative Humidity: 50\% Ground Roughness: Urban or forest

Cloud Cover: 1 tenths

SOURCE STRENGTH INFORMATION:

Puddle Area: 55 square feet

Average Puddle Depth: 1 centimeters

Soil Type: Default Ground Temperature: $29^{\circ} \mathrm{C}$

Initial Puddle Temperature: boiling point

Release Duration: 37 minutes

Max Computed Release Rate: 19.9 kilograms/min

Max Average Sustained Release Rate: 2.05 kilograms/min

(averaged over a minute or more)

Total Amount Released: 34.8 kilograms

TIME DEPENDENT INFORMATION:

Concentration Estimates at the point:

Downwind: 100 meters

Off Centerline: 0 meters

Max Concentration:

Outdoor: 207 ppm 


\section{ENGINEERING COMPUTATION SHEET}

Title of Project EPHA for THM

Reviewed by S. A. Henderson

Subject Concentration Analysis for THM Hazardous Chemicals

Works Calculation 7

Computer T. M. Franey

Date 09/16/96

Sheet No. 278 of 577

1-RD-59

For analysis on 1 ton, see hard copy. 
OSR :S.10 (Rev 6-18.42)

\section{ENGINEERING COMPUTATION SHEET}

Title of Project EPHA for THM

Subject Concentration Analysis for THM Hazardous Chemicals

Computer T. M. Franey

Date $\underline{09 / 16 / 96}$
Reviewed by $\underline{\text { S. A. Henderson }}$

Works Calculation 7

Sheet No. 279 of 577

1-RD-59

For analysis on 10 tons, see hard copy. 


\section{ENGINEERING COMPUTATION SHEET}

Title of Project EPHA for THM

Reviewed by S. A. Henderson

Works Calculation 7

Subject Concentration Analysis for THM Hazardous Chemicals

Date $\underline{09 / 16 / 96}$

Sheet No. 280 of 577

1-RD-59

For analysis on 17.5 tons, see hard copy. 
Title of Project EPHA for THM

Subject Concentration Analysis for THM Hazardous Chemicals

Computer T. M. Franey

1-RD-60

SITE DATA INFORMATION: Location: AIKEN, SOUTH CAROLINA

Building Air Exchanges Per Hour: 60 (User specified)

Date and Time: Fixed at July 1, 19960000 hours

CHEMICAL INFORMATION:

Chemical Name: CHLORINE Molecular Weight: $70.90 \mathrm{~kg} / \mathrm{kmol}$

TLV-TWA: $0.50 \mathrm{ppm}$. IDLH: $30.00 \mathrm{ppm}$

Footprint Level of Concern: 3 ppm

Boiling Point: $-34.03^{\circ} \mathrm{C}$

Vapor Pressure at Ambient Temperature: greater than $1 \mathrm{~atm}$

Ambient Saturation Concentration: $1,000,000 \mathrm{ppm}$ or $100.0 \%$

ATMOSPHERIC INFORMATION: (MANUAL INPUT OF DATA)

Wind: 1.7 meters $/ \mathrm{sec}$ from $90^{\circ}$ true

Inversion Height: 200 meters

Stability Class: E Air Temperature: $29^{\circ} \mathrm{C}$

Relative Humidity: 50\% Ground Roughness: Urban or forest

Cloud Cover: 1 tenths

\section{SOURCE STRENGTH INFORMATION:}

Puddle Area: .1 square feet

Average Puddle Depth: 1 centimeters

Soil Type: Default Ground Temperature: $29^{\circ} \mathrm{C}$

Initial Puddle Temperature: boiling point

Release Duration: 9 minutes

Max Computed Release Rate: 258 grams/min

Max Average Sustained Release Rate: 36.3 grams/min

(averaged over a minute or more)

Total Amount Released: 145 grams

TIME DEPENDENT INFORMATION:

Concentration Estimates at the point:

Downwind: 30 meters

Off Centerline: 0 meters

Max Concentration:

Outdoor: $9.38 \mathrm{ppm}$ 
Title of Project EPHA for THM

Subject Concentration Analysis for THM Hazardous Chemicals

Computer T. M. Franey

Date 09/16/96
Reviewed by $\underline{\text { S. A. Henderson }}$

Works Calculation 7

Sheet No. 282 of 577

1-RD-60

SITE DATA INFORMATION:

Location: AIKEN, SOUTH CAROLINA

Building Air Exchanges Per Hour: 60 (User specified)

Date and Time: Fixed at July 1, 19960000 hours

CHEMICAL INFORMATION:

Chemical Name: CHLORINE Molecular Weight: $70.90 \mathrm{~kg} / \mathrm{kmol}$

TLV-TWA: $0.50 \mathrm{ppm}$

IDLH: $30.00 \mathrm{ppm}$

Footprint Level of Concern: 3 ppm

Boiling Point: $-34.03^{\circ} \mathrm{C}$

Vapor Pressure at Ambient Temperature: greater than $1 \mathrm{~atm}$

Ambient Saturation Concentration: $1,000,000$ ppm or $100.0 \%$

ATMOSPHERIC INFORMATION: (MANUAL INPUT OF DATA)

Wind: 1.7 meters $/ \mathrm{sec}$ from $90^{\circ}$ true

Inversion Height: 200 meters

Stability Class: E Air Temperature: $29^{\circ} \mathrm{C}$

Relative Humidity: 50\% Ground Roughness: Urban or forest

Cloud Cover: 1 tenths

SOURCE STRENGTH INFORMATION:

Puddle Area: 1 square feet

Average Puddle Depth: 1 centimeters

Soil Type: Default Ground Temperature: $29^{\circ} \mathrm{C}$

Initial Puddle Temperature: boiling point

Release Duration: 10 minutes

Max Computed Release Rate: 2.34 kilograms/min

Max Average Sustained Release Rate: 347 grams/min

(averaged over a minute or more)

Total Amount Released: 1.45 kilograms

FOOTPRINT INFORMATION:

Model Run: Heavy Gas

User specified LOC: 3 ppm

Max Threat Zone for LOC: 180 meters

Max Threat Zone for IDLH: 23 meters

Note: The Heavy Gas footprint is an initial screening.

For short releases it may be an overestimation.

Be sure to check concentration information at specific locations.

TIME DEPENDENT INFORMATION:

Concentration Estimates at the point:

Downwind: 100 meters

Off Centerline: 0 meters

Max Concentration:

Outdoor: $7.03 \mathrm{ppm}$ 
Title of Project EPHA for THM

Subject Concentration Analysis for THM Hazardous Chemicals

Date $\underline{09 / 16 / 96}$
Reviewed by S. A. Henderson

SRS

Works Calculation 7

Sheet No. 283 of 577

1-RD-60

For analysis on 1 ton, see hard copy. 
Title of Project EPHA for THM

Reviewed by $\underline{\mathrm{S}}$. A. Hénderson

Subject Concentration Analysis for THM Hazardous Chemicals

Computer T.M. Franey

Date 09/16/96
Works Calculation 7

Sheet No. $\underline{284 \text { of } 577}$

1-RD-60

For analysis on 10 tons, see hard copy. 


\section{ENGINEERING COMPUTATION SHEET}

Title of Project EPHA for THM

Reviewed by $\underline{S}$. A. Henderson

Subject Concentration Analysis for THM Hazardous Chemicals

Date 09/16/96
Works Calculation 7

Sheet No. $\underline{285 \text { of } 577}$

1-RD-60

For analysis on $\mathbf{1 7 . 5}$ tons, see hard copy. 


\section{ENGINEERING COMPUTATION SHEET}

Title of Project EPHA for THM

Subject Concentration Analysis for THM Hazardous Chemicals

Computer T. M. Franey

Date 09/16/96

1-RD-61

SITE DATA INFORMATION: Location: AIKEN, SOUTH CAROLINA

Building Air Exchanges Per Hour: 60 (User specified)

Date and Time: Fixed at July 1, 19960000 hours

CHEMICAL INFORMATION:

Chemical Name: TRIMETHYLAMINE, ANHYDROUS

Molecular Weight: $59.11 \mathrm{~kg} / \mathrm{kmol}$

TLV-TWA: $5.00 \mathrm{ppm}$ IDLH: -unavail-

Footprint Level of Concern: $25 \mathrm{ppm}$

Boiling Point: $2.87^{\circ} \mathrm{C}$

Vapor Pressure at Ambient Temperature: greater than $1 \mathrm{~atm}$

Ambient Saturation Concentration: $1,000,000 \mathrm{ppm}$ or $100.0 \%$

ATMOSPHERIC INFORMATION: (MANUAL INPUT OF DATA)

Wind: 1.7 meters $/ \mathrm{sec}$ from $90^{\circ}$ true

Inversion Height: 200 meters

Stability Class: E Air Temperature: $29^{\circ} \mathrm{C}$

Relative Humidity: 50\% Ground Roughness: Urban or forest

Cloud Cover: 1 tenths

\section{SOURCE STRENGTH INFORMATION:}

Puddle Area: 1 square feet

Average Puddle Depth: 1 centimeters

Soil Type: Default Ground Temperature: $29^{\circ} \mathrm{C}$

Initial Puddle Temperature: boiling point

Release Duration: 15 minutes

Max Computed Release Rate: 961 grams/min

Max Average Sustained Release Rate: $90.9 \mathrm{grams} / \mathrm{min}$

(averaged over a minute or more)

Total Amount Released: 609 grams

FOOTPRINT INFORMATION:

Dispersion Module: Gaussian

User specified LOC: $25 \mathrm{ppm}$

Max Threat Zone for LOC: 32 meters

Note: Footprint was not drawn because effects of near-field patchiness make plume presentation unreliable for short distances.

TIME DEPENDENT INFORMATION:

Concentration Estimates at the point:

Downwind: 30 meters

Off Centerline: 0 meters

Max Concentration:

Outdoor: $28.1 \mathrm{ppm}$
Reviewed by S. A. Henderson

Works Calculation 7

Sheet No. 286 of 577 


\section{ENGINEERING COMPUTATION SHEET}

Title of Project EPHA for THM

Subject Concentration Analysis for THM Hazardous Chemicals

Computer T. M. Franey

Date $\underline{09 / 16 / 96}$

1-RD-61

SITE DATA INFORMATION:

Location: AIKEN, SOUTH CAROLINA

Building Air Exchanges Per Hour: 60 (User specified)

Date and Time: Fixed at July 1, 19960000 hours

CHEMICAL INFORMATION:

Chemical Name: TRIMETHYLAMINE, ANHYDROUS

Molecular Weight: $59.11 \mathrm{~kg} / \mathrm{kmol}$

TLV-TWA: $5.00 \mathrm{ppm}$ IDLH: -unavail-

Footprint Level of Concern: $25 \mathrm{ppm}$

Boiling Point: $2.87^{\circ} \mathrm{C}$

Vapor Pressure at Ambient Temperature: greater than 1 atm

Ambient Saturation Concentration: $1,000,000$ ppm or $100.0 \%$

ATMOSPHERIC INFORMATION: (MANUAL INPUT OF DATA)

Wind: 1.7 meters $/ \mathrm{sec}$ from $90^{\circ}$ true

Inversion Height: 200 meters

Stability Class: E Air Temperature: $29^{\circ} \mathrm{C}$

Relative Humidity: 50\% Ground Roughness: Urban or forest

Cloud Cover: 1 tenths

SOURCE STRENGTH INFORMATION:

Puddle Area: 5 square feet

Average Puddle Depth: 1 centimeters

Soil Type: Default Ground Temperature: $29^{\circ} \mathrm{C}$

Initial Puddle Temperature: boiling point

Release Duration: 17 minutes

Max Computed Release Rate: 4.52 kilograms/min

Max Average Sustained Release Rate: 431 grams/min

(averaged over a minute or more)

Total Amount Released: 3.04 kilograms

FOOTPRINT INFORMATION:

Model Run: Heavy Gas

User specified LOC: $25 \mathrm{ppm}$

Max Threat Zone for LOC: 113 meters

Note: The Heavy Gas footprint is an initial screening.

For short releases it may be an overestimation.

Be sure to check concentration information at specific locations.

TIME DEPENDENT INFORMATION:

Concentration Estimates at the point:

Downwind: 100 meters

Off Centerline: 0 meters

Max Concentration:

Outdoor: $31.2 \mathrm{ppm}$
Reviewed by S.A. Henderson

Works Calculation 7

Sheet No. 287 of 577 
Title of Project EPHA for THM

Reviewed by S. A. Henderson

Subject Concentration Analysis for THM Hazardous Chemicals

Computer T. M. Franey

Date $\underline{09 / 16 / 96}$
Works Calculation 7

Sheet No. 288 of 577

1-RD-61

For analysis on 1 ton, see hard copy. 


\section{ENGINEERING COMPUTATION SHEET}

Title of Project EPHA for THM

Reviewed by $\underline{S}$. A. Henderson

Subject Concentration Analysis for THM Hazardous Chemicals

Computer T. M. Franey

Date $\underline{09 / 16 / 96}$
Works Calculation 7

Sheet No. 289 of 577

1-RD.61

For analysis on 10 tons, see hard copy. 
OSR 25-10(Rev 6-18.42)

\section{ENGINEERING COMPUTATION SHEET}

Title of Project EPHA for THM

Reviewed by $\mathrm{S}$. A. Henderson

SRS

Subject Concentration Analysis for THM Hazardous Chemicals

Works Calculation 7

Computer T. M. Franey

Date $\underline{09 / 16 / 96}$

Sheet No. 290 of 577

1-RD-61

For analysis on $\mathbf{1 7 . 5}$ tons, see hard copy. 
Title of Project EPHA for THM

Reviewed by S. A. Henderson

Works Calculation 7

Subject Concentration Analysis for THM Hazardous Chemicals

Computer T.M. Franey

Date 09/16/96
Sheet No. 291 of 577

\section{$50 \%$ Average Meteorological Conditions}

\section{ALOHA Runs}




\section{ENGINEERING COMPUTATION SHEET}

Title of Project EPHA for THM

Reviewed by $\underline{\text { S. A. Henderson }}$

Subject Concentration Analysis for THM Hazardous Chemicals

Computer T. M. Franey

Date $\underline{09 / 16 / 96}$

SITE DATA INFORMATION: 1-RD-1

Location: AIKEN, SOUTH CAROLINA

Building Air Exchanges Per Hour: 60 (User specified)

Date and Time: Fixed at July 1, 19961300 hours

CHEMICAL INFORMATION:

Chemical Name: ACETALDEHYDE

Molecular Weight: $44.05 \mathrm{~kg} / \mathrm{kmol}$

TLV-TWA: $100.00 \mathrm{ppm}$ IDLH: $10000.00 \mathrm{ppm}$

Note: Potential or confirmed human carcinogen.

Footprint Level of Concern: 200 ppm

Boiling Point: $20.40^{\circ} \mathrm{C}$

Vapor Pressure at Ambient Temperature: greater than $1 \mathrm{~atm}$

Ambient Saturation Concentration: $1,000,000 \mathrm{ppm}$ or $100.0 \%$

ATMOSPHERIC INFORMATION: (MANUAL INPUT OF DATA)

Wind: 2.5 meters $/ \mathrm{sec}$ from $90^{\circ}$ true

Inversion Height: 500 meters

Stability Class: C . Air Temperature: $25^{\circ} \mathrm{C}$

Relative Humidity: $50 \%$ Ground Roughness: 100 centimeters

Cloud Cover: 7 tenths

\section{SOURCE STRENGTH INFORMATION:}

Puddle Area: 410 square feet

Average Puddle Depth: 1 centimeters

Soil Type: Default Ground Temperature: $25^{\circ} \mathrm{C}$

Initial Puddle Temperature: boiling point

Release Duration: ALOHA limited the duration to 1 hour

Max Computed Release Rate: 34.7 kilograms/min

Max Average Sustained Release Rate: 4.32 kilograms/min (averaged over a minute or more)

Total Amount Released: 205 kilograms

\section{FOOTPRINT INFORMATION:}

Dispersion Module: Gaussian

User specified LOC: $200 \mathrm{ppm}$

Max Threat Zone for LOC: 30 meters

Max Threat Zone for IDLH: less than 10 meters(10.9 yards)

Note: Footprint was not drawn because.

effects of near-field patchiness make plume

presentation unreliable for short distances.

\section{TIME DEPENDENT INFORMATION:}

Concentration Estimates at the point:

Downwind: 30 meters

Off Centerline: 0 meters

Max Concentration:

Outdoor: $200 \mathrm{ppm}$

Indoor: $200 \mathrm{ppm}$

Note: Indoor graph is shown with a dotted line. 


\section{ENGINEERING COMPUTATION SHEET}

Title of Project EPHA for THM

Reviewed by S. A. Henderson

Subject Concentration Analysis for THM Hazardous Chemicals .

Computer T. M. Franey

Date 09/16/96
Works Calculation 7

Sheet No. 293 of 577

\section{SITE DATA INFORMATION: 1-RD-1}

Location: AIKEN, SOUTH CAROLINA

Building Air Exchanges Per Hour: 60 (User specified)

Date and Time: Fixed at July 1, 19961300 hours

\section{CHEMICAL INFORMATION:}

Chemical Name: ACETALDEHYDE

Molecular Weight: $44.05 \mathrm{~kg} / \mathrm{kmol}$

TLV-TWA: $100.00 \mathrm{ppm}$ IDLH: $10000.00 \mathrm{ppm}$

Note: Potential or confirmed human carcinogen.

Footprint Level of Concern: 200 ppm

Boiling Point: $20.40^{\circ} \mathrm{C}$

Vapor Pressure at Ambient Temperature: greater than $1 \mathrm{~atm}$

Ambient Saturation Concentration: $1,000,000 \mathrm{ppm}$ or $100.0 \%$

ATMOSPHERIC INFORMATION: (MANUAL INPUT OF DATA)

Wind: 2.5 meters $/ \mathrm{sec}$ from $90^{\circ}$ true

Inversion Height: 500 meters

Stability Class: C Air Temperature: $25^{\circ} \mathrm{C}$

Relative Humidity: 50\% Ground Roughness: 100 centimeters

Cloud Cover: 7 tenths

\section{SOURCE STRENGTH INFORMATION:}

Puddle Area: 4600 square feet

Average Puddle Depth: 1 centimeters

Soil Type: Default Ground Temperature: $25^{\circ} \mathrm{C}$

Initial Puddle Temperature: boiling point

Release Duration: ALOHA limited the duration to 1 hour

Max Computed Release Rate: 366 kilograms/min

Max Average Sustained Release Rate: 48.1 kilograms/min

(averaged over a minute or more)

Total Amount Released: 2,244 kilograms

\section{FOOTPRINT INFORMATION:}

Dispersion Module: Gaussian

User specified LOC: 200 ppm

- Max Threat Zone for LOC: 100 meters

Max Threat Zone for IDLH: 12 meters

TIME DEPENDENT INFORMATION:

Concentration Estimates at the point:

Downwind: 100 meters

Off Centerline: 0 meters

Max Concentration:

Outdoor: $200 \mathrm{ppm}$

Indoor: $200 \mathrm{ppm}$

Note: Indoor graph is shown with a dotted line. 
Title of Project EPHA for THM

Subject Concentration Analysis for THM Hazardous Chemicals

Computer T. M. Franey
Reviewed by S. A. Henderson

Works Calculation 7

Sheet No. 294 of 577
SITE DATA INFORMATION: 1-RD-1

Location: AIKEN, SOUTH CAROLINA

Building Air Exchanges Per Hour: 60 (User specified)

Date and Time: Fixed at July 1, 19961300 hours

CHEMICAL INFORMATION:

Chemical Name: ACETALDEHYDE

Molecular Weight: $44.05 \mathrm{~kg} / \mathrm{kmol}$

TLV-TWA: $100.00 \mathrm{ppm}$ IDLH: $10000.00 \mathrm{ppm}$

Note: Potential or confirmed human carcinogen.

Footprint Level of Concern: 200 ppm

Boiling Point: $20.40^{\circ} \mathrm{C}$

Vapor Pressure at Ambient Temperature: greater than $1 \mathrm{~atm}$

Ambient Saturation Concentration: $1,000,000$ ppm or $100.0 \%$

ATMOSPHERIC INFORMATION: (MANUAL INPUT OF DATA)

Wind: 2.5 meters $/ \mathrm{sec}$ from $90^{\circ}$ true

Inversion Height: 500 meters

Stability Class: C Air Temperature: $25^{\circ} \mathrm{C}$

Relative Humidity: 50\% Ground Roughness: 100 centimeters

Cloud Cover: 7 tenths

SOURCE STRENGTH INFORMATION:

Puddle Area: 24420 square feet

Average Puddle Depth: 1 centimeters

Soil Type: Default Ground Temperature: $25^{\circ} \mathrm{C}$

Initial Puddle Temperature: boiling point

Release Duration: ALOHA limited the duration to 1 hour

Max Computed Release Rate: 1,870 kilograms/min

Max Average Sustained Release Rate: 252 kilograms/min

(averaged over a minute or more)

Total Amount Released: 11,747 kilograms

FOOTPRINT INFORMATION:

Model Run: Heavy Gas

User specified LOC: 200 ppm

Max Threat Zone for LOC: 601 meters

Max Threat Zone for IDLH: 36 meters

Note: The Heavy Gas footprint is an initial screening.

For short releases it may be an overestimation.

Be sure to check concentration information at specific locations.

\section{TTME DEPENDENT INFORMATION:}

Concentration Estimates at the point:

Downwind: 100 meters

Off Centerline: 0 meters

Max Concentration:

Outdoor: $2,770 \mathrm{ppm}$

Indoor: $2,770 \mathrm{ppm}$

Note: Indoor graph is shown with a dotted line. 


\section{ENGINEERING COMPUTATION SHEET}

Title of Project EPHA for THM

Subject Concentration Analysis for THM Hazardous Chemicals

Computer T. M. Franey

Date $09 / 16 / 96$

SITE DATA INFORMATION: 1-RD-1

Location: AIKEN, SOUTH CAROLINA

Building Air Exchanges Per Hour: 60 (User specified)

Date and Time: Fixed at July 1, 19961300 hours

CHEMICAL INFORMATION:

Chemical Name: ACETALDEHYDE

Molecular Weight: $44.05 \mathrm{~kg} / \mathrm{kmol}$

TLV-TWA: $100.00 \mathrm{ppm}$ IDLH: $10000.00 \mathrm{ppm}$

Note: Potential or confirmed human carcinogen.

Footprint Level of Concern: 200 ppm

Boiling Point: $20.40^{\circ} \mathrm{C}$

Vapor Pressure at Ambient Temperature: greater than $1 \mathrm{~atm}$

Ambient Saturation Concentration: $1,000,000$ ppm or $100.0 \%$

ATMOSPHERIC INFORMATION: (MANUAL INPUT OF DATA)

Wind: 2.5 meters $/ \mathrm{sec}$ from $90^{\circ}$ true

Inversion Height: 500 meters

Stability Class: C Air Temperature: $25^{\circ} \mathrm{C}$

Relative Humidity: 50\% Ground Roughness: 100 centimeters

Cloud Cover: 7 tenths

SOURCE STRENGTH INFORMATION:

Puddle Area: 40700 square feet

Average Puddle Depth: 1 centimeters

Soil Type: Default Ground Temperature: $25^{\circ} \mathrm{C}$

Initial Puddle Temperature: boiling point

Release Duration: ALOHA limited the duration to 1 hour

Max Computed Release Rate: 3,080 kilograms/min

Max Average Sustained Release Rate: 418 kilograms/min

(averaged over a minute or more)

Total Amount Released: 19,502 kilograms

FOOTPRINT INFORMATION:

Model Run: Heavy Gas

User specified LOC: $200 \mathrm{ppm}$

Max Threat Zone for LOC: 780 meters

Max Threat Zone for IDLH: 47 meters

Note: The Heavy Gas footprint is an initial screening.

For short releases it may be an overestimation.

Be sure to check concentration information at specific locations.

TIME DEPENDENT INFORMATION:

Concentration Estimates at the point:

Downwind: 100 meters

Off Centerline: 0 meters

Max Concentration:

Outdoor: $4,040 \mathrm{ppm}$

Indoor: $4,040 \mathrm{ppm}$

Note: Indoor graph is shown with a dotted line.
Reviewed by S. A. Henderson

Works Calculation 7

Sheet No. 295 of 577 


\section{ENGINEERING COMPUTATION SHEET}

Title of Project EPHA for THM

Subject Concentration Analysis for THM Hazardous Chemicals

Computer T. M. Franey Date $\underline{09 / 16 / 96}$

\section{SITE DATA INFORMATION: 1-RD-1}

Location: AIKEN, SOUTH CAROLINA

Building Air Exchanges Per Hour: 60 (User specified)

Date and Time: Fixed at July 1, 19961300 hours

CHEMICAL INFORMATION:

Chemical Name: ACETALDEHYDE

Molecular Weight: $44.05 \mathrm{~kg} / \mathrm{kmol}$

TLV-TWA: $100.00 \mathrm{ppm}$ IDLH: $10000.00 \mathrm{ppm}$

Note: Potential or confirmed human carcinogen.

Footprint Level of Concern: 200 ppm

Boiling Point: $20.40^{\circ} \mathrm{C}$

Vapor Pressure at Ambient Temperature: greater than $1 \mathrm{~atm}$

Ambient Saturation Concentration: $1,000,000 \mathrm{ppm}$ or $100.0 \%$

ATMOSPHERIC INFORMATION: (MANUAL INPUT OF DATA)

Wind: 2.5 meters $/ \mathrm{sec}$ from $90^{\circ}$ true

Inversion Height: 500 meters

Stability Class: C Air Temperature: $25^{\circ} \mathrm{C}$

Relative Humidity: 50\% Ground Roughness: 100 centimeters

Cloud Cover: 7 tenths

\section{SOURCE STRENGTH INFORMATION:}

Puddle Area: 10000 square meters

Average Puddle Depth: 1 centimeters

Soil Type: Default $\quad$ Ground Temperature: $25^{\circ} \mathrm{C}$

Initial Puddle Temperature: boiling point

Release Duration: ALOHA limited the duration to 1 hour

Max Computed Release Rate: 7,970 kilograms/min

Max Average Sustained Release Rate: 1,100 kilograms/min

(averaged over a minute or more)

Total Amount Released: 51,216 kilograms

\section{FOOTPRINT INFORMATION:}

Model Run: Heavy Gas

User specified LOC: 200 ppm

Max Threat Zone for LOC: 1.3 kilometers

Max Threat Zone for IDLH: 82 meters

Note: The Heavy Gas footprint is an initial screening.

For short releases it may be an overestimation.

Be sure to check concentration information at specific locations.

TIME DEPENDENT INFORMATION:

Concentration Estimates at the point:

Downwind: 100 meters

Off Centerline: 0 meters

Max Concentration:

Outdoor: 8,090 ppm

Indoor: $8,090 \mathrm{ppm}$

Note: Indoor graph is shown with a dotted line.
Reviewed by S. A. Henderson

Works Calculation 7

Sheet No. 296 of 577 
Title of Project EPHA for THM

Subject Concentration Analysis for THM Hazardous Chemicals

Computer T. M. Franey
Reviewed by $\underline{S}$. A. Henderson

Works Calculation 7

Sheet No. 297 of 577

SITE DATA INFORMATION: 1-RD-2

Location: AIKEN, SOUTH CAROLINA

Building Air Exchanges Per Hour: 60 (User specified)

Date and Time: Fixed at July 1, 19961300 hours

CHEMICAL INFORMATION:

- Chemical Name: ACETIC ACID

Molecular Weight: $60.05 \mathrm{~kg} / \mathrm{kmol}$

TLV-TWA: $10.00 \mathrm{ppm}$ IDLH: $1000.00 \mathrm{ppm}$

Footprint Level of Concern: $50 \mathrm{ppm}$

Boiling Point: $117.90^{\circ} \mathrm{C}$

Vapor Pressure at Ambient Temperature: 0.021 atm

Ambient Saturation Concentration: $21,051 \mathrm{ppm}$ or $2.11 \%$

ATMOSPHERIC INFORMATION: (MANUAL INPUT OF DATA)

Wind: 2.5 meters $/ \mathrm{sec}$ from $90^{\circ}$ true

Inversion Height: 500 meters

Stability Class: C Air Temperature: $25^{\circ} \mathrm{C}$

Relative Humidity: 50\% Ground Roughness: 100 centimeters

Cloud Cover: 7 tenths

SOURCE STRENGTH INFORMATION:

Puddle Area: 1587 square feet

Average Puddle Depth: 1 centimeters

Soil Type: Default $\quad$ Ground Temperature: $25^{\circ} \mathrm{C}$

Initial Puddle Temperature: Ground temperature

Release Duration: ALOHA limited the duration to 1 hour

Max Computed Release Rate: 2.4 kilograms/min

Max Average Sustained Release Rate: 2.39 kilograms/min (averaged over a minute or more)

Total Amount Released: 131 kilograms

FOOTPRINT INFORMATION:

Dispersion Module: Gaussian

User specified LOC: $50 \mathrm{ppm}$

Max Threat Zone for LOC: 30 meters

Max Threat Zone for IDLH: less than 10 meters(10.9 yards)

Note: Footprint was not drawn because effects of near-field patchiness make plume

presentation unreliable for short distances.

\section{TIME DEPENDENT INFORMATION:}

Concentration Estimates at the point:

Downwind: 30 meters

Off Centerline: 0 meters

Max Concentration:

Outdoor: $50 \mathrm{ppm}$

Indoor: $50 \mathrm{ppm}$

Note: Indoor graph is shown with a dotted line. 
Title of Project EPHA for THM

Subject Concentration Analysis for THM Hazardous Chemicals

Computer T. M. Franey Date 09/16/96
Reviewed by S. A. Henderson

Works Calculation 7

Sheet No. 298 of 577

\section{SITE DATA INFORMATION: 1-RD-2 Location: AIKEN, SOUTH CAROLINA \\ Building Air Exchanges Per Hour: 60 (User specified) \\ Date and Time: Fixed at July 1, 19961300 hours}

\author{
CHEMICAL INFORMATION: \\ Chemical Name: ACETIC ACID \\ Molecular Weight: $60.05 \mathrm{~kg} / \mathrm{kmol}$ \\ TLV-TWA: $10.00 \mathrm{ppm}$ IDLH: $1000.00 \mathrm{ppm}$ \\ Footprint Level of Concern: 50 ppm \\ Boiling Point: $117.90^{\circ} \mathrm{C}$ \\ Vapor Pressure at Ambient Temperature: 0.021 atm \\ Ambient Saturation Concentration: $21,051 \mathrm{ppm}$ or $2.11 \%$
}

\section{ATMOSPHERIC INFORMATION: (MANUAL INPUT OF DATA)}

Wind: 2.5 meters $/ \mathrm{sec}$ from $90^{\circ}$ true

Inversion Height: 500 meters

Stability Class: C Air Temperature: $25^{\circ} \mathrm{C}$

Relative Humidity: 50\% Ground Roughness: 100 centimeters

Cloud Cover: 7 tenths

\section{SOURCE STRENGTH INFORMATION:}

Puddle Area: 21400 square feet

Average Puddle Depth: 1 centimeters

Soil Type: Default Ground Temperature: $25^{\circ} \mathrm{C}$

Initial Puddle Temperature: Ground temperature

Release Duration: ALOHA limited the duration to 1 hour

Max Computed Release Rate: 29.3 kilograms/min

Max Average Sustained Release Rate: 29.1 kilograms/min

(averaged over a minute or more)

Total Amount Released: 1,579 kilograms

\section{FOOTPRINT INFORMATION:}

Dispersion Module: Gaussian

User specified LOC: $50 \mathrm{ppm}$

Max Threat Zone for LOC: 100 meters

Max Threat Zone for IDLH: 25 meters

Note: Footprint was not drawn because effects of near-field patchiness make plume presentation unreliable for short distances.

\section{TIME DEPENDENT INFORMATION:}

Concentration Estimates at the point:

Downwind: 100 meters

Off Centerline: 0 meters

Max Concentration:

Outdoor: $50 \mathrm{ppm}$

Indoor: $50 \mathrm{ppm}$

Note: Indoor graph is shown with a dotted line. 
Title of Project EPHA for THM

Subject Concentration Analysis for THM Hazardous Chemicals

Computer T. M. Franey

Date $09 / 16 / 96$

SITE DATA INFORMATION: 1-RD-2

Location: AIKEN, SOUTH CAROLINA

Building Air Exchanges Per Hour: 60 (User specified)

Date and Time: Fixed at July 1, 19961300 hours

CHEMICAL INFORMATION:

Chemical Name: ACETIC ACID

Molecular Weight: $60.05 \mathrm{~kg} / \mathrm{kmol}$

TLV-TWA: $10.00 \mathrm{ppm}$ IDLH: $1000.00 \mathrm{ppm}$

Footprint Level of Concern: $50 \mathrm{ppm}$

Boiling Point: $117.90^{\circ} \mathrm{C}$

Vapor Pressure at Ambient Temperature: $0.021 \mathrm{~atm}$

Ambient Saturation Concentration: 21,051 ppm or 2.11\%

ATMOSPHERIC INFORMATION: (MANUAL INPUT OF DATA)

Wind: 2.5 meters $/ \mathrm{sec}$ from $90^{\circ}$ true

Inversion Height: 500 meters

Stability Class: $\mathrm{C}$ Air Temperature: $25^{\circ} \mathrm{C}$

Relative Humidity: 50\% Ground Roughness: 100 centimeters

Cloud Cover: 7 tenths

\section{SOURCE STRENGTH INFORMATION:}

Puddle Area: 24420 square feet

Average Puddle Depth: 1 centimeters

Soil Type: Default Ground Temperature: $25^{\circ} \mathrm{C}$

Initial Puddle Temperature: Ground temperature

Release Duration: ALOHA limited the duration to 1 hour

Max Computed Release Rate: 33.3 kilograms/min

Max Average. Sustained Release Rate: 33 kilograms/min

(averaged over a minute or more)

Total Amount Released: 1,792 kilograms

\section{FOOTPRINT INFORMATION:}

Dispersion Module: Gaussian

User specified LOC: $50 \mathrm{ppm}$

Max Threat Zone for LOC: 107 meters

Max Threat Zone for IDLH: 27 meters

\section{TIME DEPENDENT INFORMATION:}

Concentration Estimates at the point:

Downwind: 100 meters

Off Centerline: 0 meters

Max Concentration:

Outdoor: $53.2 \mathrm{ppm}$

Indoor: $53.2 \mathrm{ppm}$

Note: Indoor graph is shown with a dotted line.
Reviewed by S. A. Henderson

Works Calculation 7

Sheet No. 299 of 577 


\section{ENGINEERING COMPUTATÍON SHEET}

Title of Project EPHA for THM

Reviewed by $\underline{S}$. A. Henderson

Subject Concentration Analysis for THM Hazardous Chemicals

Works Calculation 7

Computer T. M. Franey

Date $\underline{09 / 16 / 96}$

Sheet No. 300 of 577

SITE DATA INFORMATION: 1-RD-2

Location: AIKEN, SOUTH CAROLINA

Building Air Exchanges Per Hour: 60 (User specified)

Date and Time: Fixed at July 1, 19961300 hours

CHEMICAL INFORMATION:

Chemical Name: ACETIC ACID

Molecular Weight: $60.05 \mathrm{~kg} / \mathrm{kmol}$

TLV-TWA: $10.00 \mathrm{ppm}$ IDLH: $1000.00 \mathrm{ppm}$

Footprint Level of Concern: 50 ppm

Boiling Point: $117.90^{\circ} \mathrm{C}$

Vapor Pressure at Ambient Temperature: $0.021 \mathrm{~atm}$

Ambient Saturation Concentration: $21,051 \mathrm{ppm}$ or $2.11 \%$

ATMOSPHERIC INFORMATION: (MANUAL INPUT OF DATA)

Wind: 2.5 meters $/ \mathrm{sec}$ from $90^{\circ}$ true

Inversion Height: 500 meters

Stability Class: C Air Temperature: $25^{\circ} \mathrm{C}$

Relative Humidity: 50\% Ground Roughness: 100 centimeters

Cloud Cover: 7 tenths

SOURCE STRENGTH INFORMATION:

Puddle Area: 40700 square feet

Average Puddle Depth: 1 centimeters

Soil Type: Default Ground Temperature: $25^{\circ} \mathrm{C}$

Initial Puddle Temperature: Ground temperature

Release Duration: ALOHA limited the duration to 1 hour

Max Computed Release Rate: 54.4 kilograms/min

Max Average Sustained Release Rate: 54 kilograms/min

(averaged over a minute or more)

Total Amount Released: 2,927 kilograms

FOOTPRINT INFORMATION:

Dispersion Module: Gaussian

User specified LOC: $50 \mathrm{ppm}$

Max Threat Zone for LOC: 135 meters

Max Threat Zone for IDLH: 34 meters

\section{TIME DEPENDENT INFORMATION:}

Concentration Estimates at the point:

Downwind: 100 meters

Off Centerline: 0 meters

Max Concentration:

Outdoor: $67.5 \mathrm{ppm}$

Indoor: $67.5 \mathrm{ppm}$

Note: Indoor graph is shown with a dotted line. 
Title of Project EPHA for THM

Subject Concentration Analysis for THM Hazardous Chemicals

Computer T.M. Franey

Date $\underline{09 / 16 / 96}$
Reviewed by $\mathrm{S}$. A. Henderson

Works Calculation 7

Sheet No. $\underline{301 \text { of } 577}$

\section{SITE DATA INFORMATION: 1-RD-2}

Location: AIKEN, SOUTH CAROLINA

Building Air Exchanges Per Hour: 60 (User specified)

Date and Time: Fixed at July 1, 19961300 hours

\section{CHEMICAL INFORMATION:}

Chemical Name: ACETIC ACID

Molecular Weight: $60.05 \mathrm{~kg} / \mathrm{kmol}$

TLV-TWA: $10.00 \mathrm{ppm}$ IDLH: $1000.00 \mathrm{ppm}$

Footprint Level of Concern: $50 \mathrm{ppm}$

Boiling Point: $117.90^{\circ} \mathrm{C}$

Vapor Pressure at Ambient Temperature: $0.021 \mathrm{~atm}$

Ambient Saturation Concentration: $21,051 \mathrm{ppm}$ or $2.11 \%$

ATMOSPHERIC INFORMATION: (MANUAL INPUT OF DATA)

Wind: 2.5 meters $/ \mathrm{sec}$ from $90^{\circ}$ true

Inversion Height: 500 meters

Stability Class: C Air Temperature: $25^{\circ} \mathrm{C}$

Relative Humidity: 50\% Ground Roughness: 100 centimeters

Cloud Cover: 7 tenths

SOURCE STRENGTH INFORMATION:

Puddle Area: 10000 square meters

Average Puddle Depth: 1 centimeters

Soil Type: Default Ground Temperature: $25^{\circ} \mathrm{C}$

Initial Puddle Temperature: Ground temperature

Release Duration: ALOHA limited the duration to 1 hour

Max Computed Release Rate: 139 kilograms/min

Max Average Sustained Release Rate: 138 kilograms/min (averaged over a minute or more)

Total Amount Released: 7,458 kilograms

FOOTPRINT INFORMATION:

Dispersion Module: Gaussian

User specified LOC: $50 \mathrm{ppm}$

Max Threat Zone for LOC: 211 meters

Max Threat Zone for IDLH: 56 meters

TIME DEPENDENT INFORMATION:

Concentration Estimates at the point:

Downwind: 100 meters

Off Centerline: 0 meters

Max Concentration:

Outdoor: $117 \mathrm{ppm}$

Indoor: $117 \mathrm{ppm}$

Note: Indoor graph is shown with a dotted line. 
Title of Project EPHA for THM

Subject Concentration Analysis for THM Hazardous Chemicals

Computer T. M. Franey Date $09 / 16 / 96$
Reviewed by S. A. Henderson

Works Calculation 7

Sheet No. 302 of 577

SITE DATA INFORMATION: 1-RD-3

Location: AIKEN, SOUTH CAROLINA

Building Air Exchanges Per Hour: 60 (User specified)

Date and Time: Fixed at July 1, 19961300 hours

\title{
CHEMICAL INFORMATION:
}

Chemical Name: ACETONITRILE

Molecular Weight: $41.05 \mathrm{~kg} / \mathrm{kmol}$

TLV-TWA: $40.00 \mathrm{ppm}$ IDLH: $4000.00 \mathrm{ppm}$

Footprint Level of Concern: $35 \mathrm{ppm}$

Boiling Point: $81.60^{\circ} \mathrm{C}$

Vapor Pressure at Ambient Temperature: 0.12 atm

Ambient Saturation Concentration: 122,023 ppm or $12.2 \%$

\author{
ATMOSPHERIC INFORMATION: (MANUAL INPUT OF DATA) \\ Wind: 2.5 meters $/ \mathrm{sec}$ from $90^{\circ}$ true \\ Inversion Height: 500 meters \\ Stability Class: C Air Temperature: $25^{\circ} \mathrm{C}$ \\ Relative Humidity: 50\% Ground Roughness: 100 centimeters \\ Cloud Cover: 7 tenths
}

\section{SOURCE STRENGTH INFORMATION:}

Puddle Area: 103 square feet

Average Puddle Depth: 1 centimeters

Soil Type: Default Ground Temperature: $25^{\circ} \mathrm{C}$

Initial Puddle Temperature: Ground temperature

Release Duration: ALOHA limited the duration to 1 hour

Max Computed Release Rate: 641 grams/min

Max Average Sustained Release Rate: 589 grams/min

(averaged over a minute or more)

Total Amount Released: 33.0 kilograms

\section{FOOTPRINT INFORMATION:}

Dispersion Module: Gaussian

User specified LOC: $35 \mathrm{ppm}$

Max Threat Zone for LOC: 30 meters

Max Threat Zone for IDLH: less than 10 meters(10.9 yards)

Note: Footprint was not drawn because effects of near-field patchiness make plume presentation unreliable for short distances.

\section{TIME DEPENDENT INFORMATION:}

Concentration Estimates at the point:

Downwind: 30 meters

Off Centerline: 0 meters

Max Concentration:

Outdoor: $35.2 \mathrm{ppm}$

Indoor: $35.2 \mathrm{ppm}$

Note: Indoor graph is shown with a dotted line. 
Title of Project EPHA for THM

\begin{tabular}{lll} 
Subject & Concentration Analysis for THM Hazardous Chemicals \\
Computer T. M. Franey & Date $09 / 16 / 96$ \\
\hline
\end{tabular}

Reviewed by $\mathrm{S}$. A. Henderson

Works Calculation 7

Sheet No. 303 of 577

\section{SITE DATA INFORMATION: 1-RD-3 \\ Location: AIKEN, SOUTH CAROLINA \\ Building Air Exchanges Per Hour: 60 (User specified) \\ Date and Time: Fixed at July 1, 19961300 hours}

\section{CHEMICAL INFORMATION:}

Chemical Name: ACETONITRIE

Molecular Weight: $41.05 \mathrm{~kg} / \mathrm{kmol}$

TLV-TWA: $40.00 \mathrm{ppm}$ IDLH: $4000.00 \mathrm{ppm}$

Footprint Level of Concern: $35 \mathrm{ppm}$

Boiling Point: $81.60^{\circ} \mathrm{C}$

Vapor Pressure at Ambient Temperature: $0.12 \mathrm{~atm}$

Ambient Saturation Concentration: 122,023 ppm or $12.2 \%$

\section{ATMOSPHERIC INFORMATION: (MANUAL INPUT OF DATA)}

Wind: 2.5 meters $/ \mathrm{sec}$ from $90^{\circ}$ true

Inversion Height: 500 meters

Stability Class: C Air Temperature: $25^{\circ} \mathrm{C}$

Relative Humidity: 50\% Ground Roughness: 100 centimeters

Cloud Cover: 7 tenths

\section{SOURCE STRENGTH INFORMATION:}

Puddle Area: 1297 square feet

Average Puddle Depth: 1 centimeters

Soil Type: Default Ground Temperature: $25^{\circ} \mathrm{C}$

Initial Puddle Temperature: Ground temperature

Release Duration: ALOHA limited the duration to 1 hour

Max Computed Release Rate: 6.94 kilograms/min

Max Average Sustained Release Rate: 6.55 kilograms/min

(averaged over a minute or more)

Total Amount Released: 374 kilograms

FOOTPRINT INFORMATION:

Dispersion Module: Gaussian

User specified LOC: $35 \mathrm{ppm}$

Max Threat Zone for LOC: 100 meters

Max Threat Zone for IDLH: less than 10 meters(10.9 yards)

Note: Footprint was not drawn because effects of near-field patchiness make plume presentation unreliable for short distances.

\section{TIME DEPENDENT INFORMATION:}

Concentration Estimates at the point:

Downwind: 100 meters

Off Centerline: 0 meters

Max Concentration:

Outdoor: $35 \mathrm{ppm}$

Indoor: $35 \mathrm{ppm}$

Note: Indoor graph is shown with a dotted line. 
Title of Project EPHA for THM

Subject Concentration Analysis for THM Hazardous Chemicals

Computer T. M. Franey
Reviewed by $\underline{\text { S.A. Henderson }}$

Works Calculation 7

Sheet No. $\underline{304 \text { of } 577}$

SITE DATA INFORMATION: 1-RD-3

Location: AIKEN, SOUTH CAROLINA

Building Air Exchanges Per Hour: 60 (User specified)

Date and Time: Fixed at July 1, 19961300 hours

CHEMICAL INFORMATION:

Chemical Name: ACETONITRILE

Molecular Weight: $41.05 \mathrm{~kg} / \mathrm{kmol}$

TLV-TWA: $40.00 \mathrm{ppm}$ IDLH: $4000.00 \mathrm{ppm}$

Footprint Level of Concern: 35 ppm

Boiling Point: $81.60^{\circ} \mathrm{C}$

Vapor Pressure at Ȧmbient Temperature: $0.12 \mathrm{~atm}$

Ambient Saturation Concentration: 122,023 ppm or $12.2 \%$

ATMOSPHERIC INFORMATION: (MANUAL INPUT OF DATA)

Wind: 2.5 meters $/ \mathrm{sec}$ from $90^{\circ}$ true

Inversion Height: 500 meters

Stability Class: C Air Temperature: $25^{\circ} \mathrm{C}$

Relative Humidity: $50 \%$ Ground Roughness: 100 centimeters

Cloud Cover: 7 tenths

SOURCE STRENGTH INFORMATION:

Puddle Area: 24420 square feet

Average Puddle Depth: 1 centimeters

Soil Type: Default Ground Temperature: $25^{\circ} \mathrm{C}$

Initial Puddle Temperature: Ground temperature

Release Duration: ALOHA limited the duration to 1 hour

Max Computed Release Rate: 112 kilograms/min

Max Average Sustained Release Rate: 109 kilograms/min

(averaged over a minute or more)

Total Amount Released: 6,329 kilograms

FOOTPRINT INFORMATION:

Dispersion Module: Gaussian

User specified LOC: $35 \mathrm{ppm}$

Max Threat Zone for LOC: 408 meters

Max Threat Zone for IDLH: 27 meters

TIME DEPENDENT INFORMATION:

Concentration Estimates at the point:

Downwind: 100 meters

Off Centerline: 0 meters

Max Concentration:

Outdoor: $256 \mathrm{ppm}$

Indoor: $256 \mathrm{ppm}$

Note: Indoor graph is shown with a dotted line. 
Title of Project EPHA for THM

Subject Concentration Analysis for THM Hazardous Chemicals

Computer T. M. Franey

Date $\underline{09 / 16 / 96}$

\section{SITE DATA INFORMATION: 1-RD-3}

Location: AIKEN, SOUTH CAROLINA

Building Air Exchanges Per Hour: 60 (User specified)

Date and Time: Fixed at July 1, 19961300 hours

CHEMICAL INFORMATION:

Chemical Name: ACETONITRILE

Molecular Weight: $41.05 \mathrm{~kg} / \mathrm{kmol}$

TLV-TWA: $40.00 \mathrm{ppm}$ IDLH: $4000.00 \mathrm{ppm}$

Footprint Level of Concern: $35 \mathrm{ppm}$

Boiling Point: $81.60^{\circ} \mathrm{C}$

Vapor Pressure at Ambient Temperature: $0.12 \mathrm{~atm}$

Ambient Saturation Concentration: 122,023 ppm or $12.2 \%$

ATMOSPHERIC INFORMATION: (MANUAL INPUT OF DATA)

Wind: 2.5 meters $/ \mathrm{sec}$ from $90^{\circ}$ true

Inversion Height: 500 meters

Stability Class: C Air Temperature: $25^{\circ} \mathrm{C}$

Relative Humidity: 50\% Ground Roughness: 100 centimeters

Cloud Cover: 7 tenths

\section{SOURCE STRENGTH INFORMATION:}

Puddle Area: 40700 square feet

Average Puddle Depth: 1 centimeters

Soil Type: Default Ground Temperature: $25^{\circ} \mathrm{C}$

Initial Puddle Temperature: Ground temperature

Release Duration: ALOHA limited the duration to 1 hour

Max Computed Release Rate: 183 kilograms/min

Max Average Sustained Release Rate: 177 kilograms/min

(averaged over a minute or more)

Total Amount Released: 10,365 kilograms

\section{FOOTPRINT INFORMATION:}

Dispersion Module: Gaussian

User specified LOC: 35 ppm

Max Threat Zone for LOC: 522 meters

Max Threat Zone for IDLH: 34 meters

\section{TIME DEPENDENT INFORMATION:}

Concentration Estimates at the point:

Downwind: 100 meters

Off Centerline: 0 meters

Max Concentration:

Outdoor: $324 \mathrm{ppm}$

Indoor: $324 \mathrm{ppm}$

Note: Indoor graph is shown with a dotted line.
Reviewed by S. A. Henderson

Works Calculation 7

Sheet No. $\underline{305 \text { of } 577}$ 
Title of Project EPHA for THM

Subject Concentration Analysis for THM Hazardous Chemicals

Computer T. M. Franey Date 09/16/96
Reviewed by S. A. Henderson

Works Calculation 7

Sheet No. 306 of 577

SITE DATA INFORMATION: 1-RD-3

Location: AIKEN, SOUTH CAROLINA

Building Air Exchanges Per Hour: 60 (User specified)

Date and Time: Fixed at July 1, 19961300 hours

CHEMICAL INFORMATION:

Chemical Name: ACETONITRILE

Molecular Weight: $41.05 \mathrm{~kg} / \mathrm{kmol}$

TLV-TWA: $40.00 \mathrm{ppm}$ IDLH: $4000.00 \mathrm{ppm}$

Footprint Level of Concern: $35 \mathrm{ppm}$

Boiling Point: $81.60^{\circ} \mathrm{C}$

Vapor Pressure at Ambient Temperature: $0.12 \mathrm{~atm}$

Ambient Saturation Concentration: 122,023 ppm or $12.2 \%$

ATMOSPHERIC INFORMATION: (MANUAL INPUT OF DATA)

Wind: 2.5 meters $/ \mathrm{sec}$ from $90^{\circ}$ true

Inversion Height: 500 meters

Stability Class: C Air Temperature: $25^{\circ} \mathrm{C}$

Relative Humidity: $50 \%$ Ground Roughness: 100 centimeters

Cloud Cover: 7 tenths

\section{SOURCE STRENGTH INFORMATION:}

Puddle Area: 10000 square meters

Average Puddle Depth: 1 centimeters

Soil Type: Default Ground Temperature: $25^{\circ} \mathrm{C}$

Initial Puddle Temperature: Ground temperature

Release Duration: ALOHA limited the duration to 1 hour

Max Computed Release Rate: 462 kilograms/min

Max Average Sustained Release Rate: 452 kilograms/min

(averaged over a minute or more)

Total Amount Released: 26,542 kilograms

FOOTPRINT INFORMATION:

Model Run: Heavy Gas

User specified LOC: $35 \mathrm{ppm}$

Max Threat Zone for LOC: 2.2 kilometers

Max Threat Zone for IDLH: 80 meters

Note: The Heavy Gas footprint is an initial screening.

For short releases it may be an overestimation.

Be sure to check concentration information at specific locations.

\section{TIME DEPENDENT INFORMATION:}

Concentration Estimates at the point:

Downwind: 100 meters

Off Centerline: 0 meters

Max Concentration:

Outdoor: $3,230 \mathrm{ppm}$

Indoor: $3,230 \mathrm{ppm}$

Note: Indoor graph is shown with a dotted line. 


\section{ENGINEERING COMPUTATION SHEET、}

Title of Project EPHA for THM

Subject Concentration Analysis for THM Hazardous Chemicals

Computer T. M. Franey

Date 09/16/96

\section{1-RD-4 SITE DATA INFORMATION:}

Location: AIKEN, SOUTH CAROLINA

Building Air Exchanges Per Hour: 60 (User specified)

Date and Time: Fixed at July 1, 19961300 hours

\section{CHEMICAL INFORMATION:}

Chemical Name: TERPINOLENE / ALPHA-PINENE / TURP

Molecular Weight: $136.24 \mathrm{~kg} / \mathrm{kmol}$

TLV-TWA: -unavail- IDLH: -unavail-

Footprint Level of Concern: $500 \mathrm{ppm}$

Boiling Point: $185.00^{\circ} \mathrm{C}$

Vapor Pressure at Ambient Temperature: 7.78e-004 atm

Ambient Saturation Concentration: 792 ppm or $0.079 \%$

ATMOSPHERIC INFORMATION: (MANUAL INPUT OF DATA)

Wind: 2.5 meters $/ \mathrm{sec}$ from $90^{\circ}$ true

Inversion Height: 500 feet

Stability Class: C Air Temperature: $25^{\circ} \mathrm{C}$

Relative Humidity: 50\% Ground Roughness: Urban or forest

Cloud Cover: 7 tenths

\section{SOURCE STRENGTH INFORMATION:}

Puddle Area: 40000 square feet

Average Puddle Depth: 1 centimeters

Soil Type: Default Ground Temperature: $25^{\circ} \mathrm{C}$

Initial Puddle Temperature: Ground temperature

Release Duration: ALOHA limited the duration to 1 hour

Max Computed Release Rate: 16.7 kilograms/min

Max Average Sustained Release Rate: 16.5 kilograms/min

(averaged over a minute or more)

Total Amount Released: 858 kilograms

\section{TIME DEPENDENT INFORMATION:}

Concentration Estimates at the point:

The point selected is at the source.
Reviewed by S. A. Henderson

Works Calculation 7

Sheet No. $\underline{307 \text { of } 577}$ 


\section{ENGINEERING COMPUTATION SHEET}

Title of Project EPHA for THM

Reviewed by $\underline{\text { S. A. Henderson }}$

Subject Concentration Analysis for THM Hazardous Chemicals

Computer T.M. Franey

Date $\underline{09 / 16 / 96}$

1-RD-4 SITE DATA INFORMATION:

Location: AIKEN, SOUTH CAROLINA

Building Air Exchanges Per Hour: 60 (User specified)

Date and Time: Fixed at July 1, 19961300 hours

CHEMICAL INFORMATION:

Chemical Name: TERPINOLENE / ALPHA-PINENE / TURP

Molecular Weight: $136.24 \mathrm{~kg} / \mathrm{kmol}$

TLV-TWA: -unavail- IDLH: -unavail-

Footprint Level of Concern: 500 ppm

Boiling Point: $185.00^{\circ} \mathrm{C}$

Vapor Pressure at Ambient Temperature: 7.78e-004 atm

Ambient Saturation Concentration: 792 ppm or $0.079 \%$

ATMOSPHERIC INFORMATION: (MANUAL INPUT OF DATA)

Wind: 2.5 meters $/ \mathrm{sec}$ from $90^{\circ}$ true

Inversion Height: 500 feet

Stability Class: C Air Temperature: $25^{\circ} \mathrm{C}$

Relative Humidity: 50\% Ground Roughness: Urban or forest

Cloud Cover: 7 tenths

SOURCE STRENGTH INFORMATION:

Puddle Area: 100000 square feet

Average Puddle Depth: 1 centimeters

Soil Type: Default $\quad$ Ground Temperature: $25^{\circ} \mathrm{C}$

Initial Puddle Temperature: Ground temperature

Release Duration: ALOHA limited the duration to 1 hour

Max Computed Release Rate: $\mathbf{4 0 . 3}$ kilograms/min

Max Average Sustained Release Rate: 39.8 kilograms/min

(averaged over a minute or more)

Total Amount Released: 2,071 kilograms

TIME DEPENDENT INFORMATION:

Concentration Estimates at the point:

Downwind: 100 meters

Off Centerline: 0 meters

Max Concentration:

Outdoor: $15.1 \mathrm{ppm}$
Works Calculation 7

Sheet No. $\underline{308 \text { of } 577}$ 
Title of Project EPHA for THM

Subject Concentration Analysis for THM Hazardous Chemicals

Computer T. M. Franey

Date $09 / 16 / 96$
Reviewed by $\mathrm{S}$. A. Henderson

Works Calculation 7

Sheet No. 309 of 577

\section{1-RD-4 SITE DATA INFORMATION:}

Location: AIKEN, SOUTH CAROLINA

Building Air Exchanges Per Hour: 60 (User specified)

Date and Time: Fixed at July 1, 19961300 hours

\section{CHEMICAL INFORMATION:}

Chemical Name: TERPINOLENE / ALPHA-PINENE / TURP

Molecular Weight: $136.24 \mathrm{~kg} / \mathrm{kmol}$

TLV-TWA: -unavail- IDLH: -unavail-

Footprint Level of Concern: $500 \mathrm{ppm}$

Boiling Point: $185.00^{\circ} \mathrm{C}$

Vapor Pressure at Ambient Temperature: 7.78e-004 atm

Ambient Saturation Concentration: $792 \mathrm{ppm}$ or $0.079 \%$

\section{ATMOSPHERIC INFORMATION: (MANUAL INPUT OF DATA)}

Wind: 2.5 meters $/ \mathrm{sec}$ from $90^{\circ}$ true

Inversion Height: 500 feet

Stability Class: C Air Temperature: $25^{\circ} \mathrm{C}$

Relative Humidity: $50 \%$ Ground Roughness: Urban or forest

Cloud Cover: 7 tenths

\section{SOURCE STRENGTH INFORMATION:}

Puddle Area: 24420 square feet

Average Puddle Depth: 1 centimeters

Soil Type: Default Ground Temperature: $25^{\circ} \mathrm{C}$

Initial Puddle Temperature: Ground temperature

Release Duration: ALOHA limited the duration to 1 hour

Max Computed Release Rate: 10.4 kilograms/min

Max Average Sustained Release Rate: 10.2 kilograms/min (averaged over a minute or more)

Total Amount Released: 534 kilograms

\section{FOOTPRINT INFORMATION:}

Dispersion Module: Gaussian

User specified LOC: $500 \mathrm{ppm}$

Max Threat Zone for LOC: 27 meters

Note: Footprint was not drawn because effects of near-field patchiness make plume presentation unreliable for short distances.

\section{TIME DEPENDENT INFORMATION:}

Concentration Estimates at the point:

Downwind: 100 meters

Off Centerline: 0 meters

Max Concentration:

Outdoor: $7.27 \mathrm{ppm}$ 


\section{ENGINEERING COMPUTATION SHEET}

Title of Project EPHA for THM

Subject Concentration Analysis for THM Hazardous Chemicals

Computer T.M. Franey

Date $\underline{09 / 16 / 96}$

1-RD-4 SITE DATA INFORMATION:

Location: AIKEN, SOUTH CAROLINA

Building Air Exchanges Per Hour: 60 (User specified)

Date and Time: Fixed at July 1, 19961300 hours

\section{CHEMICAL INFORMATION:}

Chemical Name: TERPINOLENE / ALPHA-PINENE / TURP

Molecular Weight: $136.24 \mathrm{~kg} / \mathrm{kmol}$

TLV-TWA: -unavail- IDLH: -unavail-

Footprint Level of Concern: 500 ppm

Boiling Point: $185.00^{\circ} \mathrm{C}$

Vapor Pressure at Ambient Temperature: 7.78e-004 atm

Ambient Saturation Concentration: 792 ppm or $0.079 \%$

\section{ATMOSPHERIC INFORMATION: (MANUAL INPUT OF DATA)}

Wind: 2.5 meters $/ \mathrm{sec}$ from $90^{\circ}$ true

Inversion Height: 500 feet

Stability Class: C Air Temperature: $25^{\circ} \mathrm{C}$

Relative Humidity: 50\% Ground Roughness: Urban or forest

Cloud Cover: 7 tenths

\section{SOURCE STRENGTH INFORMATION:}

Puddle Area: 40700 square feet

Average Puddle Depth: 1 centimeters

Soil Type: Default Ground Temperature: $25^{\circ} \mathrm{C}$

Initial Puddle Temperature: Ground temperature

Release Duration: ALOHA limited the duration to 1 hour

Max Computed Release Rate: 16.9 kilograms/min

Max Average.Sustained Release Rate: 16.8 kilograms/min

(averaged over.a minute or more)

Total Amount Released: 872 kilograms

FOOTPRINT INFORMATION:

Dispersion Module: Gaussian

User specified LOC: $500 \mathrm{ppm}$

Max Threat Zone for LOC: 34 meters

Note: Footprint was not drawn because effects of near-field patchiness make plume presentation unreliable for short distances.

\section{TIME DEPENDENT INFORMATION:}

Concentration Estimates at the point:

Downwind: 100 meters

Off Centerline: 0 meters

Max Concentration:

Outdoor: $9.23 \mathrm{ppm}$
Reviewed by S. A. Henderson

Works Calculation 7

Sheet No. 310 of 577 
Title of Project EPHA for THM

Subject Concentration Analysis for THM Hazardous Chemicals

Computer T. M. Franey

Date $\underline{09 / 16 / 96}$

1-RD-4 SITE DATA INFORMATION:

Location: AIKEN, SOUTH CAROLINA

Building Air Exchanges Per Hour: 60 (User specified)

Date and Time: Fixed at July 1, 19961300 hours

\section{CHEMICAL INFORMATION:}

Chemical Name: TERPINOLENE / ALPHA-PINENE / TURP

Molecular Weight: $136.24 \mathrm{~kg} / \mathrm{kmol}$

TLV-TWA: -unavail- IDLH: -unavail-

Footprint Level of Concern: 500 ppm

Boiling Point: $185.00^{\circ} \mathrm{C}$

Vapor Pressure at Ambient Temperature: 7.78e-004 atm

Ambient Saturation Concentration: 792 ppm or $0.079 \%$

ATMOSPHERIC INFORMATION: (MANUAL INPUT OF DATA)

Wind: 2.5 meters $/ \mathrm{sec}$ from $90^{\circ}$ true

Inversion Height: 500 feet

Stability Class: C Air Temperature: $25^{\circ} \mathrm{C}$

Relative Humidity: 50\% Ground Roughness: Urban or forest

Cloud Cover: 7 tenths

SOURCE STRENGTH INFORMATION:

Puddle Area: 10000 square meters

Average Puddle Depth: 1 centimeters

Soil Type: Default Ground Temperature: $25^{\circ} \mathrm{C}$

Initial Puddle Temperature: Ground temperature

Release Duration: ALOHA limited the duration to 1 hour

Max Computed Release Rate: 43.3 kilograms/min

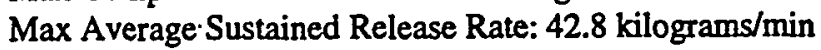
(averaged over a minute or more)

Total Amount Released: 2,223 kilograms

\section{FOOTPRINT INFORMATION:}

Dispersion Module: Gaussian

User specified LOC: $500 \mathrm{ppm}$

Max Threat Zone for LOC: 56 meters

Note: Footprint was not drawn because effects of near-field patchiness make plume presentation unreliable for short distances.

\section{TIME DEPENDENT INFORMATION:}

Concentration Estimates at the point:

Downwind: 100 meters

Off Centerline: 0 meters

Max Concentration:

Outdoor: $16 \mathrm{ppm}$
Reviewed by S. A. Henderson

Works Calculation 7

Sheet No. $\underline{311 \text { of } 577}$

of 577 
Title of Project EPHA for THM

Subject Concentration Analysis for THM Hazardous Chemicals

Computer T. M. Franey
Reviewed by S. A. Henderson

Works Calculation 7

Sheet No. $\underline{312 \text { of } 577}$

\section{CHEMICAL INFORMATION:}

Chemical Name: AMMONIA - 29\% - SAH

Molecular Weight: $17.72 \mathrm{~kg} / \mathrm{kmol}$

TLV-TWA: -unavail- IDLH: -unavail-

Footprint Level of Concern: 200 ppm

Boiling Point: $27.66^{\circ} \mathrm{C}$

Vapor Pressure at Ambient Temperature: $0.87 \mathrm{~atm}$

Ambient Saturation Concentration: 884,396 ppm or $88.4 \%$

ATMOSPHERIC INFORMATION: (MANUAL INPUT OF DATA)

Wind: 2.5 meters $/ \mathrm{sec}$ from $90^{\circ}$ true

Inversion Height: 500 meters

Stability Class: C Air Temperature: $25^{\circ} \mathrm{C}$

Relative Humidity: 50\% Ground Roughness: 100 centimeters

Cloud Cover: 7 tenths

\section{SOURCE STRENGTH INFORMATION:}

Puddle Area: 57 square feet

Average Puddle Depth: 1 centimeters

Soil Type: Default Ground Temperature: $25^{\circ} \mathrm{C}$

Initial Puddle Temperature: Ground temperature

Release Duration: 43 minutes

Max Computed Release Rate: 3.53 kilograms/min

Max Average Sustained Release Rate: 1.4 kilograms/min

(averaged over a minute or more)

Total Amount Released: 36.2 kilograms

\section{FOOTPRINT INFORMATION:}

Dispersion Module: Gaussian

User specified LOC: $200 \mathrm{ppm}$

Max Threat Zone for LOC: 30 meters

Note: Footprint was not drawn because effects of near-field patchiness make plume presentation unreliable for short distances.

\section{TIME DEPENDENT INFORMATION:}

Concentration Estimates at the point:

Downwind: 30 meters

Off Centerline: 0 meters

Max Concentration:

Outdoor: $200 \mathrm{ppm}$

Indoor: $198 \mathrm{ppm}$

Note: Indoor graph is shown with a dotted line.

INFORMATION: 1-RD-5

(User specified)

ate and Time: Fixed at July 1, 19961300 hours 


\section{ENGINEERING COMPUTATION SHEET}

Title of Project EPHA for THM

Reviewed by $\underline{\text { S. A. Henderson }}$

Subject Concentration Analysis for THM Hazardous Chemicals

Computer T. M. Franey

Date $09 / 16 / 96$
Works Calculation 7

Sheet No. $\underline{313 \text { of } 577}$

SITE DATA INFORMATION: 1-RD-5

Location: AIKEN, SOUTH CAROLINA

Building Air Exchanges Per Hour: 60 (User specified)

Date and Time: Fixed at July 1, 19961300 hours

CHEMICAL INFORMATION:

Chemical Name: AMMONIA - 29\% - SAH

Molecular Weight: $17.72 \mathrm{~kg} / \mathrm{kmol}$

TLV-TWA: -unavail- IDLH: -unavail-

Footprint Level of Concern: 200 ppm

Boiling Point: $27.66^{\circ} \mathrm{C}$

Vapor Pressure at Ambient Temperature: $0.87 \mathrm{~atm}$

Ambient Saturation Concentration: 884,396 ppm or $88.4 \%$

ATMOSPHERIC INFORMATION: (MANUAL INPUT OF DATA)

Wind: 2.5 meters $/ \mathrm{sec}$ from $90^{\circ}$ true

Inversion Height: 500 meters

Stability Class: C Air Temperature: $25^{\circ} \mathrm{C}$

Relative Humidity: $50 \%$ Ground Roughness: 100 centimeters

Cloud Cover: 7 tenths

SOURCE STRENGTH INFORMATION:

Puddle Area: 714 square feet

Average Puddle Depth: 1 centimeters

Soil Type: Default Ground Temperature: $25^{\circ} \mathrm{C}$

Initial Puddle Temperature: Ground temperature

Release Duration: 47 minutes

Max Computed Release Rate: 37.3 kilograms/min

Max Average Sustained Release Rate: 15.6 kilograms/min

(averaged over a minute or more)

Total Amount Released: 454 kilograms

FOOTPRINT INFORMATION:

Dispersion Module: Gaussian

User specified LOC: $200 \mathrm{ppm}$

Max Threat Zone for LOC: 100 meters

Note: Footprint was not drawn because

effects of near-field patchiness make plume

presentation unreliable for short distances.

\section{TIME DEPENDENT INFORMATION:}

Concentration Estimates at the point:

Downwind: 100 meters

Off Centerline: 0 meters

Max Concentration:

Outdoor: $200 \mathrm{ppm}$

Indoor: $199 \mathrm{ppm}$

Note: Indoor graph is shown with a dotted line. 
Title of Project EPHA for THM

Subject Concentration Analysis for THM Hazardous Chemicals

Computer T. M. Franey
Date $\underline{09 / 16 / 96}$
Reviewed by S. A. Henderson

Works Calculation 7

Sheet No. $\underline{314 \text { of } 577}$

SITE DATA INFORMATION: 1-RD-5

Location: AIKEN, SOUTH CAROLINA

Building Air Exchanges Per Hour: 60 (User specified)

Date and Time: Fixed at July 1, 19961300 hours

\section{CHEMICAL INFORMATION:}

Chemical Name: AMMONIA - 29\% - SAH

Molecular Weight: $17.72 \mathrm{~kg} / \mathrm{kmol}$

TLV-TWA: -unavail- IDLH: -unavail-

Footprint Level of Concern: $200 \mathrm{ppm}$

Boiling Point: $27.66^{\circ} \mathrm{C}$

Vapor Pressure at Ambient Temperature: 0.87 atm

Ambient Saturation Concentration: $884,396 \mathrm{ppm}$ or $88.4 \%$

\section{ATMOSPHERIC INFORMATION: (MANUAL INPUT OF DATA)}

Wind: 2.5 meters $/ \mathrm{sec}$ from $90^{\circ}$ true

Inversion Height: 500 meters

Stability Class: C Air Temperature: $25^{\circ} \mathrm{C}$

Relative Humidity: 50\% Ground Roughness: 100 centimeters

Cloud Cover: 7 tenths

\section{SOURCE STRENGTH INFORMATION:}

Puddle Area: 24420 square feet

Average Puddle Depth: 1 centimeters

Soil Type: Default Ground Temperature: $25^{\circ} \mathrm{C}$

Initial Puddle Temperature: Ground temperature

Release Duration: 53 minutes

Max Computed Release Rate: 1,040 kilograms/min

Max Average Sustained Release Rate: 463 kilograms/min

(averaged over a minute or more)

Total Amount Released: 15,519 kilograms

\section{FOOTPRINT INFORMATION:}

Dispersion Module: Gaussian

User specified LOC: 200 ppm

Max Threat Zone for LOC: 548 meters

\section{TIME DEPENDENT INFORMATION:}

Concentration Estimates at the point:

Downwind: 100 meters

Off Centerline: 0 meters

Max Concentration:

Outdoor: $2,530 \mathrm{ppm}$

Indoor: $2,520 \mathrm{ppm}$

Note: Indoor graph is shown with a dotted line. 


\section{ENGINEERING COMPUTATION SHEET}

Title of Project EPHA for THM

Subject Concentration Analysis for THM Hazardous Chemicals

Computer T. M. Franey

Date $\underline{09 / 16 / 96}$

SITE DATA INFORMATION: 1-RD-5

Location: AIKEN, SOUTH CAROLINA

Building Air Exchanges Per Hour: 60 (User specified)

Date and Time: Fixed at July 1, 19961300 hours

CHEMICAL INFORMATION:

Chemical Name: AMMONIA - 29\% - SAH

Molecular Weight: $17.72 \mathrm{~kg} / \mathrm{kmol}$

TLV-TWA: -unavail- IDLH: -unavail-

Footprint Level of Concern: 200 ppm

Boiling Point: $27.66^{\circ} \mathrm{C}$

Vapor Pressure at Ambient Temperature: $0.87 \mathrm{~atm}$

Ambient Saturation Concentration: 884,396 ppm or $88.4 \%$

ATMOSPHERIC INFORMATION: (MANUAL INPUT OF DATA)

Wind: 2.5 meters $/ \mathrm{sec}$ from $90^{\circ}$ true

Inversion Height: 500 meters

Stability Class: C Air Temperature: $25^{\circ} \mathrm{C}$

Relative Humidity: $50 \%$ Ground Roughness: 100 centimeters

Cloud Cover: 7 tenths

SOURCE STRENGTH INFORMATION:

Puddle Area: 40700 square feet

Average Puddle Depth: 1 centimeters

Soil Type: Default Ground Temperature: $25^{\circ} \mathrm{C}$

Initial Puddle Temperature: Ground temperature

Release Duration: 54 minutes

Max Computed Release Rate: 1,690 kilograms/min

Max Average Sustained Release Rate: 757 kilograms/min

(averaged over a minute or more)

Total Amount Released: 25,866 kilograms

FOOTPRINT INFORMATION:

Dispersion Module: Gaussian

User specified LOC: $200 \mathrm{ppm}$

Max Threat Zone for LOC: 703 meters

TIME DEPENDENT INFORMATION:

Concentration Estimates at the point:

Downwind: 100 meters

Off Centerline: 0 meters

Max Concentration:

Outdoor: $3,210 \mathrm{ppm}$

Indoor: $3,200 \mathrm{ppm}$

Note: Indoor graph is shown with a dotted line.
Reviewed by S. A. Henderson

Works Calculation 7

Sheet No. $\underline{315 \text { of } 577}$ 
Title of Project EPHA for THM

Subject Concentration Analysis for THM Hazardous Chemicals

Computer T. M. Franey Date $\underline{09 / 16 / 96}$

SITE DATA INFORMATION: 1-RD-5

Location: AIKEN, SOUTH CAROLINA

Building Air Exchanges Per Hour: 60 (User specified)

Date and Time: Fixed at July 1, 19961300 hours

CHEMICAL INFORMATION:

Chemical Name: AMMONIA - 29\% - SAH

Molecular Weight: $17.72 \mathrm{~kg} / \mathrm{kmol}$

TLV-TWA: -unavail- IDLH: -unavail-

Footprint Level of Concern: 200 ppm

Boiling Point: $27.66^{\circ} \mathrm{C}$

Vapor Pressure at Ambient Temperature: $0.87 \mathrm{~atm}$

Ambient Saturation Concentration: 884,396 ppm or $88.4 \%$

ATMOSPHERIC INFORMATION: (MANUAL INPUT OF DATA)

Wind: 2.5 meters $/ \mathrm{sec}$ from $90^{\circ}$ true

Inversion Height: 500 meters

Stability Class: C Air Temperature: $25^{\circ} \mathrm{C}$

Relative Humidity: $50 \%$ Ground Roughness: 100 centimeters

Cloud Cover: 7 tenths

\section{SOURCE STRENGTH INFORMATION:}

Puddle Area: 10000 square meters

Average Puddle Depth: 1 centimeters

Soil Type: Default Ground Temperature: $25^{\circ} \mathrm{C}$

Initial Puddle Temperature: Ground temperature

Release Duration: 56 minutes

Max Computed Release Rate: 4,250 kilograms/min

Max Average Sustained Release Rate: 1,930 kilograms/min

(averaged over a minute or more)

Total Amount Released: 68,407 kilograms

FOOTPRINT INFORMATION:

Dispersion Module: Gaussian

User specified LOC: $200 \mathrm{ppm}$

Max Threat Zone for LOC: 1.1 kilometers

TIME DEPENDENT INFORMATION:

Concentration Estimates at the point:

Downwind: 100 meters

Off Centerline: 0 meters

Max Concentration:

Outdoor: 5,550 ppm

Indoor: $5,550 \mathrm{ppm}$

Note: Indoor graph is shown with a dotted line.
Reviewed by S. A. Henderson

Works Calculation 7

Sheet No. 316 of 577 
Title of Project EPHA for THM

Subject Concentration Analysis for THM Hazardous Chemicals

Computer T.M. Franey
Reviewed by S.A. Henderson

Works Calculation 7

Sheet No. $\underline{317 \text { of } 577}$

SITE DATA INFORMATION: 1-RD-6

Location: AIKEN, SOUTH CAROLINA

Building Air Exchanges Per Hour: 60 (User specified)

Date and Time: Fixed at July 1, 19961300 hours

CHEMICAL INFORMATION:

Chemical Name: BENZENE Molecular Weight: $78.11 \mathrm{~kg} / \mathrm{kmol}$

TLV-TWA: $0.10 \mathrm{ppm}$ IDLH: $3000.00 \mathrm{ppm}$

Note: Potential or confirmed human carcinogen.

Footprint Level of Concern: $150 \mathrm{ppm}$

Boiling Point: $80.09^{\circ} \mathrm{C}$

Vapor Pressure at Ambient Temperature: 0.12 atm

Ambient Saturation Concentration: $127,171 \mathrm{ppm}$ or $12.7 \%$

ATMOSPHERIC INFORMATION: (MANUAL INPUT OF DATA)

Wind: 2.5 meters $/ \mathrm{sec}$ from $90^{\circ}$ true

Inversion Height: 500 meters

Stability Class: C Air Temperature: $25^{\circ} \mathrm{C}$

Relative Humidity: $50 \%$ Ground Roughness: 100 centimeters

Cloud Cover: 7 tenths

\section{SOURCE STRENGTH INFORMATION:}

Puddle Area: 535 square feet

Average Puddle Depth: 1 centimeters

Soil Type: Default Ground Temperature: $25^{\circ} \mathrm{C}$

Initial Puddle Temperature: Ground temperature

Release Duration: ALOHA limited the duration to 1 hour

Max Computed Release Rate: 6.6 kilograms/min

Max Average Sustained Release Rate: 6.13 kilograms/min

(averaged over a minute or more)

Total Amount Released: 347 kilograms

FOOTPRINT INFORMATION:

Dispersion Module: Gaussian

User specified LOC: $150 \mathrm{ppm}$

Max Threat Zone for LOC: 30 meters

Max Threat Zone for IDLH: less than 10 meters(10.9 yards)

Note: Footprint was not drawn because effects of near-field patchiness make plume presentation unreliable for short distances.

\section{TIME DEPENDENT INFORMATION:}

Concentration Estimates at the point:

Downwind: 30 meters

Off Centerline: 0 meters

Max Concentration:

Outdoor: $150 \mathrm{ppm}$

Indoor: $150 \mathrm{ppm}$

Note: Indoor graph is shown with a dotted line. 


\section{ENGINEERING COMPUTATION SHEET}

Title of Project EPHA for THM

Subject Concentration Analysis for THM Hazardous Chemicals

Computer T. M. Franey

Date $\underline{09 / 16 / 96}$
Reviewed by $\mathrm{S}$. A. Henderson

Works Calculation 7

- Sheet No. 318 of 577

\section{CHEMICAL INFORMATION:}

Chemical Name: BENZENE Molecular Weight: $78.11 \mathrm{~kg} / \mathrm{kmol}$

TLV-TWA: $0.10 \mathrm{ppm}$ IDLH: $3000.00 \mathrm{ppm}$

Note: Potential or confirmed human carcinogen.

Footprint Level of Concern: 150 ppm

Boiling Point: $80.09^{\circ} \mathrm{C}$

Vapor Pressure at Ambient Temperature: $0.12 \mathrm{~atm}$

Ambient Saturation Concentration: $127,171 \mathrm{ppm}$ or $12.7 \%$

\section{ATMOSPHERIC INFORMATION: (MANUAL INPUT OF DATA)}

Wind: 2.5 meters $/ \mathrm{sec}$ from $90^{\circ}$ true

Inversion Height: 500 meters

Stability Class: C Air Temperature: $25^{\circ} \mathrm{C}$

Relative Humidity: $50 \%$ Ground Roughness: 100 centimeters

Cloud Cover: 7 tenths

\section{SOURCE STRENGTH INFORMATION:}

Puddle Area: 4000 square feet

Average Puddle Depth: 1 centimeters

Soil Type: Default Ground Temperature: $25^{\circ} \mathrm{C}$

Initial Puddle Temperature: Ground temperature

Release Duration: ALOHA limited the duration to 1 hour

Max Computed Release Rate: 44.6 kilograms/min

Max Average Sustained Release Rate: 42.1 kilograms/min (averaged over a minute or more)

Total Amount Released: 2,415 kilograms

\section{FOOTPRINT INFORMATION:}

Dispersion Module: Gaussian

User specified LOC: $150 \mathrm{ppm}$

Max Threat Zone for LOC: 77 meters

Max Threat Zone for IDLH: 11 meters

Note: Footprint was not drawn because effects of near-field patchiness make plume presentation unreliable for short distances.

\section{TTME DEPENDENT INFORMATION:}

Concentration Estimates at the point:

Downwind: 100 meters

Off Centerline: 0 meters

Max Concentration:

Outdoor: $102 \mathrm{ppm}$

Indoor: $102 \mathrm{ppm}$

Note: Indoor graph is shown with a dotted line. 


\section{ENGINEERING COMPUTATION SHEET}

Title of Project EPHA for THM

Subject Concentration Analysis for THM Hazardous Chemicals

Computer T. M. Franey

Date 09/16/96
Reviewed by S. A. Henderson

Works Calculation 7

Sheet No. $\underline{319 \text { of } 577}$

SITE DATA INFORMATTON: 1-RD-6

Location: AIKEN, SOUTH CAROLINA

Building Air Exchanges Per Hour: 60 (User specified)

Date and Time: Fixed at July 1, 19961300 hours

\section{CHEMICAL INFORMATION:}

Chemical Name: BENZENE Molecular Weight: $78.11 \mathrm{~kg} / \mathrm{kmol}$

TLV-TWA: $0.10 \mathrm{ppm}$ IDLH: $3000.00 \mathrm{ppm}$

Note: Potential or confirmed human carcinogen.

Footprint Level of Concern: 150 ppm

Boiling Point: $80.09^{\circ} \mathrm{C}$

Vapor Pressure at Ambient Temperature: $0.12 \mathrm{~atm}$

Ambient Saturation Concentration: 127,171 ppm or $12.7 \%$

ATMOSPHERIC INFORMATION: (MANUAL INPUT OF DATA)

Wind: 2.5 meters $/ \mathrm{sec}$ from $90^{\circ}$ true

Inversion Height: 500 meters

Stability Class: C Air Temperature: $25^{\circ} \mathrm{C}$

Relative Humidity: 50\% Ground Roughness: 100 centimeters

Cloud Cover: 7 tenths

\section{SOURCE STRENGTH INFORMATION:}

Puddle Area: 24420 square feet

Average Puddle Depth: 1 centimeters

Soil Type: Defauit Ground Temperature: $25^{\circ} \mathrm{C}$

Initial Puddle Temperature: Ground temperature

Release Duration: ALOHA limited the duration to 1 hour

Max Computed Release Rate: 250 kilograms/min

Max Average Sustained Release Rate: 239 kilograms/min

(averaged over a minute or more)

Total Amount Released: 13,871 kilograms

\section{FOOTPRINT INFORMATION:}

Model Run: Heavy Gas

User specified LOC: $150 \mathrm{ppm}$

Max Threat Zone for LOC: 499 meters

Max Threat Zone for IDLH: 61 meters

Note: The Heavy Gas footprint is an initial screening.

For short releases it may be an overestimation.

Be sure to check concentration information at specific locations.

\section{TIME DEPENDENT INFORMATION:}

Concentration Estimates at the point:

Downwind: 100 meters

Off Centerline: 0 meters

Max Concentration:

Outdoor: $1,540 \mathrm{ppm}$

Indoor: $1,540 \mathrm{ppm}$

Note: Indoor graph is shown with a dotted line. 


\section{ENGINEERING COMPUTATION SHEET}

Title of Project EPHA for THM

Subject Concentration Analysis for THM Hazardous Chemicals

Computer T. M. Franey

Date 09/16/96

\section{SITE DATA INFORMATION: 1-RD-6}

Location: AIKEN, SOUTH CAROLINA

Building Air Exchanges Per Hour: 60 (User specified)

Date and Time: Fixed at July 1, 19961300 hours

\section{CHEMICAL INFORMATION:}

Chemical Name: BENZENE

TLV-TWA: $0.10 \mathrm{ppm}$ IDLH: $3000.00 \mathrm{ppm}$
Note: Potential or confirmed human carcinogen.

Molecular Weight: $78.11 \mathrm{~kg} / \mathrm{kmol}$

Footprint Level of Concern: $150 \mathrm{ppm}$

Boiling Point: $80.09^{\circ} \mathrm{C}$

Vapor Pressure at Ambient Temperature: $0.12 \mathrm{~atm}$

Ambient Saturation Concentration: 127,171 ppm or $12.7 \%$

\section{ATMOSPHERIC INFORMATION: (MANUAL INPUT OF DATA)}

Wind: 2.5 meters $/ \mathrm{sec}$ from $90^{\circ}$ true

Inversion Height: 500 meters

Stability Class: C Air Temperature: $25^{\circ} \mathrm{C}$

Relative Humidity: 50\% Ground Roughness: 100 centimeters

Cloud Cover: 7 tenths

\section{SOURCE STRENGTH INFORMATION:}

Puddle Area: 40700 square feet

Average Puddle Depth: 1 centimeters

Soil Type: Default Ground Temperature: $25^{\circ} \mathrm{C}$

Initial Puddle Temperature: Ground temperature

Release Duration: ALOHA limited the duration to 1 hour

Max Computed Release Rate: 408 kilograms/min

Max Average Sustained Release Rate: 392 kilograms/min (averaged over a minute or more)

Total Amount Released: 22,742 kilograms

\section{FOOTPRINT INFORMATION:}

Model Run: Heavy Gas

User specified LOC: $150 \mathrm{ppm}$

Max Threat Zone for LOC: 641 meters

Max Threat Zone for IDLH: 79 meters

Note: The Heavy Gas footprint is an initial screening.

For short releases it may be an overestimation.

Be sure to check concentration information at specific locations.

\section{TIME DEPENDENT INFORMATION:}

\section{Concentration Estimates at the point:}

Downwind: 100 meters

Off Centerline: 0 meters

Max Concentration:

Outdoor: $2,220 \mathrm{ppm}$

Indoor: 2,220 ppm

Note: Indoor graph is shown with a dotted line. 
Title of Project EPHA for THM

Subject Concentration Analysis for THM Hazardous Chemicals

Computer T. M. Franey
Reviewed by S. A. Henderson

Works Calculation 7

Sheet No. $\underline{321 \text { of } 577}$
SITE DATA INFORMATION: 1-RD-6

Location: AIKEN, SOUTH CAROLINA

Building Air Exchanges Per Hour: 60 (User specified)

Date and Time: Fixed at July 1, 19961300 hours

CHEMICAL INFORMATION:

Chemical Name: BENZENE Molecular Weight: $78.11 \mathrm{~kg} / \mathrm{kmol}$

TLV-TWA: $0.10 \mathrm{ppm}$ IDLH: $3000.00 \mathrm{ppm}$

Note: Potential or Confirmed human carcinogen.

Footprint Level of Concern: 150 ppm

Boiling Point: $80.09^{\circ} \mathrm{C}$

Vapor Pressure at Ambient Temperature: $0.12 \mathrm{~atm}$

Ambient Saturation Concentration: 127,171 ppm or $12.7 \%$

ATMOSPHERIC INFORMATION: (MANUAL INPUT OF DATA)

Wind: 2.5 meters $/ \mathrm{sec}$ from $90^{\circ}$ true

Inversion Height: 500 meters

Stability Class: C Air Temperature: $25^{\circ} \mathrm{C}$

Relative Humidity: 50\% Ground Roughness: 100 centimeters

Cloud Cover: 7 tenths

SOURCE STRENGTH INFORMATION:

Puddle Area: 10000 square meters

Average Puddle Depth: 1 centimeters

Soil Type: Default Ground Temperature: $25^{\circ} \mathrm{C}$

Initial Puddle Temperature: Ground temperature

Release Duration: ALOHA limited the duration to 1 hour

Max Computed Release Rate: 1,030 kilograms/min

Max Average.Sustained Release Rate: 1,000 kilograms/min

(averaged over a minute or more)

Total Amount Released: 58,341 kilograms

\section{FOOTPRINT INFORMATION:}

Model Run: Heavy Gas

User specified LOC: $150 \mathrm{ppm}$

Max Threat Zone for LOC: 1.0 kilometers

Max Threat Zone for IDLH: 133 meters

Note: The Heavy Gas footprint is an initial screening.

For short releases it may be an overestimation.

Be sure to check concentration information at specific locations.

\section{TIME DEPENDENT INFORMATION:}

Concentration Estimates at the point:

Downwind: 100 meters

Off Centerline: 0 meters

Max Concentration:

Outdoor: 4,400 ppm

Indoor: $4,400 \mathrm{ppm}$

Note: Indoor graph is shown with a dotted line. 
Title of Project EPHA for THM

Subject Concentration Analysis for THM Hazardous Chemicals

Computer T.M. Franey
Reviewed by S. A. Henderson

Works Calculation 7

Sheet No. 322 of 577

\section{SITE DATA INFORMATION: 1-RD-7 \\ Location: AIKEN, SOUTH CAROLINA \\ Building Air Exchanges Per Hour: 60 (User specified) \\ Date and Time: Fixed at July 1, 19961300 hours}

\section{CHEMICAL INFORMATION:}

Chemical Name: BENZYL ALCOHOL

Molecular Weight: $108.14 \mathrm{~kg} / \mathrm{kmol}$

TLV-TWA: -unavail- IDLH: -unavail-

Footprint Level of Concern: 200 ppm

Boiling Point: $204.70^{\circ} \mathrm{C}$

Vapor Pressure at Ambient Temperature: 1.07e-004 atm

Ambient Saturation Concentration: 109 ppm or $0.011 \%$

\section{ATMOSPHERIC INFORMATION: (MANUAL INPUT OF DATA) \\ Wind: 2.5 meters $/ \mathrm{sec}$ from $90^{\circ}$ true \\ Inversion Height: 500 meters \\ Stability Class: C Air Temperature: $25^{\circ} \mathrm{C}$ \\ Relative Humidity: $50 \%$ Ground Roughness: 100 centimeters \\ Cloud Cover: 7 tenths}

\section{SOURCE STRENGTH INFORMATION:}

Puddle Area: 30430 square feet

Average Puddle Depth: 1 centimeters

Soil Type: Default Ground Temperature: $25^{\circ} \mathrm{C}$

Initial Puddle Temperature: Ground temperature

Release Duration: ALOHA limited the duration to 1 hour

Max Computed Release Rate: 644 grams/min

Max Average Sustained Release Rate: 633 grams/min

(averaged over a minute or more)

Total Amount Released: 30.7 kilograms

\section{FOOTPRINT INFORMATION:}

Dispersion Module: Gaussian

User specified LOC: 200 ppm

Max Threat Zone for LOC: 30 meters

Note: Footprint was not drawn because effects of near-field patchiness make plume presentation unreliable for short distances.

\section{TIME DEPENDENT INFORMATION:}

Concentration Estimates at the point:

Downwind: 30 meters

Off Centerline: 0 meters

Max Concentration:

Outdoor: $3.07 \mathrm{ppm}$

Indoor: $3.07 \mathrm{ppm}$

Note: Indoor graph is shown with a dotted line. 
Title of Project EPHA for THM

Subject Concentration Analysis for THM Hazardous Chemicals

Computer T. M. Franey

Date 09/16/96
Reviewed by $\underline{\text { S. A. Henderson }}$

Works Calculation 7

Sheet No. 323 of 577

SITE DATA INFORMATION: $\quad$ 1-RD-7

Location: AIKEN, SOUTH CAROLINA

Building Air Exchanges Per Hour: 60 (User specified)

Date and Time: Fixed at July 1, 19961300 hours

\section{CHEMICAL INFORMATION:}

Chemical Name: BENZYL ALCOHOL

Molecular Weight: $108.14 \mathrm{~kg} / \mathrm{kmol}$

TLV-TWA: -unavail- IDLH: -unavail-

Footprint Level of Concern: 200 ppm

Boiling Point: $204.70^{\circ} \mathrm{C}$

Vapor Pressure at Ambient Temperature: 1.07e-004 atm

Ambient Saturation Concentration: $109 \mathrm{ppm}$ or $0.011 \%$

ATMOSPHERIC INFORMATION: (MANUAL INPUT OF DATA)

Wind: 2.5 meters $/ \mathrm{sec}$ from $90^{\circ}$ true

Inversion Height: 500 meters

Stability Class: C Air Temperature: $25^{\circ} \mathrm{C}$

Relative Humidity: $50 \%$ Ground Roughness: 100 centimeters

Cloud Cover: 7 tenths

SOURCE STRENGTH INFORMATION:

Puddle Area: 99999 square feet

Average Puddle Depth: 1 centimeters

Soil Type: Default Ground Temperature: $25^{\circ} \mathrm{C}$

Initial Puddle Temperature: Ground temperature

Release Duration: ALOHA limited the duration to 1 hour

Max Computed Release Rate: 2.03 kilograms/min

Max Average Sustained Release Rate: 1.99 kilograms/min

(averaged over a minute or more)

Total Amount Released: 96.2 kilograms

\section{FOOTPRINT INFORMATION:}

Dispersion Module: Gaussian

User specified LOC: $200 \mathrm{ppm}$

Max Threat Zone for LOC: 55 meters

Note: Footprint was not drawn because effects of near-field patchiness make plume presentation unreliable for short distances.

\section{TIME DEPENDENT INFORMATION:}

Concentration Estimates at the point:

\section{Downwind: 100 meters}

Off Centerline: 0 meters

Max Concentration:

Outdoor: $0.953 \mathrm{ppm}$

Indoor: $0.953 \mathrm{ppm}$

Note: Indoor graph is shown with a dotted line. 
Title of Project EPHA for THM

Subject Concentration Analysis for THM Hazardous Chemicals

Computer T.M. Franey

Date $\underline{09 / 16 / 96}$
Reviewed by S. A. Henderson

Works Calculation 7

Sheet No. $\underline{324 \text { of } 577}$

SITE DATA INFORMATION: 1-RD-7

Location: AIKEN, SOUTH CAROLINA

Building Air Exchanges Per Hour: 60 (User specified)

Date and Time: Fixed at July 1, 19961300 hours

\section{CHEMICAL INFORMATION:}

Chemical Name: BENZYL ALCOHOL

Molecular Weight: $108.14 \mathrm{~kg} / \mathrm{kmol}$

TLV-TWA: -unavail- IDLH: -unavail-

Footprint Level of Concern: 200 ppm

Boiling Point: $204.70^{\circ} \mathrm{C}$

Vapor Pressure at Ambient Temperature: 1.07e-004 atm

Ambient Saturation Concentration: 109 ppm or $0.011 \%$

\section{ATMOSPHERIC INFORMATION: (MANUAL INPUT OF DATA)}

Wind: 2.5 meters $/ \mathrm{sec}$ from $90^{\circ}$ true

Inversion Height: 500 meters

Stability Class: C Air Temperature: $25^{\circ} \mathrm{C}$

Relative Humidity: 50\% Ground Roughness: 100 centimeters

Cloud Cover: 7 tenths

\section{SOURCE STRENGTH INFORMATION:}

Puddle Area: 24420 square feet

Average Puddle Depth: 1 centimeters

Soil Type: Default Ground Temperature: $25^{\circ} \mathrm{C}$

Initial Puddle Temperature: Ground temperature

Release Duration: ALOHA limited the duration to 1 hour

Max Computed Release Rate: 521 grams/min

Max Average Sustained Release Rate: 512 grams/min

(averaged over a minute or more)

Total Amount Released: 24.8 kilograms

\section{FOOTPRINT INFORMATION:}

Dispersion Module: Gaussian

User specified LOC: 200 ppm

Max Threat Zone for LOC: 27 meters

Note: Footprint was not drawn because effects of near-field patchiness make plume presentation unreliable for short distances.

\section{TIME DEPENDENT INFORMATION:}

Concentration Estimates at the point:

Downwind: 100 meters

Off Centerline: 0 meters

Max Concentration:

Outdoor: $0.458 \mathrm{ppm}$

Indoor: $0.458 \mathrm{ppm}$

Note: Indoor graph is shown with a dotted line. 
Title of Project EPHA for THM

Subject Concentration Analysis for THM Hazardous Chemicals

Computer T. M. Franey

Date $\underline{09 / 16 / 96}$
Reviewed by $\mathrm{S}$. A. Henderson

Works Calculation 7

Sheet No. 325 of 577

SITE DATA INFORMATION: 1-RD-7

Location: AIKEN, SOUTH CAROLINA

Building Air Exchanges Per Hour: 60 (User specified)

Date and Time: Fixed at July 1, 19961300 hours

CHEMICAL INFORMATION:

Chemical Name: BENZYL ALCOHOL

Molecular Weight: $108.14 \mathrm{~kg} / \mathrm{kmol}$

TLV-TWA: -unavail- - IDLH: -unavail-

Footprint Level of Concern: 200 ppm

Boiling Point: $204.70^{\circ} \mathrm{C}$

Vapor Pressure at Ambient Temperature: 1.07e-004 atm

Ambient Saturation Concentration: 109 ppm or $0.011 \%$

ATMOSPHERIC INFORMATION: (MANUAL INPUT OF DATA)

Wind: 2.5 meters $/ \mathrm{sec}$ from $90^{\circ}$ true

Inversion Height: 500 meters

Stability Class: C Air Temperature: $25^{\circ} \mathrm{C}$

Relative Humidity: $50 \%$ Ground Roughness: 100 centimeters

Cloud Cover: 7 tenths

\section{SOURCE STRENGTH INFORMATION:}

Puddle Area: 40700 square feet

Average Puddle Depth: 1 centimeters

Soil Type: Default Ground Temperature: $25^{\circ} \mathrm{C}$

Initial Puddle Temperature: Ground temperature

Release Duration: ALOHA limited the duration to 1 hour

Max Computed Release Rate: 852 grams/min

Max Average Sustained Release Rate: 838 grams/min

(averaged over a minute or more)

Total Amount Released: 40.5 kilograms

\section{FOOTPRINT INFORMATION:}

Dispersion Module: Gaussian

User specified LOC: $200 \mathrm{ppm}$

Max Threat Zone for LOC: 34 meters

Note: Footprint was not drawn because effects of near-field patchiness make plume presentation unreliable for short distances.

\section{TIME DEPENDENT INFORMATION:}

Concentration Estimates at the point:

Downwind: 100 meters

Off Centerline: 0 meters

Max Concentration:

Outdoor: $0.582 \mathrm{ppm}$

Indoor: $0.582 \mathrm{ppm}$

Note: Indoor graph is shown with a dotted line. 


\section{ENGINEERING COMPUTATION SHEET}

Title of Project EPHA for THM

Reviewed by S. A. Henderson

Subject Concentration Analysis for THM Hazardous Chemicals

Computer T.M. Franey

Date $\underline{09 / 16 / 96}$

SITE DATA INFORMATION: 1-RD-8

Location: AIKEN, SOUTH CAROLINA

Building Air Exchanges Per Hour: 60 (User specified)

Date and Time: Fixed at July 1, 19961300 hours

CHEMICAL INFORMATION:

Chemical Name: BENZYL CHLORIDE

Molecular Weight: $126.58 \mathrm{~kg} / \mathrm{kmol}$

TLV-TWA: $1.00 \mathrm{ppm}$ IDLH: $10.00 \mathrm{ppm}$

Footprint Level of Concern: $10 \mathrm{ppm}$

Boiling Point: $179.40^{\circ} \mathrm{C}$

Vapor Pressure at Ambient Temperature: 0.0017 atm

Ambient Saturation Concentration: 1,747 ppm or $0.17 \%$

ATMOSPHERIC INFORMATION: (MANUAL INPUT OF DATA)

Wind: 2.5 meters $/ \mathrm{sec}$ from $90^{\circ}$ true

Inversion Height: 500 meters

Stability Class: C Air Temperature: $25^{\circ} \mathrm{C}$

Relative Humidity: 50\% Ground Roughness: 100 centimeters

Cloud Cover: 7 tenths

SOURCE STRENGTH INFORMATION:

Puddle Area: 3700 square feet

Average Puddle Depth: 1 centimeters

Soil Type: Default Ground Temperature: $25^{\circ} \mathrm{C}$

Initial Puddle Temperature: Ground temperature

Release Duration: ALOHA limited the duration to 1 hour

Max Computed Release Rate: 1.54 kilograms/min

Max Average Sustained Release Rate: 1.53 kilograms/min

(averaged over a minute or more)

Total Amount Released: 79.3 kilograms

FOOTPRINT INFORMATION:

Dispersion Module: Gaussian

User specified LOC: $10 \mathrm{ppm}$

Max Threat Zone for LOC: 30 meters

Max Threat Zone for IDLH: 30 meters

Note: Footprint was not drawn because

effects of near-field patchiness make plume

presentation unreliable for short distances.

\section{TIME DEPENDENT INFORMATION:}

Concentration Estimates at the point:

Downwind: 30 meters

Off Centerline: 0 meters

Max Concentration:

Outdoor: $10 \mathrm{ppm}$

Indoor: $10 \mathrm{ppm}$

Note: Indoor graph is shown with a dotted line.
Works Calculation 7

Sheet No. $\underline{327 \text { of } 577}$ 
Title of Project EPHA for THM

Subject Concentration Analysis for THM Hazardous Chemicals

Computer T. M. Franey

SITE DATA INFORMATION: 1-RD-8 Location: AIKEN, SOUTH CAROLINA

Building Air Exchanges Per Hour: 60 (User specified)

Date and Time: Fixed at July 1, 19961300 hours

CHEMICAL INFORMATION:

Chemical Name: BENZYL CHLORIDE

Molecular Weight: $126.58 \mathrm{~kg} / \mathrm{kmol}$

TLV-TWA: $1.00 \mathrm{ppm}$ IDLH: $10.00 \mathrm{ppm}$

Footprint Level of Concern: $10 \mathrm{ppm}$

Boiling Point: $179.40^{\circ} \mathrm{C}$

Vapor Pressure at Ambient Temperature: $0.0017 \mathrm{~atm}$

Ambient Saturation Concentration: $1,747 \mathrm{ppm}$ or $0.17 \%$

ATMOSPHERIC INFORMATION: (MANUAL INPUT OF DATA)

Wind: 2.5 meters $/ \mathrm{sec}$ from $90^{\circ}$ true

Inversion Height: 500 meters

Stability Class: C Air Temperature: $25^{\circ} \mathrm{C}$

Relative Humidity: 50\% Ground Roughness: 100 centimeters

Cloud Cover: 7 tenths

\section{SOURCE STRENGTH INFORMATION:}

Puddle Area: 50000 square feet

Average Puddle Depth: 1 centimeters

Soil Type: Default Ground Temperature: $25^{\circ} \mathrm{C}$

Initial Puddle Temperature: Ground temperature

Release Duration: ALOHA limited the duration to 1 hour

Max Computed Release Rate: 18.9 kilograms/min

Max Average Sustained Release Rate: 18.6 kilograms/min

(averaged over a minute or more)

Total Amount Released: 966 kilograms

FOOTPRINT INFORMATION:

Dispersion Module: Gaussian

User specified LOC: $10 \mathrm{ppm}$

Max Threat Zone for LOC: 100 meters

Max Threat Zone for IDLH: 100 meters

Note: Footprint was not drawn because effects of near-field patchiness make plume presentation unreliable for short distances.

\section{TIME DEPENDENT INFORMATION:}

Concentration Estimates at the point:

Downwind: 100 meters

Off Centerline: 0 meters

Max Concentration:

Outdoor: $10 \mathrm{ppm}$

Indoor: $10 \mathrm{ppm}$

Note: Indoor graph is shown with a dotted line. 
Title of Project EPHA for THM

Subject Concentration Analysis for THM Hazardous Chemicals

Computer T. M. Franey

Date 09/16/96

SITE DATA INFORMATION: 1-RD-8

Location: AIKEN, SOUTH CAROLINA

Building Air Exchanges Per Hour: 60 (User specified)

Date and Time: Fixed at July 1, 19961300 hours

CHEMICAL INFORMATION:

Chemical Name: BENZYL CHLORIDE

Molecular Weight: $126.58 \mathrm{~kg} / \mathrm{kmol}$

TLV-TWA: $1.00 \mathrm{ppm}$ IDLH: $10.00 \mathrm{ppm}$

Footprint Level of Concern: $10 \mathrm{ppm}$

Boiling Point: $179.40^{\circ} \mathrm{C}$

Vapor Pressure at Ambient Temperature: $0.0017 \mathrm{~atm}$

Ambient Saturation Concentration: $1,747 \mathrm{ppm}$ or $0.17 \%$

ATMOSPHERIC INFORMATION: (MANUAL INPUT OF DATA)

Wind: 2.5 meters $/ \mathrm{sec}$ from $90^{\circ}$ true

Inversion Height: 500 meters

Stability Class: C Air Temperature: $25^{\circ} \mathrm{C}$

Relative Humidity: 50\% Ground Roughness: 100 centimeters

Cloud Cover: 7 tenths

\section{SOURCE STRENGTH INFORMATION:}

Puddle Area: 24420 square feet

Average Puddle Depth: 1 centimeters

Soil Type: Default Ground Temperature: $25^{\circ} \mathrm{C}$

Initial Puddle Temperature: Ground temperature

Release Duration: ALOHA limited the duration to 1 hour

Max Computed Release Rate: 9.46 kilograms/min

Max Average Sustained Release Rate: 9.35 kilograms/min

(averaged over a minute or more)

Total Amount Released: 485 kilograms

FOOTPRINT INFORMATION:

Dispersion Module: Gaussian

User specified LOC: $10 \mathrm{ppm}$

Max Threat Zone for LOC: 72 meters

Max Threat Zone for IDLH: 72 meters

Note: Footprint was not drawn because

effects of near-field patchiness make plume

presentation unreliable for short distances.

\section{TIME DEPENDENT INFORMATION:}

Concentration Estimates at the point:

Downwind: 100 meters

Off Centerline: 0 meters

Max Concentration:

Outdoor: $7.15 \mathrm{ppm}$

Indoor: $7.15 \mathrm{ppm}$

Note: Indoor graph is shown with a dotted line.
Reviewed by $\underline{S}$. A. Henderson

Works Calculation 7

Sheet No. $\underline{329 \text { of } 577}$ 
Title of Project EPHA for THM

Subject Concentration Analysis for THM Hazardous Chemicals

Computer T.M. Franey

Date $\underline{09 / 16 / 96}$
Reviewed by S. A. Henderson

Works Calculation 7

Sheet No. $\underline{330 \text { of } 577}$

SITE DATA INFORMATION: 1-RD-8

Location: AIKEN, SOUTH CAROLINA

Building Air Exchanges Per Hour: 60 (User specified)

Date and Time: Fixed at July 1, 19961300 hours

CHEMICAL INFORMATION:

Chemical Name: BENZYL CHLORIDE

Molecular Weight: $126.58 \mathrm{~kg} / \mathrm{kmol}$

TLV-TWA: $1.00 \mathrm{ppm}$ IDLH: $10.00 \mathrm{ppm}$

Footprint Level of Concern: $10 \mathrm{ppm}$

Boiling Point: $179.40^{\circ} \mathrm{C}$

Vapor Pressure at Ambient Temperature: $0.0017 \mathrm{~atm}$

Ambient Saturation Concentration: $1,747 \mathrm{ppm}$ or $0.17 \%$

ATMOSPHERIC INFORMATION: (MANUAL INPUT OF DATA)

Wind: 2.5 meters $/ \mathrm{sec}$ from $90^{\circ}$ true

Inversion Height: 500 meters

Stability Class: C Air Temperature: $25^{\circ} \mathrm{C}$

Relative Humidity: 50\% Ground Roughness: 100 centimeters

Cloud Cover: 7 tenths

SOURCE STRENGTH INFORMATION:

Puddle Area: 40700 square feet

Average Puddle Depth: 1 centimeters

Soil Type: Default Ground Temperature: $25^{\circ} \mathrm{C}$

Initial Puddle Temperature: Ground temperature

Release Duration: ALOHA limited the duration to 1 hour

Max Computed Release Rate: 15.5 kilograms/min

Max Average Sustained Release Rate: 15.3 kilograms/min

(averaged over a minute or more)

Total Amount Released: 792 kilograms

FOOTPRINT INFORMATION:

Dispersion Module: Gaussian

User specified LOC: $10 \mathrm{ppm}$

Max Threat Zone for LOC: 91 meters

Max Threat Zone for IDLH: 91 meters

Note: Footprint was not drawn because effects of near-field patchiness make plume

presentation unreliable for short distances.

TIME DEPENDENT INFORMATION:

Concentration Estimates at the point:

Downwind: 100 meters

Off Centerline: 0 meters

Max Concentration:

Outdoor: $9.07 \mathrm{ppm}$

Indoor: $9.07 \mathrm{ppm}$

Note: Indoor graph is shown with a dotted line. 


\section{ENGINEERING COMPUTATION SHEET}

Title of Project EPHA for THM

Reviewed by S. A. Henderson

Subject Concentration Analysis for THM Hazardous Chemicals

Computer T. M. Franey

Date $09 / 16 / 96$

SITE DATA INFORMATION: 1-RD-8

Location: AIKEN, SOUTH CAROLINA

Building Air Exchanges Per Hour: 60 (User specified)

Date and Time: Fixed at July 1, 19961300 hours

CHEMICAL INFORMATION:

Chemical Name: BENZYL CHLORIDE

Molecular Weight: $126.58 \mathrm{~kg} / \mathrm{kmol}$

TLV-TWA: $1.00 \mathrm{ppm}$. IDLH: $10.00 \mathrm{ppm}$

Footprint Level of Concern: $10 \mathrm{ppm}$

Boiling Point: $179.40^{\circ} \mathrm{C}$

Vapor Pressure at Ambient Temperature: $0.0017 \mathrm{~atm}$

Ambient Saturation Concentration: 1,747 ppm or $0.17 \%$

ATMOSPHERIC INFORMATION: (MANUAL INPUT OF DATA)

Wind: 2.5 meters $/ \mathrm{sec}$ from $90^{\circ}$ true

Inversion Height: 500 meters

Stability Class: C Air Temperature: $25^{\circ} \mathrm{C}$

Relative Humidity: 50\% Ground Roughness: 100 centimeters

Cloud Cover: 7 tenths

\section{SOURCE STRENGTH INFORMATION:}

Puddle Areá: 10000 square meters

Average Puddle Depth: 1 centimeters

Soil Type: Default Ground Temperature: $25^{\circ} \mathrm{C}$

Initial Puddle Temperature: Ground temperature

Release Duration: ALOHA limited the duration to 1 hour

Max Computed Release Rate: 39.5 kilograms/min

Max Average Sustained Release Rate: 39 kilograms/min

(averaged over a minute or more)

Total Amount Released: 2,020 kilograms

FOOTPRINT INFORMATION:

Dispersion Module: Gaussian

User specified LOC: $10 \mathrm{ppm}$

Max Threat Zone for LOC: 143 meters

Max Threat Zone for IDLH: 143 meters

TIME DEPENDENT INFORMATION:

Concentration Estimates at the point:

Downwind: 100 meters

Off Centerline: 0 meters

Max Concentration:

Outdoor: $15.7 \mathrm{ppm}$

Indoor: $15.7 \mathrm{ppm}$

Note: Indoor graph is shown with a dotted line.
Works Calculation 7

Sheet No. $\underline{331 \text { of } 577}$ 
Title of Project EPHA for THM

Subject Concentration Analysis for THM Hazardous Chemicals

Computer T. M. Franey Date 09/16/96
Reviewed by $\underline{\text { S. A. Henderson }}$

Works Calculation 7

Sheet No. $\underline{32}$ of 577
SITE DATA INFORMATION: 1-RD-9

Location: AIKEN, SOUTH CAROLINA

Building Air Exchanges Per Hour: 60 (User specified)

Date and Time: Fixed at July 1, 19961300 hours

CHEMICAL INFORMATION:

Chemical Name: BUTYRALDEHYDE

Molecular Weight: $72.11 \mathrm{~kg} / \mathrm{kmol}$

TLV-TWA: -unavail- IDLH: -unavail-

Footprint Level of Concern: 50.ppm

Boiling Point: $74.80^{\circ} \mathrm{C}$

Vapor Pressure at Ambient Temperature: 0.15 atm

Ambient Saturation Concentration: 149,153 ppm or $14.9 \%$

ATMOSPHERIC INFORMATION: (MANUAL INPUT OF DATA)

Wind: 2.5 meters $/ \mathrm{sec}$ from $90^{\circ}$ true

Inversion Height: 500 meters

Stability Class: C Air Temperature: $25^{\circ} \mathrm{C}$

Relative Humidity: 50\% Ground Roughness: 100 centimeters

Cloud Cover: 7 tenths

\section{SOURCE STRENGTH INFORMATION:}

Puddle Area: 119 square feet

Average Puddle Depth: 1 centimeters

Soil Type: Default Ground Temperature: $25^{\circ} \mathrm{C}$

Initial Puddle Temperature: Ground temperature

Release Duration: ALOHA limited the duration to 1 hour

Max Computed Release Rate: 1.69 kilograms/min

Max Average Sustained Release Rate: 1.49 kilograms/min

(averaged over a minute or more)

Total Amount Released: 81.3 kilograms

FOOTPRINT INFORMATION:

Dispersion Module: Gaussian

User specified LOC: $50 \mathrm{ppm}$

Max Threat Zone for LOC: 30 meters

Note: Footprint was not drawn because effects of near-field patchiness make plume presentation unreliable for short distances.

TIME DEPENDENT INFORMATION:

Concentration Estimates at the point:

Downwind: 30 meters

Off Centerline: 0 meters

Max Concentration:

Outdoor: $50.2 \mathrm{ppm}$

Indoor: $50.2 \mathrm{ppm}$

Note: Indoor graph is shown with a dotted line. 
Title of Project EPHA for THM

Subject Concentration Analysis for THM Hazardous Chemicals

Computer T. M. Franey

Date 09/16/96
Reviewed by $\underline{S}$. A. Henderson

Works Calculation 7

Sheet No. $\underline{333 \text { of } 577}$

SITE DATA INFORMATION: 1-RD-9

Location: AIKEN, SOUTH CAROLINA

Building Air Exchanges Per Hour: 60 (User specified)

Date and Time: Fixed at July 1, 19961300 hours

CHEMICAL INFORMATION:

Chemical Name: BUTYRALDEHYDE

Molecular Weight: $72.11 \mathrm{~kg} / \mathrm{kmol}$

TLV-TWA: -unavail- IDLH: -unavail-

Footprint Level of Concern: 50 ppm

Boiling Point: $74.80^{\circ} \mathrm{C}$

Vapor Pressure at Ambient Temperature: $0.15 \mathrm{~atm}$

Ambient Saturation Concentration: $149,153 \mathrm{ppm}$ or $14.9 \%$

ATMOSPHERIC INFORMATION: (MANUAL INPUT OF DATA)

Wind: 2.5 meters $/ \mathrm{sec}$ from $90^{\circ}$ true

Inversion Height: 500 meters

Stability Class: C Air Temperature: $25^{\circ} \mathrm{C}$

Relative Humidity: 50\% Ground Roughness: 100 centimeters

Cloud Cover: 7 tenths

SOURCE STRENGTH INFORMATION:

Puddle Area: 1485 square feet

Average Puddle Depth: 1 centimeters

Soil Type: Default Ground Temperature: $25^{\circ} \mathrm{C}$

Initial Puddle Temperature: Ground temperature

Release Duration: ALOHA limited the duration to 1 hour

Max Computed Release Rate: 18.3 kilograms/min

Max Average Sustained Release Rate: 16.6 kilograms/min

(averaged over a minute or more)

Total Amount Released: 924 kilograms

\section{FOOTPRINT INFORMATION:}

Dispersion Module: Gaussian

User specified LOC: $50 \mathrm{ppm}$

Max Threat Zone for LOC: 100 meters

Note: Footprint was not drawn because

effects of near-field patchiness make plume

presentation unreliable for short distances.

\section{TIME DEPENDENT INFORMATION:}

Concentration Estimates at the point:

Downwind: 100 meters

Off Centerline: 0 meters

Max Concentration:

Outdoor: $50 \mathrm{ppm}$

Indoor: $50 \mathrm{ppm}$

Note: Indoor graph is shown with a dotted line. 
Title of Project EPHA for THM

Subject Concentration Analysis for THM Hazardous Chemicals

Computer T. M. Franey Date $\underline{09 / 16 / 96}$

SITE DATA INFORMATION: 1-RD-9

Location: AIKEN, SOUTH CAROLINA

Building Air Exchanges Per Hour: 60 (User specified)

Date and Time: Fixed at July 1, 19961300 hours

CHEMICAL INFORMATION:

Chemical Name: BUTYRALDEHYDE

Molecular Weight: $72.11 \mathrm{~kg} / \mathrm{kmol}$

TLV-TWA: -unavail- IDLH: -unavail-

Footprint Level of Concern: $50 \mathrm{ppm}$

Boiling Point: $74.80^{\circ} \mathrm{C}$

Vapor Pressure at Ambient Temperature: 0.15 atm

Ambient Saturation Concentration: 149,153 ppm or $14.9 \%$

ATMOSPHERIC INFORMATION: (MANUAL INPUT OF DATA)

Wind: 2.5 meters/sec from $90^{\circ}$ true

Inversion Height: 500 meters

Stability Class: C Air Temperature: $25^{\circ} \mathrm{C}$

Relative Humidity: $50 \%$ Ground Roughness: 100 centimeters

Cloud Cover: 7 tenths

SOURCE STRENGTH INFORMATION:

Puddle Area: 24420 square feet

Average Puddle Depth: 1 centimeters

Soil Type: Default Ground Temperature: $25^{\circ} \mathrm{C}$

Initial Puddle Temperature: Ground temperature

Release Duration: ALOHA limited the duration to 1 hour

Max Computed Release Rate: 264 kilograms/min

Max Average Sustained Release Rate: 245 kilograms/min

(averaged over a minute or more)

Total Amount Released: 13,848 kilograms

FOOTPRINT INFORMATION:

Model Run: Heavy Gas

User specified LOC: $50 \mathrm{ppm}$

Max Threat Zone for LOC: 997 meters

Note: The Heavy Gas footprint is an initial screening.

For short releases it may be an overestimation.

Be sure to check concentration information at specific locations.

TIME DEPENDENT INFORMATION:

Concentration Estimates at the point:

Downwind: 100 meters

Off Centerline: 0 meters

Max Concentration:

Outdoor: 1,690 ppm

Indoor: $1,690 \mathrm{ppm}$

Note: Indoor graph is shown with a dotted line.
Reviewed by S. A. Henderson

Works Calculation 7

Sheet No. 334 of 577 


\section{ENGINEERING COMPUTATION SHEET}

Title of Project EPHA for THM

Reviewed by $\underline{\mathrm{S}}$. A. Henderson

Subject Concentration Analysis for THM Hazardous Chemicals

Computer T.M. Franey

Date $\underline{09 / 16 / 96}$

\section{SITE DATA INFORMATION: 1-RD-9}

Location: AIKEN, SOUTH CAROLINA

Building Air Exchanges Per Hour: 60 (User specified)

Date and Time: Fixed at July 1, 19961300 hours

\section{CHEMICAL INFORMATION:}

Chemical Name: BUTYRALDEHYDE

Molecular Weight: $72.11 \mathrm{~kg} / \mathrm{kmol}$

TLV-TWA: -unavail- IDLH: -unavail-

Footprint Level of Concern: $50 \mathrm{ppm}$

Boiling Point: $74.80^{\circ} \mathrm{C}$

Vapor Pressure at Ambient Temperature: $0.15 \mathrm{~atm}$

Ambient Saturation Concentration: 149,153 ppm or $14.9 \%$

ATMOSPHERIC INFORMATION: (MANUAL INPUT OF DATA)

Wind: 2.5 meters $/ \mathrm{sec}$ from $90^{\circ}$ true

Inversion Height: 500 meters

Stability Class: C Air Temperature: $25^{\circ} \mathrm{C}$

Relative Humidity: $50 \%$ Ground Roughness: 100 centimeters

Cloud Cover: 7 tenths

\section{SOURCE STRENGTH INFORMATION:}

Puddle Area: 40700 square feet

Average Puddle Depth: 1 centimeters

Soil Type: Default Ground Temperature: $25^{\circ} \mathrm{C}$

Initial Puddle Temperature: Ground temperature

Release Duration: ALOHA limited the duration to 1 hour

Max Computed Release Rate: 429 kilograms/min

Max Average Sustained Release Rate: 400 kilograms/min

(averaged over a minute or more)

Total Amount Released: 22,716 kilograms

\section{FOOTPRINT INFORMATION:}

Model Run: Heavy Gas

User specified LOC: $50 \mathrm{ppm}$

Max Threat Zone for LOC: 1.3 kilometers

Note: The Heavy Gas footprint is an initial screening.

For short releases it may be an overestimation.

Be sure to check concentration information at specific locations.

\section{TIME DEPENDENT INFORMATION:}

Concentration Estimates at the point:

Downwind: 100 meters

Off Centerline: 0 meters

Max Concentration:

Outdoor: $2,450 \mathrm{ppm}$

Indoor: $2,450 \mathrm{ppm}$

Note: Indoor graph is shown with a dotted line.
Works Calculation 7

Sheet No. $\underline{335 \text { of } 577}$ 


\section{ENGINEERING COMPUTATION SHEET}

Title of Project EPHA for THM

Subject Concentration Analysis for THM Hazardous Chemicals

Computer T. M. Franey

Date $\underline{09 / 16 / 96}$

SITE DATA INFORMATTON: 1-RD-9

Location: AIKEN, SOUTH CAROLINA

Building Air Exchanges Per Hour: 60 (User specified)

Date and Time: Fixed at July 1, 19961300 hours

CHEMICAL INFORMATION:

Chemical Name: BUTYRALDEHYDE

Molecular Weight: $72.11 \mathrm{~kg} / \mathrm{kmol}$

TLV-TWA: -unavail- IDLH: -unavail-

Footprint Level of Concern: $50 \mathrm{ppm}$

Boiling Point: $74.80^{\circ} \mathrm{C}$

Vapor Pressure at Ambient Temperature: 0.15 atm

Ambient Saturation Concentration: $149,153 \mathrm{ppm}$ or $14.9 \%$

\section{ATMOSPHERIC INFORMATION: (MANUAL INPUT OF DATA)}

Wind: 2.5 meters $/ \mathrm{sec}$ from $90^{\circ}$ true

Inversion Height: 500 meters

Stability Class: C Air Temperature: $25^{\circ} \mathrm{C}$

Relative Humidity: 50\% Ground Roughness: 100 centimeters

Cloud Cover: 7 tenths

\section{SOURCE STRENGTH INFORMATION:}

Puddle Area: 10000 square meters

Average Puddle Depth: 1 centimeters

Soil Type: Default Ground Temperature: $25^{\circ} \mathrm{C}$

Initial Puddle Temperature: Ground temperature

Release Duration: ALOHA limited the duration to 1 hour

Max Computed Release Rate: 1,090 kilograms/min

Max Average Sustained Release Rate: 1,020 kilograms/min

(averaged over a minute or more)

Total Amount Released: 58,347 kilograms

\section{FOOTPRINT INFORMATION:}

Model Run: Heavy Gas

User specified LOC: $50 \mathrm{ppm}$

Max Threat Zone for LOC: 2.0 kilometers

Note: The Heavy Gas footprint is an initial screening.

For short releases it may be an overestimation.

$\mathrm{Be}$ sure to check concentration information at specific locations.

\section{TIME DEPENDENT INFORMATION:}

Concentration Estimates at the point:

Downwind: 100 meters

Off Centerline: 0 meters

Max Concentration:

Outdoor: 4,880 ppm

Indoor: $4,880 \mathrm{ppm}$

Note: Indoor graph is shown with a dotted line.
Reviewed by S.A. Henderson

Works Calculation 7

Sheet No. $\underline{336 \text { of } 577}$ 


\section{ENGINEERING COMPUTATION SHEET}

Title of Project EPHA for THM

Subject Concentration Analysis for THM Hazardous Chemicals

Computer T. M. Franey

Date $\underline{09 / 16 / 96}$

SITE DATA INFORMATION: 1-RD-10

Location: AIKEN, SOUTH CAROLINA

Building Air Exchanges Per Hour: 60 (User specified)

Date and Time: Fixed at July 1, 19961300 hours

CHEMICAL INFORMATION:

Chemical Name: CARBON BISULFIDE

Molecular Weight: $76.14 \mathrm{~kg} / \mathrm{kmol}$

TLV-TWA: $10.00 \mathrm{ppm}$ IDLH: $500.00 \mathrm{ppm}$

Footprint Level of Concern: 50 ppm

Boiling Point: $46.23^{\circ} \mathrm{C}$

Vapor Pressure at Ambient Temperature: $0.47 \mathrm{~atm}$

Ambient Saturation Concentration: $481,124 \mathrm{ppm}$ or $48.1 \%$

ATMOSPHERIC INFORMATION: (MANUAL INPUT OF DATA)

Wind: 2.5 meters $/ \mathrm{sec}$ from $90^{\circ}$ true

Inversion Height: 500 meters

Stability Class: C Air Temperature: $25^{\circ} \mathrm{C}$

Relative Humidity: 50\% Ground Roughness: 100 centimeters

Cloud Cover: 7 tenths

\section{SOURCE STRENGTH INFORMATION:}

Puddle Area: 32.3 square feet

Average Puddle Depth: 1 centimeters

Soil Type: Default Ground Temperature: $25^{\circ} \mathrm{C}$

Initial Puddle Temperature: Ground temperature

Release Duration: 33 minutes

Max Computed Release Rate: 2.1 kilograms/min

Max Average Sustained Release Rate: 1.48 kilograms/min

(averaged over a minute or more)

Total Amount Released: 37.7 kilograms

FOOTPRINT INFORMATION:

Dispersion Module: Gaussian

User specified LOC: $50 \mathrm{ppm}$

Max Threat Zone for LOC: 30 meters

Max Threat Zone for IDLH: less than 10 meters(10.9 yards)

Note: Footprint was not drawn because

effects of near-field patchiness make plume

presentation unreliable for short distances.

\section{TIME DEPENDENT INFORMATION:}

Concentration Estimates at the point:

Downwind: 30 meters

Off Centerline: 0 meters

Max Concentration:

Outdoor: $50 \mathrm{ppm}$

Indoor: $49.6 \mathrm{ppm}$

Note: Indoor graph is shown with a dotted line.
Reviewed by $\mathrm{S}$. A. Henderson

Works Calculation 7

Sheet No. 337 of 577 


\section{ENGINEERING COMPUTATION SHEET}

Title of Project EPHA for THM

Subject Concentration Analysis for THM Hazardous Chemicals

Computer T. M. Franey

Date $\underline{09 / 16 / 96}$

SITE DATA INFORMATION: 1-RD-10

Location: AIKEN, SOUTH CAROLINA

Building Air Exchanges Per Hour: 60 (User specified)

Date and Time: Fixed at July 1, 19961300 hours

CHEMICAL INFORMATION:

Chemical Name: CARBON BISULFIDE

Molecular Weight: $76.14 \mathrm{~kg} / \mathrm{kmol}$

TLV-TWA: $10.00 \mathrm{ppm}$ IDLH: $500.00 \mathrm{ppm}$

Footprint Level of Concern: $50 \mathrm{ppm}$

Boiling Point: $46.23^{\circ} \mathrm{C}$

Vapor Pressure at Ambient Temperature: 0.47 atm

Ambient Saturation Concentration: 481,124 ppm or $48.1 \%$

ATMOSPHERIC INFORMATION: (MANUAL INPUT OF DATA)

Wind: 2.5 meters $/ \mathrm{sec}$ from $90^{\circ}$ true

Inversion Height: 500 meters

Stability Class: C Air Temperature: $25^{\circ} \mathrm{C}$

Relative Humidity: 50\% Ground Roughness: 100 centimeters

Cloud Cover: 7 tenths

SOURCE STRENGTH INFORMATION:

Puddle Area: 260 square feet

Average Puddle Depth: 1 centimeters

Soil Type: Default Ground Temperature: $25^{\circ} \mathrm{C}$

Initial Puddle Temperature: Ground temperature

Release Duration: 36 minutes

Max Computed Release Rate: 14.9 kilograms/min

Max Average Sustained Release Rate: 10.8 kilograms/min

(averaged over a minute or more)

Total Amount Released: 303 kilograms

\section{FOOTPRINT INFORMATION:}

Dispersion Module: Gaussian

User specified LOC: 50 ppm

Max Threat Zone for LOC: 81 meters

Max Threat Zone for IDLH: 22 meters

Note: Footprint was not drawn because

effects of near-field patchiness make plume

presentation unreliable for short distances.

\section{TIME DEPENDENT INFORMATION:}

Concentration Estimates at the point:

Downwind: 100 meters

Off Centerline: 0 meters

Max Concentration:

Outdoor: $33.1 \mathrm{ppm}$

Indoor: $33 \mathrm{ppm}$

Note: Indoor graph is shown with a dotted line.
Reviewed by $\underline{S}$. A. Henderson

Works Calculation 7

Sheet No. $\underline{338 \text { of } 577}$ 
Title of Project EPHA for THM

Subject Concentration Analysis for THM Hazardous Chemicals

Computer T. M. Franey

Date $\underline{09 / 16 / 96}$

SITE DATA INFORMATION: 1-RD-10

Location: AIKEN, SOUTH CAROLINA

Building Air Exchanges Per Hour: 60 (User specified)

Date and Time: Fixed at July 1, 19961300 hours

CHEMICAL INFORMATION:

Chemical Name: CARBON BISULFIDE

Molecular Weight: $76.14 \mathrm{~kg} / \mathrm{kmol}$

TLV-TWA: $10.00 \mathrm{ppm}$ IDLH: $500.00 \mathrm{ppm}$

Footprint Level of Concern: 50 ppm

Boiling Point: $46.23^{\circ} \mathrm{C}$

Vapor Pressure at Ambient Temperature: $0.47 \mathrm{~atm}$

Ambient Saturation Concentration: 481,124 ppm or $48.1 \%$

ATMOSPHERIC INFORMATION: (MANUAL INPUT OF DATA)

Wind: 2.5 meters $/ \mathrm{sec}$ from $90^{\circ}$ true

Inversion Height: 500 meters

Stability Class: C Air Temperature: $25^{\circ} \mathrm{C}$

Relative Humidity: 50\% Ground Roughness: 100 centimeters

Cloud Cover: 7 tenths

SOURCE STRENGTH INFORMATION:

Puddle Area: 24420 square feet

Average Puddle Depth: 1 centimeters

Soil Type: Default Ground Temperature: $25^{\circ} \mathrm{C}$

Initial Puddle Temperature: Ground temperature

Release Duration: 42 minutes

Max Computed Release Rate: 1,120 kilograms/min

Max Average Sustained Release Rate: 841 kilograms/min

(averaged over a minute or more)

Total Amount Released: 28,488 kilograms

FOOTPRINT INFORMATION:

Model Run: Heavy Gas

User specified LOC: $50 \mathrm{ppm}$

Max Threat Zone for LOC: 1.8 kilometers

Max Threat Zone for IDLH: 436 meters

Note: The Heavy Gas footprint is an initial screening.

For short releases it may be an overestimation.

Be sure to check concentration information at specific locations.

\section{TIME DEPENDENT INFORMATION:}

Concentration Estimates at the point:

Downwind: 100 meters

Off Centerline: 0 meters

Max Concentration:

Outdoor: $5,760 \mathrm{ppm}$

Indoor: $5,750 \mathrm{ppm}$

Note: Indoor graph is shown with a dotted line.
Reviewed by S. A. Henderson

Works Calculation 7

Sheet No. $\underline{339 \text { of } 577}$ 


\section{ENGINEERING COMPUTATION SHEET}

Title of Project EPHA for THM

Subject Concentration Analysis for THM Hazardous Chemicals

Computer T. M. Franey

Date $09 / 16 / 96$

SITE DATA INFORMATION: 1-RD-10

Location: AIKEN, SOUTH CAROLINA

Building Air Exchanges Per Hour: 60 (User specified)

Date and Time: Fixed at July 1, 19961300 hours

CHEMICAL INFORMATION:

Chemical Name: CARBON BISULFIDE

Molecular Weight: $76.14 \mathrm{~kg} / \mathrm{kmol}$

TLV-TWA: $10.00 \mathrm{ppm}$ IDLH: $500.00 \mathrm{ppm}$

Footprint Level of Concern: 50 ppm

Boiling Point: $46.23^{\circ} \mathrm{C}$

Vapor Pressure at Ambient Temperature: $0.47 \mathrm{~atm}$

Ambient Saturation Concentration: $481,124 \mathrm{ppm}$ or $48.1 \%$

ATMOSPHERIC INFORMATION: (MANUAL INPUT OF DATA)

Wind: 2.5 meters $/ \mathrm{sec}$ from $90^{\circ}$ true

Inversion Height: 500 meters

Stability Class: C Air Temperature: $25^{\circ} \mathrm{C}$

Relative Humidity: 50\% Ground Roughness: 100 centimeters

Cloud Cover: 7 tenths

SOURCE STRENGTH INFORMATION:

Puddle Area: 40700 square feet

Average Puddle Depth: 1 centimeters

Soil Type: Default Ground Temperature: $25^{\circ} \mathrm{C}$

Initial Puddle Temperature: Ground temperature

Release Duration: 43 minutes

Max Computed Release Rate: 1,820 kilograms/min

Max Average Sustained Release Rate: 1,380 kilograms/min

(averaged over a minute or more)

Total Amount Released: 47,479 kilograms

FOOTPRINT INFORMATION:

Model Run: Heavy Gas

User specified LOC: $50 \mathrm{ppm}$

Max Threat Zone for LOC: 2.4 kilometers

Max Threat Zone for IDLH: 564 meters

Note: The Heavy Gas footprint is an initial screening.

For short releases it may be an overestimation.

Be sure to check concentration information at specific locations.

-

TIME DEPENDENT INFORMATION:

Concentration Estimates at the point:

Downwind: 100 meters

Off Centerline: 0 meters

Max Concentration:

Outdoor: $9,040 \mathrm{ppm}$

Indoor: $9,020 \mathrm{ppm}$

Note: Indoor graph is shown with a dotted line.
Reviewed by S. A. Henderson

Works Calculation 7

Sheet No. 340 of 577 
Title of Project EPHA for THM

Subject Concentration Analysis for THM Hazardous Chemicals

Computer T. M. Franey Date $\underline{09 / 16 / 96}$
Reviewed by S. A. Henderson

Works Calculation 7

Sheet No. $\underline{341 \text { of } 577}$

SITE DATA INFORMATION: 1-RD-10

Location: AIKEN, SOUTH CAROLINA

Building Air Exchanges Per Hour: 60 (User specified)

Date and Time: Fixed at July 1, 19961300 hours

CHEMICAL INFORMATION:

Chemical Name: CARBON BISULFIDE

Molecular Weight: $76.14 \mathrm{~kg} / \mathrm{kmol}$

TLV-TWA: $10.00 \mathrm{ppm}$ 'IDLH: $500.00 \mathrm{ppm}$

Footprint Level of Concern: $50 \mathrm{ppm}$

Boiling Point: $46.23^{\circ} \mathrm{C}$

Vapor Pressure at Ambient Temperature: $0.47 \mathrm{~atm}$

Ambient Saturation Concentration: 481,124 ppm or $48.1 \%$

ATMOSPHERIC INFORMATION: (MANUAL INPUT OF DATA)

Wind: 2.5 meters $/ \mathrm{sec}$ from $90^{\circ}$ true

Inversion Height: 500 meters

Stability Class: C Air Temperature: $25^{\circ} \mathrm{C}$

Relative Humidity: 50\% Ground Roughness: 100 centimeters

Cloud Cover: 7 tenths

\section{SOURCE STRENGTH INFORMATION:}

Puddle Area: 10000 square meters

Average Puddle Depth: 1 centimeters

Soil Type: Default Ground Temperature: $25^{\circ} \mathrm{C}$

Initial Puddle Temperature: Ground temperature

Release Duration: 44 minutes

Max Computed Release Rate: 4,620 kilograms/min

Max Average.Sustained Release Rate: 3,510 kilograms/min

(averaged over a minute or more)

Total Amount Released: 125,568 kilograms

\section{FOOTPRINT INFORMATION:}

Model Run: Heavy Gas

User specified LOC: $50 \mathrm{ppm}$

Max Threat Zone for LOC: 3.9 kilometers

Max Threat Zone for IDLH: 929 meters

Note: The Heavy Gas footprint is an initial screening.

For short releases it may be an overestimation.

Be sure to check concentration information at specific locations.

\section{TIME DEPENDENT INFORMATION:}

Concentration Estimates at the point:

Downwind: 100 meters

Off Centerline: 0 meters

Max Concentration:

Outdoor: $21,400 \mathrm{ppm}$

Indoor: $21,300 \mathrm{ppm}$

Note: Indoor graph is shown with a dotted line. 
Title of Project EPHA for THM

Subject Concentration Analysis for THM Hazardous Chemicals

Computer T. M. Franey

Date 09/16/96

SITE DATA INFORMATION: 1-RD-11

Location: AIKEN, SOUTH CAROLINA

Building Air Exchanges Per Hour: 60 (User specified)

Date and Time: Fixed at July 1, 19961300 hours

CHEMICAL INFORMATION:

Chemical Name: ACETYL CHLORIDE

Molecular Weight: $78.50 \mathrm{~kg} / \mathrm{kmol}$

TLV-TWA: -unavail- IDLH: -unavail-

Footprint Level of Concern: 5 ppm

Boiling Point: $50.75^{\circ} \mathrm{C}$

Vapor Pressure at Ambient Temperature: 0.38 atm

Ambient Saturation Concentration: 384,742 ppm or $38.5 \%$

ATMOSPHERIC INFORMATION: (MANUAL INPUT OF DATA)

Wind: 2.5 meters $/ \mathrm{sec}$ from $90^{\circ}$ true

Inversion Height: 500 feet

Stability Class: C Air Temperature: $25^{\circ} \mathrm{C}$

Relative Humidity: 50\% Ground Roughness: Urban or forest

Cloud Cover: 7 tenths

SOURCE STRENGTH INFORMATION:

Puddle Area: 4 square feet

Average Puddle Depth: 1 centimeters

Soil Type: Default Ground Temperature: $25^{\circ} \mathrm{C}$

Initial Puddle Temperature: Ground temperature

Release Duration: 35 minutes

Max Computed Release Rate: 215 grams/min

Max Average Sustained Release Rate: 152 grams/min (averaged over a minute or more)

Total Amount Released: 4.10 kilograms

\section{TIME DEPENDENT INFORMATION:}

Concentration Estimates at the point:

Downwind: 30 meters

Off Centerline: 0 meters

Max Concentration:

Outdoor: 5.09 ppm
Reviewed by S. A. Henderson

Works Calculation 7

Sheet No. 342 of 577 
Title of Project EPHA for THM

Subject Concentration Analysis for THM Hazardous Chemicals

Computer T. M. Franey Date 09/16/96
Reviewed by $\mathrm{S}$. A. Henderson

Works Calculation 7

Sheet No. $\underline{343 \text { of } 577}$

\section{1-RD-11SITE DATA INFORMATION: Location: AIKEN, SOUTH CAROLINA \\ Building Air Exchanges Per Hour: 60 (User specified) \\ Date and Time: Fixed at July 1, 19961300 hours}

CHEMICAL INFORMATION:

Chemical Name: ACETYL CHLORIDE

Molecular Weight: $78.50 \mathrm{~kg} / \mathrm{kmol}$

TLV-TWA: -unavail- IDLH: -unavail-

Footprint Level of Concern: 5 ppm

Boiling Point: $50.75^{\circ} \mathrm{C}$

Vapor Pressure at Ambient Temperature: 0.38 atm

Ambient Saturation Concentration: 384,742 ppm or $38.5 \%$

ATMOSPHERIC INFORMATION: (MANUAL INPUT OF DATA)

Wind: 2.5 meters $/ \mathrm{sec}$ from $90^{\circ}$ true

Inversion Height: 500 feet

Stability Class: C Air Temperature: $25^{\circ} \mathrm{C}$

Relative Humidity: 50\% Ground Roughness: Urban or forest

Cloud Cover: 7 tenths

\section{SOURCE STRENGTH INFORMATION:}

Puddle Area: 50 square feet

Average Puddle Depth: 1 centimeters

Soil Type: Default Ground Temperature: $25^{\circ} \mathrm{C}$

Initial Puddle Temperature: Ground temperature

Release Duration: 39 minutes

Max Computed Release Rate: 2.27 kilograms/min

Max Average Sustained Release Rate: 1.66 kilograms/min

(averaged over a minute or more)

Total Amount Released: 51.2 kilograms

TIME DEPENDENT INFORMATION:

Concentration Estimates at the point:

Downwind: 100 meters

Off Centerline: 0 meters

Max Concentration:

Outdoor: $5.01 \mathrm{ppm}$ 


\section{ENGINEERING COMPUTATION SHEET}

Title of Project EPHA for THM

Reviewed by $\underline{\text { S. A. Henderson }}$

Subject Concentration Analysis for THM Hazardous Chemicals

Computer T. M. Franey

Date $\underline{09 / 16 / 96}$

1-RD-11 SITE DATA INFORMATION:

Location: AIKEN, SOUTH CAROLINA

Building Air Exchanges Per Hour: 60 (User specified)

Date and Time: Fixed at July 1, 19961300 hours

CHEMICAL INFORMATION:

Chemical Name: ACETYL CHLORIDE

Molecular Weight: $78.50 \mathrm{~kg} / \mathrm{kmol}$

TLV-TWA: -unavail- IDLH: -unavail-

Footprint Level of Concern: 5 ppm

Boiling Point: $50.75^{\circ} \mathrm{C}$

Vapor Pressure at Ambient Temperature: 0.38 atm

Ambient Saturation Concentration: 384,742 ppm or $38.5 \%$

ATMOSPHERIC INFORMATION: (MANUAL INPUT OF DATA)

Wind: 2.5 meters $/ \mathrm{sec}$ from $90^{\circ}$ true

Inversion Height: 500 feet

Stability Class: C Air Temperature: $25^{\circ} \mathrm{C}$

Relative Humidity: $50 \%$ Ground Roughness: Urban or forest

Cloud Cover: 7 tenths

\section{SOURCE STRENGTH INFORMATION:}

Puddle Area: 24420 square feet

Average Puddle Depth: 1 centimeters

Soil Type: Default Ground Temperature: $25^{\circ} \mathrm{C}$

Initial Puddle Temperature: Ground temperature

Release Duration: 49 minutes

Max Computed Release Rate: 806 kilograms/min

Max Average Sustained Release Rate: 624 kilograms/min

(averaged over a minute or more)

Total Amount Released: 25,001 kilograms

FOOTPRINT INFORMATION:

Model Run: Heavy Gas

User specified LOC: 5 ppm

Max Threat Zone for LOC: 5.4 kilometers

Note: The Heavy Gas footprint is an initial screening.

For short releases it may be an overestimation.

Be sure to check concentration information at specific locations.

\section{TIME DEPENDENT INFORMATION:}

Concentration Estimates at the point:

Downwind: 100 meters

Off Centerline: 0 meters

Max Concentration:

Outdoor: $4,070 \mathrm{ppm}$
Works Calculation 7

Sheet No. 344 of 577 


\section{ENGINEERING COMPUTATION SHEET}

Title of Project EPHA for THM

Subject Concentration Analysis for THM Hazardous Chemicals

Computer T. M. Franey

Date 09/16/96

1-RD-11 SITE DATA INFORMATION:

Location: AIKEN, SOUTH CAROLINA

Building Air Exchanges Per Hour: 60 (User specified)

Date and Time: Fixed at July 1, 19961300 hours

CHEMICAL INFORMATION:

Chemical Name: ACETYL CHLORIDE

Molecular Weight: $78.50 \mathrm{~kg} / \mathrm{kmol}$

TLV-TWA: -unavail- IDLH: -unavail-

Footprint Level of Concern: 5 ppm

Boiling Point: $50.75^{\circ} \mathrm{C}$

Vapor Pressure at Ambient Temperature: $0.38 \mathrm{~atm}$

Ambient Saturation Concentration: 384,742 ppm or $38.5 \%$

ATMOSPHERIC INFORMATION: (MANUAL INPUT OF DATA)

Wind: 2.5 meters $/ \mathrm{sec}$ from $90^{\circ}$ true

Inversion Height: 500 feet

Stability Class: C Air Temperature: $25^{\circ} \mathrm{C}$

Relative Humidity: 50\% Ground Roughness: Urban or forest

Cloud Cover: 7 tenths

\section{SOURCE STRENGTH INFORMATION:}

Puddle Area: 40700 square feet

Average Puddle Depth: 1 centimeters

Soil Type: Default Ground Temperature: $25^{\circ} \mathrm{C}$

Initial Puddle Temperature: Ground temperature

Release Duration: 50 minutes

Max Computed Release Rate: 1,310 kilograms/min

Max Average Sustained Release Rate: 1,020 kilograms/min

(averaged over a minute or more)

Total Amount Released: 41,668 kilograms

FOOTPRINT INFORMATION:

Model Run: Heavy Gas

User specified LOC: 5 ppm

Max Threat Zone for LOC: 6.9 kilometers

Note: The Heavy Gas footprint is an initial screening.

For short releases it may be an overestimation.

Be sure to check concentration information at specific locations.

TIME DEPENDENT INFORMATION:

Concentration Estimates at the point:

Downwind: 100 meters

Off Centerline: 0 meters

Max Concentration:

Outdoor: 6,290 ppm
Reviewed by S. A. Henderson

Works Calculation 7

Sheet No. $\underline{345 \text { of } 577}$ 
Title of Project EPHA for THM

Subject Concentration Analysis for THM Hazardous Chemicals

Computer T. M. Franey
Reviewed by S. A. Henderson

Works Calculation 7

Sheet No. 346 of 577

\section{1-RD-11 SITE DATA INFORMATION:}

Location: AIKEN, SOUTH CAROLINA

Building Air Exchanges Per Hour: 60 (User specified)

Date and Time: Fixed at July 1, 19961300 hours

\section{CHEMICAL INFORMATION:}

Chemical Name: ACETYL CHLORIDE

Molecular Weight: $78.50 \mathrm{~kg} / \mathrm{kmol}$

TLV-TWA: -unavail- IDLH: -unavail-

Footprint Level of Concern: 5 ppm

Boiling Point: $50.75^{\circ} \mathrm{C}$

Vapor Pressure at Ambient Temperature: 0.38 atm

Ambient Saturation Concentration: 384,742 ppm or $38.5 \%$

ATMOSPHERIC INFORMATION: (MANUAL INPUT OF DATA)

Wind: 2.5 meters $/ \mathrm{sec}$ from $90^{\circ}$ true

Inversion Height: 500 feet

Stability Class: C Air Temperature: $25^{\circ} \mathrm{C}$

Relative Humidity: 50\% Ground Roughness: Urban or forest

Cloud Cover: 7 tenths

SOURCE STRENGTH INFORMATTON:

Puddle Area: 10000 square meters

Average Puddle Depth: 1 centimeters

Soil Type: Default Ground Temperature: $25^{\circ} \mathrm{C}$

Initial Puddle Temperature: Ground temperature

Release Duration: 51 minutes

Max Computed Release Rate: 3,330 kilograms/min

Max Average Sustained Release Rate: 2,610 kilograms/min

(averaged over a minute or more)

Total Amount Released: 110,199 kilograms

\section{FOOTPRINT INFORMATION:}

Model Run: Heavy Gas

User specified LOC: 5 ppm

Max Threat Zone for LOC: greater than 10 kilometers

Note: The Heavy Gas footprint is an initial screening.

For short releases it may be an overestimation.

Be sure to check concentration information at specific locations.

\section{TIME DEPENDENT INFORMATION:}

Concentration Estimates at the point:

Downwind: 100 meters

Off Centerline: 0 meters

Max Concentration:

Outdoor: $14,500 \mathrm{ppm}$ 


\section{ENGINEERING COMPUTATION.SHEET}

Title of Project EPHA for THM

Subject Concentration Analysis for THM Hazardous Chemicals

Computer T. M. Franey

Date 09/16/96
Reviewed by S. A. Henderson

Works Calculation 7

Sheet No. 347 of 577

\section{SITE DATA INFORMATION: 1-RD-12}

Location: AIKEN, SOUTH CAROLINA

Building Air Exchanges Per Hour: 60 (User specified)

Date and Time: Fixed at July 1, 19961300 hours

CHEMICAL INFORMATION:

Chemical Name: CHLOROSULFONIC ACID

Molecular Weight: $116.52 \mathrm{~kg} / \mathrm{kmol}$

TLV-TWA: not available IDLH: not available

Footprint Level of Concern: $10 \mathrm{ppm}$

Boiling Point: $330.00^{\circ} \mathrm{C}$

Vapor Pressure at Ambient Temperature: 5.37e-006 atm

Ambient Saturation Concentration: 5.47 ppm or $5.47 \mathrm{e}-004 \%$

ATMOSPHERIC INFORMATION: (MANUAL INPUT OF DATA)

Wind: 2.5 meters $/ \mathrm{sec}$ from $90^{\circ}$ true

Inversion Height: 500 meters

Stability Class: C Air Temperature: $25^{\circ} \mathrm{C}$

Relative Humidity: $50 \%$ Ground Roughness: 100 centimeters

Cloud Cover: 7 tenths

SOURCE STRENGTH INFORMATION:

Puddle Area: 30430 square feet

Average Puddle Depth: 1 centimeters

Soil Type: Default Ground Temperature: $25^{\circ} \mathrm{C}$

Initial Puddle Temperature: Ground temperature

Release Duration: ALOHA limited the duration to 1 hour

Max Computed Release Rate: 35.3 grams/min

Max Average Sustained Release Rate: 34.8 grams/min

(averaged over a minute or more)

Total Amount Released: 1.71 kilograms

\section{FOOTPRINT INFORMATION:}

Dispersion Module: Gaussian

User specified LOC: $10 \mathrm{ppm}$

Max Threat Zone for LOC: 30 meters

Max Threat Zone for IDLH: 30 meters

Note: Footprint was not drawn because effects of near-field patchiness make plume presentation unreliable for short distances.

\section{TIME DEPENDENT INFORMATION:}

Concentration Estimates at the point:

Downwind: 30 meters

Off Centerline: 0 meters

- Max Concentration:

Outdoor: $0.186 \mathrm{ppm}$

Indoor: $0.186 \mathrm{ppm}$

Note: Indoor graph is shown with a dotted line. 
Title of Project EPHA for THM

Subject Concentration Analysis for THM Hazardous Chemicals

Computer T. M. Franey Date $09 / 16 / 96$
Reviewed by S. A. Henderson

Works Calculation 7

Sheet No. 348 of 577
SITE DATA INFORMATION: 1-RD-12

Location: AIKEN, SOUTH CAROLINA

Building Air Exchanges Per Hour: 60 (User specified)

Date and Time: Fixed at July 1, 19961300 hours

CHEMICAL INFORMATION:

Chemical Name: CHLOROSULFONIC ACID

Molecular Weight: $116.52 \mathrm{~kg} / \mathrm{kmol}$

TLV-TWA: not available IDLH: not available

Footprint Level of Concern: 10 ppm

Boiling Point: $330.00^{\circ} \mathrm{C}$

Vapor Pressure at Ambient Temperature: 5.37e-006 atm

Ambient Saturation Concentration: 5.47 ppm or $5.47 \mathrm{e}-004 \%$

ATMOSPHERIC INFORMATION: (MANUAL INPUT OF DATA)

Wind: 2.5 meters $/ \mathrm{sec}$ from $90^{\circ}$ true

Inversion Height: 500 meters

Stability Class: C Air Temperature: $25^{\circ} \mathrm{C}$

Relative Humidity: 50\% Ground Roughness: 100 centimeters

Cloud Cover: 7 tenths

\section{SOURCE STRENGTH INFORMATION:}

Puddle Area: 99999 square feet

Average Puddle Depth: 1 centimeters

Soil Type: Default Ground Temperature: $25^{\circ} \mathrm{C}$

Initial Puddle Temperature: Ground temperature

Release Duration: ALOHA limited the duration to 1 hour

Max Computed Release Rate: 111 grams/min

Max Average Sustained Release Rate: 109 grams/min

(averaged over a minute or more)

Total Amount Released: 5.36 kilograms

FOOTPRINT INFORMATION:

Dispersion Module: Gaussian

User specified LOC: $10 \mathrm{ppm}$

Max Threat Zone for LOC: 55 meters

Max Threat Zone for IDLH: 54 meters

Note: Footprint was not drawn because effects of near-field patchiness make plume presentation unreliable for short distances.

TIME DEPENDENT INFORMATION:

Concentration Estimates at the point:

Dowhwind: 100 meters

Off Centerline: 0 meters

Max Concentration:

Outdoor: $0.0577 \mathrm{ppm}$

Indoor: $0.0577 \mathrm{ppm}$

Note: Indoor graph is shown with a dotted line. 


\section{ENGINEERING COMPUTATION SHEET}

Title of Project EPHA for THM

Subject Concentration Analysis for THM Hazardous Chemicals

Computer T. M. Franey Date $\underline{09 / 16 / 96}$

SITE DATA INFORMATION: 1-RD-12

Location: AIKEN, SOUTH CAROLINA

Building Air Exchanges Per Hour: 60 (User specified)

Date and Time: Fixed at July 1, 19961300 hours

CHEMICAL INFORMATION:

Chemical Name: CHLOROSULFONIC ACID

Molecular Weight: $116.52 \mathrm{~kg} / \mathrm{kmol}$

TLV-TWA: not available

Footprint Level of Concern: $10 \mathrm{ppm}$

Boiling Point: $330.00^{\circ} \mathrm{C}$

Vapor Pressure at Ambient Temperature: 5.37e-006 atm

Ambient Saturation Concentration: 5.47 ppm or $5.47 \mathrm{e}-004 \%$

ATMOSPHERIC INFORMATION: (MANUAL INPUT OF DATA)

Wind: 2.5 meters $/ \mathrm{sec}$ from $90^{\circ}$ true

Inversion Height: 500 meters

Stability Class: C Air Temperature: $25^{\circ} \mathrm{C}$

Relative Humidity: 50\% Ground Roughness: 100 centimeters

Cloud Cover: 7 tenths

\section{SOURCE STRENGTH INFORMATION:}

Puddle Area: 24420 square feet

Average Puddle Depth: 1 centimeters

Soil Type: Default Ground Temperature: $25^{\circ} \mathrm{C}$

Initial Puddle Temperature: Ground temperature

Release Duration: ALOHA limited the duration to 1 hour

Max Computed Release Rate: 28.6 grams/min

Max Average Sustained Release Rate: 28.1 grams/min

(averaged over a minute or more)

Total Amount Released: 1.38 kilograms

FOOTPRINT INFORMATION:

Dispersion Module: Gaussian

User specified LOC: $10 \mathrm{ppm}$

Max Threat Zone for LOC: 27 meters

Max Threat Zone for IDLH: 27 meters

Note: Footprint was not drawn because effects of near-field patchiness make plume presentation unreliable for short distances.

\section{TIME DEPENDENT INFORMATION:}

Concentration Estimates at the point:

Downwind: 100 meters

Off Centerline: 0 meters

Max Concentration:

Outdoor: $0.0277 \mathrm{ppm}$

Indoor: $0.0277 \mathrm{ppm}$

Note: Indoor graph is shown with a dotted line.
Reviewed by S. A. Henderson

Works Calculation 7

Sheet No. $\underline{349 \text { of } 577}$ 
Title of Project EPHA for THM

Subject Concentration Analysis for THM Hazardous Chemicals

Computer T. M. Franey
Reviewed by S. A. Henderson

Works Calculation 7

Sheet No. $\underline{350 \text { of } 577}$

SITE DATA INFORMATION: 1-RD-12

Location: AIKEN, SOUTH CAROLINA

Building Air Exchanges Per Hour: 60 (User specified)

Date and Time: Fixed at July 1, 19961300 hours

CHEMICAL INFORMATION:

Chemical Name: CHLOROSULFONIC ACID

Molecular Weight: $116.52 \mathrm{~kg} / \mathrm{kmol}$

TLV-TWA: not available IDLH: not available

Footprint Level of Concern: $10 \mathrm{ppm}$

Boiling Point: $330.00^{\circ} \mathrm{C}$

Vapor Pressure at Ambient Temperature: 5.37e-006 atm

Ambient Saturation Concentration: 5.47 ppm or $5.47 \mathrm{e}-004 \%$

.

ATMOSPHERIC INFORMATION: (MANUAL INPUT OF DATA)

Wind: 2.5 meters $/ \mathrm{sec}$ from $90^{\circ}$ true

Inversion Height: 500 meters

Stability Class: C Air Temperature: $25^{\circ} \mathrm{C}$

Relative Humidity: 50\% Ground Roughness: 100 centimeters

Cloud Cover: 7 tenths

SOURCE STRENGTH INFORMATION:

Puddle Area: 40700 square feet

Average Puddle Depth: 1 centimeters

Soil Type: Default Ground Temperature: $25^{\circ} \mathrm{C}$

Initial Puddle Temperature: Ground temperature

Release Duration: ALOHA limited the duration to 1 hour

Max Computed Release Rate: 46.7 grams/min

Max Average Sustained Release Rate: 46 grams/min

(averaged over a minute or more)

Total Amount Released: 2.26 kilograms

\section{FOOTPRINT INFORMATION:}

Dispersion Module: Gaussian

User specified LOC: $10 \mathrm{ppm}$

Max Threat Zone for LOC: 34 meters

Max Threat Zone for IDLH: 35 meters

Note: Footprint was not drawn because

effects of near-field patchiness make plume

presentation unreliable for short distances.

\section{TIME DEPENDENT INFORMATION:}

Concentration Estimates at the point:

Downwind: 100 meters

Off Centerline: 0 meters

Max Concentration:

Outdoor: $0.0352 \mathrm{ppm}$

Indoor: $0.0352 \mathrm{ppm}$

Note: Indoor graph is shown with a dotted line. 
Title of Project EPHA for THM

Subject Concentration Analysis for THM Hazardous Chemicals

Computer T. M. Franey
Reviewed by $\mathrm{S}$. A. Henderson

Works Calculation 7

Sheet No. 351 of 577

\section{SITE DATA INFORMATION: 1-RD-12 \\ Location: AIKEN, SOUTH CAROLINA \\ Building Air Exchanges Per Hour: 60 (User specified) \\ Date and Time: Fixed at July 1, 19961300 hours}

CHEMICAL INFORMATION:

Chemical Name: CHLOROSULFONIC ACID

Molecular Weight: $116.52 \mathrm{~kg} / \mathrm{kmol}$

TLV-TWA: not available ' IDLH: not available

Footprint Level of Concern: $10 \mathrm{ppm}$

Boiling Point: $330.00^{\circ} \mathrm{C}$

Vapor Pressure at Ambient Temperature: 5.37e-006 atm

Ambient Saturation Concentration: 5.47 ppm or $5.47 \mathrm{e}-004 \%$

ATMOSPHERIC INFORMATION: (MANUAL INPUT OF DATA)

Wind: 2.5 meters $/ \mathrm{sec}$ from $90^{\circ}$ true

Inversion Height: 500 meters

Stability Class: C Air Temperature: $25^{\circ} \mathrm{C}$

Relative Humidity: 50\% Ground Roughness: 100 centimeters

Cloud Cover: 7 tenths

SOURCE STRENGTH INFORMATION:

Puddle Area: 10000 square meters

Average Puddle Depth: 1 centimeters

Soil Type: Default Ground Temperature: $25^{\circ} \mathrm{C}$

Initial Puddle Temperature: Ground temperature

Release Duration: ALOHA limited the duration to 1 hour

Max Computed Release Rate: 119 grams/min

Max Average Sustained Release Rate: 117 grams/min

(averaged over a minute or more)

Total Amount Released: 5.75 kilograms

\section{FOOTPRINT INFORMATION:}

Dispersion Module: Gaussian

User specified LOC: $10 \mathrm{ppm}$

Max Threat Zone for LOC: 56 meters

Max Threat Zone for IDLH: 56 meters

Note: Footprint was not drawn because

effects of near-field patchiness make plume

presentation unreliable for short distances.

\section{TIME DEPENDENT INFORMATION:}

Concentration Estimates at the point:

Downwind: 100 meters

Off Centerline: 0 meters

Max Concentration:

Outdoor: $0.0608 \mathrm{ppm}$

Indoor: $0.0608 \mathrm{ppm}$

Note: Indoor graph is shown with a dotted line. 
Title of Project EPHA for THM

Subject Concentration Analysis for THM Hazardous Chemicals

Computer T. M. Franey Date $09 / 16 / 96$
Reviewed by $\underline{\text { S. A. Henderson }}$

Works Calculation 7

Sheet No. 352 of 577

SITE DATA INFORMATION: 1-RD-13

Location: AIKEN, SOUTH CAROLINA

Building Air Exchanges Per Hour: 60 (User specified)

Date and Time: Fixed at July 1, 19961300 hours

CHEMICAL INFORMATION:

Chemical Name: CYCLOHEXANE

Molecular Weight: $84.16 \mathrm{~kg} / \mathrm{kmol}$

TLV-TWA: $300.00 \mathrm{ppm}$ IDLH: $10000.00 \mathrm{ppm}$

Footprint Level of Concern: $1300 \mathrm{ppm}$

Boiling Point: $80.72^{\circ} \mathrm{C}$

Vapor Pressure at Ambient Temperature: $0.13 \mathrm{~atm}$

Ambient Saturation Concentration: 131,831 ppm or 13.2\%

ATMOSPHERIC INFORMATION: (MANUAL INPUT OF DATA)

Wind: 2.5 meters $/ \mathrm{sec}$ from $90^{\circ}$ true

Inversion Height: 500 meters

Stability Class: C Air Temperature: $25^{\circ} \mathrm{C}$

Relative Humidity: 50\% Ground Roughness: 100 centimeters

Cloud Cover: 7 tenths

SOURCE STRENGTH INFORMATION:

Puddle Area: 2950 square feet

Average Puddle Depth: 1 centimeters

Soil Type: Default Ground Temperature: $25^{\circ} \mathrm{C}$

Initial Puddle Temperature: Ground temperature

Release Duration: ALOHA limited the duration to 1 hour

Max Computed Release Rate: 36.9 kilograms/min

Max Average Sustained Release Rate: 34.8 kilograms/min

(averaged over a minute or more)

Total Amount Released: 1,993 kilograms

\section{FOOTPRINT INFORMATION:}

Dispersion Module: Gaussian

User specified LOC: $1300 \mathrm{ppm}$

Max Threat Zone for LOC: 13 meters

Max Threat Zone for IDLH: less than 10 meters(10.9 yards)

Note: Footprint was not drawn because

effects of near-field patchiness make plume

presentation unreliable for short distances.

\section{TIME DEPENDENT INFORMATION:}

Concentration Estimates at the point:

Downwind: 30 meters

Off Centerline: 0 meters

Max Concentration:

Outdoor: 384 ppm

Indoor: $384 \mathrm{ppm}$

Note: Indoor graph is shown with a dotted line. 


\section{ENGINEERING COMPUTATION SHEET}

Title of Project EPHA for THM

Subject Concentration Analysis for THM Hazardous Chemicals

Computer T.M. Franey

Date $\underline{09 / 16 / 96}$

SITE DATA INFORMATION: 1-RD-13

Location: AIKEN, SOUTH CAROLINA

Building Air Exchanges Per Hour: 60 (User specified)

Date and Time: Fixed at July 1, 19961300 hours

CHEMICAL INFORMATION:

Chemical Name: CYCLOHEXANE

Molecular Weight: $84.16 \mathrm{~kg} / \mathrm{kmol}$

TLV-TWA: $300.00 \mathrm{ppm}$ IDLH: $10000.00 \mathrm{ppm}$

Footprint Level of Concern: $1300 \mathrm{ppm}$

Boiling Point: $80.72^{\circ} \mathrm{C}$

Vapor Pressure at Ambient Temperature: $0.13 \mathrm{~atm}$

Ambient Saturation Concentration: 131,831 ppm or $13.2 \%$

ATMOSPHERIC INFORMATION: (MANUAL INPUT OF DATA)

Wind: 2.5 meters $/ \mathrm{sec}$ from $90^{\circ}$ true

Inversion Height: 500 meters

Stability Class: C Air Temperature: $25^{\circ} \mathrm{C}$

Relative Humidity: 50\% Ground Roughness: 100 centimeters

Cloud Cover: 7 tenths

\section{SOURCE STRENGTH INFORMATION:}

Puddle Area: 18700 square feet

Average Puddle Depth: 1 centimeters

Soil Type: Default Ground Temperature: $25^{\circ} \mathrm{C}$

Initial Puddle Temperature: Ground temperature

Release Duration: ALOHA limited the duration to 1 hour

Max Computed Release Rate: 215 kilograms/min

Max Average Sustained Release Rate: 205 kilograms/min

(averaged over a minute or more)

Total Amount Released: 11,867 kilograms

FOOTPRINT INFORMATION:

Model Run: Heavy Gas

User specified LOC: $1300 \mathrm{ppm}$

Max Threat Zone for LOC: 100 meters

Max Threat Zone for IDLH: 16 meters

Note: The Heavy Gas footprint is an initial screening.

For short releases it may be an overestimation.

Be sure to check concentration information at specific locations.

\section{TIME DEPENDENT INFORMATION:}

Concentration Estimates at the point:

Downwind: 100 meters

Off Centerline: 0 meters

Max Concentration:

Outdoor: $1,300 \mathrm{ppm}$

Indoor: $1,300 \mathrm{ppm}$

Note: Indoor graph is shown with a dotted line.
Reviewed by S. A. Henderson

Works Calculation 7

Sheet No. $\underline{353 \text { of } 577}$ 


\section{ENGINEERING COMPUTATION SHEET}

Title of Project EPHA for THM

Subject Concentration Analysis for THM Hazardous Chemicals

Computer T. M. Franey

Date $\underline{09 / 16 / 96}$

SITE DATA INFORMATION: 1-RD-13

Location: AIKEN, SOUTH CAROLINA

Building Air Exchanges Per Hour: 60 (User specified)

Date and Time: Fixed at July 1, 19961300 hours

CHEMICAL INFORMATION:

Chemical Name: CYCLOHEXANE

Molecular Weight: $84.16 \mathrm{~kg} / \mathrm{kmol}$

TLV-TWA: $300.00 \mathrm{ppm}$ IDLH: $10000.00 \mathrm{ppm}$

Footprint Level of Concern: $1300 \mathrm{ppm}$

Boiling Point: $80.72^{\circ} \mathrm{C}$

Vapor Pressure at Ambient Temperature: $0.13 \mathrm{~atm}$

Ambient Saturation Concentration: 131,831 ppm or $13.2 \%$

ATMOSPHERIC INFORMATION: (MANUAL INPUT OF DATA)

Wind: 2.5 meters $/ \mathrm{sec}$ from $90^{\circ}$ true

Inversion Height: 500 meters

Stability Class: C Air Temperature: $25^{\circ} \mathrm{C}$

Relative Humidity: 50\% Ground Roughness: 100 centimeters

Cloud Cover: 7 tenths

\section{SOURCE STRENGTH INFORMATTON:}

Puddle Area: 24420 square feet

Average Puddle Depth: 1 centimeters

Soil Type: Default Ground Temperature: $25^{\circ} \mathrm{C}$

Initial Puddle Temperature: Ground temperature

Release Duration: ALOHA limited the duration to 1 hour

Max Computed Release Rate: 277 kilograms/min

Max Average Sustained Release Rate: 265 kilograms/min

(averaged over a minute or more)

Total Amount Released: 15,363 kilograms

\section{FOOTPRINT INFORMATION: .}

Model Run: Heavy Gas

User specified LOC: 1300 ppm

Max Threat Zone for LOC: 115 meters

Max Threat Zone for IDLH: 19 meters

Note: The Heavy Gas footprint is an initial screening.

For short releases it may be an overestimation.

Be sure to check concentration information at specific locations.

\section{TIME DEPENDENT INFORMATION:}

Concentration Estimates at the point:

Downwind: 100 meters

Off Centerline: 0 meters

Max Concentration:

Outdoor: $1,580 \mathrm{ppm}$

Inḍoor: $1,580 \mathrm{ppm}$

Note: Indoor graph is shown with a dotted line.
Reviewed by $\underline{S}$. A. Henderson

Works Calculation 7

Sheet No. $\underline{354 \text { of } 577}$ 
Title of Project EPHA for THM

Subject Concentration Analysis for THM Hazardous Chemicals

Computer T. M. Franey Date $\underline{09 / 16 / 96}$
Reviewed by S. A. Henderson

Works Calculation 7

Sheet No. $\underline{355 \text { of } 577}$

SITE DATA INFORMATION: 1-RD-13

Location: AIKEN, SOUTH CAROLINA

Building Air Exchanges Per Hour: 60 (User specified)

Date and Time: Fixed at July 1, 19961300 hours

\section{CHEMICAL INFORMATION:}

Chemical Name: CYCLOHEXANE

Molecular Weight: $84.16 \mathrm{~kg} / \mathrm{kmol}$

TLV-TWA: $300.00 \mathrm{ppm}$ IDLH: $10000.00 \mathrm{ppm}$

Footprint Level of Concern: 1300 ppm

Boiling Point: $80.72^{\circ} \mathrm{C}$

Vapor Pressure at Ambient Temperature: $0.13 \mathrm{~atm}$

Ambient Saturation Concentration: 131,831 ppm or $13.2 \%$

ATMOSPHERIC INFORMATION: (MANUAL INPUT OF DATA)

Wind: 2.5 meters $/ \mathrm{sec}$ from $90^{\circ}$ true

Inversion Height: 500 meters

Stability Class: C Air Temperature: $25^{\circ} \mathrm{C}$

Relative Humidity: 50\% Ground Roughness: 100 centimeters

Cloud Cover: 7 tenths

\section{SOURCE STRENGTH INFORMATION:}

Puddle Area: 40700 square feet

Average Puddle Depth: 1 centimeters

Soil Type: Default Ground Temperature: $25^{\circ} \mathrm{C}$

Initial Puddle Temperature: Ground temperature

Release Duration: ALOHA limited the duration to 1 hour

Max Computed Release Rate: 452 kilograms/min

Max Average Sustained Release Rate: 434 kilograms/min (averaged over a minute or more)

Total Amount Released: 25,189 kilograms

\section{FOOTPRINT INFORMATION:}

Model Run: Heavy Gas

User specified LOC: $1300 \mathrm{ppm}$

Max Threat Zone for LOC: 149 meters

Max Threat Zone for IDLH: 29 meters

Note: The Heavy Gas footprint is an initial screening.

For short releases it may be an overestimation.

Be sure to check concentration information at specific locations.

\section{TIME DEPENDENT INFORMATION:}

\section{Concentration Estimates at the point:}

Downwind: 100 meters

Off Centerline: 0 meters

Max Concentration:

Outdoor: $2,300 \mathrm{ppm}$

Indoor: $2,300 \mathrm{ppm}$

Note: Indoor graph is shown with a dotted line. 
Title of Project EPHA for THM

Subject Concentration Analysis for THM Hazardous Chemicals

Computer T. M. Franey
Date 09/16/96
Reviewed by S. A. Henderson

Works Calculation 7

Sheet No. $\underline{356 \text { of } 577}$

SITE DATA INFORMATION: 1-RD-13

Location: AIKEN, SOUTH CAROLINA

Building Air Exchanges Per Hour: 60 (User specified)

Date and Time: Fixed at July 1, 19961300 hours

CHEMICAL INFORMATION:

Chemical Name: CYCLOHEXANE

Molecular Weight: $84.16 \mathrm{~kg} / \mathrm{kmol}$

TLV-TWA: $300.00 \mathrm{ppm}$ - IDLH: $10000.00 \mathrm{ppm}$.

Footprint Level of Concern: 1300 ppm

Boiling Point: $80.72^{\circ} \mathrm{C}$

Vapor Pressure at Ambient Temperature: $0.13 \mathrm{~atm}$

Ambient Saturation Concentration: 131,831 ppm or $13.2 \%$

ATMOSPHERIC INFORMATION: (MANUAL INPUT OF DATA)

Wind: 2.5 meters $/ \mathrm{sec}$ from $90^{\circ}$ true

Inversion Height: 500 meters

Stability Class: C Air Temperature: $25^{\circ} \mathrm{C}$

Relative Humidity: 50\% Ground Roughness: 100 centimeters

Cloud Cover: 7 tenths

\section{SOURCE STRENGTH INFORMATION:}

Puddle Area: 10000 square meters

Average Puddle Depth: 1 centimeters

Soil Type: Default Ground Temperature: $25^{\circ} \mathrm{C}$

Initial Puddle Temperature: Ground temperature

Release Duration: ALOHA limited the duration to 1 hour

Max Computed Release Rate: 1,150 kilograms/min

Max Average Sustained Release Rate: 1,110 kilograms/min

(averaged over a minute or more)

Total Amount Released: 64,626 kilograms

FOOTPRINT INFORMATION:

Model Run: Heavy Gas

User specified LOC: $1300 \mathrm{ppm}$

Max Threat Zone for LOC: 247 meters

Max Threat Zone for IDLH: 50 meters

Note: The Heavy Gas footprint is an initial screening.

For short releases it may be an overestimation.

Be sure to check concentration information at specific locations.

\section{TIME DEPENDENT INFORMATION:}

Concentration Estimates at the point:

Downwind: 100 meters

Off Centerline: 0 meters

Max Concentration:

Outdoor: $4,650 \mathrm{ppm}$

Indoor: $4,650 \mathrm{ppm}$

Note: Indoor graph is shown with a dotted line. 
Title of Project EPHA for THM

Subject Concentration Analysis for THM Hazardous Chemicals

Computer T. M. Franey
Reviewed by S. A. Henderson

Works Calculation 7

Sheet No. $\underline{357 \text { of } 577}$

SITE DATA INFORMATION: 1-RD-14

Location: AIKEN, SOUTH CAROLINA

Building Air Exchanges Per Hour: 60 (User specified)

Date and Time: Fixed at July 1, 19961300 hours

CHEMICAL INFORMATION:

Chemical Name: CYCLOHEXANONE

Molecular Weight: $98.14 \mathrm{~kg} / \mathrm{kmol}$

TLV-TWA: $25.00 \mathrm{ppm}$ IDLH: $5000.00 \mathrm{ppm}$

Footprint Level of Concern: $13.00 \mathrm{ppm}$

Boiling Point: $155.75^{\circ} \mathrm{C}$

Vapor Pressure at Ambient Temperature: $0.0057 \mathrm{~atm}$

Ambient Saturation Concentration: 5,792 ppm or $0.58 \%$

ATMOSPHERIC INFORMATION: (MANUAL INPUT OF DATA)

Wind: 2.5 meters $/ \mathrm{sec}$ from $90^{\circ}$ true

Inversion Height: 500 meters

Stability Class: C Air Temperature: $25^{\circ} \mathrm{C}$

Relative Humidity: 50\% Ground Roughness: 100 centimeters

Cloud Cover: 7 tenths

SOURCE STRENGTH INFORMATION:

Puddle Area: 30430 square feet

Average Puddle Depth: 1 centimeters

Soil Type: Default Ground Temperature: $25^{\circ} \mathrm{C}$

Initial Puddle Temperature: Ground temperature

Release Duration: ALOHA limited the duration to 1 hour

Max Computed Release Rate: 16.8 kilograms/min

Max Average Sustained Release Rate: 16.6 kilograms/min

(averaged over a minute or more)

Total Amount Released: 862 kilograms

\section{FOOTPRINT INFORMATION:}

Dispersion Module: Gaussian

User specified LOC: $1300 \mathrm{ppm}$

Max Threat Zone for LOC: 30 meters

Max Threat Zone for IDLH: 30 meters

Note: Footprint was not drawn because effects of near-field patchiness make plume

presentation unreliable for short distances.

\section{TIME DEPENDENT INFORMATION:}

Concentration Estimates at the point:

Downwind: 30 meters

Off Centerline: 0 meters

Max Concentration:

Outdoor: $88.7 \mathrm{ppm}$

Indoor: $88.7 \mathrm{ppm}$

Note: Indoor graph is shown with a dotted line. 
Title of Project EPHA for THM

Subject Concentration Analysis for THM Hazardous Chemicals

Computer T. M. Franey Date $09 / 16 / 96$
Reviewed by $\underline{\text { S. A. Henderson }}$

Works Calculation 7

Sheet No. $\underline{358 \text { of } 577}$

SITE DATA INFORMATION: 1-RD-14

Location: AIKEN, SOUTH CAROLINA

Building Air Exchanges Per Hour: 60 (User specified)

Date and Time: Fixed at July 1, 19961300 hours

CHEMICAL INFORMATION:

Chemical Name: CYCLOHEXANONE

Molecular Weight: $98.14 \mathrm{~kg} / \mathrm{kmol}$

TLV-TWA: $25.00 \mathrm{ppm}$ IDLH: $5000.00 \mathrm{ppm}$

Footprint Level of Concern: $1300 \mathrm{ppm}$

Boiling Point: $155.75^{\circ} \mathrm{C}$

Vapor Pressure at Ambient Temperature: $0.0057 \mathrm{~atm}$

Ambient Saturation Concentration: 5,792 ppm or $0.58 \%$

ATMOSPHERIC INFORMATION: (MANUAL INPUT OF DATA)

Wind: 2.5 meters $/ \mathrm{sec}$ from $90^{\circ}$ true

Inversion Height: 500 meters

Stability Class: C Air Temperature: $25^{\circ} \mathrm{C}$

Relative Humidity: 50\% Ground Roughness: 100 centimeters

Cloud Cover: 7 tenths

\section{SOURCE STRENGTH INFORMATION:}

Puddle Area: 99999 square feet

Average Puddle Depth: 1 centimeters

Soil Type: Default Ground Temperature: $25^{\circ} \mathrm{C}$

Initial Puddle Temperature: Ground temperature

Release Duration: ALOHA limited the duration to 1 hour

Max Computed Release Rate: 52.7 kilograms/min

Max Average Sustained Release Rate: 52.1 kilograms/min

(averaged over a minute or more)

Total Amount Released: 2,704 kilograms

\section{FOOTPRINTT INFORMATION:}

Dispersion Module: Gaussian

User specified LOC: $1300 \mathrm{ppm}$

Max Threat Zone for LOC: 55 meters

Max Threat Zone for IDLH: 54 meters

Note: Footprint was not drawn because effects of near-field patchiness make plume

presentation unreliable for short distances.

\section{TIME DEPENDENT INFORMATION:}

Concentration Estimates at the point:

Downwind: 100 meters

Off Centerline: 0 meters

Max Concentration:

Outdoor: $27.5 \mathrm{ppm}$

Indoor: $27.5 \mathrm{ppm}$

Note: Indoor graph is shown with a dotted line. 


\section{ENGINEERING COMPUTATION SHEET}

Title of Project EPHA for THM

Subject Concentration Analysis for THM Hazardous Chemicals

Computer T. M. Franey

Date 09/16/96
Reviewed by $\underline{\text { S. A. Henderson }}$

Works Calculation 7

Sheet No. $\underline{359 \text { of } 577}$

SITE DATA INFORMATION: 1-RD-14

Location: AIKEN, SOUTH CAROLINA

Building Air Exchanges Per Hour: 60 (User specified)

Date and Time: Fixed at July 1, 19961300 hours

CHEMICAL INFORMATION:

Chemical Name: CYCLOHEXANONE

Molecular Weight: $98.14 \mathrm{~kg} / \mathrm{kmol}$

TLV-TWA: $25.00 \mathrm{ppm}$ DLH: $5000.00 \mathrm{ppm}$

Footprint Level of Concern: $1300 \mathrm{ppm}$

Boiling Point: $155.75^{\circ} \mathrm{C}$

Vapor Pressure at Ambient Temperature: $0.0057 \mathrm{~atm}$

Ambient Saturation Concentration: 5,792 ppm or $0.58 \%$

ATMOSPHERIC INFORMATION: (MANUAL INPUT OF DATA)

Wind: 2.5 meters $/ \mathrm{sec}$ from $90^{\circ}$ true

Inversion Height: 500 meters

Stability Class: C Air Temperature: $25^{\circ} \mathrm{C}$

Relative Humidity: 50\% Ground Roughness: 100 centimeters

Cloud Cover: 7 tenths

SOURCE STRENGTH INFORMATION:

Puddle Area: 24420 square feet

Average Puddle Depth: 1 centimeters

Soil Type: Default Ground Temperature: $25^{\circ} \mathrm{C}$

Initial Puddle Temperature: Ground temperature

Release Duration: ALOHA limited the duration to 1 hour

Max Computed Release Rate: 13.6 kilograms/min

Max Average Sustained Release Rate: 13.4 kilograms/min

(averaged over a minute or more)

Total Amount Released: 698 kilograms

FOOTPRINT INFORMATION:

Dispersion Module: Gaussian

User specified LOC: $1300 \mathrm{ppm}$

Max Threat Zone for LOC: 27 meters

Max Threat Zone for IDLH: 27 meters

Note: Footprint was not drawn because

effects of near-field patchiness make plume

presentation unreliable for short distances.

TIME DEPENDENT INFORMATION:

Concentration Estimates at the point:

Downwind: 100 meters

Off Centerline: 0 meters

Max Concentration:

Outdoor: $13.2 \mathrm{ppm}$

Indoor: $13.2 \mathrm{ppm}$

Note: Indoor graph is shown with a dotted line. 
Title of Project EPHA for THM

Subject Concentration Analysis for THM Hazardous Chemicals

Computer T. M. Franey

Date $\underline{09 / 16 / 96}$

Reviewed by $\mathrm{S}$. A. Henderson

SITE DATA INFORMATION: 1-RD-14

Location: AIKEN, SOUTH CAROLINA

Building Air Exchanges Per Hour: 60 (User specified)

Date and Time: Fixed at July 1, 19961300 hours

CHEMICAL INFORMATION:

Chemical Name: CYCLOHEXANONE

Molecular Weight: $98.14 \mathrm{~kg} / \mathrm{kmol}$

TLV-TWA: $25.00 \mathrm{ppm}$ IDLH: $5000.00 \mathrm{ppm}$

Footprint Level of Concern: 1300 ppm

Boiling Point: $155.75^{\circ} \mathrm{C}$

Vapor Pressure at Ambient Temperature: 0.0057 atm

Ambient Saturation Concentration: 5,792 ppm or $0.58 \%$

\section{ATMOSPHERIC INFORMATION: (MANUAL INPUT OF DATA)}

Wind: 2.5 meters $/ \mathrm{sec}$ from $90^{\circ}$ true

Inversion Height: 500 meters

Stability Class: C Air Temperature: $25^{\circ} \mathrm{C}$

Relative Humidity: 50\% Ground Roughness: 100 centimeters

Cloud Cover: 7 tenths

\section{SOURCE STRENGTH INFORMATION:}

Puddle Area: 40700 square feet

Average Puddle Depth: 1 centimeters

Soil Type: Default Ground Temperature: $25^{\circ} \mathrm{C}$

Initial Puddle Temperature: Ground temperature

Release Duration: ALOHA limited the duration to 1 hour

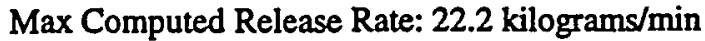

Max Average Sustained Release Rate: 21.9 kilograms/min (averaged over a minute or more)

Total Amount Released: 1,139 kilograms

\section{FOOTPRINT INFORMATION:}

Dispersion Module: Gaussian

User specified LOC: $1300 \mathrm{ppm}$

Max Threat Zone for LOC: 34 meters

Max Threat Zone for IDLH: 35 meters

Note: Footprint was not drawn because effects of near-field patchiness make plume presentation unreliable for short distances.

\section{TIME DEPENDENT INFORMATION:}

Concentration Estimates at the point:

Downwind: 100 meters

Off Centerline: 0 meters

Max Concentration:

Outdoor: $16.8 \mathrm{ppm}$

Indoor: $16.8 \mathrm{ppm}$

Note: Indoor graph is shown with a dotted line. 


\section{ENGINEERING COMPUTATION SHEET}

Title of Project EPHA for THM

Reviewed by $\underline{\text { S. A. Henderson }}$

Subject Concentration Analysis for THM Hazardous Chemicals

Computer T. M. Franey

Date $\underline{09 / 16 / 96}$

SITE DATA INFORMATION: 1-RD-14

Location: AIKEN, SOUTH CAROLINA

Building Air Exchanges Per Hour: 60 (User specified)

Date and Time: Fixed at July 1, 19961300 hours

CHEMICAL INFORMATION:

Chemical Name: CYCLOHEXANONE

Molecular Weight: $98.14 \mathrm{~kg} / \mathrm{kmol}$

TLV-TWA: $25.00 \mathrm{ppm}$ IDLH: $5000.00 \mathrm{ppm}$

Footprint Level of Concern: 1300 ppm

Boiling Point: $155.75^{\circ} \mathrm{C}$

Vapor Pressure at Ambient Temperature: $0.0057 \mathrm{~atm}$

Ambient Saturation Concentration: 5,792 ppm or $0.58 \%$

ATMOSPHERIC INFORMATION: (MANUAL INPUT OF DATA)

Wind: 2.5 meters $/ \mathrm{sec}$ from $90^{\circ}$ true

Inversion Height: 500 meters

Stability Class: C Air Temperature: $25^{\circ} \mathrm{C}$

Relative Humidity: 50\% Ground Roughness: 100 centimeters

Cloud Cover: 7 tenths

\section{SOURCE STRENGTH INFORMATION:}

Puddle Area: 10000 square meters

Average Puddle Depth: 1 centimeters

Soil Type: Default Ground Temperature: $25^{\circ} \mathrm{C}$

Initial Puddle Temperature: Ground temperature

Release Duration: ALOHA limited the duration to 1 hour

Max Computed Release Rate: 56.6 kilograms/min

Max Average Sustained Release Rate: 56 kilograms/min (averaged over a minute or more)

Total Amount Released: 2,902 kilograms

FOOTPRINT INFOQRMATION:

Dispersion Module: Gaussian

User specified LOC: 1300 ppm

Max Threat Zone for LOC: 56 meters

Max Threat Zone for IDLH: 56 meters

Note: Footprint was not drawn because effects of near-field patchiness make plume presentation unreliable for short distances.

\section{TIME DEPENDENT INFORMATION:}

Concentration Estimates at the point:

Downwind: 100 meters

Off Centerline: 0 meters

Max Concentration:

Outdoor: $29 \mathrm{ppm}$

Indoor: $29 \mathrm{ppm}$

Note: Indoor graph is shown with a dotted line.
Works Calculation 7

Sheet No. $\underline{361 \text { of } 577}$ 
Title of Project EPHA for THM

Subject Concentration Analysis for THM Hazardous Chemicals

Computer T.M. Franey
Reviewed by $\underline{\text { S. A. Henderson }}$

Works Calculation 7

Sheet No. 362 of 577

SITE DATA INFORMATION:

1-RD-15

Location: AIKEN, SOUTH CAROLINA

Building Air Exchanges Per Hour: 60 (User specified)

Date and Time: Fixed at July 1, 19961300 hours

CHEMICAL INFORMATION:

Chemical Name: DIETHYLENE GLYCOL MONOETHYL ETHER

Molecular Weight: $134.18 \mathrm{~kg} / \mathrm{kmol}$

TLV-TWA: -unavail- IDLH: -unavail-

Footprint Level of Concern: 125 ppm

Boiling Point: $202.00^{\circ} \mathrm{C}$

Vapor Pressure at Ambient Temperature: 1.66e-004 atm

Ambient Saturation Concentration: 169 ppm or $0.017 \%$

ATMOSPHERIC INFORMATION: (MANUAL INPUT OF DATA)

Wind: 2.5 meters $/ \mathrm{sec}$ from $90^{\circ}$ true

Inversion Height: 500 meters

Stability Class: C Air Temperature: $25^{\circ} \mathrm{C}$

Relative Humidity: 50\% Ground Roughness: 100 centimeters

Cloud Cover: 7 tenths

SOURCE STRENGTH INFORMATION:

Puddle Area: 30430 square feet

Average Puddle Depth: 1 centimeters

Soil Type: Default Ground Temperature: $25^{\circ} \mathrm{C}$

Initial Puddle Temperature: Ground temperature

Release Duration: ALOHA limited the duration to 1 hour

Max Computed Release Rate: 558 grams/min

Max Average Sustained Release Rate: 548 grams/min

(averaged over a minute or more)

- Total Amount Released: 26.2 kilograms

\section{TIME DEPENDENT INFORMATION:}

Concentration Estimates at the point:

Downwind: 30 meters

Off Centerline: 0 meters

Max Concentration:

Outdoor: $2.14 \mathrm{ppm}$

Indoor: $2.14 \mathrm{ppm}$

Note: Indoor graph is shown with a dotted line. 


\section{ENGINEERING COMPUTATION SHEET}

Title of Project EPHA for THM

Reviewed by S. A. Henderson

Subject Concentration Analysis for THM Hazardous Chemicals

Computer T.M. Franey Date $\underline{09 / 16 / 96}$

SITE DATA INFORMATION:

l-RD-15

Location: AIKEN, SOUTH CAROLINA

Building Air Exchanges Per Hour: 60 (User specified)

Date and Time: Fixed at July 1, 19961300 hours

CHEMICAL INFORMATION:

Chemical Name: DIETHYLENE GLYCOL MONOETHYL ETHER

Molecular Weight: $134.18 \mathrm{~kg} / \mathrm{kmol}$

TLV-TWA: -unavail- IDLH: -unavail-

Footprint Level of Concern: 125 ppm

Boiling Point: $202.00^{\circ} \mathrm{C}$

Vapor Pressure at Ambient Temperature: 1.66e-004 atm

Ambient Saturation Concentration: 169 ppm or $0.017 \%$

ATMOSPHERIC INFORMATION: (MANUAL INPUT OF DATA)

Wind: 2.5 meters $/ \mathrm{sec}$ from $90^{\circ}$ true

Inversion Height: 500 meters

Stability Class: C Air Temperature: $25^{\circ} \mathrm{C}$

Relative Humidity: 50\% Ground Roughness: 100 centimeters

Cloud Cover: 7 tenths

SOURCE STRENGTH INFORMATION:

Puddle Area: 99999 square feet

Average Puddle Depth: 1 centimeters

Soil Type: Default Ground Temperature: $25^{\circ} \mathrm{C}$

Initial Puddle Temperature: Ground temperature

Release Duration: ALOHA limited the duration to 1 hour

Max Computed Release Rate: 1.76 kilograms/min

Max Average Sustained Release Rate: 1.73 kilograms/min

(averaged over a minute or more)

Total Amount Released: 82.4 kilograms

TIME DEPENDENT INFORMATION:

Concentration Estimates at the point:

Downwind: 100 meters

Off Centerline: 0 meters

Max Concentration:

Outdoor: $0.666 \mathrm{ppm}$

Indoor: $0.666 \mathrm{ppm}$

Note: Indoor graph is shown with a dotted line. 
Title of Project EPHA for THM

Subject Concentration Analysis for THM Hazardous Chemicals

Computer T. M. Franey Date $\underline{09 / 16 / 96}$
Reviewed by S. A. Henderson

Works Calculation 7

Sheet No. $\underline{364 \text { of } 577}$

\section{1-RD-15}

SITE DATA INFORMATION:

Location: AIKEN, SOUTH CAROLINA

Building Air Exchanges Per Hour: 60 (User specified)

Date and Time: Fixed at July 1, 19961300 hours

\section{CHEMICAL INFORMATION:}

Chemical Name: DIETHYLENE GLYCOL MONOETHYL ETHER

Molecular Weight: $134.18 \mathrm{~kg} / \mathrm{kmol}$

TLV-TWA: -unavail- IDLH: -unavail-

Footprint Level of Concern: 125 ppm

Boiling Point: $202.00^{\circ} \mathrm{C}$

Vapor Pressure at Ambient Temperature: $1.66 \mathrm{e}-004 \mathrm{~atm}$

Ambient Saturation Concentration: 169 ppm or $0.017 \%$

ATMOSPHERIC INFORMATION: (MANUAL INPUT OF DATA)

Wind: 2.5 meters $/ \mathrm{sec}$ from $90^{\circ}$ true

Inversion Height: 500 meters

Stability Class: C Air Temperature: $25^{\circ} \mathrm{C}$

Relative Humidity: 50\% Ground Roughness: 100 centimeters

Cloud Cover: 7 tenths

\section{SOURCE STRENGTH INFORMATION:}

Puddle Area: 24420 square feet

Average Puddle Depth: 1 centimeters

Soil Type: Default Ground Temperature: $25^{\circ} \mathrm{C}$

Initial Puddle Temperature: Ground temperature

Release Duration: ALOHA limited the duration to 1 hour

Max Computed Release Rate: 451 grams/min

Max Average Sustained Release Rate: 443 grams/min

(averaged over a minute or more)

Total Amount Released: 21.2 kilograms

\section{FOOTPRINT INFORMATION:}

Dispersion Module: Gaussian

User specified LOC: $125 \mathrm{ppm}$

Max Threat Zone for LOC: 27 meters

Note: Footprint was not drawn because effects of near-field patchiness make plume presentation unreliable for short distances.

\section{TIME DEPENDENT INFORMATION:}

Concentration Estimates at the point:

Downwind: 100 meters

Off Centerline: 0 meters

Max Concentration:

Outdoor: $0.319 \mathrm{ppm}$

Indoor: $0.319 \mathrm{ppm}$

Note: Indoor graph is shown with a dotted line. 


\section{ENGINEERING COMPUTATION SHEET}

Title of Project EPHA for THM

Reviewed by $\underline{\text { S. A. Henderson }}$

Works Calculation 7

Sheet No. $\underline{365 \text { of } 577}$

Computer T.M. Franey

Date $09 / 16 / 96$
1-RD-15

SITE DATA INFORMATION:

Location: AIKEN, SOUTH CAROLINA

Building Air Exchanges Per Hour: 60 (User specified)

Date and Time: Fixed at July 1, 19961300 hours

\section{CHEMICAL INFORMATION:}

Chemical Name: DIETHYLENE GLYCOL MONOETHYL ETHER

Molecular Weight: $134.18 \mathrm{~kg} / \mathrm{kmol}$

TLV-TWA: -unavail- IDLH: -unavail-

Footprint Level of Concern: 125 ppm

Boiling Point: $202.00^{\circ} \mathrm{C}$

Vapor Pressure at Ambient Temperature: 1.66e-004 atm

Ambient Saturation Concentration: 169 ppm or $0.017 \%$

ATMOSPHERIC INFORMATION: (MANUAL INPUT OF DATA)

Wind: 2.5 meters $/ \mathrm{sec}$ from $90^{\circ}$ true

Inversion Height: 500 meters

Stability Class: C Air Temperature: $25^{\circ} \mathrm{C}$

Relative Humidity: 50\% Ground Roughness: 100 centimeters

Cloud Cover: 7 tenths

\section{SOURCE STRENGTH INFORMATION:}

Puddle Area: 40700 square feet

Average Puddle Depth: 1 centimeters

Soil Type: Default Ground Temperature: $25^{\circ} \mathrm{C}$

Initial Puddle Temperature: Ground temperature

Release Duration: ALOHA limited the duration to 1 hour

Max Computed Release Rate: 738 grams/min

Max Average Sustained Release Rate: 725 grams/min

(averaged over a minute or more)

Total Amount Released: 34.7 kilograms

FOOTPRINT INFORMATION:

Dispersion Module: Gaussian

User specified LOC: $125 \mathrm{ppm}$

Max Threat Zone for LOC: 34 meters

Note: Footprint was not drawn because effects of near-field patchiness make plume presentation unreliable for short distances.

\section{TIME DEPENDENT INFORMATION:}

Concentration Estimates at the point:

Downwind: 100 meters

Off Centerline: 0 meters

Max Concentration:

Outdoor: $0.406 \mathrm{ppm}$

Indoor: $0.406 \mathrm{ppm}$

Note: Indoor graph is shown with a dotted line. 
Title of Project EPHA for THM

Subject Concentration Analysis for THM Hazardous Chemicals

Computer T. M. Franey Date $\underline{09 / 16 / 96}$

SITE DATA INFORMATION:

$$
\text { 1-RD-15 }
$$

Location: AIKEN, SOUTH CAROLINA

Building Air Exchanges Per Hour: 60 (User specified)

Date and Time: Fixed at July 1, 19961300 hours

\section{CHEMICAL INFORMATION:}

Chemical Name: DIETHYLENE GLYCOL MONOETHYL ETHER

Molecular Weight: $134.18 \mathrm{~kg} / \mathrm{kmol}$

TLV-TWA: -unavail- IDLH: -unavail-

Footprint Level of Concern: 125 ppm

Boiling Point: $202.00^{\circ} \mathrm{C}$

Vapor Pressure at Ambient Temperature: 1.66e-004 atm

Ambient Saturation Concentration: $169 \mathrm{ppm}$ or $0.017 \%$

\section{ATMOSPHERIC INFORMATION: (MANUAL INPUT OF DATA)}

Wind: 2.5 meters $/ \mathrm{sec}$ from $90^{\circ}$ true

Inversion Height: 500 meters

Stability Class: C Air Temperature: $25^{\circ} \mathrm{C}$

Relative Humidity: 50\% Ground Roughness: 100 centimeters

Cloud Cover: 7 tenths

\section{SOURCE STRENGTH INFORMATION:}

Puddle Area: 10000 square meters

Average Puddle Depth: 1 centimeters

Soil Type: Default Ground Temperature: $25^{\circ} \mathrm{C}$

Initial Puddle Temperature: Ground temperature

Release Duration: ALOHA limited the duration to 1 hour

Max Computed Release Rate: 1.89 kilograms/min

Max Average Sustained Release Rate: 1.85 kilograms/min

(averaged over a minute or more)

Total Amount Released: 88.4 kilograms

\section{FOOTPRINT INFORMATION:}

Dispersion Module: Gaussian

User specified LOC: $125 \mathrm{ppm}$

Max Threat Zone for LOC: 56 meters

Note: Footprint was not drawn because effects of near-field patchiness make plume presentation unreliable for short distances.

TIME DEPENDENT INFORMATION:

Concentration Estimates at the point:

Downwind: 100 meters

Off Centerline: 0 meters

Max Concentration:

Outdoor: $0.702 \mathrm{ppm}$

Indoor: $0.702 \mathrm{ppm}$

Note: Indoor graph is shown with a dotted line. 
Title of Project EPHA for THM

Subject Concentration Analysis for THM Hazardous Chemicals

Computer T. M. Franey

Date $\underline{09 / 16 / 96}$
Reviewed by S. A. Henderson

Works Calculation 7

Sheet No. 367 of 577

SITE DATA INFORMATION: 1-RD-16

Location: AIKEN, SOUTH CAROLINA

Building Air Exchanges Per Hour: 60 (User specified)

Date and Time: Fixed at July 1, 19961300 hours

CHEMICAL INFORMATION:

Chemical Name: DIOXANE Molecular Weight: $88.11 \mathrm{~kg} / \mathrm{kmol}$

TLV-TWA: $25.00 \mathrm{ppm}$ IDLH: $2000.00 \mathrm{ppm}$

Note: Potential or confirmed human carcinogen.

Footprint Level of Concern: 125 ppm

Boiling Point: $101.32^{\circ} \mathrm{C}$

Vapor Pressure at Ambient Temperature: 0.050 atm

Ambient Saturation Concentration: $51,142 \mathrm{ppm}$ or $5.11 \%$

ATMOSPHERIC INFORMATION: (MANUAL INPUT OF DATA)

Wind: 2.5 meters $/ \mathrm{sec}$ from $90^{\circ}$ true

Inversion Height: 500 meters

Stability Class: C Air Temperature: $25^{\circ} \mathrm{C}$

Relative Humidity: 50\% Ground Roughness: 100 centimeters

Cloud Cover: 7 tenths

SOURCE STRENGTH INFORMATION:

Puddle Area: 1750 square feet

Average Puddle Depth: 1 centimeters

Soil Type: Default Ground Temperature: $25^{\circ} \mathrm{C}$

Initial Puddle Temperature: Ground temperature

Release Duration: ALOHA limited the duration to 1 hour

Max Computed Release Rate: 9.21 kilograms/min

Max Average Sustained Release Rate: 9.19 kilograms/min

(averaged over a minute or more)

Total Amount Released: 533 kilograms

FOOTPRINT INFORMATION:

Dispersion Module: Gaussian

User specified LOC: $125 \mathrm{ppm}$

Max Threat Zone for LOC: 30 meters

Max Threat Zone for IDLH: less than 10 meters(10.9 yards)

Note: Footprint was not drawn because

effects of near-field patchiness make plume

presentation unreliable for short distances.

TIME DEPENDENT INFORMATION:

Concentration Estimates at the point:

Downwind: 30 meters

Off Centerline: 0 meters

Max Concentration:

Outdoor: $125 \mathrm{ppm}$

Indoor: $125 \mathrm{ppm}$

Note: Indoor graph is shown with a dotted line. 
Title of Project EPHA for THM

Subject Concentration Analysis for THM Hazardous Chemicals

Computer T. M. Franey

Date $09 / 16 / 96$
Reviewed by S. A. Henderson

Works Calculation 7

Sheet No. 368 of 577

SITE DATA INFORMATION: 1-RD-16

Location: AIKEN, SOUTH CAROLINA

Building Air Exchanges Per Hour: 60 (User specified)

Date and Time: Fixed at July 1, 19961300 hours

CHEMICAL INFORMATION:

Chemical Name: DIOXANE Molecular Weight: $88.11 \mathrm{~kg} / \mathrm{kmol}$

TLV-TWA: $25.00 \mathrm{ppm}$ IDLH: $2000.00 \mathrm{ppm}$

Note: Potential or confirmed human carcinogen.

Footprint Level of Concern: 125 ppm

Boiling Point: $101.32^{\circ} \mathrm{C}$

Vapor Pressure at Ambient Temperature: $0.050 \mathrm{~atm}$

Ambient Saturation Concentration: 51,142 ppm or $5.11 \%$

ATMOSPHERIC INFORMATION: (MANUAL INPUT OF DATA)

Wind: 2.5 meters $/ \mathrm{sec}$ from $90^{\circ}$ true

Inversion Height: 500 meters

Stability Class: C Air Temperature: $25^{\circ} \mathrm{C}$

Relative Humidity: 50\% Ground Roughness: 100 centimeters

Cloud Cover: 7 tenths

SOURCE STRENGTH INFORMATION:

Puddle Area: 17150 square feet

Average Puddle Depth: 1 centimeters

Soil Type: Default Ground Temperature: $25^{\circ} \mathrm{C}$

Initial Puddle Temperature: Ground temperature

Release Duration: ALOHA limited the duration to 1 hour

Max Computed Release Rate: 83.5 kilograms/min

Max Average Sustained Release Rate: 83.2 kilograms/min (averaged over a minute or more)

Total Amount Released: 4,794 kilograms

\section{FOOTPRINT INFORMATION:}

Dispersion Module: Gaussian

User specified LOC: $125 \mathrm{ppm}$

Max Threat Zone for LOC: 87 meters

Max Threat Zone for IDLH: 22 meters

Note: Footprint was not drawn because effects of near-field patchiness make plume

presentation unreliable for short distances.

\section{TIME DEPENDENT INFORMATION:}

Concentration Estimates at the point:

Dowinwind: 100 meters

Off Centerline: 0 meters

Max Concentration:

Outdoor: $108 \mathrm{ppm}$

Indoor: $108 \mathrm{ppm}$

- Note: Indoor graph is shown with a dotted line. 


\section{ENGINEERING COMPUTATION SHEET}

Title of Project EPHA for THM

Subject Concentration Analysis for THM Hazardous Chemicals

Computer T. M. Franey

Date 09/16/96

SITE DATA INFORMATION: 1-RD-16

Location: AIKEN, SOUTH CAROLINA

Building Air Exchanges Per Hour: 60 (User specified)

Date and Time: Fixed at July 1, 19961300 hours

CHEMICAL INFORMATION:

Chemical Name: DIOXANE Molecular Weight: $88.11 \mathrm{~kg} / \mathrm{kmol}$

TLV-TWA: $25.00 \mathrm{ppm}$ IDLH: $2000.00 \mathrm{ppm}$

Note: Potential or confirmed human carcinogen.

Footprint Level of Concern: 125 ppm

Boiling Point: $101.32^{\circ} \mathrm{C}$

Vapor Pressure at Ambient Temperature: 0.050 atm

Ambient Saturation Concentration: 51,142 ppm or $5.11 \%$

ATMOSPHERIC INFORMATION: (MANUAL INPUT OF DATA)

Wind: 2.5 meters $/ \mathrm{sec}$ from $90^{\circ}$ true

Inversion Height: 500 meters

Stability Class: C Air Temperature: $25^{\circ} \mathrm{C}$

Relative Humidity: 50\% Ground Roughness: 100 centimeters

Cloud Cover: 7 tenths

SOURCE STRENGTH INFORMATION:

Puddle Area: 24420 square feet

Average Puddle Depth: 1 centimeters

Soil Type: Default Ground Temperature: $25^{\circ} \mathrm{C}$

Initial Puddle Temperature: Ground temperature

Release Duration: ALOHA limited the duration to 1 hour

Max Computed Release Rate: 117 kilograms/min

Max Average Sustained Release Rate: 117 kilograms/min

(averaged over a minute or more)

Total Amount Released: 6,740 kilograms

\section{FOOTPRINT INFORMATION:}

Model Run: Heavy Gas

User specified LOC: 125 ppm

Max Threat Zone for LOC: 257 meters

Max Threat Zone for IDLH: 36 meters

Note: The Heavy Gas footprint is an initial screening.

For short releases it may be an overestimation.

Be sure to check concentration information at specific locations.

TIME DEPENDENT INFORMATION:

Concentration Estimates at the point:

Downwind: 100 meters

Off Centerline: 0 meters

Max Concentration:

Outdoor: $794 \mathrm{ppm}$

Indoor: $794 \mathrm{ppm}$

Note: Indoor graph is shown with a dotted line.
Reviewed by S. A. Henderson

Works Calculation 7

Sheet No. 369 of 577 
Title of Project EPHA for THM

Subject Concentration Analysis for THM Hazardous Chemicals

Computer T. M. Franey

Date $09 / 16 / 96$

SITE DATA INFORMATION: 1-RD-16

Location: AIKEN, SOUTH CAROLINA

Building Air Exchanges Per Hour: 60 (User specified)

Date and Time: Fixed at July 1, 19961300 hours

CHEMICAL INFORMATION:

Chemical Name: DIOXANE Molecular Weight: $88.11 \mathrm{~kg} / \mathrm{kmol}$

TLV-TWA: $25.00 \mathrm{ppm}$ IDLH: $2000.00 \mathrm{ppm}$

Note: Potential or confirmed human carcinogen.

Footprint Level of Concern: $125 \mathrm{ppm}$

Boiling Point: $101.32^{\circ} \mathrm{C}$

Vapor Pressure at Ambient Temperature: $0.050 \mathrm{~atm}$

Ambient Saturation Concentration: 51,142 ppm or $5.11 \%$

ATMOSPHERIC INFORMATION: (MANUAL INPUT OF DATA)

Wind: 2.5 meters $/ \mathrm{sec}$ from $90^{\circ}$ true

Inversion Height: 500 meters

Stability Class: C Air Temperature: $25^{\circ} \mathrm{C}$

Relative Humidity: $50 \%$ Ground Roughness: 100 centimeters

Cloud Cover: 7 tenths

\section{SOURCE STRENGTH INFORMATION:}

Puddle Area: 40700 square feet

Average Puddle Depth: 1 centimeters

Soil Type: Default Ground Temperature: $25^{\circ} \mathrm{C}$

Initial Puddle Temperature: Ground temperature

Release Duration: ALOHA limited the duration to 1 hour

Max Computed Release Rate: 193 kilograms/min

Max Average Sustained Release Rate: 192 kilograms/min (averaged over a minute or more)

Total Amount Released: 11,033 kilograms

\section{FOOTPRINT INFORMATION:}

Model Run: Heavy Gas

User specified LOC: $125 \mathrm{ppm}$

Max Threat Zone for LOC: 312 meters

Max Threat Zone for IDLH: 45 meters

Note: The Heavy Gas footprint is an initial screening.

For short releases it may be an overestimation.

Be sure to check concentration information at specific locations.

\section{TIME DEPENDENT INFORMATION:}

Concentration Estimates at the point:

Downwind: 100 meters

Off Centerline: 0 meters

Max Concentration:

Outdoor: $1,060 \mathrm{ppm}$

Indoor: $1,060 \mathrm{ppm}$

Note: Indoor graph is shown with a dotted line.
Reviewed by $\underline{\text { S. A. Henderson }}$

Works Calculation 7

Sheet No. 370 of 577 
ENGINEERING COMPUTATION SHEET

Title of Project EPHA for THM

Subject Concentration Analysis for THM Hazardous Chemicals

Computer T. M. Franey

Date 09/16/96
Reviewed by S. A. Henderson

Works Calculation 7

Sheet No. $\underline{371 \text { of } 577}$

SITE DATA INFORMATION: $\quad$ 1-RD-16

Location: AIKEN, SOUTH CAROLINA

Building Air Exchanges Per Hour: 60 (User specified)

Date and Time: Fixed at July 1, 19961300 hours

CHEMICAL INFORMATION:

Chemical Name: DIOXANE Molecular Weight: $88.11 \mathrm{~kg} / \mathrm{kmol}$

TLV-TWA: $25.00 \mathrm{ppm}$ IDLH: $2000.00 \mathrm{ppm}$

Note: Potential or confirmed human carcinogen.

Footprint Level of Concern: 125 ppm

Boiling Point: $101.32^{\circ} \mathrm{C}$

Vapor Pressure at Ambient Temperature: 0.050 atm

Ambient Saturation Concentration: 51,142 ppm or $5.11 \%$

ATMOSPHERIC INFORMATION: (MANUAL INPUT OF DATA)

Wind: 2.5 meters $/ \mathrm{sec}$ from $90^{\circ}$ true

Inversion Height: 500 meters

Stability Class: C Air Temperature: $25^{\circ} \mathrm{C}$

Relative Humidity: 50\% Ground Roughness: 100 centimeters

Cloud Cover: 7 tenths

SOURCE STRENGTH INFORMATION:

Puddle Area: 10000 square meters

Average Puddle Depth: 1 centimeters

Soil Type: Default Ground Temperature: $25^{\circ} \mathrm{C}$

Initial Puddle Temperature: Ground temperature

Release Duration: ALOHA limited the duration to 1 hour

Max Computed Release Rate: 494 kilograms/min

Max Average Sustained Release Rate: 492 kilograms/min

(averaged over a minute or more)

Total Amount Released: 28,217 kilograms

FOOTPRINT INFORMATTON:

Model Run: Heavy Gas

User specified LOC: 125 ppm

Max Threat Zone for LOC: 435 meters

Max Threat Zone for IDLH: 67 meters

Note: The Heavy Gas footprint is an initial screening.

For short releases it may be an overestimation.

Be sure to check concentration information at specific locations.

TIME DEPENDENT INFORMATION:

Concentration Estimates at the point:

Downwind: 100 meters

Off Centerline: 0 meters

Max Concentration:

Outdoor: $1,680 \mathrm{ppm}$

Indoor: $1,680 \mathrm{ppm}$

Note: Indoor graph is shown with a dotted line. 


\section{ENGINEERING COMPUTATION SHEET}

Title of Project EPHA for THM

Reviewed by $\mathrm{S}$. A. Henderson

Subject Concentration Analysis for THM Hazardous Chemicals

Computer T. M. Franey

Date $\underline{09 / 16 / 96}$
Works Calculation 7

Sheet No. $\underline{372 \text { of } 577}$

\section{1-RD-17}

SITE DATA INFORMATION:

Location: AIKEN, SOUTH CAROLINA

Building Air Exchanges Per Hour: 60 (User specified)

Date and Time: Fixed at July 1, 19961300 hours

\section{CHEMICAL INFORMATION:}

Chemical Name: METHYLANILINE (DIPHEYNLMETHANE DISOCYANATE)

Molecular Weight: $250.3 \mathrm{~kg} / \mathrm{kmol}$

TLV-TWA: $0.50 \mathrm{ppm}$ IDLH: $100.00 \mathrm{ppm}$

Footprint Level of Concern: .5 ppm

Boiling Point: $195.87^{\circ} \mathrm{C}$

Vapor Pressure at Ambient Temperature: 5.96e-004 atm

Ambient Saturation Concentration: 607 ppm or $0.061 \%$

\section{ATMOSPHERIC INFORMATION: (MANUAL INPUT OF DATA)}

Wind: 2.5 meters $/ \mathrm{sec}$ from $90^{\circ}$ true

Inversion Height: 500 meters

Stability Class: C Air Temperature: $25^{\circ} \mathrm{C}$

Relative Humidity: 50\% Ground Roughness: Urban or forest

Cloud Cover: 7 tenths

\section{SOURCE STRENGTH INFORMATION:}

Puddle Area: 300 square feet

Average Puddle Depth: 1 centimeters

Soil Type: Default Ground Temperature: $25^{\circ} \mathrm{C}$

Initial Puddle Temperature: Ground temperature

Release Duration: ALOHA limited the duration to 1 hour

Max Computed Release Rate: $24.9 \mathrm{grams} / \mathrm{min}$

Max Average Sustained Release Rate: 24.6 grams/min

(averaged over a minute or more)

Total Amount Released: 1.24 kilograms

\section{TIME DEPENDENT INFORMATION:}

Concentration Estimates at the point:

Downwind: 30 meters

Off Centerline: 0 meters

Max Concentration:

Outdoor: $0.499 \mathrm{ppm}$ 
Title of Project EPHA for THM

Subject Concentration Analysis for THM Hazardous Chemicals

Computer T. M. Franey

Date 09/16/96
Reviewed by $\mathrm{S}$. A. Henderson

Works Calculation 7

Sheet No. $\underline{373 \text { of } 577}$

1-RD-17

SITE DATA INFORMATION:

Location: AIKEN, SOUTH CAROLINA

Building Air Exchanges Per Hour: 60 (User specified)

Date and Time: Fixed at July 1, 19961300 hours

CHEMICAL INFORMATION:

Chemical Name: METHYLANILINE (DIPHEYNLMETHANE DIISOCYANATE)

Molecular Weight: $250.3 \mathrm{~kg} / \mathrm{kmol}$

TLV-TWA: $0.50 \mathrm{ppm}$ IDLH: $100.00 \mathrm{ppm}$

Footprint Level of Concern: $.5 \mathrm{ppm}$

Boiling Point: $195.87^{\circ} \mathrm{C}$

Vapor Pressure at Ambient Temperature: 5.96e-004 atm

Ambient Saturation Concentration: 607 ppm or $0.061 \%$

ATMOSPHERIC INFORMATION: (MANUAL INPUT OF DATA)

Wind: 2.5 .meters $/ \mathrm{sec}$ from $90^{\circ}$ true

Inversion Height: 500 meters

Stability Class: C Air Temperature: $25^{\circ} \mathrm{C}$

Relative Humidity: 50\% Ground Roughness: Urban or forest

Cloud Cover: 7 tenths

\section{SOURCE STRENGTH INFORMATION:}

Puddle Area: 3800 square feet

Average Puddle Depth: 1 centimeters

Soil Type: Default Ground Temperature: $25^{\circ} \mathrm{C}$

Initial Puddle Temperature: Ground temperature

Release Duration: ALOHA limited the duration to 1 hour

Max Computed Release Rate: 283 grams/min

Max Average Sustained Release Rate: 279 grams/min

(averaged over a minute or more)

Total Amount Released: 14.0 kilograms

TIME DEPENDENT INFORMATION:

Concentration Estimates at the point:

Downwind: 100 meters

. Off Centerline: 0 meters

Max Concentration:

Outdoor: $0.498 \mathrm{ppm}$ 
Title of Project EPHA for THM

Subject Concentration Analysis for THM Hazardous Chemicals

Computer T. M. Franey

Date $\underline{09 / 16 / 96}$

Reviewed by $\underline{\text { S. A. Henderson }}$

Works Calculation 7

Sheet No. 374 of 577

1-RD-17

SITE DATA INFORMATION:

Location: AIKEN, SOUTH CAROLINA

Building Air Exchanges Per Hour: 60 (User specified)

Date and Time: Fixed at July 1, 19961300 hours

CHEMICAL INFORMATION:

Chemical Name: METHYLANILINE (DIPHEYNLMETHANE DIISOCYANATE)

Molecular Weight: $250.3 \mathrm{~kg} / \mathrm{kmol}$

TLV-TWA: $0.50 \mathrm{ppm}$ IDLH: $100.00 \mathrm{ppm}$

Footprint Level of Concern: $.5 \mathrm{ppm}$

Boiling Point: $195.87^{\circ} \mathrm{C}$

Vapor Pressure at Ambient Temperature: 5.96e-004 atm

Ambient Saturation Concentration: $607 \mathrm{ppm}$ or $0.061 \%$

ATMOSPHERIC INFORMATION: (MANUAL INPUT OF DATA)

Wind: 2.5 meters $/ \mathrm{sec}$ from $90^{\circ}$ true

Inversion Height: 500 meters

Stability Class: C Air Temperature: $25^{\circ} \mathrm{C}$

Relative Humidity: $50 \%$ Ground Roughness: Urban or forest

Cloud Cover: 7 tenths

SOURCE STRENGTH INFORMATION:

Puddle Area: 24420 square feet

Average Puddle Depth: 1 centimeters

Soil Type: Default Ground Temperature: $25^{\circ} \mathrm{C}$

Initial Puddle Temperature: Ground temperature

Release Duration: ALOHA limited the duration to 1 hour

Max Computed Release Rate: 1.69 kilograms/min

Max Average Sustained Release Rate: $1.67 \mathrm{kilograms} / \mathrm{min}$

(averaged over a minute or more)

Total Amount Released: 83.1 kilograms

FOOTPRINT INFORMATION:

Dispersion Module: Gaussian

User specified LOC: $.5 \mathrm{ppm}$

Max Threat Zone for LOC: 242 meters

Max Threat Zone for IDLH: 27 meters

TIME DEPENDENT INFORMATION:

Concentration Estimates at the point:

Downwind: 100 meters

Off, Centerline: 0 meters

Max Concentration:

Outdoor: $1.5 \mathrm{ppm}$ 
Title of Project EPHA for THM

Subject Concentration Analysis for THM Hazardous Chemicals

Computer T. M. Franey
Reviewed by S. A. Henderson

Works Calculation 7

Sheet No. $\underline{375 \text { of } 577}$

1-RD-17

SITE DATA INFORMATION:

Location: AIKEN, SOUTH CAROLINA

Building Air Exchanges Per Hour: 60 (User specified)

Date and Time: Fixed at July 1, 19961300 hours

\section{CHEMICAL INFORMATION:}

Chemical Name: METHYLANILINE (DIPHEYNLMETHANE DIISOCYANATE)

Molecular Weight: $250.3 \mathrm{~kg} / \mathrm{kmol}$

TLV-TWA: $0.50 \mathrm{ppm}$ IDLH: $100.00 \mathrm{ppm}$

Footprint Level of Concern: $.5 \mathrm{ppm}$

Boiling Point: $195.87^{\circ} \mathrm{C}$

Vapor Pressure at Ambient Temperature: 5.96e-004 atm

Ambient Saturation Concentration: 607 ppm or $0.061 \%$

ATMOSPHERIC INFORMATION: (MANUAL INPUT OF DATA)

Wind: 2.5 meters $/ \mathrm{sec}$ from $90^{\circ}$ true

Inversion Height: 500 meters

Stability Class: C Air Temperature: $25^{\circ} \mathrm{C}$

Relative Humidity: 50\% Ground Roughness: Urban or forest

Cloud Cover: 7 tenths

SOURCE STRENGTH INFORMATION:

Puddle Area: 40700 square feet

Average Puddle Depth: 1 centimeters

Soil Type: Default Ground Temperature: $25^{\circ} \mathrm{C}$

Initial Puddle Temperature: Ground temperature

Release Duration: ALOHA limited the duration to 1 hour

Max Computed Release Rate: 2.76 kilograms/min

Max Average Sustained Release Rate: 2.72 kilograms/min

(averaged over a minute or more)

Total Amount Released: 136 kilograms

FOOTPRINT INFORMATION:

Dispersion Module: Gaussian

User specified LOC: $.5 \mathrm{ppm}$

Max Threat Zone for LOC: 309 meters

Max Threat Zone for IDLH: 34 meters

TIME DEPENDENT INFORMATION:

Concentration Estimates at the point:

Downwind: 100 meters

Off Centerline: 0 meters

Max Concentration:

Outdoor: $1.91 \mathrm{ppm}$ 


\section{ENGINEERING COMPUTATION SHEET}

Title of Project EPHA for THM

Reviewed by S.A. Henderson

Subject Concentration-Analysis for THM Hazardous Chemicals

Computer T. M. Franey

Date 09/16/96
Works Calculation 7

Sheet No. $\underline{376 \text { of } 577}$

1-RD-17

SITE DATA INFORMATION:

Location: AIKEN, SOUTH CAROLINA

Building Air Exchanges Per Hour: 60 (User specified)

Date and Time: Fixed at July 1, 19961300 hours

\section{CHEMICAL INFORMATION:}

Chemical Name: METHYLANILINE (DIPHEYNLMETHANE DIISOCYANATE)

Molecular Weight: $250.3 \mathrm{~kg} / \mathrm{kmol}$

TLV-TWA: $0.50 \mathrm{ppm}$ IDLH: $100.00 \mathrm{ppm}$

Footprint Level of Concern: $.5 \mathrm{ppm}$

Boiling Point: $195.87^{\circ} \mathrm{C}$

Vapor Pressure at Ambient Temperature: 5.96e-004 atm

Ambient Saturation Concentration: 607 ppm or $0.061 \%$

ATMOSPHERIC INFORMATION: (MANUAL INPUT OF DATA)

Wind: 2.5 meters $/ \mathrm{sec}$ from $90^{\circ}$ true

Inversion Height: 500 meters

Stability Class: C Air Temperature: $25^{\circ} \mathrm{C}$

Relative Humidity: $50 \%$ Ground Roughness: Urban or forest.

Cloud Cover: 7 tenths

SOURCE STRENGTH INFORMATION:

Puddle Area: 10000 square meters

Average Puddle Depth: 1 centimeters

Soil Type: Default Ground Temperature: $25^{\circ} \mathrm{C}$

Initial Puddle Temperature: Ground temperature

Release Duration: ALOHA limited the duration to 1 hour

Max Computed Release Rate: 7.05 kilograms/min

Max Average Sustained Release Rate: 6.95 kilograms/min (averaged over a minute or more)

Total Amount Released: 346 kilograms

FOOTPRINT INFORMATION:

Dispersion Module: Gaussian

User specified LOC: $.5 \mathrm{ppm}$

Max Threat Zone for LOC: 492 meters

Max Threat Zone for IDLH: 56 meters

TIME DEPENDENT INFORMATION:

Concentration Estimates at the point:

Downwind: 100 meters

Off Centerline: 0 meters

Max Concentration:

Outdoor: $3.3 \mathrm{ppm}$ 


\section{ENGINEERING COMPUTATION SHEET}

Title of Project EPHA for THM

Reviewed by $\underline{\text { S. A. Henderson }}$

Subject Concentration Analysis for THM Hazardous Chemicals.

Computer T. M. Franey

Date 09/16/96

SITE DATA INFORMATION: 1-RD-18

Location: AIKEN, SOUTH CAROLINA

Building Air Exchanges Per Hour: 60 (User specified)

Date and Time: Fixed at July 1, 19961300 hours

CHEMICAL INFORMATION:

Chemical Name: ETHYL ACETATE

Molecular Weight: $88.11 \mathrm{~kg} / \mathrm{kmol}$

TLV-TWA: $400.00 \mathrm{ppm}$ IDLH: $10000.00 \mathrm{ppm}$

Footprint Level of Concern: $2000 \mathrm{ppm}$

Boiling Point: $77.06^{\circ} \mathrm{C}$

Vapor Pressure at Ambient Temperature: 0.12 atm

Ambient Saturation Concentration: 125,394 ppm or $12.5 \%$

ATMOSPHERIC INFORMATION: (MANUAL INPUT OF DATA)

Wind: 2.5 meters $/ \mathrm{sec}$ from $90^{\circ}$ true

Inversion Height: 500 meters

Stability Class: C Air Temperature: $25^{\circ} \mathrm{C}$

Relative Humidity: 50\% Ground Roughness: 100 centimeters

Cloud Cover: 7 tenths

\section{SOURCE STRENGTH INFORMATION:}

Puddle Area: 3140 square feet

Average Puddle Depth: 1 centimeters

Soil Type: Default Ground Temperature: $25^{\circ} \mathrm{C}$

Initial Puddle Temperature: Ground temperature

Release Duration: ALOHA limited the duration to.1 hour

Max Computed Release Rate: 39.7 kilograms/min

Max Average Sustained Release Rate: 37 kilograms/min

(averaged over a minute or more)

Total Amount Released: 2,098 kilograms

FOOTPRINT INFORMATION:

Model Run: Heavy Gas

User specified LOC: $2000 \mathrm{ppm}$

Max Threat Zone for LOC: 30 meters

Max Threat Zone for IDLH: less than 10 meters(10.9 yards)

Note: Footprint wasn't drawn because effects of near-field patchiness make plume presentation unreliable for short distances.

\section{TIME DEPENDENT INFORMATION:}

Concentration Estimates at the point:

Downwind: 30 meters

Off Centerline: 0 meters

Max Concentration:

Outdoor: $2,000 \mathrm{ppm}$

Indoor: $2,000 \mathrm{ppm}$

Note: Indoor graph is shown with a dotted line.
Works Calculation 7

Sheet No. $\underline{377 \text { of } 577}$ 
Title of Project EPHA for THM

Subject Concentration Analysis for THM Hazardous Chemicals

Computer T. M. Franey
Reviewed by S. A. Henderson

Works Calculation 7

Sheet No. $\underline{378 \text { of } 577}$

SITE DATA INFORMATION: 1-RD-18

Location: AIKEN, SOUTH CAROLINA

Building Air Exchanges Per Hour: 60 (User specified)

Date and Time: Fixed at July 1, 19961300 hours

\section{CHEMICAL INFORMATION:}

Chemical Name: ETHYL ACETATE

Molecular Weight: $88.11 \mathrm{~kg} / \mathrm{kmol}$

TLV-TWA: $400.00 \mathrm{ppm}$ IDLH: $10000.00 \mathrm{ppm}$

Footprint Level of Concern: $2000 \mathrm{ppm}$

Boiling Point: $77.06^{\circ} \mathrm{C}$

Vapor Pressure at Ambient Temperature: 0.12 atm

Ambient Saturation Concentration: 125,394 ppm or $12.5 \%$

ATMOSPHERIC INFORMATION: (MANUAL INPUT OF DATA)

Wind: 2.5 meters $/ \mathrm{sec}$ from $90^{\circ}$ true

Inversion Height: 500 meters

Stability Class: C Air Temperature: $25^{\circ} \mathrm{C}$

Relative Humidity: 50\% Ground Roughness: 100 centimeters

Cloud Cover: 7 tenths

\section{SOURCE STRENGTH INFORMATION:}

Puddle Area: 35500 square feet

Average Puddle Depth: 1 centimeters

Soil Type: Default Ground Temperature: $25^{\circ} \mathrm{C}$

Initial Puddle Temperature: Ground temperature

Release Duration: ALOHA limited the duration to 1 hour

Max Computed Release Rate: 401 kilograms/min

Max Average Sustained Release Rate: 381 kilograms/min

(averaged over a minute or more)

Total Amount Released: 21,939 kilograms

\section{FOOTPRINT INFORMATION:}

Model Run: Heavy Gas

User specified LOC: 2000 ppm

Max Threat Zone for LOC: 100 meters

Max Threat Zone for IDLH: 26 meters

Note: Footprint wasn't drawn because effects of near-field patchiness make plume presentation unreliable for short distances.

\section{TIME DEPENDENT INFORMATION:}

Concentration Estimates at the point:

Downwind: 100 meters

Off Centerline: 0 meters

Max Concentration:

Outdoor: $2,000 \mathrm{ppm}$

Indoor: $2,000 \mathrm{ppm}$

Note: Indoor graph is shown with a dotted line. 


\section{ENGINEERING COMPUTATION SHEET}

Title of Project EPHA for THM

Subject Concentration Analysis for THM Hazardous Chemicals

Computer T.M. Franey

Date 09/16/96
Reviewed by S. A. Henderson

Works Calculation 7

Sheet No. $\underline{379 \text { of } 577}$

SITE DATA INFORMATION: I-RD-18

Location: AIKEN, SOUTH CAROLINA

Building Air Exchanges Per Hour: 60 (User specified)

Date and Time: Fixed at July 1, 19961300 hours

CHEMICAL INFORMATION:

Chemical Name: ETHYL ACETATE

Molecular Weight: $88.11 \mathrm{~kg} / \mathrm{kmol}$

TLV-TWA: $400.00 \mathrm{ppm}$ IDLH: $10000.00 \mathrm{ppm}$

Footprint Level of Concern: 2000 ppm

Boiling Point: $77.06^{\circ} \mathrm{C}$

Vapor Pressure at Ambient Temperature: $0.12 \mathrm{~atm}$

Ambient Saturation Concentration: $125,394 \mathrm{ppm}$ or $12.5 \%$

ATMOSPHERIC INFORMATION: (MANUAL INPUT OF DATA)

Wind: 2.5 meters $/ \mathrm{sec}$ from $90^{\circ}$ true

Inversion Height: 500 meters

Stability Class: C Air Temperature: $25^{\circ} \mathrm{C}$

Relative Humidity: 50\% Ground Roughness: 100 centimeters

Cloud Cover: 7 tenths

SOURCE STRENGTH INFORMATION:

Puddle Area: 24420 square feet

Average Puddle Depth: 1 centimeters

Soil Type: Default Ground Temperature: $25^{\circ} \mathrm{C}$

Initial Puddle Temperature: Ground temperature

Release Duration: ALOHA limited the duration to 1 hour

Max Computed Release Rate: 281 kilograms/min

Max Average Sustained Release Rate: 266 kilograms/min

(averaged over a minute or more)

Total Amount Released: 15,268 kilograms

FOOTPRINT INFORMATION:

Model Run: Heavy Gas

User specified LOC: $2000 \mathrm{ppm}$

Max Threat Zone for LOC: 82 meters

Max Threat Zone for IDLH: 17 meters

Note: Footprint wasn't drawn because effects of near-field patchiness make plume presentation unreliable for short distances.

\section{TIME DEPENDENT INFORMATION:}

Concentration Estimates at the point:

Downwind: 100 meters

Off Centerline: 0 meters

Max Concentration:

Outdoor: $1,510 \mathrm{ppm}$

Indoor: $1,510 \mathrm{ppm}$

Note: Indoor graph is shown with a dotted line. 
Title of Project EPHA for THM

Subject Concentration Analysis for THM Hazardous Chemicals

Computer T. M. Franey

Date $\underline{09 / 16 / 96}$
Reviewed by $\mathrm{S}$. A. Henderson

Works Calculation 7

Sheet No. $\underline{380 \text { of } 577}$

SITE DATA INFORMATION: 1-RD-18

Location: AIKEN, SOUTH CAROLINA

Building Air Exchanges Per Hour: 60 (User specified)

Date and Time: Fixed at July 1, 19961300 hours

CHEMICAL INFORMATION:

Chemical Name: ETHYL ACETATE

Molecular Weight: $88.11 \mathrm{~kg} / \mathrm{kmol}$

TLV-TWA: $400.00 \mathrm{ppm}$ IDLH: $10000.00 \mathrm{ppm}$

Footprint Level of Concern: $2000 \mathrm{ppm}$

Boiling Point: $77.06^{\circ} \mathrm{C}$

Vapor Pressure at Ambient Temperature: 0.12 atm

Ambient Saturation Concentration: 125,394 ppm or $12.5 \%$

ATMOSPHERIC INFORMATION: (MANUAL INPUT OF DATA)

Wind: 2.5 meters $/ \mathrm{sec}$ from $90^{\circ}$ true

Inversion Height: 500 meters

Stability Class: C Air Temperature: $25^{\circ} \mathrm{C}$

Relative Humidity: $50 \%$ Ground Roughness: 100 centimeters

Cloud Cover: 7 tenths

\section{SOURCE STRENGTH INFORMATION:}

Puddle Area: 40700 square feet

Average Puddle Depth: 1 centimeters

Soil Type: Default Ground Temperature: $25^{\circ} \mathrm{C}$

Initial Puddle Temperature: Ground temperature

Release Duration: ALOHA limited the duration to 1 hour

Max Computed Release Rate: 457 kilograms/min

Max Average Sustained Release Rate: 435 kilograms/min

(averaged over a minute or more)

Total Amount Released: 25,047 kilograms

FOOTPRINT INFORMATION:

Model Run: Heavy Gas

User specified LOC: $2000 \mathrm{ppm}$

Max Threat Zone for LOC: 107 meters

Max Threat Zone for IDLH: 28 meters

Note: The Heavy Gas footprint is an initial screening.

For short releases it may be an overestimation.

Be sure to check concentration information at specific locations.

\section{TIME DEPENDENT INFORMATION:}

Concentration Estimates at the point:

Downwind: 100 meters

Off Centerline: 0 meters

Max Concentration:

Outdoor: $2,210 \mathrm{ppm}$

Indoor: $2,210 \mathrm{ppm}$

Note: Indoor graph is shown with a dotted line. 
Title of Project EPHA for THM

Subject Concentration Analysis for THM Hazardous Chemicals

Computer T. M. Franey Date $09 / 16 / 96$

SITE DATA INFORMATION: 1-RD-18

Location: AIKEN, SOUTH CAROLINA

Building Air Exchanges Per Hour: 60 (User specified)

Date and Time: Fixed at July 1, 19961300 hours

CHEMICAL INFORMATION:

Chemical Name: ETHYL ACETATE

Molecular Weight: $88.11 \mathrm{~kg} / \mathrm{kmol}$

TLV-TWA: $400.00 \mathrm{ppm}$ IDLH: $10000.00 \mathrm{ppm}$

Footprint Level of Concern: $2000 \mathrm{ppm}$

Boiling Point: $77.06^{\circ} \mathrm{C}$

Vapor Pressure at Ambient Temperature: 0.12 atm

Ambient Saturation Concentration: $125,394 \mathrm{ppm}$ or $12.5 \%$

ATMOSPHERIC INFORMATION: (MANUAL INPUT OF DATA)

Wind: 2.5 meters $/ \mathrm{sec}$ from $90^{\circ}$ true

Inversion Height: 500 meters

Stability Class: C Air Temperature: $25^{\circ} \mathrm{C}$

Relative Humidity: $50 \%$ Ground Roughness: 100 centimeters

Cloud Cover: 7 tenths

\section{SOURCE STRENGTH INFORMATION:}

Puddle Area: 10000 square meters

Average Puddle Depth: 1 centimeters

Soil Type: Default Ground Temperature: $25^{\circ} \mathrm{C}$

Initial Puddle Temperature: Ground temperature

Release Duration: ALOHA limited the duration to 1 hour

Max Computed Release Rate: 1,160 kilograms/min

Max Average.Sustained Release Rate: 1,110 kilograms/min

(averaged over a minute or more)

Total Amount Released: 64,327 kilograms

\section{FOOTPRINT INFORMATION:}

Model Run: Heavy Gas

User specified LOC: $2000 \mathrm{ppm}$

Max Threat Zone for LOC: 180 meters

Max Threat Zone for IDLH: 47 meters

Note: The Heavy Gas footprint is an initial screening.

For short releases it may be an overestimation.

Be sure to check concentration information at specific locations.

\section{TIME DEPENDENT INFORMATION:}

\section{Concentration Estimates at the point:}

Downwind: 100 meters

Off Centerline: 0 meters

Max Concentration:

Outdoor: 4,490 ppm

Indoor: $4,490 \mathrm{ppm}$

Note: Indoor graph is shown with a dotted line.
Reviewed by S. A. Henderson

Works Calculation 7

Sheet No. $\underline{381 \text { of } 577}$ 
Title of Project EPHA for THM

Subject Concentration Analysis for THM Hazardous Chemicals

Computer T. M. Franey Date 09/16/96

SITE DATA INFORMATION: 1-RD-19

Location: AIKEN, SOUTH CAROLINA

Building Air Exchanges Per Hour: 60 (User specified)

Date and Time: Fixed at July 1, 19961300 hours

CHEMICAL NNFORMATION:

Chemical Name: ETHYL BENZENE

Molecular Weight: $106.17 \mathrm{~kg} / \mathrm{kmol}$

TLV-TWA: $100.00 \mathrm{ppm}$ IDLH: $2000.00 \mathrm{ppm}$

Footprint Level of Concern: 160 ppm

Boiling Point: $136.20^{\circ} \mathrm{C}$

Vapor Pressure at Ambient Temperature: $0.013 \mathrm{~atm}$

Ambient Saturation Concentration: 12,900 ppm or $1.29 \%$

ATMOSPHERIC INFORMATION: (MANUAL INPUT OF DATA)

Wind: 2.5 meters $/ \mathrm{sec}$ from $90^{\circ}$ true

Inversion Height: 500 meters

Stability Class: C Air Temperature: $25^{\circ} \mathrm{C}$

Relative Humidity: $50 \%$ Ground Roughness: 100 centimeters

Cloud Cover: 7 tenths

\section{SOURCE STRENGTH INFORMATION:}

Puddle Area: 16700 square feet

Average Puddle Depth: 1 centimeters

Soil Type: Default Ground Temperature: $25^{\circ} \mathrm{C}$

Initial Puddle Temperature: Ground temperature

Release Duration: ALOHA limited the duration to 1 hour

Max Computed Release Rate: 32.3 kilograms/min

Max Average Sustained Release Rate: 32.1 kilograms/min (averaged over a minute or more)

Total Amount Released: 1,743 kilograms

\section{FOOTPRINT INFORMATION:}

Dispersion Module: Gaussian

User specified LOC: 160 ppm

Max Threat Zone for LOC: 30 meters

Max Threat Zone for DLH: 22 meters

Note: Footprint was not drawn because effects of near-field patchiness make plume presentation unreliable for short distances.

\section{TIME DEPENDENT INFORMATION:}

Concentration Estimates at the point:

Downwind: 30 meters

Off Centerline: 0 meters

Max Concentration:

Outdoor: $160 \mathrm{ppm}$

Indoor: $160 \mathrm{ppm}$

Note: Indoor graph is shown with a dotted line.
Reviewed by $\mathrm{S}$. A. Henderson

Works Calculation 7

Sheet No. 382 of 577 


\section{ENGINEERING COMPUTATION SHEET}

Title of Project EPHA for THM

Subject Concentration Analysis for THM Hazardous Chemicals

Computer T. M. Franey

Date 09/16/96

SITE DATA INFORMATION: $\quad$ 1-RD-19

Location: AIKEN, SOUTH CAROLINA

Building Air Exchanges Per Hour: 60 (User specified)

Date and Time: Fixed at July 1, 19961300 hours

\section{CHEMICAL INFORMATION:}

Chemical Name: ETHYL BENZENE

Molecular Weight: $106.17 \mathrm{~kg} / \mathrm{kmol}$

TLV-TWA: $100.00 \mathrm{ppm}$ IDLH: $2000.00 \mathrm{ppm}$

Footprint Level of Concern: 160 ppm

Boiling Point: $136.20^{\circ} \mathrm{C}$

Vapor Pressure at Ambient Temperature: $0.013 \mathrm{~atm}$

Ambient Saturation Concentration: 12,900 ppm or $1.29 \%$

ATMOSPHERIC INFORMATION: (MANUAL INPUT OF DATA)

Wind: 2.5 meters $/ \mathrm{sec}$ from $90^{\circ}$ true

Inversion Height: 500 meters

Stability Class: C Air Temperature: $25^{\circ} \mathrm{C}$

Relative Humidity: $50 \%$ Ground Roughness: 100 centimeters

Cloud Cover: 7 tenths

SOURCE STRENGTH INFORMATION:

Puddle Area: 99999 square feet

Average Puddle Depth: 1 centimeters

Soil Type: Default Ground Temperature: $25^{\circ} \mathrm{C}$

Initial Puddle Temperature: Ground temperature

Release Duration: ALOHA limited the duration to 1 hour

Max Computed Release Rate: 182 kilograms/min

Max Average Sustained Release Rate: 180 kilograms/min

(averaged over a minute or more)

Total Amount Released: 9,753 kilograms

FOOTPRINT INFORMATION:

Dispersion Module: Gaussian

User specified LOC: $160 \mathrm{ppm}$

Max Threat Zone for LOC: 71 meters

- Max Threat Zone for IDLH: 55 meters

Note: Footprint was not drawn because

effects of near-field patchiness make plume

presentation unreliable for short distances.

TIME DEPENDENT INFORMATION:

Concentration Estimates at the point:

Downwind: 100 meters

Off Centerline: 0 meters

Max Concentration:

Outdoor: $87.9 \mathrm{ppm}$

Indoor: $87.9 \mathrm{ppm}$

Note: Indoor graph is shown with a dotted line.
Reviewed by $\underline{\text { S. A. Henderson }}$

Works Calculation 7

Sheet No. $\underline{383 \text { of } 577}$ 
Title of Project EPHA for THM

Subject Concentration Analysis for THM Hazardous Chemicals

Computer T.M. Franey
Reviewed by $\underline{S}$. A. Henderson

Works Calculation 7

Sheet No. $\underline{384 \text { of } 577}$
SITE DATA INFORMATION: $\quad$ 1-RD-19
Location: AIKEN, SOUTH CAROLINA
Building Air Exchanges Per Hour: 60 (User specified)
Date and Time: Fixed at July 1, 19961300 hours

CHEMICAL INFORMATION:

Chemical Name: ETHYL BENZENE

Molecular Weight: $106.17 \mathrm{~kg} / \mathrm{kmol}$

TLV-TWA: $100.00 \mathrm{ppm}$ IDLH: $2000.00 \mathrm{ppm}$

Footprint Level of Concern: 160 ppm

Boiling Point: $136.20^{\circ} \mathrm{C}$

Vapor Pressure at Ambient Temperature: $0.013 \mathrm{~atm}$

Ambient Saturation Concentration: 12,900 ppm or $1.29 \%$

ATMOSPHERIC INFORMATION: (MANUAL INPUT OF DATA)

Wind: 2.5 meters $/ \mathrm{sec}$ from $90^{\circ}$ true

Inversion Height: 500 meters

Stability Class: C Air Temperature: $25^{\circ} \mathrm{C}$

Relative Humidity: $50 \%$ Ground Roughness: 100 centimeters

Cloud Cover: 7 tenths

SOURCE STRENGTH INFORMATION:

Puddle Area: 24420 square feet

Average Puddle Depth: 1 centimeters

Soil Type: Default Ground Temperature: $25^{\circ} \mathrm{C}$

Initial Puddle Temperature: Ground temperature

Release Duration: ALOHA limited the duration to 1 hour

Max Computed Release Rate: 46.6 kilograms/min

Max Average Sustained Release Rate: 46.3 kilograms $/ \mathrm{min}$ (averaged over a minute or more)

Total Amount Released: 2,511 kilograms

\section{FOOTPRINT INFORMATION:}

Dispersion Module: Gaussian

User specified LOC: $160 \mathrm{ppm}$

Max Threat Zone for LOC: 36 meters

Max Threat Zone for IDLH: 27 meters

Note: Footprint was not drawn because effects of near-field patchiness make plume presentation unreliable for short distances.

\section{TIME DEPENDENT INFORMATION:}

Concentration Estimates at the point:

Downwind: 100 meters

Off Centerline: 0 meters

Max Concentration:

Outdoor: $42.2 \mathrm{ppm}$

Indoor: $42.2 \mathrm{ppm}$

Note: Indoor graph is shown with a dotted line. 


\section{ENGINEERING COMPUTATION SHEET}

Title of Project EPHA for THM

Reviewed by S. A. Henderson

Subject Concentration Analysis for THM Hazardous Chemicals

Computer T. M. Franey

Date $09 / 16 / 96$

SITE DATA INFORMATION: 1-RD-19

Location: AIKEN, SOUTH CAROLINA

Building Air Exchanges Per Hour: 60 (User specified)

Date and Time: Fixed at July 1, 19961300 hours

CHEMICAL INFORMATION:

Chemical Name: ETHYL BENZENE

Molecular Weight: $106.17 \mathrm{~kg} / \mathrm{kmol}$

TLV-TWA: $100.00 \mathrm{ppm}$ IDLH: $2000.00 \mathrm{ppm}$

Footprint Level of Concern: 160 ppm

Boiling Point: $136.20^{\circ} \mathrm{C}$

Vapor Pressure at Ambient Temperature: $0.013 \mathrm{~atm}$

Ambient Saturation Concentration: 12,900 ppm or $1.29 \%$

\section{ATMOSPHERIC INFORMATION: (MANUAL INPUT OF DATA)}

Wind: 2.5 meters $/ \mathrm{sec}$ from $90^{\circ}$ true

Inversion Height: 500 meters

Stability Class: C Air Temperature: $25^{\circ} \mathrm{C}$

Relative Humidity: 50\% Ground Roughness: 100 centimeters

Cloud Cover: 7 tenths

\section{SOURCE STRENGTH INFORMATION:}

Puddle Area: 40700 square feet

Average Puddle Depth: 1 centimeters

Soil Type: Default Ground Temperature: $25^{\circ} \mathrm{C}$

Initial Puddle Temperature: Ground temperature

Release Duration: ALOHA limited the duration to 1 hour

Max Computed Release Rate: 76.3 kilograms/min

Max Average Sustained Release Rate: 75.7 kilograms/min

(averaged over a minute or more)

Total Amount Released: 4,104 kilograms

FOOTPRINT INFORMATION:

Dispersion Module: Gaussian

User specified LOC: 160 ppm

Max Threat Zone for LOC: 46 meters

Max Threat Zone for IDLH: 34 meters

Note: Footprint was not drawn because effects of near-field patchiness make plume

presentation unreliable for short distances.

\section{TIME DEPENDENT INFORMATION:}

\section{Concentration Estimates at the point:}

Downwind: 100 meters

Off Centerline: 0 meters

Max Concentration:

Outdoor: $53.5 \mathrm{ppm}$

Indoor: $53.5 \mathrm{ppm}$

Note: Indoor graph is shown with a dotted line.
Works Calculation 7

Sheet No. $\underline{385 \text { of } 577}$ 
Title of Project EPHA for THM

Subject Concentration Analysis for THM Hazardous Chemicals

Computer T. M. Franey

Date 09/16/96
Reviewed by S. A. Henderson

Works Calculation 7

Sheet No. $\underline{386 \text { of } 577}$

SITE DATA INFORMATION: 1-RD-19

Location: AIKEN, SOUTH CAROLINA

Building Air Exchanges Per Hour: 60 (User specified)

Date and Time: Fixed at July 1, 19961300 hours

CHEMICAL INFORMATION:

Chemical Name: ETHYL BENZENE

Molecular Weight: $106.17 \mathrm{~kg} / \mathrm{kmol}$

TLV-TWA: $100.00 \mathrm{ppm}$ IDLH: $2000.00 \mathrm{ppm}$

Footprint Level of Concern: 160 ppm

Boiling Point: $136.20^{\circ} \mathrm{C}$

Vapor Pressure at Ambient Temperature: $0.013 \mathrm{~atm}$

Ambient Saturation Concentration: 12,900 ppm or $1.29 \%$

ATMOSPHERIC INFORMATION: (MANUAL INPUT OF DATA)

Wind: 2.5 meters $/ \mathrm{sec}$ from $90^{\circ}$ true

Inversion Height: 500 meters

Stability Class: C Air Temperature: $25^{\circ} \mathrm{C}$

Relative Humidity: 50\% Ground Roughness: 100 centimeters

Cloud Cover: 7 tenths

\section{SOURCE STRENGTH INFORMATION:}

Puddle Area: 10000 square meters

Average Puddle Depth: 1 centimeters

Soil Type: Default Ground Temperature: $25^{\circ} \mathrm{C}$

Initial Puddle Temperature: Ground temperature

Release Duration: ALOHA limited the duration to 1 hour

Max Computed Release Rate: 195 kilograms/min

Max Average Sustained Release Rate: 194 kilograms/min

(averaged over a minute or more)

Total Amount Released: 10,470 kilograms

\section{FOOTPRINT INFORMATION:}

Dispersion Module: Gaussian

User specified LOC: $160 \mathrm{ppm}$

Max Threat Zone for LOC: 74 meters

Max Threat Zone for IDLH: 56 meters

Note: Footprint was not drawn because effects of near-field patchiness make plume presentation unreliable for short distances.

\section{TIME DEPENDENT INFORMATION:}

Concentration Estimates at the point:

Downwind: 100 meters

Off Centerline: 0 meters

Max Concentration:

Outdoor: $92.7 \mathrm{ppm}$

Indoor: $92.7 \mathrm{ppm}$

Note: Indoor graph is shown with a dotted line. 
Title of Project EPHA for THM

Subject Concentration Analysis for THM Hazardous Chemicals

Computer T. M. Franey.
Reviewed by S. A. Henderson

Works Calculation 7

Sheet No. $\underline{387 \text { of } 577}$

1-RD-20

SITE DATA INFORMATION:

Location: AIKEN, SOUTH CAROLINA

Building Air Exchanges Per Hour: 60 (User specified)

Date and Time: Fixed at July 1, 19961300 hours

\section{CHEMICAL INFORMATION:}

Chemical Name: ETHYLENE GLYCOL MONOBUTYL ETHER

Molecular Weight: $118.18 \mathrm{~kg} / \mathrm{kmol}$

TLV-TWA: $25.00 \mathrm{ppm}$ IDLH: $700.00 \mathrm{ppm}$

Footprint Level of Concern: $150 \mathrm{ppm}$

Boiling Point: $171.32^{\circ} \mathrm{C}$

Vapor Pressure at Ambient Temperature: $0.0011 \mathrm{~atm}$

Ambient Saturation Concentration: $1,161 \mathrm{ppm}$ or $0.12 \%$

ATMOSPHERIC INFORMATION: (MANUAL INPUT OF DATA)

Wind: 4.5 meters $/ \mathrm{sec}$ from $90^{\circ}$ true

Inversion Height: 500 meters

Stability Class: C Air Temperature: $25^{\circ} \mathrm{C}$

Relative Humidity: $50 \%$ Ground Roughness: Urban or forest

Cloud Cover: 7 tenths

\section{SOURCE STRENGTH INFORMATION:}

Puddle Area: 30000 square feet

Average Puddle Depth: 1 centimeters

Soil Type: Default Ground Temperature: $25^{\circ} \mathrm{C}$

Initial Puddle Temperature: Ground temperature

Release Duration: ALOHA limited the duration to 1 hour

Max Computed Release Rate: 5.58 kilograms/min

Max Average Sustained Release Rate: 5.51 kilograms/min

(averaged over a minute or more)

Total Amount Released: 279 kilograms

TIME DEPENDENT INFORMATION:

Concentration Estimates at the point:

Downwind: 30 meters

Off Centerline: 0 meters

Max Concentration:

Outdoor: $13.6 \mathrm{ppm}$ 


\section{ENGINEERING COMPUTATION SHEET}

Title of Project EPHA for THM

Reviewed by S: A. Henderson

Subject Concentration Analysis for THM Hazardous Chemicals

Computer T. M. Franey

Date 09/16/96

\section{1-RD-20}

SITE DATA INFORMATION:

Location: AIKEN, SOUTH CAROLINA

Building Air Exchanges Per Hour: 60 (User specified)

Date and Time: Fixed at July 1, 19961300 hours

CHEMICAL INFORMATION:

Chemical Name: ETHYLENE GLYCOL MONOBUTYL ETHER

Molecular Weight: $118.18 \mathrm{~kg} / \mathrm{kmol}$

TLV-TWA: $25.00 \mathrm{ppm}$ IDLH: $700.00 \mathrm{ppm}$

Footprint Level of Concern: 150 ppm

Boiling Point: $171.32^{\circ} \mathrm{C}$

Vapor Pressure at Ambient Temperature: $0.0011 \mathrm{~atm}$

Ambient Saturation Concentration: $1,161 \mathrm{ppm}$ or $0.12 \%$

ATMOSPHERIC INFORMATION: (MANUAL INPUT OF DATA)

Wind: 4.5 meters $/ \mathrm{sec}$ from $90^{\circ}$ true

Inversion Height: 500 meters

Stability Class: C Air Temperature: $25^{\circ} \mathrm{C}$

Relative Humidity: 50\% Ground Roughness: Urban or forest

Cloud Cover: 7 tenths

SOURCE STRENGTH INFORMATION:

Puddle Area: 100000 square feet

Average Puddle Depth: 1 centimeters

Soil Type: Default Ground Temperature: $25^{\circ} \mathrm{C}$

Initial Puddle Temperature: Ground temperature

Release Duration: ALOHA limited the duration to 1 hour

Max Computed Release Rate: 17.9 kilograms $/ \mathrm{min}$

Max Average Sustained Release Rate: 17.7 kilograms/min (averaged over a minute or more)

Total Amount Released: 893 kilograms

TIME DEPENDENT INFORMATION:

Concentration Estimates at the point:

Downwind: 100 meters

- Off Centerline: 0 meters

Max Concentration:

Outdoor: $4.3 \mathrm{ppm}$
Works Calculation 7

Sheet No. $\underline{388 \text { of } 577}$ 
Title of Project EPHA for THM

Subject Concentration Analysis for THM Hazardous Chemicals

Computer T. M. Franey

Date $\underline{09 / 16 / 96}$

Reviewed by $\underline{\text { S. A. Henderson }}$

Works Calculation 7

Sheet No. $\underline{389 \text { of } 577}$

1-RD-20

SITE DATA INFORMATION:

Location: AIKEN, SOUTH CAROLINA

Building Air Exchanges Per Hour: 60 (User specified)

Date and Time: Fixed at July 1, 19961300 hours

CHEMICAL INFORMATION:

Chemical Name: ETHYLENE GLYCOL MONOBUTYL ETHER

Molecular Weight: $118.18 \mathrm{~kg} / \mathrm{kmol}$

TLV-TWA: $25.00 \mathrm{ppm}$ IDLH: $700.00 \mathrm{ppm}$

Footprint Level of Concern: $150 \mathrm{ppm}$

Boiling Point: $171.32^{\circ} \mathrm{C}$

Vapor Pressure at Ambient Temperature: 0.0011 atm

Ambient Saturation Concentration: $1,161 \mathrm{ppm}$ or $0.12 \%$

ATMOSPHERIC INFORMATION: (MANUAL INPUT OF DATA)

Wind: 4.5 meters $/ \mathrm{sec}$ from $90^{\circ}$ true

Inversion Height: 500 meters

Stability Class: C Air Temperature: $25^{\circ} \mathrm{C}$

Relative Humidity: $50 \%$ Ground Roughness: Urban or forest

Cloud Cover: 7 tenths

\section{SOURCE STRENGTH INFORMATION:}

Puddle Area: 24420 square feet

Average Puddle Depth: 1 centimeters

Soil Type: Default Ground Temperature: $25^{\circ} \mathrm{C}$

Initial Puddle Temperature: Ground temperature

Release Duration: ALOHA limited the duration to 1 hour

Max Computed Release Rate: 4.57 kilograms/min

Max Average Sustained Release Rate: 4.52 kilograms $/ \mathrm{min}$ (averaged over a minute or more)

Total Amount Released: 229 kilograms

FOOTPRINT INFORMATION:

Dispersion Module: Gaussian

User specified LOC: $150 \mathrm{ppm}$

Max Threat Zone for LOC: 27 meters

Max Threat Zone for IDLH: 27 meters

Note: Footprint was not drawn because

effects of near-field patchiness make plume

presentation unreliable for short distances.

TIME DEPENDENT INFORMATION:

Concentration Estimates at the point:

Downwind: 100 meters

Off Centerline: 0 meters

Max Concentration:

Outdoor: 2.05 ppm 
Title of Project EPHA for THM

Subject Concentration Analysis for THM Hazardous Chemicals

Computer T.M. Franey

Date 09/16/96
Reviewed by $\mathrm{S}$. A. Henderson

Works Calculation 7

Sheet No. $\underline{390 \text { of } 577}$

1-RD-20

SITE DATA INFORMATION:

Location: AIKEN, SOUTH CAROLINA

Building Air Exchanges Per Hour: 60 (User specified)

Date and Time: Fixed at July 1, 19961300 hours

CHEMICAL INFORMATION:

Chemical Name: ETHYLENE GLYCOL MONOBUTYL ETHER

Molecular Weight: $118.18 \mathrm{~kg} / \mathrm{kmol}$

TLV-TWA: $25.00 \mathrm{ppm}$ IDLH: $700.00 \mathrm{ppm}$

Footprint Level of Concern: $150 \mathrm{ppm}$

Boiling Point: $171.32^{\circ} \mathrm{C}$

Vapor Pressure at Ambient Temperature: 0.0011 atm

Ambient Saturation Concentration: $1,161 \mathrm{ppm}$ or $0.12 \%$

ATMOSPHERIC INFORMATION: (MANUAL INPUT OF DATA)

Wind: 4.5 meters $/ \mathrm{sec}$ from $90^{\circ}$ true

Inversion Height: 500 meters

Stability Class: C Air Temperature: $25^{\circ} \mathrm{C}$

Relative Humidity: 50\% Ground Roughness: Urban or forest

Cloud Cover: 7 tenths

SOURCE STRENGTH INFORMATION:

Puddle Area: 40700 square feet

Average Puddle Depth: 1 centimeters

Soil Type: Default Ground Temperature: $25^{\circ} \mathrm{C}$

Initial Puddle Temperature: Ground temperature

Release Duration: ALOHA limited the duration to 1 hour

Max Computed Release Rate: 7.5 kilograms/min

Max Average Sustained Release Rate: 7.4 kilograms/min (averaged over a minute or more)

Total Amount Released: 375 kilograms

FOOTPRINT INFORMATION:

Dispersion Module: Gaussian

User specified LOC: $150 \mathrm{ppm}$

Max Threat Zone for LOC: 34 meters

Max Threat Zone for IDLH: 35 meters

Note: Footprint was not drawn because effects of near-field patchiness make plume presentation unreliable for short distances.

TIME DEPENDENT INFORMATION:

Concentration Estimates at the point:

Downwind: 100 meters

Off Centerline: 0 meters

Max Concentration:

Outdoor: $2.61 \mathrm{ppm}$ 
Title of Project EPHA for THM

Subject Concentration Analysis for THM Hazardous Chemicals

Computer T. M. Franey

Date $\underline{09 / 16 / 96}$
Reviewed by S. A. Henderson

Works Calculation 7

Sheet No. 391 of 577

1-RD-20

SITE DATA INFORMATION:

Location: AIKEN, SOUTH CAROLINA

Building Air Exchanges Per Hour: 60 (User specified)

Date and Time: Fixed at July 1, 19961300 hours

CHEMICAL INFORMATION:

Chemical Name: ETHYLENE GLYCOL MONOBUTYL ETHER

Molecular Weight: $118.18 \mathrm{~kg} / \mathrm{kmol}$

TLV-TWA: $25.00 \mathrm{ppm}$ IDLH: $700.00 \mathrm{ppm}$

Footprint Level of Concern: $150 \mathrm{ppm}$

Boiling Point: $171.32^{\circ} \mathrm{C}$

Vapor Pressure at Ambient Temperature: 0.0011 atm

Ambient Saturation Concentration: $1,161 \mathrm{ppm}$ or $0.12 \%$

ATMOSPHERIC INFORMATION: (MANUAL INPUT OF DATA)

Wind: 4.5 meters $/ \mathrm{sec}$ from $90^{\circ}$ true

Inversion Height: 500 meters

Stability Class: C Air Temperature: $25^{\circ} \mathrm{C}$

Relative Humidity: 50\% Ground Roughness: Urban or forest.

Cloud Cover: 7 tenths

SOURCE STRENGTH INFORMATION:

Puddle Area: 10000 square meters

Average Puddle Depth: 1 centimeters

Soil Type: Default Ground Temperature: $25^{\circ} \mathrm{C}$

Initial Puddle Temperature: Ground temperature

Release Duration: ALOHA limited the duration to 1 hour

Max Computed Release Rate: 19.2 kilograms/min

Max Average Sustained Release Rate: 19 kilograms/min

(averaged over a minute or more)

Total Amount Released: 958 kilograms

FOOTPRINT INFORMATION:

Dispersion Module: Gaussian

User specified LOC: $150 \mathrm{ppm}$

Max Threat Zone for LOC: 56 meters

Max Threat Zone for IDLH: 56 meters

Note: Footprint was not drawn because effects of near-field patchiness make plume presentation unreliable for short distances.

\section{TIME DEPENDENT INFORMATION:}

Concentration Estimates at the point:

Downwind: 100 meters

Off Centerline: 0 meters

Max Concentration:

Outdoor: $4.54 \mathrm{ppm}$ 
Title of Project EPHA for THM

Subject Concentration Analysis for THM Hazardous Chemicals

Computer T. M. Franey

Date 09/16/96

Reviewed by S.A. Henderson

Works Calculation 7

Sheet No. 392 of 577

\section{1-RD-21}

SITE DATA INFORMATION:

Location: AIKEN, SOUTH CAROLINA

Building Air Exchanges Per Hour: 60 (User specified)

Date and Time: Fixed at July 1, 19960000 hours

\section{CHEMICAL INFORMATION:}

Chemical Name: Fluorosilicic acid as HYDDUFLOURIC ACID - 70 WT\% - SAH

Molecular Weight: $20.01 \mathrm{~kg} / \mathrm{kmol}$

TLV-TWA: -unavail- IDLH: -unavail-

Footprint Level of Concern: $20 \mathrm{ppm}$

Boiling Point: $110.35^{\circ} \mathrm{C}$

Vapor Pressure at Ambient Temperature: 2.36e-004 atm

Ambient Saturation Concentration: 240 ppm or $0.024 \%$

\section{ATMOSPHERIC INFORMATION: (MANUAL INPUT OF DATA)}

Wind: 1.7 meters $/ \mathrm{sec}$ from $90^{\circ}$ true

Inversion Height: 200 meters

Stability Class: E Air Temperature: $29^{\circ} \mathrm{C}$

Relative Humidity: $50 \%$ Ground Roughness: 100 centimeters

Cloud Cover: 1 tenths

\section{SOURCE STRENGTH INFORMATION:}

Puddle Area: 24600 square feet

Average Puddle Depth: 1 centimeters

Soil Type: Default Ground Temperature: $29^{\circ} \mathrm{C}$

Initial Puddle Temperature: Ground temperature

Release Duration: ALOHA limited the duration to 1 hour

Max Computed Release Rate: 117 grams/min

Max Average Sustained Release Rate: 109 grams/min

(averaged over a minute or more)

Total Amount Released: 6.01 kilograms

\section{FOOTPRINT INFORMATION:}

Dispersion Module: Gaussian

User specified LOC: $20 \mathrm{ppm}$

Max Threat Zone for LOC: 30 meters

Note: Footprint was not drawn because effects of near-field patchiness make plume presentation unreliable for short distances.

\section{TIME DEPENDENT INFORMATION:}

\section{Concentration Estimates at the point:}

Downwind: 30 meters

Off Centerline: 0 meters

Max Concentration:

Outdoor: $20 \mathrm{ppm}$

Indoor: $20 \mathrm{ppm}$

Note: Indoor graph is shown with a dotted line. 


\section{ENGINEERING COMPUTATION SHEET}

Title of Project EPHA for THM

Reviewed by $\underline{\text { S. A. Henderson }}$

Subject Concentration Analysis for THM Hazardous Chemicals

Computer T. M. Franey

Date $\underline{09 / 16 / 96}$
Works Calculation 7

Sheet No. $\underline{393 \text { of } 577}$

1-RD-21

SITE DATA INFORMATION:

Location: AIKEN, SOUTH CAROLINA

Building Air Exchanges Per Hour: 60 (User specified)

Date and Time: Fixed at July 1, 19960000 hours

\section{CHEMICAL INFORMATION:}

Chemical Name: Fluorosilicic acid as HYDDUFLOURIC ACID - 70 WT\% - SAH

Molecular Weight: $20.01 \mathrm{~kg} / \mathrm{kmol}$

TLV-TWA: -unavail- IDLH: -unavail-

Footprint Level of Concern: $20 \mathrm{ppm}$

Boiling Point: $110.35^{\circ} \mathrm{C}$

Vapor Pressure at Ambient Temperature: 2.36e-004 atm

Ambient Saturation Concentration: 240 ppm or $0.024 \%$

\section{ATMOSPHERIC INFORMATION: (MANUAL INPUT OF DATA)}

Wind: 1.7 meters $/ \mathrm{sec}$ from $90^{\circ}$ true

Inversion Height: 200 meters

Stability Class: E Air Temperature: $29^{\circ} \mathrm{C}$

Relative Humidity: $50 \%$ Ground Roughness: 100 centimeters

Cloud Cover: 1 tenths

\section{SOURCE STRENGTH INFORMATION:}

Puddle Area: 107639 square feet

Average Puddle Depth: 1 centimeters

Soil Type: Default Ground Temperature: $29^{\circ} \mathrm{C}$

Initial Puddle Temperature: Ground temperature

Release Duration: ALOHA limited the duration to 1 hour

Max Computed Release Rate: 476 grams/min

Max Average Sustained Release Rate: 443 grams/min

(averaged over a minute or more)

Total Amount Released: 24.3 kilograms

\section{FOOTPRINT INFORMATION:}

Dispersion Module: Gaussian

User specified LOC: $20 \mathrm{ppm}$

Max Threat Zone for LOC: 61 meters

Note: Footprint was not drawn because effects of near-field patchiness make plume presentation unreliable for short distances.

\section{TIME DEPENDENT INFORMATION:}

Concentration Estimates at the point:

Downwind: 100 meters

Off Centerline: 0 meters

Max Concentration:

Outdoor: $7.69 \mathrm{ppm}$

Indoor: $7.69 \mathrm{ppm}$

Note: Indoor graph is shown with a dotted line. 
Title of Project EPHA for THM

Subject Concentration Analysis for THM Hazardous Chemicals

Computer T. M. Franey
Reviewed by $\underline{\text { S. A. Henderson }}$

Works Calculation 7

Sheet No. 394 of 577

1-RD-21

SITE DATA INFORMATION:

Location: AIKEN, SOUTH CAROLINA

Building Air Exchanges Per Hour: 60 (User specified)

Date and Time: Fixed at July 1, 19960000 hours

\section{CHEMICAL INFORMATION:}

Chemical Name: Fluorosilicic acid as HYDDUFLOURIC ACID - 70 WT\% - SAH

Molecular Weight: $20.01 \mathrm{~kg} / \mathrm{kmol}$

TLV-TWA: -unavail- IDLH: -unavail-

Footprint Level of Concern: $20 \mathrm{ppm}$

Boiling Point: $110.35^{\circ} \mathrm{C}$

Vapor Pressure at Ambient Temperature: $2.36 \mathrm{e}-004 \mathrm{~atm}$

Ambient Saturation Concentration: 240 ppm or $0.024 \%$

\section{ATMOSPHERIC INFORMATION: (MANUAL INPUT OF DATA)}

Wind: 1.7 meters $/ \mathrm{sec}$ from $90^{\circ}$ true

Inversion Height: 200 meters

Stability Class: E Air Temperature: $29^{\circ} \mathrm{C}$

Relative Humidity: 50\% Ground Roughness: 100 centimeters

Cloud Cover: 1 tenths

\section{SOURCE STRENGTH INFORMATION:}

Puddle Area: 24420 square feet

Average Puddle Depth: 1 centimeters

Soil Type: Default Ground Temperature: $29^{\circ} \mathrm{C}$

Initial Puddle Temperature: Ground temperature

Release Duration: ALOHA limited the duration to 1 hour

Max Computed Release Rate: 117 grams/min

Max Average Sustained Release Rate: 109 grams/min

(averaged over a minute or more)

Total Amount Released: 5.97 kilograms

\section{FOOTPRINT INFORMATION:}

Dispersion Module: Gaussian

User specified LOC: $20 \mathrm{ppm}$

Max Threat Zone for LOC: 30 meters

Note: Footprint was not drawn because effects of near-field patchiness make plume presentation unreliable for short distances.

\section{TIME DEPENDENT INFORMATION:}

Concentration Estimates at the point:

Downwind: 100 meters

Off Centerline: 0 meters

Max Concentration:

Outdoor: $2.18 \mathrm{ppm}$

Indoor: $2.18 \mathrm{ppm}$

Note: Indoor graph is shown with a dotted line. 


\section{ENGINEERING COMPUTATION SHEET}

Title of Project EPHA for THM

Reviewed by S. A. Henderson

Subject Concentration Analysis for THM Hazardous Chemicals

Works Calculation 7

Computer T. M. Franey

Date $\underline{09 / 16 / 96}$

\title{
1-RD-21
}

SITE DATA INFORMATION:

Location: AIKEN, SOUTH CAROLINA

Building Air Exchanges Per Hour: 60 (User specified)

Date and Time: Fixed at July 1, 19960000 hours

\section{CHEMICAL INFORMATION:}

Chemical Name: Fluorosilicic acid as HYDDUFLOURIC ACID - 70 WT\% - SAH

Molecular Weight: $20.01 \mathrm{~kg} / \mathrm{kmol}$

TLV-TWA: -unavail- IDLH: -unavail-

Footprint Level of Concern: $20 \mathrm{ppm}$

Boiling Point: $110.35^{\circ} \mathrm{C}$

Vapor Pressure at Ambient Temperature: 2.36e-004 atm

Ambient Saturation Concentration: 240 ppm or $0.024 \%$

\author{
ATMOSPHERIC INFORMATION: (MANUAL INPUT OF DATA) \\ Wind: 1.7 meters $/ \mathrm{sec}$ from $90^{\circ}$ true \\ Inversion Height: 200 meters \\ Stability Class: E Air Temperature: $29^{\circ} \mathrm{C}$ \\ Relative Humidity: $50 \% \quad$ Ground Roughness: 100 centimeters \\ Cloud Cover: 1 tenths
}

\section{SOURCE STRENGTH INFORMATION:}

Puddle Area: 40700 square feet

Average Puddle Depth: 1 centimeters

Soil Type: Default Ground Temperature: $29^{\circ} \mathrm{C}$

Initial Puddle Temperature: Ground temperature

Release Duration: ALOHA limited the duration to 1 hour

Max Computed Release Rate: 189 grams/min

Max Average Sustained Release Rate: $176 \mathrm{grams} / \mathrm{min}$

(averaged over a minute or more)

Total Amount Released: 9.68 kilograms

\section{FOOTPRINT INFORMATION:}

Dispersion Module: Gaussian

User specified LOC: $20 \mathrm{ppm}$

Max Threat Zone for LOC: 38 meters

Note: Footprint was not drawn because effects of near-field patchiness make plume presentation unreliable for short distances.

\section{TIME DEPENDENT INFORMATION:}

Concentration Estimates at the point:

Downwind: 100 meters

Off Centerline: 0 meters

Max Concentration:

Outdoor: $3.15 \mathrm{ppm}$

Indoor: $3.15 \mathrm{ppm}$

Note: Indoor graph is shown with a dotted line. 
Title of Project EPHA for THM

Subject Concentration Analysis for THM Hazardous Chemicals

Computer T. M. Franey

Date $\underline{09 / 16 / 96}$

Reviewed by S. A. Henderson

1-RD-21

SITE DATA INFORMATION

Location: AIKEN, SOUTH CAROLINA

Building Air Exchanges Per Hour: 60 (User specified)

Date and Time: Fixed at July 1, 19960000 hours

\section{CHEMICAL INFORMATION:}

Chemical Name: Fluorosilicic acid as HYDDUFLOURIC ACID - 70 WT\% - SAH

Molecular Weight: $20.01 \mathrm{~kg} / \mathrm{kmol}$

TLV-TWA: -unavail- IDLH: -unavail-

Footprint Level of Concern: 20 ppm

Boiling Point: $110.35^{\circ} \mathrm{C}$

Vapor Pressure at Ambient Temperature: 2.36e-004 atm

Ambient Saturation Concentration: 240 ppm or $0.024 \%$

ATMOSPHERIC INFORMATION: (MANUAL INPUT OF DATA)

Wind: 1.7 meters $/ \mathrm{sec}$ from $90^{\circ}$ true

Inversion Height: 200 meters

Stability Class: E Air Temperature: $29^{\circ} \mathrm{C}$

Relative Humidity: 50\% Ground Roughness: 100 centimeters

Cloud Cover: 1 tenths

\section{SOURCE STRENGTH INFORMATION:}

Puddle Area: 10000 square meters

Average Puddle Depth: 1 centimeters

Soil Type: Default Ground Temperature: $29^{\circ} \mathrm{C}$

Initial Puddle Temperature: Ground temperature

Release Duration: ALOHA limited the duration to 1 hour

Max Computed Release Rate: 476 grams/min

Max Average Sustained Release Rate: 443 grams/min (averaged over a minute or more)

Total Amount Released: 24.3 kilograms

\section{FOOTPRINT INFORMATION:}

Dispersion Module: Gaussian

User specified LOC: $20 \mathrm{ppm}$

Max Threat Zone for LOC: 61 meters

Note: Footprint was not drawn because effects of near-field patchiness make plume presentation unreliable for short distances.

\section{TIME DEPENDENT INFORMATION:}

Concentration Estimates at the point:

Downwind: 100 meters

Off Centerline: 0 meters

Max Concentration:

Outdoor: $7.69 \mathrm{ppm}$

Indoor: $7.69 \mathrm{ppm}$ 


\section{ENGINEERING COMPUTATION.SHEET}

Title of Project EPHA for THM

Reviewed by S.A. Henderson

Subject Concentration Analysis for THM Hazardous Chemicals

Computer T.M. Franey

Date $\underline{09 / 16 / 96}$
Works Calculation 7

Sheet No. $\underline{397 \text { of } 577}$

SITE DATA INFORMATION: 1-RD-22

Location: AIKEN, SOUTH CAROLINA

Building Air Exchanges Per Hour: 60 (User specified)

Date and Time: Fixed at July 1, 19961300 hours

CHEMICAL INFORMATION:

Chemical Name: FORMIC ACID

Molecular Weight: $46.03 \mathrm{~kg} / \mathrm{kmol}$

TLV-TWA: $5.00 \mathrm{ppm}$. IDLH: $30.00 \mathrm{ppm}$

Footprint Level of Concern: 20 ppm

Boiling Point: $100.56^{\circ} \mathrm{C}$

Vapor Pressure at Ambient Temperature: $0.056 \mathrm{~atm}$

Ambient Saturation Concentration: 57,013 ppm or $5.70 \%$

ATMOSPHERIC INFORMATION: (MANUAL INPUT OF DATA)

Wind: 2.5 meters $/ \mathrm{sec}$ from $90^{\circ}$ true

Inversion Height: 500 meters

Stability Class: C Air Temperature: $25^{\circ} \mathrm{C}$

Relative Humidity: 50\% Ground Roughness: 100 centimeters

Cloud Cover: 7 tenths

\section{SOURCE STRENGTH INFORMATION:}

Puddle Area: 168 square feet

Average Puddle Depth: 1 centimeters

Soil Type: Default Ground Temperature: $25^{\circ} \mathrm{C}$

Initial Puddle Temperature: Ground temperature

Release Duration: ALOHA limited the duration to 1 hour

Max Computed Release Rate: 392 grams/min

Max Average Sustained Release Rate: 391 grams/min (averaged over a minute or more)

Total Amount Released: 22.5 kilograms

FOOTPRINT INFORMATION:

Dispersion Module: Gaussian

User specified LOC: $20 \mathrm{ppm}$

Max Threat Zone for LOC: 30 meters

Max Threat Zone for IDLH: 23 meters

Note: Footprint was not drawn because effects of near-field patchiness make plume

presentation unreliable for short distances.

TIME DEPENDENT INFORMATION:

Concentration Estimates at the point:

Downwind: 30 meters

Off Centerline: 0 meters

Max Concentration:

Outdoor: $20 \mathrm{ppm}$

Indoor: $20 \mathrm{ppm}$

Note: Indoor graph is shown with a dotted line. 
Title of Project EPHA for THM

Subject Concentration Analysis for THM Hazardous Chemicals

Computer T.M. Franey
Reviewed by $\mathrm{S}$. A. Henderson

Works Calculation 7

Sheet No. $\underline{398 \text { of } 577}$

SITE DATA INFORMATION: 1-RD-22

Location: AIKEN, SOUTH CAROLINA

Building Air Exchanges Per Hour: 60 (User specified)

Date and Time: Fixed at July 1, 19961300 hours

CHEMICAL INFORMATION:

Chemical Name: FORMIC ACID

Molecular Weight: $46.03 \mathrm{~kg} / \mathrm{kmol}$

TLV-TWA: $5.00 \mathrm{ppm}$ IDLH: $30.00 \mathrm{ppm}$

Footprint Level of Concern: 20. ppm

Boiling Point: $100.56^{\circ} \mathrm{C}$

Vapor Pressure at Ambient Temperature: $0.056 \mathrm{~atm}$

Ambient Saturation Concentration: 57,013 ppm or 5.70\%

ATMOSPHERIC INFORMATION: (MANUAL INPUT OF DATA)

Wind: 2.5 meters $/ \mathrm{sec}$ from $90^{\circ}$ true

Inversion Height: 500 meters

Stability Class: C Air Temperature: $25^{\circ} \mathrm{C}$

Relative Humidity: 50\% Ground Roughness: 100 centimeters

Cloud Cover: 7 tenths

SOURCE STRENGTH INFORMATION:

Puddle Area: 2090 square feet

Average Puddle Depth: 1 centimeters

Soil Type: Default Ground Temperature: $25^{\circ} \mathrm{C}$

Initial Puddle Temperature: Ground temperature

Release Duration: ALOHA limited the duration to 1 hour

Max Computed Release Rate: 4.4 kilograms/min

Max Average Sustained Release Rate: 4.38 kilograms/min

(averaged over a minute or more)

Total Amount Released: 250 kilograms

FOOTPRINT INFORMATION:

Dispersion Module: Gaussian

User specified LOC: $20 \mathrm{ppm}$

Max Threat Zone for LOC: 100 meters

Max Threat Zone for IDLH: 79 meters

Note: Footprint was not drawn because effects of near-field patchiness make plume presentation unreliable for short distances.

\section{TIME DEPENDENT INFORMATION:}

Concentration Estimates at the point:

Downwind: 100 meters

Off Cénterline: 0 meters

Max Concentration:

Outdoor: $20 \mathrm{ppm}$

Indoor: $20 \mathrm{ppm}$

Note: Indoor graph is shown with a dotted line. 


\section{ENGINEERING COMPUTATION SHEET}

Title of Project EPHA for THM

Reviewed by $\underline{\text { S. A. Henderson }}$

Subject Concentration Analysis for THM Hazardous Chemicals

Computer T. M. Franey

Date $\underline{09 / 16 / 96}$
Works Calculation 7

Sheet No. $\underline{399 \text { of } 577}$

SITE DATA INFORMATION: 1-RD-22

Location: AIKEN, SOUTH CAROLINA

Building Air Exchanges Per Hour: 60 (User specified)

Date and Time: Fixed at July 1, 19961300 hours

CHEMICAL INFORMATION:

Chemical Name: FORMIC ACID

Molecular Weight: $46.03 \mathrm{~kg} / \mathrm{kmol}$

TLV-TWA: $5.00 \mathrm{ppm}$ IDLH: $30.00 \mathrm{ppm}$

Footprint Level of Concern: 20 ppm

Boiling Point: $100.56^{\circ} \mathrm{C}$

Vapor Pressure at Ambient Temperature: $0.056 \mathrm{~atm}$

Ambient Saturation Concentration: 57,013 ppm or 5.70\%

ATMOSPHERIC INFORMATION: (MANUAL INPUT OF DATA)

Wind: 2.5 meters $/ \mathrm{sec}$ from $90^{\circ}$ true

Inversion Height: 500 meters

Stability Class: C Air Temperature: $25^{\circ} \mathrm{C}$

Relative Humidity: 50\% Ground Roughness: 100 centimeters

Cloud Cover: 7 tenths

SOURCE STRENGTH INFORMATION:

Puddle Area: 24420 square feet

Average Puddle Depth: 1 centimeters

Soil Type: Default Ground Temperature: $25^{\circ} \mathrm{C}$

Initial Puddle Temperature: Ground temperature

Release Duration: ALOHA limited the duration to 1 hour

Max Computed Release Rate: 47 kilograms/min

Max Average Sustained Release Rate: 46.8 kilograms/min

(averaged over a minute or more)

Total Amount Released: 2,641 kilograms

FOOTPRINT INFORMATION:

Dispersion Module: Gaussian

User specified LOC: $20 \mathrm{ppm}$

Max Threat Zone for LOC: 326 meters

Max Threat Zone for IDLH: 255 meters

TIME DEPENDENT INFORMATION:

Concentration Estimates at the point:

Downwind: 100 meters

Off Centerline: 0 meters

Max Concentration:

Outdoor: $98.3 \mathrm{ppm}$

Indoor: $98.3 \mathrm{ppm}$

Note: Indoor graph is shown with a dotted line. 
Title of Project EPHA for THM

Subject Concentration Analysis for THM Hazardous Chemicals

Computer T. M. Franey Date $\underline{09 / 16 / 96}$
Reviewed by S. A. Henderson

Works Calculation 7

Sheet No. 400 of 577

SITE DATA INFORMATION: 1-RD-22

Location: AIKEN, SOUTH CAROLINA

Building Air Exchanges Per Hour: 60 (User specified)

Date and Time: Fixed at July 1, 19961300 hours

CHEMICAL INFORMATION:

Chemical Name: FORMIC ACID

Molecular Weight: $46.03 \mathrm{~kg} / \mathrm{kmol}$

TLV-TWA: $5.00 \mathrm{ppm}$ IDLH: $30.00 \mathrm{ppm}$

Footprint Level of Concern: $20 \mathrm{ppm}$

Boiling Point: $100.56^{\circ} \mathrm{C}$

Vapor Pressure at Ambient Temperature: 0.056 atm

Ambient Saturation Concentration: 57,013 ppm or 5.70\%

ATMOSPHERIC INFORMATION: (MANUAL INPUT OF DATA)

Wind: 2.5 meters $/ \mathrm{sec}$ from $90^{\circ}$ true

Inversion Height: 500 meters

Stability Class: C Air Temperature: $25^{\circ} \mathrm{C}$

Relative Humidity: 50\% Ground Roughness: 100 centimeters

Cloud Cover: 7 tenths

\section{SOURCE STRENGTH INFORMATION:}

Puddle Area: 40700 square feet

Average Puddle Depth: 1 centimeters

Soil Type: Default Ground Temperature: $25^{\circ} \mathrm{C}$

Initial Puddle Temperature: Ground temperature

Release Duration: ALOHA limited the duration to I hour

Max Computed Release Rate: 76.9 kilograms/min

Max Average Sustained Release Rate: 76.6 kilograms/min

(averaged over a minute or more)

Total Amount Released: 4,316 kilograms

FOOTPRINT INFORMATION:

Dispersion Module: Gaussian

User specified LOC: $20 \mathrm{ppm}$

Max Threat Zone for LOC: 417 meters

Max Threat Zone for IDLH: 327 meters

TIME DEPENDENT INFORMATION:

Concentration Estimates at the point:

Downwind: 100 meters

Off Centerline: 0 meters

Max Concentration:

Outdoor: 125 ppm

Indoor: $125 \mathrm{ppm}$

Note: Indoor graph is shown with a dotted line. 
Title of Project EPHA for THM

Subject Concentration Analysis for THM Hazardous Chemicals

Computer T.M. Franey

Date 09/16/96

Reviewed by $\underline{\text { S. A. Henderson }}$

Works Calculation 7

Sheet No. 401 of 577

SITE DATA INFORMATION: 1-RD-22.

Location: AIKEN, SOUTH CAROLINA

Building Air Exchanges Per Hour: 60 (User specified)

Date and Time: Fixed at July 1, 19961300 hours

CHEMICAL INFORMATION:

Chemical Name: FORMIC ACID

Molecular Weight: $46.03 \mathrm{~kg} / \mathrm{kmol}$

TLV-TWA: $5.00 \mathrm{ppm}$ IDLH: $30.00 \mathrm{ppm}$

Footprint Level of Concern: 20 ppm

Boiling Point: $100.56^{\circ} \mathrm{C}$

Vapor Pressure at Ambient Temperature: $0.056 \mathrm{~atm}$

Ambient Saturation Concentration: 57,013 ppm or $5.70 \%$

ATMOSPHERIC INFORMATION: (MANUAL INPUT OF DATA)

Wind: 2.5 meters $/ \mathrm{sec}$ from $90^{\circ}$ true

Inversion Height: 500 meters

Stability Class: C Air Temperature: $25^{\circ} \mathrm{C}$

Relative Humidity: 50\% Ground Roughness: 100 centimeters

Cloud Cover: 7 tenths

SOURCE STRENGTH INFORMATION:

Puddle Area: 10000 square meters

Average Puddle Depth: 1 centimeters

Soil Type: Default Ground Temperature: $25^{\circ} \mathrm{C}$

Initial Puddle Temperature: Ground temperature

Release Duration: ALOHA limited the duration to 1 hour

Max Computed Release Rate: 197 kilograms/min

Max Average Sustained Release Rate: 196 kilograms/min

(averaged over a minute or more)

Total Amount Released: 11,011 kilograms

FOOTPRINT INFORMATION:

Dispersion Module: Gaussian

User specified LOC: $20 \mathrm{ppm}$

Max Threat Zone for LOC: 669 meters

Max Threat Zone for IDLH: 522 meters

TIME DEPENDENT INFORMATION:

Concentration Estimates at the point:

Downwind: 100 meters

Off Centerline: 0 meters

Max Concentration:

Outdoor: $217 \mathrm{ppm}$

Indoor: $217 \mathrm{ppm}$

Note: Indoor graph is shown with a dotted line. 
Title of Project EPHA for THM

Subject Concentration Analysis for THM Hazardous Chemicals

Computer T. M. Franey

Date $09 / 16 / 96$

SITE DATA INFORMATION: 1-RD-23

Location: AIKEN, SOUTH CAROLINA

Building Air Exchanges Per Hour: 60 (User specified)

Date and Time: Fixed at July 1, 19961300 hours

CHEMICAL INFORMATION:

Chemical Name: DIETHYLENE GLYCOL DIMETHYL ETHER

Molecular Weight: $134.18 \mathrm{~kg} / \mathrm{kmol}$

TLV-TWA: -unavail- . IDLH: -unavail-

Footprint Level of Concern: $25 \mathrm{ppm}$

Boiling Point: $159.76^{\circ} \mathrm{C}$

Vapor Pressure at Ambient Temperature: $0.0039 \mathrm{~atm}$

Ambient Saturation Concentration: 3,963 ppm or $0.40 \%$

ATMOSPHERIC INFORMATION: (MANUAL INPUT OF DATA)

Wind: 2.5 meters $/ \mathrm{sec}$ from $90^{\circ}$ true

Inversion Height: 500 meters

Stability Class: C Air Temperature: $25^{\circ} \mathrm{C}$

Relative Humidity: 50\% Ground Roughness: 100 centimeters

Cloud Cover: 7 .tenths

\section{SOURCE STRENGTH INFORMATION:}

Puddle Area: 11180 square feet

Average Puddle Depth: 1 centimeters

Soil Type: Default - Ground Temperature: $25^{\circ} \mathrm{C}$

Initial Puddle Temperature: Ground temperature

Release Duration: ALOHA limited the duration to 1 hour

Max Computed Release Rate: 6.2 kilograms/min

Max Average Sustained Release Rate: 6.12 kilograms/min (averaged over a minute or more)

Total Amount Released: 316 kilograms

\section{FOOTPRINT INFORMATION:}

Dispersion Module: Gaussian

User specified LOC: $25 \mathrm{ppm}$

Max Threat Zone for LOC: 30 meters

Note: Footprint was not drawn because effects of near-field patchiness make plume presentation unreliable for short distances.

\section{TIME DEPENDENT INFORMATION:}

Concentration Estimates at the point:

Downwind: 30 meters

Off Centerline: 0 meters

Max Concentration:

Outdoor: $25 \mathrm{ppm}$

Indoor: $25 \mathrm{ppm}$

Note: Indoor graph is shown with a dotted line.
Reviewed by S. A. Henderson

Works Calculation 7

Sheet No. 402 of 577 
Title of Project EPHA for THM

Subject Concentration Analysis for THM Hazardous Chemicals

Computer T. M. Franey

Date $\underline{09 / 16 / 96}$

SITE DATA INFORMATION: 1-RD-23

Location: AIKEN, SOUTH CAROLINA

Building Air Exchanges Per Hour: 60 (User specified)

Date and Time: Fixed at July 1, 19961300 hours

\section{CHEMICAL INFORMATION:}

Chemical Name: DIETHYLENE GLYCOL DIMETHYL ETHER

Molecular Weight: $134.18 \mathrm{~kg} / \mathrm{kmol}$

TLV-TWA: -unavail- IDLH: -unavail-
Footprint Level of Concern: $25 \mathrm{ppm}$

Boiling Point: $159.76^{\circ} \mathrm{C}$

Vapor Pressure at Ambient Temperature: 0.0039 atm

Ambient Saturation Concentration: 3,963 ppm or $0.40 \%$

ATMOSPHERIC INFORMATION: (MANUAL INPUT OF DATA)

Wind: 2.5 meters $/ \mathrm{sec}$ from $90^{\circ}$ true

Inversion Height: 500 meters

Stability Class: C Air Temperature: $25^{\circ} \mathrm{C}$

Relative Humidity: 50\% Ground Roughness: 100 centimeters

Cloud Cover: 7 tenths

\section{SOURCE STRENGTH INFORMATION:}

Puddle Area: 99999 square feet

Average Puddle Depth: 1 centimeters

Soil Type: Default Ground Temperature: $25^{\circ} \mathrm{C}$

Initial Puddle Temperature: Ground temperature

Release Duration: ALOHA limited the duration to 1 hour

Max Computed Release Rate: 51.2 kilograms/min

Max Average Sustained Release Rate: 50.6 kilograms/min (averaged over a minute or more)

Total Amount Released: 2,598 kilograms

\section{FOOTPRINT INFORMATION:}

Dispersion Module: Gaussian

User specified LOC: $25 \mathrm{ppm}$

Max Threat Zone for LOC: 86 meters

Note: Footprint was not drawn because effects of near-field patchiness make plume presentation unreliable for short distances.

\section{TIME DEPENDENT INFORMATION:}

Concentration Estimates at the point:

Downwind: 100 meters

Off Centerline: 0 meters

Max Concentration:

Outdoor: $19.5 \mathrm{ppm}$

Indoor: $19.5 \mathrm{ppm}$

Note: Indoor graph is shown with a dotted line.
Reviewed by $\underline{\text { S. A. Henderson }}$

Works Calculation 7

Sheet No. 403 of 577 


\section{ENGINEERING COMPUTATION SHEET}

Title of Project EPHA for THM

Subject Concentration Analysis for THM Hazardous Chemicals

Computer T. M. Franey
Reviewed by $\mathrm{S}$. A. Henderson

Works Calculation 7

Sheet No. 404 of 577

\section{SITE DATA INFORMATION: 1-RD-23}

Location: AIKEN, SOUTH CAROLINA

Building Air Exchanges Per Hour: 60 (User specified)

Date and Time: Fixed at July 1, 19961300 hours

\section{CHEMICAL INFORMATION:}

Chemical Name: DIETHYLENE GLYCOL DIMETHYL ETHER

Molecular Weight: $134.18 \mathrm{~kg} / \mathrm{kmol}$

TLV-TWA: -unavail- IDLH: -unavail-

Footprint Level of Concern: 25 ppm

Boiling Point: $159.76^{\circ} \mathrm{C}$

Vapor Pressure at Ambient Temperature: $0.0039 \mathrm{~atm}$

Ambient Saturation Concentration: $3,963 \mathrm{ppm}$ or $0.40 \%$

\section{ATMOSPHERIC INFORMATION: (MANUAL INPUT OF DATA)}

Wind: 2.5 meters $/ \mathrm{sec}$ from $90^{\circ}$ true

Inversion Height: 500 meters

Stability Class: C Air Temperature: $25^{\circ} \mathrm{C}$

Relative Humidity: $50 \%$ Ground Roughness: 100 centimeters

Cloud Cover: 7 tenths

\section{SOURCE STRENGTH INFORMATION:}

Puddle Area: 24420 square feet

Average Puddle Depth: 1 centimeters

Soil Type: Default Ground Temperature: $25^{\circ} \mathrm{C}$

Initial Puddle Temperature: Ground temperature

Release Duration: ALOHA limited the duration to 1 hour

Max Computed Release Rate: 13.2 kilograms/min

Max Average Sustained Release Rate: 13 kilograms/min

(averaged over a minute or more)

Total Amount Released: 669 kilograms

\section{FOOTPRINT INFORMATION:}

Dispersion Module: Gaussian

User specified LOC: $25 \mathrm{ppm}$

Max Threat Zone for LOC: 44 meters

Note: Footprint was not drawn because effects of near-field patchiness make plume presentation unreliable for short distances.

\section{TIME DEPENDENT INFORMATION:}

Concentration Estimates at the point:

Downwind: 100 meters

Off Centerline: 0 meters

Max Concentration:

Outdoor: $9.37 \mathrm{ppm}$

Indoor: $9.37 \mathrm{ppm}$

Note: Indoor graph is shown with a dotted line. 


\section{ENGINEERING COMPUTATION SHEET}

Title of Project EPHA for THM

Subject Concentration Analysis for THM Hazardous Chemicals

Computer T. M. Franey

Date $\underline{09 / 16 / 96}$
Reviewed by $\mathrm{S}$. A. Henderson

Works Calculation 7

Sheet No. $\underline{405 \text { of } 577}$

SITE DATA INFORMATION: 1-RD-23

Location: AIKEN, SOUTH CAROLINA

Building Air Exchanges Per Hour: 60 (User specified)

Date and Time: Fixed at July 1, 19961300 hours

CHEMICAL INFORMATION:

Chemical Name: DIETHYLENE GLYCOL DIMETHYL ETHER

Molecular Weight: $134.18 \mathrm{~kg} / \mathrm{kmol}$

TLV-TWA: -unavail- IDLH: -unavail-

Footprint Level of Concern: 25 ppm

Boiling Point: $159.76^{\circ} \mathrm{C}$

Vapor Pressure at Ambient Temperature: $0.0039 \mathrm{~atm}$

Ambient Saturation Concentration: $3,963 \mathrm{ppm}$ or $0.40 \%$

ATMOSPHERIC INFORMATION: (MANUALL INPUT OF DATA)

Wind: 2.5 meters $/ \mathrm{sec}$ from $90^{\circ}$ true

Inversion Height: 500 meters

Stability Class: C Air Temperature: $25^{\circ} \mathrm{C}$

Relative Humidity: 50\% Ground Roughness: 100 centimeters

Cloud Cover: 7 tenths

\section{SOURCE STRENGTH INFORMATION:}

Puddle Area: 40700 square feet

Average Puddle Depth: 1 centimeters

Soil Type: Default Ground Temperature: $25^{\circ} \mathrm{C}$

Initial Puddle Temperature: Ground temperature

Release Duration: ALOHA limited the duration to 1 hour

Max Computed Release Rate: 21.5 kilograms/min

Max Average Sustained Release Rate: 21.3 kilograms/min

(averaged over a minute or more)

Total Amount Released: 1,093 kilograms

\section{FOOTPRINT INFORMATION:}

Dispersion Module: Gaussian

User specified LOC: $25 \mathrm{ppm}$

Max Threat Zone for LOC: 56 meters

Note: Footprint was not drawn because effects of near-field patchiness make plume presentation unreliable for short distances.

\section{TIME DEPENDENT INFORMATION:}

Concentration Estimates at the point:

Downwind: 100 meters

Off Centerline: 0 meters

Max Concentration:

Outdoor: $11.9 \mathrm{ppm}$

Indoor: $11.9 \mathrm{ppm}$

Note: Indoor graph is shown with a dotted line. 
Title of Project EPHA for THM

Subject Concentration Analysis for THM Hazardous Chemicals

Computer T. M. Franey
Date $\underline{09 / 16 / 96}$
Reviewed by S. A. Henderson

Works Calculation 7

Sheet No. 406 of 577

SITE DATA INFORMATION: 1-RD-23

Location: AIKEN, SOUTH CAROLINA

Building Air Exchanges Per Hour: 60 (User specified)

Date and Time: Fixed at July 1, 19961300 hours

CHEMICAL INFORMATION:

Chemical Name: DIETHYLENE GLYCOL DIMETHYL ETHER

Molecular Weight: $134.18 \mathrm{~kg} / \mathrm{kmol}$

TLV-TWA: -unavail- IDLH: -unavail-

Footprint Level of Concern: $25 \mathrm{ppm}$

Boiling Point: $159.76^{\circ} \mathrm{C}$

Vapor Pressure at Ambient Temperature: $0.0039 \mathrm{~atm}$

Ambient Saturation Concentration: 3,963 ppm or $0.40 \%$

ATMOSPHERIC INFORMATION: (MANUAL INPUT OF DATA)

Wind: 2.5 meters $/ \mathrm{sec}$ from $90^{\circ}$ true

Inversion Height: 500 meters

Stability Class: C Air Temperature: $25^{\circ} \mathrm{C}$

Relative Humidity: $50 \%$ Ground Roughness: 100 centimeters

Cloud Cover: 7 tenths

SOURCE STRENGTH INFORMATION:

Puddle Area: 10000 square meters

Average Puddle Depth: 1 centimeters

Soil Type: Default Ground Temperature: $25^{\circ} \mathrm{C}$

Initial Puddle Temperature: Ground temperature

Release Duration: ALOHA limited the duration to 1 hour

Max Computed Release Rate: 55 kilograms/min

Max Average Sustained Release Rate: 54.3 kilograms/min

(averaged over a minute or more)

Total Amount Released: 2,789 kilograms

FOOTPRINT INFORMATION:

Dispersion Module: Gaussian

User specified LOC: $25 \mathrm{ppm}$

Max Threat Zone for LOC: 89 meters

Note: Footprint was not drawn because

effects of near-field patchiness make plume

presentation unreliable for short distances.

\section{TIME DEPENDENT INFORMATION:}

Concentration Estimates at the point:

Downwind: 100 meters

Off Centerline: 0 meters

Max Concentration:

Outdoor: $20.6 \mathrm{ppm}$

Indoor: $20.6 \mathrm{ppm}$

Note: Indoor graph is shown with a dotted line. 


\section{ENGINEERING COMPUTATION SHEET}

Title of Project EPHA for THM

Subject Concentration Analysis for THM Hazardous Chemicals

Computer T. M. Franey

Date $\underline{09 / 16 / 96}$

Reviewed by $\underline{\text { S. A. Henderson }}$

Works Calculation 7

Sheet No. 407 of 577

SITE DATA INFORMATION: $\quad$ 1-RD-24

Location: AIKEN, SOUTH CAROLINA

Building Air Exchanges Per Hour: 60 (User specified)

Date and Time: Fixed at July 1, 19961300 hours

\section{CHEMICAL INFORMATTON:}

Chemical Name: HYDROGEN CHLORIDE 33-40\% / MSDS 6966

Molecular Weight: $36.46 \mathrm{~kg} / \mathrm{kmol}$

TLV-TWA: -unavail- IDLH: -unavail-

Footprint Level of Concern: 20 ppm

Boiling Point: $148.89^{\circ} \mathrm{C}$

Vapor Pressure at Ambient Temperature: $0.0012 \mathrm{~atm}$

Ambient Saturation Concentration: $1,206 \mathrm{ppm}$ or $0.12 \%$

ATMOSPHERIC INFORMATION: (MANUAL INPUT OF DATA)

Wind: 2.5 meters $/ \mathrm{sec}$ from $90^{\circ}$ true

Inversion Height: 500 meters

Stability Class: C Air Temperature: $25^{\circ} \mathrm{C}$

Relative Humidity: $50 \%$ Ground Roughness: 100 centimeters

Cloud Cover: 7 tenths

\section{SOURCE STRENGTH INFORMATION:}

Puddle Area: 13080 square feet

Average Puddle Depth: 1 centimeters

Soil Type: Default $\quad$ Ground Temperature: $25^{\circ} \mathrm{C}$

Initial Puddle Temperature: Ground temperature

Release Duration: ALOHA limited the duration to 1 hour

Max Computed Release Rate: 1.38 kilograms/min

Max Average Sustained Release Rate: 1.36 kilograms/min

(averaged over a minute or more)

Total Amount Released: 67.6 kilograms

FOOTPRINT INFORMATTON:

Dispersion Module: Gaussian

User specified LOC: $20 \mathrm{ppm}$

Max Threat Zone for LOC: 30 meters

Note: Footprint was not drawn because effects of near-field patchiness make plume presentation unreliable for short distances.

TIME DEPENDENT INFORMATION:

Concentration Estimates at the point:

Downwind: 30 meters

Off Centerline: 0 meters

Max Concentration:

Outdoor: $20 \mathrm{ppm}$

Indoor: $20 \mathrm{ppm}$

Note: Indoor graph is shown with a dotted line. 


\section{ENGINEERING COMPUTATION SHEET}

Title of Project EPHA for THM

Subject Concentration Analysis for THM Hazardous Chemicals

Computer T. M. Franey

Date $\underline{09 / 16 / 96}$

\section{SITE DATA INFORMATION: 1-RD-24}

Location: AIKEN, SOUTH CAROLINA

Building Air Exchanges Per Hour: 60 (User specified)

Date and Time: Fixed at July 1, 19961300 hours

CHEMICAL INFORMATION:

Chemical Name: HYDROGEN CHLORIDE 33-40\% / MSDS 6966

Molecular Weight: $36.46 \mathrm{~kg} / \mathrm{kmol}$

TLV-TWA: -unavail- IDLH: -unavail-

Footprint Level of Concern: $20 \mathrm{ppm}$

Boiling Point: $148.89^{\circ} \mathrm{C}$

Vapor Pressure at Ambient Temperature: 0.0012 atm

Ambient Saturation Concentration: 1,206 ppm or $0.12 \%$

ATMOSPHERIC INFORMATION: (MANUAL INPUT OF DATA)

Wind: 2.5 meters $/ \mathrm{sec}$ from $90^{\circ}$ true

Inversion Height: 500 meters

Stability Class: C Air Temperature: $25^{\circ} \mathrm{C}$

Relative Humidity: 50\% Ground Roughness: 100 centimeters

Cloud Cover: 7 tenths

SOURCE STRENGTH INFORMATION:

Puddle Area: 99999 square feet

Average Puddle Depth: 1 centimeters

Soil Type: Default Ground Temperature: $25^{\circ} \mathrm{C}$

Initial Puddle Temperature: Ground temperature

Release Duration: ALOHA limited the duration to 1 hour

Max Computed Release Rate: 9.69 kilograms/min

Max Average Sustained Release Rate: 9.54 kilograms/min

(averaged over a minute or more)

Total Amount Released: 472 kilograms

FOOTPRINT INFORMATION:

Dispersion Module: Gaussian

User specified LOC: $20 \mathrm{ppm}$

Max Threat Zone for LOC: 79 meters

Note: Footprint was not drawn because effects of near-field patchiness make plume presentation unreliable for short distances.

TIME DEPENDENT INFORMATION:

Concentration Estimates at the point:

Downwind: 100 meters

Off Centerline: 0 meters

Max Concentration:

Outdoor: $13.5 \mathrm{ppm}$

Indoor: $13.5 \mathrm{ppm}$

Note: Indoor graph is shown with a dotted line.
Reviewed by S. A. Henderson

Works Calculation 7

Sheet No. 408 of 577 


\section{ENGINEERING COMPUTATION SHEET}

Title of Project EPHA for THM

Subject Concentration Analysis for THM Hazardous Chemicals

Computer T. M. Franey

Date $\underline{09 / 16 / 96}$

SITE DATA INFORMATION: 1-RD-24

Location: AIKEN, SOUTH CAROLINA

Building Air Exchanges Per Hour: 60 (User specified)

Date and Time: Fixed at July 1, 19961300 hours

CHEMICAL INFORMATION:

Chemical Name: HYDROGEN CHLORIDE 33-40\% / MSDS 6966

Molecular Weight: $36.46 \mathrm{~kg} / \mathrm{kmol}$

TLV-TWA: -unavail- IDLH: -unavail-

Footprint Level of Concern: 20 ppm

Boiling Point: $148.89^{\circ} \mathrm{C}$

Vapor Pressure at Ambient Temperature: $0.0012 \mathrm{~atm}$

Ambient Saturation Concentration: $1,206 \mathrm{ppm}$ or $0.12 \%$

ATMOSPHERIC INFORMATION: (MANUAL INPUT OF DATA)

Wind: 2.5 meters $/ \mathrm{sec}$ from $90^{\circ}$ true

Inversion Height: 500 meters

Stability Class: C Air Temperature: $25^{\circ} \mathrm{C}$

Relative Humidity: 50\% Ground Roughness: 100 centimeters

Cloud Cover: 7 tenths

\section{SOURCE STRENGTH INFORMATION:}

Puddle Area: 24420 square feet

Average Puddle Depth: 1 centimeters

Soil Type: Default Ground Temperature: $25^{\circ} \mathrm{C}$

Initial Puddle Temperature: Ground temperature

Release Duration: ALOHA limited the duration to 1 hour

Max Computed Release Rate: 2.51 kilograms/min

Max Average Sustained Release Rate: 2.47 kilograms/min

(averaged over a minute or more)

Total Amount Released: 123 kilograms

\section{FOOTPRINT INFORMATION:}

Dispersion Module: Gaussian

User specified LOC: $20 \mathrm{ppm}$

Max Threat Zone for LOC: 41 meters

Note: Footprint was not drawn because

effects of near-field patchiness make plume

presentation unreliable for short distances.

\section{TIME DEPENDENT INFORMATION:}

Concentration Estimates at the point:

Downwind: 100 meters

Off Centerline: 0 meters

Max Concentration:

Outdoor: $6.55 \mathrm{ppm}$

Indoor: $6.55 \mathrm{ppm}$

Note: Indoor graph is shown with a dotted line.
Reviewed by $\mathrm{S}$. A. Henderson

Works Calculation 7

Sheet No. 409 of 577 
ENGINEERING COMPUTATION SHEET

Title of Project EPHA for THM

Subject Concentration Analysis for THM Hazardous Chemicals

Computer T. M. Franey

Date $\underline{09 / 16 / 96}$

SITE DATA INFORMATION: $\quad$ 1-RD-24

Location: AIKEN, SOUTH CAROLINA

Building Air Exchanges Per Hour: 60 (User specified)

Date and Time: Fixed at July 1, 19961300 hours

CHEMICAL INFORMATION:

Chemical Name: HYDROGEN CHLORIDE 33-40\% / MSDS 6966

Molecular Weight: $36.46 \mathrm{~kg} / \mathrm{kmol}$

TLV-TWA: -unavail- IDLH: -unavail-

Footprint Level of Concern: $20 \mathrm{ppm}$

Boiling Point: $148.89^{\circ} \mathrm{C}$

Vapor Pressure at Ambient Temperature: $0.0012 \mathrm{~atm}$

Ambient Saturation Concentration: $1,206 \mathrm{ppm}$ or $0.12 \%$

ATMOSPHERIC INFORMATION: (MANUAL INPUT OF DATA)

Wind: 2.5 meters $/ \mathrm{sec}$ from $90^{\circ}$ true

Inversion Height: 500 meters

Stability, Class: C Air Temperature: $25^{\circ} \mathrm{C}$

Relative Humidity: $50 \%$ Ground Roughness: 100 centimeters

Cloud Cover: 7 tenths

\section{SOURCE STRENGTH INFORMATION:}

Puddle Area: 40700 square feet

Average Puddle Depth: 1 centimeters

Soil Type: Default Ground Temperature: $25^{\circ} \mathrm{C}$

Initial Puddle Temperature: Ground temperature

Release Duration: ALOHA limited the duration to 1 hour

Max Computed Release Rate: 4.09 kilograms/min

Max Average'Sustained Release Rate: 4.03 kilograms/min

(averaged over a minute or more)

Total Amount Released: 200 kilograms

\section{FOOTPRINT INFORMATION:}

Dispersion Module: Gaussian

User specified LOC: $20 \mathrm{ppm}$

Max Threat Zone for LOC: 52 meters

Note: Footprint was not drawn because effects of near-field patchiness make plume

presentation unreliable for short distances.

\section{TIME DEPENDENT INFORMATION:}

Concentration Estimates at the point:

Downwind: 100 meters

Off Centerline: 0 meters

Max Concentration:

Outdoor: $8.29 \mathrm{ppm}$

Indoor: $8.29 \mathrm{ppm}$

Note: Indoor graph is shown with a dotted line.
Reviewed by $\mathrm{S}$. A. Henderson

Works Calculation 7

Sheet No. 410 of 577 


\section{ENGINEERING COMPUTATION SHEET}

Title of Project EPHA for THM

Subject Concentration Analysis for THM Hazardous Chemicals

Computer T. M. Franey Date $09 / 16 / 96$
Reviewed by $\underline{S}$. A. Hénderson Works Calculation 7 Sheet No. 411 of 577

\section{SITE DATA INFORMATION: 1-RD-24 \\ Location: AIKEN, SOUTH CAROLINA \\ Building Air Exchanges Per Hour: 60 (User specified) \\ Date and Time: Fixed at July 1, 19961300 hours}

\section{CHEMICAL INFORMATION:}

Chemical Name: HYDROGEN CHLORIDE 33-40\%/MSDS 6966

Molecular Weight: $36.46 \mathrm{~kg} / \mathrm{kmol}$

TLV-TWA: -unavail-

IDLH: -unavail-

Footprint Level of Concern: $20 \mathrm{ppm}$

Boiling Point: $148.89^{\circ} \mathrm{C}$

Vapor Pressure at Ambient Temperature: $0.0012 \mathrm{~atm}$

Ambient Saturation Concentration: $1,206 \mathrm{ppm}$ or $0.12 \%$

\section{ATMOSPHERIC INFORMATION: (MANUAL INPUT OF DATA)}

Wind: 2.5 meters $/ \mathrm{sec}$ from $90^{\circ}$ true

Inversion Height: 500 meters

Stability Class: C Air Temperature: $25^{\circ} \mathrm{C}$

Relative Humidity: 50\% Ground Roughness: 100 centimeters

Cloud Cover: 7 tenths

\section{SOURCE STRENGTH INFORMATION:}

Puddle Area: 10000 square meters

Average Puddle Depth: 1 centimeters

Soil Type: Default Ground Temperature: $25^{\circ} \mathrm{C}$

Initial Puddle Temperature: Ground temperature

Release Duration: ALOHA limited the duration to 1 hour

Max Computed Release-Rate: 10.4 kilograms/min

Max Average Sustained Release Rate: 10.2 kilograms/min

(averaged over a minute or more)

Total Amount Released: 507 kilograms

\section{FOOTPRINT INFORMATION:}

Dispersion Module: Gaussian

User specified LOC: $20 \mathrm{ppm}$

Max Threat Zone for LOC: 82 meters

Note: Footprint was not drawn because effects of near-field patchiness make plume presentation unreliable for short distances.

\section{TIME DEPENDENT INFORMATION:}

Concentration Estimates at the point:

Downwind: 100 meters

Off Centerline: 0 meters

Max Concentration:

Outdoor: $14.3 \mathrm{ppm}$

Indoor: $14.3 \mathrm{ppm}$

Note: Indoor graph is shown with a dotted line. 


\section{ENGINEERING COMPUTATION SHEET}

Title of Project EPHA for THM

Reviewed by $\mathrm{S}$. A. Henderson

Subject Concentration Analysis for THM Hazardous Chemicals

Computer T. M. Franey

Date $\underline{09 / 16 / 96}$

SITE DATA INFORMATION: 1-RD-25

Location: AIKEN, SOUTH CAROLINA

Building Air Exchanges Per Hour: 60 (User specified)

Date and Time: Fixed at July 1, 19961300 hours

CHEMICAL INFORMATION:

Chemical Name: HYDDUFLOURIC ACID - 70 WT\% - SAH

Molecular Weight: $20.01 \mathrm{~kg} / \mathrm{kmol}$

TLV-TWA: -unavail- IDLH: -unavail-

Footprint Level of Concern: 20 ppm

Boiling Point: $110.35^{\circ} \mathrm{C}$

Vapor Pressure at Ambient Temperature: $1.28 \mathrm{e}-004 \mathrm{~atm}$

Ambient Saturation Concentration: 130 ppm or $0.013 \%$

ATMOSPHERIC INFORMATION: (MANUAL INPUT OF DATA)

Wind: 2.5 meters $/ \mathrm{sec}$ from $90^{\circ}$ true

Inversion Height: 500 meters

Stability Class: C Air Temperature: $25^{\circ} \mathrm{C}$

Relative Humidity: 50\% Ground Roughness: 100 centimeters

Cloud Cover: 7 tenths

SOURCE STRENGTH INFORMATION:

Puddle Area: 30430 square feet

Average Puddle Depth: 1 centimeters

Soil Type: Default Ground Temperature: $25^{\circ} \mathrm{C}$

Initial Puddle Temperature: Ground temperature

Release Duration: ALOHA limited the duration to 1 hour

Max Computed Release Rate: 417 grams/min

Max Average Sustained Release Rate: 403 grams/min

(averaged over a minute or more)

Total Amount Released: 17.1 kilograms

\section{FOOTPRINT INFORMATION:}

Dispersion Module: Gaussian

User specified LOC: $20 \mathrm{ppm}$

Max Threat Zone for LOC: 30 meters

Note: Footprint was not drawn because effects of near-field patchiness make plume

presentation unreliable for short distances.

\section{TIME DEPENDENT INFORMATION:}

Concentration Estimates at the point:

Downwind: 30 meters

Off Centerline: 0 meters

Max Concentration:

Outdoor: $10.6 \mathrm{ppm}$

Indoor: $10.6 \mathrm{ppm}$

Note: Indoor graph is shown with a dotted line.
Works Calculation 7

Sheet No. 412 of 577 
Title of Project EPHA for THM

Subject Concentration Analysis for THM Hazardous Chemicals

Computer T. M. Franey
Reviewed by S.A. Henderson

Works Calculation 7

Sheet No. 413 of 577

\section{SITE DATA INFORMATION: 1-RD-25}

Location: AIKEN, SOUTH CAROLINA

Building Air Exchanges Per Hour: 60 (User specified)

Date and Time: Fixed at July 1, 19961300 hours

\section{CHEMICAL INFORMATION:}

Chemical Name: HYDDUFLOURIC ACID - 70 WT\% - SAH

Molecular Weight: $20.01 \mathrm{~kg} / \mathrm{kmol}$

TLV-TWA: -unavail- IDLH: -unavail-

Footprint Level of Concern: $20 \mathrm{ppm}$

Boiling Point: $110.35^{\circ} \mathrm{C}$

Vapor Pressure at Ambient Temperature: $1.28 \mathrm{e}-004 \mathrm{~atm}$

Ambient Saturation Concentration: $130 \mathrm{ppm}$ or $0.013 \%$

\section{ATMOSPHERIC INFORMATION: (MANUAL INPUT OF DATA) \\ Wind: 2.5 meters $/ \mathrm{sec}$ from $90^{\circ}$ true \\ Inversion Height: 500 meters \\ Stability Class: C Air Temperature: $25^{\circ} \mathrm{C}$ \\ Relative Humidity: 50\% Ground Roughness: 100 centimeters \\ Cloud Cover: 7 tenths}

\section{SOURCE STRENGTH INFORMATION:}

Puddle Area: 99999 square feet

Average Puddle Depth: 1 centimeters

Soil Type: Default Ground Temperature: $25^{\circ} \mathrm{C}$

Initial Puddle Temperature: Ground temperature

Release Duration: ALOHA limited the duration to 1 hour

Max Computed Release Rate: 1.31 kilograms/min

Max Average Sustained Release Rate: 1.26 kilograms/min.

(averaged over a minute or more)

Total Amount Released: 53.3 kilograms

\section{FOOTPRINT INFORMATION:}

Dispersion Module: Gaussian

User specified LOC: $20 \mathrm{ppm}$

Max Threat Zone for LOC: 55 meters

Note: Footprint was not drawn because effects of near-field patchiness make plume presentation unreliable for short distances.

\section{TIME DEPENDENT INFORMATION:}

Concentration Estimates at the point:

Downwind: 100 meters

Off Centerline: 0 meters

Max Concentration:

Outdoor: $3.27 \mathrm{ppm}$

Indoor: $3.27 \mathrm{ppm}$

Note: Indoor graph is shown with a dotted line. 
Title of Project EPHA for THM

Subject Concentration Analysis for THM Hazardous Chemicals

Computer T. M. Franey

Date 09/16/96
Reviewed by S. A. Henderson

Works Calculation 7

Sheet No. 414 of 577

\section{SITE DATA INFORMATION: 1-RD-25 \\ Location: AIKEN, SOUTH CAROLINA \\ Building Air Exchanges Per Hour: 60 (User specified) \\ Date and Time: Fixed at July 1, 19961300 hours}

CHEMICAL INFORMATION:

Chemical Name: HYDDUFLOURIC ACID - 70 WT\% - SAH

Molecular Weight: $20.01 \mathrm{~kg} / \mathrm{kmol}$

TLV-TWA: -unavail- IDLH: -unavail-

Footprint Level of Concern: 20 ppm

Boiling Point: $110.35^{\circ} \mathrm{C}$

Vapor Pressure at Ambient Temperature: $1.28 \mathrm{e}-004 \mathrm{~atm}$

Ambient Saturation Concentration: 130 ppm or $0.013 \%$

ATMOSPHERIC INFORMATION: (MANUAL INPUT OF DATA)

Wind: 2.5 meters $/ \mathrm{sec}$ from $90^{\circ}$ true

Inversion Height: 500 meters

Stability Class: C Air Temperature: $25^{\circ} \mathrm{C}$

Relative Humidity: 50\% Ground Roughness: 100 centimeters

Cloud Cover: 7 tenths

\section{SOURCE STRENGTH INFORMATION:}

Puddle Area: 24420 square feet

Average Puddle Depth: 1 centimeters

Soil Type: Default Ground Temperature: $25^{\circ} \mathrm{C}$

Initial Puddle Temperature: Ground temperature

Release Duration: ALOHA limited the duration to 1 hour

Max Computed Release Rate: 338 grams/min

Max Average.Sustained Release Rate: 326 grams/min (averaged over a minute or more)

Total Amount Released: 13.8 kilograms

FOOTPRINT INFORMATION:

Dispersion Module: Gaussian

User specified LOC: $20 \mathrm{ppm}$

Max Threat Zone for LOC: 27 meters

Note: Footprint was not drawn because effects of near-field patchiness make plume presentation unreliable for short distances.

TIME DEPENDENT INFORMATION:

Concentration Estimates at the point:

Downwind: 100 meters

Off Centerline: 0 meters

Max Concentration:

Outdoor: $1.58 \mathrm{ppm}$

Indoor: $1.58 \mathrm{ppm}$

Note: Indoor graph is shown with a dotted line. 
Title of Project EPHA for THM

Subject Concentration Analysis for THM Hazardous Chemicals

\section{Computer T. M. Franey} Date 09/16/96

SITE DATA INFORMATION: 1-RD-25

Location: AIKEN, SOUTH CAROLINA

Building Air Exchanges Per Hour: 60 (User specified)

Date and Time: Fixed at July 1, 19961300 hours

\section{CHEMICAL INFORMATION:}

Chemical Name: HYDDUFLOURIC ACID - 70 WT\% - SAH

Molecular Weight: $20.01 \mathrm{~kg} / \mathrm{kmol}$

TLV-TWA: -unavail- IDLH: -unavail-

Footprint Level of Concern: $20 \mathrm{ppm}$

Boiling Point: $110.35^{\circ} \mathrm{C}$

Vapor Pressure at Ambient Temperature: $1.28 \mathrm{e}-004 \mathrm{~atm}$

Ambient Saturation Concentration: 130 ppm or $0.013 \%$

\section{ATMOSPHERIC INFORMATION: (MANUAL INPUT OF DATA)}

Wind: 2.5 meters $/ \mathrm{sec}$ from $90^{\circ}$ true

Inversion Height: 500 meters

Stability Class: C Air Temperature: $25^{\circ} \mathrm{C}$

Relative Humidity: 50\% Ground Roughness: 100 centimeters

Cloud Cover: 7 tenths

\section{SOURCE STRENGTH INFORMATION:}

Puddle Area: 40700 square feet

Average Puddle Depth: 1 centimeters

Soil Type: Default Ground Temperature: $25^{\circ} \mathrm{C}$

Initial Puddle Temperature: Ground temperature

Release Duration: ALOHA limited the duration to 1 hour

Max Computed Release Rate: 552 grams/min

Max Average.Sustained Release Rate: 532 grams/min

(averaged over a minute or more)

Total Amount Released: 22.5 kilograms

\section{FOOTPRINT INFORMATION:}

Dispersion Module: Gaussian

User specified LOC: 20 ppm

Max Threat Zone for LOC: 34 meters

Note: Footprint was not drawn because effects of near-field patchiness make plume

presentation unreliable for short distances.

\section{TIME DEPENDENT INFORMATION:}

Concentration Estimates at the point:

Downwind: 100 meters

Off Centerline: 0 meters

Max Concentration:

Outdoor: 2 ppm

Indoor: $2 \mathrm{ppm}$

Note: Indoor graph is shown with a dotted line.
Reviewed by S. A. Henderson

Works Calculation 7

Sheet No. 415 of 577 


\section{ENGINEERING COMPUTATION SHEET}

Title of Project EPHA for THM

Subject Concentration Analysis for THM Hazardous Chemicals

Computer T. M. Franey

Date 09/16/96
Reviewed by S. A. Henderson

Works Calculation 7

Sheet No. 416 of 577

SITE DATA INFORMATION: 1-RD-25

Location: AIKEN, SOUTH CAROLINA

Building Air Exchanges Per Hour: 60 (User specified)

Date and Time: Fixed at July 1, 19961300 hours

CHEMICAL INFORMATION:

Chemical Name: HYDDUFLOURIC ACID - 70 WT\% - SAH

Molecular Weight: $20.01 \mathrm{~kg} / \mathrm{kmol}$

TLV-TWA: -unavail- IDLH: -unavail-

Footprint Level of Concern: $20 \mathrm{ppm}$

Boiling Point: $110.35^{\circ} \mathrm{C}$

Vapor Pressure at Ambient Temperature: $1.28 \mathrm{e}-004 \mathrm{~atm}$

Ambient Saturation Concentration: 130 ppm or $0.013 \%$

ATMOSPHERIC INFORMATION: (MANUAL INPUT OF DATA)

Wind: 2.5 meters $/ \mathrm{sec}$ from $90^{\circ}$ true

Inversion Height: 500 meters

Stability Class: C Air Temperature: $25^{\circ} \mathrm{C}$

Relative Humidity: $50 \%$ Ground Roughness: 100 centimeters

Cloud Cover: 7 tenths

- SOURCE STRENGTH INFORMATION:

Puddle Area: 10000 square meters

Average Puddle Depth: 1 centimeters

Soil Type: Default Ground Temperature: $25^{\circ} \mathrm{C}$

. Initial Puddle Temperature: Ground temperature

Release Duration: ALOHA limited the duration to 1 hour

Max Computed Release Rate: 1.41 kilograms/min

Max Average Sustained Release Rate: 1.36 kilograms/min

(averaged over a minute or more)

Total Amount Released: 57.1 kilograms

\section{FOOTPRINT INFORMATION:}

Dispersion Module: Gaussian

User specified LOC: $20 \mathrm{ppm}$

Max Threat Zone for LOC: 56 meters

Note: Footprint was not drawn because

effects of near-field patchiness make plume

presentation unreliable for short distances.

\section{TIME DEPENDENT INFORMATION:}

Concentration Estimates at the point:

Downwind: 100 meters

Off Centerline: 0 meters

Max Concentration:

Outdoor: $3.45 \mathrm{ppm}$

Indoor: $3.45 \mathrm{ppm}$

Note: Indoor graph is shown with a dotted line. 


\section{ENGINEERING COMPUTATION SHEET}

Title of Project EPHA for THM

Subject Concentration Analysis for THM Hazardous Chemicals

Computer T. M. Franey

Date 09/16/96
Reviewed by S. A. Henderson

Works Calculation 7

Sheet No. 417 of 577

SITE DATA INFORMATION: 1-RD-26

Location: AIKEN, SOUTH CAROLINA

Building Air Exchanges Per Hour: 60 (User specified)

Date and Time: Fixed at July 1, 19961300 hours

CHEMICAL INFORMATION:

Chemical Name: HYDROGEN PEROXIDE

Molecular Weight: $34.01 \mathrm{~kg} / \mathrm{kmol}$

TLV-TWA: $1.00 \mathrm{ppm}$ IDLH: $75.00 \mathrm{ppm}$

Footprint Level of Concern: $10 \mathrm{ppm}$

Boiling Point: $150.20^{\circ} \mathrm{C}$

Vapor Pressure at Ambient Temperature: $0.0026 \mathrm{~atm}$

Ambient Saturation Concentration: 2,636 ppm or $0.26 \%$

ATMOSPHERIC INFORMATION: (MANUAL INPUT OF DATA)

Wind: 2.5 meters $/ \mathrm{sec}$ from $90^{\circ}$ true

Inversion Height: 500 meters

Stability Class: C Air Temperature: $25^{\circ} \mathrm{C}$

Relative Humidity: 50\% Ground Roughness: 100 centimeters

Cloud Cover: 7 tenths

\section{SOURCE STRENGTH INFORMATION:}

Puddle Area: 3307 square feet

Average Puddle Depth: 1 centimeters

Soil Type: Default Ground Temperature: $25^{\circ} \mathrm{C}$

Initial Puddle Temperature: Ground temperature

Release Duration: ALOHA limited the duration to 1 hour

Max Computed Release Rate: 393 grams/min

Max Average Sustained Release Rate: 388 grams/min

(averaged over a minute or more)

Total Amount Released: 19.5 kilograms

FOOTPRINT INFORMATION:

Dispersion Module: Gaussian

User specified LOC: $10 \mathrm{ppm}$

Max Threat Zone for LOC: 30 meters

Max Threat Zone for DLH: 10 meters

Note: Footprint was not drawn because

effects of near-field patchiness make plume

presentation unreliable for short distances.

TIME DEPENDENT INFORMATION:

Concentration Estimates at the point:

Downwind: 30 meters

Off Centerline: 0 meters

Max Concentration:

Outdoor: $10 \mathrm{ppm}$

Indoor: $10 \mathrm{ppm}$

Note: Indoor graph is shown with a dotted line. 


\section{ENGINEERING COMPUTATION SHEET}

Title of Project EPHA for THM

Reviewed by S. A. Henderson

Subject Concentration Analysis for THM Hazardous Chemicals

Computer T. M. Franey

Date 09/16/96

SITE DATA INFORMATION: 1-RD-26

Location: AIKEN, SOUTH CAROLINA

Building Air Exchanges Per Hour: 60 (User specified)

Date and Time: Fixed at July 1, 19961300 hours

\section{CHEMICAL INFORMATION:}

Chemical Name: HYDROGEN PEROXIDE

Molecular Weight: $34.01 \mathrm{~kg} / \mathrm{kmol}$

TLV-TWA: $1.00 \mathrm{ppm}$ IDLH: $75.00 \mathrm{ppm}$

Footprint Level of Concern: $10 \mathrm{ppm}$

Boiling Point: $150.20^{\circ} \mathrm{C}$

Vapor Pressure at Ambient Temperature: $0.0026 \mathrm{~atm}$

Ambient Saturation Concentration: 2,636 ppm or 0.26\%

ATMOSPHERIC INFORMATION: (MANUAL INPUT OF DATA)

Wind: 2.5 meters $/ \mathrm{sec}$ from $90^{\circ}$ true

Inversion Height: 500 meters

Stability Class: C Air Temperature: $25^{\circ} \mathrm{C}$

Relative Humidity: 50\% Ground Roughness: 100 centimeters

Cloud Cover: 7 tenths

\section{SOURCE STRENGTH INFORMATION:}

Puddle Area: 46580 square feet

Average Puddle Depth: 1 centimeters

Soil Type: Default Ground Temperature: $25^{\circ} \mathrm{C}$

Initial Puddle Temperature: Ground temperature

Release Duration: ALOHA limited the duration to 1 hour

Max Computed Release Rate: 4.91 kilograms/min

Max Average Sustained Release Rate: 4.84 kilograms/min

(averaged over a minute or more)

Total Amount Released: 242 kilograms

\section{FOOTPRINT INFORMATION:}

Dispersion Module: Gaussian

User specified LOC: $10 \mathrm{ppm}$

Max Threat Zone for LOC: 100 meters

Max Threat Zone for IDLH: 37 meters

Note: Footprint was not drawn because effects of near-field patchiness make plume presentation unreliable for short distances.

\section{TIME DEPENDENT INFORMATION:}

Concentration Estimates at the point:

Downwind: 100 meters

Off Centerline: 0 meters

Max Concentration:

Outdoor: $10 \mathrm{ppm}$

Indoor: $10 \mathrm{ppm}$

Note: Indoor graph is shown with a dotted line.
Works Calculation 7

Sheet No. 418 of 577 


\section{ENGINEERING COMPUTATION SHEET}

Title of Project EPHA for THM

Reviewed by $\underline{\text { S. A. Henderson }}$

Subject Concentration Analysis for THM Hazardous Chemicals

Computer T.M. Franey

Date $\underline{09 / 16 / 96}$
Works Calculation 7

Sheet No. 419 of 577
SITE DATA INFORMATION: 1-RD-26

Location: AIKEN, SOUTH CAROLINA

Building Air Exchanges Per Hour: 60 (User specified)

Date and Time: Fixed at July 1, 19961300 hours

\section{CHEMICAL INFORMATION:}

Chemical Name: HYDROGEN PEROXIDE

Molecular Weight: $34.01 \mathrm{~kg} / \mathrm{kmol}$

TLV-TWA: $1.00 \mathrm{ppm}$ IDLH: $75.00 \mathrm{ppm}$

Footprint Level of Concern: $10 \mathrm{ppm}$

Boiling Point: $150.20^{\circ} \mathrm{C}$

Vapor Pressure at Ambient Temperature: $0.0026 \mathrm{~atm}$

Ambient Saturation Concentration: $2,636 \mathrm{ppm}$ or $0.26 \%$

ATMOSPHERIC INFORMATION: (MANUAL INPUT OF DATA)

Wind: 2.5 meters $/ \mathrm{sec}$ from $90^{\circ}$ true

Inversion Height: 500 meters

Stability Class: C Air Temperature: $25^{\circ} \mathrm{C}$

Relative Humidity: 50\% Ground Roughness: 100 centimeters

Cloud Cover: 7 tenths

\section{SOURCE STRENGTH INFORMATION:}

Puddle Area: 24420 square feet

Average Puddle Depth: 1 centimeters

Soil Type: Default Ground Temperature: $25^{\circ} \mathrm{C}$

Initial Puddle Temperature: Ground temperature

Release Duration: ALOHA limited the duration to 1 hour

Max Computed Release Rate: 2.65 kilograms/min

Max Average Sustained Release Rate: 2.61 kilograms/min (averaged over a minute or more)

Total Amount Released: 131 kilograms

\section{FOOTPRINT INFORMATION:}

Dispersion Module: Gaussian

User specified LOC: $10 \mathrm{ppm}$

Max Threat Zone for LOC: 74 meters

Max Threat Zone for IDLH: 27 meters

Note: Footprint was not drawn because effects of near-field patchiness make plume presentation unreliable for short distances.

\section{TIME DEPENDENT INFORMATION:}

Concentration Estimates at the point:

Downwind: 100 meters

Off Centerline: 0 meters

Max Concentration:

Outdoor: $7.42 \mathrm{ppm}$

Indoor: $7.42 \mathrm{ppm}$

Note: Indoor graph is shown with a dotted line. 
Title of Project EPHA for THM

Subject Concentration Analysis for THM Hazardous Chemicals

Computer T. M. Franey

Date $\underline{09 / 16 / 96}$
Reviewed by S. A. Henderson

Works Calculation 7

Sheet No. $\underline{420 \text { of } 577}$

SITE DATA INFORMATION: 1-RD-26

Location: AIKEN, SOUTH CAROLINA

Building Air Exchanges Per Hour: 60 (User specified)

Date and Time: Fixed at July 1, 19961300 hours

CHEMICAL INFORMATION:

Chemical Name: HYDROGEN PEROXIDE

Molecular Weight: $34.01 \mathrm{~kg} / \mathrm{kmol}$

TLV-TWA: $1.00 \mathrm{ppm}$ IDLH: $75.00 \mathrm{ppm}$

Footprint Level of Concern: $10 \mathrm{ppm}$

Boiling Point: $150.20^{\circ} \mathrm{C}$

Vapor Pressure at Ambient Temperature: 0.0026 atm

Ambient Saturation Concentration: $2,636 \mathrm{ppm}$ or $0.26 \%$

ATMOSPHERIC INFORMATION: (MANUAL INPUT OF DATA)

Wind: 2.5 meters $/ \mathrm{sec}$ from $90^{\circ}$ true

Inversion Height: 500 meters

Stability Class: C Air Temperature: $25^{\circ} \mathrm{C}$

Relative Humidity: 50\% Ground Roughness: 100 centimeters

Cloud Cover: 7 tenths

SOURCE STRENGTH INFORMATION:

Puddle Area: 40700 square feet

Average Puddle Depth: 1 centimeters

Soil Type: Default Ground Temperature: $25^{\circ} \mathrm{C}$

Initial Puddle Temperature: Ground temperature

Release Duration: ALOHA limited the duration to 1 hour

Max Computed Release Rate: 4.32 kilograms/min

Max Average Sustained Release Rate: 4.25 kilograms/min

(averaged over a minute or more)

Total Amount Released: 212 kilograms

FOOTPRINT INFORMATION:

Dispersion Module: Gaussian

User specified LOC: $10 \mathrm{ppm}$

Max Threat Zone for LOC: 94 meters

Max Threat Zone for IDLH: 34 meters

Note: Footprint was not drawn because effects of near-field patchiness make plume presentation unreliable for short distances.

TIME DEPENDENT INFORMATION:

Concentration Estimates at the point:

Downwind: 100 meters

Off Centerline: 0 meters

Max Concentration:

Outdoor: $9.39 \mathrm{ppm}$

Indoor: $9.39 \mathrm{ppm}$

Note: Indoor graph is shown with a dotted line. 


\section{ENGINEERING COMPUTATION SHEET}

Title of Project EPHA for THM

Reviewed by S. A. Henderson

Subject Concentration Analysis for THM Hazardous Chemicals

Computer T. M. Franey

Date $\underline{09 / 16 / 96}$

SITE DATA INFORMATION: $\quad$ 1-RD-26

Location: AIKEN, SOUTH CAROLINA

Building Air Exchanges Per Hour: 60 (User specified)

Date and Time: Fixed at July 1, 19961300 hours

CHEMICAL INFORMATION:

Chemical Name: HYDROGEN PEROXIDE

Molecular Weight: $34.01 \mathrm{~kg} / \mathrm{kmol}$

TLV-TWA: $1.00 \mathrm{ppm}$ IDLH: $75.00 \mathrm{ppm}$

Footprint Level of Concern: 10 ppm

Boiling Point: $150.20^{\circ} \mathrm{C}$

Vapor Pressure at Ambient Temperature: $0.0026 \mathrm{~atm}$

Ambient Saturation Concentration: $2,636 \mathrm{ppm}$ or $0.26 \%$

ATMOSPHERIC INFORMATION: (MANUAL INPUT OF DATA)

Wind: 2.5 meters $/ \mathrm{sec}$ from $90^{\circ}$ true

Inversion Height: 500 meters

Stability Class: C Air Temperature: $25^{\circ} \mathrm{C}$

Relative Humidity: 50\% Ground Roughness: 100 centimeters

Cloud Cover: 7 tenths

SOURCE STRENGTH INFORMATION:

Puddle Area: 10000 square meters

Average Puddle Depth: 1 centimeters

Soil Type: Default Ground Temperature: $25^{\circ} \mathrm{C}$

Initial Puddle Temperature: Ground temperature

Release Duration: ALOHA limited the duration to 1 hour

Max Computed Release Rate: 11 kilograms/min

Max Average Sustained Release Rate: 10.8 kilograms/min

(averaged over a minute or more)

Total Amount Released: 538 kilograms

FOOTPRINT INFORMATION:

Dispersion Module: Gaussian

User specified LOC: $10 \mathrm{ppm}$

Max Threat Zone for LOC: 147 meters

Max Threat Zone for IDLH: 56 meters

TIME DEPENDENT INFORMATION:

Concentration Estimates at the point:

Downwind: 100 meters

Off Centerline: 0 meters

Max Concentration:

Outdoor: $16.1 \mathrm{ppm}$

Indoor: $16.1 \mathrm{ppm}$

Note: Indoor graph is shown with a dotted line.
Works Calculation 7

Sheet No. 421 of 577 
Title of Project EPHA for THM

Subject Concentration Analysis for THM Hazardous Chemicals

Computer T. M. Franey

Date $\underline{09 / 16 / 96}$
Reviewed by S. A. Henderson

Works Calculation 7

Sheet No. $\underline{422 \text { of } 577}$

\section{SITE DATA INFORMATION: 1-RD-27}

Location: AIKEN, SOUTH CAROLINA

Building Air Exchanges Per Hour: 60 (User specified)

Date and Time: Fixed at July 1, 19961300 hours

\section{CHEMICAL INFORMATION:}

Chemical Name: ISOPROPANOL

Molecular Weight: $60.10 \mathrm{~kg} / \mathrm{kmol}$

TLV-TWA: $400.00 \mathrm{ppm}$ IDLH: $12000.00 \mathrm{ppm}$

Footprint Level of Concern: 400 ppm

Boiling Point: $82.26^{\circ} \mathrm{C}$

Vapor Pressure at Ambient Temperature: $0.060 \mathrm{~atm}$

Ambient Saturation Concentration: $61,017 \mathrm{ppm}$ or $6.10 \%$

\section{ATMOSPHERIC INFORMATION: (MANUAL INPUT OF DATA)}

Wind: 2.5 meters $/ \mathrm{sec}$ from $90^{\circ}$ true

Inversion Height: 500 meters

Stability Class: C Air Temperature: $25^{\circ} \mathrm{C}$

Relative Humidity: 50\% Ground Roughness: 100 centimeters

Cloud Cover: 7 tenths

SOURCE STRENGTH INFORMATION:

Puddle Area: 9600 square feet

Average Puddle Depth: 1 centimeters

Soil Type: Default Ground Temperature: $25^{\circ} \mathrm{C}$

Initial Puddle Temperature: Ground temperature

Release Duration: ALOHA limited the duration to 1 hour

Max Computed Release Rate: 42.8 kilograms/min

Max Average Sustained Release Rate: 42.5 kilograms/min

(averaged over a minute or more)

Total Amount Released: 2,542 kilograms

\section{FOOTPRINT INFORMATION:}

Dispersion Module: Gaussian

User specified LOC: 400 ppm

Max Threat Zone for LOC: 30 meters

Max Threat Zone for IDLH: 17 meters

Note: Footprint was not drawn because effects of near-field patchiness make plume presentation unreliable for short distances.

\section{TIME DEPENDENT INFORMATION:}

Concentration Estimates at the point:

Downwind: 30 meters

Off Centerline: 0 meters

Max Concentration:

Outdoor: $400 \mathrm{ppm}$

Indoor: $400 \mathrm{ppm}$

Note: Indoor graph is shown with a dotted line. 


\section{ENGINEERING COMPUTATION SHEET}

Title of Project EPHA for THM

Subject Concentration Analysis for THM Hazardous Chemicals

Computer T.M. Franey

Date $\underline{09 / 16 / 96}$

SITE DATA INFORMATION: 1-RD-27

Location: AIKEN, SOUTH CAROLINA

Building Air Exchanges Per Hour: 60 (User specified)

Date and Time: Fixed at July 1, 19961300 hours

CHEMICAL INFORMATION:

Chemical Name: ISOPROPANOL

Molecular Weight: $60.10 \mathrm{~kg} / \mathrm{kmol}$

TLV-TWA: $400.00 \mathrm{ppm}$ IDLH: $12000.00 \mathrm{ppm}$

Footprint Level of Concern: 400 ppm

Boiling Point: $82.26^{\circ} \mathrm{C}$

Vapor Pressure at Ambient Temperature: $0.060 \mathrm{~atm}$

Ambient Saturation Concentration: 61,017 ppm or $6.10 \%$

ATMOSPHERIC INFORMATION: (MANUAL INPUT OF DATA)

Wind: 2.5 meters $/ \mathrm{sec}$ from $90^{\circ}$ true

Inversion Height: 500 meters

Stability Class: C Air Temperature: $25^{\circ} \mathrm{C}$

Relative Humidity: $50 \%$ Ground Roughness: 100 centimeters

Cloud Cover: 7 tenths

\section{SOURCE STRENGTH INFORMATION:}

Puddle Area: 36000 square feet

Average Puddle Depth: 1 centimeters

Soil Type: Default Ground Temperature: $25^{\circ} \mathrm{C}$

Initial Puddle Temperature: Ground temperature

Release Duration: ALOHA limited the duration to 1 hour

Max Computed Release Rate: 152 kilograms/min

Max Average Sustained Release Rate: 152 kilograms/min (averaged over a minute or more)

Total Amount Released: 9,123 kilograms

\section{FOOTPRINT INFORMATION:}

Dispersion Module: Gaussian

User specified LOC: $400 \mathrm{ppm}$

Max Threat Zone for LOC: 56 meters

- Max Threat Zone for IDLH: 33 meters

Note: Footprint was not drawn because

effects of near-field patchiness make plume

presentation unreliable for short distances.

\section{TIME DEPENDENT INFORMATION:}

Concentration Estimates at the point:

Downwind: 100 meters

Off Centerline: 0 meters

Max Concentration:

Outdoor: $202 \mathrm{ppm}$

Indoor: $202 \mathrm{ppm}$

Note: Indoor graph is shown with a dotted line.
Reviewed by $\underline{\text { S. A. Henderson }}$

Works Calculation 7

Sheet No. 423 of 577 


\section{ENGINEERING COMPUTATION SHEET}

Title of Project EPHA for THM

Subject Concentration Analysis for THM Hazardous Chemicals

Computer T.M. Franey
Reviewed by S. A. Henderson

Works Calculation 7

Sheet No. $\underline{424 \text { of } 577}$

\section{SITE DATA INFORMATION: , 1-RD-27}

Location: AIKEN, SOUTH CAROLINA

Building Air Exchanges Per Hour: 60 (User specified)

Date and Time: Fixed at July 1, 19961300 hours

CHEMICAL INFORMATION:

Chemical Name: ISOPROPANOL

Molecular Weight: $60.10 \mathrm{~kg} / \mathrm{kmol}$

TLV-TWA: $400.00 \mathrm{ppm}$ IDLH: $12000.00 \mathrm{ppm}$

Footprint Level of Concern: 400 ppm

Boiling Point: $82.26^{\circ} \mathrm{C}$

Vapor Pressure at Ambient Temperature: $0.060 \mathrm{~atm}$

Ambient Saturation Concentration: 61,017 ppm or $6.10 \%$

ATMOSPHERIC INFORMATION: (MANUAL INPUT OF DATA)

Wind: 2.5 meters $/ \mathrm{sec}$ from $90^{\circ}$ true

Inversion Height: 500 meters

Stability Class: C Air Temperature: $25^{\circ} \mathrm{C}$

Relative Humidity: 50\% Ground Roughness: 100 centimeters

Cloud Cover: 7 tenths

SOURCE STRENGTH INFORMATION:

Puddle Area: 24420 square feet

Average Puddle Depth: 1 centimeters

Soil Type: Default Ground Temperature: $25^{\circ} \mathrm{C}$

Initial Puddle Temperature: Ground temperature

Release Duration: ALOHA limited the duration to 1 hour

Max Computed Release Rate: $105 \mathrm{kilograms} / \mathrm{min}$

Max Average-Sustained Release Rate: 105 kilograms/min

(averaged over a minute or more)

Total Amount Released: 6,267 kilograms

FOOTPRINT INFORMATION:

Dispersion Module: Gaussian

User specified LOC: $400 \mathrm{ppm}$

Max Threat Zone for LOC: 47 meters

Max Threat Zone for IDLH: 27 meters

Note: Footprint was not drawn because effects of near-field patchiness make plume

presentation unreliable for short distances.

TIME DEPENDENT INFORMATION:

Concentration Estimates at the point:

Downwind: 100 meters

Off Centerline: 0 meters

Max Concentration:

Outdoor: $168 \mathrm{ppm}$

Indoor: $168 \mathrm{ppm}$

Note: Indoor graph is shown with a dotted line. 


\section{ENGINEERING COMPUTATION SHEET}

Title of Project EPHA for THM

Subject Concentration Analysis for THM Hazardous Chemicals

Computer T. M. Franey

Date 09/16/96

SITE DATA INFORMATION: 1-RD-27

Location: AIKEN, SOUTH CAROLINA

Building Air Exchanges Per Hour: 60 (User specified)

Date and Time: Fixed at July 1, 19961300 hours

CHEMICAL INFORMATION:

Chemical Name: ISOPROPANOL

Molecular Weight: $60.10 \mathrm{~kg} / \mathrm{kmol}$

TLV-TWA: $400.00 \mathrm{ppm}$ IDLH: $12000.00 \mathrm{ppm}$

Footprint Level of Concern: 400 ppm

Boiling Point: $82.26^{\circ} \mathrm{C}$

Vapor Pressure at Ambient Temperature: $0.060 \mathrm{~atm}$

Ambient Saturation Concentration: $61,017 \mathrm{ppm}$ or $6.10 \%$

ATMOSPHERIC INFORMATION: (MANUAL INPUT OF DATA)

Wind: 2.5 meters $/ \mathrm{sec}$ from $90^{\circ}$ true

Inversion Height: 500 meters

Stability Class: C Air Temperature: $25^{\circ} \mathrm{C}$

Relative Humidity: 50\% Ground Roughness: 100 centimeters

Cloud Cover: 7 tenths

SOURCE STRENGTH INFORMATION:

Puddle Area: 40700 square feet

Average Puddle Depth: 1 centimeters

Soil Type: Default Ground Temperature: $25^{\circ} \mathrm{C}$

Initial Puddle Temperature: Ground temperature

Release Duration: ALOHA limited the duration to 1 hour

Max Computed Release Rate: 172 kilograms/min

Max Average Sustained Release Rate: 172 kilograms/min

(averaged over a minute or more)

Total Amount Released: 10,273 kilograms

FOOTPRINT INFORMATION:

Model Run: Heavy Gas

User specified LOC: $400 \mathrm{ppm}$

Max Threat Zone for LOC: 248 meters

Max Threat Zone for IDLH: IDLH level was never exceeded

Note: The Heavy Gas footprint is an initial screening.

For short releases it may be an overestimation.

Be sure to check concentration information at specific locations.

\section{TIME DEPENDENT INFORMATION:}

Concentration Estimates at the point:

Downwind: 100 meters

Off Centerline: 0 meters

Max Concentration:

Outdoor: $1,270 \mathrm{ppm}$

Indoor: $1,270 \mathrm{ppm}$

Note: Indoor graph is shown with a dotted line.
Reviewed by S. A. Henderson

Works Calculation 7

Sheet No. 425 of 577 
Title of Project EPHA for THM

Subject Concentration Analysis for THM Hazardous Chemicals

Computer T. M. Franey
Date $\underline{09 / 16 / 96}$
Reviewed by $\mathrm{S}$. A. Henderson

Works Calculation 7

Sheet No. 426 of 577

SITE DATA INFORMATION: 1-RD-27

Location: AIKEN, SOUTH CAROLINA

Building Air Exchanges Per Hour: 60 (User specified)

Date and Time: Fixed at July 1, 19961300 hours

CHEMICAL INFORMATION:

Chemical Name: ISOPROPANOL

Molecular Weight: $60.10 \mathrm{~kg} / \mathrm{kmol}$

TLV-TWA: $400.00 \mathrm{ppm}$ IDLH: $12000.00 \mathrm{ppm}$

Footprint Level of Concern: $400 \mathrm{ppm}$

Boiling Point: $82.26^{\circ} \mathrm{C}$

Vapor Pressure at Ambient Temperature: $0.060 \mathrm{~atm}$

Ambient Saturation Concentration: $61,017 \mathrm{ppm}$ or $6.10 \%$

ATMOSPHERIC INFORMATION: (MANUAL INPUT OF DATA)

Wind: 2.5 meters $/ \mathrm{sec}$ from $90^{\circ}$ true

Inversion Height: 500 meters

Stability Class: C Air Temperature: $25^{\circ} \mathrm{C}$

Relative Humidity: $50 \%$ Ground Roughness: 100 centimeters

Cloud Cover: 7 tenths

SOURCE STRENGTH INFORMATION:

Puddle Area: 10000 square meters

Average Puddle Depth: 1 centimeters

Soil Type: Default Ground Temperature: $25^{\circ} \mathrm{C}$

Initial Puddle Temperature: Ground temperature

Release Duration: ALOHA limited the duration to 1 hour

Max Computed Release Rate: 442 kilograms/min

Max Average Sustained Release Rate: $\mathbf{4 4 2}$ kilograms/min

(averaged over a minute or more)

Total Amount Released: 26,343 kilograms

FOOTPRINT INFORMATION:

Model Run: Heavy Gas

User specified LOC: $400 \mathrm{ppm}$.

Max Threat Zone for LOC: 408 meters

Max Threat Zone for IDLH: IDLH level was never exceeded

Note: The Heavy Gas footprint is an initial screening.

For short releases it may be an overestimation.

Be sure to check concentration information at specific locations.

TIME DEPENDENT INFORMATION:

Concentration Estimates at the point:

Downwind: 100 meters

Off Centerline: 0 meters

Max Concentration:

Outdoor: $2,240 \mathrm{ppm}$

Indoor: $2,240 \mathrm{ppm}$

Note: Indoor graph is shown with a dotted line. 
Title of Project EPHA for THM

Subject Concentration Analysis for THM Hazardous Chemicals

Computer T. M. Franey

Date $\underline{09 / 16 / 96}$
Reviewed by $\underline{\text { S. A. Henderson }}$

Works Calculation 7

Sheet No. 427 of 577

SITE DATA INFORMATION: 1-RD-28

Location: AIKEN, SOUTH CAROLINA

Building Air Exchanges Per Hour: 60 (User specified)

Date and Time: Fixed at July 1, 19961300 hours

CHEMICAL INFORMATION:

Chemical Name: METHYL ALCOHOL

Molecular Weight: $32.04 \mathrm{~kg} / \mathrm{kmol}$

TLV-TWA: $200.00 \mathrm{ppm}$ IDLH: $25000.00 \mathrm{ppm}$

Footprint Level of Concern: $1000 \mathrm{ppm}$

Boiling Point: $64.70^{\circ} \mathrm{C}$

Vapor Pressure at Ambient Temperature: $0.17 \mathrm{~atm}$

Ambient Saturation Concentration: 168,779 ppm or $16.9 \%$

ATMOSPHERIC INFORMATION: (MANUAL INPUT OF DATA)

Wind: 2.5 meters $/ \mathrm{sec}$ from $90^{\circ}$ true

Inversion Height: 500 meters

Stability Class: C Air Temperature: $25^{\circ} \mathrm{C}$

Relative Humidity: 50\% Ground Roughness: 100 centimeters

Cloud Cover: 7 tenths

SOURCE STRENGTH INFORMATION:

Puddle Area: 12200 square feet

Average Puddle Depth: 1 centimeters

Soil Type: Default Ground Temperature: $25^{\circ} \mathrm{C}$

Initial Puddle Temperature: Ground temperature

Release Duration: ALOHA limited the duration to 1 hour

Max Computed Release Rate: 69.2 kilograms/min

Max Average Sustained Release Rate: 59.2 kilograms/min

(averaged over a minute or more)

Total Amount Released: 3,148 kilograms

FOOTPRINT INFORMATION:

Dispersion Module: Gaussian

User specified LOC: $1000 \mathrm{ppm}$

Max Threat Zone for LOC: 30 meters

Max Threat Zone for IDLH: 19 meters

Note: Footprint was not drawn because effects of near-field patchiness make plume presentation unreliable for short distances.

\section{TIME DEPENDENT INFORMATION:}

Concentration Estimates at the point:

Downwind: 30 meters

Off Centerline: 0 meters

Max Concentration:

Outdoor: 1,000 ppm

Indoor: $1,000 \mathrm{ppm}$

Note: Indoor graph is shown with a dotted line. 
Title of Project EPHA for THM

Subject Concentration Analysis for THM Hazardous Chemicals

Computer T. M. Franey

Date 09/16/96
Reviewed by $\underline{\text { S. A. Henderson }}$

Works Calculation 7

Sheet No. $\underline{428 \text { of } 577}$

\section{SITE DATA INFORMATION: 1-RD-28 \\ Location: AIKEN, SOUTH CAROLINA \\ Building Air Exchanges Per Hour: 60 (User specified) \\ Date and Time: Fixed at July 1, 19961300 hours}

\section{CHEMICAL INFORMATION:}

Chemical Name: METHYL ALCOHOL

Molecular Weight: $32.04 \mathrm{~kg} / \mathrm{kmol}$

TLV-TWA: $200.00 \mathrm{ppm}$ IDLH: $25000.00 \mathrm{ppm}$

Footprint Level of Concern: $1000 \mathrm{ppm}$

Boiling Point: $64.70^{\circ} \mathrm{C}$

Vapor Pressure at Ambient Temperature: $0.17 \mathrm{~atm}$

Ambient Saturation Concentration: $168,779 \mathrm{ppm}$ or $16.9 \%$

\section{ATMOSPHERIC INFORMATION: (MANUAL INPUT OF DATA) \\ Wind: 2.5 meters $/ \mathrm{sec}$ from $90^{\circ}$ true Inversion Height: 500 meters \\ Stability Class: C Air Temperature: $25^{\circ} \mathrm{C}$ \\ Relative Humidity: 50\% Ground Roughness: 100 centimeters \\ Cloud Cover: 7 tenths}

\section{SOURCE STRENGTH INFORMATION:}

Puddle Area: 99999 square feet

Average Puddle Depth: 1 centimeters

Soil Type: Default Ground Temperature: $25^{\circ} \mathrm{C}$

Initial Puddle Temperature: Ground temperature

Release Duration: ALOHA limited the duration to 1 hour

Max Computed Release Rate: 512 kilograms/min

Max Average Sustained Release Rate: 448 kilograms/min (averaged over a minute or more)

Total Amount Released: 24,221 kilograms

\section{FOOTPRINT INFORMATION:}

Dispersion Module: Gaussian

User specified LOC: 1000 ppm

Max Threat Zone for LOC: 82 meters

Max Threat Zone for IDLH: 55 meters

Note: Footprint was not drawn because effects of near-field patchiness make plume presentation unreliable for short distances.

\section{TIME DEPENDENT INFORMATION:}

\section{Concentration Estimates at the point:}

Downwind: 100 meters

Off Centerline: 0 meters

Max Concentration:

Outdoor: 724 ppm

Indoor: $724 \mathrm{ppm}$

Note: Indoor graph is shown with a dotted line. 


\section{ENGINEERING COMPUTATION SHEET}

Title of Project EPHA for THM

Subject Concentration Analysis for THM Hazardous Chemicals

Computer T.M. Franey

Date 09/16/96
Reviewed by S.A. Henderson

Works Calculation 7

Sheet No. $\underline{429 \text { of } 577}$

SITE DATA INFORMATION: 1-RD-28

Location: AIKEN, SOUTH CAROLINA

Building Air Exchanges Per Hour: 60 (User specified)

Date and Time: Fixed at July 1, 19961300 hours

CHEMICAL INFORMATION:

Chemical Name: METHYL ALCOHOL

Molecular Weight: $32.04 \mathrm{~kg} / \mathrm{kmol}$

TLV-TWA: $200.00 \mathrm{ppm}$ IDLH: $25000.00 \mathrm{ppm}$

Footprint Level of Concern: 1000 ppm

Boiling Point: $64.70^{\circ} \mathrm{C}$

Vapor Pressure at Ambient Temperature: $0.17 \mathrm{~atm}$

Ambient Saturation Concentration: 168,779 ppm or $16.9 \%$

ATMOSPHERIC INFORMATION: (MANUAL INPUT OF DATA)

Wind: 2.5 meters $/ \mathrm{sec}$ from $90^{\circ}$ true

Inversion Height: 500 meters

Stability Class: C Air Temperature: $25^{\circ} \mathrm{C}$

Relative Humidity: $50 \%$ Ground Roughness: 100 centimeters

Cloud Cover: 7 tenths

SOURCE STRENGTH INFORMATION:

Puddle Area: 24420 square feet

Average Puddle Depth: 1 centimeters

Soil Type: Default $\quad$ Ground Temperature: $25^{\circ} \mathrm{C}$

Initial Puddle Temperature: Ground temperature

Release Duration: ALOHA limited the duration to 1 hour

Max Computed Release Rate: 134 kilograms/min

Max Average Sustained Release Rate: 115 kilograms/min (averaged over a minute or more)

Total Amount Released: 6,168 kilograms

FOOTPRINT INFORMATION:

Dispersion Module: Gaussian

User specified LOC: 1000 ppm

Max Threat Zone for LOC: 42 meters

Max Threat Zone for IDLH: 27 meters

Note: Footprint was not drawn because effects of near-field patchiness make plume presentation unreliable for short distances.

TIME DEPENDENT INFORMATION:

Concentration Estimates at the point:

Downwind: 100 meters

Off Centerline: 0 meters

Max Concentration:

Outdoor: $348 \mathrm{ppm}$

Indoor: $348 \mathrm{ppm}$

Note: Indoor graph is shown with a dotted line. 
Title of Project EPHA for THM

Subject Concentration Analysis for THM Hazardous Chemicals

Computer T.M. Franey
SITE DATA INFORMATION: 1-RD-28
Location: AIKEN, SOUTH CAROLINA
Building Air Exchanges Per Hour: 60 (User specified)
Date and Time: Fixed at July 1, 19961300 hours

CHEMICAL INFORMATION:

Chemical Name: METHYL ALCOHOL

Molecular Weight: $32.04 \mathrm{~kg} / \mathrm{kmol}$

TLV-TWA: $200.00 \mathrm{ppm}$ IDLH: $25000.00 \mathrm{ppm}$

Footprint Level of Concern: 1000 ppm

Boiling Point: $64.70^{\circ} \mathrm{C}$

Vapor Pressure at Ambient Temperature: 0.17 atm

Ambient Saturation Concentration: 168,779 ppm or $16.9 \%$

\section{ATMOSPHERIC INFORMATION: (MANUAL INPUT OF DATA)}

Wind: 2.5 meters $/ \mathrm{sec}$ from $90^{\circ}$ true

Inversion Height: 500 meters

Stability Class: C Air Temperature: $25^{\circ} \mathrm{C}$

Relative Humidity: $50 \%$ Ground Roughness: 100 centimeters

Cloud Cover: 7 tenths

\section{SOURCE STRENGTH INFORMATION:}

Puddle Area: 40700 square feet

Average Puddle Depth: 1 centimeters

Soil Type: Default Ground Temperature: $25^{\circ} \mathrm{C}$

Initial Puddle Temperature: Ground temperature

Release Duration: ALOHA limited the duration to 1 hour

Max Computed Release Rate: 218 kilograms/min

Max Average Sustained Release Rate: 189 kilograms/min

(averaged over a minute or more)

Total Amount Released: 10,122 kilograms

\section{FOOTPRINT INFORMATION:}

Dispersion Module: Gaussian

User specified LOC: $1000 \mathrm{ppm}$

Max Threat Zone for LOC: 53 meters

Max Threat Zone for IDLH: 34 meters

Note: Footprint was not drawn because effects of near-field patchiness make plume presentation unreliable for short distances.

\section{TIME DEPENDENT INFORMATION:}

Concentration Estimates at the point:

Downwind: 100 meters

Off Centerline: 0 meters

Max Concentration:

Outdoor: 442 ppm

Indoor: $442 \mathrm{ppm}$

Note: Indoor graph is shown with a dotted line.
Reviewed by S. A. Henderson

Works Calculation 7

Sheet No. 430 of 577 
Title of Project EPHA for THM

Subject Concentration Analysis for THM Hazardous Chemicals

Computer T. M. Franey Date 09/16/96

SITE DATA INFORMATION: 1-RD-28

Location: AIKEN, SOUTH CAROLINA

Building Air Exchanges Per Hour: 60 (User specified)

Date and Time: Fixed at July 1, 19961300 hours

CHEMICAL INFORMATION:

Chemical Name: METHYL ALCOHOL

Molecular Weight: $32.04 \mathrm{~kg} / \mathrm{kmol}$

TLV-TWA: $200.00 \mathrm{ppm}$ IDLH: $25000.00 \mathrm{ppm}$

Footprint Level of Concern: $1000 \mathrm{ppm}$

Boiling Point: $64.70^{\circ} \mathrm{C}$

Vapor Pressure at Ambient Temperature: $0.17 \mathrm{~atm}$

Ambient Saturation Concentration: $168,779 \mathrm{ppm}$ or $16.9 \%$

ATMOSPHERIC INFORMATION: (MANUAL INPUT OF DATA)

Wind: 2.5 meters $/ \mathrm{sec}$ from $90^{\circ}$ true

Inversion Height: 500 meters

Stability Class: C Air Temperature: $25^{\circ} \mathrm{C}$

Relative Humidity: $50 \%$ Ground Roughness: 100 centimeters

Cloud Cover: 7 tenths

\section{SOURCE STRENGTH INFORMATION:}

Puddle Area: 10000 square meters

Average Puddle Depth: 1 centimeters

Soil Type: Default Ground Temperature: $25^{\circ} \mathrm{C}$

Initial Puddle Temperature: Ground temperature

Release Duration: ALOHA limited the duration to 1 hour

Max Computed Release Rate: 550 kilograms/min

Max Average Sustained Release Rate: 481 kilograms/min

(averaged over a minute or more)

Total Amount Released: 26,016 kilograms

\section{FOOTPRINT INFORMATION:}

Dispersion Module: Gaussian

User specified LOC: $1000 \mathrm{ppm}$

Max Threat Zone for LOC: 85 meters

Max Threat Zone for IDLH: 56 meters

Note: Footprint was not drawn because effects of near-field patchiness make plume presentation unreliable for short distances.

\section{TIME DEPENDENT INFORMATION:}

Concentration Estimates at the point:

Downwind: 100 meters.

Off Centerline: 0 meters

Max Concentration:

Outdoor: 764 ppm

Indoor: $764 \mathrm{ppm}$

Note: Indoor graph is shown with a dotted line.
Reviewed by S. A. Henderson

Works Calculation 7

Sheet No. 431 of 577 
Title of Project EPHA for THM

Subject Concentration Analysis for THM Hazardous Chemicals

Computer T. M. Franey

Date $\underline{09 / 16 / 96}$
Reviewed by S. A. Henderson

Works Calculation 7

Sheet No. 432 of 577

\section{SITE DATA INFORMATION: 1-RD-29}

Location: AIKEN, SOUTH CAROLINA

Building Air Exchanges Per Hour: 60 (User specified)

Date and Time: Fixed at July 1, 19961300 hours

CHEMICAL INFORMATION:

Chemical Name: METHYL ETHYL KETONE

Molecular Weight: $72.11 \mathrm{~kg} / \mathrm{kmol}$

TLV-TWA: 200,00 ppm IDLH: $3000.00 \mathrm{ppm}$

Footprint Level of Concern: 1200 ppm

Boiling Point: $79.64^{\circ} \mathrm{C}$

Vapor Pressure at Ambient Temperature: $0.13 \mathrm{~atm}$

Ambient Saturation Concentration: 127,903 ppm or $12.8 \%$

ATMOSPHERIC INFORMATION: (MANUAL INPUT OF DATA)

Wind: 2.5 meters $/ \mathrm{sec}$ from $90^{\circ}$ true

Inversion Height: 500 meters

Stability Class: C Air Temperature: $25^{\circ} \mathrm{C}$

Relative Humidity: 50\% Ground Roughness: 100 centimeters

Cloud Cover: 7 tenths

\section{SOURCE STRENGTH INFORMATION:}

Puddle Area: 6250 square feet

Average Puddle Depth: 1 centimeters

Soil Type: Default Ground Temperature: $25^{\circ} \mathrm{C}$

Initial Puddle Temperature: Ground temperature

Release Duration: ALOHA limited the duration to 1 hour

Max Computed Release Rate: 58.1 kilograms/min

Max Average Sustained Release Rate: 55.3 kilograms $/ \mathrm{min}$

(averaged over a minute or more)

Total Amount Released: 3,177 kilograms

FOOTPRINT INFORMATION:

Dispersion Module: Gaussian

User specified LOC: $1200 \mathrm{ppm}$

Max Threat Zone for LOC: 17 meters

Max Threat Zone for IDLH: 14 meters

Note: Footprint was not drawn because

effects of near-field patchiness make plume

presentation unreliable for short distances.

\section{TIME DEPENDENT INFORMATION:}

Concentration Estimates at the point:

Downwind: 30 meters

Off Centerline: 0 meters

Max Concentration:

Outdoor: 499 ppm

Indoor: $499 \mathrm{ppm}$

Note: Indoor graph is shown with a dotted line. 
Title of Project EPHA for THM

Subject Concentration Analysis for THM Hazardous Chemicals

Computer T. M. Franey Date $09 / 16 / 96$

SITE DATA INFORMATION: 1-RD-29

Location: AIKEN, SOUTH CAROLINA

Building Air Exchanges Per Hour: 60 (User specified)

Date and Time: Fixed at July 1, 19961300 hours

CHEMICAL INFORMATION:

Chemical Name: METHYL ETHYL KETONE

Molecular Weight: $72.11 \mathrm{~kg} / \mathrm{kmol}$

TLV-TWA: $200.00 \mathrm{ppm}$ IDLH: $3000.00 \mathrm{ppm}$

Footprint Level of Concern: $1200 \mathrm{ppm}$

Boiling Point: $79.64^{\circ} \mathrm{C}$

Vapor Pressure at Ambient Temperature: $0.13 \mathrm{~atm}$

Ambient Saturation Concentration: 127,903 ppm or $12.8 \%^{\circ}$

ATMOSPHERIC INFORMATION: (MANUAL INPUT OF DATA)

Wind: 2.5 meters $/ \mathrm{sec}$ from $90^{\circ}$ true

Inversion Height: 500 meters

Stability Class: C Air Temperature: $25^{\circ} \mathrm{C}$

Relative Humidity: $50 \%$ Ground Roughness: 100 centimeters

Cloud Cover: 7 tenths

SOURCE STRENGTH INFORMATION:

Puddle Area: 19000 square feet

Average Puddle Depth: 1 centimeters

Soil Type: Default Ground Temperature: $25^{\circ} \mathrm{C}$

Initial Puddle Temperature: Ground temperature

Release Duration: ALOHA limited the duration to 1 hour

Max Computed Release Rate: 168 kilograms/min

Max Average Sustained Release Rate: 161 kilograms/min

(averaged over a minute or more)

Total Amount Released: 9,306 kilograms

FOOTPRINT INFORMATION:

Model Run: Heavy Gas

User specified LOC: $1200 \mathrm{ppm}$

Max Threat Zone for LOC: 100 meters

Max Threat Zone for IDLH: 49 meters

Note: Footprint wasn't drawn because effects of near-field patchiness make plume presentation unreliable for short distances.

\section{TIME DEPENDENT INFORMATION:}

Concentration Estimates at the point:

Downwind: 100 meters

Off Centerline: 0 meters

Max Concentration:

Outdoor: $1,200 \mathrm{ppm}$

Indoor: $1,200 \mathrm{ppm}$

Note: Indoor graph is shown with a dotted line.
Reviewed by $\underline{\text { S. A. Henderson }}$

Works Calculation 7

Sheet No. 433 of 577 
Title of Project EPHA for THM

Subject Concentration Analysis for THM Hazardous Chemicals

Computer T. M. Franey Date 09/16/96
Reviewed by S. A. Henderson

Works Calculation 7

Sheet No. 434 of 577

SITE DATA INFORMATION: 1-RD-29

Location: AIKEN, SOUTH CAROLINA

Building Air Exchanges Per Hour: 60 (User specified)

Date and Time: Fixed at July 1, 19961300 hours

CHEMICAL INFORMATION:

Chemical Name: METHYL ETHYL KETONE

Molecular Weight: $72.11 \mathrm{~kg} / \mathrm{kmol}$

TLV-TWA: $200.00 \mathrm{ppm}$ IDLH: $3000.00 \mathrm{ppm}$

Footprint Level of Concern: $1200 \mathrm{ppm}$

Boiling Point: $79.64^{\circ} \mathrm{C}$

Vapor Pressure at Ambient Temperature: $0.13 \mathrm{~atm}$

Ambient Saturation Concentration: 127,903 ppm or $12.8 \%$

ATMOSPHERIC INFORMATION: (MANUAL INPUT OF DATA)

Wind: 2.5 meters $/ \mathrm{sec}$ from $90^{\circ}$ true

Inversion Height: 500 meters

Stability Class: C Air Temperature: $25^{\circ} \mathrm{C}$

Relative Humidity: 50\% Ground Roughness: 100 centimeters

Cloud Cover: 7 tenths

SOURCE STRENGTH INFORMATION:

Puddle Area: 24420 square feet

Average Puddle Depth: 1 centimeters

Soil Type: Default Ground Temperature: $25^{\circ} \mathrm{C}$

Initial Puddle Temperature: Ground temperature

Release Duration: ALOHA limited the duration to 1 hour

Max Computed Release Rate: 213 kilograms/min

Max Average Sustained Release Rate: 205 kilograms/min

(averaged over a minute or more)

Total Amount Released: 11,864 kilograms

FOOTPRINT INFORMATION:

Model Run: Heavy Gas

User specified LOC: 1200 ppm

Max Threat Zone for LOC: 113 meters

Max Threat Zone for IDLH: 57 meters

Note: The Heavy Gas footprint is an initial screening.

For short releases it may be an overestimation.

Be sure to check concentration information at specific locations.

TIME DEPENDENT INFORMATION:

Concentration Estimates at the point:

Downwind: 100 meters

Off Centerline: 0 meters

Max Concentration:

Outdoor: $1,430 \mathrm{ppm}$

Indoor: $1,430 \mathrm{ppm}$

Note: Indoor graph is shown with a dotted line. 
Title of Project EPHA for THM

Subject Concentration Analysis for THM Hazardous Chemicals

Computer T. M. Franey

Date $09 / 16 / 96$
Reviewed by $\underline{\text { S. A. Henderson }}$

Works Calculation 7

Sheet No. 435 of 577

SITE DATA INFORMATION: 1-RD-29

Location: AIKEN, SOUTH CAROLINA

Building Air Exchanges Per Hour: 60 (User specified)

Date and Time: Fixed at July 1, 19961300 hours

CHEMICAL INFORMATION:

Chemical Name: METHYL ETHYL KETONE

Molecular.Weight: $72.11 \mathrm{~kg} / \mathrm{kmol}$

TLV-TWA: $200.00 \mathrm{ppm}$ IDLH: $3000.00 \mathrm{ppm}$

Footprint Level of Concern: 1200 ppm

Boiling Point: $79.64^{\circ} \mathrm{C}$

Vapor Pressure at Ambient Temperature: $0.13 \mathrm{~atm}$

Ambient Saturation Concentration: 127,903 ppm or $12.8 \%$

ATMOSPHERIC INFORMATION: (MANUAL INPUT OF DATA)

Wind: 2.5 meters $/ \mathrm{sec}$ from $90^{\circ}$ true

Inversion Height: 500 meters

Stability Class: C Air Temperature: $25^{\circ} \mathrm{C}$

Relative Humidity: 50\% Ground Roughness: 100 centimeters

Cloud Cover: 7 tenths

\section{SOURCE STRENGTH INFORMATION:}

Puddle Area: 40700 square feet

Average Puddle Depth: 1 centimeters

Soil Type: Default Ground Temperature: $25^{\circ} \mathrm{C}$

Initial Puddle Temperature: Ground temperature

Release Duration: ALOHA limited the duration to 1 hour

Max Computed Release Rate: 347 kilograms/min

Max Average Sustained Release Rate: 334 kilograms/min

(averaged over a minute or more)

Total Amount Released: 19,451 kilograms

FOOTPRINT INFORMATION:

Model Run: Heavy Gas

User specified LOC: $1200 \mathrm{ppm}$

Max Threat Zone for LOC: 147 meters

Max Threat Zone for IDLH: 74 meters

Note: The Heavy Gas footprint is an initial screening.

For short releases it may be an overestimation.

Be sure to check concentration information at specific locations.

TIME DEPENDENT INFORMATION:

Concentration Estimates at the point:

Downwind: 100 meters

Off Centerline: 0 meters

Max Concentration:

Outdoor: $2,030 \mathrm{ppm}$

Indoor: $2,030 \mathrm{ppm}$

Note: Indoor graph is shown with a dotted line. 
Title of Project EPHA for THM

Subject Concentration Analysis for THM Hazardous Chemicals

Computer T. M. Franey
Reviewed by S. A. Henderson

Works Calculation 7

Sheet No. 436 of 577

\section{SITE DATA INFORMATION: 1-RD-29}

Location: AIKEN, SOUTH CAROLINA

Building Air Exchanges Per Hour: 60 (User specified)

Date and Time: Fixed at July 1, 19961300 hours

CHEMICAL INFORMATION:

Chemical Name: METHYL ETHYL KETONE

Molecular Weight: $72.11 \mathrm{~kg} / \mathrm{kmol}$

TLV-TWA: 200.00 ppm IDLH: 3000.00 ppm

Footprint Level of Concern: $1200 \mathrm{ppm}$

Boiling Point: $79.64^{\circ} \mathrm{C}$

Vapor Pressure at Ambient Temperature: 0.13 atm

Ambient Saturation Concentration: 127,903 ppm or $12.8 \%$

ATMOSPHERIC INFORMATION: (MANUAL INPUT OF DATA)

Wind: 2.5 meters $/ \mathrm{sec}$ from $90^{\circ}$ true

Inversion Height: 500 meters

Stability Class: C Air Temperature: $25^{\circ} \mathrm{C}$

Relative Humidity: 50\% Ground Roughness: 100 centimeters

Cloud Cover: 7 tenths

\section{SOURCE STRENGTH INFORMATION:}

Puddle Area: 10000 square meters

Average Puddle Depth: 1 centimeters

Soil Type: Default Ground Temperature: $25^{\circ} \mathrm{C}$

Initial Puddle Temperature: Ground temperature

Release Duration: ALOHA limited the duration to 1 hour

Max Computed Release Rate: 881 kilograms/min

Max Average Sustained Release Rate: 854 kilograms/min

(averaged over a minute or more)

Total Amount Released: 49,900 kilograms

\section{FOOTPRINT INFORMATION:}

Model Run: Heavy Gas

User specified LOC: $1200 \mathrm{ppm}$

Max Threat Zone for LOC; 242 meters

Max Threat Zone for IDLH: 123 meters

Note: The Heavy Gas footprint is an initial screening.

For short releases it may be an overestimation.

Be sure to check concentration information at specific locations.

\section{TIME DEPENDENT INFORMATION:}

Concentration Estimates at the point:

Downwind: 100 meters

Off Centerline: 0 meters

Max Concentration:

Outdoor: $3,940 \mathrm{ppm}$

Indoor: $3,940 \mathrm{ppm}$

Note: Indoor graph is shown with a dotted line. 
Title of Project EPHA for THM

Subject Concentration Analysis for THM Hazardous Chemicals

Computer T. M. Franey
Reviewed by $\underline{S . A . ~ H e n d e r s o n ~}$

Works Calculation 7

Sheet No. 437 of 577

SITE DATA INFORMATION: 1-RD-30

Location: AIKEN, SOUTH CAROLINA

Building Air Exchanges Per Hour: 60 (User specified)

Date and Time: Fixed at July 1, 19961300 hours

CHEMICAL INFORMATION:

Chemical Name: METHYL ISOBUTYL KETONE

Molecular Weight: $100.16 \mathrm{~kg} / \mathrm{kmol}$

TLV-TWA: $50.00 \mathrm{ppm}$ IDLH: $3000.00 \mathrm{ppm}$

Footprint Level of Concern: 250 ppm

Boiling Point: $116.50^{\circ} \mathrm{C}$

Vapor Pressure at Ambient Temperature: 0.026 atm

Ambient Saturation Concentration: $26,580 \mathrm{ppm}$ or $2.66 \%$

ATMOSPHERIC INFORMATION: (MANUAL INPUT OF DATA)

Wind: 2.5 meters $/ \mathrm{sec}$ from $90^{\circ}$ true

Inversion Height: 500 meters

Stability Class: $\mathrm{C} \quad$ Air Temperature: $25^{\circ} \mathrm{C}$

Relative Humidity: 50\% Ground Roughness: 100 centimeters

Cloud Cover: 7 tenths

\section{SOURCE STRENGTH INFORMATION:}

Puddle Area: 10750 square feet

Average Puddle Depth: 1 centimeters

Soil Type: Default Ground Temperature: $25^{\circ} \mathrm{C}$

Initial Puddle Temperature: Ground temperature

Release Duration: ALOHA limited the duration to 1 hour

Max Computed Release Rate: 36.4 kilograms/min

Max Average Sustained Release Rate: 36.3 kilograms/min (averaged over a minute or more)

Total Amount Released: 2,025 kilograms

\section{FOOTPRINT INFORMATION:}

Dispersion Module: Gaussian

User specified LOC: $250 \mathrm{ppm}$

Max Threat Zone for LOC: 27 meters

Max Threat Zone for IDLH: 18 meters

Note: Footprint was not drawn because effects of near-field patchiness make plume presentation unreliable for short distances.

\section{TIME DEPENDENT INFORMATION:}

Concentration Estimates at the point:

Downwind: 30 meters

Off Centerline: 0 meters

Max Concentration:

Outdoor: $200 \mathrm{ppm}$

Indoor: $200 \mathrm{ppm}$

Note: Indoor graph is shown with a dotted line. 


\section{ENGINEERING COMPUTATION SHEET}

Title of Project EPHA for THM

Subject Concentration Analysis for THM Hazardous Chemicals

Computer T.M. Franey Date 09/16/96.

SITE DATA INFORMATION: 1-RD-30

Location: AIKEN, SOUTH CAROLINA

Building Air Exchanges Per Hour: 60 (User specified)

Date and Time: Fixed at July 1, 19961300 hours

CHEMICAL INFORMATION:

Chemical Name: METHYL ISOBUTYL KETONE

Molecular Weight: $100.16 \mathrm{~kg} / \mathrm{kmol}$

TLV-TWA: $50.00 \mathrm{ppm}$ IDLH: $3000.00 \mathrm{ppm}$

Footprint Level of Concern: $250 \mathrm{ppm}$

Boiling Point: $116.50^{\circ} \mathrm{C}$

Vapor Pressure at Ambient Temperature: $0.026 \mathrm{~atm}$

Ambient Saturation Concentration: 26,580 ppm or $2.66 \%$

ATMOSPHERIC INFORMATION: (MANUAL INPUT OF DATA)

Wind: 2.5 meters $/ \mathrm{sec}$ from $90^{\circ}$ true

Inversion Height: 500 meters

Stability Class: C Air Temperature: $25^{\circ} \mathrm{C}$

Relative Humidity: 50\% Ground Roughness: 100 centimeters

Cloud Cover: 7 tenths

\section{SOURCE STRENGTH INFORMATION:}

Puddle Area: 34500 square feet

Average Puddle Depth: 1 centimeters

Soil Type: Default Ground Temperature: $25^{\circ} \mathrm{C}$

Initial Puddle Temperature: Ground temperature

Release Duration: ALOHA limited the duration to 1 hour

Max Computed Release Rate: 112 kilograms/min

Max Average Sustained Release Rate: 112 kilograms/min

(averaged over a minute or more)

Total Amount Released: 6,222 kilograms

FOOTPRINT INFORMATION:

Dispersion Module: Gaussian

User specified LOC: $250 \mathrm{ppm}$

Max Threat Zone for LOC: 46 meters

Max Threat Zone for IDLH: 32 meters

Note: Footprint was not drawn because

effects of near-field patchiness make plume

presentation unreliable for short distances.

TIME DEPENDENT INFORMATION:

Concentration Estimates at the point:

Downwind: 100 meters

Off Centerline: 0 meters

Max Concentration:

Outdoor: $90.9 \mathrm{ppm}$

Indoor: $90.9 \mathrm{ppm}$

Note: Indoor graph is shown with a dotted line.
Reviewed by S. A. Henderson

Works Calculation 7

Sheet No. 438 of 577 


\section{ENGINEERING COMPUTATION SHEET}

Title of Project EPHA for THM

Subject Concentration Analysis for THM Hazardous Chemicals

Computer T. M. Franey

Date $\underline{09 / 16 / 96}$

SITE DATA INFORMATION: 1-RD-30

Location: AIKEN, SOUTH CAROLINA

Building Air Exchanges Per Hour: 60 (User specified)

Date and Time: Fixed at July 1, 19961300 hours

CHEMICAL INFORMATION:

Chemical Name: METHYL ISOBUTYL KETONE

Molecular Weight: $100.16 \mathrm{~kg} / \mathrm{kmol}$

TLV-TWA: $50.00 \mathrm{ppm}$ IDLH: $3000.00 \mathrm{ppm}$

Footprint Level of Concern: $250 \mathrm{ppm}$

Boiling Point: $116.50^{\circ} \mathrm{C}$

Vapor Pressure at Ambient Temperature: $0.026 \mathrm{~atm}$

Ambient Saturation Concentration: 26,580 ppm or $2.66 \%$

ATMOSPHERIC INFORMATION: (MANUAL INPUT OF DATA)

Wind: 2.5 meters $/ \mathrm{sec}$ from $90^{\circ}$ true

Inversion Height: 500 meters

Stability Class: C Air Temperature: $25^{\circ} \mathrm{C}$.

Relative Humidity: 50\% Ground Roughness: 100 centimeters

Cloud Cover: 7 tenths

SOURCE STRENGTH INFORMATION:

Puddle Area: 24420 square feet

Average Puddle Depth: 1 centimeters

Soil Type: Default Ground Temperature: $25^{\circ} \mathrm{C}$

Initial Puddle Temperature: Ground temperature

Release Duration: ALOHA limited the duration to 1 hour

Max Computed Release Rate: 80.5 kilograms/min

Max Average Sustained Release Rate: 80 kilograms/min (averaged over a minute or more)

Total Amount Released: 4,461 kilograms

FOOTPRINT INFORMATION:

Dispersion Module: Gaussian

User specified LOC: $250 \mathrm{ppm}$

Max Threat Zone for LOC: 39 meters

Max Threat Zone for IDLH: 27 meters

Note: Footprint was not drawn because effects of near-field patchiness make plume presentation unreliable for short distances.

\section{TIME DEPENDENT INFORMATION:}

Concentration Estimates at the point:

Downwind: 100 meters

Off Centerline: 0 meters

Max Concentration:

Outdoor: $77.3 \mathrm{ppm}$

Indoor: $77.3 \mathrm{ppm}$

Note: Indoor graph is shown with a dotted line.
Reviewed by S. A. Henderson

Works Calculation 7

Sheet No. 439 of 577 


\section{ENGINEERING COMPUTATION SHEET}

Title of Project EPHA for THM

Subject Concentration Analysis for THM Hazardous Chemicals

Computer T. M. Franey

Date 09/16/96

SITE DATA INFORMATION: 1-RD-30

Location: AIKEN, SOUTH CAROLINA

Building Air Exchanges Per Hour: 60 (User specified)

Date and Time: Fixed at July 1, 19961300 hours

CHEMICAL INFORMATION:

Chemical Name: METHYL ISOBUTYL KETONE

Molecular Weight: $100.16 \mathrm{~kg} / \mathrm{kmol}$

TLV-TWA: $50.00 \mathrm{ppm}$. IDLH: $3000.00 \mathrm{ppm}$

Footprint Level of Concern: $250 \mathrm{ppm}$

Boiling Point: $116.50^{\circ} \mathrm{C}$

Vapor Pressure at Ambient Temperature: $0.026 \mathrm{~atm}$

Ambient Saturation Concentration: $26,580 \mathrm{ppm}$ or $2.66 \%$

ATMOSPHERIC INFORMATION: (MANUAL INPUT OF DATA)

Wind: 2.5 meters $/ \mathrm{sec}$ from $90^{\circ}$ true

Inversion Height: 500 meters

Stability Class: C Air Temperature: $25^{\circ} \mathrm{C}$

Relative Humidity: 50\% Ground Roughness: 100 centimeters

Cloud Cover: 7 tenths

SOURCE STRENGTH INFORMATION:

Puddle Area: 40700 square feet

Average Puddle Depth: 1 centimeters

Soil Type: Default Ground Temperature: $25^{\circ} \mathrm{C}$

Initial Puddle Temperature: Ground temperature

Release Duration: ALOHA limited the duration to 1 hour

Max Computed Release Rate: 132 kilograms/min

Max Average Sustained Release Rate: 131 kilograms/min

(averaged over a minute or more)

Total Amount Released: 7,297 kilograms

FOOTPRINT INFORMATION:

Model Run: Heavy Gas

User specified LOC: $250 \mathrm{ppm}$

Max Threat Zone for LOC: 112 meters

Max Threat Zone for IDLH: IDLH level was never exceeded

Note: The Heavy Gas footprint is an initial screening.

For short releases it may be an overestimation.

Be sure to check concentration information at specific locations.

TIME DEPENDENT INFORMATION:

Concentration Estimates at the point:

Downwind: 100 meters

Off Centerline: 0 meters

Max Concentration:

Outdoor: $312 \mathrm{ppm}$

Indoor: $312 \mathrm{ppm}$

Note: Indoor graph is shown with a dotted line.
Reviewed by $\mathrm{S}$. A. Henderson

Works Calculation 7

Sheet No. 440 of 577 


\section{ENGINEERING COMPUTATION SHEET}

Title of Project EPHA for THM

Subject Concentration Analysis for THM Hazardous Chemicals

Computer T. M. Franey

Date $\underline{09 / 16 / 96}$
Reviewed by S. A. Henderson

Works Calculation 7

Sheet No. 441 of 577

SITE DATA INFORMATION: $\quad$ 1-RD-30

Location: AIKEN, SOUTH CAROLINA

Building Air Exchanges Per Hour: 60 (User specified)

Date and Time: Fixed at July 1, 19961300 hours

\section{CHEMICAL INFORMATION:}

Chemical Name: METHYL ISOBUTYL KETONE

Molecular Weight: $100.16 \mathrm{~kg} / \mathrm{kmol}$

TLV-TWA: $50.00 \mathrm{ppm}$ IDLH: $3000.00 \mathrm{ppm}$

Footprint Level of Concern: $250 \mathrm{ppm}$

Boiling Point: $116.50^{\circ} \mathrm{C}$

Vapor Pressure at Ambient Temperature: $0.026 \mathrm{~atm}$

Ambient Saturation Concentration: 26,580 ppm or $2.66 \%$

ATMOSPHERIC INFORMATION: (MANUAL INPUT OF DATA)

Wind: 2.5 meters $/ \mathrm{sec}$ from $90^{\circ}$ true

Inversion Height: 500 meters

Stability Class: C Air Temperature: $25^{\circ} \mathrm{C}$

Relative Humidity: 50\% Ground Roughness: 100 centimeters

Cloud Cover: 7 tenths

SOURCE STRENGTH INFORMATION:

Puddle Area: 10000 square meters

Average Puddle Depth: 1 centimeters

Soil Type: Default Ground Temperature: $25^{\circ} \mathrm{C}$

Initial Puddle Temperature: Ground temperature

Release Duration: ALOHA limited the duration to 1 hour

Max Computed Release Rate: 338 kilograms/min

Max Average Sustained Release Rate: 336 kilograms/min

(averaged over a minute or more)

Total Amount Released: 18,640 kilograms

FOOTPRINT INFORMATION:

Model Run: Heavy Gas

User specified LOC: $250 \mathrm{ppm}$

Max Threat Zone for LOC: 148 meters

Max Threat Zone for IDLH: IDLH level was never exceeded

Note: The Heavy Gas footprint is an initial screening.

For short releases it may be an overestimation.

Be sure to check concentration information at specific locations.

TIME DEPENDENT INFORMATION:

Concentration Estimates at the point:

Downwind: 100 meters

Off Centerline: 0 meters

Max Concentration:

Outdoor: $643 \mathrm{ppm}$

Indoor: $643 \mathrm{ppm}$

Note: Indoor graph is shown with a dotted line. 


\section{ENGINEERING COMPUTATION SHEET}

Title of Project EPHA for THM

Reviewed by S. A. Henderson

Subject Concentration Analysis for THM Hazardous Chemicals

Computer T. M. Franey

Date $\underline{09 / 16 / 96}$

SITE DATA INFORMATION: 1-RD-31

Location: AIKEN, SOUTH CAROLINA

Building Air Exchanges Per Hour: 60 (User specified)

Date and Time: Fixed at July 1, 19961300 hours

CHEMICAL INFORMATION:

Chemical Name: METHYLENE CHLORIDE

Molecular Weight: $84.90 \mathrm{~kg} / \mathrm{kmol}$

TLV-TWA: -unavail- . IDLH: -unavail-

Footprint Level of Concern: 750 ppm

Boiling Point: $40.00^{\circ} \mathrm{C}$

Vapor Pressure at Ambient Temperature: $0.55 \mathrm{~atm}$

Ambient Saturation Concentration: $561,055 \mathrm{ppm}$ or $56.1 \%$

ATMOSPHERIC INFORMATION: (MANUAL INPUT OF DATA)

Wind: 2.5 meters $/ \mathrm{sec}$ from $90^{\circ}$ true

Inversion Height: $\mathbf{5 0 0}$ meters

Stability Class: C Air Temperature: $25^{\circ} \mathrm{C}$

Relative Humidity: 50\% Ground Roughness: 100 centimeters

Cloud Cover: 7 tenths

\section{SOURCE STRENGTH INFORMATION:}

Puddle Area: 132 square feet

Average Puddle Depth: 1 centimeters

Soil Type: Default Ground Temperature: $25^{\circ} \mathrm{C}$

Initial Puddle Temperature: Ground temperature

Release Duration: 26 minutes

Max Computed Release Rate: 11.5 kilograms/min

Max Average Sustained Release Rate: 7.8 kilograms/min

(averaged over a minute or more)

Total Amount Released: 150 kilograms

FOOTPRINT INFORMATION:

Model Run: Heavy Gas

User specified LOC: 750 ppm

Max Threat Zone for LOC: 30 meters

Note: Footprint wasn't drawn because effects of near-field patchiness make plume presentation unreliable for short distances.

TIME DEPENDENT INFORMATION:

Concentration Estimates at the point:

Downwind: 30 meters

Off Centerline: 0 meters

Max Concentration:

Outdoor: $751 \mathrm{ppm}$

Indoor: $709 \mathrm{ppm}$

Note: Indoor graph is shown with a dotted line.
Works Calculation 7

Sheet No. 442 of 577 
Title of Project EPHA for THM

Subject Concentration Analysis for THM Hazardous Chemicals

Computer T. M. Franey Date $\underline{09 / 16 / 96}$

SITE DATA INFORMATION: 1-RD-31

Location: AIKEN, SOUTH CAROLINA

Building Air Exchanges Per Hour: 60 (User specified)

Date and Time: Fixed at July 1, 19961300 hours

\section{CHEMICAL INFORMATION:}

Chemical Name: METHYLENE CHLORIDE

Molecular Weight: $84.90 \mathrm{~kg} / \mathrm{kmol}$

TLV-TWA: -unavail- IDLH: -unavail-

Footprint Level of Concern: 750 ppm

Boiling Point: $40.00^{\circ} \mathrm{C}$

Vapor Pressure at Ambient Temperature: $0.55 \mathrm{~atm}$

Ambient Saturation Concentration: 561,055 ppm or $56.1 \%$

ATMOSPHERIC INFORMATION: (MANUAL INPUT OF DATA)

Wind: 2.5 meters $/ \mathrm{sec}$ from $90^{\circ}$ true

Inversion Height: 500 meters

Stability Class: C Air Temperature: $25^{\circ} \mathrm{C}$.

Relative Humidity: 50\% Ground Roughness: 100 centimeters

Cloud Cover: 7 tenths

\section{SOURCE STRENGTH INFORMATION:}

Puddle Area: 1712 square feet

Average Puddle Depth: 1 centimeters

Soil Type: Default $\quad$ Ground Temperature: $25^{\circ} \mathrm{C}$

Initial Puddle Temperature: Ground temperature

Release Duration: 28 minutes

Max Computed Release Rate: 130 kilograms/min

Max Average Sustained Release Rate: 90.6 kilograms/min

(averaged over a minute or more)

Total Amount Released: 1,948 kilograms

\section{FOOTPRINT INFORMATION:}

Model Run: Heavy Gas

User specified LOC: $750 \mathrm{ppm}$

Max Threat Zone for LOC: 100 meters

Note: Footprint wasn't drawn because effects of near-field patchiness make plume presentation unreliable for short distances.

\section{TIME DEPENDENT INFORMATION:}

Concentration Estimates at the point:

Downwind: 100 meters

Off Centerline: 0 meters

Max Concentration:

Outdoor: 750 ppm

Indoor: $708 \mathrm{ppm}$

Note: Indoor graph is shown with a dotted line.
Reviewed by S. A. Henderson

Works Calculation 7

Sheet No. 443 of 577 
Title of Project EPHA for THM

\section{ENGINEERING COMPUTATION SHEET}

Subject Concentration Analysis for THM Hazardous Chemicals

Computer T. M. Franey

Date 09/16/96

\section{SITE DATA INFORMATION: 1-RD-31}

Location: AIKEN, SOUTH CAROLINA

Building Air Exchanges Per Hour: 60 (User specified)

Date and Time: Fixed at July 1, 19961300 hours

CHEMICAL INFORMATION:

Chemical Name: METHYLENE CHLORIDE

Molecular Weight: $84.90 \mathrm{~kg} / \mathrm{kmol}$

TLV-TWA: -unavail- IDLH: -unavail-

Footprint Level of Concern: 750 ppm

Boiling Point: $40.00^{\circ} \mathrm{C}$

Vapor Pressure at Ambient Temperature: 0.55 atm

Ambient Saturation Concentration: 561,055 ppm or $56.1 \%$

ATMOSPHERIC INFORMATION: (MANUAL INPUT OF DATA)

Wind: 2.5 meters $/ \mathrm{sec}$ from $90^{\circ}$ true

Inversion Height: 500 meters

Stability Class: C Air Temperature: $25^{\circ} \mathrm{C}$

Relative Humidity: 50\% Ground Roughness: 100 centimeters

Cloud Cover: 7 tenths

SOURCE STRENGTH INFORMATION:

Puddle Area: 24420 square feet

Average Puddle Depth: 1 centimeters

Soil Type: Default Ground Temperature: $25^{\circ} \mathrm{C}$

Initial Puddle Temperature: Ground temperature

Release Duration: 31 minutes

Max Computed Release Rate: 1,640 kilograms/min

Max Average Sustained Release Rate: 1,170 kilograms/min

(averaged over a minute or more)

Total Amount Released: 27,792 kilograms

FOOTPRINT INFORMATION:

Model Run: Heavy Gas

User specified LOC: $750 \mathrm{ppm}$

Max Threat Zone for LOC: 380 meters

Note: The Heavy Gas footprint is an initial screening.

For short releases it may be an overestimation.

Be sure to check concentration information at specific locations.

\section{TIME DEPENDENT INFORMATION:}

Concentration Estimates at the point:

Downwind: 100 meters

Off Centerline: 0 meters

Max Concentration:

Outdoor: $7,410 \mathrm{ppm}$

Indoor: 7,240 ppm

Note: Indoor graph is shown with a dotted line.
Reviewed by S. A. Henderson

Works Calculation 7

Sheet No. 444 of 577 
Title of Project EPHA for THM

Subject Concentration Analysis for THM Hazardous Chemicals

Computer T. M. Franey

Date $\underline{09 / 16 / 96}$
Reviewed by $\underline{\text { S. A. Henderson }}$

Works Calculation 7

Sheet No. 445 of 577

\section{SITE DATA INFORMATION: 1-RD-31}

Location: AIKEN, SOUTH CAROLINA

Building Air Exchanges Per Hour: 60 (User specified)

Date and Time: Fixed at July 1, 19961300 hours

\section{CHEMICAĹ INFORMATION:}

Chemical Name: METHYLENE CHLORIDE

Molecular Weight: $84.90 \mathrm{~kg} / \mathrm{kmol}$

TLV-TWA: -unavail- IDLH: -unavail-

Footprint Level of Concern: 750 ppm

Boiling Point: $40.00^{\circ} \mathrm{C}$

Vapor Pressure at Ambient Temperature: $0.55 \mathrm{~atm}$

Ambient Saturation Concentration: 561,055 ppm or 56.1\%

ATMOSPHERIC INFORMATION: (MANUAL INPUT OF DATA)

Wind: 2.5 meters $/ \mathrm{sec}$ from $90^{\circ}$ true

Inversion Height: 500 meters .

Stability Class: C Air Temperature: $25^{\circ} \mathrm{C}$

Relative Humidity: 50\% Ground Roughness: 100 centimeters

Cloud Cover: 7 tenths

\section{SOURCE STRENGTH INFORMATION:}

Puddle Area: 40700 square feet

Average Puddle Depth: 1 centimeters

Soil Type: Default Ground Temperature: $25^{\circ} \mathrm{C}$

Initial Puddle Temperature: Ground temperature

Release Duration: 31 minutes

Max Computed Release Rate: 2,670 kilograms/min

Max Average. Sustained Release Rate: 1,920 kilograms/min

(averaged over a minute or more)

Total Amount Released: 46,320 kilograms

\section{FOOTPRINT INFORMATION:}

Model Run: Heavy Gas

User specified LOC: 750 ppm

Max Threat Zone for LOC: 494 meters

Note: The Heavy Gas footprint is an initial screening.

For short releases it may be an overestimation.

$\mathrm{Be}$ sure to check concentration information at specific locations.

\section{TIME DEPENDENT INFORMATION:}

Concentration Estimates at the point:

Downwind: 100 meters

Off Centerline: 0 meters

Max Concentration:

Outdoor: $11,900 \mathrm{ppm}$

Indoor: $11,700 \mathrm{ppm}$

Note: Indoor graph is shown with a dotted line. 


\section{ENGINEERING COMPUTATION SHEET}

Title of Project EPHA for THM

Subject Concentration Analysis for THM Hazardous Chemicals

Computer T. M. Franey

Date 09/16/96
Reviewed by $\underline{\text { S. A. Henderson }}$

Works Calculation 7

Sheet No. 446 of 577

\section{SITE DATA INFORMATION: 1-RD-31}

Location: AIKEN, SOUTH CAROLINA

Building Air Exchanges Per Hour: 60 (User specified)

Date and Time: Fixed at July 1, 19961300 hours

CHEMICAL INFORMATION:

Chemical Name: METHYLENE CHLORIDE

Molecular Weight: $84.90 \mathrm{~kg} / \mathrm{kmol}$

TLV-TWA: -unavail- IDLH: -unavail-

Footprint Level of Concern: $750 \mathrm{ppm}$

Boiling Point: $40.00^{\circ} \mathrm{C}$

Vapor Pressure at Ambient Temperature: $0.55 \mathrm{~atm}$

Ambient Saturation Concentration: 561,055 ppm or $56.1 \%$

ATMOSPHERIC INFORMATION: (MANUAL INPUT OF DATA)

Wind: 2.5 meters $/ \mathrm{sec}$ from $90^{\circ}$ true

Inversion Height: 500 meters

Stability Class: $\mathrm{C}$ Air Temperature: $25^{\circ} \mathrm{C}$

Relative Humidity: $50 \%$ Ground Roughness: 100 centimeters

Cloud Cover: 7 tenths

\section{SOURCE STRENGTH INFORMATION:}

Puddle Area: 10000 square meters

Average Puddle Depth: 1 centimeters

Soil Type: Default Ground Temperature: $25^{\circ} \mathrm{C}$

Initial Puddle Temperature: Ground temperature

Release Duration: 32 minutes

Max Computed Release Rate: 6,780 kilograms/min

Max Average Sustained Release Rate: 4,900 kilograms/min

(averaged over a minute or more)

Total Amount Released: 122,503 kilograms

\section{FOOTPRINT INFORMATION:}

Model Run: Heavy Gas

User specified LOC: $750 \mathrm{ppm}$

Max Threat Zone for LOC: 819 meters

Note: The Heavy Gas footprint is an initial screening.

For short releases it may be an overestimation.

Be sure to check concentration information at specific locations.

\section{TIME DEPENDENT INFORMATION:}

Concentration Estimates at the point:

Downwind: 100 meters

Off Centerline: 0 meters

Max Concentration:

Outdoor: $29,600 \mathrm{ppm}$

Indoor: $29,100 \mathrm{ppm}$

Note: Indoor graph is shown with a dotted line. 
Title of Project EPHA for THM

Subject Concentration Analysis for THM Hazardous Chemicals

Computer T. M. Franey

SITE DATA INFORMATION: 1-RD-32

Location: AIKEN, SOUTH CAROLINA

Building Air Exchanges Per Hour: 60 (User specified)

Date and Time: Fixed at July 1, 19961300 hours

\section{CHEMICAL INFORMATION:}

Chemical Name: METHYL CELLOSOLVE

Molecular Weight: $76.1 \mathrm{~kg} / \mathrm{kmol}$

TLV-TWA: not available IDLH: not available

Footprint Level of Concern: 250 ppm

Boiling Point: $171.00^{\circ} \mathrm{C}$

Vapor Pressure at Ambient Temperature: $4.85 \mathrm{e}-004 \mathrm{~atm}$

Ambient Saturation Concentration: 494 ppm or $0.049 \%$

\section{ATMOSPHERIC INFORMATION: (MANUAL INPUT OF DATA)}

Wind: 2.5 meters $/ \mathrm{sec}$ from $90^{\circ}$ true

Inversion Height: 500 meters

Stability Class: C Air Temperature: $25^{\circ} \mathrm{C}$

Relative Humidity: 50\% Ground Roughness: 100 centimeters

Cloud Cover: 7 tenths

\section{SOURCE STRENGTH INFORMATION:}

Puddle Area: 30430 square feet

Average Puddle Depth: 1 centimeters

Soil Type: Default Ground Temperature: $25^{\circ} \mathrm{C}$

Initial Puddle Temperature: Ground temperature

Release Duration: ALOHA limited the duration to 1 hour

Max Computed Release Rate: 1.26 kilograms/min

Max Average Sustained Release Rate: 1.24 kilograms/min (averaged over a minute or more)

Total Amount Released: 59.6 kilograms

\section{FOOTPRINT INFORMATION:}

Dispersion Module: Gaussian

User specified LOC: $250 \mathrm{ppm}$

Max Threat Zone for LOC: 30 meters

Max Threat Zone for IDLH: 30 meters

Note: Footprint was not drawn because effects of near-field patchiness make plume

presentation unreliable for short distances.

\section{TIME DEPENDENT INFORMATION:}

Concentration Estimates at the point:

Downwind: 30 meters

Off Centerline: 0 meters

Max Concentration:

Outdoor: $10.6 \mathrm{ppm}$

Indoor: $10.6 \mathrm{ppm}$

Note: Indoor graph is shown with a dotted line. 


\section{ENGINEERING COMPUTATION SHEET}

Title of Project EPHA for THM

Subject Concentration Analysis for THM Hazardous Chemicals

Computer T. M. Franey Date $\underline{09 / 16 / 96}$
Reviewed by $\underline{S}$. A. Henderson

Works Calculation 7

Sheet No. 448 of 577

\section{SITE DATA INFORMATION: 1-RD-32 \\ Location: AIKEN, SOUTH CAROLINA \\ Building Air Exchanges Per Hour: 60 (User specified) \\ Date and Time: Fixed at July 1, 19961300 hours

\author{
CHEMICAL INFORMATION: \\ Chemical Name: METHYL CELLOSOLVE \\ Molecular Weight: $76.1 \mathrm{~kg} / \mathrm{kmol}$ \\ TLV-TWA: not available IDLH: not available \\ Footprint Level of Concern: $250 \mathrm{ppm}$ \\ Boiling Point: $171.00^{\circ} \mathrm{C}$ \\ Vapor Pressure at Ambient Temperature: 4.85e-004 atm \\ Ambient Saturation Concentration: 494 ppm or $0.049 \%$
}
ATMOSPHERIC INFORMATION: (MANUAL INPUT OF DATA)
Wind: 2.5 meters $/ \mathrm{sec}$ from $90^{\circ}$ true
Inversion Height: 500 meters
Stability Class: C Air Temperature: $25^{\circ} \mathrm{C}$
Relative Humidity: 50\% Ground Roughness: 100 centimeters
Cloud Cover: 7 tenths

\section{SOURCE STRENGTH INFORMATION:}

Puddle Area: 99999 square feet

Average Puddle Depth: 1 centimeters

Soil Type: Default Ground Temperature: $25^{\circ} \mathrm{C}$

Initial Puddle Temperature: Ground temperature

Release Duration: ALOHA limited the duration to 1 hour

Max Computed Release Rate: 3.95 kilograms/min

Max Average Sustained Release Rate: 3.87 kilograms/min

(averaged over a minute or more)

Total Amount Released: 186 kilograms

FOOTPRINT INFORMATION:

Dispersion Module: Gaussian

User specified LOC: $250 \mathrm{ppm}$

Max Threat Zone for LOC: 55 meters

Max Threat Zone for IDLH: 54 meters

Note: Footprint was not drawn because

effects of near-field patchiness make plume

presentation unreliable for short distances.

\section{TIME DEPENDENT INFORMATION:}

Concentration Estimates at the point:

Downwind: 100 meters

Off Centerline: 0 meters

Max Concentration:

Outdoor: $3.28 \mathrm{ppm}$

Indoor: $3.28 \mathrm{ppm}$

Note: Indoor graph is shown with a dotted line. 


\section{ENGINEERING COMPUTATION SHEET}

Title of Project EPHA for THM

Subject Concentration Analysis for THM Hazardous Chemicals

Computer T. M. Franey

Date $\underline{09 / 16 / 96}$

\section{SITE DATA INFORMATION: 1-RD-32}

Location: AIKEN, SOUTH CAROLINA

Building Air Exchanges Per Hour: 60 (User specified)

Date and Time: Fixed at July 1, 19961300 hours

CHEMICAL INFORMATION:

Chemical Name: METHYL CELLOSOLVE

Molecular Weight: $76.1 \mathrm{~kg} / \mathrm{kmol}$

TLV-TWA: not available IDLH: not available

Footprint Level of Concern: 250 ppm

Boiling Point: $171.00^{\circ} \mathrm{C}$

Vapor Pressure at Ambient Temperature: $4.85 \mathrm{e}-004 \mathrm{~atm}$

Ambient Saturation Concentration: 494 ppm or $0.049 \%$

ATMOSPHERIC INFORMATION: (MANUAL INPUT OF DATA)

Wind: 2.5 meters $/ \mathrm{sec}$ from $90^{\circ}$ true

Inversion Height: 500 meters

Stability Class: C Air Temperature: $25^{\circ} \mathrm{C}$

Relative Humidity: 50\% Ground Roughness: 100 centimeters

Cloud Cover: 7 tenths

\section{SOURCE STRENGTH INFORMATION:}

Puddle Area: 24420 square feet

Average Puddle Depth: 1 centimeters

Soil Type: Default Ground Temperature: $25^{\circ} \mathrm{C}$

Initial Puddle Temperature: Ground temperature

Release Duration: ALOHA limited the duration to 1 hour

Max Computed Release Rate: 1.02 kilograms/min

Max Average Sustained Release Rate: 1 kilograms/min

(averaged over a minute or more)

Total Amount Released: 48.3 kilograms

FOOTPRINT INFORMATION:

Dispersion Module: Gaussian

User specified LOC: $250 \mathrm{ppm}$

Max Threat Zone for LOC: 27 meters

Max Threat Zone for IDLH: 27 meters

Note: Footprint was not drawn becaise effects of near-field patchiness make plume

presentation unreliable for short distances.

\section{TIME DEPENDENT INFORMATION:}

Cancentration Estimates at the point:

Downwind: 100 meters

Off Centerline: 0 meters

Max Concentration:

Outdoor: $1.59 \mathrm{ppm}$

Indoor: $1.59 \mathrm{ppm}$

Note: Indoor graph is shown with a dotted line.
Reviewed by S. A. Henderson

Works Calculation 7

Sheet No. 449 of 577 


\section{ENGINEERING COMPUTATION SHEET}

Title of Project EPHA for THM

Subject Concentration Analysis for THM Hazardous Chemicals

Computer T. M. Franey
SITE DATA INFORMATION: 1-RD-32
Location: AIKEN, SOUTH CAROLINA
Building Air Exchanges Per Hour: 60 (User specified)
Date and Time: Fixed at July 1, 19961300 hours

CHEMICAL INFORMATION:

Chemical Name: METHYL CELLOSOLVE

Molecular Weight: $76.1 \mathrm{~kg} / \mathrm{kmol}$

TLV-TWA: not available IDLH: not available

Footprint Level of Concern: 250 ppm

Boiling Point: $171.00^{\circ} \mathrm{C}$

Vapor Pressure at Ambient Temperature: $4.85 \mathrm{e}-004 \mathrm{~atm}$

Ambient Saturation Concentration: 494 ppm or $0.049 \%$

\section{ATMOSPHERIC INFORMATION: (MANUAL INPUT OF DATA)}

Wind: 2.5 meters $/ \mathrm{sec}$ from $90^{\circ}$ true

Inversion Height: 500 meters

Stability Class: C Air Temperature: $25^{\circ} \mathrm{C}$

Relative Humidity: $50 \%$ Ground Roughness: 100 centimeters

Cloud Cover: 7 tenths

\section{SOURCE STRENGTH INFORMATION:}

Puddle Area: 40700 square feet

Average Puddle Depth: 1 centimeters

Soil Type: Default Ground Temperature: $25^{\circ} \mathrm{C}$

Initial Puddle Temperature: Ground temperature

Release Duration: ALOHA limited the duration to 1 hour

Max Computed Release Rate: 1.66 kilograms/min

Max Average Sustained Release Rate: 1.63 kilograms/min

(averaged over a minute or more)

Total Amount Released: 78.7 kilograms

\section{FOOTPRINT INFORMATION:}

Dispersion Module: Gaussian

User specified LOC: $250 \mathrm{ppm}$

Max Threat Zone for LOC: 34 meters

Max Threat Zone for IDLH: 35 meters

Note: Footprint was not drawn because effects of near-field patchiness make plume presentation unreliable for short distances.

TIME DEPENDENT INFORMATION:

Concentration Estimates at the point:

Downwind: 100 meters

Off Centerline: 0 meters

Max Concentration:

Outdoor: $2.01 \mathrm{ppm}$

Indoor: $2.01 \mathrm{ppm}$

Note: Indoor graph is shown with a dotted line.
Reviewed by $\underline{S . A . ~ H e n d e r s o n}$

Works Calculation 7

Sheet No. 450 of 577 


\section{ENGINEERING COMPUTATION SHEET}

Title of Project EPHA for THM

Subject Concentration Analysis for THM Hazardous Chemicals

Computer T. M. Franey

Date $\underline{09 / 16 / 96}$

SITE DATA INFORMATION: 1-RD-32

Location: AIKEN, SOUTH CAROLINA

Building Air Exchanges Per Hour: 60 (User specified)

Date and Time: Fixed at July 1, 19961300 hours

CHEMICAL INFORMATION:

Chemical Name: METHYL CELLOSOLVE

Molecular Weight: $76.1 \mathrm{~kg} / \mathrm{kmol}$

TLV-TWA: not available. IDLH: not available

Footprint Level of Concern: 250 ppm

Boiling Point: $171.00^{\circ} \mathrm{C}$

Vapor Pressure at Ambient Temperature: 4.85e-004 atm

Ambient Saturation Concentration: 494 ppm or $0.049 \%$

ATMOSPHERIC INFORMATION: (MANUAL INPUT OF DATA)

Wind: 2.5 meters $/ \mathrm{sec}$ from $90^{\circ}$ true

Inversion Height: 500 meters

Stability Class: C Air Temperature: $25^{\circ} \mathrm{C}$

Relative Humidity: 50\% Ground Roughness: 100 centimeters

Cloud Cover: 7 tenths

\section{SOURCE STRENGTH INFORMATION:}

Puddle Area: 10000 square meters

Average Puddle Depth: 1 centimeters

Soil Type: Default $\quad$ Ground Temperature: $25^{\circ} \mathrm{C}$

Initial Puddle Temperature: Ground temperature

Release Duration: ALOHA limited the duration to 1 hour

Max Computed Release Rate: 4.24 kilograms/min

Max Average Sustained Release Rate: 4.16 kilograms/min

(averaged over a minute or more)

Total Amount Released: 200 kilograms

FOOTPRINT INFORMATION:

Dispersion Module: Gaussian

User specified LOC: $250 \mathrm{ppm}$

Max Threat Zone for LOC: 56 meters

Max Threat Zone for IDLH: 56 meters

Note: Footprint was not drawn because effects of near-field patchiness make plume

presentation unreliable for short distances.

TIME DEPENDENT INFORMATION:

Concentration Estimates at the point:

Downwind: 100 meters

Off Centerline: 0 meters

Max Concentration:

Outdoor: $3.46 \mathrm{ppm}$

Indoor: $3.46 \mathrm{ppm}$

Note: Indoor graph is shown with a dotted line.
Reviewed by S. A. Henderson

Works Calculation 7

Sheet No. 451 of 577 


\section{ENGINEERING COMPUTATION SHEET}

Title of Project EPHA for THM

Subject Concentration Analysis for THM Hazardous Chemicals

Computer T. M. Franey Date $\underline{09 / 16 / 96}$
Reviewed by S.A. Henderson

Works Calculation 7

Sheet No. $\underline{452 \text { of } 577}$

SITE DATA INFORMATION: 1-RD-33

Location: AIKEN, SOUTH CAROLINA

Building Air Exchanges Per Hour: 60 (User specified)

Date and Time: Fixed at July 1, 19961300 hours

CHEMICAL INFORMATION:

Chemical Name: MONOETHANOLAMINE

Molecular Weight: $61.08 \mathrm{~kg} / \mathrm{kmol}$

TLV-TWA: $3.00 \mathrm{ppm}$ IDLH: $1000.00 \mathrm{ppm}$

Footprint Level of Concern: $100 \mathrm{ppm}$

Boiling Point: $171.00^{\circ} \mathrm{C}$

Vapor Pressure at Ambient Temperature: $4.85 \mathrm{e}-004 \mathrm{~atm}$

Ambient Saturation Concentration: 494 ppm or $0.049 \%$

ATMOSPHERIC INFORMATION: (MANUAL INPUT OF DATA)

Wind: 2.5 meters $/ \mathrm{sec}$ from $90^{\circ}$ true

Inversion Height: 500 meters

Stability Class: C Air Temperature: $25^{\circ} \mathrm{C}$

Relative Humidity: 50\% Ground Roughness: 100 centimeters

Cloud Cover: 7 tenths

SOURCE STRENGTH INFORMATION:

Puddle Area: 30430 square feet

Average Puddle Depth: 1 centimeters

Soil Type: Default Ground Temperature: $25^{\circ} \mathrm{C}$

Initial Puddle Temperature: Ground temperature

Release Duration: ALOHA limited the duration to 1 hour

Max Computed Release Rate: 1.26 kilograms/min

Max Average Sustained Release Rate: 1.24 kilograms/min

(averaged over a minute or more)

Total Amount Released: 59.6 kilograms

\section{FOOTPRINT INFORMATION:}

Dispersion Module: Gaussian

User specified LOC: 100 ppm

Max Threat Zone for LOC: 30 meters

Max Threat Zone for IDLH: 30 meters

Note: Footprint was not drawn because effects of near-field patchiness make plume presentation unreliable for short distances.

\section{TIME DEPENDENT INFORMATION:}

Concentration Estimates at the point:

Downwind: 30 meters

Off Centerline: 0 meters

Max Concentration:

Outdoor: $10.6 \mathrm{ppm}$

Indoor: $10.6 \mathrm{ppm}$

Note: Indoor graph is shown with a dotted line. 


\section{ENGINEERING COMPUTATION SHEET}

Title of Project EPHA for THM

Subject Concentration Analysis for THM Hazardous Chemicals

Computer T. M. Franey

Date $\underline{09 / 16 / 96}$
Reviewed by $\underline{\text { S. A. Henderson }}$

Works Calculation 7

Sheet No. $\underline{453 \text { of } 577}$

SITE DATA INFORMATION: 1-RD-33

Location: AIKEN, SOUTH CAROLINA

Building Air Exchanges Per Hour: 60 (User specified)

Date and Time: Fixed at July 1, 19961300 hours

CHEMICAL INFORMATION:

Chemical Name: MONOETHANOLAMINE

Molecular Weight: $61.08 \mathrm{~kg} / \mathrm{kmol}$

TLV-TWA: $3.00 \mathrm{ppm}$ IDLH: $1000.00 \mathrm{ppm}$

Footprint Level of Concern: $100 \mathrm{ppm}$

Boiling Point: $171.00^{\circ} \mathrm{C}$

Vapor Pressure at Ambient Temperature: $4.85 \mathrm{e}-004 \mathrm{~atm}$

Ambient Saturation Concentration: 494 ppm or $0.049 \%$

ATMOSPHERIC INFORMATION: (MANUAL INPUT OF DATA)

Wind: 2.5 meters $/ \mathrm{sec}$ from $90^{\circ}$ true

Inversion Height: 500 meters

Stability Class: C Air Temperature: $25^{\circ} \mathrm{C}$

Relative Humidity: $50 \%$ Ground Roughness: 100 centimeters

Cloud Cover: 7 tenths

SOURCE STRENGTH INFORMATION:

Puddle Area: 99999 square feet

Average Puddle Depth: 1 centimeters

Soil Type: Default Ground Temperature: $25^{\circ} \mathrm{C}$

Initial Puddle Temperature: Ground temperature

Release Duration: ALOHA limited the duration to 1 hour

Max Computed Release Rate: 3.95 kilograms/min

Max Average Sustained Release Rate: 3.87 kilograms/min

(averaged over a minute or more)

Total Amount Released: 186 kilograms

FOOTPRINT INFORMATION:

Dispersion Module: Gaussian

User specified LOC: $100 \mathrm{ppm}$

Max Threat Zone for LOC: 55 meters

Max Threat Zone for IDLH: 54 meters

Note: Footprint was not drawn because

effects of near-field patchiness make plume

presentation unreliable for short distances.

TIME DEPENDENT INFORMATION:

Concentration Estimates at the point:

Downwind: 100 meters

Off Centerline: 0 meters

Max Concentration:

Outdoor: $3.28 \mathrm{ppm}$

Indoor: $3.28 \mathrm{ppm}$

Note: Indoor graph is shown with a dotted line. 


\section{ENGINEERING COMPUTATION SHEET}

Title of Project EPHA for THM

Subject Concentration Analysis for THM Hazardous Chemicals

Computer T. M. Franey Date $\underline{09 / 16 / 96}$
Reviewed by $\mathrm{S}$. A. Henderson

Works Calculation 7

Sheet No. 454 of 577

\section{SITE DATA INFORMATION: 1-RD-33}

Location: AIKEN, SOUTH CAROLINA

Building Air Exchanges Per Hour: 60 (User specified)

Date and Time: Fixed at July 1, 19961300 hours

CHEMICAL INFORMATION:

Chemical Name: MONOETHANOLAMINE

Molecular Weight: $61.08 \mathrm{~kg} / \mathrm{kmol}$

TLV-TWA: $3.00 \mathrm{ppm}$ IDLH: $1000.00 \mathrm{ppm}$

Footprint Level of Concern: 100 ppm

Boiling Point: $171.00^{\circ} \mathrm{C}$

Vapor Pressure at Ambient Temperature: 4.85e-004 atm

Ambient Saturation Concentration: 494 ppm or $0.049 \%$

ATMOSPHERIC INFORMATION: (MANUAL INPUT OF DATA)

Wind: 2.5 meters $/ \mathrm{sec}$ from $90^{\circ}$ true

Inversion Height: 500 meters

Stability Class: C Air Temperature: $25^{\circ} \mathrm{C}$

Relative Humidity: 50\% Ground Roughness: 100 centimeters

Cloud Cover: 7 tenths

\section{SOURCE STRENGTH INFORMATION:}

Puddle Area: 24420 square feet

Average Puddle Depth: 1 centimeters

Soil Type: Default Ground Temperature: $25^{\circ} \mathrm{C}$

Initial Puddle Temperature: Ground temperature

Release Duration: ALOHA limited the duration to 1 hour

Max Computed Release Rate: 1.02 kilograms/min

Max Average Sustained Release Rate: 1 kilograms/min (averaged over a minute or more)

Total Amount Released: 48.3 kilograms

\section{FOOTPRINT INFORMATION:}

Dispersion Module: Gaussian

User specified LOC: 100 ppm

Max Threat Zone for LOC: 27 meters

Max Threat Zone for IDLH: 27 meters

Note: Footprint was not drawn because effects of near-field patchiness make plume presentation unreliable for short distances.

\section{TIME DEPENDENT INFORMATION:}

Concentration Estimates at the point:

Downwind: 100 meters

Off Centerline: 0 meters

Max Concentration:

Outdoor: $1.59 \mathrm{ppm}$

Indoor: $1.59 \mathrm{ppm}$

Note: Indoor graph is shown with a dotted line. 
Title of Project EPHA for THM

Subject Concentration Analysis for THM Hazardous Chemicals

Computer T. M. Franey

Date $\underline{09 / 16 / 96}$
Reviewed by S. A. Henderson

Works Calculation 7

Sheet No. $\underline{455 \text { of } 577}$

SITE DATA INFORMATION: 1-RD-33

Location: AIKEN, SOUTH CAROLINA

Building Air Exchanges Per Hour: 60 (User specified)

Date and Time: Fixed at July 1, 19961300 hours

CHEMICAL INFORMATION:

Chemical Name: MONOETHANOLAMINE

Molecular Weight: $61.08 \mathrm{~kg} / \mathrm{kmol}$

TLV-TWA: $3.00 \mathrm{ppm}$ IDLH: $1000.00 \mathrm{ppm}$

Footprint Level of Concern: $100 \mathrm{ppm}$

Boiling Point: $171.00^{\circ} \mathrm{C}$

Vapor Pressure at Ambient Temperature: $4.85 \mathrm{e}-004 \mathrm{~atm}$

Ambient Saturation Concentration: 494 ppm or $0.049 \%$

ATMOSPHERIC INFORMATION: (MANUAL INPUT OF DATA)

Wind: 2.5 meters $/ \mathrm{sec}$ from $90^{\circ}$ true

Inversion Height: 500 meters

Stability Class: C Air Temperature: $25^{\circ} \mathrm{C}$

Relative Humidity: 50\% Ground Roughness: 100 centimeters

Cloud Cover: 7 tenths

SOURCE STRENGTH INFORMATION:

Puddle Area: 40700 square feet

Average Puddle Depth: 1 centimeters

Soil Type: Default Ground Temperature: $25^{\circ} \mathrm{C}$

Initial Puddle Temperature: Ground temperature

Release Duration: ALOHA limited the duration to 1 hour .

Max Computed Release Rate: 1.66 kilograms/min

Max Average Sustained Release Rate: 1.63 kilograms/min

(averaged over a minute or more)

Total Amount Released: 78.7 kilograms

FOOTPRINT INFORMATION:

Dispersion Module: Gaussian

User specified LOC: 100 ppm

Max Threat Zone for LOC: 34 meters

Max Threat Zone for IDLH: 35 meters

Note: Footprint was not drawn because

effects of near-field patchiness make plume

presentation unreliable for short distances.

TIME DEPENDENT INFORMATION:

Concentration Estimates at the point:

Downwind: 100 meters

Off Centerline: 0 meters

Max Concentration:

Outdoor: $2.01 \mathrm{ppm}$

Indoor: $2.01 \mathrm{ppm}$

Note: Indoor graph is shown with a dotted line. 


\section{ENGINEERING COMPUTATION SHEET}

Title of Project EPHA for THM

Subject Concentration Analysis for THM Hazardous Chemicals

Computer T.M. Franey

Date $\underline{09 / 16 / 96}$

SITE DATA INFORMATION: 1-RD-33

Location: AIKEN, SOUTH CAROLINA

Building Air Exchanges Per.Hour: 60 (User specified)

Date and Time: Fixed at July 1, 19961300 hours

CHEMICAL INFORMATION:

Chemical Name: MONOETHANOLAMINE

Molecular Weight: $61.08 \mathrm{~kg} / \mathrm{kmol}$

TLV-TWA: $3.00 \mathrm{ppm}$ IDLH: $1000.00 \mathrm{ppm}$

Footprint Level of Concern: 100 ppm

Boiling Point: $171.00^{\circ} \mathrm{C}$

Vapor Pressure at Ambient Temperature: 4.85e-004 atm

Ambient Saturation Concentration: 494 ppm or $0.049 \%$

ATMOSPHERIC INFORMATION: (MANUAL INPUT OF DATA)

Wind: 2.5 meters $/ \mathrm{sec}$ from $90^{\circ}$ true

Inversion Height: 500 meters

Stability Class: C Air Temperature: $25^{\circ} \mathrm{C}$

Relative Humidity: $50 \%$ Ground Roughness: 100 centimeters

Cloud Cover: 7 tenths

SOURCE STRENGTH INFORMATION:

Puddle Area: 10000 square meters

Average Puddle Depth: 1 centimeters

Soil Type: Default Ground Temperature: $25^{\circ} \mathrm{C}$

Initial Puddle Temperature: Ground temperature

Release Duration: ALOHA limited the duration to 1 hour

Max Computed Release Rate: 4.24 kilograms/min

Max Average Sustained Release Rate: 4.16 kilograms/min

(averaged over a minute or more)

Total Amount Released: 200 kilograms

FOOTPRINT INFORMATION:

Dispersion Module: Gaussian

User specified LOC: 100 ppm

Max Threat Zone for LOC: 56 meters

Max Threat Zone for IDLH: 56 meters

Note: Footprint was not drawn because

effects of near-field patchiness make plume

presentation unreliable for short distances.

\section{TIME DEPENDENT INFORMATION:}

Concentration Estimates at the point:

Downwind: 100 meters

Off Centerline: 0 meters

Max Concentration:

Outdoor: $3.46 \mathrm{ppm}$

Indoor: $3.46 \mathrm{ppm}$

Note: Indoor graph is shown with a dotted line.
Reviewed by S. A. Henderson

Works Calculation 7

Sheet No. 456 of 577 


\section{ENGINEERING COMPUTATION SHEET}

Title of Project EPHA for THM

Subject Concentration Analysis for THM Hazardous Chemicals

Computer T.M. Franey Date $\underline{09 / 16 / 96}$

SITE DATA INFORMATION: 1-RD-34

Location: AIKEN, SOUTH CAROLINA

Building Air Exchanges Per Hour: 60 (User specified)

Date and Time: Fixed at July 1, 19961300 hours

CHEMICAL INFORMATION:

Chemical Name: MORPHOLINE Molecular Weight: $87.12 \mathrm{~kg} / \mathrm{kmol}$

TLV-TWA: $20.00 \mathrm{ppm}$

IDLH: $8000.00 \mathrm{ppm}$

Footprint Level of Concern: $1400 \mathrm{ppm}$

Boiling Point: $128.00^{\circ} \mathrm{C}$

Vapor Pressure at Ambient Temperature: 0.013 atm

Ambient Saturation Concentration: $13,479 \mathrm{ppm}$ or $1.35 \%$

ATMOSPHERIC INFORMATION: (MANUAL INPUT OF DATA)

Wind: 2.5 meters $/ \mathrm{sec}$ from $90^{\circ}$ true

Inversion Height: 500 meters

Stability Class: C Air Temperature: $25^{\circ} \mathrm{C}$

Relative Humidity: 50\% Ground Roughness: 100 centimeters

Cloud Cover: 7 tenths

\section{SOURCE STRENGTH INFORMATION:}

Puddle Area: 30430 square feet

Average Puddle Depth: 1 centimeters

Soil Type: Default $\quad$ Ground Temperature: $25^{\circ} \mathrm{C}$

Initial Puddle Temperature: Ground temperature

Release Duration: ALOHA limited the duration to 1 hour

Max Computed Release Rate: 47.8 kilograms/min

Max Average Sustained Release Rate: 47.5 kilograms/min

(averaged over a minute or more)

Total Amount Released: 2,560 kilograms

\section{FOOTPRINT INFORMATION:}

Dispersion Module: Gaussian

User specified LOC: $1400 \mathrm{ppm}$

Max Threat Zone for LOC: 30 meters

Max Threat Zone for IDLH: 30 meters

Note: Footprint was not drawn because effects of near-field patchiness make plume

presentation unreliable for short distances.

\section{TIME DEPENDENT INFORMATION:}

Concentration Estimates at the point:

Downwind: 30 meters

Off Centerline: 0 meters

Max Concentration:

Outdoor: $286 \mathrm{ppm}$

Indoor: $286 \mathrm{ppm}$

Note: Indoor graph is shown with a dotted line.
Reviewed by $\mathrm{S}$. A. Henderson

Works Calculation 7

Sheet No. 457 of 577 


\section{ENGINEERING COMPUTATION SHEET}

Title of Project EPHA for THM

Subject Concentration Analysis for THM Hazardous Chemicals :

Computer T. M. Franey

Date $\underline{09 / 16 / 96}$

SITE DATA INFORMATION: 1-RD-34

Location: AIKEN, SOUTH CAROLINA

Building Air Exchanges Per Hour: 60 (User specified)

Date and Time: Fixed at July 1, 19961300 hours

CHEMICAL INFORMATION:

Chemical Name: MORPHOLINE Molecular Weight: $87.12 \mathrm{~kg} / \mathrm{kmol}$

TLV-TWA: $20.00 \mathrm{ppm}$ IDLH: $8000.00 \mathrm{ppm}$

Footprint Level of Concern: 1400 ppm

Boiling Point: $128.00^{\circ} \mathrm{C}$

Vapor Pressure at Ambient Temperature: $0.013 \mathrm{~atm}$

Ambient Saturation Concentration: 13,479 ppm or $1.35 \%$

ATMOSPHERIC INFORMATION: (MANUAL INPUT OF DATA)

Wind: 2.5 meters $/ \mathrm{sec}$ from $90^{\circ}$ true

Inversion Height: 500 meters

Stability Class: C Air Temperature: $25^{\circ} \mathrm{C}$

Relative Humidity: 50\% Ground Roughness: 100 centimeters

Cloud Cover: 7 tenths

\section{SOURCE STRENGTH INFORMATION:}

Puddle Area: 99999 square feet

Average Puddle Depth: 1 centimeters

Soil Type: Default Ground Temperature: $25^{\circ} \mathrm{C}$

Initial Puddle Temperature: Ground temperature

Release Duration: ALOHA limited the duration to 1 hour

Max Computed Release Rate: 151 kilograms/min

Max Average Sustained Release Rate: 149 kilograms/min

(averaged over a minute or more)

Total Amount Released: 8,041 kilograms

FOOTPRINT INFORMATION:

Dispersion Module: Gaussian

User specified LOC: $1400 \mathrm{ppm}$

Max Threat Zone for LOC: 55 meters

Max Threat Zone for IDLH: 54 meters

Note: Footprint was not drawn because effects of near-field patchiness make plume

presentation unreliable for short distances.

\section{TIME DEPENDENT INFORMATION:}

Concentration Estimates at the point:

Downwind: 100 meters

Off Centerline: 0 meters

Max Concentration:

Outdoor: $88.8 \mathrm{ppm}$

Indoor: $88.8 \mathrm{ppm}$

Note: Indoor graph is shown with a dotted line.
Reviewed by $\underline{\mathrm{S} \text {. A. Henderson }}$

Works Calculation 7

Sheet No. 458 of 577 


\section{ENGINEERING COMPUTATION SHEET}

Title of Project EPHA for THM

Reviewed by S.A. Henderson

Subject Concentration Analysis for THM Hazardous Chemicals

Works Calculation 7

Computer T.M. Franey

Date $\underline{09 / 16 / 96}$

Sheet No. 459 of 577

SITE DATA INFORMATION: 1-RD-34

Location: AIKEN, SOUTH CAROLINA

Building Air Exchanges Per Hour: 60 (User specified)

Date and Time: Fixed at July 1, 19961300 hours

CHEMICAL INFORMATION:

Chemical Name: MORPHOLINE Molecular Weight: $87.12 \mathrm{~kg} / \mathrm{kmol}$

TLV-TWA: $20.00 \mathrm{ppm}$

IDLH: $8000.00 \mathrm{ppm}$

Footprint Level of Concern: 1400 ppm

Boiling Point: $128.00^{\circ} \mathrm{C}$

Vapor Pressure at Ambient Temperature: $0.013 \mathrm{~atm}$

Ambient Saturation Concentration: $13,479 \mathrm{ppm}$ or $1.35 \%$

ATMOSPHERIC INFORMATION: (MANUAL INPUT OF DATA)

Wind: 2.5 meters $/ \mathrm{sec}$ from $90^{\circ}$ true

Inversion Height: 500 meters

Stability Class: C Air Temperature: $25^{\circ} \mathrm{C}$

Relative Humidity: 50\% Ground Roughness: 100 centimeters

Cloud Cover: 7 tenths

\section{SOURCE STRENGTH INFORMATION:}

Puddle Area: 24420 square feet

Average Puddle Depth: 1 centimeters

Soil Type: Default Ground Temperature: $25^{\circ} \mathrm{C}$

Initial Puddle Temperature: Ground temperature

Release Duration: ALOHA limited the duration to 1 hour

Max Computed Release Rate: 38.7 kilograms/min

Max Average Sustained Release Rate: 38.4 kilograms/min

(averaged over a minute or more)

Total Amount Released: 2,072 kilograms

FOOTPRINT INFORMATION:

Dispersion Module: Gaussian

User specified LOC: $1400 \mathrm{ppm}$

Max Threat Zone for LOC: 27 meters

Max Threat Zone for IDLH: 27 meters

Note: Footprint was not drawn because effects of near-field patchiness make plume presentation unreliable for short distances.

\section{TIME DEPENDENT INFORMATION:}

Concentration Estimates at the point:

Downwind: 100 meters .

Off Centerline: 0 meters

Max Concentration:

Outdoor: $42.6 \mathrm{ppm}$

Indoor: $42.6 \mathrm{ppm}$

Note: Indoor graph is shown with a dotted line. 


\section{ENGINEERING COMPUTATION SHEET}

Title of Project EPHA for THM

Reviewed by $\underline{S}$. A. Henderson

Subject Concentration Analysis for THM Hazardous Chemicals

Computer T. M. Franey

Date 09/16/96
Works Calculation 7

Sheet No. $\underline{460 \text { of } 577}$

\section{SITE DATA INFORMATION: 1-RD-34}

Location: AIKEN, SOUTH CAROLINA

Building Air Exchanges Per Hour: 60 (User specified)

Date and Time: Fixed at July 1, 19961300 hours

\section{CHEMICAL INFORMATION:}

Chemical Name: MORPHOLINE Molecular Weight: $87.12 \mathrm{~kg} / \mathrm{kmol}$
TLV-TWA: $20.00 \mathrm{ppm}$ IDLH: $8000.00 \mathrm{ppm}$

Footprint Level of Concern: 1400 ppm

Boiling Point: $128.00^{\circ} \mathrm{C}$

Vapor Pressure at Ambient Temperature: $0.013 \mathrm{~atm}$

Ambient Saturation Concentration: $13,479 \mathrm{ppm}$ or $1.35 \%$

\section{ATMOSPHERIC INFORMATION: (MANUAL INPUT OF DATA)}

Wind: 2.5 meters $/ \mathrm{sec}$ from $90^{\circ}$ true

Inversion Height: 500 meters

Stability Class: C Air Temperature: $25^{\circ} \mathrm{C}$

Relative Humidity: 50\% Ground Roughness: 100 centimeters

Cloud Cover: 7 tenths

\section{SOURCE STRENGTH INFORMATION:}

Puddle Area: 40700 square feet

Average Puddle Depth: 1 centimeters

Soil Type: Default Ground Temperature: $25^{\circ} \mathrm{C}$

Initial Puddle Temperature: Ground temperature

Release Duration: ALOHA limited the duration to 1 hour

Max Computed Release Rate: 63.3 kilograms/min

Max Average Sustained Release Rate: 62.8 kilograms/min

(averaged over a minute or more)

Total Amount Released: 3,386 kilograms

\section{FOOTPRINT INFORMATION:}

Dispersion Module: Gaussian

User specified LOC: $1400 \mathrm{ppm}$

Max Threat Zone for LOC: 34 meters

Max Threat Zone for IDLH: 35 meters

Note: Footprint was not drawn because effects of near-field patchiness make plume

presentation unreliable for short distances.

\section{TIME DEPENDENT INFORMATION:}

Concentration Estimates at the point:

Downwind: 100 meters

Off Centerline: 0 meters

Max Concentration:

Outdoor: $54.1 \mathrm{ppm}$

Indoor: $54.1 \mathrm{ppm}$

Note: Indoor graph is shown with a dotted line. 


\section{ENGINEERING COMPUTATION SHEET}

Title of Project EPHA for THM

Subject Concentration Analysis for THM Hazardous Chemicals

Computer T.M. Franey
Reviewed by $\underline{\text { S. A. Henderson }}$

Works Calculation 7

Sheet No. $\underline{461 \text { of } 577}$

SITE DATA INFORMATION: 1-RD-34

Location: AIKEN, SOUTH CAROLINA

Building Air Exchanges Per Hour: 60 (User specified)

Date and Time: Fixed at July 1, 19961300 hours

CHEMICAL INFORMATION:

Chemical Name: MORPHOLINE Molecular Weight: $87.12 \mathrm{~kg} / \mathrm{kmol}$ TLV-TWA: $20.00 \mathrm{ppm}$ IDLH: $8000.00 \mathrm{ppm}$

Footprint Level of Concern: $1400 \mathrm{ppm}$

Boiling Point: $128.00^{\circ} \mathrm{C}$

Vapor Pressure at Ambient Temperature: 0.013 atm

Ambient Saturation Concentration: $13,479 \mathrm{ppm}$ or $1.35 \%$

ATMOSPHERIC INFORMATION: (MANUAL INPUT OF DATA)

Wind: 2.5 meters $/ \mathrm{sec}$ from $90^{\circ}$ true

Inversion Height: 500 meters

Stability Class: C Air Temperature: $25^{\circ} \mathrm{C}$

Relative Humidity: 50\% Ground Roughness: 100 centimeters

Cloud Cover: 7 tenths

\section{SOURCE STRENGTH INFORMATION:}

Puddle Area: 10000 square meters

Average Puddle Depth: 1 centimeters

Soil Type: Default Ground Temperature: $25^{\circ} \mathrm{C}$

Initial Puddle Temperature: Ground temperature

Release Duration: AlOHA limited the duration to 1 hour

Max Computed Release Rate: 162 kilograms/min

Max Average Sustained Release Rate: 160 kilograms/min (averaged over a minute or more)

Total Amount Released: 8,632 kilograms

\section{FOOTPRINT INFORMATION:}

Dispersion Module: Gaussian

User specified LOC: $1400 \mathrm{ppm}$

Max Threat Zone for LOC: 56 meters

Max Threat Zone for IDLH: 56 meters

Note: Footprint was not drawn because effects of near-field patchiness make plume presentation unreliable for short distances.

\section{TIME DEPENDENT INFORMATION:}

Concentration Estimates at the point:

Downwind: 100 meters

Off Centerline: 0 meters

Max Concentration:

Outdoor: $93.7 \mathrm{ppm}$

Indoor: $93.7 \mathrm{ppm}$

Note: Indoor graph is shown with a dotted line. 


\section{ENGINEERING COMPUTATION SHEET}

Title of Project EPHA for THM

Reviewed by $\mathrm{S}$. A. Henderson

Works Calculation 7

Sheet No. $\underline{462 \text { of } 577}$

\section{CHEMICAL INFORMATION:}

Chemical Name: BUTYL ALCOHOL Molecular Weight: $74.12 \mathrm{~kg} / \mathrm{kmol}$

TLV-TWA: $50.00 \mathrm{ppm}$ IDLH: $8000.00 \mathrm{ppm}$

Footprint Level of Concern: $50 \mathrm{ppm}$

Boiling Point: $117.66^{\circ} \mathrm{C}$

Vapor Pressure at Ambient Temperature: 0.0093 atm

Ambient Saturation Concentration: 9,442 ppm or $0.94 \%$

\section{ATMOSPHERIC INFORMATION: (MANUAL INPUT OF DATA) \\ Wind: 2.5 meters $/ \mathrm{sec}$ from $90^{\circ}$ true \\ Inversion Height: 500 meters \\ Stability Class: C Air Temperature: $25^{\circ} \mathrm{C}$ \\ Relative Humidity: 50\% Ground Roughness: 100 centimeters \\ Cloud Cover: 7 tenths}

\section{SOURCE STRENGTH INFORMATION:}

Puddle Area: 1995 square feet

Average Puddle Depth: 1 centimeters

Soil Type: Default Ground Temperature: $25^{\circ} \mathrm{C}$

Initial Puddle Temperature: Ground temperature

Release Duration: ALOHA limited the duration to 1 hour

Max Computed Release Rate: 3.31 kilograms/min

Max Average Sustained Release Rate: 3.29 kilograms/min (averaged over a minute or more)

Total Amount Released: 179 kilograms

\section{FOOTPRINT INFORMATION:}

Dispersion Module: Gaussian

User specified LOC: $50 \mathrm{ppm}$

Max Threat Zone for LOC: 30 meters

Max Threat Zone for IDLH: less than 10 meters(10.9 yards)

Note: Footprint was not drawn because

effects of near-field patchiness make plume

presentation unreliable for short distances.

\section{TIME DEPENDENT INFORMATION:}

\section{Concentration Estimates at the point:}

Downwind: 30 meters

Off Centerline: 0 meters

Max Concentration:

Outdoor: $50 \mathrm{ppm}$

Indoor: $50 \mathrm{ppm}$

Note: Indoor graph is shown with a dotted line. 


\section{ENGINEERING COMPUTATION SHEET}

Title of Project EPHA for THM

Subject Concentration Analysis for THM Hazardous Chemicals

Computer T. M. Franey

Date $\underline{09 / 16 / 96}$

SITE DATA INFORMATION: 1-RD-35

Location: AIKEN, SOUTH CAROLINA

Building Air Exchanges Per Hour: 60 (User specified)

Date and Time: Fixed at July 1, 19961300 hours

CHEMICAL INFORMATION:

Chemical Name: BUTYL ALCOHOL

Molecular Weight: $74.12 \mathrm{~kg} / \mathrm{kmol}$

TLV-TWA: $50.00 \mathrm{ppm}$ IDLH: $8000.00 \mathrm{ppm}$

Footprint Level of Concern: $50 \mathrm{ppm}$

Boiling Point: $117.66^{\circ} \mathrm{C}$

Vapor Pressure at Ambient Temperature: $0.0093 \mathrm{~atm}$

Ambient Saturation Concentration: 9,442 ppm or $0.94 \%$

ATMOSPHERIC INFORMATION: (MANUAL INPUT OF DATA)

Wind: 2.5 meters $/ \mathrm{sec}$ from $90^{\circ}$ true

Inversion Height: 500 meters

Stability Class: C Air Temperature: $25^{\circ} \mathrm{C}$

Relative Humidity: 50\% Ground Roughness: 100 centimeters

Cloud Cover: 7 tenths

\section{SOURCE STRENGTH INFORMATION:}

Puddle Area: 26900 square feet

Average Puddle Depth: 1 centimeters

Soil Type: Default Ground Temperature: $25^{\circ} \mathrm{C}$

Initial Puddle Temperature: Ground temperature

Release Duration: ALOHA limited the duration to 1 hour

Max Computed Release Rate: 40.5 kilograms/min

Max Average Sustained Release Rate: 40.2 kilograms/min

(averaged over a minute or more)

Total Amount Released: 2,171 kilograms

\section{FOOTPRINT INFORMATION:}

Dispersion Module: Gaussian

User specified LOC: $50 \mathrm{ppm}$

Max Threat Zone for LOC: 100 meters

Max Threat Zone for IDLH: 28 meters

Note: Footprint was not drawn because effects of near-field patchiness make plume presentation unreliable for short distances.

\section{TIME DEPENDENT INFORMATION:}

Concentration Estimates at the point:

Downwind: 100 meters

Off Centerline: 0 meters

Max Concentration:

Outdoor: $50 \mathrm{ppm}$

Indoor: $50 \mathrm{ppm}$

Note: Indoor graph is shown with a dotted line.
Reviewed by $\underline{\text { S. A. Henderson }}$

Works Calculation 7

Sheet No. 463 of 577 
Title of Project EPHA for THM

Subject Concentration Analysis for THM Hazardous Chemicals

Computer T. M. Franey

Date 09/16/96
Reviewed by S. A. Henderson

Works Calculation 7

Sheet No. 464 of 577

SITE DATA INFORMATION: 1-RD-35

Location: AIKEN, SOUTH CAROLINA

Building Air Exchanges Per Hour: 60 (User specified)

Date and Time: Fixed at July 1, 19961300 hours

CHEMICAL INFORMATION:

Chemical Name: BUTYL ALCOHOL

Molecular Weight: $74.12 \mathrm{~kg} / \mathrm{kmol}$

TLV-TWA: $50.00 \mathrm{ppm}$ IDLH: $8000.00 \mathrm{ppm}$

Footprint Level of Concern: $50 \mathrm{ppm}$

Boiling Point: $117.66^{\circ} \mathrm{C}$

Vapor Pressure at Ambient Temperature: $0.0093 \mathrm{~atm}$

Ambient Saturation Concentration: 9,442 ppm or $0.94 \%$

ATMOSPHERIC INFORMATION: (MANUAL INPUT OF DATA)

Wind: 2.5 meters $/ \mathrm{sec}$ from $90^{\circ}$ true .

Inversion Height: 500 meters

Stability Class: C Air Temperature: $25^{\circ} \mathrm{C}$

Relative Humidity: 50\% Ground Roughness: 100 centimeters

Cloud Cover: 7 tenths

SOURCE STRENGTH INFORMATION:

Puddle Area: 24420 square feet

Average Puddle Depth: 1 centimeters

Soil Type: Default Ground Temperature: $25^{\circ} \mathrm{C}$

Initial Puddle Temperature: Ground temperature

Release Duration: ALOHA limited the duration to 1 hour

Max Computed Release Rate: 36.9 kilograms/min

Max Average Sustained Release Rate: 36.6 kilograms/min

(averaged over a minute or more)

Total Amount Released: 1,978 kilograms

FOOTPRINT INFORMATION:

Dispersion Module: Gaussian

User specified LOC: $50 \mathrm{ppm}$

Max Threat Zone for LOC: 96 meters

Max Threat Zone for IDLH: 27 meters

Note: Footprint was not drawn because effects of near-field patchiness make plume presentation unreliable for short distances.

TIME DEPENDENT INFORMATION:

Concentration Estimates at the point:

Downwind: 100 meters

Off Centerline: 0 meters

Max Concentration:

Outdoor: $47.8 \mathrm{ppm}$

Indoor: $47.8 \mathrm{ppm}$

Note: Indoor graph is shown with a dotted line. 


\section{ENGINEERING COMPUTATION SHEET}

Title of Project EPHA for THM

Subject Concentration Analysis for THM Hazardous Chemicals

Computer T. M. Franey

Date 09/16/96

Reviewed by S. A. Henderson

Works Calculation 7

Sheet No. 465 of 577

SITE DATA INFORMATION: 1-RD-35

Lócation: AIKEN, SOUTH CAROLINA

Building Air Exchanges Per Hour: 60 (User specified)

Date and Time: Fixed at July 1, 19961300 hours

CHEMICAL INFORMATION:

Chemical Name: BUTYL ALCOHOL

Molecular Weight: $74.12 \mathrm{~kg} / \mathrm{kmol}$

TLV-TWA: $50.00 \mathrm{ppm}$ IDLH: $8000.00 \mathrm{ppm}$

Footprint Level of Concern: 50 ppm

Boiling Point: $117.66^{\circ} \mathrm{C}$

Vapor Pressure at Ambient Temperature: $0.0093 \mathrm{~atm}$

Ambient Saturation Concentration: 9,442 ppm or $0.94 \%$

ATMOSPHERIC INFORMATION: (MANUAL INPUT OF DATA)

Wind: 2.5 meters $/ \mathrm{sec}$ from $90^{\circ}$ true

Inversion Height: 500 meters

Stability Class: C Air Temperature: $25^{\circ} \mathrm{C}$

Relative Humidity: 50\% Ground Roughness: 100 centimeters

Cloud Cover: 7 tenths

\section{SOURCE STRENGTH INFORMATION:}

Puddle Area: 40700 square feet

Average Puddle Depth: 1 centimeters

Soil Type: Default $\quad$ Ground Temperature: $25^{\circ} \mathrm{C}$

Initial Puddle Temperature: Ground temperature

Release Duration: ALOHA limited the duration to 1 hour

Max Computed Release Rate: 60.4 kilograms/min

Max Average Sustained Release Rate: 59.9 kilograms/min

(averaged over a minute or more)

Total Amount Released: 3,232 kilograms

FOOTPRINT INFORMATION:

Dispersion Module: Gaussian

User specified LOC: $50 \mathrm{ppm}$

Max Threat Zone for LOC: 121 meters

Max Threat Zone for IDLH: 34 meters

TIME DEPENDENT INFORMATION:

Concentration Estimates at the point:

Downwind: 100 meters

Off Centerline: 0 meters

Max Concentration:

Outdoor: $60.7 \mathrm{ppm}$

Indoor: $60.7 \mathrm{ppm}$

Note: Indoor graph is shown with a dotted line. 
Title of Project EPHA for THM

Subject Concentration Analysis for THM Hazardous Chemicals

Computer T. M. Franey
Reviewed by S. A. Henderson

Works Calculation 7

Sheet No. 467 of 577

\title{
1-RD-36 SITE DATA INFORMATION:
}

Location: AIKEN, SOUTH CAROLINA

Building Air Exchanges Per Hour: 60 (User specified)

Date and Time: Fixed at July 1, 19961300 hours

CHEMICAL INFORMATION:

Chemical Name: 1-METHYLPYRROLIDONE

Molecular Weight: $99.13 \mathrm{~kg} / \mathrm{kmol}$

TLV-TWA: -unavail- IDLH: -unavail-

Footprint Level of Concern: $1000 \mathrm{ppm}$

Boiling Point: $202.00^{\circ} \mathrm{C}$

Vapor Pressure at Ambient Temperature: 4.49e-004 atm

Ambient Saturation Concentration: 457 ppm or $0.046 \%$

\author{
ATMOSPHERIC INFORMATION: (MANUAL INPUT OF DATA) \\ Wind: 2.5 meters $/ \mathrm{sec}$ from $90^{\circ}$ true \\ Inversion Height: 500 feet \\ Stability Class: C Air Temperature: $25^{\circ} \mathrm{C}$ \\ Relative Humidity: 50\% Ground Roughness: Urban or forest \\ Cloud Cover: 7 tenths
}

\section{SOURCE STRENGTH INFORMATION:}

Puddle Area: 40000 square feet

Average Puddle Depth: 1 centimeters

Soil Type: Default Ground Temperature: $25^{\circ} \mathrm{C}$

Initial Puddle Temperature: Ground temperature

Release Duration: ALOHA limited the duration to 1 hour

Max Computed Release Rate: 3.25 kilograms/min

Max Average Sustained Release Rate: 3.2 kilograms/min

(averaged over a minute or more)

Total Amount Released: 162 kilograms

TIME DEPENDENT INFORMATION:

Concentration Estimates at the point: 
Title of Project EPHA for THM

Subject Concentration Analysis for THM Hazardous Chemicals

Computer T. M. Franey Date $\underline{09 / 16 / 96}$
Reviewed by $\underline{S}$. A. Henderson

Works Calculation 7

Sheet No. 468 of 577

1-RD-36 SITE DATA INFORMATION:

Location: AIKEN, SOUTH CAROLINA

Building Air Exchanges Per Hour: 60 (User specified)

Date and Time: Fixed at July 1, 19961300 hours

\section{CHEMICAL INFORMATION:}

Chemical Name: 1-METHYLPYRROLIDONE

Molecular Weight: $99.13 \mathrm{~kg} / \mathrm{kmol}$

TLV-TWA: -unavail- IDLH: -unavail-

Footprint Level of Concern: 1000 ppm

Boiling Point: $202.00^{\circ} \mathrm{C}$

Vapor Pressure at Ambient Temperature: $4.49 \mathrm{e}-004 \mathrm{~atm}$

Ambient Saturation Concentration: 457 ppm or $0.046 \%$

ATMOSPHERIC INFORMATION: (MANUAL INPUT OF DATA)

Wind: 2.5 meters $/ \mathrm{sec}$ from $90^{\circ}$ true

Inversion Height: 500 feet

Stability Class: C Air Temperature: $25^{\circ} \mathrm{C}$

Relative Humidity: 50\% Ground Roughness: Urban or forest

Cloud Cover: 7 tenths

\section{SOURCE STRENGTH INFORMATION:}

Puddle Area: 100000 square feet

Average Puddle Depth: 1 centimeters

Soil Type: Default Ground Temperature: $25^{\circ} \mathrm{C}$

Initial Puddle Temperature: Ground temperature

Release Duration: ALOHA limited the duration to 1 hour

Max Computed Release Rate: 7.85 kilograms/min

Max Average Sustained Release Rate: 7.74 kilograms/min (averaged over a minute or more)

Total Amount Released: 390 kilograms

\section{TIME DEPENDENT INFORMATION:}

Concentration Estimates at the point:

Downwind: 100 meters

Off Centerline: 0 meters

Max Concentration:

Outdoor: $4.04 \mathrm{ppm}$ 
Title of Project EPHA for THM

Subject Concentration Analysis for THM Hazardous Chemicals

Computer T. M. Franey
Reviewed by S. A. Henderson

Works Calculation 7

Sheet No. 469 of 577

\section{1-RD-36 SITE DATA INFORMATION:} Location: AIKEN, SOUTH CAROLINA

Building Air Exchanges Per Hour: 60 (User specifred)

Date and Time: Fixed at July 1, 19961300 hours

\section{CHEMICAL INFORMATION:}

Chemical Name: 1-METHYLPYRROLIDONE

Molecular Weight: $99.13 \mathrm{~kg} / \mathrm{kmol}$

TLV-TWA: -unavail- IDLH: -unavail-

Footprint Level of Concern: 1000 ppm

Boiling Point: $202.00^{\circ} \mathrm{C}$

Vapor Pressure at Ambient Temperature: $4.49 \mathrm{e}-004 \mathrm{~atm}$

Ambient Saturation Concentration: 457 ppm or $0.046 \%$

ATMOSPHERIC INFORMATION: (MANUAL INPUT OF DATA)

Wind: 2.5 meters $/ \mathrm{sec}$ from $90^{\circ}$ true

Inversion Height: 500 feet

Stability Class: C Air Temperature: $25^{\circ} \mathrm{C}$

Relative Humidity: 50\% Ground Roughness: Urban or forest

Cloud Cover: 7 tenths

\section{SOURCE STRENGTH INFORMATION:}

Puddle Area: 24420 square feet

Average Puddle Depth: 1 centimeters

Soil Type: Default Ground Temperature: $25^{\circ} \mathrm{C}$

Initial Puddle Temperature: Ground temperature

Release Duration: ALOHA limited the duration to 1 hour

Max Computed Release Rate: 2.02 kilograms/min

Max Average Sustained Release Rate; 1.99 kilograms/min

(averaged over a minute or more)

Total Amount Released: 101 kilograms

\section{FOOTPRINT INFORMATION:}

Dispersion Module: Gaussian

User specified LOC: $1000 \mathrm{ppm}$

Max Threat Zone for LOC: 27 meters

Note: Footprint was not drawn because effects of near-field patchiness make plume presentation unreliable for short distances.

\section{TIME DEPENDENT INFORMATION:}

Concentration Estimates at the point:

Downwind: 100 meters

Off Centerline: 0 meters

Max Concentration:

Outdoor: $1.95 \mathrm{ppm}$ 


\section{ENGINEERING COMPUTATION SHEET}

Title of Project EPHA for THM

Subject Concentration Analysis for THM Hazardous Chemicals

Computer T. M. Franey
Reviewed by S.A. Henderson

Works Calculation 7

Sheet No. 470 of 577

\section{1-RD-36 SITE DATA INFORMATION: \\ Location: AIKEN, SOUTH CAROLINA \\ Building Air Exchanges Per Hour: 60 (User specified) \\ Date and Time: Fixed at July 1, 19961300 hours \\ CHEMICAL INFORMATION: \\ Chemical Name: 1-METHYLPYRROLIDONE \\ Molecular Weight: $99.13 \mathrm{~kg} / \mathrm{kmol}$ \\ TLV-TWA: -unavail- IDLH: -unavail- \\ Footprint Level of Concern: $1000 \mathrm{ppm}$ \\ Boiling Point: $202.00^{\circ} \mathrm{C}$ \\ Vapor Pressure at Ambient Temperature: 4.49e-004 atm \\ Ambient Saturation Concentration: 457 ppm or $0.046 \%$}

ATMOSPHERIC INFORMATION: (MANUAL INPUT OF DATA)

Wind: 2.5 meters $/ \mathrm{sec}$ from $90^{\circ}$ true

Inversion Height: 500 feet

Stability Class: C Air Temperature: $25^{\circ} \mathrm{C}$

Relative Humidity: 50\% Ground Roughness: Urban or forest

Cloud Cover: 7 tenths

\section{SOURCE STRENGTH INFORMATION:}

Puddle Area: 40700 square feet

Average Puddle Depth: 1 centimeters

Soil Type: Default Ground Temperature: $25^{\circ} \mathrm{C}$

Initial Puddle Temperature: Ground temperature

Release Duration: ALOHA limited the duration to 1 hour

Max Computed Release Rate: 3.3 kilograms/min

Max Average Sustained Release Rate: $3.26 \mathrm{kilograms} / \mathrm{min}$

(averaged over a minute or more)

Total Amount Released: 164 kilograms

\section{FOOTPRINT INFORMATION:}

Dispersion Module: Gaussian

User specified LOC: $1000 \mathrm{ppm}$

Max Threat Zone for LOC: 34 meters

Note: Footprint was not drawn because

effects of near-field patchiness make plume

presentation unreliable for short distances.

\section{TIME DEPENDENT INFORMATION:}

Concentration Estimates at the point:

Downwind: 100 meters

Off Centerline: 0 meters

Max Concentration:

Outdoor: $2.47 \mathrm{ppm}$ 


\section{ENGINEERING COMPUTATION SHEET}

Title of Project EPHA for THM

Subject Concentration Analysis for THM Hazardous Chemicals

Computer T.M. Franey

Date 09/16/96

1-RD-36 SITE DATA INFORMATION:

Location: AIKEN, SOUTH CAROLINA

Building Air Exchanges Per Hour: 60 (User specified)

Date and Time: Fixed at July 1, 19961300 hours

CHEMICAL INFORMATION:

Chemical Name: 1-METHYLPYRROLIDONE

Molecular Weight: $99.13 \mathrm{~kg} / \mathrm{kmol}$

TLV-TWA: -unavail- IDLH: -unavail-

Footprint Level of Concern: $1000 \mathrm{ppm}$

Boiling Point: $202.00^{\circ} \mathrm{C}$

Vapor Pressure at Ambient Temperature: 4.49e-004 atm

Ambient Saturation Concentration: 457 ppm or $0.046 \%$

ATMOSPHERIC INFORMATION: (MANUAL INPUT OF DATA)

Wind: 2.5 meters $/ \mathrm{sec}$ from $90^{\circ}$ true

Inversion Height: 500 feet

Stability Class: C Air Temperature: $25^{\circ} \mathrm{C}$

Relative Humidity: 50\% Ground Roughness: Urban or forest

Cloud Cover: 7 tenths

\section{SOURCE STRENGTH INFORMATION:}

Puddle Area: 10000 square meters

Average Puddle Depth: 1 centimeters

Soil Type: Default Ground Temperature: $25^{\circ} \mathrm{C}$

Initial Puddle Temperature: Ground temperature

Release Duration: ALOHA limited the duration to 1 hour

Max Computed Release Rate: 8.43 kilograms/min

Max Average Sustained Release Rate: 8.31 kilograms/min (averaged over a minute or more)

Total Amount Released: 419 kilograms

\section{FOOTPRINT INFORMATION:}

Dispersion Module: Gaussian

User specified LOC: $1000 \mathrm{ppm}$

Max Threat Zone for LOC: 56 meters

Note: Footprint was not drawn because effects of near-field patchiness make plume presentation unreliable for short distances.

\section{TIME DEPENDENT INFORMATION:}

Concentration Estimates at the point:

Downwind: 100 meters

Off Centerline: 0 meters

Max Concentration:

Outdoor: $4.26 \mathrm{ppm}$
Reviewed by S. A. Henderson

Works Calculation 7

Sheet No. 471 of 577 


\section{ENGINEERING COMPUTATION SHEET}

Title of Project. EPHA for THM

Subject Concentration Analysis for THM Hazardous Chemicals

Computer T.M. Franey

Date 09/16/96

SITE DATA INFORMATION: 1-RD-37

Location: AIKEN, SOUTH CAROLINA

Building Air Exchanges Per Hour: 60 (User specified)

Date and Time: Fixed at July 1, 19961300 hours

CHEMICAL INFORMATION:

Chemical Name: NITRIC ACID; FUMING OR CONCENTRATED

Molecular Weight: $63.01 \mathrm{~kg} / \mathrm{kmol}$

TLV-TWA: $2.00 \mathrm{ppm}$ IDLH: $100.00 \mathrm{ppm}$

Footprint Level of Concern: $15 \mathrm{ppm}$

Boiling Point: $83.00^{\circ} \mathrm{C}$

Vapor Pressure at Ambient Temperature: $0.083 \mathrm{~atm}$

Ambient Saturation Concentration: 84,423 ppm or $8.44 \%$

ATMOSPHERIC INFORMATION: (MANUAL INPUT OF DATA)

Wind: 2.5 meters $/ \mathrm{sec}$ from $90^{\circ}$ true

Inversion Height: 500 meters

Stability Class: C Air Temperature: $25^{\circ} \mathrm{C}$

Relative Humidity: $50 \%$ Ground Roughness: 100 centimeters

Cloud Cover: 7 tenths

\section{SOURCE STRENGTH INFORMATION:}

Puddle Area: 82 square feet

Average Puddle Depth: 1 centimeters

Soil Type: Default Ground Temperature: $25^{\circ} \mathrm{C}$

Initial Puddle Temperature: Ground temperature

Release Duration: ALOHA limited the duration to 1 hour

Max Computed Release Rate: 390 grams/min

Max Average Sustained Release Rate: 383 grams/min

(averaged over a minute or more)

Total Amount Released: 22.6 kilograms

\section{FOOTPRINT INFORMATION:}

Dispersion Module: Gaussian

User specified LOC: $15 \mathrm{ppm}$

Max Threat Zone for LOC: 30 meters

Max Threat Zone for IDLH: less than 10 meters(10.9.yards)

Note: Footprint was not drawn because effects of near-field patchiness make plume presentation unreliable for short distances.

\section{TIME DEPENDENT INFORMATION:}

Concentration Estimates at the point:

Downwind: 30 meters

Off Centerline: 0 meters

Max Concentration:

Outdoor: $15.1 \mathrm{ppm}$

Indoor: $15.1 \mathrm{ppm}$

Note: Indoor graph is shown with a dotted line.
Reviewed by S. A. Henderson

Works Calculation 7

Sheet No. $\underline{42 \text { of } 577}$ 
Title of Project EPHA for THM

Subject Concentration Analysis for THM Hazardous Chemicals

Computer T. M. Franey

Date 09/16/96

SITE DATA INFORMATION: 1-RD-37

Location: AIKEN, SOUTH CAROLINA

Building Air Exchanges Per Hour: 60 (User specified)

Date and Time: Fixed at July 1, 19961300 hours

CHEMICAL INFORMATION:

Chemical Name: NITRIC ACID; FUMING OR CONCENTRATED

Molecular Weight: $63.01 \mathrm{~kg} / \mathrm{kmol}$

TLV-TWA: $2.00 \mathrm{ppm}$ IDLH: $100.00 \mathrm{ppm}$

Footprint Level of Concern: $15 \mathrm{ppm}$

Boiling Point: $83.00^{\circ} \mathrm{C}$

Vapor Pressure at Ambient Temperature: $0.083 \mathrm{~atm}$

Ambient Saturation Concentration: 84,423 ppm or $8.44 \%$

ATMOSPHERIC INFORMATION: (MANUAL INPUT OF DATA)

Wind: 2.5 meters $/ \mathrm{sec}$ from $90^{\circ}$ true

Inversion Height: 500 meters

Stability Class: C Air Temperature: $25^{\circ} \mathrm{C}$

Relative Humidity: 50\% Ground Roughness: 100 centimeters

Cloud Cover: 7 tenths

SOURCE STRENGTH INFORMATION:

Puddle Area: 1015 square feet

Average Puddle Depth: 1 centimeters

Soil Type: Default Ground Temperature: $25^{\circ} \mathrm{C}$

Initial Puddle Temperature: Ground temperature

Release Duration: ALOHA limited the duration to 1 hour

Max Computed Release Rate: 4.24 kilograms/min

Max Average Sustained Release Rate: 4.24 kilograms/min

(averaged over a minute or more)

Total Amount Released: 254 kilograms

\section{FOOTPRINT INFORMATION:}

Dispersion Module: Gaussian

User specified LOC: 15 ppm

Max Threat Zone for LOC: 100 meters

Max Threat Zone for IDLH: 31 meters

Note: Footprint was not drawn because

effects of near-field patchiness make plume

presentation unreliable for short distances.

\section{TIME DEPENDENT INFORMATION:}

Concentration Estimates at the point:

Downwind: 100 meters

Off Centerline: 0 meters

Max Concentration:

Outdoor: $15 \mathrm{ppm}$

Indoor: $15 \mathrm{ppm}$

Note: Indoor graph is shown with a dotted line.
Reviewed by $\underline{S}$. A. Henderson

Works Calculation 7

Sheet No. $\underline{473 \text { of } 577}$ 


\section{ENGINEERING COMPUTATION SHEET}

Title of Project EPHA for THM

Subject Concentration Analysis for THM Hazardous Chemicals

Computer T. M. Franey Date $\underline{09 / 16 / 96}$
Reviewed by $\underline{S}$. A. Henderson

Works Calculation 7

Sheet No. 474 of 577

\section{SITE DATA INFORMATION: 1-RD-37 \\ Location: AIKEN, SOUTH CAROLINA \\ Building Air Exchanges Per Hour: 60 (User specified) \\ Date and Time: Fixed at July 1, 19961300 hours}

CHEMICAL INFORMATION:

Chemical Name: NITRIC ACID; FUMING OR CONCENTRATED

Molecular Weight: $63.01 \mathrm{~kg} / \mathrm{kmol}$

TLV-TWA: $2.00 \mathrm{ppm}$

IDLH: $100.00 \mathrm{ppm}$

Footprint Level of Concern: 15 ppm

Boiling Point: $83.00^{\circ} \mathrm{C}$

Vapor Pressure at Ambient Temperature: $0.083 \mathrm{~atm}$

Ambient Saturation Concentration: 84,423 ppm or $8.44 \%$

\section{ATMOSPHERIC INFORMATION: (MANUAL INPUT OF DATA)}

Wind: 2.5 meters $/ \mathrm{sec}$ from $90^{\circ}$ true

Inversion Height: 500 meters

Stability Class: C Air Temperature: $25^{\circ} \mathrm{C}$

Relative Humidity: 50\% Ground Roughness: 100 centimeters

Cloud Cover: 7 tenths

\section{SOURCE STRENGTH INFORMATION:}

Puddle Area: 24420 square feet

Average Puddle Depth: 1 centimeters

Soil Type: Default Ground Temperature: $25^{\circ} \mathrm{C}$

Initial Puddle Temperature: Ground temperature

Release Duration: ALOHA limited the duration to 1 hour

Max Computed Release Rate: 92.4 kilograms/min

Max Average Sustained Release Rate: 92.3 kilograms/min (averaged over a minute or more)

Total Amount Released: 5,447 kilograms

FOOTPRINT INFORMATION:

Dispersion Module: Gaussian

User specified LOC: $15 \mathrm{ppm}$

Max Threat Zone for LOC: 469 meters

Max Threat Zone for IDLH: 139 meters

\section{TIME DEPENDENT INFORMATION:}

Concentration Estimates at the point:

Downwind: 100 meters

Off Centerline: 0 meters

Max Concentration:

Outdoor: $142 \mathrm{ppm}$

Indoor: $142 \mathrm{ppm}$

Note: Indoor graph is shown with a dotted line. 


\section{ENGINEERING COMPUTATION SHEET}

Title of Project EPHA for THM

Reviewed by S. A. Henderson

Subject Concentration Analysis for THM Hazardous Chemicals

Computer T. M. Franey

Date $\underline{09 / 16 / 96}$
Works Calculation 7

Sheet No. 475 of 577

\section{SITE DATA INFORMATION: 1-RD-37 \\ Location: AIKEN, SOUTH CAROLINA \\ Building Air Exchanges Per Hour: 60 (User specified) \\ Date and Time: Fixed at July 1, 19961300 hours}

\section{CHEMICAL INFORMATION:}

Chemical Name: NITRIC ACID; FUMING OR CONCENTRATED

Molecular Weight: $63.01 \mathrm{~kg} / \mathrm{kmol}$

TLV-TWA: $2.00 \mathrm{ppm}$ IDLH: $100.00 \mathrm{ppm}$

Footprint Level of Concern: 15 ppm

Boiling Point: $83.00^{\circ} \mathrm{C}$

Vapor Pressure at Ambient Temperature: $0.083 \mathrm{~atm}$

Ambient Saturation Concentration: 84,423 ppm or $8.44 \%$

ATMOSPHERIC INFORMATION: (MANUAL INPUT OF DATA)

Wind: 2.5 meters $/ \mathrm{sec}$ from $90^{\circ}$ true

Inversion Height: 500 meters

Stability Class: C Air Temperature: $25^{\circ} \mathrm{C}$

Relative Humidity: 50\% Ground Roughness: 100 centimeters

Cloud Cover: 7 tenths

\section{SOURCE STRENGTH INFORMATION:}

Puddle Area: 40700 square feet Average Puddle Depth: 1 centimeters

Soil Type: Default Ground Temperature: $25^{\circ} \mathrm{C}$

Initial Puddle Temperature: Ground temperature

Release Duration: ALOHA limited the duration to 1 hour

Max Computed Release Rate: 152 kilograms/min

Max Average Sustained Release Rate: 152 kilograms/min

(averaged over a minute or more)

Total Amount Released: 8,927 kilograms

FOOTPRINT INFORMATION:

Dispersion Module: Gaussian

User specified LOC: $15 \mathrm{ppm}$

Max Threat Zone for LOC: 603 meters

Max Threat Zone for IDLH: 177 meters

TIME DEPENDENT INFORMATION:

Concentration Estimates at the point:

Downwind: 100 meters

Off Centerline: 0 meters

Max Concentration:

Outdoor: $181 \mathrm{ppm}$

Indoor: $181 \mathrm{ppm}$

Note: Indoor graph is shown with a dotted line. 


\section{ENGINEERING COMPUTATION SHEET}

Title of Project EPHA for THM

Subject Concentration Analysis for THM Hazardous Chemicals

Computer T.M. Franey

Date $\underline{09 / 16 / 96}$

SITE DATA INFORMATION: 1-RD-37

Location: AIKEN, SOUTH CAROLINA

Building Air Exchanges Per Hour: 60 (User specified)

Date and Time: Fixed at July 1, 19961300 hours

\section{CHEMICAL INFORMATION:}

Chemical Name: NITRIC ACID; FUMING OR CONCENTRATED

Molecular Weight: $63.01 \mathrm{~kg} / \mathrm{kmol}$

TLV-TWA: $2.00 \mathrm{ppm}$ IDLH: $100.00 \mathrm{ppm}$

Footprint Level of Concern: 15 ppm

Boiling Point: $83.00^{\circ} \mathrm{C}$

Vapor Pressure at Ambient Temperature: $0.083 \mathrm{~atm}$

Ambient Saturation Concentration: 84,423 ppm or $8.44 \%$

ATMOSPHERIC INFORMATION: (MANUAL INPUT OF DATA)

Wind: 2.5 meters $/ \mathrm{sec}$ from $90^{\circ}$ true

Inversion Height: 500 meters

Stability Class: C Air Temperature: $25^{\circ} \mathrm{C}$

Relative Humidity: 50\% Ground Roughness: 100 centimeters

Cloud Cover: 7 tenths

\section{SOURCE STRENGTH INFORMATION:}

Puddle Area: 10000 square meters

Average Puddle Depth: 1 centimeters

Soil Type: Default Ground Temperature: $25^{\circ} \mathrm{C}$

Initial Puddle Temperature: Ground temperature

Release Duration: ALOHA limited the duration to 1 hour

Max Computed Release Rate: 390 kilograms/min

Max Average Sustained Release Rate: 390 kilograms/min

(averaged over a minute or more)

Total Amount Released: 22,882 kilograms

FOOTPRINT INFORMATION:

Model Run: Heavy Gas

User specified LOC: $15 \mathrm{ppm}$

Max Threat Zone for LOC: 2.2 kilometers

Max Threat Zone for IDLH: 815 meters

Note: The Heavy Gas footprint is an initial screening.

For short releases it may be an overestimation.

Be sure to check concentration information at specific locations.

\section{TIME DEPENDENT INFORMATION:}

Concentration Estimates at the point:

Downwind: 100 meters

Off Centerline: 0 meters

Max Concentration:

Outdoor: 1,970 ppm

Indoor: $1,970 \mathrm{ppm}$

Note: Indoor graph is shown with a dotted line.
Reviewed by $\mathrm{S}$. A. Henderson

Works Calculation 7

Sheet No. 476 of 577 
OSR 2S.10 (Rev 6-18-92)

ENGINEERING COMPUTATION SHEET

Title of Project EPHA for THM

Reviewed by $\underline{S}$. A. Henderson

Subject Concentration Analysis for THM Hazardous Chemicals

Computer T. M. Franey

Date 09/16/96
Works Calculation 7

Sheet No. 477 of 577

1-RD-38

This page intentionally left blank 
Title of Project EPHA for THM

Subject Concentration Analysis for THM Hazardous Chemicals

Computer T. M. Franey

Date $\underline{09 / 16 / 96}$

1-RD-38

This page intentionally left blank
Reviewed by S. A. Henderson

Works Calculation 7

Sheet No. 478 of 577 


\section{ENGINEERING COMPUTATION SHEET}

Title of Project EPHA for THM

Reviewed by S. A. Henderson

Subject Concentration Analysis for THM Hazardous Chemicals

Computer T. M. Franey

Date 09/16/96
Works Calculation 7

Sheet No. 479 of 577

1-RD-38

This page intentionally left blank 
OSR :S-10) (Rev (r-18-92)

\section{ENGINEERING COMPUTATION SHEET}

Title of Project EPHA for THM

Reviewed by S. A. Henderson

Subject Concentration Analysis for THM Hazardous Chemicals

Works Calculation 7

Computer T. M. Franey

Date 09/16/96

Sheet No. $\underline{480 \text { of } 577}$

1-RD-38

This page intentionally left blank 
OSR $2 S \cdot 10(\operatorname{Rev} 6-18.92)$

\section{ENGINEERING COMPUTATION SHEET}

Title of Project EPHA for THM

Reviewed by $\underline{\text { S. A. Henderson }}$

Works Calculation 7

Subject Concentration Analysis for THM Hazardous Chemicals

Date $\underline{09 / 16 / 96}$

\section{1-RD-38}

This page intentionally left blank 
Title of Project EPHA for THM

Subject Concentration Analysis for THM Hazardous Chemicals

Computer T.M. Franey Date $\underline{09 / 16 / 96}$
Reviewed by $\underline{\text { S. A. Henderson }}$

Works Calculation 7

Sheet No. $\underline{482 \text { of } 577}$

1-RD-39

SITE DATA INFORMATION:

Location: AIKEN, SOUTH CAROLINA

Building Air Exchanges Per Hour: 60 (User specified)

Date and Time: Fixed at July 1, 19961300 hours

\section{CHEMICAL INFORMATION:}

Chemical Name: TETRACHLOROETHYLENE

Molecular Weight: $165.83 \mathrm{~kg} / \mathrm{kmol}$

TLV-TWA: $50.00 \mathrm{ppm}$ ' IDLH: $500.00 \mathrm{ppm}$

Note: Potential or confirmed human carcinogen.

Footprint Level of Concern: 200 ppm

Boiling Point: $121.25^{\circ} \mathrm{C}$

Vapor Pressure at Ambient Temperature: $0.024 \mathrm{~atm}$

Ambient Saturation Concentration: $24,830 \mathrm{ppm}$ or $2.48 \%$

ATMOSPHERIC INFORMATION: (MANUAL INPUT OF DATA)

Wind: 2.5 meters $/ \mathrm{sec}$ from $90^{\circ}$ true

Inversion Height: 500 meters

Stability Class: C Air Temperature: $25^{\circ} \mathrm{C}$

Relative Humidity: 50\% Ground Roughness: 100 centimeters

Cloud Cover: 7 tenths

\section{SOURCE STRENGTH INFORMATION:}

Puddle Area: 9850 square feet

Average Puddle Depth: 1 centimeters

Soil Type: Default Ground Temperature: $25^{\circ} \mathrm{C}$

Initial Puddle Temperature: Ground temperature

Release Duration: ALOHA limited the duration to 1 hour

Max Computed Release Rate: 52.1 kilograms/min

Max Average Sustained Release Rate: 51.8 kilograms/min

(averaged over a minute or more)

Total Amount Released: 2,892 kilograms

\section{TIME DEPENDENT INFORMATTON:}

Concentration Estimates at the point:

Downwind: 30 meters

Off Centerline: 0 meters

Max Concentration:

Outdoor: $175 \mathrm{ppm}$

Indoor: $175 \mathrm{ppm}$

Note: Indoor graph is shown with a dotted line. 


\section{ENGINEERING COMPUTATION SHEET}

Title of Project EPHA for THM

Reviewed by $\underline{\text { S. A. Henderson }}$

Subject Concentration Analysis for THM Hazardous Chemicals

Computer T. M. Franey Date 09/16/96

1-RD-39

SITE DATA INFORMATION:

Location: AIKEN, SOUTH CAROLINA

Building Air Exchanges Per Hour: 60 (User specified)

Date and Time: Fixed at July 1, 19961300 hours

CHEMICAL INFORMATION:

Chemical Name: TETRACHLOROETHYLENE

Molecular Weight: $165.83 \mathrm{~kg} / \mathrm{kmol}$

TLV-TWA: $50.00 \mathrm{ppm}$ IDLH: $500.00 \mathrm{ppm}$

Note: Potential or confirmed human carcinogen.

Footprint Level of Concern: 200 ppm

Boiling Point: $121.25^{\circ} \mathrm{C}$

Vapor Pressure at Ambient Temperature: $0.024 \mathrm{~atm}$

Ambient Saturation Concentration: 24,830 ppm or $2.48 \%$

ATMOSPHERIC INFORMATION: (MANUAL INPUT OF DATA)

Wind: 2.5 meters $/ \mathrm{sec}$ from $90^{\circ}$ true

Inversion Height: 500 meters

Stability Class: C Air Temperature: $25^{\circ} \mathrm{C}$

Relative Humidity: 50\% Ground Roughness: 100 centimeters

Cloud Cover: 7 tenths

SOURCE STRENGTH INFORMATION:

Puddle Area: 12900 square feet

Average Puddle Depth: 1 centimeters

Soil Type: Default Ground Temperature: $25^{\circ} \mathrm{C}$

Initial Puddle Temperature: Ground temperature

Release Duration: ALOHA limited the duration to 1 hour

Max Computed Release.Rate: 67.6 kilograms/min

Max Average Sustained Release Rate: 67.2 kilograms/min

(averaged over a minute or more)

Total Amount Released: 3,751 kilograms

\section{FOOTPRINT INFORMATION:}

Model Run: Heavy Gas

User specified LOC: $200 \mathrm{ppm}$

Max Threat Zone for LOC: 89 meters

Max Threat Zone for IDLH: 46 meters

Note: Footprint wasn't drawn because effects of near-field patchiness make plume presentation unreliable for short distances.

\section{TIME DEPENDENT INFORMATION:}

Concentration Estimates at the point:

Downwind: 100 meters

Off Centerline: 0 meters

Max Concentration:

Outdoor: $200 \mathrm{ppm}$

Indoor: $200 \mathrm{ppm}$
Works Calculation 7

Sheet No. 483 of 577 


\section{ENGINEERING COMPUTATION SHEET}

Title of Project EPHA for THM

Reviewed by $\underline{\text { S. A. Henderson }}$

Subject Concentration Analysis for THM Hazardous Chemicals

Works Calculation 7

Computer T. M. Franey

Date 09/16/96

Sheet No. 484 of 577

SITE DATA INFORMATION:

1-RD-39

Location: AIKEN, SOUTH CAROLINA

Building Air Exchanges Per Hour: 60 (User specified)

Date and Time: Fixed at July l, 19961300 hours

CHEMICAL INFORMATION:

Chemical Name: TETRACHLOROETHYLENE

Molecular Weight: $165.83 \mathrm{~kg} / \mathrm{kmol}$

TLV-TWA: $50.00 \mathrm{ppm}$ IDLH: $500.00 \mathrm{ppm}$

Note: Potential or confirmed human carcinogen.

Footprint Level of Concern: 200 ppm

Boiling Point: $121.25^{\circ} \mathrm{C}$

Vapor Pressure at Ambient Temperature: 0.024 atm

Ambient Saturation Concentration: $24,830 \mathrm{ppm}$ or $2.48 \%$

ATMOSPHERIC INFORMATION: (MANUAL INPUT OF DATA)

Wind: 2.5 meters $/ \mathrm{sec}$ from $90^{\circ}$ true

Inversion Height: 500 meters

Stability Class: C Air Temperature: $25^{\circ} \mathrm{C}$

Relative Humidity: 50\% Ground Roughness: 100 centimeters

Cloud Cover: 7 tenths

\section{SOURCE STRENGTH INFORMATION:}

Puddle Area: 24420 square feet

Average Puddle Depth: 1 centimeters

Soil Type: Default Ground Temperature: $25^{\circ} \mathrm{C}$

Initial Puddle Temperature: Ground temperature

Release Duration: ALOHA limited the duration to 1 hour

Max Computed Release Rate: 125 kilograms/min

Max Average Sustained Release Rate: 125 kilograms/min

(averaged over a minute or more)

Total Amount Released: 6,941 kilograms

\section{FOOTPRINT INFORMATION:}

Model Run: Heavy Gas

User specified LOC: $200 \mathrm{ppm}$

Max Threat Zone for LOC: 113 meters

Max Threat Zone for IDLH: 57 meters

Note: The Heavy Gas footprint is an initial screening.

For short releases it may be an overestimation.

Be sure to check concentration information at specific locations.

TIME DEPENDENT INFORMATION:

Concentration Estimates at the point:

Downwind: 100 meters

Off Centerline: 0 meters

Max Concentration:

Outdoor: 372 ppm

Indoor: $372 \mathrm{ppm}$

Note: Indoor graph is shown with a dotted line. 


\section{ENGINEERING COMPUTATION SHEET}

Title of Project EPHA for THM

Subject Concentration Analysis for THM Hazardous Chemicals

Computer T. M. Franey

Date $\underline{09 / 16 / 96}$

1-RD-39

SITE DATA INFORMATION:

Location: AIKEN, SOUTH CAROLINA

Building Air Exchanges Per Hour: 60 (User specified)

Date and Time: Fixed at July 1, 19961300 hours

CHEMICAL INFORMATION:

Chemical Name: TETRACHLOROETHYLENE

Molecular Weight: $165.83 \mathrm{~kg} / \mathrm{kmol}$

TLV-TWA: $50.00 \mathrm{ppm}$ IDLH: $500.00 \mathrm{ppm}$

Note: Potential or confirmed human carcinogen.

Footprint Level of Concern: 200 ppm

Boiling Point: $121.25^{\circ} \mathrm{C}$

Vapor Pressure at Ambient Temperature: $0.024 \mathrm{~atm}$

Ambient Saturation Concentration: 24,830 ppm or $2.48 \%$

ATMOSPHERIC INFORMATION: (MANUAL INPUT OF DATA)

Wind: 2.5 meters $/ \mathrm{sec}$ from $90^{\circ}$ true

Inversion Height: 500 meters

Stability Class: C Air Temperature: $25^{\circ} \mathrm{C}$

Relative Humidity: 50\% Ground Roughness: 100 centimeters

Cloud Cover: 7 tenths

SOURCE STRENGTH INFORMATION:

Puddle Area: 40700 square feet

Average Puddle Depth: 1 centimeters

Soil Type: Default $\quad$ Ground Temperature: $25^{\circ} \mathrm{C}$

Initial Puddle Temperature: Ground temperature

Release Duration: ALOHA limited the duration to 1 hour

Max Computed Release Rate: 205 kilograms/min

Max Average Sustained Release Rate: 204 kilograms/min

(averaged over a minute or more)

Total Amount Released: 11,364 kilograms

FOOTPRINT INFORMATION:

Model Run: Heavy Gas

User specified LOC: 200 ppm

Max Threat Zone for LOC: 133 meters

Max Threat Zone for IDLH: 68 meters

Note: The Heavy Gas footprint is an initial screening.

For short releases it may be an overestimation.

Be sure to check concentration information at specific locations.

TIME DEPENDENT INFORMATION:

Concentration Estimates at the point:

Downwind: 100 meters

Off Centerline: 0 meters

Max Concentration:

Outdoor: 492 ppm

Indoor: $492 \mathrm{ppm}$

Note: Indoor graph is shown with a dotted line.
Reviewed by $\underline{\text { S. A. Henderson }}$

Works Calculation 7

Sheet No. $\underline{485 \text { of } 577}$ 


\section{ENGINEERING COMPUTATION SHEET}

Title of Project EPHA for THM

Subject Concentration Analysis for THM Hazardous Chemicals

Computer T. M. Franey Date $09 / 16 / 96$

1-RD-39

SITE DATA INFORMATION:

Location: AIKEN, SOUTH CAROLINA

Building Air Exchanges Per Hour: 60 (User specified)

Date and Time: Fixed at July 1, 19961300 hours

CHEMICAL INFORMATION:

Chemical Name: TETRACHLOROETHYLENE

Molecular Weight: $165.83 \mathrm{~kg} / \mathrm{kmol}$

TLV-TWA: $50.00 \mathrm{ppm}$ IDLH: $500.00 \mathrm{ppm}$

Note: Potential or confirmed human carcinogen.

Footprint Level of Concern: 200 ppm

Boiling Point: $121.25^{\circ} \mathrm{C}$

Vapor Pressure at Ambient Temperature: $0.024 \mathrm{~atm}$

Ambient Saturation Concentration: 24,830 ppm or $2.48 \%$

ATMOSPHERIC INFORMATION: (MANUAL INPUT OF DATA)

Wind: 2.5 meters $/ \mathrm{sec}$ from $90^{\circ}$ true

Inversion Height: 500 meters

Stability Class: C Air Temperature: $25^{\circ} \mathrm{C}$

Relative Humidity: 50\% Ground Roughness: 100 centimeters

Cloud Cover: 7 tenths

\section{SOURCE STRENGTH INFORMATION:}

Puddle Area: 10000 square meters

Average Puddle Depth: 1 centimeters

Soil Type: Default Ground Temperature: $25^{\circ} \mathrm{C}$

Initial Puddle Temperature: Ground temperature

Release Duration: ALOHA limited the duration to 1 hour

Max Computed Release Rate: 526 kilograms/min

Max Average Sustained Release Rate: 523 kilograms/min

(averaged over a minute or more)

Total Amount Released: 29,073 kilograms

\section{FOOTPRINT INFORMATION:}

Model Run: Heavy Gas

User specified LOC: $200 \mathrm{ppm}$

Max Threat Zone for LOC: 177 meters

Max Threat Zone for IDLH: 97 meters

Note: The Heavy Gas footprint is an initial screening.

For short releases it may be an overestimation.

Be sure to check concentration information at specific locations.

\section{TIME DEPENDENT INFORMATION:}

Concentration Estimates at the point:

Downwind: 100 meters

Off Centerline: 0 meters

Max Concentration:

Outdoor: 837 ppm

Indoor: $837 \mathrm{ppm}$

Note: Indoor graph is shown with a dotted line.
Reviewed by S. A. Henderson

Works Calculation 7

Sheet No. 486 of 577 
Title of Project EPHA for THM

Subject Concentration Analysis for THM Hazardous Chemicals

Computer T. M. Franey

Date $09 / 16 / 96$
Reviewed by $\underline{\text { S. A. Henderson }}$

Works Calculation 7

Sheet No. 487 of 577

SITE DATA INFORMATION: $\quad$ 1-RD-40

Location: AIKEN, SOUTH CAROLINA

Building Air Exchanges Per Hour: 60 (User specified)

Date and Time: Fixed at July 1, 19961300 hours

\section{CHEMICAL INFORMATION:}

Chemical Name: PHENOL Molecular Weight: $94.11 \mathrm{~kg} / \mathrm{kmol}$

TLV-TWA: $5.00 \mathrm{ppm}$ IDLH: $250.00 \mathrm{ppm}$

Footprint Level of Concern: 50 ppm

Boiling Point: $181.84^{\circ} \mathrm{C}$

Vapor Pressure at Ambient Temperature: $0.0038 \mathrm{~atm}$

Ambient Saturation Concentration: 3,865 ppm or $0.39 \%$

ATMOSPHERIC INFORMATION: (MANUAL INPUT OF DATA)

Wind: 2.5 meters $/ \mathrm{sec}$ from $90^{\circ}$ true

Inversion Height: 500 meters

Stability Class: C Air Temperature: $50^{\circ} \mathrm{C}$

Relative Humidity: $50 \%$ Ground Roughness: 100 centimeters

Cloud Cover: 7 tenths

SOURCE STRENGTH INFORMATION:

Puddle Area: 14550 square feet

Average Puddle Depth: 1 centimeters

Soil Type: Default Ground Temperature: $50^{\circ} \mathrm{C}$

Initial Puddle Temperature: Ground temperature

Release Duration: ALOHA limited the duration to 1 hour

Max Computed Release Rate: 8.25 kilograms/min

Max Average Sustained Release Rate: 8.15 kilograms/min

(averaged over a minute or more)

Total Amount Released: 421 kilograms

TIME DEPENDENT INFORMATION:

Concentration Estimates at the point:

Downwind: $\quad 30$ meters

Off Centerline: 0 meters

Max Concentration:

Outdoor: $50 \mathrm{ppm}$

Indoor: $50 \mathrm{ppm}$

Note: Indoor graph is shown with a dotted line. 


\section{ENGINEERING COMPUTATION SHEET}

Title of Project EPHA for THM

Subject Concentration Analysis for THM Hazardous Chemicals

Computer T. M. Franey

Date 09/16/96
Reviewed by S. A. Henderson

Works Calculation 7

Sheet No. $\underline{488 \text { of } 577}$

SITE DATA INFORMATION: $\quad$ 1-RD-40

Location: AIKEN, SOUTH CAROLINA

Building Air Exchanges Per Hour: 60 (User specified)

Date and Time: Fixed at July 1, 19961300 hours

CHEMICAL INFORMATION:

Chemical Name: PHENOL Molecular Weight: $94.11 \mathrm{~kg} / \mathrm{kmol}$

TLV-TWA: $5.00 \mathrm{ppm}$ IDLH: $250.00 \mathrm{ppm}$

Footprint Level of Concern: $50 \mathrm{ppm}$

Boiling Point: $181.84^{\circ} \mathrm{C}$

Vapor Pressure at Ambient Temperature: $0.0038 \mathrm{~atm}$

Ambient Saturation Concentration: 3,865 ppm or $0.39 \%$

ATMOSPHERIC INFORMATION: (MANUAL INPUT OF DATA)

Wind: 2.5 meters $/ \mathrm{sec}$ from $90^{\circ}$ true

Inversion Height: 500 meters

Stability Class: C Air Temperature: $50^{\circ} \mathrm{C}$

Relative Humidity: 50\% Ground Roughness: 100 centimeters

Cloud Cover: 7 tenths

SOURCE STRENGTH INFORMATION:

Puddle Area: 99999 square feet

Average Puddle Depth: 1 centimeters

Soil Type: Default Ground Temperature: $50^{\circ} \mathrm{C}$

Initial Puddle Temperature: Ground temperature

Release Duration: ALOHA limited the duration to 1 hour

Max Computed Release Rate: 52.7 kilograms/min

Max Average Sustained Release Rate: 52.1 kilograms/min

(averaged over a minute or more)

Total Amount Released: 2,682 kilograms

\section{TIME DEPENDENT INFORMATION:}

Concentration Estimates at the point:

Downwind: 100 meters

Off Centerline: 0 meters

Max Concentration:

Outdoor: $31.1 \mathrm{ppm}$

Indoor: $31.1 \mathrm{ppm}$

Note: Indoor graph is shown with a dotted line. 
Title of Project EPHA for THM

Subject Concentration Analysis for THM Hazardous Chemicals

Computer T. M. Franey Date $09 / 16 / 96$
SITE DATA INFORMATION: 1-RD-40

Location: AIKEN, SOUTH CAROLINA

Building Air Exchanges Per Hour: 60 (User specified)

Date and Time: Fixed at July 1, 19961300 hours

\section{CHEMICAL INFORMATION:}

Chemical Name: PHENOL Molecular Weight: $94.11 \mathrm{~kg} / \mathrm{kmol}$ TLV-TWA: $5.00 \mathrm{ppm}$ IDLH: $250.00 \mathrm{ppm}$

Footprint Level of Concern: 50 ppm

Boiling Point: $181.84^{\circ} \mathrm{C}$

Vapor Pressure at Ambient Temperature: $0.0038 \mathrm{~atm}$

Ambient Saturation Concentration: 3,865 ppm or $0.39 \%$

ATMOSPHERIC INFORMATION: (MANUAL INPUT OF DATA)

Wind: 2.5 meters $/ \mathrm{sec}$ from $90^{\circ}$ true

Inversion Height: 500 meters

Stability Class: C Air Temperature: $50^{\circ} \mathrm{C}$

Relative Humidity: $50 \%$ Ground Roughness: 100 centimeters

Cloud Cover: 7 tenths

\section{SOURCE STRENGTH INFORMATION:}

Puddle Area: 24420 square feet

Average Puddle Depth: 1 centimeters

Soil Type: Default Ground Temperature: $50^{\circ} \mathrm{C}$

Initial Puddle Temperature: Ground temperature

Release Duration: ALOHA limited the duration to 1 hour

Max Computed Release Rate: 13.6 kilograms/min

Max Average Sustained Release Rate: 13.4 kilograms/min

(averaged over a minute or more)

Total Amount Released: 692 kilograms

\section{FOOTPRINT INFORMATION:}

Dispersion Module: Gaussian

User specified LOC: $50 \mathrm{ppm}$

Max Threat Zone for LOC: 39 meters

Max Threat Zone for IDLH: 27 meters

Note: Footprint was not drawn because effects of near-field patchiness make plume presentation unreliable for short distances.

\section{TIME DEPENDENT INFORMATION:}

Concentration Estimates at the point:

Downwind: 100 meters

Off Centerline: 0 meters

Max Concentration:

Outdoor: $14.9 \mathrm{ppm}$

Indoor: $14.9 \mathrm{ppm}$

Note: Indoor graph is shown with a dotted line.
Reviewed by S. A. Henderson

Works Calculation 7

Sheet No. $\underline{489 \text { of } 577}$ 


\section{ENGINEERING COMPUTATION SHEET}

Title of Project EPHA for THM

Subject Concentration Analysis for THM Hazardous Chemicals

Computer T. M. Franey

Date 09/16/96

SITE DATA INFORMATION: $\quad$ 1-RD-40

Location: AIKEN, SOUTH CAROLINA

Building Air Exchanges Per Hour: 60 (User specified)

Date and Time: Fixed at July 1, 19961300 hours

\section{CHEMICAL INFORMATION:}

Chemical Name: PHENOL Molecular Weight: $94.11 \mathrm{~kg} / \mathrm{kmol}$

TLV-TWA: $5.00 \mathrm{ppm}$ IDLH: $250.00 \mathrm{ppm}$

Footprint Level of Concern: $50 \mathrm{ppm}$

Boiling Point: $181.84^{\circ} \mathrm{C}$

Vapor Pressure at Ambient Temperature: $0.0038 \mathrm{~atm}$

Ambient Saturation Concentration: 3,865 ppm or 0.39\%

ATMOSPHERIC INFORMATION: (MANUAL INPUT OF DATA)

Wind: 2.5 meters $/ \mathrm{sec}$ from $90^{\circ}$ true

Inversion Height: 500 meters

Stability Class: C Air Temperature: $50^{\circ} \mathrm{C}$

Relative Humidity: $50 \%$ Ground Roughness: 100 centimeters

Cloud Cover: 7 tenths

\section{SOURCE STRENGTH INFORMATION:}

Puddle Area: 40700 square feet

Average Puddle Depth: 1 centimeters

Soil Type: Default Ground Temperature: $50^{\circ} \mathrm{C}$

Initial Puddle Temperature: Ground temperature

Release Duration: ALOHA limited the duration to 1 hour

Max Computed Release Rate: 22.2 kilograms/min

Max Average Sustained Release Rate: 21.9 kilograms/min

(averaged over a minute or more)

Total Amount Released: 1,130 kilograms

\section{FOOTPRINT INFORMATION:}

Dispersion Module: Gaussian

User specified LOC: $50 \mathrm{ppm}$

Max Threat Zone for LOC: 49 meters

Max Threat Zone for IDLH: 34 meters

Note: Footprint was not drawn because effects of near-field patchiness make plume presentation unreliable for short distances.

\section{TIME DEPENDENT INFORMATION:}

Concentration Estimates at the point:

Downwind: 100 meters

Off Centerline: 0 meters

Max Concentration:

Outdoor: $19 \mathrm{ppm}$

Indoor: $19 \mathrm{ppm}$

Note: Indoor graph is shown with a dotted line.
Reviewed by S. A. Henderson

Works Calculation 7

Sheet No. 490 of 577 . 


\section{ENGINEERING COMPUTATION SHEET}

Title of Project EPHA for THM

Reviewed by S. A. Henderson

Subject Concentration Analysis for THM Hazardous Chemicals

Computer T. M. Franey Date $\underline{09 / 16 / 96}$

SITE DATA INFORMATION: $\quad$ 1-RD-40

Location: AIKEN, SOUTH CAROLINA

Building Air Exchanges Per Hour: 60 (User specified)

Date and Time: Fixed at July 1, 19961300 hours

CHEMICAL INFORMATION:

Chemical Name: PHENOL Molecular Weight: $94.11 \mathrm{~kg} / \mathrm{kmol}$

TLV-TWA: $5.00 \mathrm{ppm}$ IDLH: $250.00 \mathrm{ppm}$

Footprint Level of Concern: 50 ppm

Boiling Point: $181.84^{\circ} \mathrm{C}$

Vapor Pressure at Ambient Temperature: $0.0038 \mathrm{~atm}$

Ambient Saturation Concentration: 3,865 ppm or $0.39 \%$

ATMOSPHERIC INFORMATION: (MANUAL INPUT OF DATA)

Wind: 2.5 meters $/ \mathrm{sec}$ from $90^{\circ}$ true

Inversion Height: 500 meters

Stability Class: C Air Temperature: $50^{\circ} \mathrm{C}$

Relative Humidity: 50\% Ground Roughness: 100 centimeters

Cloud Cover: 7 tenths

\section{SOURCE STRENGTH INFORMATION:}

Puddle Area: 10000 square meters

Average Puddle Depth: 1 centimeters

Soil Type: Default Ground Temperature: $50^{\circ} \mathrm{C}$

Initial Puddle Temperature: Ground temperature

Release Duration: ALOHA limited the duration to 1 hour

Max Computed Release Rate: 56.6 kilograms/min

Max Average Sustained Release Rate: 55.9 kilograms/min

(averaged over a minute or more)

Total Amount Released: 2,879 kilograms

FOOTPRINT INFORMATION:

Dispersion Module: Gaussian

User specified LOC: $50 \mathrm{ppm}$

Max Threat Zone for LOC: 78 meters

Max Threat Zone for IDLH: 56 meters

Note: Footprint was not drawn because

effects of near-field patchiness make plume

presentation unreliable for short distances.

\section{TIME DEPENDENT INFORMATION:}

Concentration Estimates at the point:

Downwind: 100 meters

Off Centerline: 0 meters

Max Concentration:

Outdoor: $32.8 \mathrm{ppm}$

Indoor: $32.8 \mathrm{ppm}$

Note: Indoor graph is shown with a dotted line.
Works Calculation 7

Sheet No. 491 of 577 


\section{ENGINEERING COMPUTATION SHEET}

Title of Project EPHA for THM

Subject Concentration Analysis for THM Hazardous Chemicals

Computer T. M. Franey

Date $\underline{09 / 16 / 96}$

SITE DATA INFORMATION:

$1-R D-41$

Location: AIKEN, SOUTH CAROLINA

Building Air Exchanges Per Hour: 60 (User specified)

Date and Time: Fixed at July 1, 19961300 hours

\section{CHEMICAL INFORMATION:}

Chemical Name: PROPYL ALCOHOL

Molecular Weight: $60.10 \mathrm{~kg} / \mathrm{kmol}$

TLV-TWA: $200.00 \mathrm{ppm}$-IDLH: $4000.00 \mathrm{ppm}$

Footprint Level of Concern: 5 ppm

Boiling Point: $97.20^{\circ} \mathrm{C}$

Vapor Pressure at Ambient Temperature: $0.027 \mathrm{~atm}$

Ambient Saturation Concentration: 27,722 ppm or 2.77\%

\section{ATMOSPHERIC INFORMATION: (MANUAL INPUT OF DATA)}

Wind: 2.5 meters $/ \mathrm{sec}$ from $90^{\circ}$ true

Inversion Height: 500 meters

Stability Class: C Air Temperature: $25^{\circ} \mathrm{C}$

Relative Humidity: 50\% Ground Roughness: 100 centimeters

Cloud Cover: 7 tenths

\section{SOURCE STRENGTH INFORMATION:}

Puddle Area: 37 square feet

Average Puddle Depth: 1 centimeters

Soil Type: Default Ground Temperature: $25^{\circ} \mathrm{C}$

Initial Puddle Temperature: Ground temperature

Release Duration: ALOHA limited the duration to 1 hour

Max Computed Release Rate: 119 grams/min

Max Average Sustained Release Rate: 119 grams/min

(averaged over a minute or more)

Total Amount Released: 6.95 kilograms

TIME DEPENDENT INFORMATION:

Concentration Estimates at the point:

Downwind: 30 meters

Off Centerline: 0 meters

Max Concentration:

Outdoor: $5.08 \mathrm{ppm}$

Indoor: $5.08 \mathrm{ppm}$

Note: Indoor graph is shown with a dotted line. 
Title of Project EPHA for THM

Subject Concentration Analysis for THM Hazardous Chemicals

Computer T. M. Franey Date $09 / 16 / 96$
Reviewed by S. A. Henderson

Works Calculation 7

Sheet No. 493 of 577

SITE DATA INFORMATION:

$$
\text { 1-RD-41 }
$$

Location: AIKEN, SOUTH CAROLINA

Building Air Exchanges Per Hour: 60 (User specified)

Date and Time: Fixed at July 1, 19961300 hours

\section{CHEMICAL INFORMATION:}

Chemical Name: PROPYL ALCOHOL

Molecular Weight: $60.10 \mathrm{~kg} / \mathrm{kmol}$

TLV-TWA: $200.00 \mathrm{ppm}$ IDLH: $4000.00 \mathrm{ppm}$

Footprint Level of Concern: 5 ppm

Boiling Point: $97.20^{\circ} \mathrm{C}$

Vapor Pressure at Ambient Temperature: $0.027 \mathrm{~atm}$

Ambient Saturation Concentration: 27,722 ppm or $2.77 \%$

\section{ATMOSPHERIC INFORMATION: (MANUAL INPUT OF DATA) \\ Wind: 2.5 meters $/ \mathrm{sec}$ from $90^{\circ}$ true \\ Inversion Height: 500 meters \\ Stability Class: C Air Temperature: $25^{\circ} \mathrm{C}$ \\ Relative Humidity: 50\% Ground Roughness: 100 centimeters \\ Cloud Cover: 7 tenths}

\section{SOURCE STRENGTH INFORMATION:}

Puddle Area: 447 square feet

Average Puddle Depth: 1 centimeters

Soil Type: Default Ground Temperature: $25^{\circ} \mathrm{C}$

Initial Puddle Temperature: Ground temperature

Release Duration: ALOHA limited the duration to 1 hour

Max Computed Release Rate: 1.3 kilograms/min

Max Average Sustained Release Rate: 1.3 kilograms/min

(averaged over a minute or more)

Total Amount Released: 75.0 kilograms

\section{TIME DEPENDENT INFORMATION:}

Concentration Estimates at the point:

Downwind: 100 meters

Off Centerline: 0 meters

Max Concentration:

Outdoor: 5 ppm

Indoor: $5 \mathrm{ppm}$

Note: Indoor graph is shown with a dotted line. 


\section{ENGINEERING COMPUTATION SHEET}

Title of Project EPHA for THM

Reviewed by S.A. Henderson

Subject Concentration Analysis for THM Hazardous Chemicals

Computer T. M. Franey

Date $\underline{09 / 16 / 96}$

$1-R D-41$

SITE DATA INFORMATION:

Location: AIKEN, SOUTH CAROLINA

Building Air Exchanges Per Hour: 60 (User specified)

Date and Time: Fixed at July 1, 19961300 hours

CHEMICAL INFORMATION:

Chemical Name: PROPYL ALCOHOL

Molecular Weight: $60.10 \mathrm{~kg} / \mathrm{kmol}$

TLV-TWA: $200.00 \mathrm{ppm}$

IDLH: $4000.00 \mathrm{ppm}$

Footprint Level of Concern: 5 ppm

Boiling Point: $97.20^{\circ} \mathrm{C}$

Vapor Pressure at Ambient Temperature: $0.027 \mathrm{~atm}$

Ambient Saturation Concentration: 27,722 ppm or $2.77 \%$

ATMOSPHERIC INFORMATION: (MANUAL INPUT OF DATA)

Wind: 2.5 meters $/ \mathrm{sec}$ from $90^{\circ}$ true

Inversion Height: 500 meters

Stability Class: C Air Temperature: $25^{\circ} \mathrm{C}$

Relative Humidity: 50\% Ground Roughness: 100 centimeters

Cloud Cover: 7 tenths

SOURCE STRENGTH INFORMATION:

Puddle Area: 24420 square feet

Average Puddle Depth: 1 centimeters

Soil Type: Default Ground Temperature: $25^{\circ} \mathrm{C}$

Initial Puddle Temperature: Ground temperature

Release Duration: ALOHA limited the duration to 1 hour

Max Computed Release Rate: 61.9 kilograms/min

Max Average Sustained Release Rate: 61.6 kilograms/min

(averaged over a minute or more)

Total Amount Released: 3,499 kilograms

FOOTPRINT.INFORMATION:

Dispersion Module: Gaussian

User specified LOC: 5 ppm

Max Threat Zone for LOC: 696 meters

Max Threat Zone for IDLH: 27 meters
Works Calculation 7

Sheet No. $\underline{494 \text { of } 577}$ 
Title of Project EPHA for THM

Subject Concentration Analysis for THM Hazardous Chemicals

Computer T. M. Franey

Date $\underline{09 / 16 / 96}$
Reviewed by $\underline{\text { S. A. Henderson }}$

Works Calculation 7

Sheet No. 495 of 577

SITE DATA INFORMATION:

$$
\text { 1-RD-41 }
$$

Location: AIKEN, SOUTH CAROLINA

Building Air Exchanges Per Hour: 60 (User specified)

Date and Time: Fixed at July 1, 19961300 hours

CHEMICAL INFORMATION:

Chemical Name: PROPYL ALCOHOL

Molecular Weight: $60.10 \mathrm{~kg} / \mathrm{kmol}$

TLV-TWA: $200.00 \mathrm{ppm}$ IDLH: $4000.00 \mathrm{ppm}$

Footprint Level of Concern: 5 ppm

Boiling Point: $97.20^{\circ} \mathrm{C}$

Vapor Pressure at Ambient Temperature: 0.027 atm

Ambient Saturation Concentration: 27,722 ppm or $2.77 \%$

ATMOSPHERIC INFORMATION: (MANUAL INPUT OF DATA)

Wind: 2.5 meters $/ \mathrm{sec}$ from $90^{\circ}$ true

Inversion Height: 500 meters

Stability Class: C Air Temperature: $25^{\circ} \mathrm{C}$

Relative Humidity: 50\% Ground Roughness: 100 centimeters

Cloud Cover: 7 tenths

\section{SOURCE STRENGTH INFORMATION:}

Puddle Area: 40700 square feet

Average Puddle Depth: 1 centimeters

Soil Type: Default Ground Temperature: $25^{\circ} \mathrm{C}$

Initial Puddle Temperature: Ground temperature

Release Duration: ALOHA limited the duration to 1 hour

Max Computed Release Rate: 101 kilograms/min

Max Average Sustained Release Rate: 101 kilograms/min

(averaged over a minute or more)

Total Amount Released: 5,726 kilograms

FOOTPRINT INFORMATION:

Dispersion Module: Gaussian

User specified LOC: 5 ppm

Max Threat Zone for LOC: 895 meters

Max Threat Zone for IDLH: 34 meters 


\section{ENGINEERING COMPUTATION SHEET}

Title of Project EPHA for THM

Subject Concentration Analysis for THM Hazardous Chemicals

Computer T. M. Franey
Reviewed by S. A. Henderson

Works Calculation 7

Sheet No. 496 of 577

SITE DATA INFORMATION:

$$
\text { 1-RD-41 }
$$

Location: AIKEN, SOUTH CAROLINA

Building Air Exchanges Per Hour: 60 (User specified)

Date and Time: Fixed at July 1, 19961300 hours

\section{CHEMICAL INFORMATION:}

Chemical Name: PROPYL ALCOHOL

Molecular Weight: $60.10 \mathrm{~kg} / \mathrm{kmol}$

TLV-TWA: $200.00 \mathrm{ppm}$ IDLH: $4000.00 \mathrm{ppm}$

Footprint Level of Concern: 5 ppm

Boiling Point: $97.20^{\circ} \mathrm{C}$

Vapor Pressure at Ambient Temperature: $0.027 \mathrm{~atm}$

Ambient Saturation Concentration: 27,722 ppm or 2.77\%

ATMOSPHERIC INFORMATION: (MANUAL INPUT OF DATA)

Wind: 2.5 meters $/ \mathrm{sec}$ from $90^{\circ}$ true

Inversion Height: 500 meters

Stability Class: C Air Temperature: $25^{\circ} \mathrm{C}$

Relative Humidity: 50\% Ground Roughness: 100 centimeters

Cloud Cover: 7 tenths

\section{SOURCE STRENGTH INFORMATION:}

Puddle Area: 10000 square meters

Average Puddle Depth: 1 centimeters

Soil Type: Default Ground Temperature: $25^{\circ} \mathrm{C}$

Initial Puddle Temperature: Ground temperature

Release Duration: ALOHA limited the duration to 1 hour

Max Computed Release Rate: 260 kilograms/min

Max Average Sustained Release Rate: 259 kilograms/min

(averaged over a minute or more)

Total Amount Released: 14,636 kilograms

FOOTPRINT INFORMATION:

Dispersion Module: Gaussian

User specified LOC: 5 ppm

Max Threat Zone for LOC: 1.5 kilometers

Max Threat Zone for IDLH: 56 meters 
Title of Project EPHA for THM

Subject Concentration Analysis for THM Hazardous Chemicals

Computer T. M. Franey Date 09/16/96
Reviewed by $\mathrm{S}$. A. Henderson

Works Calculation 7

Sheet No. 497 of 577

\section{SITE DATA INFORMATION: 1-RD-42}

Location: AIKEN, SOUTH CAROLINA

Building Air Exchanges Per Hour: 60 (User specified)

Date and Time: Fixed at July 1, 19961300 hours

\section{CHEMICAL INFORMATION:}

Chemical Name: PROPIONIC ACID

Molecular Weight: $74.08 \mathrm{~kg} / \mathrm{kmol}$

TLV-TWA: $10.00 \mathrm{ppm}$ IDLH: -unavail-

Footprint Level of Concern: 50 ppm

Boiling Point: $141.17^{\circ} \mathrm{C}$

Vapor Pressure at Ambient Temperature: $0.0041 \mathrm{~atm}$

Ambient Saturation Concentration: 4,213 ppm or $0.42 \%$

ATMOSPHERIC INFORMATION: (MANUAL INPUT OF DATA)

Wind: 2.5 meters $/ \mathrm{sec}$ from $90^{\circ}$ true

Inversion Height: 500 meters

Stability Class: C Air Temperature: $25^{\circ} \mathrm{C}$

Relative Humidity: 50\% Ground Roughness: 100 centimeters

Cloud Cover: 7 tenths

\section{SOURCE STRENGTH INFORMATION:}

Puddle Area: 12850 square feet

Average Puddle Depth: 1 centimeters

Soil Type: Default Ground Temperature: $25^{\circ} \mathrm{C}$

Initial Puddle Temperature: Ground temperature

Release Duration: ALOHA limited the duration to 1 hour

Max Computed Release Rate: 6.96 kilograms/min

Max Average Sustained Release Rate: 6.88 kilograms/min

(averaged over a minute or more)

Total Amount Released: 356 kilograms

\section{FOOTPRINT INFORMATION:}

Dispersion Module: Gaussian

User specified LOC: $50 \mathrm{ppm}$

Max Threat Zone for LOC: 30 meters

Note: Footprint was not drawn because effects of near-field patchiness make plume presentation unreliable for short distances.

\section{TIME DEPENDENT INFORMATION:}

Concentration Estimates at the point:

Downwind: 30 meters

Off Centerline: 0 meters

Max Concentration:

Outdoor: $50 \mathrm{ppm}$

Indoor: $50 \mathrm{ppm}$

Note: Indoor graph is shown with a dotted line. 


\section{ENGINEERING COMPUTATION SHEET}

Title of Project EPHA for THM

Subject Concentration Analysis for THM Hazardous Chemicals

Computer T.M. Franey

Date 09/16/96
Reviewed by S. A. Henderson

Works Calculation 7

Sheet No. $\underline{498 \text { of } 577}$

SITE DATA INFORMATION: I-RD-42

Location: AIKEN, SOUTH CAROLINA

Building Air Exchanges Per Hour: 60 (User specified)

Date and Time: Fixed at July 1, 19961300 hours

CHEMICAL INFORMATION;

Chemical Name: PROPIONIC ACID

Molecular Weight: $74.08 \mathrm{~kg} / \mathrm{kmol}$

TLV-TWA: $10.00 \mathrm{ppm}$ IDLH: -unavail-

Footprint Level of Concern: $50 \mathrm{ppm}$

Boiling Point: $141.17^{\circ} \mathrm{C}$

Vapor Pressure at Ambient Temperature: $0.0041 \mathrm{~atm}$

Ambient Saturation Concentration: 4,213 ppm or $0.42 \%$

ATMOSPHERIC INFORMATION: (MANUAL INPUT OF DATA)

Wind: 2.5 meters $/ \mathrm{sec}$ from $90^{\circ}$ true

Inversion Height: 500 meters

Stability Class: C Air Temperature: $25^{\circ} \mathrm{C}$

Relative Humidity: 50\% Ground Roughness: 100 centimeters

Cloud Cover: 7 tenths

SOURCE STRENGTH INFORMATION:

Puddle Area: 99999 square feet

Average Puddle Depth: 1 centimeters

Soil Type: Default Ground Temperature: $25^{\circ} \mathrm{C}$

Initial Puddle Temperature: Ground temperature

Release Duration: ALOHA limited the duration to 1 hour

Max Computed Release Rate: 50.1 kilograms/min

Max Average Sustained Release Rate: 49.5 kilograms/min

(averaged over a minute or more)

Total Amount Released: 2,552 kilograms

FOOTPRINT INFORMATION:

Dispersion Module: Gaussian

User specified LOC: $50 \mathrm{ppm}$

Max Threat Zone for LOC: 80 meters

Note: Footprint was not drawn because effects of near-field patchiness make plume

presentation unreliable for short distances.

TIME DEPENDENT INFORMATION:

Concentration Estimates at the point:

Downwind: 100 meters

Off Centerline: 0 meters

Max Concentration:

Outdoor: $34.6 \mathrm{ppm}$

Indoor: $34.6 \mathrm{ppm}$

Note: Indoor graph is shown with a dotted line. 
Title of Project EPHA for THM

Subject Concentration Analysis for THM Hazardous Chemicals

Computer T. M. Franey

Date 09/16/96
Reviewed by $\underline{\text { S. A. Henderson }}$

Works Calculation 7

Sheet No. $\underline{499 \text { of } 577}$

\section{SITE DATA INFORMATION: 1-RD-42 Location: AIKEN, SOUTH CAROLINA \\ Building Air Exchanges Per Hour: 60 (User specified) \\ Date and Time: Fixed at July 1, 19961300 hours}

\section{CHEMICAL INFORMATION:}

Chemical Name: PROPIONIC ACID

Molecular Weight: $74.08 \mathrm{~kg} / \mathrm{kmol}$

TLV-TWA: $10.00 \mathrm{ppm}$

IDLH: -unavail-

Footprint Level of Concern: 50 ppm

Boiling Point: $141.17^{\circ} \mathrm{C}$

Vapor Pressure at Ambient Temperature: $0.0041 \mathrm{~atm}$

Ambient Saturation Concentration: $4,213 \mathrm{ppm}$ or $0.42 \%$

ATMOSPHERIC INFORMATION: (MANUAL INPUT OF DATA)

Wind: 2.5 meters $/ \mathrm{sec}$ from $90^{\circ}$ true

Inversion Height: 500 meters

Stability Class: C Air Temperature: $25^{\circ} \mathrm{C}$

Relative Humidity: 50\% Ground Roughness: 100 centimeters

Cloud Cover: 7 tenths

\section{SOURCE STRENGTH INFORMATION:}

Puddle Area: 24420 square feet

Average Puddle Depth: 1 centimeters

Soil Type: Default Ground Temperature: $25^{\circ} \mathrm{C}$

Initial Puddle Temperature: Ground temperature

Release Duration: ALOHA limited the duration to 1 hour

Max Computed Release Rate: 12.9 kilograms/min

Max Average.Sustained Release Rate: 12.7 kilograms/min

(averaged over a minute or more)

Total Amount Released: 659 kilograms

\section{FOOTPRINT INFORMATION:}

Dispersion Module: Gaussian

User specified LOC: $50 \mathrm{ppm}$

Max Threat Zone for LOC: 41 meters •

Note: Footprint was not drawn because

effects of near-field patchiness make plume

presentation unreliable for short distances.

\section{TIME DEPENDENT INFORMATION:}

Concentration Estimates at the point:

Downwind: 100 meters

Off Centerline: 0 meters

Max Concentration:

Outdoor: $16.6 \mathrm{ppm}$

Indoor: $16.6 \mathrm{ppm}$

Note: Indoor graph is shown with a dotted line. 


\section{ENGINEERING COMPUTATION SHEET}

Title of Project EPHA for THM

Subject Concentration Analysis for THM Hazardous Chemicals

Computer T. M. Franey

Date $\underline{09 / 16 / 96}$

SITE DATA INFORMATION: 1-RD-42

Location: AIKEN, SOUTH CAROLINA

Building Air Exchanges Per Hour: 60 (User specified)

Date and Time: Fixed at July 1, 19961300 hours

CHEMICAL INFORMATION:

Chemical Name: PROPIONIC ACID

Molecular Weight: $74.08 \mathrm{~kg} / \mathrm{kmol}$

TLV-TWA: $10.00 \mathrm{ppm}$ IDLH: -unavail-

Footprint Level of Concern: 50 ppm

Boiling Point: $141.17^{\circ} \mathrm{C}$

Vapor Pressure at Ambient Temperature: $0.0041 \mathrm{~atm}$

Ambient Saturation Concentration: 4,213 ppm or $0.42 \%$

ATMOSPHERIC INFORMATION: (MANUAL INPUT OF DATA)

Wind: 2.5 meters $/ \mathrm{sec}$ from $90^{\circ}$ true

Inversion Height: 500 meters

Stability Class: C Air Temperature: $25^{\circ} \mathrm{C}$

Relative Humidity: $50 \%$ Ground Roughness: 100 centimeters

Cloud Cover: 7 tenths

SOURCE STRENGTH INFORMATION:

Puddle Area: 40700 square feet

Average Puddle Depth: 1 centimeters

Soil Type: Default Ground Temperature: $25^{\circ} \mathrm{C}$

Initial Puddle Temperature: Ground temperature

Release Duration: ALOHA limited the duration to 1 hour

Max Computed Release Rate: 21.1 kilograms/min

Max Average Sustained Release Rate: 20.8 kilograms/min

(averaged over a minute or more)

Total Amount Released: 1,076 kilograms

FOOTPRINT INFORMATION:

Dispersion Module: Gaussian

- User specified LOC: $50 \mathrm{ppm}$

Max Threat Zone for LOC: 52 meters

Note: Footprint was not drawn because effects of near-field patchiness make plume presentation unreliable for short distances.

\section{TIME DEPENDENT INFORMATION:}

Concentration Estimates at the point:

Downwind: 100 meters

Off Centerline: 0 meters

Max Concentration:

Outdoor: $21.1 \mathrm{ppm}$

Indoor: $21.1 \mathrm{ppm}$

Note: Indoor graph is shown with a dotted line.
Reviewed by S. A. Henderson

Works Calculation 7

Sheet No. $\underline{500 \text { of } 577}$ 
Title of Project EPHA for THM

Subject Concentration Analysis for THM Hazardous Chemicals

Computer T. M. Franey

Date $\underline{09 / 16 / 96}$
Reviewed by $\underline{\text { S. A. Henderson }}$

Works Calculation 7

Sheet No. $\underline{501 \text { of } 577}$
SITE DATA INFORMATION: 1-RD-42

Location: AIKEN, SOUTH CAROLINA

Building Air Exchanges Per Hour: 60 (User specified)

Date and Time: Fixed at July 1, 19961300 hours

CHEMICAL INFORMATION:

Chemical Name: PROPIONIC ACID

Molecular Weight: $74.08 \mathrm{~kg} / \mathrm{kmol}$

TLV-TWA: $10.00 \mathrm{ppm}$. IDLH: -unavail-

Footprint Level of Concern: $50 \mathrm{ppm}$

Boiling Point: $141.17^{\circ} \mathrm{C}$

Vapor Pressure at Ambient Temperature: 0.0041 atm

Ambient Saturation Concentration: 4,213 ppm or $0.42 \%$

ATMOSPHERIC INFORMATION: (MANUAL INPUT OF DATA)

Wind: 2.5 meters $/ \mathrm{sec}$ from $90^{\circ}$ true

Inversion Height: 500 meters

Stability Class: C Air Temperature: $25^{\circ} \mathrm{C}$

Relative Humidity: 50\% Ground Roughness: 100 centimeters

Cloud Cover: 7 tenths

SOURCE STRENGTH INFORMATION:

Puddle Area: 10000 square meters

Average Puddle Depth: 1 centimeters

Soil Type: Default Ground Temperature: $25^{\circ} \mathrm{C}$

Initial Puddle Temperature: Ground temperature

Release Duration: ALOHA limited the duration to 1 hour

Max Computed Release Rate: 53.7 kilograms/min

Max Average Sustained Release Rate: 53.1 kilograms/min

(averaged over a minute or more)

Total Amount Released: 2,739 kilograms

\section{FOOTPRINT INFORMATION:}

Dispersion Module: Gaussian

User specified LOC: $50 \mathrm{ppm}$

Max Threat Zone for LOC: 83 meters

Note: Footprint was not drawn because effects of near-field patchiness make plume presentation unreliable for short distances.

\section{TIME DEPENDENT INFORMATION:}

Concentration Estimates at the point:

Downwind: 100 meters

Off Centerline: 0 meters

Max Concentration:

Outdoor: $36.5 \mathrm{ppm}$

Indoor: $36.5 \mathrm{ppm}$

Note: Indoor graph is shown with a dotted line. 


\section{ENGINEERING COMPUTATION SHEET}

Title of Project EPHA for THM

Reviewed by $\underline{\text { S. A. Henderson }}$

Subject Concentration Analysis for THM Hazardous Chemicals

Computer T. M. Franey

Date $\underline{09 / 16 / 96}$

SITE DATA INFORMATION: 1-RD-43

Location: AIKEN, SOUTH CAROLINA

Building Air Exchanges Per Hour: 60 (User specified)

Date and Time: Fixed at July 1, 19961300 hours

\section{CHEMICAL INFORMATION:}

Chemical Name: FORMALDEHYDE-56\%-SAH

Molecular Weight: $24.78 \mathrm{~kg} / \mathrm{kmol}$

TLV-TWA: -unavail- IDLH: -unavail-

Footprint Level of Concern: $10 \mathrm{ppm}$

Boiling Point: $100.10^{\circ} \mathrm{C}$

Vapor Pressure at Ambient Temperature: $0.018 \mathrm{~atm}$

Ambient Saturation Concentration: 17,876 ppm or $1.79 \%$

ATMOSPHERIC INFORMATION: (MANUAL INPUT OF DATA)

Wind: 2.5 meters $/ \mathrm{sec}$ from $90^{\circ}$ true

Inversion Height: 500 meters

Stability Class: C Air Temperature: $25^{\circ} \mathrm{C}$

Relative Humidity: 50\% Ground Roughness: 100 centimeters

Cloud Cover: 7 tenths

\section{SOURCE STRENGTH INFORMATION:}

Puddle Area: 116 square feet

Average Puddle Depth: 1 centimeters

Soil Type: Default Ground Temperature: $25^{\circ} \mathrm{C}$

Initial Puddle Temperature: Ground temperature

Release Duration: ALOHA limited the duration to 1 hour

Max Computed Release Rate: 103 grams/min

Max Average Sustained Release Rate: 102 grams/min

(averaged over a minute or more)

Total Amount Released: 5.80 kilograms

FOOTPRINT INFORMATION:

Dispersion Module: Gaussian

User specified LOC: $10 \mathrm{ppm}$

Max Threat Zone for LOC: $\mathbf{3 0}$ meters

Note: Footprint was not drawn because effects of near-field patchiness make plume presentation unreliable for short distances.

TIME DEPENDENT INFORMATION:

Concentration Estimates at the point:

Downwind: 30 meters

Off Centerline: 0 meters

Max Concentration:

Outdoor: $10 \mathrm{ppm}$

Indoor: $10 \mathrm{ppm}$

Note: Indoor graph is shown with a dotted line.
Works Calculation 7

Sheet No. $\underline{502 \text { of } 577}$ 
Title of Project EPHA for THM

Subject Concentration Analysis for THM Hazardous Chemicals

Computer T.M. Franey
Reviewed by S. A. Henderson

Works Calculation 7

Sheet No. $\underline{503 \text { of } 577}$

SITE DATA INFORMATION: $\quad$ 1-RD-43

Location: AIKEN, SOUTH CAROLINA

Building Air Exchanges Per Hour: 60 (User specified)

Date and Time: Fixed at July 1, 19961300 hours

CHEMICAL INFORMATION:

Chemical Name: FORMALDEHYDE-56\%-SAH

Molecular Weight: $24.78 \mathrm{~kg} / \mathrm{kmol}$

TLV-TWA: -unavail- IDLH: -unavail-

Footprint Level of Concern: 10 ppm

Boiling Point: $100.10^{\circ} \mathrm{C}$

Vapor Pressure at Ambient Temperature: 0.018 atm

Ambient Saturation Concentration: $17,876 \mathrm{ppm}$ or $1.79 \%$

ATMOSPHERIC INFORMATION: (MANUAL INPUT OF DATA)

Wind: 2.5 meters $/ \mathrm{sec}$ from $90^{\circ}$ true

Inversion Height: 500 meters

Stability Class: C Air Temperature: $25^{\circ} \mathrm{C}$

Relative Humidity: 50\% Ground Roughness: 100 centimeters

Cloud Cover: 7 tenths

SOURCE STRENGTH INFORMATION:

Puddle Area: 1440 square feet

Average Puddle Depth: 1 centimeters

Soil Type: Default Ground Temperature: $25^{\circ} \mathrm{C}$

Initial Puddle Temperature: Ground temperature

Release Duration: ALOHA limited the duration to 1 hour

Max Computed Release Rate: 1.15 kilograms/min

Max Average Sustained Release Rate: 1.14 kilograms/min

(averaged over a minute or more)

Total Amount Released: 63.8 kilograms

FOOTPRINT INFORMATION:

Dispersion Module: Gaussian

User specified LOC: $10 \mathrm{ppm}$

Max Threat Zone for LOC: 100 meters

TIME DEPENDENT INFORMATION:

Concentration Estimates at the point:

Downwind: 100 meters

Off Centerline: 0 meters

Max Concentration:

Outdoor: $10 \mathrm{ppm}$

Indoor: $10 \mathrm{ppm}$

Note: Indoor graph is shown with a dotted line. 


\section{ENGINEERING COMPUTATION SHEET}

Title of Project EPHA for THM

Reviewed by S. A. Henderson

Subject Concentration Analysis for THM Hazardous Chemicals

Computer T. M. Franey

Date $\underline{09 / 16 / 96}$
Works Calculation 7

Sheet No. $\underline{504 \text { of } 577}$

SITE DATA INFORMATION: 1-RD-43

Location: AIKEN, SOUTH CAROLINA

Building Air Exchanges Per Hour: 60 (User specified)

Date and Time: Fixed at July 1, 19961300 hours

CHEMICAL INFORMATION:

Chemical Name: FORMALDEHYDE-56\%-SAH

Molecular Weight: $24.78 \mathrm{~kg} / \mathrm{kmol}$

TLV-TWA: -unavail- IDLH: -unavail-

Footprint Level of Concern: 10 ppm

Boiling Point: $100.10^{\circ} \mathrm{C}$

Vapor Pressure at Ambient Temperature: $0.018 \mathrm{~atm}$

Ambient Saturation Concentration: 17,876 ppm or $1.79 \%$

ATMOSPHERIC INFORMATION: (MANUAL INPUT OF DATA)

Wind: 2.5 meters $/ \mathrm{sec}$ from $90^{\circ}$ true

Inversion Height: 500 meters

Stability Class: C Air Temperature: $25^{\circ} \mathrm{C}$

Relative Humidity: 50\% Ground Roughness: 100 centimeters

Cloud Cover: 7 tenths

\section{SOURCE STRENGTH INFORMATION:}

Puddle Area: 24420 square feet

Average Puddle Depth: 1 centimeters

Soil Type: Default Ground Temperature: $25^{\circ} \mathrm{C}$

Initial Puddle Temperature: Ground temperature

Release Duration: ALOHA limited the duration to 1 hour

Max Computed Release Rate: 17.5 kilograms/min

Max Average Sustained Release Rate: 17.4 kilograms/min

(averaged over a minute or more)

Total Amount Released: 961 kilograms

FOOTPRINT INFORMATION:

Dispersion Module: Gaussian

User specified LOC: $10 \mathrm{ppm}$

Max Threat Zone for LOC: 392 meters

TIME DEPENDENT INFORMATION:

Concentration Estimates at the point:

Downwind: 100 meters

Off Centerline: 0 meters

Max Concentration:

Outdoor: $67.9 \mathrm{ppm}$

Indoor: $67.9 \mathrm{ppm}$

Note: Indoor graph is shown with a dotted line. 


\section{ENGINEERING COMPUTATION SHEET}

Title of Project EPHA for THM

Subject Concentration Analysis for THM Hazardous Chemicals

Computer T. M. Franey

Date $\underline{09 / 16 / 96}$

Reviewed by $\underline{\text { S.A. Henderson }}$

Works Calculation 7

Sheet No. $\underline{505 \text { of } 577}$

SITE DATA INFORMATION: 1-RD-43

Location: AIKEN, SOUTH CAROLINA

Building Air Exchanges Per Hour: 60 (User specified)

Date and Time: Fixed at July 1, 19961300 hours

CHEMICAL INFORMATION:

Chemical Name: FORMALDEHYDE-56\%-SAH

Molecular Weight: $24.78 \mathrm{~kg} / \mathrm{kmol}$

TLV-TWA: -unavail- IDLH: -unavail-

Footprint Level of Concern: 10 ppm

Boiling Point: $100.10^{\circ} \mathrm{C}$

Vapor Pressure at Ambient Temperature: $0.018 \mathrm{~atm}$

Ambient Saturation Concentration: 17,876 ppm or $1.79 \%$

ATMOSPHERIC INFORMATION: (MANUAL INPUT OF DATA)

Wind: 2.5 meters $/ \mathrm{sec}$ from $90^{\circ}$ true

Inversion Height: 500 meters

Stability Class: C Air Temperature: $25^{\circ} \mathrm{C}$

Relative Humidity: $50 \%$ Ground Roughness: 100 centimeters

Cloud Cover: 7 tenths

SOURCE STRENGTH INFORMATION:

Puddle Area: 40700 square feet

Average Puddle Depth: 1 centimeters

Soil Type: Default Ground Temperature: $25^{\circ} \mathrm{C}$

Initial Puddle Temperature: Ground temperature

Release Duration: ALOHA limited the duration to 1 hour

Max Computed Release Rate: 28.6 kilograms/min

Max Average Sustained Release Rate: 28.5 kilograms/min

(averaged over a minute or more)

Total Amount Released: 1,570 kilograms

FOOTPRINT INFORMATION:

Dispersion Module: Gaussian

User specified LOC: $10 \mathrm{ppm}$

Max Threat Zone for LOC: 501 meters

TIME DEPENDENT INFORMATION:

Concentration Estimates at the point:

Downwind: 100 meters

Off Centerline: 0 meters

Max Concentration:

Outdoor: $86.2 \mathrm{ppm}$

Indoor: $86.2 \mathrm{ppm}$

Note: Indoor graph is shown with a dotted line. 


\section{ENGINEERING COMPUTATION SHEET}

Title of Project EPHA for THM

Subject Concentration Analysis for THM Hazardous Chemicals

Computer T. M. Franey

Date $\underline{09 / 16 / 96}$
Reviewed by S. A. Henderson

Works Calculation 7

Sheet No. $\underline{506 \text { of } 577}$

SITE DATA INFORMATION: 1-RD-43

Location: AIKEN, SOUTH CAROLINA

Building Air Exchanges Per Hour: 60 (User specified)

Date and Time: Fixed at July 1, 19961300 hours

CHEMICAL INFORMATION:

Chemical Name: FORMALDEHYDE-56\%-SAH

Molecular Weight: $24.78 \mathrm{~kg} / \mathrm{kmol}$

TLV-TWA: -unavail- IDLH: -unavail-

Footprint Level of Concern: $10 \mathrm{ppm}$

Boiling Point: $100.10^{\circ} \mathrm{C}$

Vapor Pressure at Ambient Temperature: $0.018 \mathrm{~atm}$

Ambient Saturation Concentration: $17,876 \mathrm{ppm}$ or $1.79 \%$

ATMOSPHERIC INFORMATION: (MANUAL INPUT OF DATA)

Wind: 2.5 meters $/ \mathrm{sec}$ from $90^{\circ}$ true

Inversion Height: 500 meters

Stability Class: C Air Temperature: $25^{\circ} \mathrm{C}$

Relative Humidity: 50\% Ground Roughness: 100 centimeters

Cloud Cover: 7 tenths

\section{SOURCE STRENGTH INFORMATION:}

Puddle Area: 10000 square meters

Average Puddle Depth: 1 centimeters

Soil Type: Default Ground Temperature: $25^{\circ} \mathrm{C}$

Initial Puddle Temperature: Ground temperature

Release Duration: ALOHA limited the duration to 1 hour

Max Computed Release Rate: 73.3 kilograms/min

Max Average Sustained Release Rate: 72.8 kilograms/min

(averaged over a minute or more)

Total Amount Released: 4,002 kilograms

FOOTPRINT INFORMATION:

Dispersion Module: Gaussian

User specified LOC: $10 \mathrm{ppm}$

Max Threat Zone for LOC: 805 meters

\section{TIME DEPENDENT INFORMATION:}

Concentration Estimates at the point:

Downwind: 100 meters

Off Centerline: 0 meters

Max Concentration:

Outdoor: $149 \mathrm{ppm}$

Indoor: $149 \mathrm{ppm}$

Note: Indoor graph is shown with a dotted line. 


\section{ENGINEERING COMPUTATION SHEET}

Title of Project EPHA for THM

Subject Concentration Analysis for THM Hazardous Chemicals

Computer T. M. Franey

Date $\underline{09 / 16 / 96}$
Reviewed by $\underline{\text { S. A. Henderson }}$

Works Calculation 7

Sheet No. $\underline{507 \text { of } 577}$

\author{
SITE DATA INFORMATION: 1-RD-44 \\ Location: AIKEN, SOUTH CAROLINA \\ Building Air Exchanges Per Hour: 60 (User specified) \\ Date and Time: Fixed at July 1, 19961300 hours \\ CHEMICAL INFORMATION: \\ Chemical Name: SULFURIC ACID \\ Molecular Weight: $98.08 \mathrm{~kg} / \mathrm{kmol}$ \\ TLV-TWA: $0.25 \mathrm{ppm}$ IDLH: $19.60 \mathrm{ppm}$ \\ Footprint Level of Concern: 10.ppm \\ Boiling Point: $330.00^{\circ} \mathrm{C}$ \\ Vapor Pressure at Ambient Temperature: 5.37e-006 atm \\ Ambient Saturation Concentration: 5.47 ppm or 5.47e-004\% \\ ATMOSPHERIC INFORMATION: (MANUAL INPUT OF DATA) \\ Wind: 2.5 meters $/ \mathrm{sec}$ from $90^{\circ}$ true \\ Inversion Height: 500 meters \\ Stability Class: C Air Temperature: $25^{\circ} \mathrm{C}$ \\ Relative Humidity: 50\% Ground Roughness: 100 centimeters \\ Cloud Cover: 7 tenths
}

SOURCE STRENGTH INFORMATION:

Puddle Area: 30430 square feet

Average Puddle Depth: 1 centimeters

Soil Type: Default Ground Temperature: $25^{\circ} \mathrm{C}$

Initial Puddle Temperature: Ground temperature

Release Duration: ALOHA limited the duration to 1 hour

Max Computed Release Rate: 35.3 grams/min

Max Average Sustained Release Rate: 34.8 grams/min

(averaged over a minute or more)

Total Amount Released: 1.71 kilograms

\section{FOOTPRINT INFORMATION:}

Dispersion Module: Gaussian

User specified LOC: $10 \mathrm{ppm}$

Max Threat Zone for LOC: 30 meters

Max Threat Zone for IDLH: 30 meters

Note: Footprint was not drawn because effects of near-field patchiness make plume presentation unreliable for short distances.

\section{TIME DEPENDENT INFORMATION:}

Concentration Estimates at the point:

Downwind: 30 meters

Off Centerline: 0 meters

Max Concentration:

Outdoor: $0.186 \mathrm{ppm}$

Indoor: $0.186 \mathrm{ppm}$

Note: Indoor graph is shown with a dotted line. 


\section{ENGINEERING COMPUTATION SHEET}

Title of Project EPHA for THM

Subject Concentration Analysis for THM Hazardous Chemicals

Computer T. M. Franey Date 09/16/96
Reviewed by S. A. Henderson

Works Calculation 7

Sheet No. $\underline{508 \text { of } 577}$

SITE DATA INFORMATION: 1-RD-44

Location: AIKEN, SOUTH CAROLINA

Building Air Exchanges Per Hour: 60 (User specified)

Date and Time: Fixed at July 1, 19961300 hours

CHEMICAL INFORMATION:

Chemical Name: SULFURIC ACID

Molecular Weight: $98.08 \mathrm{~kg} / \mathrm{kmol}$

TLV-TWA: $0.25 \mathrm{ppm}$ IDLH: $19.60 \mathrm{ppm}$

Footprint Level of Concern: $10 \mathrm{ppm}$

Boiling Point: $330.00^{\circ} \mathrm{C}$

Vapor Pressure at Ambient Temperature: 5.37e-006 atm

Ambient Saturation Concentration: 5.47 ppm or 5.47e-004\%

ATMOSPHERIC INFORMATION: (MANUAL INPUT OF DATA)

Wind: 2.5 meters $/ \mathrm{sec}$ from $90^{\circ}$ true

Inversion Height: 500 meters

Stability Class: C Air Temperature: $25^{\circ} \mathrm{C}$

Relative Humidity: 50\% Ground Roughness: 100 centimeters

Cloud Cover: 7 tenths

SOURCE STRENGTH INFORMATION:

Puddle Area: 99999 square feet

Average Puddle Depth: 1 centimeters

Soil Type: Default Ground Temperature: $25^{\circ} \mathrm{C}$

Initial Puddle Temperature: Ground temperature

Release Duration: ALOHA limited the duration to 1 hour

Max Computed Release Rate: 111 grams/min

Max Average Sustained Release Rate: 109 grams/min

(averaged over a minute or more)

Total Amount Released: 5.36 kilograms

FOOTPRINT INFORMATION:

Dispersion Module: Gaussian

User specified LOC: $10 \mathrm{ppm}$

Max Threat Zone for LOC: 55 meters

Max Threat Zone for IDLH: 54 meters

Note: Footprint was not drawn because

effects of near-field patchiness make plume

presentation unreliable for short distances.

TIME DEPENDENT INFORMATION:

Concentration Estimates at the point:

Downwind: 100 meters

Off Centerline: 0 meters

Max Concentration:

Outdoor: $0.0577 \mathrm{ppm}$

Indoor: $0.0577 \mathrm{ppm}$

Note: Indoor graph is shown with a dotted line. 
Title of Project EPHA for THM

Subject Concentration Analysis for THM Hazardous Chemicals

Computer T. M. Franey

Date 09/16/96
Reviewed by S. A. Henderson

Works Calculation 7

Sheet No. $\underline{509 \text { of } 577}$

SITE DATA INFORMATION: 1-RD-44

Location: AIKEN, SOUTH CAROLINA

Building Air Exchanges Per Hour: 60 (User specified)

Date and Time: Fixed at July 1, 19961300 hours

CHEMICAL INFORMATION:

Chemical Name: SULFURIC ACID

Molecular Weight: $98.08 \mathrm{~kg} / \mathrm{kmol}$

TLV-TWA: $0.25 \mathrm{ppm}$ IDLH: $19.60 \mathrm{ppm}$

Footprint Level of Concern: $10 \mathrm{ppm}$

Boiling Point: $330.00^{\circ} \mathrm{C}$

Vapor Pressure at Ambient Temperature: $5.37 \mathrm{e}-006 \mathrm{~atm}$

Ambient Saturation Concentration: 5.47 ppm or $5.47 \mathrm{e}-004 \%$

ATMOSPHERIC INFORMATION: (MANUAL INPUT OF DATA)

Wind: 2.5 meters $/ \mathrm{sec}$ from $90^{\circ}$ true

Inversion Height: 500 meters

Stability Class: C Air Temperature: $25^{\circ} \mathrm{C}$

Relative Humidity: $50 \%$ Ground Roughness: 100 centimeters

Cloud Cover: 7 tenths

\section{SOURCE STRENGTH INFORMATION:}

Puddle Area: 24420 square feet

Average Puddle Depth: 1 centimeters

Soil Type: Default Ground Temperature: $25^{\circ} \mathrm{C}$

Initial Puddle Temperature: Ground temperature

Release Duration: ALOHA limited the duration to 1 hour

Max Computed Release Rate: 28.6 grams/min

Max Average Sustained Release Rate: 28.1 grams/min

(averaged over a minute or more)

Total Amount Released: 1.38 kilograms

FOOTPRINT INFORMATION:

Dispersion Module: Gaussian

User specified LOC: $10 \mathrm{ppm}$

Max Threat Zone for LOC: 27 meters

Max Threat Zone for IDLH: 27 meters

Note: Footprint was not drawn because

effects of near-field patchiness make plume

presentation unreliable for short distances.

TIME DEPENDENT INFORMATION:

Concentration Estimates at the point:

Downwind: 100 meters

Off Centerline: 0 meters

Max Concentration:

Outdoor: $0.0277 \mathrm{ppm}$

Indoor: $0.0277 \mathrm{ppm}$

Note: Indoor graph is shown with a dotted line. 


\section{ENGINEERING COMPUTATION SḦEET}

Title of Project EPHA for THM

Subject Concentration Analysis for THM Hazardous Chemicals

Computer T. M. Franey

Date 09/16/96

SITE DATA INFORMATION: 1-RD-44

Location: AIKEN, SOUTH CAROLINA

Building Air Exchanges Per Hour: 60 (User specified)

Date and Time: Fixed at July 1, 19961300 hours

CHEMICAL INFORMATION:

Chemical Name: SULFURIC ACID

Molecular Weight: $98.08 \mathrm{~kg} / \mathrm{kmol}$

TLV-TWA: $0.25 \mathrm{ppm}$ IDLH: $19.60 \mathrm{ppm}$

Footprint Level of Concern: $10 \mathrm{ppm}$

Boiling Point: $330.00^{\circ} \mathrm{C}$

Vapor Pressure at Ambient Temperature: 5.37e-006 atm

Ambient Saturation Concentration: 5.47 ppm or $5.47 \mathrm{e}-004 \%$

ATMOSPHERIC INFORMATION: (MANUAL INPUT OF DATA)

Wind: 2.5 meters $/ \mathrm{sec}$ from $90^{\circ}$ true

Inversion Height: 500 meters

Stability Class: C Air Temperature: $25^{\circ} \mathrm{C}$

Relative Humidity: 50\% Ground Roughness: 100 centimeters

Cloud Cover: 7 tenths

SOURCE STRENGTH INFORMATION:

Puddle Area: 40700 square feet

Average Puddle Depth: 1 centimeters

Soil Type: Default Ground Temperature: $25^{\circ} \mathrm{C}$

Initial Puddle Temperature: Ground temperature

Release Duration: ALOHA limited the duration to 1 hour

Max Computed Release Rate: 46.7 grams/min

Max Average Sustained Release Rate: 46 grams/min

(averaged over a minute or more)

Total Amount Released: 2.26 kilograms

FOOTPRINT INFORMATION:

Dispersion Module: Gaussian

User specified LOC: $10 \mathrm{ppm}$

Max Threat Zone for LOC: 34 meters

Max Threat Zone for IDLH: 35 meters

Note: Footprint was not drawn because

effects of near-field patchiness make plume

presentation unreliable for short distances.

TIME DEPENDENT INFORMATION:

Concentration Estimates at the point:

Downwind: 100 meters

Off Centerline: 0 meters

Max Concentration:

Outdoor: $0.0352 \mathrm{ppm}$

Indoor: $0.0352 \mathrm{ppm}$

Note: Indoor graph is shown with a dotted line.
Reviewed by S.A. Henderson

Works Calculation 7

Sheet No. $\underline{510 \text { of } 577}$ 
Title of Project EPHA for THM

Subject Concentration Analysis for THM Hazardous Chemicals

Computer T. M. Franey

Date $09 / 16 / 96$
Reviewed by S. A. Henderson

Works Calculation 7

Sheet No. 511 of 577

\section{SITE DATA INFORMATION: 1-RD-44}

Location: AIKEN, SOUTH CAROLINA

Building Air Exchanges Per Hour: 60 (User specified)

Date and Time: Fixed at July 1, 19961300 hours

\section{CHEMICAL INFORMATION:}

Chemical Name: SULFURIC ACID

Molecular Weight: $98.08 \mathrm{~kg} / \mathrm{kmol}$

TLV-TWA: $0.25 \mathrm{ppm}$ IDLH: $19.60 \mathrm{ppm}$

Footprint Level of Concern: $10 \mathrm{ppm}$

Boiling Point: $330.00^{\circ} \mathrm{C}$

Vapor Pressure at Ambient Temperature: $5.37 \mathrm{e}-006 \mathrm{~atm}$

Ambient Saturation Concentration: 5.47 ppm or 5.47e-004\%

\section{ATMOSPHERIC INFORMATION: (MANUAL INPUT OF DATA)}

Wind: 2.5 meters $/ \mathrm{sec}$ from $90^{\circ}$ true

Inversion Height: 500 meters

Stability Class: C Air Temperature: $25^{\circ} \mathrm{C}$

Relative Humidity: 50\% Ground Roughness: 100 centimeters

Cloud Cover: 7 tenths

\section{SOURCE STRENGTH INFORMATION:}

Puddle Area: 10000 square meters

Average Puddle Depth: 1 centimeters

Soil Type: Default Ground Temperature: $25^{\circ} \mathrm{C}$

Initial Puddle Temperature: Ground temperature

Release Duration: ALOHA limited the duration to 1 hour

Max Computed Release Rate: 119 grams/min

Max Average Sustained Release Rate: 117 grams/min

(averaged over a minute or more)

Total Amount Released: 5.75 kilograms

\section{FOOTPRINT INFORMATION:}

Dispersion Module: Gaussian

User specified LOC: $10 \mathrm{ppm}$

Max Threat Zone for LOC: 56 meters

Max Threat Zone for IDLH: 56 meters

Note: Footprint was not drawn because effects of near-field patchiness make plume presentation unreliable for short distances.

\section{TIME DEPENDENT INFORMATION:}

Concentration Estimates at the point:

Downwind: 100 meters

Off Centerline: 0 meters

Max Concentration:

Outdoor: $0.0608 \mathrm{ppm}$

Indoor: $0.0608 \mathrm{ppm}$

Note: Indoor graph is shown with a dotted line. 


\section{ENGINEERING COMPUTATION SHEET}

Title of Project EPHA for THM

Subject Concentration Analysis for THM Hazardous Chemicals

Computer T.M. Franey

Date $\underline{09 / 16 / 96}$

SITE DATA INFORMATION: $\quad$ 1-RD-45

Location: AIKEN, SOUTH CAROLINA

Building Air Exchanges Per Hour: 60 (User specified)

Date and Time: Fixed at July 1, 19961300 hours

CHEMICAL INFORMATION:

Chemical Name: SULFURIC ACID, FUMING -

Molecular Weight: $98.00 \mathrm{~kg} / \mathrm{kmol}$

TLV-TWA: $0.25 \mathrm{ppm}$ IDLH: $19.60 \mathrm{ppm}$

Footprint Level of Concern: $10 \mathrm{ppm}$

Boiling Point: $93.50^{\circ} \mathrm{C}$

Vapor Pressure at Ambient Temperature: $0.012 \mathrm{~atm}$

Ambient Saturation Concentration: 12,705 ppm or $1.27 \%$

ATMOSPHERIC INFORMATION: (MANUAL INPUT OF DATA)

Wind: 2.5 meters $/ \mathrm{sec}$ from $90^{\circ}$ true

Inversion Height: 500 meters

Stability Class: C Air Temperature: $25^{\circ} \mathrm{C}$

Relative Humidity: 50\% Ground Roughness: 100 centimeters

Cloud Cover: 7 tenths

\section{SOURCE STRENGTH INFORMATION:}

Puddle Area: 189 square feet

Average Puddle Depth: 1 centimeters

Soil Type: Default Ground Temperature: $25^{\circ} \mathrm{C}$

Initial Puddle Temperature: Ground temperature

Release Duration: ALOHA limited the duration to 1 hour

Max Computed Release Rate: 426 grams/min

Max Average Sustained Release Rate: 423 grams/min

(averaged over a minute or more)

Total Amount Released: 23.3 kilograms

\section{FOOTPRINT INFORMATION:}

Dispersion Module: Gaussian

User specified LOC: $10 \mathrm{ppm}$

Max Threat Zone for LOC: 30 meters

Max Threat Zone for IDLH: 20 meters

Note: Footprint was not drawn because effects of near-field patchiness make plume presentation unreliable for short distances.

\section{TIME DEPENDENT INFORMATION:}

Concentration Estimates at the point:

Downwind: 30 meters

Off Centerline: 0 meters

Max Concentration:

Outdoor: $10 \mathrm{ppm}$

Indoor: $10 \mathrm{ppm}$

Note: Indoor graph is shown with a dotted line.

(n)
Reviewed by $\underline{\text { S. A. Henderson }}$

Works Calculation 7

Sheet No. $\underline{512 \text { of } 577}$ 


\section{ENGINEERING COMPUTATION SHEET}

Title of Project EPHA for THM

Subject Concentration Analysis for THM Hazardous Chemicals

Computer T. M. Franey Date $\underline{09 / 16 / 96}$
Reviewed by S. A. Henderson

Works Calculation 7

Sheet No. 513 of 577

\section{SITE DATA INFORMATION: 1-RD-45}

Location: AIKEN, SOUTH CAROLINA

Building Air Exchanges Per Hour: 60 (User specified)

Date and Time: Fixed at July 1, 19961300 hours

\section{CHEMICAL INFORMATION:}

Chemical Name: SULFURIC ACID, FUMING -

Molecular Weight: $98.00 \mathrm{~kg} / \mathrm{kmol}$

TLV-TWA: $0.25 \mathrm{ppm}$ IDLH: $19.60 \mathrm{ppm}$

Footprint Level of Concern: 10 ppm

Boiling Point: $93.50^{\circ} \mathrm{C}$

Vapor Pressure at Ambient Temperature: $0.012 \mathrm{~atm}$

Ambient Saturation Concentration: 12,705 ppm or $1.27 \%$

ATMOSPHERIC INFORMATION: (MANUAL INPUT OF DATA)

Wind: 2.5 meters $/ \mathrm{sec}$ from $90^{\circ}$ true

Inversion Height: 500 meters

Stability Class: C Air Temperature: $25^{\circ} \mathrm{C}$

Relative Humidity: $50 \%$ Ground Roughness: 100 centimeters

Cloud Cover: 7 tenths

\section{SOURCE STRENGTH INFORMATION:}

Puddle Area: 2300 square feet

Average Puddle Depth: 1 centimeters

Soil Type: Default Ground Temperature: $25^{\circ} \mathrm{C}$

Initial Puddle Temperature: Ground temperature

Release Duration: ALOHA limited the duration to 1 hour

Max Computed Release Rate: 4.76 kilograms/min

Max Average Sustained Release Rate: 4.73 kilograms/min

(averaged over a minute or more)

Total Amount Released: 258 kilograms

\section{FOOTPRINT INFORMATION:}

Dispersion Module: Gaussian

User specified LOC: $10 \mathrm{ppm}$

Max Threat Zone for LOC: 100 meters

Max Threat Zone for IDLH: 66 meters

Note: Footprint was not drawn because effects of near-field patchiness make plume presentation unreliable for short distances.

\section{TIME DEPENDENT INFORMATION:}

Concentration Estimates at the point:

Downwind: 100 meters

Off Centerline: 0 meters

Max Concentration:

Outdoor: $10 \mathrm{ppm}$

Indoor: $10 \mathrm{ppm}$

Note: Indoor graph is shown with a dotted line. 
Title of Project EPHA for THM

Subject Concentration Analysis for THM Hazardous Chemicals

Computer T. M. Franey Date $09 / 16 / 96$

SITE DATA INFORMATION: 1-RD-45

Location: AIKEN, SOUTH CAROLINA

Building Air Exchanges Per Hour: 60 (User specified)

Date and Time: Fixed at July 1, 19961300 hours

CHEMICAL INFORMATION:

Chemical Name: SULFURIC ACID, FUMING -

Molecular Weight: $98.00 \mathrm{~kg} / \mathrm{kmol}$

TLV-TWA: 0.25 ppm IDLH: $19.60 \mathrm{ppm}$

Footprint Level of Concern: 10 ppm

Boiling Point: $93.50^{\circ} \mathrm{C}$

Vapor Pressure at Ambient Temperature: $0.012 \mathrm{~atm}$

Ambient Saturation Concentration: 12,705 ppm or $1.27 \%$

\section{ATMOSPHERIC INFORMATION: (MANUAL INPUT OF DATA)}

Wind: 2.5 meters $/ \mathrm{sec}$ from $90^{\circ}$ true

Inversion Height: 500 meters

Stability Class: C Air Temperature: $25^{\circ} \mathrm{C}$

Relative Humidity: $50 \%$ Ground Roughness: 100 centimeters

Cloud Cover: 7 tenths

\section{SOURCE STRENGTH INFORMATION:}

Puddle Area: 24420 square feet

Average Puddle Depth: 1 centimeters

Soil Type: Default Ground Temperature: $25^{\circ} \mathrm{C}$

Initial Puddle Temperature: Ground temperature

Release Duration: ALOHA limited the duration to 1 hour

Max Computed Release Rate: 47 kilograms/min

Max Average Sustained Release Rate: 46.7 kilograms/min

(averaged over a minute or more)

Total Amount Released: 2,518 kilograms

FOOTPRINT INFORMATION:

Dispersion Module: Gaussian

User specified LOC: 10 ppm

Max Threat Zone for LOC: 313 meters

Max Threat Zone for IDLH: 205 meters

TIME DEPENDENT NNFORMATION:

Concentration Estimates at the point:

Downwind: 100 meters

Off Centerline: 0 meters

Max Concentration:

Outdoor: $46.1 \mathrm{ppm}$

Indoor: $46.1 \mathrm{ppm}$

Note: Indoor graph is shown with a dotted line.
Reviewed by S.A. Henderson

Works Calculation 7

Sheet No. 514 of 577 


\section{ENGINEERING COMPUTATION SHEET}

Title of Project EPHA for THM

Reviewed by $\underline{\text { S. A. Henderson }}$

Subject Concentration Analysis for THM Hazardous Chemicals

Computer T. M. Franey

Date $\underline{09 / 16 / 96}$

SITE DATA INFORMATION: $\quad$ 1-RD-45

Location: AIKEN, SOUTH CAROLINA

Building Air Exchanges Per Hour: 60 (User specified)

Date and Time: Fixed at July 1, 19961300 hours

\section{CHEMICAL INFORMATION:}

Chemical Name: SULFURIC ACID, FUMING -

Molecular Weight: $98.00 \mathrm{~kg} / \mathrm{kmol}$

TLV-TWA: 0.25 ppm IDLH: 19.60 ppm

Footprint Level of Concern: 10 ppm

Boiling Point: $93.50^{\circ} \mathrm{C}$

Vapor Pressure at Ambient Temperature: $0.012 \mathrm{~atm}$

Ambient Saturation Concentration: 12,705 ppm or $1.27 \%$

\section{ATMOSPHERIC INFORMATION: (MANUAL INPUT OF DATA)}

Wind: 2.5 meters $/ \mathrm{sec}$ from $90^{\circ}$ true

Inversion Height: 500 meters

Stability Class: C Air Temperature: $25^{\circ} \mathrm{C}$.

Relative Humidity: $50 \%$ Ground Roughness: 100 centimeters

Cloud Cover: 7 tenths

\section{SOURCE STRENGTH INFORMATION:}

Puddle Area: 40700 square feet

Average Puddle Depth: 1 centimeters

Soil Type: Default Ground Temperature: $25^{\circ} \mathrm{C}$

Initial Puddle Temperature: Ground temperature

Release Duration: ALOHA limited the duration to 1 hour

Max Computed Release Rate: 77.2 kilograms/min

Max Average Sustained Release Rate: 76.6 kilograms/min (averaged over a minute or more)

-Total Amount Released: 4,127 kilograms

FOOTPRINT INFORMATION:

Dispersion Module: Gaussian

User specified LOC: $10 \mathrm{ppm}$

Max Threat Zone for LOC: 402 meters

Max Threat Zone for IDLH: 263 meters

\section{TIME DEPENDENT INFORMATION:}

Concentration Estimates at the point:

Downwind: 100 meters

Off Centerline: 0 meters

Max Concentration:

Outdoor: 58.7 ppm

Indoor: $58.7 \mathrm{ppm}$

Note: Indoor graph is shown with a dotted line.
Works Calculation 7

Sheet No. 515 of 577 


\section{ENGINEERING COMPUTATION SHEET}

Title of Project EPHA for THM

Subject Concentration Analysis for THM Hazardous Chemicals

Computer T. M. Franey

Date 09/16/96

SITE DATA INFORMATION: 1-RD-45

Location: AIKEN, SOUTH CAROLINA

Building Air Exchanges Per Hour: 60 (User specified)

Date and Time: Fixed at July 1, 19961300 hours

CHEMICAL INFORMATION:

Chemical Name: SULFURIC ACID, FUMING -

Molecular Weight: $98.00 \mathrm{~kg} / \mathrm{kmol}$

TLV-TWA: $0.25 \mathrm{ppm}$. IDLH: $19.60 \mathrm{ppm}$

Footprint Level of Concern: 10 ppm

Boiling Point: $93.50^{\circ} \mathrm{C}$

Vapor Pressure at Ambient Temperature: $0.012 \mathrm{~atm}$

Ambient Saturation Concentration: 12,705 ppm or $1.27 \%$

ATMOSPHERIC INFORMATION: (MANUAL INPUT OF DATA)

Wind: 2.5 meters $/ \mathrm{sec}$ from $90^{\circ}$ true

Inversion Height: 500 meters

Stability Class: C Air Temperature: $25^{\circ} \mathrm{C}$

Relative Humidity: 50\% Ground Roughness: 100 centimeters

Cloud Cover: 7 tenths

SOURCE STRENGTH INFORMATION:

Puddle Area: 10000 square meters

Average Puddle Depth: 1 centimeters

Soil Type: Default Ground Temperature: $25^{\circ} \mathrm{C}$

Initial Puddle Temperature: Ground temperature

Release Duration: ALOHA limited the duration to 1 hour

Max Computed Release Rate: 198 kilograms/min

Max Average Sustained Release Rate: 197 kilograms/min (averaged over a minute or more)

Total Amount Released: 10,576 kilograms

FOOTPRINT INFORMATION:

Dispersion Module: Gaussian

User specified LOC: $10 \mathrm{ppm}$

Max Threat Zone for LOC: 647 meters

Max Threat Zone for IDLH: 420 meters

\section{TIME DEPENDENT INFORMATION:}

Concentration Estimates at the point:

Downwind: 100 meters

Off Centerline: 0 meters

Max Concentration:

Outdoor: $102 \mathrm{ppm}$

Indoor: $102 \mathrm{ppm}$

Note: Indoor graph is shown with a dotted line.
Reviewed by S. A. Henderson

Works Calculation 7

Sheet No. $\underline{516 \text { of } 577}$ 
Title of Project EPHA for THM

Subject Concentration Analysis for THM Hazardous Chemicals

Computer T. M. Franey

Date $\underline{09 / 16 / 96}$
Reviewed by $\underline{S . A . ~ H e n d e r s o n}$

Works Calculation 7

Sheet No. 517 of 577

SITE DATA INFORMATION: $\quad$ 1-RD-46

Location: AIKEN, SOUTH CAROLINA

Building Air Exchanges Per Hour: 60 (User specified)

Date and Time: Fixed at July 1;,1996 1300 hours

\section{CHEMICAL INFORMATION:}

Chemical Name: TETRAHYDROFURAN

Molecular Weight: $72.11 \mathrm{~kg} / \mathrm{kmol}$

TLV-TWA: 200.00 ppm IDLH: $20000.00 \mathrm{ppm}$

Footprint Level of Concern: $1000 \mathrm{ppm}$

Boiling Point: $65.97^{\circ} \mathrm{C}$

Vapor Pressure at Ambient Temperature: $0.21 \mathrm{~atm}$

Ambient Saturation Concentration: 216,394 ppm or $21.6 \%$

\section{ATMOSPHERIC INFORMATION: (MANUAL INPUT OF DATA)}

Wind: 2.5 meters/sec from $90^{\circ}$ true

Inversion Height: 500 meters

Stability Class: C Air Temperature: $25^{\circ} \mathrm{C}$

Relative Humidity: $50 \%$ Ground Roughness: 100 centimeters

Cloud Cover: 7 tenths

\section{SOURCE STRENGTH INFORMATION:}

Puddle Area: 1850 square feet

Average Puddle Depth: 1 centimeters

Soil Type: Default Ground Temperature: $25^{\circ} \mathrm{C}$

Initial Puddle Temperature: Ground temperature

Release Duration: 57 minutes

Max Computed Release Rate: 35 kilograms/min

Max Average Sustained Release Rate: 29.9 kilograms/min

(averaged over a minute or more)

Total Amount Released: 1,512 kilograms

\section{FOOTPRINT INFORMATION:}

Dispersion Module: Gaussian

User specified LOC: $1000 \mathrm{ppm}$

Max Threat Zone for LOC: 15 meters

Max Threat Zone for IDLH: less than 10 meters(10.9 yards)

Note: Footprint was not drawn because effects of near-field patchiness make plume presentation unreliable for short distances.

\section{TIME DEPENDENT INFORMATION:}

Concentration Estimates at the point:

Downwind: 30 meters

Off Centerline: 0 meters

Max Concentration:

Outdoor: 484 ppm

Indoor: $484 \mathrm{ppm}$

Note: Indoor graph is shown with a dotted line. 
Title of Project EPHA for THM

Subject Concentration Analysis for THM Hazardous Chemicals

Computer T.M. Franey

Date 09/16/96
Reviewed by $\mathrm{S}$. A. Henderson

Works Calculation 7

Sheet No. 518 of 577

SITE DATA INFORMATION: - 1-RD-46

Location: AIKEN, SOUTH CAROLINA

Building Air Exchanges Per Hour: 60 (User specified)

Date and Time: Fixed at July 1, 19961300 hours

\section{CHEMICAL INFORMATION:}

Chemical Name: TETRAHYDROFURAN

Molecular.Weight: $72.11 \mathrm{~kg} / \mathrm{kmol}$

TLV-TWA: $200.00 \mathrm{ppm}$ IDLH: $20000.00 \mathrm{ppm}$

Footprint Level of Concern: 1000 ppm

Boiling Point: $65.97^{\circ} \mathrm{C}$

Vapor Pressure at Ambient Temperature: $0.21 \mathrm{~atm}$

Ambient Saturation Concentration: 216,394 ppm or $21.6 \%$

ATMOSPHERIC INFORMATION: (MANUAL INPUT OF DATA)

Wind: 2.5 meters $/ \mathrm{sec}$ from $90^{\circ}$ true

Inversion Height: 500 meters

Stability Class: C Air Temperature: $25^{\circ} \mathrm{C}$

Relative Humidity: $50 \%$ Ground Roughness: 100 centimeters

Cloud Cover: 7 tenths

\section{SOURCE STRENGTH INFORMATION:}

Puddle Area: 7500 square feet

Average Puddle Depth: 1 centimeters

Soil Type: Default Ground Temperature: $25^{\circ} \mathrm{C}$

Initial Puddle Temperature: Ground temperature

Release Duration: ALOHA limited the duration to 1 hour

Max Computed Release Rate: 132 kilograms/min

Max Average Sustained Release Rate: 114 kilograms/min

(averaged over a minute or more)

Total Amount Released: 6,111 kilograms

FOOTPRINT INFORMATION:

Model Run: Heavy Gas

User specified LOC: $1000 \mathrm{ppm}$

Max Threat Zone for LOC: 100 meters

Max Threat Zone for IDLH: IDLH level was never exceeded

Note: The Heavy Gas footprint is an initial screening.

For short releases it may be an overestimation.

Be sure to check concentration information at specific locations.

\section{TIME DEPENDENT INFORMATION:}

Concentration Estimates at the point:

Downwind: 100 meters

Off Centerline: 0 meters

Max Concentration:

Outdoor: $1,000 \mathrm{ppm}$

Indoor: $1,000 \mathrm{ppm}$

Note: Indoor graph is shown with a dotted line. 


\section{ENGINEERING COMPUTATION SHEET}

Title of Project EPHA for THM

Reviewed by $\underline{S}$. A. Henderson

Subject Concentration Analysis for THM Hazardous Chemicals

Computer T. M. Franey

Date 09/16/96

SITE DATA INFORMATION: 1-RD-46

Location: AIKEN, SOUTH CAROLINA

Building Air Exchanges Per Hour: 60 (User specified)

Date and Time: Fixed at July 1, 19961300 hours

CHEMICAL INFORMATION:

Chemical Name: TETRAHYDROFURAN

Molecular Weight: $72.11 \mathrm{~kg} / \mathrm{kmol}$

TLV-TWA: $200.00 \mathrm{ppm}$ IDLH: $20000.00 \mathrm{ppm}$

Footprint Level of Concern: $1000 \mathrm{ppm}$

Boiling Point: $65.97^{\circ} \mathrm{C}$

Vapor Pressure at Ambient Temperature: $0.21 \mathrm{~atm}$

Ambient Saturation Concentration: 216,394 ppm or $21.6 \%$

ATMOSPHERIC INFORMATION: (MANUAL INPUT OF DATA)

Wind: 2.5 meters $/ \mathrm{sec}$ from $90^{\circ}$ true

Inversion Height: 500 meters

Stability Class: C Air Temperature: $25^{\circ} \mathrm{C}$

Relative Humidity: 50\% Ground Roughness: 100 centimeters

Cloud Cover: 7 tenths

\section{SOURCE STRENGTH INFORMATION:}

Puddle Area: 24420 square feet

Average Puddle Depth: $l$ centimeters

Soil Type: Default Ground Temperature: $25^{\circ} \mathrm{C}$

Initial Puddle Temperature: Ground temperature

Release'Duration: ALOHA limited the duration to 1 hour

Max Computed Release Rate: 408 kilograms/min

Max Average Sustained Release Rate: 355 kilograms/min

(averaged over a minute or more)

Total Amount Released: 19,189 kilograms

\section{FOOTPRINT INFORMATION:}

Model Run: Heavy Gas

User specified LOC: $1000 \mathrm{ppm}$

Max Threat Zone for LOC: 181 meters

Max Threat Zone for IDLH: IDLH level was never exceeded

Note: The Heavy Gas footprint is an initial screening.

For short releases it may be an overestimation.

Be sure to check concentration information at specific locations.

\section{TIME DEPENDENT INFORMATION:}

Concentration Estimates at the point:

Downwind: 100 meters

Off Centerline: 0 meters

Max Concentration:

Outdoor: $2,460 \mathrm{ppm}$

Indoor: $2,460 \mathrm{ppm}$

Note: Indoor graph is shown with a dotted line.
Works Calculation 7

Sheet No. 519 of 577 
Title of Project EPHA for THM

Subject Concentration Analysis for THM Hazardous Chemicals

Computer T. M. Franey

Date 09/16/96
Reviewed by $\underline{\text { S. A. Henderson }}$

Works Calculation 7

Sheet No. $\underline{520 \text { of } 577}$

SITE DATA INFORMATION: 1-RD-46

Location: AIKEN, SOUTH CAROLINA

Building Air Exchanges Per Hour: 60 (User specified)

Date and Time: Fixed at July 1, 19961300 hours

CHEMICAL INFORMATION:

Chemical Name: TETRAHYDROFURAN

Molecular Weight: $72.11 \mathrm{~kg} / \mathrm{kmol}$

TLV-TWA: $200.00 \mathrm{ppm}$ - IDLH: $20000.00 \mathrm{ppm}$

Footprint Level of Concern: $1000 \mathrm{ppm}$

Boiling Point: $65.97^{\circ} \mathrm{C}$

Vapor Pressure at Ambient Temperature: $0.21 \mathrm{~atm}$

Ambient Saturation Concentration: 216,394 ppm or 21.6\%

ATMOSPHERIC INFORMATION: (MANUAL INPUT OF DATA)

Wind: 2.5 meters $/ \mathrm{sec}$ from $90^{\circ}$ true

Inversion Height: 500 meters

Stability Class: C Air Temperature: $25^{\circ} \mathrm{C}$

Relative Humidity: 50\% Ground Roughness: 100 centimeters

Cloud Cover: 7 tenths

\section{SOURCE STRENGTH INFORMATION:}

Puddle Area: 40700 square feet

Average Puddle Depth: 1 centimeters

Soil Type: Default Ground Temperature: $25^{\circ} \mathrm{C}$

Initial Puddle Temperature: Ground temperature

Release Duration: ALOHA limited the duration to 1 hour

Max Computed Release Rate: 664 kilograms/min

Max Average Sustained Release Rate: 582 kilograms/min

(averaged over a minute or more)

Total Amount Released: 31,515 kilograms

\section{FOOTPRINT INFORMATION:}

Model Run: Heavy Gas

User specified LOC: $1000 \mathrm{ppm}$

Max Threat Zone for LOC: 236 meters

Max Threat Zone for IDLH: IDLH level was never exceeded

Note: The Heavy Gas footprint is an initial screening.

For short releases it may be an overestimation.

Be sure to check concentration information at specific locations.

\section{TIME DEPENDENT INFORMATION:}

Concentration Estimates at the point:

Downwind: 100 meters

Off Centerline: 0 meters

Max Concentration:

Outdoor: $3,650 \mathrm{ppm}$

Indoor: $3,650 \mathrm{ppm}$

Note: Indoor graph is shown with a dotted line. 


\section{ENGINEERING COMPUTATION SHEET}

Title of Project EPHA for THM

Subject Concentration Analysis for THM Hazardous Chemicals

Computer T.M. Franey Date $\underline{09 / 16 / 96}$

SITE DATA INFORMATION: 1-RD-46

Location: AIKEN, SOUTH CAROLINA

Building Air Exchanges Per Hour: 60 (User specified)

Date and Time: Fixed at July 1, 19961300 hours

CHEMICAL INFORMATION:

Chemical Name: TETRAHYDROFURAN

Molecular Weight: $72.11 \mathrm{~kg} / \mathrm{kmol}$

TLV-TWA: $200.00 \mathrm{ppm}$ IDLH: $20000.00 \mathrm{ppm}$

Footprint Level of Concern: 1000 ppm

Boiling Point: $65.97^{\circ} \mathrm{C}$

Vapor Pressure at Ambient Temperature: $0.21 \mathrm{~atm}$

Ambient Saturation Concentration: 216,394 ppm or $21.6 \%$

ATMOSPHERIC INFORMATION: (MANUAL INPUT OF DATA)

Wind: 2.5 meters $/ \mathrm{sec}$ from $90^{\circ}$ true

Inversion Height: 500 meters

Stability Class: C Air Temperature: $25^{\circ} \mathrm{C}$

Relative Humidity: $50 \%$ Ground Roughness: 100 centimeters

Cloud Cover: 7 tenths

SOURCE STRENGTH INFORMATION:

Puddle Area: 10000 square meters

Average Puddle Depth: 1 centimeters

Soil Type: Default Ground Temperature: $25^{\circ} \mathrm{C}$

Initial Puddle Temperature: Ground temperature

Release Duration: ALOHA limited the duration to 1 hour

Max Computed Release Rate: 1,680 kilograms/min

Max Average Sustained Release Rate: 1,490 kilograms/min

(averaged over a minute or more)

Total Amount Released: 81,034 kilograms

FOOTPRINT INFORMATION:

Model Run: Heavy Gas

User specified LOC: $1000 \mathrm{ppm}$

Max Threat Zone for LOC: 392 meters

Max Threat Zone for IDLH: 37 meters

Note: The Heavy Gas footprint is an initial screening.

For short releases it may be an overestimation.

Be sure to check concentration information at specific locations.

\section{TIME DEPENDENT INFORMATION:}

Concentration Estimates at the point:

Downwind: 100 meters

Off Centerline: 0 meters

Max Concentration:

Outdoor: 7,660 ppm

Indoor: 7,660 ppm

Note: Indoor graph is shown with a dotted line.
Reviewed by $\underline{\text { S. A. Henderson }}$

Works Calculation 7

Sheet No. $\underline{521 \text { of } 577}$ 


\section{ENGINEERING COMPUTATION SHEET}

Title of Project EPHA for THM

Subject Concentration Analysis for THM Hazardous Chemicals

Computer T. M. Franey

Date $\underline{09 / 16 / 96}$

SITE DATA INFORMATION: 1-RD-47

Location: AIKEN, SOUTH CAROLINA

Building Air Exchanges Per Hour: 60 (User specified)

Date and Time: Fixed at July 1, 19961300 hours

CHEMICAL INFORMATION:

Chemical Name: TOLUENE

Molecular Weight: $92.14 \mathrm{~kg} / \mathrm{kmol}$

TLV-TWA: $50.00 \mathrm{ppm}$

IDLH: $2000.00 \mathrm{ppm}$

Footprint Level of Concern: 300 ppm

Boiling Point: $110.63^{\circ} \mathrm{C}$

Vapor Pressure at Ambient Temperature: $0.037 \mathrm{~atm}$

Ambient Saturation Concentration: 38,111 ppm or $3.81 \%$

\section{ATMOSPHERIC INFORMATION: (MANUAL INPUT OF DATA)}

Wind: 2.5 meters $/ \mathrm{sec}$ from $90^{\circ}$ true

Inversion Height: 500 meters

Stability Class: C Air Temperature: $25^{\circ} \mathrm{C}$

Relative Humidity: 50\% Ground Roughness: 100 centimeters

Cloud Cover: 7 tenths

SOURCE STRENGTH INFORMATION:

Puddle Area: 11950 square feet

Average Puddle Depth: 1 centimeters

Soil Type: Default Ground Temperature: $25^{\circ} \mathrm{C}$

Initial Puddle Temperature: Ground temperature

Release Duration: ALOHA limited the duration to 1 hour

Max Computed Release Rate: 51.1 kilograms/min

Max Average Sustained Release Rate: 50.9 kilograms/min (averaged over a minute or more)

Total Amount Released: 2,894 kilograms

\section{FOOTPRINT INFORMATION:}

Dispersion Module: Gaussian

User specified LOC: $300 \mathrm{ppm}$

Max Threat Zone for LOC: 30 meters

Max Threat Zone for IDLH: 19 meters

Note: Footprint was not drawn because effects of near-field patchiness mäke plume presentation unreliable for short distances.

\section{TIME DEPENDENT INFORMATION:}

Concentration Estimates at the point:

Downwind: 30 meters

Off Centerline: 0 meters

Max Concentration:

Outdoor: 300 ppm

Indoor: $300 \mathrm{ppm}$

Note: Indoor graph is shown with a dotted line.
Reviewed by S. A. Henderson

Works Calculation 7

Sheet No. $\underline{522 \text { of } 577}$ 
Title of Project EPHA for THM

Subject Concentration Analysis for THM Hazardous Chemicals

Computer T. M. Franey

Date 09/16/96

SITE DATA INFORMATION: 1-RD-47

Location: AIKEN, SOUTH CAROLINA

Building Air Exchanges Per Hour: 60 (User specified)

Date and Time: Fixed at July 1, 19961300 hours

CHEMICAL INFORMATION:

Chemical Name: TOLUENE Molecular Weight: $92.14 \mathrm{~kg} / \mathrm{kmol}$

TLV-TWA: $50.00 \mathrm{ppm}$ IDLH: $2000.00 \mathrm{ppm}$

Footprint Level of Concern: 300 ppm

Boiling Point: $110.63^{\circ} \mathrm{C}$

Vapor Pressure at Ambient Temperature: $0.037 \mathrm{~atm}$

Ambient Saturation Concentration: $38,111 \mathrm{ppm}$ or $3.81 \%$

ATMOSPHERIC INFORMATION: (MANUAL INPUT OF DATA)

Wind: 2.5 meters $/ \mathrm{sec}$ from $90^{\circ}$ true

Inversion Height: 500 meters

Stability Class: C Air Temperature: $25^{\circ} \mathrm{C}$

Relative Humidity: 50\% Ground Roughness: 100 centimeters

Cloud Cover: 7 tenths

SOURCE STRENGTH INFORMATION:

Puddle Area: 22350 square feet

Average Puddle Depth: 1 centimeters

Soil Type: Default Ground Temperature: $25^{\circ} \mathrm{C}$

Initial Puddle Temperature: Ground temperature

Release Duration: ALOHA limited the duration to 1 hour

Max Computed Release Rate: 93.5 kilograms/min

Max Average Sustained Release Rate: 93.1 kilograms/min

(averaged over a minute or more)

Total Amount Released: 5,288 kilograms

FOOTPRINT INFORMATION:

Dispersion Module: Gaussian

User specified LOC: 300 ppm

Max Threat Zone for LOC: 41 meters

Max Threat Zone for IDLH: 26 meters

Note: Footprint was not drawn because effects of near-field patchiness make plume presentation unreliable for short distances.

TIME DEPENDENT INFORMATION:

Concentration Estimates at the point:

Downwind: 100 meters

Off Centerline: 0 meters

Max Concentration:

Outdoor: 102 ppm

Indoor: $102 \mathrm{ppm}$

Note: Indoor graph is shown with a dotted line.

Reviewed by S. A. Henderson

Works Calculation 7

Sheet No. $\underline{523 \text { of } 577}$ 


\section{ENGINEERING COMPUTATION SHEET}

Title of Project EPHA for THM

Reviewed by S.A. Henderson

Subject Concentration Analysis for THM Hazardous Chemicals

Computer T. M. Franey

Date 09/16/96

SITE DATA INFORMATION: $\quad$ 1-RD-47

Location: AIKEN, SOUTH CAROLINA

Building Air Exchanges Per Hour: 60 (User specified)

Date and Time: Fixed at July 1, 19961300 hours

CHEMICAL INFORMATION:

Chemical Name: TOLUENE Molecular Weight: $92.14 \mathrm{~kg} / \mathrm{kmol}$

TLV-TWA: $50.00 \mathrm{ppm}$ IDLH: $2000.00 \mathrm{ppm}$

Footprint Level of Concern: 300 ppm

Boiling Point: $110.63^{\circ} \mathrm{C}$

Vapor Pressure at Ambient Temperature: $0.037 \mathrm{~atm}$

Ambient Saturation Concentration: $38,111 \mathrm{ppm}$ or $3.81 \%$

ATMOSPHERIC INFORMATION: (MANUAL INPUT OF DATA)

Wind: 2.5 meters $/ \mathrm{sec}$ from $90^{\circ}$ true

Inversion Height: 500 meters

Stability Class: C Air Temperature: $25^{\circ} \mathrm{C}$

Relative Humidity: $50 \%$ Ground Roughness: 100 centimeters

Cloud Cover: 7 tenths

\section{SOURCE STRENGTH INFORMATION:}

Puddle Area: 24420 square feet

Average Puddle Depth: 1 centimeters

Soil Type: Default Ground Temperature: $25^{\circ} \mathrm{C}$

Initial Puddle Temperature: Ground temperature

Release Duration: ALOHA limited the duration to 1 hour

Max Computed Release Rate: 102 kilograms/min

Max Average Sustained Release Rate: 101 kilograms/min

(averaged over a minute or more)

Total Amount Released: 5,759 kilograms

\section{FOOTPRINT INFORMATION:}

Model Run: Heavy Gas

User specified LOC: $300 \mathrm{ppm}$

Max Threat Zone for LOC: 118 meters

Max Threat Zone for IDLH: 29 meters

Note: The Heavy Gas footprint is an initial screening.

For short releases it may be an overestimation.

Be sure to check concentration information at specific locations.

\section{TIME DEPENDENT INFORMATION:}

Concentration Estimates at the point:

Downwind: 100 meters

Off Centerline: 0 meters

Max Concentration:

Outdoor: $441 \mathrm{ppm}$

Indoor: $441 \mathrm{ppm}$

Note: Indoor graph is shown with a dotted line.
Works Calculation 7

Sheet No: $\underline{524 \text { of } 577}$ 
Title of Project EPHA for THM

Subject Concentration Analysis for THM Hazardous Chemicals

Computer T.M. Franey

Date $\underline{09 / 16 / 96}$
Reviewed by S. A. Henderson

Works Calculation 7

Sheet No. $\underline{525 \text { of } 577}$
SITE DATA INFORMATION: 1-RD-47

Location: AIKEN, SOUTH CAROLINA

Building Air Exchanges Per Hour: 60 (User specified)

Date and Time: Fixed at July 1, 19961300 hours

\section{CHEMICAL INFORMATION:}

Chemical Name: TOLUENE Molecular Weight: $92.14 \mathrm{~kg} / \mathrm{kmol}$

TLV-TWA: $50.00 \mathrm{ppm}$

Footprint Level of Concern: 300 ppm

Boiling Point: $110.63^{\circ} \mathrm{C}$

Vapor Pressure at Ambient Temperature: $0.037 \mathrm{~atm}$

Ambient Saturation Concentration: 38,111 ppm or $3.81 \%$

ATMOSPHERIC INFORMATION: (MANUAL INPUT OF DATA)

Wind: 2.5 meters $/ \mathrm{sec}$ from $90^{\circ}$ true

Inversion Height: 500 meters

Stability Class: C Air Temperature: $25^{\circ} \mathrm{C}$

Relative Humidity: $50 \%$ Ground Roughness: 100 centimeters

Cloud Cover: 7 tenths

SOURCE STRENGTH INFORMATION:

Puddle Area: 40700 square feet

Average Puddle Depth: 1 centimeters

Soil Type: Default Ground Temperature: $25^{\circ} \mathrm{C}$

Initial Puddle Temperature: Ground temperature

Release Duration: ALOHA limited the duration to 1 hour

Max Computed Release Rate: 167 kilograms/min

Max Average Sustained Release Rate: 166 kilograms/min

(averaged over a minute or more)

Total Amount Released: 9,423 kilograms

\section{FOOTPRINT INFORMATION:}

Model Run: Heavy Gas

User specified LOC: 300 ppm

Max Threat Zone for LOC: 144 meters

Max Threat Zone for IDLH: 35 meters

Note: The Heavy Gas footprint is an initial screening.

For short releases it may be an overestimation.

Be sure to check concentration information at specific locations.

\section{TIME DEPENDENT INFORMATION:}

Concentration Estimates at the point:

Downwind: 100 meters

Off Centerline: 0 meters

Max Concentration:

Outdoor: 772 ppm

Indoor: $772 \mathrm{ppm}$

Note: Indoor graph is shown with a dotted line. 
Title of Project EPHA for THM

Subject Concentration Analysis for THM Hazardous Chemicals

Computer T. M. Franey
Reviewed by S. A. Henderson

Works Calculation 7

Sheet No. $\underline{526 \text { of } 577}$

\section{SITE DATA INFORMATION: 1-RD-47}

Location: AIKEN, SOUTH CAROLINA

Building Air Exchanges Per Hour: 60 (User specified)

Date and Time: Fixed at July 1, 19961300 hours

CHEMICAL INFORMATION:

Chemical Name: TOLUENE Molecular Weight: $92.14 \mathrm{~kg} / \mathrm{kmol}$

TLV-TWA: $50.00 \mathrm{ppm}$ IDLH: $2000.00 \mathrm{ppm}$

Footprint Level of Concern: $300 \mathrm{ppm}$

Boiling Point: $110.63^{\circ} \mathrm{C}$

Vapor Pressure at Ambient Temperature: $0.037 \mathrm{~atm}$

Ambient Saturation Concentration: 38,111 ppm or $3.81 \%$

ATMOSPHERIC INFORMATION: (MANUAL INPUT OF DATA)

Wind: 2.5 meters $/ \mathrm{sec}$ from $90^{\circ}$ true

Inversion Height: 500 meters

Stability Class: C Air Temperature: $25^{\circ} \mathrm{C}$

Relative Humidity: $50 \%$ Ground Roughness: 100 centimeters

Cloud Cover: 7 tenths

SOURCE STRENGTH INFORMATION:

Puddle Area: 10000 square meters

Average Puddle Depth: 1 centimeters

Soil Type: Default Ground Temperature: $25^{\circ} \mathrm{C}$

Initial Puddle Temperature: Ground temperature

Release Duration: ALOHA limited the duration to 1 hour

Max Computed Release Rate: 427 kilograms/min

Max Average Sustained Release Rate: 426 kilograms/min

(averaged over a minute or more)

Total Amount Released: 24,080 kilograms

\section{FOOTPRINT INFORMATION:}

Model Run: Heavy Gas

User specified LOC: 300 ppm

Max Threat Zone for LOC: 191 meters

Max Threat Zone for IDLH: 55 meters

Note: The Heavy Gas footprint is an initial screening.

For short releases it may be an overestimation.

Be sure to check concentration information at specific locations.

\section{TIME DEPENDENT INFORMATION:}

\section{Concentration Estimates at the point:}

Downwind: 100 meters

Off Centerline: 0 meters

Max Concentration:

Outdoor: $1,270 \mathrm{ppm}$

Indoor: $1,270 \mathrm{ppm}$

Note: Indoor graph is shown with a dotted line. 
Title of Project EPHA for THM

Subject Concentration Analysis for THM Hazardous Chemicals

Computer T.M. Franey

Date 09/16/96

Reviewed by $\underline{\text { S. A. Henderson }}$

Works Calculation 7

Sheet No. $\underline{527 \text { of } 577}$

\section{SITE DATA INFORMATION: I-RD-48}

Location: AIKEN, SOUTH CAROLINA

Building Air Exchanges Per Hour: 60 (User specified)

Date and Time: Fixed at July 1, 19961300 hours

\section{CHEMICAL INFORMATION:}

Chemical Name: O-TOLUIDINE

Molecular Weight: $107.15 \mathrm{~kg} / \mathrm{kmol}$

TLV-TWA: $2.00 \mathrm{ppm}$ IDLH: $100.00 \mathrm{ppm}$

Note: Potential or confirmed human carcinogen.

Footprint Level of Concern: 10 ppm

Boiling Point: $200.40^{\circ} \mathrm{C}$

Vapor Pressure at Ambient Temperature: 3.39e-004 atm

Ambient Saturation Concentration: 345 ppm or $0.034 \%$

ATMOSPHERIC INFORMATION: (MANUAL INPUT OF DATA)

Wind: 2.5 meters $/ \mathrm{sec}$ from $90^{\circ}$ true

Inversion Height: 500 meters

Stability Class: C Air Temperature: $25^{\circ} \mathrm{C}$

Relative Humidity: 50\% Ground Roughness: 100 centimeters

Cloud Cover: 7 tenths

\section{SOURCE STRENGTH INFORMATION:}

Puddle Area: 23880 square feet

Average Puddle Depth: 1 centimeters

Soil Type: Default Ground Temperature: $25^{\circ} \mathrm{C}$

Initial Puddle Temperature: Ground temperature

Release Duration: ALOHA limited the duration to 1 hour

Max Computed Release Rate: 2.07 kilograms/min

Max Average Sustained Release Rate: 2.04 kilograms/min

(averaged over a minute or more)

Total Amount Released: 103 kilograms

\section{TIME DEPENDENT INFORMATION:}

Concentration Estimates at the point:

Downwind: 30 meters

Off Centerline: 0 meters

Max Concentration:

Outdoor: $10 \mathrm{ppm}$

Indoor: $10 \mathrm{ppm}$

Note: Indoor graph is shown with a dotted line. 
Title of Project EPHA for THM

Subject Concentration Analysis for THM Hazardous Chemicals

Computer T. M. Franey

Date $09 / 16 / 96$

\section{SITE DATA INFORMATION: $\quad$ 1-RD-48}

Location: AIKEN, SOUTH CAROLINA

Building Air Exchanges Per Hour: 60 (User specified)

Date and Time: Fixed at July 1, 19961300 hours

\section{CHEMICAL INFORMATION:}

Chemical Name: O-TOLUIDINE

Molecular Weight: $107.15 \mathrm{~kg} / \mathrm{kmol}$

TLV-TWA: $2.00 \mathrm{ppm}$ IDLH: $100.00 \mathrm{ppm}$

Note: Potential or confirmed human carcinogen.

Footprint Level of Concern: 10 ppm

Boiling Point: $200.40^{\circ} \mathrm{C}$

Vapor Pressure at Ambient Temperature: 3.39e-004 atm

Ambient Saturation Concentration: 345 ppm or $0.034 \%$

\section{ATMOSPHERIC INFORMATION: (MANUAL INPUT OF DATA)}

Wind: 2.5 meters $/ \mathrm{sec}$ from $90^{\circ}$ true

Inversion Height: 500 meters

Stability Class: C Air Temperature: $25^{\circ} \mathrm{C}$

Relative Humidity: 50\% Ground Roughness: 100 centimeters

Cloud Cover: 7 tenths

\section{SOURCE STRENGTH INFORMATION:}

Puddle Area: 99999 square feet

Average Puddle Depth: 1 centimeters

Soil Type: Default Ground Temperature: $25^{\circ} \mathrm{C}$

Initial Puddle Temperature: Ground temperature

Release Duration: ALOHA limited the duration to 1 hour

Max Computed Release Rate: 8.21 kilograms/min

Max Average Sustained Release Rate: 8.09 kilograms/min

(averaged over a minute or more)

Total Amount Released: 406 kilograms

\section{TIME DEPENDENT INFORMATION:}

Concentration Estimates at the point:

Downwind: 100 meters

Off Centerline: 0 meters

Max Concentration:

Outdoor: $3.91 \mathrm{ppm}$

Indoor: $3.91 \mathrm{ppm}$

Note: Indoor graph is shown with a dotted line.
Reviewed by S. A. Henderson

Works Calculation 7

Sheet No. $\underline{528 \text { of } 577}$

7


Title of Project EPHA for THM

Subject Concentration Analysis for THM Hazardous Chemicals

Computer T. M. Franey Date $\underline{09 / 16 / 96}$
Reviewed by $\mathrm{S}$. A. Henderson

Works Calculation 7

Sheet No. $\underline{529 \text { of } 577}$

SITE DATA INFORMATION: 1-RD-48

Location: AIKEN, SOUTH CAROLINA

Building Air Exchanges Per Hour: 60 (User specified)

Date and Time: Fixed at July 1, 19961300 hours

\section{CHEMICAL INFORMATION:}

Chemical Name: O-TOLUIDINE

Molecular Weight: $107.15 \mathrm{~kg} / \mathrm{kmol}$

TLV-TWA: $2.00 \mathrm{ppm}$ IDLH: $100.00 \mathrm{ppm}$

Note: Potential or confirmed human carcinogen.

Footprint Level of Concern: $10 \mathrm{ppm}$

Boiling Point: $200.40^{\circ} \mathrm{C}$

Vapor Pressure at Ambient Temperature: 3.39e-004 atm

Ambient Saturation Concentration: 345 ppm or $0.034 \%$

ATMOSPHERIC INFORMATION: (MANUAL INPUT OF DATA)

Wind: 2.5 meters $/ \mathrm{sec}$ from $90^{\circ}$ true

Inversion Height: 500 meters

Stability Class: C Air Temperature: $25^{\circ} \mathrm{C}$

Relative Humidity: $50 \%$ Ground Roughness: 100 centimeters

Cloud Cover: 7 tenths

\section{SOURCE STRENGTH INFORMATION:}

Puddle Area: 24420 square feet

Average Puddle Depth: 1 centimeters

Soil Type: Default Ground Temperature: $25^{\circ} \mathrm{C}$

Initial Puddle Temperature: Ground temperature

Release Duration: ALOHA limited the duration to 1 hour

Max Computed Release Rate: 2.11 kilograms/min

Max Average Sustained Release Rate: 2.08 kilograms/min

(averaged over a minute or more)

Total Amount Released: 105 kilograms

\section{FOOTPRINT INFORMATION:}

Dispersion Module: Gaussian

User specified LOC: $10 \mathrm{ppm}$

Max Threat Zone for LOC: 30 meters

Max Threat Zone for IDLH: 27 meters

Note: Footprint was not drawn because effects of near-field patchiness make plume presentation unreliable for short distances.

\section{TIME DEPENDENT INFORMATION:}

Concentration Estimates at the point:

Downwind: 100 meters

Off Centerline: 0 meters

Max Concentration:

Outdoor: $1.88 \mathrm{ppm}$

Indoor: $1.88 \mathrm{ppm}$

Note: Indoor graph is shown with a dotted line. 
Title of Project EPHA for THM

Subject Concentration Analysis for THM Hazardous Chemicals

Computer T. M. Franey Date 09/16/96
Reviewed by S. A. Henderson

Works Calculation 7

Sheet No. $\underline{530 \text { of } 577}$

\section{SITE DATA INFORMATION: $\quad$ 1-RD-48 \\ Location: AIKEN, SOUTH CAROLINA \\ Building Air Exchanges Per Hour: 60 (User specified) \\ Date and Time: Fixed at July 1, 19961300 hours \\ CHEMICAL INFORMATION: \\ Chemical Name: O-TOLUIDINE \\ Molecular Weight: $107.15 \mathrm{~kg} / \mathrm{kmol}$ \\ TLV-TWA: $2.00 \mathrm{ppm}$ IDLH: $100.00 \mathrm{ppm}$ \\ Note: Potential or confirmed human carcinogen. \\ Footprint Level of Concern: 10 ppm \\ Boiling Point: $200.40^{\circ} \mathrm{C}$ \\ Vapor Pressure at Ambient Temperature: 3.39e-004 atm \\ Ambient Saturation Concentration: 345 ppm or $0.034 \%$}

\section{ATMOSPHERIC INFORMATION: (MANUAL INPUT OF DATA) \\ Wind: 2.5 meters $/ \mathrm{sec}$ from $90^{\circ}$ true \\ Inversion Height: 500 meters \\ Stability Class: C Air Temperature: $25^{\circ} \mathrm{C}$ \\ Relative Humidity: $50 \%$ Ground Roughness: 100 centimeters \\ Cloud Cover: 7 tenths}

\section{SOURCE STRENGTH INFORMATION:}

Puddle Area: 40700 square feet

Average Puddle Depth: 1 centimeters

Soil Type: Default ' Ground Temperature: $25^{\circ} \mathrm{C}$

Initial Puddle Temperature: Ground temperature

Release Duration: ALOHA limited the duration to 1 hour

Max Computed Release Rate: 3.45 kilograms/min

Max Average Sustained Release Rate: 3.41 kilograms/min

(averaged over a minute or more)

Total Amount Released: 171 kilograms

FOOTPRINT INFORMATION:

Dispersion Module: Gaussian

User specified LOC: $10 \mathrm{ppm}$

Max Threat Zone for LOC: 39 meters

Max Threat Zone for IDLH: 34 meters

Note: Footprint was not drawn because effects of near-field patchiness make plume presentation unreliable for short distances.

TIME DEPENDENT INFORMATION:

Concentration Estimates at the point:

Downwind: 100 meters

Off Centerline: 0 meters

Max Concentration:

Outdoor: $2.39 \mathrm{ppm}$

Indoor: $2.39 \mathrm{ppm}$

Note: Indoor graph is shown with a dotted line. 
Title of Project EPHA for THM

Subject Concentration Analysis for THM Hazardous Chemicals

Computer T. M. Franey

Date $\underline{09 / 16 / 96}$
Reviewed by $\underline{\text { S. A. Henderson }}$

Works Calculation 7

Sheet No. $\underline{531 \text { of } 577}$

SITE DATA INFORMATION: 1-RD-49

Location: AIKEN, SOUTH CAROLINA

Building Air Exchanges Per Hour: 60 (User specified)

Date and Time: Fixed at July 1, 19961300 hours

CHEMICAL INFORMATION:

Chemical Name: 1,1,1-TRICHLOROETHANE

Molecular Weight: $133.40 \mathrm{~kg} / \mathrm{kmol}$

TLV-TWA: $350.00 \mathrm{ppm}$ IDLH: $1000.00 \mathrm{ppm}$

Footprint Level of Concern: 1000 ppm

Boiling Point: $74.08^{\circ} \mathrm{C}$

Vapor Pressure at Ambient Temperature: $0.16 \mathrm{~atm}$

Ambient Saturation Concentration: 165,660 ppm or $16.6 \%$

ATMOSPHERIC INFORMATION: (MANUAL INPUT OF DATA)

Wind: 2.5 meters $/ \mathrm{sec}$ from $90^{\circ}$ true

Inversion Height: 500 meters

Stability Class: C Air Temperature: $25^{\circ} \mathrm{C}$

Relative Humidity: $50 \%$ Ground Roughness: 100 centimeters

Cloud Cover: 7 tenths

SOURCE STRENGTH INFORMATION:

Puddle Area: 955 square feet

Average Puddle Depth: 1 centimeters

Soil Type: Default Ground Temperature: $25^{\circ} \mathrm{C}$

Initial Puddle Temperature: Ground temperature

Release Duration: 58 minutes

Max Computed Release Rate: 24 kilograms/min

Max Average Sustained Release Rate: 21.9 kilograms/min

(averaged over a minute or more)

Total Amount Released: 1,180 kilograms

FOOTPRINT INFORMATION:

Model Run: Heavy Gas

User specified LOC: $1000 \mathrm{ppm}$

Max Threat Zone for LOC: 30 meters

Max Threat Zone for IDLH: 30 meters

Note: Footprint wasn't drawn because effects of near-field patchiness make plume presentation unreliable for short distances.

\section{TIME DEPENDENT INFORMATION:}

Concentration Estimates at the point:

Downwind: 30 meters

Off Centerline: 0 meters

Max Concentration:

Outdoor: $1,000 \mathrm{ppm}$

Indoor: $1,000 \mathrm{ppm}$

Note: Indoor graph is shown with a dotted line. 


\section{ENGINEERING COMPUTATION SHEET}

Title of Project EPHA for THM

Subject Concentration Analysis for THM Hazardous Chemicals

Computer T. M. Franey

Date 09/16/96

SITE DATA INFORMATION: 1-RD-49

Location: AIKEN, SOUTH CAROLINA

Building Air Exchanges Per Hour: 60 (User specified)

Date and Time: Fixed at July 1, 19961300 hours

CHEMICAL INFORMATION:

Chemical Name: 1,1,1-TRICHLOROETHANE

Molecular Weight: $133.40 \mathrm{~kg} / \mathrm{kmol}$

TLV.TWA: $350.00 \mathrm{ppm}$ IDLH: $1000.00 \mathrm{ppm}$

Footprint Level of Concern: 1000 ppm

Boiling Point: $74.08^{\circ} \mathrm{C}$

Vapor Pressure at Ambient Temperature: $0.16 \mathrm{~atm}$

Ambient Saturation Concentration: 165,660 ppm or $16.6 \%$

ATMOSPHERIC INFORMATION: (MANUAL INPUT OF DATA)

Wind: 2.5 meters $/ \mathrm{sec}$ from $90^{\circ}$ true

Inversion Height: 500 meters

Stability Class: C Air Temperature: $25^{\circ} \mathrm{C}$

Relative Humidity: $50 \%$ Ground Roughness: 100 centimeters

Cloud Cover: 7 tenths

SOURCE STRENGTH INFORMATION:

Puddle Area: 11300 square feet

Average Puddle Depth: 1 centimeters

Soil Type: Default Ground Temperature: $25^{\circ} \mathrm{C}$

Initial Puddle Temperature: Ground temperature

Release Duration: ALOHA limited the duration to 1 hour

Max Computed Release Rate: 253 kilograms/min

Max Average Sustained Release Rate: 235 kilograms/min

(averaged over a minute or more)

Total Amount Released: 13,269 kilograms

FOOTPRINT INFORMATION:

Model Run: Heavy Gas

User specified LOC: $1000 \mathrm{ppm}$

Max Threat Zone for LOC: 100 meters

Max Threat Zone for IDLH: 99 meters

Note: The Heavy Gas footprint is an initial screening.

For short releases it may be an overestimation.

Be sure to check concentration information at specific locations.

\section{TIME DEPENDENT INFORMATION:}

Concentration Estimates at the point:

Downwind: 100 meters

Off Centerline: 0 meters

Max Concentration:

Outdoor: $1,000 \mathrm{ppm}$

Indoor: $1,000 \mathrm{ppm}$

Note: Indoor graph is shown with a dotted line.
Reviewed by $\mathrm{S}$. A. Henderson

Works Calculation 7

Sheet No. $\underline{532 \text { of } 577}$ 


\section{ENGINEERING COMPUTATION SHEET}

Title of Project EPHA for THM

Subject Concentration Analysis for THM Hazardous Chemicals

Computer T.M. Franey Date 09/16/96

\section{SITE DATA INFORMATION: 1-RD-49}

Location: AIKEN, SOUTH CAROLINA

Building Air Exchanges Per Hour: 60 (User specified)

Date and Time: Fixed at July 1, 19961300 hours

\section{CHEMICAL INFORMATION:}

Chemical Name: 1,1,1-TRICHLOROETHANE

Molecular Weight: $133.40 \mathrm{~kg} / \mathrm{kmol}$

TLV-TWA: $350.00 \mathrm{ppm}$ IDLH: $1000.00 \mathrm{ppm}$

Footprint Level of Concern: $1000 \mathrm{ppm}$

Boiling Point: $74.08^{\circ} \mathrm{C}$

Vapor Pressure at Ambient Temperature: $0.16 \mathrm{~atm}$

Ambient Saturation Concentration: 165,660 ppm or $16.6 \%$

ATMOSPHERIC INFORMATION: (MANUAL INPUT OF DATA)

Wind: 2.5 meters $/ \mathrm{sec}$ from $90^{\circ}$ true

Inversion Height: 500 meters

Stability Class: C Air Temperature: $25^{\circ} \mathrm{C}$

Relative Humidity: 50\% Ground Roughness: 100 centimeters

Cloud Cover: 7 tenths

\section{SOURCE STRENGTH INFORMATION:}

Puddle Area: 24420 square feet

Average Puddle Depth: 1 centimeters

Soil Type: Default Ground Temperature: $25^{\circ} \mathrm{C}$

Initial Puddle Temperature: Ground temperature

Release Duration: ALOHA limited the duration to 1 hour

Max Computed Release Rate: 530 kilograms/min

Max Average Sustained Release Rate: 494 kilograms/min

(averaged over a minute or more)

Total Amount Released: 28,014 kilograms

\section{FOOTPRINT INFORMATION:}

Model Run: Heavy Gas

User specified LOC: $1000 \mathrm{ppm}$

Max Threat Zone for LOC: 150 meters

Max Threat Zone for IDLH: 149 meters

Note: The Heavy Gas footprint is an initial screening.

For short releases it may be an overestimation.

Be sure to check concentration information at specific locations.

\section{TIME DEPENDENT INFORMATION:}

Concentration Estimates at the point:

Downwind: 100 meters

Off Centerline: 0 meters

Max Concentration:

Outdoor: $1,900 \mathrm{ppm}$

Indoor: $1,900 \mathrm{ppm}$

Note: Indoor graph is shown with a dotted line.
Reviewed by S. A. Henderson

Works Calculation 7

Sheet No. $\underline{533 \text { of } 577}$ 


\section{ENGINEERING COMPUTATION SHEET}

Title of Project EPHA for THM

Subject Concentration Analysis for THM Hazardous Chemicals

Computer T. M. Franey Date $\underline{09 / 16 / 96}$
Reviewed by S. A. Henderson

Works Calculation 7

Sheet No. $\underline{534 \text { of } 577}$

SITE DATA INFORMATION: $\quad$ 1-RD-49

Location: AIKEN, SOUTH CAROLINA

Building Air Exchanges Per Hour: 60 (User specified)

Date and Time: Fixed at July 1, 19961300 hours

CHEMICAL INFORMATION:

Chemical Name: 1,1,1-TRICHLOROETHANE

Molecular Weight: $133.40 \mathrm{~kg} / \mathrm{kmol}$

TLV-TWA: $350.00 \mathrm{ppm}$ IDLH: $1000.00 \mathrm{ppm}$

Footprint Level of Concern: 1000 ppm

Boiling Point: $74.08^{\circ} \mathrm{C}$

Vapor Pressure at Ambient Temperature: 0.16 atm

Ambient Saturation Concentration: 165,660 ppm or $16.6 \%$

\section{ATMOSPHERIC INFORMATION: (MANUAL INPUT OF DATA)}

Wind: 2.5 meters $/ \mathrm{sec}$ from $90^{\circ}$ true

Inversion Height: 500 meters

Stability Class: C Air Temperature: $25^{\circ} \mathrm{C}$

Relative Humidity: $50 \%$ Ground Roughness: 100 centimeters

Cloud Cover: 7 tenths

\section{SOURCE STRENGTH INFORMATION:}

Puddle Area: 40700 square feet

Average Puddle Depth: 1 centimeters

Soil Type: Default Ground Temperature: $25^{\circ} \mathrm{C}$

Initial Puddle Temperature: Ground temperature

Release Duration: ALOHA limited the duration to 1 hour

Max Computed Release Rate: 864 kilograms/min

Max Average Sustained Release Rate: 809 kilograms/min

(averaged over a minute or more)

Total Amount Released: 45,989 kilograms

\section{FOOTPRINT INFORMATION:}

Model Run: Heavy Gas

User specified LOC: $1000 \mathrm{ppm}$

Max Threat Zone for LOC: 196 meters

Max Threat Zone for IDLH: 195 meters

Note: The Heavy Gas footprint is an initial screening.

For short releases it may be an overestimation.

Be sure to check concentration information at specific locations.

\section{TIME DEPENDENT INFORMATION:}

Concentration Estimates at the point:

Downwind: 100 meters

Off Centerline: 0 meters

Max Concentration:

Outdoor: $2,920 \mathrm{ppm}$

Indoor: $2,920 \mathrm{ppm}$

Note: Indoor graph is shown with a dotted line. 


\section{ENGINEERING COMPUTATION SHEET}

Title of Project EPHA for THM

Subject Concentration Analysis for THM Hazardous Chemicals

Computer T. M. Franey

Date 09/16/96

SITE DATA INFORMATION: $\quad$ 1-RD-49

Location: AIKEN, SOUTH CAROLINA

Building Air Exchanges Per Hour: 60 (User specified)

Date and Time: Fixed at July 1, 19961300 hours

CHEMICAL INFORMATION:

Chemical Name: 1,1,1-TRICHLOROETHANE

Molecular Weight: $133.40 \mathrm{~kg} / \mathrm{kmol}$

TLV-TWA: $350.00 \mathrm{ppm}$ IDLH: $1000.00 \mathrm{ppm}$

Footprint Level of Concern: 1000 ppm

Boiling Point: $74.08^{\circ} \mathrm{C}$

Vapor Pressure at Ambient Temperature: $0.16 \mathrm{~atm}$

Ambient Saturation Concentration: 165,660 ppm or $16.6 \%$

ATMOSPHERIC INFORMATION: (MANUAL INPUT OF DATA)

Wind: 2.5 meters $/ \mathrm{sec}$ from $90^{\circ}$ true

Inversion Height: 500 meters

Stability Class: C Air Temperature: $25^{\circ} \mathrm{C}$

Relative Humidity: $50 \%$ Ground Roughness: 100 centimeters

Cloud Cover: 7 tenths

SOURCE STRENGTH INFORMATION:

Puddle Area: 10000 square meters

Average Puddle Depth: 1 centimeters

Soil Type: Default $\quad$ Ground Temperature: $25^{\circ} \mathrm{C}$

Initial Puddle Temperature: Ground temperature

Release Duration: ALOHA limited the duration to 1 hour

Max Computed Release Rate: 2,200 kilograms/min

Max Average Sustained Release Rate: 2,070 kilograms/min

(averaged over a minute or more)

Total Amount Released: 118,257 kilograms

FOOTPRINT INFORMATION:

Model Run: Heavy Gas

User specified LOC: $1000 \mathrm{ppm}$

Max Threat Zone for LOC: 329 meters

Max Threat Zone for IDLH: 326 meters

Note: The Heavy Gas footprint is an initial screening.

For short releases it may be an overestimation.

Be sure to check concentration information at specific locations.

TIME DEPENDENT INFORMATION:

Concentration Estimates at the point:

Downwind: 100 meters

Off Centerline: 0 meters

Max Concentration:

Outdoor: $6,590 \mathrm{ppm}$

Indoor: $6,580 \mathrm{ppm}$

Note: Indoor graph is shown with a dotted line.
Reviewed by S. A. Henderson

Works Calculation 7

Sheet No. $\underline{535 \text { of } 577}$ 


\section{ENGINEERING COMPUTATION SHEET}

Title of Project EPHA for THM

Reviewed by $\mathrm{S}$. A. Henderson

Subject Concentration Analysis for THM Hazardous Chemicals

Computer T. M. Franey

Date $\underline{09 / 16 / 96}$

SITE DATA INFORMATION: 1-RD-50

Location: AIKEN, SOUTH CAROLINA

Building Air Exchanges Per Hour: 60 (User specified)

Date and Time: Fixed at July 1, 19961300 hours

CHEMICAL INFORMATION:

Chemical Name: TRICHLOROETHYLENE

Molecular Weight: $131.39 \mathrm{~kg} / \mathrm{kmol}$

TLV-TWA: $50.00 \mathrm{ppm}$ IDLH: $1000.00 \mathrm{ppm}$

Note: Potential or confirmed human carcinogen.

Footprint Level of Concern: 500 ppm

Boiling Point: $86.95^{\circ} \mathrm{C}$

Vapor Pressure at Ambient Temperature: $0.097 \mathrm{~atm}$

Ambient Saturation Concentration: 98,733 ppm or $9.87 \%$

ATMOSPHERIC INFORMATION: (MANUAL INPUT OF DATA)

Wind: $2.5^{\circ}$ meters $/ \mathrm{sec}$ from $90^{\circ}$ true

Inversion Height: 500 meters

Stability Class: C Air Temperature: $25^{\circ} \mathrm{C}$

Relative Humidity: 50\% Ground Roughness: 100 centimeters

Cloud Cover: 7 tenths

SOURCE STRENGTH INFORMATION:

Puddle Area: 1700 square feet

Average Puddle Depth: 1 centimeters

Soil Type: Default Ground Temperature: $25^{\circ} \mathrm{C}$

Initial Puddle Temperature: Ground temperature

Release Duration: ALOHA limited the duration to 1 hour

Max Computed Release Rate: 22.8 kilograms/min

Max Average Sustained Release Rate: 22.5 kilograms/min (averaged over a minute or more)

Total Amount Released: 1,336 kilograms

FOOTPRINT INFORMATION:

Dispersion Module: Gaussian

User specified LOC: $500 \mathrm{ppm}$

Max Threat Zone for LOC: 13 meters

Max Threat Zone for IDLH: less than 10 meters(10.9 yards)

Note: Footprint was not drawn because

effects of near-field patchiness make plume

presentation unreliable for short distances.

TIME DEPENDENT INFORMATION:

Concentration Estimates at the point:

Downwind: 30 meters

Off Centerline: 0 meters

Max Concentration:

Outdoor: 208 ppm

Indoor: $208 \mathrm{ppm}$

Note: Indoor graph is shown with a dotted line.
Works Calculation 7

Sheet No. $\underline{536 \text { of } 577}$ 
Title of Project EPHA for THM

Subject Concentration Analysis for THM Hazardous Chemicals

Computer T. M. Franey Date $09 / 16 / 96$

SITE DATA INFORMATION: 1-RD-50

Location: AIKEN, SOUTH CAROLINA

Building Air Exchanges Per Hour: 60 (User specified)

Date and Time: Fixed at July 1, 19961300 hours

CHEMICAL INFORMATION:

Chemical Name: TRICHLOROETHYLENE

Molecular Weight: $131.39 \mathrm{~kg} / \mathrm{kmol}$

TLV-TWA: $50.00 \mathrm{ppm}$ IDLH: $1000.00 \mathrm{ppm}$

Note: Potential or confirmed human carcinogen.

Footprint Level of Concern: 500 ppm

Boiling Point: $86.95^{\circ} \mathrm{C}$

Vapor Pressure at Ambient Temperature: $0.097 \mathrm{~atm}$

Ambient Saturation Concentration: 98,733 ppm or $9.87 \%$

ATMOSPHERIC INFORMATION: (MANUAL INPUT OF DATA)

Wind: 2.5 meters $/ \mathrm{sec}$ from $90^{\circ}$ true

Inversion Height: $\mathbf{5 0 0}$ meters

Stability Class: C Air Temperature: $25^{\circ} \mathrm{C}$

Relative Humidity: $50 \%$ Ground Roughness: 100 centimeters

Cloud Cover: 7 tenths

\section{SOURCE STRENGTH INFORMATION:}

Puddle Area: 8200 square feet

Average Puddle Depth: 1 centimeters

Soil Type: Default Ground Temperature: $25^{\circ} \mathrm{C}$

Initial Puddle Temperature: Ground temperature

Release Duration: ALOHA limited the duration to 1 hour

Max Computed Release Rate: 102 kilograms/min

Max Average Sustained Release Rate: 102 kilograms/min

(averaged over a minute or more)

Total Amount Released: 6,106 kilograms

FOOTPRINT INFORMATION:

Model Run: Heavy Gas

User specified LOC: $500 \mathrm{ppm}$

Max Threat Zone for LOC: 100 meters

Max Threat Zone for IDLH: 61 meters

Note: The Heavy Gas footprint is an initial screening.

For short releases it may be an overestimation.

Be sure to check concentration information at specific locations.

TIME DEPENDENT INFORMATION:

Concentration Estimates at the point:

Downwind: 100 meters

Off Centerline: 0 meters

Max Concentration:

Outdoor: $500 \mathrm{ppm}$

Indoor: $500 \mathrm{ppm}$

Note: Indoor graph is shown with a dotted line.
Reviewed by S. A. Henderson

Works Calculation 7

Sheet No. 537 of 577 
Title of Project EPHA for THM

Subject Concentration Analysis for THM Hazardous Chemicals

Computer T. M. Franey

Date 09/16/96
Reviewed by S. A. Henderson

Works Calculation 7

Sheet No. $\underline{538 \text { of } 577}$

SITE DATA INFORMATION: 1-RD-50

Location: AIKEN, SOUTH CAROLINA

Building Air Exchanges Per Hour: 60 (User specified)

Date and Time: Fixed at July 1, 19961300 hours

CHEMICAL INFORMATION:

Chemical Name: TRICHLOROETHYLENE

Molecular Weight: $131.39 \mathrm{~kg} / \mathrm{kmol}$

TLV-TWA: $50.00 \mathrm{ppm}$ ' IDLH: $1000.00 \mathrm{ppm}$

Note: Potential or confirmed human carcinogen.

Footprint Level of Concern: 500 ppm

Boiling Point: $86.95^{\circ} \mathrm{C}$

Vapor Pressure at Ambient Temperature: $0.097 \mathrm{~atm}$

Ambient Saturation Concentration: 98,733 ppm or $9.87 \%$

ATMOSPHERIC INFORMATION: (MANUAL INPUT OF DATA)

Wind: 2.5 meters $/ \mathrm{sec}$ from $90^{\circ}$ true

Inversion Height: 500 meters

Stability Class: $\mathrm{C} \quad$ Air Temperature: $25^{\circ} \mathrm{C}$

Relative Humidity: 50\% Ground Roughness: 100 centimeters

Cloud Cover: 7 tenths

SOURCE STRENGTH INFORMATION:

Puddle Area: 24420 square feet

Average Puddle Depth: 1 centimeters

Soil Type: Default Ground Temperature: $25^{\circ} \mathrm{C}$

Initial Puddle Temperature: Ground temperature

Release Duration: ALOHA limited the duration to 1 hour

Max Computed Release Rate: 293 kilograms/min

Max Average Sustained Release Rate: 293 kilograms/min

(averaged over a minute or more)

Total Amount Released: 17,547 kilograms

FOOTPRINT INFORMATION:

Model Run: Heavy Gas

User specified LOC: 500 ppm

Max Threat Zone for LOC: 174 meters .

Max Threat Zone for IDLH: 108 meters

Note: The Heavy Gas footprint is an initial screening.

For short releases it may be an overestimation.

Be sure to check concentration information at specific locations.

TIME DEPENDENT INFORMATION:

Concentration Estimates at the point:

Downwind: 100 meters

Off Centerline: 0 meters

Max Concentration:

Outdoor: $1,140 \mathrm{ppm}$

Indoor: $1,140 \mathrm{ppm}$

Note: Indoor graph is shown with a dotted line. 
Title of Project EPHA for THM

Reviewed by S. A. Henderson

Subject Concentration Analysis for THM Hazardous Chemicals

Computer T. M. Franey

Date $\underline{09 / 16 / 96}$
Works Calculation 7

Sheet No. $\underline{539 \text { of } 577}$

SITE DATA INFORMATION: $\quad$ 1-RD-50

Location: AIKEN, SOUTH CAROLINA

Building Air Exchanges Per Hour: 60 (User specified)

Date and Time: Fixed at July 1, 19961300 hours

CHEMICAL INFORMATION:

Chemical Name: TRICHLOROETHYLENE

Molecular Weight: $131.39 \mathrm{~kg} / \mathrm{kmol}$

TLV-TWA: $50.00 \mathrm{ppm}$ IDLH: $1000.00 \mathrm{ppm}$

Note: Potential or confirmed human carcinogen.

Footprint Level of Concern: 500 ppm

Boiling Point: $86.95^{\circ} \mathrm{C}$

Vapor Pressure at Ambient Temperature: $0.097 \mathrm{~atm}$

Ambient Saturation Concentration: 98,733 ppm or $9.87 \%$

ATMOSPHERIC INFORMATION: (MANUAL INPUT OF DATA)

Wind: 2.5 meters $/ \mathrm{sec}$ from $90^{\circ}$ true

Inversion Height: 500 meters

Stability Class: C Air Temperature: $25^{\circ} \mathrm{C}$

Relative Humidity: $50 \%$ Ground Roughness: 100 centimeters

Cloud Cover: 7 tenths

SOURCE STRENGTH INFORMATION:

Puddle Area: 10000 square meters

Average Puddle Depth: 1 centimeters

Soil Type: Default Ground Temperature: $25^{\circ} \mathrm{C}$

Initial Puddle Temperature: Ground temperature

Release Duration: ALOHA limited the duration to 1 hour

Max Computed Release Rate: 1,240 kilograms/min

Max Average Sustained Release Rate: 1,240 kilograms/min

(averaged over a minute or more)

Total Amount Released: 73,841 kilograms

FOOTPRINT INFORMATION:

Model Run: Heavy Gas

User specified LOC: $500 \mathrm{ppm}$

Max Threat Zone for LOC: 375 meters

Max Threat Zone for IDLH: 237 meters

Note: The Heavy Gas footprint is an initial screening.

For short releases it may be an overestimation.

Be sure to check concentration information at specific locations.

\section{TTME DEPENDENT INFORMATION:}

Concentration Estimates at the point:

Downwind: 100 meters

Off Centerline: 0 meters

Max Concentration:

Outdoor: $3,510 \mathrm{ppm}$

Indoor: $3,510 \mathrm{ppm}$

Note: Indoor graph is shown with a dotted line. 


\section{ENGINEERING COMPUTATION SHEET}

Title of Project EPHA for THM

Subject Concentration Analysis for THM Hazardous Chemicals

Computer T. M. Franey

Date $\underline{09 / 16 / 96}$

SITE DATA INFORMATION: 1-RD-51

Location: AIKEN, SOUTH CAROLINA

Building Air Exchanges Per Hour: 60 (User specified)

Date and Time: Fixed at July 1, 19961300 hours

CHEMICAL INFORMATION:

Chemical Name: TRIETHYLAMINE

Molecular Weight: $101.19 \mathrm{~kg} / \mathrm{kmol}$

TLV-TWA: $10.00 \mathrm{ppm}$ IDLH: $1000.00 \mathrm{ppm}$

Footprint Level of Concern: 100 ppm

Boiling Point: $88.77^{\circ} \mathrm{C}$

Vapor Pressure at Ambient Temperature: $0.090 \mathrm{~atm}$

Ambient Saturation Concentration: 91,682 ppm or $9.17 \%$

ATMOSPHERIC INFORMATION: (MANUAL INPUT OF DATA)

Wind: 2.5 meters $/ \mathrm{sec}$ from $90^{\circ}$ true

Inversion Height: 500 meters

Stability Class: C Air Temperature: $25^{\circ} \mathrm{C}$

Relative Humidity: 50\% Ground Roughness: 100 centimeters

Cloud Cover: 7 tenths

SOURCE STRENGTH INFORMATION:

Puddle Area: 485 square feet

Average Puddle Depth: 1 centimeters

Soil Type: Default Ground Temperature: $25^{\circ} \mathrm{C}$

Initial Puddle Temperature: Ground temperature

Release Duration: ALOHA limited the duration to 1 hour

Max Computed Release Rate: 5.3 kilograms/min

Max Average Sustained Release Rate: 5.19 kilograms/min

(averaged over a minute or more)

Total Amount Released: 306 kilograms

FOOTPRINT INFORMATION:

Dispersion Module: Gaussian

User specified LOC: $100 \mathrm{ppm}$

Max Threat Zone for LOC: 30 meters

Max Threat Zone for IDLH: less than 10 meters(10.9 yards)

Note: Footprint was not drawn because effects of near-field patchiness make plume

presentation unreliable for short distances.

\section{TIME DEPENDENT INFORMATION:}

Concentration Estimates at the point:

Downwind: 30 meters

Off Centerline: 0 meters

Max Concentration:

Outdoor: $100 \mathrm{ppm}$

Indoor: $100 \mathrm{ppm}$

Note: Indoor graph is shown with a dotted line.
Reviewed by $\mathrm{S}$. A. Henderson

Works Calculation 7

Sheet No. $\underline{540 \text { of } 577}$

\section{7}




\section{ENGINEERING COMPUTATION SHEET}

Title of Project EPHA for THM

Reviewed by S. A. Henderson

Subject Concentration Analysis for THM Hazardous Chemicals

Computer T. M. Franey

Date $\underline{09 / 16 / 96}$

SITE DATA INFORMATION: 1-RD-51

Location: AIKEN, SOUTH CAROLINA

Building Air Exchanges Per Hour: 60 (User specified)

Date and Time: Fixed at July 1, 19961300 hours

\section{CHEMICAL INFORMATION:}

Chemical Name: TRIETHYLAMINE

Molecular Weight: $101.19 \mathrm{~kg} / \mathrm{kmol}$

TLV-TWA: $10.00 \mathrm{ppm}$ IDLH: $1000.00 \mathrm{ppm}$

Footprint Level of Concern: 100 ppm

Boiling Point: $88.77^{\circ} \mathrm{C}$

Vapor Pressure at Ambient Temperature: $0.090 \mathrm{~atm}$

Ambient Saturation Concentration: 91,682 ppm or $9.17 \%$

ATMOSPHERIC INFORMATION: (MANUAL INPUT OF DATA)

Wind: 2.5 meters $/ \mathrm{sec}$ from $90^{\circ}$ true

Inversion Height: 500 meters

Stability Class: C Air Temperature: $25^{\circ} \mathrm{C}$

Relative Humidity: 50\% Ground Roughness: 100 centimeters

Cloud Cover: 7 tenths

\section{SOURCE STRENGTH INFORMATION:}

Puddle Area: 3670 square feet

Average Puddle Depth: 1 centimeters

Soil Type: Default Ground Temperature: $25^{\circ} \mathrm{C}$

Initial Puddle Temperature: Ground temperature

Release Duration: ALOHA limited the duration to 1 hour

Max Computed Release Rate: 36.3 kilograms/min

Max Average Sustained Release Rate: 36 kilograms/min

(averaged over a minute or more)

Total Amount Released: 2,147 kilograms

\section{TIME DEPENDENT INFORMATION:}

Concentration Estimates at the point:

Downwind: 100 meters

Off Centerline: 0 meters

Max Concentration:

Outdoor: $68.5 \mathrm{ppm}$

Indoor: $68.5 \mathrm{ppm}$

Note: Indoor graph is shown with a dotted line.
Works Calculation 7

Sheet No. $\underline{541 \text { of } 577}$ 


\section{ENGINEERING COMPUTATION SHEET}

Title of Project EPHA for THM.

Subject Concentration Analysis for THM Hazardous Chemicals

Computer T.M. Franey

Date 09/16/96
SITE DATA INFORMATION: 1-RD-51

Location: AIKEN, SOUTH CAROLINA

Building Air Exchanges Per Hour: 60 (User specified)

Date and Time: Fixed at July 1, 19961300 hours

\section{CHEMICAL INFORMATION:}

Chemical Name: TRIETHYLAMINE

Molecular Weight: $101.19 \mathrm{~kg} / \mathrm{kmol}$

TLV-TWA: $10.00 \mathrm{ppm}$ IDLH: $1000.00 \mathrm{ppm}$

Footprint Level of Concern: 100 ppm

Boiling Point: $88.77^{\circ} \mathrm{C}$

Vapor Pressure at Ambient Temperature: $0.090 \mathrm{~atm}$

Ambient Saturation Concentration: 91,682 ppm or $9.17 \%$

ATMOSPHERIC INFORMATION: (MANUAL INPUT OF DATA)

Wind: 2.5 meters $/ \mathrm{sec}$ from $90^{\circ}$ true

Inversion Height: 500 meters

Stability Class: C Air Temperature: $25^{\circ} \mathrm{C}$

Relative Humidity: 50\% Ground Roughness: 100 centimeters

Cloud Cover: 7 tenths

\section{SOURCE STRENGTH INFORMATION:}

Puddle Area: 24420 square feet

Average Puddle Depth: 1 centimeters

Soil Type: Default Ground Temperature: $25^{\circ} \mathrm{C}$

Initial Puddle Temperature: Ground temperature

Release Duration: ALOHA limited the duration to 1 hour

Max Computed Release Rate: 224 kilograms/min

Max Average Sustained Release Rate: 224 kilograms/min (averaged over a minute or more)

Total Amount Released: 13,393 kilograms

\section{FOOTPRINT INFORMATION:}

Model Run: Heavy Gas

User specified LOC: $100 \mathrm{ppm}$

Max Threat Zone for LOC: 522 meters

Max Threat Zone for IDLH: 107 meters

Note: The Heavy Gas footprint is an initial screening.

For short releases it may be an overestimation.

$B e$ sure to check concentration information at specific locations.

TIME DEPENDENT INFORMATION:

Concentration Estimates at the point:

Downwind: 100 meters

Off Centerline: 0 meters

Max Concentration:

Outdoor: $1,120 \mathrm{ppm}$

Indoor: $1,120 \mathrm{ppm}$

Note: Indoor graph is shown with a dotted line.
Reviewed by S.A. Henderson

Works Calculation 7

Sheet No. $\underline{542 \text { of } 577}$ 
Title of Project EPHA for THM

Subject Concentration Analysis for THM Hazardous Chemicals

Computer T.M. Franey Date $\underline{09 / 16 / 96}$
Reviewed by S. A. Henderson

Works Calculation 7

Sheet No. 543 of 577

\section{SITE DATA INFORMATION: 1-RD-51 \\ Location: AIKEN, SOUTH CAROLINA \\ Building Air Exchanges Per Hour: 60 (User specified) \\ Date and Time: Fixed at July 1, 19961300 hours}

\section{CHEMICAL INFORMATION:}

Chemical Name: TRIETHYLAMINE

Molecular Weight: $101.19 \mathrm{~kg} / \mathrm{kmol}$

TLV-TWA: $10.00 \mathrm{ppm}$ IDLH: $1000.00 \mathrm{ppm}$

Footprint Level of Concern: 100 ppm

Boiling Point: $88.77^{\circ} \mathrm{C}$

Vapor Pressure at Ambient Temperature: $0.090 \mathrm{~atm}$

Ambient Saturation Concentration: 91,682 ppm or 9.17\%

\section{ATMOSPHERIC INFORMATION: (MANUAL INPUT OF DATA)}

Wind: 2.5 meters $/ \mathrm{sec}$ from $90^{\circ}$ true

Inversion Height: 500 meters

Stability Class: $\mathrm{C} \quad$ Air Temperature: $25^{\circ} \mathrm{C}$

Relative Humidity: $50 \%$ Ground Roughness: 100 centimeters

Cloud Cover: 7 tenths

\section{SOURCE STRENGTH INFORMATION:}

Puddle Area: 40700 square feet

Average Puddle Depth: 1 centimeters

Soil Type: Default Ground Temperature: $25^{\circ} \mathrm{C}$

Initial Puddle Temperature: Ground temperature

Release Duration: ALOHA limited the duration to 1 hour

Max Computed Release Rate: 367 kilograms/min

Max Average Sustained Release Rate: 367 kilograms/min

(averaged over a minute or more)

Total Amount Released: 21,953 kilograms

FOOTPRINT INFORMATION:

Model Run: Heavy Gas

User specified LOC: $100 \mathrm{ppm}$

Max Threat Zone for LOC: 691 meters

Max Threat Zone for IDLH: 140 meters

Note: The Heavy Gas footprint is an initial screening.

For short releases it may be an overestimation.

Be sure to check concentration information at specific locations.

\section{TIME DEPENDENT INFORMATION:}

Concentration Estimates at the point:

Downwind: 100 meters

Off Centerline: 0 meters

Max Concentration:

Outdoor: $1,620 \mathrm{ppm}$

Indoor: $1,620 \mathrm{ppm}$

Note: Indoor graph is shown with a dotted line. 


\section{ENGINEERING COMPUTATION SHEET}

Title of Project EPHA for THM

Subject Concentration Analysis for THM Hazardous Chemicals

Computer T. M. Franey

Date 09/16/96
Reviewed by $\underline{S}$. A. Henderson

Works Calculation 7

Sheet No. 544 of 577

SITE DATA INFORMATION: 1-RD-51

Location: AIKEN, SOUTH CAROLINA

Building Air Exchanges Per Hour: 60 (User specified)

Date and Time: Fixed at July 1, 19961300 hours

CHEMICAL INFORMATION:

Chemical Name: TRIETHYLAMINE

Molecular Weight: $101.19 \mathrm{~kg} / \mathrm{kmol}$

TLV-TWA: $10.00 \mathrm{ppm}$ IDLH: $1000.00 \mathrm{ppm}$

Footprint Level of Concern: $100 \mathrm{ppm}$

Boiling Point: $88.77^{\circ} \mathrm{C}$

Vapor Pressure at Ambient Temperature: $0.090 \mathrm{~atm}$

Ambient Saturation Concentration: 91,682 ppm or $9.17 \%$

ATMOSPHERIC INFORMATION: (MANUAL INPUT OF DATA)

Wind: 2.5 meters $/ \mathrm{sec}$ from $90^{\circ}$ true

Inversion Height: $\mathbf{5 0 0}$ meters

Stability Class: C Air Temperature: $25^{\circ} \mathrm{C}$

Relative Humidity: 50\% Ground Roughness: 100 centimeters

Cloud Cover: 7 tenths

\section{SOURCE STRENGTH INFORMATION:}

Puddle Area: 10000 square meters

Average Puddle Depth: 1 centimeters

Soil Type: Default Ground Temperature: $25^{\circ} \mathrm{C}$

Initial Puddle Temperature: Ground temperature

Release Duration: ALOHA limited the duration to 1 hour

Max Computed Release Rate: 945 kilograms/min

Max Average Sustained Release Rate: 944 kilograms/min

(averaged over a minute or more)

Total Amount Released: 56,291 kilograms

\section{FOOTPRINT INFORMATION:}

Model Run: Heavy Gas

User specified LOC: 100 ppm

Max Threat Zone for LOC: 1.1 kilometers

-Max Threat Zone for IDLH: 233 meters

Note: The Heavy Gas footprint is an initial screening.

For short releases it may be an overestimation.

Be sure to check concentration information at specific locations.

\section{TIME DEPENDENT INFORMATION:}

Concentration Estimates at the point:

Downwind: 100 meters

Off Centerline: 0 meters

Max Concentration:

Outdoor: $3,220 \mathrm{ppm}$

Indoor: $3,220 \mathrm{ppm}$

Note: Indoor graph is shown with a dotted line. 
Title of Project EPHA for THM

Subject Concentration Analysis for THM Hazardous Chemicals

Computer T. M. Franey
Reviewed by S. A. Henderson

Works Calculation 7

Sheet No. $\underline{545 \text { of } 577}$

SITE DATA INFORMATION: 1-RD-52

Location: AIKEN, SOUTH CAROLINA

Building Air Exchanges Per Hour: 60 (User specified)

Date and Time: Fixed at July 1, 19961300 hours

CHEMICAL INFORMATION:

Chemical Name: VINYL ACETATE

Molecular Weight: $86.09 \mathrm{~kg} / \mathrm{kmol}$

TLV-TWA: 10.00 ppm IDLH: -unavail-

Note: Potential or confirmed human carcinogen.

Footprint Level of Concern: 75 ppm

Boiling Point: $72.50^{\circ} \mathrm{C}$

Vapor Pressure at Ambient Temperature: $0.15 \mathrm{~atm}$

Ambient Saturation Concentration: $153,288 \mathrm{ppm}$ or $15.3 \%$

\section{ATMOSPHERIC INFORMATION: (MANUAL INPUT OF DATA)}

Wind: 2.5 meters $/ \mathrm{sec}$ from $90^{\circ}$ true

Inversion Height: 500 meters

Stability Class: C Air Temperature: $25^{\circ} \mathrm{C}$

Relative Humidity: 50\% Ground Roughness: 100 centimeters

Cloud Cover: 7 tenths

\section{SOURCE STRENGTH INFORMATION:}

Puddle Area: 181 square feet

Average Puddle Depth: 1 centimeters

Soil Type: Default Ground Temperature: $25^{\circ} \mathrm{C}$

Initial Puddle Temperature: Ground temperature

Release Duration: ALOHA limited the duration to 1 hour

Max Computed Release Rate: 3.15 kilograms $/ \mathrm{min}$

Max Average Sustained Release Rate: 2.76 kilograms/min

(averaged over a minute or more)

Total Amount Released: 150 kilograms

TIME DEPENDENT INFORMATION:

Concentration Estimates at the point:

Downwind: 30 meters

Off Centerline: 0 meters

Max Concentration:

Outdoor: $74.9 \mathrm{ppm}$

Indoor: $74.9 \mathrm{ppm}$

Note: Indoor graph is shown with a dotted line. 


\section{ENGINEERING COMPUTATION SHEET}

Title of Project EPHA for THM

Reviewed by S. A. Henderson

Subject Concentration Analysis for THM Hazardous Chemicals

Computer T. M. Franey

Date $\underline{09 / 16 / 96}$
Works Calculation 7

Sheet No. $\underline{546 \text { of } 577}$

SITE DÄTA INFORMATION: 1-RD-52

Location: AIKEN, SOUTH CAROLINA

Building Air Exchanges Per Hour: 60 (User specified)

Date and Time: Fixed at July 1, 19961300 hours

CHEMICAL INFORMATION:

Chemical Name: VINYL ACETATE

Molecular Weight: $86.09 \mathrm{~kg} / \mathrm{kmol}$

TLV-TWA: $10.00 \mathrm{ppm}$ IDLH: -unavail-

Note: Potential or confirmed human carcinogen.

Footprint Level of Concern: 75 ppm

Boiling Point: $72.50^{\circ} \mathrm{C}$

Vapor Pressure at Ambient Temperature: $0.15 \mathrm{~atm}$

Ambient Saturation Concentration: 153,288 ppm or $15.3 \%$

ATMOSPHERIC INFORMATION: (MANUAL INPUT OF DATA)

Wind: 2.5 meters $/ \mathrm{sec}$ from $90^{\circ}$ true

Inversion Height: 500 meters

Stability Class: C Air Temperature: $25^{\circ} \mathrm{C}$

Relative Humidity: $50 \%$ Ground Roughness: 100 centimeters

Cloud Cover: 7 tenths

SOURCE STRENGTH INFORMATION:

Puddle Area: 2130 square feet

Average Puddle Depth: 1 centimeters

Soil Type: Default $\quad$ Ground Temperature: $25^{\circ} \mathrm{C}$

Initial Puddle Temperature: Ground temperature

Release Duration: ALOHA limited the duration to 1 hour

Max Computed Release Rate: 32.6 kilograms/min

Max Average Sustained. Release Rate: 29.3 kilograms/min

(averaged over a minute or more)

Total Amount Released: 1,621 kilograms

TIME DEPENDENT INFORMATION:

Concentration Estimates at the point:

Downwind: 100 meters

Off Centerline: 0 meters

Max Concentration:

Outdoor: $71.3 \mathrm{ppm}$

Indoor: $71.3 \mathrm{ppm}$

Note: Indoor graph is shown with a dotted line. 
Title of Project EPHA for THM

Subject Concentration Analysis for THM Hazardous Chemicals

Computer T. M. Franey

\section{SITE DATA INFORMATION: 1-RD-52}

Location: AIKEN, SOUTH CAROLINA

Building Air Exchanges Per Hour: 60 (User specified)

Date and Time: Fixed at July 1', 19961300 hours

\section{CHEMICAL INFORMATION:}

Chemical Name: VINYL ACETATE

Molecular Weight: $86.09 \mathrm{~kg} / \mathrm{kmol}$

TLV-TWA: 10.00 ppm IDLH: -unavail-

Note: Potential or confirmed human carcinogen.

Footprint Level of Concern: 75 ppm

Boiling Point: $72.50^{\circ} \mathrm{C}$

Vapor Pressure at Ambient Temperature: $0.15 \mathrm{~atm}$

Ambient Saturation Concentration: 153,288 ppm or $15.3 \%$

ATMOSPHERIC INFORMATION: (MANUAL INPUT OF DATA)

Wind: 2.5 meters $/ \mathrm{sec}$ from $90^{\circ}$ true

Inversion Height: 500 meters

Stability Class: C Air Temperature: $25^{\circ} \mathrm{C}$

Relative Humidity: 50\% Ground Roughness: 100 centimeters

Cloud Cover: 7 tenths

\section{SOURCE STRENGTH INFORMATION:}

Puddle Area: 24420 square feet

Average Puddle Depth: 1 centimeters

Soil Type: Default $\quad$ Ground Temperature: $25^{\circ} \mathrm{C}$

Initial Puddle Temperature: Ground temperature

Release Duration: ALOHA limited the duration to 1 hour

Max Computed Release Rate: 333 kilograms/min

Max Average Sustained Release Rate: 306 kilograms/min (averaged over a minute or more)

Total Amount Released: 17,191 kilograms

\section{FOOTPRINT INFORMATION:}

Model Run: Heavy Gas

User specified LOC: $75 \mathrm{ppm}$

Max Threat Zone for LOC: 786 meters

Note: The Heavy Gas footprint is an initial screening.

For short releases it may be an overestimation.

Be sure to check concentration information at specific locations.

\section{TIME DEPENDENT INFORMATION:}

Concentration Estimates at the point:

Downwind: 100 meters

Off Centerline: 0 meters

Max Concentration:

Outdoor: $1,780 \mathrm{ppm}$

Indoor: $1,780 \mathrm{ppm}$

Note: Indoor graph is shown with a dotted line. 


\section{ENGINEERING COMPUTATION SHEET}

Title of Project EPHA for THM

Reviewed by S. A. Henderson

Subject Concentration Analysis for THM Hazardous Chemicals

Computer T. M. Franey

Date $\underline{09 / 16 / 96}$

SITE DATA INFORMATION: 1-RD-52

Location: AIKEN, SOUTH CAROLINA

Building Air Exchanges Per Hour: 60 (User specified)

Date and Time: Fixed at July 1, 19961300 hours

CHEMICAL INFORMATION:

Chemical Name: VINYL ACETATE

Molecular Weight: $86.09 \mathrm{~kg} / \mathrm{kmol}$

TLV-TWA: $10.00 \mathrm{ppm}$ IDLH: -unavail-

Note: Potential or confirmed human carcinogen.

Footprint Level of Concern: 75 ppm

Boiling Point: $72.50^{\circ} \mathrm{C}$

Vapor Pressure at Ambient Temperature: $0.15 \mathrm{~atm}$

Ambient Saturation Concentration: 153,288 ppm or $15.3 \%$

ATMOSPHERIC INFORMATION: (MANUAL INPUT OF DATA)

Wind: 2.5 meters $/ \mathrm{sec}$ from $90^{\circ}$ true

Inversion Height: 500 meters

Stability Class: C Air Temperature: $25^{\circ} \mathrm{C}$

Relative Humidity: $50 \%$ Ground Roughness: 100 centimeters

Cloud Cover: 7 tenths

SOURCE STRENGTH INFORMATION:

Puddle Area: 40700 square feet

Average Puddle Depth: 1 centimeters

Soil Type: Default Ground Temperature: $25^{\circ} \mathrm{C}$

Initial Puddle Temperature: Ground temperature

Release Duration: ALOHA limited the duration to 1 hour

Max Computed Release Rate: 543 kilograms/min

Max Average Sustained Release Rate: 502 kilograms/min

(averaged over a minute or more)

Total Amount Released: 28,222 kilograms

FOOTPRINT INFORMATION:

Model Run: Heavy Gas

User specified LOC: 75 ppm

Max Threat Zone for LOC: 1.0 kilometers

Note: The Heavy Gas footprint is an initial screening.

For short releases it may be an overestimation.

Be sure to check concentration information at specific locations.

TIME DEPENDENT INFORMATION:

Concentration Estimates at the point:

Downwind: 100 meters

Off Centerline: 0 meters

Max Concentration:

Outdoor: $2,630 \mathrm{ppm}$

Indoor: $2,630 \mathrm{ppm}$

Note: Indoor graph is shown with a dotted line.
Works Calculation 7

Sheet No. $\underline{548 \text { of } 577}$ 


\section{ENGINEERING COMPUTATION SHEET}

Title of Project EPHA for THM

Subject Concentration Analysis for THM Hazardous Chemicals

Computer T. M. Franey

Date 09/16/96

SITE DATA INFORMATION: 1-RD-52

Location: AIKEN, SOUTH CAROLINA

Building Air Exchanges Per Hour: 60 (User specified)

Date and Time: Fixed at July 1, 19961300 hours

\section{CHEMICAL INFORMATION:}

Chemical Name: VINYL ACETATE

Molecular Weight: $86.09 \mathrm{~kg} / \mathrm{kmol}$

TLV-TWA: $10.00 \mathrm{ppm}$ IDLH: -unavail-

Note: Potential or confirmed human carcinogen.

Footprint Level of Concern: 75 ppm

Boiling Point: $72.50^{\circ} \mathrm{C}$

Vapor Pressure at Ambient Temperature: 0.15 atm

Ambient Saturation Concentration: 153,288 ppm or $15.3 \%$

ATMOSPHERIC INFORMATION: (MANUAL INPUT OF DATA)

Wind: 2.5 meters $/ \mathrm{sec}$ from $90^{\circ}$ true

Inversion Height: 500 meters

Stability Class: C Air Temperature: $25^{\circ} \mathrm{C}$

Relative Humidity: 50\% Ground Roughness: 100 centimeters

Cloud Cover: 7 tenths

\section{SOURCE STRENGTH INFORMATION:}

Puddle Area: 10000 square meters

Average Puddle Depth: 1 centimeters

Soil Type: Default Ground Temperature: $25^{\circ} \mathrm{C}$

Initial Puddle Temperature: Ground temperature

Release Duration: ALOHA limited the duration to 1 hour

Max Computed Release Rate: 1,380 kilograms/min

Max Average Sustained Release Rate: 1,280 kilograms/min

(averaged over a minute or more)

Total Amount Released: 72,530 kilograms

\section{FOOTPRINT INFORMATION:}

Model Run: Heavy Gas

User specified LOC: $75 \mathrm{ppm}$

Max Threat Zone for LOC: 1.8 kilometers

Note: The Heavy Gas footprint is an initial screening.

For short releases it may be an overestimation.

Be sure to check concentration information at specific locations.

TIME DEPENDENT INFORMATION:

Concentration Estimates at the point:

Downwind: 100 meters

Off Centerline: 0 meters

Max Concentration:

Outdoor: 5,460 ppm

Indoor: $5,460 \mathrm{ppm}$

Note: Indoor graph is shown with a dotted line.
Reviewed by S. A. Henderson

Works Calculation 7

Sheet No. $\underline{549 \text { of } 577}$ 


\section{ENGINEERING COMPUTATION SHEET}

Title of Project EPHA for THM

Reviewed by $\mathrm{S}$. A. Henderson

Works Calculation 7

Sheet No. $\underline{50 \text { of } 577}$

Subject Concentration Analysis for THM Hazardous Chemicals

Computer T. M. Franey

SITE DATA INFORMATION: 1-RD-53
Location: AIKEN, SOUTH CAROLINA

Building Air Exchanges Per Hour: 60 (User specified)

Date and Time: Fixed at July 1, 19961300 hours

CHEMICAL INFORMATION:

Chemical Name: O-XYLENE Molecular Weight: $106.17 \mathrm{~kg} / \mathrm{kmol}$

TLV-TWA: $100.00 \mathrm{ppm}$ IDLH: $1000.00 \mathrm{ppm}$

Footprint Level of Concern: 200 ppm

Boiling Point: $144.43^{\circ} \mathrm{C}$

Vapor Pressure at Ambient Temperature: $0.0087 \mathrm{~atm}$

Ambient Saturation Concentration: $8,878 \mathrm{ppm}$ or $0.89 \%$

ATMOSPHERIC INFORMATION: (MANUAL INPUT OF DATA)

Wind: 2.5 meters $/ \mathrm{sec}$ from $90^{\circ}$ true

Inversion Height: 500 meters

Stability Class: C Air Temperature: $25^{\circ} \mathrm{C}$

Relative Humidity: 50\% Ground Roughness: 100 centimeters

Cloud Cover: 7 tenths

SOURCE STRENGTH INFORMATION:

Puddle Area: 30300 square feet

Average Puddle Depth: 1 centimeters

Soil Type: Default Ground Temperature: $25^{\circ} \mathrm{C}$

Initial Puddle Temperature: Ground temperature

Release Duration: ALOHA limited the duration to 1 hour

Max Computed Release Rate: 40.8 kilograms/min

Max Average Sustained Release Rate: 40.4 kilograms/min

(averaged over a minute or more)

Total Amount Released: 2,166 kilograms

\section{TIME DEPENDENT INFORMATION:}

Concentration Estimates at the point:

Downwind: 30 meters

Off Centerline: 0 meters

Max Concentration:

Outdoor: 200 ppm

Indoor: $200 \mathrm{ppm}$

Note: Indoor graph is shown with a dotted line. 
Title of Project EPHA for THM

Subject Concentration Analysis for THM Hazardous Chemicals

Computer T. M. Franey
Reviewed by $\mathrm{S}$. A. Henderson

Works Calculation 7

Sheet No. $\underline{551 \text { of } 577}$

\section{SITE DATA INFORMATION: 1-RD-53}

Location: AIKEN, SOUTH CAROLINA

Building Air Exchanges Per Hour: 60 (User specified)

Date and Time: Fixed at July 1, 19961300 hours

\section{CHEMICAL INFORMATION:}

Chemical Name: O-XYLENE Molecular Weight: $106.17 \mathrm{~kg} / \mathrm{kmol}$ TLV-TWA: $100.00 \mathrm{ppm}$ IDLH: $1000.00 \mathrm{ppm}$

Footprint Level of Concern: 200 ppm

Boiling Point: $144.43^{\circ} \mathrm{C}$

Vapor Pressure at Ambient Temperature: $0.0087 \mathrm{~atm}$

Ambient Saturation Concentration: $8,878 \mathrm{ppm}$ or $0.89 \%$

\section{ATMOSPHERIC INFORMATION: (MANUAL INPUT OF DATA)}

Wind: 2.5 meters $/ \mathrm{sec}$ from $90^{\circ}$ true

Inversion Height: 500 meters

Stability Class: C Air Temperature: $25^{\circ} \mathrm{C}$

Relative Humidity: 50\% Ground Roughness: 100 centimeters

Cloud Cover: 7 tenths

\section{SOURCE STRENGTH INFORMATION:}

Puddle Area: 99999 square feet

Average Puddle Depth: 1 centimeters

Soil Type: Default . Ground Temperature: $25^{\circ} \mathrm{C}$

Initial Puddle Temperature: Ground temperature

Release Duration: ALOHA limited the duration to 1 hour

Max Computed Release Rate: 129 kilograms/min

Max Average Sustained Release Rate: 128 kilograms/min (averaged over a minute or more)

Total Amount Released: 6,832 kilograms

\section{TIME DEPENDENT INFORMATION:}

Concentration Estimates at the point:

Downwind: 100 meters

Off Centerline: 0 meters

Max Concentration:

Outdoor: $62.3 \mathrm{ppm}$

Indoor: $62.3 \mathrm{ppm}$

Note: Indoor graph is shown with a dotted line. 
Title of Project EPHA for THM

Subject Concentration Analysis for THM Hazardous Chemicals

Computer T. M. Franey

Date 09/16/96

Reviewed by $\underline{\text { S. A. Henderson }}$

Works Calculation 7

Sheet No. $\underline{52 \text { of } 577}$

SITE DATA INFORMATION: 1-RD-53

Location: AIKEN, SOUTH CAROLINA

Building Air Exchanges Per Hour: 60 (User specified)

Date and Time: Fixed at July 1, 19961300 hours

CHEMICAL INFORMATION:

Chemical Name: O-XYLENE Molecular Weight: $106.17 \mathrm{~kg} / \mathrm{kmol}$

TLV-TWA: $100.00 \mathrm{ppm}$ IDLH: $1000.00 \mathrm{ppm}$

Footprint Level of Concern: 200 ppm

Boiling Point: $144.43^{\circ} \mathrm{C}$

Vapor Pressure at Ambient Temperature: 0.0087 atm

Ambient Saturation Concentration: 8,878 ppm or $0.89 \%$

ATMOSPHERIC INFORMATION: (MANUAL INPUT OF DATA)

Wind: 2.5 meters $/ \mathrm{sec}$ from $90^{\circ}$ true

Inversion Height: 500 meters

Stability Class: C Air Temperature: $25^{\circ} \mathrm{C}$

Relative Humidity: 50\% Ground Roughness: 100 centimeters

Cloud Cover: 7 tenths

\section{SOURCE STRENGTH INFORMATION:}

Puddle Area: 24420 square feet

Average Puddle Depth: 1 centimeters

Soil Type: Default Ground Temperature: $25^{\circ} \mathrm{C}$

Initial Puddle Temperature: Ground temperature

Release Duration: ALOHA limited the duration to 1 hour

Max Computed Release Rate: 33.1 kilograms/min

Max Average Sustained Release Rate: 32.9 kilograms/min

(averaged over a minute or more)

Total Amount Released: 1,760 kilograms

\section{FOOTPRINT INFORMATION:}

Dispersion Module: Gaussian

User specified LOC: $200 \mathrm{ppm}$

Max Threat Zone for LOC: 27 meters

Max Threat Zone for IDLH: 27 meters

Note: Footprint was not drawn because effects of near-field patchiness make plume presentation unreliable for short distances.

\section{TIME DEPENDENT INFORMATION:}

Concentration Estimates at the point:

Downwind: 100 meters

Off Centerline: 0 meters

Max Concentration:

Outdoor: $29.9 \mathrm{ppm}$

Indoor: $29.9 \mathrm{ppm}$

Note: Indoor graph is shown with a dotted line. 


\section{ENGINEERING COMPUTATION SHEET}

Title of Project EPHA for THM

Reviewed by $\underline{\text { S. A. Henderson }}$

Subject Concentration Analysis for THM Hazardous Chemicals

Computer T. M. Franey

Date $\underline{09 / 16 / 96}$

SITE DATA INFORMATION: 1-RD-53.

Location: AIKEN, SOUTH CAROLINA

Building Air Exchanges Per Hour: 60 (User specified)

Date and Time: Fixed at July 1, 19961300 hours

\section{CHEMICAL INFORMATION:}

Chemical Name: O-XYLENE Molecular Weight: $106.17 \mathrm{~kg} / \mathrm{kmol}$ TLV-TWA: $100.00 \mathrm{ppm} \quad$ IDLH: $1000.00 \mathrm{ppm}$

Footprint Level of Concern: 200 ppm

Boiling Point: $144.43^{\circ} \mathrm{C}$

Vapor Pressure at Ambient Temperature: $0.0087 \mathrm{~atm}$

Ambient Saturation Concentration: 8,878 ppm or $0.89 \%$

ATMOSPHERIC INFORMATION: (MANUAL INPUT OF DATA)

Wind: 2.5 meters $/ \mathrm{sec}$ from $90^{\circ}$ true

Inversion Height: 500 meters

Stability Class: C Air Temperature: $25^{\circ} \mathrm{C}$

Relative Humidity: 50\% Ground Roughness: 100 centimeters

Cloud Cover: 7 tenths

\section{SOURCE STRENGTH INFORMATION:}

Puddle Area: 40700 square feet

Average Puddle Depth: 1 centimeters

Soil Type: Default Ground Temperature: $25^{\circ} \mathrm{C}$

Initial Puddle Temperature: Ground temperature

Release Duration: ALOHA limited the duration to 1 hour

Max Computed Release Rate: 54.2 kilograms/min

Max Average Sustained Release Rate: 53.7 kilograms/min

(averaged over a minute or more)

Total Amount Released: 2,876 kilograms

\section{FOOTPRINT INFORMATION:}

Dispersion Module: Gaussian

User specified LOC: 200 ppm

Max Threat Zone for LOC: 34 meters

Max Threat Zone for IDLH: 35 meters

Note: Footprint was not drawn because effects of near-field patchiness make plume presentation unreliable for short distances. 
Title of Project EPHA for THM

Subject Concentration Analysis for THM Hazardous Chemicals

Computer T. M. Franey

Date 09/16/96
Reviewed by $\mathrm{S}$. A. Henderson

Works Calculation 7

Sheet No. $\underline{54 \text { of } 577}$

\section{SITE DATA INFORMATION:}

Location: AIKEN, SOUTH CAROLINA

Building Air Exchanges Per Hour: 60 (User specified)

Date and Time: Fixed at July 1, 19961300 hours

\section{CHEMICAL INFORMATION:}

Chemical Name: O-XYLENE Molecular Weight: $106.17 \mathrm{~kg} / \mathrm{kmol}$

TLV-TWA: $100.00 \mathrm{ppm}$ IDLH: $1000.00 \mathrm{ppm}$

Footprint Level of Concern: 200 ppm

Boiling Point: $144.43^{\circ} \mathrm{C}$

Vapor Pressure at Ambient Temperature: $0.0087 \mathrm{~atm}$

Ambient Saturation Concentration: 8,878 ppm or $0.89 \%$

ATMOSPHERIC INFORMATION: (MANUAL INPUT OF DATA)

Wind: 2.5 meters $/ \mathrm{sec}$ from $90^{\circ}$ true

Inversion Height: 500 meters

Stability Class: C Air Temperature: $25^{\circ} \mathrm{C}$

Relative Humidity: $50 \%$ Ground Roughness: 100 centimeters

Cloud Cover: 7 tenths

SOURCE STRENGTH INFORMATION:

Puddle Area: 10000 square meters

Average Puddle Depth: 1 centimeters

Soil Type: Default Ground Temperature: $25^{\circ} \mathrm{C}$

Initial Puddle Temperature: Ground temperature

Release Duration: ALOHA limited the duration to 1 hour

Max Computed Release Rate: 138 kilograms/min

Max Average Sustained Release Rate: 137 kilograms/min (averaged over a minute or more)

Total Amount Released: 7,334 kilograms

\section{FOOTPRINT INFORMATION:}

Dispersion Module: Gaussian

User specified LOC: $200 \mathrm{ppm}$

Max Threat Zone for LOC: 56 meters

Max Threat Zone for IDLH: 56 meters

Note: Footprint was not drawn because effects of near-field patchiness make plume presentation unreliable for short distances. 
Title of Project EPHA for THM

Subject Concentration Analysis for THM Hazardous Chemicals

Computer T. M. Franey Date $\underline{09 / 16 / 96}$
Reviewed by $\underline{S}$. A. Henderson

Works Calculation 7 Sheet No. $\underline{55}$ of 577
SITE DATA INFORMATION: 1-RD-54 - 1-RD-58

Location: AIKEN, SOUTH CAROLINA

Building Air Exchanges Per Hour: 60 (User specified)

Date and Time: Fixed at July 1, 19961300 hours

\section{CHEMICAL INFORMATION:}

Chemical Name: RESPIRABLE DUST

Molecular Weight: $80.00 \mathrm{~kg} / \mathrm{kmol}$

TLV-TWA: -unavail- IDLH: -unavail-

Footprint Level of Concern: 50 ppm

Boiling Point: -unavail-

Note: Not enough chemical data to use Heavy Gas option

ATMOSPHERIC INFORMATION: (MANUAL INPUT OF DATA)

Wind: 2.5 meters $/ \mathrm{sec}$ from $90^{\circ}$ true

Inversion Height: 500 meters

Stability Class: C Air Temperature: $25^{\circ} \mathrm{C}$

Relative Humidity: 50\% Ground Roughness: 100 centimeters

Cloud Cover: 7 tenths

\section{SOURCE STRENGTH INFORMATION:}

Direct Source: 3.4 pounds Source Height: 0

Release Duration: 1 minute

Release Rate: 25.7 grams/sec

Total Amount Released: 1.54 kilograms

FOOTPRINT INFORMATION:

Dispersion Module: Gaussian

User specified LOC: $50 \mathrm{ppm}$

Max Threat Zone for LOC: 30 meters

Note: Footprint was not drawn because effects of near-field patchiness make plume presentation unreliable for short distances.

TIME DEPENDENT INFORMATION:

Concentration Estimates at the point:

Downwind: 30 meters

Off Centerline: 0 meters

Max Concentration:

Outdoor: $50.6 \mathrm{ppm}$

Indoor: $30.5 \mathrm{ppm}$

Note: Indoor graph is shown with a dotted line. 
Title of Project EPHA for THM

Subject Concentration Analysis for THM Hazardous Chemicals

Computer T. M. Franey

Date $\underline{09 / 16 / 96}$

SITE DATA INFORMATION: 1-RD-54 - 1-RD-58

Location: AIKEN, SOUTH CAROLINA

Building Air Exchanges Per Hour: 60 (User specified)

Date and Time: Fixed at July 1, 19961300 hours

\section{CHEMICAL INFORMATION:}

Chemical Name: RESPIRABLE DUST

Molecular Weight: $80.00 \mathrm{~kg} / \mathrm{kmol}$

TLV-TWA: -unavail- IDLH: -unavail-

Footprint Level of Concern: 50 ppm

Boiling Point: -unavail-

Note: Not enough chemical data to use Heavy Gas option

\author{
ATMOSPHERIC INFORMATION: (MANUAL INPUT OF DATA) \\ Wind: 2.5 meters $/ \mathrm{sec}$ from $90^{\circ}$ true \\ Inversion Height: 500 meters \\ Stability Class: C Air Temperature: $25^{\circ} \mathrm{C}$ \\ Relative Humidity: 50\% Ground Roughness: 100 centimeters \\ Cloud Cover: 7 tenths
}

\section{SOURCE STRENGTH INFORMATION:}

Direct Source: 37.2 pounds

Source Height: 0

Release Duration: 1 minute

Release Rate: 281 grams/sec

Total Amount Released: 16.9 kilograms

FOOTPRINT INFORMATION:

Dispersion Module: Gaussian

User specified LOC: $50 \mathrm{ppm}$

Max Threat Zone for LOC: 100 meters

Note: Footprint was not drawn because effects of near-field patchiness make plume presentation unreliable for short distances.

TIME DEPENDENT INFORMATION:

Concentration Estimates at the point:

Downwind: 100 meters

Off Centerline: 0 meters

Max Concentration:

Outdoor: $50 \mathrm{ppm}$

Indoor: $29.7 \mathrm{ppm}$

Note: Indoor graph is shown with a dotted line.
Reviewed by $\mathrm{S}$. A. Henderson

Works Calculation 7

Sheet No. $\underline{56 \text { of } 577}$ 
Title of Project EPHA for THM

Subject Concentration Analysis for THM Hazardous Chemicals

Computer T. M. Franey Date 09/16/96
Reviewed by $\mathrm{S}$. A. Henderson

Works Calculation 7

Sheet No. $\underline{57 \text { of } 577}$
SITE DATA INFORMATION: 1-RD-54 - 1-RD-58
Location: AIKEN, SOUTH CAROLINA
Building Air Exchanges Per Hour: 60 (User specified)
Date and Time: Fixed at July 1, 19961300 hours
CHEMICAL INFORMATION:
Chemical Name: RESPIRABLE DUST
Molecular Weight: $80.00 \mathrm{~kg} / \mathrm{kmol}$
TLV-TWA: -unavail- IDLH: -unavail-
Footprint Level of Concern: 50 ppm
Boiling Point: -unavail-
Note: Not enough chemical data to use Heavy Gas option

ATMOSPHERIC INFORMATION: (MANUAL INPUT OF DATA)

Wind: 2.5 meters $/ \mathrm{sec}$ from $90^{\circ}$ true

Inversion Height: 500 meters

Stability Class: C Air Temperature: $25^{\circ} \mathrm{C}$

Relative Humidity: 50\% Ground Roughness: 100 centimeters

Cloud Cover: 7 tenths

\section{SOURCE STRENGTH INFORMATION:}

Direct Source: 37.6 pounds

Source Height: 0

Release Duration: 1 minute

Release Rate: 284 grams/sec

Total Amount Released: 17.1 kilograms

FOOTPRINT INFORMATION:

Dispersion Module: Gaussian

User specified LOC: $50 \mathrm{ppm}$

Max Threat Zóne for LOC: 100 meters

TIME DEPENDENT INFORMATION:

Concentration Estimates at the point:

Downwind: 100 meters

Off Centerline: 0 meters

Max Concentration:

Outdoor: $50.6 \mathrm{ppm}$

Indoor: $30 \mathrm{ppm}$

Note: Indoor graph is shown with a dotted line. 


\section{ENGINEERING COMPUTATION SHEET}

Title of Project EPHA for THM

Subject Concentration Analysis for THM Hazardous Chemicals

Computer T. M. Franey

Date 09/16/96

SITE DATA INFORMATION: 1-RD-54 - 1-RD-58

Location: AIKEN, SOUTH CAROLINA

Building Air Exchanges Per Hour: 60 (User specified)

Date and Time: Fixed at July 1, 19961300 hours

CHEMICAL INFORMATION:

Chemical Name: RESPIRABLE DUST

Molecular Weight: $80.00 \mathrm{~kg} / \mathrm{kmol}$

TLV-TWA: -unavail- ' IDLH: -unavail-

Footprint Level of Concern: $50 \mathrm{ppm}$

Boiling Point: -unavail-

Note: Not enough chemical data to use Heavy Gas option

ATMOSPHERIC INFORMATION: (MANUAL INPUT OF DATA)

Wind: 2.5 meters $/ \mathrm{sec}$ from $90^{\circ}$ true

Inversion Height: 500 meters

Stability Class: C Air Temperature: $25^{\circ} \mathrm{C}$

Relative Humidity: 50\% Ground Roughness: 100 centimeters

Cloud Cover: 7 tenths

SOURCE STRENGTH INFORMATION:

Direct Source: 135 pounds Source Height: 0

Release Duration: 1 minute

Release Rate: 1.02 kilograms/sec

Total Amount Released: 61.2 kilograms

FOOTPRINT INFORMATION:

Dispersion Module: Gaussian

User specified LOC: $50 \mathrm{ppm}$

Max Threat Zone for LOC: 191 meters

TIME DEPENDENT INFORMATION:

Concentration Estimates at the point:

Downwind: 100 meters

Off Centerline: 0 meters

Max Concentration:

Outdoor: $182 \mathrm{ppm}$

Indoor: $108 \mathrm{ppm}$

Note: Indoor graph is shown with a dotted line.
Reviewed by $\underline{\text { S. A. Henderson }}$

Works Calculation 7

Sheet No. $\underline{58 \text { of } 577}$ 


\section{ENGINEERING COMPUTATION SHEET}

Title of Project EPHA for THM

Subject Concentration Analysis for THM Hazardous Chemicals

Computer T. M. Franey Date 09/16/96

SITE DATA INFORMATION: 1-RD-54 - 1-RD-58

Location: AIKEN, SOUTH CAROLINA

Building Air Exchanges Per Hour: 60 (User specified)

Date and Time: Fixed at July 1, 19961300 hours

CHEMICAL INFORMATION:

Chemical Name: RESPIRABLE DUST

Molecular Weight: $80.00 \mathrm{~kg} / \mathrm{kmol}$

TLV-TWA: -unavail- IDLH: -unavail-

Footprint Level of Concern: 50 ppm

Boiling Point: -unavail-

Note: Not enough chemical data to use Heavy Gas option

ATMOSPHERIC INFORMATION: (MANUAL INPUT OF DATA)

Wind: 2.5 meters $/ \mathrm{sec}$ from $90^{\circ}$ true

Inversion Height: 500 meters

Stability Class: C Air Temperature: $25^{\circ} \mathrm{C}$

Relative Humidity: $50 \%$ Ground Roughness: 100 centimeters

Cloud Cover: 7 tenths

SOURCE STRENGTH INFORMATION:

Direct Source: 263 pounds Source Height: 0

Release Duration: 1 minute

Release Rate: 1.99 kilograms/sec

Total Amount Released: 119 kilograms

FOOTPRINT INFORMATION:

Dispersion Module: Gaussian

User specified LOC: $50 \mathrm{ppm}$

Max Threat Zone for LOC: 267 meters

TIME DEPENDENT INFORMATION:

Concentration Estimates at the point:

Downwind: 100 meters

Off Centerline: 0 meters

Max Concentration:

- Outdoor: 354 ppm

Indoor: $210 \mathrm{ppm}$

Note: Indoor graph is shown with a dotted line.
Reviewed by S. A. Henderson

Works Calculation 7

Sheet No. $\underline{599 \text { of } 577}$ 


\section{ENGINEERING COMPUTATION SHEET}

Title of Project EPHA for THM

Subject Concentration Analysis for THM Hazardous Chemicals

Computer T. M. Franey
Reviewed by $\underline{\text { S. A. Henderson }}$

Works Calculation 7

Sheet No. $\underline{500 \text { of } 577}$

\section{1-RD-59}

SITE DATA INFORMATION:

Location: AIKEN, SOUTH CAROLINA

Building Air Exchanges Per Hour: 60 (User specified)

Date and Time: Fixed at July 1, 19961300 hours

CHEMICAL INFORMATION:

Chemical Name: AMMONIA, ANHYDROUS

Molecular Weight: $17.03 \mathrm{~kg} / \mathrm{kmol}$

TLV-TWA: $25.00 \mathrm{ppm}$ IDLH: $500.00 \mathrm{ppm}$

Footprint Level of Concern: 200 ppm

Boiling Point: $-33.43^{\circ} \mathrm{C}$

Vapor Pressure at Ambient Temperature: greater than 1 atm

Ambient Saturation Concentration: $1,000,000 \mathrm{ppm}$ or $100.0 \%$

ATMOSPHERIC INFORMATION: (MANUAL INPUT OF DATA)

Wind: 2.5 meters $/ \mathrm{sec}$ from $90^{\circ}$ true

Inversion Height: 500 meters

Stability Class: C Air Temperature: $25^{\circ} \mathrm{C}$

Relative Humidity: 50\% Ground Roughness: Urban or forest

Cloud Cover: 7 tenths

\section{SOURCE STRENGTH INFORMATION:}

Puddle Area: 34 square feet

Average Puddle Depth: 1 centimeters

Soil Type: Default Ground Temperature: $25^{\circ} \mathrm{C}$

Initial Puddle Temperature: boiling point

Release Duration: 31 minutes

Max Computed Release Rate: 12.4 kilograms/min

Max Average Sustained Release Rate: 1.35 kilograms/min (averaged over a minute or more)

Total-Amount Released: 21.5 kilograms

TIME DEPENDENT INFORMATION:

Concentration Estimates at the point:

Downwind: 30 meters

Off Centerline: 0 meters

Max Concentration:

Outdoor: 203 ppm 


\section{ENGINEERING COMPUTATION SHEET}

Title of Project EPHA for THM

Subject Concentration Analysis for THM Hazardous Chemicals

Computer T. M. Franey

Date $\underline{09 / 16 / 96}$
Reviewed by S. A. Henderson

Works Calculation 7

Sheet No. $\underline{561 \text { of } 577}$

\section{1-RD-59}

SITE DATA INFORMATION:

Location: AIKEN, SOUTH CAROLINA

Building Air Exchanges Per Hour: 60 (User specified)

Date and Time: Fixed at July 1, 19961300 hours

\section{CHEMICAL INFORMATION:}

Chemical Name: AMMONIA, ANHYDROUS

Molecular Weight: $17.03 \mathrm{~kg} / \mathrm{kmol}$

TLV-TWA: $25.00 \mathrm{ppm}$

IDLH: $500.00 \mathrm{ppm}$

Footprint Level of Concern: 200 ppm

Boiling Point: $-33.43^{\circ} \mathrm{C}$

Vapor Pressure at Ambient Temperature: greater than $1 \mathrm{~atm}$

Ambient Saturation Concentration: $1,000,000$ ppm or $100.0 \%$

ATMOSPHERIC INFORMATION: (MANUAL INPUT OF DATA)

Wind: 2.5 meters $/ \mathrm{sec}$ from $90^{\circ}$ true

Inversion Height: 500 meters

Stability Class: C Air Temperature: $25^{\circ} \mathrm{C}$

Relative Humidity: $50 \%$ Ground Roughness: Urban or forest

Cloud Cover: 7 tenths

\section{SOURCE STRENGTH INFORMATION:}

Puddle Area: 400 square feet

Average Puddle Depth: 1 centimeters

Soil Type: Default . Ground Temperature: $25^{\circ} \mathrm{C}$

Initial Puddle Temperature: boiling point

Release Duration: 34 minutes

Max Computed Release Rate: 133 kilograms/min

Max Average Sustained Release Rate: 14.9 kilograms/min

(averaged over a minute or more)

Total Amount Released: 253 kilograms

\section{TIME DEPENDENT INFORMATION:}

Concentration Estimates at the point:

Downwind: 100 meters

Off Centerline: 0 meters

Max Concentration:

Outdoor: 202 ppm 
Title of Project EPHA for THM

Subject Concentration Analysis for THM Hazardous Chemicals

Computer T. M. Franey

Date $\underline{09 / 16 / 96}$

1-RD-59

SITE DATA INFORMATION:

Location: AIKEN, SOUTH CAROLINA

Building Air Exchanges Per Hour: 60 (User specified)

Date and Time: Fixed at July 1, 19961300 hours

\section{CHEMICAL INFORMATION:}

Chemical Name: AMMONIA, ANHYDROUS

Molecular Weight: $17.03 \mathrm{~kg} / \mathrm{kmol}$

TLV-TWA: $25.00 \mathrm{ppm}$ IDLH: $500.00 \mathrm{ppm}$

Footprint Level of Concern: 200 ppm

Boiling Point: $-33.43^{\circ} \mathrm{C}$

Vapor Pressure at Ambient Temperature: greater than 1 atm

Ambient Saturation Concentration: $1,000,000$ ppm or $100.0 \%$

\section{ATMOSPHERIC INFORMATION: (MANUAL INPUT OF DATA)}

Wind: 2.5 meters $/ \mathrm{sec}$ from $90^{\circ}$ true

Inversion Height: 500 meters

Stability Class: C Air Temperature: $25^{\circ} \mathrm{C}$

Relative Humidity: 50\% Ground Roughness: Urban or forest

Cloud Cover: 7 tenths

\section{SOURCE STRENGTH INFORMATION:}

Puddle Area: 2000 square feet

Puddle Mass: 1 tons

Soil Type: Default Ground Temperature: $25^{\circ} \mathrm{C}$

Initial Puddle Temperature: boiling point

Release Duration: 23 minutes

Max Computed Release Rate: 745 kilograms/min

Max Average Sustained Release Rate: 87.4 kilograms/min (averaged over a minute or more)

Total Amount Released: 907 kilograms

FOOTPRINT INFORMATION:

Dispersion Module: Gaussian

User specified LOC: $200 \mathrm{ppm}$

Max Threat Zone for LOC: 245 meters

Max Threat Zone for IDLH: 152 meters

TIME DEPENDENT INFORMATION:

Concentration Estimates at the point:

Downwind: 100 meters

Off Centerline: 0 meters

Max Concentration:

Outdoor: $1,080 \mathrm{ppm}$
Reviewed by S. A. Henderson

Works Calculation 7

Sheet No. $\underline{562 \text { of } 577}$ 


\section{ENGINEERING COMPUTATION SHEET}

Title of Project EPHA for THM

Subject Concentration Analysis for THM Hazardous Chemicals

Computer T. M. Franey

Date $\underline{09 / 16 / 96}$
Reviewed by $\underline{\text { S. A. Henderson }}$

Works_Calculation 7

Sheet No. $\underline{53 \text { of } 577}$

1-RD-59

SITE DATA INFORMATION:

Location: AIKEN, SOUTH CAROLINA

Building Air Exchanges Per Hour: 60 (User specified)

Date and Time: Fixed at July 1, 19961300 hours

CHEMICAL INFORMATION:

Chemical Name: AMMONIA, ANHYDROUS

Molecular Weight: $17.03 \mathrm{~kg} / \mathrm{kmol}$

TLV-TWA: $25.00 \mathrm{ppm}$ IDLH: $500.00 \mathrm{ppm}$

Footprint Level of Concern: 200 ppm

Boiling Point: $-33.43^{\circ} \mathrm{C}$

Vapor Pressure at Ambient Temperature: greater than $1 \mathrm{~atm}$

Ambient Saturation Concentration: $1,000,000$ ppm or $100.0 \%$

ATMOSPHERIC INFORMATION: (MANUAL INPUT OF DATA)

Wind: 2.5 meters $/ \mathrm{sec}$ from $90^{\circ}$ true

Inversion Height: 500 meters

Stability Class: C Air Temperature: $25^{\circ} \mathrm{C}$

Relative Humidity: 50\% Ground Roughness: Urban or forest

Cloud Cover: 7 tenths

SOURCE STRENGTH INFORMATION:

Puddle Area: 20000 square feet

Puddle Mass: 10 tons

Soil Type: Default Ground Temperature: $25^{\circ} \mathrm{C}$

Initial Puddle Temperature: boiling point

Release Duration: 24 minutes

Max Computed Release Rate: 6,980 kilograms/min

Max Average Sustained Release Rate: 842 kilograms/min

(averaged over a minute or more)

Total Amount Released: 9,072 kilograms

FOOTPRINT INFORMATION:

Dispersion Module: Gaussian

User specified LOC: $200 \mathrm{ppm}$

Max Threat Zone for LOC: 767 meters

Max Threat Zone for IDLH: 475 meters

TIME DEPENDENT INFORMATION:

Concentration Estimates at the point:

Downwind: 100 meters

Off Centerline: 0 meters

Max Concentration:

Outdoor: 5,270 ppm 
Title of Project EPHA for THM

Subject Concentration Analysis for THM Hazardous Chemicals

Computer T.M. Franey
Reviewed by S. A. Henderson

Works Calculation 7

Sheet No. $\underline{564 \text { of } 577}$

\author{
1-RD-59 \\ SITE DATA INFORMATION: \\ Location: AIKEN, SOUTH CAROLINA \\ Building Air Exchanges Per Hour: 60 (User specified) \\ Date and Time: Fixed at July 1, 19961300 hours \\ CHEMICAL INFORMATION: \\ Chemical Name: AMMONIA, ANHYDROUS \\ Molecular Weight: $17.03 \mathrm{~kg} / \mathrm{kmol}$ \\ TLV-TWA: $25.00 \mathrm{ppm} \quad$ IDLH: $500.00 \mathrm{ppm}$ \\ Footprint Level of Concern: 200 ppm \\ Boiling Point: $-33.43^{\circ} \mathrm{C}$ \\ Vapor Pressure at Ambient Temperature: greater than 1 atm \\ Ambient Saturation Concentration: $1,000,000$ ppm or $100.0 \%$ \\ ATMOSPHERIC INFORMATION: (MANUAL INPUT OF DATA) \\ Wind: 2.5 meters/sec from $90^{\circ}$ true \\ Inversion Height: 500 meters \\ Stability Class: $\mathrm{C} \quad$ Air Temperature: $25^{\circ} \mathrm{C}$ \\ Relative Humidity: $50 \%$ Ground Roughness: Urban or forest \\ Cloud Cover: 7 tenths \\ SOURCE STRENGTH INFORMATION: \\ Puddle Area: 35000 square feet \\ Puddle Mass: 17.5 tons \\ Soil Type: Default Ground Temperature: $25^{\circ} \mathrm{C}$ \\ Initial Puddle Temperature: boiling point \\ Release Duration: 25 minutes \\ Max Computed Release Rate: 12,000 kilograms/min \\ Max Average Sustained Release Rate: 1,460 kilograms/min \\ (averaged over a minute or more) \\ Total Amount Released: 15,876 kilograms \\ FOOTPRINT INFORMATION: \\ Dispersion Module: Gaussian \\ User specified LOC: 200 ppm \\ Max Threat Zone for LOC: 997 meters \\ Max Threat Zone for IDLH: 628 meters \\ TIME DEPENDENT INFORMATION: \\ - Concentration Estimates at the point: \\ Downwind: 100 meters \\ Off Centerline: 0 meters \\ Max Concentration: \\ Outdoor: 6,940 ppm
}


OSR $\geq s-10$ (REV 6- $(8-42)$

\section{ENGINEERING COMPUTATION SHEET}

Title of Project EPHA for THM

Reviewed by S. A. Henderson

Subject Concentration Analysis for THM Hazardous Chemicals

Computer T. M. Franey

Date 09/16/96

1-RD-59

For analysis on 1 ton, see hard copy.
Works Calculation 7

Sheet No. $\underline{565 \text { of } 577}$ 


\section{ENGINEERING COMPUTATION SHEET}

Title of Project EPHA for THM

Reviewed by $\underline{\text { S. A. Henderson }}$

Subject Concentration Analysis for THM Hazardous Chemicals

Works Calculation 7

Computer T.M. Franey

Date $\underline{09 / 16 / 96}$

Sheet No. 566 of 577

1-RD-59

For analysis on 10 tons, see hard copy. 


\section{ENGINEERING COMPUTATION SHEET}

Title of Project EPHA for THM

Reviewed by S. A. Henderson

Subject Concentration Analysis for THM Hazardous Chemicals Works Calculation 7

Computer T. M. Franey

Date 09/16/96

1-RD-59

For analysis on 17.5 tons, see hard copy. 


\section{ENGINEERING COMPUTATION SHEET}

Title of Project EPHA for THM

Reviewed by S. A. Henderson

Subject Concentration Analysis for THM Hazardous Chemicals

Computer T. M. Franey
Works Calculation 7

Sheet No. $\underline{568 \text { of } 577}$

\section{1-RD-60}

SITE DATA INFORMATION:

Location: AIKEN, SOUTH CAROLINA

Building Air Exchanges Per Hour: 60 (User specified)

Date and Time: Fixed at July 1, 19961300 hours

CHEMICAL INFORMATION:

Chemical Name: CHLORINE Molecular Weight: $70.90 \mathrm{~kg} / \mathrm{kmol}$

TLV-TWA: $0.50 \mathrm{ppm}$

IDLH: $30.00 \mathrm{ppm}$

Footprint Level of Concern: 3 ppm

Boiling Point: $-34.03^{\circ} \mathrm{C}$

Vapor Pressure at Ambient Temperature: greater than $1 \mathrm{~atm}$

Ambient Saturation Concentration: $1,000,000 \mathrm{ppm}$ or $100.0 \%$

ATMOSPHERIC INFORMATION: (MANUAL INPUT OF DATA)

Wind: 2.5 meters $/ \mathrm{sec}$ from $90^{\circ}$ true

Inversion Height: 500 meters

Stability Class: C Air Temperature: $25^{\circ} \mathrm{C}$

Relative Humidity: 50\% Ground Roughness: Urban or forest

Cloud Cover: 7 tenths

SOURCE STRENGTH INFORMATION:

Puddle Area: 0.5 square feet

Average Puddle Depth: 1 centimeters

Soil Type: Default Ground Temperature: $25^{\circ} \mathrm{C}$

Initial Puddle Temperature: boiling point

Release Duration: 8 minutes

Max Computed Release Rate: 1.27 kilograms/min

Max Average Sustained Release Rate: 181 grams/min

(averaged over a minute or more)

Total Amount Released: 726 grams

TIME DEPENDENT INFORMATION:

Concentration Estimates at the point:

Downwind: 30 meters

Off Centerline: 0 meters

Max Concentration:

Outdoor: $6.71 \mathrm{ppm}$ 


\section{ENGINEERING COMPUTATION SHEET}

Title of Project EPHA for THM

Subject Concentration Analysis for THM Hazardous Chemicals

Computer T. M. Franey
Reviewed by S. A. Henderson

Works Calculation 7

Sheet No. $\underline{569 \text { of } 577}$

1-RD-60

SITE DATA INFORMATION:

Location: AIKEN, SOUTH CAROLINA

Building Air Exchanges Per Hour: 60 (User specified)

Date and Time: Fixed at July 1, 19961300 hours

CHEMICAL INFORMATION:

Chemical Name: CHLORINE Molecular Weight: $70.90 \mathrm{~kg} / \mathrm{kmol}$

TLV-TWA: $0.50 \mathrm{ppm}$ IDLH: $30.00 \mathrm{ppm}$

Footprint Level of Concern: 3 ppm

Boiling Point: $-34.03^{\circ} \mathrm{C}$

Vapor Pressure at Ambient Temperature: greater than 1 atm

Ambient Saturation Concentration: $1,000,000$ ppm or $100.0 \%$

ATMOSPHERIC INFORMATION: (MANUAL INPUT OF DATA)

Wind: 2.5 meters $/ \mathrm{sec}$ from $90^{\circ}$ true

Inversion Height: 500 meters

Stability Class: C Air Temperature: $25^{\circ} \mathrm{C}$

Relative Humidity: 50\% Ground Roughness: Urban or forest

Cloud Cover: 7 tenths

SOURCE STRENGTH INFORMATION:

Puddle Area: 3 square feet

Average Puddle Depth: 1 centimeters

Soil Type: Default Ground Temperature: $25^{\circ} \mathrm{C}$

Initial Puddle Temperature: boiling point

Release Duration: 9 minutes

Max Computed Release Rate: 7.09 kilograms/min

Max Average Sustained Release Rate: 1.03 kilograms/min

(averaged over a minute or more)

Total Amount Released: 4.35 kilograms

TIME DEPENDENT INFORMATION:

Concentration Estimates at the point:

Downwind: 100 meters

Off Centerline: 0 meters

Max Concentration:

Outdoor: $3.43 \mathrm{ppm}$ 
Title of Project EPHA for THM

Subject Concentration Analysis for THM Hazardous Chemicals

Computer T. M. Franey

Date $\underline{09 / 16 / 96}$

1-RD-60

For analysis on 1 ton, see hard copy.
Reviewed by $\mathrm{S}$. A. Henderson

SRS Works Calculation 7

Sheet No. $\underline{570 \text { of } 577}$ 
ENGINEERING COMPUTATION SHEET

Title of Project EPHA for THM

Reviewed by S. A. Henderson

Subject Concentration Analysis for THM Hazardous Chemicals

Computer T. M. Franey

Date 09/16/96
Works Calculation 7

Sheet No. $\underline{571 \text { of } 577}$

1-RD-60

For analysis on 10 tons, see hard copy. 


\section{ENGINEERING COMPUTATION SHEET}

Title of Project EPHA for THM

Subject Concentration Analysis for THM Hazardous Chemicals

Computer T. M. Franey

Date $\underline{09 / 16 / 96}$
Reviewed by S. A. Henderson

Works Calculation 7

Sheet No. $\underline{572 \text { of } 577}$

1-RD-60

For analysis on 17.5 tons, see hard copy. 
OSR 2S.10 (Rev 6-18-92)

\section{ENGINEERING COMPUTATION SHEET}

Title of Project EPHA for THM

Reviewed by $\underline{\text { S. A. Henderson }}$

Subject Concentration Analysis for THM Hazardous Chemicals

Works Calculation 7

Computer T. M. Franey

Date $\underline{09 / 16 / 96}$

Sheet No. $\underline{573 \text { of } 577}$

\section{1-RD-61}

For analysis on quantity for ERPG-2 at $30 \mathrm{~m}$, see hard copy. 


\section{ENGINEERING COMPUTATION SHEET}

Title of Project EPHA for THM

Reviewed by. S. A. Henderson

Subject Concentration Analysis for THM Hazardous Chemicals

Computer T. M. Franey

Date 09/16/96

1-RD-61

For analysis on quantity for ERPG-2 At $100 \mathrm{~m}$, see hard copy.
Works Calculation 7

Sheet No. $\underline{574 \text { of } 577}$ 


\section{ENGINEERING COMPUTATION SHEET}

Title of Project EPHA for THM

Reviewed by $\mathrm{S}$. A. Henderson

Subject Concentration Analysis for THM Hazardous Chemicals

Computer T. M. Franey

Date $\underline{09 / 16 / 96}$

1-RD-61

For analysis on 1 ton, see hard copy.
Works Calculation 7

\section{- Sheet No. $\underline{575 \text { of } 577}$}


Title of Project EPHA for THM

Subject Concentration Analysis for THM Hazardous Chemicals

Computer T. M. Franey

Date 09/16/96
Reviewed by S. A. Henderson

Works Calculation 7

Sheet No. $\underline{576 \text { of } 577}$

1-RD-61

For analysis on 10 tons, see hard copy. 
Title of Project EPHA for THM

Reviewed by $\underline{\text { S. A. Henderson }}$

Subject Concentration Analysis for THM Hazardous Chemicals

Computer T. M. Franey

Date 09/16/96
Works Calculation 7

Sheet No. $\underline{577 \text { of } 577}$

1-RD-61

For analysis on 17.5 tons, see hard copy. 
Title of Project EPHA for THM

Subject Concentration Analysis for THM Hazardous Chemicals

Computer N.E. Baucom B. K. Tharakan Date 09/16/96 INTRODUCTION
Reviewed by S. A. Henderson

Works Calculation 8

Sheet No. 1 of 314

Calculation 8 is for chemicals that underwent a full hazard analysis in calculation 7 and had ERPG values that have changed since Revision 0 of Transportation EPHA. This calculation estimates the vapor concentrations of chemicals requiring full hazards analysis for the transportation EPHA, at 30 and 100 meter receptor points and the maximum distance to ERPG-2 concentration for each of the THM EPHA segments for adverse and average meteorological conditions. Concentrations for ALOHA default distances for maximum segment source terms are also analyzed.

\section{ASSUMPTIONS}

Releases are ground level and immediately spread to $1-\mathrm{cm}$ depth. (Ref 1 )

SRS established $95 \%$ adverse meteorological conditions: (Ref 2)

E-stability, $1.7 \mathrm{~m} / \mathrm{s}$ wind, $200 \mathrm{~m}$ inversion and $29^{\circ} \mathrm{C}$ ambient temperature.

SRS established $50 \%$ average meteorological conditions: (Ref 2)

C-stability, $2.5 \mathrm{~m} / \mathrm{s}$ wind, $500 \mathrm{~m}$ inversion and $25^{\circ} \mathrm{C}$ ambient temperature.

Receptor points are at ground level. (Ref 2)

\section{CALCULATION}

The Areal Locations of Hazardous Atmospheres (ALOHA), dispersion model, Version 5.2.1s, (Ref. 3) is utilized to calculate and/or back calculate, as appropriate, concentration information for the following chemicals and associated ERPG-2 values (Ref. 4).

Table 1 Chemicals, Associated ERPG, and State

\begin{tabular}{|c|c|c|c|}
\hline $\begin{array}{l}\text { Chemical Name / } \\
\text { Molecular Weight }\end{array}$ & $\begin{array}{c}\text { ERPG-2 or } \\
\text { Equiv } \\
\text { ppm }\end{array}$ & $\begin{array}{l}\text { State } \\
\text { (S)olfd } \\
\text { (L)lquid } \\
\text { (G)as }\end{array}$ & $\begin{array}{c}\text { RELEASE } \\
\text { DESIGNATION }\end{array}$ \\
\hline Acetic Acid & 35 & L & 1-RD-2 \\
\hline Acetonitrile /41.1 & 60 & $\mathbf{L}$ & $1-R D-3$ \\
\hline Alpha-Pinene / 136.3 & $300\left(\mathrm{mg} / \mathrm{m}^{3}\right)$ & $\bar{L}$ & 1-RD-4 \\
\hline Benzyl Alcohol & 100 & $\mathbf{L}$ & 1-RD-7 \\
\hline Butyraldehyde & $500\left(\mathrm{mg} / \mathrm{m}^{3}\right)$ & $\mathbf{L}$ & 1-RD-9 \\
\hline Chloracetone /92.5 & 1 & $\mathbf{L}$ & 1-RD-11 \\
\hline Cyclohexanone / 98.2 & 125 & $\mathrm{~L}$ & 1-RD-14 \\
\hline Diethylene Glycol / 106.1 & 100 & L & 1-RD-15 \\
\hline Dioxane / 88.1 & 75 & $\mathbf{L}$ & 1-RD-16 \\
\hline Diphenylmethane-4,4-Diisocyanate & $2\left(\mathrm{mg} / \mathrm{m}^{3}\right)$ & $\mathbf{L}$ & 1-RD-17 \\
\hline Ethyl benzene / 106.2 & 125 & $\mathbf{L}$ & 1-RD-19 \\
\hline Ethylene Glycol Monobutyl Ether & 125 & L & 1-RD-20 \\
\hline Formic Acid / 46.0 & 10 & $\mathbf{L}$ & 1-RD-22 \\
\hline Diethylene Gycol Dimethyl Ether & $500\left(\mathrm{mg} / \mathrm{m}^{3}\right)$ & $\mathbf{L}$ & 1-RD-23 \\
\hline Hydrogen Peroxide / 34.0 & 50 & $\mathbf{L}$ & 1-RD-26 \\
\hline Methyl Ethyl Ketone /72.1 & 300 & $\mathbf{L}$ & 1-RD-29 \\
\hline Methyl Isobutyl Ketone / 100.2 & 500 & $\mathbf{L}$ & 1-RD-30 \\
\hline Ethylene Glycol Monomethyl Ether & 25 & $\mathbf{L}$ & 1-RD-32 \\
\hline
\end{tabular}




\section{ENGINEERING COMPUTATION SHEET}

Title of Project EPHA for THM

Subject Concentration Analysis for THM Hazardous Chemicals

Computer N.E. Baucom B. K. Tharakan Date 09/16/96

Table 1 cont. Chemicals, Associated ERPG, and State

\begin{tabular}{|c|c|c|c|}
\hline $\begin{array}{l}\text { Chemical Name / } \\
\text { Molecular Weight } \\
\text { - }\end{array}$ & $\begin{array}{c}\text { ERPG-2 or } \\
\text { Equiv } \\
\text { ppm }\end{array}$ & $\begin{array}{l}\text { State } \\
\text { (S)olid } \\
\text { (L)lquid } \\
\text { (G)es }\end{array}$ & $\begin{array}{c}\text { RELEASE } \\
\text { DESIGNATION }\end{array}$ \\
\hline Monothanolamine /61.1 & 30 & L & 1-RD-33 \\
\hline Morpholine / 87.1 & 100 & $\mathbf{L}$ & 1-RD-34 \\
\hline N Methyl Pyrrolidone /99.15 & 500 & $\mathrm{~L}$ & 1-RD-36 \\
\hline Propionic Acid / 74.1 & 15 & $\mathbf{L}$ & 1-RD-42 \\
\hline Tetrahydrofuran /72.1 & 2000 & L & 1-RD-46 \\
\hline Trichloroethane-1,1,1/133.4 & 700 & L & 1-RD-49 \\
\hline Triethylamine / 101.22 & 3 & $\mathbf{L}$ & 1-RD-51 \\
\hline Benzoic Acid & $75\left(\mathrm{mg} / \mathrm{m}^{3}\right)$ & $\mathbf{S}$ & 1-RD-54 \\
\hline $\begin{array}{l}\text { Silicia, Crystalline Quartz (Resp. dust) / } \\
60.09\end{array}$ & $0.5\left(\mathrm{mg} / \mathrm{m}^{3}\right)$ & $\mathbf{S}$ & 1-RD-56 \\
\hline Sodium Sulfhydrate & $4\left(\mathrm{mg} / \mathrm{m}^{3}\right)$ & $\mathbf{S}$ & 1-RD-57 \\
\hline
\end{tabular}

The ALOHA computer code was utilized to develop ground level concentrations at specified receptor points. Liquids at ambient temperature are input as puddles, solids at ambient temperature are input as direct releases after applying appropriate release fractions, and gases are input as instantaneous direct releases. The ALOHA code is then allowed to calculate the ground level concentration at the specified receptor point and these results are compared with the target concentration of ERPG-2. If the ALOHA projected concentration is greater than the target concentration the source term (puddle area, release rate as applicable) is reduced and the concentration re-calculated by ALOHA. If the ALOHA projected concentration is less than the target concentration the source term (puddle area, release rate as applicable) is increased and the concentration re-calculated by ALOHA. This process continues until the source term producing the target concentration at the target receptor point is established.

Additional ALOHA calculations were performed to determine the footprint of the ERPG-2 concentration for the maximum allowed source term per segment for each of the materials at risk. The segment maximums were also utilized to allow ALOHA to calculate the maximum concentration at 6,000 meters for $95 \%$ meteorological conditions (ALOHA default distance based on wind speed for one hour) and 10,000 meters for 50\% meteorological conditions (ALOHA default distance). 
Title of Project EPHA for THM

Subject Concentration Analysis for THM Hazardous Chemicals

Reviewed by S..A. Henderson

Computer N.E. Baucom B.K. Tharakan Date 09/16/96

RESULTS

Works Calculation 8

Sheet No. 3 of 314

All results shown are centerline distance downwind.

Table $295 \%$ Adverse met Consequence Assessment Results - Chemical

\begin{tabular}{|c|c|c|c|c|c|}
\hline & $\begin{array}{l}\text { Piddle Size Required } \\
\text { to produce ERPG-2 } \\
\text { Concentration @ } \\
30 \text { meters (ALERT) } \\
\text { (FT }{ }^{2} \text { ) }\end{array}$ & $\begin{array}{c}\text { Puddle Size Required } \\
\text { to produce ERPG-2 } \\
\text { Concentration } Q 100 \\
\text { meters (SAE) } \\
\left(\mathrm{FT}^{2}\right)\end{array}$ & $\begin{array}{c}\text { Maximum Distance to } \\
\text { PAC (GE) for Segment } \\
1 \text { Moximum Source } \\
\text { Term }\left(24,420 \mathrm{ft}^{2}\right) \\
\text { (meters) }\end{array}$ & $\begin{array}{c}\text { Maximum Distance to } \\
\text { PAC (GE) for } \\
\text { Segment } 2 \text { Maximum } \\
\text { Source Term }(40,700 \\
\left.\mathrm{t}^{2}\right) \\
\text { (meters) } \\
\end{array}$ & $\begin{array}{l}\text { Madmum Distance to } \\
\text { PAC (GE) for Segment } \\
3 \text { Maximum Source } \\
\text { Term (170,940 } \mathrm{ft}^{3} \text { ) } \\
\text { (meters) }\end{array}$ \\
\hline 1-RD-2 & 115 & 1425 & 428 & 568 & 1200 \\
\hline 1-RD-3 & 21 & 265 & 1100 & 1400 & 2200 \\
\hline 1-RD-4 & 491 & 5941 & 202 & 261 & 432 \\
\hline 1-RD-7 & $>30000$ & $>100000$ & 27 & 34 & 56 \\
\hline 1-RD-9 & 84 & 777 & 532 & 686 & 1100 \\
\hline 1-RD-11 & 3 & 39 & 3400 & 4800 & $>10000$ \\
\hline 1-RD-14 & 7750 & NA & 52 & 67 & 109 \\
\hline 1-RD-15 & $>30000$ & $>100000$ & 27 & 34 & 56 \\
\hline 1-RD-16 & 98 & 1400 & 501 & 643 & 1000 \\
\hline 1-RD-17 & 17237 & $>100000$ & 35 & 45 & 72 \\
\hline 1-RD-19 & 3300 & 24086 & 147 & 189 & 306 \\
\hline 1-RD-20 & $>30000$ & $>100000$ & 27 & 34 & 56 \\
\hline 1-RD-22 & 7 & 101 & 2400 & 3100 & 5000 \\
\hline I-RD-23 & 8750 & $>100000$ & 50 & 64 & 103 \\
\hline 1-RD-26 & 5175 & 62200 & 63 & 81 & 131 \\
\hline 1-RD-29 & 250 & 2125 & 334 & 436 & 679 \\
\hline 1-RD-30 & 6034 & 32500 & 87 & 112 & 187 \\
\hline 1-RD-32 & 145 & 1810 & 377 & 498 & 1200 \\
\hline 1-RD-33 & 18500 & $>100000$ & 34 & 44 & 70 \\
\hline 1-RD-34 & $>30000$ & $>100000$ & 27 & 26 & 42 \\
\hline 1-RD-36 & $>30000$ & NA & 27 & 34 & 56 \\
\hline 1-RD-42 & 283 & 3600 & 263 & 346 & 597 \\
\hline 1-RD-46 & 1080 & 13300 & 138 & 183 & 310 \\
\hline 1-RD-49 & 472 & 6060 & 208 & 275 & 466 \\
\hline 1-RD-51 & 1.7 & 20 & 5300 & 6900 & $>10000$ \\
\hline
\end{tabular}

Table 2 cont. 95\% Adverse met Consequence Assesment Results - Chemical

\begin{tabular}{|c|c|c|c|c|c|}
\hline & $\begin{array}{l}\text { Pounds required to } \\
\text { prodace ERPG-2 } \\
\text { Concentration at } 30 \\
\text { meters (ALERT) } \\
\left(\mathrm{b}_{\mathrm{m}}\right) \\
\end{array}$ & $\begin{array}{c}\text { Poands required to } \\
\text { produce BRPG-2 } \\
\text { Concentration at } 30 \\
\text { meters (SAE) } \\
\text { (lbe) }\end{array}$ & $\begin{array}{l}\text { Madmom Distance to } \\
\text { PAC for Segment } 1 \\
\text { Maximum Source } \\
\text { Term (37,600 lbm)) } \\
\text { (meters) }\end{array}$ & $\begin{array}{l}\text { Maximum Distance to } \\
\text { PAC for Segment } 2 \\
\text { Marimum Sonrce } \\
\text { Term (135,000 lbm) } \\
\text { (meters) }\end{array}$ & $\begin{array}{l}\text { Maximum Distance to } \\
\text { PAC for Segment } 3 \\
\text { Maximum Source } \\
\text { Term (263,000 lbm) } \\
\text { (meters) }\end{array}$ \\
\hline 1-RD-54 & 285 & 3000 & 344 & 580 & 755 \\
\hline 1-RD-56 & 1.9 & 20 & 2500 & 4300 & 5800 \\
\hline 1-RD-57 & 15 & 160 & 1100 & 1800 & 2400 \\
\hline
\end{tabular}


Title of Project EPHA for THM

Subject Concentration Analysis for THM Hazardous Chemicals

Computer N.E. Baucom B. K. Tharakan Date 09/16/96

Table $350 \%$ Average met Consequence Assessment Results - Chemical

\begin{tabular}{|c|c|c|c|c|c|}
\hline & $\begin{array}{l}\text { Puddle Size Required } \\
\text { to produce ERPG-2 } \\
\text { Concentration } 9 \\
30^{0} \text { meters (ALERT) } \\
\left(\text { FT }^{2} \text { ) }\right.\end{array}$ & $\begin{array}{c}\text { Puddle Size Required } \\
\text { to produce ERPG-2 } \\
\text { Concentration } \odot 100 \\
\text { meters (SAE) } \\
\left(\mathrm{FT}^{2}\right)\end{array}$ & $\begin{array}{l}\text { Maximum Distance to } \\
\text { PAC (GE) for Segment } \\
1 \text { Maximum Source } \\
\left.\text { Term (24,420 } \mathrm{t}^{2}\right) \\
\text { (meters) }\end{array}$ & $\begin{array}{c}\text { Maximum Distance to } \\
\text { PAC (GE) for } \\
\text { Segment } 2 \text { Maximam } \\
\text { Source Term }(40,700 \\
\left.n^{2}\right) \\
\text { (meters) }\end{array}$ & $\begin{array}{l}\text { Maximum Distance to } \\
\text { PAC (GE) for Segment } \\
3 \text { Maximum Source } \\
\text { Term (170,940 } \mathrm{n}^{2} \text { ) } \\
\text { (meters) }\end{array}$ \\
\hline 1-RD-2 & 895 & 11850 & 140 & 178 & 280 \\
\hline $1-R D-3$ & 177 & 2325 & 303 & 386 & 1600 \\
\hline 1-RD-4 & 3341 & 42582 & 77 & 98 & 155 \\
\hline 1-RD-7 & $>30000$ & $>100000$ & 27 & 34 & 56 \\
\hline 1-RD-9 & 737 & 6485 & 471 & 603 & 994 \\
\hline $1-R D-11$ & 37 & 477 & 651 & 832 & 1300 \\
\hline 1-RD-14 & $>30000$ & $>100000$ & 27 & 34 & 56 \\
\hline 1-RD-15 & $>30000$ & $\geq 100000$ & 27 & 34 & 56 \\
\hline 1-RD-16 & 997 & 13000 & 135 & 556 & 907 \\
\hline 1-RD-17 & $>30000$ & $>100000$ & 16 & 20 & 32 \\
\hline 1-RD-19 & 17700 & $>100000$ & 35 & 45 & 72 \\
\hline $1-R D-20$ & $>30000$ & $>100000$ & 27 & 34 & 56 \\
\hline 1-RD-22 & 59 & 760 & 528 & 677 & 1100 \\
\hline 1-RD-23 & $>30000$ & $>100000$ & 27 & 34 & 56 \\
\hline 1-RD-26 & 26800 & $>100000$ & 29 & 37 & 58 \\
\hline 1-RD-29 & 3165 & 9384 & 313 & 400 & 640 \\
\hline 1-RD-30 & $>30000$ & 61426 & 27 & 34 & 173 \\
\hline 1-RD-32 & 1950 & 27750 & 94 & 119 & 186 \\
\hline 1-RD-33 & $>30000$ & $>100000$ & 27 & 34 & 56 \\
\hline 1-RD-34 & 1450 & 19600 & 111 & 140 & 220 \\
\hline $1-\mathrm{RD}-36$ & $>30000$ & $>100000$ & 27 & 34 & 56 \\
\hline 1-RD-42 & 2050 & 28500 & 93 & 118 & 185 \\
\hline 1-RD-46 & 3010 & 21100 & 108 & 140 & 232 \\
\hline 1-RD-49 & 875 & 7840 & 176 & 229 & 381 \\
\hline \multirow[t]{2}{*}{ 1-RD-51 } & 11.3 & 143 & 3200 & 4100 & 6700 \\
\hline & $\begin{array}{c}\text { Pounds required to } \\
\text { produce ERPG-2 } \\
\text { Concentration at } 30 \\
\text { meters (ALERT) } \\
\left(\mathrm{b}_{\mathrm{m}}\right) \\
\end{array}$ & $\begin{array}{l}\text { Pounds required to } \\
\text { produce ERPG-2 } \\
\text { Concentration at } 30 \\
\text { meters (SAE) } \\
\text { (lb })\end{array}$ & $\begin{array}{l}\text { Maximum Distance to } \\
\text { PAC for Segment } 1 \\
\text { Maximum Source Tem } \\
(37,600 \mathrm{bm}) \\
\text { (meters) }\end{array}$ & $\begin{array}{l}\text { Maximum Distance for } \\
\text { PAC for Segmeat } 2 \\
\text { Maximum Source Term } \\
(135,000 \mathrm{bm}) \\
\text { (meters) }\end{array}$ & $\begin{array}{c}\text { Maximum Distance to } \\
\text { PAC for Segment } 3 \\
\text { Maximum Source Term } \\
\text { (263,000 lbm) } \\
\text { (meters) }\end{array}$ \\
\hline 1-RD-54 & 1900 & 21300 & 133 & 252 & 353 \\
\hline 1-RD-56 & 12.8 & 142 & 1300 & 2000 & 2600 \\
\hline 1-RD-57 & 100 & 1135 & 569 & 948 & 1200 \\
\hline
\end{tabular}

Reviewed by S.A. Henderson

Works Calculation 8

Sheot No. 4 of 314 
Title of Project EPHA for THM

\section{ENGINEERING COMPUTATION SHEET}

Subject Concentration Analysis for THM Hazardous Chemicals

Computer N.E. Baucom B. K. Tharakan Date 09/16/96

REFERENCES
Reviewed by S. A. Henderson

Works Calculation 8

Sheet No. 5 of 314

1. Hadlock, D. J., Standards for Development and Maintenance of a Facility Hazards Assessment, $6 Q$ Vol. 2, EMPP 6Q-001, Rev. 1, Westinghouse Savannah River Company, Savannah River Site, Aiken, SC, November 1, 1995.

2. Hadlock, D. J., Consequence Assessment for Emergency Preparedness Hazards Assessments, TP-95002, Rev. 3, January 20, 1997.

3. ALOHA, Areal Locations Of Hazardous Atmospheres, Version 5.2.1S, User's Manuel, National Oceanic and Atmospheric Administration and U.S. Environmental Protection Agency, June, 1995.

4. Craig, D. K., ERPGs and TEELs for Chemicals of Concern, WSMS-SAE-98-00101, Rev. 14, Augusta 4, 1998. Addendum to Rev. 14, ERPGs and TEELs for Chemicals of Concem, WSMS Inter-Office Memorandum, WSMS-SAE-M-98-00100, September 3, 1998. 
Title of Project EPHA for THM

Subject Concentration Analysis for THM Hazardous Chemicals

Computer N.E. Baucom B. K. Tharakan Date 09/16/96

1-RD-2 ADVERSE / ALERT

\section{SITE DATA INFORMATION:}

Location: AIKEN, SOUTH CAROLINA

Building Air Exchanges Per Hour: 60 (User specified)

Time: June 30, 19982359 hours EDT (User specified)

\section{CHEMICAL INFORMATION:}

Chemical Name: ACETIC ACID

Molecular Weight: $60.05 \mathrm{~kg} / \mathrm{kmol}$

TLV-TWA: $10 \mathrm{ppm}$ IDLH: $50 \mathrm{ppm}$

Footprint Level of Concern: 35 ppm

Boiling Point: $117.90^{\circ} \mathrm{C}$

Vapor Pressure at Ambient Temperature: $0.026 \mathrm{~atm}$

Ambient Saturation Concentration: 26,132 ppm or 2.61\%

ATMOSPHERIC INFORMATION: (MANUAL INPUT OF DATA)

Wind: 1.7 meters $/ \mathrm{sec}$ from $90^{\circ}$ true at 2 meters

Inversion Height: 200 meters

Stability Class: E Air Temperature: $29^{\circ} \mathrm{C}$

Relative Humidity: 50\% Ground Roughness: Urban or forest

Cloud Cover: 1 tenths

\section{SOURCE STRENGTH INFORMATION:}

Puddle Area: 115 square feet

Average Puddle Depth: 1 centimeters

Soil Type: Default Ground Temperature: $29^{\circ} \mathrm{C}$

Initial Puddle Temperature: Ground temperature

Release Duration: ALOHA limited the duration to 1 hour

Max Computed Release Rate: 201 grams/min

Max Average Sustained Release Rate: 186 grams/min

(averaged over a minute or more)

Total Amount Released: 10.1 kilograms

\section{TIME DEPENDENT INFORMATION:}

Concentration Estimates at the point:

Downwind: 30 meters

Off Centerline: 0 meters

Max Concentration:

Outdoor: 35 ppm
Reviewed by S. A. Henderson

Works Calculation 8

Sheet No. $\underline{6 \text { of } 314}$ 
Title of Project EPHA for THM

Reviewed by S. A. Henderson

Computer N.E. Baucom B. K. Tharakan Date 09/16/96

\section{SITE DATA INFORMATION:}

Location: AIKEN, SOUTH CAROLINA

Building Air Exchanges Per Hour: 60 (User specified)

Time: June 30, 19982359 hours EDT (User specified)

CHEMICAL INFORMATION:

Chemical Name: ACETIC ACID

Molecular Weight: $60.05 \mathrm{~kg} / \mathrm{kmol}$

TLV-TWA: $10 \mathrm{ppm}$ IDLH: $50 \mathrm{ppm}$

Footprint Level of Concern: 35 ppm

Boiling Point: $117.90^{\circ} \mathrm{C}$

Vapor Pressure at Ambient Temperature: 0.026 atm

Ambient Saturation Concentration: 26,132 ppm or $2.61 \%$

ATMOSPHERIC INFORMATION: (MANUAL INPUT OF DATA)

Wind: 1.7 meters $/ \mathrm{sec}$ from $90^{\circ}$ true at 2 meters

Inversion Height: 200 meters

Stability Class: E Air Temperature: $29^{\circ} \mathrm{C}$

Relative Humidity: 50\% Ground Roughness: Urban or forest

Cloud Cover: 1 tenths

SOURCE STRENGTH INFORMATION:

Puddle Area: 1425 square feet

Average Puddle Depth: 1 centimeters

Soil Type: Default Ground Temperature: $29^{\circ} \mathrm{C}$

Initial Puddle Temperature: Ground temperature

Release Duration: ALOHA limited the duration to 1 hour

Max Computed Release Rate: 2.17 kilograms/min

Max Average Sustained Release Rate: 2.02 kilograms/min

(averaged over a minute or more)

Total Amount Released: 110 kilograms

\section{TIME DEPENDENT INFORMATION:}

Concentration Estimates at the point:

Downwind: 100 meters

Off Centerline: 0 meters

Max Concentration:

Outdoor: 35 ppm 
Title of Project EPHA for THM

Subject Concentration Analysis for THM Hazardous Chemicals

Computer N.E. Baucom B. K. Tharakan Date 09/16/96

1-RD-2 ADVERSE / MAX DIST SEG 1

SITE DATA INFORMATION:

Location: AIKEN, SOUTH CAROLINA

Building Air Exchanges Per Hour: 60 (User specified)

Time: June 30, 19982359 hours EDT (User specified)

CHEMICAL INFORMATION:

Chemical Name: ACETIC ACID

Molecular Weight: $60.05 \mathrm{~kg} / \mathrm{kmol}$

TLV-TWA: $10 \mathrm{ppm}$ IDLH: $50 \mathrm{ppm}$

Footprint Level of Concern: 35 ppm

Boiling Point: $117.90^{\circ} \mathrm{C}$

Vapor Pressure at Ambient Temperature: $0.026 \mathrm{~atm}$

Ambient Saturation Concentration: 26,132 ppm or $2.61 \%$

ATMOSPHERIC INFORMATION: (MANUAL INPUT OF DATA)

Wind: 1.7 meters $/ \mathrm{sec}$ from $90^{\circ}$ true at 2 meters

Inversion Height: 200 meters

Stability Class: E Air Temperature: $29^{\circ} \mathrm{C}$

Relative Humidity: 50\% Ground Roughness: Urban or forest

Cloud Cover: 1 tenths

\section{SOURCE STRENGTH INFORMATION:}

Puddle Area: 24420 square feet

Average Puddle Depth: 1 centimeters

Soil Type: Default Ground Temperature: $29^{\circ} \mathrm{C}$

Initial Puddle Temperature: Ground temperature

Release Duration: ALOHA limited the duration to 1 hour

Max Computed Release Rate: 32.2 kilograms/min

Max Average Sustained Release Rate: 30.2 kilograms/min

(averaged over a minute or more)

Total Amount Released: 1,665 kilograms

FOOTPRINT INFORMATION:

Dispersion Module: Gaussian

User-specified LOC: $35 \mathrm{ppm}$

Max Threat Zone for LOC: 428 meters
Reviewed by S. A. Henderson

Works Calculation 8

\section{Sheet No. 8 of 314}


Title of Project EPHA for THM

Subject Concentration Analysis for THM Hazardous Chemicals

Computer N.E. Baucom B. K. Tharakan Date 09/16/96

\section{1-RD-2 ADVERSE / MAX DIST SEG 2}

\section{SITE DATA INFORMATION:}

Location: AIKEN, SOUTH CAROLINA

Building Air Exchanges Per Hour: 60 (User specified)

Time: June 30, 19982359 hours EDT (User-specified)

\section{CHEMICAL INFORMATION:}

Chemical Name: ACETIC ACID

Molecular Weight: $60.05 \mathrm{~kg} / \mathrm{kmol}$

TLV-TWA: $10 \mathrm{ppm}$ IDLH: $50 \mathrm{ppm}$

Footprint Level of Concern: 35 ppm

Boiling Point: $117.90^{\circ} \mathrm{C}$

Vapor Pressure at Ambient Temperature: $0.026 \mathrm{~atm}$

Ambient Saturation Concentration: 26,132 ppm or 2.61\%

\section{ATMOSPHERIC INFORMATION: (MANUAL INPUT OF DATA)}

Wind: 1.7 meters $/ \mathrm{sec}$ from $90^{\circ}$ true at 2 meters

Inversion Height: 200 meters

Stability Class: E Air Temperature: $29^{\circ} \mathrm{C}$

Relative Humidity: 50\% Ground Roughness: Urban or forest

Cloud Cover: 1 tenths

\section{SOURCE STRENGTH INFORMATION:}

Puddle Area: 40700 square feet

Average Puddle Depth: 1 centimeters

Soil Type: Default Ground Temperature: $29^{\circ} \mathrm{C}$

Initial Puddle Temperature: Ground temperature

Release Duration: ALOHA limited the duration to 1 hour

Max Computed Release Rate: 52.5 kilograms/min

Max Average Sustained Release Rate: 49.2 kilograms/min

(averaged over a minute or more)

Total Amount Released: 2,716 kilograms

\section{FOOTPRINT INFORMATION:}

Dispersion Module: Gaussian

User-specified LOC: $35 \mathrm{ppm}$

Max Threat Zone for LOC: 568 meters
Reviewed by S.A. Henderson

Works Calculation 8

Sheet No. 9 of 314 
Title of Project EPHA for THM

Subject Concentration Analysis for THM Hazardous Chemicals

Computer N.E. Baucom B. K. Tharakan Date 09/16/96

1-RD-2 ADVERSE / MAX DIST SEG 3

\section{SITE DATA INFORMATION:}

Location: AIKEN, SOUTH CAROLINA

Building Air Exchanges Per Hour: 60 (User specified)

Time: June 30, 19982359 hours EDT (User specified)

\section{CHEMICAL INFORMATION:}

Chemical Name: ACETIC ACID

Molecular Weight: $60.05 \mathrm{~kg} / \mathrm{kmol}$

TLV-TWA: $10 \mathrm{ppm}$, IDLH: $50 \mathrm{ppm}$

Footprint Level of Concern: 35 ppm

Boiling Point: $117.90^{\circ} \mathrm{C}$

Vapor Pressure at Ambient Temperature: $0.026 \mathrm{~atm}$

Ambient Saturation Concentration: $26,132 \mathrm{ppm}$ or $2.61 \%$

ATMOSPHERIC INFORMATION: (MANUAL INPUT OF DATA)

Wind: 1.7 meters $/ \mathrm{sec}$ from $90^{\circ}$ true at 2 meters

Inversion Height: 200 meters

Stability Class: E Air Temperature: $29^{\circ} \mathrm{C}$

Relative Humidity: 50\% Ground Roughness: Urban or forest

Cloud Cover: 1 tenths

SOURCE STRENGTH INFORMATION:

Puddle Area: 10000 square meters

Average Puddle Depth: 1 centimeters

Soil Type: Default Ground Temperature: $29^{\circ} \mathrm{C}$

Initial Puddle Temperature: Ground temperature

Release Duration: ALOHA limited the duration to 1 hour

Max Computed Release Rate: 133 kilograms/min

Max Average Sustained Release Rate: 125 kilograms/min (averaged over a minute or more)

Total Amount Released: 6,903 kilograms

FOOTPRINT INFORMATION:

Model Run: Heavy Gas

User-specified LOC: $35 \mathrm{ppm}$

Max Threat Zone for LOC: 1.2 kilometers
Reviewed by S: A. Henderson

Works Calculation 8

Sheet No. 10 of 314 4 


\section{1-RD-3 ADVERSE / ALERT}

SITE DATA INFORMATION:

Location: AIKEN, SOUTH CAROLINA

Building Air Exchanges Per Hour: 60 (User specified)

Time: June 30, 19982359 hours EDT (User specified)

\section{CHEMICAL INFORMATION:}

Chemical Name: ACETONITRILE

Molecular Weight: $41.05 \mathrm{~kg} / \mathrm{kmol}$

TLV-TWA: $40 \mathrm{ppm}$ IDLH: $500 \mathrm{ppm}$

Footprint Level of Concern: 60 ppm

Boiling Point: $81.60^{\circ} \mathrm{C}$

Vapor Pressure at Ambient Temperature: 0.14 atm

Ambient Saturation Concentration: 145,864 ppm or $14.6 \%$

\section{ATMOSPHERIC INFORMATION: (MANUAL INPUT OF DATA)}

Wind: 1.7 meters $/ \mathrm{sec}$ from $90^{\circ}$ true at 2 meters

Inversion Height: 200 meters

Stability Class: E Air Temperature: $29^{\circ} \mathrm{C}$

Relative Humidity: 50\% Ground Roughness: Urban or forest

Cloud Cover: 1 tenths

\section{SOURCE STRENGTH INFORMATION:}

Puddle Area: 21 square feet

Average Puddle Depth: 1 centimeters

Soil Type: Default Ground Temperature: $29^{\circ} \mathrm{C}$

Initial Puddle Temperature: Ground temperature

Release Duration: ALOHA limited the duration to 1 hour

Max Computed Release Rate: 183 grams/min

Max Average Sustained Release Rate: 176 grams/min

(averaged over a minute or more)

Total Amount Released: 6.81 kilograms

TIME DEPENDENT INFORMATION:

Concentration Estimates at the point:

Downwind: 30 meters

Off Centerline: 0 meters

Max Concentration:

Outdoor: $58.5 \mathrm{ppm}$ 
Title of Project EPHA for THM

Subject Concentration Analysis for THM Hazardous Chemicals

Works Calculation 8

Computer N.E. Baucom B. K. Tharakan Date 09/16/96

1-RD-3 ADVERSE / SAE

SITE DATA INFORMATION:

Location: AIKEN, SOUTH CAROLINA

Building Air Exchanges Per Hour: 60 (User specified)

Time: June 30, 19982359 hours EDT (User specified)

CHEMICAL INFORMATION:

Chemical Name: ACETONITRILE

Molecular Weight: $41.05 \mathrm{~kg} / \mathrm{kmol}$

TLV-TWA: $40 \mathrm{ppm}$ IDLH: $500 \mathrm{ppm}$

Footprint Level of Concern: 60 ppm

Boiling Point: $81.60^{\circ} \mathrm{C}$

Vapor Pressure at Ambient Temperature: $0.14 \mathrm{~atm}$

Ambient Saturation Concentration: 145,864 ppm or $14.6 \%$

ATMOSPHERIC INFORMATION: (MANUAL INPUT OF DATA)

Wind: 1.7 meters $/ \mathrm{sec}$ from $90^{\circ}$ true at 2 meters

Inversion Height: 200 meters

Stability Class: E Air Temperature: $29^{\circ} \mathrm{C}$

Relative Humidity: 50\% Ground Roughness: Urban or forest

Cloud Cover: 1 tenths

SOURCE STRENGTH INFORMATION:

Puddle Area: 265 square feet

Average Puddle Depth: 1 centimeters

Soil Type: Default Ground Temperature: $29^{\circ} \mathrm{C}$

Initial Puddle Temperature: Ground temperature

Release Duration: ALOHA limited the duration to 1 hour

Max Computed Release Rate: 1.95 kilograms/min

Max Average Sustained Release Rate: 1.95 kilograms/min

(averaged over a minute or more)

Total Amount Released: 75.9 kilograms

TTME DEPENDENT INFORMATTON:

Concentration Estimates at the point:

Downwind: 100 meters

Off Centerline: 0 meters

Max Concentration:

Outdoor: 59.9 ppm 
Title of Project EPHA for THM

Subject Concentration Anatysis for THM Hazardous Chemicals

Computer N.E. Baucom B.K. Tharakan Date 09/16/96
Reviewed by S. A. Henderson

Works Calculation 8

Sheet No. 13 of 314

\section{1-RD-3 ADVERSE / MAX DIST SEG 1}

SITE DATA INFORMATION:

Location: AIKEN, SOUTH CAROLINA

Building Air Exchanges Per Hour: 60 (User specified)

Time: June 30, 19982359 hours EDT (User specified)

CHEMICAL INFORMATION:

Chemical Name: ACETONITRILE

Molecular Weight: $41.05 \mathrm{~kg} / \mathrm{kmol}$

TLV-TWA: $40 \mathrm{ppm}$ IDLH: $500 \mathrm{ppm}$

Footprint Level of Concern: $60 \mathrm{ppm}$

Boiling Point: $81.60^{\circ} \mathrm{C}$

Vapor Pressure at Ambient Temperature: 0.14 atm

Ambient Saturation Concentration: $145,864 \mathrm{ppm}$ or $14.6 \%$

ATMOSPHERIC INFORMATION: (MANUAL INPUT OF DATA)

Wind: 1.7 meters $/ \mathrm{sec}$ from $90^{\circ}$ true at 2 meters

Inversion Height: 200 meters

Stability Class: E Air Temperature: $29^{\circ} \mathrm{C}$

Relative Humidity: 50\% Ground Roughness: Urban or forest

Cloud Cover: 1 tenths

\section{SOURCE STRENGTH INFORMATION:}

Puddle Area: 24420 square feet

Average Puddle Depth: 1 centimeters

Soil Type: Default Ground Temperature: $29^{\circ} \mathrm{C}$

Initial Puddle Temperature: Ground temperature

Release Duration: ALOHA limited the duration to 1 hour

Max Computed Release Rate: 140 kilograms/min

Max Average Sustained Release Rate: 140 kilograms/min

(averaged over a minute or more)

Total Amount Released: 5,790 kilograms

FOOTPRINT INFORMATION:

Model Run: Heavy Gas

User-specified LOC: $60 \mathrm{ppm}$

Max Threat Zone for LOC: 1.1 kilometers 
Title of Project EPHA for THM

Subject Concentration Analysis for THM Hazardous Chemicals

Computer N.E. Baucom B. K. Tharakan Date 09/16/96

\section{1-RD-3 ADVERSE / MAX DIST SEG 2}

\section{SITE DATA INFORMATION:}

Location: AIKEN, SOUTH CAROLINA

Building Air Exchanges Per Hour: 60 (User specified)

Time: June 30, 19982359 hours EDT (User specified)

CHEMICAL INFORMATION:

Chemical Name: ACETONITRIIE

Molecular Weight: $41.05 \mathrm{~kg} / \mathrm{kmol}$

TLV-TWA: $40 \mathrm{ppm}$ IDLH: $500 \mathrm{ppm}$

Footprint Level of Concern: $60 \mathrm{ppm}$

Boiling Point: $81.60^{\circ} \mathrm{C}$

Vapor Pressure at Ambient Temperature: $0.14 \mathrm{~atm}$

Ambient Saturation Concentration: 145,864 ppm or $14.6 \%$

ATMOSPHERIC INFORMATION: (MANUAL INPUT OF DATA)

Wind: 1.7 meters $/ \mathrm{sec}$ from $90^{\circ}$ true at 2 meters

Inversion Height: 200 meters

Stability Class: E Air Temperature: $29^{\circ} \mathrm{C}$

Relative Humidity: 50\% Ground Roughness: Urban or forest

Cloud Cover: 1 tenths

\section{SOURCE STRENGTH INFORMATION:}

Puddle Area: 40700 square feet

Average Puddle Depth: 1 centimeters

Soil Type: Default Ground Temperature: $29^{\circ} \mathrm{C}$

Initial Puddle Temperature: Ground temperature

Release Duration: ALOHA limited the duration to 1 hour

Max Computed Release Rate: 228 kilograms/min

Max Average Sustained Release Rate: 228 kịlograms/min

(averaged over a minute or more)

Total Amount Released: 9,467 kilograms

FOOTPRINT INFORMATION:

Model Run: Heavy Gas

User-specified LOC: $60 \mathrm{ppm}$

Max Threat Zone for LOC: 1.4 kilometers
Reviewed by S. A. Henderson

Works Calculation 8

Sheet No. 14 of 314 
Titie of Project EPHA for THM

Subject Concentration Analysis for THM Hazardous Chemicals

Computer N.E. Baucom B. K. Tharakan Date 09/16/96

1-RD-3 ADVERSE / MAX DIST SEG 3

SITE DATA INFORMATION:

Location: AIKEN, SOUTH CAROLINA

Building Air Exchanges Per Hour: 60 (User specified)

Time: June 30, 19982359 hours EDT (User specified)

CHEMICAL INFÓRMATION:

Chemical Name: ACETONITRILE

Molecular Weight: $41.05 \mathrm{~kg} / \mathrm{kmol}$

TLV-TWA: 40 ppm IDLH: 500 ppm

Footprint Level of Concern: $60 \mathrm{ppm}$

Boiling Point: $81.60^{\circ} \mathrm{C}$

Vapor Pressure at Ambient Temperature: $0.14 \mathrm{~atm}$

Ambient Saturation Concentration: 145,864 ppm or $14.6 \%$

ATMOSPHERIC INFORMATION: (MANUAL INPUT OF DATA)

Wind: 1.7 meters $/ \mathrm{sec}$ from $90^{\circ}$ true at 2 meters

Inversion Height: 200 meters

Stability Class: E Air Temperature: $29^{\circ} \mathrm{C}$

Relative Humidity: $50 \%$ Ground Roughness: Urban or forest

Cloud Cover: 1 tenths

SOURCE STRENGTH INFORMATION:

Puddle Area: 10000 square meters

Average Puddle Depth: 1 centimeters

Soil Type: Default Ground Temperature: $29^{\circ} \mathrm{C}$

Initial Puddle Temperature: Ground temperature

Release Duration: ALOHA limited the duration to 1 hour

Max Computed Release Rate: 575 kilograms/min

Max Average Sustained Release Rate: 575 kilograms/min

(averaged over a minute or more)

Total Amount Released: 24,165 kilograms

FOOTPRINT INFORMATION:

Model Run: Heavy Gas

User-specified LOC: $60 \mathrm{ppm}$

Max Threat Zone for LOC: 2.2 kilometers
Reviewed by S. A. Henderson

Works Calculation 8

Sheet No. 15 of 314 
Title of Project EPHA for THM

Subject Concentration Analysis for THM Hazardous Chemicals

Computer N.E, Baucom B. K. Tharakan Date 09/16/96 1-RD-4 ADVERSE / ALERT

\section{SITE DATA INFORMATION:}

Location: AIKEN, SOUTH CAROLINA

Building Air Exchanges Per Hour: 0.44 (Sheltered single storied)

Time: June 30, 19982359 hours EDT (User specified)

\section{CHEMICAL INFORMATION:}

Chemical Name: ALPHA-PINENE

Molecular Weight: $136,26 \mathrm{~kg} / \mathrm{kmol}$

TLV-TWA: -unavail- IDLH: -unavail-

Footprint Level of Concern: $300 \mathrm{mg} /(\mathrm{cu} \mathrm{m})$

Boiling Point: $155.00^{\circ} \mathrm{C}$

Vapor Pressure at Ambient Temperature: $0.0085 \mathrm{~atm}$

Ambient Saturation Concentration: 8,689 ppm or $0.87 \%$

Note: Not enough chemical data to use Heavy Gas option.

ATMOSPHERIC INFORMATION: (MANUAL INPUT OF DATA)

Wind: 1.7 meters $/ \sec$ from $90^{\circ}$ true at 2 meter

Inversion Height: 200 meters

Stability Class: E

Relative Humidity: 50\%

Air Temperature: $29^{\circ} \mathrm{C}$

Cloud Cover: 1 tenths

Ground Roughness: Urban or forest
Reviewed by S. A. Henderson

Works Calculation 8

Sheet No. 16 of 314

SOURCE STRENGTH INFORMATION:

Direct Source: .0086 kilograms / sec

Source Height: 0

Release Duration: 60 minutes

Release Rate: 516 grams / min

Total Amount Released: 31.0 kilograms

TIME DEPENDENT INFORMATION:

Concentration Estimates at the point:

Downwind: $\quad 30$ meters

Off centerline: $\quad 0$ meters

Max concentration:

Outdoor: $299 \mathrm{mg} /(\mathrm{cu} \mathrm{m})$

Indoor: $106 \mathrm{mg} /(\mathrm{cu} \mathrm{m}$ )

Note: Indoor graph is shown with a dotted line. 
Title of Project EPHA for THM

Reviewed by S. A. Henderson

Subject Concentration Analysis for THM Hazardous Chemicals

Works Calculation 8

Computer N.E. Baucom B.K. Tharakan Date 09/16/96

Sheot No. 17 of 314

SPLL AREA CALCULATION FOR 1-RD-4 ADVERSE/ ALERT CONDITIONS

\begin{tabular}{|c|c|c|c|c|}
\hline Parameter & Value & Dimensions & Value & $\begin{array}{c}\text { Dimensions } \\
\text { (USA) }\end{array}$ \\
\hline E (Evaporation Rate) & 8.64E-3 & $\mathrm{kg} / \mathrm{s}$ & & \\
\hline MW (Molecular Weight) & 136 & $\mathrm{~kg} / \mathrm{kg}$-mole & & \\
\hline Pv (Vapor Pressure) & 6.49 & $\mathrm{~mm} \mathrm{Hg}$ & & \\
\hline Pv (Vapor Pressure) & 865 & $\mathbf{P a}$ & & \\
\hline Rg (Gas Law Constant) & 8314 & J/kgmole-K & & \\
\hline Temperature & 29 & ${ }^{\circ} \mathrm{C}$ & & \\
\hline Volume of Spill & 120.6 & gal & & \\
\hline d (Depth of Pool) & 0.01 & $\mathbf{m}$ & & \\
\hline A (Surface Area of Spill) & 45.7 & $\mathbf{m}^{\wedge} \mathbf{2}$ & 491 & $\mathrm{ft}^{\wedge} \mathbf{2}$ \\
\hline Km (Mass Transfer Coeff) & $4.03 \mathrm{E}-3$ & $\mathrm{~m} / \mathrm{s}$ & & \\
\hline $\mathrm{Z}$ (Pool Diameter in Wind Direction) & 8 & $\mathbf{m}$ & & \\
\hline Sc (Schmidt Number) & 1.72 & & & \\
\hline $\mathrm{U}$ (Wind velocity) & 1.7 & $\mathrm{~m} / \mathrm{s}$ & & \\
\hline nu (kinematic viscosity of air) & $1.50 \mathrm{E}-5$ & $\mathrm{~m}^{\wedge} 2 / \mathrm{sec}$ & & \\
\hline mu (viscosity of air) & $1.90 \mathrm{E}-5$ & $\mathrm{~kg} / \mathrm{m}-\mathrm{s}$ & & \\
\hline $\begin{array}{l}\text { Dm (Molecular Diffusivity of Solute } \\
\text { in Air }\end{array}$ & $8.72 \mathrm{E}-6$ & $\mathrm{~m}^{\wedge} 2 / \mathrm{sec}$ & & \\
\hline $\begin{array}{l}\text { D_H20 (Molecular Diffusivity of } \\
\text { Water) }\end{array}$ & $2.40 \mathrm{E}-5$ & & & - \\
\hline MW_H20 & 18 & $\mathrm{~kg} / \mathbf{k g} \cdot \mathbf{m o l e}$ & & \\
\hline \multicolumn{5}{|l|}{ Conversions } \\
\hline$T$ (Absolute) & 302.15 & 'K & & \\
\hline $\mathrm{V}$ (Volume) & 0.46 & $m^{\wedge} 3$ & & \\
\hline Evaporation Rate & 518 & $\mathrm{~g} / \mathrm{min}$ & & \\
\hline $60 \mathrm{~min} l \mathrm{lb}$ released & 68.56 & & & \\
\hline
\end{tabular}


Title of Project EPHA for THM

Subject Concentration Analysis for THM Hazardous Chemicals

Computer N.E. Baucom B. K. Tharakan Date 09/16/96

\section{1-RD-4 ADVERSE / SAE}

\section{SITE DATA INFORMATION:}

Location: AIKEN, SOUTH CAROLINA

Building Air Exchanges Per Hour: 0.44 (Sheltered single storied)

Time: June 30, 19982359 hours EDT (User specified)

\section{CHEMICAL INFORMATION:}

Chemical Name: ALPHA-PINENE

Molecular Weight: $136.26 \mathrm{~kg} / \mathrm{kmol}$

TLV.TWA: -unavail-

ILL: -unavail-

Footprint Level of Concern: $300 \mathrm{mg} /(\mathrm{cu} \mathrm{m})$

Boiling Point: $155.00^{\circ} \mathrm{C}$

Vapor Pressure at Ambient Temperatures: $0.0085 \mathrm{~atm}$

Ambient Saturation Concentration: 8,689 ppm or 0.87\%

Note: Not enough chemical data to use Heavy Gas option

\section{ATMOSPHERIC INFORMATION: (MANUAL INPUT OF DATA)}

Wind: 1.7 meters $/ \sec$ from $90^{\circ}$ true at 2 meters

Inversion Height: 200 meters

Stability Class: $\mathbf{E}$

Air Temperature: $29^{\circ} \mathrm{C}$

Relative Humidity: $50 \%$

Ground Roughness: Urban or forest
Reviewed by S. A. Henderson

Works Calculation 8

Sheet No. 18 of 314

- Cloud Cover: 1 tenths

\section{SOURCE STRENGTH INFORMATION:}

Direct Source: .091 kilograms/ sec

Source Height: 0

Release Duration: 60 minutes

Release Rate: 5.46 kilograms/min

Total Amount Released: 328 kilograms

\section{TIME DEPENDENT INFORMATION:}

Concentration Estimates at the point:

Downwind: 100 meters

Off Centerline: 0 meters

Max Concentration:

Outdoor: $300 \mathrm{mg} /(\mathrm{cu} \mathrm{m})$

Indoor: $106 \mathrm{mg} /(\mathrm{cu} \mathrm{m})$

Note: Indoor graph is shown with a dotted line. 
Title of Project EPHA for THM

Reviewed by S. A. Henderson

Subject Concentration Analysis for THM Hazardous Chemicals

Works :Calculation 8

Computer N.E. Baucom B. K. Tharakan Date 09/16/96

Sheet No. 19 of 314

SPILL AREA CALCULATION FiOR 1-RD-4 ADVERSE / SAE CONDITIONS

\begin{tabular}{|c|c|c|c|c|}
\hline Parameter & Value & Dimensions & Value & $\begin{array}{c}\text { Dimensions } \\
\text { (USA) }\end{array}$ \\
\hline E (Evaporation Rate) & $9.09 \mathrm{E}-2$ & $\mathbf{k g} / \mathbf{s}$ & & \\
\hline MW (Molecular Weight) & 136 & kg/kg-mole & & \\
\hline Pv (Vapor Pressure) & 6.49 & $\mathrm{~mm} \mathrm{Hg}$ & & \\
\hline Pv (Vapor Pressure) & 865 & $\mathbf{P a}$ & & \\
\hline Rg (Gas Law Constant) & 8314 & J/kgmole-K & & \\
\hline Temperature & 29 & $\mathrm{C}$ & & \\
\hline Volume of Spill & 1458 & gal & & \\
\hline d (Depth of Pool) & 0.01 & $\mathbf{m}$ & & \\
\hline A (Surface Area of Spill) & 551.9 & $\mathbf{m}^{\wedge} \mathbf{2}$ & 5941 & $\mathrm{ft}^{\wedge} \mathbf{2}$ \\
\hline Km (Mass Transfer Coeff) & $3.51 \mathrm{E}-3$ & $\mathrm{~m} / \mathrm{s}$ & & \\
\hline Z (Pool Diameter in Wind Direction) & 27 & $\mathbf{m}$ & & - \\
\hline Sc (Schmidt Number) & 1.72 & & & \\
\hline $\mathrm{U}$ (Wind velocity) & 1.7 & $\mathrm{~m} / \mathrm{s}$ & & \\
\hline nu (kinematic viscosity of air) & $1.50 \mathrm{E}-5$ & $m^{\wedge} 2 / \sec$ & & \\
\hline mu (viscosity of air) & $1.90 \mathrm{E}-5$ & $\mathrm{~kg} / \mathrm{m}-\mathrm{s}$ & & \\
\hline $\begin{array}{l}\text { Dm (Molecular Diffusivity of Solute } \\
\text { in Air }\end{array}$ & $8.72 \mathrm{E}-6$ & $\mathrm{~m}^{\wedge} 2 / \mathrm{sec}$ & & \\
\hline $\begin{array}{l}\text { D_H20 (Molecular Diffusivity of } \\
\text { Water) }\end{array}$ & $2.40 \mathrm{E}-5$ & & & \\
\hline MW_H20 & 18 & $\mathrm{~kg} / \mathrm{kg}$-mole & & \\
\hline \multicolumn{5}{|l|}{ Conversions } \\
\hline$T$ (Absolute) & 302.15 & ${ }^{\cdot} \mathbf{K}$ & & \\
\hline V (Volume) & 5.52 & $m^{\wedge} 3$ & & \\
\hline Evaporation Rate & 5456 & $\mathrm{~g} / \mathrm{min}$ & & \\
\hline $60 \mathrm{~min} \mathrm{lb}$ released & 721.67 & & & \\
\hline
\end{tabular}


Title of Project EPHA for THM

Subject Concentration Analysis for THM Hazardous Chemicals

Computer N.E. Baucom B. K. Tharakan Date 09/16/96

1-RD-4 ADVERSE / MAX DIST SEG 1

SITE DATA INFORMATION:

Location: AIKEN, SOUTH CAROLINA

Building Air Exchanges Per Hour: 0.44 (Sheltered single storied)

Time: June 30, 19982359 hours EDT (User specified)

\section{CHEMICAL INFORMATION:}

Chemical Name: ALPHA-PINENE

Molecular Weight: $136.26 \mathrm{~kg} / \mathrm{kmol}$

TLV-TWA: -unavailIDLH: -unavail-

Footprint Level of Concern: $300 \mathrm{mg} /(\mathrm{cu} \mathrm{m})$

Boiling Point: $155.00^{\circ} \mathrm{C}$

Vapor Pressure at Ambient Temperatures: 0.0085 atm Ambient Saturation Concentration: 8,689 ppm or $0.87 \%$

Note: Not enough chemical data to use Heavy Gas option

ATMOSPHERIC INFORMATION: (MANUAL INPUT OF DATA)

Wind: 1.7 meters $/ \sec$ from $90^{\circ}$ true at 2 meters

Inversion Height: 200 meters

Stability Class: E

Air Temperature: $29^{\circ} \mathrm{C}$

Relative Humidity: $\mathbf{5 0 \%}$

Ground Roughness: Urban or forest
Reviewed by S. A. Henderson

Works Calculation 8

Sheet No. 20 of 314

Cloud Cover: 1 tenths

\section{SOURCE STRENGTH INFORMATION:}

Direct Source: .346 kilograms/ sec

Source Height: 0

Release Duration: 60 minutes

Release Rate: 20.8 kilograms/min

Total Amount Released: 1,246 kilograms

FOOTPRINT INFORMATION:

Dispersion Module: Gaussian

User-specified LOC: $300 \mathrm{mg} / \mathrm{cu} \mathrm{m}$ )

Max Threat Zone for LOC: 202 meters 
Tịtle of Project EPHA for THM

Reviewed by S. A. Henderson

Subject Concentration Analysis for THM Hazardous Chemicals

Works Calculation 8

Computer N.E. Baucom B. K. Tharakan Date 09/16/96

Sheet No. 21 of 314

Determination of Evaporation Rate for Segment 1 Maximum Source Term of $24420 \mathrm{ft}^{2}$

\begin{tabular}{|c|c|c|c|c|}
\hline Parameter & Value & Dimensions & Value & $\begin{array}{c}\text { Dimensions } \\
\text { (USA) }\end{array}$ \\
\hline E (Evaporation Rate) & $3.46 \mathrm{E}-1$ & $\mathrm{~kg} / \mathrm{s}$ & & \\
\hline MW (Molecular Weight) & 136 & $\mathrm{~kg} / \mathrm{kg} \cdot \mathrm{mole}$ & & \\
\hline Pv (Vapor Pressure) & 6.49 & $\operatorname{mm~Hg}$ & & \\
\hline Pv (Vapor Pressure) & 865 & $\mathbf{P a}$ & & \\
\hline Rg (Gas Law Constant) & 8314 & J/kgmole-K & & \\
\hline Temperature & 29 & ${ }^{\circ} \mathrm{C}$ & & \\
\hline Volume of Spill & 5993 & gal & & \\
\hline d (Depth of Pool) & 0.01 & $\mathbf{m}$ & & \\
\hline A (Surface Area of Spill) & 2268.5 & $\mathrm{~m}^{\wedge} \mathbf{2}$ & 24421 & $\mathrm{ft}^{\wedge} \mathbf{2}$ \\
\hline Km (Mass Transfer Coeff) & 3.25E-3 & $\mathbf{m} / \mathbf{s}$ & & \\
\hline Z(Pool Diameter in Wind Direction) & 54 & $\mathbf{m}$ & & \\
\hline Sc (Schmidt Number) & 1.72 & & & \\
\hline $\mathrm{U}$ (Wind velocity) & 1.7 & $\mathrm{~m} / \mathrm{s}$ & & \\
\hline nu (kinematic viscosity of air) & $1.50 \mathrm{E}-5$ & $\mathrm{~m}^{\wedge} 2 / \mathrm{sec}$ & & \\
\hline mu (viscosity of air) & $1.90 \mathrm{E}-5$ & $\mathrm{~kg} / \mathbf{m}-\mathrm{s}$ & & \\
\hline $\begin{array}{l}\text { Dm (Molecular Diffusivity of Solute } \\
\text { in Air }\end{array}$ & $8.72 \mathrm{E}-6$ & $\mathrm{~m}^{\wedge} 2 / \mathrm{sec}$ & & \\
\hline $\begin{array}{l}\text { D_H20 (Molecular Diffusivity of } \\
\text { Water) }\end{array}$ & $2.40 \mathrm{E}-5$ & & & \\
\hline MW_H20 & 18 & $\mathrm{~kg} / \mathrm{kg}$-mole & & \\
\hline \multicolumn{5}{|l|}{ Conversions } \\
\hline T (Absolute) & 302.15 & 'K & & \\
\hline $\mathrm{V}$ (Volume) & 22.69 & $\mathbf{m}^{\wedge} \mathbf{3}$ & & \\
\hline Evaporation Rate & 20732 & g/min & & \\
\hline $60 \mathrm{~min} \mathrm{lb}$ released & 2742.32 & 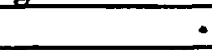 & & \\
\hline
\end{tabular}


Title of Project EPHA for THM

Subject Concentration Anatysis for THM Hazardous Chemicals

Computer N.E. Baucom B. K. Tharakan Date 09/16/96

1-RD-4 ADVERSE / MAX DIST SEG 2

\section{SITE DATA INFORMATION:}

Location: AIKEN, SOUTH CAROLINA

Building Air Exchanges Per Hour: 0.44 (Sheltered single storied)

Time: June 30, 19982359 hours EDT (User specified)

CHEMICAL INFORMATION:

Chemical Name: ALPHA-PINENE

Molecular Weight: $136.26 \mathrm{~kg} / \mathrm{kmol}$

TLV-TWA: -unavail- ILH: -unavail-

Footprint Level of Concern: $300 \mathrm{mg} /(\mathrm{cu} \mathrm{m})$

Boiling Point: $155.00^{\circ} \mathrm{C}$

Vapor Pressure at Ambient Temperatures: 0.0085 atm

Ambient Saturation Concentration: 8,689 ppm or $0.87 \%$

Note: Not enough chemical data to use Heavy Gas option

ATMOSPHERIC INFORMATION: (MANUAL INPUT OF DATA)

Wind: 1.7 meters $/ \sec$ from $90^{\circ}$ true at 2 meters

Inversion Height: 200 meters

Stability Class: E

Air Temperature: $29^{\circ} \mathrm{C}$

Relative Humidity: 50\%

Ground Roughness: Urban or forest
Reviewed by S. A. Henderson

Works Calculation 8

Sheet No. 22 of 314

Cloud Cover: 1 tenths

\section{SOURCE STRENGTH INFORMATION:}

Direct Source: .56 kilograms/ sec

Source Height: 0

Release Duration: 60 minutes

Release Rate: 33.6 kilograms/min

Total Amount Released: 2,016 kilograms

\section{FOOTPRINT INFORMATION:}

Dispersion Module: Gaussian

User-specified LOC: $300 \mathrm{mg} /(\mathrm{cu} \mathrm{m})$

Max Threat Zone for LOC: 261 meters 
Title of Projèct EPHA for THM

Subject Concentration Analysis for THM Hazardous Chemicals

Computer N.E. Baucom B. K. Tharakan Date 09/16/96
Reviewed by S. A. Henderson

Works Calculation 8

Sheet No. 23 of 314

\begin{tabular}{|c|c|c|c|c|}
\hline Parameter & Value & Dimensions & Value & $\begin{array}{l}\text { Dimensions } \\
\text { (USA) }\end{array}$ \\
\hline E (Evaporation Rate) & $5.60 \mathrm{E}-1$ & $\mathrm{~kg} / \mathrm{s}$ & & \\
\hline MW (Molecular Weight) & 136 & $\mathrm{~kg} / \mathrm{kg}$-mole & & \\
\hline Pv (Vapor Pressure) & 6.49 & $\mathrm{~mm} \mathrm{Hg}$ & & \\
\hline Pv (Vapor Pressure) & 865 & $\mathbf{P a}$ & & \\
\hline $\mathrm{Rg}$ (Gas Law Constant) & 8314 & $\mathrm{~J} / \mathrm{kgmole}-\mathrm{K}$ & & \\
\hline Temperature & 29 & $\mathrm{C}$ & & \\
\hline Volume of Spill & 9988 & gal & & \\
\hline d (Depth of Pool) & 0.01 & $\mathbf{m}$ & & \\
\hline A (Surface Area of Spill) & 3780.8 & $\mathbf{m}^{\wedge} \mathbf{2}$ & 40700 & $\mathrm{ft}^{\wedge} \mathbf{2}$ \\
\hline Km (Mass Transfer Coeff) & $3.15 \mathrm{E}-3$ & $\mathbf{m} / \mathbf{s}$ & & \\
\hline Z (Pool Diameter in Wind Direction) & 69 & $\mathbf{m}$ & & \\
\hline Sc (Schmidt Number) & 1.72 & & & \\
\hline U (Wind velocity) & 1.7 & $\mathbf{m} / \mathbf{s}$ & & \\
\hline nu (kinematic viscosity of air) & $1.50 \mathrm{E}-5$ & $\mathrm{~m}^{\wedge} 2 / \mathrm{sec}$ & & \\
\hline mu (viscosity of air) & $1.90 \mathrm{E}-5$ & $\mathbf{k g} / \mathbf{m}-\mathrm{s}$ & & \\
\hline $\begin{array}{l}\text { Dm (Molecular Diffusivity of Solute } \\
\text { in Air }\end{array}$ & $8.72 \mathrm{E}-6$ & $\mathrm{~m}^{\wedge} 2 / \sec$ & & \\
\hline $\begin{array}{l}\text { D_H20 (Molecular Diffusivity of } \\
\text { Water) }\end{array}$ & $2.40 \mathrm{E}-5$ & & & \\
\hline MW_H20 & 18 & kg/kg-mole & & \\
\hline \multicolumn{5}{|l|}{ Conversions } \\
\hline T (Absolute) & 302.15 & $\cdot \mathbf{K}$ & & \\
\hline V (Volume) & 37.81 & $\mathbf{m}^{\wedge} \mathbf{3}$ & & \\
\hline Evaporation Rate & 33585 & $\mathrm{~g} / \mathrm{min}$ & & \\
\hline $60 \mathrm{~min} l \mathrm{lb}$ released & 4442.51 & & & \\
\hline
\end{tabular}


Title of Project EPHA for THM

\section{ENGINEERING COMPUTATION SHEET}

Subject Concentration Analysis for THM Hazardous Chemicals

Computer N.E. Baucom B. K. Tharakan Date 09/16/96

1-RD-4 ADV ERSE / MAX DIST SEG 3

\section{SITE DATA INFORMATION:}

\section{Location: AIKEN, SOUTH CAROLINA}

Building Air Exchanges Per Hour: 0.44 (Sheltered single storied)

Time: June 30, 19982359 hours EDT (User specified)

\section{CHEMICAL INFORMATION:}

Chemical Name: ALPHA-PINENE

Molecular Weight: $136.26 \mathrm{~kg} / \mathrm{kmol}$

TLV-TWA: -unavail-

IDLH: -unavail-

Footprint Level of Concern: $300 \mathrm{mg} /(\mathrm{cu} \mathrm{m})$

Boiling Point: $155.00^{\circ} \mathrm{C}$

Vapor Pressure at Ambient Temperatures: 0.0085 atm Ambient Saturation Concentration: 8,689 ppm or 0.87\%

Note: Not enough chemical data to use Heavy Gas option

ATMOSPHERIC INFORMATION: (MANUAL INPUT OF DATA) Wind: 1.7 meters / sec from $90^{\circ}$ true at 2 meters

Inversion Height: 200 meters

Stability Class: E

Air Temperature: $29^{\circ} \mathrm{C}$

Relative Humidity: $50 \%$

Ground Roughness: Urban or lorest
Reviewed by S.A. Henderson

Works Calculation 8

Sheet No. 24 of 314

SOURCE STRENGTH INFORMATION:

Direct Source: 1.4 kilograms/ sec

Source Height: 0

Release Duration: 60 minutes

Release Rate: 84 kilograms/min

- Total Amount Released: 5,040 kilograms

FOOTPRINT INFORMATION:

Dispersion Module: Gaussian

User-specified LOC: $300 \mathrm{mg} /(\mathrm{cu} \mathrm{m})$

Max Threat Zone for LOC: 432 meters 
Title of Project EPHA for THM

Subject Concentration Analysis for THM Hazardous Chemicals

Computer N.E. Baucom B. K. Tharakan Date 09/16/96
Reviewed by S. A. Henderson

Works Calculation 8

Sheet No. 25 of 314

Determination of Evaporation Rate for Segment 3 Maximum Source Term of $10000 \mathrm{~m}^{2}$

\begin{tabular}{|c|c|c|c|c|}
\hline Parameter & Value & Dimensions & Value & $\begin{array}{l}\text { Dimensions } \\
\text { (USA) }\end{array}$ \\
\hline E (Evaporation Rate) & $1.40 \mathrm{E}+0$ & $\mathrm{~kg} / \mathrm{s}$ & & \\
\hline MW (Molecular Weight) & 136 & $\mathrm{~kg} / \mathrm{kg}-\mathrm{mole}$ & & \\
\hline Pv (Vapor Pressure) & 6.49 & $\mathrm{~mm} \mathrm{Hg}$ & & \\
\hline Pv (Yapor Pressure) & 865 & $\mathbf{P a}$ & & \\
\hline Rg (Gas Law Constant) & 8314 & J/kgmole-K & & \\
\hline Temperature & 29 & $\mathrm{C}$ & & \\
\hline Volume of Spill & 26418 & gal & & $\therefore$ \\
\hline d (Depth of Pool) & 0.01 & $\mathbf{m}$ & & \\
\hline A (Surface Area of Spill) & 10000.0 & $\mathbf{m}^{\wedge} \mathbf{2}$ & 107650 & $\mathrm{ft}^{\wedge} \mathbf{2}$ \\
\hline Km (Mass Transfer Coefin) & $2.99 \mathrm{E}-3$ & $\mathbf{m} / \mathbf{s}$ & & \\
\hline $\mathrm{Z}$ (Pool Diameter in Wind Direction) & 113 & $\mathbf{m}$ & & \\
\hline Sc (Schmidt Number) & 1.72 & & & \\
\hline $\mathrm{U}$ (Wind velocity) & 1.7 & $\mathbf{m} / \mathbf{s}$ & & \\
\hline nu (kinematic viscosity of air) & $1.50 \mathrm{E}-5$ & $\mathrm{~m}^{\wedge} 2 / \mathrm{sec}$ & & \\
\hline mu (viscosity of air) & $1.90 \mathrm{E}-5$ & $\mathbf{k g} / \mathbf{m}-\mathbf{s}$ & & \\
\hline $\begin{array}{l}\text { Dm (Molecular Diffusivity of Solute } \\
\text { in Air }\end{array}$ & $8.72 \mathrm{E}-6$ & $\mathrm{~m}^{\wedge} 2 / \mathrm{sec}$ & & \\
\hline $\begin{array}{l}\text { D_H20 (Molecular Diffusivity of } \\
\text { Water) }\end{array}$ & $2.40 \mathrm{E}-5$ & & & * \\
\hline MW H20 & 18 & $\mathrm{~kg} / \mathrm{kg}$-mole & & \\
\hline 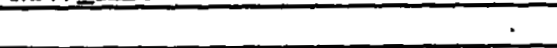 & & & 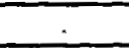 & \\
\hline \multicolumn{5}{|l|}{ Conversions } \\
\hline T (Absolute) & 302.15 & 'K & & \\
\hline $\mathbf{V}$ (Volume) & 100.00 & $\mathbf{m}^{\wedge} \mathbf{3}$ & & \\
\hline Evaporation Rate & 84160 & g/min & & \\
\hline $60 \mathrm{~min} / \mathrm{b}$ released & 11132.23 & & & \\
\hline
\end{tabular}


Title of Project EPHA for THM

Subject Concentration Analysis for THM Hazardous Chemicals

Computer N.E. Baucom B. K. Tharakan Date 09/16/96

\section{1-RD.7 ADVERSE / ALERT}

\section{SITE DATA INFORMATION:}

\section{Location: AIKEN, SOUTH CAROLINA}

Building Air Exchanges Per Hour: 0.44 (Sheltered single storied)

Time: June 30, 19982359 hours EDT (User specified)

\section{CHEMICAL INFORMATION:}

Chemical Name: BENZYL ALCOHOL

Molecular Weight: $108.14 \mathrm{~kg} / \mathrm{kmol}$

TLV-TWA: -unavail-

IDLH: -unavail

Footprint Level of Concern: $100 \mathrm{ppm}$

Boiling Point: $204.70^{\circ} \mathrm{C}$

Vapor Pressure at Ambient Temperature: $1.54 \mathrm{e}-004 \mathrm{~atm}$

Ambient Saturation Concentration: 157 ppm or 0.016\%

ATMOSPHERIC INFORMATION: (MANUAL INPUT OF DATA)

Wind: 1.7 meters $/ \sec$ from $90^{\circ}$ true at 2 meters

Inversion Height: 200 meters

Stability Class: E

Relative Humidity: 50\%

Cloud Cover: 1 tenths

Air Temperature: $29^{\circ} \mathrm{C}$

Ground Roughness: Urban or forest
Reviewed by S. A. Henderson

Works Calculation 8

Sheet No. 26 of 314

\section{SOURCE STRENGTH INFORMATION:}

Puddle Area: 30000 square feet

Average Puddle Depth: 1 centimeters

Soil Type: Default Ground Temperature: $29^{\circ} \mathrm{C}$

Initial Puddle Temperature: Ground temperature

Release Duration: ALOHA limited the duration to 1 hour

Max Computed Release Rate: $\mathbf{3 7 5}$ grams / min

Max Average Sustained Release Rate: 363 grams / min

(averaged over a minute or more)

Total amount Released: 20.8 kilograms

TIME DEPENDENT INFORMATION:

Concentration Estimates at the point:

Downwind: $\quad 30$ meters

Off Centerline: 0 meters

Max Concentration:

Outdoor: $9.63 \mathrm{ppm}$ 
Title of Projèct EPHA for THM

Subject Concentration Analysis for THM Hazardous Chemicals

Computer N.E. Baucom B. K. Tharakan Date 09/16/96 1-RD-7 ADVERSE / SAE

\section{SITE DATA INFORMATION:}

Location: AIKEN, SOUTH CAROLINA

Building Air Exchanges Per Hour: 0.44 (Sheltered single storied)

Time: June 30, 19982359 hours EDT (User specified)

\section{CHEMICAL INFORMATION:}

Chemical Name: BENZYL ALCOHOL

Molecular Weight: $108.14 \mathrm{~kg} / \mathrm{kmol}$

TLV-TWA: -unavail-

DLH: -unavail

Footprint Level of Concern: $100 \mathrm{ppm}$

Boiling Point: $204.70^{\circ} \mathrm{C}$

Vapor Pressure at Ambient Temperature: 1.54e-004 atm

Ambient Saturation Concentration: 157 ppm or 0.016\%

\section{ATMOSPHERIC INFORMATION: (MANUAL INPUT OF DATA)}

Wind: 1.7 meters $/ \sec$ from $90^{\circ}$ true at 2 meters

Inversion Height: 200 meters

Stability Class: E

Relative Humidity: 50\%

Air Temperature: $2^{\circ} \mathrm{C}$

Cloud Cover: 1 tenths

\section{SOURCE STRENGTH INFORMATION:}

Puddle Area: 100000 square feet

Average Puddle Depth: 1 centimeters

Soil Type: Default $\quad$ Ground Temperature: $29^{\circ} \mathrm{C}$

Initial Puddle Temperature: Ground temperature

Release Duration: ALOHA limited the duration to 1 hour

Max Computed Release Rate: 1.19 kilograms / min

Max Average Sustained Release Rate: 1.15 grams/ min (averaged over a minute or more)

Total amount Released: 65.9 kilograms

\section{TMME DEPENDENT INFORMATION:}

Concentration Estimates at the point:

Downwind: 100 meters

Off Centerline: 0 meters

Max Concentration:

Outdoor: 2.9 ppm
Reviewed by S. A. Henderson

Works Calculation 8

Sheet No. 27 of 314 
Title of Project EPHA for THM

Subject Concentration Anăysis for THM Hazardous Chemicals

Computer N.E. Baucom B. K. Tharakan Date 09/16/96
Reviewed by S. A. Henderson

Works Calculation 8

Sheet No. 28 of 314

\section{1-RD-7 ADVERSE / MAX DIST SEG 1}

\section{SITE DATA INFORMATION:}

Location: AIKEN, SOUTH CAROLINA

Building Air Exchanges Per Hour: 0.44 (Sheltered single storied)

Time: June 30, 19982359 hours EDT (User specified)

\section{CHEMICAL INFORMATION:}

Chemical Name: BENZYL ALCOHOL

Molecular Weight: $108.14 \mathrm{~kg} / \mathrm{kmol}$

TLV-TWA: -unavail- IDLH: -unavail

Footprint Level of Concern: 100 ppm

Boiling Point: $204.70^{\circ} \mathrm{C}$

Vapor Pressure at Ambient Temperature: $1.54 \mathrm{e}-004 \mathrm{~atm}$

Ambient Saturation Concentration: 157 ppm or 0.016\%

\section{ATMOSPHERIC INFORMATION: (MANUAL INPUT OF DATA)}

Wind: 1.7 meters $/ \sec$ from $90^{\circ}$ true at 2 meters

Inversion Height: 200 meters

Stability Class: $\mathbf{E}$

Air Temperature: $29^{\circ} \mathrm{C}$

Relative Humidity: 50\% Ground Roughness: Urban or forest

Cloud Cover: 1 tenths

\section{SOURCE STRENGTH INFORMATION:}

Puddle Area: 24420 square feet

Average Puddle Depth: 1 centimeters

Soil Type: Default Ground Temperature: $29^{\circ} \mathrm{C}$

Initial Puddle Temperature: Ground temperature

Release Duration: ALOHA limited the duration to 1 hour

Max Computed Release Rate: 308 grams / min

Max Average Sustained Release Rate: 298 grams / min

(averaged over a minute or more)

Total amount Released: 17.0 kilograms

\section{FOOTPRINT INFORMATION:}

Dispersion Module: Gaussian

User-specified LOC: $100 \mathrm{ppm}$

Max Threat Zone for LOC: 27 meters 
Title of Project EPHA for THM

Subject Concentration Analysis for THM Hazardous Chemicals

Computer N.E. Baucom B. K. Tharakan Date 09/16/96

1-RD-7 ADVERSE/MAX DIST SEG 2

SITE DATA INFORMATION:

Location: AIKEN, SOUTH CAROLINA

Building Air Exchanges Per Hour: 0.44 (Sheltered single storied)

Time: June 30, 19982359 hours EDT (User specified)

\section{CHEMICAL INFORMATION:}

Chemical Name: BENZYL ALCOHOL

Molecular Weight: $108.14 \mathrm{~kg} / \mathrm{kmol}$

TLV.TWA: -unavail- IDLH: -unavail

Footprint Level of Concern: 100 ppm

Boiling Point: $204.70^{\circ} \mathrm{C}$

Vapor Pressure at Ambient Temperature: $1.54 \mathrm{e}-004 \mathrm{~atm}$

Ambient Saturation Concentration: 157 ppm or $0.016 \%$

ATMOSPHERIC INFORMATION: (MANUAL INPUT OF DATA)

Wind: 1.7 meters / $\sec$ from $90^{\circ}$ true at 2 meters

Inversion Height: 200 meters

Stability Class: $\mathbf{E}$

Air Temperature: $2^{\circ} \mathrm{C}$

Relative Humidity: 50\% Ground Roughness: Urban or forest

Reviewed by S. A. Hénderson

Works Calculation 8

Sheet No. 29 of 314

Cloud Cover: 1 tenths

SOURCE STRENGTH INFORMATION:

Puddle Area: 40700 square feet

Average Puddle Depth: 1 centimeters

Soil Type: Default $\quad$ Ground Temperature: $29^{\circ} \mathrm{C}$

Initial Puddle Temperature: Ground temperature

Release Duration: ALOHA limited the duration to 1 hour

Max Computed Release Rate: 503 grams / min

Max Average Sustained Release Rate: 486 grams / min

(averaged over a minute or more)

Total amount Released: 27.8 kilograms

FOOTPRINT INFORMATION:

Dispersion Module: Gaussian

User-specified LOC: 100 ppm

Max Threat Zone for LOC: 34 meters 
Title of Project EPHA for THM

Subject Concentration Analysis for THM Hazardous Chemicals

Computer N.E. Baucom B. K. Tharakan Date 09/16/96

1-RD-7 ADVERSE / MAX DIST SEG 3

\section{SITE DATA INFORMATION:}

Location: AIKEN, SOUTH CAROLINA

Building Air Exchanges Per Hour: 0.44 (Sheltered single storied)

Time: June 30, 19982359 hours EDT (User specified)

\section{CHEMICAL INFORMATION:}

Chemical Name: BENZYL ALCOHOL

Molecular Weight: $108.14 \mathrm{~kg} / \mathrm{kmol}$

TLV-TWA: -unavail-

IDLH: -unavail

Footprint Level of Concern: $100 \mathrm{ppm}$

Boiling Point: $204.70^{\circ} \mathrm{C}$

Vapor Pressure at Ambient Temperature: $1.54 \mathrm{e}-004 \mathrm{~atm}$

Ambient Saturation Concentration: 157 ppm or $0.016 \%$

\section{ATMOSPHERIC INFORMATION: (MANUAL INPUT OF DATA)}

Wind: 1.7 meters $/ \sec$ from $90^{\circ}$ true at 2 meters

Inversion Height: 200 meters

Stability Class: E

Relative Humidity: 50\%

Air Temperature: $29^{\circ} \mathrm{C}$

Cloud Cover: 1 tenths
Reviewed by S. A. Henderson

Works Calculation 8

Sheet No. 30 of 314

\section{SOURCE STRENGTH INFORMATION:}

Puddle Area: 10000 square meters

Average Puddle Depth: 1 centimeters

Soil Type: Default $\quad$ Ground Temperature: $29^{\circ} \mathrm{C}$

Initial Puddle Temperature: Ground temperature

Release Duration: ALOHA limited the duration to 1 hour

Max Computed Release Rate: 1.28 kilograms / min

Max Average Sustained Release Rate: 1.24 kilograms / min (averaged over a minute or more)

Total amount Released: 70.7 kilograms

FOOTPRINT INFORMATION:

Dispersion Module: Gaussian

User-specified LOC: 100 ppm

Max Threat Zone for LOC: 56 meters 
Title of Project EPHA for THM

Subject Concentration Analysis for THM Hazardous Chemicals

Computer N.E. Baucom B. K. Tharakan Date 09/16/96

\section{1-RD-9 ADVERSE / ALERT}

\section{SITE DATA INFORMATION:}

Location: AIKEN, SOUTH CAROLINA

Building Air Exchanges Per Hour: 60 (User specified)

Time: June 30, 19982359 hours EDT (User specified)

CHEMICAL INFORMATION:

Chemical Name: BUTYRALDEHYDE

Molecular Weight: $72.11 \mathrm{~kg} / \mathrm{kmol}$

TLV-TWA: -unavail-

IDLH: -unavail

Footprint Level of Concern: $500 \mathrm{mg} /(\mathrm{cu} \mathrm{m}$ )

Boiling Point: $74.80^{\circ} \mathrm{C}$

Vapor Pressure at Ambient Temperature: $0.18 \mathrm{~atm}$

Ambient Saturation Concentration: 179,846 ppm or $18.0 \%$

ATMOSPHERIC INFORMATION: (MANUAL INPUT OF DATA)

Wind: 1.7 meters $/ \mathrm{sec}$ from $90^{\circ}$ true at 2 meters

Inversion Height: 200 meters

Stability Class: E

Air Temperature: $29^{\circ} \mathrm{C}$

Relative Humidity: $50 \% \quad$ Ground Roughness: Urban or forest

Cloud Cover: 1 tenths

SOURCE STRENGTH INFORMATION:

Puddle Area: 84 square feet

Average Puddle Depth: 1 centimeters

Soil Type: Default $\quad$ Ground Temperature: $29^{\circ} \mathrm{C}$

Initial Puddle Temperature: Ground temperature

Release Duration: ALOHA limited the duration to 1 hour

Max Computed Release Rate: 1.29 kilograms / min

Max Average Sustained Release Rate: 1.03 kilograms/min

(averaged over a minute or more)

Total amount Released: $\mathbf{4 8 . 5}$ kilograms

\section{TIME DEPENDENT INFORMATION:}

Concentration Estimates at the point:

Downwind: 30 meters

Off Centerline: 0 meters

Max Concentration:

Outdoor: $497 \mathrm{mg} /(\mathrm{cu} \mathrm{m})$
Reviewed by S. A. Henderson

Works Calculation 8

Sheet No. 31 of 314 
Title of Project EPHA for THM

Subject Concentration Analysis for THM Hazardous Chemicals

Computer N.E. Baucom B. K. Tharakan Date 09/16/96
Reviewed by S. A. Henderson

Works Calculation 8

Sheet No. $\underline{32 \text { of } 314}$

\section{1-RD-9 ADVERSE / SAE}

SITE DATA INFORMATION:

Location: AIKEN, SOUTH CAROLINA

Building Air Exchanges Per Hour: 60 (User specified)

Time: June 30, 19982359 hours EDT (User specified)

\section{CHEMICAL INFORMATION:}

Chemical Name: BUTYRALDEHYDE

Molecular Weight: $72.11 \mathrm{~kg} / \mathrm{kmol}$

TLV-TWA: -unavail- IDLH: -unavail

Footprint Level of Concern: $500 \mathrm{mg} /(\mathrm{cu} \mathrm{m}$ )

Boiling Point: $74.80^{\circ} \mathrm{C}$

Vapor Pressure at Ambient Temperature: $0.18 \mathrm{~atm}$

Ambient Saturation Concentration: 179,846 ppm or 18.0\%

ATMOSPHERIC INFORMATION: (MANUAL INPUT OF DATA)

Wind: 1.7 meters $/ \sec$ from $90^{\circ}$ true at 2 meters

Inversion Height: 200 meters

Stability Class: E

Relative Humidity: 50\%

Air Temperature: $29^{\circ} \mathrm{C}$

Cloud Cover: 1 tenths

Ground Roughness: Urban or forest

\section{SOURCE STRENGTH INFORMATION:}

Puddle Area: 777 square feet

Average Puddle Depth: 1 centimeters

Soil Type: Default Ground Temperature: $29^{\circ} \mathrm{C}$

Initial Puddle Temperature: Ground temperature

Release Duration: ALOHA limited the duration to 1 hour

Max Computed Release Rate: 10.5 kilograms / min

Max Average Sustained Release Rate: 8.53 kilograms / min (averaged over a minute or more)

Total amount Released: 409 kilograms

FOOTPRINT INFORMATION:

Model Run: Heavy Gas

User-specified LOC: $500 \mathrm{mg} /(\mathrm{cu} \mathrm{m})$

Max Threat Zone for LOC: 100 meters

Note: Footprint was not drawn because effects of near-field patchiness make dispersion predictions unreliable for short distances.

TIME DEPENDENT INFORMATION:

Concentration Estimates at the point:

Downwind: 100 meters

Off Centerline: 0 meters

Max Concentration:

Outdoor: $500 \mathrm{mg} /(\mathrm{cu} \mathrm{m})$ 
Title of Project EPHA for THM

Subject Concentration Analysis for THM Hazardous Chemicals

Computer N.E. Baucom B. K. Tharakan Date 09/16/96
Reviewed by S. A. Henderson Works Calculation 8

Sheet No. 33 of 314

\section{1-RD-9 ADVERSE / MAX DIST SEG 1}

\section{SITE DATA INEORMATION:}

Location: AIKEN, SOUTH CAROLINA

Building Air Exchanges Per Hour: 60 (User specified)

Time: June 30, 19982359 hours EDT (User specified)

\section{CHEMICAL INFORMATION:}

Chemical Name: BUTYRALDEHYDE

Molecular Weight: $72.11 \mathrm{~kg} / \mathrm{kmol}$

TLV.TWA: -unavail- IDLH: -unavail

Footprint Level of Concern: $500 \mathrm{mg} /(\mathrm{cu} \mathrm{m}$ )

Boiling Point: $74.80^{\circ} \mathrm{C}$

Vapor Pressure at Ambient Temperature: 0.18 atm

Ambient Saturation Concentration: $179,846 \mathrm{ppm}$ or $18.0 \%$

\section{ATMOSPHERIC INFORMATION: (MANUAL INPUT OF DATA)}

Wind: 1.7 meters / sec from $90^{\circ}$ true at 2 meters

Inversion Height: 200 meters

Stability Class: E

Air Temperature: $29^{\circ} \mathrm{C}$

Relative Humidity: 50\%

Ground Roughness: Urban or forest

Cloud Cover: 1 tenths

\section{SOURCE STRENGTH INFORMATION:}

Puddle Area: 24420 square feet

Average Puddle Depth: 1 centimeters

Soil Type: Default $\quad$ Ground Temperature: $29^{\circ} \mathrm{C}$

Initial Puddle Temperature: Ground temperature

Release Duration: ALOHA limited the duration to 1 hour

Max Computed Release Rate: 280 kilograms / min

Max Average Sustained Release Rate: 233 kilograms / min (averaged over a minute or more)

Total amount Released: 11,338 kilograms

\section{FOOTPRINT INFORMATION:}

Model Run: Heavy Gas

User-specified LOC: $500 \mathrm{mg} /(\mathrm{cu} \mathrm{m})$

Max Threat Zone for LOC: 532 meters 
Title of Project EPHA for THM

Subject Concentration Analysis for THM Hazardous Chemicals

Computer N.E. Baucom B. K. Tharakan Date 09/16/96
Reviewed by S. A. Henderson Works Calculation 8

Sheet No. 34 of 314

\title{
1-RD-9 ADVERSE / MAX DIST SEG 2
}

\section{SITE DATA INFORMATION:}

Location: AIKEN, SOUTH CAROLINA

Building Air Exchanges Per Hour: 60 (User specified)

Time: June 30, 19982359 hours EDT (User specified)

\author{
CHEMICAL INFORMATION: \\ Chemical Name: BUTYRALDEHYDE \\ Molecular Weight: $72.11 \mathrm{~kg} / \mathrm{kmol}$ \\ TLV-TWA: -unavail- IDLH: -unavail \\ Footprint Level of Concern: $500 \mathrm{mg} /(\mathrm{cu} \mathrm{m}$ ) \\ Boiling Point: $74.80^{\circ} \mathrm{C}$ \\ Vapor Pressure at Ambient Temperature: $0.18 \mathrm{~atm}$ \\ Ambient Saturation Concentration: 179,846 ppm or $18.0 \%$
}

\section{ATMOSPHERIC INFORMATION: (MANUAL INPUT OF DATA)}

Wind: 1.7 meters / sec from $90^{\circ}$ true at 2 meters

Inversion Height: 200 meters

Stability Class: E

Air Temperature: $29^{\circ} \mathrm{C}$

Relative Humidity: 50\% Ground Roughness: Urban or forest

Cloud Cover: 1 tenths

\section{SOURCE STRENGTH INFORMATION:}

Puddle Area: 40700 square feet

Average Puddle Depth: 1 centimeters

Soil Type: Default Ground Temperature: $29^{\circ} \mathrm{C}$

Initial Puddle Temperature: Ground temperature

Release Duration: ALOHA limited the duration to 1 hour

Max Computed Release Rate: 456 kilograms / min

Max Average Sustained Release Rate: 379 kilograms / min

(averaged over a minute or more)

Total amount Released: 18,579 kilograms

\section{FOOTPRINT INFORMATION:}

Model Run: Heavy Gas

User-specified LOC: $500 \mathrm{mg} /(\mathrm{cu} \mathrm{m})$

Max Threat Zone for LOC: 686 meters 
Title of Project EPHA for THM

Subject Concentration Analysis for THM Hazardous Chemicals

Computer N.E. Baucom B. K. Tharakan Date 09/16/96

\section{1-RD.9 ADVERSE / MAX DIST SEG 3}

\section{SITE DATA INFORMATION:}

\section{Location: AIKEN, SOUTH CAROLINA}

Building Air Exchanges Per Hour: 60 (User specified)

Time: June 30, 19982359 hours EDT (User specified)

\section{CHEMICAL INFORMATION:}

Chemical Name: BUTYRALDEHYDE

Molecular Weight: $72.11 \mathrm{~kg} / \mathrm{kmol}$

TLV-TWA: -unavail- TDLH: -unavail

Footprint Level of Concern: $500 \mathrm{mg} /(\mathrm{cu} \mathrm{m}$ )

Boiling Point: $74.80^{\circ} \mathrm{C}$

Vapor Pressure at Ambient Temperature: $0.18 \mathrm{~atm}$

Ambient Saturation Concentration: 179,846 ppm or $18.0 \%$

ATMOSPHERIC INFORMATION: (MANUAL INPUT OF DATA)

Wind: 1.7 meters $/ \sec$ from $90^{\circ}$ true at 2 meters

Inversion Height: 200 meters

Stability Class: E

Relative Humidity: 50\%

Air Temperature: $29^{\circ} \mathrm{C}$

Cloud Cover: 1 tenths

Ground Roughness: Urban or forest
Reviewed by S.. A. Henderson

Works Calculation 8

Sheet No. 35 of 314

\section{SOURCE STRENGTH INFORMATION:}

Puddle Area: 10000 square meters

Average Puddle Depth: 1 centimeters

Soil Type: Default $\quad$ Ground Temperature: $29^{\circ} \mathrm{C}$

Initial Puddle Temperature: Ground temperature

Release Duration: ALOHA limited the duration to 1 hour

Max Computed Release Rate: 1,160 kilograms / min

Max Average Sustained Release Rate: 965 kilograms / min (averaged over a minute or more)

Total amount Released: 47,589 kilograms

\section{FOOTPRINT INFORMATION:}

Model Run: Heavy Gas

User-specified LOC: $500 \mathrm{mg} /(\mathrm{cu} \mathrm{m})$

Max Threat Zone for LOC: 1.1 kilometers 
Title of Project EPHA for THM

Subject Concentration Analysis for THM Hazardous Chemicals

Computer N.E. Baucom B. K. Tharakan Date 09/16/96

\section{1-RD-11 ADVERSE / ALERT}

\section{SITE DATA INFORMATION:}

Location: AIKEN, SOUTH CAROLINA

Building Air Exchanges Per Hour: 0.44 (sheltered single storied)

Time: June 30, 19982359 hours EDT (User specified)

\section{CHEMICAL INFORMATION:}

Chemical Name: CHLORACETONE

Molecular Weight: $92.52 \mathrm{~kg} / \mathrm{kmol}$

TLV-TWA: -unavailILH: -unavail

Footprint Level of Concern: 1 ppm

Boiling Point: $119.00^{\circ} \mathrm{C}$

Vapor Pressure at Ambient Temperature: $0.026 \mathrm{~atm}$

Ambient Saturation Concentration: 26,221 ppm or $2.62 \%$

Note: Not enough chemical data to use Heavy Gas option

ATMOSPHERIC INFORMATION: (MANUAL INPUT OF DATA)

Wind: 1.7 meters $/ \sec$ from $90^{\circ}$ true at 2 meters

Inversion Height: 200 meters

Stability Class: E

Air Temperature: $29^{\circ} \mathrm{C}$

Relative Humidity: $50 \%$

Ground Roughness: Urban or forest
Reviewed by S.A. Henderson

Works Calculation 8

Sheet No. 36 of 314

Cloud Cover: 1 tenths

\section{SOURCE STRENGTH INFORMATION:}

Direct Source: 1.08E-4 kilograms / sec

Source Height: 0

Release Duration : 60 minutes

Release Rate: 6.47 grams/min

Total amount Released: $\mathbf{3 8 8}$ grams

\section{TIME DEPENDENT INFORMATION:}

Concentration Estimates at the point:

Downwind: 30 meters

Off Centerline: 0 meters

Max Concentration:

Outdoor: 1 ppm

Indoor: $0.356 \mathrm{ppm}$

Note: Indoor graph is shown with a dotted line. 
Title of Project EPHA for THM

Subject Concentration Analysis for THM Hazardous Chemicals

Computer N.E. Baucom B. K. Tharakan Date 09/16/96
Reviewed by S. A. Henderson Works Calculation 8

Sheet No. 37 of 314

\section{SPILL AREA CALCULATION FOR 1-RD-11 ADVERSE / ALERT CONDITIONS}

\begin{tabular}{|c|c|c|c|c|}
\hline $\begin{array}{ll}\text { Parameter } & \\
\end{array}$ & Value & Dimensions & Value & $\begin{array}{c}\text { Dimensions } \\
\text { (USA) }\end{array}$ \\
\hline E (Evaporation Rate) & $1.08 \mathrm{E}-4$ & $\mathrm{~kg} / \mathrm{s}$ & & \\
\hline MW (Molecular Weight) & 93 & $\mathrm{~kg} / \mathrm{kg}$-mole & & \\
\hline Pv (Vapor Pressure) & 12.00 & $\mathrm{~mm} \mathrm{Hg}$ & & \\
\hline Pv (Vapor Pressure) & 1600 & $\mathbf{P a}$ & & \\
\hline Rg (Gas Law Constant) & 8314 & J/kgmole-K & & \\
\hline Temperature & 29 & $\mathrm{C}$ & & \\
\hline Volume of Spill & 0.8 & gal & & \\
\hline d (Depth of Pool) & 0.01 & m & & \\
\hline A (Surface Area of Spill) & 0.3 & $\mathbf{m}^{\wedge} \mathbf{2}$ & 3 & $\mathrm{ft}^{\wedge} 2$ \\
\hline Km (Mass Transfer Coeff) & $6.06 \mathrm{E}-3$ & $\mathrm{~m} / \mathrm{s}$ & & \\
\hline Z (Pool Diameter in Wind Direction) & 1 & $\mathbf{m}$ & & \\
\hline Sc (Schmidt Number) & 1.42 & & & \\
\hline $\mathrm{U}$ (Wind velocity) & 1.7 & $\mathbf{m} / \mathbf{s}$ & & \\
\hline nu (kinematic viscosity of air) & $1.50 \mathrm{E}-5$ & $\mathrm{~m}^{\wedge} 2 / \mathrm{sec}$ & & \\
\hline mu (viscosity of air) & $1.90 \mathrm{E}-5$ & $\mathbf{k g} / \mathbf{m}-\mathbf{s}$ & & \\
\hline $\begin{array}{l}\text { Dm (Molecular Diffusivity of Solute } \\
\text { in Air) }\end{array}$ & $1.06 \mathrm{E}-5$ & $\mathrm{~m}^{\wedge} 2 / \mathrm{sec}$ & & \\
\hline $\begin{array}{l}\text { D_H20 (Molecular Diffusivity of } \\
\text { Water) }\end{array}$ & $2.40 \mathrm{E}-5$ & & & \\
\hline MW_H20 & 18 & kg/kg-mole & & \\
\hline \multicolumn{5}{|l|}{ Conversions } \\
\hline T (Absolute) & 302.15 & ${ }^{\cdot} \mathbf{K}$ & & \\
\hline V (Volume) & 0.003 & $\mathbf{m}^{\wedge} \mathbf{3}$ & & \\
\hline Evaporation Rate & 6 & g/min & & \\
\hline $60 \mathrm{~min} / \mathrm{lb}$ released & 0.86 & & & \\
\hline
\end{tabular}


Title of Project EPHA for THM

Subject Concentration Analysis for THM Hazardous Chemicals

Computer N.E. Baucom B. K. Tharakan Date 09/16/96

\section{1-RD-11 ADVERSE / SAE}

\section{SITE DATA INFORMATION:}

Location: AIKEN, SOUTH CAROLINA

Building Air Exchanges Per Hour: 0.44 (Sheltered single storied)

Time: June 30, 19982359 hours EDT (User specified)

CHEMICAL INFORMATION:

Chemical Name: CHLORACETONE

Molecular Weight: $92.52 \mathrm{~kg} / \mathrm{kmol}$

TLV-TWA: -unavail-

Footprint Level of Concern: 1 ppm

Boiling Point: $119.00^{\circ} \mathrm{C}$

Vapor Pressure at Ambient Temperature: $0.026 \mathrm{~atm}$

Ambient Saturation Concentration: 26,221 ppm or 2.62\%

Note: Not enough chemical data to use Heavy Gas option

ATMOSPHERIC INFORMATION: (MANUAL INPUT OF DATA)

Wind: 1.7 meters $/ \sec$ from $90^{\circ}$ true at 2 meters

Inversion Height: 200 meters

Stability Class: E

Air Temperature: $29^{\circ} \mathrm{C}$

Relative Humidity: 50\%

Ground Roughness: Urban or forest
Reviewed by S.A. Henderson

Works Calculation 8

Sheet No. $\underline{38 \text { of } 314}$

\section{SOURCE STRENGTH INFORMATION:}

Direct Source: 1.13E-3 kilograms / sec

Source Height: 0

Release Duration : 60 minutes

Release Rate: 67.8 grams/min

Total amount Released: 4.07 kilograms

TIME DEPENDENT INFORMATION:

Concentration Estimates at the point:

Downwind: 100 meters

Off Centerline: 0 meters

Max Concentration:

Outdoor: $0.997 \mathrm{ppm}$

Indoor: $0.353 \mathrm{ppm}$

Note: Indoor graph is shown with a dotted line. 
Title of Project EPHA for THM

Subject Concentration Analysis for THM Hazardous Chemicals

Computer N.E. Baucom B.K. Tharakan Date 09/16/96
Reviewed by S. A. Henderson Works Calculation 8

Sheet No. 39 of 314

\section{SPILL AREA CALCULATION FOR 1-RD-11 ADVERSE / SAE CONDITIONS}

\begin{tabular}{|c|c|c|c|c|}
\hline Parameter & Value & Dimensions & Value & $\begin{array}{l}\text { Dimensions } \\
\text { (USA) }\end{array}$ \\
\hline E (Evaporation Rate) & 1.13E-3 & $\mathrm{kg} / \mathrm{s}$ & & \\
\hline MW (Molecular Weight) & 93 & $\mathrm{~kg} / \mathrm{kg}$-mole & & \\
\hline Py (Vapor Pressure) & 12.00 & $\mathrm{~mm} \mathrm{Hg}$ & & \\
\hline Pv (Vapor Pressure) & 1600 & $\mathbf{P a}$ & & \\
\hline $\mathbf{R g}$ (Gas Law Constant) & 8314 & J/kgmole-K & & \\
\hline Temperature & 29 & ${ }^{\circ} \mathrm{C}$ & & \\
\hline Volume of Spill & 9.6 & gal & & 7 \\
\hline d (Depth of Pool) & 0.01 & m & & \\
\hline A (Surface Area of Spill) & 3.6 & $\mathbf{m}^{\wedge} \mathbf{2}$ & 39 & $\mathrm{ft}^{\wedge} \mathbf{2}$ \\
\hline Km (Mass Transfer Coeff) & $5.28 \mathrm{E}-3$ & $\mathrm{~m} / \mathrm{s}$ & & \\
\hline Z (Pool Diameter in Wind Direction) & 2 & $\mathbf{m}$ & & \\
\hline Sc (Schmidt Number) & 1.42 & & & \\
\hline $\mathrm{U}$ (Wind velocity) & 1.7 & $\mathrm{~m} / \mathrm{s}$ & & \\
\hline nu (kinematic viscosity of air) & $1.50 \mathrm{E}-5$ & $\mathrm{~m}^{\wedge} 2 / \mathrm{sec}$ & & \\
\hline mu (viscosity of air) & $1.90 \mathrm{E}-5$ & $\mathrm{~kg} / \mathrm{m}-\mathrm{s}$ & & \\
\hline $\begin{array}{l}\text { Dm (Molecular Diffusivity of Solute } \\
\text { in Air) }\end{array}$ & $1.06 \mathrm{E}-5$ & $\mathrm{~m}^{\wedge} 2 / \mathrm{sec}$ & & \\
\hline $\begin{array}{l}\text { D_H20 (Molecular Diffusivity of } \\
\text { Water) }\end{array}$ & $2.40 \mathrm{E}-5$ & & & \\
\hline MW_H20 & 18 & $\mathrm{~kg} / \mathrm{kg}$-mole & & \\
\hline Conversions & . & & & \\
\hline T(Absolute) & $\mathbf{3 0 2 . 1 5}$ & ${ }^{\circ} \mathbf{K}$ & & \\
\hline $\mathrm{V}$ (Volume) & 0.036 & $\mathbf{m}^{\wedge} \mathbf{3}$ & & \\
\hline Evaporation Rate & 68 & $\mathrm{~g} / \mathrm{min}$ & & \\
\hline $60 \mathrm{~min} / \mathrm{b}$ released & 8.97 & & & \\
\hline
\end{tabular}


Title of Project EPHA for THM

Subject Concentration Analysis for THM Hazardous Chemicals

Computer N.E. Baucom B. K. Tharakan Date 09/16/96

\section{1-RD-11 ADVERSE / MAX DIST SEG 1}

\section{SITE DATA INEORMATION:}

Location: AIKEN, SOUTH CAROLINA

Building Air Exchanges Per Hour: 0.44 (Sheltered single storied)

Time: June 30, 19982359 hours EDT (User specified)

\section{CHEMICAL INFORMATION:}

Chemical Name: CHLORACETONE

Molecular Weight: $92.52 \mathrm{~kg} / \mathrm{kmol}$

TLV-TWA: -unavailIDLH: -unavail

Footprint Level of Concern: 1 ppm

Boiling Point: $119.00^{\circ} \mathrm{C}$

Vapor Pressure at Ambient Temperature: $0.026 \mathrm{~atm}$

Ambient Saturation Concentration: 26,221 ppm or $2.62 \%$

Note: Not enough chemical data to use Heavy Gas option

\section{ATMOSPHERIC INFORMATION: (MANUAL INPUT OF DATA)}

Wind: 1.7 meters / sec from $90^{\circ}$ true at 2 meters

Inversion Height: 200 meters

Stability Class: E

Relative Humidity: $50 \%$

Air Temperature: $29^{\circ} \mathrm{C}$

Cloud Cover: 1 tenths
Reviewed by S. A. Henderson

Works Calculation 8

Sheet No. 40 of 314

\section{SOURCE STRENGTH INFORMATION:}

Direct Source: 4.94E-1 kilograms / sec

Source Height: 0

Release Duration: 60 minutes

Release Rate: 29.6 kilograms / min

Total amount Released: 1,778 kilograms

FOOTPRINT INFORMATION:

Dispersion Module: Gaussian

User-specified LOC: 1 ppm

Max Threat Zone for LOC: 3.4 kilometers 
Title of Project EPHA for THM

Subject Concentration Analysis for THM Hazardous Chemicals

Computer N.E. Baucom B. K. Tharakan Date 09/16/96
Reviewed by S. A. Henderson Works Calculation 8

Sheet No. 41 of 314

Determination of Evaporation Rate for Segment 1 Maximum Source Term of $24420 \mathrm{ft}^{2}$

\begin{tabular}{|c|c|c|c|c|}
\hline Parameter & Value & Dimensions & Value & $\begin{array}{c}\text { Dimensions } \\
\text { (USA) }\end{array}$ \\
\hline E (Evaporation Rate) & $4.94 \mathrm{E}-1$ & $\mathrm{~kg} / \mathrm{s}$ & & \\
\hline MW (Molecular Weight) & 93 & $\mathrm{~kg} / \mathrm{kg}$-mole & & . \\
\hline Pv (Vapor Pressure) & 12.00 & $\mathrm{~mm} \mathrm{Hg}$ & & \\
\hline Pv (Vapor Pressure) & 1600 & $\mathbf{P a}$ & & \\
\hline Rg (Gas Law Constant) & 8314 & J/kgmole-K & & \\
\hline Temperature & 29 & $\mathrm{C}$ & & \\
\hline Volume of Spill & 5993 & gal & & \\
\hline d (Depth of Pool) & 0.01 & $\mathbf{m}$ & & \\
\hline A (Surface Area of Spill) & 2268.5 & $m^{\wedge} \mathbf{2}$ & 24421 & $\mathrm{ft}^{\wedge} \mathbf{2}$ \\
\hline Km (Mass Transfer Coefi) & $3.69 \mathrm{E}-3$ & $\mathrm{~m} / \mathrm{s}$ & & \\
\hline Z (Pool Diameter in Wind Direction) & 54 & $\mathbf{m}$ & & \\
\hline Sc (Schmidt Number) & 1.42 & & & \\
\hline U (Wind velocity) & 1.7 & $\mathbf{m} / \mathbf{s}$ & & \\
\hline nu (kinematic viscosity of air) & $1.50 \mathrm{E}-5$ & $m^{\wedge} 2 / \sec$ & & \\
\hline mu (viscosity of air) & $1.90 \mathrm{E}-5$ & $\mathrm{~kg} / \mathrm{m}-\mathrm{s}$ & & \\
\hline $\begin{array}{l}\text { Dm (Molecular Diffusivity of Solute } \\
\text { in Air) }\end{array}$ & $1.06 \mathrm{E}-5$ & $\mathrm{~m}^{\wedge} 2 / \mathrm{sec}$ & & \\
\hline $\begin{array}{l}\text { D_H20 (Molecular Diffusivity of } \\
\text { Water) }\end{array}$ & $2.40 \mathrm{E}-5$ & & & \\
\hline MW_H20 & 18 & kg/kg-mole & & \\
\hline \multicolumn{5}{|l|}{ Conversions } \\
\hline T (Absolute) & 302.15 & $\cdot \mathbf{K}$ & & \\
\hline V (Volume) & 22.685 & $m^{\wedge} 3$ & & \\
\hline Evaporation Rate & 29613 & $\mathrm{~g} / \mathrm{min}$ & & \\
\hline $60 \mathrm{~min} 1 \mathrm{~b}$ released & 3917.12 & & & \\
\hline
\end{tabular}


Title of Project EPHA for THM

Subject Concentration Analysis for THM Hazardous Chemicals

Computer N.E. Baucom B. K. Tharakan Date 09/16/96

1-RD-11 ADVERSE / MAX DIST SEG 2

SITE DATA INFORMATION:

Location: AIKEN, SOUTH CAROLINA

Building Air Exchanges Per Hour: 0.44 (Sheltered single storied)

Time: June 30, 19982359 hours EDT (User specified)

CHEMICAL INFORMATION:

Chemical Name: CHLORACETONE

Molecular Weight: $92.52 \mathrm{~kg} / \mathrm{kmol}$

TLV-TWA: -unavail-

Footprint Level of Concern: 1 ppm

Boiling Point: $119.00^{\circ} \mathrm{C}$

Vapor Pressure at Ambient Temperature: $0.026 \mathrm{~atm}$

Ambient Saturation Concentration: 26,221 ppm or 2.62\%

Note: Not enough chemical data to use Heavy Gas option

ATMOSPHERIC INFORMATION: (MANUAL INPUT OF DATA)

Wind: 1.7 meters / sec from $90^{\circ}$ true at 2 meters

Inversion Height: 200 meters

Stability Class: E

Air Temperature: $29^{\circ} \mathrm{C}$

Relative Humidity: $50 \%$ - Ground Roughness: Urban or forest

Cloud Cover: 1 tenths

\section{SOURCE STRENGTH INFORMATION:}

Direct Source: 8E-1 kilograms / sec

Source Height: 0

Release Duration: 60 minutes

Release Rate: 48 kilograms / min

Total amount Released: 2,880 kilograms

FOOTPRINT INFORMATION:

Dispersion Module: Gaussian

User-specified LOC: 1 ppm

Max Threat Zone for LOC: 4.8 kilometers
Reviewed by S. A. Henderson

Works Calculation 8

Sheet No. 42 of 314 
Title of Project EPHA for THM

Subject Cencentration Analysis for THM Hazardous Chemicals

Computer N.E. Baucom B. K. Tharakan Date 09/16/96
Reviewed by S. A. Henderson

Works Calculation 8

Sheet No. $\underline{43 \text { of } 314}$

Determination of Evaporation Rate for Segment 2 Maximum Source Term of $40700 \mathrm{ft}^{2}$

\begin{tabular}{|c|c|c|c|c|}
\hline Parameter : & Value & Dimensions & Value & $\begin{array}{c}\text { Dimensions } \\
\text { (USA) }\end{array}$ \\
\hline E (Evaporation Rate) & $8.00 \mathrm{E}-1$ & $\mathrm{~kg} / \mathrm{s}$ & & \\
\hline MW (Molecular Weight) & 93 & $\mathrm{~kg} / \mathrm{kg}$-mole & & \\
\hline Pv (Vapor Pressure) & 12.00 & $\mathrm{~mm} \mathrm{Hg}$ & & \\
\hline Pv (Vapor Pressure) & 1600 & $\mathbf{P a}$ & & \\
\hline Rg (Gas Law Constant) & 8314 & J/kgmole-K & & \\
\hline Temperature & 29 & C & & \\
\hline Volume of Spill & 9988 & gal & & \\
\hline d (Depth of Pool) & 0.01 & $\mathbf{m}$ & & \\
\hline A (Surface Area of Spill) & 3780.8 & $\mathrm{~m}^{\wedge} \mathbf{2}$ & 40700 & $\mathrm{ft}^{\wedge} \mathbf{2}$ \\
\hline $\mathrm{Km}$ (Mass Transfer Coeff) & $3.59 \mathrm{E}-3$ & $\mathrm{~m} / \mathrm{s}$ & & . \\
\hline $\mathrm{Z}$ (Pool Diameter in Wind Direction) & 69 & $\mathbf{m}$ & & \\
\hline Sc (Schmidt Number) & 1.42 & & & \\
\hline $\mathrm{U}$ (Wind velocity) & 1.7 & $\mathbf{m} / \mathbf{s}$ & & \\
\hline nu (kinematic viscosity of air) & $1.50 \mathrm{E}-5$ & $m^{\wedge} 2 /$ sec & & \\
\hline mu (viscosity of air) & $1.90 \mathrm{E}-5$ & $\mathrm{~kg} / \mathrm{m}-\mathrm{s}$ & & \\
\hline $\begin{array}{l}\text { Dm (Molecular Diffusivity of Solute } \\
\text { in Air) }\end{array}$ & $1.06 \mathrm{E}-5$ & $\mathrm{~m}^{\wedge} 2 / \mathrm{sec}$ & & \\
\hline $\begin{array}{l}\text { D_H20 (Molecular Diffusivity of } \\
\text { Water) }\end{array}$ & $2.40 \mathrm{E}-5$ & & & \\
\hline MW_H2O & 18 & kg/kg-mole & & \\
\hline \multicolumn{5}{|l|}{ Conversions } \\
\hline $\mathbf{T}$ (Absolute) & 302.15 & $\bar{K}$ & & \\
\hline V (Volume) & 37.808 & $\mathbf{m}^{\wedge} \mathbf{3}$ & & \\
\hline Evaporation Rate & 47973 & $\mathrm{~g} / \mathrm{min}$ & & \\
\hline 60 min $1 \mathrm{~b}$ released & 6345.66 & & & \\
\hline
\end{tabular}


Title of Project EPHA for THM

Subject Concentration Analysis for THM Hazardous Chemicals

Computer N.E. Baucom B. K. Tharakan Date 09/16/96

\section{1-RD-11 ADVERSE / MAX DIST SEG 3}

\section{SITE DATA INFORMATION:}

Location: AIKEN, SOUTH CAROLINA

Building Air Exchanges Per Hour: 0.44 (Sheltered single storied)

Time: June 30, 19982359 hours EDT (User specified)

\section{CHEMICAL INFORMATION:}

Chemical Name: CHLORACETONE

Molecular Weight: $92.52 \mathrm{~kg} / \mathrm{kmol}$

TLV-TWA: -unavailDLH: -unavail

Footprint Level of Concern: 1 ppm

Boiling Point: $119.00^{\circ} \mathrm{C}$

Vapor Pressure at Ambient Temperature: $0.026 \mathrm{~atm}$

Ambient Saturation Concentration: 26,221 ppm or 2.62\%

Note: Not enough chemical data to use Heavy Gas option

ATMOSPHERIC INFORMATION: (MANUAL INPUT OF DATA)

Wind: 1.7 meters $/ \sec$ from $90^{\circ}$ true at 2 meters

Inversion Height: 200 meters

Stability Class: E

Air Temperature: $29^{\circ} \mathrm{C}$

Relative Humidity: $50 \%$

Ground Roughness: Urban or forest
Reviewed by S. A. Henderson

Works Calculation 8

Sheet No. 44 of 314

Cloud Cover: 1 tenths

SOURCE STRENGTH INFORMATION:

Direct Source: 2 kilograms / sec

Source Height: 0

Release Duration: 60 minutes

Release Rate: 120 kilograms / min

Total amount Released: 7,200 kilograms

FOOTPRINT INFORMATION:

Dispersion Module: Gaussian

User-specified LOC: 1 ppm

Max Threat Zone for LOC: greater than $10 \mathrm{~km}$ 
Title of Project EPHA for THM

Subject Concentration Analysis for THM Hazardous Chemicals

Computer N.E. Baucom B. K. Tharakan Date 09/16/96
Reviewed by S.A. A. Henderson

Works Calculation 8

Sheet No. 45 of 314

Determination of Evaporation Rate for Segment 3 Maximum Source Term of $10000 \mathrm{~m}^{2}$

\begin{tabular}{|c|c|c|c|c|}
\hline Parameter & Value & Dimensions & Value & $\begin{array}{c}\text { Dimensions } \\
\text { (USA) }\end{array}$ \\
\hline E (Evaporation Rate) & $2.00 \mathrm{E}+0$ & $\mathrm{~kg} / \mathrm{s}$ & & \\
\hline MW (Molecular Weight) & 93 & $\mathrm{~kg} / \mathrm{kg}$-mole & & \\
\hline Pv (Vapor Pressure) & 12.00 & $\mathrm{~mm} \mathrm{Hg}$ & & \\
\hline Py (Vapor Pressure) & 1600 & $\mathbf{P a}$ & & \\
\hline Rg (Gas Law Constant) & 8314 & J/kgmole-K & & \\
\hline Temperature & 29 & $\mathrm{C}$ & & \\
\hline Volume of Spill & 26418 & gal & & \\
\hline d (Depth of Pool) & 0.01 & m & & \\
\hline A (Surface Area of Spill) & 10000.0 & $\mathbf{m}^{\wedge} \mathbf{2}$ & 107650 & $\mathrm{ft}^{\wedge} \mathbf{2}$ \\
\hline $\mathrm{Km}$ (Mass Transfer Coeff) & $3.40 \mathrm{E}-3$ & $\mathbf{m} / \mathbf{s}$ & & \\
\hline $\mathbf{Z}$ (Pool Diameter in Wind Direction) & 113 & $\mathbf{m}$ & & \\
\hline Sc (Schmidt Number) & 1.42 & & & \\
\hline $\mathrm{U}$ (Wind velocity) & 1.7 & $\mathrm{~m} / \mathrm{s}$ & & \\
\hline nu (kinematic viscosity of air) & $1.50 \mathrm{E}-5$ & $\mathrm{~m}^{\wedge} \mathbf{2} / \mathrm{sec}$ & & \\
\hline mu (viscosity of air) & $1.90 \mathrm{E}-5$ & $\mathrm{~kg} / \mathrm{m}-\mathbf{s}$ & & \\
\hline $\begin{array}{l}\text { Dm (Molecular Diffusivity of Solute } \\
\text { in Air) }\end{array}$ & $1.06 \mathrm{E}-5$ & $\mathrm{~m}^{\wedge} 2 / \mathrm{sec}$ & & \\
\hline $\begin{array}{l}\text { D_H20 (Molecular Diffusivity of } \\
\text { Water) }\end{array}$ & $2.40 \mathrm{E}-5$ & * & & \\
\hline MW_H20 & 18 & kg/kg.mole & & \\
\hline \multicolumn{5}{|l|}{ Conversions } \\
\hline T (Absolute) & 302.15 & 'K & & \\
\hline$V$ (Volume) & 100.000 & $\mathrm{~m}^{\wedge} \mathbf{3}$ & & \\
\hline Evaporation Rate & 120213 & $\mathrm{~g} / \mathrm{min}$ & & \\
\hline $60 \mathrm{~min}$ Ib released & 15901.21 & & & \\
\hline
\end{tabular}


Title of Project EPHA for THM

Subject Concentration Analvsis for THM Hazardous Chemicals

Computer N.E. Baucom B. K. Tharakan Date 09/16/96
Reviewed by S. A. Henderson Works Calculation 8

Sheet No. 46 of 314

\section{1-RD-14 ADVERSE / ALERT}

\section{SITE DATA INFORMATION:}

Location: AIKEN, SOUTH CAROLINA

Building Air Exchanges Per Hour: 60 (User specified)

Time: June 30, 19982359 hours EDT (User specified)

\section{CHEMICAL INFORMATION:}

Chemical Name: CYCLOHEXANONE

Molecular Weight: $98.14 \mathrm{~kg} / \mathrm{kmol}$

TLV-TWA: $25 \mathrm{ppm}$ IDLH: $700 \mathrm{ppm}$

Footprint Level of Concern: 125 ppm

Boiling Point: $155.75^{\circ} \mathrm{C}$

Vapor Pressure at Ambient Temperature: 0.0071 atm

Ambient Saturation Concentration: 7,276 ppm or $0.73 \%$

ATMOSPHERIC INFORMATION: (MANUAL INPUT OF DATA)

Wind: 1.7 meters $/ \mathrm{sec}$ from $90^{\circ}$ true at 2 meters

Inversion Height: 200 meters

Stability Class: E Air Temperature: $29^{\circ} \mathrm{C}$

Relative Humidity: 50\% Ground Roughness: Urban or forest

Cloud Cover: 1 tenths

\section{SOURCE STRENGTH INFORMATION:}

Puddle Area: 7750 square feet

Average Puddle Depth: 1 centimeters

Soil Type: Default Ground Temperature: $29^{\circ} \mathrm{C}$

Initial Puddle Temperature: Ground temperature

Release Duration: ALOHA limited the duration to 1 hour

Max Computed Release Rate: 4.43 kilograms/min

Max Average Sustained Release Rate: 4.27 kilograms/min

(averaged over a minute or more)

Total Amount Released: 244 kilograms

\section{TIME DEPENDENT INFORMATION:}

Concentration Estimates at the point:

Downwind: 30 meters

Off Centerline: 0 meters

Max Concentration:

Outdoor: 125 ppm 
Title of Projoct EPHA for THM

\section{ENGINEERING COMPUTATION SHEET}

Subject Concentration Analysis for THM Hazardous Chemicals

Computer N.E. Baucom B.K. Tharakan Date 09/16/96

1-RD-14 ADVERSE / SAE

Not applicable. See run for 1-RD-14 MAX DIST SEG 3.
Reviewed by S.. A. Henderson

Works Calculation 8

Sheet No. 47 of 314 
Title of Project EPHA for THM

Subject Concentration Analysis for THM Hazardous Chemicals

Computer N.E. Baucom B. K. Tharakan Date 09/16/96

\section{1-RD-14 ADVERSE / MAX DIST SEG 1}

\section{SITE DATA INFORMATION:}

Location: AIKEN, SOUTH CAROLINA

Building Air Exchanges Per Hour: 60 (User specified)

Time: June 30, 19982359 hours EDT (User specified)

CHEMICAL INFORMATION:

Chemical Name: CYCLOHEXANONE

Molecular Weight: $98.14 \mathrm{~kg} / \mathrm{kmol}$

TLV-TWA: 25 ppm IDLH: $700 \mathrm{ppm}$

Footprint Level of Concern: $125 \mathrm{ppm}$

Boiling Point: $155.75^{\circ} \mathrm{C}$

Vapor Pressure at Ambient Temperature: $0.0071 \mathrm{~atm}$

Ambient Saturation Concentration: 7,276 ppm or $0.73 \%$

ATMOSPHERIC INFORMATION: (MANUAL INPUT OF DATA)

Wind: 1.7 meters $/ \mathrm{sec}$ from $90^{\circ}$ true at 2 meters

Inversion Height: 200 meters

Stability Class: E Air Temperature: $29^{\circ} \mathrm{C}$

Relative Humidity: 50\% Ground Roughness: Urban or forest

Cloud Cover: 1 tenths

SOURCE STRENGTH INFORMATION:

Puddle Area: 24420 square feet

Average Puddle Depth: 1 centimeters

Soil Type: Default Ground Temperature: $29^{\circ} \mathrm{C}$

Initial Puddle Temperature: Ground temperature

Release Duration: ALOHA limited the duration to 1 hour

Max Computed Release Rate: 13.3 kilograms/min

Max Average Sustained Release Rate: 12.8 kilograms/min (averaged over a minute or more)

Total Amount Released: 733 kilograms

FOOTPRINT INFORMATION:

Dispersion Module: Gaussian

User-specified LOC: 125 ppm

Max Threat Zone for LOC: 52 meters
Reviewed by S. A. Henderson

Works Calculation 8

Sheet No. 48 of 314 
Title of Project EPHA for THM

Subject Concentration Analysis for THM Hazardous Chemicals

Computer N.E. Baucom B. K. Tharakan Date 09/16/96

\section{1-RD-14 ADVERSE / MAX DIST SEG 2}

SITE DATA INFORMATION:

Location: AIKEN, SOUTH CAROLINA

Building Air Exchanges Per Hour: 60 (User specified)

Time: June 30, 19982359 hours EDT (User specified)

CHEMICAL INFORMATION:

Chemical Name: CYCLOHEXANONE

Molecular Weight: $98.14 \mathrm{~kg} / \mathrm{kmol}$

TLV-TWA: 25 ppm IDLH: 700 ppm

Footprint Level of Concern: 125 ppm

Boiling Point: $155.75^{\circ} \mathrm{C}$

Vapor Pressure at Ambient Temperature: $0.0071 \mathrm{~atm}$

Ambient Saturation Concentration: 7,276 ppm or $0.73 \%$

ATMOSPHERIC INFORMATION: (MANUAL INPUT OF DATA)

Wind: 1.7 meters $/ \mathrm{sec}$ from $90^{\circ}$ true at 2 meters

Inversion Height: 200 meters

Stability Class: E Air Temperature: $29^{\circ} \mathrm{C}$

Relative Humidity: $50 \%$ Ground Roughness: Urban or forest

Cloud Cover: 1 tenths

\section{SOURCE STRENGTH INFORMATION:}

Puddle Area: 40700 square feet

Average Puddle Depth: 1 centimeters

Soil Type: Default Ground Temperature: $29^{\circ} \mathrm{C}$

Initial Puddle Temperature: Ground temperature

Release Duration: ALOHA limited the duration to 1 hour

Max Computed Release Rate: 21.6 kilograms/min

Max Average Sustained Release Rate: 20.9 kilograms/min (averaged over a minute or more)

Total Amount Released: 1,197 kilograms

FOOTPRINT INFORMATION:

Dispersion Module: Gaussian

User-specified LOC: 125 ppm

Max Threat Zone for LOC: 67 meters
Reviewed by S. A. Henderson

Works Calculation 8

Sheet No. 49 of 314 
Title of Project EPHA for THM

Subject Concentration Analysis for THM Hazardous Chemicals

Computer N.E. Baucom B. K. Tharakan Date 09/16/96

\section{1-RD-14 ADVERSE / MAX DIST SEG 3}

SITE DATA INFORMATION: Location: AIKEN, SOUTH CAROLINA

Building Air Exchanges Per Hour: 60 (User specified)

Time: June 30, 19982359 hours EDT (User specified)

\section{CHEMICAL INFORMATION:}

Chemical Name: CYCLOHEXANONE

Molecular Weight: $98.14 \mathrm{~kg} / \mathrm{kmol}$

TLV-TWA: $25 \mathrm{ppm}$ IDLH: $700 \mathrm{ppm}$

Footprint Level of Concern: 125 ppm

Boiling Point: $155.75^{\circ} \mathrm{C}$

Vapor Pressure at Ambient Temperature: $0.0071 \mathrm{~atm}$

Ambient Saturation Concentration: $7,276 \mathrm{ppm}$ or $0.73 \%$

ATMOSPHERIC INFORMATION: (MANUAL INPUT OF DATA)

Wind: 1.7 meters/sec from $90^{\circ}$ true at 2 meters

Inversion Height: 200 meters

Stability Class: E Air Temperature: $29^{\circ} \mathrm{C}$

Relative Humidity: 50\% Ground Roughness: Urban or forest

Cloud Cover: 1 tenths

\section{SOURCE STRENGTH INFORMATION:}

Puddle Area: 10000 square meters

Average Puddle Depth: 1 centimeters

Soil Type: Default Ground Temperature: $29^{\circ} \mathrm{C}$

Initial Puddle Temperature: Ground temperature

Release Duration: ALOHA limited the duration to 1 hour

Max Computed Release Rate: 55 kilograms/min

Max Average Sustained Release Rate: 53.1 kilograms/min

(averaged over a minute or more)

Total Amount Released: 3,045 kilograms

FOOTPRINT INFORMATION:

Dispersion Module: Gaussian

User-specified LOC: 125 ppm

Max Threat Zone for LOC: 109 meters

Max Threat Zone for IDLH: 56 meters

Note: Footprint was not drawn because dispersion predictions are unreliable for lengths less than the maximum diameter of the puddle. Maximum diameter of the puddle: 113 meters
Reviewed by S. A. Henderson

Works Galculation 8

Sheet No. 50 of 314 
Title of Project EPHA for THM

Subject Concentration Analysis for THM Hazardous Chemicals

Computer N.E. Baucom B.K. Tharakan Date 09/16/96

\section{1-RD-15 ADVERSE / ALERT}

\section{SITE DATA INFORMATION:}

Location: AIKEN, SOUTH CAROLINA

Building Air Exchanges Per Hour: 0.44 (Sheltered single storied)

Time: June 30, 19982359 hours EDT (User specified)

\section{CHEMICAL INFORMATION:}

Chemical Name: DIETHYLENE GLYCOL DIMETHYL ETHER

Molecular Weight: $134.18 \mathrm{~kg} / \mathrm{kmol}$

TLV-TWA: -unavail- IDLH: -unavail-

Footprint Level of Concern: 100 ppm

Boiling Point: $202.00^{\circ} \mathrm{C}$

Vapor Pressure at Ambient Temperature: 2.38e-004 atm

Ambient Saturation Concentration: 242 ppm or $0.24 \%$

\section{ATMOSPHERIC INFORMATION: (MANUAL INPUT OF DATA)}

Wind: 1.7 meters $/ \mathrm{sec}$ from $90^{\circ}$ true at 2 meters

Inversion Height: 200 meters

Stability Class: E Air Temperature: $29^{\circ} \mathrm{C}$

Relative Humidity: 50\% Ground Roughness: Urban or forest

Cloud Cover: 1 tenths

\section{SOURCE STRENGTH INFORMATION:}

Puddle Area: 30001 square feet

Average Puddle Depth: 1 centimeters

Soil Type: Default Ground Temperature: $29^{\circ} \mathrm{C}$

Initial Puddle Temperature: Ground temperature

Release Duration: ALOHA limited the duration to 1 hour

Max Computed Release Rate: 690 grams / min

Max Average Sustained Release Rate: 666 grams/min

(averaged over a minute or more)

Total Amount Released: 38.2 kilograms

\section{TIME DEPENDENT INFORMATION:}

Concentration Estimates at the point:

Downwind: 30 meters

Off Centerline: 0 meters

Max Concentration:

Outdoor: $14.3 \mathrm{ppm}$
Reviewed by S. A. Henderson

Works Calculation 8

Sheet No. $\underline{51 \text { of } 314}$ 
Title of Project EPHA for THM

Subject. Concentration Analysis for THM Hazardous Chemicals

Computer N.E. Baucom B. K. Tharakan Date 09/16/96

\section{1-RD-15 ADVERSE / SAE}

\section{SITE DATA INFORMATION:}

Location: AIKEN, SOUTH CAROLINA

Building Air Exchanges Per Hour: 0.44 (Sheltered single storied)

Time: June 30, 19982359 hours EDT (User specified)

\section{CHEMICAL INFORMATION:}

Chemical Name: DIETHYLENE GLYCOL DIMETHYL ETHER

Molecular Weight: $134.18 \mathrm{~kg} / \mathrm{kmol}$

TLV-TWA: -unavail- IDLH: -unavail-

Footprint Level of Concern: 100 ppm

Boiling Point: $202.00^{\circ} \mathrm{C}$

Vapor Pressure at Ambient Temperature: 2.38e-004 atm

Ambient Saturation Concentration: 242 ppm or 0.24\%

ATMOSPHERIC INFORMATION: (MANUAL INPUT OF DATA)

Wind: 1.7 meters $/ \mathrm{sec}$ from $90^{\circ}$ true at 2 meters

Inversion Height: 200 meters

Stability Class: E Air Temperature: $29^{\circ} \mathrm{C}$

Relative Humidity: 50\% Ground Roughness: Urban or forest

Cloud Cover: 1 tenths

\section{SOURCE STRENGTH INFORMATION:}

Puddle Area: 100001 square feet

Average Puddle Depth: 1 centimeters

Soil Type: Default $\quad$ Ground Temperature: $29^{\circ} \mathrm{C}$

Initial Puddle Temperature: Ground temperature

Release Duration: ALOHA limited the duration to 1 hour

Max Computed Release Rate: 2.2 kilograms / min

Max Average Sustained Release Rate: 2.12 kilograms/min

(averaged over a minute or more)

Total Amount Released: 121 kilograms

\section{TIME DEPENDENT INFORMATION:}

Concentration Estimates at the point:

Downwind: 100 meters

Off Centerline: 0 meters

Max Concentration:

Outdoor: $4.3 \mathrm{ppm}$
Reviewed by S. A. Henderson

Works Calculation 8

Sheet No. $\underline{52 \text { of } 314}$

52.01314 
Title of Project EPHA for THM

Subject Concentration Analysis for THM Hazardous Chemicals

Computer N.E. Baucom B. K. Tharakan

Date 09/16/96
Reviewed by S. A. Henderson

Works Calculation 8

Sheet No. $\underline{53 \text { of } 314}$

\section{1-RD-15 ADVERȘE / MAX DIST SEG 1}

SITE DATA INFORMATION:

Location: AIKEN, SOUTH CAROLINA

Building Air Exchanges Per Hour: 0.44 (Sheltered single storied)

Time: June 30, 19982359 hours EDT (User specified)

\section{CHEMICAL INFORMATION:}

Chemical Name: DIETHYLENE GLYCOL DIMETHYL ETHER

Molecular Weight: $134.18 \mathrm{~kg} / \mathrm{kmol}$

TLV-TWA: -unavail- IDLH: -unavail-

Footprint Level of Concern: 100 ppm

Boiling Point: $202.00^{\circ} \mathrm{C}$

Vapor Pressure at Ambient Temperature: $2.38 \mathrm{e}-004 \mathrm{~atm}$

Ambient Saturation Concentration: 242 ppm or $0.24 \%$

ATMOSPHERIC INFORMATION: (MANUAL INPUT OF DATA)

Wind: 1.7 meters $/ \mathrm{sec}$ from $90^{\circ}$ true at 2 meters

Inversion Height: 200 meters

Stability Class: E Air Temperature: $29^{\circ} \mathrm{C}$

Relative Humidity: 50\% Ground Roughness: Urban or forest

Cloud Cover: 1 tenths

\section{SOURCE STRENGTH INFORMATION:}

Puddle Area: 24420 square feet

Average Puddle Depth: 1 centimeters

Soil Type: Default Ground Temperature: $29^{\circ} \mathrm{C}$

Initial Puddle Temperature: Ground temperature

Release Duration: ALOHA limited the duration to 1 hour

Max Computed Release Rate: 566 grams/min

Max Average Sustained Release Rate: 547 grams/min

(averaged over a minute or more)

Total Amount Released: 31.3 kilograms

FOOTPRINT INFORMATION:

Dispersion Module: Gaussian

.User-specified LOC: $100 \mathrm{ppm}$

Max Threat Zone for LOC: 27 meters

Note: Footprint was not drawn because

effects of near-field patchiness make dispersion

Predictions unreliable for short distances. 
Title of Projoct EPHA for THM

Subject Concentration Analysis for THM Hazardous Chemicals

Computer N.E. Baucom B. K. Tharakan Date 09/16/96
Reviewed by S. A. Henderson

Works Calculation 8

Sheet No. 54 of 314

\section{1-RD-15 ADVERSE / MAX DIST SEG 2}

\section{SITE DATA INFORMATION:}

Location: AIKEN, SOUTH CAROLINA

Building Air Exchanges Per Hour: 0.44 (Sheltered single storied)

Time: June 30, 19982359 hours EDT (User specified)

\section{CHEMICAL INFORMATION:}

Chemical Name: DIETHYLENE GLYCOL DIMETHYL ETHER

Molecular Weight: $134.18 \mathrm{~kg} / \mathrm{kmol}$

TLV-TWA: -unavail- IDLH: -unavail-

Footprint Level of Concern: 100 ppm

Boiling Point: $202.00^{\circ} \mathrm{C}$

Vapor Pressure at Ambient Temperature: 2.38e-004 atm

Ambient Saturation Concentration: 242 ppm or $0.24 \%$

\section{ATMOSPHERIC INFORMATION: (MANUAL INPUT OF DATA) \\ Wind: 1.7 meters $/ \mathrm{sec}$ from $90^{\circ}$ true at 2 meters \\ Inversion Height: 200 meters \\ Stability Class: E Air Temperature: $29^{\circ} \mathrm{C}$ \\ Relative Humidity: 50\% Ground Roughness: Urban or forest \\ Cloud Cover: 1 tenths}

\section{SOURCE STRENGTH INFORMATION:}

Puddle Area: 40700 square feet

Average Puddle Depth: 1 centimeters

Soil Type: Default Ground Temperature: $29^{\circ} \mathrm{C}$

Initial Puddle Temperature: Ground temperature

Release Duration: ALOHA limited the duration to 1 hour

Max Computed Release Rate: 925 grams/min

Max Average Sustained Release Rate: 893 grams/min

(averaged over a minute or more)

Total Amount Released: 51.2 kilograms

\section{FOOTPRINT INFORMATION:}

Dispersion Module: Gaussian

User-specified LOC: $100 \mathrm{ppm}$

Max Threat Zone for LOC: 34 meters

Note: Footprint was not drawn because

effects of near-field patchiness make dispersion

predictions unreliable for short distances. 
Title of Project EPHA for THM

Subject Concentration Analysis for THM Hazardous Chemicals

Computer N.E. Baucom B. K. Tharakan Date 09/16/96

1-RD-15 ADVERSE / MAX DIST SEG 3

SITE DATA INFORMATION:

Location: AIKEN, SOUTH CAROLINA

Building Air Exchanges Per Hour: 0.44 (Sheltered single storied)

Time: June 30, 19982359 hours EDT (User specified)

CHEMICAL INFORMATION:

Chemical Name: DIETHYLENE GLYCOL DIMETHYL ETHER

Molecular Weight: $134.18 \mathrm{~kg} / \mathrm{kmol}$

TLV-TWA: -unavail- IDLH: -unavail-

Footprint Level of Concern: 100 ppm

Boiling Point: $202.00^{\circ} \mathrm{C}$

Vapor Pressure at Ambient Temperature: $2.38 \mathrm{e}-004$ atm

Ambient Saturation Concentration: 242 ppm or $0.24 \%$

ATMOSPHERIC INFORMATION: (MANUAL INPUT OF DATA)

Wind: 1.7 meters $/ \mathrm{sec}$ from $90^{\circ}$ true at 2 meters

Inversion Height: 200 meters

Stability Class: E Air Temperature: $29^{\circ} \mathrm{C}$

Relative Humidity: 50\% Ground Roughness: Urban or forest

Cloud Cover: 1 tenths

SOURCE STRENGTH INFORMATION:

Puddle Area: 10000 square meters

Average Puddle Depth: 1 centimeters

Soil Type: Default Ground Temperature: $29^{\circ} \mathrm{C}$

Initial Puddle Temperature: Ground temperature

Release Duration: ALOHA limited the duration to 1 hour

Max Computed Release Rate: 2.36 kilograms/min

Max Average Sustained Release Rate: 2.28 kilograms/min

(averaged over a minute or more)

Total Amount Released: 130 kilograms

FOOTPRINT INFORMATION:

Dispersion Module: Gaussian

User-specified LOC: $100 \mathrm{ppm}$

Max Threat Zone for LOC: 56 meters
Reviewed by $\underline{S}$. A. Henderson

Works Calculation 8

Sheet No. $\underline{55 \text { of } 314}$ 
Title of Project EPHA for THM

Subject Concentration Analysis for THM Hazardous Chemicals

Computer N.E. Baucom B. K. Tharakan Date 09/16/96

\section{1-RD-16 ADVERSE / ALERT}

\section{SITE DATA INFORMATION:}

Location: AIKEN, SOUTH CAROLINA

Building Air Exchanges Per, Hour: 60 (User specified)

Time: June 30, 19982359 hours EDT (User specified)

\section{CHEMICAL INFORMATION:}

Chemical Name: DIOXANE Molecular Weight: $88.11 \mathrm{~kg} / \mathrm{kmol}$

TLV-TWA: 25 ppm

IDLH: 500 ppm

Warning: Potential or confirmed human carcinogen.

Footprint Level of Concern: 75 ppm

Boiling Point: $101.32^{\circ} \mathrm{C}$

Vapor Pressure at Ambient Temperature: $0.061 \mathrm{~atm}$

Ambient Saturation Concentration: 62,411 ppm or $6.24 \%$

ATMOSPHERIC INFORMATION: (MANUAL INPUT OF DATA)

Wind: 1.7 meters $/ \mathrm{sec}$ from $90^{\circ}$ true at 2 meters

Inversion Height: 200 meters

Stability Class: E Air Temperature: $29^{\circ} \mathrm{C}$

Relative Humidity: 50\% Ground Roughness: Urban or forest

Cloud Cover: 1 tenths

SOURCE STRENGTH INFORMATION:

Puddle Area: 98 square feet

Average Puddle Depth: 1 centimeters

Soil Type: Default Ground Temperature: $29^{\circ} \mathrm{C}$

Initial Puddle Temperature: Ground temperature

Release Duration: ALOHA limited the duration to 1 hour

Max Computed Release Rate: 564 grams/min

Max Average Sustained Release Rate: 564 grams/min

(averaged over a minute or more)

Total Amount Released: 26.5 kilograms

TIME DEPENDENT. INFORMATION:

Concentration Estimates at the point:

Downwind: 30 meters

Off Centerline: 0 meters

Max Concentration:

Outdoor: 74.7 ppm
Reviewed by S. A. Henderson

Works Calculation 8

Sheet No. 56 of 314 
Title of Project EPHA for THM

Subject Concentration Analysis for THM Hazardous Chemicals

Computer N.E. Baucom B. K. Tharakan Date 09/16/96 1-RD-16 ADVERSE / SAE

\section{SITE DATA INFORMATION:}

Location: AIKENं, SOUTH CAROLINA

Building Air Exchanges Per Hour: 60 (User specified)

Time: June 30, 19982359 hours EDT (User specified)

CHEMICAL INFORMATION:

Chemical Name: DIOXANE Molecular Weight: $88.11 \mathrm{~kg} / \mathrm{kmol}$

TLV-TWA: 25 ppm IDLH: 500 ppm

Warning: Potential or confirmed human carcinogen.

Footprint Level of Concern: 75 ppm

Boiling Point: $101.32^{\circ} \mathrm{C}$

Vapor Pressure at Ambient Temperature: $0.061 \mathrm{~atm}$

Ambient Saturation Concentration: 62,411 ppm or $6.24 \%$

\section{ATMOSPHERIC INFORMATION: (MANUAL INPUT OF DATA)}

Wind: 1.7 meters $/ \mathrm{sec}$ from $90^{\circ}$ true at 2 meters

Inversion Height: 200 meters

Stability Class: E Air Temperature: $29^{\circ} \mathrm{C}$

Relative Humidity: 50\% Ground Roughness: Urban or forest

Cloud Cover: 1 tenths

\section{SOURCE STRENGTH INFORMATION:}

Puddle Area: 1400 square feet Average Puddle Depth: 1 centimeters Soil Type: Default Ground Temperature: $29^{\circ} \mathrm{C}$ Initial Puddle Temperature: Ground temperature Release Duration: ALOHA limited the duration to 1 hour Max Computed Release Rate: 7.01 kilograms/min Max Average Sustained Release Rate: 6.31 kilograms/min (averaged over a minute or more)

Total Amount Released: 336 kilograms

\section{TIME DEPENDENT INFORMATION:}

Concentration Estimates at the point:

Downwind: 100 meters

Off Centerline: 0 meters

Max Concentration:

Outdoor: 75 ppm
Reviewed by S. A. Henderson

Works Calculation 8

Sheet No. $\underline{57 \text { of } 314}$ 
Title of Project EPHA for THM

Subject Concentration Analysis for THM Hazardous Chemicals

Computer N.E. Baucom B.K. Tharakan Date 09/16/96

1-RD-16 ADVERSE / MAX DIST SEG 1

\section{SITE DATA INFORMATION:}

Location: AIKEN, SOUTH CAROLINA

Building Air Exchanges Per Hour: 60 (User specified)

Time: June 30, 19982359 hours EDT (User specified)

\section{CHEMICAL INFORMATION:}

Chemical Name: DIOXANE

TLV-TWA: $25 \mathrm{ppm}$

Molecular Weight: $88.11 \mathrm{~kg} / \mathrm{kmol}$

Warning: Potential or confirmed human carcinogen.

Footprint Level of Concern: 75 ppm

Boiling Point: $101.32^{\circ} \mathrm{C}$

Vapor Pressure at Ambient Temperature: $0.061 \mathrm{~atm}$

Ambient Saturation Concentration: 62,411 ppm or $6.24 \%$

\section{ATMOSPHERIC INFORMATION: (MANUAL INPUT OF DATA)}

Wind: 1.7 meters $/ \mathrm{sec}$ from $90^{\circ}$ true at 2 meters

Inversion Height: 200 meters

Stability Class: E Air Temperature: $29^{\circ} \mathrm{C}$

Relative Humidity: 50\% Ground Roughness: Urban or forest

Cloud Cover: 1 tenths

\section{SOURCE STRENGTH INFORMATION:}

Puddle Area: 24420 square feet

Average Puddle Depth: 1 centimeters

Soil Type: Default. Ground Temperature: $29^{\circ} \mathrm{C}$

Initial Puddle Temperature: Ground temperature

Release Duration: ALOHA limited the duration to 1 hour

Max Computed Release Rate: 107 kilograms/min

Max Average Sustained Release Rate: 97.5 kilograms/min

(averaged over a minute or more)

Total Amount Released: 5,233 kilograms

\section{FOOTPRINT INFORMATION:}

Model Run: Heavy Gas

User-specified LOC: 75 ppm

Max Threat Zone for LOC: 501 meters
Reviewed by S. A. Henderson

Works Calculation 8

Sheet No. $\underline{58 \text { of } 314}$ 
Title of Project EPHA for THM

Subject Concentration Analysis for THM Hazardous Chemicals

Computer N.E. Baucom B. K. Tharakan Date 09/16/96

1-RD-16 ADVERSE / MAX DIST SEG 2

SITE DATA INFORMATION:

Location: AIKEN, SOUTH CAROLINA

Building Air Exchanges Per Hour: 60 (User specified)

Time: June 30, 19982359 hours EDT (User specified)

\section{CHEMICAL INFORMATION:}

Chemical Name: DIOXANE Molecular Weight: $88.11 \mathrm{~kg} / \mathrm{kmol}$

TLV-TWA: 25 ppm

IDLH: 500 ppm

Warning: Potential or confirmed human carcinogen.

Footprint Level of Concern: 75 ppm

Boiling Point: $101.32^{\circ} \mathrm{C}$

Vapor Pressure at Ambient Temperature: $0.061 \mathrm{~atm}$

Ambient Saturation Concentration: 62,411 ppm or $6.24 \%$

\section{ATMOSPHERIC INFORMATION: (MANUAL INPUT OF DATA)}

Wind: 1.7 meters $/ \mathrm{sec}$ from $90^{\circ}$ true at 2 meters

Inversion Height: 200 meters

Stability Class: E Air Temperature: $29^{\circ} \mathrm{C}$

Relative Humidity: $50 \%$ Ground Roughness: Urban or forest

Cloud Cover: 1 tenths

\section{SOURCE STRENGTH INFORMATION:}

Puddle Area: 40700 square feet

Average Puddle Depth: 1 centimeters

Soil Type: Default Ground Temperature: $29^{\circ} \mathrm{C}$

Initial Puddle Temperature: Ground temperature

Release Duration: ALOHA limited the duration to 1 hour

Max Computed Release Rate: 175 kilograms/min

Max Average Sustained Release Rate: 159 kilograms/min

(averaged over a minute or more)

Total Amount Released: 8,557 kilograms

\section{FOOTPRINT INFORMATION:}

Model Run: Heavy Gas

User-specified LOC: 75 ppm

Max Threat Zone for LOC: 643 meters
Reviewed by S. A. Henderson

Works Calculation 8

Sheet No. 59 of 314 
Title of Project EPHA for THM

Subject Concentration Analysis for THM Hazardous Chemicals

Computer N.E. Baucom B. K. Tharakan Date 09/16/96

\section{1-RD-16 ADVERSE / MAX DIST SEG 3}

\section{SITE DATA INFORMATION:}

\section{Location: AIKEN, SOUTH CAROLINA}

Building Air Exchanges Per Hour: 60 (User specified)

Time: June 30, 19982359 hours EDT (User specified)

\section{CHEMICAL INFORMATION:}

Chemical Name: DIOXANE Molecular Weight: $88.11 \mathrm{~kg} / \mathrm{kmol}$

TLV-TWA: 25 ppm

IDLH: $500 \mathrm{ppm}$

Warning: Potential or confirmed human carcinogen.

Footprint Level of Concern: 7,5 ppm

Boiling Point: $101.32^{\circ} \mathrm{C}$

Vapor Pressure at Ambient Temperature: $0.061 \mathrm{~atm}$

Ambient Saturation Concentration: 62,411 ppm or $6.24 \%$

\section{ATMOSPHERIC INFORMATION: (MANUAL INPUT OF DATA)}

Wind: 1.7 meters $/ \mathrm{sec}$ from $90^{\circ}$ true at 2 meters

Inversion Height: 200 meters

Stability Class: E Air Temperature: $29^{\circ} \mathrm{C}$

Relative Humidity: 50\% Ground Roughness: Urban or forest

Cloud Cover: 1 tenths

\section{SOURCE STRENGTH INFORMATION:}

Puddle Area: 10000 square meters

Average Puddle Depth: 1 centimeters

Soil Type: Default Ground Temperature: $29^{\circ} \mathrm{C}$

Initial Puddle Temperature: Ground temperature

Release Duration: ALOHA limited the duration to 1 hour

Max Computed Release Rate: 444 kilograms/min

Max Average Sustained Release Rate: 405 kilograms/min (averaged over a minute or more)

Total Amount Released: 21,843 kilograms

FOOTPRINT INFORMATION:

Model Run: Heavy Gas

User-specified LOC: 75 ppm

Max Threat Zone for LOC: 1.0 kilometers
Reviewed by S. A. Henderson

Works Calculation 8

Sheet No. 60 of 314 
Title of Project EPHA for THM

Subject Concentration Analysis for THM Hazardous Chemicals

Computer N.E. Baucom B. K. Tharakan Date 09/16/96

1-RD-17 ADVERSE / ALERT

\section{SITE DATA INFORMATION:}

Location: AIKEN, SOUTH CAROLINA

Building Air Exchanges Per Hour: 0.44 (Sheltered single storied)

Time: June 30, 19982359 hours EDT (User specified)

\section{CHEMICAL INFORMATION:}

Chemical Name: DIPHENYLMETHANE-4, 4-DUSOCYANATE

Molecular Weight: $250.27 \mathrm{~kg} / \mathrm{kmol}$

TLV-TWA: -unavail- IDH: -unavail-

Footprint Level of Concern: $2 \mathrm{mg} /$ (cu m)

Boiling Point: $184.00^{\circ} \mathrm{C}$

Note: Not enough chemical data to use Heavy Gas Option

ATMOSPHERIC INFORMATION: (MANUAL INPUT OF DATA)

Wind: 1.7 meters/sec from $90^{\circ}$ true at 2 meters

Inversion Height: 200 meters

Stability Class: E

Air Temperature: $29^{\circ} \mathrm{C}$

Relative Humidity: $50 \%$ Ground Roughness: Urban or forest

Cloud Cover: 1 tenths

SOURCE STRENGTH INFORMATION:

Direct Source: $5.75 \mathrm{e}-5$ kilograms / sec

Source Height: 0

Release Duration: 60 minutes

Release Rate: 3.45 grams / min

Total Amount Released: 207 grams

\section{TIME DEPENDENT INFORMATION:}

Concentration Estimates at the point"

Downwind: $\mathbf{3 0}$ meters

Off Centerline: 0 meters

Max Concentration:

Outdoor: $2 \mathrm{mg} /$ (cu m)

Indoor: $\quad 0.707 \mathrm{mg} /(\mathrm{cu} \mathrm{m})$

Note: Indoor graph is shown with a dotted line.
Reviewed by S. A. Henderson

Works Calculation 8

Sheet No. 61 of 314

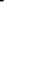


Title of Project EPHA for THM

Reviewed by S. A. Henderson

Subject Concentration Analysis for THM Hazardous Chemicals Works Calculation 8

Computer N.E. Baucom B. K. Tharakan Date 09/16/96

SPILL AREA CALCULATION FOR 1-RD-17 ADVERSE / ALERT CONDITIONS

\begin{tabular}{|c|c|c|c|c|}
\hline Parameter & Value & Dimensions & Value & $\begin{array}{c}\text { Dimensions } \\
\text { (USA) }\end{array}$ \\
\hline E (Evaporation Rate) & $5.75 \mathrm{E}-5$ & $\mathrm{~kg} / \mathrm{s}$ & & \\
\hline MW (Molecular Weight) & 250 & $\mathrm{~kg} / \mathrm{kg}-\mathrm{mole}$ & & \\
\hline Pv (Vapor Pressure) & 0.001 & $\mathrm{~mm} \mathrm{Hg}$ & & \\
\hline Pv (Vapor Pressure) & 0 & $\mathbf{P a}$ & & \\
\hline Rg (Gas Law Constant) & 8314 & J/kgmole-K & & \\
\hline Temperature & 29 & $\mathrm{C}$ & & \\
\hline Volume of Spill & 4230 & gal & & \\
\hline d (Depth of Pool) & 0.01 & $\mathbf{m}$ & & \\
\hline A (Surface Area of Spill) & 1601.2 & $\mathrm{~m}^{\wedge} \mathbf{2}$ & 17237 & $\mathrm{ft}^{\wedge} \mathbf{2}$ \\
\hline Km (Mass Transfer Coeff) & $2.70 \mathrm{E}-3$ & $\mathrm{~m} / \mathrm{s}$ & & \\
\hline Z (Pool Diameter in Wind Direction) & 45 & $\mathbf{m}$ & & \\
\hline Sc (Schmidt Number) & 2.33 & & & \\
\hline $\mathrm{U}$ (Wind velocity) & 1.7 & $\mathbf{m} / \mathbf{s}$ & & \\
\hline nu (kinematic viscosity of air) & $1.50 \mathrm{E}-5$ & $\mathrm{~m}^{\wedge} 2 / \mathrm{sec}$ & & \\
\hline mu (viscosity of air) & $1.90 \mathrm{E}-5$ & $\mathrm{~kg} / \mathrm{m}-\mathrm{s}$ & & \\
\hline $\begin{array}{l}\text { Dm (Molecular Diffusivity of Solute } \\
\text { in Air) }\end{array}$ & 644E-6 & $\mathrm{m}^{\wedge} 2 / \sec$ & & \\
\hline $\begin{array}{l}\text { D_H20 (Molecular Diffusivity of } \\
\text { Water) }\end{array}$ & $2.40 \mathrm{E}-5$ & & & \\
\hline MW_H20 & 18 & kg/kg-mole & & \\
\hline \multicolumn{5}{|l|}{ Conversions } \\
\hline T (Absolute) & 302.15 & 'K & & \\
\hline V (Volume) & 16.01 & $m^{\wedge} \mathbf{3}$ & & \\
\hline Evaporation Rate & 3 & $\mathrm{~g} / \mathrm{min}$ & & \\
\hline $60 \mathrm{~min}$ lb released & 0.46 & & & \\
\hline
\end{tabular}




\section{ENGINEERING COMPUTATION SHEET}

Title of Project EPHA for THM

Subject Concentration Analysis for THM Hazardous Chemicals

Computer N.E. Baucom B. K. Tharakan Date 09/16/96

1-RD-17 ADVERSE / SAE

SITE DATA INFORMATION:

Location: AIKEN, SOUTH CAROLINA

Building Air Exchanges Per Hour: 0.44 (Sheltered single storied)

Time: June 30, 19982359 hours EDT (User specified)

CHEMICAL INFORMATION:

Chemical Name: DIPHENYLMETHANE-4, 4-DISOCYANATE

Molecular Weight: $250.27 \mathrm{~kg} / \mathrm{kmol}$

TLV-TWA: -unavail-

DLH: -unavail-

Footprint Level of Concern: $2 \mathrm{mg} /(\mathrm{cu} \mathrm{m})$

Boiling Point: $184.00^{\circ} \mathrm{C}$

Note: Not enough chemical data to use Heavy Gas Option

ATMOSPHERIC INFORMATION: (MANUAL INPUT OF DATA)

Wind: 1.7 meters/sec from $90^{\circ}$ true at 2 meters

Inversion Height: 200 meters

Stability Class: E Air Temperature: $29^{\circ} \mathrm{C}$

Relative Humidity: $50 \%$ Ground Roughness: Urban or forest

Cloud Cover: 1 tenths

SOURCE STRENGTH INFORMATION:

Direct Source: $6.08 \mathrm{E}-4$ kilograms / sec

Source Height: 0

Release Duration: 60 minutes

Release Rate: 36.5 grams / min

Total Amount Released: 2.19 kilograms

TIME DEPENDENT INFORMATION:

Concentration Estimates at the point"

Downwind: 100 meters

Off Centerline: 0 meters

Max Concentration:

Outdoor: $2 \mathrm{mg} /(\mathrm{cu} \mathrm{m})$

Indoor: $\quad 0.708 \mathrm{mg} /(\mathrm{cu} \mathrm{m})$

Note: Indoor graph is shown with a dotted line.
Reviewed by S. A. Henderson

Works Calculation 8

Sheet No. 63 of 314 
Title of Projèct EPHA for THM

Subject Concentration Analysis for THM Hazardous Chemicals

Computer N.E. Baucom B.K. Tharakan Date 09/16/96
Reviewed by S. A. Henderson

Works Calcuiation 8

Sheet No. 64 of 314

\section{SPILL AREA CALCULATION FOR 1-RD-17 ADVERSE / ALERT CONDITIONS}

\begin{tabular}{|c|c|c|c|c|}
\hline Parameter & Value & Dimensions & Value & $\begin{array}{c}\text { Dimensions } \\
\text { (USA) }\end{array}$ \\
\hline E (Evaporation Rate) & $6.08 \mathrm{E}-4$ & $\mathrm{~kg} / \mathrm{s}$ & & \\
\hline MW (Molecular Weight) & 250 & kg/kg-mole & & \\
\hline Pv (Vapor Pressure) & 0.001 & $\mathrm{~mm} \mathrm{Hg}$ & & \\
\hline Pv (Vapor Pressure) & 0 & $\mathbf{P a}$ & & \\
\hline Rg (Gas Law Constant) & 8314 & $\mathrm{~J} / \mathrm{kgmole}-\mathrm{K}$ & & \\
\hline Temperature & 29 & $\cdot \mathrm{C}$ & & \\
\hline Volume of Spill & 51450 & gal & & \\
\hline d (Depth of Pool) & 0.01 & m & & \\
\hline A (Surface Area of Spill) & 1947504 & $\mathrm{~m}^{\wedge} \mathbf{2}$ & 209652 & $\mathrm{ft}^{\wedge} 2$ \\
\hline Km (Mass Transfer Coeff) & $2.35 \mathrm{E}-3$ & $\mathbf{m} / \mathbf{s}$ & & \\
\hline $\mathrm{Z}$ (Pool Diameter in Wind Direction) & 157 & $\mathbf{m}$ & & \\
\hline Sc (Schmidt Number) & 2.33 & & & \\
\hline $\mathrm{U}$ (Wind velocity) & 1.7 & $\mathrm{~m} / \mathrm{s}$ & & \\
\hline nu (kinematic viscosity of air) & $1.50 \mathrm{E}-5$ & $m^{\wedge} 2 /$ sec & & \\
\hline mu (viscosity of air) & $1.90 \mathrm{E}-5$ & $\mathrm{~kg} / \mathrm{m}-\mathrm{s}$ & & \\
\hline $\begin{array}{l}\text { Dm (Molecular Diffusivity of Solute } \\
\text { in Air) }\end{array}$ & 6.44E-6 & $\mathrm{m}^{\wedge} 2 / \mathrm{sec}$ & & . \\
\hline $\begin{array}{l}\text { D_H20 (Molecular Diffusivity of } \\
\text { Water) }\end{array}$ & $2.40 \mathrm{E}-5$ & & & \\
\hline MW_H20 & 18 & kg/kg-mole & & \\
\hline \multicolumn{5}{|l|}{ Conversions } \\
\hline $\mathbf{T}$ (Absolute) & 302.15 & ${ }^{\circ} \mathbf{K}$ & & \\
\hline V (Volume) & 194.75 & $\mathbf{m}^{\wedge} \mathbf{3}$ & & \\
\hline Evaporation Rate & 36 & $\mathrm{~g} / \mathrm{min}$ & & \\
\hline $60 \mathrm{~min} l \mathrm{lb}$ released & 4.83 & & & \\
\hline
\end{tabular}


Title of Project EPHA for THM

Subject Concentration Analysis for THM Hazardous Chemicals

Computer N.E. Baucom B. K. Tharakan Date 09/16/96 1-RD.17 ADVERSE / MAX DIST SEG 1

\section{SITE DATA INFORMATION:}

Location: AIKEN, SOUTH CAROLINA

Building Air Exchanges Per Hour: 0.44 (Sheltered single storied)

Time: June 30, 19982359 hours EDT (User specified)

\section{CHEMICAL INFORMATION:}

Chemical Name: DIPHENYLMETHANE-4, 4-DISOCYANATE

Molecular Weight: $250.27 \mathrm{~kg} / \mathrm{kmol}$

TLV-TWA: -unavail- IDLH: -unavail-

Footprint Level of Concern: 2.mg / (cu m )

Boiling Point: $184.00^{\circ} \mathrm{C}$

Note: Not enough chemical data to use Heavy Gas option

ATMOSPHERIC INFORMATION: (MAÑUAL INPUT OF DATA)

Wind: 1.7 meters $/ \mathrm{sec}$ from $90^{\circ}$ true at 2 meters

Inversion Height: 200 meters

Stability Class: E Air Temperature: $29^{\circ} \mathrm{C}$

Relative Humidity: 50\% Ground Roughness: Urban or forest

Cloud Cover: 1 tenths

\section{SOURCE STRENGTH INFORMATION:}

Direct Source: 7.98E-5 kilograms / sec

Source Height: 0

Release Duration: 60 minutes

Release Rate: 4.79 grams / $\mathrm{min}$

Total Amount Released: 287 grams

FOOTPRINT INFORMATION:

Dispersion Module: Gaussian

User-specified LOC: $2 \mathrm{mg} /$ (cu m)

Max Threat Zone for LOC: 35 meters

Note: Footprint was not drawn because effects of near-field patchiness make dispersion predictions unreliable for short distances.
Reviewed by S. A. Henderson

Works Calculation 8

Sheet No. 65 of 314 
Title of Project EPHA for THM

Subject Concentration Analysis for THM Hazardous Chemicals

Computer N.E. Baucom B. K. Tharakan Date 09/16/96

Determination of Evaporation Rate for Segment 1 Maximum Source Term of $24420 \mathrm{ft}^{2}$
Reviewed by S. A. Hienderson

Works Calculation 8

Sheet No. 66 of 314

\begin{tabular}{|c|c|c|c|c|}
\hline Parameter & Value & Dimensions & Value & $\begin{array}{c}\text { Dimensions } \\
\text { (USA) }\end{array}$ \\
\hline E (Evaporation Rate) & $7.98 \mathrm{E}-5$ & $\mathrm{~kg} / \mathrm{s}$ & & \\
\hline MW (Molecular Weight) & 250 & $\mathrm{~kg} / \mathrm{kg}-\mathrm{mole}$ & & \\
\hline Py (Vapor Pressure) & 0.001 & $\mathrm{~mm} \mathrm{Hg}$ & & \\
\hline Py (Vapor Pressure) & 0 & $\mathbf{P a}$ & & \\
\hline Rg (Gas Law Constant) & 8314 & J/kgmole-K & & \\
\hline Temperature & 29 & ${ }^{\circ} \mathrm{C}$ & & \\
\hline Volume of Spill & 5993 & gal & & \\
\hline d (Depth of Pool) & 0.01 & $\mathbf{m}$ & & \\
\hline A (Surface Area of Spill) & 2268.5 & $m^{\wedge} 2$ & 24421 & $\mathrm{ft} \mathbf{t}^{\wedge}$ \\
\hline Km (Mass Transfer Coeff) & $2.65 \mathrm{E}-3$ & $\mathrm{~m} / \mathrm{s}$ & & \\
\hline Z (Pool Diameter in Wind Direction) & 54 & $\mathbf{m}$ & & \\
\hline Sc (Schmidt Number) & 2.33 & & & \\
\hline $\mathrm{U}$ (Wind velocity) & 1.7 & $\mathbf{m} / \mathbf{s}$ & & \\
\hline nu (kinematic viscosity of air) & $1.50 \mathrm{E}-5$ & $m^{\wedge} 2 / \sec$ & & \\
\hline mu (viscosity of air) & $1.90 \mathrm{E}-5$ & $\mathbf{k g} / \mathbf{m}-\mathbf{s}$ & & \\
\hline $\begin{array}{l}\text { Dm (Molecular Diffusivity of Solute } \\
\text { in Air) }\end{array}$ & $6.44 \mathrm{E}-6$ & $m^{\wedge} 2 /$ sec & & . \\
\hline $\begin{array}{l}\text { D_H20 (Molecular Diffusivity of } \\
\text { Water) }\end{array}$ & $2.40 \mathrm{E}-5$ & & & \\
\hline MW_H2O & 18 & kg/kg-mole & & \\
\hline \multicolumn{5}{|l|}{ Conversions } \\
\hline T (Absolute) & 302.15 & $\bar{K}$ & & \\
\hline V(Volume) & 22.69 & $\mathbf{m}^{\wedge} \mathbf{3}$ & & \\
\hline Evaporation Rate & 5 & $\mathrm{~g} / \mathrm{min}$ & & \\
\hline $60 \mathrm{~min} l \mathrm{lb}$ released & 0.63 & & & \\
\hline
\end{tabular}


Title of Project EPHA for THM

Reviewed by S. A. Henderson

Works Calculation 8

Subject Concentration Analysis for THM Hazardous Chemicals

Computer N.E. Baucom B. K. Tharakan Date 09/16/96

1-RD-17 ADVERSE / MAX DIST SEG 2

SITE DATA INFORMATION:

Location: AIKEN, SOUTH CAROLINA

Building Air Exchanges Per Hour: 0.44 (Sheltered single storied)

Time: June 30, 19982359 hours EDT (User specified)

CHEMICAL INFORMATION:

Chemical Name: DIPHENYLMETHANE-4, 4-DIISOCYANATE

Molecular Weight: $250.27 \mathrm{~kg} / \mathrm{kmol}$

TLV-TWA: -unavail- IDLH: -unavail-

Footprint Level of Concern: $2 \mathrm{mg} /$ (cu m)

Boiling Point: $184.00^{\circ} \mathrm{C}$

Note: Not enough chemical data to use Heavy Gas option

ATMOSPHERIC INFORMATION: (MANUAL INPUT OF DATA)

Wind: 1.7 meters $/ \mathrm{sec}$ from $90^{\circ}$ true at 2 meters

Inversion Height: 200 meters

Stability Class: E Air Temperature: $29^{\circ} \mathrm{C}$

Relative Humidity: 50\% Ground Roughness: Urban or forest

Cloud Cover: 1 tenths

SOURCE STRENGTH INFORMATION:

Direct Source: $1.29 \mathrm{E}-4$ kilograms / sec

Source Height: 0

Release Duration: 60 minutes

Release Rate: 7.74 grams / min

Total Amount Released: 464 grams

FOOTPRINT INFORMATION:

Dispersion Module: Gaussian

User-specified LOC: $2 \mathrm{mg} /$ (cu m)

Max Threat Zone for LOC: 45 meters

Note: Footprint was not drawn because

effects of near-field patchiness make dispersion

predictions unreliable for short distances. 
ENGINEERING COMPUTATION SHEET

Title of Project EPHA for THM

Reviewed by S. A. Henderson

Works Calculation 8

Sheet No. 68 of 314

Computer N.E. Baucom B. K. Tharakan Date 09/16/96

Determination of Evaporation Rate for Segment 2 Maximum Source Term of $40700 \mathrm{ft}^{2}$.

\begin{tabular}{|c|c|c|c|c|}
\hline Parameter & Value & Dimensions & Value & $\begin{array}{l}\text { Dimensions } \\
\text { (USA) }\end{array}$ \\
\hline E (Evaporation Rate) & $1.29 \mathrm{E}-4$ & $\mathrm{~kg} / \mathrm{s}$ & & \\
\hline MW (Molecular Weight) & 250 & $\mathrm{~kg} / \mathrm{kg}$-mole & & \\
\hline Pv (Vapor Pressure) & 0.001 & $\mathrm{~mm} \mathrm{Hg}$ & & \\
\hline Pv (Vapor Pressure) & 0 & $\mathbf{P a}$ & & \\
\hline Rg (Gas Law Constant) & 8314 & J/kgmole-K & & \\
\hline Temperature & 29 & ${ }^{\circ} \mathrm{C}$ & & \\
\hline Volume of Spill & 9988 & gal & & \\
\hline d (Depth of Pool) & 0.01 & $\mathbf{m}$ & & \\
\hline A (Surface Area of Spill) & $\mathbf{3 7 8 0 . 8}$ & $\mathrm{m}^{\wedge} \mathbf{2}$ & 40700 & $\mathrm{ft}^{\wedge} \mathbf{2}$ \\
\hline Km (Mass Transfer Coefi) & $2.58 \mathrm{E}-3$ & $\dot{\mathrm{m}} / \mathbf{s}$ & & \\
\hline Z (Pool Diameter in Wind Direction) & 69 & $\mathbf{m}$ & & \\
\hline Sc (Schmidt Number) & 2.33 & & & \\
\hline $\mathrm{U}$ (Wind velocity) & 1.7 & $\mathrm{~m} / \mathrm{s}$ & & \\
\hline nu (kinematic viscosity of air) & $1.50 \mathrm{E}-5$ & $\mathrm{~m}^{\wedge} 2 / \mathrm{sec}$ & & \\
\hline mu (viscosity of air) & $1.90 \mathrm{E}-5$ & $\mathbf{k g} / \mathbf{m}-\mathbf{s}$ & & \\
\hline $\begin{array}{l}\text { Dm (Molecular Diffusivity of Solute } \\
\text { in Air) }\end{array}$ & $6.44 E-6$ & $\mathrm{~m}^{\wedge} 2 / \mathrm{sec}$ & & \\
\hline $\begin{array}{l}\text { D_H20 (Molecular Diffusivity of } \\
\text { Water) }\end{array}$ & $2.40 \mathrm{E}-5$ & & & \\
\hline MW_H20 & 18 & $\mathrm{~kg} / \mathrm{kg}-\mathrm{mole}$ & & \\
\hline \multicolumn{5}{|l|}{ Conversions } \\
\hline T (Absolute) & 302.15 & $\cdot \mathbf{K}$ & & \\
\hline V (Volume) & 37.81 & $m^{\wedge} 3$ & & \\
\hline Evaporation Rate & 8 & $\mathrm{~g} / \mathrm{min}$ & & \\
\hline $60 \mathrm{~min} / \mathrm{lb}$ released & 1.03 & & & \\
\hline
\end{tabular}


Title of Project EPHA for THM

Subject Concentration Analysis for THM Hazardous Chemicals

Computer N.E. Baucom B. K. Tharakan Date 09/16/96
Reviewed by S. A. Henderson

Works Calculation 8

Sheet No. 69 of 314

\section{1-RD.17 ADVERSE / MAX DIST SEG 3 \\ SITE DATA INFORMATION: \\ Location: AIKEN, SOUTH CAROLINA \\ Building Air Exchanges Per Hour: 0.44 (Sheltered single storied) \\ Time: June 30, 19982359 hours EDT, (User specified)}

\section{CHEMICAL INFORMATION:}

Chemical Name: DIPHENYLMETHANE-4, 4-DISSOCYANATE

Molecular Weight: $250.27 \mathrm{~kg} / \mathrm{kmol}$

TLV-TWA: -unavail- IDLH: -unavail-

Footprint Level of Concern: $2 \mathrm{mg} /(\mathrm{cu} \mathrm{m}$ )

Boiling Point: $184.00^{\circ} \mathrm{C}$

Note: Not enough chemical data to use Heavy Gas option

ATMOSPHERIC INFORMATION: (MANUAL INPUT OF DATA)

Wind: 1.7 meters $/ \mathrm{sec}$ from $90^{\circ}$ true at 2 meters

Inversion Height: 200 meters

Stability Class: E Air Temperature: $29^{\circ} \mathrm{C}$

Relative Humidity: 50\% Ground Roughness: Urban or forest

Cloud Cover: 1 tenths

\section{SOURCE STRENGTH INFORMATION:}

Direct Source: $3.24 \mathrm{E}-4$ kilograms / sec

Source Height: 0

Release Duration: 60 minutes

Release Rate: 19.4 grams / min

Total Amount Released: 1.17 kilograms

\section{FOOTPRINT INFORMATION:}

Dispersion Module: Gaussian

User-specified.LOC: $2 \mathrm{mg} /$ (cu m)

Max Threat Zone for LOC: 72 meters

Note: Footprint was not drawn because effects of near-field patchiness make dispersion predictions unreliable for short distances. 
Title of Project EPHA for THM

Reviewed by S. A. Henderson

Subject Concentration Analysis for THM Hazardous Chemicals

Works Calculation 8

Computer N.E. Baucom B. K. Tharakan Date 09/16/96

Sheot No. 70 of 314

Determination of Evaporation Rate for Segment 3 Maximum Source Term of $10000 \mathrm{~m}^{2}$

\begin{tabular}{|c|c|c|c|c|}
\hline Parameter & Value & Dimensions & Value & $\begin{array}{c}\text { Dimensions } \\
\text { (USA) }\end{array}$ \\
\hline E(Evaporation Rate) & $3.24 \mathrm{E}-4$ & $\mathrm{~kg} / \mathrm{s}$ & & \\
\hline MW (Molecular Weight) & 250 & $\mathrm{~kg} / \mathrm{kg}$-mole & & \\
\hline Pv (Vapor Pressure) & 0.001 & $\mathrm{~mm} \mathrm{Hg}$ & & \\
\hline Pv (Vapor Pressure) & 0 & $\mathbf{P a}$ & & \\
\hline $\mathrm{Rg}$ (Gas Law Constant) & 8314 & J/kgmole-K & & \\
\hline Temperature & 29 & $\mathrm{C}$ & & \\
\hline Volume of Spill & 26418 & gal & & \\
\hline d (Depth of Pool) & 0.01 & $\mathbf{m}$ & & \\
\hline A (Surface Area of Spill) & 10000.0 & $m^{\wedge} 2$ & 107650 & $\mathrm{ft}^{\wedge} \mathbf{2}$ \\
\hline Km (Mass Transfer Coeff) & $2.44 \mathrm{E} \cdot 3$ & $\mathrm{~m} / \mathrm{s}$ & & \\
\hline Z (Pool Diameter in Wind Direction) & 113 & $\mathbf{m}$ & & \\
\hline Sc (Schmidt Number) & 2.33 & & & \\
\hline U (Wind velocity) & 1.7 & $\mathbf{m} / \mathbf{s}$ & & \\
\hline nu (kinematic viscosity of air) & $1.50 \mathrm{E}-5$ & $\mathrm{~m}^{\wedge} 2 / \mathrm{sec}$ & & \\
\hline mu (viscosity of air) & $1.90 \mathrm{E}-5$ & $\mathrm{~kg} / \mathrm{m}-\mathrm{s}$ & & \\
\hline $\begin{array}{l}\text { Dm (Molecular Diffusivity of Solute } \\
\text { in Air) }\end{array}$ & $6.44 \mathrm{E}-6$. & $\mathrm{m}^{\wedge} 2 / \mathrm{sec}$ & & \\
\hline $\begin{array}{l}\text { D_H20 (Molecular Diffusivity of } \\
\text { Water) }\end{array}$ & $2.40 \mathrm{E}-5$ & & & \\
\hline MW_H20 & 18 & kg/kg-mole & & \\
\hline \multicolumn{5}{|l|}{ Conversions } \\
\hline$T$ (Absolute) & 302.15 & $\bar{K}$ & & \\
\hline V (Volume) & 37.81 & $\mathbf{m}^{\wedge} \mathbf{3}$ & & \\
\hline Evaporation Rate & 19 & $\mathrm{~g} / \mathrm{min}$ & & \\
\hline $60 \mathrm{~min} / \mathrm{lb}$ released & 2.57 & & & \\
\hline
\end{tabular}




\section{1-RD-19 ADVERSE / ALERT}

\section{SITE DATA INFORMATION:}

Location: AIKEN, SOUTH CAROLINA

Building Air Exchanges Per Hour: 60 (User specified)

Time: June 30, 19982359 hours EDT (User specified)

CHEMICAL INFORMATION:

Chemical Name: ETHYL BENZENE

Molecular Weight: $106.17 \mathrm{~kg} / \mathrm{kmol}$

TLV-TWA: $100 \mathrm{ppm}$ IDLH: $800 \mathrm{ppm}$

Footprint Level of Concern: 125 ppm

Boiling Point: $136.20^{\circ} \mathrm{C}$

Vapor Pressure at Ambient Temperature: $0.016 \mathrm{~atm}$

Ambient Saturation Concentration: $16,129 \mathrm{ppm}$ or $1.61 \%$

\section{ATMOSPHERIC INFORMATION: (MANUAL INPUT OF DATA)}

Wind: 1.7 meters $/ \mathrm{sec}$ from $90^{\circ}$ true at 2 meters

Inversion Height: 200 meters

Stability Class: E Air Temperature: $29^{\circ} \mathrm{C}$

Relative Humidity: 50\% Ground Roughness: Urban or forest

Cloud Cover: 1 tenths

\section{SOURCE STRENGTH INFORMATION:}

Puddle Area: 3300 square feet

Average Puddle Depth: 1 centimeters

Soil Type: Default Ground Temperature: $29^{\circ} \mathrm{C}$

Initial Puddle Temperature: Ground temperature

Release Duration: ALOHA limited the duration to 1 hour

Max Computed Release Rate: 4.64 kilograms/min

Max Average Sustained Release Rate: 4.42 kilograms/min (averaged over a minute or more)

Total Amount Released: 250 kilograms

TIME DEPENDENT INFORMATION:

Concentration Estimates at the point:

Downwind: 30 meters

Off Centerline: 0 meters

Max Concentration:

Outdoor: 125 ppm 
Title of Project EPHA for THM

Reviewed by S.A. Henderson

Subject Concentration Analysis for THM Hazardous Chemicals

Works Calculation 8

Computer N.E. Baucom B. K. Tharakan Date 09/16/96

1-RD-19, ADVERSE/SAE

\section{SITE DATA INFORMATION:}

Location: AIKEN, SOUTH CAROLINA

Building Air Exchanges Per Hour: 60 (User specified)

Time: June 30, 19982359 hours EDT (User specified)

\section{CHEMICAL INFORMATION:}

Chemical Name: ETHYL BENZENE

Molecular Weight: $106.17 \mathrm{~kg} / \mathrm{kmol}$

TLV-TWA: 100 ppm IDLH: 800 ppm

Footprint Level of Concern: 125 ppm

Boiling Point: $136.20^{\circ} \mathrm{C}$

Vapor Pressure at Ambient Temperature: 0.016 atm

Ambient Saturation Concentration: 16,129 ppm or $1.61 \%$

ATMOSPHERIC INFORMATION: (MANUAL INPUT OF DATA)

Wind: 1.7 meters $/ \mathrm{sec}$ from $90^{\circ}$ true at 2 meters

Inversion Height: 200 meters

Stability Class: E Air Temperature: $29^{\circ} \mathrm{C}$

Relative Humidity: 50\% Ground Roughness: Urban or forest

Cloud Cover: 1 tenths

\section{SOURCE STRENGTH INFORMATION:}

Puddle Area: 24086 square feet

Average Puddle Depth: 1 centimeters

Soil Type: Default Ground Temperature: $29^{\circ} \mathrm{C}$

Initial Puddle Temperature: Ground temperature

Release Duration: ALOHA limited the duration to 1 hour

Max Computed Release Rate: 31.1 kilograms/min

Max Average Sustained Release Rate: 29.7 kilograms/min

(averaged oyer a minute or more)

Total Amount Released: 1,678 kilograms

\section{TIME DEPENDENT INFORMATION:}

Concentration Estimates at the point:

Downwind: 100 meters

Off Centerline: 0 meters

Max Concentration:

Outdoor: 88.5 ppm 
Title of Project EPHA for THM

Subject Concentration Analysis for THM Hazardous Chemicals

Computer N.E. Baucom B. K. Tharakan Date 09/16/96

1-RD-19 ADVERSE / MAX DIST SEG 1

SITE DATA INFORMATION:

Location: AIKEN, SOUTH CAROLINA

Building Air Exchanges Per Hour: 60 (User specified)

Time: June 30, 19982359 hours EDT (User specified)

CHEMICAL INFORMATION:

Chemical Name: ETHYL BENZENE

Molecular Weight: $106.17 \mathrm{~kg} / \mathrm{kmol}$

TLV-TWA: 100 ppm IDLH: 800 ppm

Footprint Level of Concern: 125 ppm

Boiling Point: $136.20^{\circ} \mathrm{C}$

Vapor Pressure at Ambient Temperature: $0.016 \mathrm{~atm}$

Ambient Saturation Concentration: 16,129 ppm or $1.61 \%$

ATMOSPHERIC INFORMATION: (MANUAL INPUT OF DATA)

Wind: 1.7 meters $/ \mathrm{sec}$ from $90^{\circ}$ true at 2 meters

Inversion Height: 200 meters

Stability Class: E Air Temperature: $29^{\circ} \mathrm{C}$

Relative Humidity: 50\% Ground Roughness: Urban or forest

Cloud Cover: 1 tenths

SOURCE STRENGTH INFORMATION:

Puddle Area: 24420 square feet

Average Puddle Depth: 1 centimeters

Soil Type: Default $\quad$ Ground Temperature: $29^{\circ} \mathrm{C}$

Initial Puddle Temperature: Ground temperature

Release Duration: ALOHA limited the duration to 1 hour

Max Computed Release Rate: 31.5 kilograms/min

Max Average Sustained Release Rate: 30.1 kilograms/min (averaged over a minute or more)

Total Amount Released: 1,700 kilograms

FOOTPRINT INFORMATION:

Model Run: Heavy Gas

User-specified LOC: 125 ppm

Max Threat Zone for LOC: 147 meters
Reviewed by S. A. Henderson

Works Calculation 8

Sheet No. 73 of 314 
Title of Project EPHA for THM

Reviewed by S. A. Henderson

Subject Concentration Analysis for THM Hazardous Chemicals

Computer N.E. Baucom B. K. Tharakan Date 09/16/96

1-RD-19 ADVERSE / MAX DIST SEG 2

SITE DATA INFORMATION:

Location: AIKEN, SOUTH CAROLINA

Building Air Exchanges Per Hour: 60 (User specified)

Time: June 30, 19982359 hours EDT (User specified)

\section{CHEMICAL INFORMATION:}

Chemical Name: ETHYL BENZENE

Molecular Weight: $106.17 \mathrm{~kg} / \mathrm{kmol}$

TLV-TWA: 100 ppm IDLH: 800 ppm

Footprint Level of Concern: 125 ppm

Boiling Point: $136.20^{\circ} \mathrm{C}$

Vapor Pressure at Ambient Temperature: $0.016 \mathrm{~atm}$

Ambient Saturation Concentration: 16,129 ppm or $1.61 \%$

ATMOSPHERIC INFORMATION: (MANUAL INPUT OF DATA)

Wind: 1.7 meters $/ \mathrm{sec}$ from $90^{\circ}$ true at 2 meters

Inversion Height: 200 meters

Stability Class: E Air Temperature: $29^{\circ} \mathrm{C}$

Relative Humidity: 50\% Ground Roughness: Urban or forest

Cloud Cover: 1 tenths

\section{SOURCE STRENGTH INFORMATION:}

Puddle Area: 40700 square feet

Average Puddle Depth: 1 centimeters

Soil Type: Default Ground Temperature: $29^{\circ} \mathrm{C}$

Initial Puddle Temperature: Ground temperature

Release Duration: ALOHA limited the duration to 1 hour

Max Computed Release Rate: 51.4 kilograms/min

Max Average Sustained Release Rate: 49.1 kilograms/min

(averaged over a minute or more)

Total Amount Released: 2,777 kilograms

\section{FOOTPRINT INFORMATION:}

Model Run: Heavy Gas

User-specified LOC: $125 \mathrm{ppm}$

Max Threat Zone for LOC: 189 meters

Works Calculation 8
Sheet No. 74 of 314

Works Calculation 8 
Title of Project EPHA for THM

Subject Concentration Analvsis for THM Hazardous Chemicals

Computer N.E. Baucom B. K. Tharakan Date 09/16/96

1-RD-19 ADVERSE / MAX DIST SEG 3

SITE DATA INFORMATION:

Location: AIKEN, SOUTH CAROLINA

Building Air Exchanges Per Hour: 60 (User specified)

Time: June 30, 19982359 hours EDT (User specified)

CHEMICAL INFORMATION:

Chemical Name: ETHYL BENZENE

Molecular Weight: $106.17 \mathrm{~kg} / \mathrm{kmol}$

TLV-TWA: 100 ppm IDLH: 800 ppm

Footprint Level of Concern: 125 ppm

Boiling Point: $136.20^{\circ} \mathrm{C}$

Vapor Pressure at Ambient Temperature: $0.016 \mathrm{~atm}$

Ambient Saturation Concentration: $16,129 \mathrm{ppm}$ or $1.61 \%$

ATMOSPHERIC INFORMATION: (MANUAL INPUT OF DATA)

Wind: 1.7 meters/sec from $90^{\circ}$ true at 2 meters

Inversion Height: 200 meters

Stability Class: E Air Temperature: $29^{\circ} \mathrm{C}$

Relative Humidity: 50\% Ground Roughness: Urban or forest

Cloud Cover: 1 tenths

\section{SOURCE STRENGTH INFORMATION:}

Puddle Area: 10000 square meters

Average Puddle Depth: 1 centimeters

Soil Type: Default Ground Temperature: $29^{\circ} \mathrm{C}$

Initial Puddle Temperature: Ground temperature

Release Duration: ALOHA limited the duration to 1 hour

Max Computed Release Rate: 131 kilograms/min

Max Average Sustained Release Rate: 125 kilograms/min

(averaged over a minute or more)

Total Amount Released: 7,076 kilograms

\section{FOOTPRINT INFORMATION:}

Model Run: Heavy Gas

User-specified LOC: $125 \mathrm{ppm}$

Max Threat Zone for LOC: 306 meters
Reviewed by S. A. Henderson

Works Calculation 8

Sheet No. 75 of 314 
Title of Projoct EPHA for THM

Subject Concentration Analysis for THM Hazardous Chemicals

Computer N.E. Baucom B. K. Tharakan Date 09/16/96 1-RD-20 ADVERSE / ALERT

\section{SITE DATA INFORMATION:}

Location: AIKEN, SOUTH CAROLINA

Building Air Exchanges Per Hour: 0.44 (Sheltered single storied)

Time: June 30, 19982359 hours EDT (User specified)

\section{CHEMICAL INFORMATION:}

Chemical Name: ETHYLENE GLYCOL MONOBUTYL ETHER

Molecular Weight: $118.18 \mathrm{~kg} / \mathrm{kmol}$

TLV-TWA: $25 \mathrm{ppm}$ IDLH: $700 \mathrm{ppm}$

Footprint Level of Concern: $125 \mathrm{ppm}$

Boiling Point: $171.32^{\circ} \mathrm{C}$

Vapor Pressure at Ambient Temperature: 0.0015 atm

Ambient Saturation Concentration: $1,570 \mathrm{ppm}$ or $0.16 \%$

\section{ATMOSPHERIC INFORMATION: (MANUAL INPUT OF DATA)}

Wind: 1.7 meters $/ \mathrm{sec}$ from $90^{\circ}$ true at 2 meters

Inversion Height: 200 meters

Stability Class: E Air Temperature: $29^{\circ} \mathrm{C}$

Relative Humidity: $50 \%$ Ground Roughness: Urban or forest

Cloud Cover: 1 tenths

\section{SOURCE STRENGTH INFORMATION:}

Puddle Area: 30001 square feet

Average Puddle Depth: 1 centimeters

Soil Type: Default Ground Temperature: $29^{\circ} \mathrm{C}$

Initial Puddle Temperature: Ground temperature

Release Duration: ALOHA limited the duration to 1 hour

Max Computed Release Rate: 4.04 kilograms/min

Max Average Sustained Release Rate: 3.91 kilograms/min

(averaged over a minute or more)

Total Amount Released: 224 kilograms

\section{TIME DEPENDENT INFORMATION:}

Concentration Estimates at the point:

Downwind: 30 meters

Off Centerline: 0 meters

Max Concentration:

Outdoor: $95.1 \mathrm{ppm}$

Indoor: $32.1 \mathrm{ppm}$

Note: Indoor graph is shown with a dotted line.
Reviewed by S. A. Henderson

Works Calculation 8

Sheet No. 76 of 314 
Title of Project EPHA for THM

Subject Concentration Analysis for THM Hazardous Chemicals

Computer N.E. Baucom B. K. Tharakan Date 09/16/96

1-RD-20 ADVERSE / SAE

SITE DATA INFORMATION:

Location: AIKEN, SOUTH CAROLINA

Building Air Exchanges Per Hour: 0.44 (Sheltered single storied)

Time: June 30, 19982359 hours EDT (User specified)

CHEMICAL INFORMATION:

Chemical Name: ETHYLENE GLYCOL MONOBUTYL ETHER

Molecular Weight: $118.18 \mathrm{~kg} / \mathrm{kmol}$

TLV-TWA: $25 \mathrm{ppm}$ IDLH: $700 \mathrm{ppm}$

Footprint Level of Concern: 125 ppm

Boiling Point: $171.32^{\circ} \mathrm{C}$

Vapor Pressure at Ambient Temperature: $0.0015 \mathrm{~atm}$

Ambient Saturation Concentration: $1,570 \mathrm{ppm}$ or $0.16 \%$

ATMOSPHERIC INFORMATION: (MANUAL INPUT OF DATA)

Wind: 1.7 meters $/ \mathrm{sec}$ from $90^{\circ}$ true at 2 meters

Inversion Height: 200 meters

Stability Class: E Air Temperature: $29^{\circ} \mathrm{C}$

Relative Humidity: 50\% Ground Roughness: Urban or forest

Cloud Cover: 1 tenths

\section{SOURCE STRENGTH INFORMATION:}

Puddle Area: 100001 square feet

Average Puddle Depth: 1 centimeters

Soil Type: Default Ground Temperature: $29^{\circ} \mathrm{C}$

Initial Puddle Temperature: Ground temperature

Release Duration: ALOHA limited the duration to 1 hour

Max Computed Release Rate: 12.8 kilograms/min

Max Average Sustained Release Rate: 12.4 kilograms/min

(averaged over a minute or more)

Total Amount Released: 713 kilograms

\section{TIME DEPENDENT INFORMATION:}

Concentration Estimates at the point:

Downwind: 100 meters

Off Centerline: 0 meters

Max Concentration:

Outdoor: $28.6 \mathrm{ppm}$

Indoor: $9.66 \mathrm{ppm}$

Note: Indoor graph is shown with a dotted line.
Reviewed by S.A. Henderson

Works Calculation 8

Sheet No. 77 of 314 
Title of Project EPHA for THM

Reviewed by S.A. Henderson

Subject Concentration Analysis for THM Hazardous Chemicals

Works Calculation 8

Computer N.E. Baucom B. K. Tharakan Date 09/16/96

1-RD-20 ADVERSE / MAX DIST SEG 1

Sheet No. 78 of 314

SITE DATA INFORMATION:

Location: AIKEN, SOUTH CARÒLINA

Building Air Exchanges Per Hour: 0.44 (Sheltered single storied)

Time: June 30, 19982359 hours EDT (User specified)

CHEMICAL INFORMATION:

Chemical Name: ETHYLENE GLYCOL MONOBUTYL ETHER

Molecular Weight: $118.18 \mathrm{~kg} / \mathrm{kmol}$

TLV-TWA: 25 ppm IDLH: 700 ppm

Footprint Level of Concern: 125 ppm

Boiling Point: $171.32^{\circ} \mathrm{C}$

Vapor Pressure at Ambient Temperature: $0.0015 \mathrm{~atm}$

Ambient Saturation Concentration: $1,570 \mathrm{ppm}$ or $0.16 \%$

ATMOSPHERIC INFORMATION: (MANUAL INPUT OF DATA)

Wind: 1.7 meters $/ \mathrm{sec}$ from $90^{\circ}$ true at 2 meters

Inversion Height: 200 meters

Stability Class: E Air Temperature: $29^{\circ} \mathrm{C}$

Relative Humidity: 50\% Ground Roughness: Urban or forest

Cloud Cover: 1 tenths

SOURCE STRENGTH INFORMATION:

Puddle Area: 24420 square feet

Average Puddle Depth: 1 centimeters

Soil Type: Default Ground Temperature: $29^{\circ} \mathrm{C}$

Initial Puddle Temperature: Ground temperature

Release Duration: ALOHA limited the duration to 1 hour

Max Computed Release Rate: 3.32 kilograms/min

Max Average Sustained Release Rate: 3.21 kilograms/min

(averaged over a minute or more)

Total Amount Released: 184 kilograms

FOOTPRINT INFORMATION:

Dispersion Module: Gaussian

User-specified LOC: 125 ppm

Max Threat Zone for LOC: 27 meters

Max Threat Zone for IDLH: 27 meters

Note: Footprint was not drawn because

effects of near-field patchiness make dispersion

Predictions unreliable for short distances. 
Title of Project EPHA for THM

Subject Concentration Analysis for THM Hazardous Chemicals

Computer N.E. Baucom B. K. Tharakan Date 09/16/96

\section{1-RD-20 ADVERSE / MAX DIST SEG 2}

\section{SITE DATA INFORMATION:}

\section{Location: AIKEN, SOUTH CAROLINA}

Building Air Exchanges Per Hour: 0.44 (Sheltered single storied)

Time: June 30, 19982359 hours EDT (User specified)

\section{CHEMICAL INFORMATION:}

Chemical Name: ETHYLENE GLYCOL MONOBUTYL ETHER

Molecular Weight: $118.18 \mathrm{~kg} / \mathrm{kmol}$

TLV-TWA: 25 ppm IDLH: $700 \mathrm{ppm}$

Footprint Level of Concern: 125 ppm

Boiling Point: $171.32^{\circ} \mathrm{C}$

Vapor Pressure at Ambient Temperature: $0.0015 \mathrm{~atm}$

Ambient Saturation Concentration: 1,570 ppm or $0.16 \%$

\section{ATMOSPHERIC INFORMATION: (MANUAL INPUT OF DATA)}

Wind: 1.7 meters $/ \mathrm{sec}$ from $90^{\circ}$ true at 2 meters

Inversion Height: 200 meters

Stability Class: E Air Temperature: $29^{\circ} \mathrm{C}$

Relative Humidity: 50\% Ground Roughness: Urban or forest

Cloud Cover: 1 tenths

\section{SOURCE STRENGTH INFORMATION:}

Puddle Area: 40700 square feet

Average Puddle Depth: 1 centimeters

Soil Type: Default Ground Temperature: $29^{\circ} \mathrm{C}$

Initial Puddle Temperature: Ground temperature

Release Duration: ALOHA limited the duration to 1 hour

Max Computed Release Rate: 5.42 kilograms/min

Max Average Sustained Release Rate: 5.25 kilograms/min (averaged over a minute or more)

Total Amount Released: 301 kilograms

\section{FOOTPRINT INFORMATION:}

Dispersion Module: Gaussian

User-specified LOC: $125 \mathrm{ppm}$

Max Threat Zone for LOC: 34 meters

Max Threat Zone for IDLH: 35 meters

Note: Footprint was not drawn because

effects of near-field patchiness make dispersion

Predictions unreliable for short distances.
Reviewed by S. A. Henderson

Works Calculation 8

Sheet No. 79 of 314 
Title of Project EPHA for THM

Subject Concentration Analysis for THM Hazardous Chemicals

Computer N.E. Baucom B. K. Tharakan Date 09/16/96 1-RD-20 ADVERSE / MAX DIST SEG 3

\section{SITE DATA INFORMATION:}

Location: AIKEN, SOUTH CAROLINA

Building Air Exchanges Per Hour: 0.44 (Sheltered single storied)

Time: June 30, 19982359 hours EDT (User specified)

\section{CHEMICAL INFORMATION:}

Chemical Name: ETHYLENE GLYCOL MONOBUTYL ETHER

Molecular Weight: $118.18 \mathrm{~kg} / \mathrm{kmol}$

TLV-TWA: $25 \mathrm{ppm}$ IDLH: $700 \mathrm{ppm}$

Footprint Level of Concern: 125 ppm

Boiling Point: $171.32^{\circ} \mathrm{C}$

Vapor Pressure at Ambient Temperature: $0.0015 \mathrm{~atm}$

Ambient Saturation Concentration: $1,570 \mathrm{ppm}$ or $0.16 \%$

\section{ATMOSPHERIC INFORMATION: (MANUAL INPUT OF DATA)}

Wind: 1.7 meters $/ \mathrm{sec}$ from $90^{\circ}$ true at 2 meters

Inversion Height: 200 meters

Stability Class: E Air Temperature: $29^{\circ} \mathrm{C}$

Relative Humidity: 50\% Ground Roughness: Urban or forest

Cloud Cover: 1 tenths

\section{SOURCE STRENGTH INFORMATION:}

Puddle Area: 10000 square meters

Average Puddle Depth: 1 centimeters

Soil Type: Default Ground Temperature: $29^{\circ} \mathrm{C}$

Initial Puddle Temperature: Ground temperature

Release Duration: ALOHA limited the duration to 1 hour

Max Computed Release Rate: 13.8 kilograms/min

Max Average Sustained Release Rate: 13.4 kilograms/min (averaged over a minute or more)

Total Amount Released: 766 kilograms

\section{FOOTPRINT INFORMATION:}

Dispersion Module: Gaussian

User-specified LOC: $125 \mathrm{ppm}$

Max Threat Zone for LOC: 56 meters

Max Threat Zone for IDLH: 56 meters

Note: Footprint was not drawn because

effects of near-field patchiness make dispersion

Predictions unreliable for short distances.
Reviewed by S. A. Henderson

Works Calculation 8

Sheet No. $\underline{80 \text { of } 314}$ 
Title of Project EPHA for THM

Subject Concentration Analysis for THM Hazardous Chemicals

Computer N.E. Baucom B. K. Tharakan Date 09/16/96
Reviewed by S. A. Henderson

Works Calculation 8

Sheet No. 81 of 314

\section{1-RD-22 ADVERSE / ALERT}

\section{SITE DATA INFORMATION:}

Location: AIKEN, SOUTH CAROLINA

Building Air Exchanges Per Hour: 60 (User Specified)

Time: June 30, 19982359 hours EDT (User specified)

CHEMICAL INFORMATION:

Chemical Name: FORMIC ACID

Molecular Weight: $46.03 \mathrm{~kg} / \mathrm{kmol}$

TLV-TWA: 5 ppm IDLH: 30 ppm

Footprint Level of Concern: $10 \mathrm{ppm}$

Boiling Point: $100.56^{\circ} \mathrm{C}$

Vapor Pressure at Ambient Temperature: 0.068 atm

Ambient Saturation Concentration: 69,160 ppm or $6.92 \%$

ATMOSPHERIC INFORMATTON: (MANUAL INPUT OF DATA)

Wind: 1.7 meters $/ \mathrm{sec}$ from $90^{\circ}$ true at 2 meters

Inversion Height: 200 meters

Stability Class: E Air Temperature: $29^{\circ} \mathrm{C}$

Relative Humidity: 50\% Ground Roughness: Urban or forest

Cloud Cover: 1 tenths

\section{SOURCE STRENGTH INFORMATION:}

Puddle Area: 7 square feet

Average Puddle Depth: 1 centimeters

Soil Type: Default Ground Temperature: $29^{\circ} \mathrm{C}$

Initial Puddle Temperature: Ground temperature

Release Duration: ALOHA limited the duration to 1 hour

Max Computed Release Rate: $\mathbf{3 2 . 8}$ grams/min

Max Average Sustained Release Rate: 32.8 grams/min

(averaged over a minute or more)

Total Amount Released: 1.44 kilograms

TIME DEPENDENT INFORMATION:

Concentration Estimates at the point:

Downwind: 30 meters

Off Centerline: 0 meters

Max Concentration:

Outdoor: $10.1 \mathrm{ppm}$ 
Title of Project EPHA for THM

Subject Concentration Analysis for THM Hazardous Chemicals

Computer N.E. Baucom B. K. Tharakan Date 09/16/96

1.RD-22 ADVERSE / SAE

SITE DATA INFORMATION:

Location: AIKEN, SOUTH CAROLINA

Building Air Exchanges Per Hour: 60 (User Specified)

Time: June 30, 19982359 hours EDT (User specified)

CHEMICAL INFORMATION:

Chemical Name: FORMIC ACID

Molecular Weight: $46.03 \mathrm{~kg} / \mathrm{kmol}$

TLV-TWA: $5 \mathrm{ppm} \quad$ IDLH: $30 \mathrm{ppm}$

Footprint Level of Concern: $10 \mathrm{ppm}$

Boiling Point: $100.56^{\circ} \mathrm{C}$

Vapor Pressure at Ambient Temperature: $0.068 \mathrm{~atm}$

Ambient Saturation Concentration: 69,160 ppm or $6.92 \%$

ATMOSPHERIC INFORMATION: (MANUAL INPUT OF DATA)

Wind: 1.7 meters $/ \mathrm{sec}$ from $90^{\circ}$ true at 2 meters

Inversion Height: 200 meters

Stability Class: E Air Temperature: $29^{\circ} \mathrm{C}$

Relative Humidity: $50 \%$ Ground Roughness: Urban or forest

Cloud Cover: 1 tenths

\section{SOURCE STRENGTH INFORMATION:}

Puddle Area: 101 square feet

Average Puddle Depth: 1 centimeters

Soil Type: Default Ground Temperature: $29^{\circ} \mathrm{C}$

Initial Puddle Temperature: Ground temperature

Release Duration: ALOHA limited the duration to 1 hour

Max Computed Release Rate: 393 grams/min

Max Average Sustained Release Rate: 345 grams/min

(averaged over a minute or more)

Total Amount Released: 71.8 kilograms

TIME DEPENDENT INFORMATION:

Concentration Estimates at the point:

Downwind: 100 meters

Off Centerline: 0 meters

Max Concentration:

Outdoor: $10 \mathrm{ppm}$
Reviewed by S. A. Henderson

Works Calculation 8

Sheet No. 82 of 314 
Title of Project EPHA for THM

Subject Concentration Analysis for THM Hazardous Chemicals

Computer N.E. Baucom B. K. Tharakan Date 09/16/96

-1-RD-22 ADVERSE / MAX DIST SEG 1

\section{SITE DATA INFORMATION:}

Location: AIKEN, SOUTH CAROLINA.

Building Air Exchanges Per Hour: 60 (User specified)

Time: June 30, 19982359 hours EDT (User specified)

\section{CHEMICAL INFORMATION:}

Chemical Name: FORMIC ACID

Molecular Weight: $46.03 \mathrm{~kg} / \mathrm{kmol}$

TLV-TWA: 5 ppm IDLH: $30 \mathrm{ppm}$

Footprint Level of Concern: $10 \mathrm{ppm}$

Boiling Point: $100.56^{\circ} \mathrm{C}$

Vapor Pressure at Ambient Temperature: $0.068 \mathrm{~atm}$

Ambient Saturation Concentration: 69,160 ppm or $6.92 \%$

\section{ATMOSPHERIC INFORMATION: (MANUAL INPUT OF DATA)}

Wind: 1.7 meters $/ \mathrm{sec}$ from $90^{\circ}$ true at 2 meters

Inversion Height: 200 meters

Stability Class: E Air Temperature: $29^{\circ} \mathrm{C}$

Relative Humidity: $50 \%$ Ground Roughness: Urban or forest

Cloud Cover: 1 tenths

\section{SOURCE STRENGTH INFORMATION:}

Puddle Area: 24420 square feet

Average Puddle Depth: 1 centimeters

Soil Type: Default * Ground Temperature: $29^{\circ} \mathrm{C}$

Initial Puddle Temperature: Ground temperature

Release Duration: ALOHA limited the duration to 1 hour

Max Computed Release Rate: 70.1 kilograms/min

Max Average Sustained Release Rate: 63.1 kilograms/min

(averaged over a minute or more)

Total Amount Released: 3,331 kilograms

FOOTPRINT INFORMATION:

Model Run: Heavy Gas

User-specified LOC: $10 \mathrm{ppm}$

Max Threat Zone for LOC: 2.4 kilometers
Reviewed by S. A. Henderson

Works Calculation 8

Sheet No. 83 of 314 
Title of Project EPHA for THM

Subject Concentration Analysis for THM Hazardous Chemicals

Computer N.E. Baucom B. K. Tharakan Date 09/16/96

1-RD-22 ADVERSE / MAX DIST SEG 2

\section{SITE DATA INFORMATION:}

Location: AIKEN, SOUTH CAROLINA

Building Air Exchanges Per Hour: 60 (User specified)

Time: June 30, 19982359 hours EDT (User specified)

\section{CHEMICAL INFORMATION:}

Chemical Name: FORMIC ACID

Molecular Weight: $46.03 \mathrm{~kg} / \mathrm{kmol}$

TLV-TWA: 5 ppm IDLH: $30 \mathrm{ppm}$

Footprint Level of Concern: $10 \mathrm{ppm}$

Boiling Point: $100.56^{\circ} \mathrm{C}$

Vapor Pressure at Ambient Temperature: $0.068 \mathrm{~atm}$

Ambient Saturation Concentration: 69,160 ppm or $6.92 \%$

ATMOSPHERIC INFORMATION: (MANUAL INPUT OF DATA)

Wind: 1.7 meters $/ \mathrm{sec}$ from $90^{\circ}$ true at 2 meters

Inversion Height: 200 meters

Stability Class: E Air Temperature: $29^{\circ} \mathrm{C}$

Relative Humidity: 50\% Ground Roughness: Urban or forest

Cloud Cover: 1 tenths

\section{SOURCE STRENGTH INFORMATTON:}

Puddle Area: 40700 square feet

Average Puddle Depth: 1 centimeters

Soil Type: Default Ground Temperature: $29^{\circ} \mathrm{C}$

Initial Puddle Temperature: Ground temperature

Release Duration: ALOHA limited the duration to 1 hour

Max Computed Release Rate: 114 kilograms/min

Max Average Sustained Release Rate: 103 kilograms/min

(averaged over a minute or more)

Total Amount Released: 5,437 kilograms

FOOTPRINT INFORMATION:

Model Run: Heavy Gas

User-specified LOC: $10 \mathrm{ppm}$

Max Threat Zone for LOC: 3.1 kilometers
Reviewed by S. A. Henderson

Works Calculation 8

Sheet No. 84 of 314 
Title of Project EPHA for THM

Subject Concentration Analysis for THM Hazardous Chemicals

Computer N.E. Baucom B. K. Tharakan Date 09/16/96

1.RD-22 ADVERSE / MAX DIST SEG 3

\section{SITE DATA INFORMATION:}

Location: AIKEN, SOUTH CAROLINA

Building Air Exchanges Per Hour: 60 (User specified)

Time: June 30, 19982359 hours EDT (User specified)

\section{CHEMICAL INFORMATION:}

Chemical Name: FORMIC ACID

Molecular Weight: $46.03 \mathrm{~kg} / \mathrm{kmol}$

TLV-TWA: 5 ppm IDLH: $30 \mathrm{ppm}$

Footprint Level of Concern: $10 \mathrm{ppm}$

Boiling Point: $100.56^{\circ} \mathrm{C}$

Vapor Pressure at Ambient Temperature: $0.068 \mathrm{~atm}$

Ambient Saturation Concentration: 69,160 ppm or $6.92 \%$

ATMOSPHERIC INFORMATION: (MANUAL INPUT OF DATA)

Wind: 1.7 meters $/ \mathrm{sec}$ from $90^{\circ}$ true at 2 meters

Inversion Height: 200 meters

Stability Class: E Air Temperature: $29^{\circ} \mathrm{C}$

Relative Humidity: 50\% Ground Roughness: Urban or forest

Cloud Cover: 1 tenths

\section{SOURCE STRENGTH INFORMATION:}

Puddle Area: 10000 square meters

Average Puddle Depth: 1 centimeters

Soil Type: Default Ground Temperature: $29^{\circ} \mathrm{C}$

Initial Puddle Temperature: Ground temperature

Release Duration: ALOHA limited the duration to 1 hour

Max Computed Release Rate: 288 kilograms/min

Max Average Sustained Release Rate: 261 kilograms/min

(averaged over a minute or more)

Total Amount Released: 13,836 kilograms

\section{FOOTPRINT INFORMATION:}

Model Run: Heavy Gas

User-specified LOC: $10 \mathrm{ppm}$

Max Threat Zone for LOC: 5.0 kilometers
Reviewed by S.A. Henderson

Works Calculation 8

Sheet No. 85 of 314 
Title of Project EPHA for THM

Subject Concentration Analysis for THM Hazardous Chemicals

Computer N.E. Baucom B. K. Tharakan Date 09/16/96

1-RD-23 ADVERSE / ALERT

\section{SITE DATA INFORMATION:}

Location: AIKEN, SOUTH CAROLINA

Building Air Exchanges Per Hour: 0.44 (Sheltered single storied)

Time: June 30, 19982359 hours EDT (User specified)

\section{CHEMICAL INFORMATION:}

Chemical Name: DIETHYLENE GLYCOL DIMETHYL ETHER

Molecular Weight: $134.18 \mathrm{~kg} / \mathrm{kmol}$

TLV-TWA: -unavail- IDLH: -unavail-

Footprint Level of Concern: $500 \mathrm{mg} /$ (cu m)

Boiling Point: $159.76^{\circ} \mathrm{C}$

Vapor Pressure at Ambient Temperature: $0.0050 \mathrm{~atm}$

Ambient Saturation Concentration: 5,107 ppm or $0.51 \%$

\section{ATMOSPHERIC INFORMATION: (MANUAL INPUT OF DATA)}

Wind: 1.7 meters $/ \mathrm{sec}$ from $90^{\circ}$ true at 2 meters

Inversion Height: 200 meters

Stability Class: E Air Temperature: $29^{\circ} \mathrm{C}$

Relative Humidity: 50\% Ground Roughness: Urban or forest

Cloud Cover: 1 tenths

\section{SOURCE STRENGTH INFORMATION:}

Puddle Area: 8750 square feet

Average Puddle Depth: 1 centimeters

Soil Type: Default Ground Temperature: $29^{\circ} \mathrm{C}$

Initial Puddle Temperature: Ground temperature

Release Duration: ALOHA limited the duration to 1 hour

Max Computed Release Rate: 4.47 kilograms/min

Max Average Sustained Release Rate: 4.32 kilograms/min

(averaged over a minute or more)

Total Amount Released: 248 kilograms

\section{TIME DEPENDENT INFORMATION:}

Concentration Estimates at the point:

Downwind: 30 meters

Off Centerline: 0 meters

Max Concentration:

Outdoor: $500 \mathrm{mg} /(\mathrm{cu} \mathrm{m})$

Indoor: $169 \mathrm{mg} /$ (cu m)

Note: Indoor graph is shown with a dotted line.
Reviewed by S. A. Henderson

Works Calculation 8

Sheet No. 86 of 314 
Title of Project EPHA for THM

Reviewed by S.A. Henderson

Subject Concentration Analysis for THM Hazardous Chemicals

Computer N.E. Baucom B. K. Tharakan Date 09/16/96 1-RD-23 ADVERSE / SAE

SITE DATA INFORMATION:

Location: AIKEN, SOUTH CAROLINA

Building Air Exchanges Per Hour: 0.44 (Sheltered single storied)

Time: June 30, 19982359 hours EDT (User specified)

\section{CHEMICAL INFORMATION:}

Chemical Name: DIETHYLENE GLYCOL DIMETHYL ETHER

Molecular Weight: $134.18 \mathrm{~kg} / \mathrm{kmol}$

TLV-TWA: -unavail- IDLH: -unavail-

Footprint Level of Concern: $500 \mathrm{mg} /$ (cu m)

Boiling Point: $159.76^{\circ} \mathrm{C}$

Vapor Pressure at Ambient Temperature: $0.0050 \mathrm{~atm}$

Ambient Saturation Concentration: 5,107 ppm or $0.51 \%$

ATMOSPHERIC INFORMATION: (MANUAL INPUT OF DATA)

Wind: 1.7 meters $/ \mathrm{sec}$ from $90^{\circ}$ true at 2 meters

Inversion Height: 200 meters

Stability Class: E Air Temperature: $29^{\circ} \mathrm{C}$

Relative Humidity: 50\% Ground Roughness: Urban or forest

Cloud Cover: 1 tenths

SOURCE STRENGTH INFORMATION:

Puddle Area: 100001 square feet

Average Puddle Depth: 1 centimeters

Soil Type: Default Ground Temperature: $29^{\circ} \mathrm{C}$

Initial Puddle Temperature: Ground temperature

Release Duration: ALOHA limited the duration to 1 hour

Max Computed Release Rate: 46.4 kilograms/min

Max Average Sustained Release Rate: $\mathbf{4 4 . 9}$ kilograms/min (averaged over a minute or more)

Total Amount Released: 2,574 kilograms

TIME DEPENDENT INFORMATION:

Concentration Estimates at the point:

Downwind: 100 meters

Off Centerline: 0 meters

Max Concentration:

Outdoor: $492 \mathrm{mg} /$ (cu m)

Indoor: $166 \mathrm{mg} /$ (cu m)

Note: Indoor graph is shown with a dotted line.
Works Calculation 8

Sheet No. 87 of 314 
Title of Project EPHA for THM

Subject Concentration Analysis for THM Hazardous Chemicals

Computer N.E. Baucom B. K. Tharakan Date 09/16/96

\section{1-RD-23 ADVERSE / MAX DIST SEG 1}

\section{SITE DATA INFORMATION:}

Location: AIKEN, SOUTH CAROLINA

Building Air Exchanges Per Hour: 0.44 (Sheltered single storied)

Time: June 30, 19982359 hours EDT (User specified)

\section{CHEMICAL INFORMATION:}

( Chemical Name: DIETHYLENE GLYCOL DIMETHYL ETHER

Molecular Weight: $134.18 \mathrm{~kg} / \mathrm{kmol}$

TLV-TWA: - unavail- IDLH: -unavail-

Footprint Level of Concern: $500 \mathrm{mg} /$ ( $\mathrm{cu} \mathrm{m}$ )

Boiling Point: $159.76^{\circ} \mathrm{C}$

Vapor Pressure at Ambient Temperature: $0.0050 \mathrm{~atm}$

Ambient Saturation Concentration: 5,107 ppm or 0.51\%

ATMOSPHERIC INFORMATION: (MANUAL INPUT OF DATA)

Wind: 1.7 meters $/ \mathrm{sec}$ from $90^{\circ}$ true at 2 meters

Inversion Height: 200 meters

Stability Class: E Air Temperature: $29^{\circ} \mathrm{C}$

Relative Humidity: $50 \%$ Ground Roughness: Urban or forest

Cloud Cover: 1 tenths

SOURCE STRENGTH INFORMATION:

Puddle Area: 24420 square feet

Average Puddle Depth: 1 centimeters

Soil Type: Default Ground Temperature: $29^{\circ} \mathrm{C}$

Initial Puddle Temperature: Ground temperature

Release Duration: ALOHA limited the duration to 1 hour

Max Computed Release Rate: 12 kilograms/min

Max Average Sustained Release Rate: 11.6 kilograms/min (averaged over a minute or more)

Total Amount Released: 663 kilograms

\section{FOOTPRINT INFORMATION:}

Dispersion Module: Gaussian

User-specified LOC: $500 \mathrm{mg} /$ (cu m)

Max Threat Zone for LOC: 50 meters

Note: Footprint was not drawn because

effects of near-field patchiness make dispersion

predictions unreliable for short distances.
Reviewed by $\underline{\text { S. A. Henderson }}$

Works Calculation 8

Sheet No. 88 of 314 
Title of Project EPHA for THM

Subject Concentration Analysis for THM Hazardous Chemicals

Computer N.E. Baucom B. K. Tharakan Date 09/16/96
Reviewed by $\mathbf{S}$. A. Henderson

Works Calculation 8

Sheet No. $\underline{89 \text { of } 314}$

\section{1-RD-23 ADVERSE / MAX DIST SEG 2}

SITE DATA INFORMATION:

Location: AIKEN, SOUTH CAROLINA

Building Air Exchanges Per Hour: 0.44 (Sheltered single storied)

Time: June 30, 19982359 hours EDT (User specified)

CHEMICAL INFORMATION:

Chemical Name: DIETHYLENE GLYCOL DIMETHYL ETHER

Molecular Weight: $134.18 \mathrm{~kg} / \mathrm{kmol}$

TLV-TWA: -unavail- IDLH: -unavail-

Footprint Level of Concern: $500 \mathrm{mg} /$ (cu m)

Boiling Point: $159.76^{\circ} \mathrm{C}$

Vapor Pressure at Ambient Temperature: $0.0050 \mathrm{~atm}$

Ambient Saturation Concentration: 5,107 ppm or 0.51\%

ATMOSPHERIC INFORMATION: (MANUAL INPUT OF DATA)

Wind: 1.7 meters $/ \mathrm{sec}$ from $90^{\circ}$ true at 2 meters

Inversion Height: 200 meters

Stability Class: E Air Temperature: $29^{\circ} \mathrm{C}$

Relative Humidity: 50\% Ground Roughness: Urban or forest

Cloud Cover: 1 tenths

SOURCE STRENGTH INFORMATION:

Puddle Area: 40700 square feet

Average Puddle Depth: 1 centimeters

Soil Type: Default Ground Temperature: $29^{\circ} \mathrm{C}$

Initial Puddle Temperature: Ground temperature

Release Duration: ALOHA limited the duration to 1 hour

Max Computed Release Rate: 19.6 kilograms/min

Max Average Sustained Release Rate: 18.9 kilograms/min

(averaged over a minute or more)

Total Amount Released: 1,084 kilograms

FOOTPRINT INFORMATION:

Dispersion Module: Gaussian

User-specified LOC: $500 \mathrm{mg} /$ (cu m)

Max Threat Zone for LOC: 64 meters

Note: Footprint was not drawn because

effects of near-field patchiness make dispersion

predictions unreliable for short distances. 
Subject Concentration Analvsis for THM Hazardous Chemicals Works Calculation 8

Computer N.E. Baucom B. K. Tharakan Date 09/16/96 1-RD-23 ADVERSE / MAX DIST SEG 3

\section{SITE DATA INFORMATION:}

Location: AIKEN, SOUTH CAROLINA

Building Air Exchanges Per Hour: 0.44 (Sheltered single storied)

Time: June 30, 19982359 hours EDT (User specified) .

\section{CHEMICAL INFORMATION:}

Chemical Name: DIETHYLENE GLYCOL DIMETHYL ETHER

Molecular Weight: $134.18 \mathrm{~kg} / \mathrm{kmol}$

TLV-TWA: -unavail- IDLH: -unavail-

Footprint Level of Concern: $500 \mathrm{mg} /$ ( cu m)

Boiling Point: $159.76^{\circ} \mathrm{C}$

Vapor Pressure at Ambient Temperature: $0.0050 \mathrm{~atm}$

Ambient Saturation Concentration: 5,107 ppm or $0.51 \%$

\section{ATMOSPHERIC INFORMATION: (MANUAL INPUT OF DATA)}

Wind: 1.7 meters $/ \mathrm{sec}$ from $90^{\circ}$ true at 2 meters

Inversion Height: 200 meters

Stability Class: E Air Temperature: $29^{\circ} \mathrm{C}$

Relative Humidity: 50\% Ground Roughness: Urban or forest

Cloud Cover: 1 tenths

\section{SOURCE STRENGTH INFORMATION:}

Puddle Area: 10000 square meters

Average Puddle Depth: 1 centimeters

Soil Type: Default Ground Temperature: $29^{\circ} \mathrm{C}$

Initial Puddle Temperature: Ground temperature

Release Duration: ALOHA limited the duration to 1 hour

Max Computed Release Rate: 49.8 kilograms/min

Max Average Sustained Release Rate: 48.29 kilograms/min

(averaged over a minute or more)

Total Amount Released: 2,763 kilograms

\section{FOOTPRINT INFORMATION:}

Dispersion Module: Gaussian

User-specified LOC: $500 \mathrm{mg} /$ (cu m)

Max Threat Zone for LOC: 103 meters

Note: Footprint was not drawn because dispersion preditions are unreliable for lengths less than the maximum diameter of the puddle. Maximum diameter of the puddle: 113 meters 
Title of Project EPHA for THM

Subject Concentration Analysis for THM Hazardous Chemicals

Computer N.E. Baucom B. K. Tharakan Date 09/16/96

\section{1-RD-26 ADVERSE / ALERT}

\section{SITE DATA INFORMATION:}

Location: AIKEN, SOUTH CAROLINA

Building Air Exchanges Per Hour: 60 (User specified)

Time: June 30, 19982359 hours EDT (User specified)

\section{CHEMICAL INFORMATION:}

Chemical Name: HYDROGEN PEROXIDE

Molecular Weight: $34.01 \mathrm{~kg} / \mathrm{kmol}$

TLV-TWA: 1 ppm IDLH: 75 ppm

Footprint Level of Concern: $50 \mathrm{ppm}$

Boiling Point: $150.20^{\circ} \mathrm{C}$

Vapor Pressure at Ambient Temperature: $0.0034 \mathrm{~atm}$

Ambient Saturation Concentration: 3,462 ppm or $0.35 \%$

ATMOSPHERIC INFORMATION: (MANUAL INPUT OF DATA)

Wind: 1.7 meters $/ \mathrm{sec}$ from $90^{\circ}$ true at 2 meters

Inversion Height: 200 meters

Stability Class: E Air Temperature: $29^{\circ} \mathrm{C}$

Relative Humidity: $50 \%$ Ground Roughness: Urban or forest

Cloud Cover: 1 tenths

\section{SOURCE STRENGTH INFORMATION:}

Puddle Area: 5175 square feet

Average Puddle Depth: 1 centimeters

Soil Type: Default Ground Temperature: $29^{\circ} \mathrm{C}$

Initial Puddle Temperature: Ground temperature

Release Duration: ALOHA limited the duration to 1 hour

Max Computed Release Rate: 607 grams/min

Max Average Sustained Release Rate: 589 grams/min (averaged over a minute or more)

Total Amount Released: 33.6 kilograms

\section{TIME DEPENDENT INFORMATION:}

Concentration Estimates at the point:

Downwind: 30 meters

Off Centerline: 0 meters

Max Concentration:

Outdoor: 50 ppm
Reviewed by S. A. Henderson

Works Calculation 8

Sheet No. 91 of 314 
Title of Project EPHA for THM

Reviewed by S. A. Henderson

Subject Concentration Analysis for THM Hazardous Chemicals

Computer N.E. Baucom B. K. Tharakan Date 09/16/96

1-RD-26 ADVERSE / SAE

SITE DATA INFORMATION:

Location: AIKEN, SOUTH CAROLINA

Building Air Exchanges Per Hour: 60 (User specified)

Time: June 30, 19982359 hours EDT (User specified)

\section{CHEMICAL INFORMATION:}

Chemical Name: HYDROGEN PEROXIDE

Molecular Weight: $34.01 \mathrm{~kg} / \mathrm{kmol}$

TLV-TWA: 1 ppm IDLH: 75 ppm

Footprint Level of Concern: $50 \mathrm{ppm}$

Boiling Point: $150.20^{\circ} \mathrm{C}$

Vapor Pressure at Ambient Temperature: $0.0034 \mathrm{~atm}$

Ambient Saturation Concentration: 3,462 ppm or $0.35 \%$

ATMOSPHERIC INFORMATION: (MANUAL INPUT OF DATA)

Wind: 1.7 meters $/ \mathrm{sec}$ from $90^{\circ}$ true at 2 meters

Inversion Height: 200 meters

Stability Class: E Air Temperature: $29^{\circ} \mathrm{C}$

Relative Humidity: 50\% Ground Roughness: Urban or forest

Cloud Cover: 1 tenths

\section{SOURCE STRENGTH INFORMATION:}

Puddle Area: 62200 square feet

Average Puddle Depth: 1 centimeters

Soil Type: Default Ground Temperature: $29^{\circ} \mathrm{C}$

Initial Puddle Temperature: Ground temperature

Release Duration: ALOHA limited the duration to 1 hour

Max Computed Release Rate: 6.42 kilograms/min

Max Average Sustained Release Rate: 6.23 kilograms/min

(averaged over a minute or more)

Total Amount Released: 356 kilograms

\section{TIME DEPENDENT INFORMATION:}

Concentration Estimates at the point:

Downwind: 100 meters

Off Centerline: 0 meters

Max Concentration:

Outdoor: $50 \mathrm{ppm}$
Works Calculation 8

Sheet No. 92 of 314 
Titie of Project EPHA for THM

Subject Concentration Analysis for THM Hazardous Chemicals

Computer N.E. Baucom B. K. Tharakan Date 09/16/96

1-RD-26 ADVERSE / MAX DIST SEG 1

\section{SITE DATA INFORMATION:}

Location: AIKEN, SOUTH CAROLINA

Building Air Exchanges Per Hour: 60 (User specified)

Time: June 30, 19982359 hours EDT (User specified)

\section{CHEMICAL INFORMATION:}

Chemical Name: HYDROGEN PEROXIDE

Molecular Weight: $34.01 \mathrm{~kg} / \mathrm{kmol}$

TLV-TWA: $1 \mathrm{ppm}$ IDLH: $75 \mathrm{ppm}$

Footprint Level of Concern: $50 \mathrm{ppm}$

Boiling Point: $150.20^{\circ} \mathrm{C}$

Vapor Pressure at Ambient Temperature: $0.0034 \mathrm{~atm}$

Ambient Saturation Concentration: 3,462 ppm or $0.35 \%$

\section{ATMOSPHERIC INFORMATION: (MANUAL INPUT OF DATA)}

Wind: 1.7 meters $/ \mathrm{sec}$ from $90^{\circ}$ true at 2 meters

Inversion Height: 200 meters

Stability Class: E Air Temperature: $29^{\circ} \mathrm{C}$

Relative Humidity: $50 \%$ Ground Roughness: Urban or forest

Cloud Cover: 1 tenths

\section{SOURCE STRENGTH INFORMATION:}

Puddle Area: 24420 square feet

Average Puddle Depth: 1 centimeters

Soil Type: Default Ground Temperature: $29^{\circ} \mathrm{C}$

Initial Puddle Temperature: Ground temperature

Release Duration: ALOHA limited the duration to 1 hour

Max Computed Release Rate: 2.64 kilograms/min

Max Average Sustained Release Rate: 2.56 kilograms/min

(averaged over a minute or more)

Total Amount Released: 146 kilograms

\section{FOOTPRINT INFORMATION:}

Dispersion Module: Gaussian

User-specified LOC: $50 \mathrm{ppm}$

Max Threat Zone for LOC: 63 meters
Reviewed by S. A. Henderson

Works Calculation 8

Sheet No. 93 of 314 
Title of Project EPHA for THM

Subject Concentration Analysis for THM Hazardous Chemicals

Computer N.E. Baucom B. K. Tharakan Date 09/16/96
Reviewed by S. A. Henderson

Works Calculation 8

Sheet No. 94 of 314

\section{1-RD-26 ADVERSE / MAX DIST SEG 2}

SITE DATA INFORMATION:

Location: AIKEN, SOUTH CAROLINA

Building Air Exchanges Per Hour: 60 (User specified)

Time: June 30, 19982359 hours EDT (User specified)

\section{CHEMICAL INFORMATION:}

Chemical Name: HYDROGEN PEROXIDE

Molecular Weight: $34.01 \mathrm{~kg} / \mathrm{kmol}$

TLV-TWA: 1 ppm IDLH: 75 ppm

Footprint Level of Concern: $50 \mathrm{ppm}$

Boiling Point: $150.20^{\circ} \mathrm{C}$

Vapor Pressure at Ambient Temperature: 0.0034 atm

Ambient Saturation Concentration: 3,462 ppm or $0.35 \%$

ATMOSPHERIC INFORMATION: (MANUAL INPUT OF DATA)

Wind: 1.7 meters $/ \mathrm{sec}$ from $90^{\circ}$ true at 2 meters

Inversion Height: 200 meters

Stability Class: E Air Temperature: $29^{\circ} \mathrm{C}$

Relative Humidity: 50\% Ground Roughness: Urban or forest

Cloud Cover: 1 tenths

SOURCE STRENGTH INFORMATION:

Puddle Area: 40700 square feet

Average Puddle Depth: 1 centimeters

Soil Type: Default Ground Temperature: $29^{\circ} \mathrm{C}$

Initial Puddle Temperature: Ground temperature

Release Duration: ALOHA limited the duration to 1 hour

Max Computed Release Rate: 4.29 kilograms/min

Max Average Sustained Release Rate: 4.16 kilograms/min

(averaged over a minute or more)

Total Amount Released: 238 kilograms

FOOTPRINT INFORMATION:

Dispersion Module: Gaussian

User-specified LOC: $50 \mathrm{ppm}$

Max Threat Zone for LOC: 81 meters 
Title of Project EPHA for THM

Subject Concentration Analysis for THM Hazardous Chemicals

Computer N.E. Baucom B.K. Tharakan Date 09/16/96

1-RD.26 ADVERSE/MAX DIST SEG 3

\section{SITE DATA INFORMATION:}

Location: AIKEN, SOUTH CAROLINA

Building Air Exchanges Per Hour: 60 (User specified)

Time: June 30, 19982359 hours EDT (User specified)

\section{CHEMICAL INFORMATION:}

Chemical Name: HYDROGEN PEROXIDE

Molecular Weight: $34.01 \mathrm{~kg} / \mathrm{kmol}$

TLV-TWA: $1 \mathrm{ppm}$ IDLH: $75 \mathrm{ppm}$

Footprint Level of Concern: 50 ppm

Boiling Point: $150.20^{\circ} \mathrm{C}$

Vapor Pressure at Ambient Temperature: $0.0034 \mathrm{~atm}$

Ambient Saturation Concentration: 3,462 ppm or $0.35 \%$

ATMOSPHERIC INFORMATION: (MANUAL INPUT OF DATA)

Wind: 1.7 meters $/ \mathrm{sec}$ from $90^{\circ}$ true at 2 meters

Inversion Height: 200 meters

Stability Class: E Air Temperature: $29^{\circ} \mathrm{C}$

Relative Humidity: $50 \%$ Ground Roughness: Urban or forest

Cloud Cover: 1 tenths

\section{SOURCE STRENGTH INFORMATION:}

Puddle Area: 10000 square meters

Average Puddle Depth: 1 centimeters

Soil Type: Default Ground Temperature: $29^{\circ} \mathrm{C}$

Initial Puddle Temperature: Ground temperature

Release Duration: ALOHA limited the duration to 1 hour

Max Computed Release Rate: 10.8 kilograms/min

Max Average Sustained Release Rate: 10.5 kilograms/min

(averaged over a minute or more)

Total Amount Released: 600 kilograms

\section{FOOTPRINT INFORMATION:}

Dispersion Module: Gaussian

User-specified LOC: 50 ppm

Max Threat Zone for LOC: 131 meters
Reviewed by S. A. Henderson

Works Calculation 8

Sheet No. 95 of 314 
Title of Project EPHA for THM

Subject Concentration Analysis for THM Hazardous Chemicals

Computer N.E. Baucom B. K. Tharakan Date 09/16/96

\section{1-RD-29 ADVERSE / ALERT}

\section{SITE DATA INFORMATION:}

Location: AIKEN, SOUTH CAROLINA

Building Air Exchanges Per Hour: 60 (User specified)

Time: June 30, 19982359 hours EDT (User specified)

\section{CHEMICAL INFORMATION:}

Chemical Name: METHYL ETHYL KETONE

Molecular Weight: $72.11 \mathrm{~kg} / \mathrm{kmol}$

TLV-TWA: $200 \mathrm{ppm}$ IDLH: $3000 \mathrm{ppm}$

Footprint Level of Concern: 300 ppm

Boiling Point: $79.64^{\circ} \mathrm{C}$

Vapor Pressure at Ambient Temperature: $0.15 \mathrm{~atm}$

Ambient Saturation Concentration: 148,928 ppm or $14.9 \%$

ATMOSPHERIC INFORMATION: (MANUAL INPUT OF DATA)

Wind: 1.7 meters $/ \mathrm{sec}$ from $90^{\circ}$ true at 2 meters

Inversion Height: 200 meters

Stability Class: E Air Temperature: $29^{\circ} \mathrm{C}$

Relative Humidity: 50\% Ground Roughness: Urban or forest

Cloud Cover: 1 tenths

\section{SOURCE STRENGTH INFORMATION:}

Puddle Area: 250 square feet

Average Puddle Depth: 1 centimeters

Soil Type: Default Ground Temperature: $29^{\circ} \mathrm{C}$

Initial Puddle Temperature: Ground temperature

Release Duration: ALOHA limited the duration to 1 hour

Max Computed Release Rate: 2.93 kilograms/min

Max Average Sustained Release Rate: 2.4 kilograms/min

(averaged over a minute or more)

Total Amount Released: 117 kilograms

TIME DEPENDENT INFORMATION:

Concentration Estimates at the point:

Downwind: 30 meters

Off Centerline: 0 meters

Max Concentration:

Outdoor: 300 ppm
Reviewed by S. A. Henderson

Works Calculation 8

Sheet No. 96 of 314 
Title of Project EPHA for THM

Subject Concentration Analysis for THM Hazardous Chemicals

Computer N.E. Baucom B. K. Tharakan Date 09/16/96

1-RD-29 ADVERSE / SAE

SITE DATA INFORMATION:

Location: AIKEN, SOUTH CAROLINA

Building Air Exchanges Per Hour: 60 (User specified)

Time: June 30, 19982359 hours EDT (User specified)

CHEMICAL INFORMATION:

Chemical Name: METHYL ETHYL KETONE

Molecular Weight: $72.11 \mathrm{~kg} / \mathrm{kmol}$

TLV-TWA: $200 \mathrm{ppm}$ IDLH: $3000 \mathrm{ppm}$

Footprint Level of Concern: 300 ppm

Boiling Point: $79.64^{\circ} \mathrm{C}$

Vapor Pressure at Ambient Temperature: $0.15 \mathrm{~atm}$

Ambient Saturation Concentration: 148,928 ppm or $14.9 \%$

\section{ATMOSPHERIC INFORMATION: (MANUAL INPUT OF DATA)}

Wind: 1.7 meters $/ \mathrm{sec}$ from $90^{\circ}$ true at 2 meters

Inversion Height: 200 meters

Stability Class: E Air Temperature: $29^{\circ} \mathrm{C}$

Relative Humidity: 50\% Ground Roughness: Urban or forest

Cloud Cover: 1 tenths

\section{SOURCE STRENGTH INFORMATION:}

Puddle Area: 2125 square feet

Average Puddle Depth: 1 centimeters

Soil Type: Default Ground Temperature: $29^{\circ} \mathrm{C}$

Initial Puddle Temperature: Ground temperature

Release Duration: ALOHA limited the duration to 1 hour

Max Computed Release Rate: 2.22 kilograms/min

Max Average Sustained Release Rate: 18.6 kilograms/min

(averaged over a minute or more)

Total Amount Released: 910 kilograms

FOOTPRINT INFORMATION:

Model Run: Heavy Gas

User-specified LOC: 300 ppm

Max Threat Zone for LOC: 100 meters

Note: Footprint was not drawn because effects of near-field patchiness make dispersion predictions unrealible for short distances.

TIME DEPENDENT INFORMATION:

Concentration Estimates at the point:

Downwind: 100 meters

Off Centerline: 0 meters

Max Concentration:

Outdoor: $300 \mathrm{ppm}$
Reviewed by S. A. Heinderson

Works Calculation 8

Sheet No. 97 of 314 
Title of Project EPHA for THM

Subject Concentration Analysis for THM Hazardous Chemicals

Computer N.E. Baucom B. K. Tharakan Date 09/16/96

1-RD-29 ADVERSE / MAX DIST SEG 1

SITE DATA INFORMATION:

Location: AIKEN, SOUTH CAROLINA

Building Air Exchanges Per Hour: 60 (User specified)

Time: June 30, 19982359 hours EDT (User specified)

CHEMICAL INFORMATION:

Chemical Name: METHYL ETHYL KETONE

Molecular Weight: $72.11 \mathrm{~kg} / \mathrm{kmol}$

TLV-TWA: 200 ppm IDLH: 3000 ppm

Footprint Level of Concern: 300 ppm

Boiling Point: $79.64^{\circ} \mathrm{C}$

Vapor Pressure at Ambient Temperature: $0.15 \mathrm{~atm}$

Ambient Saturation Concentration: 148,928 ppm or $14.9 \%$

ATMOSPHERIC INFORMATION: (MANUAL INPUT OF DATA)

Wind: 1.7 meters $/ \mathrm{sec}$ from $90^{\circ}$ true at 2 meters

Inversion Height: 200 meters

Stability Class: E Air Temperature: $29^{\circ} \mathrm{C}$

Relative Humidity: $50 \%$ Ground Roughness: Urban or forest

Cloud Cover: 1 tenths

SOURCE STRENGTH INFORMATION:

Puddle Area: 24420 square feet

Average Puddle Depth: 1 centimeters

Soil Type: Default Ground Temperature: $29^{\circ} \mathrm{C}$

Initial Puddle Temperature: Ground temperature

Release Duration: ALOHA limited the duration to 1 hour

Max Computed Release Rate: 228 kilograms/min

Max Average Sustained Release Rate: 194 kilograms/min

(averaged over a minute or more)

Total Amount Released: 9,559 kilograms

FOOTPRINT INFORMATION:

Model Run: Heavy Gas

User-specified LOC: 300 ppm

Max Threat Zone for LOC: 334 meters

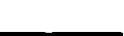

Reviewed by S. A. Henderson

Works Calculation 8

Sheet No. 98 of 314 
Title of Project EPHA for THM

Subject Concentration Analysis for THM Hazardous Chemicals

Computer N.E. Baucom B. K. Tharakan Date 09/16/96

1-RD-29 ADVERSE / MAX DIST SEG 2

SITE DATA INFORMATTON:

Location: AIKEN, SOUTH CAROLINA

Building Air Exchanges Per Hour: 60 (User specified)

Time: June 30, 19982359 hours EDT (User specified)

CHEMICAL INFORMATION:

Chemical Name: METHYL ETHYL KETONE

Molecular Weight: $72.11 \mathrm{~kg} / \mathrm{kmol}$

TLV-TWA: 200 ppm IDLH: 3000 ppm

Footprint Level of Concern: 300 ppm

Boiling Point: $79.64^{\circ} \mathrm{C}$

Vapor Pressure at Ambient Temperature: $0.15 \mathrm{~atm}$

Ambient Saturation Concentration: 148,928 ppm or $14.9 \%$

ATMOSPHERIC INFORMATION: (MANUAL INPUT OF DATA)

Wind: 1.7 meters $/ \mathrm{sec}$ from $90^{\circ}$ true at 2 meters

Inversion Height: 200 meters

Stability Class: E Air Temperature: $29^{\circ} \mathrm{C}$

Relative Humidity: 50\% Ground Roughness: Urban or forest

Cloud Cover: 1 tenths

\section{SOURCE STRENGTH INFORMATION:}

Puddle Area: 40700 square feet

Average Puddle Depth: 1 centimeters

Soil Type: Default Ground Temperature: $29^{\circ} \mathrm{C}$

Initial Puddle Temperature: Ground temperature

Release Duration: ALOHA limited the duration to 1 hour

Max Computed Release Rate: 371 kilogramsimin

Max Average Sustained Release Rate: 362 kilograms/min

(averaged over a minute or more)

Total Amount Released: 15,653 kilograms

FOOTPRINT INFORMATION:

Model Run: Heavy Gas

User-specified LOC: $300 \mathrm{ppm}$

Max Threat Zone for LOC: 436 meters
Reviewed by S. A. Henderson

Works Calculation 8

Sheet No. 29 of 314
( 
Title of Project EPHA for THM

Subject Concentration Analysis for THM Hazardous Chemicals

Computer N.E. Baucom B. K. Tharakan Date 09/16/96

1-RD-29 ADVERSE / MAX DIST SEG 3

\section{SITE DATA INFORMATION:}

Location: AIKEN, SOUTH CAROLINA

Building Air Exchanges Per Hour: 60 (User specified)

Time: June 30, 19982359 hours EDT (User specified)

\section{CHEMICAL INFORMATION:}

Chemical Name: METHYL ETHYL KETONE

Molecular Weight: $72.11 \mathrm{~kg} / \mathrm{kmol}$

TLV-TWA: 200 ppm IDLH: $3000 \mathrm{ppm}$

Footprint Level of Concern: $300 \mathrm{ppm}$

Boiling Point: $79.64^{\circ} \mathrm{C}$

Vapor Pressure at Ambient Temperature: $0.15 \mathrm{~atm}$

Ambient Saturation Concentration: 148,928 ppm or $14.9 \%$

\section{ATMOSPHERIC INFORMATION: (MANUAL INPUT OF DATA)}

Wind: 1.7 meters $/ \mathrm{sec}$ from $90^{\circ}$ true at 2 meters

Inversion Height: 200 meters

Stability Class: E Air Temperature: $29^{\circ} \mathrm{C}$

Relative Humidity: 50\% Ground Roughness: Urban or forest

Cloud Cover: 1 tenths

\section{SOURCE STRENGTH INFORMATION:}

Puddle Area: 10000 square meters

Average Puddle Depth: 1 centimeters

Soil Type: Default Ground Temperature: $29^{\circ} \mathrm{C}$

Initial Puddle Temperature: Ground temperature

Release Duration: ALOHA limited the duration to 1 hour

Max Computed Release Rate: 941 kilograms/min

Max Average Sustained Release Rate: 917 kilograms/min

(averaged over a minute or more)

Total Amount Released: 40,061 kilograms

FOOTPRINT INFORMATION:

Model Run: Heavy Gas

User-specified LOC: 300 ppm

Max Threat Zone for LOC: 679 meters
Reviewed by S. A. Henderson

Works Calculation 8

Sheet No. 100 of 314 
Title of Project EPHA for THM

Reviewed by S. A. Henderson

Subject Concentration Analysis for THM Hazardous Chemicals

Works Calculation 8

Computer N.E. Baucom B.K. Tharakan Date 09/16/96

\section{1-RD-30 ADVERSE / ALERT}

\section{SITE DATA INFORMATION:}

Location: AIKEN, SOUTH CAROLINA

Building Air Exchanges Per Hour: 60 (User specified)

Time: June 30, 19982359 hours EDT (User specified)

\section{CHEMICAL INFORMATION:}

Chemical Name: METHYL ISOBUTYL KETONE

Molecular Weight: $100.16 \mathrm{~kg} / \mathrm{kmol}$

TLV-TWA: $50 \mathrm{ppm}$ IDLH: $500 \mathrm{ppm}$

Footprint Level of Concern: $500 \mathrm{ppm}$

Boiling Point: $116.50^{\circ} \mathrm{C}$

Vapor Pressure at Ambient Temperature: $0.032 \mathrm{~atm}$

Ambient Saturation Concentration: 33,051 ppm or 3.31\%

\section{ATMOSPHERIC INFORMATION: (MANUAL INPUT OF DATA)}

Wind: 1.7 meters $/ \mathrm{sec}$ from $90^{\circ}$ true at 2 meters

Inversion Height: 200 meters

Stability Class: E Air Temperature: $29^{\circ} \mathrm{C}$

Relative Humidity: 50\% Ground Roughness: Urban or forest

Cloud Cover: 1 tenths

\section{SOURCE STRENGTH INFORMATION:}

Puddle Area: 6034 square feet

Average Puddle Depth: 1 centimeters

Soil Type: Default Ground Temperature: $29^{\circ} \mathrm{C}$

Initial Puddle Temperature: Ground temperature

Release Duration: ALOHA limited the duration to 1 hour

Max Computed Release Rate: 16.3 kilograms/min

Max Average Sustained Release Rate: 15.2 kilograms/min

(averaged over a minute or more)

Total Amount Released: 834 kilograms

\section{TIME DEPENDENT INFORMATION:}

Concentration Estimates at the point:

Downwind: 30 meters

Off Centerline: 0 meters

Max Concentration:

Outdoor: 435 ppm 
Title of Project EPHA for THM

Reviewed by S. A. Henderson

Subject Concentration Analysis for THM Hazardous Chemicals

Computer N.E. Baucom B. K. Tharakan Date 09/16/96

\section{1-RD-30 ADVERSE / SAE}

\section{SITE DATA INFORMATION:}

Location: AIKEN, SOUTH CAROLINA

Building Air Exchanges Per Hour: 60 (User specified)

Time: June 30, 19982359 hours EDT (User specified)

\section{CHEMICAL INFORMATION:}

Chemical Name: METHYL ISOBUTYL KETONE

Molecular Weight: $100.16 \mathrm{~kg} / \mathrm{kmol}$

TLV-TWA: $50 \mathrm{ppm}$ IDLH: $500 \mathrm{ppm}$

Footprint Level of Concern: $500 \mathrm{ppm}$

Boiling Point: $116.50^{\circ} \mathrm{C}$

Vapor Pressure at Ambient Temperature: $0.032 \mathrm{~atm}$

Ambient Saturation Concentration: 33,051 ppm or $3.31 \%$

ATMOSPHERIC INFORMATION: (MANUAL INPUT OF DATA)

Wind: 1.7 meters $/ \mathrm{sec}$ from $90^{\circ}$ true at 2 meters

Inversion Height: 200 meters

Stability Class: E Air Temperature: $29^{\circ} \mathrm{C}$

Relative Humidity: $50 \%$ Ground Roughness: Urban or forest

Cloud Cover: 1 tenths

SOURCE STRENGTH INFORMATION:

Puddle Area: 32500 square feet

Average Puddle Depth: 1 centimeters

Soil Type: Default Ground Temperature: $29^{\circ} \mathrm{C}$

Initial Puddle Temperature: Ground temperature

Release Duration: ALOHA limited the duration to 1 hour

Max Computed Release Rate: 81.6 kilograms/min

Max Average Sustained Release Rate: 76.2 kilograms/min

(averaged over a minute or more)

Total Amount Released: 4,203 kilograms

FOOTPRINT INFORMATION:

Model Run: Heavy Gas

User-specified LOC: equals IDLH (500 ppm)

Max Threat Zone for LOC: 100 meters

\section{TIME DEPENDENT INFORMATION:}

Concentration Estimates at the point:

Downwind: 100 meters

Off Centerline: 0 meters

Max Concentration:

Outdoor: 501 ppm
Works Calculation 8

Sheet No. 102 of 314 
Title of Project EPHA for THM

Subject Concentration Analysis for THM Hazardous Chemicais

Computer N.E. Baucom B. K. Tharakan Date 09/16/96
Reviewed by S. A. Henderson

Works Calculation 8

Sheet No. 103 of 314

\section{1-RD-30 ADVERSE / MAX DIST SEG 1}

SITE DATA INFORMATION:

Location: AIKEN, SOUTH CAROLINA

Building Air Exchanges Per Hour: 60 (User specified)

Time: June 30, 19982359 hours EDT (User specified)

CHEMICAL INFORMATION:

Chemical Name: METHYL ISOBUTYL KETONE

Molecular Weight: $100.16 \mathrm{~kg} / \mathrm{kmol}$

TLV-TWA: $50 \mathrm{ppm}$ IDLH: $500 \mathrm{ppm}$

Footprint Level of Concern: 500 ppm

Boiling Point: $116.50^{\circ} \mathrm{C}$

Vapor Pressure at Ambient Temperature: $0.032 \mathrm{~atm}$

Ambient Saturation Concentration: $33,051 \mathrm{ppm}$ or $3.31 \%$

ATMOSPHERIC INFORMATION: (MANUAL INPUT OF DATA)

Wind: 1.7 meters $/ \mathrm{sec}$ from $90^{\circ}$ true at 2 meters

Inversion Height: 200 meters

Stability Class: E Air Temperature: $29^{\circ} \mathrm{C}$

Relative Humidity: $50 \%$ Ground Roughness: Urban or forest

Cloud Cover: 1 tenths

SOURCE STRENGTH INFORMATION:

Puddle Area: 24420 square feet

Average Puddle Depth: 1 centimeters

Soil Type: Default Ground Temperature: $29^{\circ} \mathrm{C}$

Initial Puddle Temperature: Ground temperature

Release Duration: ALOHA limited the duration to 1 hour

Max Computed Release Rate: 62.1 kilograms/min

Max Average Sustained Release Rate: $\mathbf{5 7 . 9}$ kilograms/min

(averaged over a minute or more)

Total Amount Released: 3,193 kilograms

FOOTPRINT INFORMATION:

Model Run: Heavy Gas

User-specified LOC: equals IDLH (500 ppm)

Max Threat Zone for LOC: 87 meters

Note: Footprint was not drawn because effects of near-field patchiness make dispersion predictions unreliable for short distances. 
Title of Project EPHA for THM

Subject Concentration Analysis for THM Hazardous Chemicals

Computer N.E. Baucom B. K. Tharakan Date 09/16/96
Reviewed by S. A. Henderson Works Calculation 8

Sheet No. 104 of 314

\section{RD-30 ADVERSE / MAX DIST SEG 2}

\section{SITE DATA INFORMATION:}

Location: AIKEN, SOUTH CAROLINA

Building Air Exchanges Per Hour: 60 (User specified)

Time: June 30, 19982359 hours EDT (User specified)

\section{CHEMICAL INFORMATION:}

Chemical Name: METHYL ISOBUTYL KETONE

Molecular Weight: $100.16 \mathrm{~kg} / \mathrm{kmol}$

TLV-TWA: $50 \mathrm{ppm}$ IDLH: $500 \mathrm{ppm}$

Footprint Level of Concern: 500 ppm

Boiling Point: $116.50^{\circ} \mathrm{C}$

Vapor Pressure at Ambient Temperature: $0.032 \mathrm{~atm}$

Ambient Saturation Concentration: $33,051 \mathrm{ppm}$ or $3.31 \%$

ATMOSPHERIC INFORMÁTION: (MANUAL INPUT OF DATA)

Wind: 1.7 meters $/ \mathrm{sec}$ from $90^{\circ}$ true at 2 meters

Inversion Height: 200 meters

Stability Class: E Air Temperature: $29^{\circ} \mathrm{C}$

Relative Humidity: 50\% Ground Roughness: Urban or forest

Cloud Cover: 1 tenths

\section{SOURCE STRENGTH INFORMATION:}

Puddle Area: 40700 square feet

Average Puddle Depth: 1 centimeters

Soil Type: Default Ground Temperature: $29^{\circ} \mathrm{C}$

Initial Puddle Temperature: Ground temperature

Release Duration: ALOHA limited the duration to 1 hour

Max Computed Release Rate: 101 kilograms/min

Max Average Sustained Release Rate: 94.5 kilograms/min

(averaged over a minute or more)

Total Amount Released: 5,219 kilograms

FOOTPRINT INFORMATION:

Model Run: Heavy Gas

User-specified LOC: equals IDLH (500 ppm)

. Max Threat Zone for LOC: 112 meters 
- Title of Project EPHA for THM

Subject Concentration Analysis for THM Hazardous Chemicals

Computer N.E. Baucom B. K. Tharakan Date 09/16/96

\section{1-RD-30 ADVERSE / MAX DIST SEG 3}

SITE DATA INFORMATION:

Location: AIKEN, SOUTH CAROLINA

Building Air Exchanges Per Hour: 60 (User specified)

Time: June 30, 19982359 hours EDT (User specified)

\section{CHEMICAL INFORMATION:}

Chemical Name: METHYL ISOBUTYL KETONE

Molecular Weight: $100.16 \mathrm{~kg} / \mathrm{kmol}$

TLV-TWA: $50 \mathrm{ppm}$ IDLH: $500 \mathrm{ppm}$

Footprint Level of Concern: 500 ppm

Boiling Point: $116.50^{\circ} \mathrm{C}$

Vapor Pressure at Ambient Temperature: 0.032 atm

Ambient Saturation Concentration: 33,051 ppm or $3.31 \%$

\section{ATMOSPHERIC INFORMATION: (MANUAL INPUT OF DATA)}

Wind: 1.7 meters $/ \mathrm{sec}$ from $90^{\circ}$ true at 2 meters

Inversion Height: 200 meters

Stability Class: E Air Temperature: $29^{\circ} \mathrm{C}$

Relative Humidity: 50\% Ground Roughness: Urban or forest

Cloud Cover: 1 tenths

\section{SOURCE STRENGTH INFORMATION:}

Puddle Area: 10000 square meters

Average Puddle Depth: 1 centimeters

Soil Type: Default Ground Temperature: $29^{\circ} \mathrm{C}$

Initial Puddle Temperature: Ground temperature

Release Duration: ALOHA limited the duration to 1 hour

Max Computed Release Rate: 257 kilograms/min

Max Average Sustained Release Rate: 241 kilograms/min

(averaged over a minute or more)

Total Amount Released: 13,311 kilograms

FOOTPRINT INFORMATION:

Model Run: Heavy Gas

User-specified LOC: equals IDLH (500 gpm)

Max Threat Zone for LOC: 187 meters
Reviewed by S. A. Henderson

Works Calculation 8

Sheet No. 105 of 314 


\section{ENGINEERING COMPUTATION SHEET}

Title of Project EPHA for THM

Reviewed by S. A. Henderson

Subject Concentration Analysis for THM Hazardous Chemicals

Computer N.E. Baucom B. K. Tharakan Date 09/16/96

1-RD-32 ADVERSE / ALERT

SITE DATA INFORMATION:

Location: AIKEN, SOUTH CAROLINA

Building Air Exchanges Per Hour: 0.44 (Sheltered single storied)

Time: June 30, 19982359 hours EDT (User specified)

CHEMICAL INFORMATION:

Chemical Name: ETHYLENE GLYCOL MONOMETHYL ETHER

Molecular Weight: $76.10 \mathrm{~kg} / \mathrm{kmol}$

TLV-TWA: 5 ppm IDLH: 2000 ppm

Footprint Level of Concern: 25 ppm

Boiling Point: $124.40^{\circ} \mathrm{C}$

Vapor Pressure at Ambient Temperature: $0.015 \mathrm{~atm}$

Ambient Saturation Concentration: 15,583 ppm 1.56\%

ATMOSPHERIC INFORMATION: (MANUAL INPUT OF DATA)

Wind: 1.7 meters $/ \mathrm{sec}$ from $90^{\circ}$ true at 2 meters

Inversion Height: 200 meters

Stability Class: E Air Temperature: $29^{\circ} \mathrm{C}$

Relative Humidity: 50\% Ground Roughness: Urban or forest

Cloud Cover: 1 tenths

\section{SOURCE STRENGTH INFORMATION:}

Puddle Area: 145 square feet

Average Puddle Depth: 1 centimeters

Soil Type: Default Ground Temperature: $29^{\circ} \mathrm{C}$

Initial Puddle Temperature: Ground temperature

Release Duration: ALOHA limited the duration to 1 hour

Max Computed Release Rate: 178 grams/min

Max Average Sustained Release Rate: 178 grams/min

(averaged over a minute or more)

Total Amount Released: 10.5 kilograms

TIME DEPENDENT INFORMATION:

Concentration Estimates at the point:

Downwind: 30 meters

Off Centerline: 0 meters

Max Concentration:

Outdoor: $25 \mathrm{ppm}$

Indoor: $8.72 \mathrm{ppm}$

Note: Indoor graph is shown with a dotted line.

\section{Works Calculation 8}

Sheet No. 106 of 314 
Title of Project EPHA for THM

Subject Concentration Analysis for THM Hazardous Chemicals

Computer N.E. Baucom B. K. Tharakan Date 09/16/96
Reviewed by S. A. Henderson

Works Calculation 8

Sheet No. 107 of 314

\section{' 1-RD-32 ADVERSE / SAE}

\section{SITE DATA INFORMATION:}

Location: AIKEN, SOUTH CAROLINA

Building Air Exchanges Per Hour: 0.44 (Sheltered single storied)

Time: June 30, 19982359 hours EDT (User specified)

\section{CHEMICAL INFORMATION:}

Chemical Name: ETHYLENE GLYCOL MONOMETHYL ETHER

Molecular Weight: $76.10 \mathrm{~kg} / \mathrm{kmol}$

TLV-TWA: 5 ppm IDLH: 2000 ppm

Footprint Level of Concern: 25 ppm

Boiling Point: $124.40^{\circ} \mathrm{C}$

Vapor Pressure at Ambient Temperature: $0.015 \mathrm{~atm}$

Ambient Saturation Concentration: 15,583 ppm $1.56 \%$

\section{ATMOSPHERIC INFORMATION: (MANUAL INPUT OF DATA)}

Wind: 1.7 meters $/ \mathrm{sec}$ from $90^{\circ}$ true at 2 meters

Inversion Height: 200 meters

Stability Class: E Air Temperature: $29^{\circ} \mathrm{C}$

Relative Humidity: 50\%. Ground Roughness: Urban or forest

Cloud Cover: 1 tenths

\section{SOURCE STRENGTH INFORMATION:}

Puddle Area: 1810 square feet

Average Puddle Depth: 1 centimeters

Soil Type:Default Ground Temperature: $29^{\circ} \mathrm{C}$

Initial Puddle Temperature: Ground temperature

Release Duration: ALOHA limited the duration to 1 hour

Max Computed Release Rate: 1.94 kilograms/min

Max Average Sustained Release Rate: 1.94 kilograms/min

(averaged over a minute or more)

Total Amount Released: 115 kilograms

\section{TIME DEPENDENT INFORMATION:}

Concentration Estimates at the point:

Downwind: 100 meters

Off Centerline: 0 meters

Max Concentration:

Outdoor: $25 \mathrm{ppm}$

Indoor: $8.73 \mathrm{ppm}$

Note: Indoor graph is shown with a dotted line. 
Title of Project EPHA for THM

Subject Concentration Analysis for THM Hazardous Chemicals

Computer N.E. Baucom B. K. Tharakan Date 09/16/96
Reviewed by S. A. Henderson

Works Calculation 8

Sheet No. 108 of 314

\section{1-RD-32 ADVERSE / MAX DIST SEG 1}

\section{SITE DATA INFORMATION:}

Location: AIKEN, SOUTH CAROLINA

Building Air Exchanges Per Hour: 0.44 (Sheltered single storied)

Time: June 30, 19982359 hours EDT (User specified)

CHEMICAL INFORMATION:

Chemical Name: ETHYLENE GLYCOL MONOMETHYL ETHER

Molecular Weight: $76.10 \mathrm{~kg} / \mathrm{kmol}$

TLV-TWA: 5 ppm IDLH: $2000 \mathrm{ppm}$

Footprint Level of Concern: 25 ppm

Boiling Point: $124.40^{\circ} \mathrm{C}$

Vapor Pressure at Ambient Temperature: 0.015 atm

Ambient Saturation Concentration: 15,583 ppm 1.56\%

ATMOSPHERIC INFORMATION: (MANUAL INPUT OF DATA)

Wind: 1.7 meters $/ \mathrm{sec}$ from $90^{\circ}$ true at 2 meters

Inversion Height: 200 meters

Stability Class: E Air Temperature: $29^{\circ} \mathrm{C}$

Relative Humidity: 50\% Ground Roughness: Urban or forest

Cloud Cover: 1 tenths

SOURCE STRENGTH INFORMATION:

Puddle Area: 24420 square feet

Average Puddle Depth: 1 centimeters

Soil Type: Default Ground Temperature: $29^{\circ} \mathrm{C}$

Initial Puddle Temperature: Ground temperature

Release Duration: ALOHA limited the duration to 1 hour

Max Computed Release Rate: 23.2 kilograms/min

Max Average Sustained Release Rate: 23.2 kilograms/min

(averaged over a minute or more)

Total Amount Released: 1,376 kilograms

FOOTPRINT INFORMATION:

Dispersion Module: Gaussian

User-specified LOC: 25 ppm

Max Threat Zone for LOC: 377 meters

Max Threat Zone for LDLH: 27 m eters 
Title of Project EPHA for THM

Subject Concentration Analysis for THM Hazardous Chemicals

Computer N.E. Baucom B.K. Tharakan Date 09/16/96

\section{1-RD-32 ADVERSE / MAX DIST SEG 2}

\section{SITE DATA INFÓRMATION:}

Location: AIKEN, SOUTH CAROLINA

Building Air Exchanges Per Hour: 0.44 (Sheltered single storied)

Time: June 30, 19982359 hours EDT (User specified)

\section{CHEMICAL INFORMATION:}

Chemical Name: ETHYLENE GLYCOL MONOMETHYL ETHER

Molecular Weight: $76.10 \mathrm{~kg} / \mathrm{kmol}$

TLV-TWA: $5 \mathrm{ppm}$ IDLH: $2000 \mathrm{ppm}$

Footprint Level of Concern: 25 ppm

Boiling Point: $124.40^{\circ} \mathrm{C}$

Vapor Pressure at Ambient Temperature: $0.015 \mathrm{~atm}$

Ambient Saturation Concentration: 15,583 ppm $1.56 \%$

\section{ATMOSPHERIC INFORMATION: (MANUAL INPUT OF DATA)}

Wind: 1.7 meters $/ \mathrm{sec}$ from $90^{\circ}$ true at 2 meters

Inversion Height: 200 meters

Stability Class: E Air Temperature: $29^{\circ} \mathrm{C}$

Relative Humidity: $50 \%$ Ground Roughness: Urban or forest

Cloud Cover: 1 tenths

\section{SOURCE STRENGTH INFORMATION:}

Puddle Area: 40700 square feet

Average Puddle Depth: 1 centimeters

Soil Type: Default Ground Temperature: $29^{\circ} \mathrm{C}$

Initial Puddle Temperature: Ground temperature

Release Duration: ALOHA limited the duration to 1 hour

Max Computed Release Rate: 37.8 kilograms/min

Max Average Sustained Release Rate: 37.7 kilograms/min

(averaged over a minute or more)

Total Amount Released: 2,242 kilograms

\section{FOOTPRINT INFORMATION:}

Dispersion Module: Gaussian

User-specified LOC: 25 ppm

Max Threat Zone for LOC: 498 meters

Max Threat Zone for IDLH: 34 meters
Reviewed by S. A. Henderson

\section{Works Calculation 8
- Sheet No. 109 of 314 \\ Works Calculation 8
Sheet No. 109 of 314}


Title of Project EPHA for THM

Subject Concentration Analysis for THM Hazardous Chemicals

Computer N.E. Baucom B. K. Tharakan Date 09/16/96
Reviewed by S. A. Henderson

Works Calculation 8

Sheet No. 110 of 314

\section{1-RD-32 ADVERSE / MAX DIST SEG 3}

\section{SITE DATA INFORMATION:}

Location: AIKEN, SOUTH CAROLINA

Building Air Exchanges Per Hour: 0.44 (Sheltered single storied)

Time: June 30, 19982359 hours EDT (User specified)

\section{CHEMICAL INFORMATION:}

Chemical Name: ETHYLENE GLYCOL MONOMETHYL ETHER

Molecular Weight: $76.10 \mathrm{~kg} / \mathrm{kmol}$

TLV-TWA: $5 \mathrm{ppm} \quad$ IDLH: $2000 \mathrm{ppm}$

Footprint Level of Concern: 25 ppm

Boiling Point: $124.40^{\circ} \mathrm{C}$

Vapor Pressure at Ambient Temperature: 0.015 atm

Ambient Saturation Concentration: 15,583 ppm 1.56\%

ATMOSPHERIC INFORMATION: (MANUAL INPUT OF DATA)

Wind: 1.7 meters $/ \mathrm{sec}$ from $90^{\circ}$ true at 2 meters

Inversion Height: 200 meters

Stability Class: E Air Temperature: $29^{\circ} \mathrm{C}$

Relative Humidity: 50\% Ground Roughness: Urban or forest

Cloud Cover: 1 tenths

\section{SOURCE STRENGTH INFORMATION:}

Puddle Area: 10000 square meters

Average Puddle Depth: 1 centimeters

Soil Type: Default Ground Temperature: $29^{\circ} \mathrm{C}$

Initial Puddle Temperature: Ground temperature

Release Duration: ALOHA limited the duration to 1 hour

Max Computed Release Rate: 96 kilograms/min

Max Average Sustained Release Rate: 95.8 kilograms/min

(averaged over a minute or more)

Total Amount Released: 5,691 kilograms

FOOTPRINT INFORMATION:

Model Run: Heavy Gas

User-specified LOC: 25 , ppm

Max Threat Zone for LOC: 1.2 kilometers 
Title of Project EPHA for THM

Subject Concentration Analysis for THM Hazardous Chemicals

Computer N.E. Baucom B. K. Tharakan Date 09/16/96 1-RD-33 ADVERSE/ALERT .

SITE'DATA INFORMATION:

Location: AIKEN, SOUTH CAROLINA

Building Air Exchanges Per Hour: 0.44 (Sheltered single storied)

Time: June 30, 19982359 hours EDT (User specified)

CHEMICAL INFORMATION:

Chemical Name: MONOETHANOLAMINE

Molecular Weight: $61.08 \mathrm{~kg} / \mathrm{kmol}$

TLV-TWA:3 ppm IDLH: 1000 ppm

Footprint Level of Concern: 30 ppm

Boiling Point: $171.00^{\circ} \mathrm{C}$

Vapor Pressure at Ambient Temperature: 6.82e-004 atm

Ambient Saturation Concentration: 694 ppm or $0.069 \%$

ATMOSPHERIC INFORMATION: (MANUAL INPUT OF DATA)

Wind: 1.7 meters $/ \mathrm{sec}$ from $90^{\circ}$ true at 2 meters

Inversion Height: 200 meters

Stability Class: E Air Temperature: $29^{\circ} \mathrm{C}$

Relative Humidity: $50 \%$ Ground Roughness: Urban or forest

Cloud Cover: 1 tenths

\section{SOURCE STRENGTH INFORMATION:}

Puddle Area: 18500 square feet

Average Puddle Depth: 1 centimeters

Soil Type: Default Ground Temperature: $29^{\circ} \mathrm{C}$

Initial Puddle Temperature: Ground temperature

Release Duration: ALOHA limited the duration to 1 hour

Max Computed Release Rate: 658 grams/min

Max Average Sustained Release Rate: 638 grams/min

(averaged over a minute or more)

Total Amount Released: 36.5 kilograms

TIME DEPENDENT INFORMATION:

Concentration Estimates at the point:

Downwind: 30 meters

Off Centerline: 0 meters

Max Concentration:

Outdoor: 30 ppm
Reviewed by S. A. Henderson

Works Calculation 8

Sheot No. 111 of 314 
Title of Project EPHA for THM

Subject Concentration Analysis for THM Hazardous Chemicals

Computer N.E. Baucom B. K. Tharakan Date 09/16/96 1-RD-33 ADVERSE / SAE

SITE DATA INFORMATION:

Location: AIKEN, SOUTH CAROLINA

Building Air Exchanges Per Hour: 0.44 (Sheltered single storied)

Time: June 30, 19982359 hours EDT (User specified)

\section{CHEMICAL INFORMATION:}

Chemical Name: MONOETHANOLAMINE

Molecular Weight: $61.08 \mathrm{~kg} / \mathrm{kmol}$

TLV-TWA:3 ppm IDLH: $1000 \mathrm{ppm}$

Footprint Level of Concern: $30 \mathrm{ppm}$

Boiling Point: $171.00^{\circ} \mathrm{C}$

Vapor Pressure at Ambient Temperature: 6.82e-004 atm

Ambient Saturation Concentration: 694 ppm or $0.069 \%$

ATMOSPHERIC INFORMATION: (MANUAL INPUT OF DATA)

Wind: 1.7 meters $/ \mathrm{sec}$ from $90^{\circ}$ true at 2 meters

Inversion Height: 200 meters

Stability Class: E Air Temperature: $29^{\circ} \mathrm{C}$

Relative Humidity: $50 \%$ Ground Roughness: Urban or forest

Cloud Cover: 1 tenths

\section{SOURCE STRENGTH INFORMATION:}

Puddle Area: 100000 square feet

Average Puddle Depth: 1 centimeters

Soil Type: Default Ground Temperature: $29^{\circ} \mathrm{C}$

Initial Puddle Temperature: Ground temperature

Release Duration: ALOHA limited the duration to 1 hour

Max Computed Release Rate: 3.3 kilograms/min

Max Average Sustained Release Rate: 3.2 kilograms/min

(averaged over a minute or more)

Total Amount Released: 183 kilograms

\section{TIME DEPENDENT INFORMATION:}

Concentration Estimates at the point:

Downwind: 100 meters

Off Centerline: 0 meters

Max Concentration:

Outdoor:14.2 ppm
Reviewed by S. A. Henderson

Works Calculation 8

Sheet No. 112 of 314 
Title of Project EPHA for THM

Reviewed by S. A. Henderson

Works Calculation 8

Sheet No. 113 of 314

Computer N.E. Baucom B. K. Tharakan Date 09/16/96

1-RD-33 ADVERSE / MAX DIST SEG 1

\section{SITE DATA INFORMATION:}

Location: AIKEN, SOUTH CAROLINA

Building Air Exchanges Per Hour: 0.44 (Sheltered single storied)

Time: June 30, 19982359 hours EDT (User specified)

\section{CHEMICAL INFORMATION:}

Chemical Name: MONOETHANOLAMINE

Molecular Weight: $61.08 \mathrm{~kg} / \mathrm{kmol}$

TLV-TWA:3 ppm IDLH: 1000 ppm

Footprint Level of Concern: 30 ppm

Boiling Point: $171.00^{\circ} \mathrm{C}$

Vapor Pressure at Ambient Temperature: 6.82e-004 atm

Ambient Saturation Concentration: 694 ppm or 0.069\%

ATMOSPHERIC INFORMATION: (MANUAL INPUT OF DATA)

Wind: 1.7 meters $/ \mathrm{sec}$ from $90^{\circ}$ true at 2 meters

Inversion Height: 200 meters

Stability Class: E . Air Temperature: $29^{\circ} \mathrm{C}$

Relative Humidity: $50 \%$ Ground Roughness: Urban or forest

Cloud Cover: 1 tenths

\section{SOURCE STRENGTH INFORMATION:}

Puddle Area: 24420 square feet

Average Puddle Depth: 1 centimeters

Soil Type: Default Ground Temperature: $29^{\circ} \mathrm{C}$

Initial Puddle Temperature: Ground temperature

Release Duration: ALOHA limited the duration to 1 hour

Max Computed Release Rate: 858 grams/min

Max Average Sustained Release Rate: 832 grams/min

(averaged over a minute or more)

Total Amount Released: 47.6 kilograms

FOOTPRINT INFORMATION:

Dispersion Module: Gaussian

User-specified LOC: $30 \mathrm{ppm}$

Max Threat Zone for LOC: 34 meters 
Title of Project EPHA for THM

Subject Concentration Analysis for THM Hazardous Chemicals

Computer N.E. Baucom B. K. Tharakan Date $\underline{09 / 16 / 96}$
Reviewed by S. A. Henderson

Works Calculation 8

Sheet No. 114 of 314

\section{1-RD-33 ADVERSE / MAX DIST SEG 2}

\section{SITE DATA INFORMATION:}

Location: AIKEN, SOUTH CAROLINA

Building Air Exchanges Per Hour: 0.44 (Sheltered single storied)

Time: June 30, 19982359 hours EDT (User specified)

\section{CHEMICAL INFORMATION:}

Chemical Name: MONOETHANOLAMINE

Molecular Weight: $61.08 \mathrm{~kg} / \mathrm{kmol}$

TLV-TWA:3 ppm IDLH: 1000 ppm

Footprint Level of Concern: 30 ppm

Boiling Point: $171.00^{\circ} \mathrm{C}$

Vapor Pressure at Ambient Temperature: 6.82e-004 atm

Ambient Saturation Concentration: 694 ppm or 0.069\%

ATMOSPHERIC INFORMATION: (MANUAL INPUT OF DATA)

Wind: 1.7 meters $/ \mathrm{sec}$ from $90^{\circ}$ true at 2 meters

Inversion Height: 200 meters

Stability Class: E Air Temperature: $29^{\circ} \mathrm{C}$

Relative Humidity: $50 \%$ Ground Roughness: Urban or forest

Cloud Cover: 1 tenths

\section{SOURCE STRENGTH INFORMATION:}

Puddle Area: 40700 square feet

- Average Puddle Depth: 1 centimeters

Soil Type: Default Ground Temperature: $29^{\circ} \mathrm{C}$

Initial Puddle Temperature: Ground temperature

Release Duration: ALOHA limited the duration to 1 hour

Max Computed Release Rate: 1.4 kilograms/min

Max Average Sustained Release Rate: 1.35 kilograms/min

(averaged over a minute or more)

Total Amount Released: 77.5 kilograms

FOOTPRINT INFORMATION:

Dispersion Module: Gaussian

User-specified LOC: $30 \mathrm{ppm}$

- Max Threat Zone for LOC: 44 meters 
Title of Project EPHA for THM

Subjoct Concentration Analysis for THM Hazardous Chemicals

Computer N.E. Baucom B. K. Tharakan Date 09/16/96
Reviewed by S. A. Henderson

Works Calculation 8

Sheet No. 115 of 314

\section{1-RD-33 ADVERSE / MAX DIST SEG 3}

SITE DATA INFORMATTON:

Location: AIKEN, SOUTH CAROLINA

Building Air Exchanges Per Hour: 0.44 (Sheltered single storied)

Time: June 30, 19982359 hours EDT (User specified)

\section{CHEMICAL INFORMATION:}

Chemical Name: MONOETHANOLAMINE

Molecular Weight: $61.08 \mathrm{~kg} / \mathrm{kmol}$

TLV-TWA:3 ppm IDLH: $1000 \mathrm{ppm}$

Footprint Level of Concern: $30 \mathrm{ppm}$

Boiling Point: $171.00^{\circ} \mathrm{C}$

Vapor Pressure at Ambient Temperature: 6.82e-004 atm

Ambient Saturation Concentration: 694 ppm or 0.069\%

ATMOSPHERIC INFORMATION: (MANUAL INPUT OF DATA)

Wind: 1.7 meters $/ \mathrm{sec}$ from $90^{\circ}$ true at 2 meters

Inversion Height: 200 meters

Stability Class: E Air Temperature: $29^{\circ} \mathrm{C}$

Relative Humidity: 50\% Ground Roughness: Urban or forest

Cloud Cover: 1 tenths

\section{SOURCE STRENGTH INFORMATION:}

Puddle Area: 10000 square meters

Average Puddle Depth: 1 centimeters

Soil Type: Default Ground Temperature: $29^{\circ} \mathrm{C}$

Initial Puddle Temperature: Ground temperature

Release Duration: ALOHA limited the duration to 1 hour

Max Computed Release Rate: 3.54 kilograms/min

Max Average Sustained Release Rate: 3.43 kilograms/min

(averaged over a minute or more)

Total Amount Released: 196 kilograms

FOOTPRINT INFORMATION:

Dispersion Module: Gaussian

User-specified LOC: $30 \mathrm{ppm}$

Max Threat Zone for LOC: 70 meters 
Title of Project EPHA for THM

Subject Concentration Analysis for THM Hazardous Chemicals

Computer N.E. Baucom B. K. Tharakan Date 09/16/96

\section{1-RD-34 ADVERSE / ALERT}

SITE DATA INFORMATION:

Location: AIKEN, SOUTH CAROLINA

Building Air Exchanges Per Hour: 0.44 (Sheltered single storied)

Time: June 30, 19982359 hours EDT (User specified)

\section{CHEMICAL INFORMATION:}

Chemical Name: MORPHOLINE

Molecular Weight: $87.12 \mathrm{~kg} / \mathrm{kmol}$

TLV-TWA: $20 \mathrm{ppm}$ IDLH: $8000 \mathrm{ppm}$

Footprint Level of Concern: $30 \mathrm{ppm}$

Boiling Point: $128.00^{\circ} \mathrm{C}$

Vapor Pressure at Ambient Temperanure: 0.017 atm

Ambient Saturation Concentration: $16,979 \mathrm{ppm}$ or $1.70 \%$

ATMOSPHERIC INFORMATION: (MANUAL INPUT OF DATA)

Wind: 1.7 meters $/ \mathrm{sec}$ from $90^{\circ}$ true at 2 meters

Inversion Height: 200 meters

Stability Class: E Air Temperature: $29^{\circ} \mathrm{C}$

Relative Humidity: 50\% Ground Roughness: Urban or forest

Cloud Cover: 1 tenths

\section{SOURCE STRENGTH INFORMATION:}

Puddle Area: 191 square feet

Average Puddle Depth: 1 centimeters

Soil Type: Default Ground Temperature: $29^{\circ} \mathrm{C}$

Initial Puddle Temperature: Ground temperature

Release Duration: ALOHA limited the duration to 1 hour

Max Computed Release Rate: 279 grams/min

Max Average Sustained Release Rate: 264 grams/min

(averaged over a minute or more)

Total Amount Released: 14.8 kilograms

\section{FOOTPRINT INFORMATION:}

Dispersion Module: Gaussian

User-specified LOC: $30 \mathrm{ppm}$

Max Threat Zone for LOC: 30 meters

Max Threat Zone for IDLH: less than 10 meters (10.9 yards)

Note: Footprint was not drawn because

Effects of near-field patchiness make dispersion

Predictions unrealiable for short distances.

\section{TIME DEPENDENT INFORMATION:}

Concentration Estimates at the point:

Downwind: 30 meters

Off Centerline: 0 meters

Max Concentration:

Outdoor: $30 \mathrm{ppm}$
Reviewed by S. A. Henderson

Works Calculation 8

Sheet No. 116 of 314 
Title of Project EPHA for THM

Subject Concentration Analysis for THM Hazardous Chemicals

Computer N.E. Baucom B. K. Tharakan Date 09/16/96 1-RD-34 ADVERSE / SAE

\section{SITE DATA INFORMATION:}

Location: AIKEN, SOUTH CAROLINA

Building Air Exchanges Per Hour: 0.44 (Sheltered single storied)

Time: June 30, 19982359 hours EDT (User specified)

\section{CHEMICAL INFORMATION:}

Chemical Name: MORPHOLINE

Molecular Weight: $87.12 \mathrm{~kg} / \mathrm{kmol}$

TLV-TWA: 20 ppm IDLH: $8000 \mathrm{ppm}$

Footprint Level of Concern: $30 \mathrm{ppm}$

Boiling Point: $128.00^{\circ} \mathrm{C}$

Vapor Pressure at Ambient Temperature: $0.017 \mathrm{~atm}$

Ambient Saturation Concentration: 16,979 ppm or $1.70 \%$

\section{ATMOSPHERIC INFORMATION: (MANUAL INPUT OF DATA)}

Wind: 1.7 meters $/ \mathrm{sec}$ from $90^{\circ}$ true at 2 meters

Inversion Height: 200 meters

Stability Class: E Air Temperature: $29^{\circ} \mathrm{C}$

Relative Humidity: 50\% Ground Roughness: Urban or forest

Cloud Cover: 1 tenths

\section{SOURCE STRENGTH INFORMATION:}

Puddle Area: 2380 square feet

Average Puddle Depth: 1 centimeters

Soil Type: Default Ground Temperature: $29^{\circ} \mathrm{C}$

Initial Puddle Temperature: Ground temperature

Release Duration: ALOHA limited the duration to 1 hour

Max Computed Release Rate: 3.06 kilograms/min

Max Average Sustained Release Rate: 2.91 kilograms/min

(averaged over a minute or more)

Total Amount Released: 163 kilograms

TIME DEPENDENT INFORMATION:

Concentration Estimates at the point:

Downwind: 100 meters

Off Centerline: 0 meters

Max Concentration:

Outdoor: $30 \mathrm{ppm}$
Reviewed by S. A. Henderson

Works Calculation 8

Sheet No. 117 of 314 
Title of Project EPHA for THM

Subject Concentration Analysis for THM Hazardous Chemicals

Computer N.E. Baucom B. K. Tharakan Date 09/16/96 1-RD-34 ADVERSE / MAX DIST SEG 1

\section{SITE DATA INFORMATION:}

\section{Location: AIKEN, SOUTH CAROLINA}

Building Air Exchanges Per Hour: 0.44 (Sheltered single storied)

Time: June 30, 19982359 hours EDT (User specified)

\section{CHEMICAL INFORMATION:}

Chemical Name: MORPHOLINE

Molecular Weight: $87.12 \mathrm{~kg} / \mathrm{kmol}$

TLV-TWA: 20 ppm IDLH: 8000 ppm

Footprint Level of Concern: $30 \mathrm{ppm}$

Boiling Point: $128.00^{\circ} \mathrm{C}$

Vapor Pressure at Ambient Temperature: $0.017 \mathrm{~atm}$

Ambient Saturation Concentration: 16,979 ppm or $1.70 \%$

\section{ATMOSPHERIC INFORMATION: (MANUAL INPUT OF DATA)}

Wind: 1.7 meters $/ \mathrm{sec}$ from $90^{\circ}$ true at 2 meters

Inversion Height: 200 meters

Stability Class: E Air Temperature: $29^{\circ} \mathrm{C}$

Relative Humidity: 50\% Ground Roughness: Urban or forest

Cloud Cover: 1 tenths

\section{SOURCE STRENGTH INFORMATION:}

Puddle Area: 24420 square feet Average Puddle Depth: 1 centimeters Soil Type: Default Ground Temperature: $29^{\circ} \mathrm{C}$ Initial Puddle Temperature: Ground temperature Release Duration: ALOHA limited the duration to 1 hour Max Computed Release Rate: 28.3 kilograms/min Max Average Sustained Release Rate: 26.9 kilograms/min (averaged over a minute or more)

Total Amount Released: 1,512 kilograms

FOOTPRINT INFORMATION:

Dispersion Module: Gaussian

User-specified LOC: $30 \mathrm{ppm}$

Max Threat Zone for LOC: 328 meters

Max Threat Zone for IDLH: 27 meters
Reviewed by S. A. Henderson

Works Calculation 8

Sheet No. 118 of 314 
Title of Project EPHA for THM

Subject Concentration Analysis for THM Hazardous Chemicals

Computer N.E. Baucom B. K. Tharakan Date 09/16/96
Reviewed by S. A. Henderson

Works Calculation 8

Sheet No. 119 of 314

\section{1-RD-34 ADVERSE / MAX DIST SEG 2}

\section{SITE DATA INFORMATION:}

Location: AIKEN, SOUTH CAROLINA

Building Air Exchanges Per Hour: 0.44 (Sheltered single storied)

Time: June 30, 19982359 hours EDT (User specified)

\section{CHEMICAL INFORMATION:}

Chemical Name: MORPHOLINE

Molecular Weight: $87.12 \mathrm{~kg} / \mathrm{kmol}$

TLV-TWA: $20 \mathrm{ppm}$ IDLH: $8000 \mathrm{ppm}$

Footprint Level of Concern: $30 \mathrm{ppm}$

Boiling Point: $128.00^{\circ} \mathrm{C}$

Vapor Pressure at Ambient Temperature: $0.017 \mathrm{~atm}$

Ambient Saturation Concentration: 16,979 ppm or $1.70 \%$

ATMOSPHERIC INFORMATION: (MANUAL INPUT OF DATA)

Wind: 1.7 meters $/ \mathrm{sec}$ from $90^{\circ}$ true at 2 meters

Inversion Height: 200 meters

Stability Class: E Air Temperature: $29^{\circ} \mathrm{C}$

Relative Humidity: 50\% Ground Roughness: Urban or forest

Cloud Cover: 1 tenths

\section{SOURCE STRENGTH INFORMATION:}

Puddle Area: 40700 square feet

Average Puddle Depth: 1 centimeters

Soil Type: Default Ground Temperature: $29^{\circ} \mathrm{C}$

Initial Puddle Temperature: Ground temperature

Release Duration: ALOHA limited the duration to 1 hour

Max Computed Release Rate: 46.1 kilograms/min

Max Average Sustained Release Rate: 43.9 kilograms/min

(averaged over a minute or more)

Total Amount Released: 2,468 kilograms

FOOTPRINT INFORMATION:

Model Run: Heavy Gas

User-specified LOC: $30 \mathrm{ppm}$

- Max Threat Zone for LOC: 586 meters 
Title of Project EPHA for THM

Reviewed by $\mathbf{S}$. A. Henderson

Subject Concentration Analysis for THM Hazardous Chemicals

Computer N.E. Baucom B. K. Tharakan

Date 09/16/96
Works Calculation 8

Sheet No. 120 of 314

\section{1-RD-34 ADVERSE / MAX DIST SEG 3}

\section{SITE DATA INFORMATION:}

Location: AIKEN, SOUTH CAROLINA

Building Air Exchanges Per Hour: 0.44 (Sheltered single storied)

Time: June 30, 19982359 hours EDT (User specified)

\section{CHEMICAL INFORMATION:}

Chemical Name: MORPHOLINE

Molecular Weight: $87.12 \mathrm{~kg} / \mathrm{kmol}$

TLV-TWA: $20 \mathrm{ppm}$ IDLH: $8000 \mathrm{ppm}$

Footprint Level of Concern: 30 ppm

Boiling Point: $128.00^{\circ} \mathrm{C}$

Vapor Pressure at Ambient Temperature: 0.017 atm

Ambient Saturation Concentration: 16,979 ppm or $1.70 \%$

ATMOSPHERIC INFORMATION: (MANUAL INPUT OF DATA)

Wind: 1.7 meters $/ \mathrm{sec}$ from $90^{\circ}$ true at 2 meters

Inversion Height: 200 meters

Stability Class: E Air Temperature: $29^{\circ} \mathrm{C}$

Relative Humidity: 50\% Ground Roughness: Urban or forest

Cloud Cover: 1 tenths

\section{SOURCE STRENGTH INFORMATION:}

Puddle Area: 10000 square meters

Average Puddle Depth: 1 centimeters

Soil Type: Default Ground Temperature: $29^{\circ} \mathrm{C}$

Initial Puddle Temperature: Ground temperature

Release Duration: ALOHA limited the duration to 1 hour

Max Computed Release Rate: 117 kilograms/min

Max Average Sustained Release Rate: 112 kilograms/min (averaged over a minute or more)

Total Amount Released: 6,281 kilograms

FOOTPRINT INFORMATION:

Model Run: Heavy Gas

User-specified LOC: $30 \mathrm{ppm}$

Max Threat Zone for LOC: 936 meters 
Title of Project EPHA for THM

Subject Concentration Analysis for THM Hazardous Chemicals

Computer N.E. Baucom B. K. Tharakan Date 09/16/96

\section{1-RD-36 ADVERSE / ALERT}

\section{SITE DATA INFORMATION:} Location: AIKEN, SOUTH CAROLINA

Building Air Exchanges Per Hour: 0.44 (Sheltered single storied)

Time: June 30, 19982359 hours EDT (User specified)

\section{CHEMICAL INFORMATION:}

Chemical Name: 1-METHYLPYRROLIDONE

Molecular Weight: $99.13 \mathrm{~kg} / \mathrm{kmol}$

TLV-TWA: -unavail- IDLH: -unavail-

Footprint Level of Concern: $500 \mathrm{mg} /$ (cu m)

Boiling Point: $202.00^{\circ} \mathrm{C}$

Vapor Pressure at Ambient Temperature: $6.02 \mathrm{e}-004 \mathrm{~atm}$

Ambient Saturation Concentration: 613 ppm or $0.061 \%$

\section{ATMOSPHERIC INFORMATION: (MANUAL INPUT OF DATA)}

Wind: 1.7 meters $/ \mathrm{sec}$ from $90^{\circ}$ true at 2 meters

Inversion Height: 200 meters

Stability Class: E Air Temperature: $29^{\circ} \mathrm{C}$

Relative Humidity: 50\% Ground Roughness: Urban or forest

Cloud Cover: 1 tenths

\section{SOURCE STRENGTH INFORMATION:}

Puddle Area: 30440 square feet

Average Puddle Depth: 1 centimeters

Soil Type: Default Ground Temperature: $29^{\circ} \mathrm{C}$

Initial Puddle Temperature: Ground temperature

Release Duration: ALOHA limited the duration to 1 hour

Max Computed Release Rate: 1.39 kilograms/min

Max Average Sustained Release Rate: 1.35 kilograms/min

(averaged over a minute or more)

Total Amount Released: 77.7 kilograms

TIME DEPENDENT INFORMATION:

Concentration Estimates at the point:

Downwind: 30 meters

Off Centerline: 0 meters

The point selected is at the source.

The concentration and dose are mathematically infinite at the source.
Reviewed by S. A. Henderson

Works Calculation 8

Sheet No. 121 of 314 


\section{ENGINEERING COMPUTATION SHEET}

Title of Project EPHA for THM

Subject Concentration Analysis for THM Hazardous Chemicals

Computer N.E. Baucom B. K. Tharakan Date 09/16/96

1.RD.36 ADVERSE / SAE

Not applicable. See run for 1-RD-36 MAX DIST SEG 3.
Reviewed by S. A. Henderson

Works Calculation 8

Sheet No. 122 of 314 
Subject Concentration Analysis for THM Hazardous Chemicals

\section{1-RD-36 ADVERSE / MAX DIST SEG 1}

\section{SITE DATA INFORMATION:}

Location: AIKEN, SOUTH CAROLINA

Building Air Exchanges Per Hour: 0.44 (Sheltered single storied)

Time: June 30, 19982359 hours EDT (User specified)

\section{CHEMICAL INFORMATION:}

Chemical Name: 1-METHYLPYRROLIDONE

Molecular Weight: $99.13 \mathrm{~kg} / \mathrm{kmol}$

TLV-TWA: -unavail- ' IDLH: -unavail-

Footprint Level of Concern: $500 \mathrm{mg} /$ (cu m)

Boiling Point: $202.00^{\circ} \mathrm{C}$

Vapor Pressure at Ambient Temperature: 6.02e-004 atm

Ambient Saturation Concentration: 613 ppm or $0.061 \%$

\section{ATMOSPHERIC INFORMATION: (MANUAL INPUT OF DATA)}

Wind: 1.7 meters $/ \mathrm{sec}$ from $90^{\circ}$ true at 2 meters

Inversion Height: 200 meters

Stability Class: E Air Temperature: $29^{\circ} \mathrm{C}$

Relative Humidity: 50\% Ground Roughness: Urban or forest

Cloud Cover: 1 tenths

\section{SOURCE STRENGTH INFORMATION:}

Puddle Area: 24420 square feet

Average Puddle Depth: 1 centimeters

Soil Type: Default Ground Temperature: $29^{\circ} \mathrm{C}$

Initial Puddle Temperature: Ground temperature

Release Duration: ALOHA limited the duration to 1 hour

Max Computed Release Rate: 1.12 kilograms/min

Max Average Sustained Release Rate: 1.09 kilograms/min

(averaged over a minute or more)

Total Amount Released: 62.9 kilograms

FOOTPRINT INFORMATION:

Dispersion Module: Gaussian

User-specified LOC: $500 \mathrm{mg} /$ (cu m)

Max Threat Zone for LOC: 27 meters 
Title of Project EPHA for THM

Subject Concentration Analysis for THM Hazardous Chemicals

Computer N.E. Baucom B. K. Tharakan Date 09/16/96
Reviewed by S. A. Henderson

Works Calculation 8

Sheet No. 124 of 314

\section{1-RD-36 ADVERSE / MAX DIST SEG 2}

SITE DATA INFORMATION:

Location: AIKEN, SOUTH CAROLINA

Building Air Exchanges Per Hour: 0.44 (Sheltered single storied)

Time: June 30, 1998 2359 hours EDT (User specified)

\section{CHEMICAL INFORMATION:}

Chemical Name: 1-METHYLPYRROLIDONE

Molecular Weight: $99.13 \mathrm{~kg} / \mathrm{kmol}$

TLV-TWA: -unavail- $\quad$ IDLH: -unavail-

Footprint Level of Concern: $500 \mathrm{mg} /$ (cu m)

Boiling Point: $202.00^{\circ} \mathrm{C}$

Vapor Pressure at Ambient Temperature: 6.02e-004 atm

Ambient Saturation Concentration: 613 ppm or $0.061 \%$

ATMOSPHERIC INFORMATION: (MANUAL INPUT OF DATA)

Wind: $1.7^{\circ}$ meters $/ \mathrm{sec}$ from $90^{\circ}$ true at 2 meters

Inversion Height: 200 meters

Stability Class: E Air Temperature: $29^{\circ} \mathrm{C}$

Relative Humidity: 50\% Ground Roughness: Urban or forest

Cloud Cover: 1 tenths

SOURCE STRENGTH INFORMATION:

Puddle Area: 40700 square feet

Average Puddle Depth: 1 centimeters

Soil Type: Default Ground Temperature: $29^{\circ} \mathrm{C}$

Initial Puddle Temperature: Ground temperature

Release Duration: ALOHA limited the duration to 1 hour

Max Computed Release Rate: 1.83 kilograms/min

Max Average Sustained Release Rate: 1.78 kilograms/min

(averaged over a minute or more)

Total Amount Released: 103 kilograms

\section{FOOTPRINT INFORMATION:}

Dispersion Module: Gaussian

User-specified LOC: $500 \mathrm{mg} /$ (cu m)

- Max Threat Zone for LOC: 34 meters 
Title of Project EPHA for THM

\section{ENGINEERING COMPUTATION SHEET}

Subject Concentration Analysis for THM Hazardous Chemicals

Computer N.E. Baucom B. K. Tharakan Date 09/16/96

\section{1-RD-36 ADVERSE / MAX DIST SEG 3}

\section{SITE DATA INFORMATION:}

Location: AIKEN, SOUTH CAROLINA

Building Air Exchanges Per Hour: 0.44 (Sheltered single storied)

Time: June 30, 19982359 hours EDT (User specified)

\section{CHEMICAL INFORMATION:}

Chemical Name: 1-METHYLPYRROLIDONE

Molecular Weight: $99.13 \mathrm{~kg} / \mathrm{kmol}$

TLV-TWA: -unavail- IDLH: -unavail-

Footprint Level of Concern: $500 \mathrm{mg} /$ (cu m)

Boiling Point: $202.00^{\circ} \mathrm{C}$

Vapor Pressure at Ambient Temperature: 6.02e-004 atm

Ambient Saturation Concentration: 613 ppm or $0.061 \%$

\section{ATMOSPHERIC INFORMATION: (MANUAL INPUT OF DATA)}

Wind: 1.7 meters $/ \mathrm{sec}$ from $90^{\circ}$ true at 2 meters

Inversion Height: 200 meters

Stability Class: E Air Temperature: $29^{\circ} \mathrm{C}$

Relative Humidity: 50\% Ground Roughness: Urban or forest

Cloud Cover: 1 tenths

\section{SOURCE STRENGTH INFORMATION:}

Puddle Area: 10000 square meters

Average Puddle Depth: 1 centimeters

Soil Type: Default Ground Temperature: $29^{\circ} \mathrm{C}$

Initial Puddle Temperature: Ground temperature

Release Duration: ALOHA limited the duration to 1 hour

Max Computed Release Rate: 4.66 kilograms/min

Max Average Sustained Release Rate: 4.52 kilograms/min (averaged over a minute or more)

Total Amount Released: 261 kilograms

\section{FOOTPRINT INFORMATTON:}

Dispersion Module: Gaussian

User-specified LOC: $500 \mathrm{mg} /$ (cu m)

Max Threat Zone for LOC: 56 meters
Reviewed by S. A. Henderson

Works Calculation 8

Sheet No. 125 of 314 
Title of Project EPHA for THM

Reviewed by S. A. Henderson

Subject Concentration Analysis for THM Hazardous Chemicals

Works Calculation 8

Computer N.E. Baucom B. K. Tharakan Date 09/16/96

1-RD-42 ADVERSE / ALERT

\section{SITE DATA INFORMATION: \\ Location: AIKEN, SOUTH CAROLINA \\ Building Air Exchanges Per Hour: 60 (User specified) \\ Time: June 30, 19982359 hours EDT (User specified)}

\section{CHEMICAL INFORMATION:}

Chemical Name: PROPIONIC ACID

Molecular Weight: $74.08 \mathrm{~kg} / \mathrm{kmol}$

TLV-TWA: 10 ppm IDLH: -unavail-

Footprint Level of Concern: $15 \mathrm{ppm}$

Boiling Point: $141.17^{\circ} \mathrm{C}$

Vapor Pressure at Ambient Temperature: $0.0063 \mathrm{~atm}$

Ambient Saturation Concentration: 6,444 ppm or $0.64 \%$

ATMOSPHERIC INFORMATION: (MANUAL INPUT OF DATA)

Wind: 1.7 meters $/ \mathrm{sec}$ from $90^{\circ}$ true at 2 meters

Inversion Height: 200 meters

Stability Class: E Air Temperature: $29^{\circ} \mathrm{C}$

Relative Humidity: 50\% Ground Roughness: Urban or forest

Cloud Cover: 1 tenths

\section{SOURCE STRENGTH INFORMATION:}

Puddle Area: 283 square feet

Average Puddle Depth: 1 centimeters

Soil Type: Default Ground Temperature: $29^{\circ} \mathrm{C}$

Initial Puddle Temperature: Ground temperature

Release Duration: ALOHA limited the duration to 1 hour

Max Computed Release Rate: 135 grams/min

Max Average Sustained Release Rate: 130 grams/min

(averaged over a minute or more)

Total Amount Released: 7.35 kilograms

\section{TIME DEPENDENT INFORMATION:}

Concentration Estimates at the point:

Downwind: 30 meters

Off Centerline: 0 meters

Max Concentration:

Outdoor: $15 \mathrm{ppm}$ 
Title of Project EPHA for THM

Subject Cencentration Analysis for THM Hazardous Chemicals

Computer N.E. Baucom B. K. Tharakan Date 09/16/96

1-RD-42 ADVERSE / SAE

SITE DATA INFORMATION:

Location: AIKEN, SOUTH CAROLINA

Building Air Exchanges Per Hour: 60 (User specified)

Time: June 30, 19982359 hours EDT (User specified)

CHEMICAL INFORMATION:

Chemical Name: PROPIONIC ACID

Molecular Weight: $74.08 \mathrm{~kg} / \mathrm{kmol}$

TLV-TWA: 10 ppm IDLH: -unavail-

Footprint Level of Concern: $15 \mathrm{ppm}$

Boiling Point: $141.17^{\circ} \mathrm{C}$

Vapor Pressure at Ambient Temperature; $0.0063 \mathrm{~atm}$

Ambient Saturation Concentration: 6,444 ppm or $0.64 \%$

ATMOSPHERIC INFORMATION: (MANUAL INPUT OF DATA)

Wind: 1.7 meters $/ \mathrm{sec}$ from $90^{\circ}$ true at 2 meters

Inversion Height: 200 meters

Stability Class: E Air Temperature: $29^{\circ} \mathrm{C}$

Relative Humidity: $50 \%$ Ground Roughness: Urban or forest

Cloud Cover: 1 tenths

SOURCE STRENGTH INFORMATION:

Puddle Area: 3600 square feet

Average Puddle Depth: 1 centimeters

Soil Type: Default Ground Temperature: $29^{\circ} \mathrm{C}$

Initial Puddle Temperature: Ground temperature

Release Duration: ALOHA limited the duration to 1 hour

Max Computed Release Rate: 1.51 kilograms/min

Max Average Sustained Release Rate: 1.45 kilograms/min

(averaged over a minute or more)

Total Amount Released: 82.3 kilograms

TIME DEPENDENT INFORMATION:

Concentration Estimates at the point:

Downwind: 100 meters

Off Centerline: 0 meters

Max Concentration:

Outdoor: 15 ppm
Reviewed by S. A. Henderson

Works Calculation 8

Sheet No. 127 of 314 
Title of Project EPHA for THM

Subject Concentration Analysis for THM Hazardous Chemicals

Computer N.E. Baucom B. K. Tharakan Date 09/16/96

\section{1-RD-42 ADVERSE / MAX DIST SEG 1}

\section{SITE DATA INFORMATION:} Location: AIKEN, SOUTH CAROLINA

Building Air Exchanges Per Hour: 60 (User specified)

Time: June 30, 19982359 hours EDT (User specified)

\section{CHEMICAL INFORMATION:}

Chemical Name: PROPIONIC ACD

Molecular Weight: $74.08 \mathrm{~kg} / \mathrm{kmol}$

TLV-TWA: 10 ppm IDLH: -unavail-

Footprint Level of Concern: 15 ppm

Boiling Point: $141.17^{\circ} \mathrm{C}$

Vapor Pressure at Ambient Temperature: $0.0063 \mathrm{~atm}$

Ambient Saturation Concentration: 6,444 ppm or $0.64 \%$

\section{ATMOSPHERIC INFORMATION: (MANUAL INPUT OF DATA)}

Wind: 1.7 meters $/ \mathrm{sec}$ from $90^{\circ}$ true at 2 meters

Inversion Height: 200 meters

Stability Class: E Air Temperature: $29^{\circ} \mathrm{C}$

Relative Humidity: 50\% Ground Roughness: Urban or forest

Cloud Cover: 1 tenths

\section{SOURCE STRENGTH INFORMATION:}

Puddle Area: 24420 square feet

Average Puddle Depth: 1 centimeters

Soil Type: Default Ground Temperature: $29^{\circ} \mathrm{C}$

Initial Puddle Temperature: Ground temperature

Release Duration: ALOHA limited the duration to 1 hour

Max Computed Release Rate: 9.35 kilograms/min

Max Average Sustained Release Rate: 8.99 kilograms/min

(averaged over a minute or more)

Total Amount Released: 512 kilograms

\section{FOOTPRINT INFORMATION:}

Dispersion Module: Gaussian

User-specified LOC: 15 ppm

Max Threat Zone for LOC: 263 meters
Reviewed by S. A. Henderson

Works Calculation 8

Sheet No. 128 of 314 
Title of Project EPHA for THM

Subject Concentration Analysis for THM Hazardous Chemicals

Computer N.E. Baucom B. K. Tharakan Date 09/16/96
Reviewed by S. A. Henderson

Works Calculation 8

Sheet No. 129 of 314

\section{1-RD-42 ADVERSE / MAX DIST SEG 2}

SITE DATA INFORMATION:

Location: AIKEN, SOUTH CAROLINA

Building Air Exchanges Per Hour: 60 (User specified)

Time: June 30, 19982359 hours EDT (User specified)

\section{CHEMICAL INFORMATION:}

Chemical Name: PROPIONIC ACID

Molecular Weight: $74.08 \mathrm{~kg} / \mathrm{kmol}$

TLV-TWA: 10 ppm IDLH: -unavail-

Footprint Level of Concern: $15 \mathrm{ppm}$

Boiling Point: $141.17^{\circ} \mathrm{C}$

Vapor Pressure at Ambient Temperature: $0.0063 \mathrm{~atm}$

Ambient Saturation Concentration: $6,444 \mathrm{ppm}$ or $0.64 \%$

\section{ATMOSPHERIC INFORMATION: (MANUAL INPUT OF DATA)}

Wind: 1.7 meters $/ \mathrm{sec}$ from $90^{\circ}$ true at 2 meters

Inversion Height: 200 meters

Stability Class: E Air Temperature: $29^{\circ} \mathrm{C}$

Relative Humidity: 50\% Ground Roughness: Urban or forest

Cloud Cover: 1 tenths

\section{SOURCE STRENGTH INFORMATION:}

Puddle Area: 40700 square feet

Average Puddle Depth: 1 centimeters

Soil Type: Default Ground Temperature: $29^{\circ} \mathrm{C}$

Initial Puddle Temperature: Ground temperature

Release Duration: ALOHA limited the duration to 1 hour

Max Computed Release Rate: 15.2 kilograms/min

Max Average Sustained Release Rate: 14.7 kilograms/min

(averaged over a minute or more)

Total Amount Released: 835 kilograms

\section{FOOTPRINT INFORMATION:}

Dispersion Module: Gaussian

User-specified LOC: $15 \mathrm{ppm}$

Max Threat Zone for LOC: 346 meters 
Title of Project EPHA for THM

Subject Concentration Analysis for THM Hazardous Chemicals

Computer N.E. Baucom B. K. Tharakan Date 09/16/96
Reviewed by S. A. Henderson

Works Calculation 8

Sheet No. 130 of 314

\section{1-RD-42 ADVERSE / MAX DIST SEG 3}

\section{SITE DATA INFORMATION:}

Location: AIKEN, SOUTH CAROLINA

Building Air Exchanges Per Hour: 60 (User specified)

Time: June 30, 19982359 hours EDT (User specified)

\section{CHEMICAL INFORMATION:}

Chemical Name: PROPIONIC ACID

Molecular Weight: $74.08 \mathrm{~kg} / \mathrm{kmol}$

TLV-TWA: $10 \mathrm{ppm}$ IDLH: -unavail-

- Footprint Level of Concern: 15 ppm

Boiling Point: $141.17^{\circ} \mathrm{C}$

Vapor Pressure at Ambient Temperature: $0.0063 \mathrm{~atm}$

Ambient Saturation Concentration: 6,444 ppm or $0.64 \%$

\section{ATMOSPHERIC INFORMATION: (MANUAL INPUT OF DATA)}

Wind: 1.7 meters $/ \mathrm{sec}$ from $90^{\circ}$ true at 2 meters

Inversion Height: 200 meters

Stability Class: E Air Temperature: $29^{\circ} \mathrm{C}$

Relative Humidity: 50\% Ground Roughness: Urban or forest

Cloud Cover: 1 tenths

\section{SOURCE STRENGTH INFORMATION:}

Puddle Area:" 10000 square meters

Average Puddle Depth: 1 centimeters

Soil Type: Default Ground Temperature: $29^{\circ} \mathrm{C}$

Initial Puddle Temperature: Ground temperature

Release Duration: ALOHA limited the duration to 1 hour

Max Computed Release Rate: 38.6 kilograms/min

Max Average Sustained Release Rate: 37.2 kilograms/min

(averaged over a minute or more)

Total Amount Released: 2,120 kilograms

FOOTPRINT INFORMATION:

Dispersion Module: Gaussian

User-specified LOC: $15 \mathrm{ppm}$

Max Threat Zone for LOC: 597 meters 
Title of Project EPHA for THM

Subject Concentration Analysis for THM Hazardous Chemicals

Computer N.E. Baucom B. K. Tharakan Date 09/16/96

\section{1-RD-46 ADVERSE / ALERT}

SITE DATA INFORMATION:

Location: AIKEN, SOUTH CAROLINA

Building Air Exchanges Per Hour: 60 (User specified)

Time: June 30, 19982359 hours EDT (User specified)

CHEMICAL INFORMATION:

Chemical Name: TETRAHYDROFURAN

Molecular Weight: $72.11 \mathrm{~kg} / \mathrm{kmol}$

TLV-TWA: $200 \mathrm{ppm}$

IDLH: $2000 \mathrm{ppm}$

Footprint Level of Concern: 2000 ppm

Boiling Point: $65.97^{\circ} \mathrm{C}$

Vapor Pressure at Ambient Temperature: $0.25 \mathrm{~atm}$

Ambient Saturation Concentration: 257,248 ppm or $25.7 \%$

ATMOSPHERIC INFORMATION: (MANUAL INPUT OF DATA)

Wind: 1.7 meters $/ \mathrm{sec}$ from $90^{\circ}$ true at 2 meters

Inversion Height: 200 meters

Stability Class: E Air Temperature: $29^{\circ} \mathrm{C}$

Relative Humidity: $50 \%$ Ground Roughness: Urban or forest

Cloud Cover: 1 tenths

SOURCE STRENGTH INFORMATION:

Puddle Area: 1080 square feet

Average Puddle Depth: 1 centimeters

Soil Type: Default Ground Temperature: $29^{\circ} \mathrm{C}$

Initial Puddle Temperature: Ground temperature

Release Duration: ALOHA limited the duration to 1 hour

Max Computed Release Rate: 21.6 kilograms/min

Max Average Sustained Release Rate: 16.6 kilograms/min

(averaged over a minute or more)

Total Amount Released: 767 kilograms

\section{FOORTPRINT INFORMATION:}

Model Run: Heavy Gas

User-specified LOC: equals IDLH (2000 ppm)

Max Threat Zone for LOC: 30 meters

Note: Footprint was not drawn because effects of near-field patchiness make dispersion predictions unreliable for short distances.

TIME DEPENDENT INFORMATION:

Concentration Estimates at the point:

Downwind: 30 meters

Off Centerline: 0 meters

Max Concentration:

Outdoor: $2,000 \mathrm{ppm}$
Reviewed by S. A. Henderson

Works Calculation 8

Sheet No. 131 of 314 
Title of Project EPHA for THM

Subject Concentration Analysis for THM Hazardous Chemicals

Computer N.E. Baucom B. K. Tharakan Date 09/16/96

\section{1-RD-46 ADVERSE / SAE}

SITE DATA INFORMATION:

Location: AIKEN, SOUTH CAROLINA

Building Air Exchanges Per Hour: 60 (User specified)

Time: June 30, 19982359 hours EDT (User specified)

CHEMICAL INFORMATION:

Chemical Name: TETRAHYDROFURAN

Molecular Weight: $72.11 \mathrm{~kg} / \mathrm{kmol}$

TLV-TWA: $200 \mathrm{ppm}$ IDLH: $2000 \mathrm{ppm}$

Footprint Level of Concern: 2000 ppm

Boiling Point: $65.97^{\circ} \mathrm{C}$

Vapor Pressure at Ambient Temperature: $0.25 \mathrm{~atm}$

Ambient Saturation Concentration: 257,248 ppm or 25.7\%

ATMOSPHERIC INFORMATION: (MANUAL INPUT OF DATA)

Wind: 1.7 meters $/ \mathrm{sec}$ from $90^{\circ}$ true at 2 meters

Inversion Height: 200 meters

Stability Class: E Air Temperature: $29^{\circ} \mathrm{C}$

Relative Humidity: 50\% Ground Roughness: Urban or forest

Cloud Cover: 1 tenths

\section{SOURCE STRENGTH INFORMATION:}

Puddle Area: 13300 square feet

Average Puddle Depth: 1 centimeters

Soil Type: Default Ground Temperature: $29^{\circ} \mathrm{C}$

Initial Puddle Temperature: Ground temperature

Release Duration: ALOHA limited the duration to 1 hour

Max Computed Release Rate: 235 kilograms/min

Max Average Sustained Release Rate: 184 kilograms/min (averaged over a minute or more)

Total Amount Released: 8,654 kilograms

FOORTPRINT INFORMATION:

Model Run: Heavy Gas

User-specified LOC: equals IDLH (2000 ppm)

Max Threat Zone for LOC: 100 meters

Note: Footprint was not drawn because effects of near-field patchiness make dispersion predictions unreliable for short distances.

TIME DEPENDENT INFORMATION:

Concentration Estimates at the point:

Downwind: 100 meters

Off Centerline: 0 meters

Max Concentration:

Outdoor: $2000 \mathrm{ppm}$
Reviewed by S. A. Henderson

Works Calculation 8

Sheet No. 132 of 314 
Title of Project EPHA for THM

Subject Concentration Analysis for THM Hazardous Chemicals

Computer N.E. Baucom B. K. Tharakan Date 09/16/96
Reviewed by S. A. Henderson

Works Calculation 8

Sheet No. 133 of 314

\section{1-RD-46 ADVERSE / MAX DIST SEG 1}

SITE DATA INFORMATION:

Location: AIKEN, SOUTH CAROLINA

Building Air Exchanges Per Hour: 60 (User specified)

Time: June 30, 19982359 hours EDT (User specified)

\section{CHEMICAL INFORMATION:}

Chemical Name: TETRAHYDROFURAN

Molecular Weight: $72.11 \mathrm{~kg} / \mathrm{kmol}$

TLV-TWA: $200 \mathrm{ppm}$-IDLH: $2000 \mathrm{ppm}$

Footprint Level of Concern: 2000 ppm

Boiling Point: $65.97^{\circ} \mathrm{C}$

Vapor Pressure at Ambient Temperature: 0.25 atm

Ambient Saturation Concentration: 257,248 ppm or $25.7 \%$

ATMOSPHERIC INFORMATION: (MANUAL INPUT OF DATA)

Wind: $1.7^{\circ}$ meters $/ \mathrm{sec}$ from $90^{\circ}$ true at 2 meters

Inversion Height: 200 meters

Stability Class: E Air Temperature: $29^{\circ} \mathrm{C}$

Relative Humidity: $50 \%$ Ground Roughness: Urban or forest

Cloud Cover: 1 tenths

\section{SOURCE STRENGTH INFORMATION:}

Puddle Area: 24420 square feet

Average Puddle Depth: 1 centimeters

Soil Type: Default Ground Temperature: $29^{\circ} \mathrm{C}$

Initial Puddle Temperature: Ground temperature

Release Duration: ALOHA limited the duration to 1 hour

Max Computed Release Rate: 419 kilograms/min

Max Average Sustained Release Rate: 331 kilograms/min

(averaged over a minute or more)

Total Amount Released:15,579 kilograms

FOORTPRINT INFORMATION:

Model Run: Heavy Gas

User-specified LOC: equals IDLH (2000 ppm)

- Max Threat Zone for LOC: 138 meters 
Title of Project EPHA for THM

Subject Concentration Analysis for THM Hazardous Chemicals

Computer N.E. Baucom B. K. Tharakan Date 09/16/96
Reviewed by S.A. Henderson

Works Calculation 8

Sheet No. 134 of 314

\section{1-RD-46 ADVERSE / MAX DIST SEG 2}

\section{SITE DATA INFO'RMATION:}

Location: AIKEN, SOUTH CAROLINA

Building Air Exchanges Per Hour: 60 (User specified)

Time: June 30, 19982359 hours EDT (User specified)

\section{CHEMICAL INFORMATION:}

Chemical Name: TETRAHYDROFURAN

Molecular Weight: $72.11 \mathrm{~kg} / \mathrm{kmol}$

TLV-TWA: $200 \mathrm{ppm}$ IDLH: $2000 \mathrm{ppm}$

Footprint Level of Concern: 2000 ppm

Boiling Point: $65.97^{\circ} \mathrm{C}$

Vapor Pressure at Ambient Temperature: $0.25 \mathrm{~atm}$

Ambient Saturation Concentration: $257,248 \mathrm{ppm}$ or $25.7 \%$

ATMOSPHERIC INFORMATION: (MANUAL INPUT OF DATA)

Wind: 1.7 meters $/ \mathrm{sec}$ from $90^{\circ}$ true at 2 meters

Inversion Height: 200 meters

Stability Class: E Air Temperature: $29^{\circ} \mathrm{C}$

Relative Humidity: 50\% Ground Roughness: Urban or forest

Cloud Cover: 1 tenths

\section{SOURCE STRENGTH INFORMATION:}

Puddle Area: 40700 square feet

Average Puddle Depth: 1 centimeters

Soil Type: Default Ground Temperature: $29^{\circ} \mathrm{C}$

Initial Puddle Temperature: Ground temperature

Release Duration: ALOHA limited the duration to 1 hour

Max Computed Release Rate: 683 kilograms/min

Max Average Sustained Release Rate: 541 kilograms $/ \mathrm{min}$ (averaged over a minute or more)

Total Amount Released: 25,542 kilograms

\section{FOORTPRINT INFORMATION:}

Model Run: Heavy Gas

User-specified LOC: equals IDLH (2000 ppm)

Max Threat Zone for LOC: 183 meters 
Title of Project EPHA for THM

Subject Concentration Analysis for THM Hazardous Chemicals

Computer N.E. Baucom B. K. Tharakan Date 09/16/96

\section{1-RD-46 ADVERSE / MAX DIST SEG 3}

\section{SITE DATA INFORMATION:}

Location: AIKEN, SOUTH CAROLINA

Building Air Exchanges Per Hour: 60 (User specified)

Time: June 30, 19982359 hours EDT (User specified)

\section{CHEMICAL INFORMATION:}

Chemical Name: TETRAHYDROFURAN

Molecular Weight: $72.11 \mathrm{~kg} / \mathrm{kmol}$

TLV-TWA: $200 \mathrm{ppm}$

IDLH: $2000 \mathrm{ppm}$

Footprint Level of Concern: $2000 \mathrm{ppm}$

Boiling Point: $65.97^{\circ} \mathrm{C}$

Vapor Pressure at Ambient Temperature: $0.25 \mathrm{~atm}$

Ambient Saturation Concentration: 257,248 ppm or 25.7\%

ATMOSPHERIC INFORMATION: (MANUAL INPUT OF DATA)

Wind: 1.7 meters $/ \mathrm{sec}$ from $90^{\circ}$ true at 2 meters

Inversion Height: 200 meters

Stability Class: E Air Temperature: $29^{\circ} \mathrm{C}$

Relative Humidity: $50 \%$ Ground Roughness: Urban or forest

Cloud Cover: 1 tenths

\section{SOURCE STRENGTH INFORMATION:}

Puddle Area: 10000 square meters

Average Puddle Depth: 1 centimeters

Soil Type: Default Ground Temperature: $29^{\circ} \mathrm{C}$

Initial Puddle Temperature: Ground temperature

Release Duration: ALOHA limited the duration to 1 hour

Max Computed Release Rate: 1,730 kilograms/min

Max Average Sustained Release Rate: 1,380 kilograms/min

(averaged over a minute or more)

Total Amount Released: 65,545 kilograms

\section{FOORTPRINT INFORMATION:}

Model Run: Heavy Gas

User-specified LOC: equals IDLH (2000 ppm)

Max Threat Zone for LOC: 310 meters
Reviewed by S. A. Henderson

Works Calculation 8

Sheet No. 135 of 314 
Title of Project EPHA for THM

Subject Concentration Analysis for THM Hazardous Chemicals

Computer N.E. Baucom B.K. Tharakan Date 09/16/96

\section{1-RD-49 ADVERSE / ALERT}

\section{SITE DATA INFORMATION:}

Location: AIKEN, SOUTH CAROLINA

Building Air Exchanges Per Hour: 60 (User specified)

Time: June 30, 19982359 hours EDT (User specified)

\section{CHEMICAL INFORMATION:}

Chemical Name: 1,1,1-TRICHLOROETHANE

Molecular Weight: $133.40 \mathrm{~kg} / \mathrm{kmol}$

TLV.TWA: $350 \mathrm{ppm}$ IDLH: $700 \mathrm{ppm}$

Footprint Level of Concern: 700 ppm

Boiling Point: $74.08^{\circ} \mathrm{C}$

Vapor Pressure at Ambient Temperature: $0.20 \mathrm{~atm}$

Ambient Saturation Concentration: 198,693 ppm or $19.9 \%$

\section{ATMOSPHERIC INFORMATION: (MANUAL INPUT OF DATA)}

Wind: 1.7 meters $/ \mathrm{sec}$ from $90^{\circ}$ true at 2 meters

Inversion Height: 200 meters

Stability Class: E. Air Temperature: $29^{\circ} \mathrm{C}$

Relative Humidity: 50\% Ground Roughness: Urban or forest

Cloud Cover: 1 tenths

\section{SOURCE STRENGTH INFORMATION:}

Puddle Area: 472 square feet

Average Puddle Depth: 1 centimeters

Soil Type: Default Ground Temperature: $29^{\circ} \mathrm{C}$

Initial Puddle Temperature: Ground temperature

Release Duration: ALOHA 11.8 kilograms/min

Max Average Sustained Release Rate: 9.68 kilograms/min

(averaged over a minute or more)

Total Amount Released: 473 kilograms

FOORTPRINT INFORMATION:

Model Run: Heavy Gas

User-specified LOC: equals IDLH (700 ppm)

Max Threat Zone for LOC: 30 meters

Note: Footprint was not drawn because effects of near-field patchiness make dispersion predictions unreliable for short distances.

\section{TIME DEPENDENT INFORMATION:}

Concentration Estimates at the point:

Downwind: 30 meters

Off Centerline: 0 meters

Max Concentration:

Outdoor: $699 \mathrm{ppm}$
Reviewed by S. A. Henderson

Works Calculation 8

Sheet No. 136 of 314 
Title of Project EPHA for THM

Subject Concentration Analysis for THM Hazardous Chemicals

Computer N.E. Baucom B. K. Tharakan Date 09/16/96

1-RD-49 ADVERSE / SAE

\section{SITE DATA INFORMATION:}

Location: AIKEN, SOUTH CAROLINA

Building Air Exchanges Per Hour: 60 (User specified)

Time: June 30, 19982359 hours EDT (User specified)

\section{CHEMICAL INFORMATION:}

Chemical Name: 1,1,1-TRICHLOROETHANE

Molecular Weight: $133.40 \mathrm{~kg} / \mathrm{kmol}$

TLV-TWA: $350 \mathrm{ppm}$

IDLH: 700 ppm

Footprint Level of Concern: $700 \mathrm{ppm}$

Boiling Point: $74.08^{\circ} \mathrm{C}$

Vapor Pressure at Ambient Temperature: $0.20 \mathrm{~atm}$

Ambient Saturation Concentration: 198,693 ppm or $19.9 \%$

\section{ATMOSPHERIC INFORMATION: (MANUAL INPUT OF DATA)}

Wind: 1.7 meters $/ \mathrm{sec}$ from $90^{\circ}$ true at 2 meters

Inversion Height: 200 meters

Stability Class: E Air Temperature: $29^{\circ} \mathrm{C}$

Relative Humidity: $50 \%$ Ground Roughness: Urban or forest

Cloud Cover: 1 tenths

\section{SOURCE STRENGTH INFORMATION:}

Puddle Area: 6060 square feet

Average Puddle Depth: 1 centimeters

Soil Type: Default Ground Temperature: $29^{\circ} \mathrm{C}$

Initial Puddle Temperature: Ground temperature

Release Duration: ALOHA limited the duration to 1 hour

Max Computed Release Rate: 135 kilograms/min

Max Average Sustained Release Rate: 112 kilograms/min (averaged over a minute or more)

Total Amount Released: 5,563 kilograms

\section{FOORTPRINT INFORMATION:}

Model Run: Heavy Gas

User-specified LOC: equals IDLH (700 ppm)

Max Threat Zone for LOC: 100 meters

Note: Footprint was not drawn because effects of near-field patchiness make dispersion predictions unreliable for short distances.

\section{TIME DEPENDENT INFORMATION:}

Concentration Estimates at the point:

Downwind: 100 meters

Off Centerline: 0 meters

Max Concentration:

Outdoor: 700 ppm
Reviewed by S. A. Henderson

Works Calculation 8

Sheet No. 137 of 314 
Title of Project EPHA for THM

Subject Concentration Analysis for THM Hazardous Chemicals

Computer N.E. Baucom B. K. Tharakan Date 09/16/96
Reviewed by S. A. Henderson

Works Calculation 8

Sheet No. 138 of 314

\section{1-RD-49 ADVERSE / MAX DIST SEG 1}

SITE DATA INFORMATION:

Location: AIKEN, SOUTH CAROLINA

Building Air Exchanges Per Hour: 60 (User specified)

Time: June 30, 19982359 hours EDT (User specified)

\section{CHEMICAL INFORMATION:}

Chemical Name: 1,1,1-TRICHLOROETHANE

Molecular Weight: $133.40 \mathrm{~kg} / \mathrm{kmol}$

TLV-TWA: $350 \mathrm{ppm}$

- IDLH: $700 \mathrm{ppm}$

Footprint Level of Concern: 700 ppm

Boiling Point: $74.08^{\circ} \mathrm{C}$

Vapor Pressure at Ambient Temperature: $0.20 \mathrm{~atm}$

Ambient Saturation Concentration: 198,693 ppm or $19.9 \%$

ATMOSPHERIC INFORMATION: (MANUAL INPUT OF DATA)

Wind: 1.7 meters $/ \mathrm{sec}$ from $90^{\circ}$ true at 2 meters

Inversion Height: 200 meters

Stability Class: E Air Temperature: $29^{\circ} \mathrm{C}$

Relative Humidity: 50\% Ground Roughness: Urban or forest

Cloud Cover: 1 tenths

\section{SOURCE STRENGTH INFORMATION:}

Puddle Area: 24420 square feet

Average Puddle Depth: 1 centimeters

Soil Type: Default Ground Temperature: $29^{\circ} \mathrm{C}$

Initial Puddle Temperature: Ground temperature

Release Duration: ALOHA limited the duration to 1 hour

Max Computed Release Rate: 514 kilograms/min

Max Average Sustained Release Rate: 432 kilograms/min

(averaged over a minute or more)

Total Amount Released: 21,443 kilograms

\section{FOORTPRINT INFORMATION:}

Model Run: Heavy Gas

User-specified LOC: equals IDLH (700 ppm)

- Max Threat Zone for LOC: 208 meters 


\section{1-RD-49 ADVERSE / MAX DIST SEG 2}

SITE DATA INFORMATION:

Location: AIKEN, SOUTH CAROLINA

Building Air Exchanges Per Hour: 60 (User specified)

Time: June 30, 19982359 hours EDT (User specified)

\section{CHEMICAL INFORMATION:}

Chemical Name: 1,1,1-TRICHLOROETHANE

Molecular Weight: $133.40 \mathrm{~kg} / \mathrm{kmol}$

TLV-TWA: $350 \mathrm{ppm}$ IDLH: $700 \mathrm{ppm}$

Footprint Level of Concern: 700 ppm

Boiling Point: $74.08^{\circ} \mathrm{C}$

Vapor Pressure at Ambient Temperature: 0.20 atm

Ambient Saturation Concentration: 198,693 ppm or $19.9 \%$

\section{ATMOSPHERIC INFORMATION: (MANUAL INPUT OF DATA)}

Wind: 1.7 meters $/ \mathrm{sec}$ from $90^{\circ}$ true at 2 meters

Inversion Height: 200 meters

Stability Class: E Air Temperature: $29^{\circ} \mathrm{C}$

Relative Humidity: 50\% Ground Roughness: Urban or forest

Cloud Cover: 1 tenths

\section{SOURCE STRENGTH INFORMATION:}

Puddle Area: 40700 square feet

Average Puddle Depth: 1 centimeters

Soil Type: Default Ground Temperature: $29^{\circ} \mathrm{C}$

Initial Puddle Temperature: Ground temperature

Release Duration: ALOHA limited the duration to 1 hour

Max Computed Release Rate: 840 kilograms/min

Max Average Sustained Release Rate: 706 kilograms/min

(averaged over a minute or more)

Total Amount Released: 35,177 kilograms

FOORTPRINT INFORMATION:

Model Run: Heavy Gas

User-specified LOC: equals IDLH (700 ppm)

Max Threat Zone for LOC: 275 meters 
SITE DATA INFORMATION:

Location: AIKEN, SOUTH CAROLINA

Building Air Exchanges Per Hour: 60 (User specified)

Time: June 30, 19982359 hours EDT (User specified)

CHEMICAL INFORMATION:

Chemical Name: 1,1,1-TRICHLOROETHANE

Molecular Weight: $133.40 \mathrm{~kg} / \mathrm{kmol}$

TLV-TWA: $350 \mathrm{ppm}$ IDLH: $700 \mathrm{ppm}$

Footprint Level of Concern: $700 \mathrm{ppm}$

Boiling Point: $74.08^{\circ} \mathrm{C}$

Vapor Pressure at Ambient Temperature: 0.20 atm

Ambient Saturation Concentration: 198,693 ppm. or 19.98

ATMOSPHERIC INFORMATION: (MANUAL INPUT OF DATA)

Wind: 1.7 meters $/ \mathrm{sec}$ from $90^{\circ}$ true at 2 meters

Inversion Height: 200 meters

Stability Class: E

Air Temperature: $29^{\circ} \mathrm{C}$

Relative Humidity: $50 \%$

Ground Roughness: Urban or forest

Cloud Cover: 1 tenths

SOURCE STRENGTH INFORMATION:

Puddle Area: 10000 square meters Average Puddle Depth: 1 centimeters

Soil Type: Default Ground Temperature: $29^{\circ} \mathrm{C}$

Initial Puddle Temperature: Ground temperature

Release Duration: ALOHA limited the duration to 1 hour

Max Computed Release Rate: 2,140 kilograms/min

Max Average Sustained Release Rate: $1,810 \mathrm{kilogxams} / \mathrm{min}$ (averaged over a minute or more)

Total Amount Released: 90,343 kilograms

FOOTPRINT INFORMATION:

Model Run: Heavy Gas

User-specified LOC: equals IDLH $(700 \mathrm{ppm})$

Max Threat Zone for LOC: 466 meters 
Title of Project EPHA for THM

Subject Concentration Analysis for THM Hazardous Chemicals Works Calculation 8

Computter N.E. Baucom B. K. Tharakan Date 09/16/96

Sheet No. 141 of 314

\section{1-RD-51 ADVERGE ATERT}

SITE DATA INEORMATION: Location: AIKEN, SOUTH CAROLINA

Building Air Exchanges Per Hour: 0.44 (Sheltered single storied) Time: June 30, 19982359 hours EDT (User specified)

CHEMICAL INFORMATION:

Chemical Name: TRIETHYLAMINE

Molecular Weight: $101.19 \mathrm{~kg} / \mathrm{kmol}$

TLV-TWA: $10 \mathrm{ppm}$ IDLH: $1000 \mathrm{ppm}$

Footprint Level of Concern: $3 \mathrm{ppm}$

Boiling Point: $88.77^{\circ} \mathrm{C}$

Vapor Pressure at Arbient Temperature: 0.11 atm

Ambient Saturation Concentration: 110,492 ppm or 11.08

ATMOSPHERIC INFORMATION: (MANUAL INPUT OF DATA)

Wind: 1.7 meters/sec from 90 true at 2 meters

Inversion Height: 200 meters

Stability Class: $\mathrm{E}$ Air Temperature: $29^{\circ} \mathrm{C}$

Relative Humidity: $50 \%$ Ground Roughness: Urban or forest

Cloud Cover: 1 tenths

SOURCE STRENGTH INFORMATION: Puddle Area: 1.7 square feet

Average Puddle Depth: 1 centimeters

Soil Type: Default Ground Temperature: $29^{\circ} \mathrm{C}$

Initial puddle Temperature: Ground temperature

Release Duration: ALOHA limited the duration to 1 hour

Max Computed Release Rate: 25.2 grams/min

Max Average Sustained Release Rate: 20.9 grams/min (averaged over a minute or more)

Total Amount Released: 1.04 kilograms

TIME DEPENDENT INFORMATION:

Concentration Estimates at the point:

Downwind: $\quad 30$ meters

Off Centerline: 0 meters

Max Concentration:

Outdoor: 2.96 ppm.

Indoor: 0.858 ppm. Note: Indoor graph is shown with a dotted line. 
Title of Próject: EPHA for THM

Subject Concentration Anaiysis for THM Hazardous Chemicals

Computer N.E. Baucom B. K. Tharakan Date 09/16/96 1-RD-51 ADVERSE / SAE

SITE DATA. INFORMATION: Location: 'AIKEN, SOUTH CAROLINA Building Air Exchanges Per Hour: 0.44 (Sheltered single storied) Time: June 30, 19982359 hours EDT (User specified)

CHEMICAL INFORMATION:

Chemical Name: TRIETHYLAMINE Molecular Weight: $101.19 \mathrm{~kg} / \mathrm{kmol}$ TLV-TWA: $10 \mathrm{ppm}$ IDLH: $1000 \mathrm{ppm}$ Footprint Level of Concern: $3 \mathrm{ppm}$ Boiling Point: $88.77^{\circ} \mathrm{C}$ Vapor Pressure at Ambient Temperature: 0.11 atm Ambient Saturation Concentration: $110,492 \mathrm{ppm}$ or $11.0 \%$

ATMOSPHERIC INFORMATION: (MANUAL INPUT OF DATA) Wind: 1.7 meters/sec from $90^{\circ}$ true at 2 meters Inversion Height: 200 meters Stability Class: E Relative Humidity: 508 Cloud Cover: 1 tenths

SOURCE STRENGTH INFORMATION: Puddle Area: 20 square feet Average Puddle Depth: 1 centimeters Soil Type: Default Ground Temperature: $29^{\circ} \mathrm{C}$ Initial Puddle Temperature: Ground temperature Release Duration: ALOHA limited the duration to 1 hour Max Computed Release Rate: 254 grams/min

Max Average Sustained Release Rate: 215 grams/min (averaged over a minute or more)

Total Amount Released: 10.8 kilograms

Air Temperature: $29^{\circ} \mathrm{C}$

Ground Roughness: Urban or forest

TIME DEPENDENT INFORMATION:

Concentration Estimates at the point:

Downwind: 100 meters

off Centerline: 0 meters

Max Concentration:

outdoor: $2.87 \mathrm{ppm}$

Indoor: $0.847 \mathrm{ppm}$

Note: Indoor graph is shown with a dotted line.
Reviewed by S. A. Henderson Works Calculation 8

Sheet No. 142 of 314 
Title of Project EPHA for THM

Subject Concentration Analysis for THM Hazardous Chemicals

Computer N.E. Baucom B. K. Tharakan Date 09/16/96

1-RD-51 ADVERSE MNX DIST SEG 1

\section{SITE DATA INEORMATION:}

Location: AIKEN, SOUTH CAROLINA

Building 'Air Exchanges Per Hour: 0.44 (Sheltered single storied)

Time: June 30, 19982359 hours EDT (User specified)

CHEMICAL INFORMATION:

Chemical Name: TRIETHYLAMINE

Molecular Weight: $101.19 \mathrm{~kg} / \mathrm{kmol}$

TLV-TWA: $10 \mathrm{ppm}$ IDLH: $1000 \mathrm{ppm}$

Footprint Level of Concern: $3 \mathrm{ppm}$

Boiling Point: $88.77^{\circ} \mathrm{C}$

Vapor Pressure at Ambient Temperature: 0.11 atm

Ambient Saturation Concentration: 110,492 ppm or 11.08

ATMOSPHERIC INFORMATION: (MANUAL INPUT OF DATA)

Wind: 1.7 meters/sec from $90^{\circ}$ true at 2 meters

Inversion Height: 200 meters

Stability Class: E

Relative Humidity: $50 \%$

Air Temperature: $29^{\circ} \mathrm{C}$

Cloud Cover: 1 tenths
Reviewed by S.A. Henderson

Works Calculation 8

Sheet No. 143 of 314

SOURCE STRENGTH INFORMATION:

Pudale Area: 24420 square feet

Average Pudale Depth: 1 centimeters

Soil Type: Default

Ground Temperature: $29^{\circ} \mathrm{C}$

Initial Pudale Temperature: Ground termerature

Release Duration: ALOHA limited the duration to 1 hour

Max Computed Release Rate: 218 kilograms/min

Max Average Sustained Release Rate: $192 \mathrm{kilograms/min}$ (averaged over a minute or more)

Total Amount Released: 9,932 kilograms

FOOTPRINT INFORMATION:

Model Run: Heavy Gas

User-specified LOC: $3 \mathrm{ppm}$

Max Threat Zone for LOC: 5.3 kilometers 
Title of Project EPHA for THM

Reviewed by S. A. Henderson

Subject Concentration Analysis for THM Hazardous Chemicals

Works Calculation 8

Computer N.E. Baucom B. K. Tharakan Date 09/16/96

1-RD-51 ADVERSE UNX DIST SEG 2

SITE DATA INFORMATION:

LOCation: AIREN, SOUTH CAROLINA

Building Air Exchanges Per Hour: 0.44 (Sheltered single storied)

Time: June 30, 19982359 hours EDT (User specified)

CHEMICAI INFORMATION:

Chemical Name: TRIETHYLAMINE

Molecular Weight: $101.19 \mathrm{~kg} / \mathrm{kmol}$

TLV-TWA: $10 \mathrm{ppm}$ IDLH: $1000 \mathrm{ppm}$

Footprint Level of Concern: $3 \mathrm{ppm}$

Boiling Point: $88.77^{\circ} \mathrm{C}$

Vapor Pressure at Ambient Temperature: 0.11 atm

Ambient Saturation Concentration: $110,492 \mathrm{ppm}$ or 11.08

ATMOSPHERIC INFORMATION: (MANUAL INPUT OF DATA)

Wind: 1.7 meters $/ \mathrm{sec}$ from $90^{\circ}$ true at 2 meters

Inversion Height: 200 meters

Stability Class: $E$

Air Temperature: $29^{\circ} \mathrm{C}$

Relative Humidity: $50 \%$

Cloud Cover: 1 tenths

Ground Roughness: Urban or forest

SOURCE STRENGTH INEORMATION:

Puddle Area: 40700 square feet

Average Puddle Depth: 1 centimeters

Soil Type: Default Ground Temperature: $29^{\circ} \mathrm{C}$

Initial Puddle Temperature: Ground temperature

Release Duration: ALOHA limited the duration to 1 hour

Max Computed Release Rate: 355 kilograms/min

Max Average Sustained Release Rate: 313 kilograms/min (averaged over a minute or more)

Total Amount Released: 16,263 kilograms

FOOTPRINT INFORMATION:

Model Run: Heavy Gas

User-specified LOC: 3 ppm

Max Threat Zone for LOC: 6.9 kilometers 


\section{ENGINEERING COMPUTATION SHEET}

Title of Project EPHA for THM

Reviewed by S. A. Henderson

Works Calculation 8

Sheet No. 145 of 314

Computer N.E. Baucom B. K. Tharakan Date 09/16/96

1-RD-51 ADVERSE MNX DIST. BEG 3

SITE' DATA INFORMATION:

Location:'AIKEN, SOUTH CAROLINA

Building Air Exchanges Per Hour: 0.44 (Sheltered single storied)

Time: June 30, 19982359 hours EDT (User specified)

CHEMICAI, INFORMATION:

Chemical Name: TRIETHYLAMINE

Molecular Weight: $101.19 \mathrm{~kg} / \mathrm{kmol}$

TLV-TWA: $10 \mathrm{ppm}$

IDLH: $1000 \mathrm{ppm}$

Footprint Level of Concern: $3 \mathrm{ppm}$

Boiling Point: $88.77^{\circ} \mathrm{C}$

Vapor Pressure at Ambient Temperature: $0.11 \mathrm{~atm}$

Ambient Saturation Concentration: $110,492 \mathrm{ppm}$ or 11.08

ATMOSPHERIC INFORMATION: (MANUAL INPUT OF DATA)

Wind: 1.7 meters $/ \mathrm{sec}$ from $90^{\circ}$ true at 2 meters

Inversion Height: 200 meters

Stability Class: $\mathrm{E}$

Air Temperature: $29^{\circ} \mathrm{C}$

Relative Humidity: 508

Ground Roughness: Urban or forest

Cloud Cover: 1 tenths

SOURCE STRENGTH INFORMATION:

Puddle Area: 10000 square meters

Average Puddle Depth: 1 centimeters

Soil Type: Default Ground Temperature: $29^{\circ} \mathrm{C}$

Initial Puddle Temperature: Ground temperature

Release Duration: ALOHA limited the duration to 1 hour

Max Computed Release Rate: $904 \mathrm{kilograms/min}$

Max Average Sustained Release Rate: 801 kilograms/min (averaged over a minute or more)

Total Amount Released: 41,624 kilograms

FOOTPRINT INFORMATION :

Model Run: Heavy Gas

User-specified LOC: $3 \mathrm{ppm}$

Max Threat Zone for LOC: greater than 10 kilometers 
Title of Project EPHA for THM

Subject Concentration Analysis for THM Hazardous Chemicals

Computer N.E. Baucom B. K. Tharakan Date 09/16/96

1.RD-54 ADVERSE / ALERT

\section{SITE DATA INFORMATION:}

Location: AIKEN, SOUTH CAROLINA

Building Air Exchanges Per Hour: 0.44 (Sheltered single storied)

Time: June 30, 19982359 hours EDT (User specified)

\section{CHEMICAL INFORMATION:}

Chemical Name: BENZOIC ACID

Molecular Weight: $122.13 \mathrm{~kg} / \mathrm{kmol}$

TLV-TWA: -unavail- IDLH: -unavail-

Footprint Level of Concern: $75 \mathrm{mg} /(\mathrm{cu} \mathrm{m})$

Boiling Point: $249.00^{\circ} \mathrm{C}$

Freezing Point: $122.00^{\circ} \mathrm{C}$

Note: Not enough chemical data to use Heavy Gas option

ATMOSPHERIC INFORMATION: (MANUAL INPUT OF DATA)

Wind: 1.7 meters $/ \mathrm{sec}$ from $90^{\circ}$ true at 2 meters

Inversion Height: 200 meters

Stability Class: E Air Temperature: $29^{\circ} \mathrm{C}$

Relative Humidity: 50\% Ground Roughness: Urban or forest

Cloud Cover: 1 tenths

\section{SOURCE STRENGTH INFORMATION:}

Direct Source: .285 pounds

Source height: 0

Release Duration: 1 minute

Release Rate: 2.15 grams / sec

Total Amount Released: 129 grams

TIME DEPENDENT INFORMATION:

Concentration Estimates at the point:

Downwind: 30 meters

Off Centerline: 0 meters

Max Concentration:

Outdoor: $74.8 \mathrm{mg} /$ (cu m)

Indoor: $0.535 \mathrm{mg}$ ! ( cu m)

Note: Indoor graph is shown with a dotted line.
Reviewed by S. A. Henderson

Works Calculation 8

Sheet No. 146 of 314 
SITE DATA INFORMATION: Location: AIREN, SOUTH CAROLINA Building Air Exchanges Per Hour: 0.44 (Sheltered single storied) Time: June 30, 19982359 hours EDT (User specified)

CHEMICAI INFORMATION:

Chemical Name: BENZOIC ACID.

Molecular Weight: $122.13 \mathrm{~kg} / \mathrm{kmol}$

TLV-TWA: -unavail- IDLH: -unavail

Footprint Level of Concern: $75 \mathrm{mg} /(\mathrm{cu} \mathrm{m}$ )

Boiling Point: $249.00^{\circ} \mathrm{C}$

Freezing Point: $122.00^{\circ} \mathrm{C}$

Note: Not enough chemical data to use Heavy Gas option

ATMOSPHERIC INFORMATION: (MANUAL INPUT OF DATA)

Wind: 1.7 meters $/ \mathrm{sec}$ from $90^{\circ}$ true at 2 meters

Inversion Height: 200 meters

Stability Class: $\mathbf{E}$

Air Temperature: $29^{\circ} \mathrm{C}$

Relative Humidity: $50 \%$

Ground Roughness: Urban or forest

Cloud Cover: 1 tenths

SOURCE STRENGTH INFORMATION:

Direct Source: 3 pounds

Source Height: 0

Release Duration: 1 minute

Release Rate: 22.7 grams/sec

Total Amount Released: 1.36 kilograms

TIME DEPENDENT INFORMATION:

Concentration Estimates at the point:

Downwind: 100 meters

off Centerline: 0 meters

Max Concentration:

outdoor: $74.6 \mathrm{mg} /(\mathrm{cu} \mathrm{m})$

Indoor: $0.545 \mathrm{mg} /(\mathrm{cu} \mathrm{m})$

Note: Indoor graph is shown with a dotted line. 


\section{1-RD-54 ADVARSE MAX DIST SEG 1}

SITE DATA INFORMATION: Location: AIKEN, SOUTH CAROLINA

Building Air Exchanges Per Hour: 0.44 (Sheltered single storied) Time: June 30, 19982359 hours EDT (User specified)

\section{CHEMICAL INFORMATION:}

Chemical Name: BENZOIC ACID

Molecular Weight: $122.13 \mathrm{~kg} / \mathrm{kmol}$

TLV-TWA: -unavail-

IDLH: -unavail

Footprint Level of Concern: $75 \mathrm{mg} /(\mathrm{cu} \mathrm{m})$

Boiling Point: $249.00^{\circ} \mathrm{C}$

Freezing Point: $122.00^{\circ} \mathrm{C}$

Note: Not enough chemical data to use Heavy Gas option

ATMOSPHERIC INFORMATION: (MANUAL INPUT OF DATA)

Wind: 1.7 meters $/ \mathrm{sec}$ from $90^{\circ}$ true at 2 meters

Inversion Height: 200 meters

Stability Class: E

Relative Humidity: $50 \%$

Air Temperature: $29^{\circ} \mathrm{C}$

Cloud Cover: 1 tenths

Ground Roughness: Urban or forest

SOURCE STRENGTH INFORMATION:

Direct Source: 37.6 pounds

Source Height: 0

Release Duration: 1 minute

Release Rate: 284 grams/sec

Total Amount Released: 17.1 kilograms

FOOTPRINT INFORMATION:

Dispersion Module: Gaussian

User-specified LOC: $75 \mathrm{mg} /(\mathrm{cu} \mathrm{m})$

Max Threat Zone for LOC: 344 meters 
Title of Project EPHA for THM

Subject Concentration Analysis for THM Hazardous Chemicals

Computer N.E. Baucom B. K. Tharakan Date 09/16/96
Reviewed by S. A. Henderson

Works Calculation 8

Sheet No. 149 of 314

\section{1-RD-54 ADVARST wXX DIST SEC 2}

SITE DATA INFORMATION:

LOCation: AIKEN, SOUTH CAROLINA

Building Air Exchanges Per Hour: 0.44 (Sheltered single storied)

Time: June 30, 19982359 hours EDT (User specified)

CHEMICAL INFORMATION :

Chemical Name: BENZOIC ACID

Molecular Weight: $122.13 \mathrm{~kg} / \mathrm{kmol}$

TLV-TWA: -unavail- IDLH: -unavail

Footprint Level of Concern: $75 \mathrm{mg} /(\mathrm{cu} \mathrm{m})$

Boiling Point: $249.00^{\circ} \mathrm{C}$

Freezing Point: $122.00^{\circ} \mathrm{C}$

Note: Not enough chemical data to use Heavy Gas option

ATMOSPHERIC INFORMATION: (MANUAL INPUT OF DATA)

Wind: 1.7 meters $/ \mathrm{sec}$ from $90^{\circ}$ true at 2 meters

Inversion Height: 200 meters

Stability Class: E

Air Temperature: $29^{\circ} \mathrm{C}$

Relative Humidity: $50 \%$

Ground Roughness: Urban or forest

Cloud Cover: 1 tenths

SOURCE STRENGTH INFORMATION:

Direct Source: 135 pounds Source Height: 0

Release Duration: 1 minute

Release Rate: 1.02 kilograms/sec

Total Amount Released: 61.2 kilograms

FOOTPRINT INFORMATION:

Dispersion Module: Gaussian

User-specified LOC: $75 \mathrm{mg} /(\mathrm{cu} \mathrm{m})$

Max Threat Zone for LOC: 580 meters 
Title of Project EPHA for THM

Subject Concentration Anahysis for THM Hazardous Chemicals

Computer N.E. Baucom B. K. Tharakan Date 09/16/96

1-RD-54 ADVARSE BAXX DIST SEG 3

SITE DATA INEORMATION:

Location: "AIKEN, SOUTH CAROLINA

Building Air Exchanges Per Hour: 0.44 (Sheltered single-storied)

Time: June 30, 19982359 hours EDT (User specified)

CHEMICAL INFORMATION:

Chemical Name: BENZOIC ACID

Molecular Weight: $122.13 \mathrm{~kg} / \mathrm{kmol}$

TLV-TWA: -unavail- IDLH: -unavail

Footprint Level of Concern: $75 \mathrm{mg} /(\mathrm{cu} \mathrm{m})$

Boiling Point: $249.00^{\circ} \mathrm{C}$

Freezing Point: $122.00^{\circ} \mathrm{C}$

Note: Not enough chemical data to use Heavy Gas option

ATMOSPHERIC INFORMATION: (MANUAL INPUT OF DATA)

Wind: 1.7 meters $/ \mathrm{sec}$ from $90^{\circ}$ true at 2 meters

Inversion Height: 200 meters

Stability Class: E

Relative Humidity: $50 \%$

Air Temperature: $29^{\circ} \mathrm{C}$

Cloud Cover: 1 tenths

SOURCE STRENGTH INFORMATION:

Direct Source: 263 pounds Source Height: 0

Release Duration: 1 minute

Release Rate: $1.99 \mathrm{kilograms} / \mathrm{sec}$

Total Amount Released: 119 kilograms

FOOTPRINT INFORMATION:

Dispersion Module: Gaussian

User-specified LOC: $75 \mathrm{mg} /(\mathrm{cu} \mathrm{m}$ )

Max Threat Zone for LOC: 755 meters
Reviewed by S. A. Henderson

Works Calculation 8

Sheet No. 150 of 314 


\section{ENGINEERING COMPUTATION SHEET}

Title of Project EPHA for THM

Reviewed by S. A. Henderson

Works Calculation 8

Sheet No. 151 of 314

Computer N.E. Baucom B. K. Tharakan Date 09/16/96

I-RD-56 ADVERSE / AIERT

SITE DATA INFORMATION:

Location:: AIKEN, SOUTH CAROLINA

Building Air Exchanges Per Hour: 0.44 (Sheltered single storied)

Time: June 30, 19982359 hours EDT (User specified)

CHEMICAL INFORMATION:

Chemical Name: SILICA, CRYSTALLINE QUARTZ (RESP. DUST)

Molecular Weight: $60.09 \mathrm{~kg} / \mathrm{kmol}$

TLV-TWA: -unavail- IDLH: -unavail

Footprint Level of Concern: $0.5 \mathrm{mg} /(\mathrm{cu} \mathrm{m})$

Boiling Point: -unavail

Note: Not enough chemical data to use Heavy Gas option

ATMOSPHERIC INFORMATION: (MANUAL INPUT OF DATA)

Wind: 1.7 meters $/ \mathrm{sec}$ from $90^{\circ}$ true at 2 meters

Inversion Height: 200 meters

Stability Class: E

Relative Humidity: $50 \%$

Air Temperature: $29^{\circ} \mathrm{C}$

Cloud Cover: 1 tenths

Ground Roughness: Urban or forest

SOURCE STRENGTH INFORMATION:

Direct Source: .0019 pounds

Source Height: 0

Release Duration: 1 minute

Release Rate: 0.0144 grams/sec

Total Amount Released: 0.86 grams

Air Temperature: $29^{\circ} \mathrm{C}$

Ground Roughness: Urban or forest

TIME DEPENDENT INFORMATION:

Concentration Estimates at the point:

Downwind: $\quad 30$ meters

off Centerline: 0 meters

Max Concentration:

Outdoor: $0.499 \mathrm{mg} /$ (cu m)

Indoor: $0.00357 \mathrm{mg} /(\mathrm{cu} \mathrm{m})$

Note: Indoor graph is shown with a dotted line. 
Title of Project EPHA for THM

Subject Concentration Analysis for THM. Hazardous Chemicals

Computer N.E. Baucom B. K. Tharakan Date 09/16/96

1-RD-56 ADVERSI SAE

\section{SITE DATA INFORMATION:} Location: - AIKEN, SOUTH CAROLINA

Building Air Exchanges Per Hour: 0.44 (Sheltered single storied) Time: June 30, 19982359 hours EDT (User specified)

CHEMICAL, INFORMATION : Chemical Name: SILICA, CRYSTALIINE QUARTZ (RESP. DUST) Molecular Weight: $60.09 \mathrm{~kg} / \mathrm{kmol}$ TLV-TWA: -unavailIDLH : -unavail Footprint Ievel of Concern: $0.5 \mathrm{mg} /(\mathrm{cu} \mathrm{m})$ Boiling Point: -unavail Note: Not enough chemical data to use Heavy Gas option

ATMOSPHERIC INFORMATION: (MANUAL INPUT OF DATA)

Wind: 1.7 meters/sec from $90^{\circ}$ true at 2 meters Inversion Height: 200 meters Stability Class: E Air Temperature: $29^{\circ} \mathrm{C}$ Relative Humidity: 508 Ground Roughness: Urban or forest cloud Cover: 1 tenths

SOURCE STRENGTH INFORMATION: Direct Source: .02 pounds Release Duration: 1 minute Source Height: 0 Release Rate: 0.151 grams/sec Total Amount Released: 9.07 grams

TIME DEPENDENT INFORMATION: Concentration Estimates at the point: Downwind: 100 meters off Centerline: 0 meters Max Concentration: outdoor: $0.498 \mathrm{mg} /(\mathrm{cu} \mathrm{m}$ ) Indoor: $0.00363 \mathrm{mg} /(\mathrm{cu} \mathrm{m}$ ) Note: Indoor graph is shown with a dotted line. 
Title of Project EPHA for THM

ENGINEERING COMPUTATION SHEET

Subject Concentration Analysis for THM Hazardous Chemicals

Computer N.E. Baucom B. K. Tharakan Date 09/16/96

1-RD-56 ADVERSE MAX DIST SEO 1

SITE DATA INFORMATION:

Location: AIKEN, SOUTH CAROLINA

Building Air Exchanges Per Hour: 0.44 (Sheltered single storied)

Time: June 30, 19982359 hours EDT (User specified)

CHEMICAL INFORMATION:

Chemical Name: SILICA, CRYSTALLINE QUARTZ (RESP. DUST)

Molecular Weight: $60.09 \mathrm{~kg} / \mathrm{kmol}$

TLV-TWA: -unavail- IDLH: -unavail

Footprint Level of Concern: $0.5 \mathrm{mg} /(\mathrm{cu} \mathrm{m})$

Boiling Point: -unavail

Note: Not enough chemical data to use Heavy Gas option

ATMOSPHERIC INFORMATION: (MANUAL INPUT OF DATA)

Wind: 1.7 meters $/ \mathrm{sec}$ from $90^{\circ}$ true at 2 meters

Inversion Height: 200 meters

Stability Class: E

Relative Humidity: $50 \%$

Air Temperature: $29^{\circ} \mathrm{C}$

Cloud Cover: 1 tenths

SOURCE STRENGTH INFORMATION:

Direct Source: 37.6 pounds

Source Height: 0

Release Duration: 1 minute

Release Rate: 284 grams/sec

Total Amount Released: 17.1 kilograms

FOOTPRINT INFORMATION:

Dispersion Module: Gaussian

User-specified LOC: $0.5 \mathrm{mg} /(\mathrm{cu} \mathrm{m}$ )

Max Threat Zone for LOC: 2.5 kilometers
Reviewed by S. A. Henderson

Works Calculation 8

Sheet No. 153 of 314
Ground Roughness : Urban or forest 
SITE DATA, INFORMATION :

Location: AIKEN, SOUTH CAROLINA

Building Air Exchanges Per Hour: 0.44 (Sheltered single storied)

Time: June 30, 19982359 hours EDT (User specified)

CHEMICAL INEORMATION:

Chemical Name: SILICA, CRYSTALLINE QUARTZ (RESP. DUST)

Molecular Weight: $60.09 \mathrm{~kg} / \mathrm{kmol}$

TLV-TWA: -unavail- IDLH: -unavail

Footprint Level of Concern: $0.5 \mathrm{mg} /(\mathrm{cu} \mathrm{m}$ )

Boiling Point: -unavail

Note: Not enough chemical data to use Heavy Gas option

ATMOSPHERIC INFORMATION: (MANUAL INPUT OF DATA)

Wind: 1.7 meters/sec from $90^{\circ}$ true at 2 meters

Inversion Height: 200 meters

Stability class: E

Air Temperature: $29^{\circ} \mathrm{C}$

Relative Humidity: 508

Ground Roughness: Urban or forest

cloud Cover: 1 tenths

SOURCE STRENGTH INFORMATION:

Direct Source: 135 pounds Source Height: 0

Release Duration: 1 minute

Release Rate: 1.02 kilograms/sec

Total Amount Released: 61.2 kilograms

FOOTPRINT INFORMATION:

Dispersion Module: Gaussian

User-specified LOC: $0.5 \mathrm{mg} /$ (cu m)

Max Threat Zone for LOC: 4.3 kilometers 


\section{ENGINEERING COMPUTATION SHEET}

Title of Project EPHA for THM

Subject Concentration Analysis for THM Hazardous Chemicals

Computer N.E. Baucom B. K. Tharakan Date 09/16/96

1-RD-56 ADVERSE MAX DIST SEO 3

SITE DATA INFORMATION:

Location: : AIKEN, SOUTH CAROLINA

Building Air Exchanges Per Hour: 0.44 (Sheltered single storied)

Time: June 30, 19982359 hours EDT (User specified)

CHEMICAL INFORMATION:

Chemical Name: SILICA, CRYSTALLINE QUARTZ (RESP. DUST)

Molecular Weight: $60.09 \mathrm{~kg} / \mathrm{kmol}$

TLV-TWA: -unavail- IDLH: -unavail

Footprint Level of Concern: $0.5 \mathrm{mg} /(\mathrm{cu} \mathrm{m})$

Boiling Point: -unavail

Note: Not enough chemical data to use Heavy Gas option

ATMOSPHERIC INFORMATION: (MANUAL INPUT OF DATA)

Wind: 1.7 meters $/ \mathrm{sec}$ from $90^{\circ}$ true at 2 meters

Inversion Height: 200 meters

Stability Class: E

Relative Humidity: 508

Air Temperature: $29^{\circ} \mathrm{C}$

Cloud Cover: 1 tenths

SOURCE STRENGTH INFORMATION:

Direct Source: 263 pounds

Release Duration: 1 minute

Release Rate: 1.99 kilograms/sec

Total Amount Released: 119 kilograms

FOOTPRINT INFORMATION :

Dispersion Module: Gaussian

User-specified LOC: $0.5 \mathrm{mg} /(\mathrm{cu} \mathrm{m})$

Max Threat Zone for LOC: 5.8 kilometers
Reviewed by S. A. Henderson

Works Calculation 8

Sheet No. 155 of 314 
Title of Project EPHA for THM

\section{ENGINEERING COMPUTATION SHEET}

Subject Concentration Analysis for THM Hazardous Chemicals

Computer N.E. Baucom B. K. Tharakan Date 09/16/96

1-RD-57 ADVERSE ARERT

SITE DATA INFORMATION:

Location:'AIREN, SOUTH CAROLINA

Building Air Exchanges Per Hour: 0.44 (Sheltered single storied)

Time: June 30, 19982359 hours EDT (User specified)

CHEMICAL INFORMATION:

Chemical Name: SODIUM SULFHYDRATE

Molecular Weight: $56.06 \mathrm{~kg} / \mathrm{kmol}$

TLV-TWA: -unavail-

IDLH: -unavail

Footprint Level of Concern: $4 \mathrm{mg} /(\mathrm{cu} \mathrm{m})$

Boiling Point: -unavail

Freezing Point: $350.00^{\circ} \mathrm{C}$

Note: Not enough chemical data to use Heavy Gas option

ATMOSPHERIC INFORMATION: (MANUAL INPUT OF DATA)

Wind: 1.7 meters $/ \mathrm{sec}$ from $90^{\circ}$ true at 2 meters

Inversion Height: 200 meters

Stability Class: E

Relative Humidity: $50 \%$

Cloud Cover: 1 tenths

Air Temperature: $29^{\circ} \mathrm{C}$

Ground Roughness: Urban or forest

SOURCE STRENGTH INFORMATION:

Direct Source: .015 pounds

Source Height: 0

Release Duration: 1 minute

Release Rate: 0.113 grams/sec

Total Amount Released: 6.80 grams

TIME DEPENDENT INFORMATION:

Concentration Estimates at the point:

Downwind: $\quad 30$ meters

off Centerline: 0 meters

Max Concentration:

Outdoor: $3.94 \mathrm{mg} /(\mathrm{cu} \mathrm{m})$

Indoor: $0.0282 \mathrm{mg} /(\mathrm{cu} \mathrm{m})$

Note: Indoor graph is shown with a dotted line. 
Title of Project EPHA for THM

Subject Concentration Analysis for THM Hazardous Chemicals

Computer N.E. Baucom B. K. Tharakan 'Date 09/16/96

1-RD-57 ADVERSE SAE

\section{SITE DATA INFORMATION:}

Location: "AIKEN, SOUTH CAROLINA

Building Air Exchanges Per Hour: 0.44 (Sheltered single storied)

Time: June 30, 19982359 hours EDT (User specified)

CHEMICAL INFORMATION:

Chemical Name: SODIUM SULFHYDRATE

Molecular Weight: $56.06 \mathrm{~kg} / \mathrm{kmol}$

TLV-TWA: -unavail-

IDLH : -unavail

Footprint Level of Concern: $4 \mathrm{mg} /(\mathrm{cu} \mathrm{m})$

Boiling Point: -unavail

Freezing Point: $350.00^{\circ} \mathrm{C}$

Note: Not enough chemical data to use Heavy Gas option

ATMOSPHERIC INFORMATION: (MANUAL INPUT OF DATA)

Wind: 1.7 meters $/ \mathrm{sec}$ from $90^{\circ}$ true at 2 meters

Inversion Height: 200 meters

Stability Class: E

Air Temperature: $29^{\circ} \mathrm{C}$

Relative Humidity: 508

Cloud Cover: 1 tenths
Reviewed by S.A. Henderson

Works Calculation 8

Sheet No. 157 of 314

SOURCE STRENGTH INFORMATION:

Direct Source: .16 pounds Source Height: 0

Release Duration: 1 minute

Release Rate: 1.21 grams/sec

Total Amount Released: 72.6 grams

TIME DEPENDENT INFORMATION:

Concentration Estimates at the point:

Downwind: $\quad 100$ meters

off Centerline: 0 meters

Max Concentration:

Outdoor: $3.98 \mathrm{mg} /(\mathrm{cu} \mathrm{m})$

Indoor: $0.0291 \mathrm{mg} /(\mathrm{cu} \mathrm{m})$

Note: Indoor graph is shown with a dotted line. 
Title of Project EPHA for THM

\section{ENGINEERING COMPUTATION SHEET}

Subject Concentration Analysis for THM Hazardous Chemicals

Computer N.E. Baucom B. K. Tharakan Date 09/16/96

1-RD-57 ADVERSE / MAX DIST SEO 1
Reviewed by S.A. Henderson

Works Calculation 8

Sheet No. 158 of 314

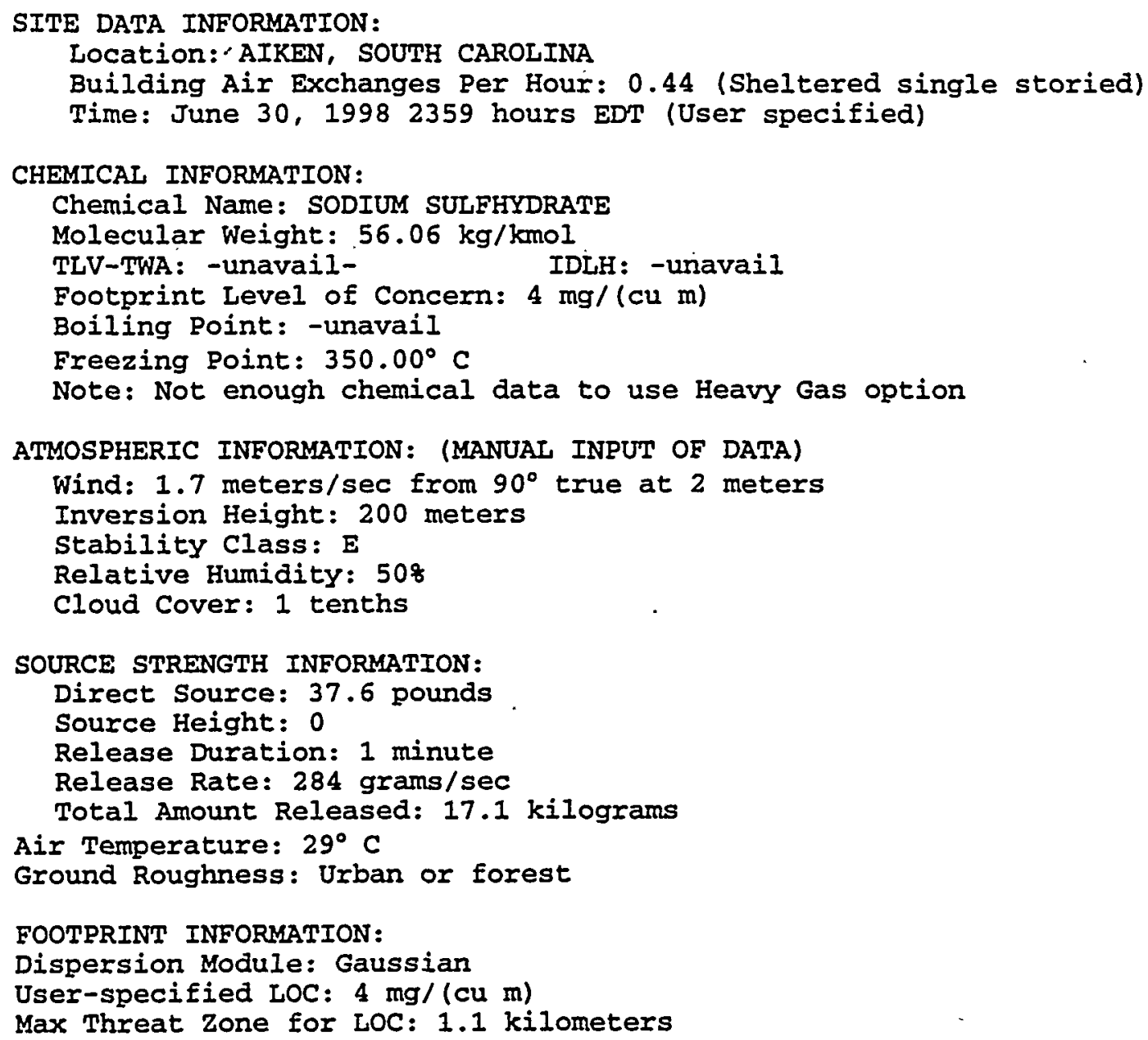

SOURCE STRENGTH INFORMATION:

Direct Source: 37.6 pounds Source Height: 0

Release Duration: 1 minute Release Rate: 284 grams/sec Total Amount Released: 17.1 kilograms

Air Temperature: $29^{\circ} \mathrm{C}$

Ground Roughness: Urban or forest

FOOTPRINT INFORMATION:

Dispersion Module: Gaussian

User-specified LOC: $4 \mathrm{mg} /(\mathrm{cu} \mathrm{m}$ )

Max Threat Zone for LOC: 1.1 kilometers 
Title of Project EPHA for THM

Subject Concentration Analysis for THM Hazardous Chemicals

Computer N.E, Baucom B. K. Tharakan Date 09/16/96

1-RD.57 ADVERSE / MAX DIST SEG 2

SITE DATA INFORMATION:

Location: AIKEN, SOUTH CAROLINA

Building Air Exchanges Per Hour: 0.44 (Sheltered single storied)

Time: June 30, 19982359 hours EDT (User specified)

\section{CHEMICAL INFORMATION:}

Chemical Name: SODIUM SULFFYDRATE

Molecular Weight: $56.06 \mathrm{~kg} / \mathrm{kmol}$

TLV-TWA: -unavail-

IDLH: -unavail

Footprint Level of Concern: $4 \mathrm{mg} /(\mathrm{cu} \mathrm{m})$

Boiling Point: -unavail

Freezing Point: $350.00^{\circ} \mathrm{C}$

Note: Not enough chemical data to use Heavy Gas option

\section{ATMOSPHERIC INFORMATION: (MANUAL INPUT OF DATA)}

Wind: 1.7 meters $/ \mathrm{sec}$ from $90^{\circ}$ true at 2 meters

Inversion Height: 200 meters

Stability Class: E

Relative Humidity: 50\%

Cloud Cover: 1 tenths

Air Temperature: $29^{\circ}$

Ground Roughness: Urban or forest

\section{SOURCE STRENGTH INFORMATION:}

Direct Source: 135 pounds

Release Duration: 1 minute

Release Rate: 1.02 kilograms/sec

Total Amount Released: 61.2 kilograms

FOOTPRINT INFORMATION:

Dispersion Module: Gaussian

User-specified LOC: $4 \mathrm{mg} /(\mathrm{cu} \mathrm{m})$

Max Threat Zone for LOC: 1.8 kilometers
Reviewed by S. A. Henderson

Works Calculation 8

Sheet No. 159 of 314 


\section{ENGINEERING COMPUTATION SHEET}

Title of Project EPHA for THM

Reviewed by S. A. Henderson

Works Caiculation 8

Sheet No. 160 of 314

Computer N.E. Baucom B. K. Tharakan Date 09/16/96

1-RD-57 ADVERSE MAX DIST SEG 3

SITE DATA INFORMATION:

Location: : AIKEN, SOUTH CAROLINA

Building Air Exchanges Per Hour: 0.44 (Sheltered single storied)

Time: June 30, 19982359 hours EDT (User specified)

CHEMICAL INFORMATION:

Chemical Name: SODIUM SULFHYDRATE

Molecular Weight: $56.06 \mathrm{~kg} / \mathrm{kmol}$

TLV-TWA: -unavail- IDLH: -unavail

Footprint Level of Concern: $4 \mathrm{mg} /(\mathrm{cu} \mathrm{m})$

Boiling Point: -unavail

Freezing Point: $350.00^{\circ} \mathrm{C}$

Note: Not enough chemical data to use Heavy Gas option

ATMOSPHERIC INFORMATION: (MANUAL INPUT OF DATA)

Wind: 1.7 meters $/ \mathrm{sec}$ from $90^{\circ}$ true at 2 meters

Inversion Height: 200 meters

Stability Class: E

Air Temperature: $29^{\circ} \mathrm{C}$

Relative Humidity: 508

Ground Roughness: Urban or forest

Cloud Cover: 1 tenths

SOURCE STRENGTH INFORMATION:

Direct Source: 263 pounds Source Height: 0

Release Duration: 1 minute

Release Rate: $1.99 \mathrm{kilograms} / \mathrm{sec}$

Total Amount Released: 119 kilograms

FOOTPRINT INFORMATION:

Dispersion Module: Gaussian

User-specified LOC: $4 \mathrm{mg} /(\mathrm{cu} \mathrm{m})$

Max Threat Zone for LOC: 2.4 kilometers 
Title of Project EPHA for THM

Subject Concentration Analysis for THM Hazardous Chemicals

Computer N.E. Baucom B. K. Tharakan Date 09/16/96
Reviewed by S. A. Henderson Works Calculation 8

Sheet No. 161 of 314

\section{1-RD-2 AVKRAGE / AIERT}

SITE DATA INFORMATION: Location: AIKEN, SOUTH CAROLINA

Building Air Exchanges Per Hour: 60 (User specified)

Time: June 30, 19981200 hours EDT (User specified)

CHEMICAL INFORMATION :

Chemical Name: ACETIC ACID

Molecular Weight: $60.05 \mathrm{~kg} / \mathrm{kmol}$

TLV-TWA: $10 \mathrm{ppm}$ IDLH: $50 \mathrm{ppm}$

Footprint Level of Concern: $35 \mathrm{ppm}$

Boiling Point: $117.90^{\circ} \mathrm{C}$

Vapor Pressure at Ambient Temperature: $0.021 \mathrm{~atm}$

Ambient Saturation Concentration: 20,919 ppm or 2.098

ATMOSPHERIC INFORMATION: (MANUAL INPUT OF DATA)

Wind: 2.5 meters/sec from $90^{\circ}$ true at 2 meters

Inversion Height: 500 meters

Stability class: C (user override)

Air Temperature: $25^{\circ} \mathrm{C}$

Relative Humidity: 50\% Ground Roughness: Urban or forest

Cloud Cover: 8 tenths

SOURCE STRENGTH INFORMATION:

Puddle Area: 895 square feet

Average Puddle Depth: 1 centimeters

Soil Type: Default Ground Temperature: $25^{\circ} \mathrm{C}$

Initial Puddle Temperature: Ground temperature

Release Duration: ALOHA limited the duration to $i$ hour

Max Computed Release Rate: $1.64 \mathrm{kilograms/min}$

Max Average Sustained Release Rate: $1.63 \mathrm{kilograms} / \mathrm{min}$ (averaged over a minute or more)

Total Amount Released: 93.8 kilograms

FOOTPRINT INFORMATION:

Dispersion Module: Gaussian

User-specified LOC: 35 ppm

Max Threat Zone for LOC: 30 meters

Max Threat Zone for IDLH: 22 meters

Note: Footprint was not drawn because effects of near-field patchiness make dispersion predictions unreliable for short distances.

TIME DEPENDENT INFORMATION:

Concentration Estimates at the point:

Downwind: $\quad 30$ meters

off Centerline: 0 meters

Max Concentration:

Outdoor: $35 \mathrm{ppm}$

Indoor: $35 \mathrm{ppm}$

Note: Indoor graph is shown with a dotted line. 
Title of Project EPHA for THM

Reviewed by S. A. Henderson

Subject Concentration Analysis for THM Hazardous Chemicals

Works Calculation 8

Computer N.E. Baucom B. K. Tharakan Date 09/16/96

1-RD-2 AVERAGE / SAE

Sheet No. 162 of 314

\section{SITE DATA INFORMATION:}

Location: AIREN, SOUTH CAROLINA

Building Air Exchanges Per Hour: 60 (User specified)

Time: June 30, 19981200 hours EDT (User specified)

CHEMICAL INFORMATION :

Chemical Name: ACETIC ACID

Molecular Weight: $60.05 \mathrm{~kg} / \mathrm{kmol}$

TLV-TWA: $10 \mathrm{ppm}$ IDLH: $50 \mathrm{ppm}$

Footprint Level of Concern: 35 ppm

Boiling Point: $117.90^{\circ} \mathrm{C}$

Vapor Pressure at Ambient Temperature: 0.021 atm

Ambient Saturation Concentration: 20,919 ppm or 2.098

ATMOSPHERIC INFORMATION: (MANUAC INPUT OF DATA)

Wind: 2.5 meters/sec from $90^{\circ}$ true at 2 meters

Inversion Height: 500 meters

Stability Class: C (user override)

Air Temperature: $25^{\circ} \mathrm{C}$

Relative Humidity: 50\% Ground Roughness: Urban or forest

cloud Cover: 8 tenths

SOURCE STRENGTH INFORMATION:

Puadle Area: 11850 square feet

Average Puddle Depth: 1 centimeters

Soil Type: Default Ground Temperature: $25^{\circ} \mathrm{C}$

Initial puddle Temperature: Ground temperature

Release Duration: ALOHA limited the duration to 1 hour

Max Computed Release Rate: $19.6 \mathrm{kilograms} / \mathrm{min}$

Max 4.verage Sustained Release Rate: $19.4 \mathrm{kilograms} / \mathrm{min}$

(averaged over a minute or more)

Total Amount Released: 1,109 kilograms

FOOTPRINT INFORMATION:

Dispersion Module: Gaussian

User-specified LOC: $35 \mathrm{ppm}$

Max Threat Zone for LOC: 100 meters

Max Threat Zone for IDLH: 72 meters

Note: Footprint was not drawn because effects of near-field patchiness make dispersion predictions unreliable for short distances.

TIME DEPENDENT INFORMATION:

Concentration Estimates at the point:

Downwind: $\quad 100$ meters

Off Centerline: 0 meters

Max Concentration:

Outdoor: $35 \mathrm{ppm}$

Indoor: $35 \mathrm{ppm}$

Note: Indoor graph is shown with a dotted line. 


\section{ENGINEERING COMPUTATION SHEET}

Title of Project EPHA for THM

Reviewed by S. A. Henderson

Works Calculation 8

Sheot No. 163 of 314

Computer N.E. Baucom B. K. Tharakan Date 09/16/96

Subject Concentration Analysis for THM Hazardous Chemicals

1-RD-2 AVARAOT / MAX DIST SEO 1

SITE DATA INEORMATION:

LOCation: AIKEN, SOUTH CAROLINA

Building Air Exchanges Per Hour: 60 (User specified)

Time: June 30, 19981200 hours EDT (User specified)

CHEMICAL INFORMATION:

Chemical Name: ACETIC ACID

Molecular Weight: $60.05 \mathrm{~kg} / \mathrm{kmol}$

TLV-TWA: $10 \mathrm{ppm}$ IDLH: $50 \mathrm{ppm}$

Footprint Level of Concern: 35 ppm

Boiling Point: $117.90^{\circ} \mathrm{C}$

Vapor Pressure at Ambient Temperature: $0.021 \mathrm{~atm}$

Ambient Saturation Concentration: 20,919 ppm or 2.098

ATMOSPHERIC INFORMATION: (MANUAL INPUT OF DATA)

Wind: 2.5 meters/sec from $90^{\circ}$ true at 2 meters

Inversion Height: 500 meters

Stability Class: C (user override)

Air Temperature: $25^{\circ} \mathrm{C}$

Relative Humidity: 508 Ground Roughness: Urban or forest

cloud cover: 8 tenths

SOURCE STRENGTH INFORMATION:

Puddle Area: 24420 square feet

Average Puddle Depth: 1 centimeters

Soil Type: Default Ground Temperature: $25^{\circ} \mathrm{C}$

Initial Puddle Temperature: Ground temperature

Release Duration: ALOHA limited the duration to 1 hour

Max Computed Release Rate: 39.2 kilograms/min

Max Average Sustained Release Rate: $38.9 \mathrm{kilograms} / \mathrm{min}$

(averaged over a minute or more)

Total Amount Released: 2,218 kilograms

FOOTPRINT INFORMATION:

Dispersion Module: Gaussian

User-specified IOC: $35 \mathrm{ppm}$

Max Threat zone for LOC: 140 meters

Max Threat Zone for IDLH: 100 meters 


\section{ENGINEERING COMPUTATION SHEET}

Title of Project EPHA for THM

Subject Concentration Analysis for THM Hazardous Chemicals

Computer N.E. Baucom B. K. Tharakan Date 09/16/96

1-RD-2 AVERAOL / MaX DIST BEO 2

SITE DATA INFORMATION:

Location: AIREN, SOUTH CAROLINA

Building Air Exchanges Per Hour: 60 (User specified)

Time: June 30, 19981200 hours EDT (User specified)

CHEMICAL INFORMATION:

Chemical Name: ACETIC ACID

Molecular Weight: $60.05 \mathrm{~kg} / \mathrm{kmol}$

TLV-TWA: $10 \mathrm{ppm}$

Footprint Level of Concern: $35 \mathrm{ppm}$

Boiling Point: $117.90^{\circ} \mathrm{C}$

Vapor Pressure at Ambient Temperature: $0.021 \mathrm{~atm}$

Ambient Saturation Concentration: $20,919 \mathrm{ppm}$ or 2.098

ATMOSPHERIC INFORMATION: (MANUAL INPUT OF DATA)

Wind: 2.5 meters $/ \mathrm{sec}$ from $90^{\circ}$ true at 2 meters

Inversion Height: 500 meters

stability Class: C (user override)

Air Temperature: $25^{\circ} \mathrm{C}$

Relative Humidity: 508 Ground Roughness: Urban or forest

cloud Cover: 8 tenths

SOURCE STRENGTH INFORMATION:

Puddle Area: 40700 square feet

Average Puddie Depth: 1 centimeters

Soil Type: DefaultGround Temperature: $25^{\circ} \mathrm{C}$

Initial puddle Temperature: Ground temperature

ReleaseDuration: ALOFA limited the duration to 1 hour

Max Computed Release Rate: 64.1 kilograms/min

MaxAverage Sustained Release Rate: $63.7 \mathrm{kilograms} / \mathrm{min}$

(averaged over a minute or more)

Total Amount Released: 3,622 kilograms

FOOTPRINT INFORMATION:

Dispersion Module: Gaussian

User-specified LOC: 35 ppm

Max Threat Zone for LOC: 178 meters

Max Threat Zone for IDLH: 127 meters
Reviewed by S. A. Henderson

Works Calculation 8

Sheet No. 164 of 314 
Title of Project EPHA for THM

Reviewed by S. A. Henderson

Subject Concentration Analysis for THM Hazardous Chemicals

Works Calculation 8

Computer N.E. Baucom B. K. Tharakan Date 09/16/96

1-RD-3 AVRRACI / ATERT

Sheet No. 165 of 314

SITE DATA INEORMATION:

LOCation: AIKEN, SOUTH CAROLINA

Building Air Exchanges Per Hour: 60 (User specified)

Time: June 30, 19981200 hours EDT (User specified)

CHEMICAI INFORMATION:

Chemical Name: ACETONITRILE

Molecular Weight: $41.05 \mathrm{~kg} / \mathrm{kmol}$

TLV-TWA: 40 ppm IDLH: $500 \mathrm{ppm}$

Footprint Level of Concern: $60 \mathrm{ppm}$

Boiling Point: $81.60^{\circ} \mathrm{C}$

Vapor Pressure at Ambient Temperature: $0.12 \mathrm{~atm}$

Ambient Saturation Concentration: $121,986 \mathrm{ppm}$ or 12.28

ATMOSPHERIC INFORMATION: (MANUAL INPUT OF DATA)

Wind: 2.5 meters/sec from $90^{\circ}$ true at 2 meters

Inversion Height: 500 meters

Stability Class: C (user override)

Air Temperature: $25^{\circ} \mathrm{C}$

Relative Humidity: 508

Ground Roughness: Urban or forest

Cloud Cover: 8 tenths

SOURCE STRENGTH INFORMATION:

Puddle Area: 177 square feet

Average Puddle Depth: 1 centimeters

Soil Type: Default Ground Temperature: $25^{\circ} \mathrm{C}$

Initial Puddle Temperature: Ground temperature

Release Duration: ALOHA Iimited the duration to 1 hour

Max Computed Release Rate: 1.32 kilograms/min

Max Average Sustained Release Rate: 1.31 kilograms/min

(averaged over a minute or more)

Total Amount Released: 61.6 kilograms

FOOTPRINT INFORMATION: Dispersion Module: Gaussian

User-specified LOC: $60 \mathrm{ppm}$

Max Threat Zone for LOC: 30 meters

Max Threat Zone for IDLH: less than 10 meters ( 10.9 yards)

Note: Footprint was not drawn because effects of near-field patchiness make dispersion predictions unreliable for short distances.

TIME DEPENDENT INFORMATION:

Concentration Estimates at the point:

Downwind: $\quad 30$ meters

off Centerline: 0 meters

Max Concentration:

Outdoor: $59.8 \mathrm{ppm}$

Indoor: $51 \mathrm{ppm}$

Note: Indoor graph is shown with a dotted line. 
Title of Project EPHA for THM

Subject Concentration Analysis for THM Hazardous Chemicals

Computer N.E. Baucom B. K. Tharakan Date 09/16/96
Reviewed by S.. A. Henderson

Works Calculation 8

Shoet No. 166 of 314

\section{1-RD-3 AVERACI / BAx}

\section{SITE DATA INFORMATION:}

LOCation: AIKEN, SOUTH CAROLINA

Building Air Exchanges Per Hour: 60 (User specified)

Time: June 30, 19981200 hours EDT (User specified)

CHEMICAI INFORMATION:

Chemical Name: ACETONITRILE

Molecular Weight: $41.05 \mathrm{~kg} / \mathrm{kmol}$

TLV-TWA : $40 \mathrm{ppm}$

Footprint Level of.Concern: $60 \mathrm{ppm}$

IDLH: $500 \mathrm{ppm}$

Boiling point: $81.60^{\circ} \mathrm{C}$

Vapor Pressure at Ambient Temperature: $0.12 \mathrm{~atm}$

Ambient Saturation Concentration: $121,986 \mathrm{ppm}$ or 12.28

ATMOSPHERIC INFORMATION: (MANUAL INPUT OF DATA)

Wind: 2.5 meters $/ \mathrm{sec}$ from $90^{\circ}$ true at 2 meters

Inversion Height: 500 meters

Stability class: C (user override)

Air Temperature: $25^{\circ} \mathrm{C}$

Relative Humidity: 508

Cloud Cover: 8 tenths

SOURCE STRENGTH INFORMATION:

Ground Roughness: Urban or forest

Puddle Area: 2325 square feet

Average Pudale Depth: 1 centimeters

Soil Type: Default Ground Temperature: $25^{\circ} \mathrm{C}$

Initial Puddle Temperature: Ground temperature

Release Duration: ALOHA limited the duration to 1 hour

Max Computed Release Rate: 14.9 kilograms/min

Max Average Sustained Release Rate:

$14.9 \mathrm{kilograms} / \mathrm{min}$

(averaged over a minute or more)

Total Amount Released: 729 kilograns

FOOTPRINT INEORMATION:

Dispersion Module: Gaussian

User-specified LOC: 60 ppm

Max Threat zone for LOC: 100 meters

Max Threat Zone for IDLH: 19 meters

Note: Footprint was not drawn because effects of near-field patchiness make dispersion predictions unreliable for short distances.

TIME DEPENDENT INFORMATION:

Concentration Estimates at the point:

Downwind: 100 meters

off Centerline: 0 meters

Max Concentration:

Outdoor: $59.9 \mathrm{ppm}$

Indoor: $52.4 \mathrm{ppm}$

Note: Indoor graph is shown with a dotted line. 
Title of Projoct EPHA for THM

Reviewed by S. A. Henderson

Subject Concentration Analysis for THM Hazardous Chemicals

Works Calculation 8

Computer N.E. Baucom B. K. Tharakan Date 09/16/96

1-RD-3 AVTRAOT / INX DIST SEO I

SITE DATA INFORMATION:

Location: AIKEN, SOUTH CAROLINA

Building Air Exchanges Per Hour: 60 (User specified)

Time: June 30, 19981200 hours EDT (User specified)

CHEMICAL INFORMATION:

Chemical Name: ACETONITRILE

Molecular Weight: $41.05 \mathrm{~kg} / \mathrm{kmol}$

TLV-TWA: $40 \mathrm{ppm}$

IDLH : $500 \mathrm{ppm}$

Footprint Level of Concern:

Boiling Point: $81.60^{\circ} \mathrm{C}$

Vapor Pressure at Ambient Temperature: $0.12 \mathrm{~atm}$

Ambient Saturation Concentration: $121,986 \mathrm{ppm}$ or 12.28

ATMOSPHERIC INFORMATION: (MANUAL INPUT OF DATA)

Wind: 2.5 meters/sec from $90^{\circ}$ true at 2 meters

Inversion Height: 500 meters

Stability class: C (user override)

Air Temperature: $25^{\circ} \mathrm{C}$

Relative Humidity: 508

Cloud Cover: 8 tenths

Ground Roughness: Urban or forest

SOURCE STRENGTH INFORMATION:

Puddle Area: 24420 square feet

Average Puddle Depth: 1 centimeters

Soil Type: Default Ground Temperature: $25^{\circ} \mathrm{C}$

Initial Pudale Temperature: Ground temperature

Release Duration: ALOHA Iimited the duration to 1 hour

Max Computed Release Rate: $138 \mathrm{kilograms/min}$

Max Average Sustained Release Rate: $138 \mathrm{kilograms/min}$

(averaged over a minute or more)

Total Amount Released: 7,024 kilograms

FOOTPRINT INFORMATION:

Dispersion Module: Gaussian

User-specified LOC: 60 ppm

Max Threat Zone for LOC: 303 meters

Max Threat Zone for IDLH: 54 meters 
Title of Project EPHA for THM

Subject Concentration Analysis for THM Hazardous Chemicals

Computer N.E. Baucom B. K. Tharakan Date 09/16/96

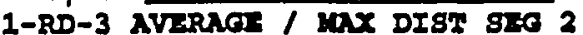

SITE DATA INEORMATION:

Location: AIKEN, SOUTH CAROLINA

Building Air Exchanges Per Hour: 60 (User specified)

Time: June 30, 19981200 hours EDT (User specified)

CHEMICAL INFORMATION:

Chemical Name: ACETONITRILE

Molecular Weight: $41.05 \mathrm{~kg} / \mathrm{kmol}$

TLV-TWA: $40 \mathrm{ppm}$

Footprint Level of Concern:

Boiling Point: $81.60^{\circ} \mathrm{C}$

Vapor Pressure at Ambient Temperature: 0.12 atm

Ambient Saturation Concentration: 121,986 ppm or $12.2 \%$

ATMOSPHERIC INFORMATION: (MANUAL INPUT OF DATA)

Wind: 2.5 meters $/ \mathrm{sec}$ from $90^{\circ}$ true at 2 meters

Inversion Height: 500 meters

Stability Class: C (user override)

Air Temperature: $25^{\circ} \mathrm{C}$

Relative Humidity: 508

Cloud Cover: 8 tenths

Ground Roughness: Urban or forest

SOURCE STRENGTH INFORMATION:

Pudale Area: 40700 square feet

Average Puddle Depth: 1 centimeters

Soil Type: Default Ground Temperature: $25^{\circ} \mathrm{C}$

Initial Puddle Temperature: Ground temperature

Release Duration: ALOHA limited the duration to 1 hour

Max Computed Release Rate: $225 \mathrm{kilograms/min}$

Max Average Sustained Release Rate: $225 \mathrm{kilograms} / \mathrm{min}$ (averaged over a minute or more)

Total Amount Released: 11,502 kilograms

FOOTPRINT INFORMATION:

Dispersion Module: Gaussian

User-specified IOC: $60 \mathrm{ppm}$

Max Threat Zone for LOC: 386 meters

Max Threat Zone for IDLH: 69 meters
IDLH: 500 ppm

$60 \mathrm{ppm}$
Reviewed by S. A. Henderson

Works Calculation 8

Sheet No. 168 of 314 
Title of Project EPHA for THM

Subject Cencentration Analysis for THM Hazardous Chemicals

Computer N.E. Baucom B. K. Tharakan Date 09/16/96

1-RD-3 AVARAG / MXX DIST SEO 3

SITE DATA INFORMATION:

Location: AIREN, SOUTH CAROLINA

Building Air Exchanges Per Hour: 60 (User specified)

Time: June 30, 19981200 hours EDT (User specified)

CHEMICAL INFORMATION :

Chemical Name: ACETONITRIIE

Molecular Weight: $41.05 \mathrm{~kg} / \mathrm{kmol}$

TLV-TWA: 40 ppm

Footprint Level of Concern:

Boiling Point: $81.60^{\circ} \mathrm{C}$

Vapor Pressure at Ambient Temperature: 0.12 atm

Ambient Saturation Concentration: 121,986 ppm or 12.28

ATMOSPHERIC INEORMATION: (MANUAI INPUT OF DATA)

Wind: 2.5 meters $/ \mathrm{sec}^{\prime}$ from $90^{\circ}$ true at 2 meters

Inversion Height: 500 meters

Stability class: C (user override)

Air Temperature: $25^{\circ} \mathrm{C}$

Relative Humidity: 508

Ground Roughness: Urban or forest

Cloud Cover: 8 tenths

SOURCE STRENGTH INFORMATION:

Puddle Area: 10000 square meters

Average Puddle Depth: 1 centimeters

Soil Type: Default

Ground Temperature: $25^{\circ}$

Initial Puddle Temperature: Ground temperature

Release Duration: AlOHA limited the duration to 1 hour

Max Computed Release Rate: 569 kilograms/min

Max Average Sustained Release Rate: 569 kilograms/min

(averaged over a minute or more)

Total Amount Released: 29,439 kilograms

FOOTPRINT INFORMATION:

Model Run: Heavy Gas

User-specified LOC: 60 ppm

Max Threat Zone for LOC: 1.6 kilometers
IDLH: $500 \mathrm{ppm}$

60 ppm
Reviewed by S. A. Henderson

Works Calculation 8

Sheet No. 169 of 314 
Subject Concentration Analysis for THM Hazardous Chemicals

\section{1-RD-4 AVERAGE / ALERT}

\section{SITE DATA INFORMATION:}

Location: AIKEN, SOUTH CAROLINA

Building Air Exchanges Per Hour: 60 (User specified)

Time: June 30, 19981200 hours EDT (User specified)

\section{CHEMICAL INFORMATION:}

Chemical Name: ALPHA-PINENE

Molecular Weight: $136.26 \mathrm{~kg} / \mathrm{kmol}$

TLV-TWA: -unavail-

IDLH: -unavail

Footprint Level of Concern: $300 \mathrm{mg} /(\mathrm{cu} \mathrm{m}$ )

Boiling Point: $155.00^{\circ} \mathrm{C}$

Vapor Pressure at Ambient Temperature: $0.0069 \mathrm{~atm}$

Ambient Saturation Concentration: $6,993 \mathrm{ppm}$ or $0.70 \%$

Note: Not enough chemical data to use Heavy Gas option

ATMOSPHERIC INFORMATION: (MANUAL INPUT OF DATA)

Wind: 2.5 meters $/ \mathrm{sec}$ from $90^{\circ}$ true at 2 meters

Inversion Height: 500 meters

Stability Class: C (user override)

Air Temperature: $25^{\circ} \mathrm{C}$

Relative Humidity: $50 \%$

Ground Roughness: Urban or forest

Cloud Cover: 8 tenths

SOURCE STRENGTH INFORMATION:

Direct Source: .0583 kilograms/sec

Source Height: 0

Release Duration: 60 minutes

Release Rate: 3.5 kilograms/min

Total Amount Released: 210 kilograms

\section{TIME DEPENDENT INFORMATION:}

Concentration Estimates at the point:

Downwind: $\quad 30$ meters

Off Centerline: 0 meters

Max Concentration:

Outdoor: $300 \mathrm{mg} /(\mathrm{cu} \mathrm{m})$

Indoor: $300 \mathrm{mg} /(\mathrm{cu} \mathrm{m})$

Note: Indoor graph is shown with a dotted line. 
Title of Project EPHA for THM

Subject Concentration Analysis for THM Hazardous Chemicals

Computer N.E. Baucom B. K. Tharakan Date 09/16/96

SPILL AREA CAICULATION FOR 1-RD-4
Reviewed by S. A. Henderson

Works Calculation 8

Sheet No. 171 of 314

I ALERT CONDITIONS

\begin{tabular}{|c|c|c|c|c|}
\hline Parameter : & Value & Dimensions & Value & $\begin{array}{l}\text { Dimensions } \\
\text { (USA) }\end{array}$ \\
\hline E (Evaporation Rate) & $5.83 \mathrm{E}-2$ & $\mathrm{~kg} / \mathrm{s}$ & & \\
\hline MW (Molecular Weight) & 136.26 & $\mathrm{~kg} / \mathrm{kg}$-mole & & \\
\hline Pv (Vapor Pressure) & 5.24 & $\mathrm{~mm} \mathrm{Hg}$ & & \\
\hline PV (Vapor Pressure) & 698.60 & $\mathrm{~Pa}$ & & \\
\hline Rg (Gas Law Constant) & 8314 & $\mathrm{~J} / \mathrm{kgmole}-\mathrm{K}$ & & \\
\hline Temperature & 25 & ${ }^{\circ} \mathrm{C}$ & & \\
\hline Volume of Spil1 & 820 & gal & & \\
\hline d (Depth of Pool) & 0.01 & $\mathrm{~m}$ & & \\
\hline A (Surface Area of Spill) & 310.4 & $m^{\wedge} 2$ & 3341 & ft 2 \\
\hline Km (Mass Transfer Coeff) & $4.89 \mathrm{E}-3$ & $\mathrm{~m} / \mathrm{s}$ & & \\
\hline $\begin{array}{l}\text { Z (Pool Diameter in wind } \\
\text { Direction) }\end{array}$ & 20 & $\mathrm{~m}$ & & \\
\hline Sc (Schmidt Number) & 1.72 & & & \\
\hline U (wind velocity) & 2.5 & $\mathrm{~m} / \mathrm{s}$ & & \\
\hline $\begin{array}{l}\text { nu (kinematic viscosity } \\
\text { of } \\
\text { air) }\end{array}$ & $1.50 \mathrm{E}-5$ & $\mathrm{~m}^{\wedge} 2 / \mathrm{sec}$ & & \\
\hline mu (viscosity of air) & $1.90 \mathrm{E}-5$ & $\mathrm{~kg} / \mathrm{m}-\mathrm{s}$ & & \\
\hline $\begin{array}{l}\text { Dm (Molecular Diffusivity } \\
\text { of } \\
\text { Solute in Air) }\end{array}$ & $8.72 E-6$ & $\mathrm{~m}^{\wedge} 2 / \mathrm{sec}$ & & \\
\hline $\begin{array}{l}\text { D_H20 " (Molecular } \\
\text { Diffusivity } \\
\text { of Water) }\end{array}$ & $2.40 \mathrm{E}-5$ & & & . \\
\hline MW_H2O & 18 & $\mathrm{~kg} / \mathrm{kg}$-mole & & \\
\hline Conversions & & & & \\
\hline T (Absolute) & 298.15 & ${ }^{\circ} \mathrm{K}$ & & \\
\hline$V$ (Volume) & 3.10 & $m^{\wedge} 3$ & & \\
\hline Evaporation Rate & 3499 & $\mathrm{~g} / \mathrm{min}$ & & \\
\hline $60 \mathrm{~min} 1 \mathrm{~b}$ released & 462.83 & & & \\
\hline
\end{tabular}


Title of Project EPHA for THM

Subject Concentration Analysis for THM Hazardous Chemicals

Computer N.E. Baucom B. K. Tharakan Date 09/16/96

1-RD-4 AVERAGE / SAE

\section{SITE DATA INFORMATION:}

Location: AIKEN, SOUTH CAROLINA

Building Air Exchanges Per Hour: 60 (User specified)

Time: June 30, 19981200 hours EDT (User specified)
Reviewed by S.A. Henderson

Works Calculation 8

Sheet No. 172 of 314

\section{CHEMICAL INFORMATION:}

Chemical Name: ALPHA-PINENE

Molecular Weight: $136.26 \mathrm{~kg} / \mathrm{kmol}$

TLV-TWA: -unavail-

IDLH: -unavail

Footprint Level of Concern: $300 \mathrm{mg} /(\mathrm{cu} \mathrm{m}$ )

Boiling Point: $155.00^{\circ} \mathrm{C}$

Vapor Pressure at Ambient Temperature: $0.0069 \mathrm{~atm}$

Ambient Saturation Concentration: 6,993 ppm or 0.70\%

Note: Not enough chemical data to use Heavy Gas option

ATMOSPHERIC INFORMATION: (MANUAL INPUT OF DATA)

Wind: 2.5 meters $/ \mathrm{sec}$ from $90^{\circ}$ true at 2 meters

Inversion Height: 500 meters

Stability Class: $C$ (user override)

Air Temperature: $25^{\circ} \mathrm{C}$

Relative Humidity: 50\%

Ground Roughness: Urban or forest

Cloud Cover: 8 tenths

SOURCE STRENGTH INFORMATION:

Direct Source: .645 kilograms/sec

Source Height: 0

Release Duration: 60 minutes

Release Rate: 38.7 kilograms/min

Total Amount Released: 2,322 kilograms

\section{,}

\section{TIME DEPENDENT INFORMATION:}

Concentration Estimates at the point:

Downwind: $\quad 100$ meters

Off Centerline: 0 meters

Max Concentration:

Outdoor: $300 \mathrm{mg} /(\mathrm{cu} \mathrm{m}$ )

Indoor: $300 \mathrm{mg} /(\mathrm{cu} \mathrm{m})$

Note: Indoor graph is shown with a dotted line. 
Title of Project EPHA for THM

Subject Concentration Analysis for THM Hazardous Chemicals

Computer N.E. Baucom B. K. Tharakan Date 09/16/96

SRIII AREA CAICULATION FOR 1-RD-4 AVERAGE / SAE CONDITIONS

\begin{tabular}{|c|c|c|c|c|}
\hline Parameter & Value & Dimensions & Value & $\begin{array}{l}\text { Dimensions } \\
\text { (USA) }\end{array}$ \\
\hline E (Evaporation Rate) & $6.45 \mathrm{E}-1$ & $\mathrm{~kg} / \mathrm{s}$ & & \\
\hline MW (Molecular Weight) & 136.26 & $\mathrm{~kg} / \mathrm{kgmole}$ & & \\
\hline Pv (Vapor Pressure) & 5.24 & $\mathrm{~mm} \mathrm{Hg}$ & & \\
\hline PV (Vapor Pressure) & 698.60 & $\mathrm{~Pa}$ & & \\
\hline Rg (Gas Law Constant) & 8314 & $\mathrm{~J} / \mathrm{kgmole}-\mathrm{K}$ & & \\
\hline Temperature & 25 & ${ }^{\circ} \mathrm{c}$ & & \\
\hline Volume of spill & 10450 & gal & & \\
\hline d (Depth of Pool) & 0.01 & $m$ & & \\
\hline A (Surface Area of Spill) & 3955.6 & $\mathrm{~m}^{\wedge} 2$ & 42582 & Et^2 \\
\hline $\mathrm{Km}$ (Mass Transfer Coeff) & $4.25 \mathrm{E}-3$ & $\mathrm{~m} / \mathrm{s}$ & & \\
\hline $\begin{array}{l}\text { Z (Pool Diameter in wind } \\
\text { Direction) }\end{array}$ & 71 & $\mathbf{m}$ & & \\
\hline Sc (Schmidt Number) & 1.72 & & & \\
\hline $\mathrm{U}$ (wind velocity) & 2.5 & $\mathrm{~m} / \mathrm{s}$ & & \\
\hline $\begin{array}{l}\text { nu (kinematic viscosity } \\
\text { of air) }\end{array}$ & $1.50 \mathrm{E}-5$ & $m^{\wedge} 2 / \sec$ & & \\
\hline mu (viscosity of air) & $1.90 \mathrm{E}-5$ & $\mathrm{~kg} / \mathrm{m}-\mathrm{s}$ & & \\
\hline $\begin{array}{l}\text { Dm (Molecular Diffusivity } \\
\text { ofsolute in Air) }\end{array}$ & $8.72 \mathrm{E}-6$ & $\mathrm{~m}^{\wedge} 2 / \mathrm{sec}$ & & \\
\hline $\begin{array}{l}\text { D H20 (Molecular } \\
\text { Diffusivity of Water) }\end{array}$ & $2.40 \mathrm{E}-5$ & & & \\
\hline MW_H2O & 18 & $\mathrm{~kg} / \mathrm{kg}-\mathrm{mole}$ & & \\
\hline \multicolumn{5}{|l|}{ Conversions } \\
\hline T (Absolute) & 298.15 & ${ }^{\circ} \mathrm{K}$ & & \\
\hline $\mathrm{V}$ (Volume) & 39.56 & $m^{\wedge} 3$ & & \\
\hline Evaporation Rate & 38712 & g/min & & \\
\hline 60 min $1 b$ released & $5120 \cdot 60$ & & & \\
\hline
\end{tabular}

Reviewed by S. A. Henderson

Works Calculation 8

Sheet No. 173 of 314 (USA) 
Title of Project EPHA for THM

Subject Concentration Analysis for THM Hazardous Chemicals

Computer N.E. Baucom B. K. Tharakan Date 09/16/96

1-RD-4 AVtract / Max DI8T 8I-0 1

SITE DATA INFORMATION:

Location: AIKEN, SOUTH CAROLINA

Building Air Exchanges Per Hour: 60 (User specified)

Time: June 30, 19981200 hours EDT (User specified)

CHEMICAI INEORMATION:

Chemical Name: ALPHA-PINEANE

Molecular Weight: $136.26 \mathrm{~kg} / \mathrm{kmol}$

TLV-TWA: -unavail-

Footprint Level of Concern: $300 \mathrm{mg} /(\mathrm{cu} \mathrm{m})$

Boiling Point: $155.00^{\circ} \mathrm{C}$

Vapor Pressure at Ambient Temperature: 0.0069 atm

Ambient Saturation Concentration: $6,993 \mathrm{ppm}$ or 0.708

Note: Not enough chemical data to use Heavy Gas option

ATMOSPHERIC INFORMATION: (MANUAI INPUT OF DATA)

Wind: 2.5 meters/sec from $90^{\circ}$ true at 2 meters

Inversion Height: 500 meters

stability class: C (user override)

Air Temperature: $25^{\circ} \mathrm{C}$

Relative Humidity: 508

cloud cover: 8 tenths

Ground Roughness: Urban or forest

SOURCE STRENGTH INFORMATION:

Direct Source: .382 kilograms/sec

Source Height: 0

Release Duration: 60 minutes

Release Rate: $22.9 \mathrm{kilograms/min}$

Total Amount Released: 1,375 kilograms

FOOTPRINT INFORMATION: Dispersion MOdule: Gaussian

User-specified $\mathrm{TOC}: 300 \mathrm{mg} /(\mathrm{cu} \mathrm{m})$

Max Threat Zone for LOC: 77 meters

Note: Footprint was not drawn because effects of near-field patchiness make dispersion predictions unreliable for short distances.
Reviewed by S. A. Henderson

Works Calculation 8

Sheet No. 174 of 314 


\section{ENGINEERING COMPUTATION SHEET}

Title of Project EPHA for THM

Reviewed by S. A. Henderson

Works Calculation 8

Sheet No. 175 of 314

Computer N.E. Baucom B. K. Tharakan Date 09/16/96

Determination of Evaporation Rate for Segment I Maximum source Term of $24420 \mathrm{ft}^{2}$

\begin{tabular}{|c|c|c|c|c|}
\hline Parameter : & Value & Dimensions & Value & $\begin{array}{l}\text { Dimensions } \\
\text { (USA) }\end{array}$ \\
\hline E (Evaporation Rate) & $3.82 \mathrm{E}-1$ & $\mathrm{Kg} / \mathrm{s}$ & & \\
\hline MW (Molecular Weight) & 136.26 & $\mathrm{~kg} / \mathrm{kg}-\mathrm{mole}$ & & \\
\hline Pv (Vapor Pressure) & 5.24 & $\mathrm{~mm} \mathrm{Hg}$ & & \\
\hline Pv (Vapor Pressure) & 698.60 & $\mathrm{~Pa}$ & & \\
\hline Rg (Gas Law Constant) & 8314 & $\mathrm{~J} / \mathrm{kgmole}-\mathrm{K}$ & & \\
\hline Temperature & 25 & ${ }^{\circ} \mathrm{C}$ & & \\
\hline Volume of Spill & 5993 & gal & & \\
\hline $\mathrm{d}$ (Depth of Pool) & 0.01 & $\mathrm{~m}$ & & \\
\hline A (Surface Area of Spil1) & 2268.5 & $m^{\wedge} 2$ & 24421 & ft^2 \\
\hline $\mathrm{Km}$ (Mass Transfer Coeff) & $4.38 E-3$ & $\mathrm{~m} / \mathrm{s}$ & & \\
\hline $\begin{array}{l}\text { Z (Pool Diameter in wind } \\
\text { Direction) }\end{array}$ & 54 & $m$ & & \\
\hline Sc (Schmidt Number) & 1.72 & & & \\
\hline $\mathrm{U}$ (wind velocity) & 2.5 & $\mathrm{~m} / \mathrm{s}$ & & \\
\hline $\begin{array}{l}\text { nu (kinematic viscosity } \\
\text { of } \\
\text { air) }\end{array}$ & $1.50 \mathrm{E}-5$ & $m^{\wedge} 2 / \mathrm{sec}$ & & \\
\hline mu (viscosity of air) & $1.90 E-5$ & $\mathrm{~kg} / \mathrm{m}-\mathrm{s}$ & & \\
\hline $\begin{array}{l}\text { Dm (Molecular Diffusivity } \\
\text { of } \\
\text { Solute in Air) }\end{array}$ & $8.72 \mathrm{E}-6$ & $m^{\wedge} 2 / \sec$ & & \\
\hline $\begin{array}{l}\text { D H20 (Molecular } \\
\text { Diffusivity } \\
\text { of water) }\end{array}$ & $2.40 \mathrm{E}-5$ & & & \\
\hline $\mathrm{MW} \_\mathrm{H} 20$ & 18 & $\mathrm{~kg} / \mathrm{kg}-\mathrm{mole}$ & & \\
\hline \multicolumn{5}{|l|}{ Conversions } \\
\hline T (Absolute) & 298.15 & ${ }^{\circ} \mathrm{K}$ & & \\
\hline$V$ (Volume) & 22.69 & $m^{\wedge} 3$ & & \\
\hline Evaporation Rate & 22897 & $\mathrm{~g} / \mathrm{min}$ & & \\
\hline $60 \mathrm{~min} 1 \mathrm{~b}$ released & 3028.75 & & & \\
\hline
\end{tabular}




\section{1-RD-4 AVERAGE / MAX DIST SEG 2}

\section{SITE DATA INFORMATION:}

Location: AIKEN, SOUTH CAROLINA

Building Air Exchanges Per Hour: 60 (User specified)

Time: June 30, 19981200 hours EDT (User specified)

\section{CHEMICAL INFORMATION:}

Chemical Name: ALPHA-PINENE

Molecular Weight: $136.26 \mathrm{~kg} / \mathrm{kmol}$

TLV-TWA: -unavail- IDLH: -unavail

Footprint Level of Concern: $300 \mathrm{mg} /(\mathrm{cu} \mathrm{m}$ )

Boiling Point: $155.00^{\circ} \mathrm{C}$

Vapor Pressure at Ambient Temperature: $0.0069 \mathrm{~atm}$

Ambient Saturation Concentration: $6,993 \mathrm{ppm}$ or $0.70 \%$

Note: Not enough chemical data to use Heavy Gas option

ATMOSPHERIC INFORMATION: (MANUAL INPUT OF DATA)

Wind: 2.5 meters $/ \mathrm{sec}$ from $90^{\circ}$ true at 2 meters

Inversion Height: 500 meters

Stability Class: $\mathrm{C}$ (user override)

Air Temperature: $25^{\circ} \mathrm{C}$

Relative Humidity: $50 \%$

Ground Roughness: Urban or forest

Cloud Cover: 8 tenths

SOURCE STRENGTH INFORMATION:

Direct Source: .618 kilograms/sec

Source Height: 0

Release Duration: 60 minutes

Release Rate: 37.1 kilograms/min

Total Amount Released: 2,225 kilograms

FOOTPRINT INFORMATION:

Dispersion Module: Gaussian

User-specified LOC: $300 \mathrm{mg} /(\mathrm{cu} \mathrm{m})$

Max Threat Zone for LOC: 98 meters

Note: Footprint was not drawn because effects of near-field patchiness make dispersion predictions unreliable for short distances. 
Title of Project EPHA for THM

Reviewed by S. A. Henderson

Subject Concentration Analysis for THM Hazardous Chemicals

Works Calculation 8

Computer N.E. Baucom B. K. Tharakan Date 09/16/96

Sheet No. 177 of 314

Determination of Evaporation Rate for Segment 2 Maximum source Term of $40700 \mathrm{Et}^{2}$

\begin{tabular}{|c|c|c|c|c|}
\hline Parameter. & Value & Dimensions & Value & $\begin{array}{l}\text { Dimensio } \\
\text { ns } \\
\text { (USA) }\end{array}$ \\
\hline E (Evaporation Rate) & $6.18 \mathrm{E}-1$ & $\mathrm{~kg} / \mathrm{s}$ & & \\
\hline MW (Molecular Weight) & 136.26 & $\mathrm{~kg} / \mathrm{kg}-\mathrm{mole}$ & & \\
\hline Pv (Vapor Pressure) & 5.24 & $\mathrm{~mm} \mathrm{Hg}$ & & \\
\hline Pv (Vapor Pressure) & 698.60 & $\mathrm{~Pa}$ & & \\
\hline Rg (Gas Law Constant) & 8314 & J/kgmole-K & & \\
\hline Temperature & 25 & ${ }^{\circ} \mathrm{c}$ & & \\
\hline Volume of Spill & 9988 & gal & & \\
\hline d (Depth of Pool) & 0.01 & $\mathrm{~m}$ & & \\
\hline A (Surface Area of Spill) & 3780.8 & $m^{\wedge} 2$ & 40700 & $f t^{\wedge} 2$ \\
\hline $\mathrm{Km}$ (Mass Transfer Coeff) & $4.26 \mathrm{E}-3$ & $\mathrm{~m} / \mathrm{s}$ & & \\
\hline $\begin{array}{l}\mathrm{Z} \text { (Pool Diameter in wind } \\
\text { Direction) }\end{array}$ & 69 & $\mathrm{~m}$ & & \\
\hline Sc (Schmidt Number) & 1.72 & & & \\
\hline U (wind velocity) & 2.5 & $\mathrm{~m} / \mathrm{s}$ & & \\
\hline $\begin{array}{l}\text { nu (kinematic viscosity } \\
\text { of air) }\end{array}$ & $1.50 \mathrm{E}-5$ & $m^{\wedge} 2 / \sec$ & & \\
\hline $\mathrm{mu}$ (viscosity of air) & $1.90 \mathrm{E}-5$ & $\mathrm{~kg} / \mathrm{m}-\mathrm{s}$ & & \\
\hline $\begin{array}{l}\text { Dm (Molecular Diffusivity } \\
\text { of solute in Air) }\end{array}$ & $8.72 E-6$ & $m^{\wedge} 2 /$ sec & & \\
\hline $\begin{array}{l}\text { D H20 (Molecular } \\
\text { Diffusivity of Water) }\end{array}$ & $2.40 \mathrm{E}-5$ & & & \\
\hline $\mathrm{MW} \_\mathrm{H} 2 \mathrm{O}$ & 18 & $\mathrm{~kg} / \mathrm{kg}$-mole & & \\
\hline \multicolumn{5}{|l|}{ Convergions } \\
\hline T (Absolute) & 298.15 & ${ }^{\circ} \mathrm{K}$ & & \\
\hline V (Volume) & 37.81 & $m^{\wedge} 3$ & & \\
\hline Evaporation Rate & 37093 & $\mathrm{~g} / \mathrm{min}$ & & \\
\hline $60 \mathrm{~min} 1 \mathrm{~b}$ released & 4906.52 & & & \\
\hline
\end{tabular}


Title of Project EPHA for THM

Subject Concentration Analysis for THM Hazardous Chemicals

Computer N.E. Baucom B. K. Tharakan Date 09/16/96

1-RD.4 AVERAGE / MAX DIST SEG 3

SITE DATA INFORMATION:

Location: AIKEN, SOUTH CAROLINA

Building Air Exchanges Per Hour: 60 (User specified)

Time: June 30, 19981200 hours EDT (User specified)

CHEMICAL INFORMATION:

Chemical Name: ALPHA-PINENE

Molecular Weight: $136.26 \mathrm{~kg} / \mathrm{kmol}$

TLV-TWA: -unavail-

IDLH: -unavail

Footprint Level of Concern: $300 \mathrm{mg} /(\mathrm{cu} \mathrm{m})$

Boiling Point: $155.000^{\circ} \mathrm{C}$

Vapor Pressure at Ambient Temperature: $0.0069 \mathrm{~atm}$

Ambient Saturation Concentration: 6,993 ppm or $0.70 \%$

Note: Not enough chemical data to use Heavy Gas option

ATMOSPHERIC INFORMATION: (MANUAL INPUT OF DATA)

Wind: 2.5 meters $/ \mathrm{sec}$ from $90^{\circ}$ true at 2 meters

Inversion Height: 500 meters

Stability Class: C (user override)

Air Temperature: $25^{\circ} \mathrm{C}$

Relative Humidity: 50\%

Cloud Cover: 8 tenths

SOURCE STRENGTH INFORMATION:

Direct Source: 1.55 kilograms/sec

Source Height: 0

Release Duration: 60 minutes

Release Rate: 93 kilograms/min

Total Amount Released: 5,580 kilograms

$\cdot$

FOOTPRINT INFORMATION:

Dispersion Module: Gaussian

User-specified LOC: $300 \mathrm{mg} /(\mathrm{cu} \mathrm{m}$ )

Max Threat Zone for LOC: 155 meters
Reviewed by S. A. Henderson

Works Calculation 8 .

Sheet No. 178 of 314 
Title of Project EPHA for THM

Subject Concentration Analysis for THM Hazardous Chemicals

Computer N.E. Baucom B. K. Tharakan Date 09/16/96

Determination of Evaporation Rate for Segment 3 Maximum Source Term of $10000 \mathrm{~m}^{2}$

\begin{tabular}{|c|c|c|c|c|}
\hline Parameter & Value & Dimensions & Value & $\begin{array}{l}\text { Dimensi } \\
\text { ons } \\
\text { (USA) }\end{array}$ \\
\hline E (Evaporation Rate) & $1.55 \mathrm{E}+0$ & $\mathrm{~kg} / \mathrm{s}$ & & \\
\hline MW (Molecular Weight) & 136.26 & $\mathrm{~kg} / \mathrm{kg}-\mathrm{mole}$ & & \\
\hline PV (Vapor Pressure) & 5.24 & $\mathrm{~mm} \mathrm{Hg}$ & & \\
\hline PV (Vapor Pressure) & 698.60 & $\mathrm{~Pa}$ & & \\
\hline Rg (Gas Law Constant) & 8314 & $\mathrm{~J} / \mathrm{kgmole}-\mathrm{K}$ & & \\
\hline Temperature & 25 & ${ }^{\circ} \mathrm{c}$ & & \\
\hline Volume of Spi11 & 26418 & gal & & \\
\hline d (Depth of Pool) & 0.01 & $m$ & & \\
\hline A (Surface Area of Spill) & 10000.0 & $\mathrm{~m}^{\wedge} 2$ & 107650 & ft^2 \\
\hline $\mathrm{Km}$ (Mass Transfer Coeff) & $4.03 \mathrm{E}-3$ & $\mathrm{~m} / \mathrm{s}$ & & \\
\hline $\begin{array}{l}\mathrm{Z} \text { (Pool Diameter in Wind } \\
\text { Direction) }\end{array}$ & 113 & $m$ & & \\
\hline Sc (Schmidt Number) & 1.72 & & & \\
\hline U (wind velocity) & 2.5 & $\mathrm{~m} / \mathrm{s}$ & & \\
\hline $\begin{array}{l}\text { nu (kinematic viscosity } \\
\text { ofair) }\end{array}$ & $1.50 \mathrm{E}-5$ & $m^{\wedge} 2 / \sec$ & & \\
\hline mu (viscosity of air) & $1.90 \mathrm{E}-5$ & $\mathrm{~kg} / \mathrm{m}-\mathrm{s}$ & & \\
\hline $\begin{array}{l}\text { Dm (Molecular Diffusivity } \\
\text { of Solute in Air) }\end{array}$ & $8.72 \mathrm{E}-6$ & $\mathrm{~m}^{\wedge} 2 / \mathrm{sec}$ & & \\
\hline $\begin{array}{l}\text { D_H20 (Molecular } \\
\text { Diffusivity of Water) }\end{array}$ & $2.40 \mathrm{E}-5$ & & & \\
\hline MW_H2O & 18 & $\mathrm{~kg} / \mathrm{kg}-\mathrm{mole}$ & & \\
\hline \multicolumn{5}{|l|}{ Conversions } \\
\hline T (Absolute) & 298.15 & ${ }^{\circ} \mathrm{K}$ & & \\
\hline $\mathrm{V}$ (Volume) & 100.00 & $m^{\wedge} 3$ & & \\
\hline Evaporation Rate & 92950 & $\mathrm{~g} / \mathrm{min}$ & & \\
\hline $60 \mathrm{~min} 1 \mathrm{~b}$ released & 12294.97 & & & \\
\hline
\end{tabular}

Sheet No 179 of 314
Reviewed by S. A. Henderson 
Title of Project EPHA for THM

Reviewed by S.A. Henderson

Subject Concentration Analysis for THM Hazardous Chemicals

Works Calculation 8

Computer. N.E. Baucom B.K. Tharakan Date 09/16/96

Sheet No. 180 of 314

\section{1-RD-7 AVERAGE / ALERT .}

SITE DATA INFORMATION:

Location: AIKEN, SOUTH CAROLINA

Building Air Exchanges Per Hour: 60 (User specified)

Time: June 30, 19981200 hours EDT (User specified)

CHEMICAL INFORMATION:

Chemical Name: BENZYL ALCOHOL

Molecular Weight: $108.14 \mathrm{~kg} / \mathrm{kmol}$

TLV-TWA: -unavail-

IDLH: -unavail

Footprint Level of Concern: 100 ppm

Boiling Point: $204.70^{\circ} \mathrm{C}$

Vapor Pressure at Ambient Temperature: 1.07e-004 atm

Ambient Saturation Concentration: 109 ppm or 0.011\%

ATMOSPHERIC INFORMATION: (MANUAL INPUT OF DATA)

Wind: 2.5 meters $/ \mathrm{sec}$ from $90^{\circ}$ true at 2 meters

Inversion Height: 500 meters

Stability Class: $\mathrm{C}$ (user override)

Air Temperature: $25^{\circ} \mathrm{C}$

Relative Humidity: 50\%

Ground Roughness: Urban or forest

Cloud Cover: 8 tenths

SOURCE STRENGTH INFORMATION:

Puddle Area: 30001 square feet

Average Puddle Depth: 1 centimeters

Soil Type: Default Ground Temperature: $25^{\circ} \mathrm{C}$

Initial Puddle Temperature: Ground temperature

ReleaseDuration: ALOHA limited the duration to 1 hour

Max Computed Release Rate: 654 grams/min

MaxAverage Sustained Release Rate: 637 grams/min

(averaged over a minute or more)

Total Amount Released: 32.5 kilograms

FOOTPRINT INFORMATION: Dispersion Module: Gaussian

User-specified LOC: $100 \mathrm{ppm}$

Max Threat Zone for LOC: 30 meters

Note: Footprint was not drawn because effects of near-field patchiness make dispersion predictions unreliable for short distances.

TIME DEPENDENT INFORMATION:

Concentration Estimates at the point:

Downwind: $\quad 30$ meters

Off Centerline: 0 meters.

Max Concentration:

Outdoor: $2.47 \mathrm{ppm}$

Indoor: $2.47 \mathrm{ppm}$

Note: Indoor graph is shown with a dotted line.

\section{1-RD-7 AVERAGE / SAE}


Title of Projejct EPHA for THM

Subject Concentration Analysis for THM Hazardous Chemicals

Computer N.E. Baucom B. K. Tharakan Date 09/16/96

SITE DATA INFORMATION:

Location: AIKEN, SOUTH CAROLINA

Building Air Exchanges Per Hour: 60 (User specified)

Time: June 30, 19981200 hours EDT (User specified)

\section{CHEMICAL INFORMATION:}

Chemical Name: BENZYL ALCOHOL

Molecular Weight: $108.14 \mathrm{~kg} / \mathrm{kmol}$

TLV-TWA: -unavail-

Footprint Level of Concern: 100 ppm

Boiling Point: $204.70^{\circ} \mathrm{C}$

Vapor Pressure at Ambient Temperature: 1.07e-004 atm

Ambient Saturation Concentration: 109 ppm or $0.011 \%$

ATMOSPHERIC INFORMATION: (MANUAL INPUT OF DATA)

Wind: 2.5 meters $/ \mathrm{sec}$ from $90^{\circ}$ true at 2 meters

Inversion Height: 500 meters

Stability Class: C (user override)

Air Temperature: $25^{\circ} \mathrm{C}$

Relative Humidity: $50 \%$

Cloud Cover: 8 tenths

\section{SOURCE STRENGTH INFORMATION:}

Puddle Area: 100001 square feet

Average Puddle Depth: 1 centimeters

Ground Roughness: Urban or forest

Soil Type: Default

Ground Temperature: $25^{\circ}$

Initial Puddle Temperature: Ground temperature

Release Duration: ALOHA limited the duration to 1 hour

Max Computed Release Rate: 2.08 kilograms/min

Max 4verage Sustained Release Rate: 2.03 kilograms/min

(averaged over a minute or more)

Total Amount Released: 103 kilograms

FOOTPRINT INFORMATION:

Dispersion Module: Gaussian

User-specified LOC: $100 \mathrm{ppm}$

Max Threat Zone for LOC: 55 meters

Note: Footprint was not drawn because effects of near-field patchiness make dispersion predictions unreliable for short distances.

\section{TIME DEPENDENT INFORMATION:}

Concentration Estimates at the point:

Downwind: $\quad 100$ meters

Off Centerline: $\quad 0$ meters

Max Concentration: outdoor: 0.775 ppm Indoor: $0.775 \mathrm{ppm}$

Note: Indoor graph is shown with a dotted line.
Reviewed by S. A. Henderson

Works Calculation 8

Sheet No. 181 of 314 
Title of Project EPHA for THM

Subject Concentration Analysis for THM Hazardous Chemicals

Computer N.E. Baucom B. K. Tharakan Date 09/16/96

1-RD-7 AVERAGE / MAX DIST SEG 1

SITE DATA INFORMATION:

Location: AIKEN, SOUTH CAROLINA

Building Air Exchanges Per Hour: 60 (User specified)

Time: June 30, 19981200 hours EDT (User specified)

\section{CHEMICAL INFORMATION:}

Chemical Name: BENZYL ALCOHOL

Molecular Weight: $108.14 \mathrm{~kg} / \mathrm{kmol}$

TLV-TWA: -unavail-

IDLH: -unavail

Footprint Level of Concern: 100 ppm

Boiling Point: $204.70^{\circ} \mathrm{C}$

Vapor Pressure at Ambient Temperature: $1.07 \mathrm{e}-004 \mathrm{~atm}$

Ambient Saturation Concentration: 109 ppm or $0.011 \%$

\section{ATMOSPHERIC INFORMATION: (MANUAL INPUT OF DATA)}

Wind: 2.5 meters $/ \mathrm{sec}$ from $90^{\circ}$ true at 2 meters

Inversion Height: 500 meters

Stability Class: $\mathrm{C}$ (user override)

Air Temperature: $25^{\circ} \mathrm{C}$

Relative Humidity: $50 \%$

Cloud Cover: 8 tenths

Ground Roughness: Urban or forest
Reviewed by S. A. Henderson

Works Calculation 8

Sheet No. 182 of 314

SOURCE STRENGTH INFORMATION:

Puddle Area: 24420 square feet

Average Puddle Depth: 1 centimeters

Soil Type: Default Ground Temperature: $25^{\circ} \mathrm{C}$

Initial Puddle Temperature: Ground temperature

ReleaseDuration: ALOHA limited the duration to 1 hour

Max Computed Release Rate: 536 grams/min

MaxAverage Sustained Release Rate: 523 grams/min

(averaged over a minute or more)

Total Amount Released: 26.7 kilograms

FOOTPRINT INFORMATION: Dispersion Module: Gaussian

User-specified LOC: $100 \mathrm{ppm}$

Max Threat Zone for LOC: 27 meters

Note: Footprint was not drawn because effects of near-field patchiness make dispersion predictions unreliable for short distances. 
Title of Project EPHA for THM

Subject Concentration Analysis for THM Hazardous Chemicals

Computer N.E. Baucom B. K. Tharakan Date 09/16/96

1-RD-7 A VERAGE / MAX DIST SEG 2

SITE DATA INFORMATION:

Location: AIKEN, SOUTH CAROLINA

Building Air Exchanges Per Hour: 60 (User specified)

Time: June 30, 19981200 hours EDT (User specified)

CHEMICAL INFORMATION:

Chemical Name: BENZYL ALCOHOL

Molecular Weight: 108.14 kg/kmol

TLV-TWA: -unavail-

IDLH: -unavail

Footprint Level of Concern: $100 \mathrm{ppm}$

Boiling Point: $204.70^{\circ} \mathrm{C}$

Vapor Pressure at Ambient Temperature: 1.07e-004 atm

Ambient Saturation Concentration: 109 ppm or $0.011 \%$

\section{ATMOSPHERIC INFORMATION: (MANUAL INPUT OF DATA)}

Wind: 2.5 meters $/ \mathrm{sec}$ from $90^{\circ}$ true at 2 meters

Inversion Height: 500 meters

Stability Class: $\mathrm{C}$ (user override)

Air Temperature: $25^{\circ} \mathrm{C}$

Relative Humidity: 50\%

Ground Roughness: Urban or forest

Cloud Cover: 8 tenths

SOURCE STRENGTH INFORMATION:

Puddle Area: 40700 square feet

Average Puddle Depth: 1 centimeters

Soil Type: Default

Ground Temperature: $25^{\circ} \mathrm{C}$

Initial Puddle Temperature: Ground temperature

Release Duration: ALOHA limited the duration to 1 hour

Max Computed Release Rate: 876 grams/min

Max Average Sustained Release Rate: 854 grams/min

(averaged over a minute or more)

Total Amount Released: 43.5 kilograms

FOOTPRINT INFORMATION: Dispersion Module: Gaussian

User-specified LOC: $100 \mathrm{ppm}$

Max Threat Zone for LOC: 34 meters

Note: Footprint was not drawn because effects of near-field patchiness make dispersion predictions unreliable for short distances.
Reviewed by S. A. Henderson

Works Calculation 8

Sheet No. 183 of 314 
Title of Project EPHA for THM

Subject Concentration Analysis for THM Hazardous Chemicals

Computer N.E. Baucom B. K. Tharakan Date 09/16/96

\section{1-RD-7 AVERAGE / MAX DIST SEG 3}

\section{SITE DATA INFORMATION:}

Location: AIKEN, SOUTH CAROLINA

Building Air Exchanges Per Hour: 60 (User specified)

Time: June 30, 19981200 hours EDT (User specified)

\section{CHEMICAL INFORMATION:}

Chemical Name: BENZYL ALCOHOL

Molecular Weight: $108.14 \mathrm{~kg} / \mathrm{kmol}$

TLV-TWA: -unavail-

IDLH: -unavail

Footprint Level of Concern: 100 ppm

Boiling Point: $204.70^{\circ} \mathrm{C}$

Vapor Pressure at Ambient Temperature: 1.07e-004 atm

Ambient Saturation Concentration: 109 ppm or $0.011 \%$

\section{ATMOSPHERIC INFORMATION: (MANUAL INPUT OF DATA)}

Wind: 2.5 meters $/ \mathrm{sec}$ from $90^{\circ}$ true at 2 meters

Inversion Height: 500 meters

Stability Class: $\mathrm{C}$ (user override)

Air Temperature: $25^{\circ} \mathrm{C}$

Relative Humidity: 50\%

Ground Roughness: Urban or forest

Cloud Cover: 8 tenths

\section{SOURCE STRENGTH INFORMATION:}

Puddle Area: 10000 square meters

Average Puddle Depth: 1 centimeters

Soil Type: Default

Ground Temperature: $25^{\circ} \mathrm{C}$

Initial Puddle Temperature: Ground temperature

Release Duration: ALOHA limited the duration to 1 hour

Max Computed Release Rate: 2.23 kilograms/min

Max Average Sustained Release Rate: 2.18 kilograms/min

(averaged over a minute or more)

Total Amount Released: 111 kilograms

FOOTPRINT INFORMATION: Dispersion Module:

Gaussian User-specified LOC: 100 ppm

Max Threat Zone for LOC: 56 meters

Note: Footprint was not drawn because effects of near-field patchiness make dispersion predictions unreliable for short distances. 
Title of Project EPHA for THM

Subject Concentration Analysis for THM Hazardous Chemicals

Computer N.E. Baucom B. K. Tharakan Date 09/16/96 1-RD-9 AVERAGI / ALRART
Reviewed by S.A. Henderson

Works Calculation 8

Sheet No. 185 of 314

\section{SITE DATA INFORMATION:}

Location: AIKEN, SOUTH CAROLINA

Building Air Exchanges Per Hour: 60 (User specified)

Time: June 30, 19981200 hours EDT (User specified)

CHEMICAL INFORMATION:

Chemical Name: BUTYRALDEHYDE

Molecular Weight: $72.11 \mathrm{~kg} / \mathrm{kmol}$

TLV-TWA: -unavail-

IDLH: -unavail

Footprint Level of Concern: $500 \mathrm{mg} /(\mathrm{cu} \mathrm{m})$

Boiling Point: $74.80^{\circ} \mathrm{C}$

Vapor Pressure at Ambient Temperature: 0.15 atm

Ambient Saturation Concentration: $149,153 \mathrm{ppm}$ or 14.98

ATMOSPHERIC INFORMATION: (MANUAL INPUT OF DATA)

Wind: 2.5 meters $/ \mathrm{sec}$ from $90^{\circ}$ true at 2 meters

Inversion Height: 500 meters

stability Class: $C$ (user override)

Air Temperature: $25^{\circ} \mathrm{C}$

Relative Humidity: 508

Cloud Cover: 8 tenths

Ground Roughness: Urban or forest

SOURCE STRENGTH INFORMATION:

Puddle Area: 737 square feet

Average Puddle Depth: 1 centimeters

Soil Type: Default

Ground Temperature: $25^{\circ}$

Initial Puddle Temperature: Ground temperature

Release Duration: ALOHA limited the duration to 1 hour

Max Computed Release Rate: $10.1 \mathrm{kilograms/min}$

Max Average Sustained Release Rate: 8.85 kilograms/min (averaged over a minute or more)

Total Amount Released: 472 kilograms

FOOTPRINT INFORMATION:

Dispersion Module: Gaussian

User-specified LOC: $500 \mathrm{mg} /(\mathrm{cu} \mathrm{m})$

Max Threat Zone for LOC: 30 meters

Note: Footprint was not drawn because effects of near-field patchiness make dispersion predictions unreliable for short distances.

TIME DEPENDENT INFORMATION:

Concentration Estimates at the point:

Downwind: 30 meters

off Centerline: 0 meters

Max Concentration: Outdoor: $500 \mathrm{mg} /(\mathrm{cu} \mathrm{m})$

Indoor: $500 \mathrm{mg} /$ (cu m)

Note: Indoor graph is shown with a dotted line. 
Title of Project EPHA for THM

Reviewed by S.A. Henderson

Subject Concentration Analysis for THM Hazardous Chemicals

Works_Calculation 8

Computer N.E. Baucom B. K. Tharakan Date $\underline{09 / 16 / 96}$

Sheet No. 186 of 314

\section{1-RD-9 AVWRAG / SAT}

SITE DATA INFORMATION:

LOCation: AIREN, SOUTH CAROLINA

Building Air Exchanges Per Hour: 60 (User specified)

Time: June 30, 19981200 hours EDT (User specified)

CHEMICAL INFORMATION :

Chemical Name: BUTYRALDEHYDE

Molecular Weight: $72.11 \mathrm{~kg} / \mathrm{kmol}$

TLV-TWA: - Unavail-

IDEH: -unavail

Footprint Level of Concern: $500 \mathrm{mg} /(\mathrm{cu} \mathrm{m})$

Boiling Point: $74.80^{\circ} \mathrm{C}$

Vapor Pressure at Ambient Temperature: $0.15 \mathrm{~atm}$

Ambient Saturation Concentration: 149,153 ppm or 14.98

ATMOSPHERIC INFORMATION: (MANUAL INPUT OF DATA)

Wind: 2.5 meters $/ \mathrm{sec}$ from $90^{\circ}$ true at 2 meters

Inversion Height: 500 meters

Stability class: C (user override)

Air Temperature: $25^{\circ} \mathrm{C}$

Relative Humidity: $50 \%$

Ground Roughness: Urban or forest

Cloud Cover: 8 tenths

SOURCE STRENGTH INFORHATION:

Puddle Area: 6485 square feet

Average Pudale Depth: 1 centimeters

Soil Type: Default Ground Temperature: $25^{\circ} \mathrm{C}$

Initial puddle Temperature: Ground temperature

Release Duration: ALOHA limited the duration to 1 hour

Max Computed Release Rate: 79.9 kilograms/min

Max Average Sustained Release Rate: 71 kilograms/min

(averaged over a minute or more)

Total Amount Released: 3,846 kilograms

FOOTPRINT INFORMATION: Dispersion Module: Gaussian

User-specified LOC: $500 \mathrm{mg} /(\mathrm{cu} \mathrm{m})$

Max Threat Zone for LOC: 83 meters

Note: Footprint was not drawn because effects of near-field patchiness make dispersion predictions unreliable for short distances.

TIME DEPENDENT INFORMATION:

Concentration Estimates at the point:

Downwind:

100 meters

off Centerline: 0 meters

Max Concentration:

Outdoor: $389 \mathrm{mg} /$ (cu m)

Indoor: $389 \mathrm{mg} /$ (cu m)

Note: Indoor graph is shown with a dotted line. 


\section{1-RD-9 AVERAGI / WaX DIST SEO 1}

SITE DATA INEORMATION:

Location: AIREN, SOUTH CAROLINA

Building Air Exchanges Per Hour: 60 (User specified)

Time: June 30, 19981200 hours EDT (User specified)

CHEMICAL INFORMATION:

Chemical Name: BUTYRALDEHYDE

Molecular Weight: $72.11 \mathrm{~kg} / \mathrm{kmol}$

TLV-TWA: -unavail-

IDLH: -unavail

Footprint Level of Concern: $500 \mathrm{mg} /(\mathrm{cu} \mathrm{m})$

Boiling Point: $74.80^{\circ} \mathrm{C}$

Vapor Pressure at Ambient Temperature: 0.15 atm

Ambient Saturation Concentration: $149,153 \mathrm{ppm}$ or 14.98

ATMOSPHERIC INEORMATION: (MANUAI INPUT OF DATA)

Wind: 2.5 meters/sec from $90^{\circ}$ true at 2 meters

Inversion Height: 500 meters

stability Class: C (user override)

Air Temperature: $25^{\circ} \mathrm{C}$

Relative Humidity: 508

Ground Roughness : Urban or forest

cloud Cover: 8 tenths

SOURCE STRENGTH INFORMATION:

Puddle Area: 24420 square feet

Average Puddle Depth: 1 centimeters

Soil Type: Default

Ground Temperature: $25^{\circ} \mathrm{C}$

Initial Puddle Temperature: Ground temperature

Release Duration: AIOHA limited the duration to 1 hour

Max Computed Release Rate: 283 kilograms/min

Max Average Sustained Release Rate: 254 kilograms/min

(averaged over a minute or more)

Total Amount Released: 13,863 kilograms

FOOTPRINT INFORMATION:

Model Run: Heavy Gas

User-specified LOC: $500 \mathrm{mg} /(\mathrm{cu} \mathrm{m}$ )

Max Threat Zone for LOC: 471 meters 
Title of Projoct EPHA for THM

Subject Concentration Analysis for THM Hazardous Chemicals

Computer N.E. Baucom B.K. Tharakan Date 09/16/96

1-RD-9 AVERACE / YAX DIST SEC 2

SITE DATA INFORMATION:

LOCation: AIKEN, SOUTH CAROUINA

Building Air Exchanges Per Hour: 60 (User specified)

Time: June 30, 19981200 hours EDT (User specified)

CHEMICAL INFORMATION:

Chemical Name: BUTYRALDEHYDE

Molecular Weight: $72.11 \mathrm{~kg} / \mathrm{kmol}$

TLV-TWA: -unavai1-

IDLH : -unavaiI

Footprint Level of Concern: $500 \mathrm{mg} /(\mathrm{cu} \mathrm{m})$

Boiling Point: $74.80^{\circ} \mathrm{C}$

Vapor Pressure at Ambient Temperature: 0.15 atm

Ambient Saturation Concentration: $149,153 \mathrm{ppm}$ or 14.98

ATMOSPHERIC INFORMATION: (MANUAL INPUT OF DATA)

Wind: 2.5 meters/sec from $90^{\circ}$ true at 2 meters

Inversion Height: 500 meters

stability Class: C (user override)

Air Temperature: $25^{\circ} \mathrm{C}$

Relative Humidity: 508

Cloud Cover: 8 tenths

Ground Roughness: Urban or forest

SOURCE STRENGTH INFORMATION:

Puddle Area: 40700 square feet

Average Puddle Depth: 1 centimeters

Soil Type: Default Ground Temperature: $25^{\circ} \mathrm{C}$

Initial puddle Temperature: Ground temperature

Release Duration: ALOHA limited the duration to 1 hour

Max Computed Release Rate: $460 \mathrm{kilograms} / \mathrm{min}$

Max Average Sustained Release Rate: $415 \mathrm{kilograms} / \mathrm{min}$

(averaged over a minute or more)

Total Amount Released: 22,732 kilograms

FOOTPRINT INFORMATION:

Model Run: Heavy Gas

User-specified LOC: $500 \mathrm{mg} /(\mathrm{cu} \mathrm{m})$

Max Threat zone for LOC: 603 meters
Reviewed by S. A. Henderson

Works_Calculation 8

Sheet No. 188 of 314

\section{.}


SITE DATA INFORMATION:

Location: AIREN, SOUTH CAROLINA

Building Air Exchanges Per Hour: 60 (User specified)

Time: June 30, 19981200 hours EDT (User specified)

CHEMICAL INFORMATION:

Chemical Name: BUTYRALDERYDE

Molecular Weight: $72.11 \mathrm{~kg} / \mathrm{kmol}$

TLV-TWA: -unavail-

IDLH: - unavail

Footprint Level of Concern: $500 \mathrm{mg} /(\mathrm{Cu} \mathrm{m})$

Boiling Point: $74.80^{\circ} \mathrm{C}$

Vapor Pressure at Ambient Temperature: 0.15 atm

Ambient Saturation Concentration: 149,153 ppm or 14.98

ATMOSPHERIC INFORMATION: (MANUAL INPUT OF DATA)

Wind: 2.5 meters $/ \mathrm{sec}$ from $90^{\circ}$ true at 2 meters

Inversion Height: 500 meters

stability Class: C (user override)

Air Temperature: $25^{\circ} \mathrm{C}$

Relative Humidity: 508

Ground Roughness: Urban or forest

Cloud Cover: 8 tenths

SOURCE STRENGTH INEORMATION:

Puddle Area: 10000 square meters

Average Pudale Depth: 1 centimeters

Soil Type: Default

Ground Temperature: $25^{\circ} \mathrm{C}$

Initial puddle Temperature: Ground temperature

Release Duration: ALOHA limited the duration to 1 hour

Max Computed Release Rate: 1,170 kilograms/min

Max Average Sustained Release Rate: 1,060 kilograms/min (averaged over a minute or more)

Total Amount Released: 58,337 kilograms

FOOTPRINT INFORMATION:

Model Run: Heavy Gas

User-specified LOC: $500 \mathrm{mg} /(\mathrm{cu} \mathrm{m}$ )

Max Threat zone for LOC: 994 meters 
Title of Project EPHA for THM

Subject Concentration Analysis for THM Hazardous Chemicals

Computer N.E. Baucom B. K. Tharakan Date 09/16/96

1-RD-11 AVERAG: ATERT

SITE DATA INFORMATION:

Location: AIKEN, SOUTH CAROLINA

Building Air Exchanges Per Hour: 60 (User specified)

Time: June 30, 19981200 hours EDT (User specified)

CHEMICAL INFORMATION:

Chemical Name: AIPHA-PINENE

Molecular Weight: $136.26 \mathrm{~kg} / \mathrm{kmol}$

$\begin{array}{ll}\text { TLV TWA: - unavail- } & \text { IDLH: } \\ \text { Footprint Level of Concern: } & 1 \text { ppm }\end{array}$

Boiling Point: $155.00^{\circ} \mathrm{C}$

Vapor Pressure at Ambient Temperature: 0.0069 atm

Ambient Saturation Concentration: $6,993 \mathrm{ppm}$ or 0.708

Note: Not enough chemical data to use Heavy Gas option

ATMOSPHERIC INFORMATION: (MANUAL INPUT OF DATA)

Wind: 2.5 meters/sec from $90^{\circ}$ true at 2 meters

Inversion Height: 500 meters

Stability Class: C (user override)

Air Temperature: $25^{\circ} \mathrm{C}$

Relative Humidity: 508

Cloud Cover: 8 tenths

Ground Roughness: Urban or forest

SOURCE STRENGTH INFORMATION:

Direct Source: $1.08 \mathrm{E}-3 \mathrm{kilograms} / \mathrm{sec}$

Source Height: 0

Release Duration: 60 minutes

Release Rate: 64.8 grams/min

Total Amount Released: 3.89 kilograms

TIME DEPENDENT INFORMATION:

Concentration Estimates at the point:

Downwind: 30 meters

off Centerline: 0 meters

Max Concentration:

Outdoor: $0.998 \mathrm{ppm}$

Indoor: $0.998 \mathrm{ppm}$

Note: Indoor graph is shown with a dotted line.

Reviewed by S. A. Henderson

Works Calculation 8

Sheet No. 190 of 314 
Title of Project EPHA for THM

Subject Concentration Analysis for THM Hazardous Chemicals

Computer N.E. Baucom B. K. Tharakan Date 09/16/96 SPIIT AREA CXICULATION TOR 1-RD-11 AVERAGE / ATERT
Reviewed by S. A. Heriderson

Works Calculation 8

Sheet No. 191 of 314

CONDITIONS

\begin{tabular}{|c|c|c|c|c|}
\hline Parameter & Value & Dinensions & Value & $\begin{array}{l}\text { Dimencions } \\
\text { (סSA) }\end{array}$ \\
\hline E (Evaporation Rate) & $1.08 \mathrm{E}-3$ & $\mathrm{~kg} / \mathrm{s}$ & & \\
\hline MW (Molecular Weight) & 93 & $\mathrm{~kg} / \mathrm{kg}-\mathrm{mole}$ & & \\
\hline Pv (Vapor Pressure) & 12.00 & $\mathrm{mg}$ & & \\
\hline Pv (Vapor Pressure) & 1600 & $\mathrm{~Pa}$ & & \\
\hline Rg (Gas Law Constant) & 8314 & $\mathrm{~J} / \mathrm{kgmole-K}$ & & \\
\hline Temperature & 29 & ${ }^{\circ} \mathrm{C}$ & & \\
\hline Volume of spill & 9.15 & gal & & \\
\hline d (Depth of Pool) & 0.01 & $m$ & & \\
\hline A (Surface Area of Spill) & 3.5 & $\mathrm{~m}^{\wedge} 2$ & 37 & $\mathrm{ft \wedge 2}$ \\
\hline $\mathrm{Km}$ (Mass Transfer Coeff) & $5.29 \mathrm{E}-3$ & $\mathrm{~m} / \mathrm{s}$ & & \\
\hline $\begin{array}{l}\text { (Pool Diameter in Wind } \\
\text { Direction) }\end{array}$ & 2 & 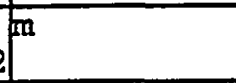 & & \\
\hline Sc (Schmidt Number) & 1.42 & & & \\
\hline$U$ (wind velocity) & 1.7 & $\mathrm{~m} / \mathrm{s}$ & & \\
\hline $\begin{array}{l}\text { nu (kinematic viscosity of } \\
\text { air) }\end{array}$ & $1.50 \mathrm{E}-5$ & $\mathrm{~m}^{\wedge} 2 / \sec$ & & \\
\hline mu (viscosity of air) & $1.90 \mathrm{E}-5$ & $\mathrm{~kg} / \mathrm{m}-\mathrm{s}$ & & $=$ \\
\hline $\begin{array}{l}\text { Dm (Molecular Diffusivity of } \\
\text { solute in Air) }\end{array}$ & $1.06 \mathrm{E}-5$ & $\mathrm{~m}^{\wedge} 2 / \mathrm{sec}$ & & \\
\hline $\begin{array}{l}\text { D_H20 (Molecular Diffusivity } \\
\text { of Water) }\end{array}$ & $2.40 \mathrm{E}-5$ & & & \\
\hline $\mathrm{MW} \_\mathrm{H} 2 \mathrm{O}$ & 18 & $\mathrm{~kg} / \mathrm{kg}-\mathrm{mole}$ & & \\
\hline \multicolumn{5}{|l|}{ Conversions } \\
\hline T (Absolute) & 302.15 & ${ }^{\circ} \mathrm{K}$ & & \\
\hline$V$ (Volume) & 0.035 & $m^{\wedge} 3$ & & \\
\hline Evaporation Rate & 65 & $g / m i n$ & & \\
\hline $60 \mathrm{~min} 1 \mathrm{~b}$ released & 8.57 & & & \\
\hline
\end{tabular}


Title of Project EPHA for THM

Subject Concentration Analysis for THM Hazardous Chemicals

Computer N.E. Baucom B. K. Tharakan Date 09/16/96 1-RD-11 AVARAGL SAE

SITE DATA INFORMATION: LOCation: AIKEN, SOUTH CAROLINA Building Air Exchanges Per Hour: 60 (User specified) Time: June 30, 19981200 hours EDT (User specified)

CHEMICAI INFORMATION : Chemical Name: AIPHA-PINENE Molecular Weight: $136.26 \mathrm{~kg} / \mathrm{kmol}$ TLV-TWA: -unavail- IDLH: -unavail Footprint Level of Concern: 1 ppm

Boiling Point: $155.00^{\circ} \mathrm{C}$

Vapor Pressure at Ambient Temperature: 0.0069 atm Ambient Saturation Concentration: 6,993 ppm or $0.70 \%$

Note: Not enough chemical data to use Heavy Gas option

ATMOSPHERIC INFORMATION: (MANUAL INPUT OF DATA)

Wind: 2.5 meters $/ \mathrm{sec}$ from $90^{\circ}$ true at 2 meters Inversion Height: 500 meters stability class: C (user override)

Air Temperature: $25^{\circ} \mathrm{C}$ Relative Humidity: 508 Cloud Cover: 8 tenths

Ground Roughness: Urban or forest

SOURCE STRENGTH INFORMATION:

Direct Source: $1.2 \mathrm{E}-2 \mathrm{kilograms} / \mathrm{sec}$

Source Height: 0

Release Duration: 60 minutes

Release Rate: 720 grams/min

Total Amount Released: 43.2 kilograms

TIME DEPENDENT INFORMATION:

Concentration Estimates at the point:

Downwind: 100 meters

off Centerline: 0 meters

Max Concentration:

Outdoor: 1. ppm

Indoor: $1 \mathrm{ppm}$

Note: Indoor graph is shown with a dotted line.
Reviewed by S. A. Henderson Works Calculation 8 Sheet No. 192 of 314 


\section{ENGINEERING COMPUTATION SHEET}

Title of Project EPHA for THM

Subject Concentration Analysis for THM Hazardous Chemicals

Computer N.E. Baucom B. K. Tharakan Date 09/16/96

SPIII AREA CALCUIATION FOR 1-RD-11 AVERAGE / SAE.CONDITIONS
Reviewed by S. A. Henderson

Works Calculation 8

Sheet No. 193 of 314

\begin{tabular}{|c|c|c|c|c|}
\hline Parameter & Value & Dimeneions & Value & $\begin{array}{l}\text { Dimenaions } \\
\text { (USA). }\end{array}$ \\
\hline E (Evaporation Rate) & $1.20 \mathrm{E}-2$ & $\mathrm{~kg} / \mathrm{s}$ & & \\
\hline MW (Molecular Weight) & 93 & $\mathrm{~kg} / \mathrm{kg}$-mole & & \\
\hline PV (Vapor Pressure) & 12.00 & $\operatorname{mm} \mathrm{Hg}$ & & \\
\hline Pv (Vapor Pressure) & 1600 & $\mathrm{~Pa}$ & & \\
\hline Rg (Gas Law Constant) & 8314 & $\mathrm{~J} / \mathrm{kgmole}-\mathrm{K}$ & & \\
\hline Temperature & 29 & ${ }^{\circ} \mathrm{C}$ & & \\
\hline Volume of spill & 117 & gal & & \\
\hline d (Depth of Pool) & 0.01 & $\mathrm{~m}$ & & \\
\hline A (Surface Area of Spill) & 44.3 & $m^{\wedge} 2$ & 477 & Ft^2 \\
\hline $\mathrm{Km}$ (Mass Transfer Coeff) & $4.59 \mathrm{E}-3$ & $\mathrm{~m} / \mathrm{s}$ & & \\
\hline $\begin{array}{l}\text { Z (Pool Diameter in wind } \\
\text { Direction) }\end{array}$ & 8 & $\mathbf{m}$ & & \\
\hline Sc (Schmidt Number) & 1.42 & & & \\
\hline $\mathrm{U}$ (wind velocity) & 1.7 & $\mathrm{~m} / \mathrm{s}$ & & \\
\hline $\begin{array}{l}\text { nu (kinematic viscosity of } \\
\text { air) }\end{array}$ & $1.50 \mathrm{E}-5$ & $m^{\wedge} 2 / \sec$ & & \\
\hline $\mathrm{mu}$ (viscosity of air) & $1.90 \mathrm{E}-5$ & $\mathrm{~kg} / \mathrm{m}-\mathrm{s}$ & & \\
\hline $\begin{array}{l}\text { Dm (Molecular Diffusivity of } \\
\text { Solute in Air) }\end{array}$ & $1.06 \mathrm{E}-5$ & $m^{\wedge} 2 / \sec$ & & \\
\hline $\begin{array}{l}\text { D_H20 (Molecular Diffusivity } \\
\text { of Water) }\end{array}$ & $2.40 \mathrm{E}-5$ & & & \\
\hline MW_H2O & 18 & $\mathrm{~kg} / \mathrm{kg}-\mathrm{mole}$ & & \\
\hline \multicolumn{5}{|l|}{ Conversions } \\
\hline T (Absolute) & 302.15 & ${ }^{\circ} \mathrm{K}$ & & \\
\hline V (Volume) & 0.443 & $\mathfrak{m}^{\wedge} 3$ & & \\
\hline Evaporation Rate & 719 & $\mathrm{~g} / \mathrm{min}$ & & \\
\hline $60 \mathrm{~min} 1 \mathrm{~b}$ released & 95.17 & & & \\
\hline
\end{tabular}


Title of Project EPHA for THM

Subject Concentration Analysis for THM Hazardous Chemicals

Computer N.E. Baucom B. K. Tharakan Date 09/16/96
Reviewed by S. A. Henderson

Works Calculation 8

Sheet No. 194 of 314

1-RD-11 AVARAOE unX DIGY GEO 1

SITE DATA INEORMATION:

Location: AIKEN, SOUTH CAROLINA

Building Air Exchanges Per Hour: 60 (User specified)

Time: June 30, 19981200 hours EDT (User specified)

CHEMICAL INFORMATION:

Chemical Name: ALPHA-PINENE

Molecular Weight: $136.26 \mathrm{~kg} / \mathrm{kmol}$

TLV-TWA: -unavail- IDLH: -unavail

Footprint Level of Concern: 1 ppm

Boiling Point: $155.00^{\circ} \mathrm{C}$

Vapor Pressure at Ambient Temperature: 0.0069 atm

Ambient Saturation Concentration: $6,993 \mathrm{ppm}$ or $0.70 \%$

Note: Not enough chemical data to use Heavy Gas option

ATMOSPHERIC INFORMATION: (MANUAL INPUT OR DATA)

Wind: 2.5 meters/sec from $90^{\circ}$ true at 2 meters

Inversion Height: 500 meters

Stability Class: C (user override)

Air Temperature: $25^{\circ} \mathrm{C}$

Relative Humidity: 50\% . Ground Roughness: Urban or forest

Cloud Cover: 8 tenths

SOURCE STRENGTH INEORMATION:

Direct Source: $4.94 \mathrm{E}-1$ kilograms/sec

Source Height: 0

Release Duration: 60 minutes

Release Rate: $29.6 \mathrm{kilograms} / \mathrm{min}$

Total Amount Released: 1,778 kilograms

FOOTPRINT INFORMATION:

Dispersion Module: Gaussian

User-specified LOC: $1 \mathrm{ppm}$

Max Threat Zone for LOC: 651 meters 
Title of Project EPHA for THM

Reviewed by S. A. Henderson

Subject Concentration Analysis for THM Hazardous Chemicals

Works Calculation 8

Computer N.E. Baucom B. K. Tharakan Date 09/16/96

Sheet No. 195 of 314

Determination of Iraporation rate for segment 1 uaximum_Source rern of $24620 \mathrm{ft}^{2}$

\begin{tabular}{|c|c|c|c|c|}
\hline Parameter & Value & Dimeneions & Value & $\begin{array}{c}\text { Dimensions } \\
\text { (OSA) }\end{array}$ \\
\hline E (Evaporation Rate) & $4.94 \mathrm{E}-1$ & $\mathrm{~kg} / \mathrm{s}$ & & \\
\hline MW (Molecular Weight) & 93 & $\mathrm{~kg} / \mathrm{kg}$-mole & & \\
\hline Pv (Vapor Pressure) & 12.00 & $\operatorname{min~} \mathrm{Hg}$ & & \\
\hline Pv (Vapor Pressure) & 1600 & $\mathrm{~Pa}$ & - & \\
\hline Rg (Gas Law Constant) & 8314 & J/kgmole-K & & \\
\hline Temperature & 29 & ${ }^{\circ} \mathrm{C}$ & & \\
\hline Volume of Spill & 5993 & gal & & \\
\hline$d$ (Depth of Pool) & 0.01 & $m$ & & \\
\hline A (Surface Area of Spill) & 2268.5 & $m^{\wedge} 2$ & 24421 & Et^2 \\
\hline $\mathrm{Km}$ (Mass Transfer Coeff) & $3.69 \mathrm{E}-3$ & $\mathrm{~m} / \mathrm{s}$ & & \\
\hline $\begin{array}{l}2 \text { (Pool Diameter in wind } \\
\text { Direction) }\end{array}$ & 54 & $\mathrm{~m}$ & & \\
\hline Sc (Schmidt Number) & 1.42 & & & \\
\hline $\mathrm{U}$ (wind velocity) & 1.7 & $\mathrm{~m} / \mathrm{s}$ & & $\therefore$ \\
\hline $\begin{array}{l}\text { nu (kinematic viscosity of } \\
\text { air) }\end{array}$ & $1.50 \mathrm{E}-5$ & $m^{\wedge} 2 / \sec$ & & \\
\hline mu (viscosity of air) & 1. $90 \mathrm{E}-5$ & $\mathrm{~kg} / \mathrm{m}-\mathrm{s}$ & & \\
\hline $\begin{array}{l}\text { Dm (Molecular Diffusivity of } \\
\text { Solute Air) }\end{array}$ & $1.06 \mathrm{E}-5$ & $m^{\wedge} 2 / \sec$ & & \\
\hline $\begin{array}{l}\text { D_H20 (Molecular Diffusivity } \\
\text { of Water) }\end{array}$ & $2.40 \mathrm{E}-5$ & & & \\
\hline MW_H20 & 18 & $\mathrm{~kg} / \mathrm{kg}$-mole & & \\
\hline \multicolumn{5}{|l|}{ Converaione } \\
\hline T (Absolute) & 302.15 & ${ }^{\circ} \mathrm{K}$ & & \\
\hline V (Volume) & 22.685 & $m^{\wedge} 3$ & & \\
\hline Evaporation Rate & 29613 & $\mathrm{~g} / \mathrm{min}$ & & \\
\hline $60 \mathrm{~min} 1 \mathrm{~b}$ released & 3917.12 & & & \\
\hline
\end{tabular}


Title of Project EPHA for THM

Reviewed by S. A. Henderson

Subject Concentration Analysis for THM Hazardous Chemicals

Works Calculation 8

Computer N.E. Baucom B. K. Tharakan Date 09/16/96

Sheet No. 196 of 314

1-RD-11 AVERAGE MAX DIST SEG 2

SITE DATA INFORMATION:

Location: AIKEN, SOUTH CAROLINA

Building Air Exchanges Per Hour: 60 (User specified)

Time: June 30, 19981200 hours EDT (User specified)

\section{CHEMICAL INFORMATION:}

Chemical Name: ALPHA-PINENE

Molecular Weight: $136.26 \mathrm{~kg} / \mathrm{kmol}$

TLV-TWA: -unavail-

IDLH: -unavail

Footprint Level of Concern: 1 ppm

Boiling Point: $155.00^{\circ} \mathrm{C}$

Vapor Pressure at Ambient Temperature: $0.0069 \mathrm{~atm}$

Ambient Saturation Concentration: 6,993 ppm or $0.70 \%$

Note: Not enough chemical data to use Heavy Gas option

ATMOSPHERIC INFORMATION: (MANUAL INPUT OF DATA)

Wind: 2.5 meters $/ \mathrm{sec}$ from $90^{\circ}$ true at 2 meters

Inversion Height: 500 meters

Stability Class: $\mathbf{C}$ (user override)

Air Temperature: $25^{\circ} \mathrm{C}$

Relative Humidity: 50\%

Ground Roughness: Urban or forest

Cloud Cover: 8 tenths

\section{SOURCE STRENGTH INFORMATION:}

Direct Source: 8E-1 kilograms/sec

Source Height: 0

Release Duration: 60 minutes

Release Rate: 48 kilograms/min

Total Amount Released: 2,880 kilograms r

FOOTPRINT INFORMATION:

Dispersion Module: Gaussian

User-specified LOC: 1 ppm

Max Threat Zone for LOC: 832 meters 
Title of Project EPHA for THM

Reviewed by S. A. Heniderson

Subject Concentration Analysis for THM Hazardous Chemicals

Works Calculation 8

Computer N.E. Baucom B. K. Tharakan Date 09/16/96

Determination of Evaporation Rate for Segment 2 Maximum Source Term of 10700 $\pm t^{2}$

\begin{tabular}{|c|c|c|c|c|}
\hline Parameter & Va1ue & Dimencions & Value & $\begin{array}{l}\text { Dimensions } \\
\text { (USA) }\end{array}$ \\
\hline E (Evaporation Rate) & $8.00 \mathrm{E}-1$ & $\mathrm{~kg} / \mathrm{s}$ & 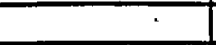 & \\
\hline MW (Molecular Weight) & 93 & $\mathrm{~kg} / \mathrm{kg}$-mole & & \\
\hline Pv (Vapor Pressure) & 12.00 & $\mathrm{~mm} \mathrm{Hg}$ & & \\
\hline Pv (Vapor Pressure) & 1600 & $\mathrm{~Pa}$ & & \\
\hline Rg (Gas Law Constant) & 8314 & J/kgmole-K & & \\
\hline Temperature & 29 & ${ }^{\circ} \mathrm{C}$ & & \\
\hline Volume of Spill & 9988 & Gal & & \\
\hline $\mathrm{d}$ (Depth of Pool) & 0.01 & $\mathbf{M}$ & & \\
\hline A (Surface Area of Spill) & 3780.8 & $\mathrm{~m}^{\wedge} 2$ & 40700 & $E t^{\wedge} 2$ \\
\hline $\mathrm{Km}$ (Mass Transfer Coeff) & $3.59 \mathrm{E}-3$ & $\mathrm{~m} / \mathrm{s}$ & & \\
\hline $\begin{array}{l}\mathrm{Z} \text { (Pool Diameter in Wind } \\
\text { Direction) }\end{array}$ & 69 & $M$ & & \\
\hline Sc (Schmidt Number) & 1.42 & & & \\
\hline $\mathrm{U}$ (wind velocity) & 1.7 & $\mathrm{~m} / \mathrm{s}$ & & \\
\hline $\begin{array}{l}\text { nu (kinematic viscosity of } \\
\text { air) }\end{array}$ & $1.50 \mathrm{E}-5$ & $\mathrm{~m}^{\wedge} 2 / \mathrm{sec}$ & & \\
\hline mu (viscosity of air) & $1.90 \mathrm{E}-5$ & $\mathrm{~kg} / \mathrm{m}-\mathrm{s}$ & & \\
\hline $\begin{array}{l}\text { Dm (Molecular Diffusivity of } \\
\text { Solute in Air) }\end{array}$ & $1.06 \mathrm{E}-5$ & $\mathrm{~m}^{\wedge} 2 / \mathrm{sec}$ & & \\
\hline $\begin{array}{l}\text { D_H20 (Molecular Diffusivity } \\
\text { of Water) }\end{array}$ & $2.40 E-5$ & & & \\
\hline $\mathrm{MW} / \mathrm{H2O}$ & 18 & $\mathrm{~kg} / \mathrm{kg}$-mole & & \\
\hline Converejone & & & & \\
\hline T (Absolute) & 302.15 & ${ }^{\circ} \mathbf{K}$ & & \\
\hline $\mathrm{V}$ (Volume) & 37.808 & $m^{\wedge} 3$ & & \\
\hline Evaporation Rate & 47973 & $\mathrm{~g} / \mathrm{min}$ & & \\
\hline $60 \mathrm{~min} 1 \mathrm{~b}$ released & 6345.66 & & & \\
\hline
\end{tabular}


Title of Project EPHA for THM

Reviewed by S. A. Henderson

Subject Concentration Analysis for THM Hazardous Chemicals

Works Calculation 8

Computer N.E. Baucom B. K. Tharakan Date 09/16/96

Sheet No. 198 of 314

1-RD-11 AVERAGE / MAX DIST SEG 3

SITE DATA INFORMATION:

Location: AIKEN, SOUTH CAROLINA

Building Air Exchanges Per Hour: 60 (User specified)

Time: June 30, 19981200 hours EDT (User specified)

CHEMICAL INFORMATION:

Chemical Name: ALPHA-PINENE

Molecular Weight: $136.26 \mathrm{~kg} / \mathrm{kmol}$

TLV-TWA: -unavail-

IDLH: -unavai

Footprint Level of Concern: 1 ppm

Boiling Point: $155.00^{\circ} \mathrm{C}$

Vapor Pressure at Ambient Temperature: 0.0069 atm

Ambient Saturation Concentration: 6,993 ppm or $0.70 \%$

Note: Not enough chemical data to use Heavy Gas option

ATMOSPHERIC INFORMATION: (MANUAL INPUT OF DATA)

Wind: 2.5 meters $/ \mathrm{sec}$ from $90^{\circ}$ true at 2 meters

Inversion Height: 500 meters

Stability Class: $\mathrm{C}$ (user override)

Air Temperature: $25^{\circ} \mathrm{C}$

Relative Humidity: 50\% Ground Roughness: Urban or forest

Cloud Cover: 8 tenths

\section{SOURCE STRENGTH INFORMATION:}

Direct Source: 2 kilograms/sec

Source Height: 0

Release Duration: 60 minutes

Release Rate: 120 kilograms/min

Total Amount Released: 7,200 kilograms

FOOTPRINT INFORMATION:

Dispersion Module: Gaussian

User-specified LOC: 1 ppm

Max Threat Zone for LOC: 1.3 kilometers 
Title of Project EPHA for THM

Subject Concentration Analysis for THM Hazardous Chemicals

\section{Computer N.E. Baucom B. K. Tharakan Date 09/16/96 \\ Determination of Evaporation Rate for Segment 3 maximum Source Term of 10000 $\mathrm{m}^{2}$}

\begin{tabular}{|c|c|c|c|c|}
\hline Parameter & Value & Dimensions: & Value & $\begin{array}{c}\text { Dimensions } \\
\text { (USA) }\end{array}$ \\
\hline E (Evaporation Rate) & $2.00 \mathrm{E}+0$ & $\mathrm{~kg} / \mathrm{s}$ & & \\
\hline MW (Molecular Weight) & 93 & $\mathrm{~kg} / \mathrm{kg}-\mathrm{mole}$ & & \\
\hline PV (Vapor Pressure) & 12.00 & $\mathrm{~mm} \mathrm{Hg}$ & & \\
\hline Pv (Vapor Pressure) & 1600 & $\mathrm{~Pa}$ & & \\
\hline Rg (Gas Law Constant) & 8314 & J/kgmole-K & & \\
\hline Temperature & 29 & ${ }^{\circ} \mathrm{C}$ & & \\
\hline Volume of Spill & 26418 & gal & & \\
\hline d (Depth of Pool) & 0.01 & $\mathrm{~m}$ & & \\
\hline A (Surface Area of Spill) & 10000.0 & $m^{\wedge} 2$ & 107650 & $f t^{\wedge} 2$ \\
\hline $\mathrm{Km}$ (Mass Transfer Coeff) & $3.40 \mathrm{E}-3$ & $\mathrm{~m} / \mathrm{s}$ & & \\
\hline $\begin{array}{l}2 \text { (PooI Diameter in Wind } \\
\text { Direction) }\end{array}$ & 113 & $\mathfrak{m}$ & & \\
\hline Sc (Schmidt Number) & 1.42 & & & \\
\hline$U$ (wind velocity) & 1.7 & $\mathrm{~m} / \mathrm{s}$ & & \\
\hline $\begin{array}{l}\text { nu (kinematic viscosity of } \\
\text { air) }\end{array}$ & $1.50 \mathrm{E}-5$ & $m^{\wedge} 2 / \sec$ & & \\
\hline mu (viscosity of air) & $1.90 \mathrm{E}-5$ & $\mathrm{~kg} / \mathrm{m}-\mathrm{s}$ & $\cdot$ & \\
\hline $\begin{array}{l}\text { Dm (Molecular Diffusivity of } \\
\text { Solute in Air) }\end{array}$ & $1.06 \mathrm{E}-5$ & $m^{\wedge} 2 / \mathrm{sec}$ & & \\
\hline $\begin{array}{l}\text { D_H20 (Molecular Diffusivity } \\
\text { of Water) }\end{array}$ & $2.40 \mathrm{E}-5$ & & & \\
\hline MW_H2O & 18 & $\mathrm{~kg} / \mathrm{kg}$-mole & & \\
\hline \multicolumn{5}{|l|}{ Converaions } \\
\hline T (Absolute) & 302.15 & ${ }^{\circ} \mathbf{R}$ & & \\
\hline V (Volume) & 100.000 & $m^{\wedge} 3$ & & \\
\hline Evaporation Rate & 120213 & $\mathrm{~g} / \mathrm{min}$ & & \\
\hline $60 \mathrm{~min} 1 \mathrm{~b}$ released & 15901.21 & & & \\
\hline
\end{tabular}


Title of Project EPHA for THM

Reviewed by S. A. Henderson

SITE DATA INFORMATION:

Location: AIREN, SOUTH CAROLINA

Building Air Exchanges Per Hour: 60 (User specified)

Time: June 30, 19981200 hours EDT (User specified)

CHEMICAL INFORMATION:

Chemical Name: CYCLOHEXANONE

Molecular Weight: $98.14 \mathrm{~kg} / \mathrm{kmol}$

TLV-TWA: $25 \mathrm{ppm}$ IDLH: $700 \mathrm{ppm}$

Footprint Level of Concern: $125 \mathrm{ppm}$

Boiling point: $155.75^{\circ} \mathrm{C}$

Vapor Pressure at Ambient Temperature: $0.0056 \mathrm{~atm}$

Ambient Saturation Concentration: 5,722 ppm or $0.57 \%$

ATMOSPHERIC INFORMATION: (MANUAL INPUT OF DATA)

Wind: 2.5 meters/sec from $90^{\circ}$ true at 2 meters

Inversion Height: 500 meters

Stability class: C (user override)

Air Temperature: $25^{\circ} \mathrm{C}$

Relative Humidity: $508 \quad$ Ground Roughness: Urban or forest

Cloud Cover: 8 tenths

SOURCE STRENGTH INFORMATION:

Pudale Area: 30001 square feet

Average Puddie Depth: 1 centimeters

Soil Type: Default Ground Temperature: $25^{\circ} \mathrm{C}$

Initial Puddle Temperature: Ground temperature

Release Duration: ALOHA limited the duration to 1 hour

Max Computed Release Rate: $23.6 \mathrm{kilograms/min}$

Max Average Sustained Release Rate: $23.3 \mathrm{kilograms} / \mathrm{min}$

(averaged over a minute or more)

Total Amount Released: 1,274 kilograms

FOOTRRINT INFORMATION:

Dispersion Module: Gaussian

User-specified LOC: 125 ppm

Max Threat Zone for LOC: 30 meters

Max Threat Zone for IDLF: 30 meters
Note: Footprint was not drawn because effects of near-field patchiness make dispersion predictions unreliable for short distances.

TIME DEPENDENT INFORMATION:

Concentration Estimates at the point:

Downwind: $\quad 30$ meters

off Centerline: 0 meters

Max Concentration:

Outdoor: $99.6 \mathrm{ppm}$

Indoor: $99.6 \mathrm{ppm}$

Note: Indoor graph is shown with a dotted line. 
Title of Project EPHA for THM

Subject Concentration Analysis for THM Hazardous Chemicals

Computer N.E. Baucom B. K. Tharakan Date 09/16/96

1-RD-IL AVERAGE / SAR

SITE DATA INEORMATION:

Location: AIREN, SOUTH CAROLINA

Building Ailr Exchanges Per Hour: 60 (User specified)

Time: June 30, 19981200 hours EDT (User specified)

CHEMICAL INFORMATION :

Chemical Name: CYCLOHEXANONE

Moleculax Weight: $98.14 \mathrm{~kg} / \mathrm{kmol}$

TLV-TWA: 25 ppm

Footprint Level of Concern: 125 ppm

Boiling Point: $155.75^{\circ} \mathrm{C}$

Vapor Pressure at Ambient Temperature: $0.0056 \mathrm{~atm}$

Ambient Saturation Concentration: 5,722 ppm or 0.578

ATMOSPHERIC INFORMATION: (MANUAL INPUT OF DATA)

wind: 2.5 meters/sec from $90^{\circ}$ true at 2 meters

Inversion Height: 500 meters

Stability Class: C (user override)

Air Temperature: $25^{\circ} \mathrm{C}$

Relative Humidity: 508

cloud Cover: 8 tenths

SOURCE STRENGTH INFORMATION:

Puddle Area: 100001 square feet

Average Puddle Depth: 1 centimeters

Soil Type: Default Ground Temperature: $25^{\circ} \mathrm{C}$

Initial Pudale Temperature: Ground temperature

Release Duration: ALOHA limited the duration to 1 hour

Max Computed Release Rate: 75.2 kilograms/min

Max Average Sustained Release Rate: $74.2 \mathrm{kilograms} / \mathrm{min}$

(averaged over a minute or more)

Total Amount Released: 4,049 kilograms

FOOTPRINT INFORMATION:

Dispersion Module: Gaussian

User-specified LOC: 125 ppm

Max Threat Zone for IOC: 55 meters

Max Threat Zone for IDLF: 54 meters

Note: Footprint was not drawn because effects of near-field patchiness make dispersion predictions unreliable for short distances.

TIME DEPENDENT INFORMATION:

Concentration Estimates at the point:

Downwind: $\quad 100$ meters

off Centerline: 0 meters

Max Concentration:

outdoor: $31.3 \mathrm{ppm}$

Indoor: $31.3 \mathrm{ppm}$

Note: Indoor graph is shown with a dotted line.
Reviewed by S. A. Henderson

Works Calculation 8

Sheet No. 201 of 314

Note: Indoor graph is shown with a dotted 1 ine. 
Title of Project EPHA for THM

\section{ENGINEERING COMPUTATION SHEET}

Subject Concentration Analysis for THM Hazardous Chemicals

Computer N.E. Baucom B. K. Tharakan Date 09/16/96

1-RD-14 AVERAGE waX DIST SE:Q I

SITE DATA INFORMATION:

Location: AIKEN, SOUTH CAROLINA

Building Air Exchanges Per Hour: 60 (User specified)

Time: June 30, 19981200 hours EDT (User specified)

CHEMICAL INFORMATION:

Chemical Name: CYCLOHEXANONE

Molecular Weight: $98.14 \mathrm{~kg} / \mathrm{kmol}$

TLV-TWA: $25 \mathrm{ppm}$

Footprint Level of Concern: 125 ppm

Boiling Point: $155.75^{\circ} \mathrm{C}$

Vapor Pressure at Ambient Temperature: 0.0056 atm

Ambient Saturation Concentration: $5,722 \mathrm{ppm}$ or 0.578

ATMOSPHERIC INFORMATION: (MANUAL INPUT OF DATA)

wind: 2.5 meters $/ \mathrm{sec}$ from $90^{\circ}$ true at 2 meters

Inversion Height: 500 meters

stability Class: C (user override)

Aix Temperature: $25^{\circ} \mathrm{C}$

Relative Humidity: 508

Cloud Cover: 8 tenths

Ground Roughness: Urban or forest

SOURCE STRENGTH INFORMATION:

Puddle Area: 24420 square feet

Average Pudale Depth: 1 centimeters

Soil Type: Default

Ground Temperature: $25^{\circ} \mathrm{C}$

Initial puddle Temperature: Ground temperature

Release Duration: ALOHA limited the duration to 1 hour

Max Computed Release Rate: 19.4 kilograms/min

Max Average Sustained Release Rate: 19.1 kilograms/min (averaged over a minute or more)

Total Amount Released: 1,046 kilograms

FOOTPRINT INFORMATION:

Dispersịon Module: Gaussian

User-specified LOC: 125 ppm

Max Threat zone for LOC: 27 meters

Max Threat zone for IDLH: 27 meters
Reviewed by S.A. Henderson

Works Calculation 8

Sheet No. 202 of 314 
Title of Project EPHA for THM

Reviewed by S. A. Henderson

SITE DATA INFORMATION:

Location: AIKEN ' SOUTH CAROLINA

Building Air Exchanges Per Hour: 60 (User specified)

Time: June 30, 19981200 hours EDT (User specified)

CHEMICAL INFORMATION:

Chemical Name: CYCLOHEXANONE

Molecular Weight: $98.14 \mathrm{~kg} / \mathrm{kmol}$

TLV-TWA: $25 \mathrm{ppm}$ IDLH: $700 \mathrm{ppm}$

Footprint Level of Concern: 125 ppm

Boiling Point: $155.75^{\circ} \mathrm{C}$

Vapor Pressure at Ambient Temperature: 0.0056 atm

Ambient Saturation Concentration: 5,722 ppm or $0.57 \%$

ATMOSPHERIC INFORMATION: (MANUAL INPUT OF DATA)

Wind: 2.5 meters/sec from $90^{\circ}$ true at 2 meters

Inversion Height: 500 meters

stabilitty class: C (user override)

Air Temperature: $25^{\circ} \mathrm{C}$

Relative Humidity: 508 Ground Roughness: Urban or forest

Cloud Cover: 8 tenths

SOURCE STRENGTH INFORMATION:

Puddle Area: 40700 square feet

Average Puddle Depth: 1 centimeters

Soil Type: Default Ground Temperature: $25^{\circ}$

Initial puddle Temperature: Ground temperature

Release Duration: ALOHA limited the duration to 1 hour

Max Computed Release Rate: $31.7 \mathrm{kilograms/min}$

Max Average Sustained Release Rate: $31.2 \mathrm{kilograms/min}$

(averaged over a minute or more)

Total Amount Released: 1,707 kilograms

FOOTPRINT INFORMATION:

Dispersion Module: Gaussian

User-specified LOC: 125 ppm

Max Threat Zone for LOC: 34 meters

Max Threat Zone for IDLH: 35 meters 
Title of Project EPHA for THM

\section{ENGINEERING COMPUTATION SHEET}

Subject Concentration Analysis for THM Hazardous Chemicals

Computer N.E. Baucom B. K. Tharakan Date 09/16/96

\section{1-RD-14 AVERAGE MDX DIST SEO 3}

SITE DATA INFORMATION:

LOCation: AIREN, SOUTH CAROLINA

Building Air Exchanges Per Hour: 60 (User specified)

Time: June 30, 19981200 hours EDT (User specified)

CHEMICAI INFORMATION :

Chemical Name: CYCLOHEXANONE

Molecular Weight: $98.14 \mathrm{~kg} / \mathrm{kmol}$

TLV-TWA: $25 \mathrm{ppm}$ IDLH: $700 \mathrm{ppm}$

Footprint Level of Concern: 125 ppm

Boiling Point: $155.75^{\circ} \mathrm{C}$

Vapor Pressure at Ambient Temperature: 0.0056 atm

Ambient Saturation Concentration: 5,722 ppm or $0.57 \%$

ATMOSPHERIC INFORMATION: (MANUAL INPUT OF DATA)

Wind: 2.5 meters/sec from $90^{\circ}$ true at 2 meters

Inversion Height: 500 meters

stability Class: C (user override)

Air Temperature: $25^{\circ} \mathrm{C}$

Relative Humidity: 508

Cloud Cover: 8 tenths

Ground Roughness: Urban or forest

SOURCE STRENGTH INFORMATION:

Puddle Area: 10000 square meters

Average Puddle Depth: 1 centimeters

Soil Type: Default Ground Temperature: $25^{\circ} \mathrm{C}$

Initial Puddle Temperature: Ground temperature

Release Duration: ALOHA limited the duration to 1 hour

Max Computed Release Rate: $80.7 \mathrm{kilograms/min}$

Max Average Sustained Release Rate: $79.6 \mathrm{kilograms/min}$

(averaged over a minute or more)

Total Amount Released: 4,346 kilograms

FOOTPRINT INFORMATION:

Dispersion Module: Gaussian

User-specified LOC: 125 ppm

Max Threat Zone for LOC: 56 meters

Max Threat zone for IDLH: 56 meters
Reviewed by S. A. Henderson

Works Calculation 8

Sheet No. 204 of 314 
Title of Project EPHA for THM

Subject Concentration Analysis for THM Hazardous Chemicals

Computer N.E. Baucom B. K. Tharakan Date 09/16/96 I-RD-15 AVERACT / ANERT

SITE DATA INFORMATION:

Location: AIKEN, SOUTH CAROLINA

Building Air Exchanges Per Hour: 60 (User specified)

Time: June 30, 19981200 hours EDT (User specified)

CHEMICAL INFORMATION:

Chemical Name: DIETHYLENE GLYCOL MONOETHYI ETHER

Molecular Weight: $134.18 \mathrm{~kg} / \mathrm{kmol}$

TLV-TWA: - unavail- IDLH: -unavail

Footprint Level of Concern: $100 \mathrm{ppm}$

Boiling Point: $202.00^{\circ} \mathrm{C}$

Vapor Pressure at Ambient Temperature: $1.66 \mathrm{e}-004$ atm

Ambient Saturation Concentration: $169 \mathrm{ppm}$ or $0.017 \%$

ATMOSPHERIC INFORMATION: (MANUAC INPUT OF DATA)

Wind: 2.5 meters/sec from $90^{\circ}$ true at 2 meters

Inversion Height: 500 meters

stability class: C (user override

Air Temperature: $25^{\circ} \mathrm{C}$

Relative Humidity: $50 \%$

Cloud Cover: 8 tenths

Ground Roughness: Urban or forest

SOURCE STRENGTH INFORMATION:

Puddle Area: 30001 square feet

Average Puddle Depth: 1 centimeters

Soil Type: Default

Ground Temperature: $25^{\circ}$

Initial Puddle Temperature: Ground temperature

Release Duration: ALOHA limited the duration to 1 hour

Max Computed Release Rate: $1.21 \mathrm{kilograms/min}$

Max Average Sustained Release Rate: $1.18 \mathrm{kilograms} / \mathrm{min}$

(Averaged over a minute or more)

Total Amount Released: 60.3 kilograms

FOOTPRINT INEORMATION:

Dispersion Module: Gaussian

User-specified LOC: $100 \mathrm{ppm}$

Max Threat Zone for LOC: 30 meters

Note: Footprint was not drawn because effects of near-field patchiness make dispersion predictions unreliable for short distances.

TIME DEPENDENT INFORMATION:

Concentration Estimates at the point:

Downwind:

30 meters

off Centerline: 0 meters

Max Concentration:

Outdoor: $3.68 \mathrm{ppm}$

Indoor: $3.68 \mathrm{ppm}$

Note: Indoor graph is shown with a dotted line.
Reviewed by S. A. Henderson

Works Calculation 8

Sheet No. 205 of 314 
Title of Project EPHA for THM

Subject Concentration Analysis for THM Hazardous Chemicals

Reviewed by S. A. Henderson

Computer N.E. Baucom B. K. Tharakan Date 09/16/96

SITE DATA INFORMATION:

Location: AIKEN, SOUTH CAROLINA

Building Air Exchanges Per Hour: 60 (User specified)

Time: June 30, 19981200 hours EDT (User specified)

CHEMICAI INFORMATION:

Chemical Name: DIETHYLENE GLYCOL MONOETHYL ETHER

Molecular Weight: $134.18 \mathrm{~kg} / \mathrm{kmol}$

TLV-TWA: - unavail-

Footprint Level of Concern: $100 \mathrm{ppm}$

Boiling Point: $202.00^{\circ} \mathrm{C}$

Vapor Pressure at Ambient Temperature: $1.66 \mathrm{e}-004$ atm

Ambient Saturation Concentration: 169 ppm or $0.017 \%$

ATMOSPHERIC INFORMATION: (MANUAL INPUT OF DATA)

Wind: 2.5 meters/sec from $90^{\circ}$ true at 2 meters

Inversion Height: 500 meters

Stability Class: C (user override)

Air Temperature: $25^{\circ} \mathrm{C}$

Relative Humidity: 508. Ground Roughness: Urban or forest

Cloud Cover: 8 tenths

SOURCE STRENGTH INFORMATION:

Puddle Area: 100001 square feet

Average Pudale Depth: 1 centimeters

Soil Type: Default Ground Temperature: $25^{\circ} \mathrm{C}$

Initial Puddle Temperature: Ground temperature

Release Duration: ALOHA limited the duration to 1 hour

Max Computed Release Rate: $3.85 \mathrm{kilograms/min}$

Max Average Sustained Release Rate: $3.75 \mathrm{kilograms} / \mathrm{min}$

(averaged over a minute or more)

Total Amount Released: 192 kilograms

FOOTPRINT INFORMATION:

Dispersion Module: Gaussian

User-specified LOC: 100 ppm

Max Threat zone for LOC: 55 meters

Note: Footprint was not drawn because effects of near-field patchiness make dispersion predictions urireliable for short distances.

TIME DEPENDENT INFORMATION:

Concentration Estimates at the point:

Downwind: $\quad 100$ meters

off Centerline: 0 meters

Max Concentration:

outdoor: $1.16 \mathrm{ppm}$

Indoor: $1.16 \mathrm{ppm}$

Note: Indoor graph is shown with a dotted line. 
Title of Project EPHA for THM

Subject Concentration Analysis for THM Hazardous Chemicals

Computer N.E. Baucom B. K. Tharakan Date $\underline{09 / 16 / 96}$

I-RD-15 AVARACI / Max DIST , SESO 1

SITE DATA INFORMATION:

Location: AIREN, SOUTH CAROLINA

Building Air Exchanges Per Hour: 60 (User specified)

Time: June 30, 19981200 hours EDT (User specified)

CHEMICAL INFORMATION :

Chemical Name: DIETHYLENE GLYCOL MONOETFYL ETHER

Molecular Weight: $134.18 \mathrm{~kg} / \mathrm{kmol}$

TLV-TWA: -unavail-

Footprint Level of Concern: $100 \mathrm{ppm}$

Boiling Point: $202.00^{\circ} \mathrm{C}$

Vapor Pressure at Ambient Temperature: $1.66 \mathrm{e}-004 \mathrm{~atm}$

Ambient Saturation Concentration: $169 \mathrm{ppm}$ or 0.0178

ATMOSPHERIC INEORMATION: (MANUAL INPUT OF DATA)

Wind: 2.5 meters/sec from $90^{\circ}$ true at 2 meters

Inversion Height: 500 meters

Stability Class: C (user override)

Air Temperature: $25^{\circ} \mathrm{C}$

Relative Humidity: $50 \%$

Cloud Cover: 8 tenths

Ground Roughness: Urban or forest

SOURCE STRENGTH INFORMATION:

Puddle Area: 24420 square feet

Average Puddle Depth: 1 centimeters

Soll Type: Default

Initial Puddle Temperature: Ground temperature

Releaseburation: AIOHA limited the duration to 1 hour

Max Computed Release Rate: 990 grams/min

MaxAverage Sustained Release Rate: 966 grams/min

(averaged over a minute or more)

Total Amount Released: 49.5 kilograms

FOOTPRINT INFORMATION:

Dispersion Module: Gaussian

User-specified LOC: $100 \mathrm{ppm}$

Max Threat zone for LOC: 27 meters

Note: Footprint was not drawn because effects of near-field patchiness make dispersion predictions unxeliable for short distances.
Reviewed by S. A. Henderson

Works Calculation 8

Sheet No. 207 of 314
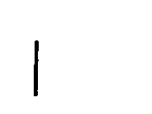
Title of Project EPHA for THM

Subject Concentration Analysis for THM Hazardous Chemicals

Computer N.E. Baucom B. K. Tharakan Date 09/16/96

1-RD.15 AVERAGE MAX DIST SEG 2

SITE DATA INFORMATION:

Location: AIKEN, SOUTH CAROLINA

Building Air Exchanges Per Hour: 60 (User specified)

Time: June 30, 19981200 hours EDT (User specified)

CHEMICAL INFORMATION:

Chemical Name: DIETHYLENE GLYCOL MONOETHYL ETHER

Molecular Weight: $134.18 \mathrm{~kg} / \mathrm{kmol}$

TLV-TWA: -unavail-

Footprint Level of Concern:

Boiling Point: $202.00^{\circ} \mathrm{C}$

Vapor Pressure at Ambient Temperature: $1.66 \mathrm{e}-004 \mathrm{~atm}$

Ambient Saturation Concentration: 169 ppm or $0.017 \%$

ATMOSPHERIC INFORMATION: (MANUAL INPUT OF DATA)

Wind: 2.5 meters $/ \mathrm{sec}$ from $90^{\circ}$ true at 2 meters

Inversion Height: 500 meters

Stability Class: $\mathrm{C}$ (user override)

Air Temperature: $25^{\circ} \mathrm{C}$

Relative Humidity: $50 \%$

Cloud Cover: 8 tenths

SOURCE STRENGTH INFORMATION:

Puddle Area: 40700 square feet

Average Puddle Depth: 1 centimeters

Soil Type: Default

IDLH: -unavail

$100 \mathrm{ppm}$

Initial Puddle Temperature: Ground temperature

Release Duration: ALOHA limited the duration to 1 hour

Max Computed Release Rate: 1.62 kilograms/min

Max Average Sustained Release Rate: 1.58 kilograms/min

(averaged over a minute or more)

Total Amount Released: 80.8 kilograms

FOOTPRINT INFORMATION:

Dispersion Module: Gaussian

User-specified LOC: 100 ppm

Max Threat Zone for LOC: 34 meters

Note: Footprint was not drawn because effects of near-field patchiness make dispersion predictions unreliable for short distances.
Reviewed by S. A. Henderson

Works Calculation 8

Sheet No. 208 of 314 


\section{1-RD-15 AVERAGE MAX DIST SEG 3}

\section{SITE DATA INFORMATION:}

Location: AIKEN, SOUTH CAROLINA

Building Air Exchanges Per Hour: 60 (User specified)

Time: June 30, 19981200 hours EDT (User specified)

\section{CHEMICAL INFORMATION:}

Chemical Name: DIETHYLENE GLYCOL MONOETHYL ETHER

Molecular Weight: $134.18 \mathrm{~kg} / \mathrm{kmol}$

TLV-TWA: -unavail-

IDLH: -unavail

Footprint Level of Concern: 100 ppm

Boiling Point: $202.00^{\circ} \mathrm{C}$

Vapor Pressure at Ambient Temperature: 1.66e-004 atm

Ambient Saturation Concentration: 169 ppm or $0.017 \%$

\section{ATMOSPHERIC INFORMATION: (MANUAL INPUT OF DATA)}

Wind: 2.5 meters $/ \mathrm{sec}$ from $90^{\circ}$ true at 2 meters

Inversion Height: 500 meters

Stability Class: $\mathrm{C}$ (user override)

Air Temperature: $25^{\circ} \mathrm{C}$

Relative Humidity: $50 \%$

Ground Roughness: Urban or forest

Cloud Cover: 8 tenths

\section{SOURCE STRENGTH INFORMATION:}

Puddle Area: 10000 square meters

Average Puddle Depth: 1 centimeters

Soil Type: Default

Ground Temperature: $25^{\circ} \mathrm{C}$

Initial Puddle Temperature: Ground temperature

Release Duration: ALOHA limited the duration to 1 hour

Max Computed Release Rate: 4.13 kilograms/min

Max Average Sustained Release Rate: 4.03 kilograms/min

(averaged over a minute or more)

Total Amount Released: 206 kilograms

\section{FOOTPRINT INFORMATION:}

Dispersion Module: Gaussian

User-specified LOC: 100 ppm

Max Threat Zone for LOC: 56 meters

Note: Footprint was not drawn because effects of near-field patchiness make dispersion predictions unreliable for short distances. 
Title of Project EPHA for THM

Subject Concentration Analysis for THM Hazardous Chemicals

Computer N.E, Baucom B. K. Tharakan Date 09/16/96 1-RD-16 AVARACE ATIRT
Reviewed by S. A. Henderson

Works Calculation 8

Sheet No. 210 of 314

SITE DATA INFORMATION:

LOcation: AIKEN, SOUTH CAROLINA

Building Air Exchanges Per Hour: 60 (User specified)

Time: June 30, 19981200 hours EDT (User specified)

CHEMICAL INFORMATION:

Chemical Name: DIOXANE Molecular Weight: $88.11 \mathrm{~kg} / \mathrm{kmol}$

TLV-TWA: $25 \mathrm{ppm}$ IDLH: $500 \mathrm{ppm}$

Warning: Potential or confirmed human carcinogen.

Footprint Level of Concern: $75 \mathrm{ppm}$

Boiling point: $101.32^{\circ} \mathrm{C}$

Vapor Pressure at Ambient Temperature: 0.050 atm

Ambient Saturation Concentration: $51,172 \mathrm{ppm}$ or 5.128

ATMOSPHERIC INFORMATION: (MANUAL INPUT OF DATA)

Wind: 2.5 meters $/ \mathrm{sec}$ from $90^{\circ}$ true at 2 meters

Inversion Height: 500 meters

Stability Class: C (user override)

Air Temperature: $25^{\circ} \mathrm{C}$

Relative Humidity: $50 \%$

Ground Roughness: Urban or forest

Cloud Cover: 8 tenths

SOURCE STRENGTH INFORMATION:

Puddle Area: 997 square feet

Average Puddle Depth: 1 centimeters

Soil Type: Default Ground Temperature: $25^{\circ} \mathrm{C}$

Initial Puddle Temperature: Ground temperature

Release Duration: ALOHA limited the duration to 1 hour

Max Computed Release Rate: 5.36 kilograms/min

Max Average Sustained Release Rate: 5.36 kilograms/min

(averaged over a minute or more)

Total Amount Released: 319 kilograms

FOOTPRINT INFORMATION :

Dispersion Module: Gaussian

User-specified LOC: $75 \mathrm{ppm}$

Max Threat zone for LOC: 30 meters

Max Threat Zone for IDLH: less than 10 meters (10.9 yards)

Note: Footprint was not drawn because effects of near-field patchiness make dispersion predictions unreliable for short distances.

TIME DEPENDENT INFORMATION:

Concentration Estimates at the point:

Downwind:

30 meters

off Centerline: 0 meters

Max Concentration:

outdoor: $75 \mathrm{ppm}$

Indoor: $75 \mathrm{ppm}$

Note: Indoor graph is shown with a dotted line. 
Title of Project EPHA for THM

Subject Concentration Analysis for THM Hazardous Chemicals

Computer N.E. Baucom B. K. Tharakan Date 09/16/96

1-RD-16 AVARAT SAE
Reviewed by S. A. Henderson

Works Calculation 8

Sheet No. 211 of 314

SITE DATA INFORMATION:

Location: AIKEN, SOUTH CAROLINA

Building Air Exchanges Per Hour: 60 (User specified)

Time: June 30, 19981200 hours EDT (User specified)

CHEMICAL INFORMATION:

Chemical Name: DIOXANE Molecular Weight: $88.11 \mathrm{~kg} / \mathrm{kmol}$

TLV-TWA: $25 \mathrm{ppm}$

IDLF: $500 \mathrm{ppm}$

Warning: Potential or confirmed human carcinogen.

Footprint Level of Concern: $75 \mathrm{ppm}$

Boiling Point: $101.32^{\circ} \mathrm{C}$

Vapor Pressure at Ambient Temperature: 0.050 atm

Ambient Saturation Concentration: 51,172 ppm or 5.128

ATMOSPHERIC INFORMATION: (MANUAL INPUT OF DATA)

Wind: 2.5 meters/sec from $90^{\circ}$ true at 2 meters

Inversion Height: 500 meters

Stability Class: C (user override)

Air Temperature: $25^{\circ} \mathrm{C}$

Relative Humidity: 508 .

Ground Roughness: Urban or forest

Cloud Cover: 8 tenths

SOURCE STRENGTH INFORMATION:

Puddle Area: 13000 square feet

Average Puddle Depth: 1 centimeters

Soil Type: Default Ground Temperature: $25^{\circ} \mathrm{C}$

Initial Pudale Temperature: Ground temperature

Release Duration: AlOHA limited the duration to I hour

Max Computed Release Rate: $63.7 \mathrm{kilograms} / \mathrm{min}$

Max Average Sustained Release Rate: $63.6 \mathrm{kilograms} / \mathrm{min}$ (averaged over a minute or more)

Total Amount Released: 3,765 kilograms

FOOTPRINT INFORMATION:

Dispexsion Module: Gaussian

User-specified LOC: 75 ppm

Max Threat Zone for LOC: 100 meters

Max Threat Zone for IDLH: 23 meters

Note: Footprint was not drawn because effects of near-field patchiness make dispersion predictions unreliable for short distances.

TIME DEPENDENT INFORMATION:

Concentration Estimates at the point:

Downwind: $\quad 100$ meters

off Centerline: 0 meters

Max Concentration:

Outdoor: $75 \mathrm{ppm}$

Indoor: 75 ppm 


\section{ENGINEERING COMPUTATION SHEET}

Title of Project EPHA for THM

Subject Concentration Analysis for THM Hazardous Chemicals

Computer N.E. Baucom B. K. Tharakan Date 09/16/96 1-RD-16 AVARACE MAX DIST SEO 1

SITE DATA INFORMATION:

LOCation: AIKEN, SOUTH CAROLINA

Building Air Exchanges Per Hour: 60 (User specified)

Time: June 30, 19981200 hours EDT (User specified)

CHEMICAL INFORMATION:

Chemical Name: DIOXANE Molecular Weight: $88.11 \mathrm{~kg} / \mathrm{kmol}$

TLV-TWA: $25 \mathrm{ppm}$

Molecular Weight: $88.11 \mathrm{~kg} / \mathrm{kmol}$
IDLH: $500 \mathrm{ppm}$

Warning: Potential or confirmed human carcinogen.

Footprint Level of Concern: 75 ppm

Boiling Point: $101.32^{\circ} \mathrm{C}$

Vapor Pressure at Ambient Temperature: $0.050 \mathrm{~atm}$

Ambient Saturation Concentration: 51,172 ppm or $5.12 \%$

ATMOSPHERIC INFORMATION: (MANUAL INPUT OF DATA)

Wind: 2.5 meters $/ \mathrm{sec}$ from $90^{\circ}$ true at 2 meters

Inversion Height: 500 meters

Stability Class: $c$ (user override)

Air Temperature: $25^{\circ} \mathrm{C}$

Relative Humidity: $508 \quad$ Ground Roughness: Urban or forest

Cloud Cover: 8 tenths

SOURCE STRENGTH INFORMATION:

Puddle Area: 24420 square feet

Average Puddle Depth: 1 centimeters

Soil Type: Default

Ground Temperature: $25^{\circ} \mathrm{C}$

Initial puddle Temperature: Ground temperature

Release Duration: ALOHA limited the duration to 1 hour

Max Computed Release Rate: 117 kilograms/min

Max Average Sustained Release Rate: 117 kilograms/min

(averaged over a minute or more)

Total Amount Released: 6,910 kilograms

FOOTPRINT INEORMATION:

Dispersion Module: Gaussian

User-specified. LOC: 75 ppm

Max Threat Zone for LOC: 135 meters

Max Threat Zone for IDLH: 31 meters
Reviewed by S. A. Henderson

Works Calculation 8

Sheet No. $\underline{212 \text { of } 314}$

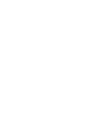


Titte of Project EPHA for THM

Subject Concentration Analysis for THM Hazardous Chemicals

Computer N.E. Baucom B. K. Tharakan Date 09/16/96 1-RD-16 AVGRAG MAX DIST SEC 2

SITE DATA INEORMATION:

Location: AIKEN, SOUTH CAROLINA

Building Air Exchanges Per Hour: 60 (User specified)

Time: June 30, 19981200 hours EDT (User specified)

CHEMICAL INFORMATION:

Chemical Name: DIOXANE Molecular Weight: $88.11 \mathrm{~kg} / \mathrm{kmol}$

TLV-TWA: 25 ppm

IDLH: $500 \mathrm{ppm}$

Warning: Potential or confirmed human carcinogen.

Footprint Level of Concern: $75 \mathrm{ppm}$

Boiling point: $101.32^{\circ} \mathrm{C}$

Vapor Pressure at Ambient Temperature: 0.050 atm

Ambient Saturation Concentration: 51,172 ppm or 5.128

ATMOSPHERIC INFORMATION: (MANUAL INPUT OF DATA)

Wind: 2.5 meters/sec from $90^{\circ}$ true at 2 meters

Inversion Height: 500 meters

stability class: C (user override)

Air Temperature: $25^{\circ} \mathrm{C}$

Relative Humidity: 508

Cloud Cover: 8 tenths

Ground Roughness: Urban or forest

SOURCE STRENGTH INFORMATION:

Puddle Area: 40700 square feet

Average Puddle Depth: 1 centimeters

Soil Type: Default Ground Temperature: $25^{\circ} \mathrm{C}$
Initial Puddle Temperature: Ground temperature

Release Duration: AIOHA limited the duration to 1 hour

Max Computed Release Rate: 192 kilograms/min

Max Average Sustained Release Rate: 191 kilograms/min

(averaged over a minute or more)

Total Amount Released: 11,305 kilograms

FOOTPRINT INFORMATION:

Model Run: Heavy Gas

User-specified LOC: $75 \mathrm{ppm}$

Max Threat zone for LOC: 556 meters
Reviewed by S. A. Henderson

Works Calculation 8

Sheet No. 213 of 314

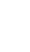
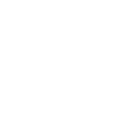


\section{ENGINEERING COMPUTATION SHEET}

Title of Project EPHA for THM

Subject Concentration Analysis for THM Hazardous Chemicals

Computer N.E. Baucom B. K. Tharakan Date 09/16/96

1-RD-16 AVARAGL wax DIS2 gEo 3

SITE DATA INFORMATION:

Location: AIREN, SOUTH CAROLINA

Building Air Exchanges Per Hour: 60 (User specified)

Time: June 30,19981200 hours EDT (User specified)

CHEMICAL INEORMATION:

Chemical Name: DIOXANE Molecular Weight: $88.11 \mathrm{~kg} / \mathrm{kmol}$

TLV-TWA: 25 ppm

Molecular Weight: $88.11 \mathrm{~kg} / \mathrm{kmol}$
IDIH: 500 ppm

Warning: Potential or confirmed human carcinogen.

Footprint Level of Concern: $75 \mathrm{ppm}$

Boiling Point: $101.32^{\circ} \mathrm{C}$

Vapor Pressure at Ambient Temperature: $0.050 \mathrm{~atm}$

Ambient Saturation Concentration: 51,172 ppm or $.5 .12 \%$

ATMOSPHERIC INFORMATION: (MANUAI INPUT OF DATA)

Wind: 2.5 meters $/ \mathrm{sec}$ from $90^{\circ}$ true at 2 meters

Inversion Height: 500 meters

stability class: C (user override)

Air Temperature: $25^{\circ} \mathrm{C}$

Relative Humidity: 508

Cloud Cover: 8 tenths

Ground Roughness: Urban or forest

SOURCE STRENGTH INFORMATION:

Puddle Area: 10000 square meters

Average Pudale Depth: 1 centimeters

Soil Type: Default Ground Temperature: $25^{\circ} \mathrm{C}$

Initial Puddle Temperature: Ground temperature

Release Duration: ALOHA limited the duration to 1 hour

Max Computed Release Rate: 492 kilograms/min

Max Average Sustained Release Rate: $491 \mathrm{kilograms/min}$

(averaged over a minute or more)

Total Amount Released: 28,893 kilograms

FOOTPRINT INFORMATION:

Model Run: Heavy Gas

User-specified LOC: $75 \mathrm{ppm}$

Max Threat Zone for LOC: 907 meters 
Title of Project EPHA for THM

Subject Concentration Analysis for THM Hazardous Chemicals

Computer N.E. Baucom B. K. Tharakan Date 09/16/96

1-RD-17 AVERAGE ALERT

SITE DATA INFORMATION:

Location: AIKEN, SOUTH CAROLINA

Building Air Exchanges Per Hour: 60 (User specified)

Time: June 30, 19981200 hours EDT (User specified)

\section{CHEMICAL INFORMATION:}

Chemical Name: DIPHENYLMETHANE-4,4-DISOCYANATE

Molecular Weight: $250.27 \mathrm{~kg} / \mathrm{kmol}$

TLV-TWA: -unavail-

IDLH: -unavail

Footprint Level of Concern: $2 \mathrm{mg} /(\mathrm{cu} \mathrm{m})$

Boiling Point: $184.00^{\circ} \mathrm{C}$

Note: Not enough chemical data to use Heavy Gas option

\section{ATMOSPHERIC INFORMATION: (MANUAL INPUT OF DATA)}

Wind: 2.5 meters $/ \mathrm{sec}$ from $90^{\circ}$ true at 2 meters

Inversion Height: 500 meters

Stability Class: $\mathrm{C}$ (user override)

Air Temperature: $25^{\circ} \mathrm{C}$

Relative Humidity: $50 \%$

Ground Roughness: Urban or forest
Reviewed by S. A. Henderson

Works Calculation 8

Sheèt No. 215 of 314

Cloud Cover: 8 tenths

\section{SOURCE STRENGTH INFORMATION:}

Direct Source: 3.88E-4 kilograms/sec

Source Height: 0

Release Duration: 60 minutes

Release Rate: 23.3 grams/min

Total Amount Released: 1.40 kilograms

\section{TIME DEPENDENT INFORMATION:}

Concentration Estimates at the point:

Downwind: $\quad 30$ meters

Off Centerline: 0 meters

Max Concentration:

Outdoor: $2 \mathrm{mg} /(\mathrm{cu} \mathrm{m})$.

Indoor: $2 \mathrm{mg} /(\mathrm{cu} \mathrm{m})$

Note: Indoor graph is shown with a dotted line. 
Title of Project EPHA for THM

Reviewed by S. A. Henderson

Subject Concentration Analysis for THM Hazardous Chemicals

Works Calculation 8

Computer N.E. Baucom B.K. Tharakan Date 09/16/96

SPILI AREA CALCULATION FOR 1-RD-17 AVERAGE

Sheet No. 216 of 314

ATERT CONDITIONS

\begin{tabular}{|c|c|c|c|c|}
\hline Parametex : & Value & Dimension: & Value & $\begin{array}{l}\text { Dimensions } \\
\text { (OSA) }\end{array}$ \\
\hline E (Evaporation Rate) & $3.88 \mathrm{E}-4$ & $\mathrm{~kg} / \mathrm{s}$ & & \\
\hline MW (Molecular Weight) & 250 & $\mathrm{~kg} / \mathrm{kg}$-mole & & \\
\hline Pv (Vapor Pressure) & 0.001 & $\mathrm{~mm} \mathrm{Hg}$ & & \\
\hline Pv (Vapor Pressure) & 0 & $\mathrm{~Pa}$ & & \\
\hline Rg (Gas Law Constant) & 8314 & J/kgmole-K & & \\
\hline Temperature & 25 & ${ }^{\circ} \mathrm{C}$ & & \\
\hline Volume of Spill & 22950 & gal & & \\
\hline d (Depth of Pool) & 0.01 & $\mathrm{~m}$ & & \\
\hline A (Surface Area of Spill) & 8687.3 & $\overline{m \wedge} 2$ & 93518 & Et^2 \\
\hline $\mathrm{Km}$ (Mass Transfer Coeff) & $3.32 E-3$ & $\mathrm{~m} / \mathrm{s}$ & & \\
\hline $\begin{array}{l}\text { Z (Pool Diameter in wind } \\
\text { Direction) }\end{array}$ & 105 & $m$ & & \\
\hline Sc (Schmidt Number) & 2.33 & & & \\
\hline $\mathrm{U}$ (wind velocity) & 2.5 & $\mathrm{~m} / \mathrm{s}$ & & \\
\hline $\begin{array}{l}\text { nu (kinematic viscosity of } \\
\text { air) }\end{array}$ & $1.50 \mathrm{E}-5$ & $\mathrm{~m}^{\wedge} 2 / \mathrm{sec}$ & & \\
\hline mu (viscosity of air) & $1.90 \mathrm{E}-5$ & $\mathrm{~kg} / \mathrm{m}-\mathrm{s}$ & & \\
\hline $\begin{array}{l}\text { Dm (Molecular Diffusivity of } \\
\text { Solute in Air) }\end{array}$ & $6.44 E-6$ & $\mathrm{~m}^{\wedge} 2 / \mathrm{sec}$ & & \\
\hline $\begin{array}{l}\text { D H20 (Molecular Diffusivity } \\
\text { of Water) }\end{array}$ & $2.40 E-5$ & & & \\
\hline MW_H20 & 18 & $\mathrm{~kg} / \mathrm{kg}$-mole & & \\
\hline \multicolumn{5}{|l|}{ Converaion: } \\
\hline T (Absolute) & 298.15 & ${ }^{\circ} \mathrm{R}$ & & \\
\hline V (Volume) & 86.87 & $m^{\wedge} 3$ & & \\
\hline Evaporation Rate & 23 & $\mathrm{~g} / \mathrm{min}$ & & \\
\hline $60 \mathrm{~min}$ Ib released & 3.08 & & & \\
\hline
\end{tabular}


Title of Project EPHA for THM

\section{ENGINEERING COMPUTATION SHEET}

Subject Concentration Analysis for THM Hazardous Chemicals

Computer N.E. Baucom B. K. Tharakan Date 09/16/96 1-RD-17 AVERAGE SAE

\section{SITE DATA INFORMATION:}

Location: AIKEN, SOUTH CAROLINA

Building Air Exchanges Per Hour: 60 (User specified)

Time: June 30, 19981200 hours EDT (User specified)

\section{CHEMICAL INFORMATION:}

Chemical Name: DIPHENYLMETHANE-4,4-DIISOCYANATE

Molecular Weight: $250.27 \mathrm{~kg} / \mathrm{kmol}$

TLV-TWA: -unavail-

Footprint Level of Concern: $2 \mathrm{mg} /(\mathrm{cu} \mathrm{m}$ )

Boiling Point: $184.00^{\circ} \mathrm{C}$

Note: Not enough chemical data to use Heavy Gas option

ATMOSPHERIC INFORMATION: (MANUAL INPUT OF DATA)

Wind: 2.5 meters $/ \mathrm{sec}$ from $90^{\circ}$ true at 2 meters

Inversion Height: 500 meters

Stability Class: C (user override)

Air Temperature: $25^{\circ} \mathrm{C}$

Relative Humidity: 50\%

Ground Roughness: Urban or forest
Reviewed by S. A. Henderson

Works Calculation 8

Sheet No. 217 of 314

Cloud Cover: 8 tenths

\section{SOURCE STRENGTH INFORMATION:}

Direct Source: 4.30E-3 kilograms/sec

Source Height: 0

Release Duration: 60 minutes

Release Rate: 258 grams/min

Total Amount Released: 15.5 kilograms

\section{TIME DEPENDENT INFORMATION:}

Concentration Estimates at the point:

Downwind:

100 meters

Off Centerline: 0 meters

Max Concentration:

Outdoor: $2 \mathrm{mg} /(\mathrm{cu} \mathrm{m}$ )

Indoor: $2 \mathrm{mg} /(\mathrm{cu} \mathrm{m}$ )

Note: Indoor graph is shown with a dotted line. 
Title of Project EPHA for THM

Reviewed by S. A. Henderson

Subject Concentration Analysis for THM Hazardous Chemicals

Works Calculation 8

Computer N.E. Baucom B. K. Tharakan Date 09/16/96

SPIII AREA CAICULATION FOR 1-RD-17 AVERAGE / SAB CONDITIONS

\begin{tabular}{|c|c|c|c|c|}
\hline Parameter & Value & Dimensions & Value & $\begin{array}{l}\text { Dimension: } \\
\text { (DSA) }\end{array}$ \\
\hline E (Evaporation Rate) & $4.30 \mathrm{E}-3$ & $\mathrm{~kg} / \mathrm{s}$ & & \\
\hline MW (Molecular Weight) & 250 & $\mathrm{~kg} / \mathrm{kg}$-mole & & \\
\hline Pv (Vapor Pressure) & 0.001 & $\mathrm{~mm} \mathrm{Hg}$ & & \\
\hline Pv (Vapor Pressure) & 0 & $\mathrm{~Pa}$ & & \\
\hline Rg (Gas Law Constant) & 8314 & J/kgmole-K & & \\
\hline Temperature & 25 & ${ }^{\circ} \mathrm{C}$ & & \\
\hline Volume of spill & 292500 & gaI & & \\
\hline d (Depth of Pool) & 0.01 & $\mathrm{~m}$ & & \\
\hline A (Surface Area of Spill) & 110720.0 & $m^{\wedge} 2$ & 1191901 & EtA2 \\
\hline $\mathrm{Km}$ (Mass Transfer Coeff) & $2.88 E-3$ & $\mathrm{~m} / \mathrm{s}$ & & \\
\hline $\begin{array}{l}\text { Z (Pool Diameter in Wind } \\
\text { Direction) }\end{array}$ & 375 & $\mathbf{m}$ & & \\
\hline Sc (Schmidt Number) & 2.33 & & & \\
\hline U (wind velocity) & 2.5 & $\mathrm{~m} / \mathrm{s}$ & & \\
\hline $\begin{array}{l}\text { nu (kinematic viscosity of } \\
\text { air) }\end{array}$ & $1.50 \mathrm{E}-5$ & $m^{\wedge} 2 / \sec$ & & \\
\hline mu (viscosity of air) & $1.90 E-5$ & $\mathrm{~kg} / \mathrm{m}-\mathrm{s}$ & & \\
\hline $\begin{array}{l}\text { Din (Molecular Diffusivity of } \\
\text { Solute in Air) }\end{array}$ & $6.44 \mathrm{E}-6$ & $\mathrm{~m}^{\wedge} 2 / \mathrm{sec}$ & & \\
\hline $\begin{array}{l}\text { D H20 (Molecular Diffusivity } \\
\text { of Water) }\end{array}$ & $2.40 \mathrm{E}-5$ & & & \\
\hline MW_H2O & 18 & $\mathrm{~kg} / \mathrm{kg}-\mathrm{mole}$ & & \\
\hline \multicolumn{5}{|l|}{ Convereions } \\
\hline T (Absolute) & 298.15 & ${ }^{\circ} \mathrm{K}$ & & \\
\hline $\mathrm{V}$ (Volume) & 1107.20 & $m^{\wedge} 3$ & & \\
\hline Evaporation Rate & 258 & g/min & & \\
\hline $60 \mathrm{~min} 1 \mathrm{~b}$ released & 34.09 & & & \\
\hline
\end{tabular}


Title of Project EPHA for THM

Subject Concentration Analysis for THM Hazardous Chemicals

Computer N.E. Baucom B. K. Tharakan Date 09/16/96 1-RD-17 AVARACE MAX DIST SEO 1

SITE DATA INFORMATION: Location: AIKEN, SOUTH CAROLINA

Building Air Exchanges Per Hour: 60 (User specified) Time: June 30, 19981200 hours EDT (User specified)

CHEMICAI INEORMATION:

Chemical Name: DIPHENYLMETHANE-4, 4-DIISOCYANATE

Molecular Weight: $250.27 \mathrm{~kg} / \mathrm{kmol}$

TLV-TWA: - unavail-

Footprint Level of Concern: $2 \mathrm{mg} /(\mathrm{cu} \mathrm{m})$

Boiling Point: $184.00^{\circ} \mathrm{C}$

Note: Not enough chemical data to use Heavy Gas option

ATMOSPHERIC INFORMATION: (MANUAL INPUT OF DATA)

Wind: 2.5 meters/sec from $90^{\circ}$ true at 2 meters

Inversion Height: 500 meters

Stability Class: C (user override)

Air Temperature: $25^{\circ} \mathrm{C}$

Relative Humidity: 508

Cloud Cover: 8 tenths

Ground Roughness: Urban or forest

SOURCE STRENGTH INFORMATION:

Direct Source: $1.09 \mathrm{E}-4 \mathrm{kilograms} / \mathrm{sec}$

Source Height: 0

Release Duration: 60 minutes

Release Rate: 6.54 grams/min

Total Amount Released: 392 grams

FOOTPRINT IMRORMATION:

Dispersion Module: Gaussian

User-specified LOC: $2 \mathrm{mg} /(\mathrm{cu} \mathrm{m}$ )

Max Threat Zone for LOC: 16 meters

Note: Footprint was not drawn because effects of near-field patchiness make dispersion predictions unreliable for short distances.
Reviewed by S. A. Henderson

Works Calculation 8

Sheet No. 219 of 314 
Title of Project EPHA for THM

Subject Concentration Analysis for THM Hazardous Chemicals
Reviewed by S. A. Henderson

Works Calculation 8

Sheet No. 220 of 314

\section{Determination of Eraporation Rate for Segment 1 Maximum Source Term of 24420} $\mathbf{s t}$

\begin{tabular}{|c|c|c|c|c|}
\hline Paramater & Value & Dimenatons & Value & $\begin{array}{l}\text { Dimengions } \\
\text { (DSA) }\end{array}$ \\
\hline E (Evaporation Rate) & $1.09 \mathrm{E}-4$ & $\mathrm{~kg} / \mathrm{s}$ & & \\
\hline MW (Molecular Weight) & 250 & $\mathrm{~kg} / \mathrm{kg}-\mathrm{mol}$ & & \\
\hline Pv (Vapor Pressure) & 0.001 & $\mathrm{~mm} \mathrm{Hg}$ & & \\
\hline Py (Vapor Pressure) & 0 & $\mathrm{~Pa}$ & & \\
\hline Rg (Gas Law Constant) & 8314 & $\mathrm{~J} / \mathrm{kgmole}-\mathrm{K}$ & & \\
\hline Temperature & 25 & ${ }^{\circ} \mathrm{C}$ & & \\
\hline Volume of Spill & 5993 & gal & & \\
\hline d (Depth of Pool) & 0.01 & $\mathbf{m}$ & & \\
\hline A (Surface Area of Spill) & 2268.5 & $m^{\wedge} 2$ & 24421 & Et^2 \\
\hline $\mathrm{Km}$ (Mass Transfer Coeff) & $3.58 \mathrm{E}-3$ & $\mathrm{~m} / \mathrm{s}$ & & \\
\hline $\begin{array}{l}\text { Z (Pool Diameter in Wind } \\
\text { Direction) }\end{array}$ & 54 & $\bar{m}$ & & \\
\hline Sc (Schmidt Number) & 2.33 & & & \\
\hline $\mathrm{U}$ (wind velocity) & 2.5 & $\mathrm{~m} / \mathrm{s}$ & & \\
\hline $\begin{array}{l}\text { nu (kinematic viscosity of } \\
\text { air) }\end{array}$ & $1.50 \mathrm{E}-5$ & $m^{\wedge} 2 / \sec$ & & \\
\hline mu. (viscosity of air) & $1.90 \mathrm{E}-5$ & $\mathrm{~kg} / \mathrm{m}-\mathrm{s}$ & & \\
\hline $\begin{array}{l}\text { Dm (Molecular Diffusivity of } \\
\text { Solute in Air) }\end{array}$ & $6.44 \mathrm{E}-6$ & $\mathrm{~m}^{\wedge} 2 / \mathrm{sec}$ & & \\
\hline $\begin{array}{l}\text { D_H20 (Molecular Diffusivity } \\
\text { of Water) }\end{array}$ & $2.40 \mathrm{E}-5$ & & & \\
\hline MW_H20 & 18 & $\mathrm{~kg} / \mathrm{kg}$-mole & & \\
\hline \multicolumn{5}{|l|}{ Converaion: } \\
\hline T (Absolute) & 298.15 & ${ }^{\circ} \mathrm{K}$ & & \\
\hline $\mathrm{V}$ (Volume) & 22.69 & $m^{\wedge} 3$ & & \\
\hline Evaporation Rate & 7 & g/min & & \\
\hline $60 \mathrm{~min} 1 \mathrm{~b}$ released & 0.87 & & & \\
\hline
\end{tabular}


Title of Project EPHA for THM

Subject Concentration Analysis for THM Hazardous Chemicals

Computer N.E. Baucom B. K. Tharakan Date 09/16/96

1-RD-17 AVERAGE MAX DIST SEG 2

SITE DATA INFORMATION:

Location: AIKEN, SOUTH CAROLINA

Building Air Exchanges Per Hour: 60 (User specified)

Time: June 30, 19981200 hours EDT (User specified)

\section{CHEMICAL INFORMATION:}

Chemical Name: DIPHENYLMETHANE-4,4-DISOCYANATE

Molecular Weight: $250.27 \mathrm{~kg} / \mathrm{kmol}$

TLV-TWA: -unavail-

IDLH: -unavail

Footprint Level of Concern: $2 \mathrm{mg} /(\mathrm{cu} \mathrm{m})$

Boiling Point: $184.00^{\circ} \mathrm{C}$

- Note: Not enough chemical data to use Heavy Gas option

ATMOSPHERIC INFORMATION: (MANUAL INPUT OF DATA)

Wind: 2.5 meters/ $\mathrm{sec}$ from $90^{\circ}$ true at 2 meters

Inversion Height: 500 meters

Stability Class: $\mathbf{C}$ (user override)

Air Temperature: $25^{\circ} \mathrm{C}$

Relative Humidity: $50 \%$

Ground Roughness: Urban or forest

Reviewed by S. A. Henderson

Works Calculation 8

Sheet No. 221 of 314

Cloud Cover: 8 tenths

\section{SOURCE STRENGTH INFORMATION:}

Direct Source: $1.77 \mathrm{E}-4$ kilograms/sec

Source Height: 0

Release Duration: 60 minutes

Release Rate: 10.6 grams/min

Total Amount Released: 637 grams

FOOTPRINT INFORMATION:

Dispersion Module: Gaussian

User-specified LOC: $2 \mathrm{mg} /(\mathrm{cu} \mathrm{m}$ )

Max Threat Zone for LOC: 20 meters

Note: Footprint was not drawn because effects of near-field patchiness make dispersion predictions unreliable for short distances. 
Title of Project EPHA for THM

Subject Concentration Analysis for THM Hazardous Chemicals

Computer N.E. Baucom B. K. Tharakan Date 09/16/96

Determination of Evaporation Rate for segment 2 Maximum Source Term of 40700 $\mathbf{f t}$

\begin{tabular}{|c|c|c|c|c|}
\hline Parameter & Value & Dimenstons & Value & $\begin{array}{l}\text { Dimeniona } \\
\text { (USA) }\end{array}$ \\
\hline E (Evaporation Rate) & $1.77 \mathrm{E}-4$ & $\mathrm{~kg} / \mathrm{s}$ & & \\
\hline MW (Molecular Weight) & 250 & $\mathrm{~kg} / \mathrm{kg}$-mole & & \\
\hline Pv (Vapor Pressure) & 0.001 & $\mathrm{~mm} \mathrm{Hg}$ & & \\
\hline Pv (Vapor Pressure) & 0 & $\mathrm{~Pa}$ & & \\
\hline Rg (Gas Law Constant) & 8314 & J/kgmole-K & & \\
\hline Temperature & 25 & ${ }^{\circ} \mathrm{C}$ & & \\
\hline Volume of spill & 9988 & gal & & \\
\hline $\mathrm{d}$ (Depth of Pool) & 0.01 & $\mathrm{~m}$ & & \\
\hline A (Surface Area of Spill) & 3780.8 & $m^{\wedge} 2$ & 40700 & Et^2 \\
\hline $\mathrm{Km}$ (Mass Transfer Coeff) & $3.48 \mathrm{E}-3$ & $\mathrm{~m} / \mathrm{s}$ & & \\
\hline $\begin{array}{l}\text { Z (Pool Diameter in Wind } \\
\text { Direction) }\end{array}$ & 69 & $m$ & & \\
\hline Sc (Schmidt Number) & 2.33 & & & \\
\hline U (wind velocity) & 2.5 & $\mathrm{~m} / \mathrm{s}$ & & \\
\hline $\begin{array}{l}\text { nu (kinematic viscosity of } \\
\text { air) }\end{array}$ & $1.50 \mathrm{E}-5$ & $m^{\wedge} 2 / \sec$ & & \\
\hline mu (viscosity of air) & $1.90 \mathrm{E}-5$ & $\mathrm{~kg} / \mathrm{m}-\mathrm{s}$ & & \\
\hline $\begin{array}{l}\text { Dm (Molecular Diffusivity of } \\
\text { Solute in Air) }\end{array}$ & $6.44 E-6$ & $m^{\wedge} 2 / \sec$ & & \\
\hline $\begin{array}{l}\text { D_H20 (Molecular Diffusivity } \\
\text { of Water) }\end{array}$ & $2.40 \mathrm{E}-5$ & & & \\
\hline MW_H2O & 18 & $\mathrm{~kg} / \mathrm{kg}$-mole & & \\
\hline \multicolumn{5}{|l|}{ Conversions } \\
\hline T (Absolute) & 298.15 & ${ }^{\circ} \mathrm{K}$ & & \\
\hline $\mathrm{V}$ (Volume) & 37.81 & $m^{\wedge} 3$ & & \\
\hline Evaporation Rate & 11 & $g / m i n$ & & \\
\hline $60 \mathrm{~min}$ 1b released & 1.40 & & & \\
\hline
\end{tabular}


Title of Project EPHA for THM

Subject cencentration Analysis for THM Hazardous Chemicals

Computer N.E. Baucom B. K. Tharakan

I-RD-17 AVERACI MAX DIST SEO 3
Reviewed by S. A. Henderson

Works Calculation 8

Sheet No. 223 of 314

\section{SITE DATA INFORMATION:}

Location: AIKEN, SOUTH CAROLINA

Building Air Exchanges Per Hour: 60 (User specified)

Time: June 30, 19981200 hours EDT (User specified)

CHEMICAL INFORMATION:

Chemical Name: DIPHENYLMETHANE-4,4-DIISOCYANATE

Molecular Weight: $250.27 \mathrm{~kg} / \mathrm{kmol}$

TLV-TWA: - unavail-

IDLH: -unavail

Footprint Level of Concern: $2 \mathrm{mg} /(\mathrm{cu} \mathrm{m}$ )

Boiling Point: $184.00^{\circ} \mathrm{C}$

Note: Not enough chemical data to use Heavy Gas option

ATMOSPHERIC INFORMATION: (MANUAL INPUT OF DATA)

Wind: 2.5 meters/sec from $90^{\circ}$ true at 2 meters

Inversion Height: 500 meters

Stability Class: C (user override)

Air Temperature: $25^{\circ} \mathrm{C}$

Relative Humidity: 50\% Ground Roughness: Urban or forest

Cloud Cover: 8 tenths

SOURCE STRENGTH INEORMATION:

Direct Source: 4.43E-4 kilograms/sec

Source Height: 0

Release Duration: 60 minutes

Release Rate: 26.6 grams/min

Total Amount Released: 1.59 kilograms

FOOTPRINT INFORMATION:

Dispersion Module: Gaussian

User-specified LOC: $2 \mathrm{mg} /(\mathrm{cu} \mathrm{m}$ )

Max Threat Zone for LOC: 32 meters

Note: Footprint was not drawn because effects of near-field patchiness make dispersion predictions unreliable for short distances. 


\section{ENGINEERING COMPUTATION SHEET}

Title of Project EPHA for THM

Subject Concentration Analysis for THM Hazardous Chemicals

Computer N.E. Baucom B. K. Tharakan Date 09/16/96

Determination of Iraporation Rate for Segment 3 Maximim Source rerm of $10000 \mathrm{~m}^{2}$
Reviewed by S. A. Henderson

Works Calculation 8

Sheet No. 224 of 314

\begin{tabular}{|c|c|c|c|c|}
\hline Parameter & Value & Dimensions & Value & $\begin{array}{l}\text { Dimensions } \\
\text { (סSA) }\end{array}$ \\
\hline E (Evaporation Rate) & $4.43 \mathrm{E}-4$ & $\mathrm{~kg} / \mathrm{s}$ & & \\
\hline MW (Molecular Weight) & 250 & $\mathrm{~kg} / \mathrm{kg}$-mole & & \\
\hline Pv (Vapor Pressure) & 0.001 & $\mathrm{~mm} \mathrm{Hg}$ & & \\
\hline PV (Vapor Pressure) & 0 & $\mathrm{~Pa}$ & & \\
\hline Rg (Gas Law Constant) & 8314 & J/kgmole-K & & \\
\hline Temperature & 25 & ${ }^{\circ} \mathrm{C}$ & & \\
\hline Volume of spill & 26418 & gal & & \\
\hline d (Depth of Pool) & 0.01 & $\mathrm{~m}$ & & \\
\hline A (Surface Area of Spill) & 10000.0 & $m^{\wedge} 2$ & 107650 & EtA2 \\
\hline $\mathrm{Km}$ (Mass Transfer Coeff) & $3.29 \mathrm{E}-3$ & $\mathrm{~m} / \mathrm{s}$ & & \\
\hline $\begin{array}{l}\mathrm{Z} \text { (Pool Diameter in wind } \\
\text { Direction) }\end{array}$ & 113 & $\mathbf{m}$ & & \\
\hline Sc (Schmidt Number) & 2.33 & & & \\
\hline U (wind velocity) & 2.5 & $\mathrm{~m} / \mathrm{s}$ & & \\
\hline $\begin{array}{l}\text { nu (kinematic viscosity of } \\
\text { air) }\end{array}$ & $1.50 \mathrm{E}-5$ & $\mathrm{~m}^{\wedge} 2 / \mathrm{sec}$ & & \\
\hline mu (viscosity of air) & $1.90 \mathrm{E}-5$ & $\mathrm{~kg} / \mathrm{m}-\mathrm{s}$ & & \\
\hline $\begin{array}{l}\text { Dm (Molecular Diffusivity of } \\
\text { Solute in Air) }\end{array}$ & $6.44 \mathrm{E}-6$ & $m^{\wedge} 2 / \sec$ & & \\
\hline $\begin{array}{l}\text { D H20 (Molecular Diffusivity } \\
\text { of water) }\end{array}$ & $2.40 \mathrm{E}-5$ & & & \\
\hline $\mathrm{MW}$ H2O & 18 & $\mathrm{~kg} / \mathrm{kg}$-mole & & \\
\hline Converalons & & & & \\
\hline T (Absolute) & 298.15 & ${ }^{\circ} \mathrm{K}$ & & \\
\hline V (Volume) & 100.00 & $m^{\wedge} 3$ & & \\
\hline Evaporation Rate & 27 & g/min & & \\
\hline $60 \mathrm{~min} 1 \mathrm{~b}$ released & 3.52 & & & \\
\hline
\end{tabular}


Title of Project EPHA for THM

Subject Concentration Analysis for THM Hazardous Chemicals

Computer N.E. Baucom B. K. Tharakan Date 09/16/96

1-RD-19 AVARAGE AIERT

SITE DATA INFORMATION: LOCation: 'AIKEN, SOUTH CAROLINA

Building Air Exchanges Per Hour: 60 (User specified)

Time: June 30, 19981200 hours EDT (User specified)

CHEMICAL INFORMATION:

Chemical Name: ETHYL BENZENE

Molecular Weight: $106.17 \mathrm{~kg} / \mathrm{kmol}$

TLV-TWA: $100 \mathrm{ppm} \quad$ IDLH: $800 \mathrm{ppm}$

Footprint Level of Concern: $125 \mathrm{ppm}$

Boiling Point: $136.20^{\circ} \mathrm{C}$

Vapor Pressure at Ambient Temperature: 0.013 atm

Ambient Saturation Concentration: $12,876 \mathrm{ppm}$ or 1.298

ATMOSPHERIC INFORMATION: (MANUAL INPUT OF DATA)

Wind: 2.5 meters $/ \mathrm{sec}$ from $90^{\circ}$ true at 2 meters

Inversion Height: 500 meters

Stability Class: $C$ (user override)

Air Temperature: $25^{\circ} \mathrm{C}$

Relative Humidity: $508 \quad$ Ground Roughness: Urban or forest

Cloud Cover: 8 tenths

SOURCE STRENGTH INFORMATION:

Pudale Area: 17700 square feet

Average Puddle Depth: 1 centimeters

Soil Type: Default Ground Temperature: $25^{\circ} \mathrm{C}$

Initial Puddle Temperature: Ground temperature

Release Duration: ALOHA Iimited the duration to 1 hour

Max Computed Release Rate: $31.8 \mathrm{kilograms/min}$

Max Average Sustained Release Rate: $31.5 \mathrm{kilograms} / \mathrm{min}$

(ayeraged over a minute or more)

Total Amount Released: 1,758 kilograms

FOOTPRINT INFORMATION :

Dispersion Module: Gaussian

User-specified LOC: $125 \mathrm{ppm}$

Max Threat Zone for LOC: 30 meters

Max Threat Zone for IDLH: 23 meters

Note: Footprint was not drawn because effects of near-field patchiness make dispersion predictions unreliable for short distances.

TIME DEPENDENT INFORMATION:

Concentration Estimates at the point:

Downwind:0 $\quad 30$ meters

off Centerline: 0 meters

Max Concentration:

Outdoor: $125 \mathrm{ppm}$

Indoor: $125 \mathrm{ppm}$

Note: Indoor graph is shown with a dotted line. 


\section{ENGINEERING COMPUTATION SHEET}

Title of Project EPHA for THM

Subject Concentration Analysis for THM Hazardous Chemicals

Computer N.E. Baucom B. K. Tharakan Date 09/16/96
Reviewed by S. A. Henderson

Works Calculation 8

Sheet No. 226 of 314

\section{1-RD-19 AVERAGT SAE}

SITE DATA INFORMATION:

Location: AIKEN, SOUTH CAROLINA

Building Air Exchanges Per Hour: 60 (User specified)

Time: June 30, 19981200 hours EDT (User specified)

CHEMICAL INFORMATION:

Chemical Name: ETHYL BENZENE

Molecular Weight: $106.17 \mathrm{~kg} / \mathrm{kmol}$

TLV-TWA: $100 \mathrm{ppm}$ IDLH: $800 \mathrm{ppm}$

Footprint Level of Concern: $125 \mathrm{ppm}$

Boiling Point: $136.20^{\circ} \mathrm{C}$

Vapor Pressure at Ambient Temperature: $0.013 \mathrm{~atm}$

Ambient Saturation Concentration: $12,876 \mathrm{ppm}$ or $1.29 \%$

ATMOSPHERIC INFORMATION: (MANUAL INPUT OF DATA) -

Wind: 2.5 meters $/ \mathrm{sec}$ from $90^{\circ}$ true at 2 meters

Inversion Height: 500 meters

Stability Class: C (user override)

Air Temperature: $25^{\circ} \mathrm{C}$

Relative Humidity: 508

Ground Roughness: Urban or forest

cloud Cover: 8 tenths

SOURCE STRENGTH INFORMATION:

Puddle Area: 100001 square feet

Average Pudale Depth: 1 centimeters

Soil Type: Default Ground Temperature: $25^{\circ} \mathrm{C}$

Initial Puddle Temperature: Ground temperature

Release Duration: ALOHA limited the duration to 1 hour

Max Computed Release Rate: 169 kilograms/min

Max Average Sustained Release Rate: $167 \mathrm{kilograms/min}$

(averaged over a minute or more)

Total Amount Released: 9,292 kilograms

FOOTPRINT INFORMATION :

Dispersion Module: Gaussian

User-specified LOC: $125 \mathrm{ppm}$

Max Threat Zone for LOC: 69 meters

Max Threat Zone for IDLH: 55 meters

Note: Footprint was not drawn because effects of near-field patchiness make dispersion predictions unreliable for short distances.

TIME DEPENDENT INFORMATION:

Concentration Estimates at the point:

Downwind: $\quad 100$ meters

off Centerline: 0 meters

Max Concentration:

Outdoor: $65 \mathrm{ppm}$

Indoor: $65 \mathrm{ppm}$

Note: Indoor graph is shown with a dotted line. 


\section{ENGINEERING COMPUTATION SHEET}

Title of Project EPHA for THM

Reviewed by $\underline{\text { S. A. Henderson }}$

Subject Concentration Analysis for THM Hazardous Chemicals

Works Calculation 8

Computer N.E. Baucom B. K. Tharakan Date 09/16/96

1-RD-19 AVERACI unX DIgP SLO 1

SITE DATA INFORMATION:

Location: AIKEN, SOUTH CAROLINA

Building Air Exchanges Per Hour: 60 (User specified)

Time: June 30, 19981200 hours EDT (User specified)

CHEMICAL INFORMATION :

Chemical Näme: ETHYL BENZENE

Molecular Weight: $106.17 \mathrm{~kg} / \mathrm{kmol}$

TLV-TWA: $100 \mathrm{ppm}$

Footprint Level of Concern: $125 \mathrm{ppm}$

Boiling Point: $136.20^{\circ} \mathrm{C}$

Vapor Pressure at Ambient Temperature: $0.013 \mathrm{~atm}$

Ambient Saturation Concentration: 12,876 ppm or 1.298

ATMOSPHERIC INFORMATION: (MANUAL INPUT OF DATA)

Wind: 2.5 meters/sec from $90^{\circ}$ true at 2 meters

Inversion Height: 500 meters

stability Class: C (user override)

Air Temperature: $25^{\circ} \mathrm{C}$

Relative Humidity: 508 Ground Roughness: Urban or forest

Cloud Cover: 8 tenths

SOURCE STRENGTH INFORMATION:

Puddle Area: 24420 square feet

Average Puddle Depth: 1 centimeters

Soil Type: DefaultGround Temperature: $25^{\circ} \mathrm{C}$

Initial Puddle Temperature: Ground temperature

ReleaseDuration: ALOHA limited the duration to 1 hour

Max Computed Release Rate: $43.4 \mathrm{kilograms/min}$

MaxAverage Sustained Release Rate: $42.9 \mathrm{kilograms} / \mathrm{min}$

(averaged over a minute or more)

Total Amount Released: 2,395 kilograms

FOOTPRINT INEORMATION:

Dispersion Module: Gaussian

User-specified LOC: $125 \mathrm{ppm}$

Max Threat Zone for LOC: 35 meters

Max Threat Zone for IDHH: 27 meters 
ENGINEERING COMPUTATION SHEET

Title of Project EPHA for THM

Reviewed by S. A. Henderson

Subject Concentration Analysis for THM Hazardous Chemicals

Computer N.E. Baucom B. K. Tharakan Date 09/16/96 1-RD-19 AVERAGE IAX DIST BLo 2

SITE DATA INFORMATION:

LOcation: AIKEN, SOUTH CAROLINA

Building Air Exchanges Per Hour: 60 (User specified)

Time: June 30, 19981200 hours EDT (User specified)

CHEMICÁL INFORMATION:

Chemical Name: ETHYL BENZENE

Molecular Weight: $106.17 \mathrm{~kg} / \mathrm{kmol}$

IDLH: $800 \mathrm{ppm}$

Level of Concern: 125 ppm

Boiling Point: $136.20^{\circ} \mathrm{C}$

Vapor Pressure at Ambient Temperature: 0.013 atm

Ambient Saturation Concentration: 12,876 ppm or $1.29 \%$

ATMOSPHERIC INFORMATION: (MANUAL INPUT OF DATA)

Wind: 2.5 meters $/ \mathrm{sec}$ from $90^{\circ}$ true at 2 meters

Inversion Height: 500 meters

Stability class: C (user override)

Air Temperature: $25^{\circ} \mathrm{C}$

Relative Humidity: 508 Ground Roughness: Urban or forest

Cloud Cover: 8 tenths

SOURCE STRENGTH INFORMATION:

Puddle Area: 40700 square feet

Average Puddle Depth: 1 centimeters

Soil Type: Default Ground Temperature: $25^{\circ} \mathrm{C}$

Initial Puddle Temperature: Ground temperature

Release Duration: ALOHA limited the duration to 1 hour

Max Computed Release Rate: $70.9 \mathrm{kilograms} / \mathrm{min}$

Max Average Sustained Release Rate: $70.2 \mathrm{kilograms} / \mathrm{min}$

(averaged over a minute or more)

Total Amount Released: 3,913 kilograms

FOOTPRINT INFORMATION:

Dispersion Module: Gaussian

User-specified LOC: 125 ppm

Max Threat Zone for LOC: 45 meters

Max Threat Zone for IDLH: 34 meters
Works Calculation 8

Sheet No. 228 of 314 
Title of Project EPHA for THM

Subject Concentration Analysis for THM Hazardous Chemicals

Computer N.E. Baucom B. K. Tharakan Date 09/16/96 1-RD-19 AVERAGE MAX DIST SEG 3

SITE DATA INFORMATION: Location:- AIKEN, SOUTH CAROLINA

Building Air Exchanges Per Hour: 60 (User specified)

Time: June 30, 19981200 hours EDT (User specified)

CHEMICAL INFORMATION:

Chemical Name: ETHYL BENZENE

Molecular Weight: $106.17 \mathrm{~kg} / \mathrm{kmol}$

TLV-TWA: $100 \mathrm{ppm}$ IDLH: $800 \mathrm{ppm}$

Footprint Level of Concern: $125 \mathrm{ppm}$

Boiling Point: $136.20^{\circ} \mathrm{C}$

Vapor, Pressure at Ambient Temperature: $0.013 \mathrm{~atm}$

Ambient Saturation Concentration: 12,876 ppm or $1.29 \%$

ATMOSPEERIC INFORMATION: (MANUAL INPUT OF DATA)

Wind: 2.5 meters/sec from $90^{\circ}$ true at 2 meters

Inversion Height: 500 meters

Stability Class: C (user override)

Air Temperature: $25^{\circ} \mathrm{C}$

Relative Humidity: $50 \%$

Ground Roughness: Urban or forest
Reviewed by S. A. Henderson

Works Calculation 8

Sheet No. 229 of 314

SOURCE STRENGTH INFORMATION:

Pudale Area: 10000 square meters

Average Pudale Depth: 1 centimeters

Soil Type: Default Ground Temperature: $25^{\circ} \mathrm{C}$

Initial Pudale Temperature: Ground temperature

Release Duration: ALOHA limited the duration to 1 hour

Max Computed Release Rate: 181 kilograms/min

Max Average Sustained Release Rate: $179 \mathrm{kilograms/min}$

(averaged over a minute or more)

Total Amount Released: 9,974 kilograms

FOOTPRINT INFORMATION:

Dispersion Module: Gaussian

User-specified LOC: $125 \mathrm{ppm}$

Max Threat Zone for IOC: 72 meters

Max Threat Zone for IDIH: 56 meters 


\section{SITE DATA INFORMATION:}

LOCation: - AIKEN, SOUTH CAROLINA

Building Air Exchanges Per Hour: 60 (User specified)

Time: June 30, 19981200 hours EDT (User specified)

CHEMICAL INFORMATION:

Chemical Name: ETHYLENE GLYCOL MONOBUTYL ETHER

Molecular Weight: $118.18 \mathrm{~kg} / \mathrm{kmol}$

TLV-TWA: $25 \mathrm{ppm}$ IDLH: $700 \mathrm{ppm}$

Footprint Level of Concern: 125 ppm

Boiling Point: $171.32^{\circ} \mathrm{C}$

Vapor Pressure at Ambient Temperature: 0.0011 atm

Ambient Saturation Concentration: $1,161 \mathrm{ppm}$ or 0.128

ATMOSPHERIC INFORMATION: (MANUAL INPUT OF DATA)

Wind: 2.5 meters $/ \mathrm{sec}$ from $90^{\circ}$. true at 2 meters

Inversion Height: 500 meters

Stability class: $c$ (user override)

Air Temperature: $25^{\circ} \mathrm{C}$

Relative Humidity: 508

Ground Roughness: Urban or forest

cloud Cover: 8 tenths

SOURCE STRENGTH INFORMATION:

Puddle Area: 30001 square feet

Average Puadle Depth: 1 centimeters

Soil Type: Default

Ground Temperature: $25^{\circ} \mathrm{C}$

Initial puddle Temperature: Ground temperature

Release Duration: ALOHA limited the duration to 1 hour

Max Computed Release Rate: 6.54 kilograms/min

Max Average Sustained Release Rate: $6.41 \mathrm{kilograms} / \mathrm{min}$

(averaged over a minute or more)

Total Amount Released: $338 \mathrm{kilograms}$

TIME DEPENDENT INFORMATION:

Concentration Estimates at the point:

Downwind: $\quad 30$ meters

off Centerline: 0 meters

Max Concentration:

Outdoor: $22.8 \mathrm{ppm}$

Indoor: $22.8 \mathrm{ppm}$

Note: Indoor graph is shown with a dotted line. 
Title of Project EPHA for THM

Subject Concentration Analysis for THM Hazardous Chemicals

Computer N.E. Baucom B. K. Tharakan Date 09/16/96 1-RD-20 AVERACX SAE.

SITE DATA INFORMATION: Location: AIREN, SOUTH CAROLINA Building Air Exchanges Per Hour: 60 (User specified) Time: June 30, 19981200 hours EDT (User specified)

CHEMICAL INFORMATION: Chemical Name: ETHYLENE GLYCOL MONOBUTYL ETHER Molecular Weight: $118.18 \mathrm{~kg} / \mathrm{kmol}$ TLV-TWA: $25 \mathrm{ppm}$ Footprint Level of Concern: $125 \mathrm{ppm}$ Boiling Point: $171.32^{\circ} \mathrm{C}$ Vapor Pressure at Ambient Temperature: $0.0011 \mathrm{~atm}$ Ambient Saturation Concentration: 1,161 ppm or 0.128

ATMOSPHERIC INFORMATION: (MANUAL INPUT OF DATA) wind: 2.5 meters $/ \mathrm{sec}$ from $90^{\circ}$ true at 2 meters Inversion Height: 500 meters Stability Class: C (user override)

Air Temperature: $25^{\circ} \mathrm{C}$ Relative Humidity: 508 Ground Roughness: Urban or forest Cloud Cover: 8 tenths

SOURCE STRENGTH INFORMATION: Puddle Area: 100001 square feet Average Puddle Depth: 1 centimeters

Soil Type: Default. Ground Temperature: $25^{\circ} \mathrm{C}$ Initial Puddle Temperature: Ground temperature

Release Duration: ALOHA limited the duration to 1 hour Max Computed Release Rate: 20.8 kilograms/min

Max Average Sustained Release Rate: 20.4 kilograms/min (averaged over a minute or more)

Total Amount Released: 1,073 kilograms

TIME DEPENDENT INFORMATION:

Concentration Estimates at the point:

Downwind: $\quad 100$ meters

Off Centerline: 0 meters

Max Concentration:

Outdoor: $7.14 \mathrm{ppm}$

Indoor: 7.14 ppm

Note: Indoor graph is shown with a dotted Iine.
Reviewed by S. A. Henderson

Works Calculation 8

Sheet No. 231 of 314 
Title of Project EPHA for THM

Subject Concentration Analysis for THM Hazardous Chemicals

Computer N.E. Baucom B. K. Tharakan Date 09/16/96 1-RD-20 AVARAOE MAX DIST BEO 1

SITE DATA INEORMATION:

LOCation: AIKEN, SOUTH CAROLINA

Building Air Exchanges Per Hour: 60 (User specified)

Time: June 30, 19981200 hours EDT (User specified)

CHEMICAL INFORMATION :

Chemical Name: ETHYLENE GLYCOL MONOBUTYL ETHER

Molecular Weight: $118.18 \mathrm{~kg} / \mathrm{kmol}$

TLV-TWA: $25 \mathrm{ppm}$ IDLF: $700 \mathrm{ppm}$

Footprint Level of Concern: $125 \mathrm{ppm}$

Boiling Point: $171.32^{\circ} \mathrm{C}$

Vapor Pressure at Ambient Temperature: 0.0011 atm

Ambient Saturation Concentration: $1,161 \mathrm{ppm}$ or 0.128

ATMOSPHERIC INFORMATION: (MANUAL INPUT OF DATA)

Wind: 2.5 meters/sec from $90^{\circ}$ true at 2 meters

Inversion Height: 500 meters

Stability Class: C (user override)

Air Temperature: $25^{\circ} \mathrm{C}$

Relative Humidity: 508 Ground Roughness: Urban or forest

Cloud Cover: 8 tenths

SOURCE STRENGTH INFORMATION:

Pudale Area: 24420 square feet

Average Puddle Depth: 1 centimeters

Soil Type: Default

Ground Temperature: $25^{\circ} \mathrm{C}$

Initial Puddle Temperature: Ground temperature

Release Duration: ALOHA limited the duration to 1 hour

Max Computed Release Rate: $5.36 \mathrm{kilograms/min}$

Max Average Sustained Release Rate: $5.26 \mathrm{kilograms} / \mathrm{min}$

(averaged over a minute or more)

Total Amount Released: 277 kilograms

FOOTPRINT INFORMATION:

Dispersion Module: Gaussian

User-specified LOC: 125 ppm

Max Threat Zone for LOC: 27 meters

Max Threat Zone for IDLH: 27 meters

Note: Footprint was not drawn because effects of near-field patchiness make dispersion predictions unreliable for short distances.
Reviewed by S. A. Henderson

Works Calculation 8

Sheet No. $\underline{232 \text { of } 314}$ 
Title of Project EPHA for THM

Reviewed by S. A. Henderson

Subject Concentration Analysis for THM Hazardous Chemicals

Works Calculation 8

Computer N.E. Baucom B. K. Tharakan Date 09/16/96

1-RD-20 AVERAGE HAX DIST SEO 2

SITE DATA INFORMATION:

Location:-AIKEN, SOUTH CAROLINA

Building Air Exchanges Per Hour: 60 (User specified)

Time: June 30, 19981200 hours EDT (User specified)

CHEMICAL INFORMATION :

Chemical Name: ETHYLENE GLYCOL MONOBUTYL ETHER

Molecular Weight: $118.18 \mathrm{~kg} / \mathrm{kmol}$

TLV-TWA: $25 \mathrm{ppm}$. IDLH: $7.00 \mathrm{ppm}$

Footprint Level of Concern: 125 ppm

Boiling Point: $171.32^{\circ} \mathrm{C}$

Vapor Pressure at Ambient Temperature: 0.0011 atm

Ambient Saturation Concentration: 1,161 ppm or 0.128

ATMOSPHERIC INFORMATION: (MANUAL INPUT OF DATA)

Wind: 2.5 meters/sec from $90^{\circ}$ true at 2 meters

Inversion Height: 500 meters

Stability Class: C (user override)

Air Temperature: $25^{\circ} \mathrm{C}$.

Relative Humidity: $50 \%$

Cloud Cover: 8 tenths

Ground Roughness: Urban or forest

SOURCE STRENGTH INFORMATION:

Pudale Area: 40700 square feet

Average Puadle Depth: 1 centimeters

Soil Type: Default

Ground Temperature: $25^{\circ} \mathrm{C}$

Initial Puddle Temperature: Ground temperature

Release Duration: ALOHA Iimited the duration to 1 hour

Max Computed Release Rate: $8.77 \mathrm{kilograms/min}$

Max Average Sustained Release Rate: $8.59 \mathrm{kilograms} / \mathrm{min}$

(averaged over a minute or more)

Total Amount Released: 453 kilograms

FOOTPRINT INFORMATION:

Dispersion Module: Gaussian

User-specified LOC: 125 ppm

Max Threat Zone for LOC: 34 meters

Max Threat Zone for IDLH: 35 meters

Note: Footprint was not drawn because effects of near-field patchiness make dispersion predictions unreliable for short distances. 
Title of Project EPHA for THM

Reviewed by S. A. Henderson

Subject Concentration Analysis for THM Hazardous Chemicals

Computer N.E. Baucom B. K. Tharakan Date 09/16/96

1-RD-20 AVERAGE MAX DIST SEO 3

SITE DATA INFORMATION:

Location:-AIKEN, SOUTH CAROLINA

Building Air Exchanges Per Hour: 60 (User specified)

Time: June 30, 19981200 hours EDT (User specified)

CHEMICAI INFORMATION:

Chemical Name: ETHYLENE GLYCOL MONOBUTYL ETHER

Molecular Weight: $118.18 \mathrm{~kg} / \mathrm{kmol}$

TLV-TWA: 25 ppm

Footprint Level of Concern: $125 \mathrm{ppm}$

Boiling Point: $171.32^{\circ} \mathrm{C}$

Vapor Pressure at Ambient Temperature: $0.0011 \mathrm{~atm}$

Ambient Saturation Concentration: $1,161 \mathrm{ppm}$ or 0.128

ATMOSPHERIC INFORMATION: (MANUAL INPUT OF DATA)

Wind: 2.5 meters $/ \mathrm{sec}$ from $90^{\circ}$ true at 2 meters

Inversion Height: 500 meters

Stability Class: $C$ (user override)

Air Temperature: $25^{\circ} \mathrm{C}$

Relative Humidity: $508 \quad$ Ground Roughness: Urban or forest.

Cloud Cover: 8 tenths

SOURCE STRENGTH INFORMATION:

Puddle Area: 10000 square meters

Average Puddle Depth: 1 centimeters

Soil Type: Default Ground Temperature: $25^{\circ} \mathrm{C}$

Initial Puddle Temperature: Ground temperature

Release Duration: ALOHA limited the duration to 1 hour

Max Computed Release Rate: 22.4 kilograms/min

Max -Average Sustained Release Rate: 21.9 kilograms/min

(averaged over a minute or more)

Total Amount Released: 1,152 kilograms

FOOTPRINT INFORMATION:

Dispersion Module: Gaussian

User-specified LOC: $125 \mathrm{ppm}$

Max Threat Zone for LOC: 56 meters

Max Threat zone for IDLH: 56 meters

Note: Footprint was not drawn because effects of near-field patchiness make dispersion predictions unreliable for short distances. 
Title of Project

Title of Project EPHA for THM

Subject Concentration Analysis for THM Hazardous Chemicals

ENGINEERING COMPUTATION SHEET

Computer N.E. Baucom B. K. Tharakan Date 09/16/96

1-RD-22 AVERAGE ATERT

SITE DATA INFORMATION: Location:-AIKEN, SOUTH CAROLINA

Building Air Exchanges Per Hour: 60 (User specified)

Time: June 30, 19981200 hours EDT (User specified)

CHEMICAL INFORMATION :

Chemical Name: FORMIC ACID

Molecular Weight: $46.03 \mathrm{~kg} / \mathrm{kmol}$

TLV-TWA: $5 \mathrm{ppm}$ IDLH: $30 \mathrm{ppm}$

Footprint Level of Concern: $10 \mathrm{ppm}$

Boiling Point: $100.56^{\circ} \mathrm{C}$

Vapor Pressure at Ambient Temperature: 0.056 atm

Ambient Saturation Concentration: 57,128 ppm or $5.71 \%$

ATMOSPHERIC INFORMATION: (MANUAL INPUT OF DATA)

Wind: 2.5 meters/sec from $90^{\circ}$ true at 2 meter's

Inversion Height: 500 meters

Stability Class: C (user override)

Air Temperature: $25^{\circ} \mathrm{C}$

Relative Humidity: 508

Cloud Cover: 8 tenths

Ground Roughness: Urban or forest

SOURCE STRENGTH INFORMATION:

Pudale Area: 59 square feet

Average Puddle Depth: 1 centimeters

Soil Type: Default Ground Temperature: $25^{\circ} \mathrm{C}$

Initial Puddle Temperature: Ground temperature

Release Duration: ALOHA limited the duration to 1 hour

Max Computed Release Rate: 235 grams/min

Max Average Sustained Release Rate: 227 grams/min (averaged over a minute or more)

Total Amount Released: 13.2 kilograms

FOOTPRINT INFORMATION:

Dispersion Module: Gaussian

User-specified LOC: $10 \mathrm{ppm}$

Max Threat Zone for LOC: 30 meters

Max Threat zone for IDLH: 17 meters

Note: Footprint was not drawn because effects of near-field patchiness make dispersion predictions unreliable for short distances.

TIME DEPENDENT INFORMATION:

Concentration Estimates at the point:

Downwind: $\quad 30$ meters

off Centerline: 0 meters

Max Concentration:

Outaoor: $9.96 \mathrm{ppm}$

Indoor: $9.96 \mathrm{ppm}$

Note: Indoor graph is shown with a dotted line. 
Title of Project EPHA for THM

Reviewed by S. A. Henderson

Subject Concentration Analysis for THM Hazardous Chemicals

Computer N.E. Baucom B. K. Tharakan Date 09/16/96 Sheet No. 236 of 314

I-RD-22 AVERAGE SAE

SITE DATA INFORMATION: Location:- AIKEN, SOUTH CAROLINA

Building Air Exchanges Per Hour: 60 (User specified)

Time: June 30, 19981200 hours EDT (User specified)

CHEMICAL INFORMATION:

Chemical Name: FORMIC ACID

Molecular Weight: $46.03 \mathrm{~kg} / \mathrm{kmol}$

TLV-TWA: $5 \mathrm{ppm}$

IDLH: $30 \mathrm{ppm}$

Footprint Level of Concern: $10 \mathrm{ppm}$

Boiling Point: $100.56^{\circ} \mathrm{C}$

Vapor Pressure at Ambient Temperature: 0.056 atm

Ambient Saturation Concentration: $57,128 \mathrm{ppm}$ or 5.718

ATMOSPHERIC INFORMATION: (MANUAL INPUT OF DATA)

Wind: 2.5 meters $/ \mathrm{sec}$ from $90^{\circ}$ true at 2 meters

Inversion Height: 500 meters

Stability Class: C (user override)

Air Temperature: $25^{\circ} \mathrm{C}$

Relative Humidity: $508 \quad$ Ground Roughness: Urban or forest

Cloud Cover: 8 tenths

SOURCE STRENGTH INFORMATION:

Puddle Area: 760 square feet

Average Puddle Depth: 1 centimeters

Soil Type: Default

Ground Temperature: $25^{\circ} \mathrm{C}$

Initial Puddle Temperature: Ground temperature

Release Duration: ALOHA limited the duration to 1 hour

Max Computed Release Rate: $2.59 \mathrm{kilograms/min}$

Max Average Sustained Release Rate: 2.55 kilograms/min (averaged over a minute or more)

Total Amount Released: 151 kilograms

FOOTPRINT INFORMATION:

Dispersion Module: Gaussian

User-specified LOC: $10 \mathrm{ppm}$

Max Threat Zone for LOC: 100 meters

Max Threat zone for IDLH: 55 meters

Note: Footprint was not drawn because effects of near-field patchiness make dispersion predictions unreliable for short distances.

TIME DEPENDENT INFORMATION:

Concentration Estimates at the point:

Downwind: $\quad 100$ meters

off Centerline: 0 meters

Max Concentration:

Outdoor: $10 \mathrm{ppm}$

Indoor: $10 \mathrm{ppm}$

Note: Indoor graph is shown with a dotted line. 
Title of Project EPHA for THM

\section{ENGINEERING COMPUTATION SHEET}

Subject Concentration Analysis for THM Hazardous Chemicals

Computer N.E. Baucom B. K. Tharakan Date 09/16/96

1-RD-22 AVERAGE MAX DIST SEO 1

SITE DATA INFORMATION:

Location:-AIKEN, SOUTH CAROLINA

Building Air Exchanges Per Hour: 60 (User specified)

Time: June 30, 19981200 hours EDT (User specified)

CHEMICAL INFORMATION:

Chemical Name: FORMIC ACID

Molecular Weight: $46.03 \mathrm{~kg} / \mathrm{kmol}$

TLV-TWA: 5 ppm

IDLH: $30 \mathrm{ppm}$

Footprint Level of Concern: $10 \mathrm{ppm}$

Boiling Point: $100.56^{\circ} \mathrm{C}$

Vapor Pressure at Ambient Temperature: 0.056 atm

Ambient Saturation Concentration: $57,128 \mathrm{ppm}$ or 5.718

ATMOSPHERIC INFORMATION: (MANUAL INPUT OF DATA)

Wind: 2.5 meters $/ \mathrm{sec}$ from $90^{\circ}$ true at 2 meters

Inversion Height: 500 meters

Stability Class: C (user override)

Air Temperature: $25^{\circ} \mathrm{C}$

Relative Humidity: 508 Ground Roughness: Urban or forest

Cloud Cover: 8 tenths

SOURCE STRENGTH INFORMATION:

Puddle Area: 24420 square feet

Average Puddle Depth: 1 centimeters

Soil Type: Default

Ground Temperature: $25^{\circ} \mathrm{C}$

Initial Puddle Temperature: Ground temperature

ReleaseDuration: ALOHA limited the duration to 1 hour

Max Computed Release Rate: 70 kilograms/min

MaxAverage Sustained Release Rate: 69.9 kilograms/min

(averaged over a minute or more)

Total Amount Released: 4,187 kilograms

FOOTPRINT INFORMATION:

Dispersion Module: Gaussian

User-specified LOC: $10 \mathrm{ppm}$

Max Threat Zone for LOC: 528 meters

Max Threat Zone for IDLH: 285 meters.
Reviewed by S. A. Henderson

Works Calculation 8

Sheet No. 237 of 314 
Title of Project EPHA for THM

\section{ENGINEERING COMPUTATION SHEET}

Subject Concentration Analysis for THM Hazardous Chemicals

Computer N.E. Baucom B. K. Tharakan Date 09/16/96

1-RD-22 AVERAGE / LAX DIST SEG 2
Reviewed by S. A. Henderson

Works Calculation 8

Sheet No. $\underline{238 \text { of } 314}$

\section{SITE DATA INFORMATION:}

Location: :AIKEN, SOUTH CAROLINA

Building Air Exchanges Per Hour: 60 (User specified)

Time: June 30, 19981200 hours EDT (User specified)

CHEMICAL INFORMATION:

Chemical Name: FORMIC ACID

Molecular Weight: $46.03 \mathrm{~kg} / \mathrm{kmol}$

TLV-TWA: $5 \mathrm{ppm}$

IDLH: $30 \mathrm{ppm}$

Footprint Level of Concern: $10 \mathrm{ppm}$

Boiling Point: $100.56^{\circ} \mathrm{C}$

Vapor Pressure at Ambient Temperature: $0.056 \mathrm{~atm}$

Ambient Saturation Concentration: $57,128 \mathrm{ppm}$ or 5.718

ATMOSPHERIC INFORMATION: (MANUAL INPUT OF DATA)

Wind: 2.5 meters/sec from $90^{\circ}$ true at 2 meters

Inversion Height: 500 meters

Stability Class: C (user override)

Air Temperature: $25^{\circ} \mathrm{C}$

Relative Humidity: $50 \%$ Ground Roughness: Urban or forest

Cloud Cover: 8 tenths

SOURCE STRENGTH INFORMATION:

Puddle Area: 40700 square feet

Average Puddle Depth: 1 centimeters

Soil Type: Default Ground Temperature: $25^{\circ} \mathrm{C}$

Initial Pudale Temperature: Ground temperature

Release Duration: ALOHA limited the duration to 1 hour

Max Computed Release Rate: $115 \mathrm{kilograms/min}$

Max Average Sustained Release Rate: 114 kilograms/min (averaged over a minute or more)

Total Amount Released: 6,844 kilograms

FOOTPRINT INFORMATION:

Dispersion Module: Gaussian

User-specified LOC: $10 \mathrm{ppm}$

Max Threat Zone for LOC: 677 meters

Max Threat Zone for IDLH: 364 meters 
Title of Project EPHA for THM

Subject Concentration Analysis for THM Hazardous Chemicals

Computer N.E. Baucom B. K. Tharakan Date 09/16/96

1-RD-22 AVERAGE MAX DIBT SEG 3
Reviewed by S. A. Henderson

Works Calculation 8

Sheet No. 239 of 314

\section{SITE DATA INEORMATION:}

LOCation: AIKEN, SOUTH CAROLINA

Building Air Exchanges Per Hour: 60 (User specified)

Time: June 30, 19981200 hours EDT (User specified)

CHEMICAL INFORMATION:

Chemical Name: FORMIC ACID

Molecular Weight: $46.03 \mathrm{~kg} / \mathrm{kmol}$

TLV-TWA: $5 \mathrm{ppm}$ IDLH: $30 \mathrm{ppm}$

Footprint Level of Concern: $10 \mathrm{ppm}$

Boiling point: $100.56^{\circ} \mathrm{C}$

Vapor Pressure at Ambient Temperature: 0.056 atm

Ambient Saturation Concentration: $57,128 \mathrm{ppm}$ or 5.718

ATMOSPHERIC INFORMATION: (MANUAL INPUT OF DATA)

Wind: 2.5 meters $/ \mathrm{sec}$ from $90^{\circ}$ true at 2 meters

Inversion Height: 500 meters

Stability Class: C (user override)

Air Temperature: $25^{\circ} \mathrm{C}$

Relative Humidity: $508 \quad$ Ground Roughness: Urban or forest

Cloud Cover: 8 tenths

SOURCE STRENGTH INFORMATION:

Puddle Area: 10000 square meters

Average Puddle Depth: 1 centimeters

Soil Type: DefaultGround Temperature: $25^{\circ} \mathrm{C}$

Initial Puddle Temperature: Ground temperature

ReleaseDuration: ALOHA Iimited the duration to 1 hour

Max Computed Release Rate: 293 kilograms/min

MaxAverage Sustained Release Rate: 293 kilograms/min

(averaged over a minute or more)

Total Amount Released: 17,458 kilograms

FOOTPRINT INFORMATION:

Dispersion Module: Gaussian

User-specified LOC: $10 \mathrm{ppm}$

Max Threat Zone for LOC: 1.1 kilometers

Max Threat Zone for IDLH: 583 meters 
Title of Project EPHA for THM

Reviewed by S. A. Henderson

Subject Concentration Analysis for THM Hazardous Chemicals

Computer N.E. Baucom B. K. Tharakan Date 09/16/96

1-RD-23 AVERAGE AIERT

SITE DATA INFORMATION:

Location: AIKEN, SOUTH CAROLINA

Building Air Exchanges Per Hour: 60 (User specified)

Time: June 30, 19981200 hours EDT (User specified)

CHEMICAL INFORMATION :

Chemical Name: DIETHYLENE GLYCOL DIMETHYL ETHER

Molecular Weight: $134.18 \mathrm{~kg} / \mathrm{kmol}$

TLV-TWA: -unavail- IDLH: -unavail

Footprint Level of Concern: $500 \mathrm{mg} /(\mathrm{cu} \mathrm{m}$ )

Boiling Point: $159.76^{\circ} \mathrm{C}$

Vapor Pressure at Ambient Temperature: $0.0039 \mathrm{~atm}$

Ambient Saturation Concentration: 3,963 ppm or 0.408

ATMOSPHERIC INFORMATION: (MANUAL INPUT OF DATA)

Wind: 2.5 meters $/ \mathrm{sec}$ from $90^{\circ}$ true at 2 meters

Inversion Height: 500 meters

Stability Class: $C$ (user override)

Air Temperature: $25^{\circ}, \mathrm{C}$

Relative Humidity: $50 \%$ Ground Roughness : Urban or forest

Cloud Cover: 8 tenths

SOURCE STRENGTH INFORMATION:

Puddle Area: 30001 square feet

Average Pudale Depth: 1 centimeters

Soil Type: Default Ground Temperature: $25^{\circ} \mathrm{C}$

Initial Puddle Temperature: Ground temperature

Release Duration: ALOHA limited the duration to 1 hour

Max Computed Release Rate: 22.1 kilograms/min

Max Average Sustained Release Rate: 21.8 kilograms/min

(averaged over a minute or more)

Total Amount Released: 1,185 kilograms

TIME DEPENDENT INFORMATION:

Concentration Estimates at the point:

Downwind: 30 meters

Off Centerline: 0 meters

Max Concentration:

outdoor: $374 \mathrm{mg} /(\mathrm{cu} \mathrm{m})$

Indoor: $374 \mathrm{mg} /(\mathrm{cu} \mathrm{m})$

Note: Indoor graph is shown with a dotted line. 


\section{ENGINEERING COMPUTATION SHEET}

Title of Project EPHA for THM

Subject Concentration Analysis for THM Hazardous Chemicals

Computer N.E. Baucom B. K. Tharakan Date 09/16/96

1-RD-23 AVERAGE / BAE

SITE DATA INFORMATION:

Location:'AIKEN, SOUTH CAROLINA

Building Air Exchanges Per Hour: 60 (User specified)

Time: June 30, 19981200 hours EDT (User specified)

CHEMICAL INFORMATION:

Chemical Name: DIETHYLENE GLYCOL DIMETHYL ETHER

Molecular Weight: $134.18 \mathrm{~kg} / \mathrm{kmol}$

TLV-TWA: -unavail- IDLH: -unavail

Footprint Level of Concern: $500 \mathrm{mg} /(\mathrm{cu} \mathrm{m})$

Boiling Point: $159.76^{\circ} \mathrm{C}$

Vapor Pressure at Ambient Temperature: $0.0039 \mathrm{~atm}$

Ambient Saturation Concentration: $3,963 \mathrm{ppm}$ or 0.408

ATMOSPHERIC INFORMATION: (MANUAL INPUT OF DATA)

Wind: 2.5 meters $/ \mathrm{sec}$ from $90^{\circ}$ true at 2 meters

Inversion Height: 500 meters

Stability Class: C (user override)

Air Temperature: $25^{\circ} \mathrm{C}$

Relative Humidity: $50 \%$

Cloud Cover: 8 tenths

Ground Roughness: Urban or forest

SOURCE STRENGTH INFORMATION:

Puddle Area: 100001 square feet

Average Puddle Depth: 1 centimeters

Soil Type: Default Ground Temperature: $25^{\circ} \mathrm{C}$

Initial Puddle Temperature: Ground temperature

Release Duration: ALOHA limited the duration to 1 hour

Max Computed Release Rate: $70.6 \mathrm{kilograms} / \mathrm{min}$

Max Average Sustained Release Rate: $.69 .6 \mathrm{kilograms} / \mathrm{min}$

(, averaged over a minute or more)

Total Amount Released: 3,770 kilograms

TIME DEPENDENT INEORMATION:

Concentration Estimates at the point:

Downwind: $\quad 100$ meters

off Centerline: 0 meters

Max Concentration:

Outdoor: $118 \mathrm{mg} /(\mathrm{cu} \mathrm{m})$

Indoor: $118 \mathrm{mg} /(\mathrm{cu} \mathrm{m})$

Note: Indoor graph is shown with a dotted line 
Title of Project EPHA for THM

Subject Concentration Analysis for THM Hazardous Chemicals

Computer N.E. Baucom B. K. Tharakan Date 09/16/96

1-RD-23 AVERAGE / MNX DIST SEQ 1

SITE DATA INFORMATION:

Location: AIKEN, SOUTH CAROLINA

Building Air Exchanges Per Hour: 60 (User specified)

Time: June 30, 19981200 hours EDT (User specified)

CHEMICAL INFORMATION:

Chemical Name: DIETHYLENE GLYCOL DIMETHYL ETHER

Molecular Weight: $134.18 \mathrm{~kg} / \mathrm{kmol}$

TLV-TWA: -unavail-

IDLH: -unavaiI

Footprint Level of Concern: $500 \mathrm{mg} /(\mathrm{cu} \mathrm{m})$

Boiling Point: $159.76^{\circ} \mathrm{C}$

Vapor Pressure at Ambient Temperature: 0.0039 atm

Ambient Saturation Concentration: $3,963 \mathrm{ppm}$ or 0.408

ATMOSPHERIC INFORMATION: (MANUAL INPUT OF DATA)

Wind: 2.5 meters $/ \mathrm{sec}$ from $90^{\circ}$ true at 2 meters.

Inversion Height: 500 meters

Stability Class: $C$ (user override)

Air Temperature: $25^{\circ} \mathrm{C}$

Relative Humidity: 508

Ground Roughness: Urban or forest
Reviewod by S. A. Henderson

Works Calculation 8

Sheet No. 242 of 314

SOURCE STRENGTH INFORMATION:

Pudale Area: 24420 square feet

Average Puddle Depth: 1 centimeters

Soil Type: Default Ground Temperature: $25^{\circ} \mathrm{C}$

Initial Puddle Temperature: Ground temperature

Release Duration: ALOHA limited the duration to 1 hour

Max Computed Release Rate: $18.2 \mathrm{kilograms} / \mathrm{min}$

Max Average Sustained Release Rate: 17.9 kilograms/min

(averaged over a minute or more)

Tota Amount Released: 972 kilograms

FOOTPRINT INFORMATION:

Dispersion Module: Gaussian

User-specified LOC: $500 \mathrm{mg} /(\mathrm{cu} \mathrm{m})$

Max Threat Zone for LOC: 27 meters

Note: Footprint was not drawn because effects of near-field patchiness make dispersion predictions unreliable for short distances. 
Title of Project EPHA for THM

Reviewed by S.A. Heniderson

Subject Concentration Analysis for THM Hazardous Chemicals

Computer N.E. Baucom B. K. Tharakan Date 09/16/96

1-RD.23 AVERAGE MAX DIST SEG 2

Sheet No. 243 of 314

\section{SITE DATA INFORMATION:}

Location: AIKEN, 'SOUTH CAROLINA

Building Air Exchanges Per Hour: 60 (User specified)

Time: June 30, 19981200 hours EDT (User specified)

\section{CHEMICAL INFORMATION:}

Chemical Name: DIETHYLENE GLYCOL DIMETHYL ETHER

Molecular Weight: $134.18 \mathrm{~kg} / \mathrm{kmol}$

TLV-TWA: -unavailIDLH: -unavail

Footprint Level of Concern: $500 \mathrm{mg} /(\mathrm{cu} \mathrm{m}$ )

Boiling Point: $159.76^{\circ} \mathrm{C}$

Vapor Pressure at Ambient Temperature: $0.0039 \mathrm{~atm}$

Ambient Saturation Concentration: 3,963 ppm or $0.40 \%$

ATMOSPHERIC INFORMATION: (MANUAL INPUT OF DATA)

Wind: 2.5 meters $/ \mathrm{sec}$ from $90^{\circ}$ true at 2 meters

Inversion Height: 500 meters

Stability Class: C (user override)

Air Temperature: $25^{\circ} \mathrm{C}$

Relative Humidity: 50\%

Ground Roughness: Urban or forest

Cloud Cover: 8 tenths

\section{SOURCE STRENGTH INFORMATION:}

Puddle Area: 40700 square feet

Average Puddle Depth: 1 centimeters

Soil Type: Default

Ground Temperature: $25^{\circ} \mathrm{C}$

Initial Puddle Temperature: Ground temperature

Release Duration: ALOHA limited the duration to 1 hour

Max Computed Release Rate: 29.7 kilograms/min

Max Average Sustained Release Rate: 29.3 kilograms/min

(averaged over a minute or more)

Total Amount Released: 1,588 kilograms

FOOTPRINT INFORMATION:

Dispersion Module: Gaussian

User-specified LOC: $500 \mathrm{mg} /(\mathrm{cu} \mathrm{m})$

Max Threat Zone for LOC: 34 meters

Note: Footprint was not drawn because effects of near-field patchiness make dispersion predictions unreliable for short distances. 
Title of Project EPHA for THM

Subject Concentration Analysis for THM Hazardous Chemicals

Computer N.E. Baucom B. K. Tharakan Date 09/16/96

1-RD-23 AVERAGI / unX DIST SEO 3

SITE DATA INEORMATION:

Location: AIKEN, SOUTH CAROLINA

Building Air Exchanges Per Hour: 60 (User specified)

Time: June 30, 19981200 hours EDT (User specified)

CHEMICAI INFORMATION :

Chemical Name: DIETHYLENE GLYCOL DIMETHYL ETHER

Molecular Weight: $134.18 \mathrm{~kg} / \mathrm{kmol}$

TLV-TWA: -unavail- IDLH: -unavail

Footprint Level of Concern: $500 \mathrm{mg} /(\mathrm{cu} \mathrm{m}$ )

Boiling Point: $159.76^{\circ} \mathrm{C}$

Vapor Pressure at Ambient Temperature: 0.0039 atm

Ambient Saturation Concentration: 3,963 ppm or 0.408

ATMOSPHERIC INFORMATION: (MANUAL INPUT OF DATA)

Wind: 2.5 meters/sec from $90^{\circ}$ true at 2 meters

Inversion Height: 500 meters

Stability Class: C (user override)

Air Temperature: $25^{\circ} \mathrm{C}$

Relative Humidity: $50 \%$ Ground Roughness: Urban or forest

Cloud Cover: 8 tenths

SOURCE STRENGTH INEORMATION:

Pudale Area: 10000 square meters

Average Pudale Depth: 1 centimeters

Soil Type: Default Ground Temperature: $25^{\circ} \mathrm{C}$

Initial Pudale Temperature: Ground temperature

Release Duration: ALOHA limited the duration to 1 hour

Max Computed Release Rate: $75.8 \mathrm{kilograms} / \mathrm{min}$

Max Average Sustained Release Rate: $74.7 \mathrm{kilograms} / \mathrm{min}$ (averaged over a minute or more)

Total Amount Released: 4,047 kilograms

FOOTPRINT INFORMATION:

Dispersion Module: Gaussian

User-specified LOC: $500 \mathrm{mg} /(\mathrm{cu} \mathrm{m}$ )

Max Threat Zone for LOC: 56 meters

Note: Footprint was not drawn because effects of near-field patchiness make dispersion predictions unreliable for short distances. 
Title of Project EPHA for THM

Subject Concentration Analysis for THM Hazardous Chemicals

\section{Computer N.E. Baucom B.K. Tharakan Date 09/16/96} I-RD-26 AVIRAOI AIIRI
Reviewed by S. A. Heinderson Works Calculation 8

Sheet No. 245 of 314

SITE DATA INFORMATION:

Location: AIKEN, SOUTH CAROLINA

Building Air Exchanges Per Hour: 60 (User specified)

Time: June 30, 19981200 hours EDT (User specified)

CHEMICAL INFORMATION :

Chemical Name: HYDROGEN PEROXIDE

Molecular Weight: $34.01 \mathrm{~kg} / \mathrm{kmol}$

TLV-TWA: 1 ppm IDLH: 75 ppm

Footprint Level of Concern: $50 \mathrm{ppm}$

Boiling point: $150.20^{\circ} \mathrm{C}$

Vapor Pressure at Ambient Temperature: 0.0026 atm

Ambient Saturation Concentration: 2,612 ppm or $0.26 \%$

ATMOSPHERIC INFORMATION: (MANUAI INPUT OF DATA)

Wind: 2.5 meters/sec from $90^{\circ}$ true at 2 meters

Inversion Height: 500 meters

stability Class: C (user overxide)

Air Temperature: $25^{\circ} \mathrm{C}$

Relative Humiaity: 508

Ground Roughness: Urban or forest

Cloud Cover: 8 tenths

SOURCE STRENGTH INFORMATION:

Puddle Area: 26800 square feet

Average Puddle Depth: 1 centimeters

Soil Type: Default Ground Temperature: $25^{\circ} \mathrm{C}$

Initial pudale Temperature: Ground temperature

Release Duration: ALOHA limited the duration to 1 hour

Max Computed Release Rate: 4.13. kilograms/min

Max Average Sustained Release Rate: 4.05 kilograms/min

(averaged over a minute or more)

Total Amount Released: 215 kilograms

FOOTPRINT INEORMATION:

Dispersion Module: Gaussian

User-specified LOC: $50 \mathrm{ppm}$

Max Threat Zone for LOC: 30 meters

Max Threat Zone for IDLH: 28 meters

Note: Footprint was not drawn because effects of near-field patchiness make

dispersion predictions unreliable for short distances.

TIME DEPENDENTI INFORMATION:

Concentration Estimates at the point:

Downwind: $\quad 30$ meters

off Centerline: 0 meters

Max Concentration:

Outdoor: $50 \mathrm{ppm}$

Indoor: $50 \mathrm{ppm}$

Note: Indoor graph is shown with a dotted line. 
Title of Project EPHA for THM

Subject Concentration Analysis for THM Hazardous Chemicals

\section{1-RD-26 AVERAGE / SAT}

SITE DATA INFORMATION:

Location: AIKEN, SOUTH CAROLINA

Building Air Exchanges Per Hour: 60 (User specified)

Time: June 30, 19981200 hours EDT (User specified)

CHEMICAL INFORMATION:

Chemical Name: HYDROGEN PEROXIDE

Molecular Weight: $34.01 \mathrm{~kg} / \mathrm{kmol}$

TLV-TWA: $1 \mathrm{ppm}$ IDLH: $75 \mathrm{ppm}$

Footprint Level of Concern: $50 \mathrm{ppm}$

Boiling Point: $150.20^{\circ} \mathrm{C}$

Vapor Pressure at Ambient Temperature: 0.0026 atm

Ambient Saturation Concentration: 2,612 ppm or 0.268

ATMOSPHERIC INFORMATION: (MANUAL INPUT OF DATA)

Wind: 2.5 meters $/ \mathrm{sec}$ from $90^{\circ}$ true at 2 meters

Inversion Height: 500 meters

Stability class: C (user override)

Air Temperature: $25^{\circ} \mathrm{C}$

Relative Humidity: 50

Ground Roughness: Urban or forest

Cloud Cover: 8 tenths

SOURCE STRENGTH INFORMATION:

Puddle Area: 100001 square feet

Average Puddle Depth: 1 centimeters

Soil Type: Default Ground Temperature: $25^{\circ} \mathrm{C}$

Initial Puddle Temperature: Ground temperature

Release Duration: ALOHA limited the duration to 1 hour

Max Computed Release Rate: $14.6 \mathrm{kilograms} / \mathrm{min}$

Max Average Sustained Release Rate: $14.3 \mathrm{kilograms} / \mathrm{min}$

(averaged over a minute or more)

Total Amount Released: 755 kilograms

FOOTPRINT INFORMATION:

Dispersion Module: Gaussian

User-specified. LOC: $50 \mathrm{ppm}$

Max Threat Zone for LOC: 56 meters

Max Threat Zone for IDLH: 55 meters

Note: Footprint was not drawn because effects of near-field patchiness make dispersion predictions unreliable for short distances.

TIME DEPENDENT INFORMATION:

Concentration Estimates at the point:

Downwind: 100 meters

off Centerline: 0 meters

Max Concentration:

Outdoor: $17.4 \mathrm{ppm}$

Indoor: $17.4 \mathrm{ppm}$

Note: Indoor graph is shown with a dotted Iine. 


\section{ENGINEERING COMPUTATION SHEET}

Title of Project EPHA for THM

Reviewed by S. A. Henderson

Works Calculation 8

Sheet No. 247 of 314

SITE DATA INFORMATION:

Location: AIREN, SOUTH CAROLINA

Building Air Exchanges Per Hour: 60 (User specified)

Time: June 30, 19981200 hours EDT (User specified)

CHEMICAL INFORMATION:

Chemical Name: HYDROGEN PEROXIDE

Molecular Weight: $34.01 \mathrm{~kg} / \mathrm{kmol}$

TLV-TWA: $1 \mathrm{ppm}$

Footprint Level of Concern: $50 \mathrm{ppm}$

Boiling Point: $150.20^{\circ} \mathrm{C}$

Vapor Pressure at Ambient Temperature: 0.0026 atm

Ambient Saturation Concentration: 2,612 ppm or $0.26 \%$

ATMOSPHERIC INFORMATION: (MANUAL INPUT OF DATA)

Wind: 2.5 meters $/ \mathrm{sec}$ from $90^{\circ}$ true at 2 meters

Inversion Height: 500 meters

stability class: C (user override)

Air Temperature: $25^{\circ} \mathrm{C}$

Relative Humidity: 508

Ground Roughness: Urban or forest

cloud Cover: 8 tenths

SOURCE STRENGTH INFORMATION:

Puddle Area: 24420 square feet

Average Puddle Depth: 1 centimeters

Soil Type: Default Ground Temperature: $25^{\circ} \mathrm{C}$

Initial Puddle Temperature: Ground temperature

Release Duration: ALOHA limited the duration to 1 hour

Max Computed Release Rate: $3.78 \mathrm{kilograms/min}$

Max Average Sustained Release Rate: 3.71 kilograms/min

(averaged over a minute or more)

Total Amount Released: 197 kilograms

FOOTPRINT INFORMATION:

Dispersion Module: Gaussian

User-specified LOC: $50 \mathrm{ppm}$

Max Threat Zone for LOC: 29 meters

Max Threat Zone for IDLH: 27 meters 
Title of Project EPHA for THM

Subject Concentration Analysis for THM Hazardous Chemicals

Computer N.E. Baucom B. K. Tharakan Date 09/16/96

1-RD-26 AVERAGE MAX DIST SEG 2

SITE DATA INFORMATION:

Location: AIKEN, SOUTH CAROLNA

Building Air Exchanges Per Hour: 60 (User specified)

Time: June 30, 19981200 hours EDT (User specified)

\section{CHEMICAL INFORMATION:}

Chemical Name: HYDROGEN PEROXIDE

Molecular Weight: $34.01 \mathrm{~kg} / \mathrm{kmol}$

TLV-TWA: 1 ppm

IDLH: 75 ppm

Footprint Level of Concern: $50 \mathrm{ppm}$

Boiling Point: $150.20^{\circ} \mathrm{C}$

Vapor Pressure at Ambient Temperature: $0.0026 \mathrm{~atm}$

Ambient Saturation Concentration: 2,612 ppm or $0.26 \%$

ATMOSPHERIC INFORMATION: (MANUAL INPUT OF DATA)

Wind: 2.5 meters $/ \mathrm{sec}$ from $90^{\circ}$ true at 2 meters

Inversion Height: 500 meters

Stability Class: $\mathbf{C}$ (user override)

Air Temperature: $25^{\circ} \mathrm{C}$

Relative Humidity: $50 \%$

Cloud Cover: 8 tenths

Ground Roughness: Urban or forest

\section{SOURCE STRENGTH INFORMATION:}

Puddle Area: 40700 square feet

Average Puddle Depth: 1 centimeters

Soil Type: Default

Initial Puddle Temperature: Ground temperature

Release Duration: ALOHA limited the duration to 1 hour

Max Computed Release Rate: 6.16 kilograms/min

Max Average Sustained Release Rate: 6.04 kilograms/min

(averaged over a minute or more)

Total Amount Released: 320 kilograms

\section{FOOTPRINT INFORMATION:}

Dispersion Module: Gaussian

User-specified LOC: $50 \mathrm{ppm}$

Max Threat Zone for LOC: 37 meters

Max Threat Zone for IDLH: 34 meters

Note: Footprint was not drawn because effects of near-field patchiness make dispersion predictions unreliable for short distances.
Reviewed by S. A. Henderson 
Title of Project EPHA for THM

Reviewed by S. A. Henderson

Subject Concentration Analysis for THM Hazardous Chemicals

\section{1-RD-26 AVERAGE MAX DIST SEG 3}

\section{SITE DATA INFORMATION:}

Location: AIKEN, SOUTH CAROLINA

Building Air Exchanges Per Hour: 60 (User specified)

Time: June 30, 19981200 hours EDT (User specified)

\section{CHEMICAL INFORMATION:}

Chemical Name: HYDROGEN PEROXIDE

Molecular Weight: $34.01 \mathrm{~kg} / \mathrm{kmol}$

TLV-TWA: $1 \mathrm{ppm}$

IDLH: 75 ppm

Footprint Level of Concern: 50 ppm

Boiling Point: $150.20^{\circ} \mathrm{C}$

Vapor Pressure at Ambient Temperature: $0.0026 \mathrm{~atm}$

Ambient Saturation Concentration: 2,612 ppm or $0.26 \%$

\section{ATMOSPHERIC INFORMATION: (MANUAL INPUT OF DATA)}

Wind: 2.5 meters $/ \mathrm{sec}$ from $90^{\circ}$ true at 2 meters

Inversion Height: 500 meters

Stability Class: $\mathrm{C}$ (user override)

Air Temperature: $25^{\circ} \mathrm{C}$

Relative Humidity: $50 \%$

Ground Roughness: Urban or forest

Cloud Cover: 8 tenths

\section{SOURCE STRENGTH INFORMATION:}

Puddle Area: 10000 square meters

Average Puddle Depth: 1 centimeters

Soil Type: Default

Ground Temperature: $25^{\circ} \mathrm{C}$

Initial Puddle Temperature: Ground temperature

Release Duration: AlOHA limited the duration to 1 hour

Max Computed Release Rate: 15.6 kilograms/min

Max Average Sustained Release Rate: 15.3 kilograms/min (averaged over a minute or more)

Total Amount Released: 810 kilograms

\section{FOOTPRINT INFORMATION:}

Dispersion Module: Gaussian User-specified LOC: $50 \mathrm{ppm}$

Max Threat Zone for LOC: 58 meters

Max Threat Zone for IDLH: 56 meters 
Titlo of Project EPHA for THM

Subject Concentration Analysis for THM Hazardous Chemicals

Computer N.E. Baucom B. K. Tharakan Date 09/16/96

1-RD-29 AVERAGE / ALERT

SITE DATA INFORMATION:

Location: AIKEN, SOUTH CAROLINA

Building Air Exchanges Per Hour: 60 (User specified)

Time: June 30, 19981200 hours EDT (User specified)

CHEMICAL INFORMATION:

Chemical Name: METHYL ETHYL KETONE

Molecular Weight: $72.11 \mathrm{~kg} / \mathrm{kmol}$

TLV-TWA: 200 ppm

IDLH: $3000 \mathrm{ppm}$

Footprint Level of Concern: 300 ppm

Boiling Point: $79.64^{\circ} \mathrm{C}$

Vapor Pressure at Ambient Temperature: $0.12 \mathrm{~atm}$

Ambient Saturation Concentration: 123,729 ppm or $12.4 \%$

ATMOSPHERIC INFORMATION: (MANUAL INPUT OF DATA)

Wind: 2.5 meters $/ \mathrm{sec}$ from $90^{\circ}$ true at 2 meters

Inversion Height: 500 meters

Stability Class: $\mathrm{C}$ (user override)

Air Temperature: $25^{\circ} \mathrm{C}$

Relative Humidity: 50\% Ground Roughness: Urban or forest

Cloud Cover: 8 tenths

\section{SOURCE STRENGTH INFORMATION:}

Puddle Area: 3165 square feet

Average Puddle Depth: 1 centimeter

Soil Type: Default

Ground Temperature: $25^{\circ} \mathrm{C}$

Initial Puddle Temperature: Ground temperature

Release Duration: ALOHA limited the duration to 1 hour

Max Computed Release Rate: 33 kilograms/min

Max Average Sustained Release Rate: 30.2 kilograms/min

(averaged over a minute or more)

Total Amount Released: 1,663 kilograms

FOOTPRINT INFORMATION:

Dispersion Module: Gaussian

User-specified LOC: $300 \mathrm{ppm}$

Max Threat Zone for LOC: 30 meters

Max Threat.Zone for IDLH: less than 10 meters (10.9 yards)

Note: Footprint was not drawn because effects of near-field patchiness make dispersion predictions unreliable for short distances.

TIME DEPENDENT INFORMATION:

Concentration Estimates at the point:

Downwind: $\quad 30$ meters

Off Centerline: 0 meters

Max Concentration:

Outdoor: $300 \mathrm{ppm}$

Indoor: $300 \mathrm{ppm}$

Note: Indoor graph is shown with a dotted line.
Reviewed by S. A. Henderson

Works Calculation 8

- Sheet No. 250 of 314 
Titie of Project EPHA for THM

Reviewed by S. A. Henderson

Subject Concentration Analysis for THM Hazardous Chemicals

Works Calculation 8

Computer N.E. Baucom B. K. Tharakan Date 09/16/96

1-RD-29 AVERAGs / SAS.

Sheet No. 251 of 314

SITE DATA INFORMATION:

Location: AIREN, SOUTH CAROLINA

Building Air Exchanges Per Hour: 60 (User specified)

Time: June 30, 19981200 hours EDT (User specified)

CHEMICAI INFORMATION :

Chemical Name: METHYL ETHYL KETONE

Molecular Weight: $72.11 \mathrm{~kg} / \mathrm{kmol}$

TLV-TWA: $200 \mathrm{ppm}$ IDLH: $3000 \mathrm{ppm}$

Footprint Level of Concern: $300 \mathrm{ppm}$

Boiling point: $79.64^{\circ} \mathrm{C}$

Vapor Pressure at Ambient Temperature: 0.12 atm

Ambient Saturation Concentration: 123,729 ppm or $12.4 \%$

ATMOSPHERIC INFORMATION: (MANUAL INPUT OF DATA)

Wind: 2.5 meters $/ \mathrm{sec}$ from $90^{\circ}$ true at 2 meters

Inversion Hẻight: 500 meters

stability class: C (user override)

Air Temperature: $25^{\circ} \mathrm{C}$

Relative Humidity: 508 Ground Roughness:Urban or forest

Cloud Cover: 8 tenths

SOURCE STRENGTH INFORMATION:

Puddle Area: 9384 square feet

Average Puddle Depth: 1 centimeters

Ground Roughness:' Urban or forest

Soil Type: Default Ground Temperature: $25^{\circ} \mathrm{C}$

Initial Pudale Temperature: Ground temperature

Release Duration: ALOHA limited the duration to 1 hour

Max Computed Release Rate: $92.9 \mathrm{kilograms} / \mathrm{min}$

Max Average Sustained Release Rate: $85.6 \mathrm{kilograms} / \mathrm{min}$

(averaged over a minute or more)

Total Amount Released: 4,747 kilograms

FOOTPRINT INFORMATION:

Dispersion Module: Gaussian

User-specified LOC: 300 ppm

Max Threat Zone for LOC: 49 meters

Max Threat zone for IDLH: 17 meters

Note: Footprint was not drawn because effects of near-field patchiness make

dispersion predictions unreliable for short distances.

TIME DEPENDENT INFORMATION:

Concentration Estimates at the point:

Downwind: 100 meters

off Centerline: 0 meters

Max Concentration:

Outdoor: $141 \mathrm{ppm}$

Indoor: $141 \mathrm{ppm}$

Note: Indoor graph is shown with a dotted line. 
Title of Project EPHA for THM

Subject Concentration Analysis for THM Hazardous Chemicals

Computer N.E. Baucom B. K. Tharakan Date 09/16/96

1-RD-29 AVERAGE MDX DIST SEO 1

SITE DATA INFORMATION:

Location: AIKEN, SOUTH CAROLINA

Building Air Exchanges Per Hour: 60 (User specified)

Time: June 30, 19981200 hours EDT (User specified)

CHEMICAL INFORMATION:

Chemical Name: METHYL ETHYL RETONE

Molecular Weight: $72.11 \mathrm{~kg} / \mathrm{kmol}$

TLV-TWA: $200 \mathrm{ppm}$

Footprint Level of Concern: 300 ppm

Boiling Point: $79.64^{\circ} \mathrm{C}$

Vapor Pressure at Ambient Temperature: 0.12 atm

Ambient Saturation Concentration: 123,729 ppm or 12.48

ATMOSPHERIC INFORMATION: (MANUAC INPUT OF DATA)

Wind: 2.5 meters $/ \mathrm{sec}$ from $90^{\circ}$ true at 2 meters

Inversion Height: 500 meters

Stability Class: C (user override)

Air Temperature: $25^{\circ} \mathrm{C}$

Relative Humidity: $50 \%$

Cloud Cover: 8 tenths

Ground Roughness: Urban of forest

SOURCE STRENGTH INFORMATION: Puddle Area: 24420 square feet Average Puddle Depth: 1 centimeters Soil Type: Default Ground Temperature: $25^{\circ} \mathrm{C}$ Initial Puddle Temperature: Ground temperature Release Duration: ALOHA limited the duration to 1 hour Max Computed Release Rate: 231 kilograms/min

Max Average Sustained Release Rate: $229 \mathrm{kilograms/min}$ (averaged over a minute or more)

Total Amount Released: 11,962 kilograms

FOOTPRINT INFORMATION:

Model Run: Heavy Gas

User-specified IOC: 300 ppm

Max Threat zone for LOC: 313 meters 
Title of Project EPHA for THM

Subject Concentration Analysis for THM Hazardous Chemicals

Computer N.E. Baucom B. K. Tharakan Date 09/16/96 1-RD-29 AVERAGE / MAX DIST SEG 2

SITE DATA INFORMATION: Location: AIKEN, SOUTH CAROLINA

Building Air Exchanges Per Hour: 60 (User specified)

Time: June 30, 19981200 hours EDT (User specified)

CHEMICAL INFORMATION:

Chemical Name: METHYL ETHYL KETONE

Molecular Weight: $72.11 \mathrm{~kg} / \mathrm{kmol}$

TLV-TWA: 200 ppm

IDLH: $3000 \mathrm{ppm}$

Footprint Level of Concem: $300 \mathrm{ppm}$

Boiling Point: $79.64^{\circ} \mathrm{C}$

Vapor Pressure at Ambient Temperature: $0.12 \mathrm{~atm}$

Ambient Saturation Concentration:123,729 ppm or $12.4 \%$

ATMOSPHERIC INFORMATION: (MANUAL INPUT OF DATA)

wind: $2.5 \mathrm{~meters} / \mathrm{sec}$ from $90^{\circ}$ true at 2 meters

Inversion Height: 500 meters

Stability Class: $\mathrm{C}$ (user override)

Air Temperature: $25^{\circ} \mathrm{C}$

Relative Humidity: $50 \%$

Cloud Cover. 8 tenths

SOURCE STRENGTH INFORMATION:

Puddle Area: 40700 square feet

Average Puddle Depth: 1 centimeters

Soil Type: Default

Initial Puddle Temperature: Ground temperature

Release Duration: ALOHA limited the duration to 1 hour

Max Computed Release Rate: 377 kilograms/min

Max Average Sustained Release Rate: 372 kilograms/min

(averaged over a minute or more)

Total Amount Released: 19,605 kilograms

FOOTPRINT INFORMATION:

Model Run: Heavy Gas

User-specified LOC: 300 ppm

Max Threat Zone for LOC: 400 meters
Reviewed by S. A. Henderson

Works Calculation 8

Sheet No. 253 of 314 
SITE DATA INFORMATION: Location: AIKEN, SOUTH CAROLINA

Building Air Exchanges Per Hour: 60 (User specified)

Time: June 30, 19981200 hours EDT (User specified)

CHEMICAL INFORMATION :

Chemical Name: METHYL ETHYL KETONE

Molecular Weight: $72.11 \mathrm{~kg} / \mathrm{kmol}$

TLV-TWA: $200 \mathrm{ppm}$ IDLH: $3000 \mathrm{ppm}$

Footprint Level of Concern: 300 ppm

Boiling point: $79.64^{\circ} \mathrm{C}$

Vapor Pressure at Ambient Temperature: $0.12 \mathrm{~atm}$

Ambient Saturation Concentration: 123,729 ppm or 12.48

ATMOSPHERIC INFORMATION: (MANUAL INPUT OF DATA)

Wind: 2.5 meters/sec from $90^{\circ}$ true at 2 meters

Inversion Height: 500 meters

Stability class: C (user override)

Air Temperature: $25^{\circ} \mathrm{C}$

Relative Humidity: $50 \%$

Ground Roughness: Urban or forest

Cloud Cover: 8 tenths

SOURCE STRENGTH INFORMATION:

Puddle Area: 10000 square meters

Average Puddle Depth: 1 centimeters

Soil Type: Default Ground Temperature: $25^{\circ} \mathrm{C}$

Initial puddle Temperature: Ground temperature

Release Duration: ALOHA limited the duration to 1 hour

Max Computed Release Rate: 954 kilograms/min

Max Average Sustained Release Rate: 944 kilograms/min (averaged over a minute or more)

Total Amount Released: 50,264 kilograms

FOOTPRINT INFORMATION:

Model Run: Heavy Gas

User-specified LOC: 300 ppm

Max Threat Zone for LOC: 640 meters 
Title of Project EPHA for THM

Subject Concentration Analysis for THM Hazardous Chemicals

Computer N.E. Baucom B. K. Tharakan Date 09/16/96 1-RD-30 AVERAGE / ALERT

SITE DATA INFORMATION: Location: AIKEN, SOUTH CAROLINA

Building Air Exchanges Per Hour: 60 (User specified)

Time: June 30, 19981200 hours EDT (User specified)

CHEMICAL INFORMATION:

Chemical Name: METHYL ISOBUTYL KETONE

Molecular Weight: $100.16 \mathrm{~kg} / \mathrm{kmol}$

TLV-TWA: $50 \mathrm{ppm}$

IDLH: $3000 \mathrm{ppm}$

Footprint Level of Concern: 500 ppm

Boiling Point: $116.50^{\circ} \mathrm{C}$

Vapor Pressure at Ambient Temperature: $0.026 \mathrm{~atm}$

Ambient Saturation Concentration: 26,580 ppm or $2.66 \%$

ATMOSPHERIC INFORMATION: (MANUAL INPUT OF DATA)

Wind: 2.5 meters $/ \mathrm{sec}$ from $90^{\circ}$ true at 2 meters

Inversion Height: 500 meters

Stability Class: $\mathrm{C}$ (user override)

Air Temperature: $25^{\circ} \mathrm{C}$

Relative Humidity: 50\%

Cloud Cover. 8 tenths

SOURCE STRENGTH INFORMATION:

Puddle Area: 30001 square feet

Average Puddle Depth: 1 centimeters

Soil Type: Default

Initial Puddle Temperature: Ground temperature

Release

Duration: ALOHA limited the duration to 1 hour

Max Computed Release Rate: 94.3 kilograms/min

Max Average Sustained Release Rate:

(laveraged over a minute or more)

Total Amount Released: 5,360 kilograms

FOOTPRINT INFORMATION:

Dispersion Module: Gaussian

User-specified LOC: 500 ppm

Max Threat Zone for LOC: 30 meters

Max Threat Zone for IDLH: 30 meters

Note: Footprint was not drawn because effects of near-field patchiness make dispersion predictions unreliable for short distances. .

TIME DEPENDENT INFORMATION:

Concentration Estimates at the point:

Downwind:

30 meters

Off Centerline: 0 meters

Max Concentration:

Outdoor: $393 \mathrm{ppm}$

Indoor: 393 ppm

Note: Indoor graph is shown with a dotted line.
Reviewed by S. A. Henderson

Works Calculation 8

Sheet No: 255 of 314 
Title of Project EPHA for THM

Subject Concentration Analysis for THM Hazardous Chemicals

Computer N.E. Baucom B. K. Tharakan Date 09/16/96

1-RD-30 AVtRACE / SA.

SITE DATA INFORMATION:

Location: AIKEN, SOUTH CAROLINA

Building Air Exchanges Per Hour: 60 (User specified)

Time: June 30, 19981200 hours EDT (User specified)

CHEMICAL INFORMATION:

Chemical Name: METHYL ISOBUTYL KETONE

Molecular Weight: $100.16 \mathrm{~kg} / \mathrm{kmol}$

TLV-TWA: 50 ppm 50 IDLE: $3000 \mathrm{ppm}$

Footprint Level of Concern: $500 \mathrm{ppm}$

Boiling point: $116.50^{\circ} \mathrm{C}$

Vapor Pressure at Ambient Temperature: $0.026 \mathrm{~atm}$

Ambient Saturation Concentration: 26,580 ppm or $2.66 \%$

ATMOSPHERIC INFORMATION: (MANUAL INPUT OF DATA)

Wind: 2.5 meters $/ \mathrm{sec}$ from $90^{\circ}$ true at 2 meters

Inversion Height: 500 meters

Stability Class: $C$ (user override)

Air Temperature: $25^{\circ} \mathrm{C}$

Relative Humidity: 508

Cloud Cover: 8 tenths

Ground Roughness: Urban or forest

SOURCE STRENGTH INFORMATION:

Puddle Area: 61426 square feet

Average Pudale Depth: 1 centimeters

Soil Type: Default

Ground Temperature: $25^{\circ}$

Releaseduration: ALOHA limited the duration to 1 hour

Max Computed Release Rate: $188 \mathrm{kilograms/min}$

MaxAverage Sustained Release Rate: $187 \mathrm{kilograms/min}$

(averaged over a minute or more)

Total Amount Released: 10,688 kilograms

FOOTPRINT INFORMATION:

Dispersion Module: Gaussian

User-specified LOC: 500 ppm

Max Threat Zone for LOC: 42 meters

Max Threat Zone for IDLH: 43 meters

Note: Footprint was not drawn because effects of near-field patchiness make dispersion predictions unreliable for short distances.

TIME DEPENDENT INFORMATION:

Concentration Estimates at the point:

Downwind: 100 meters

off Centerline: 0 meters

Max Concentration:

outdoor: $92.2 \mathrm{ppm}$

Indoor: $92.2 \mathrm{ppm}$

Note: Indoor graph is shown with a dotted line.
Reviewed by S. A. Henderson

Works Calculation 8

Sheet No. $\underline{256 \text { of } 314}$ 
Title of Project EPHA for THM

Subject Concentration Analysis for THM Hazardous Chemicals

Computer N.E. Baucom B. K. Tharakan Date 09/16/96

1-RD-30 AVERAGE MAX DIST SEG 1

SITE DATA INFORMATION:

Location: AIKEN, SOUTH CAROLINA

Building Air Exchanges Per Hour: 60 (User specified)

Time: June 30, 19981200 hours EDT (User specified)

CHEMICAL INFORMATION:

Chemical Name: METHYL ISOBUTYL KETONE

Molecular Weight: $100.16 \mathrm{~kg} / \mathrm{kmol}$

TLV-TWA: $50 \mathrm{ppm}$

IDLH: 3000 ppm

Footprint Level of Concern: 500 ppm

Boiling Point: $116.50^{\circ} \mathrm{C}$

Vapor Pressure at Ambient Temperature: $0.026 \mathrm{~atm}$

Ambient Saturation Concentration: $26,580 \mathrm{ppm}$ or $2.66 \%$

ATMOSPHERIC INFORMATION: (MANUAL INPUT OF DATA)

Wind: 2.5 meters $/ \mathrm{sec}$ from $90^{\circ}$ true at 2 meters

Inversion Height: 500 meters

Stability Class: $\mathrm{C}$ (user override)

Air Temperature: $25^{\circ} \mathrm{C}$

Relative Humidity: 50\%

Cloud Cover: 8 tenths

Ground Roughness: Urban or forest

SOURCE STRENGTH INFORMATION:

Puddle Area: 24420 square feet Average Puddle Depth: 1 centimeters

Soil Type: Default

Ground Temperature: $25^{\circ} \mathrm{C}$

Initial Puddle Temperature: Ground temperature

Release Duration: ALOHA limited the duration to 1 hour

Max Computed Release Rate: 77.3 kilograms/min

Max Average Sustained Release Rate: 76.8 kilograms/min

(averaged over a minute or more)

Total Amount Released: 4,397 kilograms

\section{FOOTPRINT INFORMATION:}

Dispersion Module: Gaussian

User-specified LOC: 500 ppm

Max Threat Zone for LOC: 27 meters

Max Threat Zone for IDLH: 27 meters

Note: Footprint was not drawn because effects of near-field patchiness make dispersion predictions unreliable for short distances.
Reviewed by S. A. Henderson

Works Calculation 8

Sheet No. 257 of 314 
Title of Project EPHA for THM

Reviewed by S. A. Henderson

Subject Concentration Analysis for THM Hazardous Chemicals

Works Calculation 8

Computer N.E. Baucom B.K. Tharakan Date 09/16/96

1-RD-30 AVERAGE MAX DIST SEG 2

SITE DATA INFORMATION:

Location: AIKEN, SOUTH CAROLINA

Building Air Exchanges Per Hour: 60 (User specified)

Time: June 30, 19981200 hours EDT (User specified)

CHEMICAL INFORMATION:

Chemical Name: METHYL ISOBUTYL KETONE

Molecular Weight: $100.16 \mathrm{~kg} / \mathrm{kmol}$

TLV-TWA: $50 \mathrm{ppm}$

IDLH: $3000 \mathrm{ppm}$

Footprint Level of Concern: 500 ppm

Boiling Point: $116.50^{\circ} \mathrm{C}$

Vapor Pressure at Ambient Temperature: $0.026 \mathrm{~atm}$

Ambient Saturation Concentration: 26,580 ppm or $2.66 \%$

ATMOSPHERIC INFORMATION: (MANUAL INPUT OF DATA)

Wind: 2.5 meters $/ \mathrm{sec}$ from $90^{\circ}$ true at 2 meters

Inversion Height: 500 meters

Stability Class: C (user override)

Air Temperature: $25^{\circ} \mathrm{C}$

Relative Humidity: $50 \%$

Ground Roughness: Urban or forest

Cloud Cover: 8 tenths

SOURCE STRENGTH INFORMATION:

Puddle Area: 40700 square feet

Average Puddle Depth: 1 centimeters

Soil Type: Default Ground Temperature: $25^{\circ} \mathrm{C}$

Initial Puddle Temperature: Ground temperature

ReleaseDuration: ALOHA limited the duration to 1 hour

Max Computed Release Rate: 127 kilograms/min

MaxAverage Sustained Release Rate: 126 kilograms/min

(averaged over a minute or more)

Total Amount Released: 7,189 kilograms

FOOTPRINT INFORMATION:

Dispersion Module: Gaussian

User-specified LOC: $500 \mathrm{ppm}$

Max Threat Zone for LOC: 34 meters

Max Threat Zone for IDLH: 35 meters

Note: Footprint was not drawn because effects of near-field patchiness make dispersion predictions unreliable for short distances. 


\section{ENGINEEAING COMPUTATION SHEET}

Title of Project EPHA for THM

Subject Concentration Analysis for THM Hazardous Chemicals

Computer N.E. Baucom B. K. Tharakan Date 09/16/96

1-RD-30 AVARACI / unX DISI SEO 3

SITE DATA INFORMATION:

Location: AIKEN, SOUTH CAROLINA

Building Air Exchanges Per Hour: 60 (User specified)

Time: June 30, 19981200 hours EDT (User specified)

CHEMICAL INFORMATION:

Chemical Name: METHYL ISOBUTYL KETONE

Molecular Weight: $100.16 \mathrm{~kg} / \mathrm{kmol}$

TLV-TWA: 50 ppm

Footprint Level of Concern: $500 \mathrm{ppm}$

Boiling Point: $116.50^{\circ} \mathrm{C}$

Vapor Pressure at Ambient Temperature: $0.026 \mathrm{~atm}$

Ambient Saturation Concentration: 26,580 ppm or 2.668

ATMOSPHERIC INFORMATION: (MANUAL INPUT OF DATA)

Wind: 2.5 meters $/ \mathrm{sec}$ from $90^{\circ}$ true at 2 meters

Inversion Height: 500 meters

stability Class: C (user override)

Air Temperature: $25^{\circ} \mathrm{C}$

Relative Humidity: 508

Cloud Cover: 8 tenths

SOURCE STRENGTH INFORMATION:

Puddle Area: 10000 square meters

Average Pudale Depth: 1 centimeters

Soil Type: Default Ground Temperature: $25^{\circ} \mathrm{C}$

Initial puddle Temperature: Ground temperature

Release Duration: ALOHA limited the duration to 1 hour

Max Computed Release Rate: 324 kilograms/min

Max Average Sustained Release Rate: 322 kilograms/min (averaged over a minute or more)

Total Amount Released: 18,353 kilograms

Ground Roughness: Urban or forest

FOOTPRINT INFORMATION:

Model Run: Heavy Gas

User-specified IOC: 500 ppm

Max Threat Zone for LOC: 173 meters
Reviewed by S. A. Henderson

Works Calculation 8

Sheet No. 259 of 314 
Title of Project EPHA for THM

Subject Concentration Analysis for THM Hazardous Chemicals

Computer N.E. Baucom B. K. Tharakan Date 09/16/96

1-RD-32 AVERAGE ATRRT

SITE DATA INFORMATION:

LOCation: AIKEN, SOUTH CAROLINA

Building Aix Exchanges Per Hour: 60 (User specified)

Time: June 30, 19981200 hours EDT (User specified)

CHEMICAL INFORMATION:

Chemical Name: ETHYLENE GLYCOL MONOMETHYL ETHER

Molecular Weight: $76.10 \mathrm{~kg} / \mathrm{kmol}$

TLV-TWA: 5 ppm

Footprint Level of Concern: 25 ppm

Boiling Point: $124.40^{\circ} \mathrm{C}$

Vapor Pressure at Ambient Temperature: $0.012 \mathrm{~atm}$

Ambient Saturation Concentration: 12,061 ppm or 1.218

ATMOSPHERIC INFORMATION: (MANUAL INPUT OF DATA)

Wind: 2.5 meters $/ \mathrm{sec}$ from $90^{\circ}$ true at 2 meters

Inversion Height: 500 meters

Stability class: $c$ (user override)

Air Temperature: $25^{\circ} \mathrm{C}$.

Relative Humidity: $50 \%$

Cloud Cover: 8 tenths

Ground Roughness: Urban or forest

SOURCE STRENGTH INFORMATION:

Puddle Area: 1950 square feet

Average Puddle Depth: 1 centimeters

Soil Type: Default Ground Temperature: $25^{\circ} \mathrm{C}$

Initial Puddle Temperature: Ground temperature

Release Duration: ALOHA limited the duration to 1 hour

Max Computed Release Rate: $2.09 \mathrm{kilograms/min}$

Max Average Sustained Release Rate: 2.09 kilograms/min

(averaged over a minute or more)

Total Amount Released: 123 kilograms

TIME DEPENDENT INFORMATION:

Concentration Estimates at the point:

Downwind: 30 meters

off Centerline: 0 meters

Max Concentration:

Outdoor: $25 \mathrm{ppm}$

Indoor: 25 ppm

Note: Indoor graph is. shown with a dotted line. 


\section{1-RD-32 AVARACE: SAE}

SITE DATA INFORMATION:

Location: AIREN, SOUTH CAROLINA

Building Air Exchanges Per Hour: 60 (User specified)

Time: June 30, 19981200 hours EDT (User specified)

CHEMICAI INFORMATION:

Chemical Name: ETHYLENE GLYCOL MONOMETHYL ETHER

Molecular Weight: $76.10 \mathrm{~kg} / \mathrm{kmol}$

TLV-TWA: 5 ppm

Footprint Level of Concern: 25 ppm

Boiling Point: $124.40^{\circ} \mathrm{C}$

Vapor Pressure at Ambient Temperature: 0.012 atm

Ambient Saturation Concentration: 12,061 ppm or 1.218

ATMOSPHERIC INFORMATION: (MANUAL INPUT OF DATA)

Wind: 2.5 meters/sec from $90^{\circ}$ true at 2 meters

Inversion Height: 500 meters

stability Class: C (user override)

Air Temperature: $25^{\circ} \mathrm{C}$

Relative Humidity: $50 \%$ Ground Roughness: Urban or forest

Cloud Cover: 8 tenths

SOURCE STRENGTH INFORMATION:

Puddle Area: 27750 square feet

Average Pudale Depth: 1 centimeters

Soil Type: Default Ground Temperature: $25^{\circ} \mathrm{C}$

Initial Puddle'Temperature: Ground temperature

Release Duration: ALOFA limited the duration to 1 hour

Max Computed Release Rate: 26.3 kilograms/min

Max Average Sustained Release Rate: $26.2 \mathrm{kilograms} / \mathrm{min}$

(averaged over a minute or more)

Total Amount Released: 1,544 kilograms

TIME DEPENDENT INFORMATION:

Concentration Estimates at the point:

Downwind: 100 meters

off Centerline: 0 meters

Max Concentration:

Outdoor: 25 ppm

Indoor: 25 ppm Note: Indoor graph is shown with a dotted line. 
Title of Project EPHA for THM

Subject Concentration Analysis for THM Hazardous Chemicals

Computer N.E. Baucom B. K. Tharakan Date 09/16/96

1-RD-32 AVIRACE IAX DI8T SL:C 1

SITE DATA INFORMATION:

Location: AIREN, SOUTH CAROLINA

Building Air Exchanges Per Hour: 60 (User specified)

Time: June 30, 19981200 hours EDT (User specified)

CHEMICAI INFORMATION:

Chemical Name: ETHYLENE GLYCOL MONOMETHYL ETHER

Molecular Weight: $76.10 \mathrm{~kg} / \mathrm{kmol}$

TLV-TWA: $5 \mathrm{ppm}$

IDLH: $2000 \mathrm{ppm}$

Footprint Level of Concern: $25 \mathrm{ppm}$

Boiling Point: $124.40^{\circ} \mathrm{C}$

Vapor Pressure at Ambient Temperature: 0.012 atm

Ambient Saturation Concentration: $12,061 \mathrm{ppm}$ or 1.218

ATMOSPHERIC INFORMATION: (MANUAL INPUT OF DATA)

Wind: 2.5 meters $/ \mathrm{sec}$ from $90^{\circ}$ true at 2 meters

Inversion Height: 500 meters

stability class: C (user override)

Air Temperature: $25^{\circ} \mathrm{C}$

Relative Humidity: 508 Ground Roughness: Urban or forest

Cloud Cover: 8 tenths

SOURCE STRENGTH INFORMATION:

Pudale Area: 24420 square feet

Average Puddle Depth: 1 centimeters

Soil Type: Default

Ground Temperature: $25^{\circ} \mathrm{C}$

Initial puddle Temperature: Ground temperature

Release Duration: ALOHA limited the duration to 1 hour

Max Computed Release Rate: $23.3 \mathrm{kilograms} / \mathrm{min}$

Max Average Sustained Release Rate: $23.2 \mathrm{kilograms} / \mathrm{min}$

(averaged over a minute or more)

Total Amount Released: 1,367 kilograms

FOOTPRINT INFORMATION :

Dispersion Module: Gaussian

User-specified LOC: 25 ppm

Max Threat Zone for LOC: 94 meters

Max Threat Zone for IDHH: 27 meters

Note: Footprint was not drawn because effects of near-field patchiness make dispersion predictions unreliable for short distances.
Reviewed by S. A. Henderson

Works Calculation 8

Sheet No. 262 of 314 


\section{ENGINEERING COMPUTATION SHEET}

Title of Project EPHA for THM

Subject Concentration Analysis for THM Hazardous Chemicals

Computer N.E. Baucom B. K. Tharakan Date 09/16/96

1-RD-32 AVERACA / wax DIg2 gIEC 2

SITE DATA INFORMATION:

Location: AIKEN, SOUTH CAROLINA

Building Air Exchanges Per Hour: 60 (User specified)

Time: June 30,19981200 hours EDT (User specified)

CHEMICAL INEORMATION:

Chemical Name: ETHYLENE GLYCOL MONOMETHYL ETHER.

Molecular Weight: $76.10 \mathrm{~kg} / \mathrm{kmol}$

TLV-TWA: 5 ppm

Footprint Level of Concern: $25 \mathrm{ppm}$

Boiling Point: $124.40^{\circ} \mathrm{C}$

Vapor Pressure at Ambient Temperature: $0.012 \mathrm{~atm}$

Ambient Saturation Concentration: 12,061 ppm or 1.218

ATMOSPHERIC INFORMATION: (MANUAC INPUT OF DATA)

Wind: 2.5 meters $/ \mathrm{sec}$ from $90^{\circ}$ true at 2 meters

Inversion Height: 500 meters

stability class: c (user override)

Air Temperature: $25^{\circ} \mathrm{C}$

Relative Humidity: $50 \%$

Cloud Cover: 8 tenths

Ground Roughness : Urban or forest

SOURCE STRENGTH INFORMATION:

Puddle Area: 40700 square feet

Average Puddle Depth: 1 centimeters

Soil Type: Default Ground Temperature: $25^{\circ} \mathrm{C}$

Initial Puddle Temperature: Ground temperature

Release Duration: AIOHA limited the duration to 1 hour

Max Computed Release Rate: $37.9 \mathrm{kilograms} / \mathrm{min}$

Max Average Sustained Release Rate: $37.8 \mathrm{kilograms} / \mathrm{min}$ (avexaged over a minute or more)

Total Amourit Released: 2,226 kilograms

FOOTPRINT INFORMATION:

Dispersion Module: Gaussian

User-specified IOC: 25 ppm

Max Threat Zone for LOC: 119 meters

Max Threat Zone for IDLH: 34 meters
Reviewed by S. A. Henderson

Works Calculation 8

Sheet No. $\underline{263 \text { of } 314}$ 
SITE DATA INFORMATION:

Location: AIREN, SOUTH CAROLINA

Building Air Exchanges Per Hour: 60 (User specified)

Time: June 30, 19981200 hours EDT (User specified)

CHEMICAL INFORMATION:

Chemical Name: ETHYLENE GLYCOL MONOMETHYL ETHER

Molecular Weight: $76.10 \mathrm{~kg} / \mathrm{kmol}$

TLV-TWA: 5 ppm

Footprint Level of Concern: 25 ppm

Boiling Point: $124.40^{\circ} \mathrm{C}$

Vapor Pressure at Ambient Temperature: 0.012 atm

Ambient Saturation Concentration: $12,061 \mathrm{ppm}$ or 1.218

ATMOSPHERIC INFORMATION: (MANUAL INPUT OF DATA)

Wind: 2.5 meters/sec from $90^{\circ}$ true at 2 meters

Inversion Height: 500 meters

Stability Class: C (user override)

Air Temperature: $25^{\circ} \mathrm{C}$

Relative Humidity: 508

Ground Roughness: Urban or forest

Cloud Cover: 8 tenths

SOURCE STRENGTH INFORMATION:

Puddle Area: 10000 square meters

Average Puddle Depth: 1 centimeters

Soil Type: Default
Initial puddle Temperature: Ground temperature

Release Duration: ALOHA limited the duration to 1 hour

Max Computed Release Rate: 96.1 kilograms/min

Max Average Sustained Release Rate: $95.8 \mathrm{kilograms} / \mathrm{min}$

(averaged over a minute or more)

Total Amount Released: 5,646 kilograms

FOOTPRINT INEORMATION:

Dispersion Module: Gaussian

User-specified LOC: 25 ppm

Max Threat Zone for LOC: 186 meters

Max Threat Zone for IDLH: 56 meters 
Title of Project EPHA for THM

Subject Concentration Analysis for THM Hazardous Chemicals

Computer N.E. Baucom B. K. Tharakan Date 09/16/96 1-RD-33 AVERAGE ALERT

\section{SITE DATA INFORMATION:}

Location: AIKEN, SOUTH CAROLINA

Building Air Exchanges Per Hour: 60 (User specified)

Time: June 30, 19981200 hours EDT (User specified)

\section{CHEMICAL INFORMATION:}

Chemical Name: MONOETHANOLAMINE

Molecular Weight: $61.08 \mathrm{~kg} / \mathrm{kmol}$

\section{TLV-TWA: 3 ppm}

Footprint Level of Concern: $30 \mathrm{ppm}$

Boiling Point: $171.00^{\circ} \mathrm{C}$

Vapor Pressure at Ambient Temperature: 4.85e-004 atm

Ambient Saturation Concentration: 494 ppm or 0.049\%

\section{ATMOSPHERIC INFORMATION: (MANUAL INPUT OF DATA)}

Wind: 2.5 meters $/ \mathrm{sec}$ from $90^{\circ}$ true at 2 meters

Inversion Height: 500 meters

Stability Class: C (user override)

Air Temperature: $25^{\circ} \mathrm{C}$

Relative Humidity: 50\%

Cloud Cover. 8 tenths

Ground Roughness: Urban or forest

\section{SOURCE STRENGTH INFORMATION:}

Puddle Area: 30001 square feet

Average Puddle Depth: 1 centimeters

Soil Type: Default

Initial Puddle Temperature: Ground temperature

Release Duration: ALOHA limited the duration to 1 hour

Max Computed Release Rate: 1.69 kilograms/min

Max Average Sustained Release Rate: 1.65 kilograms/min (averaged over a minute or more)

Total Amount Released: 84.6 kilograms

FOOTPRINT INFORMATION:

Dispersion Module: Gaussian

User-specified LOC: 30 ppm

Max Threat Zone for LOC: 30 meters

Max Threat Zone for IDLH: 30 meters

Note: Footprint was not drawn because effects of near-field patchiness make dispersion predictions unreliable for short distances.

\section{TIME DEPENDENT INFORMATION:}

Concentration Estimates at the point:

Downwind:

30 meters

Off Centerline: 0 meters

Max Concentration:

Outdoor: $11.3 \mathrm{ppm}$

Indoor: $11.3 \mathrm{ppm}$

- Note: Indoor graph is shown with a dotted line.
Reviewed by S.. A. Henderson

Works Calculation 8

Sheet No. 265 of 314 
Title of Project EPHA for THM

Subject Concentration Analysis for THM Hazardous Chemicals

Computer N.E. Baucom B. K. Tharakan Date 09/16/96

1-RD-33 AVERAGE SAE

SITE DATA INFORMATION:

Location: AIKEN, SOUTH CAROLINA

Building Air Exchanges Per Hour: 60 (User specified)

Time: June 30, 19981200 hours EDT (User specified)

CHEMICAL INFORMATION:

Chemical Name: MONOETHANOLAMINE

Molecular Weight: $61.08 \mathrm{~kg} / \mathrm{kmol}$

TLV-TWA: 3 ppm

Footprint Level of Concern: $30 \mathrm{ppm}$

Boiling Point: $171.00^{\circ} \mathrm{C}$.

Vapor Pressure at Ambient Temperature: 4.85e-004 atm

Ambient Saturation Concentration: 494 ppm or 0.0498

ATMOSPHERIC INFORMATION: (MANUAC INPUT OF DATA)

Wind: 2.5 meters $/ \mathrm{sec}$ from $90^{\circ}$ true at 2 meters

Inversion height: 500 meters

Stability Class: C (user override)

Air Temperature: $25^{\circ} \mathrm{C}$

Relative Humidity: 508

Cloud Cover: 8 tenths

Ground Roughness: Urban or forest

SOURCE STRENGTH INFORMATION:

Puddle Area: 100001 square feet

Average Puddle Depth: 1 centimeters

Soil Type: Default Ground Temperature: $25^{\circ} \mathrm{C}$

Initial Puddle Temperature: Ground temperature

ReleaseDuration: ALOHA limited the duration to 1 hour

Max Computed Release Rate: 5.36 kilograms/min

MaxAverage Sustained Release Rate: $5.23 \mathrm{kilograms/min}$

laveraged

over a minute or more)

Total Amount Released: 268 kilograms

FOOTPRINT INFORMATION:

Dispersion Module: Gaussian

User-specified LOC: $30 \mathrm{ppm}$

Max Threat Zone for LOC: 55 meters

Max Threat Zone for IDLH: 54 meters

Note: Footprint was not drawn because effects of near-field patchiness make dispersion predictions unreliable for short distances.

TIME DEPENDENT INFORMATION:

Concentration Estimates at the point:

Downwind:

100 meters

off Centerline: 0 meters

Max Concentration:

Outdoor: $3.54 \mathrm{ppm}$

Indoor: $3.54 \mathrm{ppm}$

Note: Indoor graph is shown with a dotted line.
Reviewed by S. A. Henderson

Works Calculation 8

Sheet No. 266 of 314 
Title of Project EPHA for THM

Subject Concentration Analysis for THM Hazardous Chemicals

Computer N.E. Baucom B. K. Tharakan Date 09/16/96

1-RD-33 AVERAGE MAX DIST SEO 1

SITE DATA INFORMATION:

LOCATION: AIKEN, SOUTH CAROLINA

Building Air Exchanges Per Hour: 60 (User specified)

Time: June 30, 19981200 hours EDT (User specified)

CHEMICAL INFORMATION:

Chemical Name: MONOETHANOLAMINE

Molecular Weight: $61.08 \mathrm{~kg} / \mathrm{kmol}$

TLV-TWA: 3 ppm

Footprint Level of Concern: 30 ppm

Boiling Point: $171.00^{\circ} \mathrm{C}$

Vapor Pressure at Ambient Temperature: 4.85e-004 atm

Ambient Saturation Concentration: 494 ppm or 0.0498

ATMOSPHERIC INFORMATION: (MANUAI INPUT OF DATA)

Wind: 2.5 meters $/ \mathrm{sec}$ from $90^{\circ}$ true at 2 meters

Inversion Height: 500 meters

stability Class: C (user override)

Air Temperature: $25^{\circ} \mathrm{C}$

Relative Humidity: 508

Cloud Cover: 8 tenths

Ground Roughness: Urban or forest

SOURCE STRENGTH INFORMATION:

Puddle Area: 24420 square feet

Average Puddle Depth: 1 centimeters

Soil Type: Default

Ground Temperature: $25^{\circ} \mathrm{C}$

Initial puddle Temperature: Ground temperature

Release Duration: ALOHA limited the duration to 1 hour

Max Computed Release Rate: $1.39 \mathrm{kilograms} / \mathrm{min}$

Max Average Sustained Release Rate: 1.35 kilograms/min

(averaged over a minute or more)

Total Amount Released: 69.5 kilograms

FOOTPRINT INFORMATION:

Dispersion Module: Gaussian

User-specified LOC: 30 ppm

Max Threat Zone for LOC: 27 meters

Max Threat zone for IDLH: 27 meters

Note: Footprint was not drawn because effects of near-field patchiness make dispersion predictions unreliable for short distances.
Reviewed by S. A. Henderson

Works Calculation 8

Sheet No. 267 of 314 
Title of Project EPHA for THM

Reviewed by S. A. Henderson

Subject Concentration Analysis for THM Hazardous Chemicals

Works Calculation 8

Computer N.E. Baucom B. K. Tharakan Date 09/16/96

Sheet No. 268 of 314

1-RD-33 AVERAGE MAX DIST SEG 2

SITE DATA INFORMATION:

Location: AIKEN, SOUTH CAROLINA

Building Air Exchanges Per Hour: 60 (User specified)

Time: June 30, 19981200 hours EDT (User specified)

CHEMICAL INFORMATION:

Chemical Name: MONOETHANOLAMINE

Molecular Weight: $61.08 \mathrm{~kg} / \mathrm{kmol}$

TLV-TWA: 3 ppm

IDLH: $1000 \mathrm{ppm}$

Footprint Level of Concern: 30 ppm

Boiling Point: $171.00^{\circ} \mathrm{C}$

Vapor Pressure at Ambient Temperature: $4.85 \mathrm{e}-004 \mathrm{~atm}$

Ambient Saturation Concentration: 494 ppm or $0.049 \%$

ATMOSPHERIC INFORMATION: (MANUAL INPUT OF DATA)

Wind: 2.5 meters $/ \mathrm{sec}$ from $90^{\circ}$ true at 2 meters

Inversion Height: 500 meters

Stability Class: C (user override)

Air Temperature: $25^{\circ} \mathrm{C}$

Relative Humidity: 50\%

Ground Roughness: Urban or forest

Cloud Cover: 8 tenths

\section{SOURCE STRENGTH INFORMATION:}

Puddle Area: 40700 square feet

Average Puddle Depth: 1 centimeters

Soil Type: Default

Ground Temperature: $25^{\circ} \mathrm{C}$

Initial Puddle Temperature: Ground temperature

Release Duration: ALOHA limited the duration to 1 hour

Max Computed Release Rate: 2.26 kilograms/min

Max Average Sustained Release Rate: 2.21 kilograms/min

(averaged over a minute or more)

Total Amount Released: 113 kilograms

FOOTPRINT INFORMATION:

Dispersion Module: Gaussian

User-specified LOC: $30 \mathrm{ppm}$

Max Threat Zone for LOC: 34 meters

Max Threat Zone for IDLH: 35 meters

Note: Footprint was not drawn because effects of near-field patchiness make dispersion predictions unreliable for short distances. 


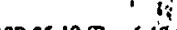
i:

Subject Concentration Analysis for THM Hazardous Chemicals

Reviewed by S. A. Henderson Works Calculation 8

Computer N.E. Baucom B. K. Tharakan Date 09/16/96

Sheet No. $\underline{269 \text { of } 314}$

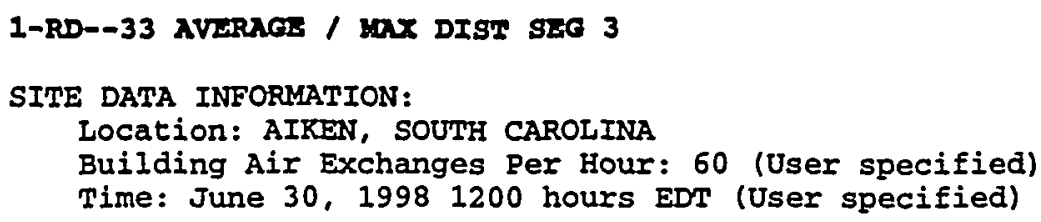

CHEMICAL INFORMATION :

Chemical Name: MONOETHANOLAMINE

Molecular Weight: $61.08 \mathrm{~kg} / \mathrm{kmol}$

TLV-TWA: 3 ppm

IDLH: 1000 ppm

Footprint Level of Concern: $30 \mathrm{ppm}$

Boiling Point: $171.00^{\circ} \mathrm{C}$

Vapor Pressure at Ambient Temperature: $4.85 \mathrm{e}-004 \mathrm{~atm}$

Ambient Saturation Concentration: $494 \mathrm{ppm}$ or 0.0498

ATMOSPHERIC INFORMATION: (MANUAL INPUT OF DATA)

Wind: 2.5 meters $/ \mathrm{sec}$ from $90^{\circ}$ true at 2 meters

Inversion Height: 500 meters

stability Class: C (user override)

Air Temperature: $25^{\circ} \mathrm{C}$

Relative Humidity: 508

Cloud Cover: 8 tenths

Ground Roughness: Urban or forest

SOURCE STRENGTH INFORMATION:

Puddle Area: 10000 square meters

Average Puddle Depth: 1 centimeters

Soil Type: Default Ground Temperature: $25^{\circ} \mathrm{C}$

Initial puddle Temperature: Ground temperature

Release Duration: ALOHA limited the duration to 1 hour

Max Computed Release Rate: 5.75 kilograms/min

Max Average Sustained Release Rate: $5.61 \mathrm{kilograms/min}$

(averaged over a minute or more)

Total Amount Released: 287 kilograms

FOOTPRINT INEORMATION:

Dispersion Module: Gaussian

User-specified LOC: $30 \mathrm{ppm}$

Max Threat Zone for LOC: 56 meters

Max Threat Zone for IDLH: 56 meters

Note: Footprint was not drawn because effects of near-field patchiness make dispersion predictions unreliable for short distances. 
Title of Project EPHA for THM

Subject Concentration Analysis for THM Hazardous Chemicals

Computer N.E. Baucom B. K. Tharakan Date 09/16/96

1-RD-34 AVERAGE ALERT

SITE DATA INFORMATION:

Location: AIKEN, SOUTH CAROLINA

Building Air Exchanges Per Hour: 60 (User specified)

Time: June 30, 19981200 hours EDT (User specified)

\section{CHEMICAL INFORMATION:}

Chemical Name: MORPHOLINE

TLV-TWA: $20 \mathrm{ppm}$

IDLH: $8000 \mathrm{ppm}$

Footprint Level of Concern: $30 \mathrm{ppm}$

Boiling Point: $128.00^{\circ} \mathrm{C}$

Vapor Pressure at Ambient Temperature: $0.013 \mathrm{~atm}$

Ambient Saturation Concentration: 13,479 ppm or $1.35 \%$

\section{ATMOSPHERIC INFORMATION: (MANUAL INPUT OF DATA)}

Wind: 2.5 meters $/ \mathrm{sec}$ from $90^{\circ}$ true at 2 meters

Inversion Height: 500 meters

Stability Class: $\mathrm{C}$ (user override)

Air Temperature: $25^{\circ} \mathrm{C}$

Relative Humidity: $50 \%$

Cloud Cover: 8 tenths

\section{SOURCE STRENGTH INFORMATION:}

Puddle Area: 1450 square feet Average Puddle Depth: 1 centimeters

Soil Type: Default Ground Temperature: $25^{\circ} \mathrm{C}$

Initial Puddle Temperature: Ground temperature

Release Duration: ALOHA limited the duration to 1 hour

Max Computed Release Rate: 2.52 kilograms/min

Max Average Sustained Release Rate: 2.5 kilograms/min (averaged over a minute or more)

Total Amount Released: 140 kilograms

\section{FOOTPRINT INFORMATION:}

Dispersion Module: Gaussian

User-specified LOC: $30 \mathrm{ppm}$

Max Threat Zone for LOC: 30 meters

Max Threat Zone for IDLH: less than 10 meters (10.9 yards)

Note: Footprint was not drawn because effects of near-field patchiness make dispersion predictions unreliable for short distances.

\section{TIME DEPENDENT INFORMATION:}

Concentration Estimates at the point:

Downwind: $\quad 30$ meters

- Off Centerline: 0 meters

Max Concentration:

Outdoor: $30 \mathrm{ppm}$

Indoor: $30 \mathrm{ppm}$

Note: Indoor graph is shown with a dotted line. 
Title of Project EPPHA for THM

Reviewed by S. A. Henderson

Subject Concentration Analysis for THM Hazardous Chemicals

Works Calculation 8

Computer N.E. Baucom B. K. Tharakan Date 09/16/96

Sheet No. 271 of 314

1-RD.34 AVERAGE / SAE

SITE DATA INFORMATION:

Location: AIKEN, SOUTH CAROLINA

Building Air Exchanges Per Hour: 60 (User specified)

Time: June 30, 19981200 hours EDT (User specified)

CHEMICAL INFORMATION:

Chemical Name: MORPHOLINE Molecular Weight: $87.12 \mathrm{~kg} / \mathrm{kmol}$

TLV-TWA: $20 \mathrm{ppm}$

Footprint Level of Concern: $30 \mathrm{ppm}$

Boiling Point: $128.00^{\circ} \mathrm{C}$

Vapor Pressure at Ambient Temperature: $0.013 \mathrm{~atm}$

Ambient Saturation Concentration: $13,479 \mathrm{ppm}$ or $1.35 \%$

ATMOSPHERIC INFORMATION: (MANUAL INPUT OF DATA)

wind: 2.5 meters $/ \mathrm{sec}$ from $90^{\circ}$ true at 2 meters

Inversion Height: 500 meters

Stability Class: $\mathrm{C}$ (user override)

Air Temperature: $25^{\circ} \mathrm{C}$

Relative Humidity: 50\%

Ground Roughness: Urban or forest

Cloud Cover: 8 tenths

SOURCE STRENGTH INFORMATION:

Puddle Area: 19600 square feet

Average Puddle Depth: 1 centimeters

Soil Type: Default

Ground Temperature: $25^{\circ}$

Initial Puddle Temperature: Ground temperature

Release Duration: ALOHA limited the duration to 1 hour

Max Computed Release Rate: 30.7 kilograms/min

Max Average Sustained Release Rate: 30.4 kilograms/min

(averaged over à minute or more)

Total Amount Released: 1,697 kilograms

\section{FOOTPRINT INFORMATION:}

Dispersion Module: Gaussian

User-specified LOC: $30 \mathrm{ppm}$

Max Threat Zone for LOC: 100 meters

Max Threat Zone for IDLH: 24 meters

Note: Footprint was not drawn because effects of near-field patchiness make dispersion predictions unreliable for short distances.

\section{TIME DEPENDENT INFORMATION:}

Concentration Estimates at the point:

Downwind: $\quad 100$ meters

Off Centerline: 0 meters

Max Concentration:

Outdoor: $30 \mathrm{ppm}$

Indoor: $30 \mathrm{ppm}$

Note: Indoor graph is shown with a dotted line. 


\section{ENGINEERING COMPUTATION SHEET}

Title of Project EPHA for THM

Reviewed by S. A. Henderson

Works Calculation 8

Sheet No. $\underline{272 \text { of } 314}$

Computer N.E. Baucom B. K. Tharakan Date 09/16/96

1-RD-34 AVERACE VAX DIST SEO 1

SITE DATA INFORMATION:

LOCation: AIKEN, SOUTH CAROLINA

Building Air Exchanges Per Hour: 60 (User specified)

Time: June 30, 19981200 hours EDT (User specified)

CHEMICAL INEORMATION:

Chemical Name: MORPHOLINE Molecular Weight: $87.12 \mathrm{~kg} / \mathrm{kmol}$

TLV-TWA: $20 \mathrm{ppm}$ IDLH: $8000 \mathrm{ppm}$

Footprint Level of Concern: $30 \mathrm{ppm}$

Boiling Point: $128.00^{\circ} \mathrm{C}$

Vapor Pressure at Ambient Temperature: $0.013 \mathrm{~atm}$

Ambient Saturation Concentration: $13,479 \mathrm{ppm}$ or $1.35 \%$

AMMOSPHERIC INFORMATION: (MANUAL INPUT OF DATA)

Wind: 2.5 meters $/ \mathrm{sec}$ from $90^{\circ}$ true at 2 meters

Inversion Height: 500 meters

Stability class: c (user override)

Air Temperature: $25^{\circ} \mathrm{C}$

Relative Humidity: 508 Ground Roughness: Urban or forest

cloud Cover: 8 tenths

SOURCE STRENGTH INFORMATION:

Puddle Area: 24420 square feet

Average Pudale Depth: 1 centimeters

Soil Type: Default

Ground Temperature: $25^{\circ} \mathrm{C}$

Initial Puddle Temperature: Ground temperature

Release Duration: ALOHA limited the duration to 1 hour

Max Computed Release Rate: 37.9 kilograms/min

Max Average Sustained Release Rate: $37.5 \mathrm{kilograms/min}$

(averaged over a minute or more)

Total Amount Released: 2,095 kilograms

FOOTPRINT INEORMATION:

Dispersion Module: Gaussian

User-specified LOC: $30 \mathrm{ppm}$

Max Threat Zone for LOC: 111 meters

Max Threat zone for IDLH: 27 meters 
Title of Project EPHA for THM

Subject Cencentration Analysis for THM Hazardous Chemicals

Computer N.E. Baucom B. K. Tharakan Date 09/16/96

1-RD-34 AVERAGE / MAX DIST SEG 2

SITE DATA INFORMATION:

Location: AIKEN, SOUTH CAROLINA

Building Air Exchanges Per Hour: 60 (User specified)

Time: June 30, 19981200 hours EDT (User specified)
Reviewed by S..A. Henderson

Works Calculation 8

Sheet No. 273 of 314

\section{CHEMICAL INFORMATION:}

Chemical Name: MORPHOLINE

TLV-TWA: 20 ppm

Molecular Weight: $87.12 \mathrm{~kg} / \mathrm{kmol}$

Footprint Level of Concern: 30 ppm

IDLH: 8000 ppm

Boiling Point: $128.00^{\circ} \mathrm{C}$

Vapor Pressure at Ambient Temperature: $0.013 \mathrm{~atm}$

Ambient Saturation Concentration: 13,479 ppm or $1.35 \%$

\section{ATMOSPHERIC INFORMATION: (MANUAL INPUT OF DATA)}

Wind: 2.5 meters $/ \mathrm{sec}$ from $90^{\circ}$ true at 2 meters

Inversion Height: 500 meters

Stability Class: $\mathrm{C}$ (user override)

Air Temperature: $25^{\circ} \mathrm{C}$

Relative Humidity: 50\%

Ground Roughness: Urban or forest

Cloud Cover: 8 tenths

\section{SOURCE STRENGTH INFORMATION: \\ Puddle Area: 40700 square feet \\ Average Puddle Depth: 1 centimeters \\ Soil Type: Default Ground Temperature: $25^{\circ} \mathrm{C}$ \\ Initial Puddle Temperature: Ground temperature \\ ReleaseDuration: ALOHA limited the duration to 1 hour \\ Max Computed Release Rate: 62 kilograms/min \\ MaxAverage Sustained Release Rate: 61.3 kilograms/min \\ (averaged over a minute or more) \\ Total Amount Released: 3,422 kilograms}

\section{FOOTPRINT INFORMATION:}

Dispersion Module: Gaussian

User-specified LOC: $30 \mathrm{ppm}$

Max Threat Zone for LOC: 140 meters

Max Threat Zone for IDLH: 34 meters 
Title of Project EPHA for THM

Reviewed by S. A. Henderson

Subject Concentration Analysis for THM Hazardous Chemicals

Works Calculation 8

Computer N.E. Baucom B. K. Tharakan Date 09/16/96

Sheet No. 274 of 314

1-RD-34 AVtRACE wNX DIST SEO 3

SITE DATA INFORMATION:

Location: AIKEN, SOUTH CAROLINA

Building Air Exchanges Per Hour: 60 (User specified)

Time: June 30, 19981200 hours EDT (User specified)

CHEMICAL INFORMATION:

Chemical Name: MORPHOLINE " Molecular Weight: $87.12 \mathrm{~kg} / \mathrm{kmol}$

TLV-TWA: $20 \mathrm{ppm}$

IDLH: $8000 \mathrm{ppm}$

Footprint Level of Concern: $30 \mathrm{ppm}$

Boiling Point: $128.00^{\circ} \mathrm{C}$

Vapor Pressure at Ambient Temperature: 0.013 atm

Ambient Saturation Concentration: $13,479 \mathrm{ppm}$ or $1.35 \%$

ATMOSPHERIC INFORMATION: (MANUAL INPUT OF DATA)

Wind: 2.5 meters $/ \mathrm{sec}$ from $90^{\circ}$ true at 2 meters

Inversion Height: 500 meters

stability class: C (user override)

Air Temperature: $25^{\circ} \mathrm{C}$

Relative Humidity: 508

Ground Roughness: Urban or forest

cloud Cover: 8 tenths

SOURCE STRENGTH INFORMATION:

Pudale Area: 10000 square meters

Average Puddle Depth: 1 centimeters

Soil Type: Default

Ground Temperature: $25^{\circ} \mathrm{C}$

Initial Puddle Temperature: Ground temperature

Release Duration: ALOHA limited the duration to 1 hour

Max Computed Release Rate: $158 \mathrm{kilograms/min}$

Max Average Sustained Release Rate: 156 kilograms/min

(averaged over a minute or more)

Total Amount Released: 8,717 kilograms

FOOTPRINT INFORMATION:

Dispersion Module: Gaussian

User-specified LOC: $30 \mathrm{ppm}$

Max Threat Zone for IOC: 220 meters

Max Threat zone for IDLH: 56 meters 
Subject Concentration Analysis for THM Hazardous Chemicals

\section{1-RD-36 AVERAGE ALERT}

\section{SITE DATA INFORMATION:}

Location: AIKEN, SOUTH CAROLINA

Building Air Exchanges Per Hour: 60 (User specified)

Time: June 30, 19981200 hours EDT (User specified)

\section{CHEMICAL INFORMATION:}

Chemical Name: 1-METHYLPYRROLIDONE

Molecular Weight: $99.13 \mathrm{~kg} / \mathrm{kmol}$

TLV-TWA: - unavail-

IDLH: -unavail

Footprint Level of Concern: $500 \mathrm{mg} /(\mathrm{cu} \mathrm{m}$ )

Boiling Point: $202.00^{\circ} \mathrm{C}$

Vapor Pressure at Ambient Temperature: 4.49e-004 atm

Ambient Saturation Concentration: 457 ppm or $0.046 \%$

\section{ATMOSPHERIC INFORMATION: (MANUAL INPUT OF DATA)}

Wind: 2.5 meters $/ \mathrm{sec}$ from $90^{\circ}$. true at 2 meters

Inversion Height: 500 meters

Stability Class: $C$ (user override)

Air Temperature: $25^{\circ} \mathrm{C}$

Relative Humidity: $50 \%$

Ground Roughness: Urban or forest

Cloud Cover: 8 tenths

SOURCE STRENGTH INFORMATION:

Puddle Area: 30001 square feet

Average Puddle Depth: 1 centimeters

Soil Type: Default Ground Temperature: $25^{\circ} \mathrm{C}$

Initial Puddle Temperature: Ground temperature

ReleaseDuration: ALOHA limited the duration to 1 hour

Max Computed Release Rate: 2.23 kilograms/min

MaxAverage Sustained Release Rate: 2.18 kilograms/min

(averaged

over a minute or more)

Total Amount Released:

115 kilograms

\section{FOOTPRINT INFORMATION:}

Dispersion Module: Gaussian

User-specified LOC: $500 \mathrm{mg} /(\mathrm{cu} \mathrm{m})$

Max Threat Zone for LOC: 30 meters

Note: Footprint was not drawn because effects of near-field patchiness make dispersion predictions unreliable for short distances.

\section{TIME DEPENDENT INFORMATION:}

Concentration Estimates at the point:

. Downwind: $\quad 30$ meters

Off Centerline: 0 meters

Max Concentration:

Outdoor: $37.4 \mathrm{mg} /(\mathrm{cu} \mathrm{m})$

Indoor: $37.4 \mathrm{mg} /(\mathrm{cu} \mathrm{m}$ )

Note: Indoor graph is shown with a dotted line. 
Title of Project EPHA for THM

Reviewed by S. A. Henderson

Subject Concentration Analysis for THM Hazardous Chemicals Works Calculation 8

Computer N.E. Baucom B. K. Tharakan Date 09/16/96 Sheet No. 276 of 314

\section{1-RD-36 AVERAGE / SAE}

\section{SITE DATA INFORMATION:} Location: AIKEN, SOUTH CAROLINA

Building Air Exchanges Per Hour: 60 (User specified)

Time: June 30, 19981200 hours EDT (User specified)

\section{CHEMICAL INFORMATION:}

Chemical Name: 1-METHYLPYRROLIDONE

Molecular Weight: $99.13 \mathrm{~kg} / \mathrm{kmol}$

TLV-TWA: -unavail-

IDLH: -unavail

Footprint Level of Concern: $500 \mathrm{mg} /(\mathrm{cu} \mathrm{m}$ )

Boiling Point: $202.00^{\circ} \mathrm{C}$

Vapor Pressure at Ambient Temperature: 4.49e-004 atm

Ambient Saturation Concentration: 457 ppm or $0.046 \%$

ATMOSPHERIC INFORMATION: (MANUAL INPUT OF DATA)

Wind: 2.5 meters $/ \mathrm{sec}$ from $90^{\circ}$ true at 2 meters

Inversion Height: 500 meters

Stability Class: C (user override)

Air Temperature: $25^{\circ} \mathrm{C}$

Relative Humidity: $50 \%$

Ground Roughness: Urban or forest

Cloud Cover: 8 tenths

SOURCE STRENGTH INFORMATION:

Puddle Area: 100001 square feet

Average Puddle Depth: 1 centimeters

Soil Type: Default

Initial Puddle Temperature: Ground temperature

Release Duration: ALOHA limited the duration to 1 hour

Max Computed Release Rate: 7.08 kilograms/min

Max Average Sustained Release Rate: 6.94 kilograms/min

(averaged over a minute or more)

Total Amount Released: 365 kilograms

\section{FOOTPRINT INFORMATION:}

Dispersion Module: Gaussian

User-specified LOC: $500 \mathrm{mg}$ (cu m)

Max Threat Zone for LOC: 55 meters

Note: Footprint was not drawn because effects of near-field patchiness make dispersion predictions unreliable for short distances.

\section{TIME DEPENDENT INFORMATION:}

Concentration Estimates at the point:

Downwind:

Off Centerline:

100 meters

0 meters

Max Concentration:

$$
\text { Outdoor. } 11.7 \mathrm{mg} /(\mathrm{cu} \mathrm{m})
$$

Indoor: $11.7 \mathrm{mg} /(\mathrm{cu} \mathrm{m})$

Note: Indoor graph is shown with a dotted line. 
Title of Project EPHA for THM

Subject Concentration Analysis for THM Hazardous Chemicals

Computer N.E. Baucom B. K. Tharakan Date 09/16/96

1-RD-36 AVERAGE / MAX DIST SEG 1

SITE DATA INFORMATION:

Location: AIKEN, SOUTH CAROLINA

Building Air Exchanges Per Hour: 60 (User specified)

Time: June 30, 19981200 hours EDT (User specified)

\section{CHEMICAL INFORMATION:}

Chemical Name: 1-METHYLPYRROLIDONE

Molecular Weight: $99.13 \mathrm{~kg} / \mathrm{kmol}$

TLV-TWA: -unavailIDLH: - Unavail

Footprint Level of Concern: $500 \mathrm{mg} /(\mathrm{cu} \mathrm{m})$

Boiling Point: $202.00^{\circ} \mathrm{C}$

Vapor Pressure at Ambient Temperature: $4.49 \mathrm{e}-004 \mathrm{~atm}$

Ambient Saturation Concentration: 457 ppm or $0.046 \%$

\section{ATMOSPHERIC INFORMATION: (MANUAL INPUT OF DATA)}

Wind: 2.5 meters $/ \mathrm{sec}$ from $90^{\circ}$ true at 2 meters

Inversion Height: 500 meters

Stability Class: $\mathrm{C}$ (user override

Air Temperature: $25^{\circ} \mathrm{C}$

Relative Humidity: 50\%

Ground Roughness: Urban or forest

Reviewed by S. A. Henderson

Works Calculation 8

Sheet No. 277 of 314

Cloud Cover: 8 tenths

\section{SOURCE STRENGTH INFORMATION:}

Puddle Area: 24420 square feet

Average Puddle Depth: 1 centimeters

Soil Type: Default

Ground Temperature: $25^{\circ} \mathrm{C}$

Initial Puddle Temperature: Ground temperature

Release Duration: ALOHA limited the duration to 1 hour

Max Computed Release Rate: 1.83 kilograms/min

Max Average Sustained Release Rate: 1.79 kilograms/min

(averaged over a minute or more)

Total Amount Released: 94.3 kilograms

FOOTPRINT INFORMATION:

Dispersion Module: Gaussian

User-specified LOC: $500 \mathrm{mg} /(\mathrm{cu} \mathrm{m})$

Max Threat Zone for LOC: 27 meters

Note: Footprint was not drawn because effects of near-field patchiness make dispersion predictions unreliable for short distances. 
Title of Project EPHA for THM

Reviewed by S. A. Henderson

Subject Concentration Analysis for THM Hazardous Chemicals Works Calculation 8

Computer N.E. Baucom B. K. Tharakan Date 09/16/96 Sheet No. 278 of 314

1-RD-36 AVERAGE MAX DIST SEG 2

SITE DATA INFORMATION:

Location: AIKEN, SOUTH CAROLINA

Building Air Exchanges Per Hour: 60 (User specified)

Time: June 30, 19981200 hours EDT (User specified)

\section{CHEMICAL INFORMATION:}

Chemical Name: 1-METHYLPYRROLIDONE

Molecular Weight: $99.13 \mathrm{~kg} / \mathrm{kmol}$

TLV-TWA: -unavail-

IDLH: -unavail

Footprint Level of Concern: $500 \mathrm{mg} /(\mathrm{cu} \mathrm{m}$ )

Boiling Point: $202.00^{\circ} \mathrm{C}$

Vapor Pressure at Ambient Temperature: 4.49e-004 atm

Ambient Saturation Concentration: 457 ppm or $0.046 \%$

ATMOSPHERIC INFORMATION: (MANUAL INPUT OF DATA)

Wind: 2.5 meters $/ \mathrm{sec}$ from $90^{\circ}$ true at 2 meters

Inversion Height: 500 meters

Stability Class: $\mathrm{C}$ (user override)

Air Temperature: $25^{\circ} \mathrm{C}$

Relative Humidity: 50\%

Ground Roughness: Urban or forest

Cloud Cover: 8 tenths

\section{SOURCE STRENGTH INFORMATTON:}

Puddle Area: 40700 square feet

Average Puddle Depth: 1 centimeters

Soil Type: Default

Ground Temperature: $25^{\circ} \mathrm{C}$

Initial Puddle Temperature: Ground temperature

Release Duration: ALOHA limited the duration to 1 hour

Max Computed Release Rate: 2.99 kilograms/min

Max Average Sustained Release Rate: 2.92 kilograms/min

(averaged over a minute or more)

Total Amount Released: 154 kilograms

\section{FOOTPRINT INFORMATION:}

Dispersion Module: Gaussian

User-specified LOC: $500 \mathrm{mg} /(\mathrm{cu} \mathrm{m})$

Max Threat Zone for LOC: 34 meters

Note: Footprint was not drawn because effects of near-field patchiness make dispersion predictions unreliable for short distances. 
Title of Project EPHA for THM

Reviewed by $\mathbf{S}$. A. Henderson

Subject Concentration Analysis for THM Hazardous Chemicals

Works Calculation 8

Computer N.E. Baucom B. K. Tharakan Date 09/16/96

Sheet No. 279 of 314

1.RD.36 AVERAGE MAX DIST SEG 3

\section{SITE DATA INFORMATION:}

Location: AIKEN, SOUTH CAROLINA

Building Air Exchanges Per Hour: 60 (User specified)

Time: June 30, 19981200 hours EDT (User specified)

\section{CHEMICAL INFORMATION:}

Chemical Name: 1-METHYLPYRROLIDONE

Molecular Weight: $99.13 \mathrm{~kg} / \mathrm{kmol}$

TLV-TWA: -unavail-

IDLH: -unavail

Footprint Level of Concern: $500 \mathrm{mg} /(\mathrm{cu} \mathrm{m}$ )

Boiling Point: $202.00^{\circ} \mathrm{C}$

Vapor Pressure at Ambient Temperature: 4.49e-004 atm

Ambient Saturation Concentration: 457 ppm or $0.046 \%$

ATMOSPHERIC INFORMATION: (MANUAL INPUT OF DATA)

Wind: 2.5 meters $/ \mathrm{sec}$ from $90^{\circ}$ true at 2 meters

Inversion Height: 500 meters

Stability Class: C (user override)

Air Temperature: $25^{\circ} \mathrm{C}$

Relative Humidity: 50\%

Ground Roughness: Urban or forest

Cloud Cover: 8 tenths

\section{SOURCE STRENGTH INFORMATION:}

Puddle Area: 10000 square meters

Average Puddle Depth: 1 centimeters

Soil Type: Default

Ground Temperature: $25^{\circ} \mathrm{C}$

Initial Puddle Temperature: Ground temperature

Release Duration: ALOHA limited the duration to 1 hour

Max Computed Release Rate: 7.6 kilograms/min

Max Wverage Sustained Release Rate: 7.45 kilograms/min

(averaged over a minute or more)

Total Amount Released: 391 kilograms

FOOTPRINT INFORMATION:

Dispersion Module: Gaussian

User-specified LOC: $500 \mathrm{mg} /(\mathrm{cu} \mathrm{m})$

Max Threat Zone for LOC: 56 meters

Note: Footprint was not drawn because effects of near-field patchiness make dispersion predictions unreliable for short distances. 
Title of Project EPHA for THM

Subject Concentration Analysis for THM Hazardous Chemicals

Computer N.E. Baucom B. K. Tharakan Date 09/16/96

\section{1-RD-42 AVARACS / ATIRI}

SITE DATA INFORMATION:

Location: AIKEN, SOUTH CAROLINA

Building Air Exchanges Per Hour: 60 (User specified)

Time: June 30, 19981200 hours EDT (User specified)

CHEMICAL INFORMATION:

Chemical Name: PROPIONIC ACID

Molecular Weight: $74.08 \mathrm{~kg} / \mathrm{kmol}$

TLV-TWA: $10 \mathrm{ppm}$

IDLH: -unavail

Footprint Level of Concern: $15 \mathrm{ppm}$

Boiling Point: $141.17^{\circ} \mathrm{C}$

Vapor Pressure at Ambient Temperature: $0.0049 \mathrm{~atm}$

Ambient Saturation Concentration: 4,960 ppm or 0.508

ATMOSPHERIC INFORMATION: (MANUAL INPUT OP DATA)

Wind: 2.5 meters $/ \mathrm{sec}$ from $90^{\circ}$ true at 2 meters

Inversion Height: 500 meters

stability class: C (user override

Air Temperature: $25^{\circ} \mathrm{C}$

Relative Humidity: $50 \%$

Cloud Cover: 8 tenths

SOURCE STRENGTH INFORMATION:

Puddle Area: 2050 square feet

Average Pudale Depth: 1 centimeters

Ground Roughness: Urban or forest

Soil Type: Default Ground Temperature: $25^{\circ} \mathrm{C}$

Initial pudale Temperature: Ground temperature

Release Duration: ALOHA limited the duration to 1 hour

Max Computed Release Rate: $1.27 \mathrm{kilograms} / \mathrm{min}$

Max Average Sustained Release Rate: $1.25 \mathrm{kilograms/min}$

(averaged over a minute or more)

Total Amount Released: 68.0 kilograms

FOOTPRINT INFORMATION:

Dispersion Module: Gaussian

User-specified LOC: 15 ppm

Max Threat zone for Loc: 30 meters

Note: Footprint was not drawn because effects of near-field patchiness make dispersion predictions unreliable for short distances.

TIME DEPENDENT INFORMATION:

Concentration Estimates at the point:

Downwind: $\quad 30$ meters

Off Centerline: 0 meters.

Max Concentration:

Outdoor: $15 \mathrm{ppm}$

Indoor: $15 \mathrm{ppm}$

Note: Indoor graph is shown with a dotted line.
Reviewed by S. A. Henderson

Works Calculation 8

Sheet No. $\underline{280 \text { of } 314}$ 
Title of Project EPHA for THM

Subject Concentration Analysis for THM Hazardous Chemicals

Computer N.E. Baucom B. K. Tharakan Date 09/16/96

1-RD-12 AVERAGL

SITE DATA INFORMATION:

Location: AIKEN, SOUTH CAROLINA

Building Air Exchanges Per Hour: 60 (User specified)

Time: June 30, 19981200 hours EDT (User specified)

CHEMICAL INFORMATION:

Chemical Name: PROPIONIC ACID

Molecular Weight: $74.08 \mathrm{~kg} / \mathrm{kmol}$

TLV-TWA: $10^{\prime} \mathrm{ppm}$

Footprint Level of Concern: $15 \mathrm{ppm}$

Boiling Point: $141.17^{\circ} \mathrm{C}$

Vapor Pressure at Ambient Temperature: 0.0049 atm

Ambient Saturation Concentration: 4,960 ppm or 0.508

ATMOSPHERIC INFORMATION: (MANUAL INPUT OF DATA)

Wind: 2.5 meters/sec from $90^{\circ}$ true at 2 meters

Inversion Height: 500 meters

Stability class: C (user override)

Air Temperature: $25^{\circ} \mathrm{C}$

Relative Humidity: 508

Cloud Cover: 8 tenths

Ground Roughness: Urban or forest

SOURCE STRENGTH INEORMATION:

Puddle Area: 28500 square feet

Average Puddle Depth: 1 centimeters

Soil Type: Default Ground Temperature: $25^{\circ} \mathrm{C}$

Initial Puddle Temperature: Ground temperature

Release Duration: ALOHA limited the duration to 1 hour

Max Computed Release Rate: $15.8 \mathrm{kilograms/min}$

Max Average Sustained Release Rate: 15.5 kilograms/min

(averaged over a minute or moxe)

Total Amount Released: 841 kilograms

FOOTPRINT INEORMATION :

Dispersion Module: Gaussian

User-specified LOC: $15 \mathrm{ppm}$

Max Threat Zone for LOC: 100 meters

Note: Footprint was not drawn because effects of near-field patchiness make dispersion predictions unreliable for short distances.

TIME DEPENDENT INFORMATION:

Concentration Estimates at the point:

Downwind:

100 meters

off Centerline: 0 meters

Max Concentration:

outdoor: $15 \mathrm{ppm}$

Indoor: $15 \mathrm{ppm}$

Note: Indoor graph is shown with a dotted line.
Reviewed by S. A. Henderson

Works Calculation 8

Sheet No. 281 of 314 
Title of Project EPHA for THM

Subject Concentration Analysis for THM Hazardous Chemicals

Computer N.E. Baucom B. K. Tharakan Date 09/16/96

1-RD-42 AVERACE MAX DIST SEO 1

SITE DATA INFORMATION: Location: AIKEN, SOUTH CAROLINA Building Air Exchanges Per Hour: 60 (User specified) Time: June 30, 19981200 hours EDT (User specified)

CHEMICAL INFORMATION : Chemical Name: PROPIONIC ACID Molecular Weight: $74.08 \mathrm{~kg} / \mathrm{kmol}$ TLV-TWA: $10 \mathrm{ppm}$ Footprint Level of Concern: $15 \mathrm{ppm}$ Boiling Point: $141.17^{\circ} \mathrm{C}$ Vapor Pressure at Ambient Temperature: $0.0049 \mathrm{~atm}$ Ambient Saturation Concentration: $4,960 \mathrm{ppm}$ or 0.508

ATMOSPHERIC INFORMATION: (MANUAL INPUT OF DATA)

Wind: 2.5 meters/sec from $90^{\circ}$ true at 2 meters Inversion Height: 500 meters Stability Class: C (user override)

Air Temperature: $25^{\circ} \mathrm{C}$ Relative Humidity: $50 \%$ Cloud Cover: 8 tenths

SOURCE STRENGTH INFORMATION: Puddle Area: 24420 square feet Average Puddle Depth: 1 centimeters Soil Type: Default Ground Temperature: $25^{\circ} \mathrm{C}$
Initial Puddle Temperature: Ground temperature Release Duration: ALOHA limited the duration to 1 hour Max Computed Release Rate: $13.6 \mathrm{kilograms/min}$ Max Average Sustained Release Rate: $13.4 \mathrm{kilograms} / \mathrm{min}$ (averaged over a minute or more) Total Amount Released: 725 kilograms

FOOTPRINT INFORMATION:

Dispersion Module: Gaussian User-specified LOC: $15 \mathrm{ppm}$

Max Threat Zone for LOC: 93 meters Note: Footprint was not drawn because effects of near-field patchiness make dispersion predictions unreliable for short distances.
Reviewed by S.A. Henderson Works Calculation 8

Sheet No. 282 of 314 


\section{ENGINEERING COMPUTATION SHEET}

Title of Project EPHA for THM

Subject Concentration Analysis for THM Hazardous Chemicals

Reviewed by S. A. Henderson

Works Calculation 8

Computer N.E. Baucom B. K. Tharakan Date 09/16/96

1-RD-42 AVERAGE / max DIST SESC 2

SITE DATA INFORMATION:

Location: AIKEN' SOUTH CAROLINA

Building Air Exchanges Per Hour: 60 (User specified)

Time: June 30, 19981200 hours EDT (User specified)

CHEMICAI INFORMATION:

Chemical Name: PROPIONIC ACID

Molecular Weight: $74.08 \mathrm{~kg} / \mathrm{kmol}$

TLV-TWA: $10 \mathrm{ppm}$

Footprint Level of Concern: 15 ppm

Boiling Point: $141.17^{\circ} \mathrm{C}$

Vapor Pressure at Ambient Temperature: $0.0049 \mathrm{~atm}$

Ambient Saturation Concentration: 4,960 ppm or 0.508

ATMOSPHERIC INFORMATION: (MANUAL INPUT OF DATA)

Wind: 2.5 meters $/ \mathrm{sec}$ from $90^{\circ}$ true at 2 meters

Inversion Height: 500 meters

Stability Class: $C$ (user override)

Aix Temperature: $25^{\circ} \mathrm{C}$

Relative Humidity: 508

Cloud Cover: 8 tenths

Ground Roughness: Urban or forest

SOURCE STRENGTH INFORMATION:

Puddle Area: 40700 square feet

Average Puddle Depth: 1 centimeters

Soil Type: Default "Ground Temperature: $25^{\circ} \mathrm{C}$

Initial puddle Temperature: Ground temperature

Release Duration: ALOHA limited the duration to 1 hour

Max Computed Release Rate: 22.2 kilograms/min

Max Average Sustained Release Rate: 21.8 kilograms/min

(averaged over a minute or more)

Total Amount Released: 1,183 kilograms

FOOTPRINT INFORMATION:

Dispersion Module: Gaussian

User-specified LOC: $15 \mathrm{ppm}$

Max Threat Zone for LOC: 118 meters 
Title of Project EPHA for THM

Subject Concentration Analysis for THM Hazardous Chemicals

Computer N.E. Baucom B. K. Tharakan Date $09 / 16 / 96$

1-RD-33 AVERAGE / unX DIST SEO 3

SITE DATA INFORMATION:

LOCation: AIKEN, SOUTH CAROLINA

Building Air Exchanges Per Hour: 60 (User specified)

Time: June 30, 19981200 hours EDr (User specified)

CHEMICAL INFORMATION:

Chemical Name: MONOETHANOLAMINE

Molecular Weight: $61.08 \mathrm{~kg} / \mathrm{kmol}$

TLV-TWA: 3 ppm

Footprint Level of Concern: $30 \mathrm{ppm}$

Boiling Point: $171.00^{\circ} \mathrm{C}$

Vapor Pressure at Ambient Temperature: $4.85 e-004$ atm

Ambient Saturation Concentration: $494 \mathrm{ppm}$ or 0.0498

ATMOSPHERIC INFORMATION: (MANUAL INPUT OF DATA)

Wind: 2.5 meters/sec from $90^{\circ}$ true at 2 meters

Inversion Height: 500 meters

stability Class: $C$ (user override)

Air Temperature: $25^{\circ} \mathrm{C}$

Relative Humidity: 508

Cloud Cover: 8 tenths

Ground Roughness: Urban or forest

SOURCE STRENGTH INFORMATION:

Puddle Area: 10000 square meters

Average Puddle Depth: 1 centimeters

Soil Type: Default Ground Temperature: $25^{\circ} \mathrm{C}$

Initial Puddle Temperature: Ground temperature

Release Duration: ALOHA limited the duration to I hour

Max Computed Release Rate: $5.75 \mathrm{kilograms/ \textrm {min }}$

Max Average Sustained Release Rate: 5.61 kilograms/min (averaged over a minute or more)

Total Amount Released: 287 kilograms

FOOTPRINT INFORMATION:

Dispersion Module: Gaussian

User-specified LOC: $30 \mathrm{ppm}$

Max Threat zone for LOC: 56 meters

Max Threat Zone for IDLH: 56 meters

Note: Footprint was not drawn because effects of near-field patchiness make dispersion predictions unreliable for short distances.
Reviewed by S. A. Henderson

Works Calculation 8

Sheet No. 284 of 314 
Title of Project EPHA for THM

Subject Concentration Analysis for THM Hazardous Chemicals

Computer N.E. Baucom B.K. Tharakan Date 09/16/96

1-RD-46 AVERAGE ALERT

SITE DATA INFORMATION:

Location: AIKEN, SOUTH CAROLINA

Building Air Exchanges Per Hour: 60 (User specified)

Time: June 30, 19981200 hours EDT (User specified)

\section{CHEMICAL INFORMATION:}

Chemical Name: TETRAHYDROFURAN -

Molecular Weight: $72.11 \mathrm{~kg} / \mathrm{kmol}$

TLV-TWA: 200 ppm

IDLH: 2000 ppm

Footprint Level of Concern: 2000 ppm

Boiling Point: $65.97^{\circ} \mathrm{C}$

Vapor Pressure at Ambient Temperature: 0.21 atm

Ambient Saturation Concentration: 216,395 ppm or $21.6 \%$

ATMOSPHERIC INFORMATION: (MANUAL INPUT OF DATA)

Wind: 2.5 meters $/ \mathrm{sec}$ from $90^{\circ}$ true at 2 meters

Inversion Height: 500 meters

Stability Class: C (user override)

Air Temperature: $25^{\circ} \mathrm{C}$

Relative Humidity: 50\%

Cloud Cover: 8 tenths

\section{SOURCE STRENGTH INFORMATION:}

Puddle Area: 3010 square feet

Average Puddle Depth: 1 centimeters

Soil Type: Default

Ground Temperature: $25^{\circ} \mathrm{C}$

Initial Puddle Temperature: Ground temperature

Release Duration: 59 minutes

Max Computed Release Rate: 58.1 kilograms/min

Max Average Sustained Release Rate: 48.3 kilograms/min

(averaged over a minute or more)

Total Amount Released: 2,461 kilograms

FOOTPRINT INFORMATION:

Dispersion Module: Gaussian

User-specified LOC: $2000 \mathrm{ppm}$

Max Threat Zone for LOC: 11 meters.

Max Threat Zone for IDLH: 11 meters

Note: Footprint was not drawn because effects of near-field patchiness make dispersion predictions unreliable for short distances.

\section{TIME DEPENDENT INFORMATION:}

Concentration Estimates at the point:

Downwind: $\quad 30$ meters

Off Centerline: 0 meters

Max Concentration:

Outdoor: $492 \mathrm{ppm}$

Indoor: $492 \mathrm{ppm}$

Note: Indoor graph is shown with a dotted line.
Reviewed by S. A. Henderson.

Works Calculation 8

Sheet No. $\underline{285 \text { of } 314}$ 
Title of Project EPHA for THM

Reviewed by $\underline{S}$. A. Henderson

Subject Concentration Analysis for THM Hazardous Chemicals Works Calculation 8

Computer N.E. Baucom B. K. Tharakan Date 09/16/96 Sheet No. 286 of 314

1-RD-46 AVERAGE / SAE

SITE DATA INFORMATION:

Location: AIKEN, SOUTH CAROLINA

Building Air Exchanges Per Hour: 60 (User specified)

Time: June 30, 19981200 hours EDT (User specified)

CHEMICAL INFORMATION:

Chemical Name: TETRAHYDROFURAN

Molecular Weight: $72.11 \mathrm{~kg} / \mathrm{kmol}$

TLV-TWA: $200 \mathrm{ppm}$

IDLH: $2000 \mathrm{ppm}$

Footprint Level of Concern: 2000 ppm

Boiling Point: $65.97^{\circ} \mathrm{C}$

Vapor Pressure at Ambient Temperature: $0.21 \mathrm{~atm}$

Ambient Saturation Concentration: 216,395 ppm or 21.6\%

ATMOSPHERIC INFORMATION: (MANUAL INPUT OF DATA)

Wind: 2.5 meters $/ \mathrm{sec}$ from $90^{\circ}$ true at 2 meters

Inversion Height: 500 meters

Stability Class: $\mathrm{C}$ (user override)

Air Temperature: $25^{\circ} \mathrm{C}$

Relative Humidity: 50\%

Ground Roughness: Urban or forest

Cloud Cover: 8 tenths

\section{SOURCE STRENGTH INFORMATION:}

Puddle Area: 21100 square feet

Average Puddle Depth: 1 centimeters

Soil Type: Default

Ground Temperature: $25^{\circ} \mathrm{C}$

Initial Puddle Temperature: Ground temperature

Release Duration: ALOHA limited the duration to 1 hour

Max Computed Release Rate: 371 kilograms/min

Max Average Sustained Release Rate: 314 kilograms/min (averaged over a minute or more)

Total Amount Released: 16,403 kilograms

\section{FOOTPRINT INFORMATION:}

Model Run: Heavy Gas

User-specified LOC: 2000 ppm

Max Threat Zone for LOC: 100 meters

Note: Footprint was not drawn because effects of near-field patchiness make dispersion predictions unreliable for short distances.

TIME DEPENDENT INFORMATION:

Concentration Estimates at the point:

Downwind: $\quad 100$ meters

Off Centerline: 0 meters

Max Concentration:

Outdoor: $2,000 \mathrm{ppm}$

Indoor: $2,000 \mathrm{ppm}$

Note: Indoor graph

Note: Indoor graph is shown with a dotted line. 
ENGINEERING COMPUTATION SHEET

Title of Project EPHA for THM

Reviewed by S. A. Henderson

Subject Concentration Analysis for THM Hazardous Chemicals

Computer N.E. Baucom B. K. Tharakan Date 09/16/96

1-RD-46 AVERAGE/MAX DIST SEG 1

SITE DATA INFORMATION:

Location: AIKEN, SOUTH CAROLINA

Building Air Exchanges Per Hour: 60 (User specified)

Time: June 30, 19981200 hours EDT (User specified)

CHEMICAL INFORMATION:

Chemical Name: TETRAHYDROFURAN

Molecular Weight: $72.11 \mathrm{~kg} / \mathrm{kmol}$

TLV-TWA: 200 ppm

IDLH: 2000 ppm

Footprint Level of Concern: 2000 ppm

Boiling Point: $65.97^{\circ} \mathrm{C}$

Vapor Pressure at Ambient Temperature: 0.21 atm

Ambient Saturation Concentration: 216,395 ppm or $21.6 \%$

ATMOSPHERIC INFORMATION: (MANUAL INPUT OF DATA)

Wind: 2.5 meters $/ \mathrm{sec}$ from $90^{\circ}$ true at 2 meters

Inversion Height: 500 meters

Stability Class: $\mathrm{C}$ (user override)

Air Temperature: $25^{\circ} \mathrm{C}$

Relative Humidity: $50 \%$

Ground Roughness: Urban or forest

Cloud Cover. 8 tenths

SOURCE STRENGTH INFORMATION:

Puddle Area: 24420 square feet

Average Puddle Depth: 1 centimeters

Soil Type: Default

Initial Puddle Temperature: Ground temperature

Ground Temperature: $25^{\circ} \mathrm{C}$

Max Computed Release Rate: 426 kilograms/min

Max Average Sustained Release Rate: 361 kilograms/min (averaged over a minute or more)

Total Amount Released: 18,900 kilograms

FOOTPRINT INFORMATION:

Model Run: Heavy Gas

User-specified LOC: $2000 \mathrm{ppm}$

Max Threat Zone for LOC: 108 meters 
Title of Project EPHA for THM

Subject Concentration Analysis for THM Hazardous Chemicals

Computer N.E. Baucom B. K. Tharakan Date 09/16/96

1-RD-46 AVERAGE MAX DIST SEG 2

SITE DATA INFORMATION:

Location: AIKEN, SOUTH CAROLINA

Building Air Exchanges Per Hour: 60 (User specified)

Time: June 30, 19981200 hours EDT (User specified)

\section{CHEMICAL INFORMATION:}

Chemical Name: TETRAHYDROFURAN

Molecular Weight: $72.11 \mathrm{~kg} / \mathrm{kmol}$

TLV-TWA: $200 \mathrm{ppm}$

IDLH: $2000 \mathrm{ppm}$

Footprint Level of Concern: $2000 \mathrm{ppm}$

Boiling Point: $65.97^{\circ} \mathrm{C}$

Vapor Pressure at Ambient Temperature: 0.21 atm

Ambient Saturation Concentration: 216,395 ppm or 21.6\%

ATMOSPHERIC INFORMATION: (MANUAL INPUT OF DATA)

Wind: 2.5 meters $/ \mathrm{sec}$ from $90^{\circ}$ true at 2 meters

Inversion Height: 500 meters

Stability Class: $\mathrm{C}$ (user override)

Air Temperature: $25^{\circ} \mathrm{C}$

Relative Humidity: $50 \%$

Cloud Cover: 8 tenths

Ground Roughness: Urban or forest

\section{SOURCE STRENGTH INFORMATION:}

Puddle Area: 40700 square feet Average Puddle Depth: 1 centimeters

Soil Type: Default Ground Temperature: $25^{\circ} \mathrm{C}$

Initial Puddle Temperature: Ground temperature

Release Duration: AlOHA limited the duration to 1 hour

Max Computed Release Rate: 694 kilograms/min

Max Average Sustained Release Rate: 592 kilograms/min

(averaged over a minute or more)

Total Amount Released: 31,015 kilograms

FOOTPRINT INFORMATION:

Model Run: Heavy Gas

User-specified LOC: 2000 ppm

Max Threat Zone for LOC: 140 meters
Reviewed by S. A. Henderson

Works Calculation 8

Sheet No. 288 of 314 
Title of Project EPHA for THM

Subject Concentration Analysis for THM Hazardous Chemicals

Computer N.E. Baucom B. K. Tharakan Date 09/16/96

1-RD-46 AVERAGE MAX DIST SEG 3

SITE DATA INFORMATION:

Location: AIKEN, SOUTH CAROLINA

Building Air Exchanges Per Hour: 60 (User specified)

Time: June 30, 19981200 hours EDT (User specified)

\section{CHEMICAL INFORMATION:}

Chemical Name: TETRAHYDROFURAN

Molecular Weight: $72.11 \mathrm{~kg} / \mathrm{kmol}$

TLV-TWA: $200 \mathrm{ppm}$

IDLH: 2000 ppm

Footprint Level of Concern: 2000 ppm

Boiling Point: $65.97^{\circ} \mathrm{C}$

Vapor Pressure at Ambient Temperature: 0.21 atm

Ambient Saturation Concentration: 216,395 ppm or $21.6 \%$

ATMOSPHERIC INFORMATION: (MANUAL INPUT OF DATA)

Wind: 2.5 meters $/ \mathrm{sec}$ from $90^{\circ}$ true at 2 meters

Inversion Height: 500 meters

Stability Class: $\mathrm{C}$ (user override)

Air Temperature: $25^{\circ} \mathrm{C}$

Relative Humidity: 50\%

Cloud Cover: 8 tenths

Ground Roughness: Urban or forest

\section{SOURCE STRENGTH INFORMATION:}

Puddle Area: 10000 square meters

Average Puddle Depth: 1 centimeters

Soil Type: Default

Initial Puddle Temperature: Ground temperature

Release Duration: ALOHA limited the duration to 1 hour

Max Computed Release Rate: 1,760 kilograms/min

Max Average Sustained Release Rate: 1,510 kilograms/min

(averaged over a minute or more)

Total Amount Released: 79,754 kilograms

FOOTPRINT INFORMATION:

Model Run: Heavy Gas

User-specified LOC: 2000 ppm

Max Threat Zone for LOC: 232 meters
Reviewed by S. A. Henderson

Works Calculation 8

Sheet No. $\underline{289 \text { of } 314}$ 
Title of Project EPHA for THM

Subject Concentration Analysis for THM Hazardous Chemicals

Computer N.E. Baucom B. K. Tharakan Date 09/16/96

1-RD.49 AVERAGE / ALERT

SITE DATA INFQRMATION:

Location: AIKEN, SOUTH CAROLINA

Building Air Exchanges Per Hour: 60 (User specified)

Time: June 30, 19981200 hours EDT (User specified)

CHEMICAL INFORMATION:

Chemical Name: 1,1,1-TRICHLOROETHANE

Molecular Weight: $133.40 \mathrm{~kg} / \mathrm{kmol}$

TLV-TWA: $350 \mathrm{ppm}$

Footprint Level of Concern: 700 ppm

Boiling Point: $74.08^{\circ} \mathrm{C}$

Vapor Pressure at Ambient Temperature: 0.16 atm

Ambient Saturation Concentration: 167,040 ppm or $16.7 \%$

ATMOSPHERIC INFORMATION: (MANUAL INPUT OF DATA)

Wind: 2.5 meters $/ \mathrm{sec}$ from $90^{\circ}$ true at 2 meters

Inversion Height: 500 meters

Stability Class: C (user override)

Air Temperature: $25^{\circ} \mathrm{C}$

Relative Humidity: 50\%

Cloud Cover: 8 tenths

IDLH: $700 \mathrm{ppm}$
Reviewed by S. A. Henderson

Works Calculation 8

Sheet No. 290 of 314

\section{SOURCE STRENGTH INFORMATION:}

Puddle Area: 875 square feet

Average Puddle Depth: 1 centimeters

Soil Type: Default

Ground Temperature: $25^{\circ} \mathrm{C}$

Initial Puddle Temperature: Ground temperature

Release Duration: 59 minutes

Max Computed Release Rate: 22.8 kilograms/min

Max Average Sustained Release Rate: 20.2 kilograms/min

(averaged over a minute or more)

Total Amount Released: 1,081 kilograms

\section{FOOTPRINT INFORMATION:}

Dispersion Module: Gaussian

ser-specified LOC: 700 ppm

$x$ Threat Zone for LOC: less than 10 meters (10.9 yards)

Max Threat Zone for IDLH: less than 10 meters (10.9 yards)

Note: Footprint was not drawn because effects of near-field patchiness make dispersion predictions unreliable for short distances.

\section{TIME DEPENDENT INFORMATION}

Concentration Estimates at the point:

Downwind: $\quad 30$ meters

Off Centerline: , 0 meters

Max Concentration:

Outdoor: ' $197 \mathrm{ppm}$

Indoor: $\quad 197 \mathrm{ppm}$

Note: Indoor graph is shown with a dotted line 
Title of Project EPHA for THM

Subject Concentration Analysis for THM Hazardous Chemicals

Computer N.E. Baucom B. K. Tharakan Date 09/16/96

I-RD-49 AVERAGE SAE

\section{SITE DATA INFORMATION:}

Location: AIKEN, SOUTH CAROLINA

Building Air Exchanges Per Hour: 60 (User specified)

Time: June 30, 19981200 hours EDT (User specified)

\section{CHEMICAL INFORMATION:}

Chemical Name: 1,1,1-TRICHLOROETHANE

Molecular Weight: $133.40 \mathrm{~kg} / \mathrm{kmol}$

TLV-TWA: $350 \mathrm{ppm}$

Footprint Level of Concern: 700 ppm

Boiling Point: $74.08^{\circ} \mathrm{C}$

Vapor Pressure at Ambient Temperature: $0.16 \mathrm{~atm}$

Ambient Saturation Concentration: 167,040 ppm or $16.7 \%$

ATMOSPHERIC INFORMATION: (MANUAL INPUT OF DATA)

Wind: 2.5 meters $/ \mathrm{sec}$ from $90^{\circ}$ true at 2 meters

Inversion Height: 500 meters

Stability Class: $\mathbf{C}$ (user override)

Air Temperature: $25^{\circ} \mathrm{C}$

Relative Humidity: 50\%

Ground Roughness: Urban or forest

Cloud Cover: 8 tenths

\section{SOURCE STRENGTH INFORMATION:}

Puddle Area: 7840 square feet Average Puddle Depth: 1 centimeters

Soil Type: Default

IDLH: 700 ppm

Initial Puddle Temperature: Ground temperature

Release Duration: ALOHA limited the duration to 1 hour

Max Computed Release Rate: 185 kilograms/min

Max Average Sustained Release Rate: 167 kilograms/min

(averaged over a minute or more)

Total Amount Released: 9,101 kilograms

FOOTPRINT INFORMATION:

Model Run: Heavy Gas

User-specified LOC: $700 \mathrm{ppm}$

Max Threat Zone for LOC: 100 meters

Note: Footprint was not drawn because effects of near-field patchiness make dispersion predictions unreliable for short distances.

\section{TIME DEPENDENT INFORMATION:}

Concentration Estimates at the point:

Downwind: 100 meters

Off Centerline: 0 meters

Max Concentration:

outdoor: $700 \mathrm{ppm}$

Indoor: $700 \mathrm{ppm}$

Note: Indoor graph is shown with a dotted line.
Reviewed by S. A. Henderson

Works Calculation 8

Sheet No. 291 of 314 
Title of Project EPHA for THM

Subjoct Concentration Analysis for THM Hazardous Chemicals

Computer N.E. Baucom B. K. Tharakan Date 09/16/96

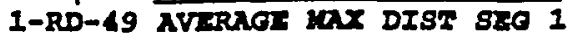

SITE DATA INFORMATION:

Location: AIKEN, SOUTH CAROLINA

Building Air Exchanges Per Hour: 60 (User specified)

Time: June 30, 19981200 hours EDT (User specified)

CHEMICAL INFORMATION:

Chemical Name: 1,1,1-TRICHLOROETHANE

Molecular Weight: $133.40 \mathrm{~kg} / \mathrm{kmol}$

TLV-TWA: $350 \mathrm{ppm}$ IDLH: $700 \mathrm{ppm}$

Footprint Level of Concern: $700 \mathrm{ppm}$

Boiling Point: $74.08^{\circ} \mathrm{C}$

Vapor Pressure at Ambient Temperature: $0.16 \mathrm{~atm}$

Ambient Saturation Concentration: $167,040 \mathrm{ppm}$ or 16.78

ATMOSPHERIC INFORMATION: (MANUAL INPUT OF DATA)

Wind: 2.5 meters/sec from $90^{\circ}$ true at 2 meters

Inversion Height: 500 meters

Stability Class: C (user override)

Air Temperature: $25^{\circ} \mathrm{C}$

Relative Humidity: 508

Cloud Cover: 8 tenths

Ground Roughness: Urban or forest

SOURCE STRENGTH INFORMATION:

Puddle Area: 24420 square feet

Average Puddle Depth: 1 centimeters

Soil Type: Default Ground Temperature: $25^{\circ} \mathrm{C}$

Initial puddle Temperature: Ground temperature

Release Duration: ALOFA Iimited the duration to 1 hour

Max Computed Release Rate: 548 kilograms/min

Max Average Sustained Release Rate: 497 kilograms/min

(averaged over a minute or more)

Total Amount Released: 27,359 kilograms

FOOTPRINT INEORMATION:

Model Run: Heavy Gas

User-specified LOC: 700 ppm

Max Threat Zone for LOC: 176 meters
Reviewed by S.A. Henderson

Works Catculation 8

Sheot No. 292 of 314 
Title of Project EPHA for THM

Subject Concentration Analysis for THM Hazardous Chemicals

Computer N.E. Baucom B. K. Tharakan Date 09/16/96

1-RD-49 AVERAGE MAX DIST SEG 2

SITE DATA INFORMATION:

Location: AIKEN, SOUTH CAROLINA

Building Air Exchanges Per Hour: 60 (User specified)

Time: June 30, 19981200 hours EDT (User specified)

\section{CHEMICAL INFORMATION:}

Chemical Name: 1,1,1-TRICHLOROETHANE

Molecular Weight: $133.40 \mathrm{~kg} / \mathrm{kmol}$

TLV-TWA: $350 \mathrm{ppm}$

IDLH: $700 \mathrm{ppm}$

Footprint Level of Concern: 700 ppm

Boiling Point: $74.08^{\circ} \mathrm{C}$

Vapor Pressure at Ambient Temperature: $0.16 \mathrm{~atm}$

Ambient Saturation Concentration: 167,040 ppm or $16.7 \%$

ATMOSPHERIC INFORMATION: (MANUAL INPUT OF DATA)

Wind: 2.5 meters $/ \mathrm{sec}$ from $90^{\circ}$ true at 2 meters

Inversion Height: 500 meters

Stability Class: C (user override)

Air Temperature: $25^{\circ} \mathrm{C}$

Relative Humidity: $50 \%$

Ground Roughness: Urban or forest

Cloud Cover: 8 tenths

\section{SOURCE STRENGTH INFORMATION:}

Puddle Area: 40700 square feet

Average Puddle Depth: 1 centimeters

Soil Type: Default

Ground Temperature: $25^{\circ} \mathrm{C}$

Initial Puddle Temperature: Ground temperature

Release Duration: ALOHA limited the duration to 1 hour

Max Computed Release Rate: 894 kilograms/min

Max Average Sustained Release Rate: 812 kilograms/min

(averaged over a minute or more)

Total Amount Released: 44,897 kilograms

FOOTPRINT INFORMATION:

Model Run: Heavy Gas

User-specified LOC: 700 ppm

Max Threat Zone for LOC: 229 meters
Reviewed by S. A. Henderson

Works Calculation 8

Sheet No. 293 of 314 
Title of Project EPHA for THM

Subject Concentration Analysis for THM Hazardous Chemicals

Computer N.E. Baucom B. K. Tharakan Date 09/16/96

1-RD-49 AVERAGE / MAX DIST SEG 3

SITE DATA INFORMATION:

Location: AIKEN, SOUTH CAROLINA

Building Air Exchanges Per Hour: 60 (User specified)

Time: June 30, 19981200 hours EDT (User specified)

CHEMICAL INFORMATION:

Chemical Name: 1,1,1-TRICHLOROETHANE

Molecular Weight: $133.40 \mathrm{~kg} / \mathrm{kmol}$

TLV-TWA: 350 ppm

Footprint Level of Concern: 700 ppm

Boiling Point: $74.08^{\circ} \mathrm{C}$

Vapor Pressure at Ambient Temperature: 0.16 atm

Ambient Saturation Concentration: 167,040 ppm or $16.7 \%$

ATMOSPHERIC INFORMATION: (MANUAL INPUT OF DATA)

Wind: 2.5 meters $/ \mathrm{sec}$ from $90^{\circ}$ true at 2 meters

Inversion Height: 500 meters

Stability Class: C (user override)

Air Temperature: $25^{\circ} \mathrm{C}$

Relative Humidity: 50\%

Cloud Cover: 8 tenths

\section{SOURCE STRENGTH INFORMATION:}

Puddle Area: 10000 square meters

Average Puddle Depth: 1 centimeters

Soil Type: Default

Ground Temperature: $25^{\circ} \mathrm{C}$

Initial Puddle Temperature: Ground temperature

Release Duration: ALOHA limited the duration to 1 hour

Max Computed Release Rate: 2,270 kilograms/min

Max Average Sustained Release Rate: 2,080 kilograms/min

(averaged over a minute or more)

Total Amount Released: 115,384 kilograms

FOOTPRINT INFORMATION:

Model Run: Heavy Gas

User-specified LOC: 700 ppm

Max Threat Zone for LOC: 381 meters
Reviewed by S. A. Henderson

Works Calculation 8

Sheet No. 294 of 314 


\section{ENGINEERING COMPUTATION SHEET}

Title of Project EPHA for THM

Subject Concentration Analysis for THM Hazardous Chemicals

Computer N.E. Baucom B. K. Tharakan Date 09/16/96

I-RD-51, AVARACI ATHRT

SITE DATA INFORMATION:

Location: AIKEN, SOUTH CAROLINA

Building Air Exchanges Per Hour: 60 (User specified)

Time: June 30, 19981200 hours EDT (User specified)

CHEMICAL INFORMATION:

Chemical Name: TRIETHYLAMINE

Molecular Weight: $101.19 \mathrm{~kg} / \mathrm{kmol}$

TLV-TWA: 10 ppm

IDLF: $1000 \mathrm{ppm}$

Footprint Level of Concern: $3 \mathrm{ppm}$

Boiling Point: $88.77^{\circ} \mathrm{C}$

Vapor Pressure at Ambient Temperature: $0.090 \mathrm{~atm}$

Ambient Saturation Concentration: 91,682 ppm or 9.178

ATMOSPHERIC INFORMATION: (MANUAL INPUT OF DATA)

Wind: 2.5 meters/sec from $90^{\circ}$ true at 2 meters

Inversion Height: 500 meters

stability class: C (user override)

Air Temperature: $25^{\circ} \mathrm{C}$

Relative Humidity: $50 \%$

Cloud Cover: 8 tenths

Ground Roughness: Urban or forest

SOURCE STRENGTF INFORMATION:

Puddle Area: 11.3 square feet

Average Puddle Depth: 1 centimeters

Soil Type: Default Ground Temperature: $25^{\circ} \mathrm{C}$

Initial Puddle Temperature: Ground temperature.

Release Duration: 56 minutes

Max Computed Release Rate: 157 grams/min

Max Average Sustained Release Rate: 145 grams/min (averaged over a minute or more)

Total Amount Released: 7.61 kilograms

TIME DEPENDENT INFORMATION:

Concentration Estimates at the point:

Downwind: 30 meters

off Centerline: 0 meters

Max Concentration:

Outdoor: $2.98 \mathrm{ppm}$

Indoor: $2.98 \mathrm{ppm}$

Note: Indoor graph is shown with a dotted line. 
Title of Project EPHA for THM

ENGINEERING COMPUTATION SHEET

Subject Concentration Analysis for THM Hazardous Chemicals

Computer N.E. Baucom B. K. Tharakan Date 09/16/96

1-RD-51 AVARIGE / SNT

SITE DATA INFORMATION:

Location: AIKEN, SOUTH CAROLINA

Building Air Exchanges Per Hour: 60 (User specified)

Time: June 30, 19981200 hours EDT (User specified)

CHEMICAI INFORMATION:

Chemical Name: TRIETHYLAMINE

Molecular Weight: $101.19 \mathrm{~kg} / \mathrm{kmol}$

TLV-TWA: 10 ppm

Footprint Level of Concern: 3 ppm

Boiling Point: $88.77^{\circ} \mathrm{C}$

Vapor Pressure at Ambient Temperature: 0.090 atm

Ambient Saturation Concentration: $91,682 \mathrm{ppm}$ or $9.17 \%$

ATMOSPHERIC INFORMATION: (MANUAL INPUT OF DATA)

Wind: 2.5 meters $/ \mathrm{sec}$ from $90^{\circ}$ true at 2 meters

Inversion Height: 500 meters

Stability Class: C (user override)

Air Temperature: $25^{\circ} \mathrm{C}$

Relative Humidity: $50 \%$

Ground Roughness: Urban or forest

IDLH: $1000 \mathrm{ppm}$
Reviewed by S. A. Henderson

Works Calculation 8

Sheet No. 296 of 314

SOURCE STRENGTH INFORMATION:

Puddle Area: 143 square feet

Average Puddle Depth: 1 centimeters

Soil Type: Default

Ground Temperature: $25^{\circ}$

Initial Puddle Temperature: Ground temperature

Release Duration: ALOHA limited the duration to 1 hour

Max Computed Release Rate: 1.71 kilograms/min

Max Average Sustained Release Rate: $1.61 \mathrm{kilograms/min}$

(averaged over a minute or more)

Total Amount Released: 91.6 kilograms

TIME DEPENDENT INFORMATION:

Concentration Estimates at the point:

Downwind: 100 meters

off Centerline: 0 meters

Max Concentration:

Outdoor: 2.99 ppm

Indoor: $2.99 \mathrm{ppm}$

Note: Indoor graph is shown with a dotted line. 
SITE DATA INFORMATION:

Location: AIKEN, SOUTH CAROLINA

Building Air Exchanges Per Hour: 60 (User specified)

Time: June 30, 19981200 hours EDT (User specified)

CHEMICAL INFORMATION:

Chemical Name: TRIETHYLAMINE

Molecular Weight: $101.19 \mathrm{~kg} / \mathrm{kmol}$

TLV-TWA: $10 \mathrm{ppm}$

IDLH: $1000 \mathrm{ppm}$

Footprint Level of Concern: $3 \mathrm{ppm}$

Boiling Point: $88.77^{\circ} \mathrm{C}$

Vapor Pressure at Ambient Temperature: 0.090 atm.

Ambient Saturation Concentration: $91,682 \mathrm{ppm}$ or 9.178

ATMOSPHERIC INFORMATION: (MANUAL INPUT OF DATA)

Wind: 2.5 meters/sec from $90^{\circ}$ true at 2 meters

Inversion Height: 500 meters

stability class: $C$ (user override)

Air Temperature: $25^{\circ} \mathrm{C}$

Relative Humidity: $50 \%$

Ground Roughness: Urban or forest

Cloud Cover: 8 tenths

SOURCE STRENGTH INEORMATION:

Puddile Area: 24420 square feet

Average Pudale Depth: 1 centimeters

Soil Type: Default Ground Temperature: $25^{\circ} \mathrm{C}$

Initial puddle Temperature: Ground temperature

Release Duration: ALOHA limited the duration to 1 hour

Max Computed Release Rate: 227 kilograms/min

Max Average Sustained Release Rate: 221 kilograms/min (averaged over a minute or more)

Total Amount Released: 12,901 kilograms

FOOTPRINT INFORMATION:

Model Run: Heavy Gas

User-specified LoC: $3 \mathrm{ppm}$

Max Threat Zone for IOC: 3.2 kilometers 
Title of Project EPHA for THM

Subject Concentration Analysis for THM Hazardous Chemicals

Computer N.E. Baucom B. K. Tharakan Date 09/16/96

1-RD-51 AVARAGT / MaX DIST GEC 2

SITE DATA INFORMATION:

LOCation: AIREN, SOUTH CAROLINA

Building Air Exchanges Per Hour: 60 (User specified)

Time: June 30, 19981200 hours EDT (User specified)

CHEMICAI INFORMATION:

Chemical Name: TRIETHYLAMINE

Molecular Weight: $101.19 \mathrm{~kg} / \mathrm{kmol}$

TLV-TWA: $10 \mathrm{ppm}$ of Concerm: $3 \mathrm{ppm}$

Boiling Point: $88.77^{\circ} \mathrm{C}$

Vapor Pressure at Ambient Temperature: 0.090 atm

Ambient Saturation Concentration: $91,682 \mathrm{ppm}$ or $9.17 \%$

ATMOSPHERIC INFORMATION: (MANUAL INPUT OF DATA)

Wind: 2.5 meters/sec from $90^{\circ}$ true at 2 meters

Inversion Height: 500 meters

stability Class: C (user override)

Air Temperature: $25^{\circ} \mathrm{C}$

Relative Humidity: $50 \%$

Ground Roughness: Urban or forest

Cloud Cover: 8 tenths

SOURCE STREANGTH INFORMATION:

Puddle Area: 40700 square feet

Average Pudale Depth: 1 centimeters

Soil Type: Default Ground Temperature: $25^{\circ} \mathrm{C}$

Initial Pudale Temperature: Ground temperature

Release Duration: ALOHA limited the duration to 1 hour

Max Computed Release Rate: $370 \mathrm{kilograms/min}$

Max Average Sustained Release Rate: 361 kilograms/min (averaged over a minute or more)

Total Amount Releaged: 21,136 kilograms

FOOTPRINT INGFORMATION:

Model Run: Heavy Gas

User-specified LOC: $3 \mathrm{ppm}$

Max Threat Zone for LOC: 4.1 kilometers
Reviewed by S. A. Henderson

Works Calculation 8

Sheet No. 298 of 314 


\section{ENGINEERING COMPUTATION SHEET}

Title of Project EPHA for THM

Subject Concentration Analysis for THM Hazardous Chemicals

Computer N.E. Baucom B. K. Tharakan Date $09 / 16 / 96$

1-RD-51 AVARAGs / WAX DIST STO 3

SITE DATA INFORMATION:

Location: AIKEN, SOUTH CAROLINA

Building Air Exchanges Per Hour: 60 (User specified)

Time: June 30, 19981200 hours EDT (User specified)

CHEMICAI INFORMATION:

Chemical Name: TRIETHYLAMINE

Molecular Weight: $101.19 \mathrm{~kg} / \mathrm{kmol}$

TLV-TWA: $10 \mathrm{ppm}$

IDLH: $1000 \mathrm{ppm}$

Footprint Level of Concern: 3 ppm

Boiling Point: $88.77^{\circ} \mathrm{C}$

Vapor Pressure at Ambient Temperature: 0.090 atm

Ambient Saturation Concentration: 91,682 ppm or $9.17 \%$

ATMOSPHERIC INFORMATION: (MANUAL INPUT OF DATA)

Wind: 2.5 meters/sec from $90^{\circ}$ true at 2 meters

Inversion Height: 500 meters

Stability Class: C (user override

Air Temperature: $25^{\circ} \mathrm{C}$

Relative Humidity: 508

Cloud Cover: 8 tenths

Ground Roughness: Urban or forest

SOURCE STRENGTH INFORMATION:

Puddle Area: 10000 square meters

Average Puddie Depth: 1 centimeters

Soil Type: Default Ground Temperature: $25^{\circ} \mathrm{C}$

Initial puddle Temperature:' Ground temperature

Release Duration: ALOHA limited the duration to 1 hour

Max Computed Release Rate: $938 \mathrm{kilograms/min}$

Max Average Sustained Release Rate: 919 kilograms/min

(averaged over a minute or more)

Total Amount Released: 54,152 kilograms

FOOTPRINT INFORMATION:

Model Run: Heavy Gas

User-specified LOC: 3 ppm

Max Threat Zone for LOC: $6.7 \mathrm{kilometers}$
Reviewed by S. A. Henderson

Works Calculation 8

Sheet No. 299 of 314 
Title of Project EPHA for THM

\section{ENGINEERING COMPUTATION SHEET}

\section{Subject Concentration Analysis for THM Hazardous Chemicals}

Computer N.E. Baucom B. K. Tharakan Date 09/16/96 1-RD-54 AVSRAGE / ATIFRI

SITE DATA INFORMATION:

Location: AIKEN, SOUTH CAROLINA

Building Air Exchanges Per Hour: 60 (User specified)

Time: June 30, 19981200 hours EDT (User specified)

CHEMICAL INFORMATION:

Chemical Name: BENZOIC ACID

Molecular Weight: $122.13 \mathrm{~kg} / \mathrm{kmol}$

TLV-TWA: -unavail- IDLH: -unavail

Footprint Level of Concern: $75 \mathrm{mg} /(\mathrm{cu} \mathrm{m})$

Boiling Point: $249.00^{\circ} \mathrm{C}$

Freezing Point: $122.00^{\circ} \mathrm{C}$

Note: Not enough chemical data to use Heavy Gas option

ATMOSPHERIC INFORMATION: (MANUAL INPUT OF DATA)

Wind: 2.5 meters/sec from $90^{\circ}$ true at 2 meters

Inversion Height: 500 meters

Stability Class: C (user override)

Air Temperature: $25^{\circ} \mathrm{C}$

Relative Humidity: $50 \%$

Cloud Cover: 8 tenths

Ground Roughness: Urban or forest

SOURCE STRENGTH INFORMATION:

Direct Source: 1.9 pounds

Release Duration: 1 minute

Release Rate: 14.4 grams/sec

Total Amount Released: 862 grams

TIME DEPENDENTT INFORMATION:

Concentration Estimates at the point:

Downwind: $\quad 30$ meters

off Centerline: 0 meters

Max Concentration:

Outdoor: $73.9 \mathrm{mg} /(\mathrm{cu} \mathrm{m})$

Indoor: $44.1 \mathrm{mg} /(\mathrm{cu} \mathrm{m})$

Note: Indoor graph is shown with a dotted line.
Reviewed by S. A. Henderson

Works Calculation 8

Sheet No. 300 of 314 
Title of Project EPHA for THM

Subject Concentration Analysis for THM Hazardous Chemicals

Computer N.E. Baucom B. K. Tharakan Date 09/16/96

1-RD-54 AVARACE SAE

SITE DATA INFORMATION:

Location: AIKEN, SOUTHE CAROLINA

Building Air Exchanges Per Hour: 60 (User specified)

Time: June 30, 19981200 hours EDT (User specified)

CHEMICAL INFORMATION:

Chemical Name: BENZOIC ACID

Molecular Weight: $122.13 \mathrm{~kg} / \mathrm{kmol}$

TLV-TWA: - unavail-

Footprint Level of Concern: $75 \mathrm{mg} /(\mathrm{cu} \mathrm{m})$

Boiling Point: $249.00^{\circ} \mathrm{C}$

Freezing point: $122.00^{\circ} \mathrm{C}$

Note: Not enough chemical data to use Heavy Gas option

ATMOSPHERIC INFORMATION: (MANUAC INPUT OF DATA)

Wind: 2.5 meters $/ \mathrm{sec}$ from $90^{\circ}$ true at 2 meters

Inversion Height: 500 meters

Stability Class: C (user override)

Air Temperature: $25^{\circ} \mathrm{C}$

Relative Humidity: 508

Cloud Cover: 8 tenths

Ground Roughness: Urban or forest

SOURCE STRENGTH INFORMATION:

Direct Source: 21.3 pounds

Source Height: 0

Release Duration: 1 minute

Release Rate: 161 grams/sec

Total Amount Released: 9.66 kilograms

TIME DEPENDENT INFORMATION:

Concentration Estimates at the point:

Downwind: 100 meters

off Centerline: 0 meters

Max Concentration:

Outdoor: $\quad 74.8 \mathrm{mg} /(\mathrm{cu} \mathrm{m}$ )

Indoor: $\quad 44.1 \mathrm{mg} /(\mathrm{cu} \mathrm{m})$

Note: Indoor graph is shown with a dotted line.

Reviewed by S. A. Henderson

Works Calculation 8

Sheet No. 301 of 314 
SITE DATA INFORMATION:

Location: AIKEN, SOUTH CAROLINA

Building Air Exchanges Per Hour: 60 (User specified)

Time: June 30,19981200 hours EDT (User specified)

CHEMICAI INFORMATION:

Chemical Name: BENZOIC ACID

Molecular Weight: $122.13 \mathrm{~kg} / \mathrm{kmol}$

TLV-TWA: -unavail-
Footprint Level of Concern: $75 \mathrm{mg} /(\mathrm{cu} \mathrm{m})$

Boiling Point: $249.00^{\circ} \mathrm{C}$

Freezing Point: $122.00^{\circ} \mathrm{C}$

Note: Not enough chemical data to use Heavy Gas option

ATMOSPHERIC INFORMATION: (MANUAI INPUT OF DATA)

Wind: 2.5 meters $/ \mathrm{sec}$ from $90^{\circ}$ true at 2 meters

Inversion Height: 500 meters

stability Class: C (user override)

Air Temperature: $25^{\circ} \mathrm{C}$

Relative Humidity: 508

Ground Roughness: Urban or forest

Cloud Cover: 8 tenths

SOURCE STRENGTH INFORMATION:

Direct Source: 37.6 pounds

Source Height: 0

Release Duration: 1 minute

Release Rate: 284 grams/sec

Total Amount Released: 17.1 kilograms

FOOTRRINT INFORMATION:

Dispersion Module: Gaussian

User- specified LOC: $75 \mathrm{mg} /(\mathrm{cu} \mathrm{m})$

Max Threat zone for IOC: 133 meters 


\section{ENGINEERING COMPUTATION SHEET}

Title of Project EPHA for THM

Reviewed by S. A. Henderson

Works Calculation 8

Sheet No. 303 of 314

Computer N.E. Baucom B. K. Tharakan Date $09 / 16 / 96$

1-RD-54 AVERAGE UNX DIST SEO 2

SITE DATA INFORMATION:

Location: AIKEN, SOUTH CAROLINA

Building Air Exchanges Per Hour: 60 (User specified)

Time: June 30, 19981200 hours EDT (User specified)

CHEMICAL INFORMATION :

Chemical Name: BENZOIC ACID

Molecular Weight: $122.13 \mathrm{~kg} / \mathrm{kmol}$

TLV-TWA: -unavail-

IDLH: -unavail

Footprint Level of Concern: $75 \mathrm{mg} /(\mathrm{cu} \mathrm{m})$

Boiling Point: $249.00^{\circ} \mathrm{C}$

Freezing point: $122.00^{\circ} \mathrm{C}$

Note: Not enough chemical data to use Heavy Gas option

ATMOSPFERIC INFORMATION: (MANUAL INPUT OF DATA)

Wind: 2.5 meters $/ \mathrm{sec}$ from $90^{\circ}$ true at 2 meters

Inversion Height: 500 meters

stability class: C (user override)

Air Temperature: $25^{\circ} \mathrm{C}$

Relative Humidity: 508 Ground Roughness: Urban or forest

Cloud Cover: 8 tenths

SOURCE STRENGTH INFORMATION:

Direct Source: 135 pounds Source Height: 0

- Release Duration: 1 minute

Release Rate: 1.02 kilograms/sec

Total Amount Released: 61.2 kilograms

EOOTPRINT INFORMATION:

Dispersion Module: Gaussian

User-specified LOC: $75 \mathrm{mg} /(\mathrm{cu} \mathrm{m})$

Max Threat Zone for LOC: 252 meters 


\section{ENGINEERING COMPUTATION SHEET}

Titie of Project EPHA for THM

Reviewed by S. A. Henderson Works Calculation 8

Subject Concentration Analysis for THM Hazardous Chemicals

Computer N.E. Baucom B. K. Tharakan Date 09/16/96

1-RD-54 AVARACI MDX DIST SEG 3

SITE DATA INFORMATION:

Location: AIREN, SOUTH CAROLINA

Building Air Exchanges Per Hour: 60 (User specified)

Time: June 30, 19981200 hours EDT (User specified)

CHEMICAL INFORMATION:

Chemical Name: BENZOIC ACID

Molecular Weight: $122.13 \mathrm{~kg} / \mathrm{kmol}$

TLV-TWA: -unavail-

Boiling Point: $249.00^{\circ} \mathrm{C}$

Freezing Point: $122.00^{\circ} \mathrm{C}$

Note: Not enough chemical data to use Heavy Gas option

ATMOSPHERIC INFORMATION: (MANUAL INPUT OF DATA)

wind: 2.5 meters/sec from $90^{\circ}$ true at 2 meters

Inversion Height: 500 meters

stability Class: C (user override)

Air Temperature: $25^{\circ} \mathrm{C}$

Relative Humidity: 508

Ground Roughness: Urban or forest

cloud Cover: 8 tenths

SOURCE STRENGTH INFORMATION:

Direct Source: 263 pounds

Release Duration: 1 minute

Release Rate: $1.99 \mathrm{kilograms} / \mathrm{sec}$

Total Amount Released: 119 kilograms

FOOTPRINT INFORMATION:

Dispersion Module: Gaussian

User-specified LOC: $75 \mathrm{mg} /(\mathrm{cu} \mathrm{m})$

Max Threat Zone for LOC: 353 meters 
Title of Project EPHA for THM

Subject Concentration Analysis for THM Hazardous Chemicals

Computer N.E. Baucom B. K. Tharakan Date 09/16/96 1-RD-56 AVSRAGS ATERT
Reviewed by S. A. Henderson

Works Calculation 8

Sheet No. 305 of 314

SITE DATA INFORMATION:

LOCation: AIKEN, SOUTH CAROLINA

Building Air Exchanges Per Hour: 60 (User specified)

Time: June 30, 19981200 hours EDT (User specified)

CHEMICAL INFORMATION :

Chemical Name: SILICA, CRYSTALIINE QUARTZ (RESP. DUST)

Molecular Weight: $60.09 \mathrm{~kg} / \mathrm{kmol}$

TLV-TWA: -unavail-

IDLH: -unavail

Footprint Level of Concern: $0.5 \mathrm{mg} /(\mathrm{cu} \mathrm{m})$

Boiling Point: -unavail

Note: Not enough chemical data to use Heavy Gas option

ATMOSPHERIC INFORMATION: (MANUAI INPUT OF DATA)

Wind: 2.5 meters/sec from $90^{\circ}$ true at 2 meters

Inversion Height: 500 meters

Stability Class: C (user override)

Air Temperature: $25^{\circ} \mathrm{C}$

Relative Humidity: 508

Cloud Cover: 8 tenths.

Ground Roughness: Uxban or forest

SOURCE STRENGTH INEORMATION:

Direct Source: .0128 pounds

Source Height: 0

Release Duration: 1 minute

Release Rate: 0.0968 grams/sec

Total Amount Released: 5.81 grams

TIME DEPENDENT INFORMATION:

Concentration Estimates at the point:

Downwind: 30 meters

off Centexline: 0 meters

Max Concentration:

Outdoor: $0.498 \mathrm{mg} /(\mathrm{cu} \mathrm{m})$

Indoor: $0.297 \mathrm{mg} /(\mathrm{cu} \mathrm{m})$

Note: Indoor graph is shown with a dotted line. 
Title of Project EPHA for THM

Reviewed by S. A. Henderson

Subject Concentration Analysis for THM Hazardous Chemicals

Works Calculation 8

Computer N.E. Baucom B. K. Tharakan Date 09/16/96

1-RD-56 AVERACE SAE

Sheet No. 306 of 314

SITE DATA INFORMATION:

LOCation: AIKEN, SOUTH CAROLINA

Building Air Exchanges Per Hour: 60 (User specified)

Time: June 30, 19981200 hours EDT (User specified)

CHEMICAL INFORMATION:

Chemical Name: SILICA, CRYSTAIIINE QUARTZ (RESP. DUST)

Molecular Weight: $60.09 \mathrm{~kg} / \mathrm{kmol}$

TLV-TWA: -unavail-

IDLH : -unavaiI

Footprint Level of Concern: $0.5 \mathrm{mg} /(\mathrm{cu} \mathrm{m})$

Boiling Point: -unavail

Note: Not enough chemical data to use Heavy Gas option

ATMOSPHERIC INFORMATION: (MANUAI INPUT OF DATA)

Wind: 2.5 meters/sec from $90^{\circ}$ true at 2 meters

inversion Height: 500 meters

Stability Class: C (user override)

Air Temperature: $25^{\circ} \mathrm{C}$

Relative Humidity: $50 \%$

Cloud. Cover: 8 tenths

Ground Roughness: Urban or forest

SOURCE STRENGTH INFORMATION:

Direct Source: .142 pounds

Source Height: 0

Release Duration: 1 minute

Release Rate: 1.07 grams/sec

Total Amount Released: 64.4 grams.

TIME DEPENDENT INFORMATION:

Concentration Estimates at the point:

Downwind: 100 meters

off Centerline: 0 meters

Max Concentration:

Outdoor: $0.499 \mathrm{mg} /(\mathrm{cu} \mathrm{m})$

Indoor: $0.294 \mathrm{mg} /(\mathrm{cu} \mathrm{m})$

Note: Indoor graph is shown with a dotted line. 
ENGINEERING COMPUTATION SHEET

Title of Project EPHA for THM

Reviewed by S. A. Henderson

Works Calculation 8

Sheet No. 307 of 314

Computer N.E. Baucom B. K. Tharakan Date $09 / 16 / 96$

1-RD-56 AVERAGE / MAX DIST SEG 1

SITE DATA INFORMATION:

Location: AIKEXN, SOUTH CAROLINA

Building Air Exchanges Per Hour: 60 (User specified)

Time: June 30, 19981200 hours EDT (User specified)

CHEMICAL INFORMATION:

Chemical Name: SILICA, CRYSTALLINE QUARTZ (RESP. DUST)

Molecular Weight: $60.09 \mathrm{~kg} / \mathrm{kmol}$

TLV-TWA: -unavail-

IDLH: -unavail

Footprint Level of Concern: $0.5 \mathrm{mg} /(\mathrm{cu} \mathrm{m})$

Boiling Point: -unavail

Note: Not enough chemical data to use Heavy Gas option

ATMOSPHERIC INFORMATION: (MANUAL INPUT OF DATA)

Wind: 2.5 meters $/ \mathrm{sec}$ from $90^{\circ}$ true at 2 meters

Inversion Height: 500 meters

Stability Class: $\mathrm{C}$ (user override)

Air Temperature: $25^{\circ} \mathrm{C}$

Relative Humidity: $50 \%$

Cloud Cover: 8 tenths

SOURCE STRENGTH INFORMATION:

Direct Source: 37.6 pounds

Source Height: 0

Release Duration: 1 minute

Release Rate: 284 grams/sec

Total Amount Released: 17.1 kilograms

Ground Roughness: Urban or forest

FOOTPRINT INFORMATION:

Dispersion Module: Gaussian

User-specified LOC: $0.5 \mathrm{mg} /(\mathrm{cu} \mathrm{m})$

Max Threat Zone for LOC: 1.3 kilometers 
Title of Project EPHA for THM

Reviewed by S. A. Henderson

Subject Concentration Analysis for THM Hazardous Chemicals

Computer N.E. Baucom B. K. Tharakan Date 09/16/96

1.RD-56 AVERAGE MAX DIST SEG 2

SITE DATA INFORMATION:

Location: AIKEN, SOUTH CAROLINA

Building Air Exchanges Per Hour: 60 (User specified)

Time: June 30, 19981200 hours EDT (User specified)

CHEMICAL INFORMATION:

Chemical Name: SILICA, CRYSTALLINE QUARTZ (RESP. DUST)

Molecular Weight: $60.09 \mathrm{~kg} / \mathrm{kmol}$

TLV-TWA: -unavail--

IDLH: -unavail

Footprint Level of Concern: $0.5 \mathrm{mg} /(\mathrm{cu} \mathrm{m})$

Boiling Point: -unavail

Note: Not enough chemical data to use Heavy Gas option

ATMOSPHERIC INFORMATION: (MANUAL INPUT OF DATA)

Wind: 2.5 meters $/ \mathrm{sec}$ from $90^{\circ}$ true at 2 meters

Inversion Height: 500 meters

Stability Class: C (user override)

Air Temperature: $25^{\circ} \mathrm{C}$

Relative Humidity: $50 \%$

Cloud Cover. 8 tenths

Ground Roughness: Urban or forest

Source Height: 0
Works Calculation 8

Sheet No. 308 of 314
RCE STRENGTH INFORMATION:

Direct Source: 135 pounds

Release Duration: 1 minute

Release Rate: 1.02 kilograms/sec

Total Amount Released: 61.2 kilograms

FOOTPRINT INFORMATION:

Dispersion Module: Gaussian

User specified LOC: $0.5 \mathrm{mg} /(\mathrm{cu} \mathrm{m})$

Max Threat Zone for LOC: 2.0 kilometers 
ENGINEERING COMPUTATION SHEET

Title of Project EPHA for THM

Reviewed by S. A. Henderson

Works Calculation 8

Sheet No. 309 of 314

Date 09/16/96

Computer N.E. Baucom B. K. Tharakan
1-RD-56 AVERAGE / MAX DIST SEG 3

SITE DATA INFORMATION:

Location: AIKENN, SOUTH CAROLINA

Building Air Exchanges Per Hour. 60 (User specified)

Time: June 30, 19981200 hours EDT (User specified)

CHEMICAL INFORMATION:

Chemical Name: SILICA, CRYSTALLINE QUARTZ (RESP. DUST)

Molecular Weight: $60.09 \mathrm{~kg} / \mathrm{kmol}$

TLV-TWA: -unavail-

IDLH: -unavail

Footprint Level of Concern: $0.5 \mathrm{mg} /(\mathrm{cu} \mathrm{m}$ )

Boiling Point: -unavail

Note: Not enough chemical data to use Heavy Gas option

ATMOSPHERIC INFORMATION: (MANUAL INPUT OF DATA)

Wind: 2.5 meters $/ \mathrm{sec}$ from $90^{\circ}$ true at 2 meters

Inversion Height: 500 meters

Stability Class: C (user override)

Air Temperature: $25^{\circ} \mathrm{C}$

Relative Humidity: $50 \%$

Cloud Cover: 8 tenths

Ground Roughness: Urban or forest

SOURCE STRENGTH INFORMATION:

Direct Source: 263 pounds

Source Height: 0

Release Duration: 1 minute

Release Rate: 1.99 kilograms/sec

Total Amount Released: 119 kilograms

FOOTPRINT INFORMATION:

Dispersion Module: Gaussian

User-specified LOC: $0.5 \mathrm{mg} /(\mathrm{cu} \mathrm{m})$

Max Threat Zone for LOC: 2.6 kilometers 
Title of Project EPHA for THM

Subject Concentration Analysis for THM Hazardous Chemicals

Computer N.E. Baucom B. K. Tharakan Date 09/16/96

1-RD-57 AVARAGI ARERT

SITE DATA INFORMATION:

LOCation: AIREN, SOUTH CAROLINA

Building Air Exchanges Per Hour: 60 (User specified)

Time: June 30, 19981200 hours EDT (User specified)

CHEMICAI INFORMATION:

Chemical Name: SODIUM SULFHYDRATE

Molecular Weight: $56.06 \mathrm{~kg} / \mathrm{kmol}$

TLV-TWA: - Unavail-

IDLH: -unavail

Footprint Level of Concern: $4 \mathrm{mg} /(\mathrm{cu} \mathrm{m})$

Boiling Point: -unavail

Freezing Point: $350.00^{\circ} \mathrm{C}$

Note: Not enough chemical data to use Heavy Gas option

ATMOSPHERIC INFORMATION: (MANUAL INPUT OF DATA)

Wind: 2.5 meters/sec from $90^{\circ}$ true at 2 meters

Inversion Height: 500 meters

Stability Class: C (user override)

Air Temperature: $25^{\circ} \mathrm{C}$

Relative Humidity: $50 \%$

Cloud Cover: 8 tenths

Ground Roughness: Urban or forest

Source Height: 0

Direct Source: 1 pounds

Release Duration: 1 minute

Release Rate: 0.756 grams/sec

Total Amount Released: 45.4 grams

TIME DEPENDENT INFORMATION:

Concentration Estimates at the point:

Downwind: $\quad 30$ meters

off Centerline: 0 meters

Max Concentration:

Outdoor: $3.89 \mathrm{mg} /(\mathrm{cu} \mathrm{m}$ )

Indoor: $2.32 \mathrm{mg} /(\mathrm{cu} \mathrm{m})$

Note: Indoor graph is shown with a dotted line. 


\section{ENGINEERING COMPUTATION SHEET}

Title of Project EPHA for THM

Subject Concentration Analysis for THM Hazardous Chemicals

Computer N.E. Baucom B. K. Tharakan Date 09/16/96

1-RD-57 AVERACI SAE

SITE DATA INEORMATION:

LOCation: AIKEN, SOUTH CAROLINA

Building Air Exchanges Per Hour: 60 (User specified)

Time: June 30, 19981200 hours EDT (User specified)

CHEMICAI INFORMATION :

Chemical Name: SODIUM SULFHYDRATE

Molecular Weight: $56.06 \mathrm{~kg} / \mathrm{kmol}$

TLV-TWA: - unavail-

IDLH: -unavail

Footprint Level of Concern: $4 \mathrm{mg} /(\mathrm{cu} \mathrm{m})$

Boiling Point: -unavail

Freezing Point: $350: 00^{\circ} \mathrm{C}$

Note: Not enough chemical data to use Heavy Gas option

ATMOSPHERIC INFORMATION: (MANUAL INPUT OF DATA)

wind: 2.5 meters/sec from $90^{\circ}$ true at 2 meters

Inversion Height: 500 meters

stability Class: $C$ (user override)

Ait Temperature: $25^{\circ} \mathrm{C}$

Relative Humidity: 508

cloud Cover: 8 tenths

Ground Roughness: Urban or forest

SOURCE STRENGTH INRORMATION:

Direct Source: 1.135 pounds

Source Height: 0

Release Duration: 1 minute

Release Rate: $8.58 \mathrm{grams} / \mathrm{sec}$

Total Amount Released: 515 grams

TIME DEPENDENT INFORMATION:

ConcentrationEstimates at the point:

Downwind: 100 meters

off Centerline: 0 meters

Max Concentration:

Outdoor: $\quad 3.99 \mathrm{mg} /(\mathrm{cu} \mathrm{m})$

Indoor: $\quad 2.35 \mathrm{mg} /(\mathrm{cu} \mathrm{m})$

Note: Indoor graph is shown with a dotted line.
Reviewed by S. A. Henderson

Works Calculation 8

Sheet No. 311 of 314 
Title of Project EPHA for THM

Reviewed by S. A. Henderson

\section{1-RD-57 AVERAGE / MAX DIST SEG I}

\section{SITE DATA INFORMATION:}

Location: AIKEN, SOUTH CAROLINA

Building Air Exchanges Per Hour: 60 (User specified)

Time: June 30, 19981200 hours EDT (User specified)

\section{CHEMICAL INFORMATION:}

Chemical Name: SODIUM SULFHYDRATE

Molecular Weight: $56.06 \mathrm{~kg} / \mathrm{kmol}$

TLV-TWA: -unavail-

IDLH: -unavail

Footprint Level of Concern: $4 \mathrm{mg} /(\mathrm{cu} \mathrm{m})$

Boiling Point: -unavail

Freezing Point: $350.00^{\circ} \mathrm{C}$

Note: Not enough chemical data to use Heavy Gas option

ATMOSPHERIC INFORMATION: (MANUAL INPUT OF DATA)

Wind: 2.5 meters $/ \mathrm{sec}$ from $90^{\circ}$ true at 2 meters

Inversion Height: 500 meters

Stability Class: $\mathrm{C}$ (user override)

Air Temperanure: $25^{\circ} \mathrm{C}$

Relative Humidity: $50 \%$

Cloud Cover. 8 tenths

\section{SOURCE STRENGTH INFORMATION:}

Direct Source: 37.6 pounds

Source Height: 0

Release Duration: 1 minute

Release Rate: 284 grams/sec

Total Amount Released: 17.1 kilograms

FOOTPRINT INFORMATION:

Dispersion Module: Gaussian

User-specified LOC: $4 \mathrm{mg} /(\mathrm{cu} \mathrm{m})$

Max Threat Zone for LOC: 569 meters 
Title of Project EPHA for THM

\section{ENGINEERING COMPUTATION SHEET}

Subject Concentration Analysis for THM Hazardous Chemicals

Computer N.E. Baucom B. K. Tharakan Date 09/16/96

1-RD-57 AVERAGE / MAX DIST SEG 2

SITE DATA INFORMATION:

Location: AIKEN, SOUTH CAROLINA

Building Air Exchanges Per Hour: 60 (User specified)

Time: June 30, 19981200 hours EDT (User specified)

\section{CHEMICAL INFORMATION:}

Chemical Name: SODIUM SULFHYDRATE

Molecular Weight: $56.06 \mathrm{~kg} / \mathrm{kmol}$.

TLV-TWA-unavailFootprint Level of Concern: $4 \mathrm{mg} /(\mathrm{cu} \mathrm{m}$ )

Boiling Point: -unavail

Freezing Point: $350.00^{\circ} \mathrm{C}$

Note: Not enough chemical data to use Heavy Gas option

ATMOSPHERIC INFORMATION: (MANUAL INPUT OF DATA)

Wind: 2.5 meters $/ \mathrm{sec}$ from $90^{\circ}$ true at 2 meters

Inversion Height: 500 meters

Stability Class: $C$ (user override)

Air Temperature: $25^{\circ} \mathrm{C}$

Relative Humidity: 50\%

Cloud Cover: 8 tenths

Ground Roughness: Urban or forest

SOURCE STRENGTH INFORMATION:

Direct Source: 135 pounds

Release Duration: 1 minute

Release Rate: 1.02 kilograms/sec

Total Amount Released: 61.2 kilograms

FOOTPRINT INFORMATION:

Dispersion Module: Gaussian

User-specified LOC: $4 \mathrm{mg} /(\mathrm{cu} \mathrm{m}$ )

Max Threat zone for LOC: 948 meters
Reviewed by S. A. Henderson

Works Calculation 8

Sheet No. $\underline{313 \text { of } 314}$ 
Title of Project EPHA for THM

Reviewed by S. A. Henderson

Subject Concentration Analysis for THM Hazardous Chemicals

Computer N.E.Baucom B. K. Tharakan Date 09/16/96

1-RD-57 AVERAGE MAX DIST SEG 3

\section{SITE DATA INFORMATION:}

Location: AIKEN, SOUTH CAROLINA

Building Air Exchanges Per Hour: 60 (User specified)

Time: June 30, 19981200 hours EDT (User specified)

\section{CHEMICAL INFORMATION:}

Chemical Name: SODIUM SULFHYDRATE

Molecular Weight: $56.06 \mathrm{~kg} / \mathrm{kmol}$

TLV-TWA: -unavail-

IDLH: -unavail

Footprint Level of Concern: $4 \mathrm{mg}$ (cu m)

Boiling Point: -unavail

Freezing Point: $350.00^{\circ} \mathrm{C}$

Note: Not enough chemical data to use Heavy Gas option

ATMOSPHERIC INFORMATION: (MANUAL INPUT OF DATA)

Wind: 2.5 meters $/ \mathrm{sec}$ from $90^{\circ}$ true at 2 meters

Inversion Height: 500 meters

Stability Class: $C$ (user override)

Air Temperature: $25^{\circ} \mathrm{C}$

Relative Humidity: 50\%

Cloud Cover: 8 tenths

Ground Roughness: Urban or forest

Source Height: 0
Works Galculation 8

Sheet No. 314 of 314
SOURCE STRENGTH INFORMATION:

Direct Source: 263 pounds

Release Duration: 1 minute

Release Rate: 1.99 kilograms/sec

Total Amount Released: 119 kilograms

FOOTPRINT INFORMATION:

Dispersion Module: Gaussian

User-specified LOC: $4 \mathrm{mg} /(\mathrm{cu} \mathrm{m})$ 


\section{APPENDIX C}

\section{CORRESPONDENCE}


S-EHA-G-00003

EPHA for the Transportation of Hazardous Materials

Rev. 1

October 11, 1996

Page C-2 of 2

This page intentionally left blank. 


\section{APPENDIX D}

\section{EMERGENCY PREPAREDNESS HAZARDS ASSESSMENT}

\section{DEFINITIONS}


This page intentionally left blank. 


\section{EPHA DEFINITIONS}

1. Accident. A deviation from normal operations or activities associated with a hazard which has the potential to result in an emergency.

2. Alert. An Alert represents events in progress or having occurred that involve an actual or potential substantial reduction in the level of facility safety and protection. At SRS, an Alert has occurred if unplanned events result in hazardous material being released to the environment in concentrations that will result in exposures greater than the protective action criteria (PAC) at 30 meters from the source.

3. Assess. The internal process by which an organization evaluates its ability to comply with DOE and other applicable regulations, orders, plans, and procedures, conducted within a single, cognizant Program Office.

4. Breach A fracture or penetration, undesirable opening of a tank, container, or line that results in the release of a hazardous material. The release of hazardous material must be of a magnitude to satisfy the EAL criteria to be considered a breach. Small leaks or seepage are not to be considered a breach.

5. Committed Effective Dose Equivalent (CEDE). The sum of the committed dose equivalents over a fifty-year period to various organs or tissues in the body, each multiplied by the appropriate weighting factor. CEDE is applicable to exposure from internally deposited radionuclides.

6. Common Hazardous Material. The material is commonly used by the general public. This includes any substance used for personal, family, or household purposes or is present in the same form and concentration as a product packaged for distribution and use by the general public (e.g., motor oil, gasoline, diesel fuel). Additionally, material screened as per the criteria set forth in this procedure is common hazardous material.

7. Consequence. The result or effect (especially projected doses or dose rates) of a release of radioactive or hazardous materials to the environment.

8. Consequence Assessment. The evaluation and interpretation of radiological or other hazardous materials measurements and other information to provide a basis for decision making.

9. Emergency Action Level (EAL). Specific, predetermined, observable criteria used to detect, recognize, and determine the emergency class of Operational Emergencies. An EAL can be an instrument reading; an equipment status indicator; a measurable parameter, onsite or offsite; a discrete, observable event; results of analyses; or another observed phenomenon that indicates entry into a particular emergency class. 
10. Emergency Planning Zone (EPZ). A geographic area surrounding a specific DOE facility for which special planning and preparedness efforts are carried out to ensure that prompt and effective protective actions can be taken to reduce or minimize the impact to onsite personnel, public health and safety, and the environment in the event of an Operational Emergency.

11. Emergency Response Planning Guidelines (ERPGs). A hazardous material personnel exposure level or range which, when exceeded by a short term or acute exposure, will cause irreversible or other serious health effects in humans. The ERPGs are approved by a committee of the American Industrial Hygiene Association.

12. Event. Any real-time occurrence or significant deviation from planned or expected behavior that could endanger or adversely affect people, property, or the environment.

13. Facility. Any equipment, structure, system, process, or activity that fulfills a specific purpose.

14. Facility Boundary. This boundary represents the receptor location that differentiates between an Alert and Site Area Emergency declaration.

15. General Emergency. A General Emergency represents events that are in progress or have occurred that involve actual or imminent catastrophic failure of facility safety systems with potential for loss of confinement integrity, catastrophic degradation of facility protection systems, or catastrophic failure in safety or protection systems threatening the integrity of a weapon or test device that could lead to substantial offsite impacts. Environmental releases of hazardous materials can reasonably be expected to exceed the appropriate protective action criteria at the site boundary.

16. Hazard. A process, condition, or asset which has the potential to adversely impact the health and safety of personnel, the public, the environment, or national security. Hazards are divided into three classes:

Low - Hazards which present minor onsite and negligible offsite impacts to people, the environment, or national security.

Moderate - Hazards which represent considerable potential onsite impacts to the people or the environment, but at most only minor offsite impacts to people, the environment, or national security.

High - Hazards with the potential for onsite and offsite impacts to large numbers of persons or with the potential for major impacts to the environment or national security.

17. Hazard Assessment. A Hazards Assessment identifies hazards significant enough to include in an emergency preparedness program and forms the technical basis for applicable emergency classification procedures. 
18. Hazardous Materials. Any solid, liquid, or gaseous material that is toxic, flammable, radioactive, corrosive, chemically reactive, or unstable upon prolonged storage in quantities that could pose a threat to life, property, or the environment.

19. Material-at-Risk (MAR). The amount of hazardous material that is available to be acted on by a given physical stress. Multiply the MAR by the appropriate release fraction to determine the source term.

20. Maximum Fire A fire analyzed for the maximum consequence. The largest at risk hazardous material inventory is assumed to be involved and the worst fire situation is utilized.

21. Maximum Inventory. For a process, the maximum quantity of a hazardous material that a process produces during the process cycle. For storage tanks the maximum inventory is equivalent to the physical capacity of the tank.

22. Notification of Unusual Event (NoUE). The NoUE emergency classification is below an Alert and is not recognized by DOE. The states of Georgia and South Carolina require the identification and reporting of radiological NoUEs at a level of $0.1 \mathrm{mrem}$ TEDE at the site boundary. The NoUE aligns with the Unusual Occurrence category in DOE Order 5000.3B.

23. Offsite. The area beyond the boundaries of the site.

24. Onsite. The facility/site area over which DOE has access control authority.

25. Release. An airborne effluent release to the environment as this pathway typically represents the most time-urgent situation. Releases to aquatic and ground pathways in most instances do not have the same time urgency as airborne releases. The hazard assessment considers releases to an aquatic or ground pathway having a time-urgent affect on the workers or the public (e.g., through a community water supply).

26. Release Fraction. The coefficient used to estimate the amount of a hazardous material (material at risk) suspended in air and made available for airborne transport under a specific set of induced physical stress. The release fraction is a combination of the fraction of the material released and the fraction of the material that is respirable.

27. Safety Analysis. A documented process to systematically identify the hazards of a DOE operation; to describe and analyze the adequacy of the measures taken to eliminate, control, or mitigate identified hazards; and to analyze and evaluate potential accidents and their associated risks.

28. Safety Analysis Report (SAR). A documented process to systematically identify the hazards of a DOE operation; to describe and analyze the adequacy of the measures taken to eliminate, control, or mitigate identified hazards; and to analyze and evaluate potential accidents and their associated risks. SARs are completed according to the WSRC $9 Q$ manual.

29. Segment. a demarcation used.in hazards assessment where the system, section, building, etc., is not affected by the failure of other systems, sections, buildings, etc. 
30. Site. The area over which DOE has access control authority. This includes any area that has been designated as a National Security Area.

31. Site Area Emergency (SAE). An SÄE represents events that are in progress or have occurred involving actual or likely major failure(s) of facility safety or safeguard systems needed for the protection of onsite personnel, the public health and safety, the environment, or national security. Environmental releases of hazardous materials are expected to exceed the appropriate $\mathrm{PAC}$ at or beyond the facility boundary but not expected to exceed the appropriate $\mathrm{PAC}$ at or beyond the site boundary.

32. Site Boundary. In general, the perimeter of the DOE-owned and controlled land at SRS is the site boundary. Areas where the general public has uncontrolled access to areas of SRS are offsite for purposes of emergency classification unless it can be assured (e.g., procedures in place) that those areas can be evacuated and access control established within one hour of event declaration.

33. Source Term. The amount of respirable material released to the environment. The source term is equal to the amount of material at risk (MAR) multiplied by an appropriate source release fraction $(R F)$. Source term $=(M A R)(R F)$.

34. Total Effective Dose Equivalent (TEDE). The sum of the deep dose equivalent (from external exposure) and the committed effective dose equivalent (from internal exposure). 